Supporting Information

\title{
Stereoselectivity in Glycosylation with Deoxofluorinated Glucosazide and Galactosazide Thiodonors
}

Martin Kurfiřt, ${ }^{\dagger}$ Lucie Červenková Št’astná, ${ }^{\dagger}$ Martin Dračínský, ${ }^{\dagger}$ Monika Müllerová, ${ }^{\dagger}$ Vojtěch Hamala, ${ }^{\dagger}$ Petra Cuř́nová, ${ }^{\dagger}$ and Jindřich Karban ${ }^{\dagger}{ }^{*}$

†Institute of the Chemical Process Fundamentals of the CAS, v.v.i, Rozvojová 135, 16502 Praha, Czech Rep. ${ }^{\ddagger}$ Institute of Organic Chemistry and Biochemistry of the CAS, Flemingovo náměstí 542/2, 16610 Praha, Czech Rep.

E-mail: karban@icpf.cas.cz

Table S1. Selected NMR data and decomposition temperatures for intermediates formed on reaction with $\mathrm{Tf}_{2} \mathrm{O} / \mathrm{Ph}_{2} \mathrm{SO}$

Table S2. Relative energies $(\mathrm{kcal} / \mathrm{mol})$ of geometry optimized conformers of oxocarbenium ion derived from $O$-methyl analogues of compounds 1-4.

Table S3. Relative calculated energies $(\mathrm{kcal} / \mathrm{mol})$ of geometry optimized $\alpha$ - and $\beta$-triflates derived from O-methyl analogues of compounds 1-4

Table S4. Relative calculated energies $(\mathrm{kcal} / \mathrm{mol})$ of geometry optimized $\alpha$-triflates derived from Omethyl analogues of compounds 1-4 with respect to separated oxocarbenium ion and triflate anion.

Cartesian coordinates, computed total energy values $E\left(E_{h}\right)$, computed sum of electronic and thermal free energies $G\left(E_{h}\right)$.

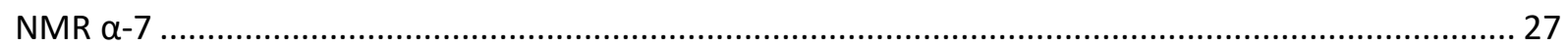

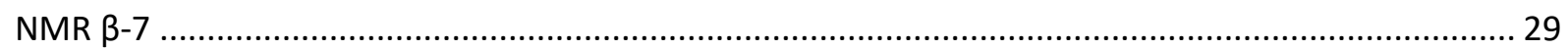

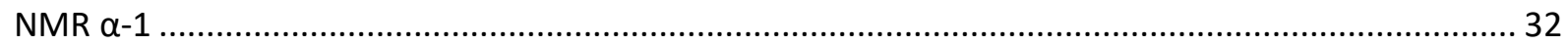

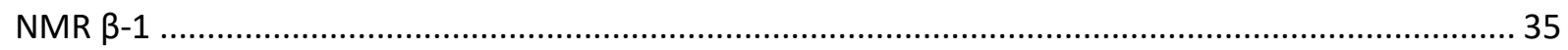

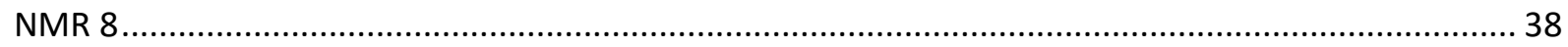

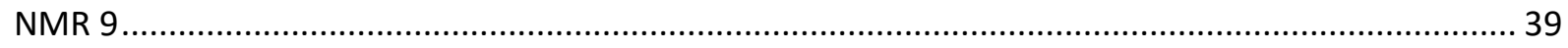

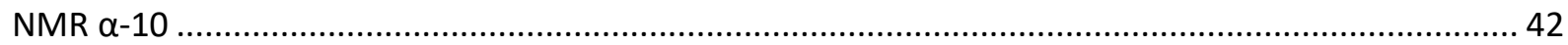

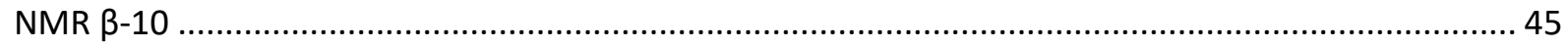

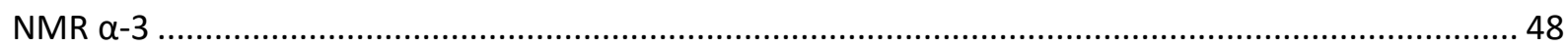

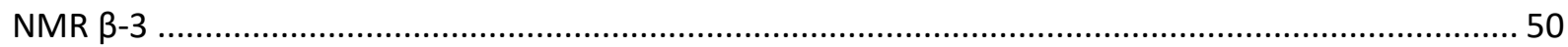

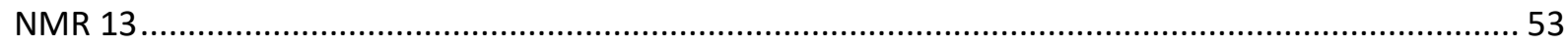

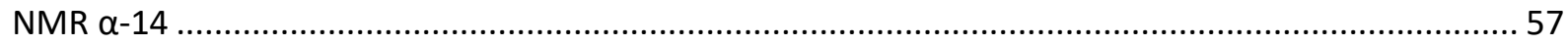

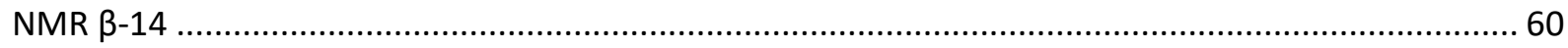

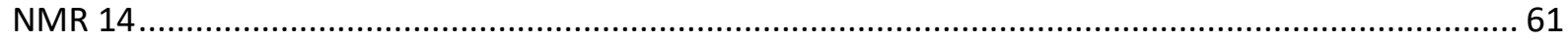




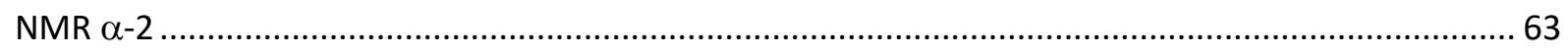

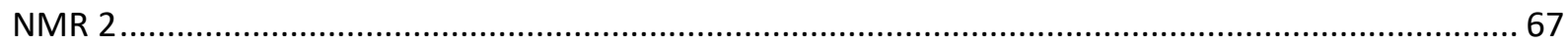

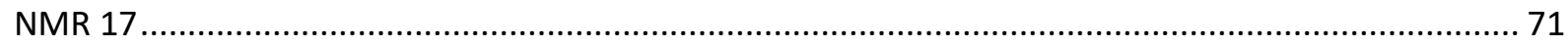

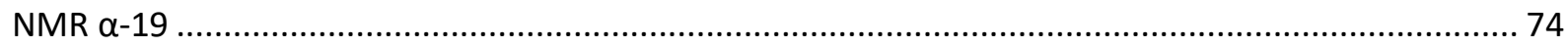

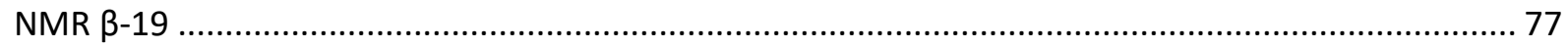

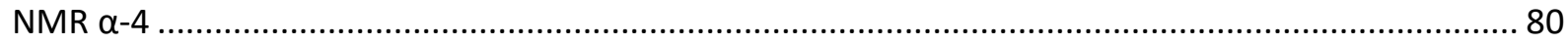

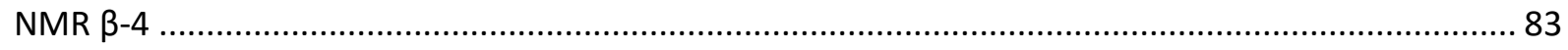

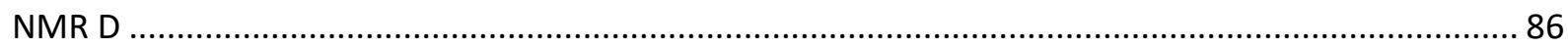

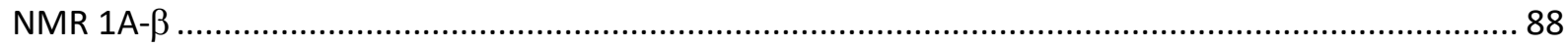

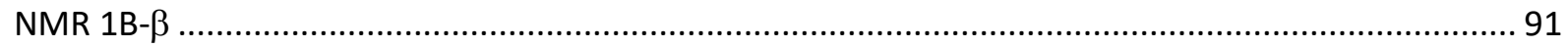

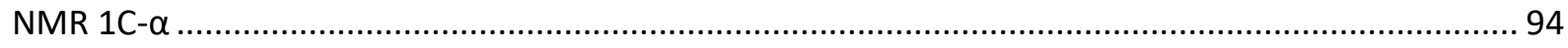

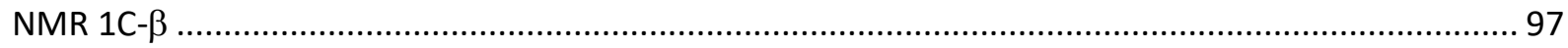

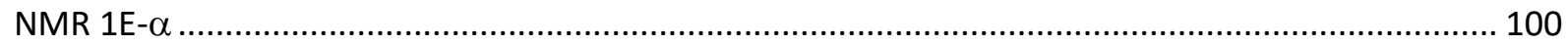

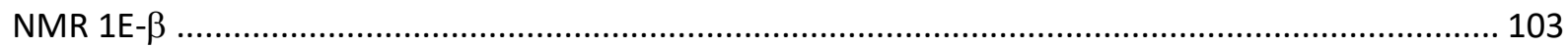

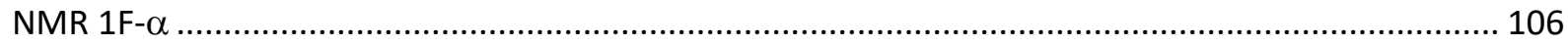

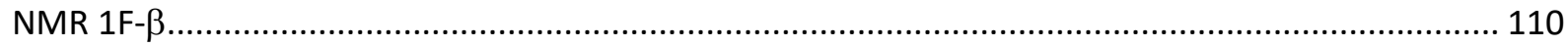

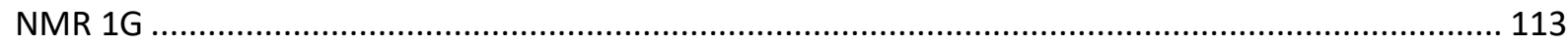

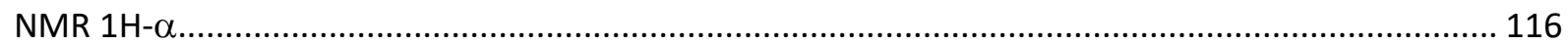

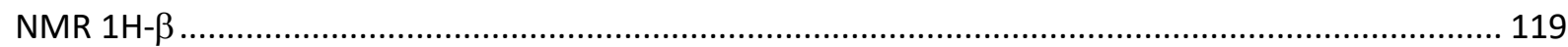

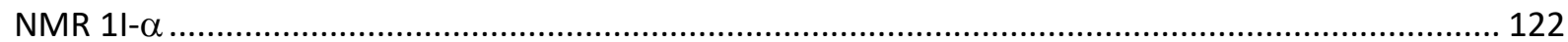

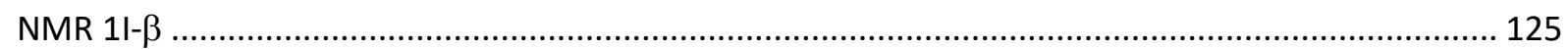

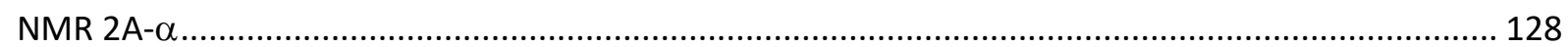

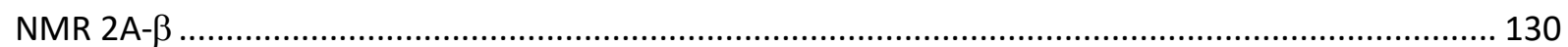

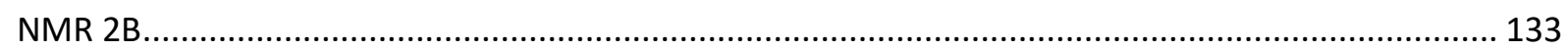

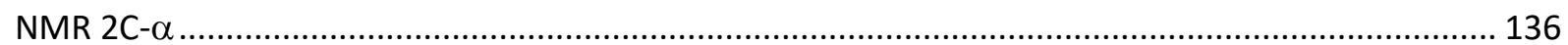

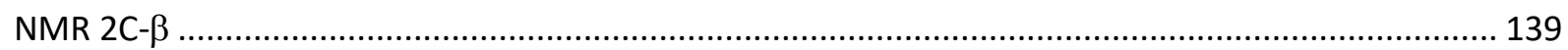

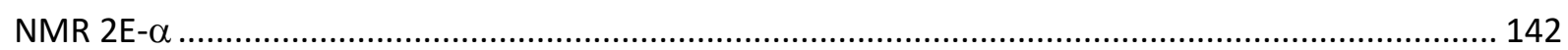

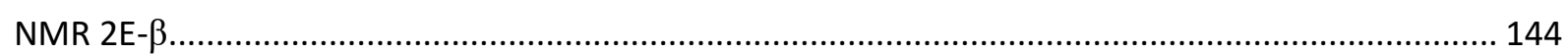

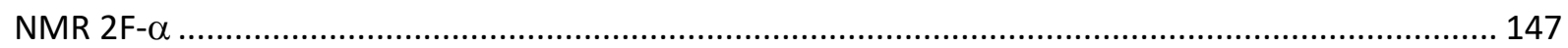

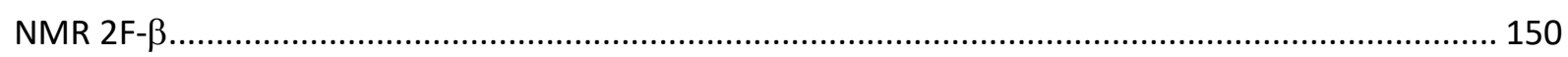

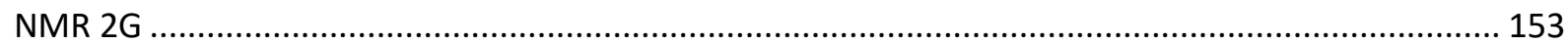

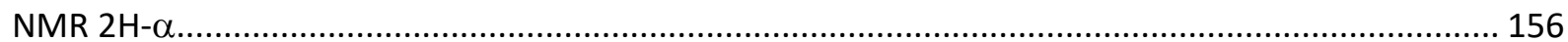

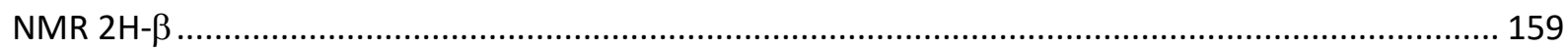

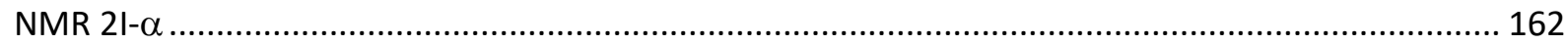

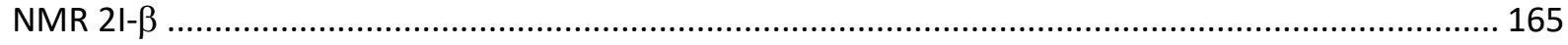




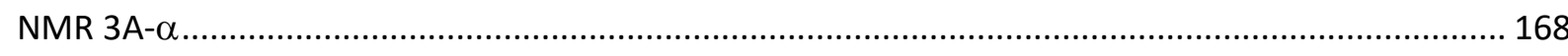

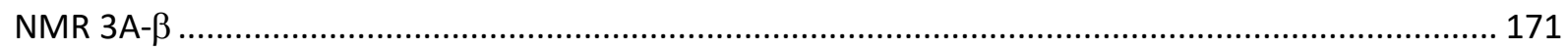

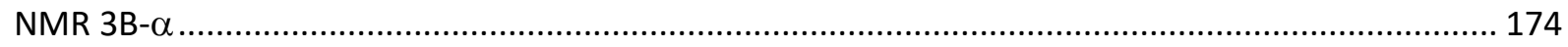

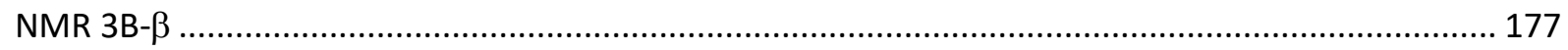

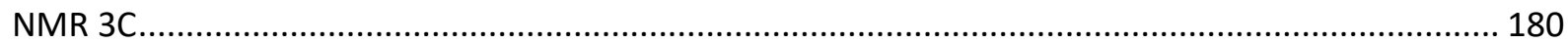

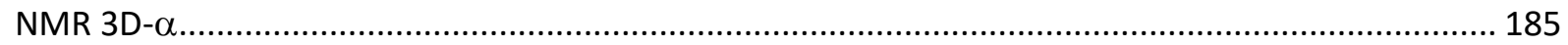

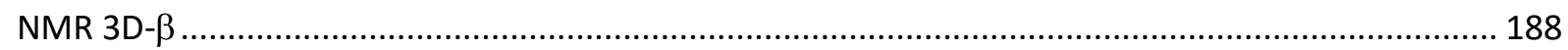

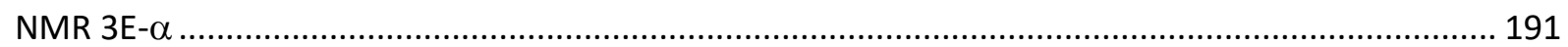

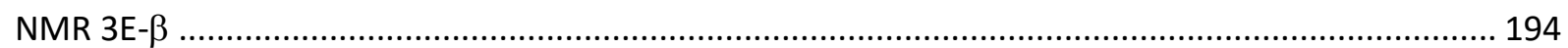

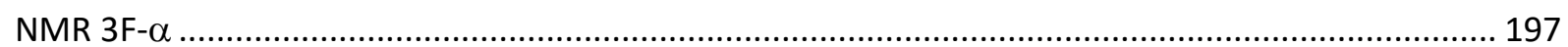

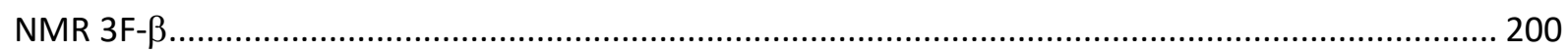

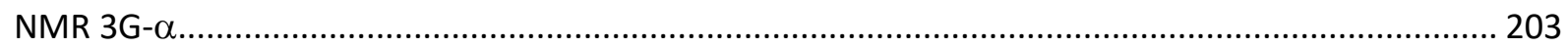

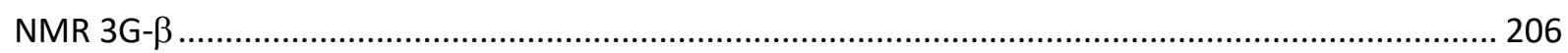

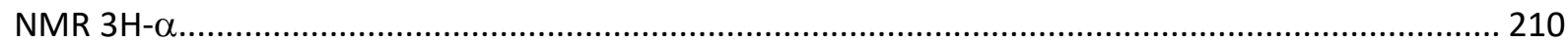

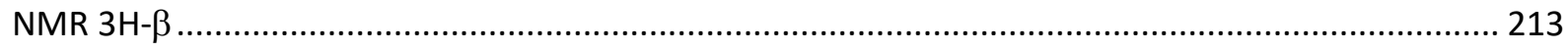

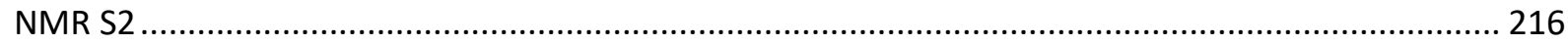

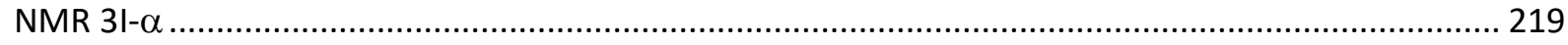

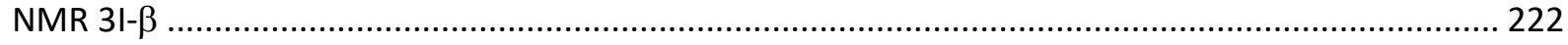

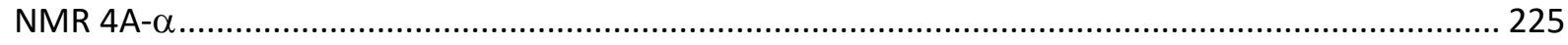

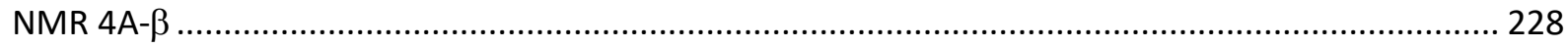

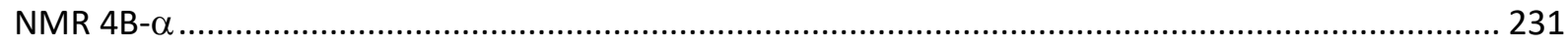

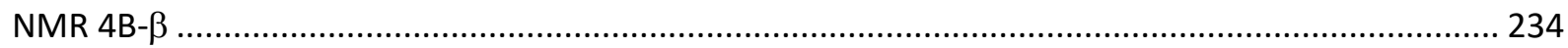

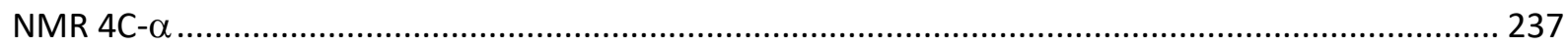

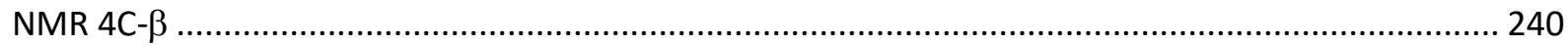

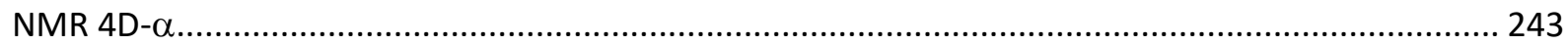

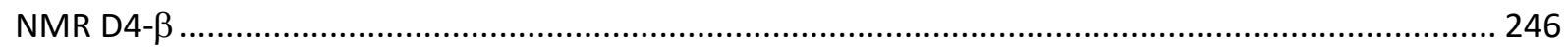

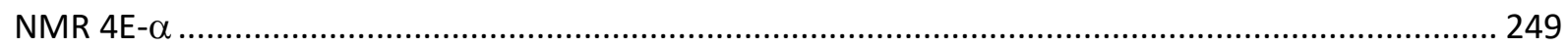

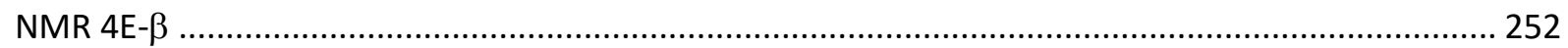

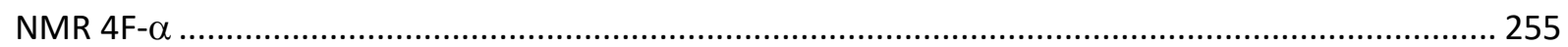

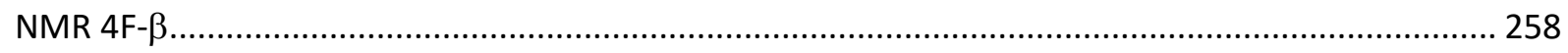

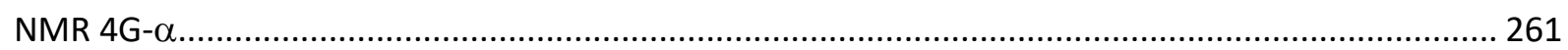

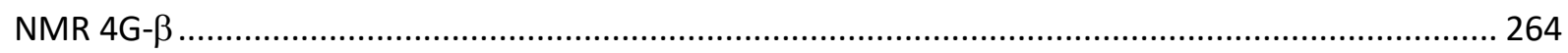

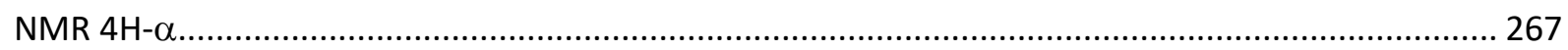

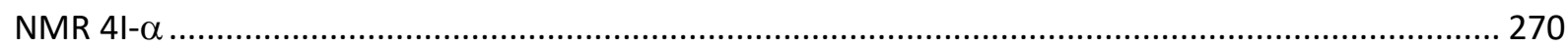

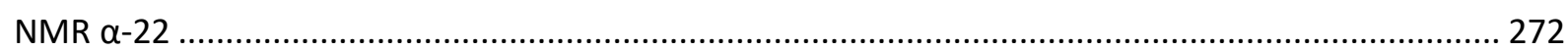


NMR $\beta-22$

NMR 23

NMR $\alpha-26$ 284

NMR $\beta-26$ 287

Observation of anomeric covalent intermediates on preactivation 291

Figure S1. Low temperature ${ }^{1} \mathrm{H}$ NMR of $\beta-1$ activated by $\mathrm{Tf}_{2} \mathrm{O}$ 291

Figure S2. Low temperature ${ }^{1} \mathrm{H}$ NMR of $\alpha-1$ activated by $\mathrm{Tf}_{2} \mathrm{O}$ 292

Figure S3. Low temperature ${ }^{1} \mathrm{H}$ NMR of $\alpha-1$ activated by $\mathrm{Tf}_{2} \mathrm{O}$ (increased amount of $\mathrm{Ph}_{2} \mathrm{SO}$ ) 292

Figure S4. Low temperature ${ }^{1} \mathrm{H}$ NMR of $\alpha-3$ activated by $\mathrm{Tf}_{2} \mathrm{O}$ 293

Figure S5. Low temperature ${ }^{1} \mathrm{H}$ NMR of $\beta-3$ activated by $\mathrm{Tf}_{2} \mathrm{O}$ 293

Figure S6. Low temperature ${ }^{1} \mathrm{H}$ NMR of 2 activated by $\mathrm{Tf}_{2} \mathrm{O}$ 294

Figure S7. Low temperature ${ }^{1} \mathrm{H}$ NMR of $\alpha-4$ activated by $\mathrm{Tf}_{2} \mathrm{O}$ 295

Figure S8. Low temperature ${ }^{1} \mathrm{H}$ NMR of $\beta-4$ activated by $\mathrm{Tf}_{2} \mathrm{O}$ 295 
Table S1. Selected NMR data and decomposition temperatures for intermediates formed on reaction with $\mathrm{Tf}_{2} \mathrm{O} / \mathrm{Ph}_{2} \mathrm{SO}$

\begin{tabular}{|c|c|c|c|}
\hline $\begin{array}{l}\text { glycosyl } \\
\text { donor }\end{array}$ & $\begin{array}{l}\boldsymbol{\alpha} \text {-triflate } \\
{ }_{\mathrm{N}_{3}}^{-\mathrm{O}}{ }_{\mathrm{OTf}} \mathrm{H} \delta(\mathrm{ppm}) \\
{ }^{3} J_{\mathrm{H} 1, \mathrm{H} 2}(\mathrm{~Hz}) \\
\mathrm{T}_{\text {decomp }}(\mathrm{K})\end{array}$ & $\begin{array}{c}\alpha \text {-oxosulfonium triflate } \\
{ }_{\mathrm{N}_{3}} \mathrm{OSPO}_{2}{ }^{+} \\
{ }^{1} \mathrm{H} \delta(\mathrm{ppm}) \\
{ }^{3} J_{\mathrm{H} 1, \mathrm{H} 2}(\mathrm{~Hz}) \\
\mathrm{T}_{\text {decomp }}(\mathrm{K})\end{array}$ & $\begin{array}{c}\beta \text {-oxosulfonium triflate } \\
{ }_{\mathrm{N}_{3} \mathrm{TfO}^{-}}{ }^{+} \\
{ }^{1} \mathrm{H} \delta(\mathrm{ppm}) \\
{ }^{3} J_{\mathrm{H} 1, \mathrm{H} 2}(\mathrm{~Hz}) \\
{ }^{\mathrm{T}} \text { decomp }(\mathrm{K})\end{array}$ \\
\hline BnO- & $\begin{array}{c}\delta_{\mathrm{H} 1}=6.09 \mathrm{t} \\
{ }^{3} J_{\mathrm{H} 1, \mathrm{H} 2}={ }^{3} J_{\mathrm{H} 1, \mathrm{~F}}=3.6 \\
\delta_{\mathrm{C} 1}=104.6 \mathrm{~d} \\
{ }^{3} J_{\mathrm{C} 1, \mathrm{~F}}=10.4 \\
\mathrm{~T}_{\text {decomp }} \sim 233-243\end{array}$ & $\begin{array}{c}\delta_{\mathrm{H} 1}=6.38 \mathrm{t} \\
{ }^{3} J_{\mathrm{H} 1, \mathrm{H} 2}={ }^{3} J_{\mathrm{H} 1, \mathrm{~F}}=3.5 \\
\delta_{\mathrm{C} 1}=104.8 \mathrm{~d} \\
{ }^{3} J_{\mathrm{C} 1, \mathrm{~F}}=10.1 \\
\mathrm{~T}_{\text {decomp }} \sim 233-243\end{array}$ & $\begin{array}{c}\delta_{\mathrm{H} 1}=5.65 \mathrm{~d} \\
3 J_{\mathrm{H} 1, \mathrm{H} 2}=8.3 \\
\delta_{\mathrm{C} 1}=102.2 \mathrm{~d} \\
{ }^{3} J_{\mathrm{C} 1, \mathrm{~F}}=11.4 \\
\mathrm{~T}_{\text {decomp }} \sim 213-233\end{array}$ \\
\hline 2 & $\begin{array}{c}\delta_{\mathrm{H} 1}=6.09 \mathrm{t} \\
{ }^{3} J_{\mathrm{H} 1, \mathrm{H} 2}={ }^{3} J_{\mathrm{H} 1, \mathrm{~F}}=4.0 \\
\delta_{\mathrm{C} 1}=106.1 \mathrm{~d} \\
{ }^{3} J_{\mathrm{C} 1, \mathrm{~F}}=10.1 \\
\mathrm{~T}_{\text {decomp }} \sim 243-253\end{array}$ & $\begin{array}{c}\delta_{\mathrm{H} 1}=6.30 \mathrm{t} \\
{ }^{3} J_{\mathrm{H} 1, \mathrm{H} 2}={ }^{3} J_{\mathrm{H} 1, \mathrm{~F}}=4.0 \\
\mathrm{~T}_{\text {decomp }} \sim 233-253\end{array}$ & $\begin{array}{c}\delta_{\mathrm{H} 1}=5.66 \mathrm{~d} \\
{ }^{3} J_{\mathrm{H} 1, \mathrm{H} 2}=8.3 \\
\mathrm{~T}_{\text {decomp }} \sim 213-233\end{array}$ \\
\hline${ }_{3}^{\mathrm{N}_{3}^{2} \mathrm{SPh}}$ & $\begin{array}{c}\delta_{\mathrm{H} 1}=6.05 \mathrm{t} \\
{ }^{3} J_{\mathrm{H} 1, \mathrm{H} 2}={ }^{5} J_{\mathrm{H} 1, \mathrm{~F}}=3.1 \\
\delta_{\mathrm{C} 1}=104.4 \mathrm{~s} \\
\mathrm{~T}_{\text {decomp }} \sim 233-253\end{array}$ & $\begin{array}{c}\delta_{\mathrm{H} 1}=6.28 \mathrm{t} \\
{ }^{3} J_{\mathrm{H} 1, \mathrm{H} 2}={ }^{5} J_{\mathrm{H} 1, \mathrm{~F}}=3.1 \\
\delta_{\mathrm{C} 1}=104.4 \mathrm{~s} \\
\mathrm{~T}_{\text {decomp }} \sim 233-243\end{array}$ & $\begin{array}{c}\delta_{\mathrm{H} 1}=5.65 \mathrm{~d} \\
3_{\mathrm{H} 1, \mathrm{H} 2}=8.4 \\
\delta_{\mathrm{C} 1}=102.6 \mathrm{~s} \\
\mathrm{~T}_{\text {decomp }} \sim 213-233\end{array}$ \\
\hline${ }_{4}^{S_{N_{3}^{2} \mathrm{OBh}}^{\mathrm{OBn}}}$ & $\begin{array}{c}\delta_{\mathrm{H} 1}=6.11 \mathrm{~d} \\
3_{\mathrm{H} 1, \mathrm{H} 2}=3.3 \\
\delta_{\mathrm{C} 1}=105.0 \mathrm{~s} \\
\mathrm{~T}_{\text {decomp }} \sim 243-253\end{array}$ & $\begin{array}{c}\delta_{\mathrm{H} 1}=6.24 \mathrm{~d} \\
3_{\mathrm{H}_{1}, \mathrm{H} 2}=3.5 \\
\delta_{\mathrm{C} 1}=105.4 \mathrm{~s} \\
\mathrm{~T}_{\text {decomp }} \sim 233-253\end{array}$ & $\begin{array}{c}\delta_{\mathrm{H} 1}=5.65 \mathrm{~d} \\
3_{\mathrm{H} 1, \mathrm{H} 2}=8.4 \\
\delta_{\mathrm{C} 1}=102.9 \mathrm{~s} \\
\mathrm{~T}_{\text {decomp }} \sim 213-223\end{array}$ \\
\hline
\end{tabular}


Table S2. Relative energies ( $\mathrm{kcal} / \mathrm{mol}$ ) of geometry optimized conformers of oxocarbenium ion derived from $\mathbf{O}$-methyl analogues of compounds 1-4.

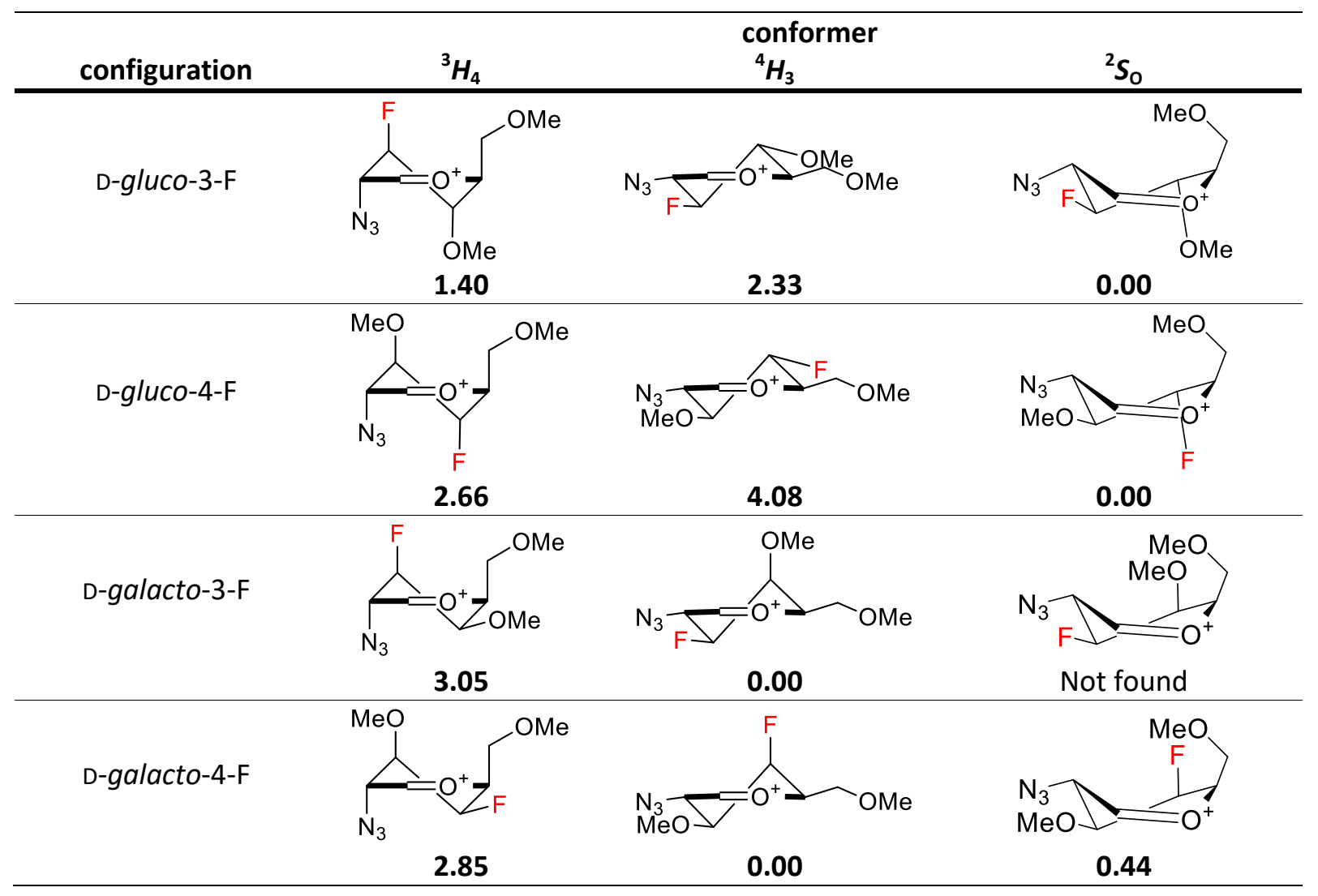

Table S3. Relative calculated energies ( $\mathrm{kcal} / \mathrm{mol}$ ) of geometry optimized $\alpha$ - and $\beta$-triflates derived from 0-methyl analogues of compounds 1-4.

\begin{tabular}{ccc}
\hline configuration & $\boldsymbol{\alpha}$-triflate & $\boldsymbol{\beta}$-triflate \\
\hline D-gluco-3-F & 0.00 & 4.46 \\
D-gluco-4-F & 0.00 & 4.24 \\
D-galacto-3-F & 0.00 & 5.55 \\
D-galacto-4-F & 0.00 & 4.69 \\
\hline
\end{tabular}


Table S4. Relative calculated energies $(\mathrm{kcal} / \mathrm{mol})$ of geometry optimized $\alpha$-triflates derived from 0-methyl analogues of compounds 1-4 with respect to separated oxocarbenium ion and triflate anion.

\begin{tabular}{cc}
\hline configuration & $\boldsymbol{\alpha}$-triflate \\
\hline D-gluco-3-F & -46.6 \\
D-gluco-4-F & -44.7 \\
D-galacto-3-F & -42.3 \\
D-galacto-4-F & -43.0 \\
\hline
\end{tabular}


Cartesian coordinates, computed total energy values $E\left(E_{h}\right)$, computed sum of electronic and thermal free energies $G\left(E_{h}\right)$

Configuration: D-gluco-4-F, conformation: ${ }^{4} \mathrm{H}_{3}$

\begin{tabular}{cccc}
\hline Atom & $X$ & $y$ & $Z$ \\
\hline $\mathrm{C}$ & 0.527319 & 0.886418 & -0.099896 \\
$\mathrm{C}$ & 1.282335 & -0.125507 & -0.968421 \\
$\mathrm{O}$ & 0.885916 & -1.545313 & -0.631265 \\
$\mathrm{C}$ & -0.104937 & -1.844759 & 0.073524 \\
$\mathrm{C}$ & -1.033836 & -0.862477 & 0.712398 \\
$\mathrm{C}$ & -0.951950 & 0.533906 & 0.060341 \\
$\mathrm{H}$ & 1.004627 & 0.994758 & 0.877757 \\
$\mathrm{~F}$ & 0.646085 & 2.091516 & -0.783336 \\
$\mathrm{H}$ & 0.984542 & -0.025491 & -2.013774 \\
$\mathrm{C}$ & 2.785240 & -0.080554 & -0.810125 \\
$\mathrm{H}$ & -0.268205 & -2.915739 & 0.205251 \\
$\mathrm{~N}$ & -2.367223 & -1.468170 & 0.832739 \\
$\mathrm{H}$ & -0.666833 & -0.765793 & 1.748236 \\
$\mathrm{H}$ & -1.409544 & 0.510298 & -0.941772 \\
$\mathrm{O}$ & -1.628060 & 1.408207 & 0.916696 \\
$\mathrm{C}$ & -2.171562 & 2.579427 & 0.286250 \\
$\mathrm{O}$ & 3.067544 & -0.293335 & 0.551868 \\
$\mathrm{H}$ & 3.112228 & 0.911292 & -1.160736 \\
$\mathrm{H}$ & 3.250223 & -0.840060 & -1.454669 \\
$\mathrm{H}$ & -2.864183 & 2.301152 & -0.518068 \\
$\mathrm{H}$ & -1.381216 & 3.218406 & -0.115258 \\
$\mathrm{H}$ & -2.718516 & 3.111396 & 1.064958 \\
$\mathrm{C}$ & 4.461879 & -0.234147 & 0.847176 \\
$\mathrm{~N}$ & -3.160935 & -1.222143 & -0.094678 \\
$\mathrm{~N}$ & -3.998769 & -1.070984 & -0.847031 \\
$\mathrm{H}$ & 4.563958 & -0.406075 & 1.919453 \\
$\mathrm{H}$ & 4.879917 & 0.749462 & 0.595072 \\
$\mathrm{H}$ & 5.016612 & -1.008471 & 0.300497 \\
\hline & $\mathrm{E}$ & $\mathrm{G}$ & \\
& -802.150398 & -801.956546 & \\
\hline & & & \\
& & & \\
& &
\end{tabular}


Configuration: D-gluco-4-F, conformation: S

\begin{tabular}{cccc}
\hline Atom & $X$ & $y$ & $Z$ \\
\hline $\mathrm{C}$ & 0.574093 & 1.369084 & 0.130853 \\
$\mathrm{C}$ & 1.770651 & 0.826939 & -0.674709 \\
$\mathrm{O}$ & 1.365021 & -0.145456 & -1.759865 \\
$\mathrm{C}$ & 0.352733 & -0.879166 & -1.650882 \\
$\mathrm{C}$ & -0.623670 & -0.748815 & -0.546979 \\
$\mathrm{C}$ & -0.789468 & 0.758143 & -0.222034 \\
$\mathrm{H}$ & 0.756040 & 1.200910 & 1.195768 \\
$\mathrm{~F}$ & 0.500527 & 2.741418 & -0.089764 \\
$\mathrm{H}$ & 2.233360 & 1.633418 & -1.241549 \\
$\mathrm{C}$ & 2.785850 & 0.066050 & 0.155693 \\
$\mathrm{H}$ & 0.208855 & -1.595193 & -2.461814 \\
$\mathrm{~N}$ & -1.861105 & -1.394561 & -0.969897 \\
$\mathrm{H}$ & -0.157349 & -1.232007 & 0.329333 \\
$\mathrm{H}$ & -1.173295 & 1.272782 & -1.114907 \\
$\mathrm{O}$ & -1.690721 & 0.826974 & 0.844079 \\
$\mathrm{C}$ & -2.430723 & 2.055902 & 0.920415 \\
$\mathrm{O}$ & 2.081445 & -0.967894 & 0.807440 \\
$\mathrm{H}$ & 3.232279 & 0.779475 & 0.864494 \\
$\mathrm{H}$ & 3.585780 & -0.326050 & -0.487481 \\
$\mathrm{H}$ & -1.763204 & 2.909587 & 1.071594 \\
$\mathrm{H}$ & -3.019991 & 2.209851 & 0.008207 \\
$\mathrm{C}$ & 2.904876 & -1.776473 & 1.652128 \\
$\mathrm{~N}$ & -2.654984 & -1.664038 & -0.052006 \\
$\mathrm{~N}$ & -3.473670 & -1.976368 & 0.669700 \\
$\mathrm{H}$ & 3.356333 & -1.174225 & 2.450048 \\
$\mathrm{H}$ & 3.698898 & -2.265526 & 1.074235 \\
$\mathrm{H}$ & 2.254268 & -2.533301 & 2.090974 \\
$\mathrm{H}$ & -3.100427 & 1.953305 & 1.774255 \\
\cline { 2 - 4 } & $\mathrm{E}$ & $\mathrm{G}$ & \\
& -802.156906 & -801.962970 & \\
\hline & & & \\
\hline
\end{tabular}


Configuration: D-gluco-4-F, conformation: ${ }^{3} \mathrm{H}_{4}$

\begin{tabular}{cccc}
\hline Atom & $X$ & $y$ & $Z$ \\
\hline $\mathrm{C}$ & 0.702524 & 1.008709 & 0.573759 \\
$\mathrm{C}$ & -0.227682 & 0.183565 & 1.467845 \\
$\mathrm{C}$ & -1.232917 & -0.644456 & 0.686404 \\
$\mathrm{O}$ & -0.562659 & -1.349227 & -0.477062 \\
$\mathrm{C}$ & 0.528670 & -0.984758 & -0.978504 \\
$\mathrm{C}$ & 1.414953 & 0.101218 & -0.447915 \\
$\mathrm{O}$ & -0.120618 & 1.919290 & -0.114712 \\
$\mathrm{H}$ & 1.457150 & 1.497492 & 1.200103 \\
$\mathrm{~F}$ & 0.558464 & -0.699155 & 2.201296 \\
$\mathrm{H}$ & -0.766069 & 0.843828 & 2.152858 \\
$\mathrm{H}$ & 0.864322 & -1.596983 & -1.816780 \\
$\mathrm{~N}$ & 2.492326 & -0.712242 & 0.169893 \\
$\mathrm{H}$ & 1.774142 & 0.705023 & -1.287294 \\
$\mathrm{C}$ & -2.444954 & 0.069244 & 0.113447 \\
$\mathrm{H}$ & -1.554236 & -1.493846 & 1.289773 \\
$\mathrm{~N}$ & 3.550846 & -0.781431 & -0.489926 \\
$\mathrm{~N}$ & 4.571867 & -0.922014 & -0.963018 \\
$\mathrm{C}$ & 0.514728 & 3.147256 & -0.493651 \\
$\mathrm{O}$ & -3.320934 & -0.925058 & -0.361377 \\
$\mathrm{H}$ & 1.358678 & 2.976776 & -1.173241 \\
$\mathrm{H}$ & 0.866682 & 3.689666 & 0.391139 \\
$\mathrm{H}$ & -0.243779 & 3.736162 & -1.009104 \\
$\mathrm{C}$ & -4.511734 & -0.379441 & -0.920990 \\
$\mathrm{H}$ & -2.138413 & 0.769463 & -0.673994 \\
$\mathrm{H}$ & -2.891936 & 0.659628 & 0.929570 \\
$\mathrm{H}$ & -4.291120 & 0.272821 & -1.777409 \\
$\mathrm{H}$ & -5.077581 & 0.196366 & -0.175125 \\
$\mathrm{H}$ & -5.117136 & -1.222257 & -1.257963 \\
\cline { 2 - 4 } & $\mathrm{E}$ & $\mathrm{G}$ & \\
& -802.152662 & -801.958838 & \\
\hline & & & \\
& & & \\
& &
\end{tabular}


Configuration: D-gluco-3-F, conformation: ${ }^{3} \mathrm{H}_{4}$

\begin{tabular}{cccc}
\hline Atom & $X$ & $y$ & $Z$ \\
\hline $\mathrm{C}$ & -0.714826 & 0.133217 & 1.230845 \\
$\mathrm{C}$ & 0.221312 & 1.182498 & 0.628854 \\
$\mathrm{C}$ & 1.281018 & 0.541454 & -0.268768 \\
$\mathrm{O}$ & 0.580043 & -0.309352 & -1.314875 \\
$\mathrm{C}$ & -0.544159 & -0.826704 & -1.123099 \\
$\mathrm{C}$ & -1.363653 & -0.729215 & 0.130086 \\
$\mathrm{H}$ & -1.494912 & 0.613172 & 1.823252 \\
$\mathrm{H}$ & 0.737427 & 1.713176 & 1.439457 \\
$\mathrm{H}$ & -0.944698 & -1.373325 & -1.978321 \\
$\mathrm{~N}$ & -2.656592 & -0.177624 & -0.316254 \\
$\mathrm{H}$ & -1.448174 & -1.752724 & 0.523104 \\
$\mathrm{C}$ & 2.349673 & -0.326328 & 0.369486 \\
$\mathrm{H}$ & 1.758209 & 1.287069 & -0.904125 \\
$\mathrm{~N}$ & -3.616491 & -0.973659 & -0.277366 \\
$\mathrm{~N}$ & -4.581335 & -1.570506 & -0.289340 \\
$\mathrm{O}$ & 3.288835 & -0.629939 & -0.633448 \\
$\mathrm{C}$ & 4.348916 & -1.454210 & -0.156979 \\
$\mathrm{H}$ & 1.908187 & -1.229246 & 0.809964 \\
$\mathrm{H}$ & 2.792328 & 0.258717 & 1.192013 \\
$\mathrm{H}$ & 3.971949 & -2.417965 & 0.212804 \\
$\mathrm{H}$ & 4.908502 & -0.960500 & 0.649678 \\
$\mathrm{H}$ & 5.016290 & -1.629872 & -1.002005 \\
$\mathrm{~F}$ & 0.031811 & -0.713089 & 2.044305 \\
$\mathrm{O}$ & -0.620379 & 2.017306 & -0.131655 \\
$\mathrm{C}$ & -0.142431 & 3.352374 & -0.335933 \\
$\mathrm{H}$ & 0.800230 & 3.369739 & -0.895786 \\
$\mathrm{H}$ & -0.909490 & 3.863281 & -0.917892 \\
$\mathrm{H}$ & -0.004314 & 3.866185 & 0.622711 \\
\cline { 2 - 4 } & $\mathrm{E}$ & $\mathrm{G}$ & \\
& -802.151570 & -801.958050 & \\
\hline & & & \\
\hline
\end{tabular}


Configuration: D-gluco-3-F, conformation: ${ }^{4} \mathrm{H}_{3}$

\begin{tabular}{cccc}
\hline Atom & $X$ & $y$ & $Z$ \\
\hline $\mathrm{C}$ & -0.291272 & 0.912824 & -0.071431 \\
$\mathrm{C}$ & -1.297198 & -0.154468 & 0.377274 \\
$\mathrm{O}$ & -0.750606 & -1.563837 & 0.226306 \\
$\mathrm{C}$ & 0.455993 & -1.836785 & 0.042183 \\
$\mathrm{C}$ & 1.527244 & -0.822788 & -0.153871 \\
$\mathrm{C}$ & 1.121373 & 0.553703 & 0.389172 \\
$\mathrm{H}$ & -1.476514 & -0.093895 & 1.452566 \\
$\mathrm{C}$ & -2.603476 & -0.132204 & -0.383084 \\
$\mathrm{H}$ & 0.693183 & -2.901430 & 0.002356 \\
$\mathrm{~N}$ & 2.763834 & -1.348732 & 0.422989 \\
$\mathrm{H}$ & 1.593756 & -0.740645 & -1.256439 \\
$\mathrm{H}$ & 1.186685 & 0.570956 & 1.481147 \\
$\mathrm{O}$ & -3.501203 & -0.993693 & 0.268278 \\
$\mathrm{H}$ & -2.432274 & -0.423864 & -1.431854 \\
$\mathrm{H}$ & -2.944095 & 0.915114 & -0.375618 \\
$\mathrm{C}$ & -4.782109 & -1.023790 & -0.355778 \\
$\mathrm{~N}$ & 3.807251 & -0.924851 & -0.109406 \\
$\mathrm{~N}$ & 4.835706 & -0.628567 & -0.486292 \\
$\mathrm{H}$ & -4.714298 & -1.382258 & -1.392316 \\
$\mathrm{H}$ & -5.250717 & -0.029960 & -0.354579 \\
$\mathrm{H}$ & -5.398817 & -1.712942 & 0.222945 \\
$\mathrm{~F}$ & 2.030116 & 1.468527 & -0.121389 \\
$\mathrm{H}$ & -0.293016 & 0.991035 & -1.169157 \\
$\mathrm{O}$ & -0.754645 & 2.093710 & 0.533036 \\
$\mathrm{C}$ & -0.445316 & 3.299903 & -0.184308 \\
$\mathrm{H}$ & -0.874071 & 3.272683 & -1.193581 \\
$\mathrm{H}$ & -0.903019 & 4.110920 & 0.382472 \\
$\mathrm{H}$ & 0.634801 & 3.454792 & -0.248385 \\
\hline & $\mathrm{E}$ & $\mathrm{G}$ & \\
& -802.150085 & -801.956610 & \\
\hline & & & \\
& & & \\
& &
\end{tabular}


Configuration: D-gluco-3-F, conformation: S

\begin{tabular}{cccc}
\hline Atom & $X$ & $y$ & $Z$ \\
\hline $\mathrm{C}$ & 0.893504 & -0.977807 & -0.102266 \\
$\mathrm{C}$ & 1.592511 & 0.082237 & 0.799215 \\
$\mathrm{O}$ & 0.675433 & 0.694588 & 1.841590 \\
$\mathrm{C}$ & -0.554333 & 0.833310 & 1.644880 \\
$\mathrm{C}$ & -1.244790 & 0.259820 & 0.466532 \\
$\mathrm{C}$ & -0.606218 & -1.109692 & 0.154344 \\
$\mathrm{H}$ & 2.348324 & -0.394128 & 1.421878 \\
$\mathrm{C}$ & 2.150177 & 1.279531 & 0.053412 \\
$\mathrm{H}$ & -1.098142 & 1.367152 & 2.426279 \\
$\mathrm{~N}$ & -2.670662 & 0.181094 & 0.763505 \\
$\mathrm{H}$ & -1.007478 & 0.939674 & -0.370377 \\
$\mathrm{H}$ & -0.788565 & -1.807388 & 0.977591 \\
$\mathrm{O}$ & 1.075724 & 1.850130 & -0.661350 \\
$\mathrm{H}$ & 2.947045 & 0.912953 & -0.611065 \\
$\mathrm{H}$ & 2.594680 & 1.994865 & 0.759257 \\
$\mathrm{C}$ & 1.435709 & 3.020045 & -1.400759 \\
$\mathrm{~N}$ & -3.408944 & 0.096006 & -0.233899 \\
$\mathrm{~N}$ & -4.203706 & 0.017604 & -1.040690 \\
$\mathrm{H}$ & 2.194060 & 2.786722 & -2.158040 \\
$\mathrm{H}$ & 1.818555 & 3.802894 & -0.734247 \\
$\mathrm{H}$ & 0.527545 & 3.371137 & -1.891378 \\
$\mathrm{~F}$ & -1.226567 & -1.596407 & -0.979788 \\
$\mathrm{H}$ & 1.002243 & -0.643980 & -1.143221 \\
$\mathrm{O}$ & 1.448237 & -2.253736 & 0.095487 \\
$\mathrm{C}$ & 2.661045 & -2.492710 & -0.626195 \\
$\mathrm{H}$ & 3.482133 & -1.856062 & -0.271907 \\
$\mathrm{H}$ & 2.923144 & -3.535935 & -0.448122 \\
$\mathrm{H}$ & 2.517966 & -2.329430 & -1.701750 \\
\cline { 2 - 4 } & $\mathrm{E}$ & $\mathrm{G}$ & \\
& -802.153805 & -801.959177 & \\
\hline & & & \\
& & & \\
& &
\end{tabular}


Configuration: D-galacto-4-F, conformation: ${ }^{4} \mathrm{H}_{3}$

\begin{tabular}{cccc}
\hline Atom & $X$ & $y$ & $Z$ \\
\hline $\mathrm{C}$ & -0.359976 & 1.092400 & -0.022050 \\
$\mathrm{C}$ & -1.507020 & 0.250968 & 0.505368 \\
$\mathrm{O}$ & -1.178990 & -1.237630 & 0.462276 \\
$\mathrm{C}$ & -0.035302 & -1.697033 & 0.236303 \\
$\mathrm{C}$ & 1.148262 & -0.869403 & -0.102692 \\
$\mathrm{C}$ & 1.007432 & 0.568626 & 0.436372 \\
$\mathrm{H}$ & -1.670096 & 0.409717 & 1.572596 \\
$\mathrm{C}$ & -2.802219 & 0.395263 & -0.266880 \\
$\mathrm{H}$ & 0.045008 & -2.784518 & 0.286034 \\
$\mathrm{~N}$ & 2.337546 & -1.574716 & 0.360839 \\
$\mathrm{H}$ & 1.107090 & -0.795535 & -1.207414 \\
$\mathrm{H}$ & 1.044665 & 0.554354 & 1.535278 \\
$\mathrm{O}$ & 2.069242 & 1.288482 & -0.118566 \\
$\mathrm{C}$ & 2.520355 & 2.398685 & 0.668253 \\
$\mathrm{O}$ & -3.805958 & -0.271523 & 0.453975 \\
$\mathrm{H}$ & -2.669059 & -0.010988 & -1.279675 \\
$\mathrm{H}$ & -3.004546 & 1.474456 & -0.367908 \\
$\mathrm{H}$ & 1.731823 & 3.148802 & 0.799005 \\
$\mathrm{H}$ & 2.870903 & 2.063010 & 1.651669 \\
$\mathrm{C}$ & -5.056868 & -0.279964 & -0.229669 \\
$\mathrm{~N}$ & 3.396660 & -1.241026 & -0.201277 \\
$\mathrm{~N}$ & 4.435282 & -1.048968 & -0.616159 \\
$\mathrm{H}$ & -4.979994 & -0.795468 & -1.197011 \\
$\mathrm{H}$ & -5.427501 & 0.740188 & -0.400223 \\
$\mathrm{H}$ & -5.760769 & -0.815647 & 0.408809 \\
$\mathrm{H}$ & 3.349085 & 2.843321 & 0.117449 \\
$\mathrm{H}$ & -0.513409 & 2.126371 & 0.302523 \\
$\mathrm{~F}$ & -0.385772 & 1.055892 & -1.417384 \\
\cline { 2 - 4 } & $\mathrm{E}$ & $\mathrm{G}$ & \\
& -802.154983 & -801.960780 & \\
\hline & & & \\
& & & \\
& &
\end{tabular}


Configuration: D-galacto-4-F, conformation: ${ }^{3} \mathrm{H}_{4}$

\begin{tabular}{cccc}
\hline Atom & $X$ & $y$ & $Z$ \\
\hline $\mathrm{C}$ & 0.762286 & 1.068360 & 0.290833 \\
$\mathrm{C}$ & -0.108593 & 0.386219 & 1.339521 \\
$\mathrm{C}$ & -1.072998 & -0.646470 & 0.731133 \\
$\mathrm{O}$ & -0.360120 & -1.486028 & -0.303764 \\
$\mathrm{C}$ & 0.733500 & -1.174638 & -0.837384 \\
$\mathrm{C}$ & 1.585165 & -0.009233 & -0.433582 \\
$\mathrm{H}$ & 1.437698 & 1.774359 & 0.788292 \\
$\mathrm{H}$ & 1.097859 & -1.895417 & -1.570367 \\
$\mathrm{~N}$ & 2.517849 & -0.699851 & 0.492436 \\
$\mathrm{H}$ & 2.081565 & 0.411447 & -1.311835 \\
$\mathrm{C}$ & -2.330449 & -0.111884 & 0.067429 \\
$\mathrm{H}$ & -1.353414 & -1.406133 & 1.461003 \\
$\mathrm{~N}$ & 3.656692 & -0.941428 & 0.037471 \\
$\mathrm{~N}$ & 4.723957 & -1.208065 & -0.234166 \\
$\mathrm{O}$ & -3.170952 & -1.218274 & -0.168314 \\
$\mathrm{C}$ & -4.414524 & -0.839562 & -0.748529 \\
$\mathrm{H}$ & -2.076360 & 0.426403 & -0.854200 \\
$\mathrm{H}$ & -2.776091 & 0.608846 & 0.768086 \\
$\mathrm{H}$ & -4.271788 & -0.350409 & -1.722382 \\
$\mathrm{H}$ & -4.972856 & -0.158540 & -0.090723 \\
$\mathrm{H}$ & -4.990910 & -1.755528 & -0.888554 \\
$\mathrm{H}$ & 0.539289 & -0.109259 & 2.069314 \\
$\mathrm{~F}$ & -0.872492 & 1.331161 & 2.000444 \\
$\mathrm{O}$ & -0.075474 & 1.692193 & -0.653127 \\
$\mathrm{C}$ & 0.488096 & 2.842808 & -1.295366 \\
$\mathrm{H}$ & 1.391674 & 2.592080 & -1.864626 \\
$\mathrm{H}$ & -0.273677 & 3.212114 & -1.981929 \\
$\mathrm{H}$ & 0.727421 & 3.618265 & -0.558953 \\
\cline { 2 - 4 } & $\mathrm{E}$ & $\mathrm{G}$ & \\
& -802.150442 & -801.955482 & \\
\hline & & & \\
\hline
\end{tabular}


Configuration: D-galacto-4-F, conformation: S

\begin{tabular}{cccc}
\hline Atom & $X$ & $y$ & $Z$ \\
\hline $\mathrm{C}$ & 0.646502 & 0.603391 & -0.055232 \\
$\mathrm{C}$ & 1.473043 & -0.532907 & -0.627565 \\
$\mathrm{O}$ & 0.777236 & -1.862129 & -0.458819 \\
$\mathrm{C}$ & -0.441040 & -1.979768 & -0.180194 \\
$\mathrm{C}$ & -1.347325 & -0.847189 & 0.126230 \\
$\mathrm{C}$ & -0.829150 & 0.483014 & -0.455337 \\
$\mathrm{H}$ & 1.571373 & -0.440375 & -1.710834 \\
$\mathrm{C}$ & 2.836726 & -0.712124 & 0.030335 \\
$\mathrm{H}$ & -0.809949 & -3.006440 & -0.152007 \\
$\mathrm{~N}$ & -2.691079 & -1.217912 & -0.303630 \\
$\mathrm{H}$ & -1.270610 & -0.756038 & 1.228626 \\
$\mathrm{H}$ & -0.910969 & 0.458820 & -1.552004 \\
$\mathrm{O}$ & -1.632810 & 1.476468 & 0.112414 \\
$\mathrm{C}$ & -1.732107 & 2.681326 & -0.657302 \\
$\mathrm{O}$ & 3.516714 & 0.487292 & -0.252565 \\
$\mathrm{H}$ & 3.339185 & -1.591679 & -0.396373 \\
$\mathrm{H}$ & 2.703312 & -0.869664 & 1.109419 \\
$\mathrm{H}$ & -0.757446 & 3.169493 & -0.771186 \\
$\mathrm{H}$ & -2.155788 & 2.477709 & -1.648285 \\
$\mathrm{C}$ & 4.797876 & 0.555049 & 0.371563 \\
$\mathrm{~N}$ & -3.609405 & -0.585390 & 0.249796 \\
$\mathrm{~N}$ & -4.549006 & -0.099469 & 0.660284 \\
$\mathrm{H}$ & 5.452203 & -0.255466 & 0.023795 \\
$\mathrm{H}$ & 4.710126 & 0.499513 & 1.464830 \\
$\mathrm{H}$ & 5.232695 & 1.515512 & 0.091756 \\
$\mathrm{H}$ & -2.399577 & 3.341108 & -0.103115 \\
$\mathrm{H}$ & 1.089942 & 1.544638 & -0.390340 \\
$\mathrm{~F}$ & 0.715074 & 0.546051 & 1.337484 \\
\cline { 2 - 4 } & $\mathrm{E}$ & $\mathrm{G}$ & \\
& -802.154280 & -801.960401 & \\
\hline & & & \\
\hline
\end{tabular}


Configuration: D-galacto-3-F, conformation: ${ }^{4} \mathrm{H}_{3}$

\begin{tabular}{cccc}
\hline Atom & $X$ & $y$ & $Z$ \\
\hline $\mathrm{C}$ & -0.290894 & 0.903289 & 0.591240 \\
$\mathrm{C}$ & -1.355598 & -0.185539 & 0.612678 \\
$\mathrm{O}$ & -0.897268 & -1.415655 & -0.174410 \\
$\mathrm{C}$ & 0.297458 & -1.642019 & -0.472055 \\
$\mathrm{C}$ & 1.418771 & -0.704730 & -0.206701 \\
$\mathrm{C}$ & 1.070965 & 0.279566 & 0.916821 \\
$\mathrm{H}$ & -1.499275 & -0.604632 & 1.609718 \\
$\mathrm{C}$ & -2.680961 & 0.206235 & -0.000207 \\
$\mathrm{H}$ & 0.480417 & -2.585914 & -0.989083 \\
$\mathrm{~N}$ & 2.633386 & -1.482083 & 0.015635 \\
$\mathrm{H}$ & 1.458901 & -0.106955 & -1.139831 \\
$\mathrm{H}$ & 1.060262 & -0.223216 & 1.888770 \\
$\mathrm{O}$ & -3.602208 & -0.813511 & 0.295516 \\
$\mathrm{H}$ & -2.556220 & 0.365399 & -1.079997 \\
$\mathrm{H}$ & -2.969305 & 1.173242 & 0.444546 \\
$\mathrm{C}$ & -4.881449 & -0.583446 & -0.288157 \\
$\mathrm{~N}$ & 3.691067 & -0.880862 & -0.249637 \\
$\mathrm{~N}$ & 4.729370 & -0.465626 & -0.443369 \\
$\mathrm{H}$ & -4.818616 & -0.530183 & -1.383941 \\
$\mathrm{H}$ & -5.331198 & 0.346798 & 0.086003 \\
$\mathrm{H}$ & -5.513458 & -1.426533 & -0.005040 \\
$\mathrm{H}$ & -0.553786 & 1.627603 & 1.374443 \\
$\mathrm{~F}$ & 2.060332 & 1.247459 & 0.930342 \\
$\mathrm{O}$ & -0.285261 & 1.482966 & -0.695051 \\
$\mathrm{C}$ & 0.096443 & 2.869313 & -0.741762 \\
$\mathrm{H}$ & 0.028382 & 3.163573 & -1.789210 \\
$\mathrm{H}$ & -0.594342 & 3.477534 & -0.146200 \\
$\mathrm{H}$ & 1.117976 & 3.009745 & -0.381232 \\
\cline { 2 - 4 } & $\mathrm{E}$ & $\mathrm{G}$ & \\
& -802.155428 & -801.962669 & \\
\hline & & & \\
\hline
\end{tabular}


Configuration: D-galacto-3-F, conformation: ${ }^{3} \mathrm{H}_{4}$

\begin{tabular}{cccc}
\hline Atom & $X$ & $y$ & $Z$ \\
\hline $\mathrm{C}$ & -0.763391 & 0.761506 & 0.825385 \\
$\mathrm{C}$ & 0.097014 & 1.085347 & -0.396108 \\
$\mathrm{C}$ & 1.035705 & -0.078805 & -0.749806 \\
$\mathrm{O}$ & 0.267324 & -1.387077 & -0.756631 \\
$\mathrm{C}$ & -0.804608 & -1.570739 & -0.131443 \\
$\mathrm{C}$ & -1.587034 & -0.502131 & 0.573651 \\
$\mathrm{H}$ & -1.424079 & 1.592394 & 1.081943 \\
$\mathrm{H}$ & -1.213564 & -2.577758 & -0.216123 \\
$\mathrm{~N}$ & -2.626190 & -0.232891 & -0.448838 \\
$\mathrm{H}$ & -1.986791 & -0.888642 & 1.515714 \\
$\mathrm{C}$ & 2.245540 & -0.299670 & 0.142788 \\
$\mathrm{H}$ & 1.360816 & -0.016940 & -1.787712 \\
$\mathrm{~N}$ & -3.747657 & -0.739836 & -0.224848 \\
$\mathrm{~N}$ & -4.811304 & -1.122281 & -0.151601 \\
$\mathrm{O}$ & 3.056115 & -1.260724 & -0.493549 \\
$\mathrm{C}$ & 4.250202 & -1.530671 & 0.233436 \\
$\mathrm{H}$ & 1.931866 & -0.620346 & 1.144458 \\
$\mathrm{H}$ & 2.746386 & 0.674108 & 0.240354 \\
$\mathrm{H}$ & 4.030438 & -1.930824 & 1.233333 \\
$\mathrm{H}$ & 4.866039 & -0.626997 & 0.343620 \\
$\mathrm{H}$ & 4.806331 & -2.276711 & -0.336583 \\
$\mathrm{~F}$ & 0.057995 & 0.486105 & 1.916209 \\
$\mathrm{H}$ & -0.587973 & 1.235678 & -1.243936 \\
$\mathrm{O}$ & 0.924888 & 2.200987 & -0.194932 \\
$\mathrm{C}$ & 0.314890 & 3.457433 & -0.514969 \\
$\mathrm{H}$ & -0.556917 & 3.659726 & 0.118307 \\
$\mathrm{H}$ & 1.073202 & 4.218477 & -0.330207 \\
$\mathrm{H}$ & 0.011823 & 3.488845 & -1.568744 \\
\cline { 2 - 4 } & $\mathrm{E}$ & $\mathrm{G}$ & \\
& -802.150575 & -801.956275 & \\
\hline & & & \\
\hline
\end{tabular}


Configuration: D-gluco-4-F $\alpha$-triflate

\begin{tabular}{cccc}
\hline Atom & $X$ & $y$ & $Z$ \\
\hline $\mathrm{C}$ & -2.258380 & -0.342867 & 0.286326 \\
$\mathrm{C}$ & -1.008174 & -1.220109 & 0.198314 \\
$\mathrm{O}$ & -0.303646 & -0.951056 & -1.034904 \\
$\mathrm{C}$ & 0.061482 & 0.362692 & -1.227463 \\
$\mathrm{C}$ & -1.124871 & 1.336569 & -1.158357 \\
$\mathrm{C}$ & -1.922891 & 1.141385 & 0.141206 \\
$\mathrm{H}$ & -2.991937 & -0.640244 & -0.468046 \\
$\mathrm{~F}$ & -2.819836 & -0.564712 & 1.551578 \\
$\mathrm{H}$ & -0.357345 & -0.985107 & 1.048453 \\
$\mathrm{C}$ & -1.319050 & -2.703059 & 0.231245 \\
$\mathrm{O}$ & 0.979483 & 0.819282 & -0.156640 \\
$\mathrm{H}$ & 0.585330 & 0.429290 & -2.180459 \\
$\mathrm{~N}$ & -0.693829 & 2.719746 & -1.418670 \\
$\mathrm{H}$ & -1.773492 & 1.080474 & -1.999744 \\
$\mathrm{H}$ & -1.304961 & 1.434748 & 1.004722 \\
$\mathrm{O}$ & -3.072560 & 1.952916 & 0.045567 \\
$\mathrm{C}$ & -3.642779 & 2.334856 & 1.299557 \\
$\mathrm{O}$ & -2.230609 & -3.005872 & -0.805113 \\
$\mathrm{H}$ & -1.741389 & -2.940323 & 1.219704 \\
$\mathrm{H}$ & -0.378968 & -3.263582 & 0.116564 \\
$\mathrm{~S}$ & 2.568059 & 0.505816 & -0.259419 \\
$\mathrm{H}$ & -2.903734 & 2.852626 & 1.926554 \\
$\mathrm{H}$ & -4.033020 & 1.471988 & 1.847165 \\
$\mathrm{H}$ & -4.458136 & 3.023154 & 1.069520 \\
$\mathrm{C}$ & -2.615960 & -4.371866 & -0.810065 \\
$\mathrm{~N}$ & -0.278331 & 3.360715 & -0.445714 \\
$\mathrm{~N}$ & 0.110728 & 4.066091 & 0.360672 \\
$\mathrm{O}$ & 2.943594 & 0.199567 & -1.633097 \\
$\mathrm{O}$ & 3.239393 & 1.525310 & 0.527788 \\
$\mathrm{C}$ & 2.658351 & -1.089758 & 0.728057 \\
$\mathrm{~F}$ & 2.116307 & -2.084380 & 0.030126 \\
$\mathrm{~F}$ & 1.995633 & -0.933063 & 1.874707 \\
$\mathrm{~F}$ & 3.937575 & -1.350413 & 0.974563 \\
$\mathrm{H}$ & -3.317557 & -4.505690 & -1.636027 \\
$\mathrm{H}$ & -3.110535 & -4.656191 & 0.130727 \\
$\mathrm{H}$ & -1.751890 & -5.035182 & -0.962941 \\
\hline & $\mathrm{E}$ & $\mathrm{G}$ & \\
\hline & -1763.810381 & -1763.592015 & \\
\hline & & & \\
\hline
\end{tabular}


Configuration: D-gluco-4-F $\beta$-triflate

\begin{tabular}{cccc}
\hline Atom & $X$ & $y$ & $Z$ \\
\hline $\mathrm{C}$ & -1.687144 & 1.609875 & -0.019626 \\
$\mathrm{C}$ & -0.470542 & 1.741278 & -0.939759 \\
$\mathrm{O}$ & 0.355774 & 0.576661 & -0.806103 \\
$\mathrm{C}$ & -0.263021 & -0.639342 & -1.092446 \\
$\mathrm{C}$ & -1.478508 & -0.881463 & -0.191570 \\
$\mathrm{C}$ & -2.444507 & 0.312338 & -0.288865 \\
$\mathrm{H}$ & -1.381021 & 1.661065 & 1.028271 \\
$\mathrm{~F}$ & -2.527665 & 2.699126 & -0.286516 \\
$\mathrm{H}$ & -0.821472 & 1.830461 & -1.978674 \\
$\mathrm{C}$ & 0.402574 & 2.938680 & -0.611564 \\
$\mathrm{O}$ & 0.748431 & -1.657056 & -0.943042 \\
$\mathrm{H}$ & -0.545570 & -0.727362 & -2.150252 \\
$\mathrm{~N}$ & -2.118783 & -2.124541 & -0.655646 \\
$\mathrm{H}$ & -1.139739 & -0.968850 & 0.844472 \\
$\mathrm{H}$ & -2.863250 & 0.357274 & -1.306600 \\
$\mathrm{O}$ & -3.459687 & 0.069987 & 0.662632 \\
$\mathrm{C}$ & -4.722855 & 0.667033 & 0.361537 \\
$\mathrm{O}$ & 0.743329 & 2.886374 & 0.758286 \\
$\mathrm{H}$ & -0.151231 & 3.858400 & -0.854091 \\
$\mathrm{H}$ & 1.299271 & 2.897647 & -1.247983 \\
$\mathrm{~S}$ & 1.746981 & -1.670199 & 0.350343 \\
$\mathrm{H}$ & -5.099687 & 0.321851 & -0.610962 \\
$\mathrm{H}$ & -4.658649 & 1.759175 & 0.353856 \\
$\mathrm{H}$ & -5.410697 & 0.344643 & 1.145226 \\
$\mathrm{C}$ & 1.732458 & 3.837413 & 1.116577 \\
$\mathrm{~N}$ & -2.902120 & -2.655421 & 0.139985 \\
$\mathrm{~N}$ & -3.633295 & -3.260284 & 0.771091 \\
$\mathrm{O}$ & 1.070530 & -1.238288 & 1.566538 \\
$\mathrm{O}$ & 2.423897 & -2.953206 & 0.257250 \\
$\mathrm{C}$ & 3.039492 & -0.349063 & -0.097016 \\
$\mathrm{~F}$ & 2.763149 & 0.790076 & 0.519042 \\
$\mathrm{~F}$ & 3.073253 & -0.172757 & -1.412981 \\
$\mathrm{~F}$ & 4.214347 & -0.813811 & 0.326810 \\
$\mathrm{H}$ & 1.906195 & 3.730609 & 2.189288 \\
$\mathrm{H}$ & 1.404268 & 4.866255 & 0.907128 \\
$\mathrm{H}$ & 2.675651 & 3.653745 & 0.581511 \\
\cline { 2 - 4 } & $\mathrm{E}$ & $\mathrm{G}$ & \\
& -1763.80362 & -1763.58545 & \\
\hline & & &
\end{tabular}


Configuration: D-gluco-3-F $\alpha$-triflate

\begin{tabular}{cccc}
\hline Atom & $x$ & \multicolumn{1}{l}{$l$} \\
\hline $\mathrm{C}$ & -2.222911 & 0.417353 & 0.268152 \\
$\mathrm{C}$ & -1.259725 & -0.768302 & 0.114961 \\
$\mathrm{O}$ & -0.470844 & -0.625542 & -1.092060 \\
$\mathrm{C}$ & 0.261502 & 0.533313 & -1.186864 \\
$\mathrm{C}$ & -0.597500 & 1.802671 & -1.058532 \\
$\mathrm{C}$ & -1.456366 & 1.736760 & 0.205026 \\
$\mathrm{H}$ & -2.944109 & 0.388187 & -0.558306 \\
$\mathrm{H}$ & -0.589555 & -0.798334 & 0.981379 \\
$\mathrm{C}$ & -1.978995 & -2.099493 & 0.026646 \\
$\mathrm{O}$ & 1.240681 & 0.634145 & -0.076547 \\
$\mathrm{H}$ & 0.811003 & 0.511506 & -2.127633 \\
$\mathrm{~N}$ & 0.195429 & 3.033263 & -1.208388 \\
$\mathrm{H}$ & -1.267189 & 1.796934 & -1.921772 \\
$\mathrm{H}$ & -0.846455 & 1.847753 & 1.108929 \\
$\mathrm{O}$ & -2.938410 & -2.041248 & -1.010855 \\
$\mathrm{H}$ & -2.454476 & -2.293105 & 0.999069 \\
$\mathrm{H}$ & -1.235832 & -2.889618 & -0.159186 \\
$\mathrm{~S}$ & 2.674423 & -0.118909 & -0.188331 \\
$\mathrm{C}$ & -3.690207 & -3.239175 & -1.124007 \\
$\mathrm{~N}$ & 0.881433 & 3.381378 & -0.236954 \\
$\mathrm{~N}$ & 1.548539 & 3.828351 & 0.571212 \\
$\mathrm{O}$ & 2.986505 & -0.420400 & -1.578892 \\
$\mathrm{O}$ & 3.586355 & 0.603755 & 0.680943 \\
$\mathrm{C}$ & 2.272721 & -1.739526 & 0.673666 \\
$\mathrm{~F}$ & 1.487324 & -2.481725 & -0.102272 \\
$\mathrm{~F}$ & 1.651941 & -1.480444 & 1.824827 \\
$\mathrm{~F}$ & 3.415532 & -2.375906 & 0.906990 \\
$\mathrm{H}$ & -4.401758 & -3.097818 & -1.940302 \\
$\mathrm{H}$ & -4.244368 & -3.458475 & -0.199047 \\
$\mathrm{H}$ & -3.046650 & -4.100802 & -1.355579 \\
$\mathrm{~F}$ & -2.363192 & 2.799614 & 0.173374 \\
$\mathrm{O}$ & -2.864578 & 0.263598 & 1.519776 \\
$\mathrm{C}$ & -4.212568 & 0.736307 & 1.550378 \\
$\mathrm{H}$ & -4.585118 & 0.548560 & 2.559323 \\
$\mathrm{H}$ & -4.265348 & 1.808091 & 1.334274 \\
$\mathrm{H}$ & -4.836540 & 0.190960 & 0.829321 \\
\cline { 2 - 4 } & $\mathrm{E}$ & $\mathrm{G}$ & \\
\hline & -1763.81024 & -1763.59215 & \\
\hline & & &
\end{tabular}


Configuration: D-gluco-3-F $\beta$-triflate

\begin{tabular}{cccc}
\hline Atom & $x$ & $y$ & $Z$ \\
\hline $\mathrm{C}$ & 2.088384 & 1.019953 & 0.145767 \\
$\mathrm{C}$ & 0.897039 & 1.414645 & 1.034337 \\
$\mathrm{O}$ & -0.185761 & 0.484797 & 0.866671 \\
$\mathrm{C}$ & 0.101768 & -0.854306 & 1.113938 \\
$\mathrm{C}$ & 1.244954 & -1.354780 & 0.223563 \\
$\mathrm{C}$ & 2.455278 & -0.437563 & 0.405253 \\
$\mathrm{H}$ & 1.792538 & 1.133244 & -0.904590 \\
$\mathrm{H}$ & 1.220437 & 1.414986 & 2.085321 \\
$\mathrm{C}$ & 0.341719 & 2.786142 & 0.694246 \\
$\mathrm{O}$ & -1.123011 & -1.583757 & 0.893981 \\
$\mathrm{H}$ & 0.320397 & -1.052847 & 2.171975 \\
$\mathrm{~N}$ & 1.551594 & -2.739860 & 0.624180 \\
$\mathrm{H}$ & 0.923925 & -1.300787 & -0.820362 \\
$\mathrm{H}$ & 2.879539 & -0.542102 & 1.410455 \\
$\mathrm{O}$ & 0.067844 & 2.834888 & -0.692008 \\
$\mathrm{H}$ & 1.080817 & 3.547132 & 0.983156 \\
$\mathrm{H}$ & -0.572670 & 2.944985 & 1.285286 \\
$\mathrm{~S}$ & -2.059311 & -1.274765 & -0.410215 \\
$\mathrm{C}$ & -0.637656 & 4.004981 & -1.070483 \\
$\mathrm{~N}$ & 2.168556 & -3.409819 & -0.212488 \\
$\mathrm{~N}$ & 2.721148 & -4.139021 & -0.892100 \\
$\mathrm{O}$ & -1.264332 & -0.960340 & -1.590265 \\
$\mathrm{O}$ & -3.035975 & -2.351322 & -0.402490 \\
$\mathrm{C}$ & -2.994730 & 0.300049 & 0.102483 \\
$\mathrm{~F}$ & -2.435496 & 1.366826 & -0.448149 \\
$\mathrm{~F}$ & -3.008810 & 0.410089 & 1.425785 \\
$\mathrm{~F}$ & -4.239429 & 0.162079 & -0.352760 \\
$\mathrm{H}$ & -0.775889 & 3.960951 & -2.152835 \\
$\mathrm{H}$ & -0.078926 & 4.918335 & -0.817470 \\
$\mathrm{H}$ & -1.623970 & 4.051880 & -0.585975 \\
$\mathrm{~F}$ & 3.431480 & -0.857436 & -0.507196 \\
$\mathrm{O}$ & 3.151286 & 1.889688 & 0.484119 \\
$\mathrm{C}$ & 4.043540 & 2.173261 & -0.595216 \\
$\mathrm{H}$ & 4.795853 & 2.861088 & -0.204076 \\
$\mathrm{H}$ & 4.533028 & 1.264573 & -0.959851 \\
$\mathrm{H}$ & 3.512939 & 2.655738 & -1.427245 \\
\cline { 2 - 4 } & $\mathrm{E}$ & $\mathrm{G}$ & \\
\hline & -1763.80313 & -1763.58492 & \\
\hline & & & \\
\hline
\end{tabular}


Configuration: D-galacto-4-F $\alpha$-triflate

\begin{tabular}{cccc}
\hline Atom & $X$ & $y$ & $Z$ \\
\hline $\mathrm{C}$ & 2.253554 & 0.499735 & 0.486223 \\
$\mathrm{C}$ & 0.935354 & 1.264271 & 0.384541 \\
$\mathrm{O}$ & 0.281831 & 0.977147 & -0.875764 \\
$\mathrm{C}$ & 0.052162 & -0.354818 & -1.128882 \\
$\mathrm{C}$ & 1.309580 & -1.233386 & -1.020381 \\
$\mathrm{C}$ & 2.000778 & -1.009197 & 0.331646 \\
$\mathrm{H}$ & 0.294888 & 0.914624 & 1.204807 \\
$\mathrm{C}$ & 1.015406 & 2.775202 & 0.512543 \\
$\mathrm{O}$ & -0.881266 & -0.924424 & -0.119131 \\
$\mathrm{H}$ & -0.417326 & -0.437314 & -2.108699 \\
$\mathrm{~N}$ & 1.000085 & -2.634295 & -1.345207 \\
$\mathrm{H}$ & 1.988770 & -0.892103 & -1.803683 \\
$\mathrm{H}$ & 1.332663 & -1.329664 & 1.146769 \\
$\mathrm{O}$ & 1.822825 & 3.325962 & -0.503291 \\
$\mathrm{H}$ & 1.409115 & 3.013461 & 1.515030 \\
$\mathrm{H}$ & -0.012146 & 3.168418 & 0.458687 \\
$\mathrm{~S}$ & -2.480355 & -0.741734 & -0.298685 \\
$\mathrm{C}$ & 1.852542 & 4.743289 & -0.452472 \\
$\mathrm{~N}$ & 0.616533 & -3.345048 & -0.408930 \\
$\mathrm{~N}$ & 0.268334 & -4.110235 & 0.361095 \\
$\mathrm{O}$ & -2.816630 & -0.487882 & -1.693135 \\
$\mathrm{O}$ & -3.102229 & -1.800770 & 0.477428 \\
$\mathrm{C}$ & -2.755430 & 0.855957 & 0.652315 \\
$\mathrm{~F}$ & -2.316500 & 1.890144 & -0.059418 \\
$\mathrm{~F}$ & -2.095739 & 0.796545 & 1.810967 \\
$\mathrm{~F}$ & -4.058641 & 0.980297 & 0.880320 \\
$\mathrm{H}$ & 2.491865 & 5.081311 & -1.270687 \\
$\mathrm{H}$ & 2.267468 & 5.108111 & 0.499568 \\
$\mathrm{H}$ & 0.848497 & 5.175716 & -0.580362 \\
$\mathrm{H}$ & 2.731718 & 0.719064 & 1.447367 \\
$\mathrm{~F}$ & 3.124228 & 0.883538 & -0.532349 \\
$\mathrm{O}$ & 3.188248 & -1.766942 & 0.327554 \\
$\mathrm{C}$ & 3.700619 & -2.060269 & 1.624948 \\
$\mathrm{H}$ & 4.582846 & -2.685120 & 1.477097 \\
$\mathrm{H}$ & 3.997937 & -1.154630 & 2.169376 \\
$\mathrm{H}$ & 2.964974 & -2.611224 & 2.227130 \\
\cline { 2 - 4 } & $\mathrm{E}$ & $\mathrm{G}$ & \\
& -1763.80568 & -1763.58737 & \\
\hline & & &
\end{tabular}


Configuration: D-galacto-3-F $\alpha$-triflate

\begin{tabular}{cccc}
\hline Atom & $x$ & \multicolumn{1}{l}{} & \multicolumn{1}{l}{ X } \\
\hline $\mathrm{C}$ & 2.196339 & 0.574315 & -0.685226 \\
$\mathrm{C}$ & 1.277886 & -0.639205 & -0.464013 \\
$\mathrm{O}$ & 0.612778 & -0.527584 & 0.820774 \\
$\mathrm{C}$ & -0.136503 & 0.609989 & 0.993800 \\
$\mathrm{C}$ & 0.632166 & 1.916802 & 0.717080 \\
$\mathrm{C}$ & 1.326321 & 1.836708 & -0.643419 \\
$\mathrm{H}$ & 0.514793 & -0.629343 & -1.253316 \\
$\mathrm{C}$ & 1.918965 & -2.013209 & -0.521941 \\
$\mathrm{O}$ & -1.268451 & 0.640350 & 0.024136 \\
$\mathrm{H}$ & -0.554964 & 0.593666 & 1.999765 \\
$\mathrm{~N}$ & -0.206460 & 3.106675 & 0.926081 \\
$\mathrm{H}$ & 1.411683 & 1.976831 & 1.478198 \\
$\mathrm{H}$ & 0.592090 & 1.822074 & -1.457504 \\
$\mathrm{O}$ & 2.919009 & -2.158902 & 0.462747 \\
$\mathrm{H}$ & 2.330010 & -2.154230 & -1.535535 \\
$\mathrm{H}$ & 1.121662 & -2.759407 & -0.380981 \\
$\mathrm{~S}$ & -2.635959 & -0.164648 & 0.347747 \\
$\mathrm{C}$ & 3.484500 & -3.460691 & 0.461670 \\
$\mathrm{~N}$ & -1.017690 & 3.389490 & 0.033742 \\
$\mathrm{~N}$ & -1.797218 & 3.776625 & -0.701851 \\
$\mathrm{O}$ & -2.769154 & -0.398213 & 1.779522 \\
$\mathrm{O}$ & -3.678711 & 0.465868 & -0.443191 \\
$\mathrm{C}$ & -2.264296 & -1.817798 & -0.466220 \\
$\mathrm{~F}$ & -1.413717 & -2.511689 & 0.284877 \\
$\mathrm{~F}$ & -1.729652 & -1.605752 & -1.670247 \\
$\mathrm{~F}$ & -3.408519 & -2.481992 & -0.590295 \\
$\mathrm{H}$ & 4.239030 & -3.483756 & 1.250508 \\
$\mathrm{H}$ & 3.964854 & -3.694329 & -0.500524 \\
$\mathrm{H}$ & 2.726340 & -4.231158 & 0.667178 \\
$\mathrm{~F}$ & 2.127451 & 2.959672 & -0.808154 \\
$\mathrm{O}$ & 3.173964 & 0.710848 & 0.326417 \\
$\mathrm{C}$ & 4.506771 & 0.427323 & -0.084552 \\
$\mathrm{H}$ & 4.816121 & 1.083935 & -0.909161 \\
$\mathrm{H}$ & 5.145476 & 0.619830 & 0.780114 \\
$\mathrm{H}$ & 4.616373 & -0.621592 & -0.379536 \\
$\mathrm{H}$ & 2.653016 & 0.495820 & -1.680901 \\
\cline { 2 - 4 } & $\mathrm{E}$ & $\mathrm{G}$ & \\
\hline & -1763.80507 & -1763.58598 & \\
\hline & & &
\end{tabular}


Configuration: D-galacto-4-F $\beta$-triflate

\begin{tabular}{|c|c|c|c|}
\hline Atom & $x$ & $y$ & $z$ \\
\hline$C$ & 1.550199 & 1.845345 & 0.061464 \\
\hline$C$ & 0.328239 & 1.833771 & 0.981021 \\
\hline 0 & -0.371053 & 0.584970 & 0.854965 \\
\hline$C$ & 0.384020 & -0.555213 & 1.125010 \\
\hline C & 1.639949 & -0.643540 & 0.249792 \\
\hline C & 2.453855 & 0.651929 & 0.400548 \\
\hline $\mathrm{H}$ & 0.698896 & 1.940391 & 2.013114 \\
\hline C & -0.698301 & 2.934097 & 0.765171 \\
\hline 0 & -0.494869 & -1.684824 & 0.939494 \\
\hline $\mathrm{H}$ & 0.656675 & -0.632277 & 2.186879 \\
\hline $\mathrm{N}$ & 2.399959 & -1.816377 & 0.714571 \\
\hline $\mathrm{H}$ & 1.342718 & -0.736839 & -0.796926 \\
\hline $\mathrm{H}$ & 2.791389 & 0.747220 & 1.444874 \\
\hline 0 & 3.552984 & 0.534145 & -0.474847 \\
\hline$C$ & 4.682901 & 1.325498 & -0.116806 \\
\hline 0 & -1.183277 & 2.909978 & -0.557506 \\
\hline $\mathrm{H}$ & -0.231315 & 3.903450 & 1.006563 \\
\hline $\mathrm{H}$ & -1.512412 & 2.767739 & 1.488094 \\
\hline$S$ & -1.454850 & -1.796422 & -0.376640 \\
\hline $\mathrm{H}$ & 5.048957 & 1.065622 & 0.885888 \\
\hline $\mathrm{H}$ & 4.459611 & 2.399800 & -0.145890 \\
\hline $\mathrm{H}$ & 5.460559 & 1.108333 & -0.850760 \\
\hline$C$ & -2.351505 & 3.696133 & -0.720707 \\
\hline$N$ & 3.253088 & -2.249023 & -0.067768 \\
\hline $\mathrm{N}$ & 4.059891 & -2.760589 & -0.689493 \\
\hline $\mathrm{O}$ & -0.820930 & -1.241553 & -1.564807 \\
\hline 0 & -1.954149 & -3.161351 & -0.332982 \\
\hline C & -2.920679 & -0.677213 & 0.082564 \\
\hline $\mathrm{F}$ & -2.794712 & 0.505213 & -0.498069 \\
\hline $\mathrm{F}$ & -2.994421 & -0.543265 & 1.402606 \\
\hline $\mathrm{F}$ & -4.016892 & -1.285552 & -0.370645 \\
\hline $\mathrm{H}$ & -2.638672 & 3.628008 & -1.772102 \\
\hline $\mathrm{H}$ & -2.175281 & 4.752251 & -0.466340 \\
\hline $\mathrm{H}$ & -3.178715 & 3.320314 & -0.100031 \\
\hline $\mathrm{F}$ & 1.165388 & 1.709325 & -1.269788 \\
\hline \multirow[t]{3}{*}{$\mathrm{H}$} & 2.088651 & 2.793417 & 0.171883 \\
\hline & $E$ & $\mathrm{G}$ & \\
\hline & -1763.7982 & -1763.57996 & \\
\hline
\end{tabular}


Configuration: D-galacto-3-F $\beta$-triflate

\begin{tabular}{|c|c|c|c|}
\hline Atom & $x$ & $y$ & $z$ \\
\hline$C$ & 1.600631 & 1.860205 & 0.351046 \\
\hline C & 0.336547 & 1.733338 & 1.217099 \\
\hline 0 & -0.319226 & 0.476945 & 0.966628 \\
\hline C & 0.459982 & -0.649529 & 1.220476 \\
\hline C & 1.759699 & -0.638050 & 0.404158 \\
\hline C & 2.505225 & 0.668171 & 0.689075 \\
\hline $\mathrm{H}$ & 0.658386 & 1.760122 & 2.270979 \\
\hline C & -0.715595 & 2.821797 & 1.069586 \\
\hline 0 & -0.360442 & -1.802029 & 0.942784 \\
\hline $\mathrm{H}$ & 0.682949 & -0.769707 & 2.290078 \\
\hline $\mathrm{N}$ & 2.551150 & -1.813137 & 0.808679 \\
\hline $\mathrm{H}$ & 1.511698 & -0.654296 & -0.659087 \\
\hline $\mathrm{H}$ & 2.837764 & 0.705185 & 1.733203 \\
\hline 0 & -1.222866 & 2.880358 & -0.243507 \\
\hline $\mathrm{H}$ & -0.266179 & 3.782529 & 1.372141 \\
\hline $\mathrm{H}$ & -1.518037 & 2.591959 & 1.788506 \\
\hline$S$ & -1.224174 & -1.927621 & -0.437515 \\
\hline C & -2.425021 & 3.626257 & -0.323649 \\
\hline $\mathrm{N}$ & 3.418595 & -2.169539 & 0.003183 \\
\hline $\mathrm{N}$ & 4.242363 & -2.608747 & -0.651007 \\
\hline $\mathrm{O}$ & -0.520132 & -1.357307 & -1.577973 \\
\hline $\mathrm{O}$ & -1.694413 & -3.303414 & -0.430792 \\
\hline C & -2.742279 & -0.841318 & -0.085239 \\
\hline $\mathrm{F}$ & -2.588108 & 0.352989 & -0.631796 \\
\hline $\mathrm{F}$ & -2.931471 & -0.735923 & 1.225686 \\
\hline $\mathrm{F}$ & -3.783666 & -1.460277 & -0.642385 \\
\hline $\mathrm{H}$ & -2.731654 & 3.627582 & -1.371836 \\
\hline $\mathrm{H}$ & -2.287862 & 4.666486 & 0.009109 \\
\hline $\mathrm{H}$ & -3.224001 & 3.170235 & 0.280721 \\
\hline $\mathrm{H}$ & 2.111273 & 2.795398 & 0.619939 \\
\hline $\mathrm{F}$ & 3.645604 & 0.682050 & -0.117366 \\
\hline 0 & 1.271518 & 1.822744 & -1.021871 \\
\hline$C$ & 2.082569 & 2.654839 & -1.845941 \\
\hline $\mathrm{H}$ & 3.140868 & 2.380902 & -1.779867 \\
\hline $\mathrm{H}$ & 1.734836 & 2.509132 & -2.870604 \\
\hline \multirow[t]{3}{*}{$\mathrm{H}$} & 1.964687 & 3.714189 & -1.578091 \\
\hline & $E$ & $G$ & \\
\hline & -1763.796232 & -1763.578098 & \\
\hline
\end{tabular}


NMR $\alpha-7$

${ }^{1} \mathrm{HNMR} 400 \mathrm{MHz}, \mathrm{CDCl}_{3} \boldsymbol{\alpha}-\mathbf{7}$

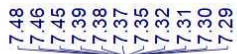

โค่์

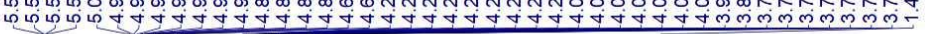

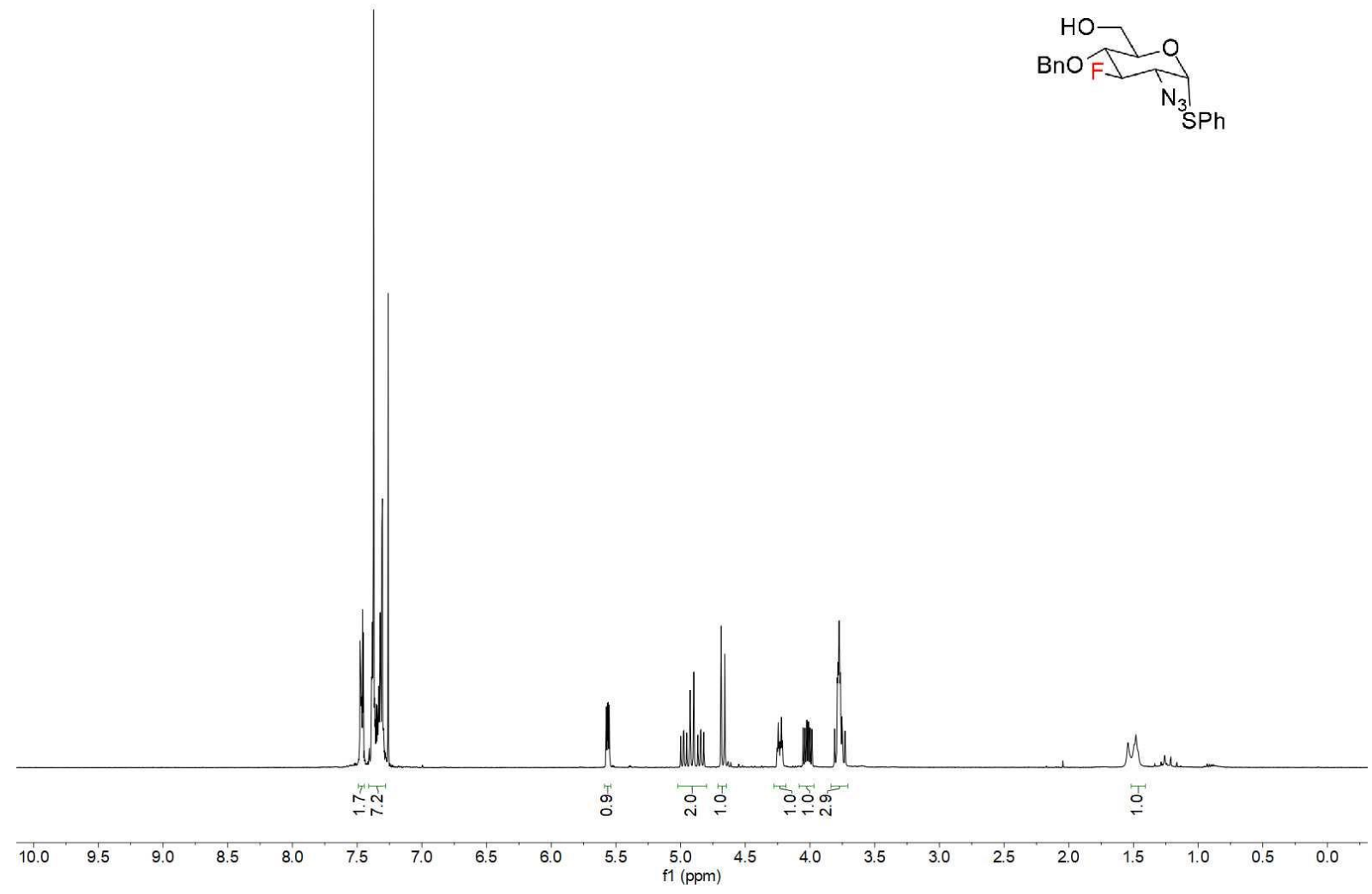

${ }^{13} \mathrm{C}\left\{{ }^{1} \mathrm{H}\right\}$ APT NMR $100 \mathrm{MHz}, \mathrm{CDCl}_{3} \boldsymbol{\alpha}-\mathbf{7}$

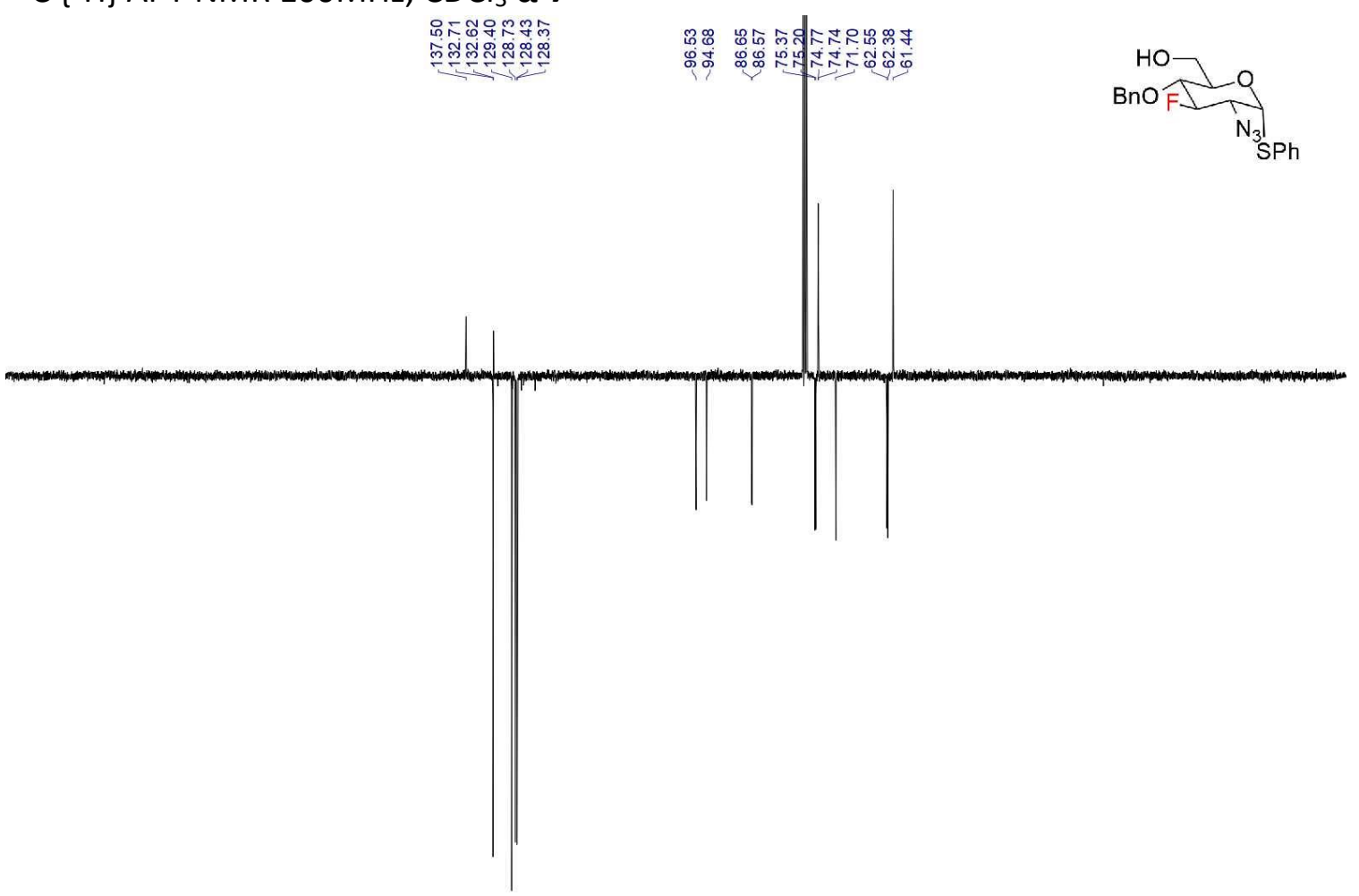

$\begin{array}{llllllllllllllllllllllllll}210 & 200 & 190 & 180 & 170 & 160 & 150 & 140 & 130 & 120 & 110 & \begin{array}{c}100 \\ \mathrm{f} 1(\mathrm{ppm})\end{array} & 90 & 80 & 70 & 60 & 50 & 40 & 30 & 20 & 10 & 0 & -10\end{array}$ 
${ }^{1} \mathrm{H}-{ }^{1} \mathrm{H}$ COSY NMR $\boldsymbol{\alpha - 7}$

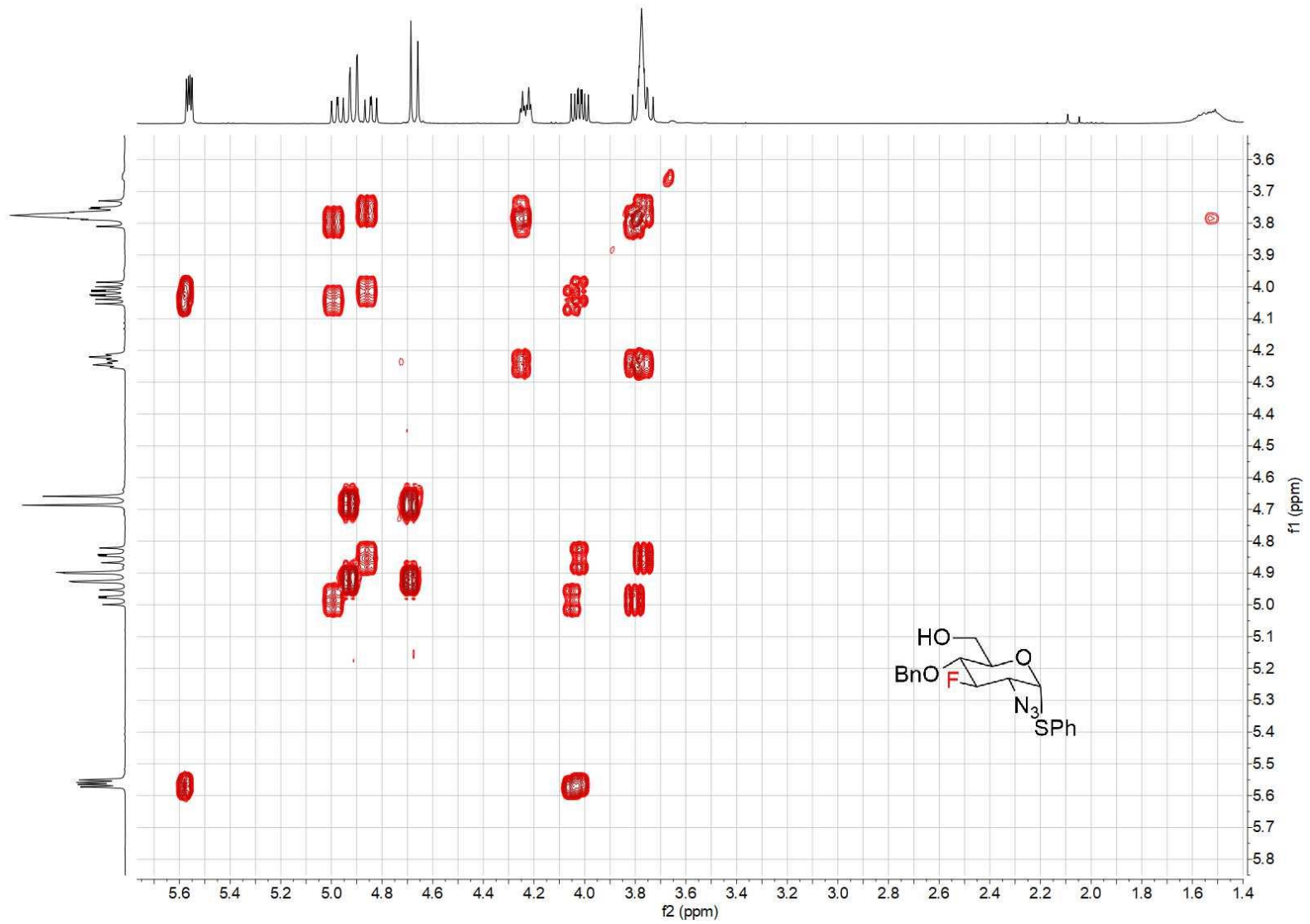

${ }^{1} \mathrm{H}^{13} \mathrm{C}$ HSQC NMR $\alpha-7$

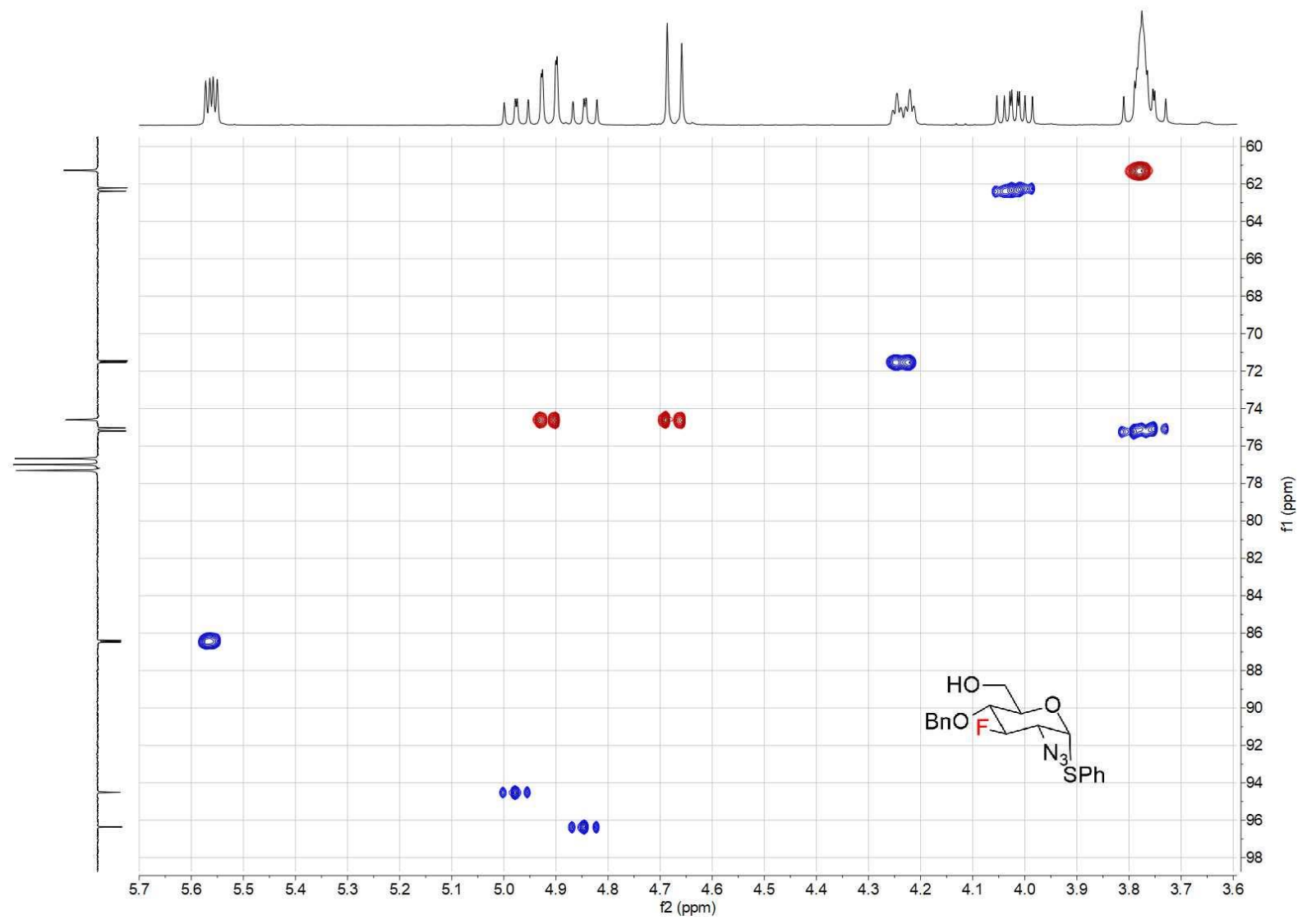


${ }^{19} \mathrm{~F} \mathrm{NMR} 376 \mathrm{MHz}, \mathrm{CDCl}_{3} \boldsymbol{\alpha}-\mathbf{7}$

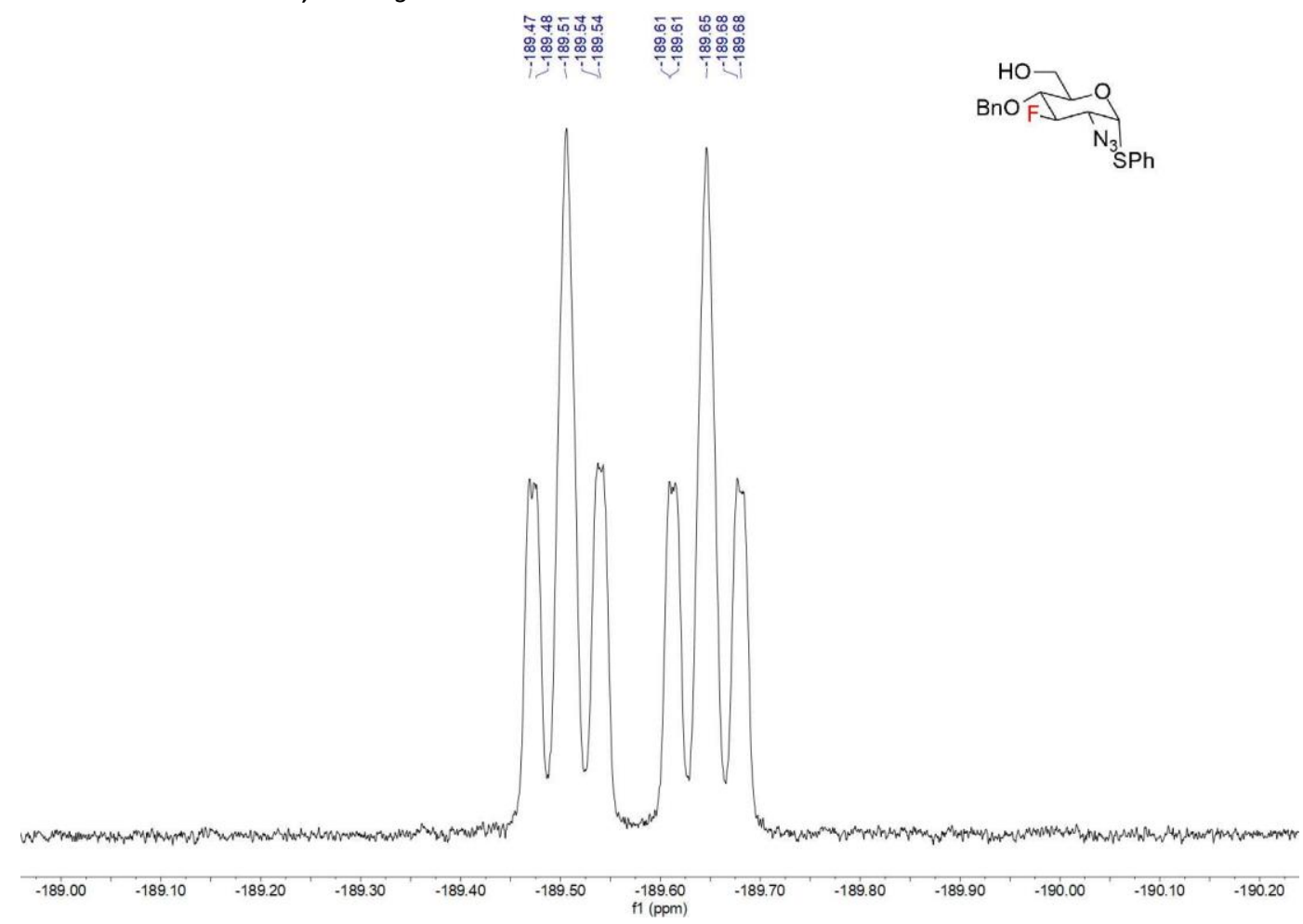

NMR $\beta$-7

${ }^{1} \mathrm{H} \mathrm{NMR} 400 \mathrm{MHz}, \mathrm{CDCl}_{3} \boldsymbol{\beta}-7$

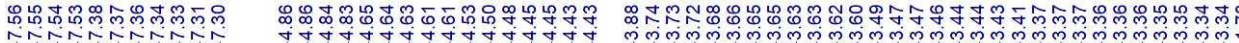

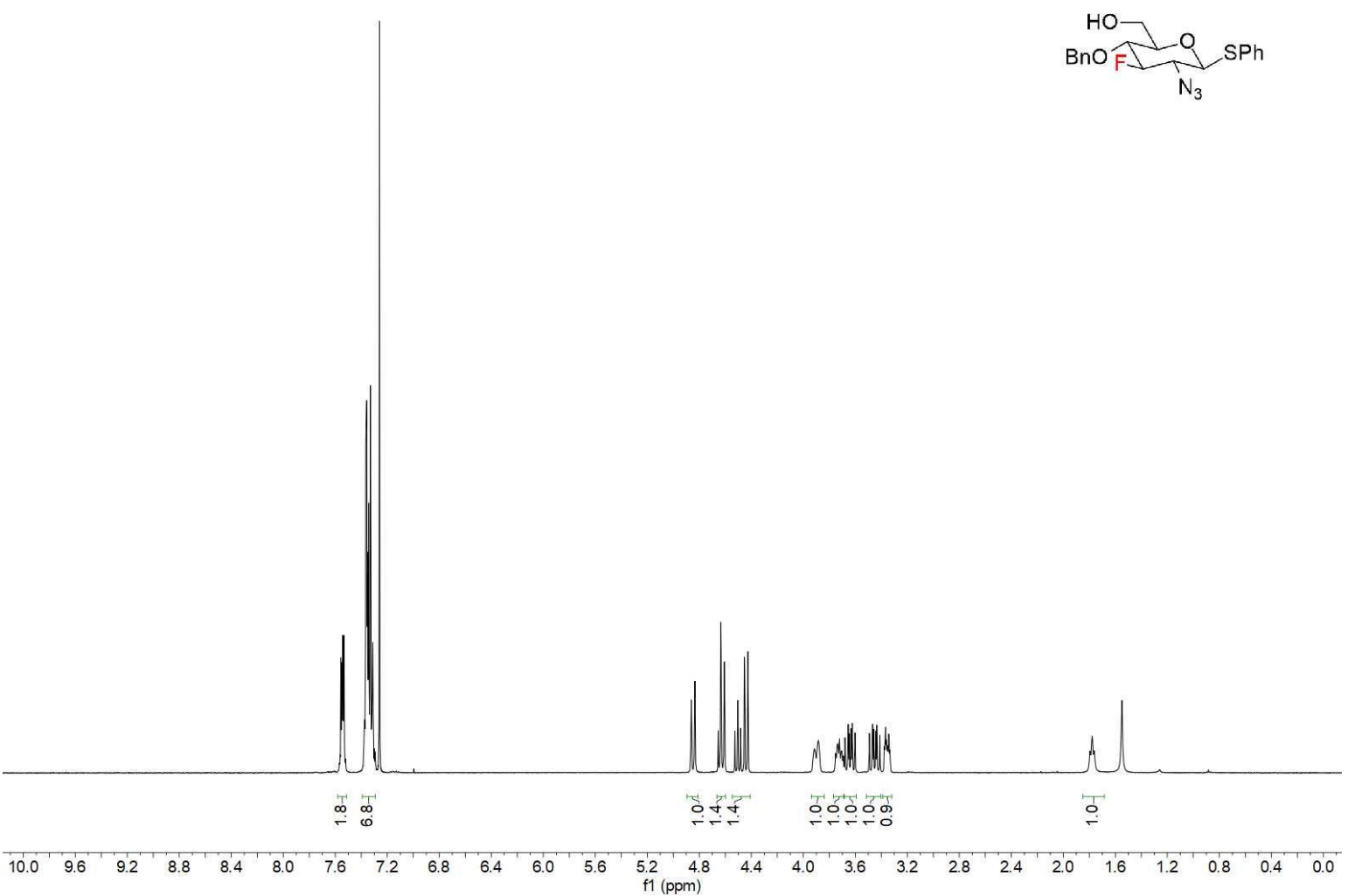




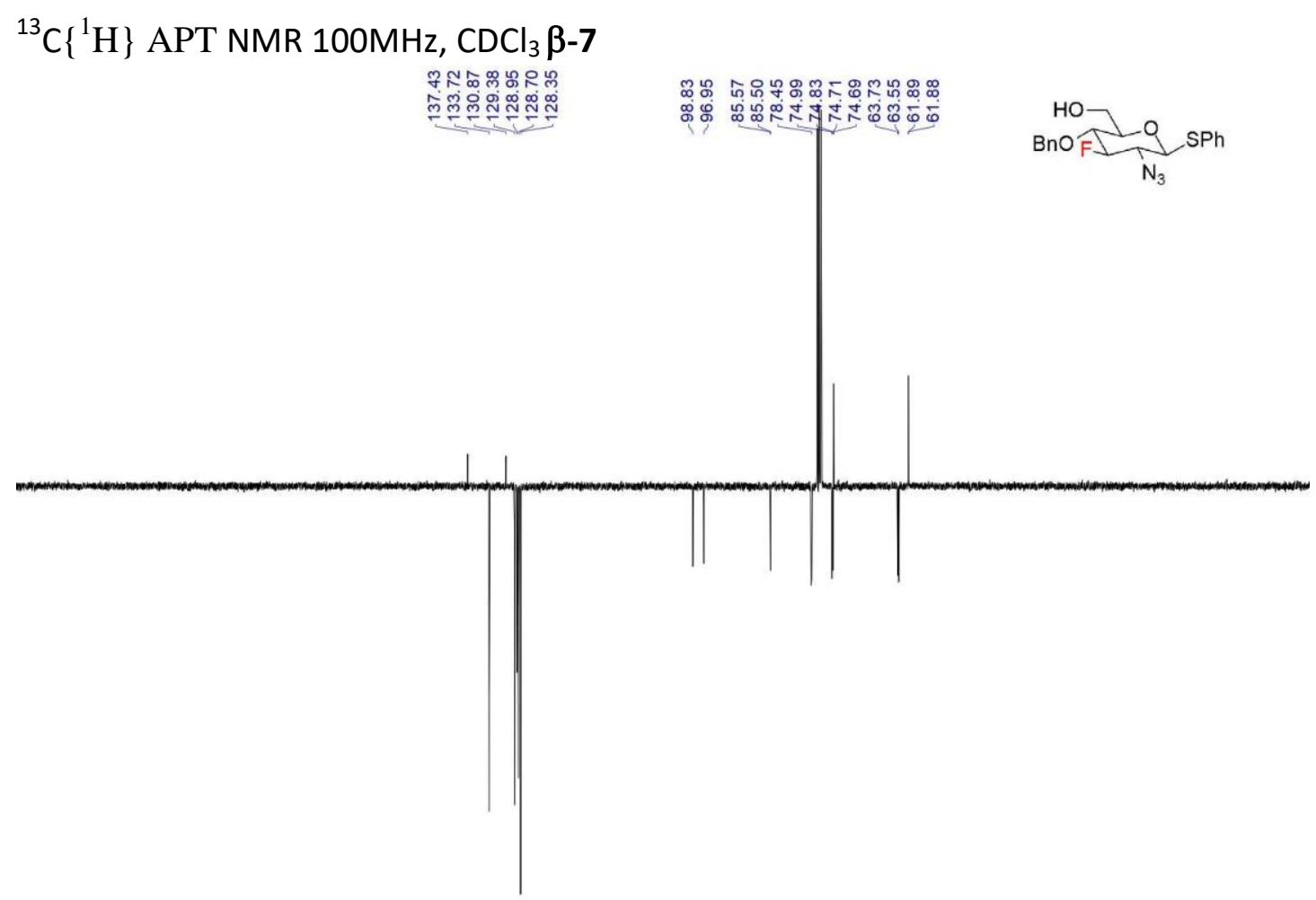

${ }^{1} \mathrm{H}-{ }^{1} \mathrm{H}$ COSY NMR $\beta-7$

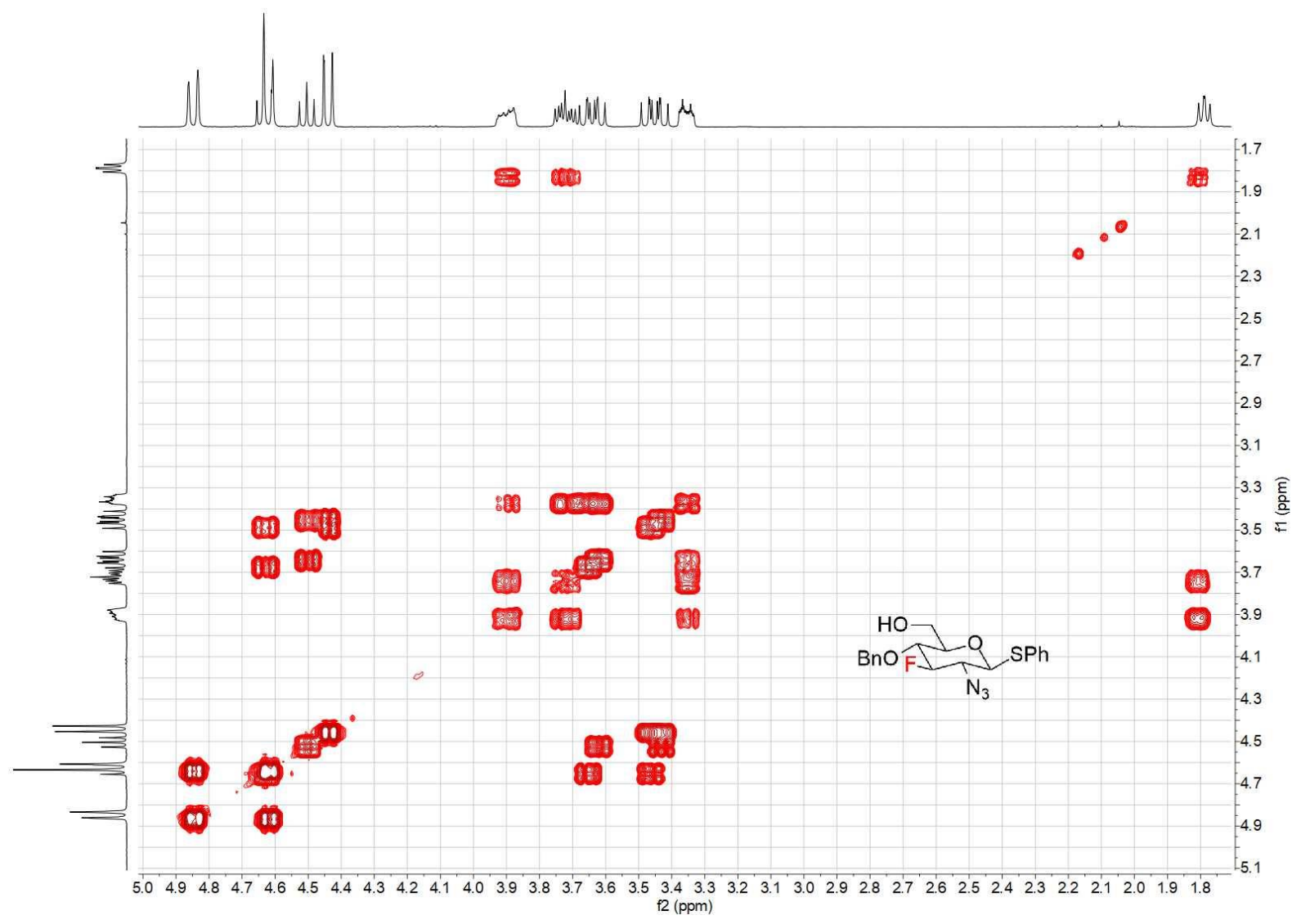




\section{${ }^{1} \mathrm{H}^{13} \mathrm{C}$ HSQC NMR $\beta$-7}

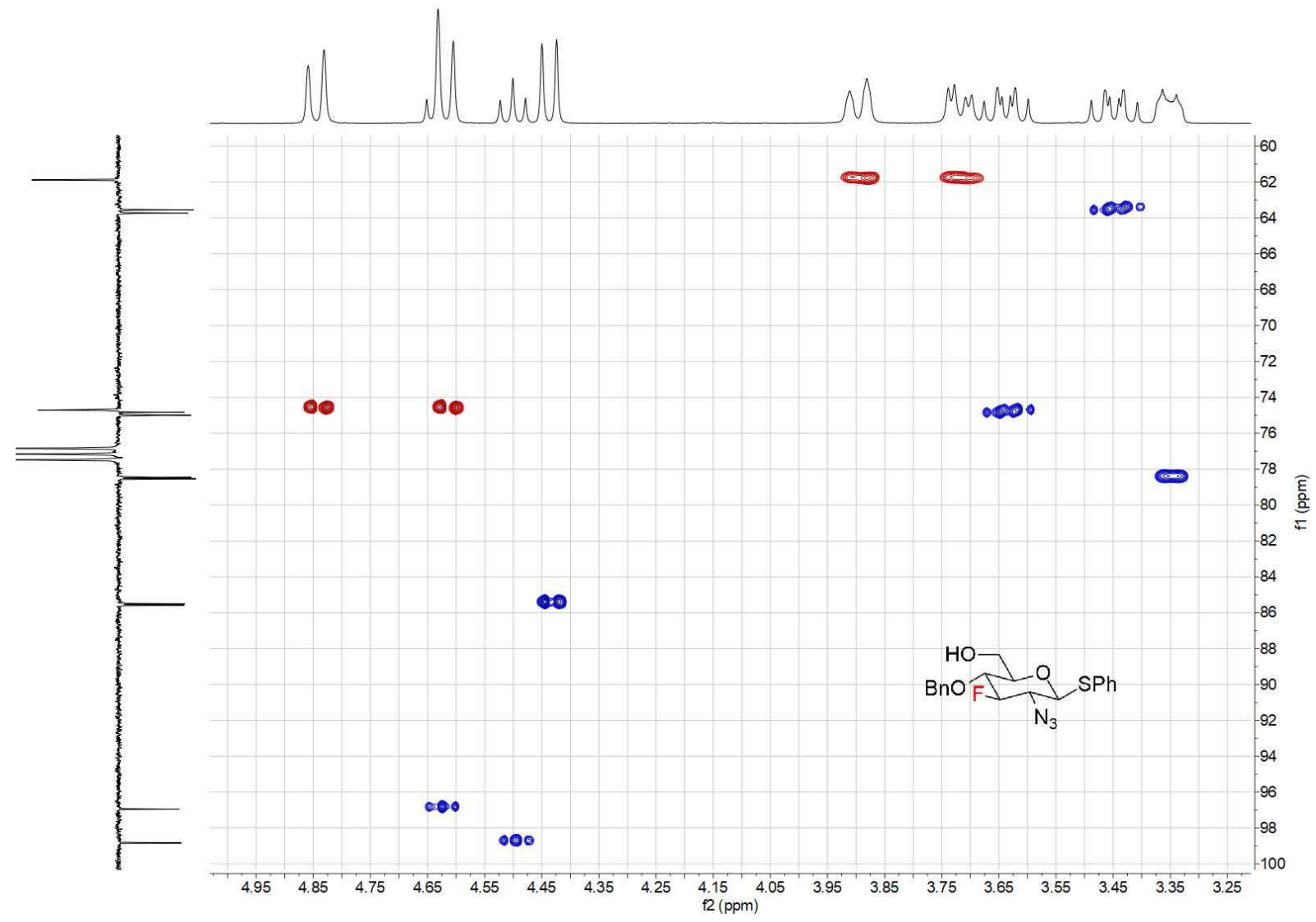

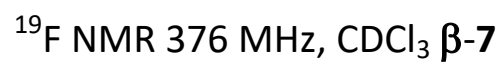

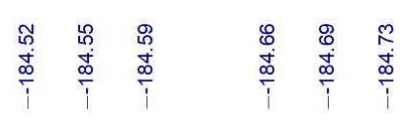
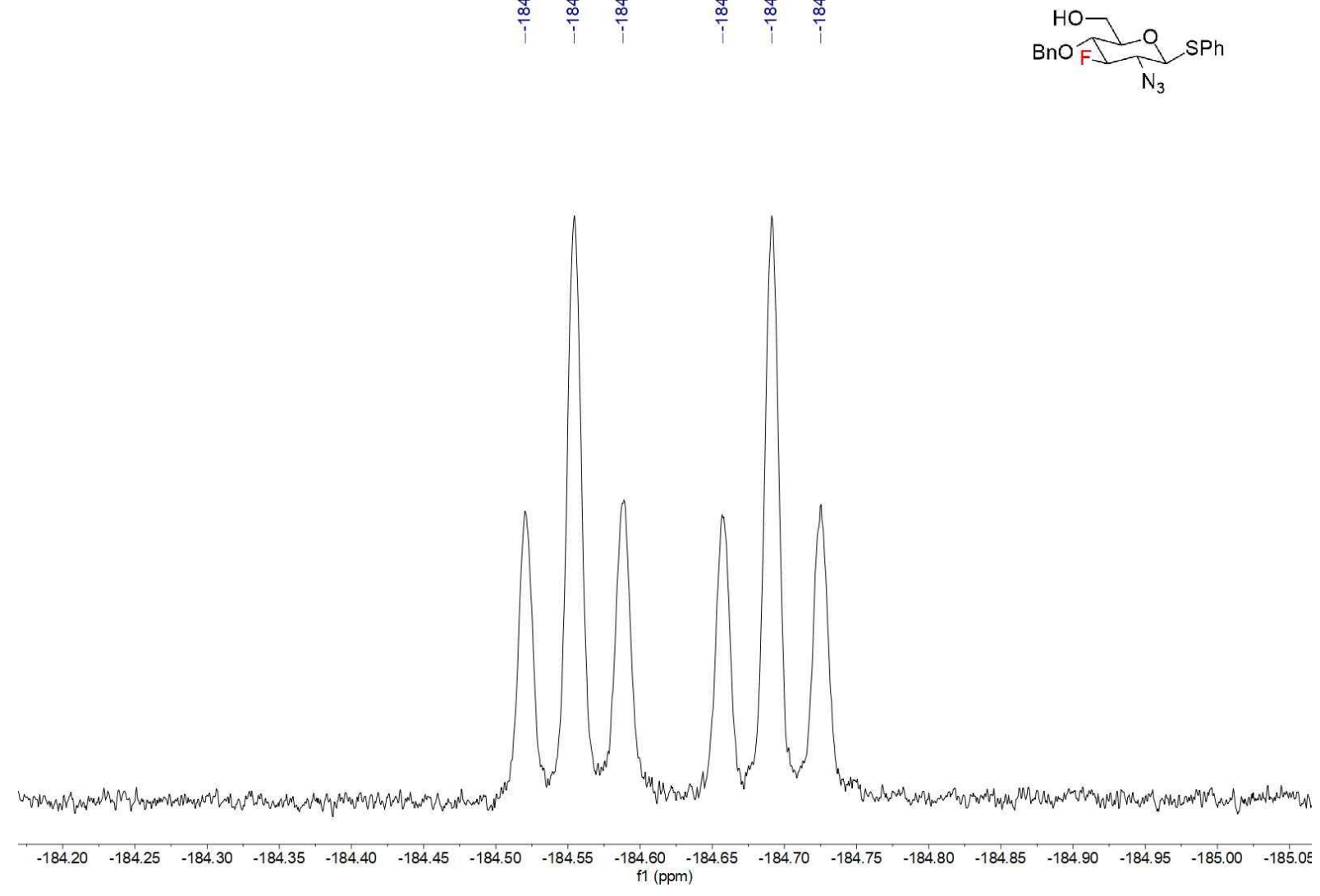
NMR $\alpha-1$

${ }^{1} \mathrm{HNMR} 400 \mathrm{MHz}, \mathrm{CDCl}_{3} \boldsymbol{\alpha}-1$

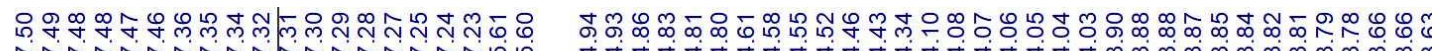

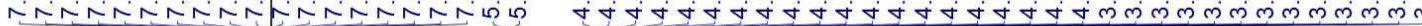

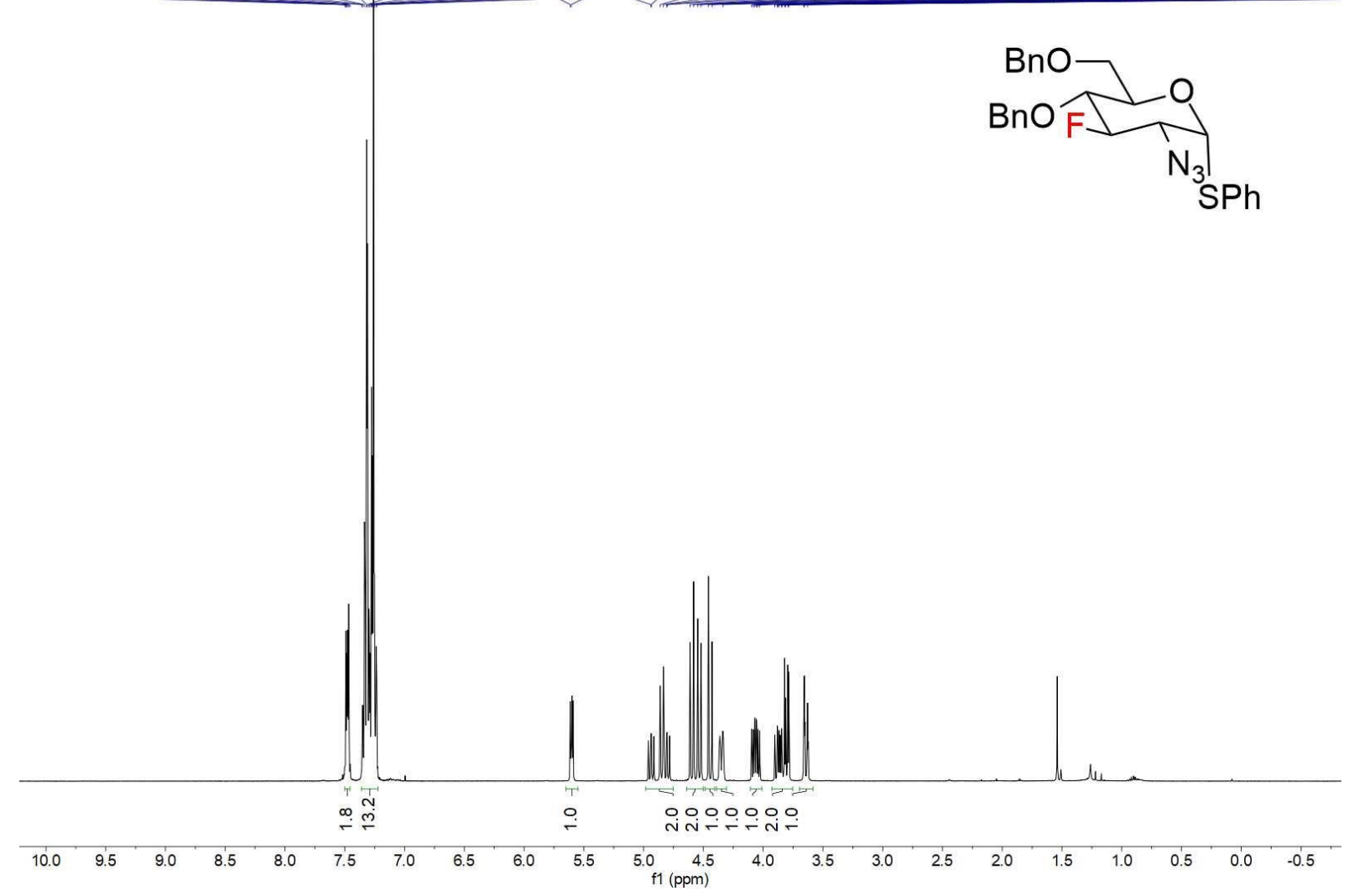

${ }^{13} \mathrm{C}\left\{{ }^{1} \mathrm{H}\right\}$ APT NMR 100MHz, $\mathrm{CDCl}_{3} \boldsymbol{\alpha}-1$

๘

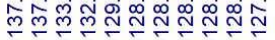

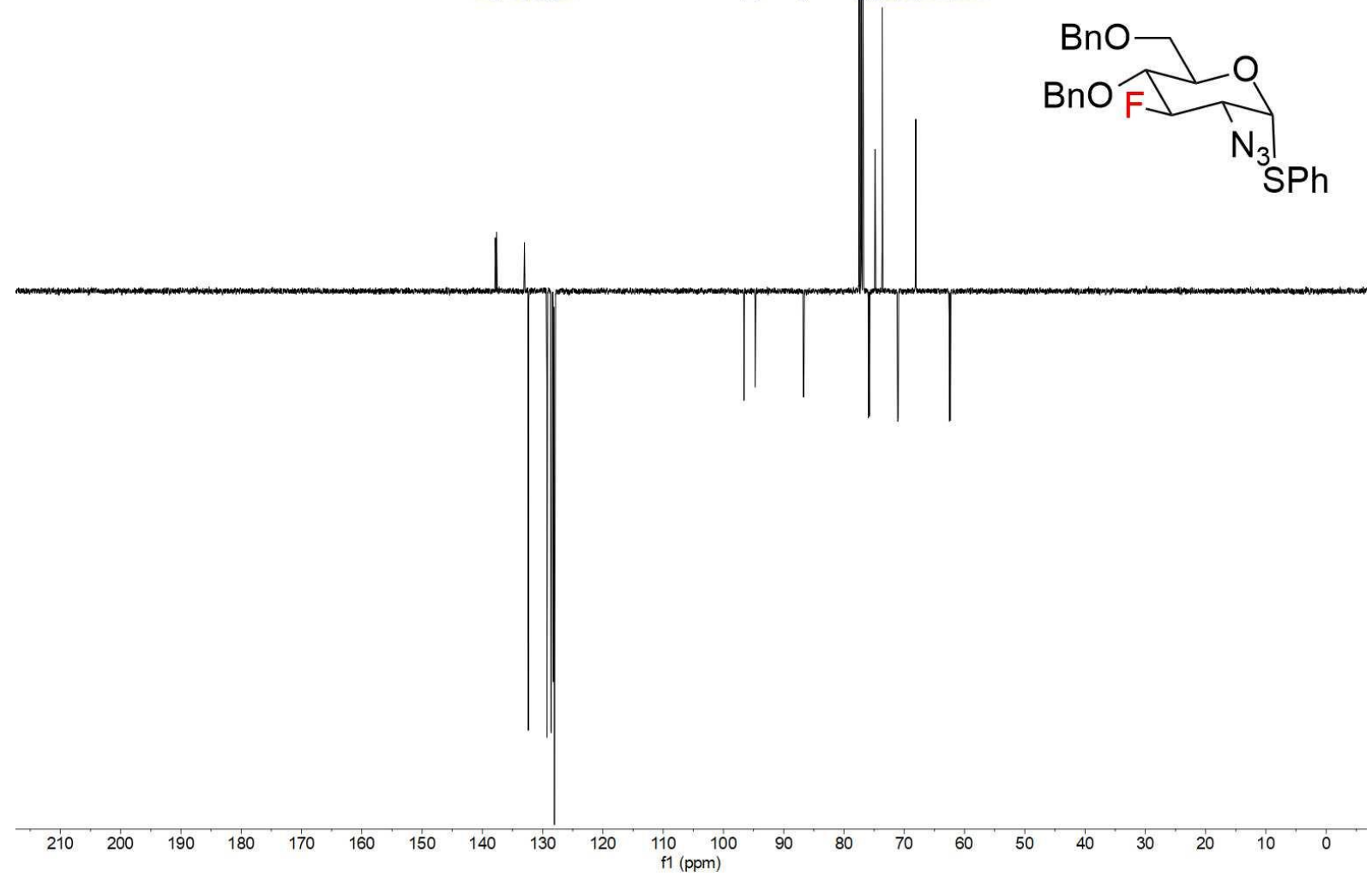


${ }^{1} \mathrm{H}-{ }^{1} \mathrm{H}$ COSY NMR $\boldsymbol{\alpha - 1}$

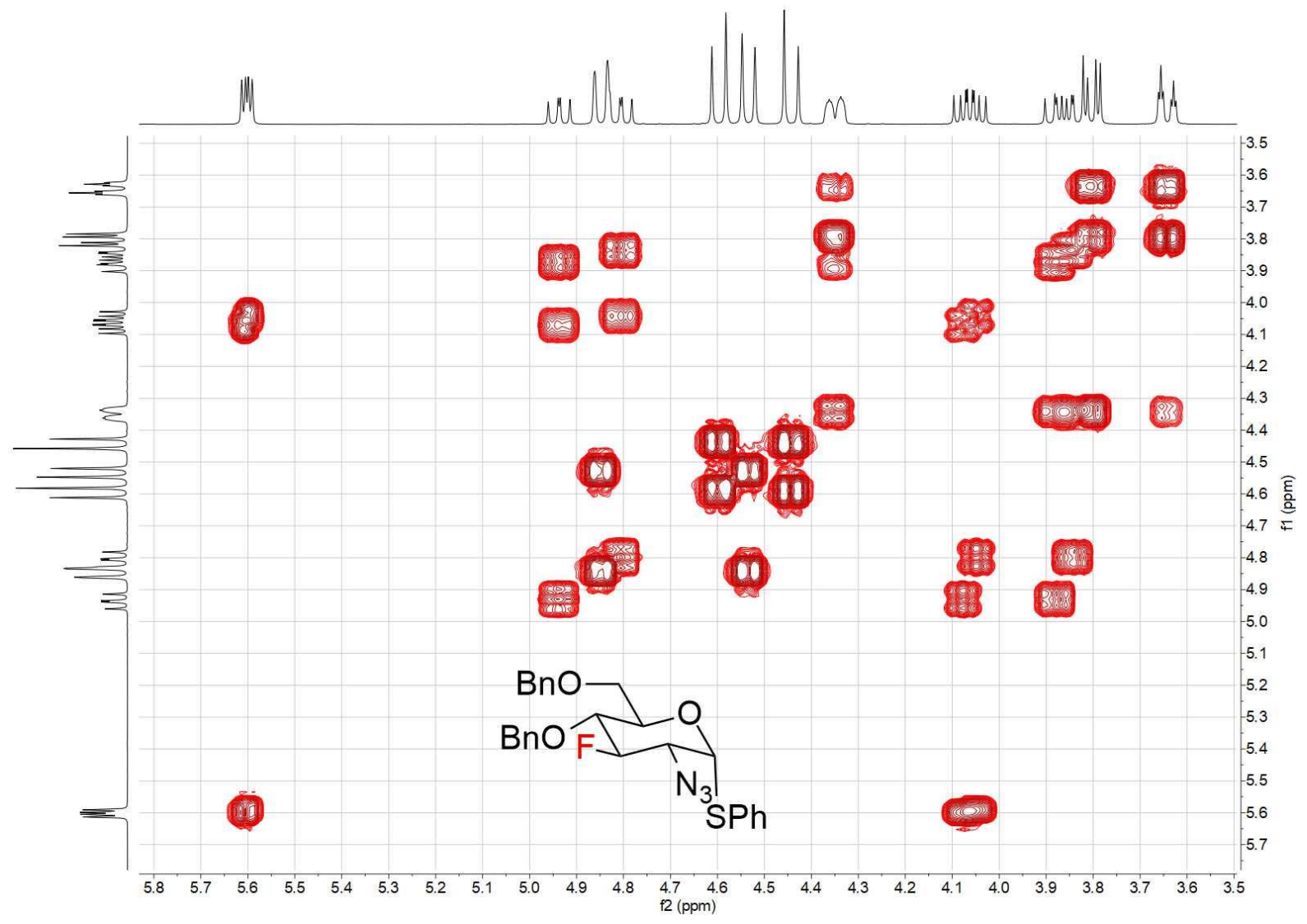

${ }^{1} \mathrm{H}-{ }^{13} \mathrm{C}$ HSQC NMR $\boldsymbol{\alpha}-\mathbf{1}$

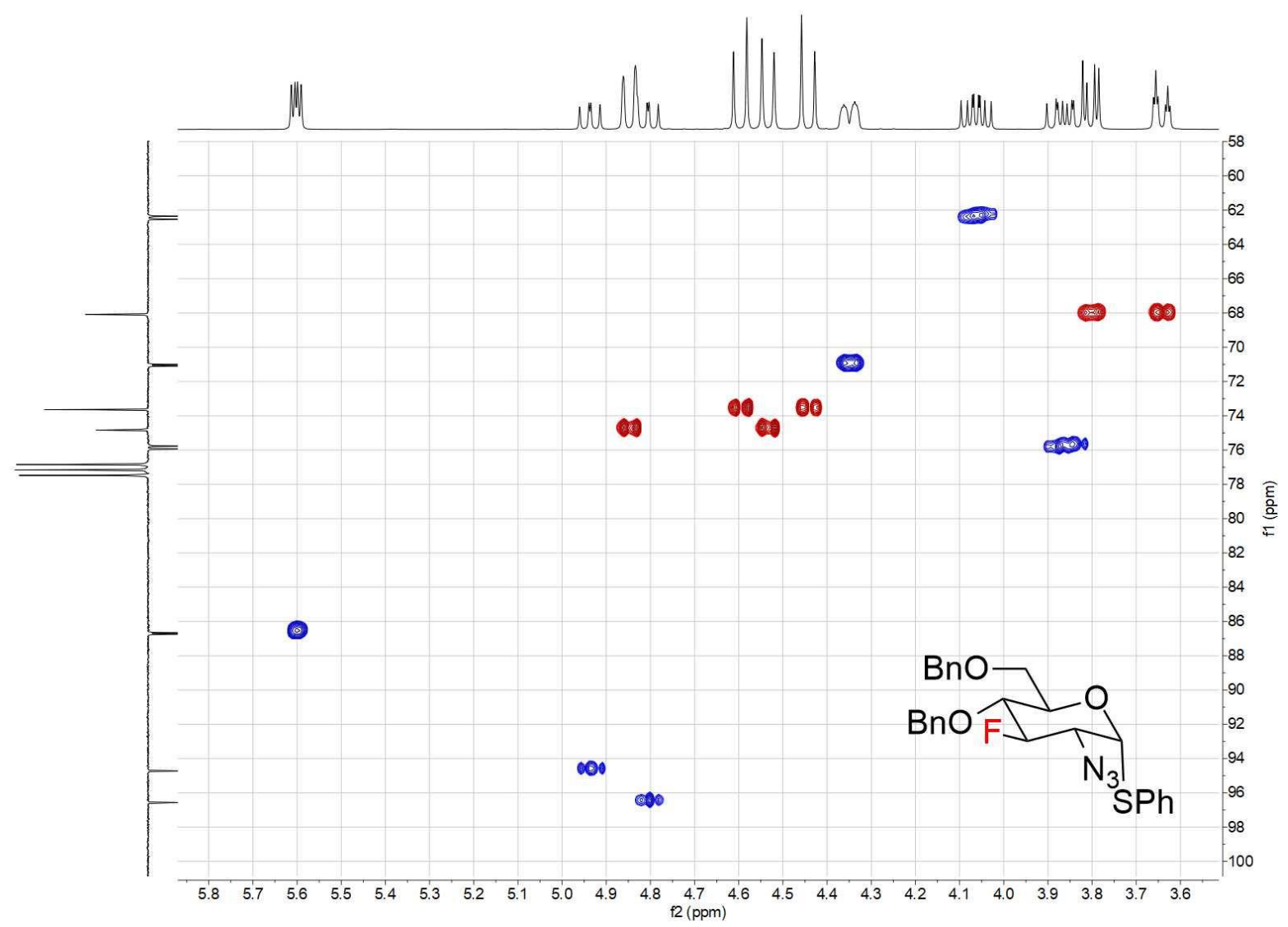




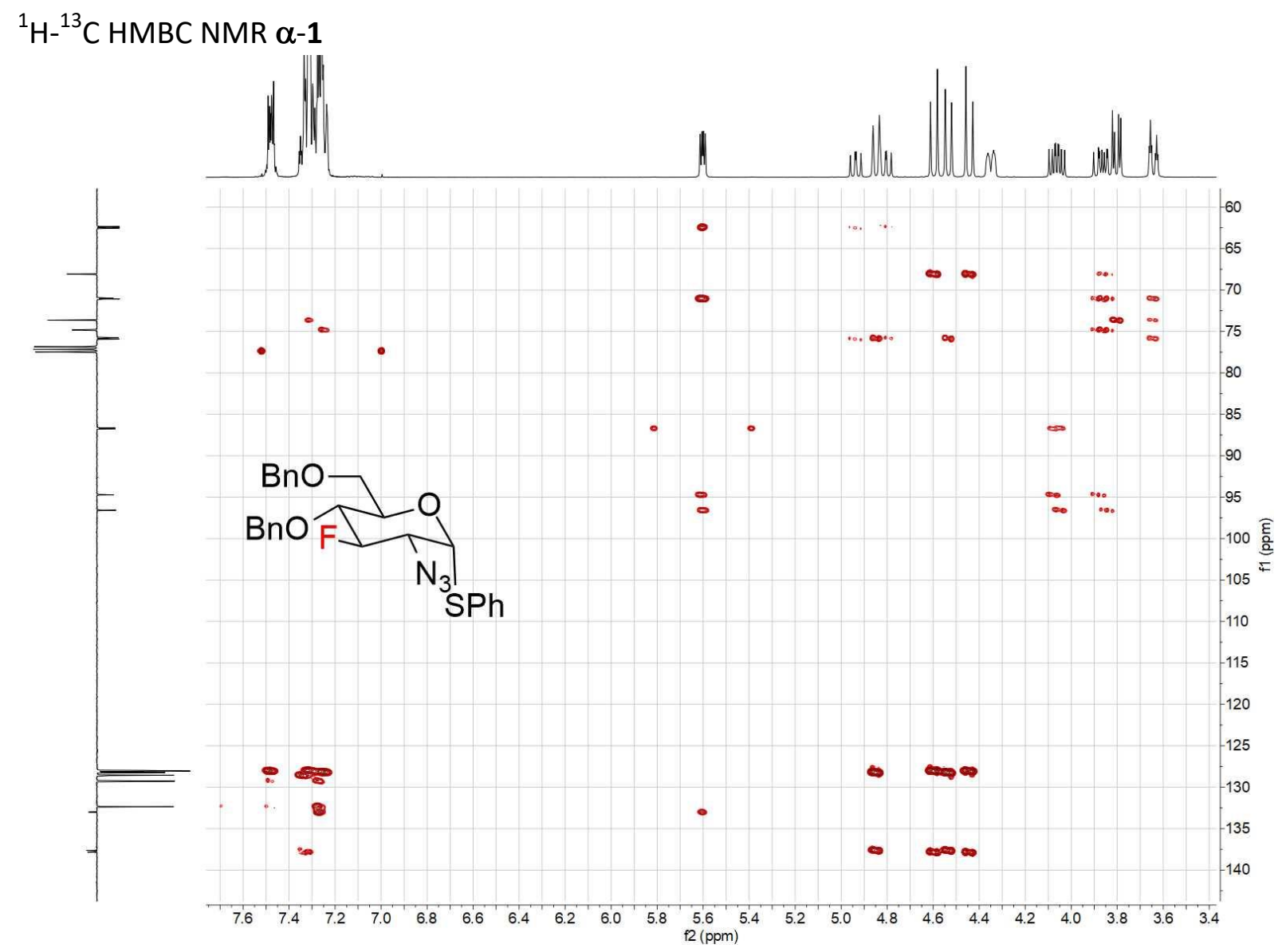

${ }^{19} \mathrm{~F} \mathrm{NMR} 376 \mathrm{MHz}, \mathrm{CDCl}_{3} \boldsymbol{\alpha}-\mathbf{1}$

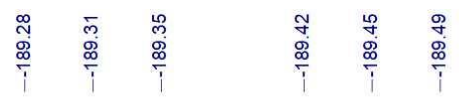
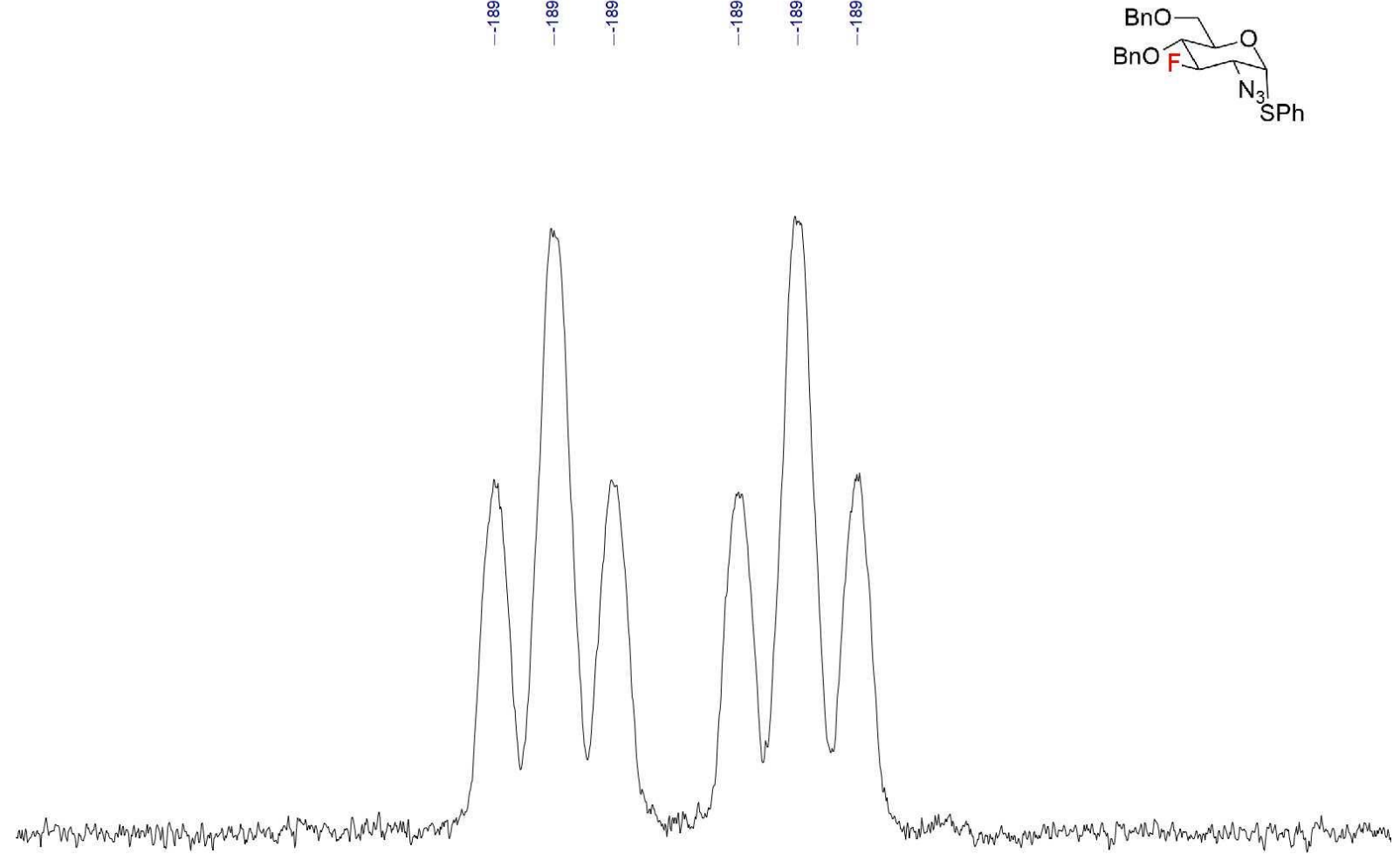
NMR $\beta-1$

${ }^{1} \mathrm{HNMR} 400 \mathrm{MHz}, \mathrm{CDCl}_{3} \boldsymbol{\beta}-1$

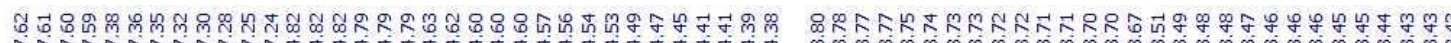

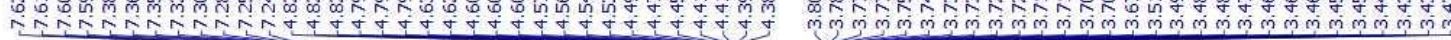

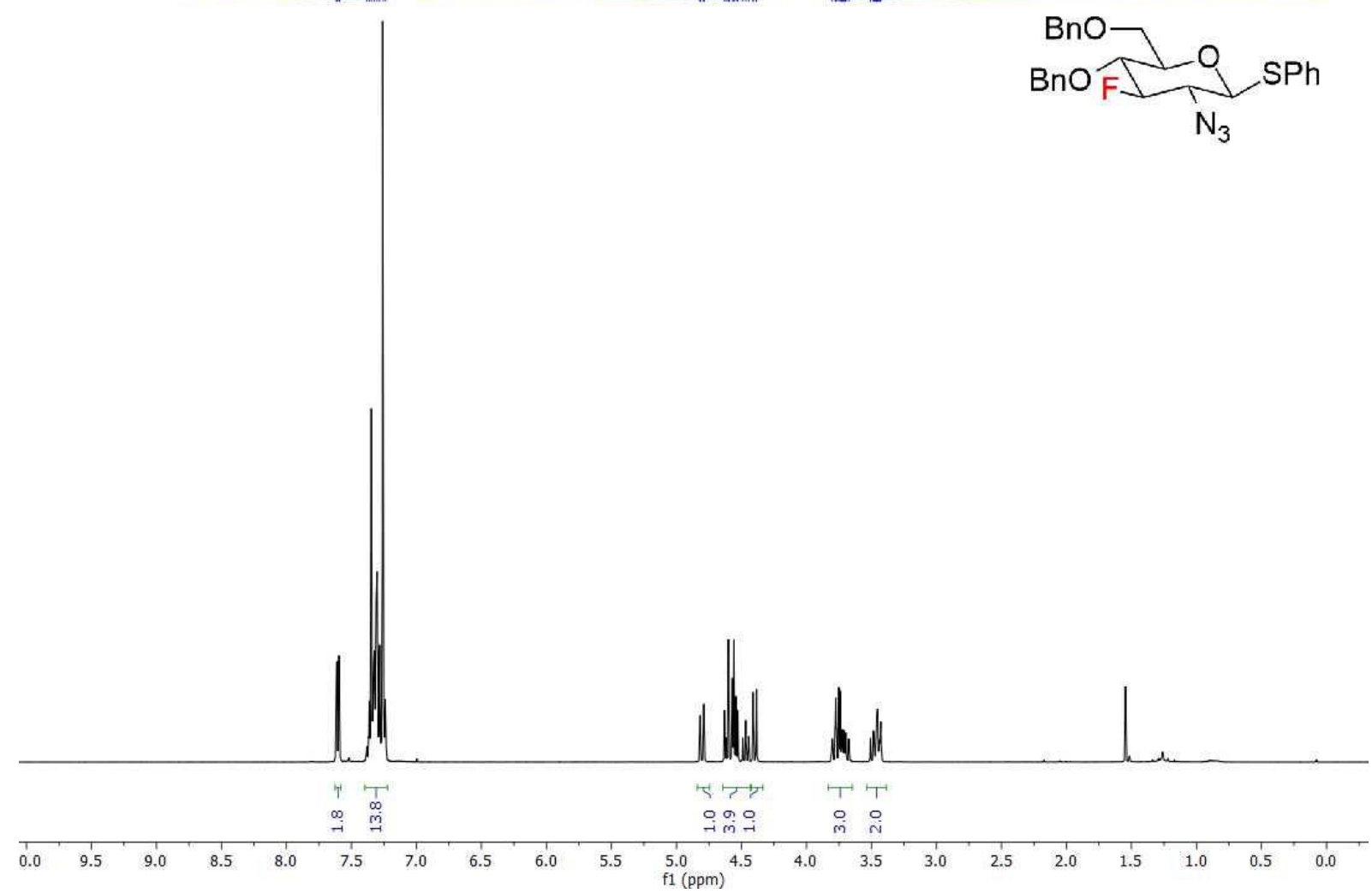

${ }^{13} \mathrm{C}\left\{{ }^{1} \mathrm{H}\right\}$ APT NMR $100 \mathrm{MHz}, \mathrm{CDCl}_{3} \beta-1$

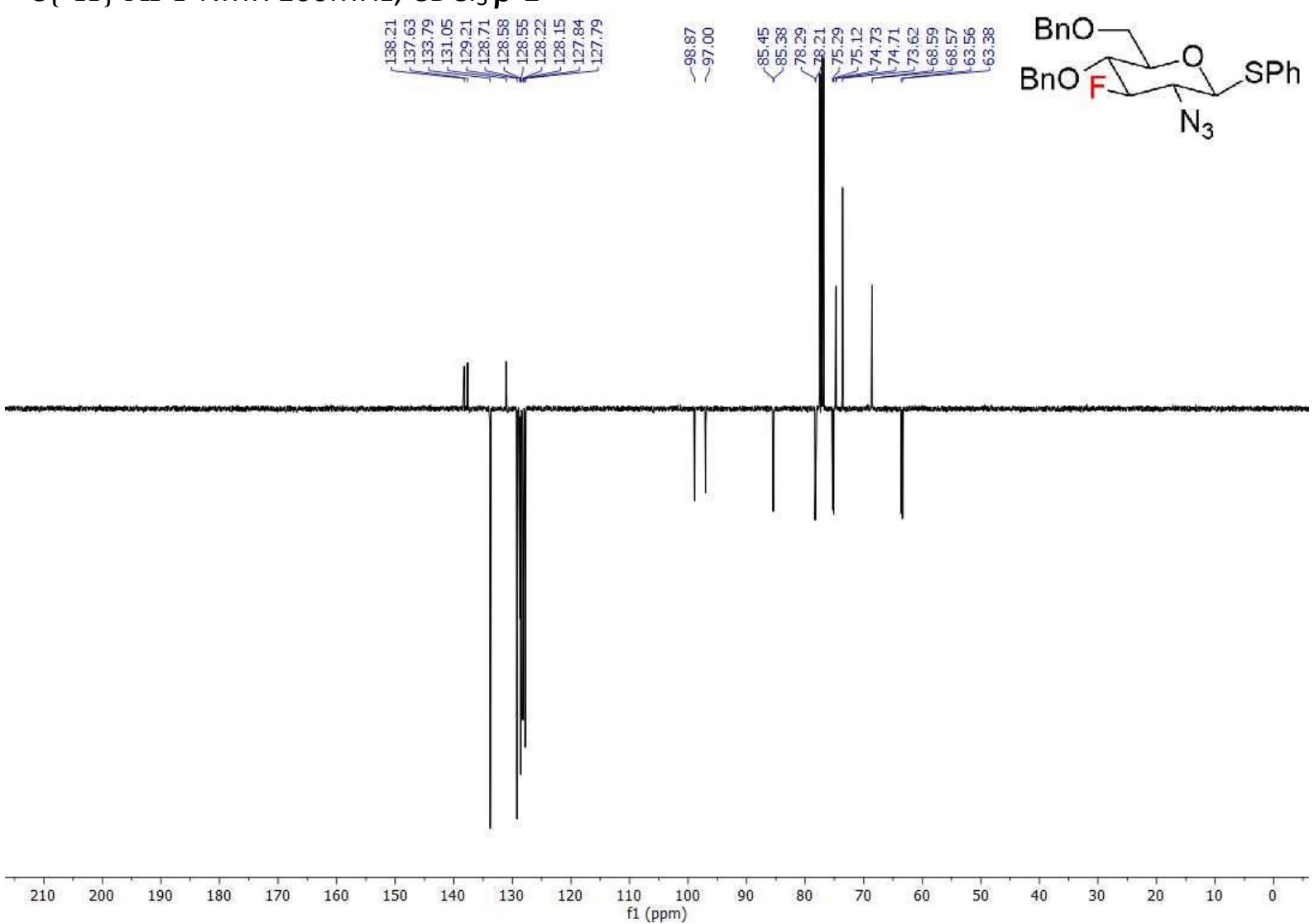


${ }^{1} \mathrm{H}-{ }^{1} \mathrm{H}$ COSY NMR $\boldsymbol{\beta}-1$

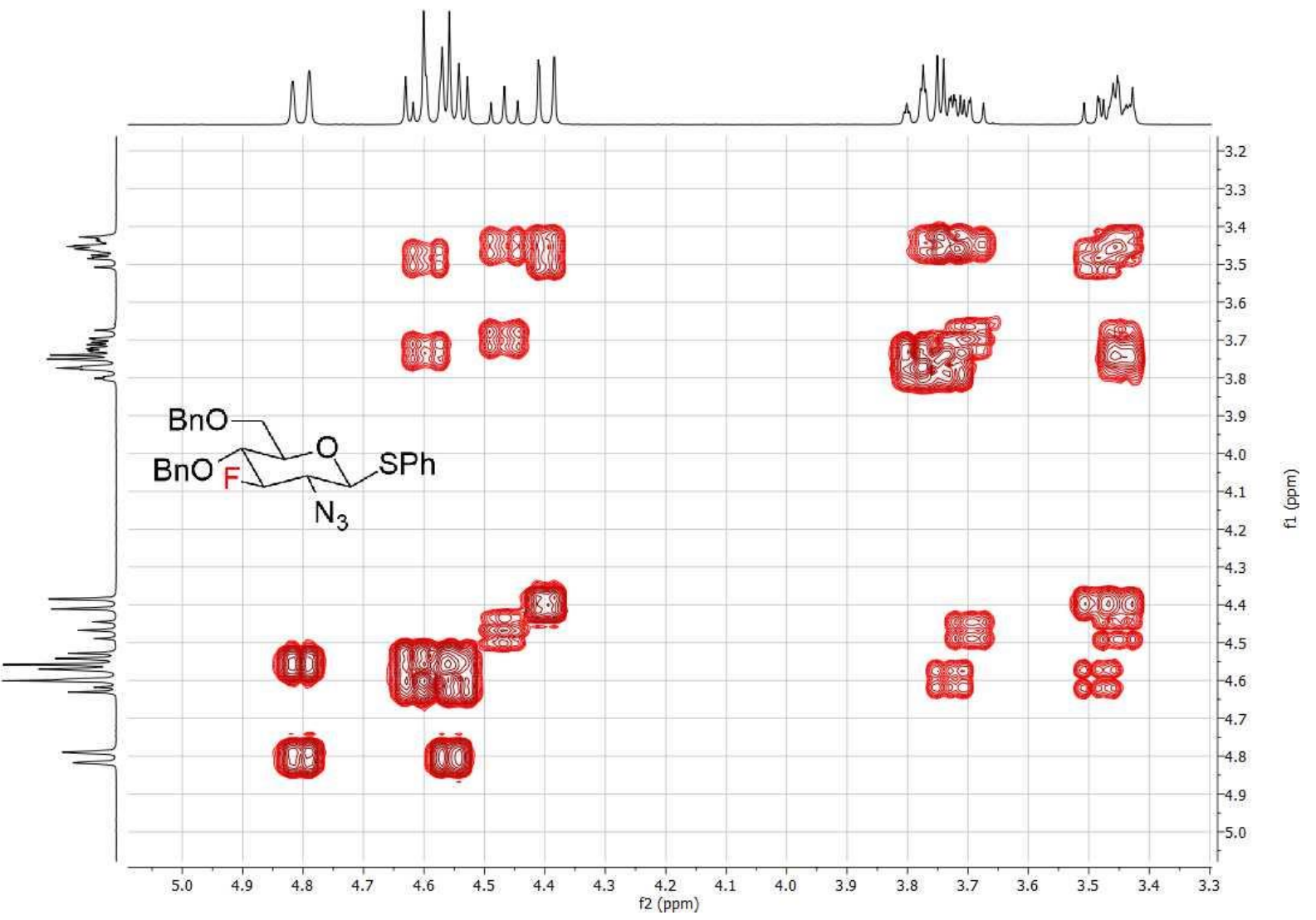

${ }^{1} \mathrm{H}-{ }^{13} \mathrm{C}$ HSQC NMR $\boldsymbol{\beta}-1$

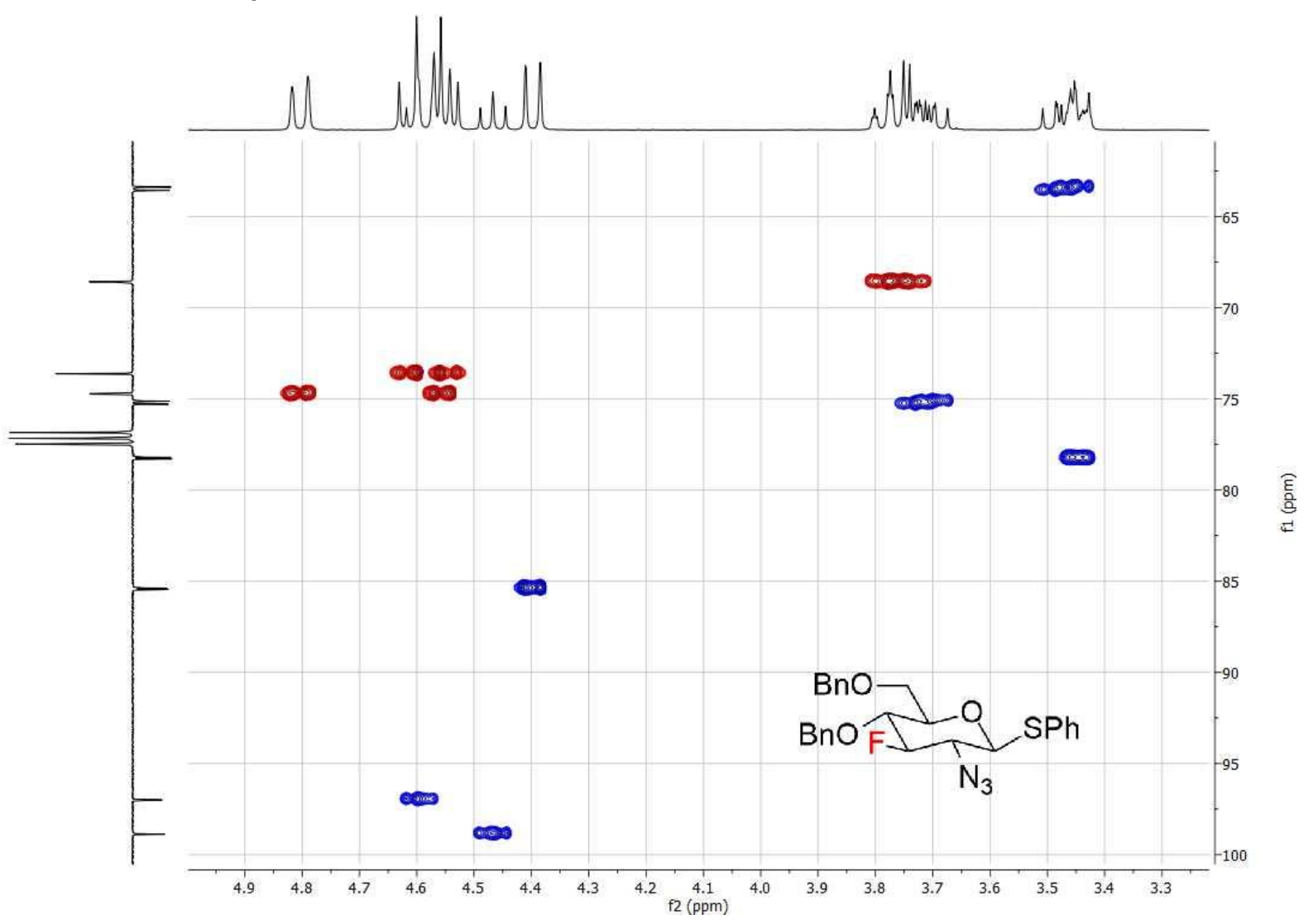

S36 
${ }^{1} \mathrm{H}-{ }^{13} \mathrm{CHMBC}$ NMR $\boldsymbol{\beta}-\mathbf{1}$

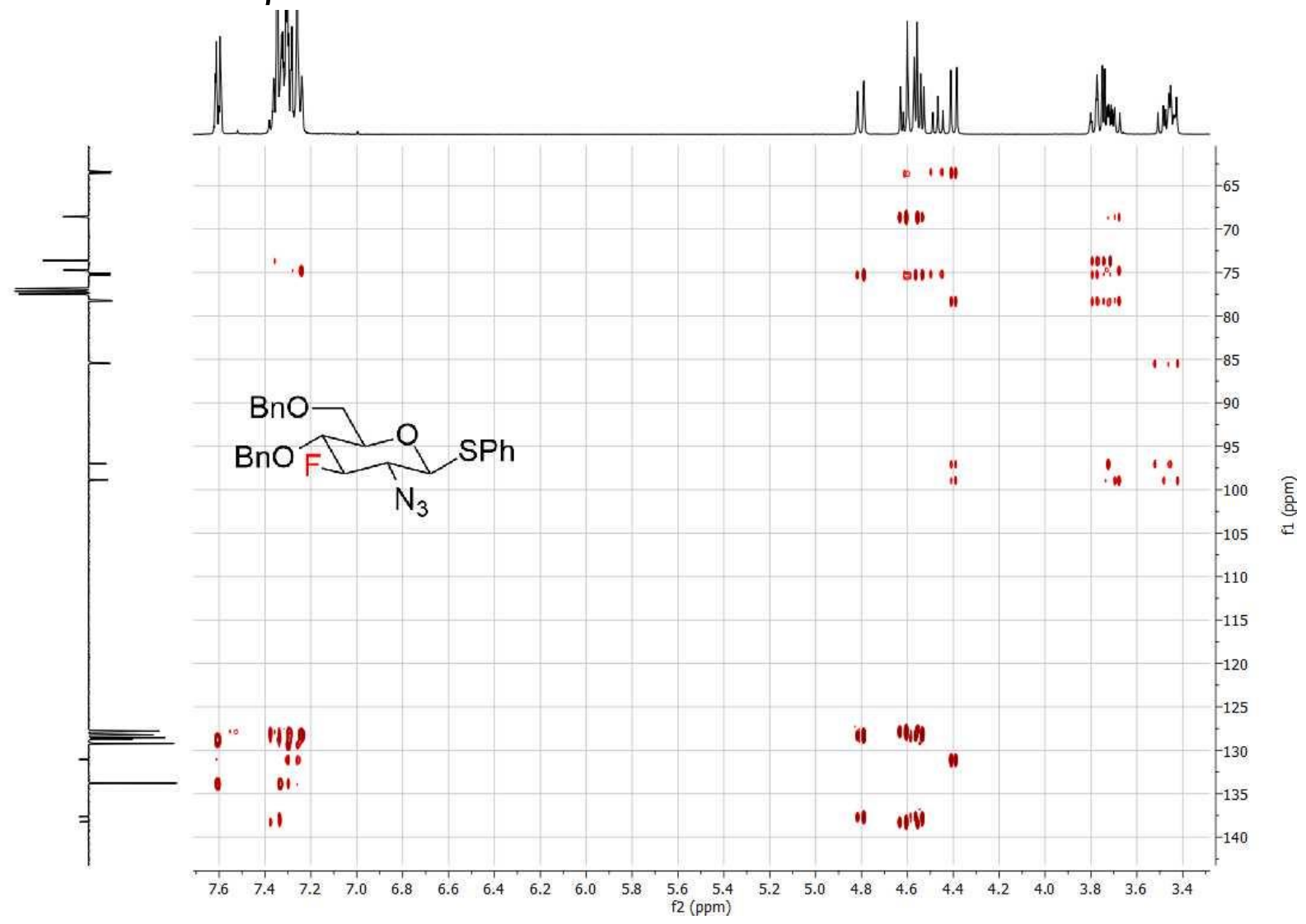

${ }^{19} \mathrm{~F}$ NMR $376 \mathrm{MHz}, \mathrm{CDCl}_{3} \boldsymbol{\beta}-\mathbf{1}$

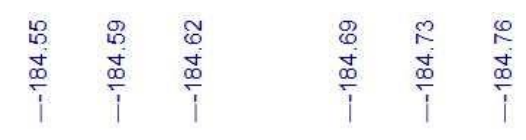
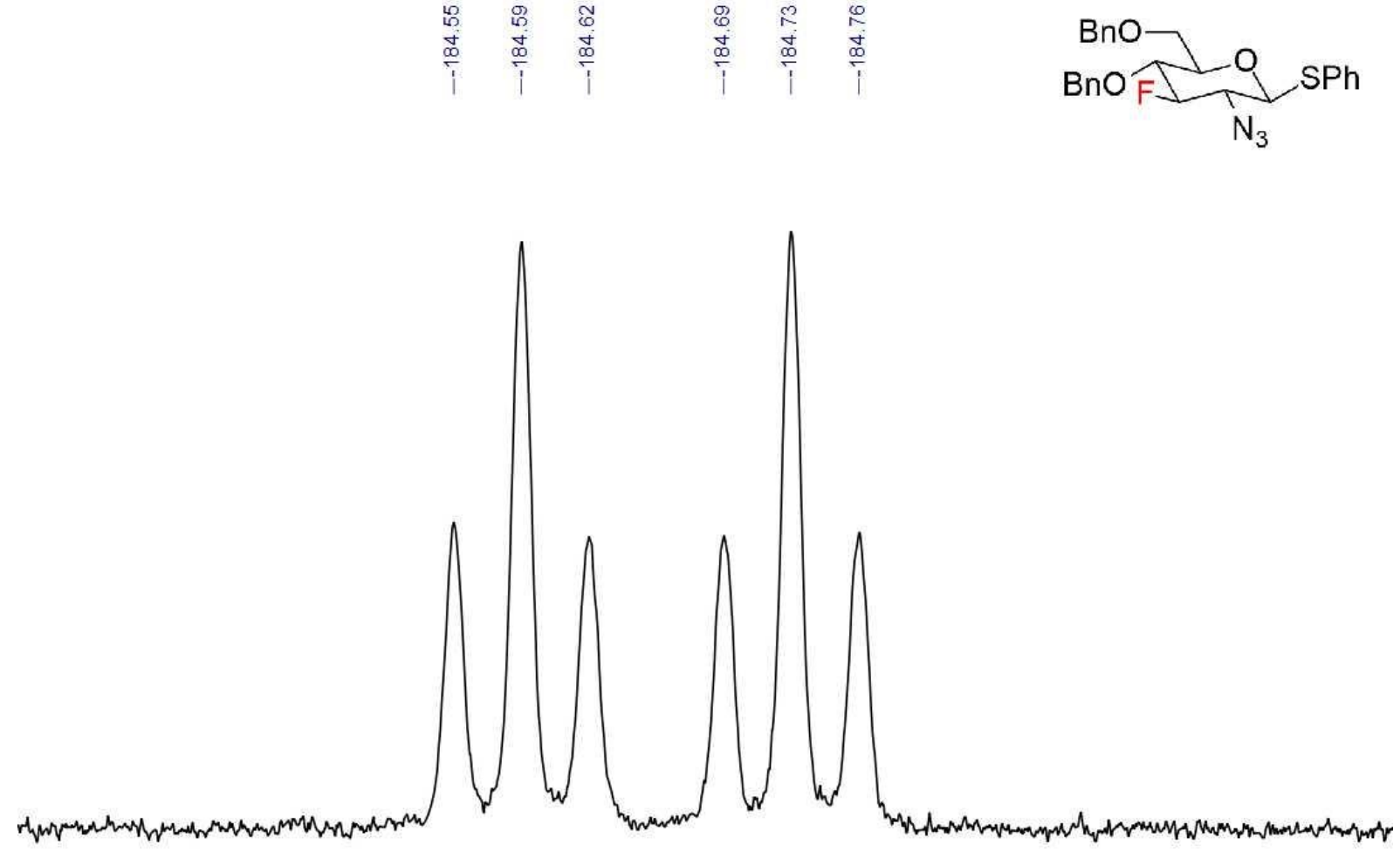

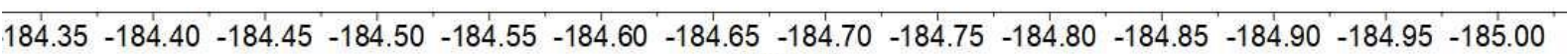
f1 (ppm) 


\section{NMR 8}

${ }^{1} \mathrm{HNMR} 400 \mathrm{MHz}, \mathrm{CDCl}_{3} 8$

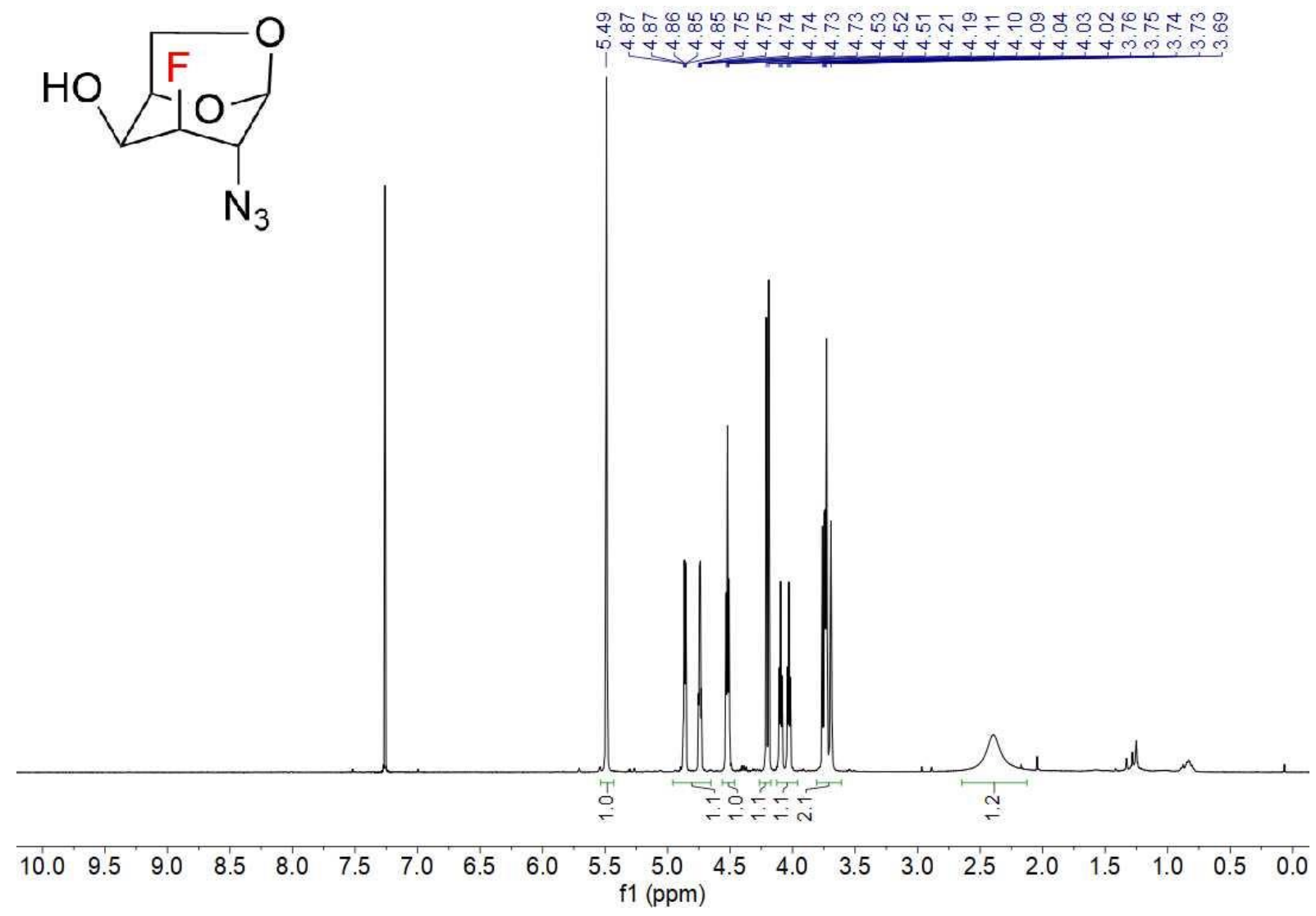

${ }^{13} \mathrm{C}\left\{{ }^{1} \mathrm{H}\right\}$ NMR $100 \mathrm{MHz}, \mathrm{CDCl}_{3} 8$

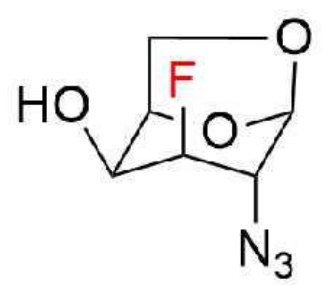


${ }^{19} \mathrm{~F}$ NMR $376 \mathrm{MHz}, \mathrm{CDCl}_{3} 8$<smiles>NC1C2COC(O2)C1C(O)F</smiles>

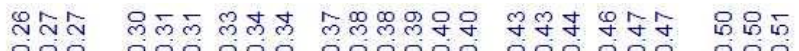

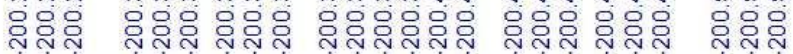

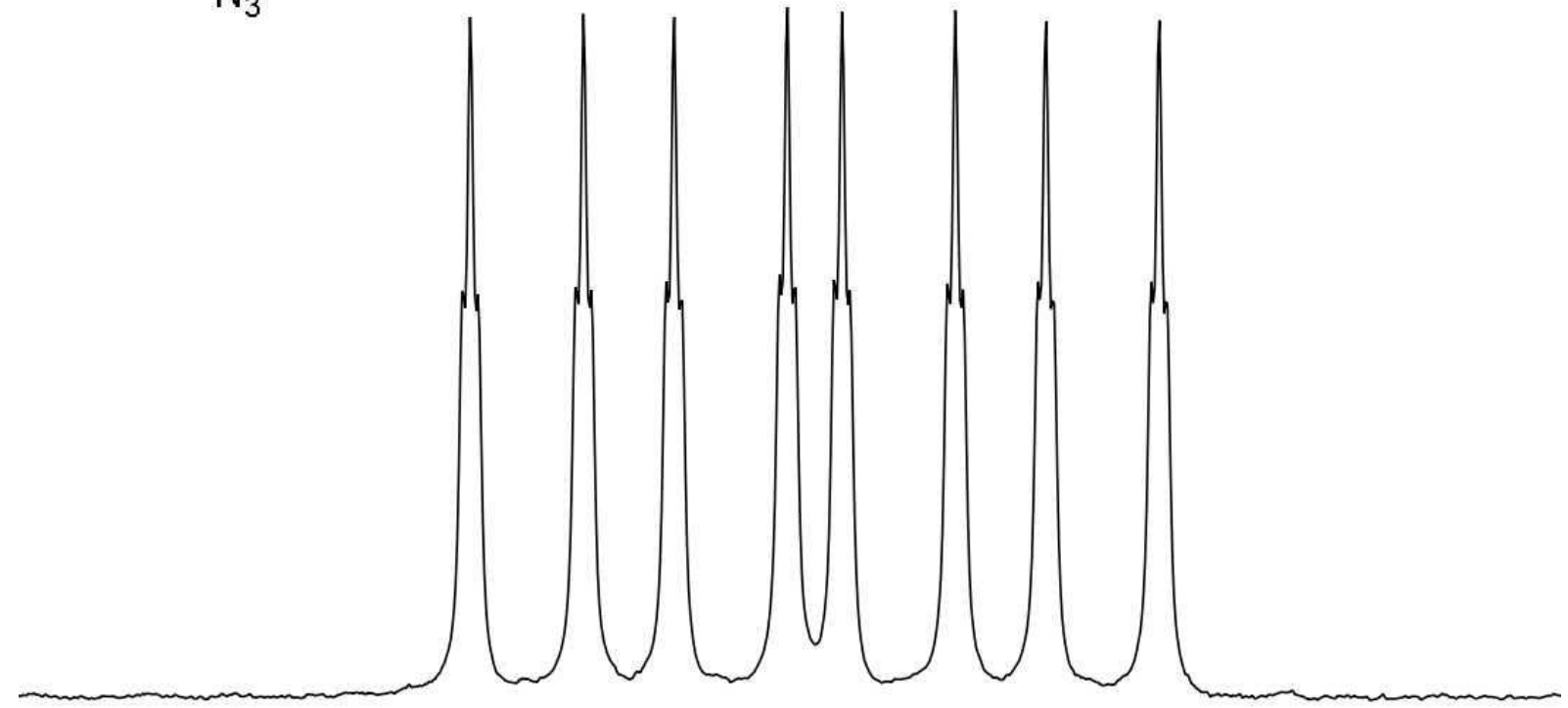

$\begin{array}{llllllllllllllll}0 & 00.12 & -200.16 & -200.20 & -200.24 & -200.28 & -200.32 & -200.36 & -200.40 & -200.44 & -200.48 & -200.52 & -200.56 & -200.60 & -200\end{array}$ f1 (ppm)

\section{NMR 9}

${ }^{1} \mathrm{HNMR} 400 \mathrm{MHz}, \mathrm{CDCl}_{3} 9$

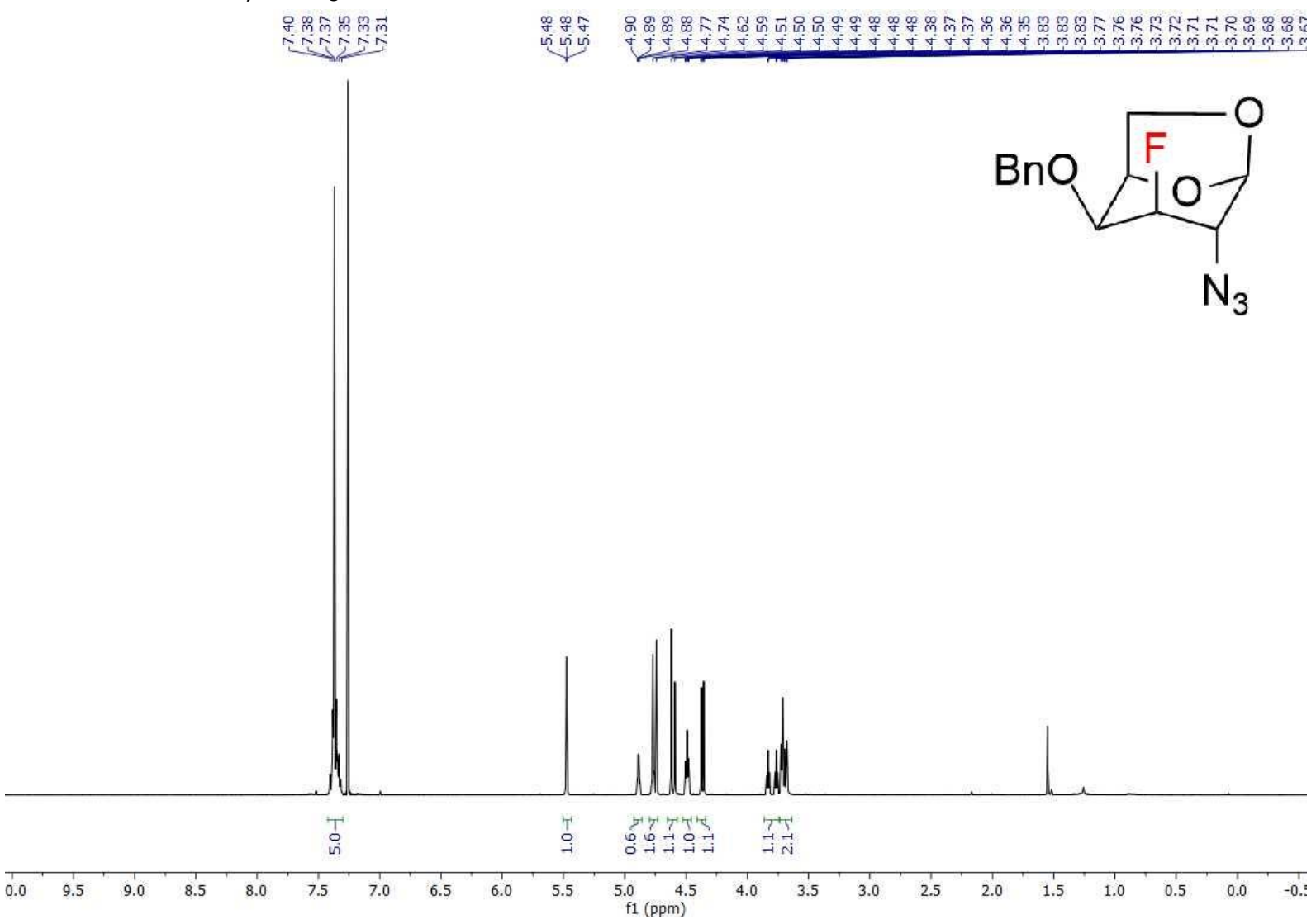


${ }^{13} \mathrm{C}\left\{{ }^{1} \mathrm{H}\right\}$ APT NMR $100 \mathrm{MHz}, \mathrm{CDCl}_{3} 9$

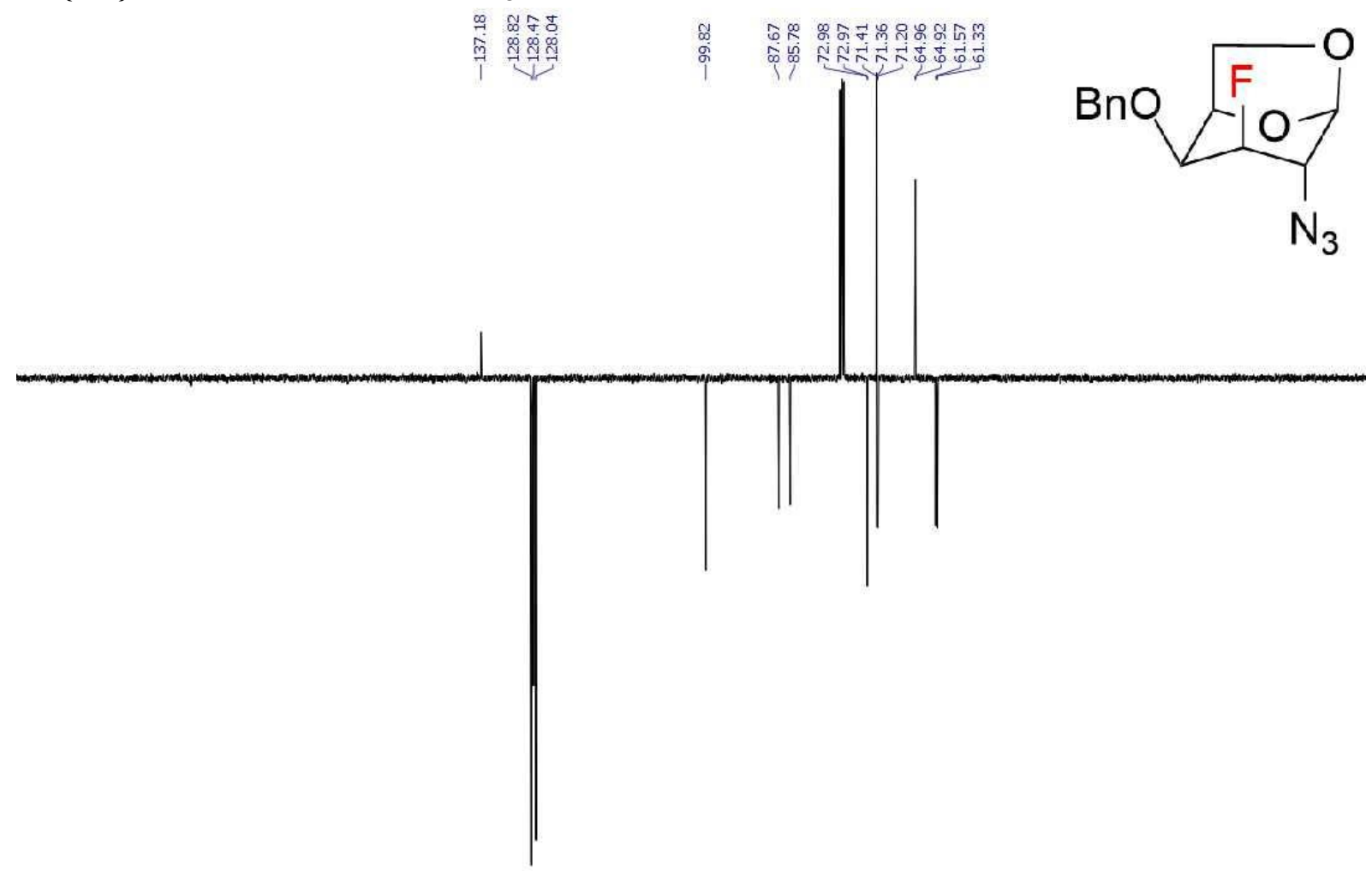

\begin{tabular}{lllllllllllllllllllllllllll}
\hline 210 & 200 & 190 & 180 & 170 & 160 & 150 & 140 & 130 & 120 & 110 & 100 & 90 & 80 & 70 & 60 & 50 & 40 & 30 & 20 & 10 & 0
\end{tabular}

${ }^{1} \mathrm{H}-{ }^{1} \mathrm{H}$ COSY NMR 9

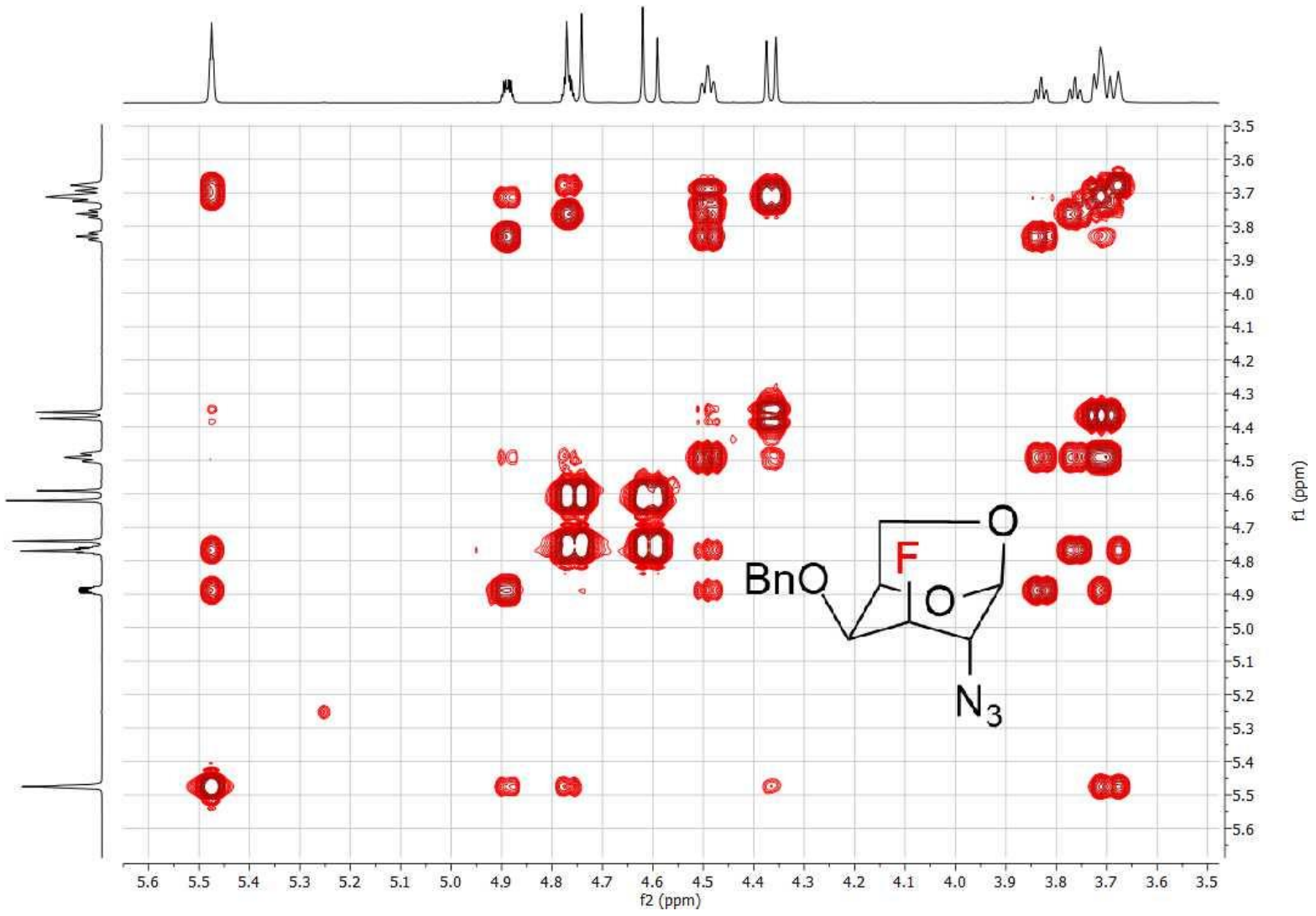


${ }^{1} \mathrm{H}^{13} \mathrm{C}$ HSQC NMR 9

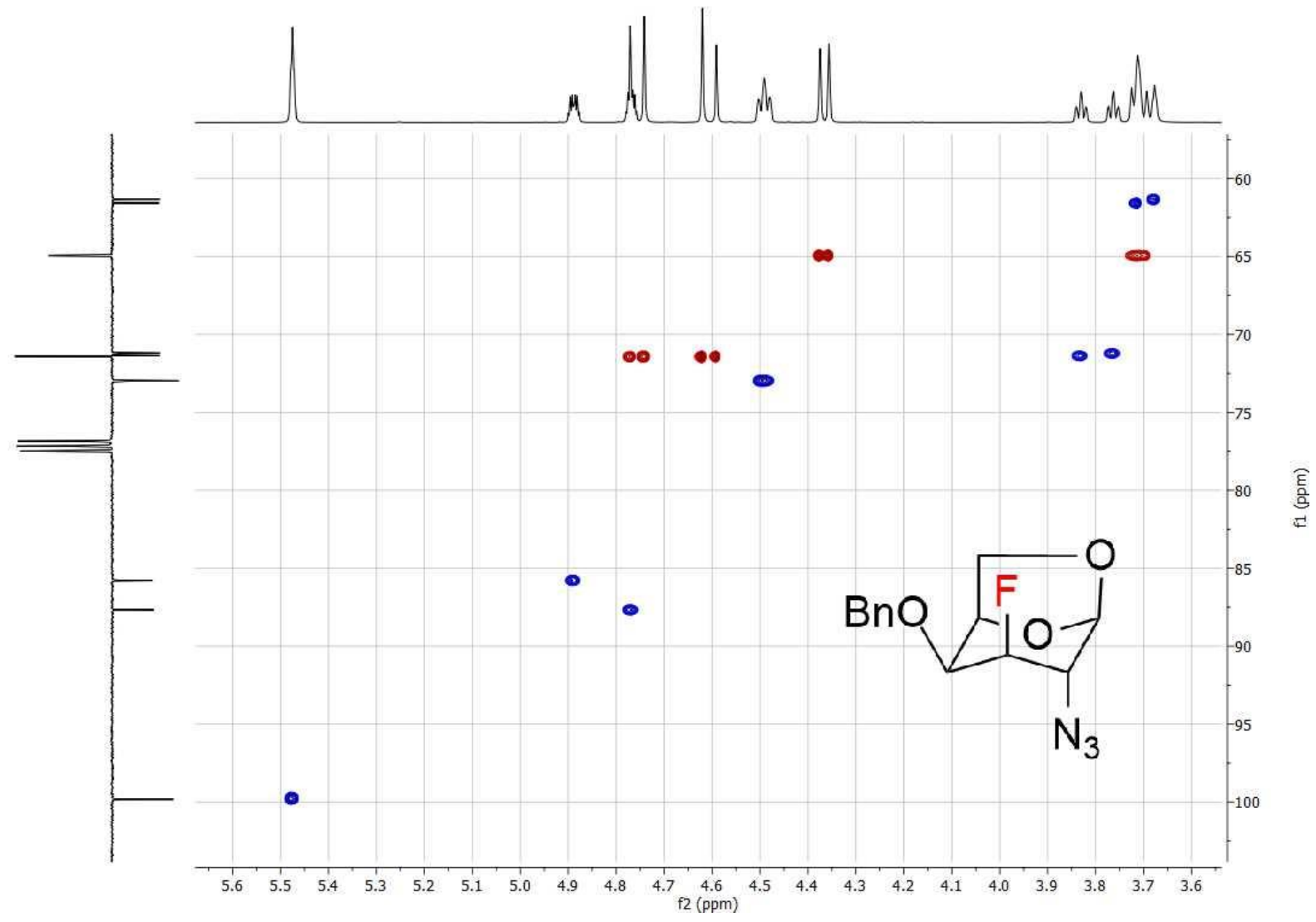

${ }^{19} \mathrm{~F} \mathrm{NMR} 376 \mathrm{MHz}, \mathrm{CDCl}_{3} 9$

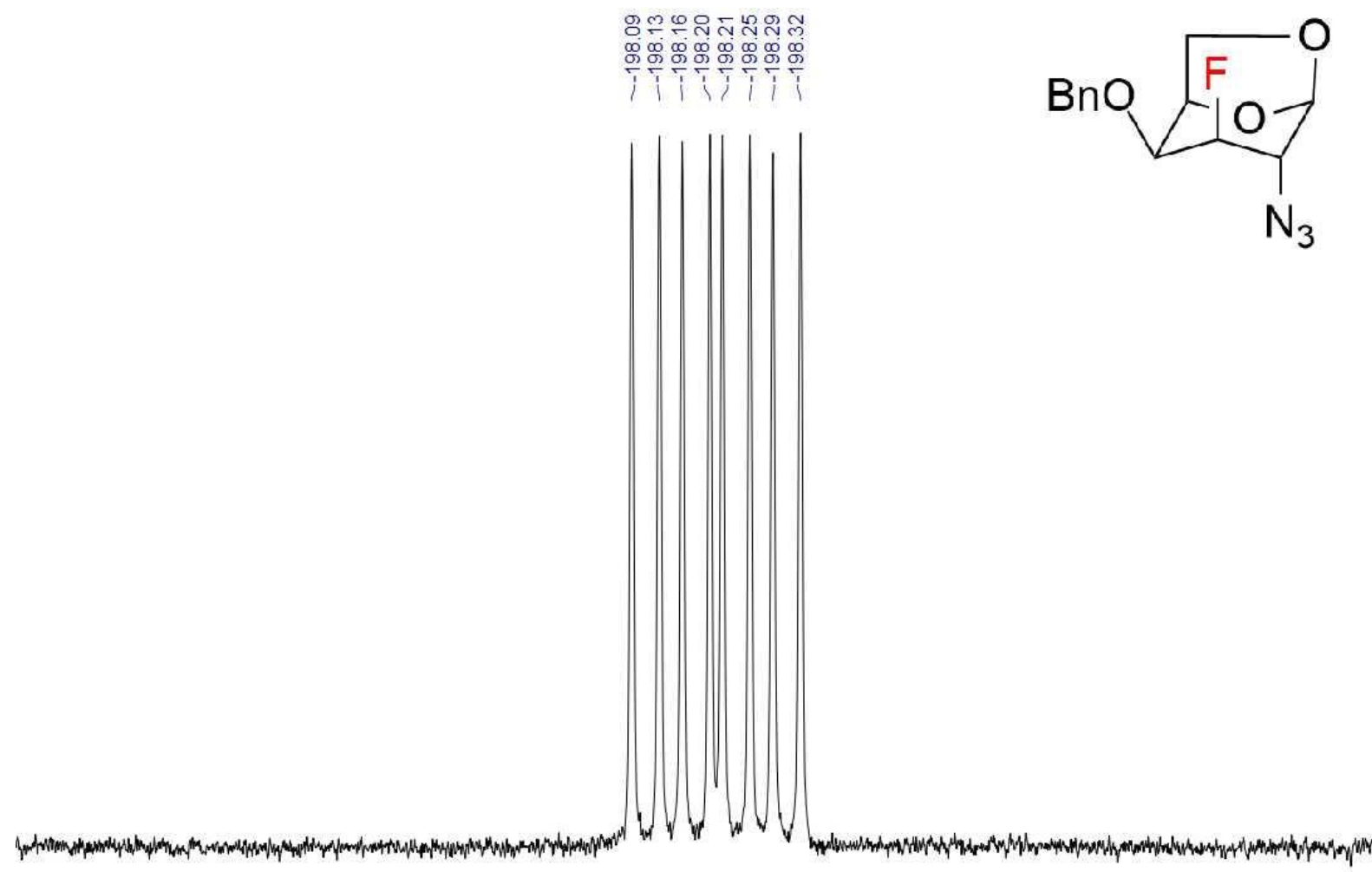

$-197.3-197.4-197.5-197.6-197.7-197.8-197.9-198.0-198.1-198.2-198.3-198.4-198.5-198.6-198.7-198.8-198.9-199.0-199.1$ f1 (ppm) 
NMR $\boldsymbol{\alpha}-10$

${ }^{1} \mathrm{H}$ NMR $400 \mathrm{MHz}, \mathrm{CDCl}_{3} \boldsymbol{\alpha}-10$

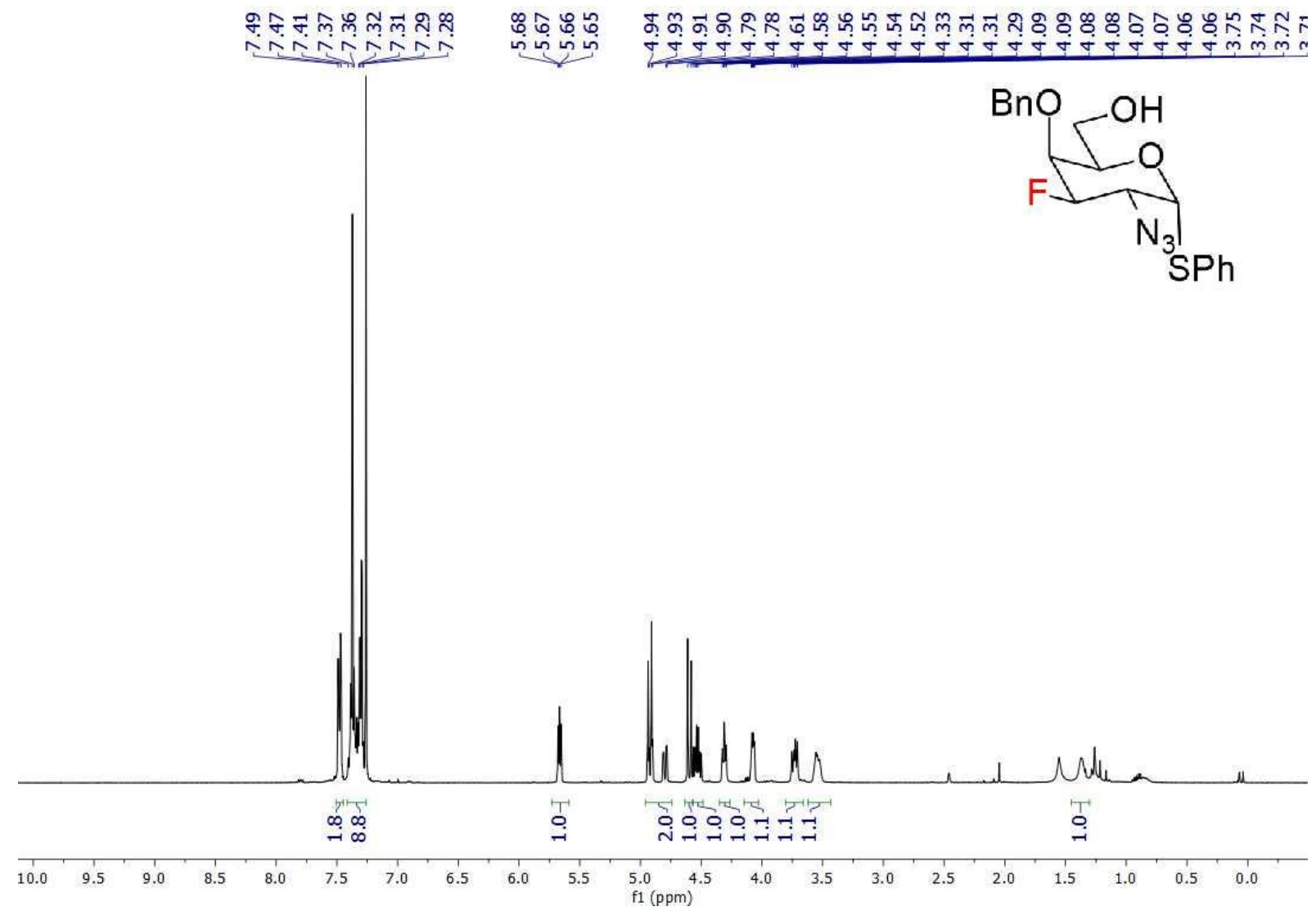

${ }^{13} \mathrm{C}\left\{{ }^{1} \mathrm{H}\right\}$ APT NMR $100 \mathrm{MHz}, \mathrm{CDCl}_{3} \boldsymbol{\alpha}-\mathbf{1 0}$

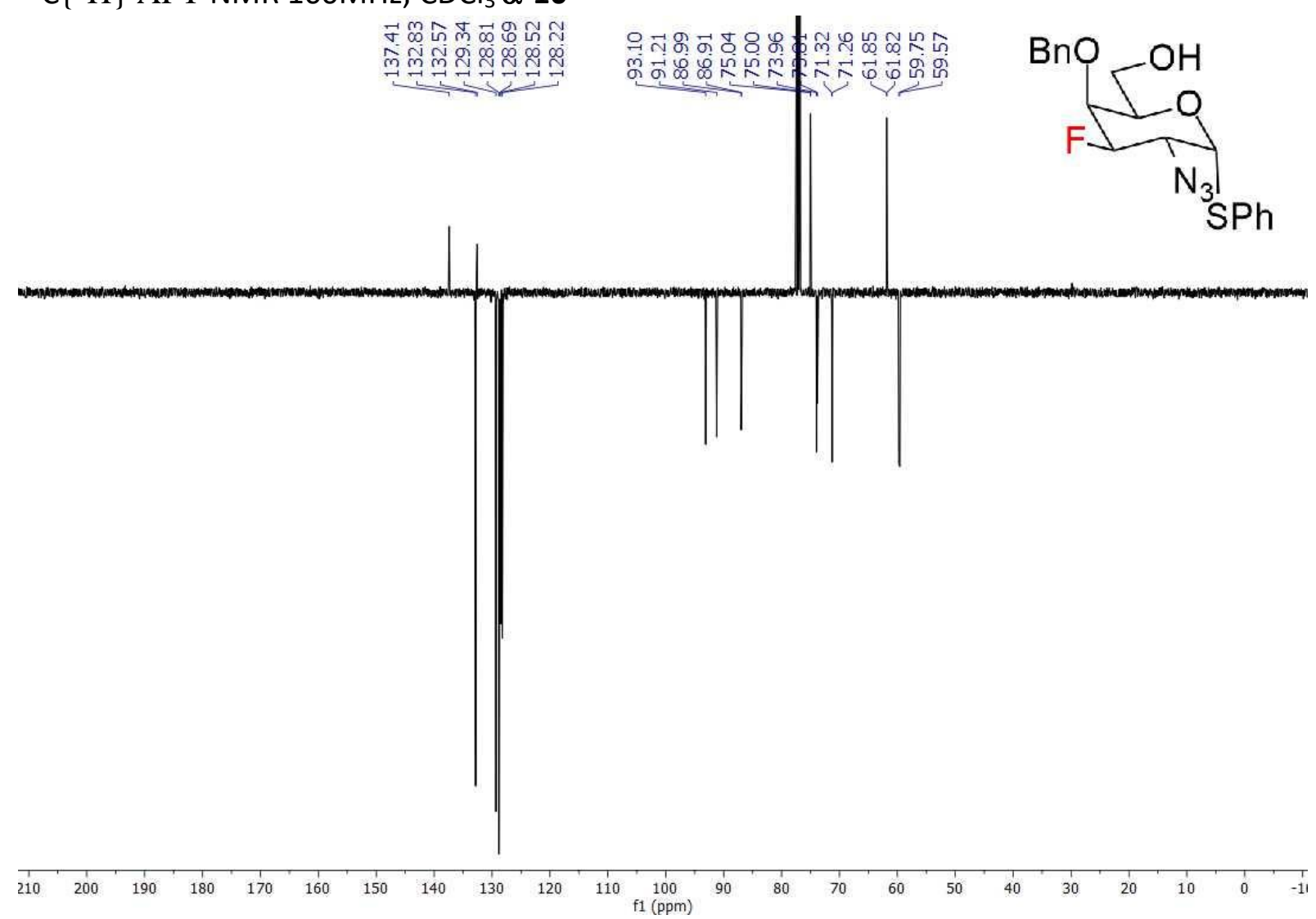


${ }^{1} \mathrm{H}-{ }^{1} \mathrm{H}$ COSY NMR $\boldsymbol{\alpha}-\mathbf{1 0}$

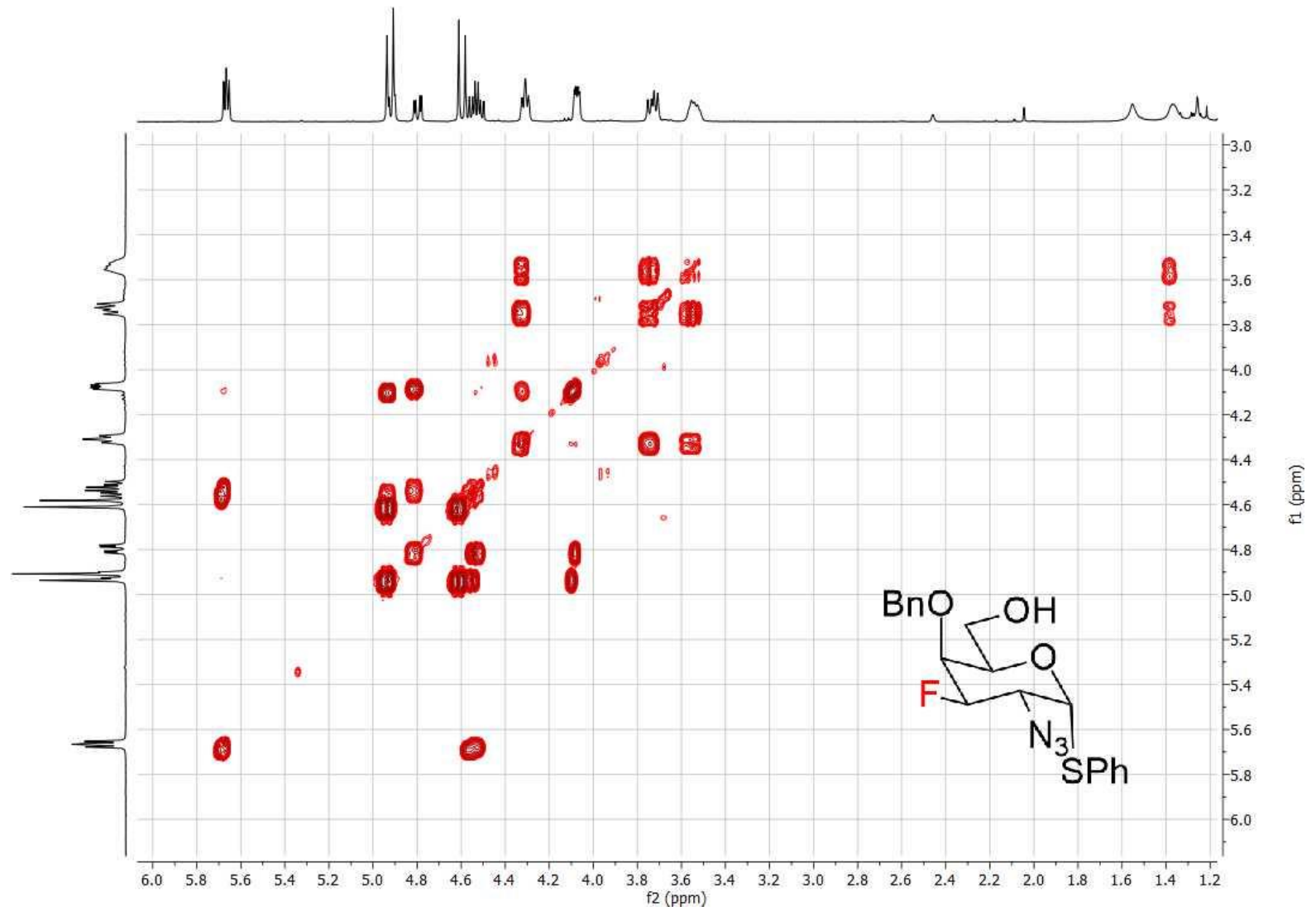

${ }^{1} \mathrm{H}^{-13} \mathrm{C}$ HSQC NMR $\boldsymbol{\alpha}-\mathbf{1 0}$

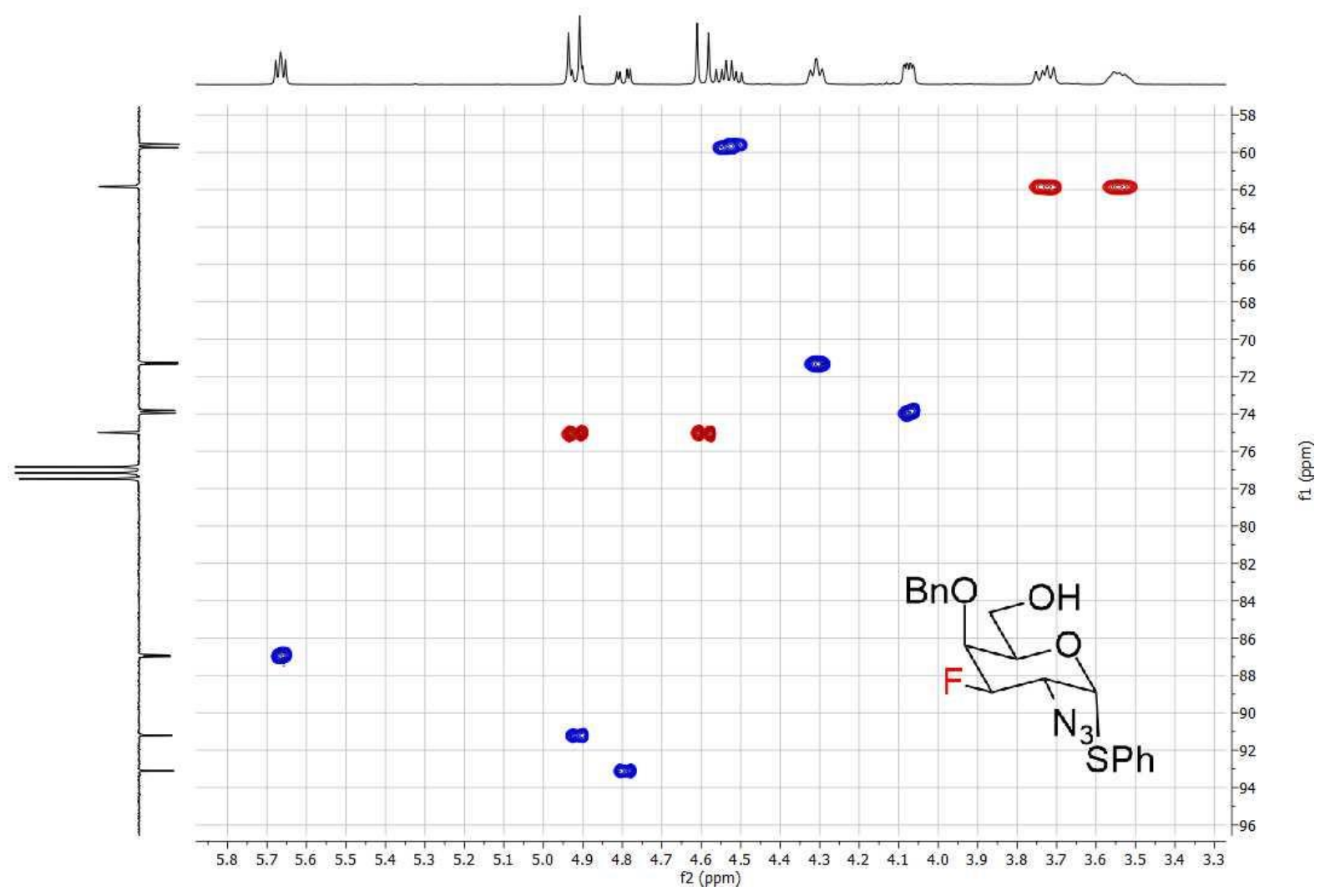




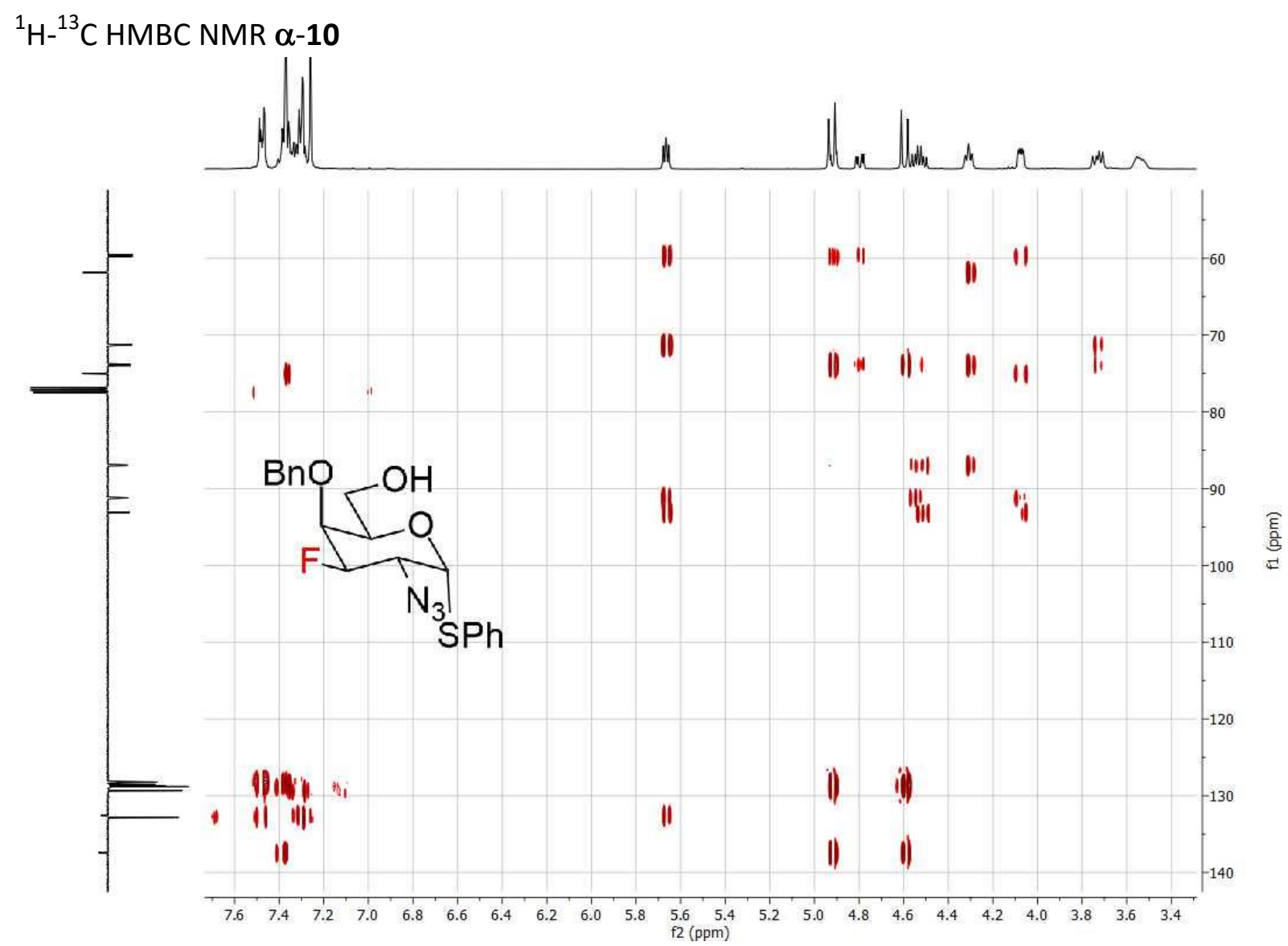

${ }^{19} \mathrm{~F}$ NMR $376 \mathrm{MHz}, \mathrm{CDCl}_{3} \boldsymbol{\alpha - 1 0}$

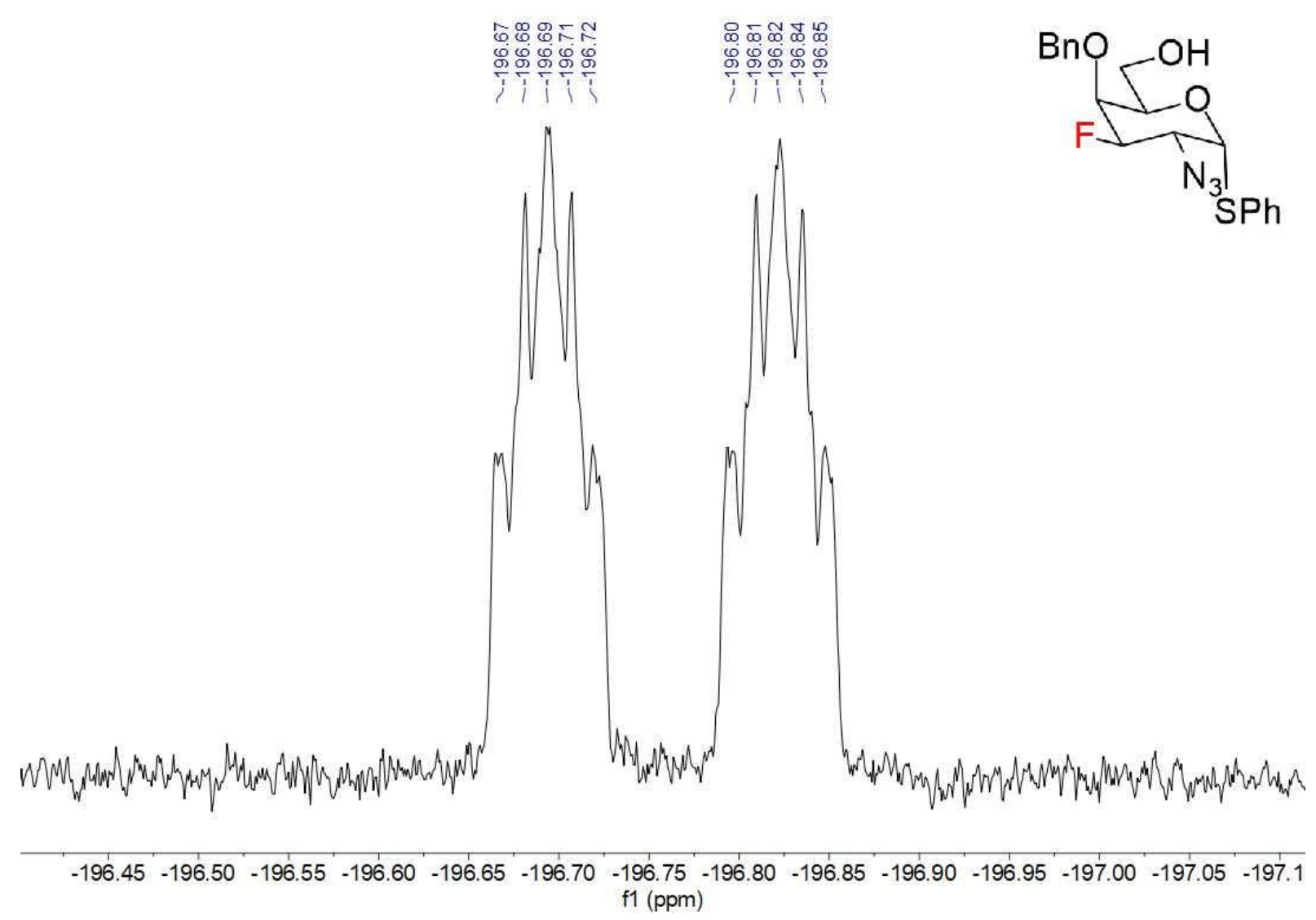


NMR $\beta-10$

${ }^{1} \mathrm{H}$ NMR $400 \mathrm{MHz}, \mathrm{CDCl}_{3} \boldsymbol{\beta}-10$

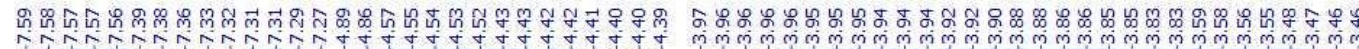

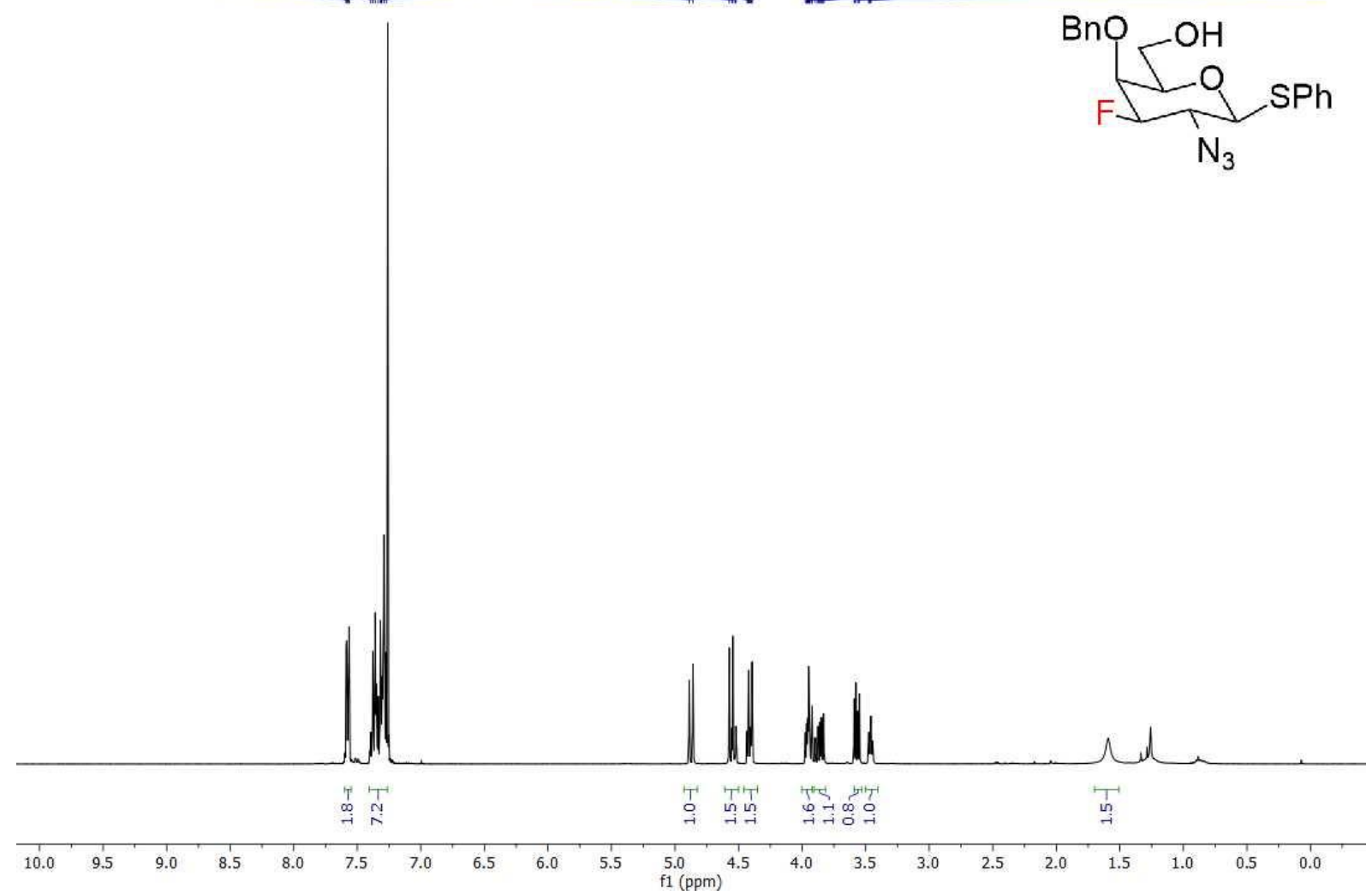

${ }^{13} \mathrm{C}\left\{{ }^{1} \mathrm{H}\right\}$ APT NMR $100 \mathrm{MHz}, \mathrm{CDCl}_{3} \boldsymbol{\beta}-10$

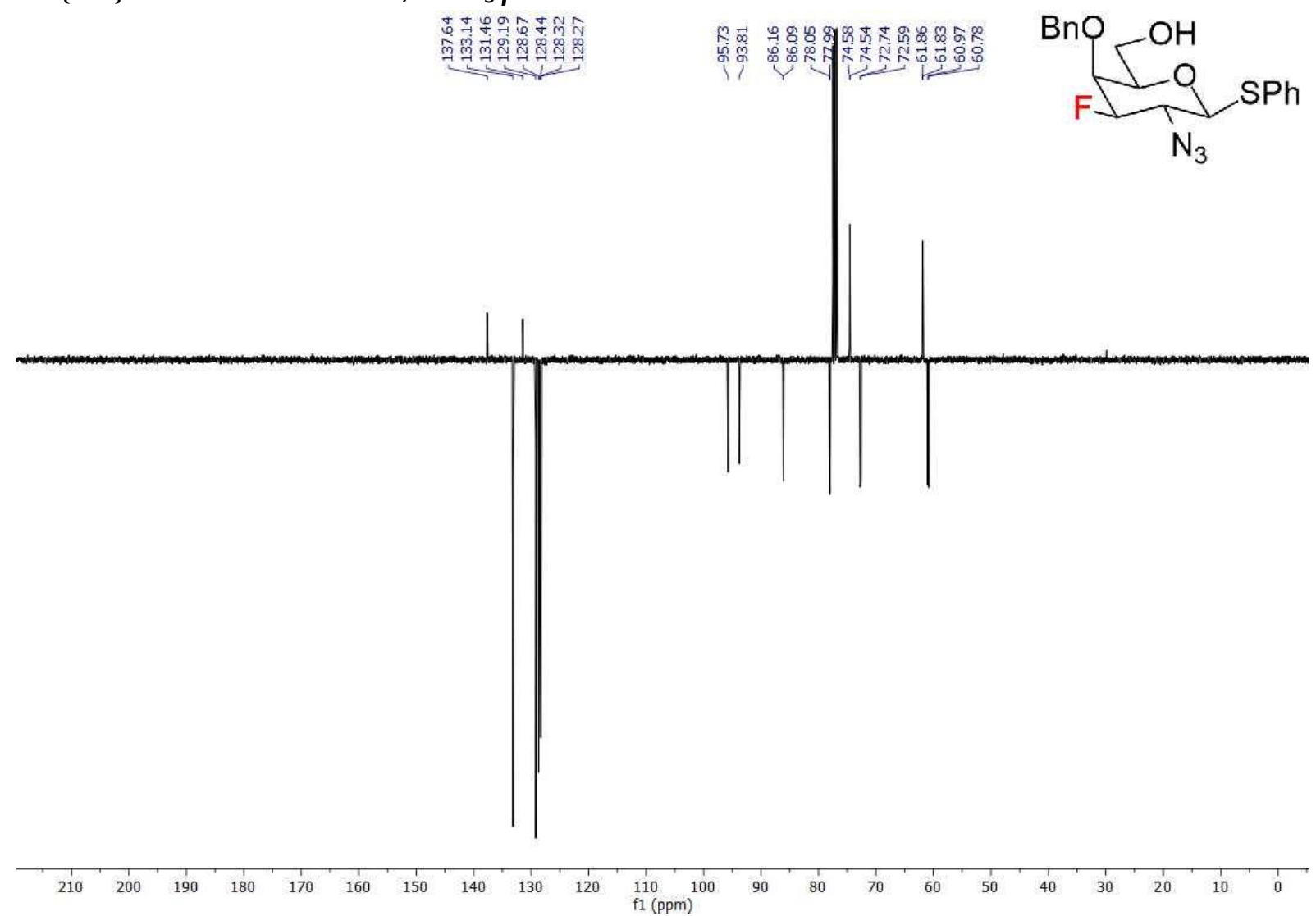


${ }^{1} \mathrm{H}-{ }^{1} \mathrm{H}$ COSY NMR $\beta-10$

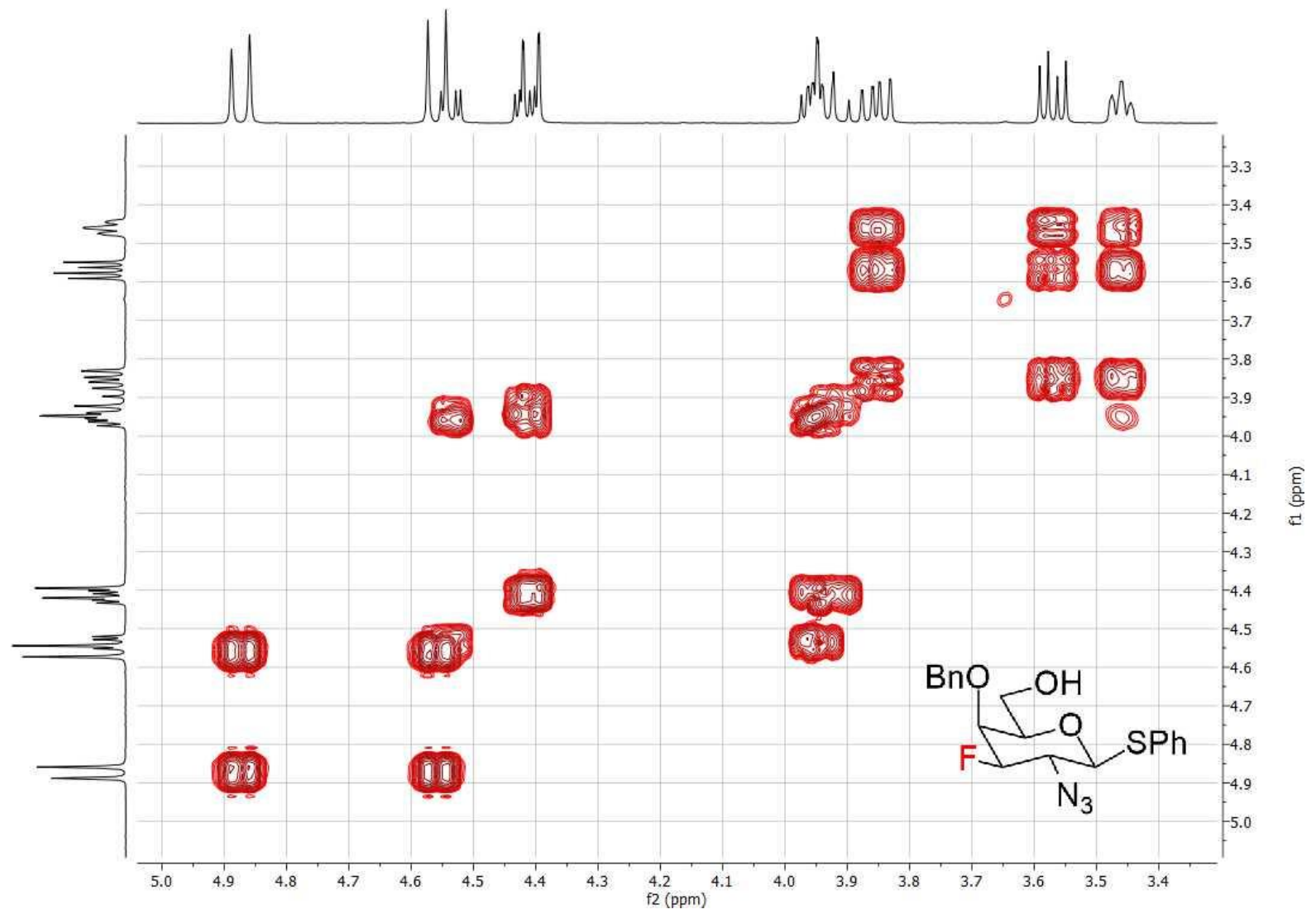

${ }^{1} \mathrm{H}-{ }^{13} \mathrm{C}$ HSQC NMR $\beta-10$

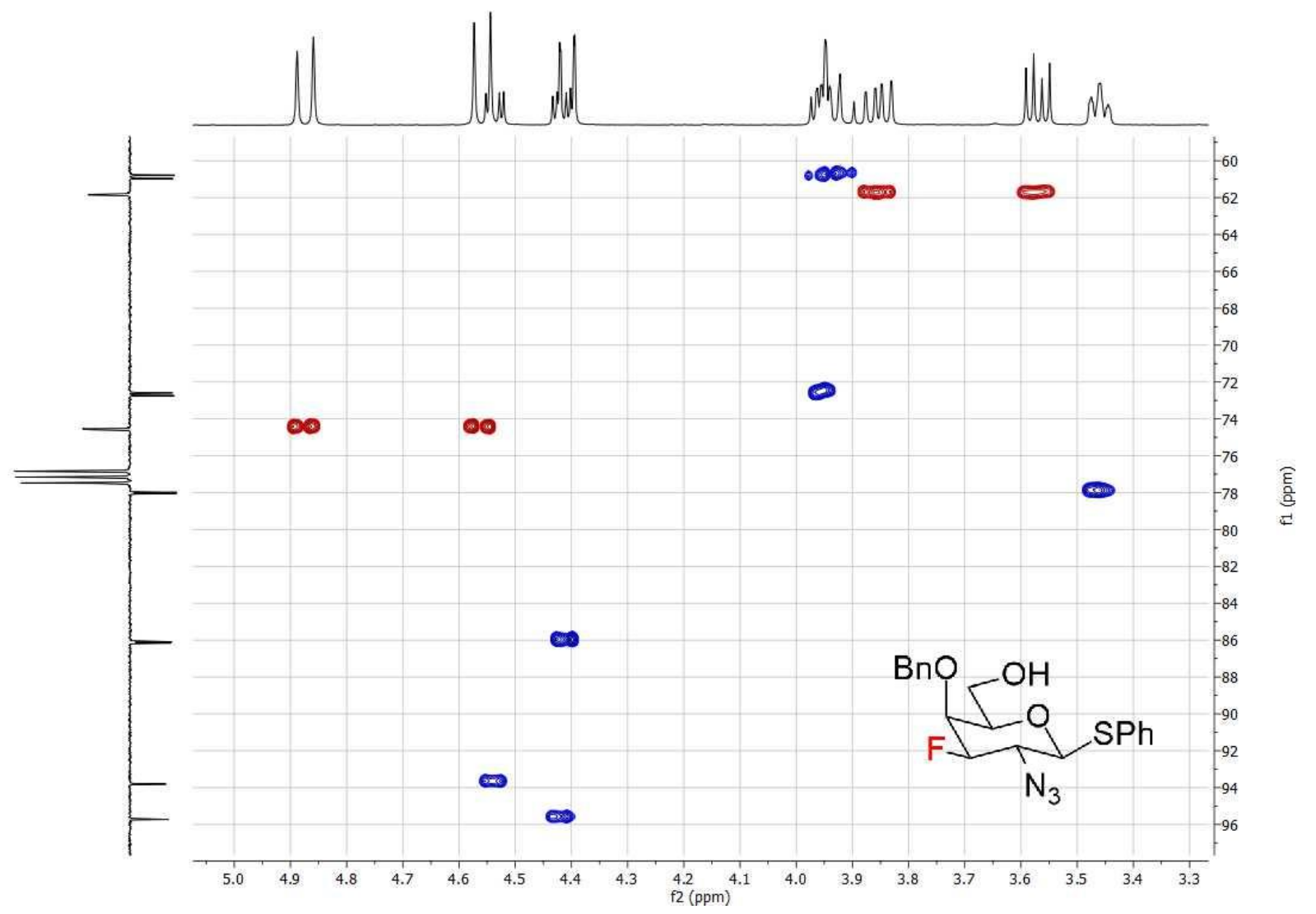


${ }^{19} \mathrm{~F} \mathrm{NMR} 376 \mathrm{MHz}, \mathrm{CDCl}_{3} \boldsymbol{\beta}-10$

œ1.

舟

융둥

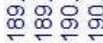

$\mathrm{BnO}-\mathrm{OH}$

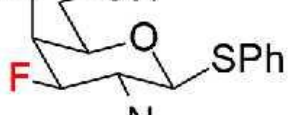

$\mathrm{N}_{3}$

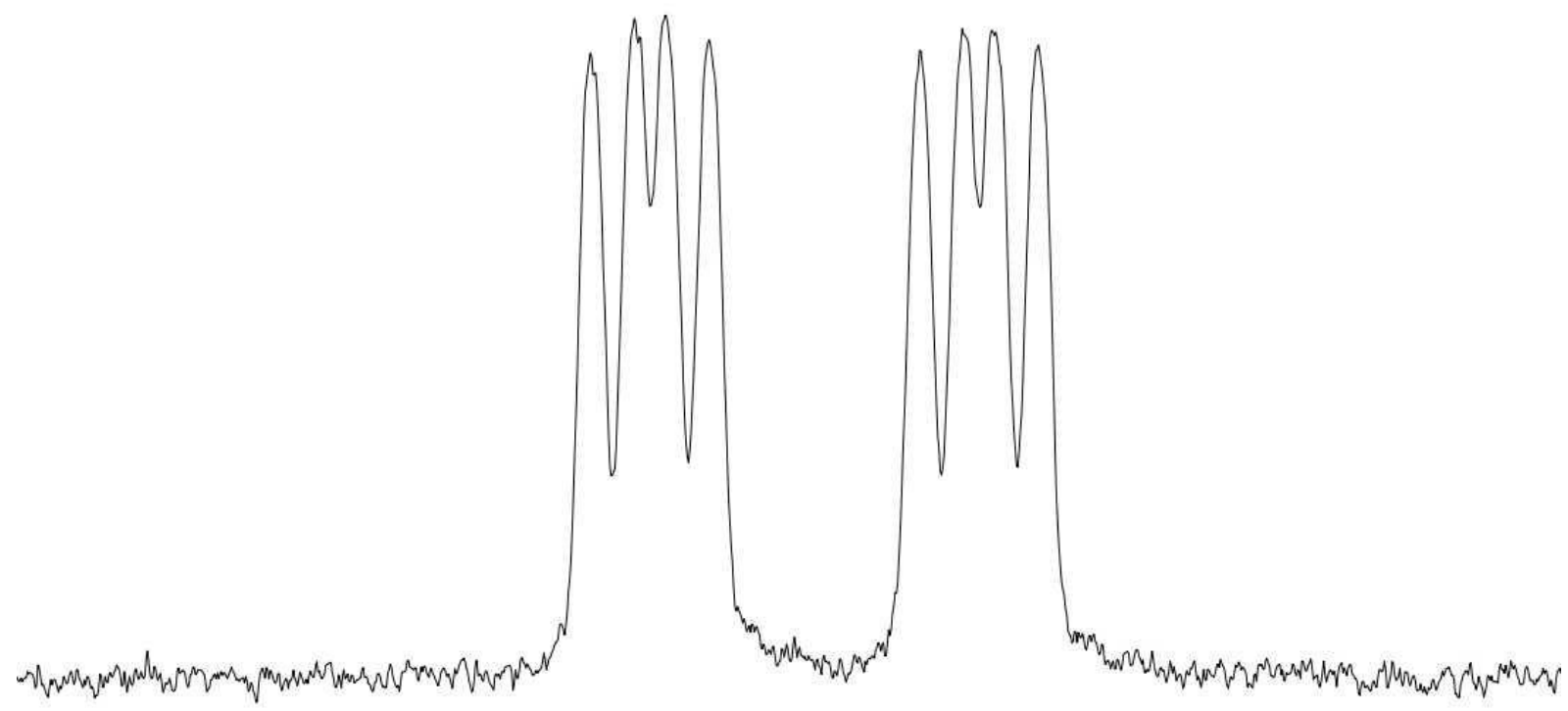

$-189.66-189.70-189.74-189.78-189.82-189.86-189.90-189.94-189.98-190.02-190.06-190.10-190.14-190.18-190$. f1 (ppm) 
NMR $\alpha-3$

${ }^{1} \mathrm{HNMR} 400 \mathrm{MHz}, \mathrm{CDCl}_{3} \boldsymbol{\alpha}-3$

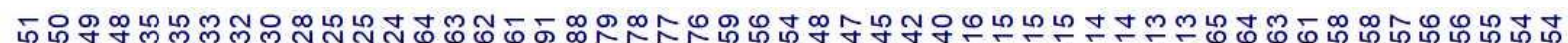

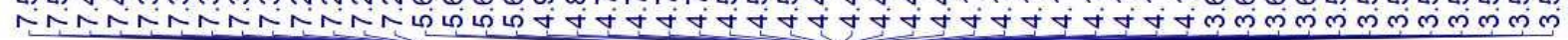

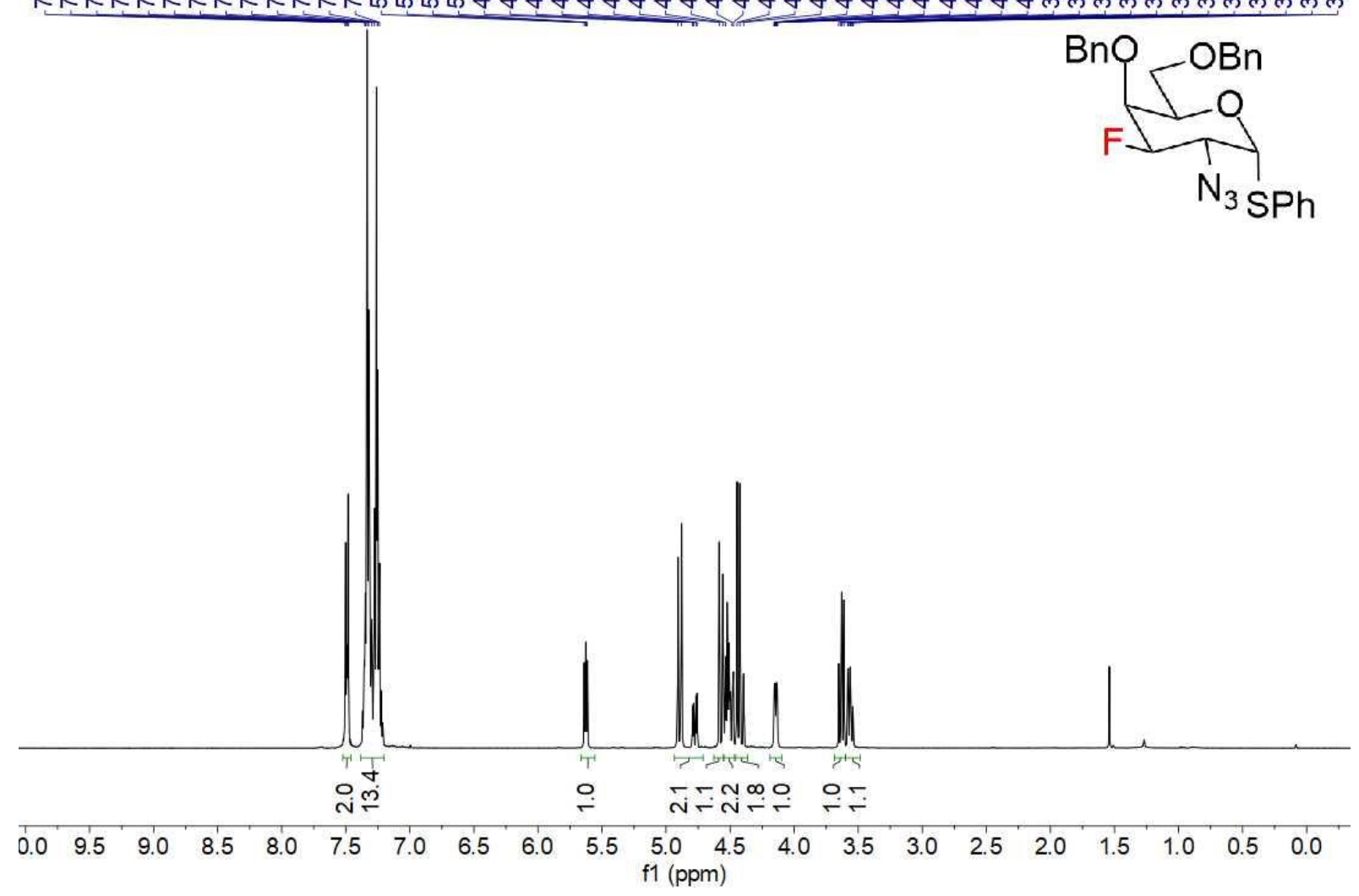

${ }^{13} \mathrm{C}\left\{{ }^{1} \mathrm{H}\right\} \mathrm{NMR} 100 \mathrm{MHz}, \mathrm{CDCl}_{3} \boldsymbol{\alpha}-\mathbf{3}$

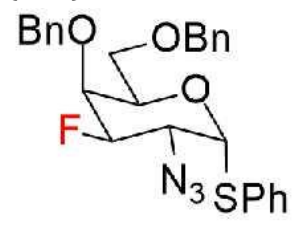

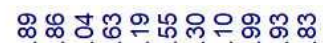

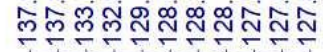

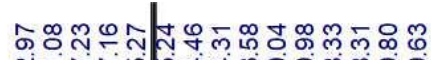

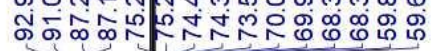

$3 \mathrm{SPh}$
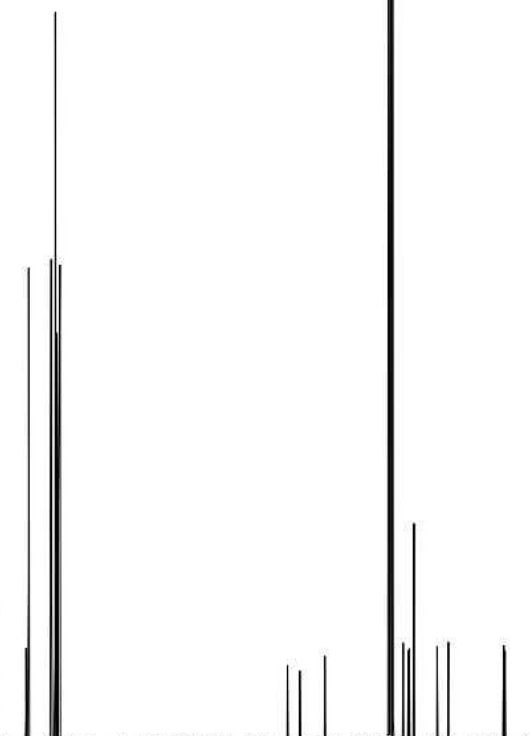

20110100

80

$\mathrm{f1}(\mathrm{ppm})$ 
${ }^{1} \mathrm{H}-{ }^{1} \mathrm{H} \cos Y$ NMR $\boldsymbol{\alpha}-3$

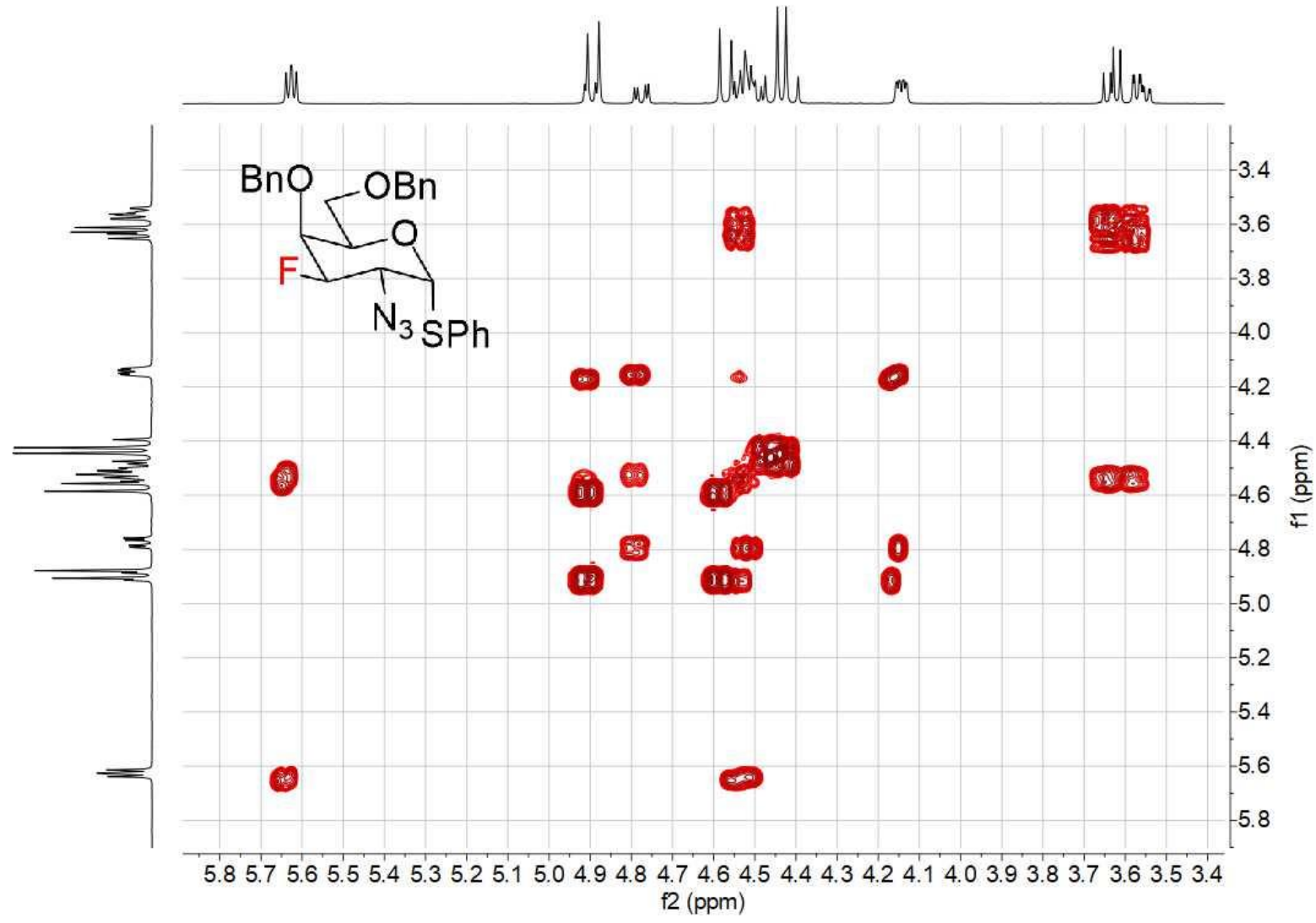

${ }^{1} \mathrm{H}-{ }^{13} \mathrm{C}$ HSQC NMR $\boldsymbol{\alpha}-3$

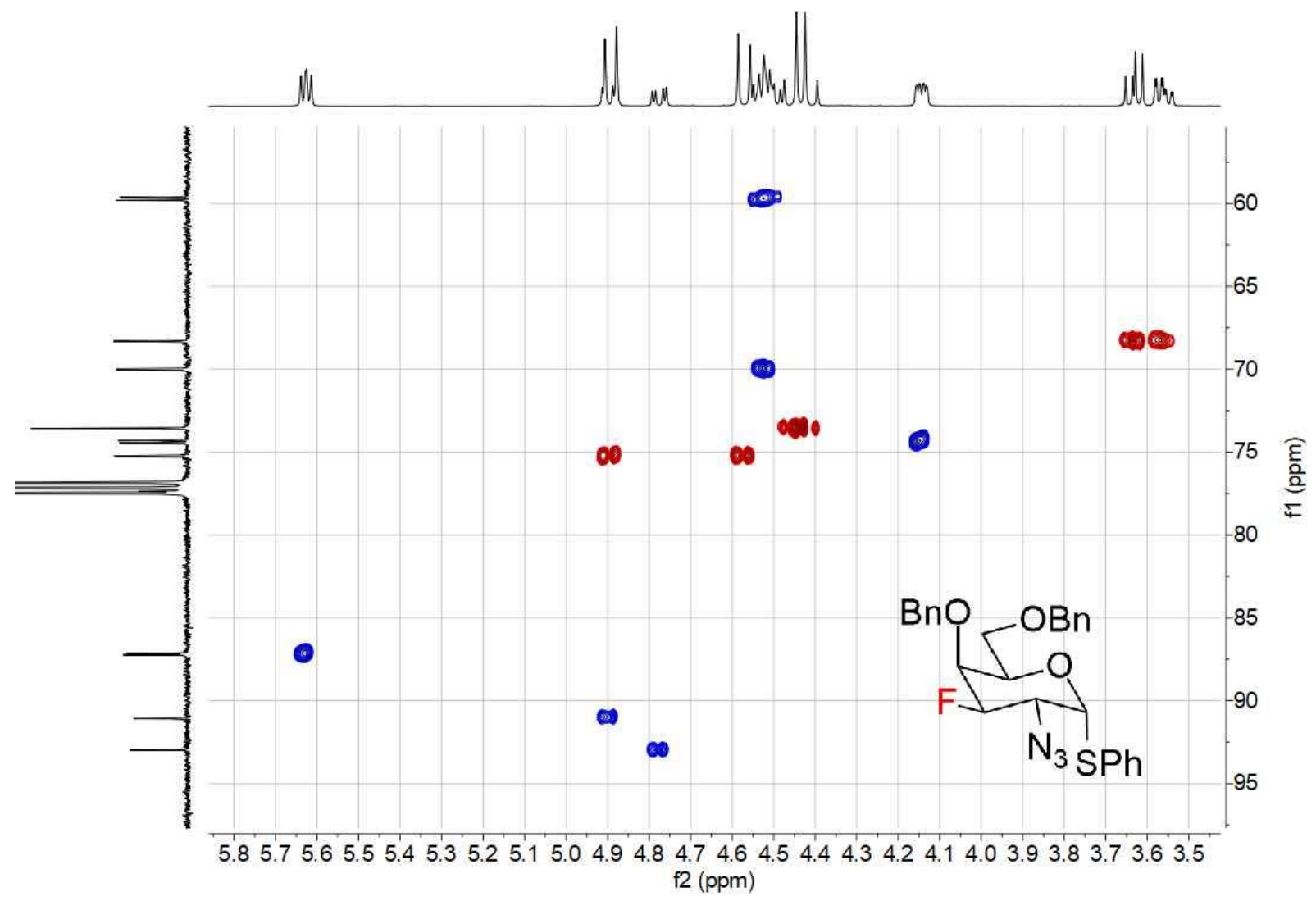


${ }^{19} \mathrm{~F} \mathrm{NMR} 376 \mathrm{MHz}, \mathrm{CDCl}_{3} \boldsymbol{\alpha}-3$

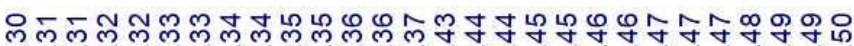

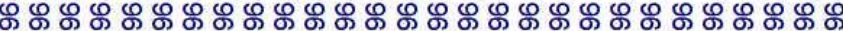
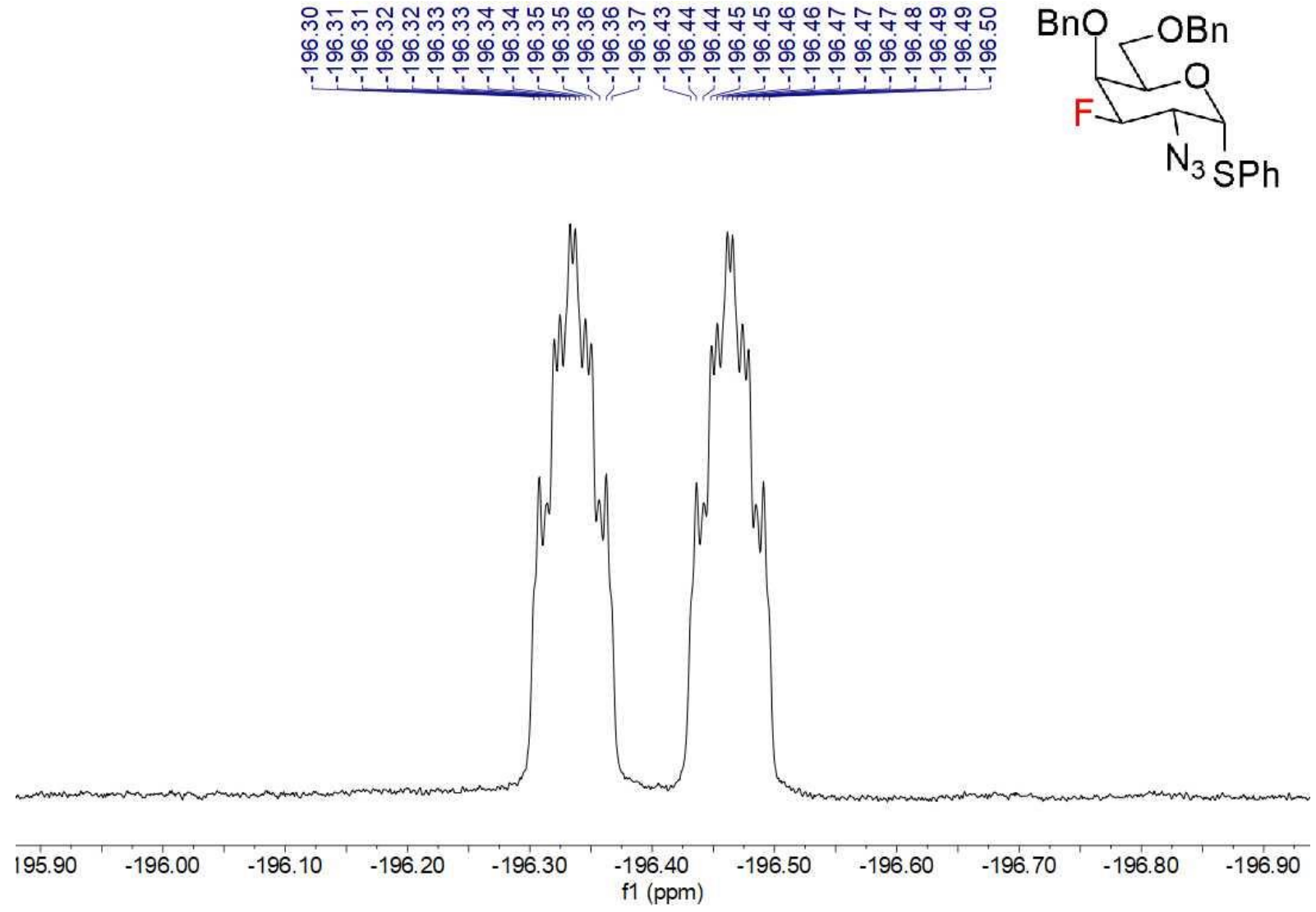

\section{NMR $\beta$-3}

${ }^{1} \mathrm{HNMR} 400 \mathrm{MHz}, \mathrm{CDCl}_{3} \boldsymbol{\beta}-3$

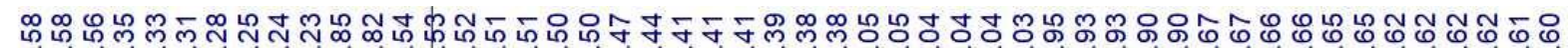

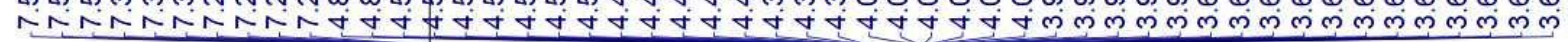

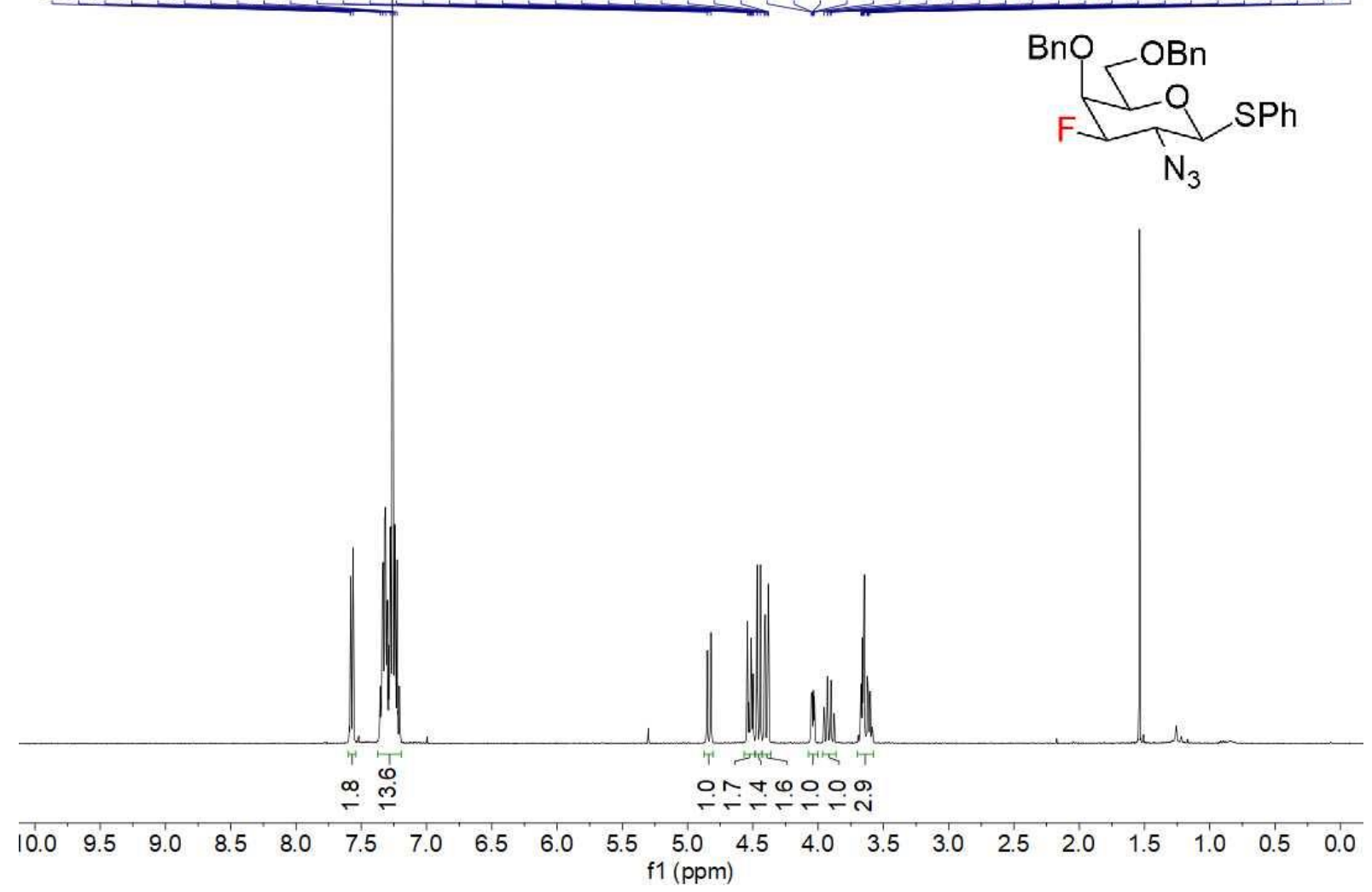


${ }^{13} \mathrm{C}\left\{{ }^{1} \mathrm{H}\right\}$ NMR $100 \mathrm{MHz}, \mathrm{CDCl}_{3} \boldsymbol{\beta}-3$

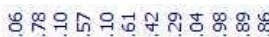

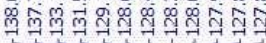

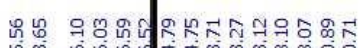

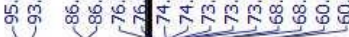
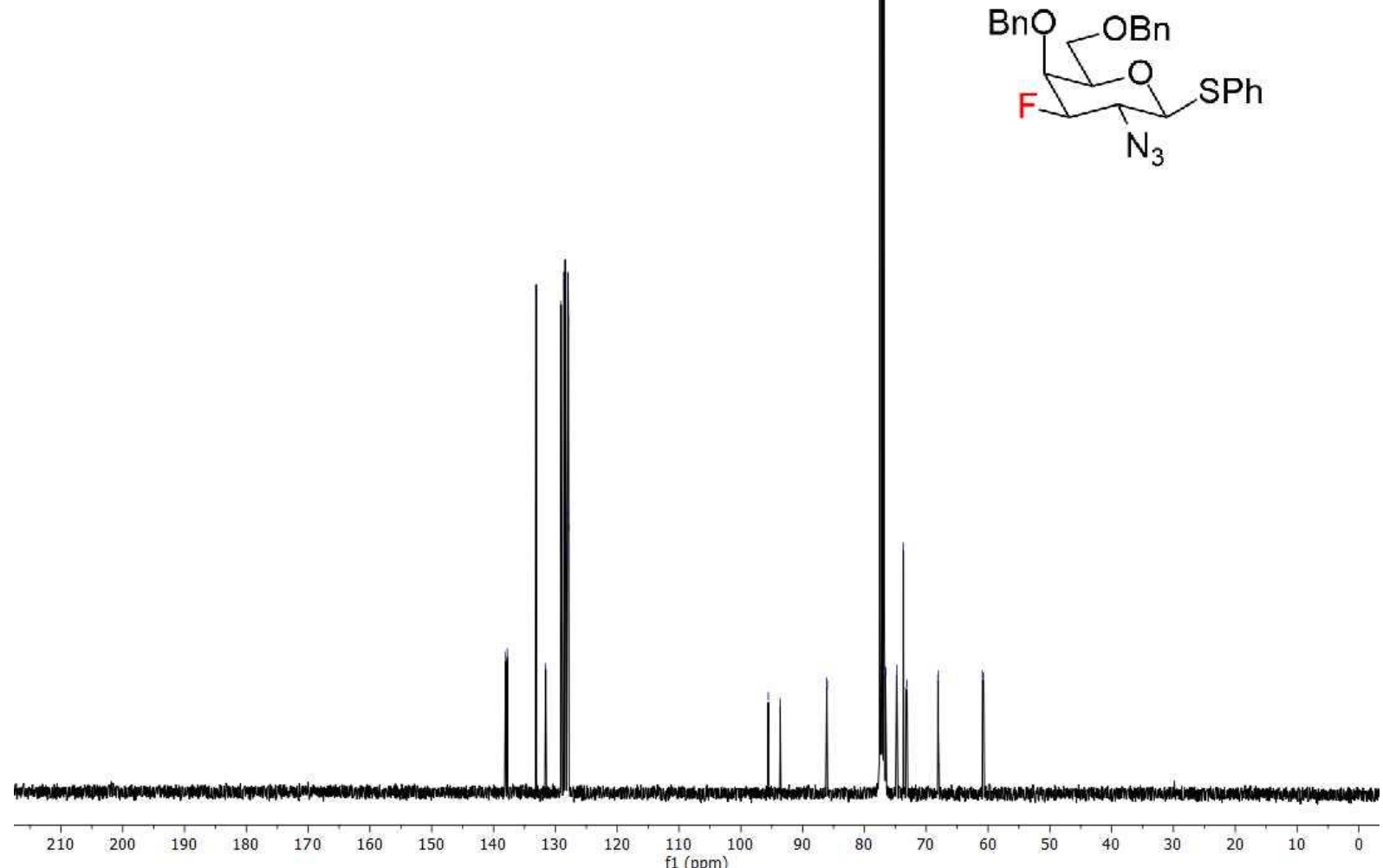

${ }^{1} \mathrm{H}-{ }^{1} \mathrm{H}$ COSY NMR $\boldsymbol{\beta}-3$

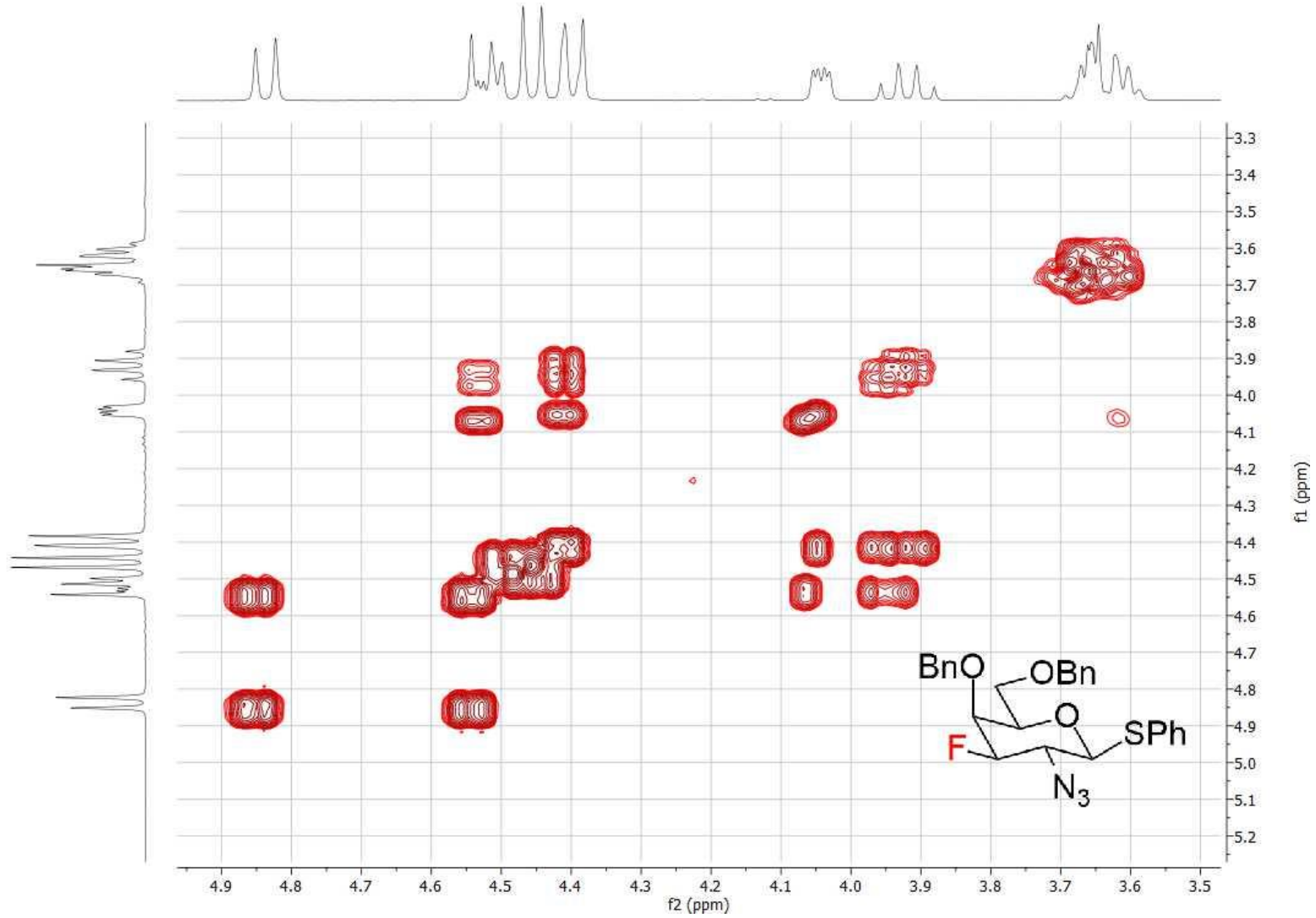


${ }^{1} \mathrm{H}-{ }^{13} \mathrm{C}$ HSQC NMR $\beta-3$

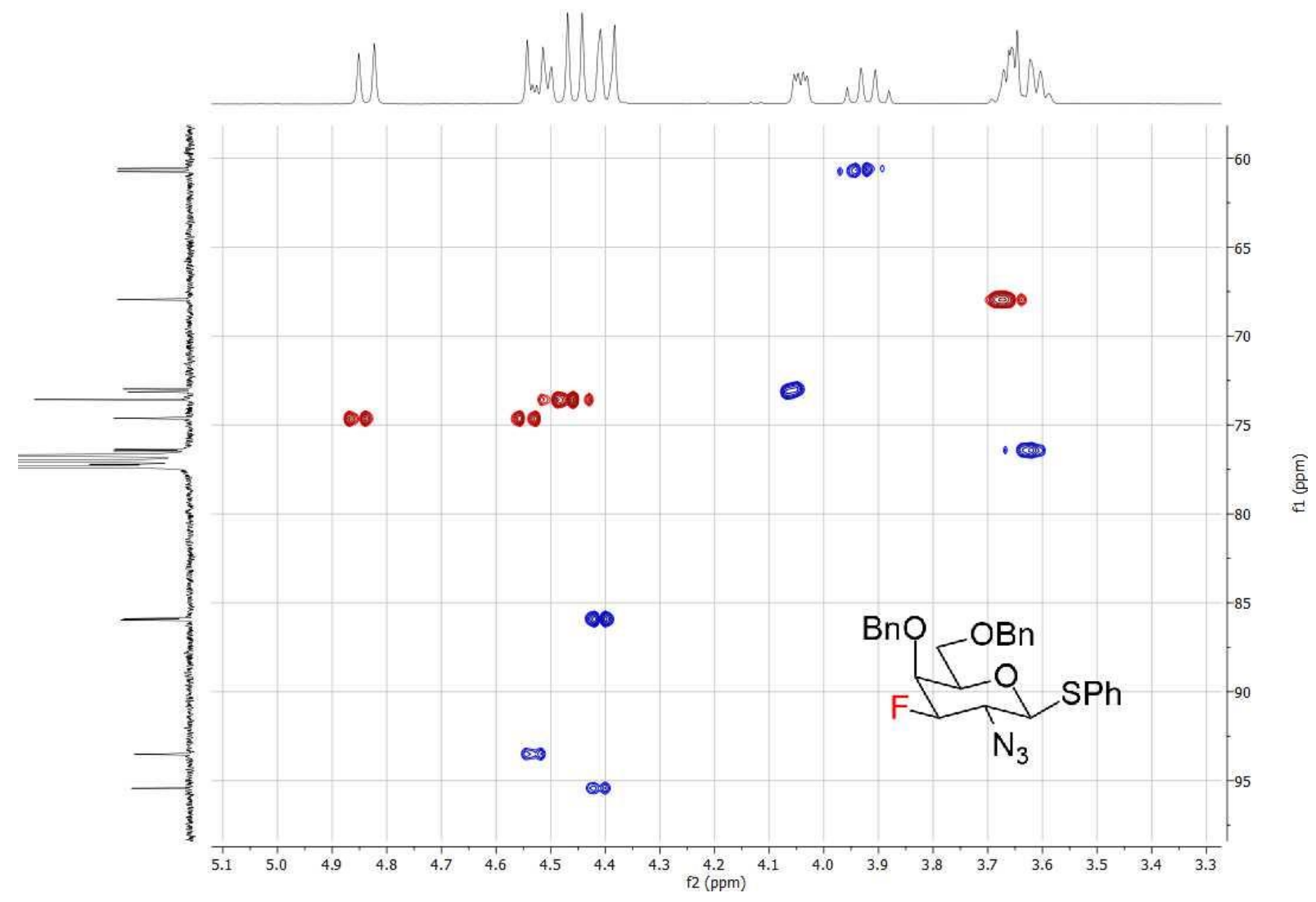

${ }^{1} \mathrm{H}-{ }^{13} \mathrm{C}$ HMBC NMR $\beta-3$

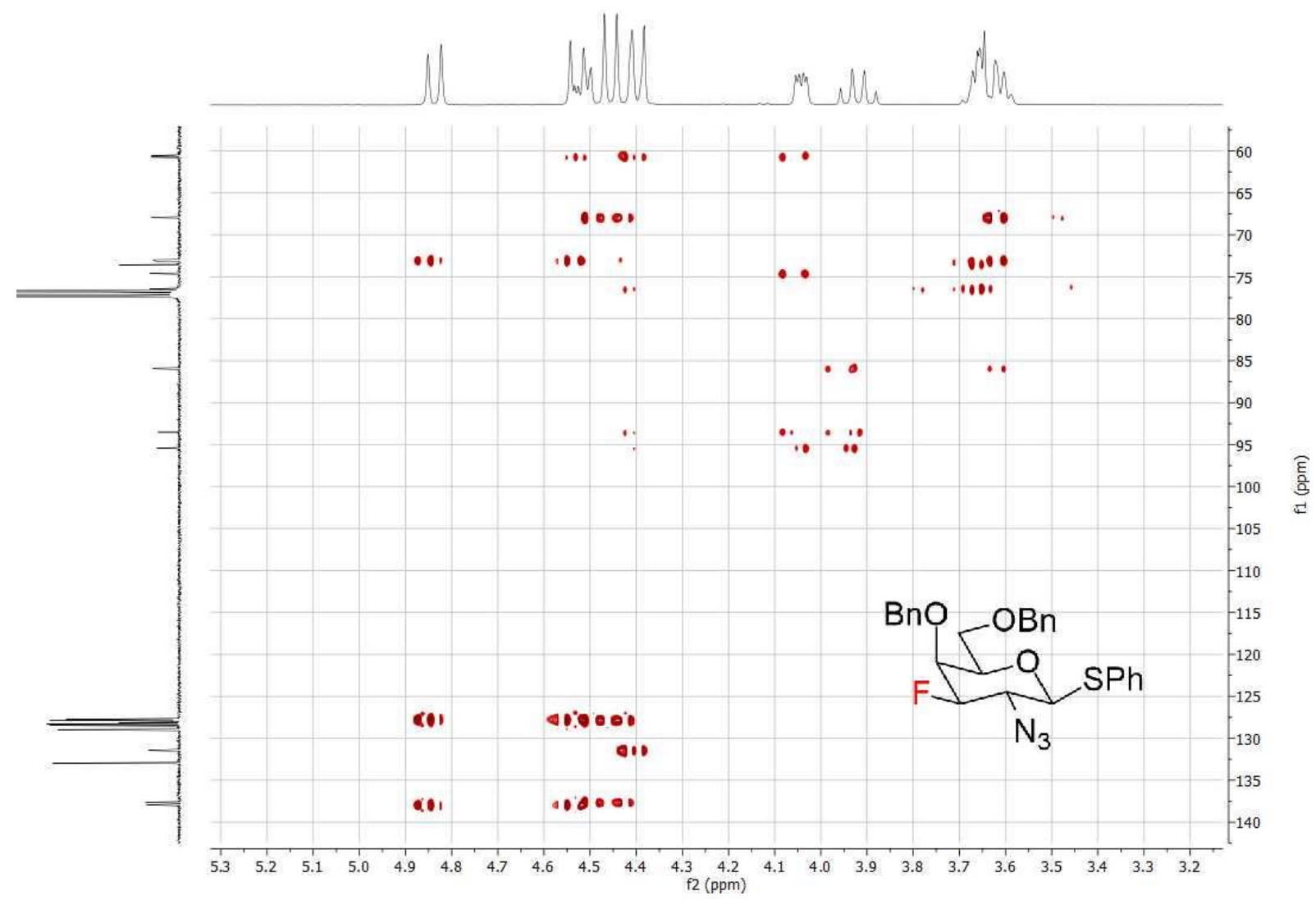


${ }^{19} \mathrm{~F} \mathrm{NMR} 376 \mathrm{MHz}, \mathrm{CDCl}_{3} \boldsymbol{\beta}-3$

Кㄷำ

๑ ஐ ஐ ஐ

$\div \div \div \div$
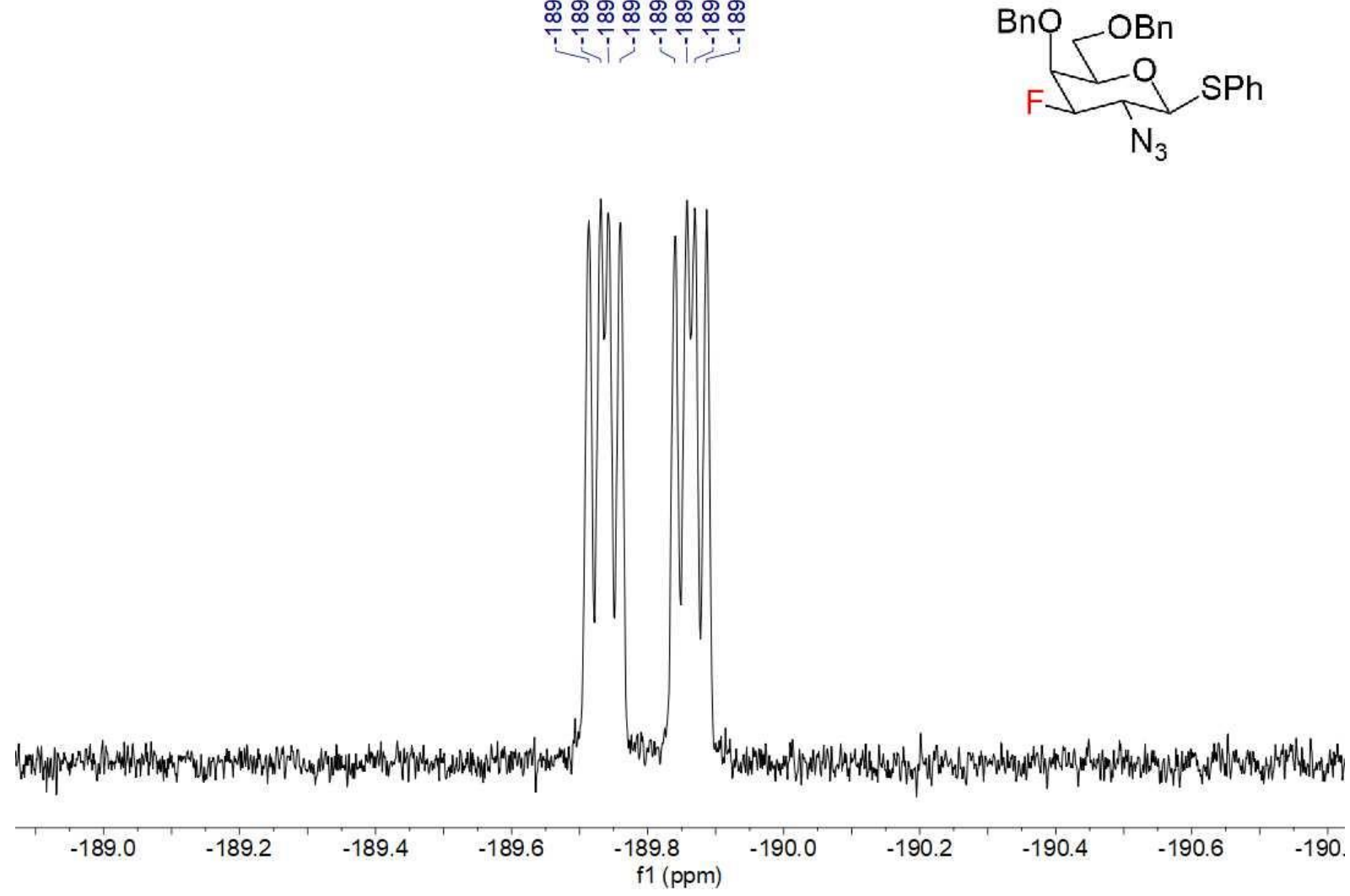

NMR 13

${ }^{1} \mathrm{HNMR} 400 \mathrm{MHz}, \mathrm{CDCl}_{3} 13$

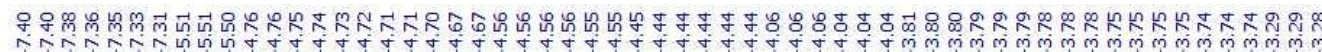

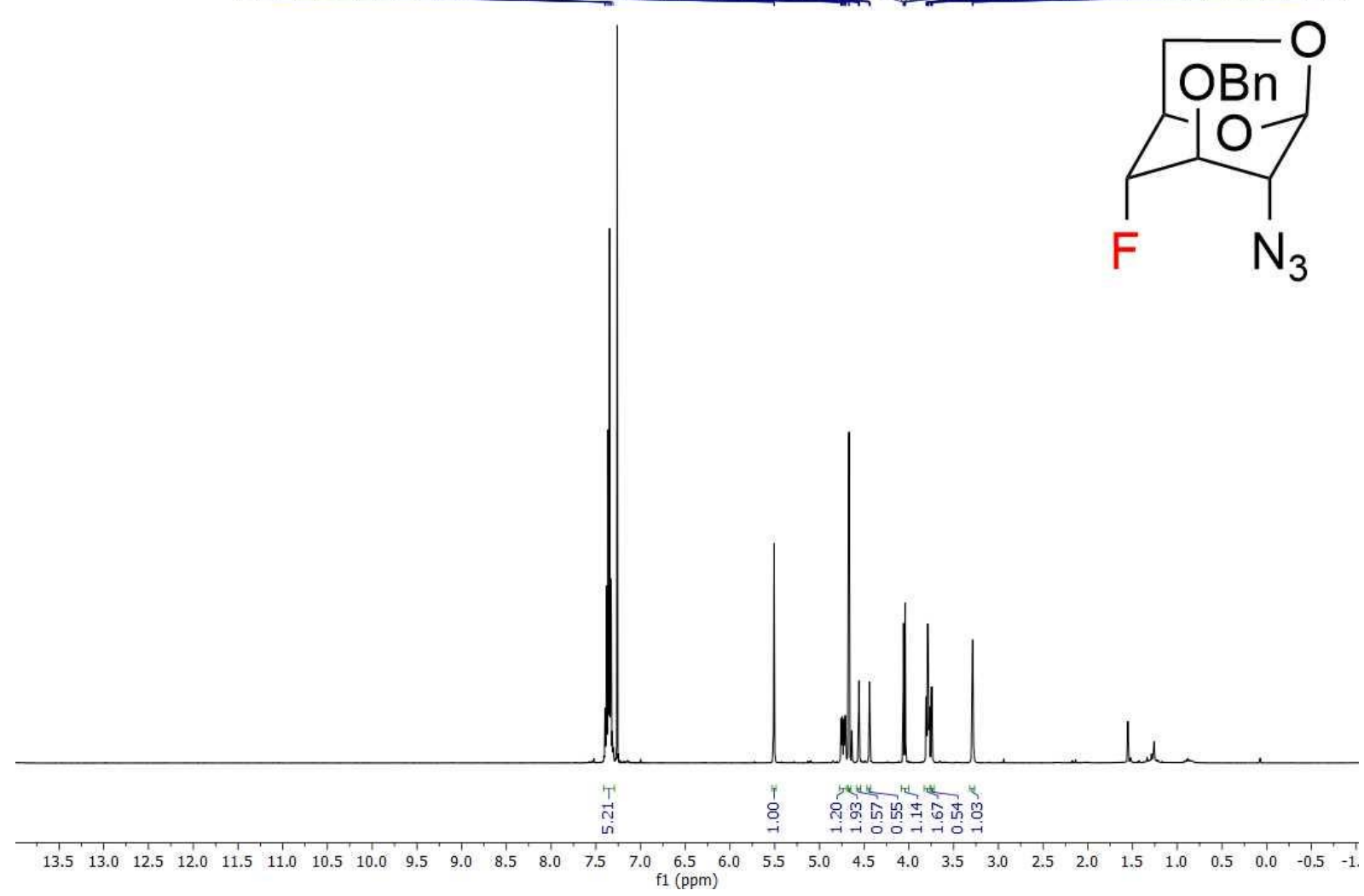


${ }^{13} \mathrm{C}\left\{{ }^{1} \mathrm{H}\right\}$ APT NMR 100MHz, $\mathrm{CDCl}_{3} 13$

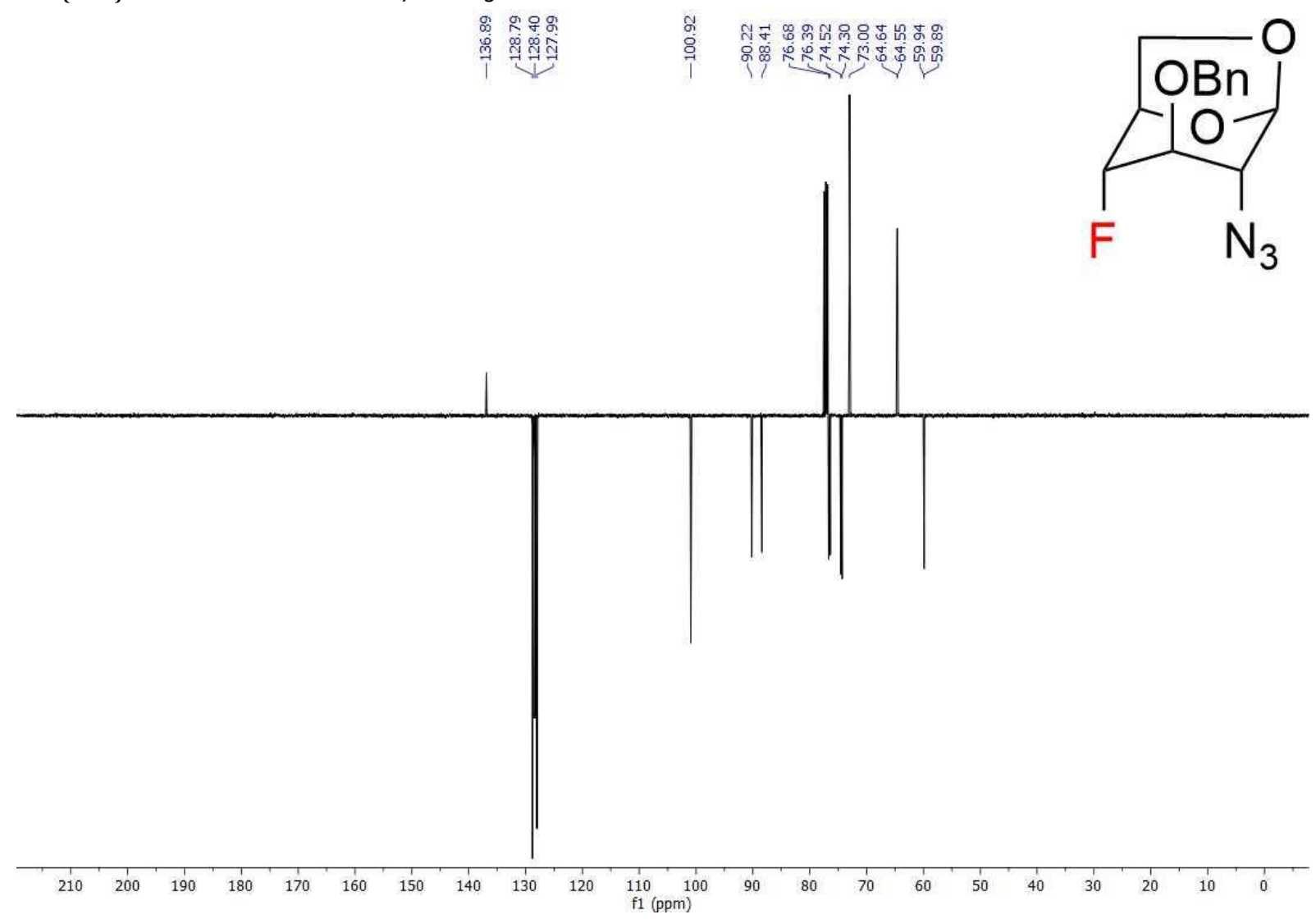

${ }^{1} \mathrm{H}-{ }^{1} \mathrm{H}$ COSY NMR 13

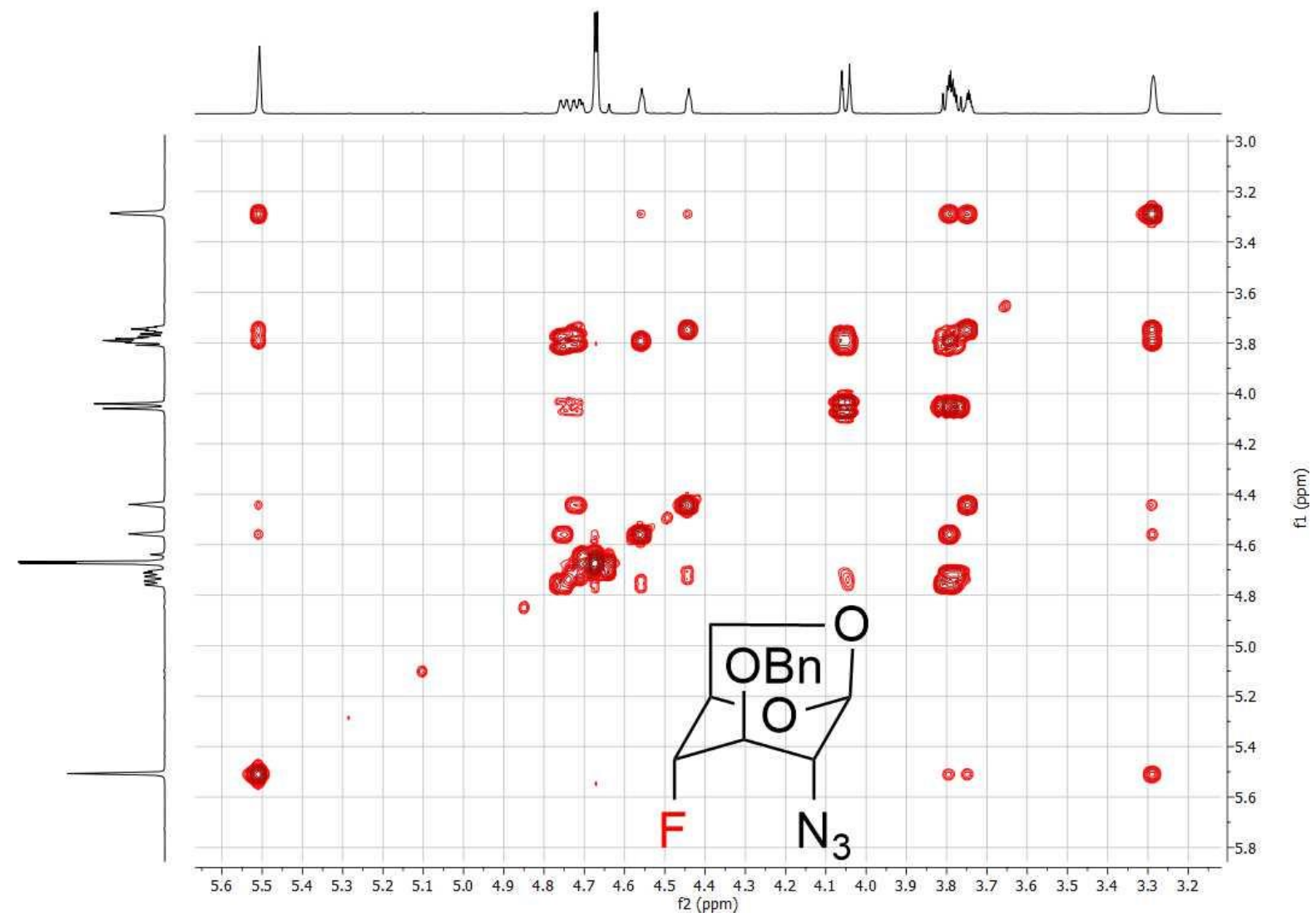


${ }^{1} \mathrm{H}^{13} \mathrm{C}$ HSQC NMR 13

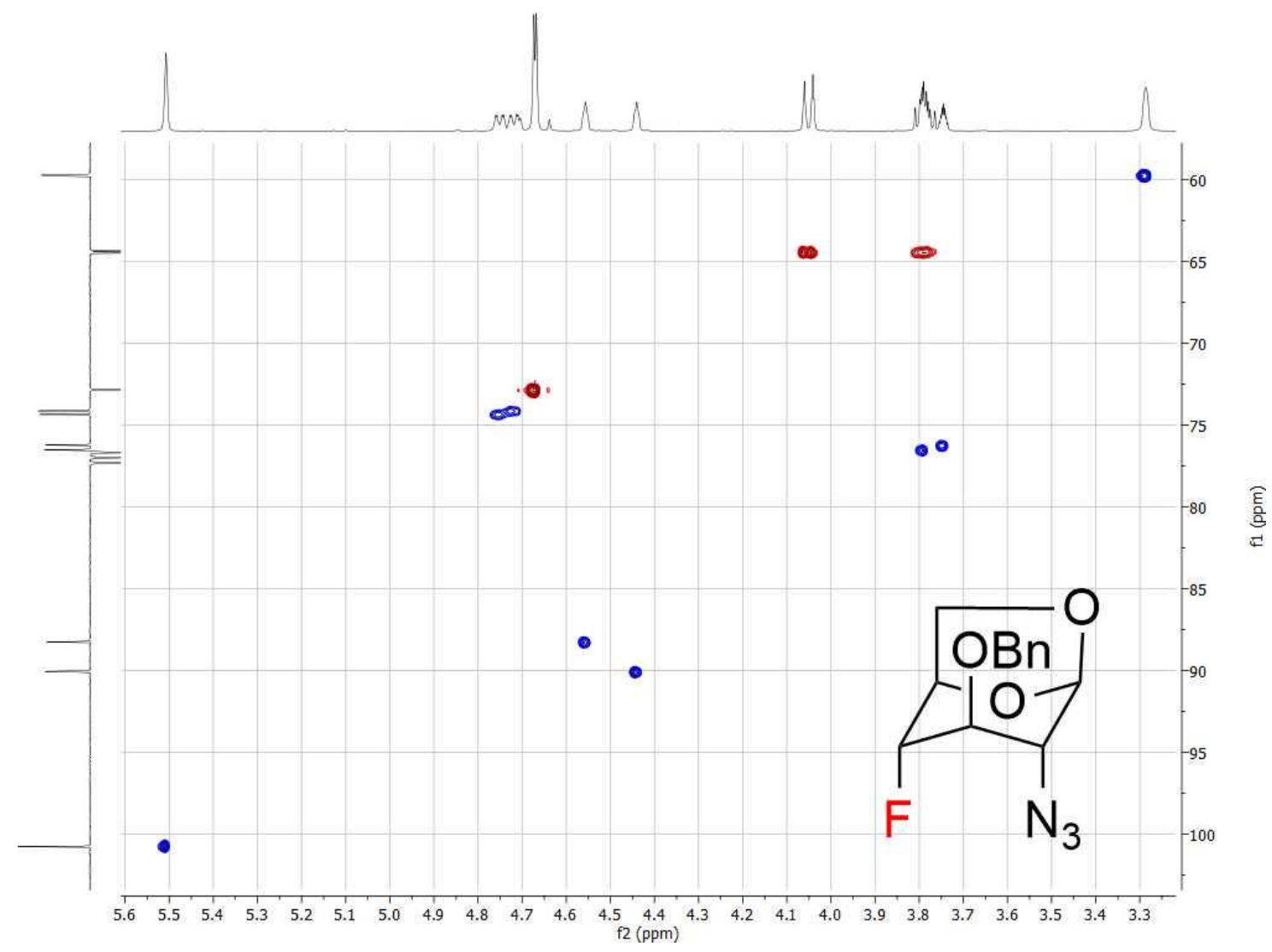

${ }^{1} \mathrm{H}-{ }^{13} \mathrm{C}$ HMBC NMR 13

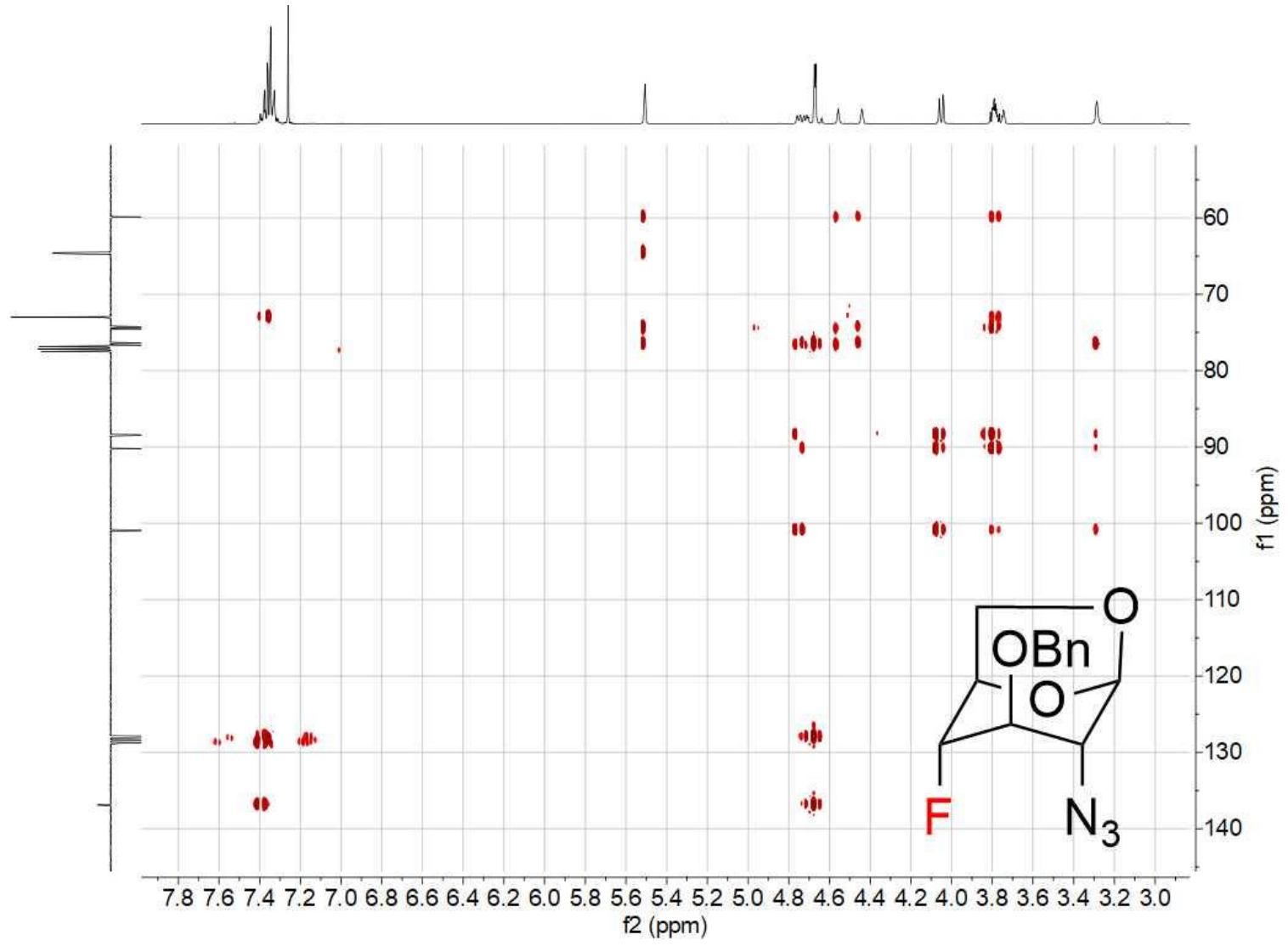


${ }^{19} \mathrm{~F} \mathrm{NMR} 376 \mathrm{MHz}, \mathrm{CDCl}_{3} 13$

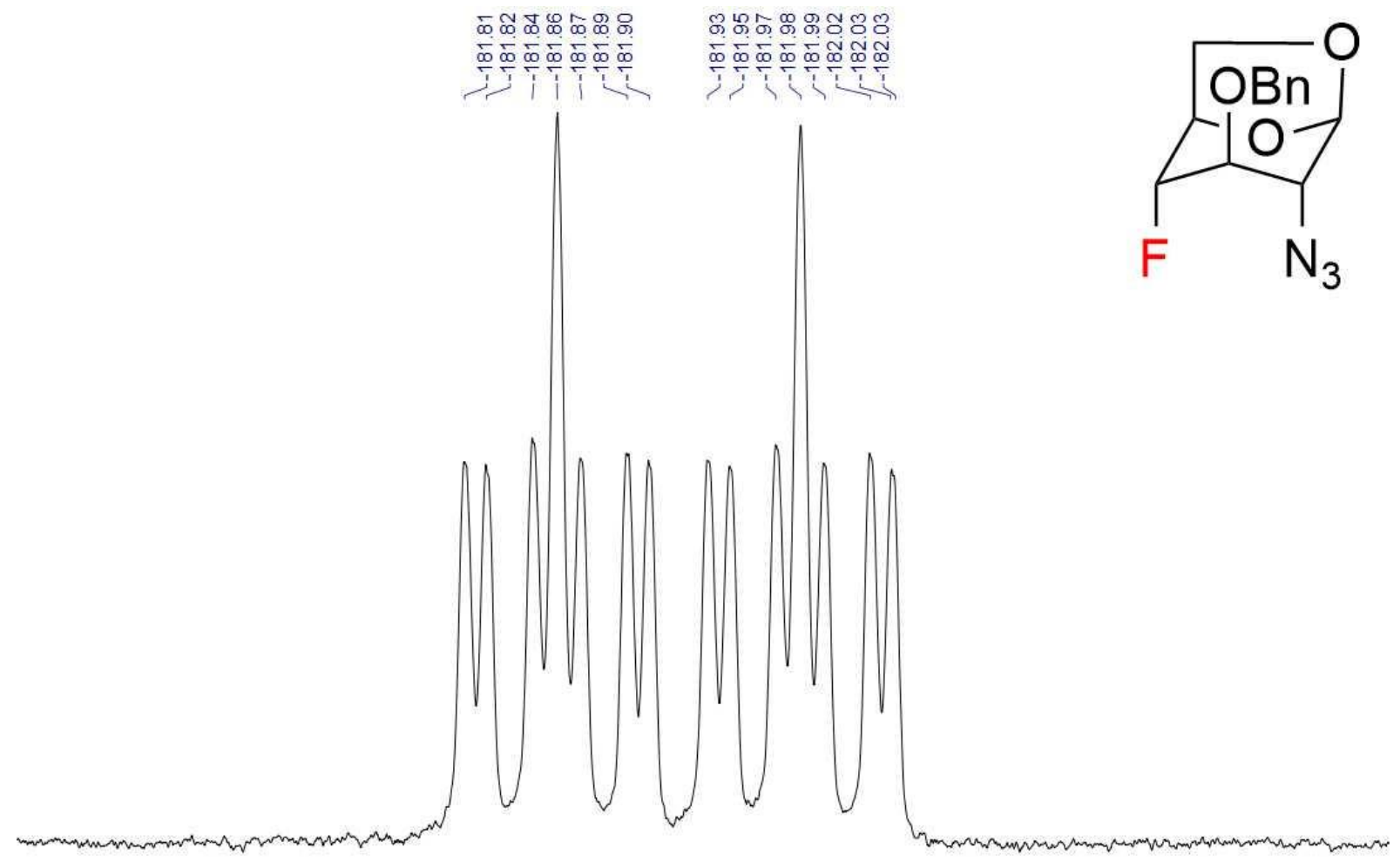

\begin{tabular}{lllllllllllllll}
\hline 181.60 & -181.65 & -181.70 & -181.75 & -181.80 & -181.85 & -181.90 & -181.95 & -182.00 & -182.05 & -182.10 & -182.15 & -182.20 & -182.25
\end{tabular} f1 (ppm) 
NMR $\alpha-14$

${ }^{1} \mathrm{H} \mathrm{NMR} 400 \mathrm{MHz}, \mathrm{CDCl}_{3} \boldsymbol{\alpha}-14$ and cca $9 \% \boldsymbol{\beta}-14$

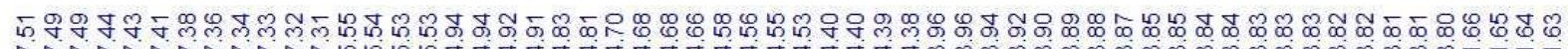

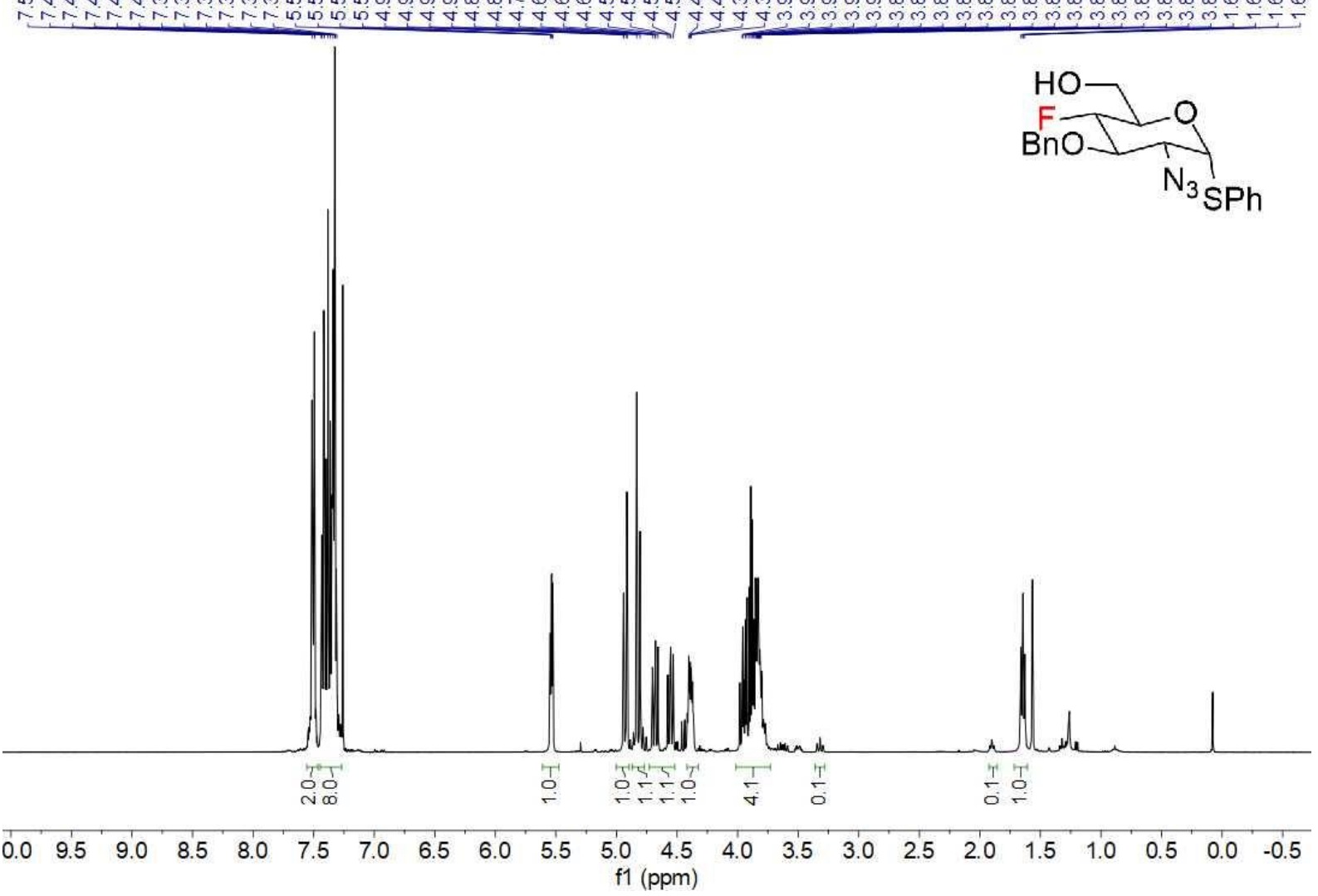

${ }^{13} \mathrm{C}\left\{{ }^{1} \mathrm{H}\right\}$ APT NMR $100 \mathrm{MHz}, \mathrm{CDCl}_{3} \boldsymbol{\alpha}-\mathbf{1 4}$ and cca $\mathbf{9 \%} \boldsymbol{\beta}-\mathbf{1 4}$

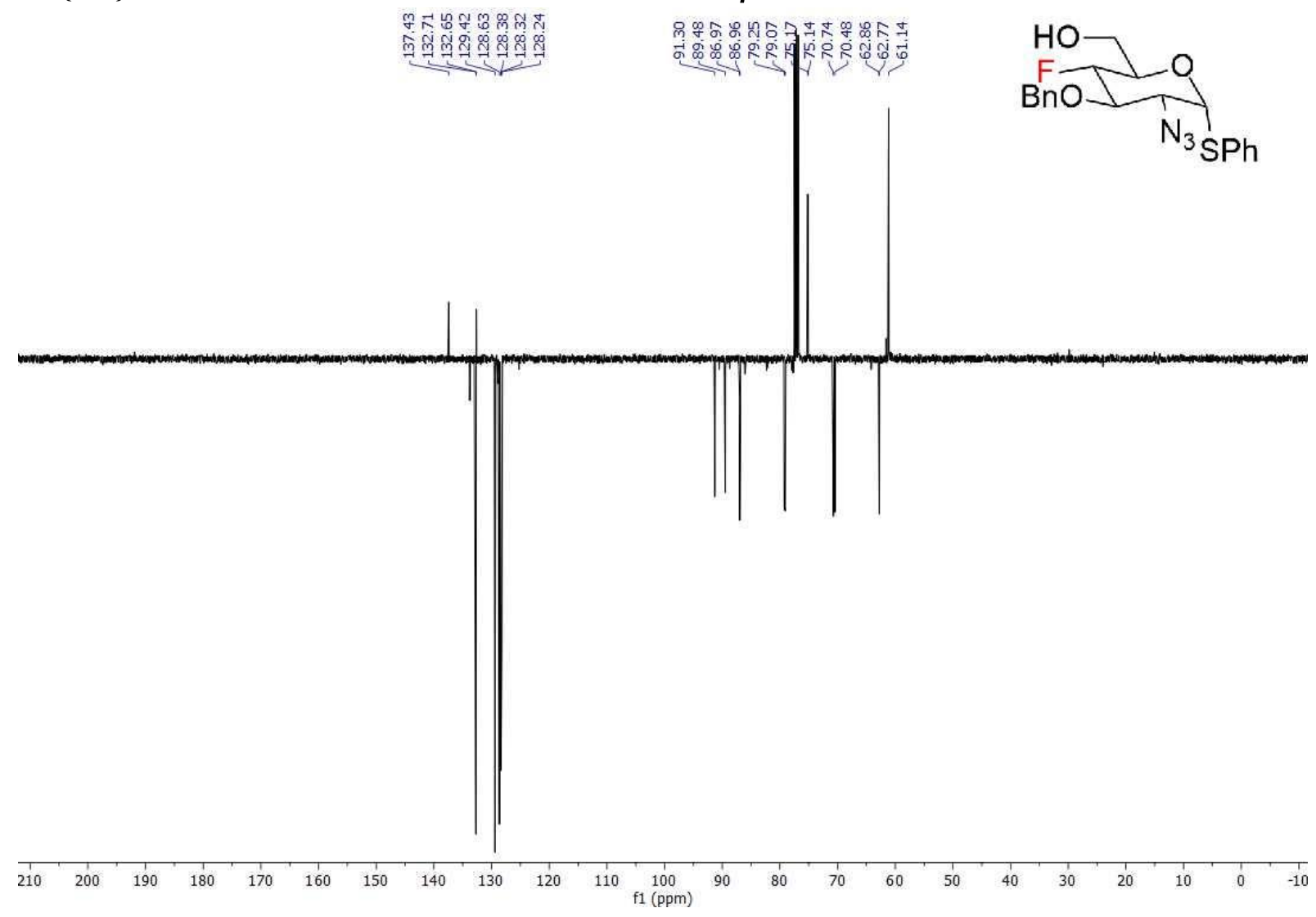


${ }^{1} \mathrm{H}-{ }^{1} \mathrm{H}$ COSY NMR $\alpha-14$ and cca $\mathbf{9 \%} \beta-14$

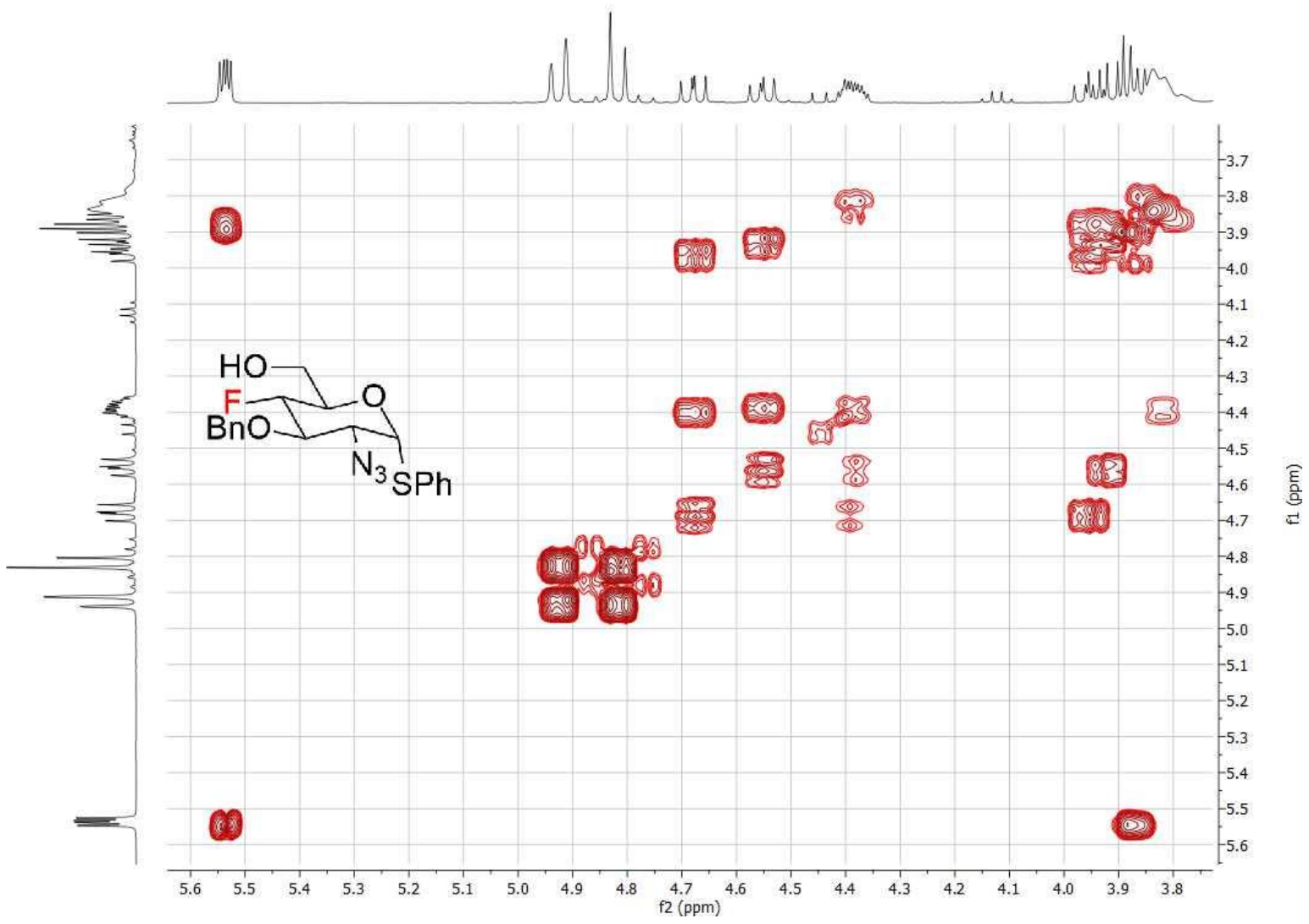

${ }^{1} \mathrm{H}-{ }^{13} \mathrm{C}$ HSQC NMR $\boldsymbol{\alpha}-14$ and cca $\mathbf{9 \%} \beta-14$

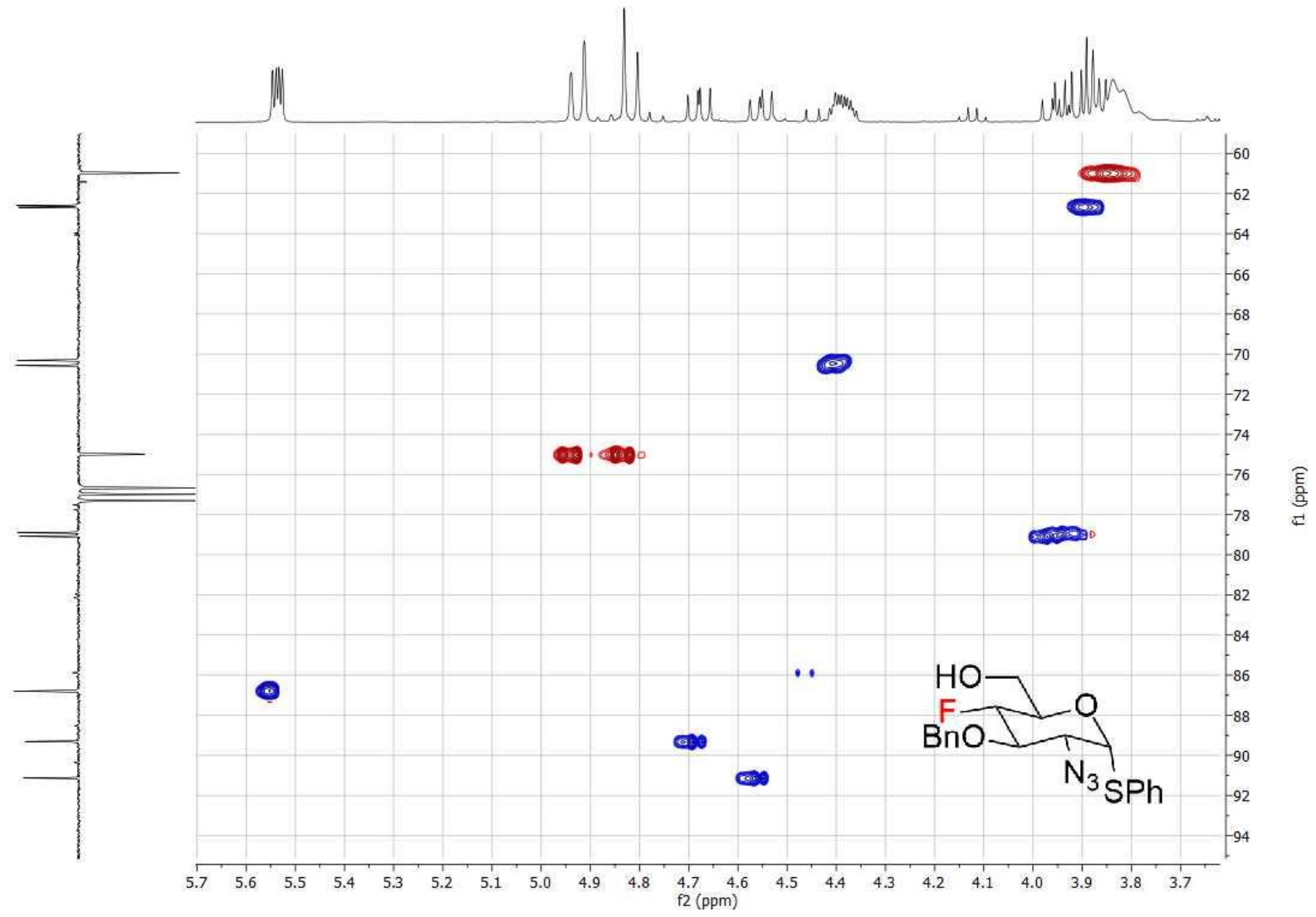


${ }^{1} \mathrm{H}_{-}{ }^{13} \mathrm{C}$ HMBC NMR $\alpha-14$ and cca $\mathbf{9 \%} \boldsymbol{\beta}-14$

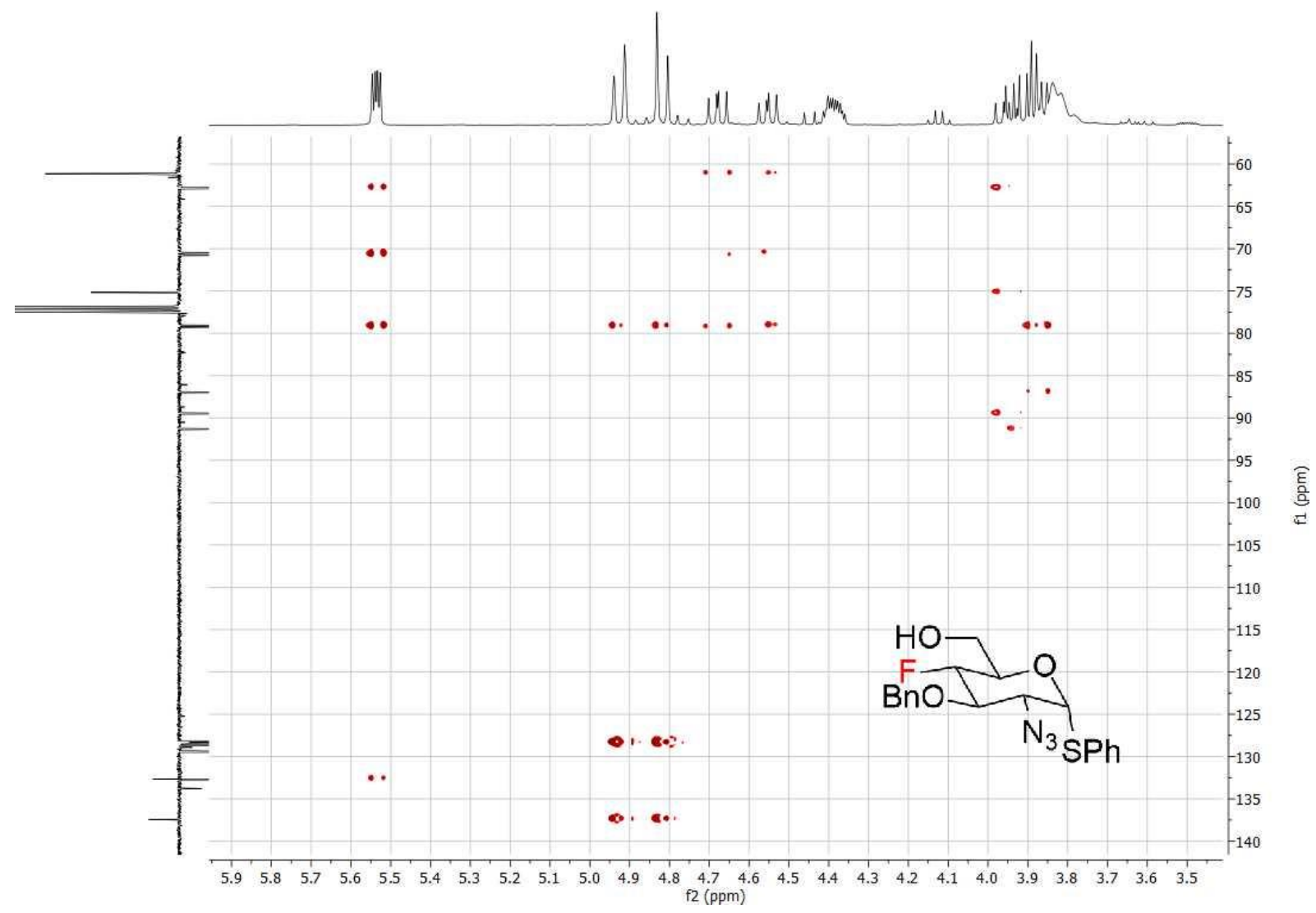

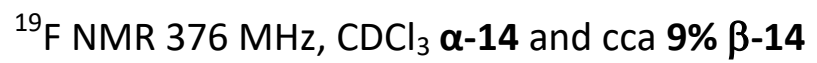

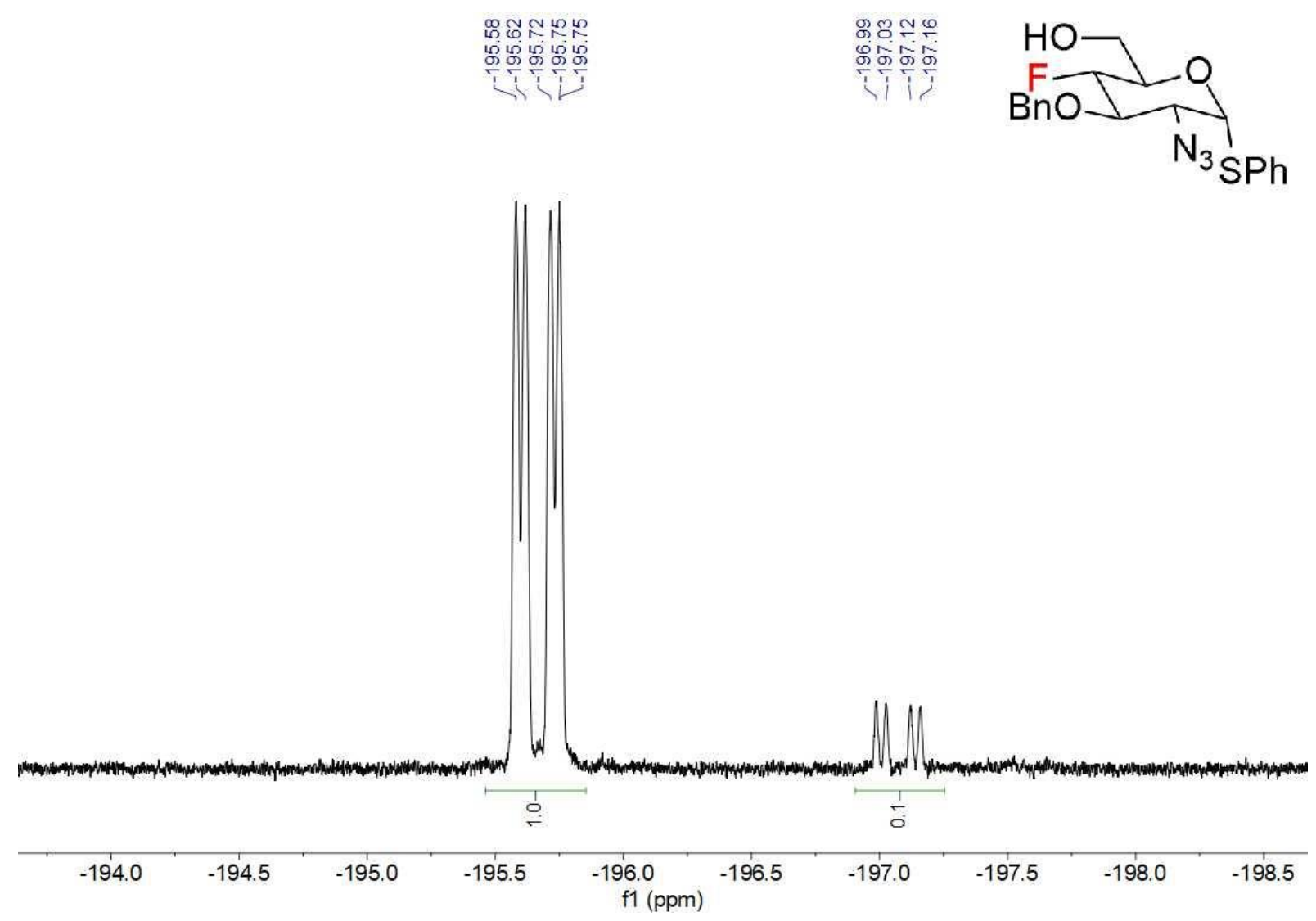




\section{NMR $\beta-14$}

${ }^{1} \mathrm{HNMR} 400 \mathrm{MHz}, \mathrm{CDCl}_{3} \beta-14$

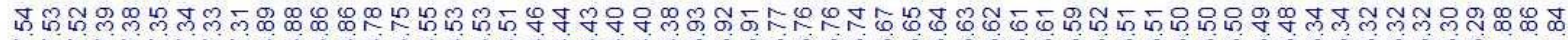

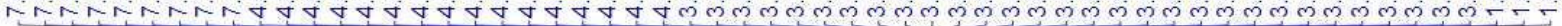

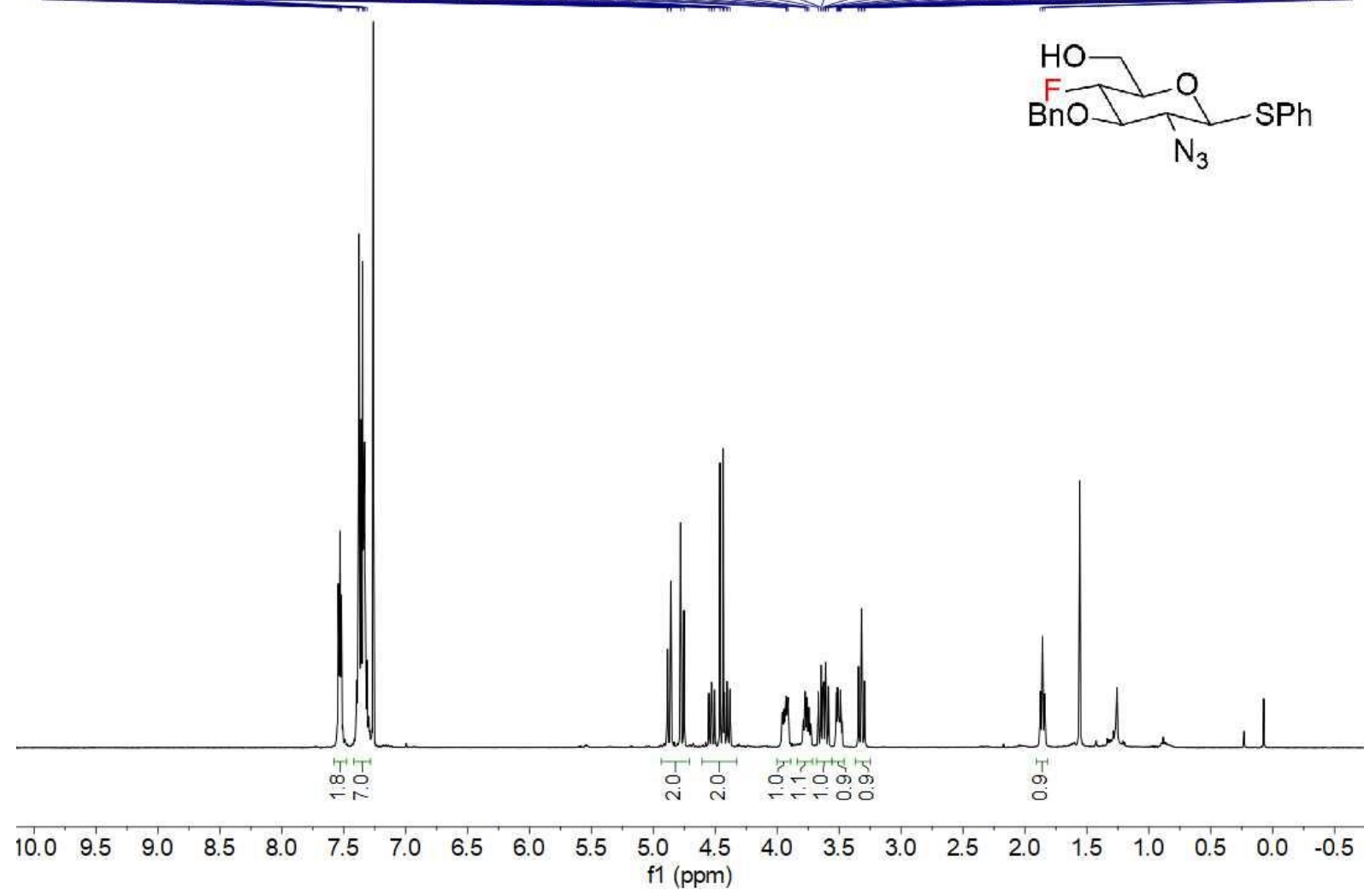

${ }^{13} \mathrm{C}\left\{{ }^{1} \mathrm{H}\right\}$ NMR $100 \mathrm{MHz}, \mathrm{CDCl}_{3} \boldsymbol{\beta}-14$

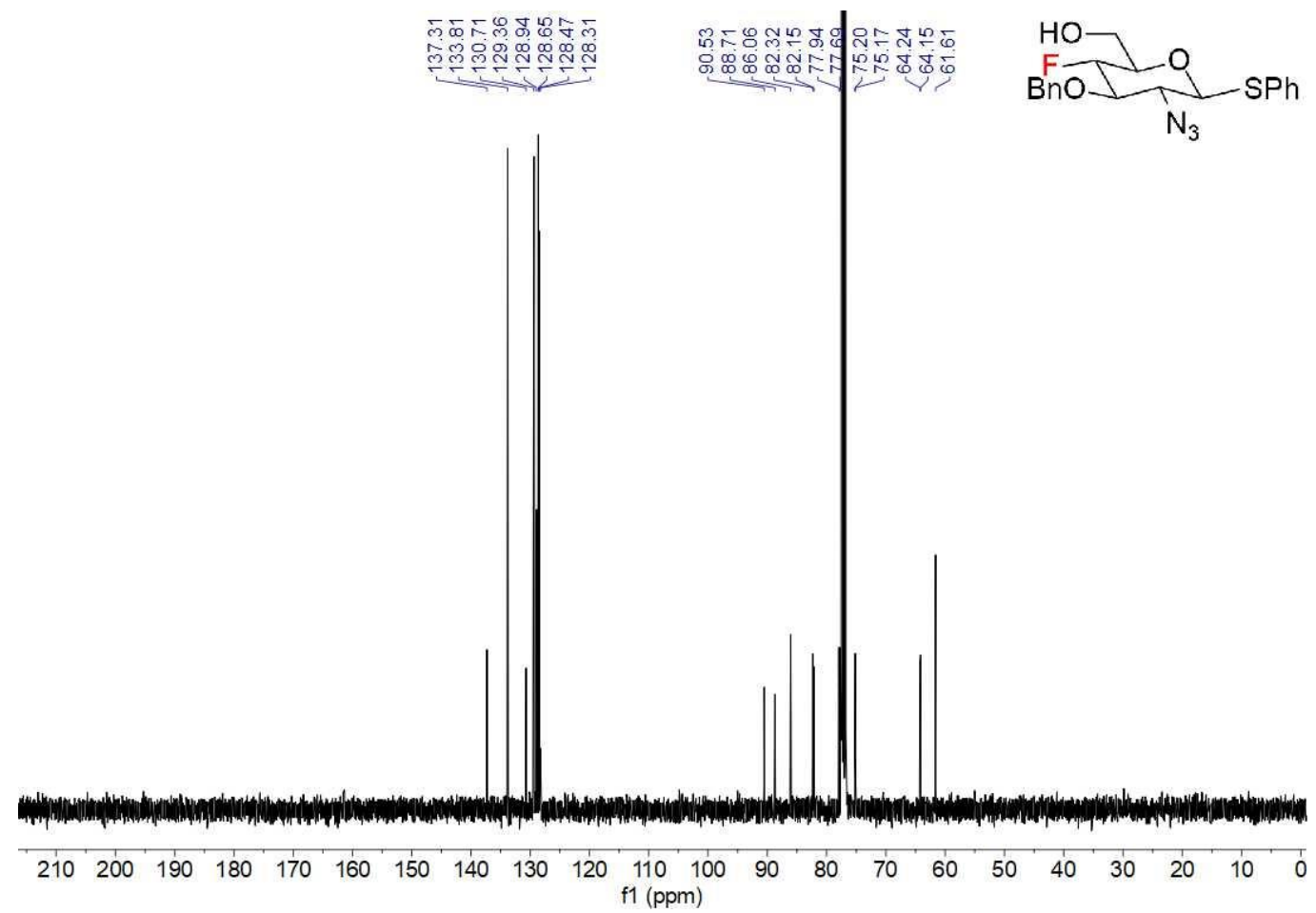


${ }^{19} \mathrm{~F} N M R 376 \mathrm{MHz}, \mathrm{CDCl}_{3} \boldsymbol{\beta}-14$

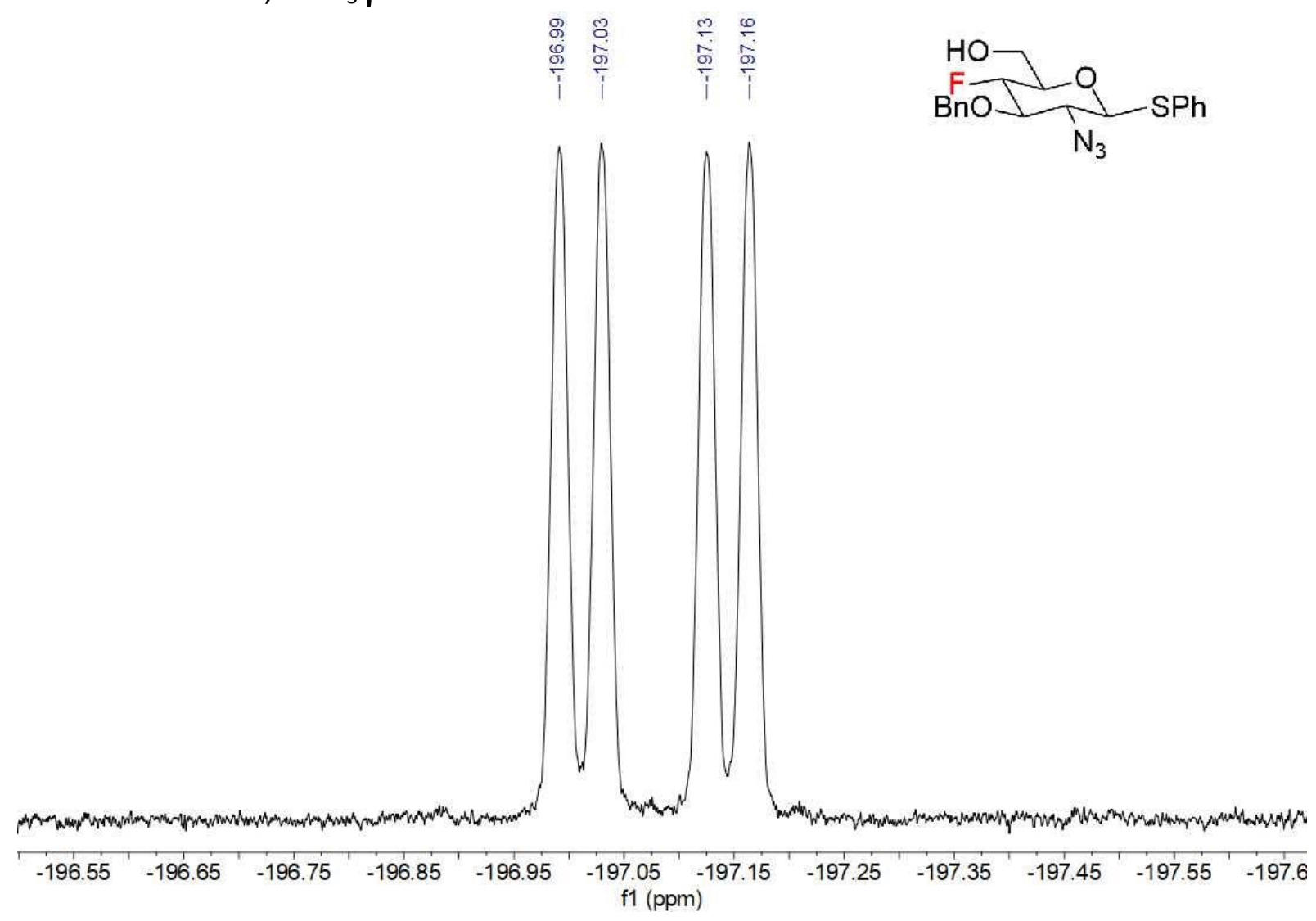

\section{NMR 14}

${ }^{1} \mathrm{H}-{ }^{1} \mathrm{H}$ COSY NMR $14(\alpha: \beta=0.6: 1.0)$

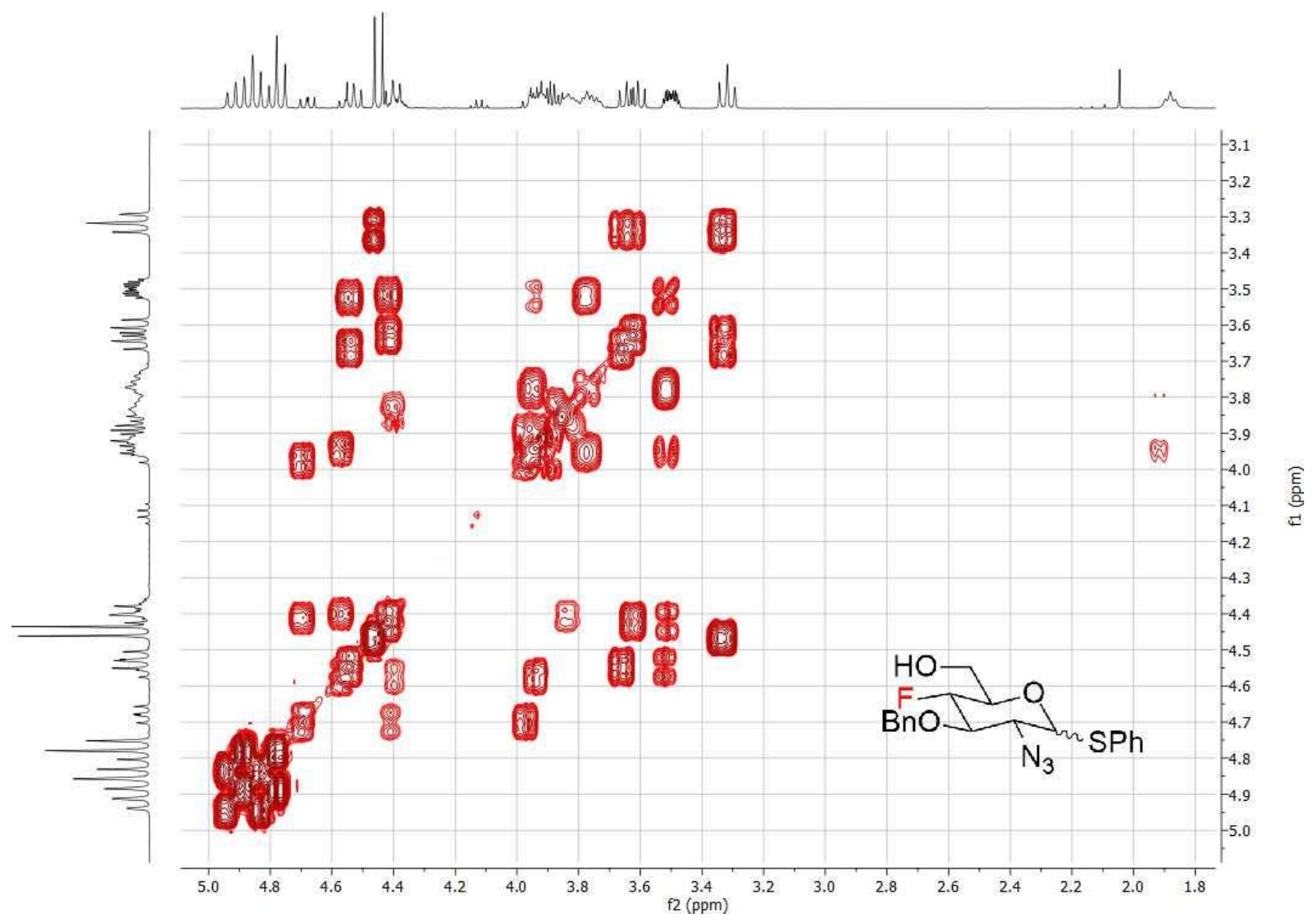


${ }^{1} \mathrm{H}-{ }^{13} \mathrm{C}$ HSQC NMR $14(\alpha: \beta=0.6: 1.0)$

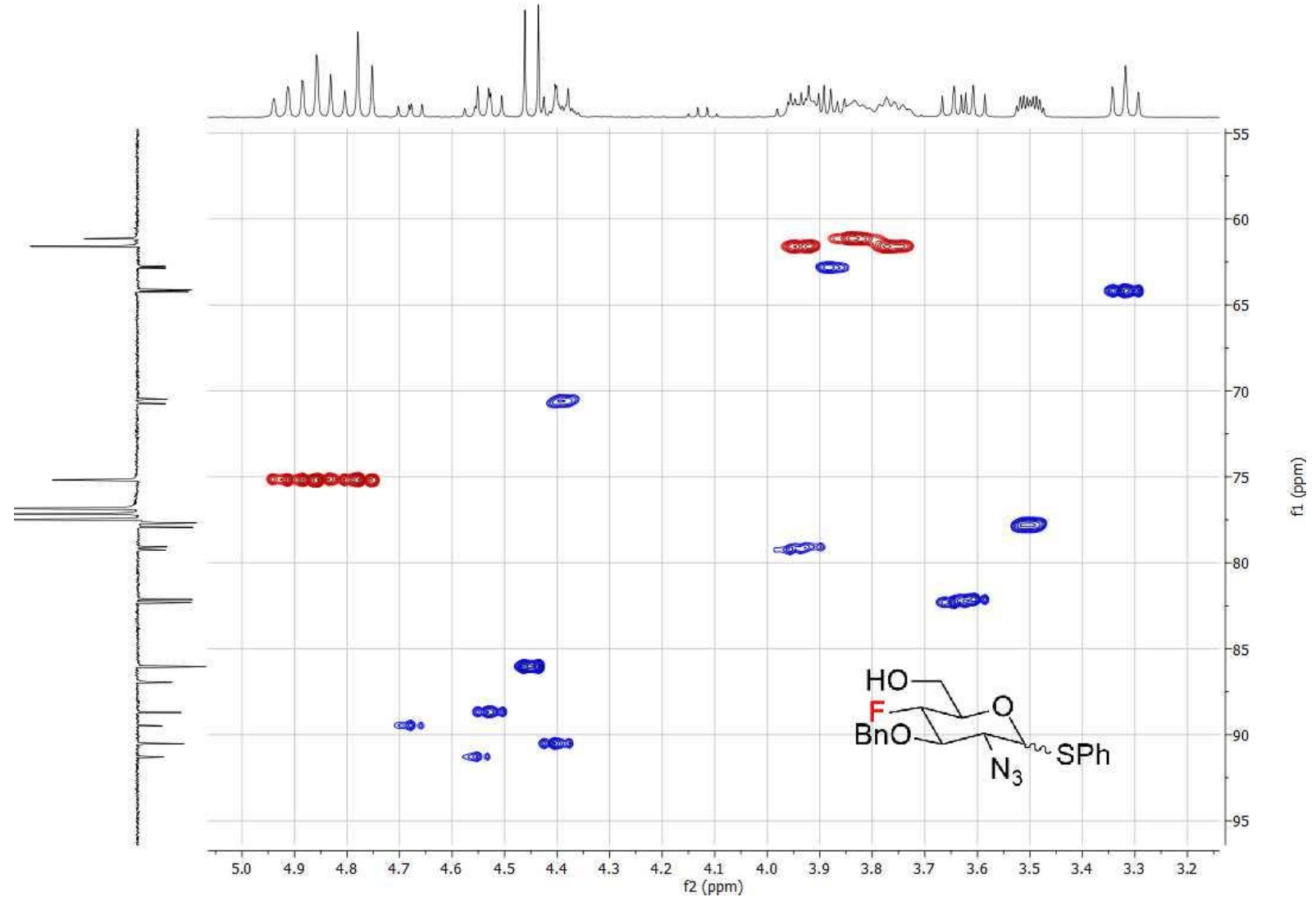

${ }^{1} \mathrm{H}-{ }^{13} \mathrm{C}$ HMBC NMR $14(\alpha: \beta=0.6: 1.0)$

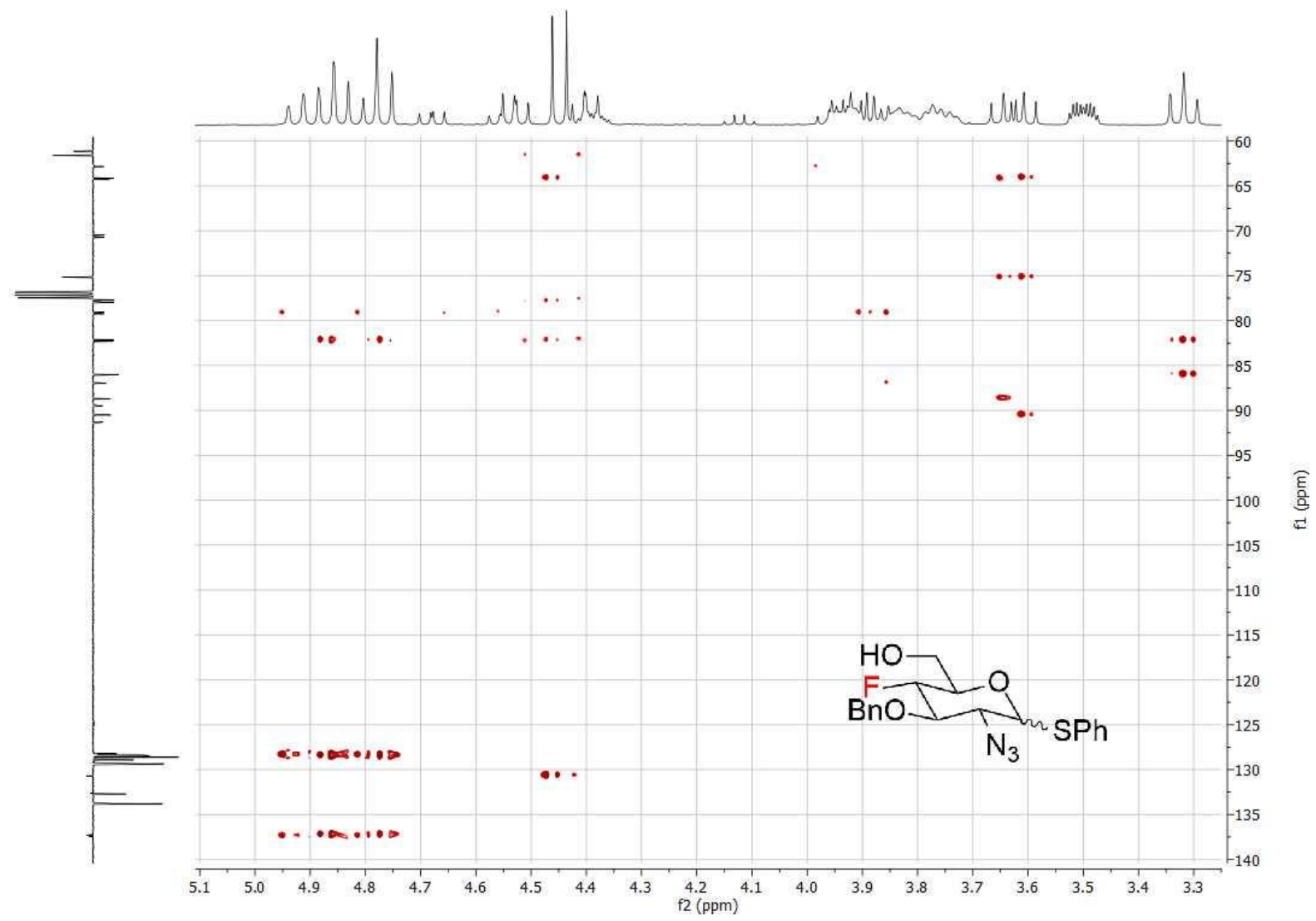


NMR $\alpha-2$

${ }^{1} \mathrm{H} N M R 400 \mathrm{MHz}, \mathrm{CDCl}_{3} \boldsymbol{\alpha}-\mathbf{2}$ and cca $9 \% \beta-2$

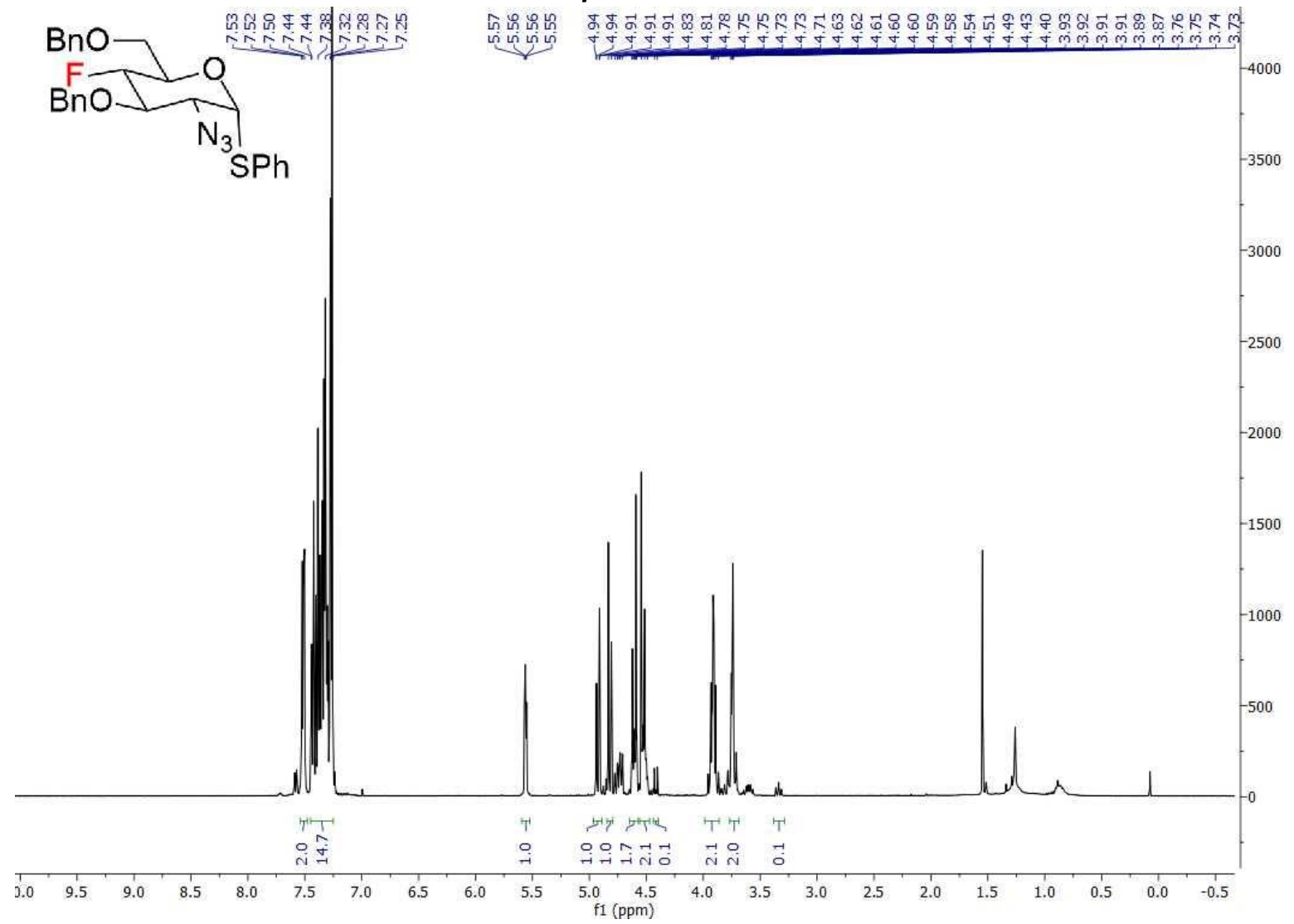

Simulated ${ }^{1} \mathrm{H}$ NMR of $\alpha-2$ anomer in Mnova and ${ }^{1} \mathrm{H}$ NMR $400 \mathrm{MHz}, \mathrm{CDCl}_{3} \alpha-2$ and cca $9 \% \beta-2$

Simulated $1 \mathrm{H}$ NMR spectrum

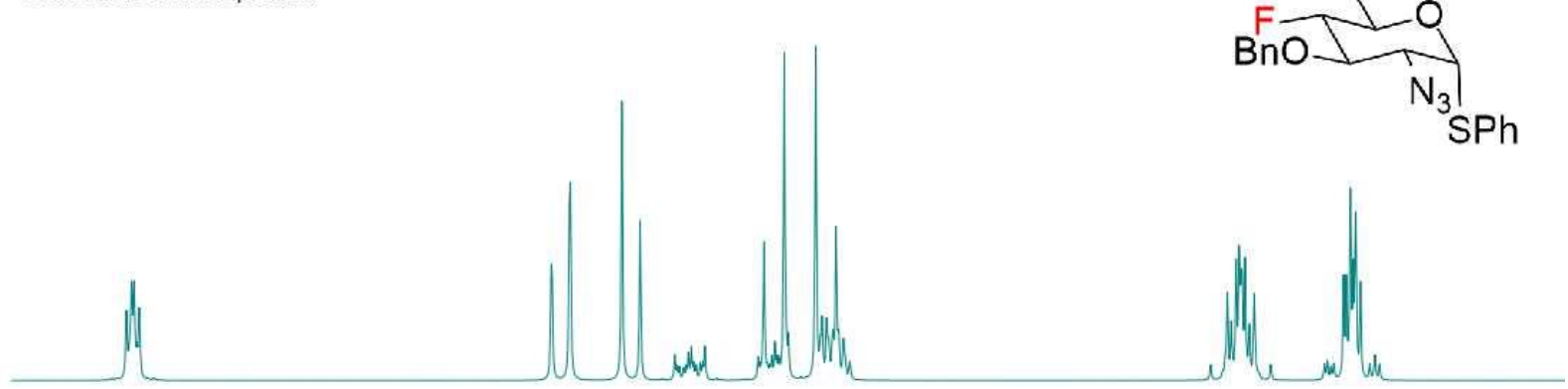

$1 \mathrm{H}$ NMR spectrum

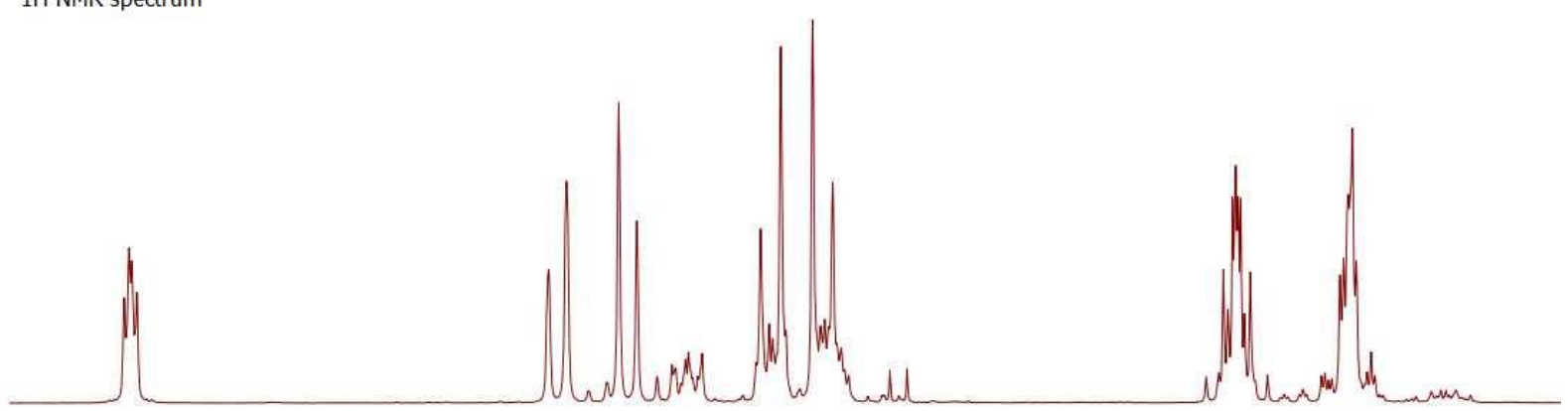

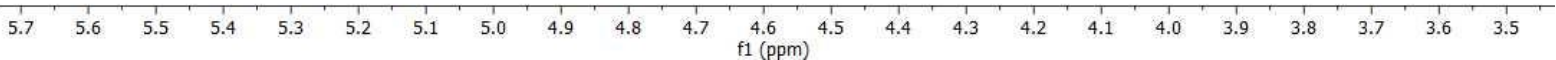


${ }^{13} \mathrm{C}\left\{{ }^{1} \mathrm{H}\right\}$ APT NMR $100 \mathrm{MHz}, \mathrm{CDCl}_{3} \boldsymbol{\alpha}-\mathbf{2}$ and cca $9 \% \boldsymbol{\beta}-\mathbf{2}$

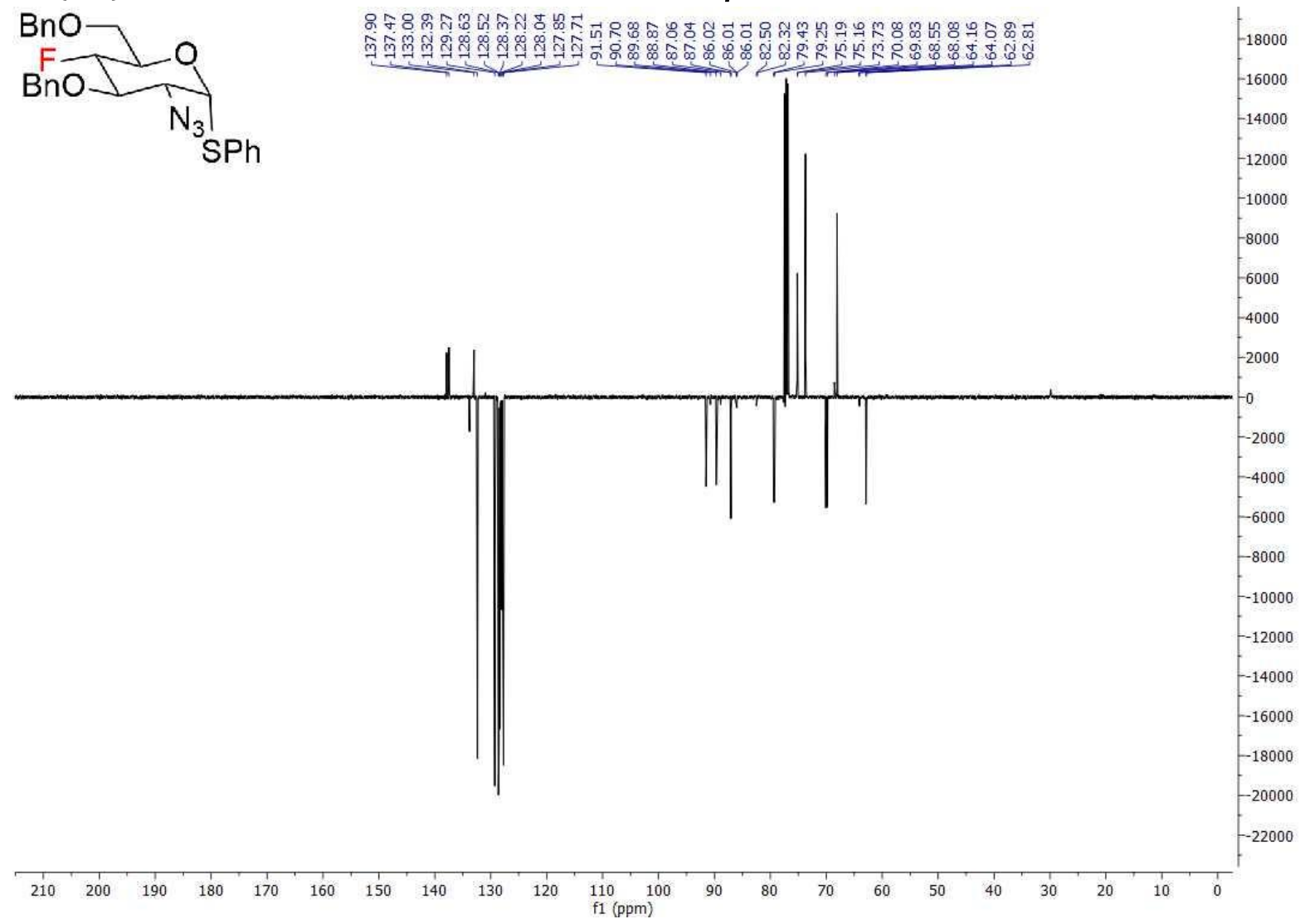

${ }^{1} \mathrm{H}-{ }^{1} \mathrm{H}$ COSY NMR $\alpha-2$ and cca $9 \% \beta-2$

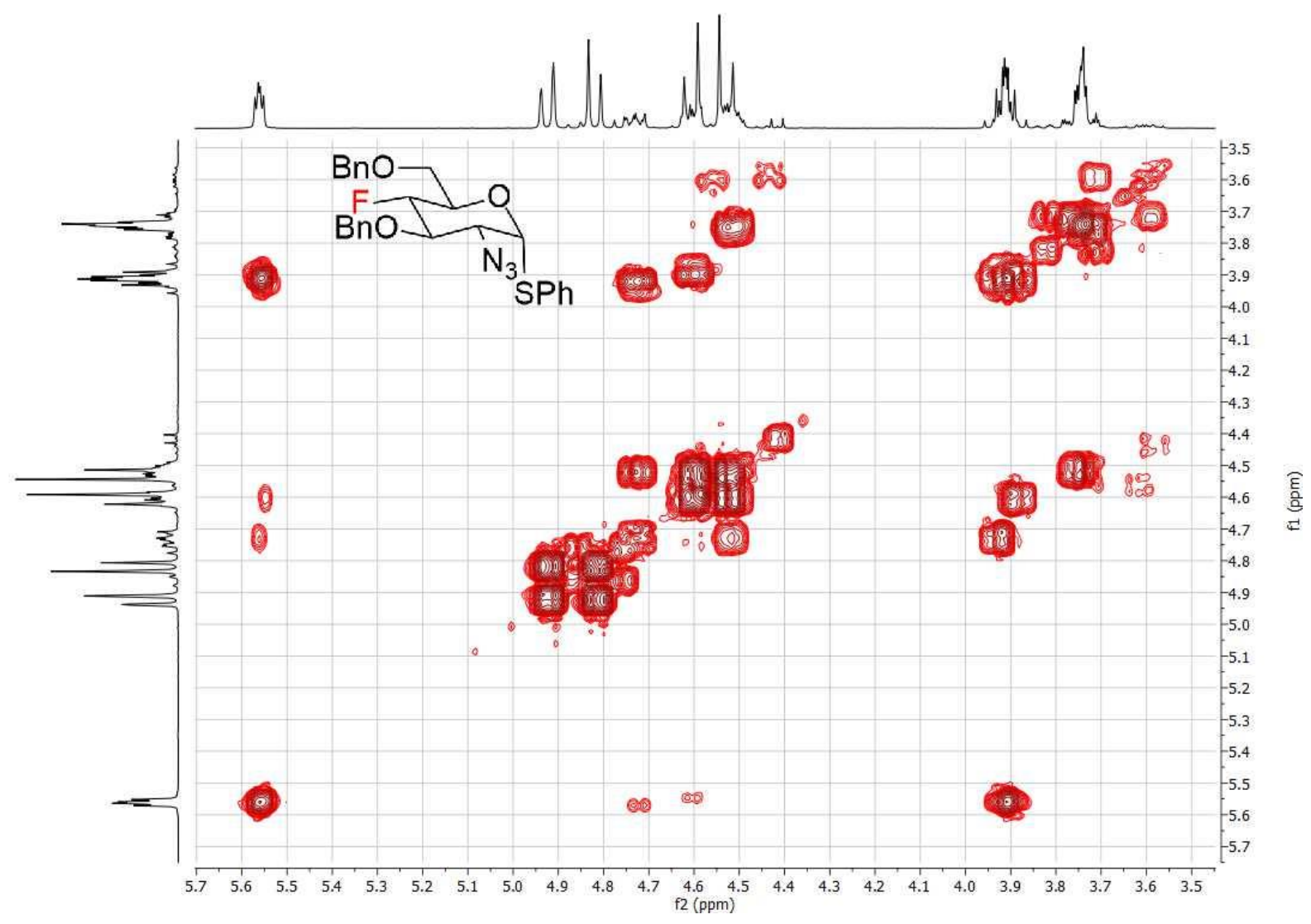




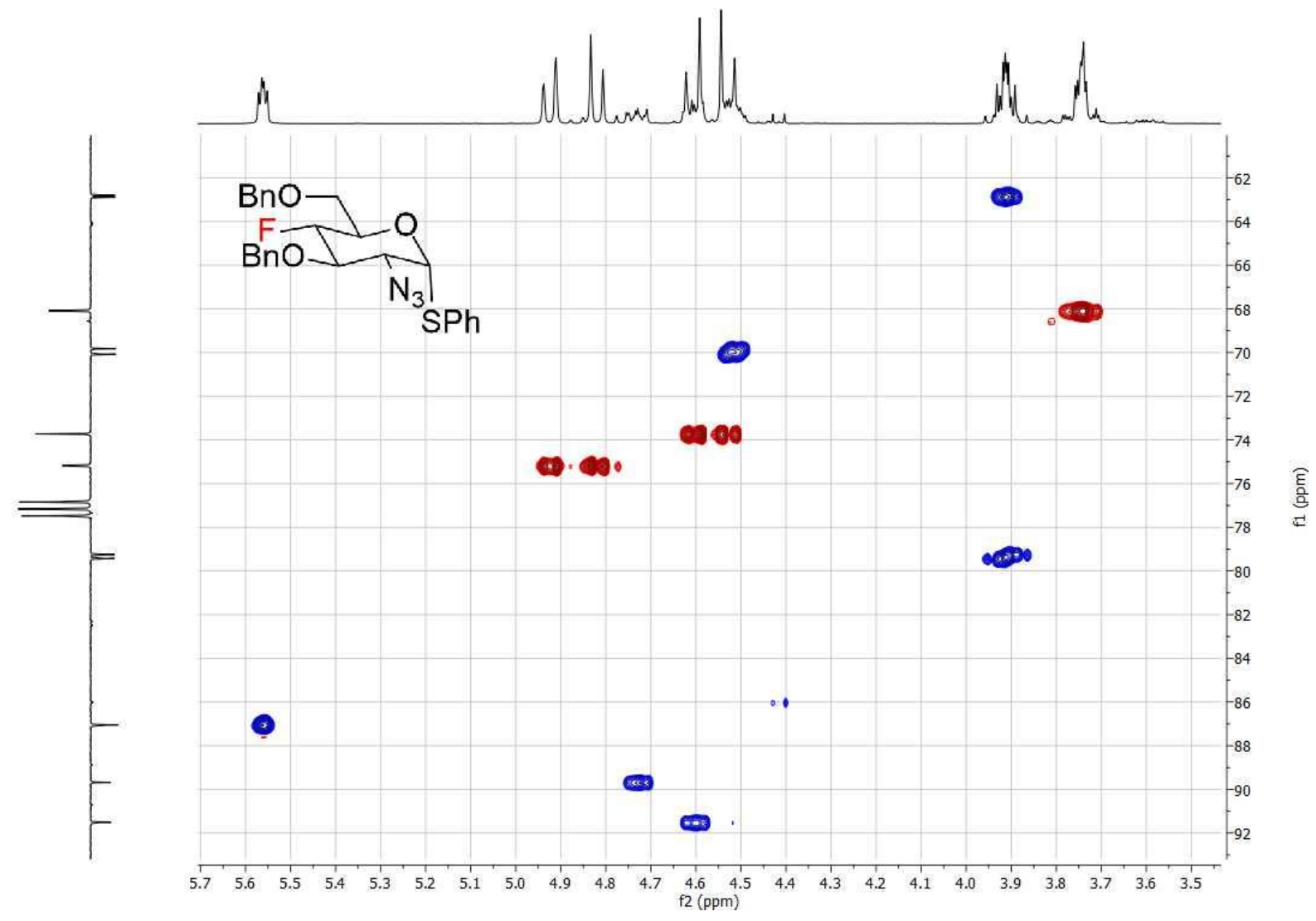

${ }^{1} \mathrm{H}-{ }^{13} \mathrm{C}$ HMBC NMR $\alpha-2$ and cca $9 \% \beta-2$

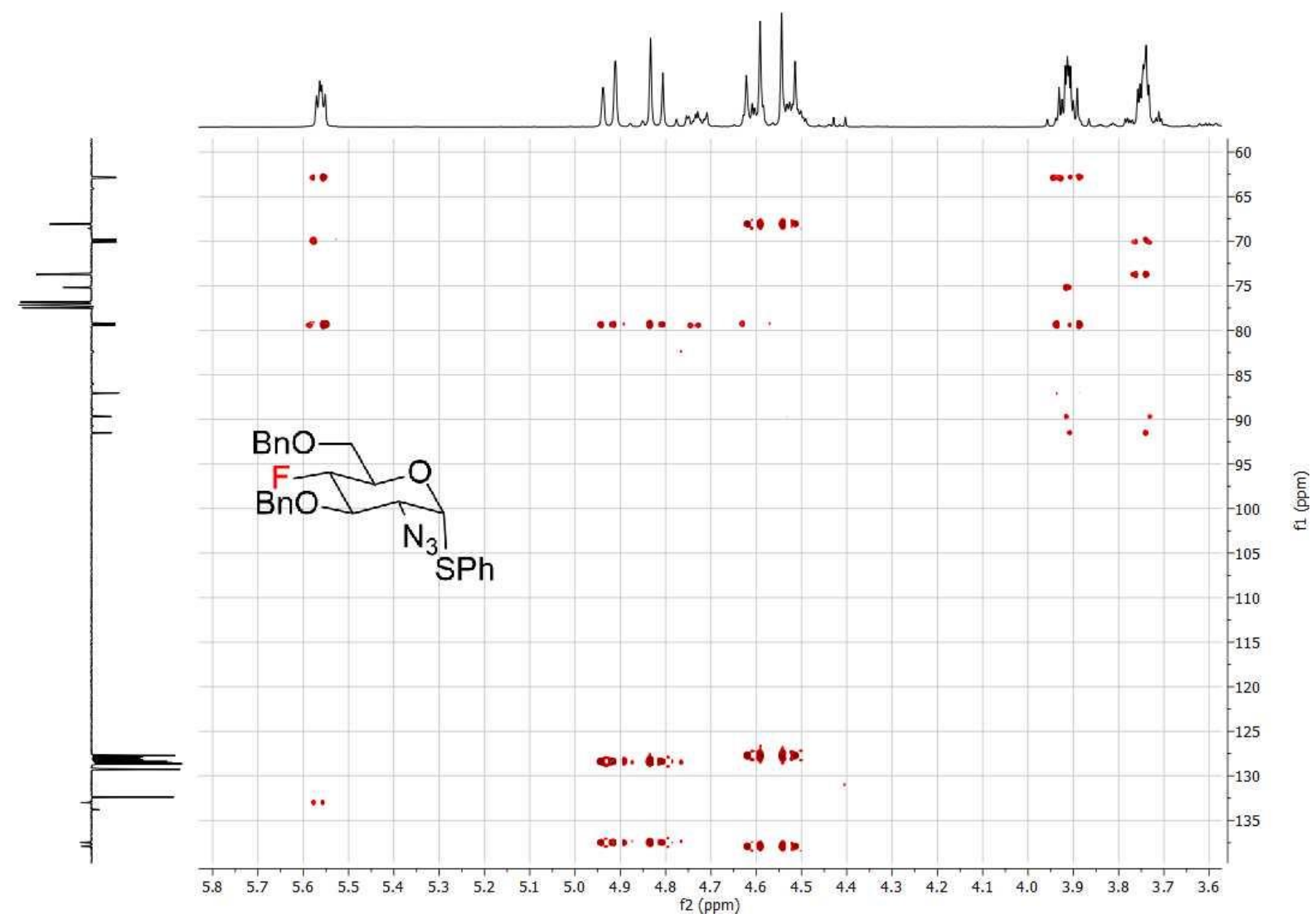


${ }^{19} \mathrm{~F} \mathrm{NMR} 376 \mathrm{MHz}, \mathrm{CDCl}_{3} \boldsymbol{\alpha}-2$ and cca $9 \% \boldsymbol{\beta}-2$

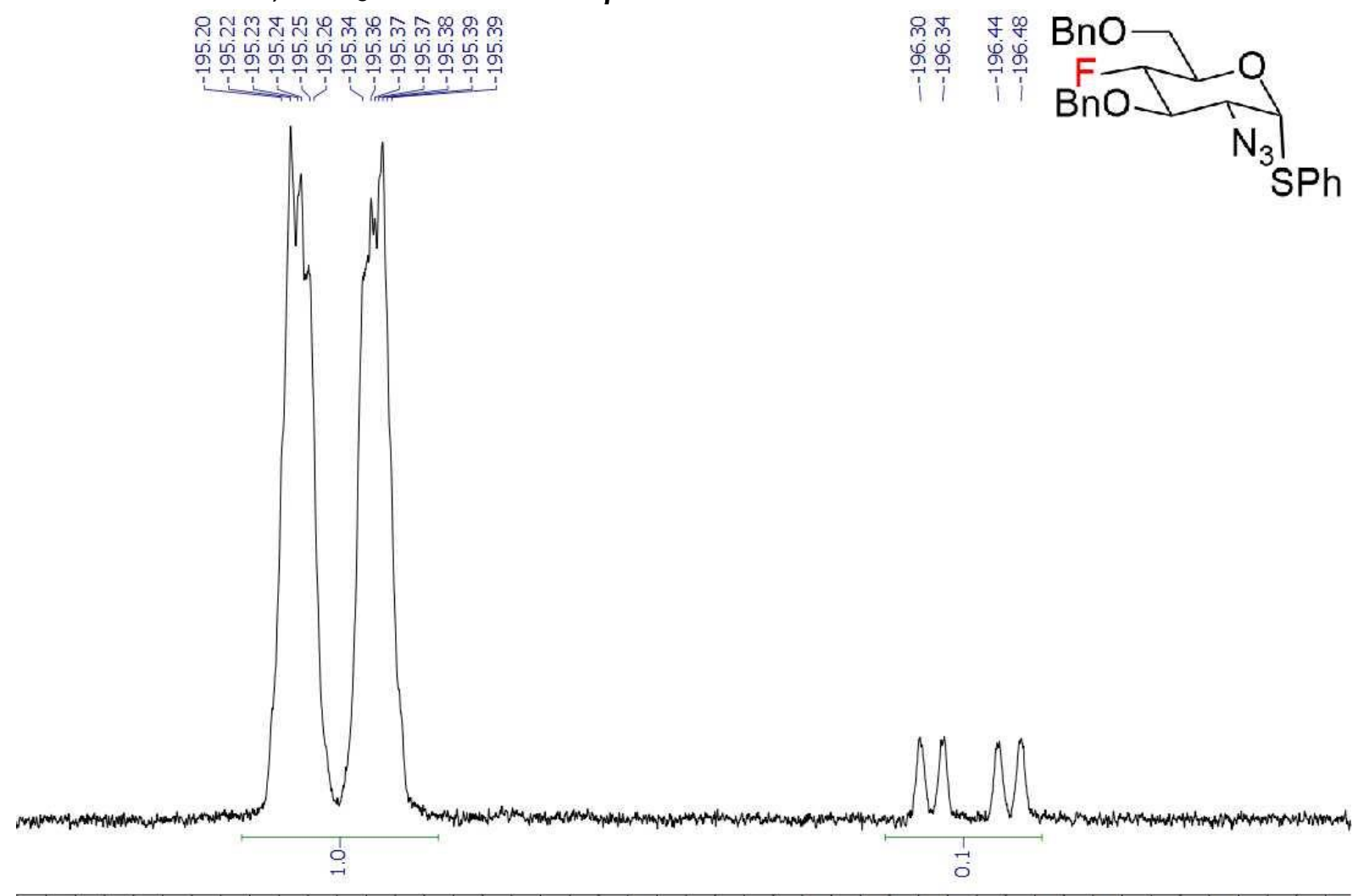

\begin{tabular}{lllllllllllllllllllllllllllllll}
\hline-194.8 & -194.9 & -195.0 & -195.1 & -195.2 & -195.3 & -195.4 & -195.5 & -195.6 & -195.7 & -195.8 & -195.9 & -196.0 & -196.1 & -196.2 & -196.3 & -196.4 & -196.5 & -196.6 & -196.7 & -196.8 & -196.9 & -197.0
\end{tabular} $\mathrm{f} 1(\mathrm{ppm})$ 


\section{NMR 2}

${ }^{1} \mathrm{H} \mathrm{NMR} 400 \mathrm{MHz}, \mathrm{CDCl}_{3} \boldsymbol{2}(\boldsymbol{\alpha}: \boldsymbol{\beta}=0.5: 1.0)$

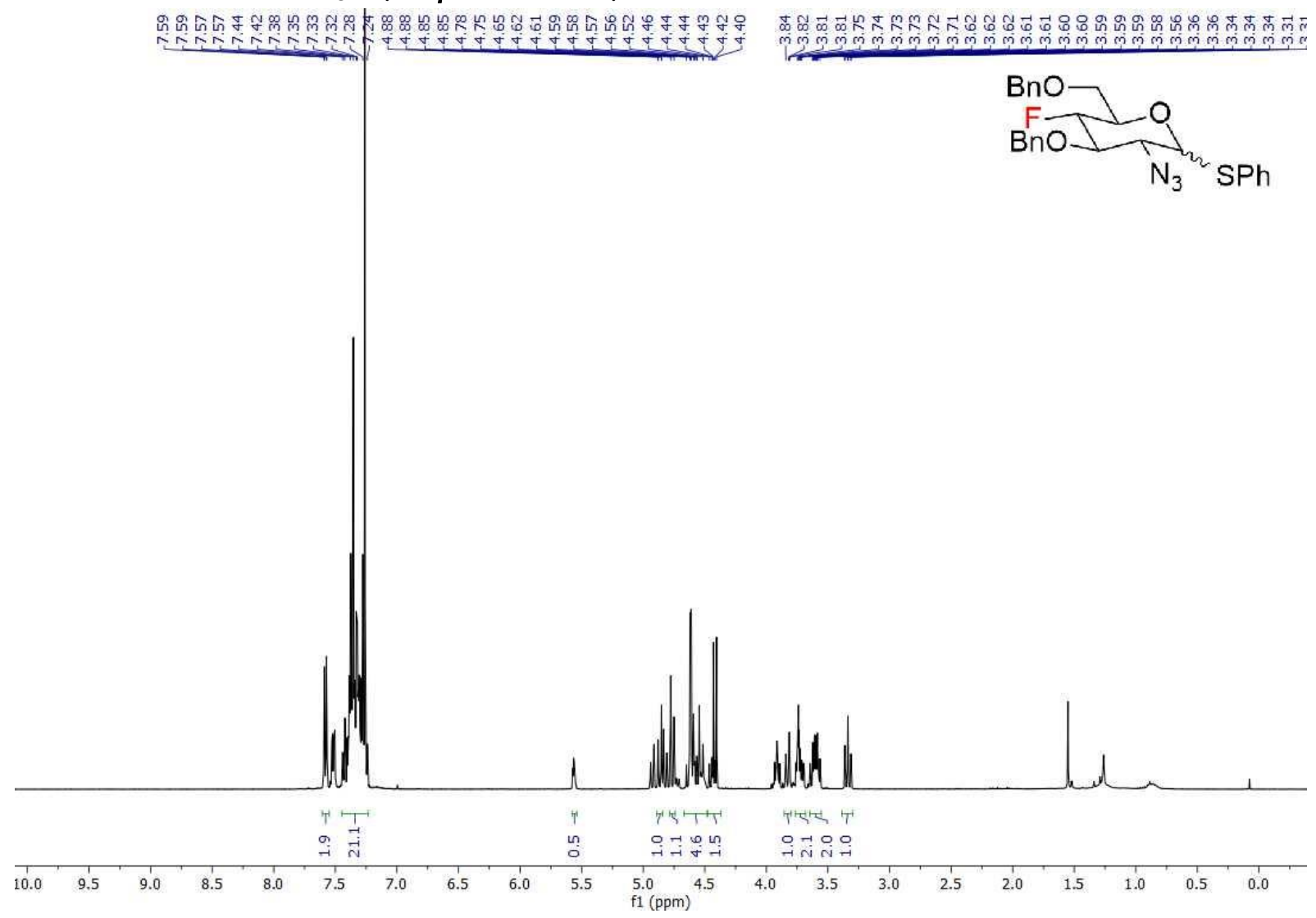

Simulated ${ }^{1} \mathrm{H} N M R$ of $\boldsymbol{\beta}-2$ anomer in Mnova and ${ }^{1} \mathrm{H} N \mathrm{MR} 400 \mathrm{MHz}, \mathrm{CDCl}_{3} \mathbf{2}(\boldsymbol{\alpha}: \boldsymbol{\beta}=0.5: 1.0)$

Simulated $1 \mathrm{H}$ NMR spectrum
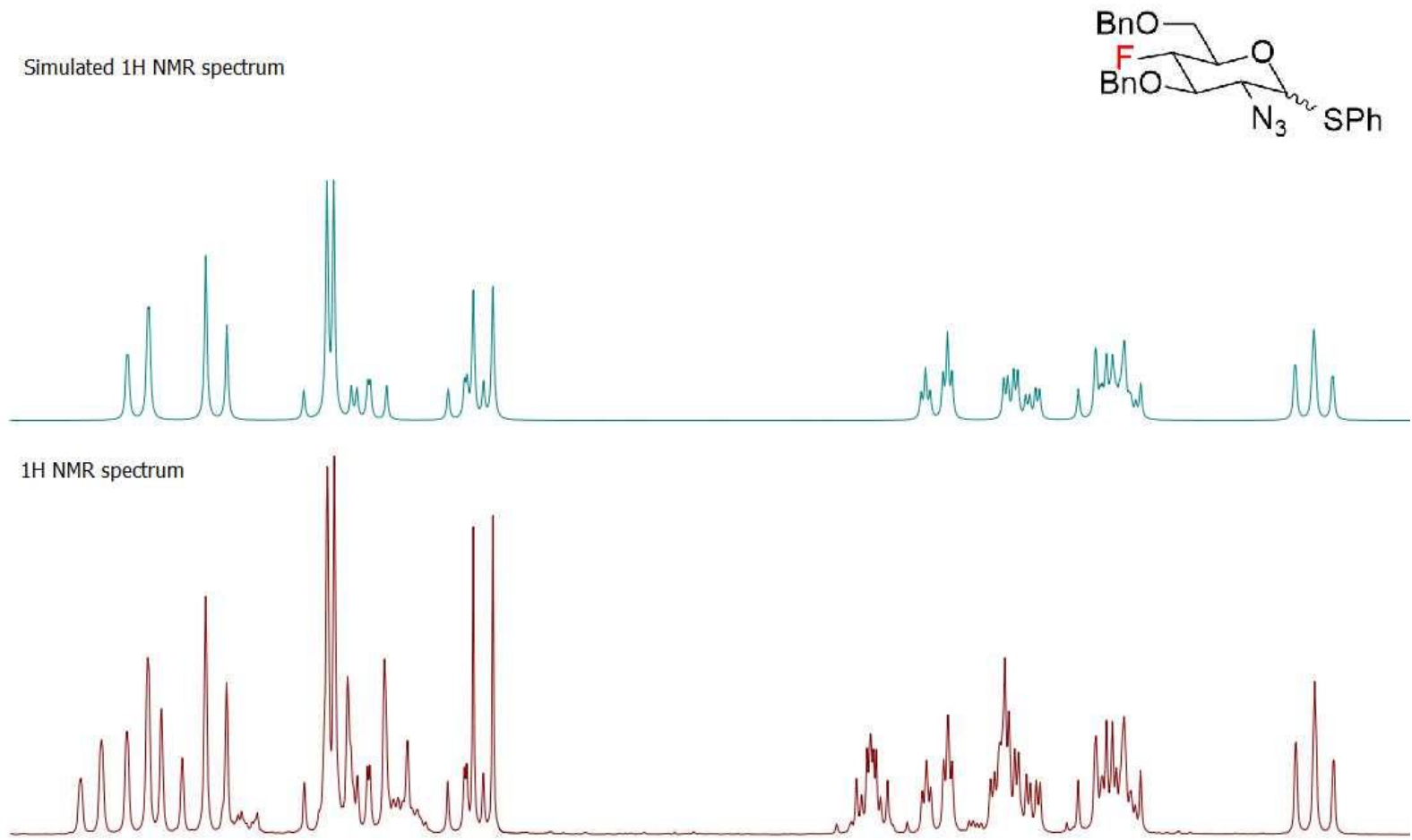

$5.0 \quad 4.9 \quad 4.8$ 4.2
f1 $1(\mathrm{ppm})$ 
${ }^{13} \mathrm{C}\left\{{ }^{1} \mathrm{H}\right\}$ APT NMR $100 \mathrm{MHz}, \mathrm{CDCl}_{3} \mathbf{2}(\boldsymbol{\alpha}: \boldsymbol{\beta}=0.5: 1.0)$

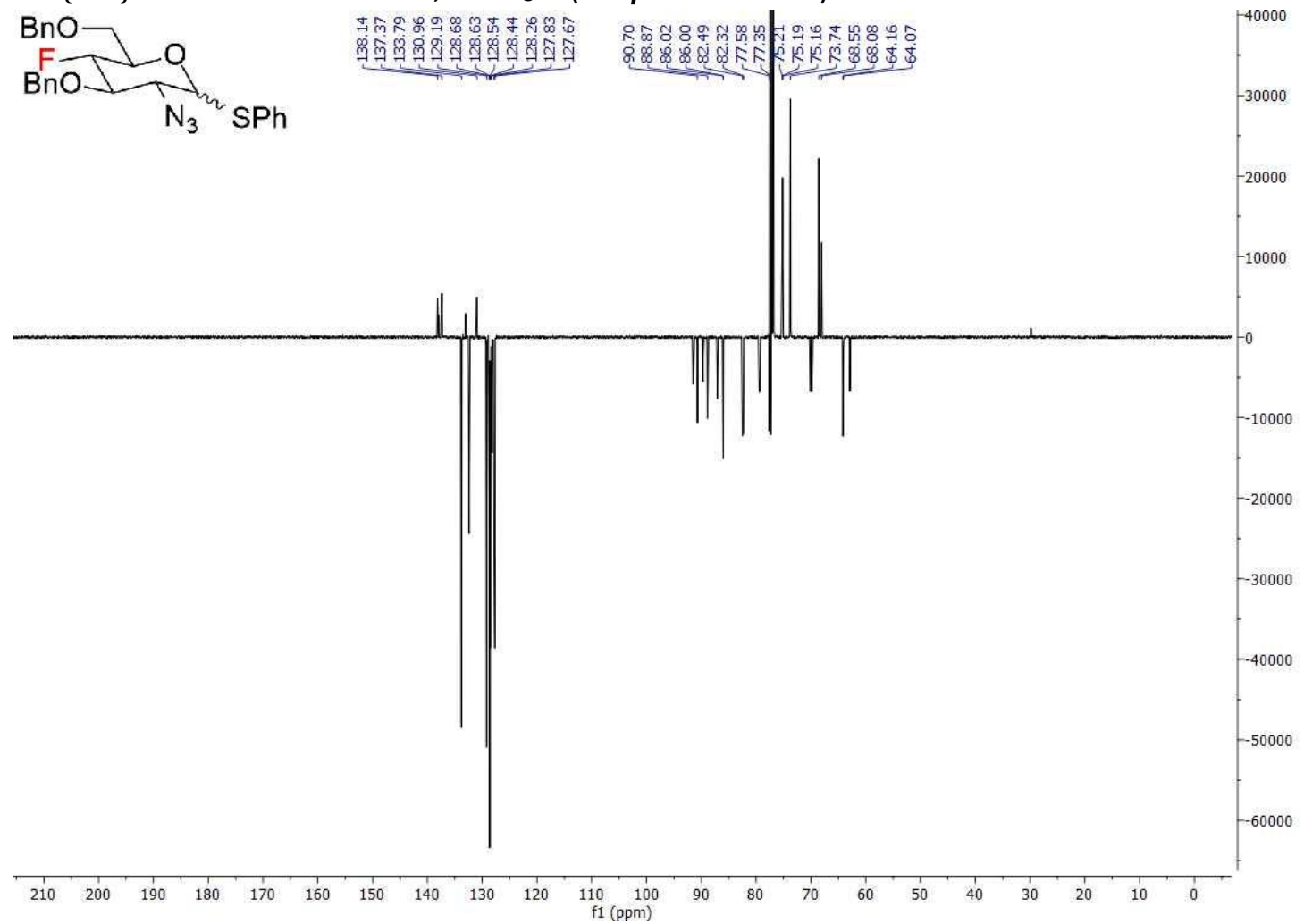

${ }^{1} \mathrm{H}-{ }^{1} \mathrm{H}$ COSY NMR $2(\alpha: \beta=0.5: 1.0)$

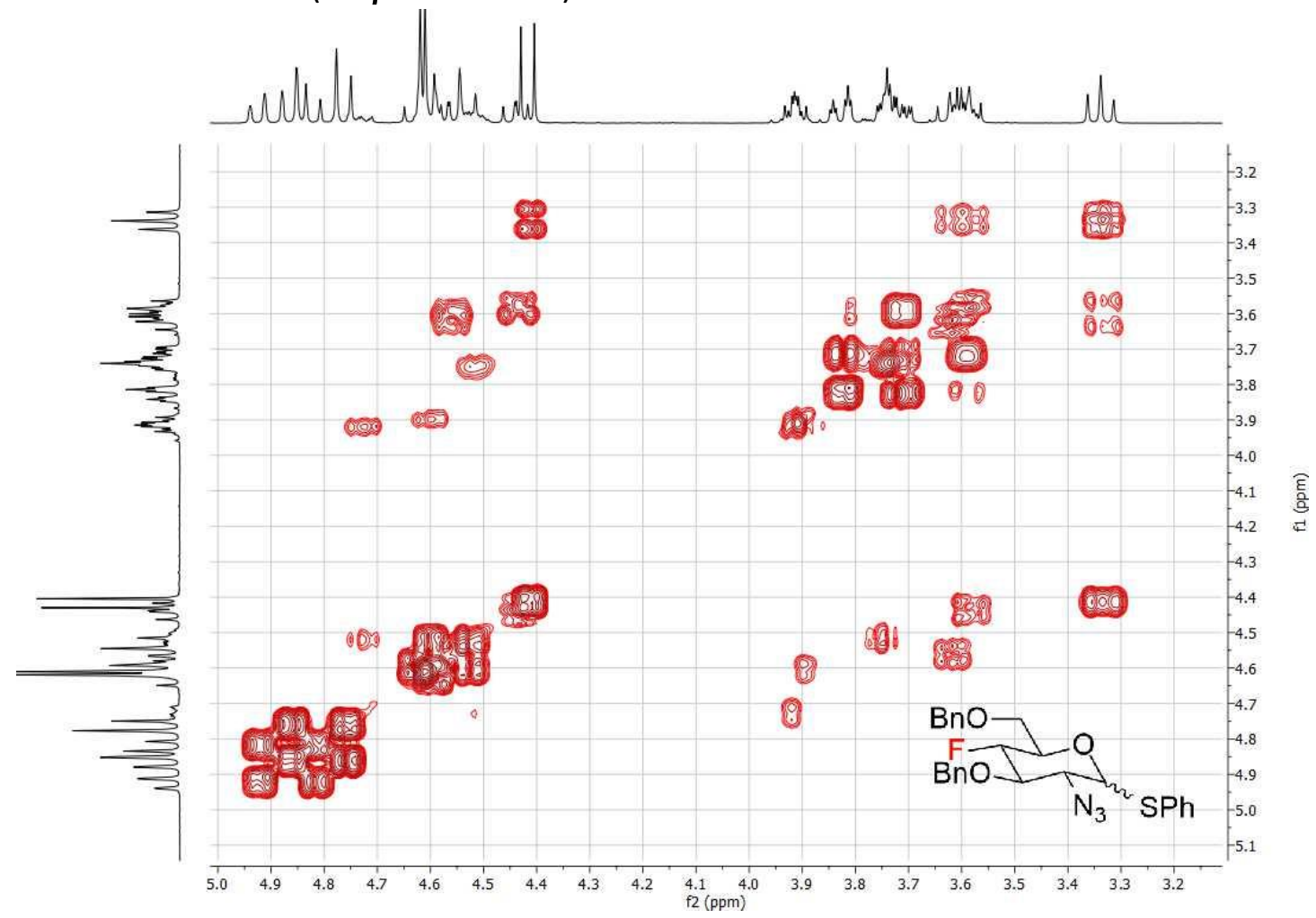



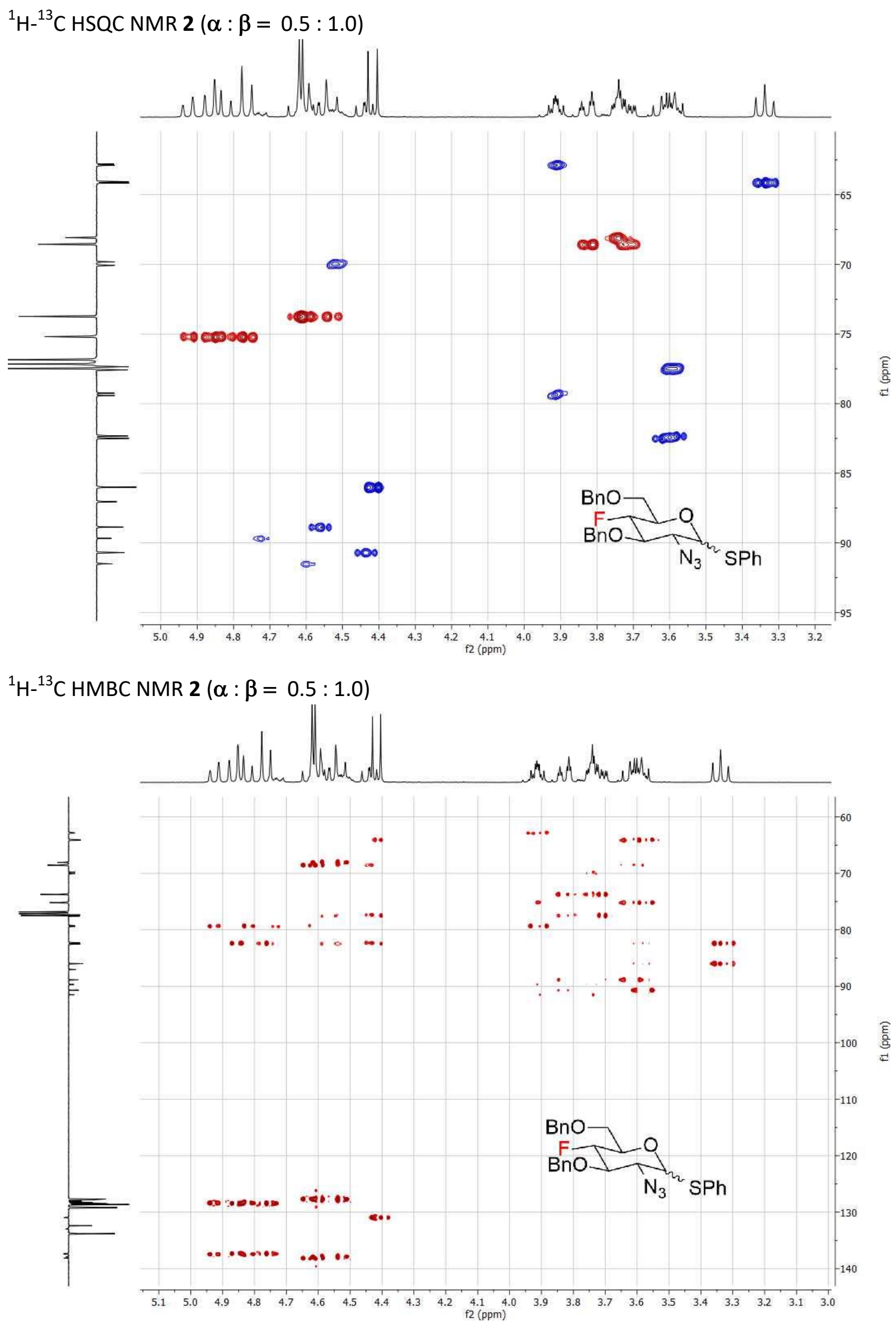

S69 


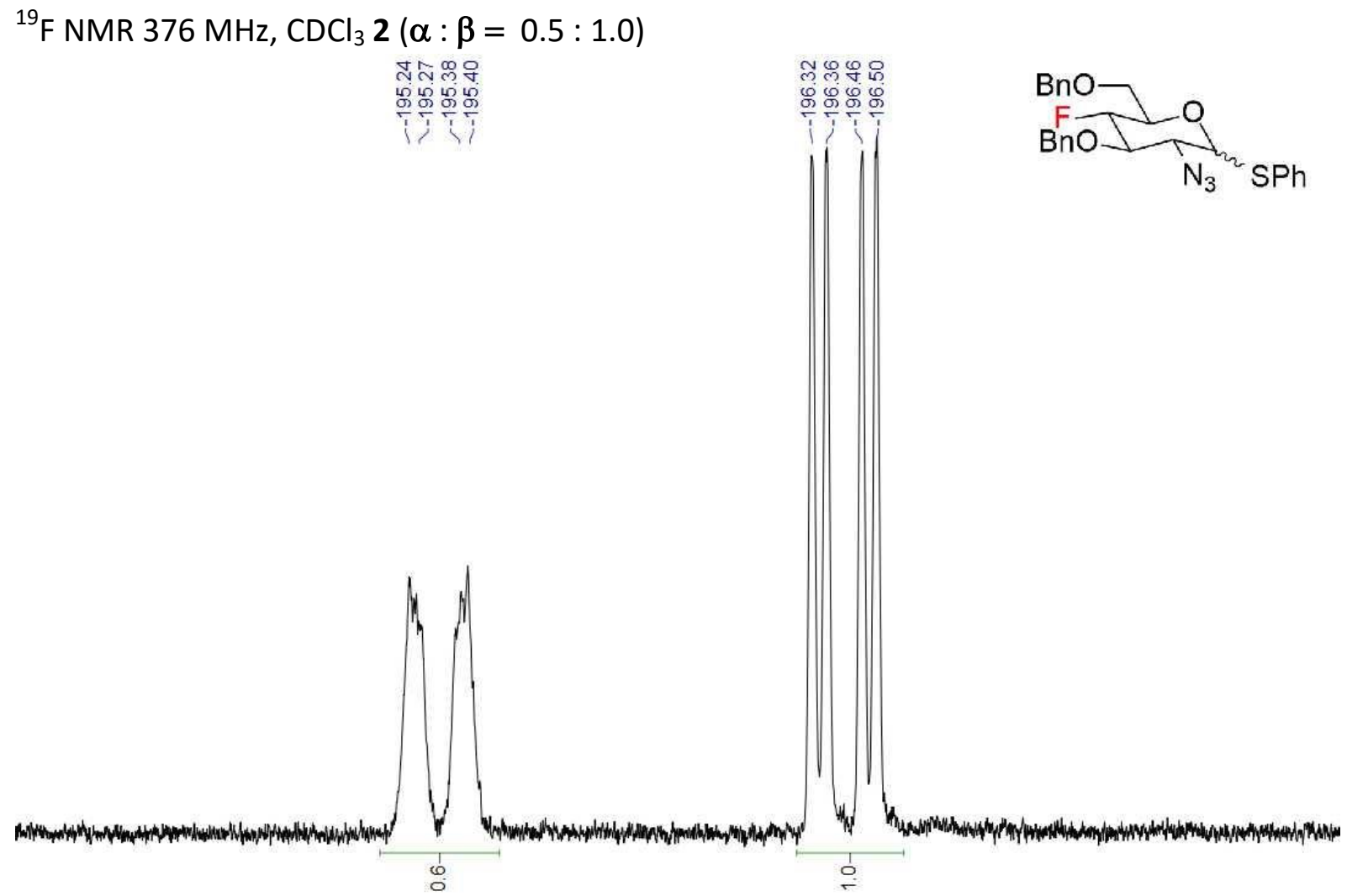

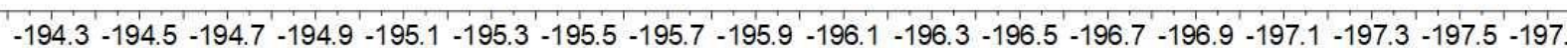
f1 (ppm) 
NMR 17

${ }^{1} \mathrm{H}$ NMR $400 \mathrm{MHz}, \mathrm{CDCl}_{3} 17$

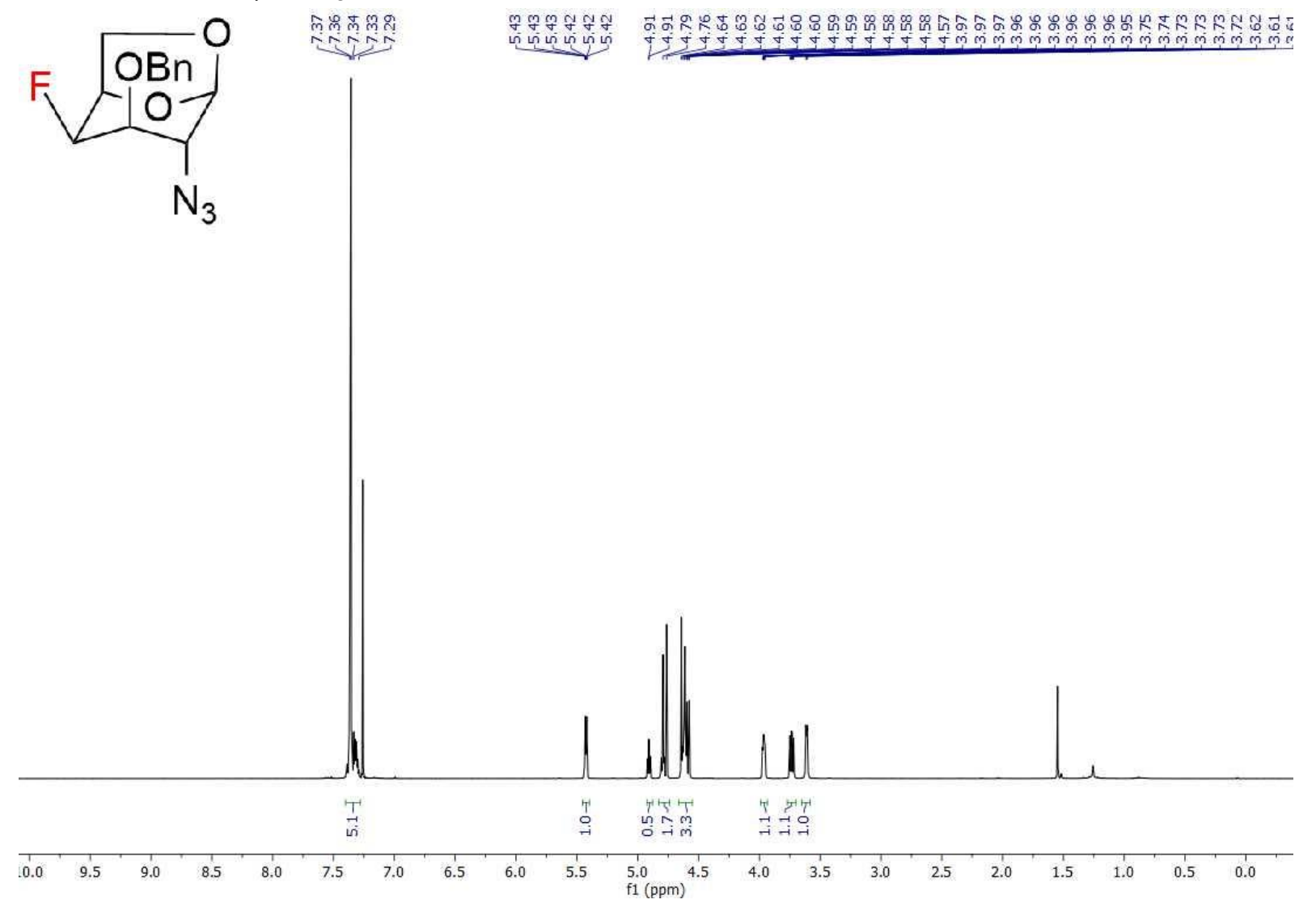

${ }^{13} \mathrm{C}\left\{{ }^{1} \mathrm{H}\right\}$ APT NMR 100MHz, $\mathrm{CDCl}_{3} 17$

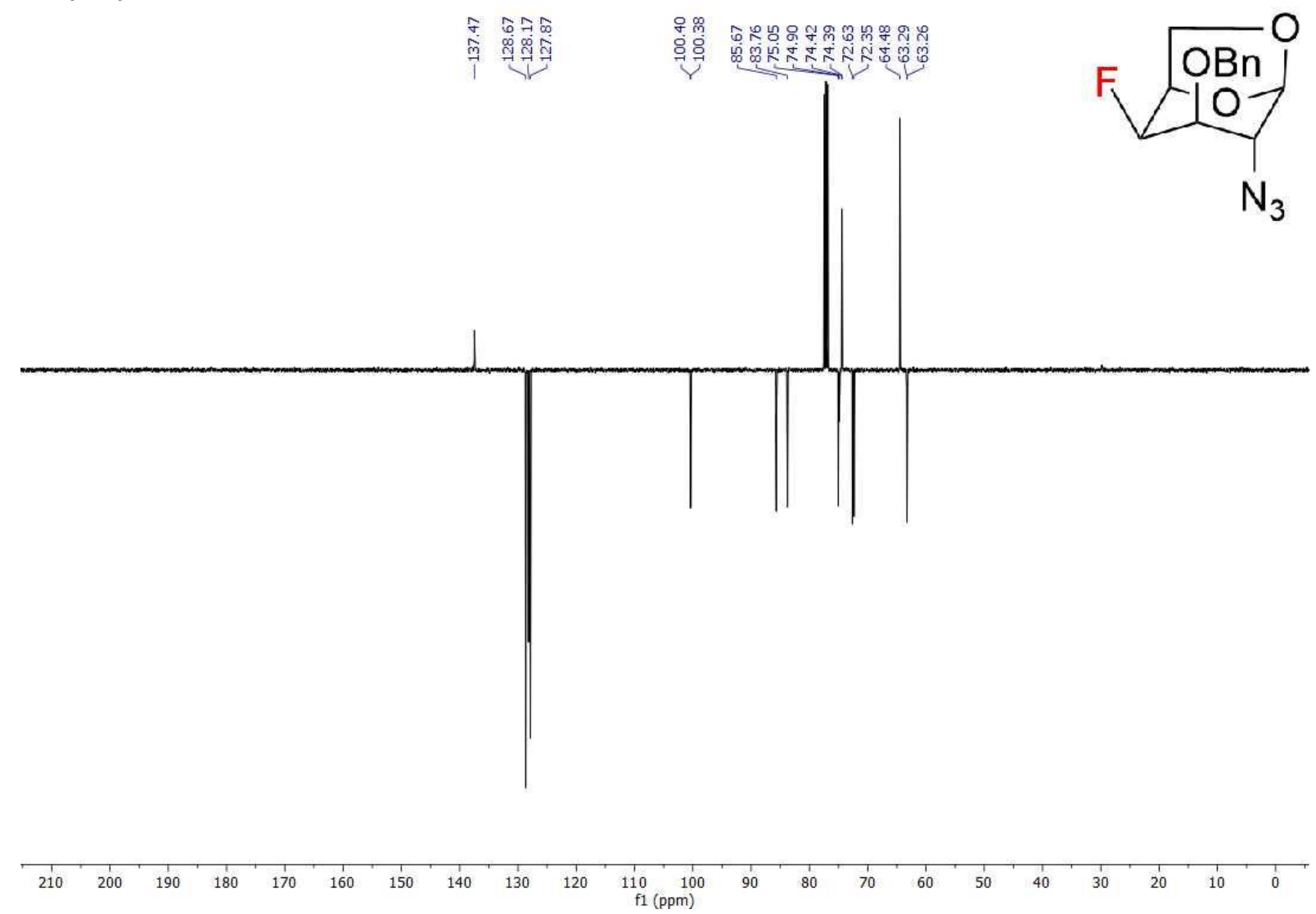


${ }^{1} \mathrm{H}-{ }^{1} \mathrm{H}$ COSY NMR 17

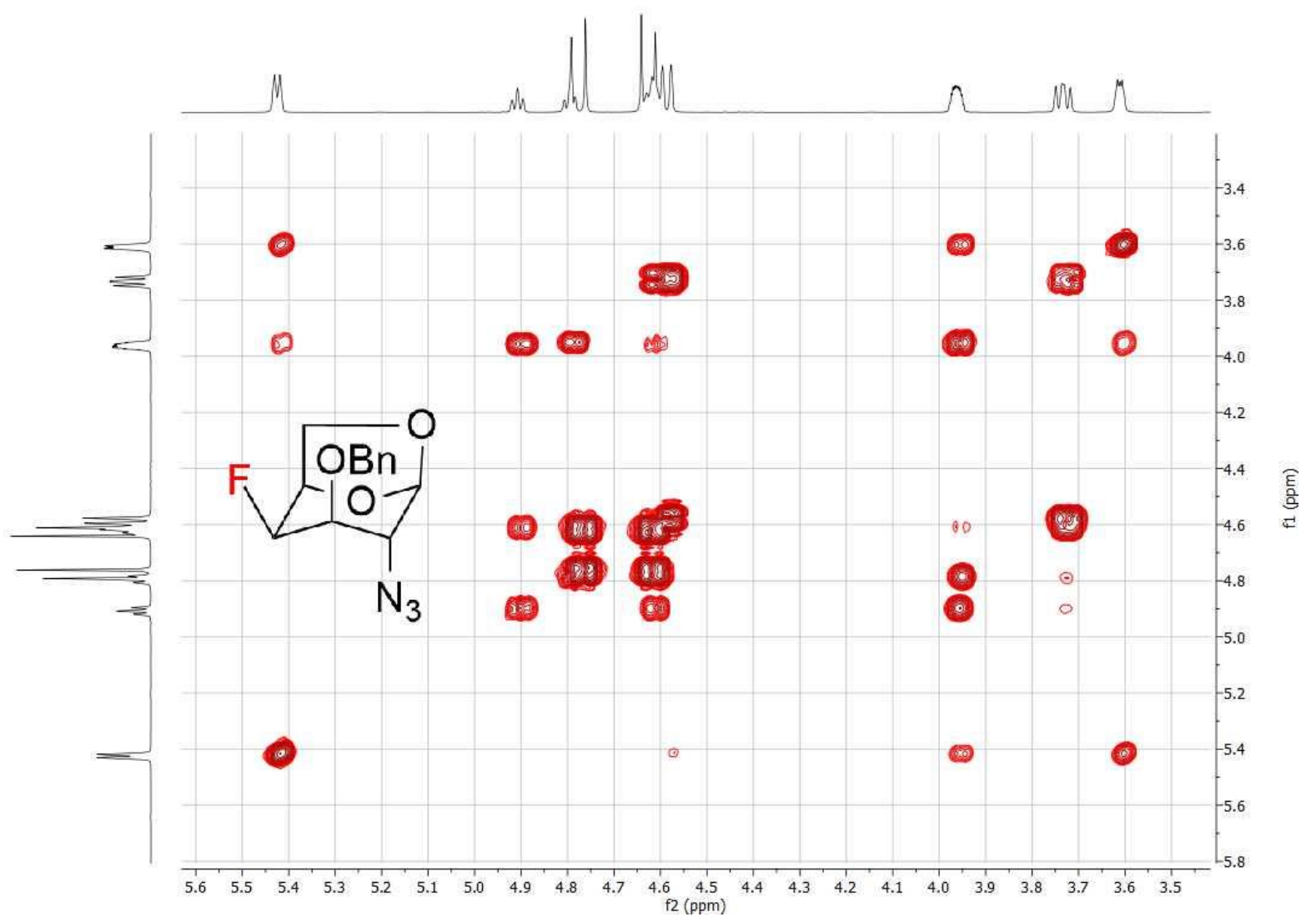

${ }^{1} \mathrm{H}-{ }^{13} \mathrm{C}$ HSQC NMR 17

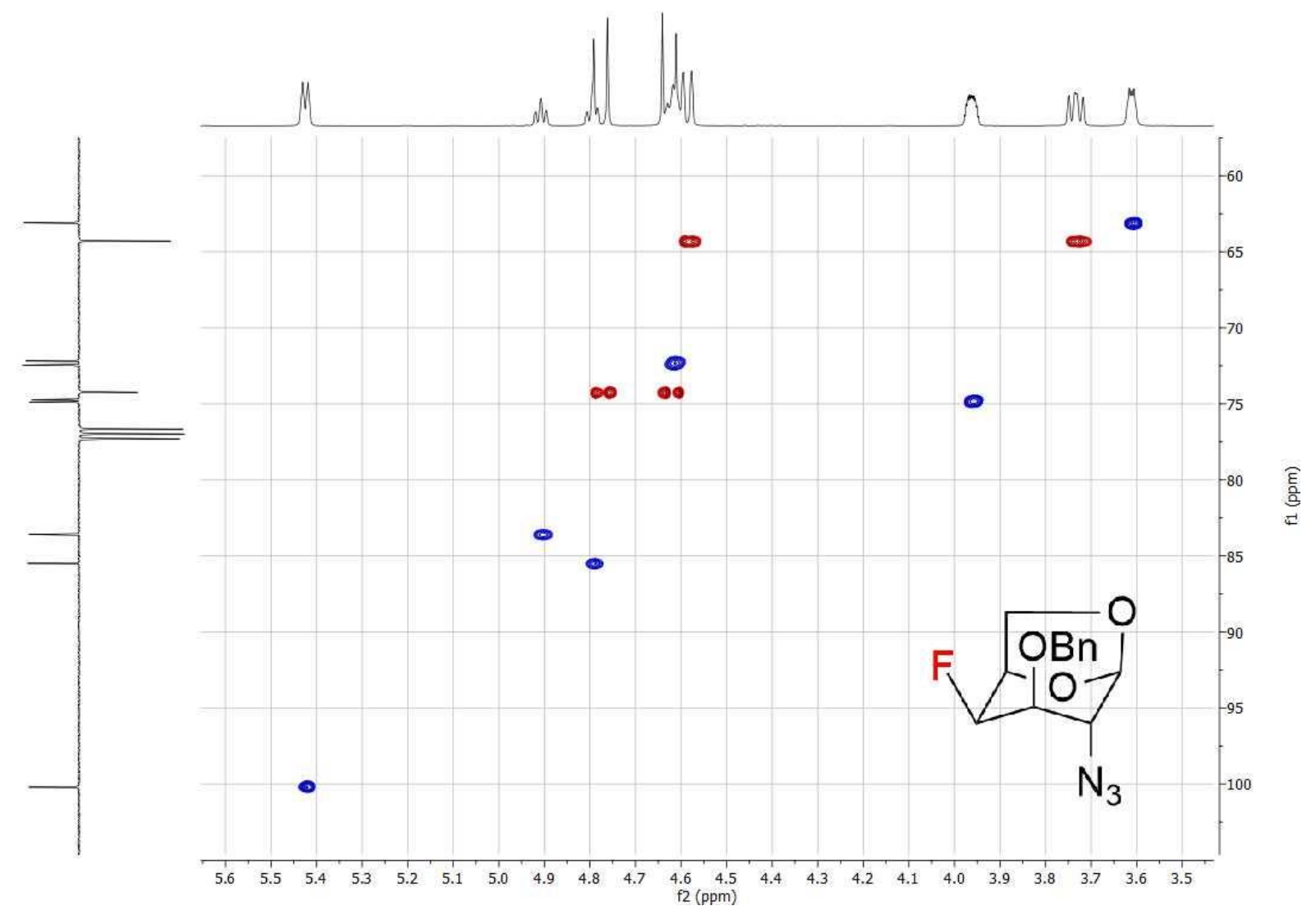

S72 


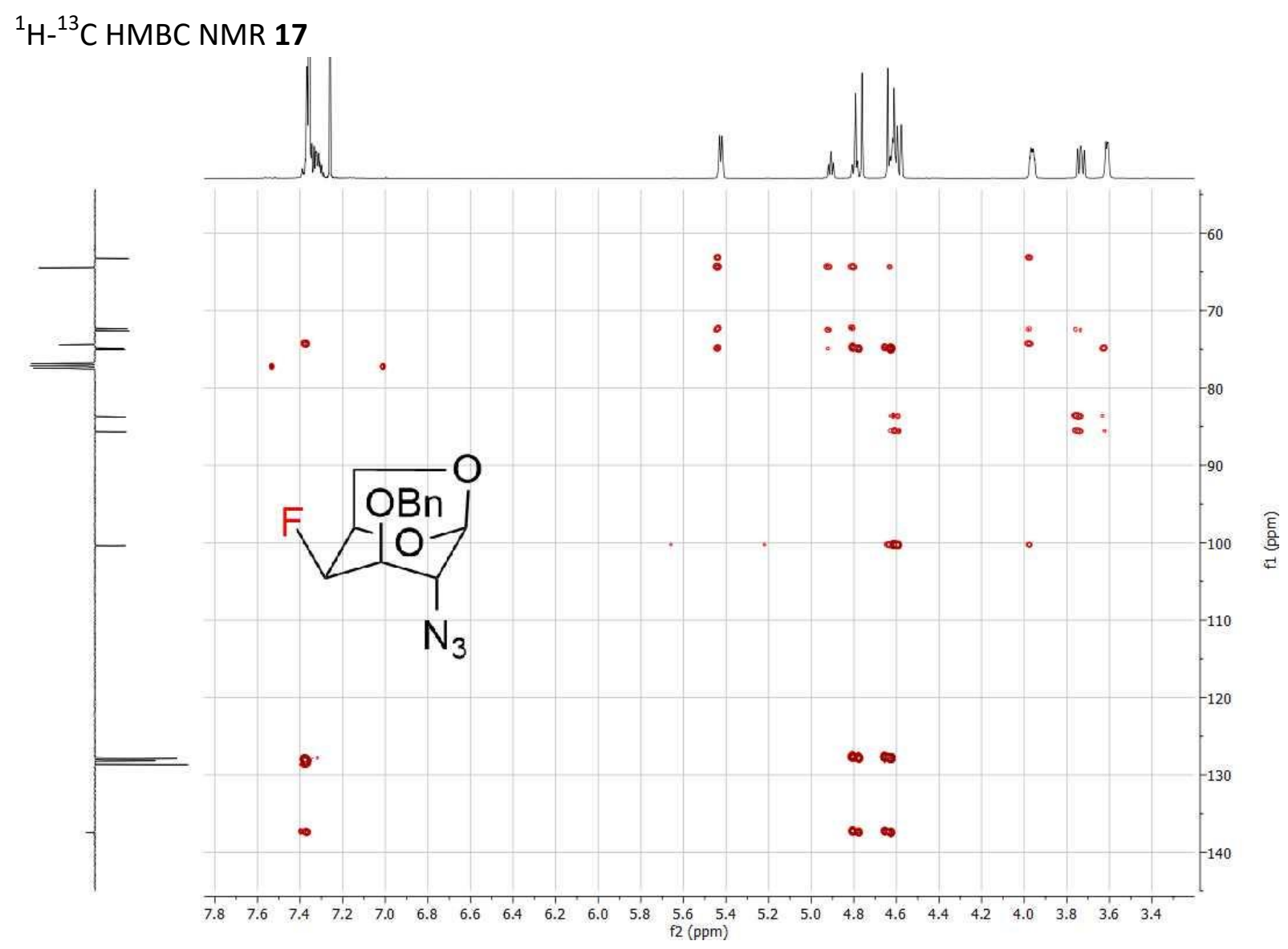

${ }^{19} \mathrm{~F} \mathrm{NMR} 376 \mathrm{MHz}, \mathrm{CDCl}_{3} 17$

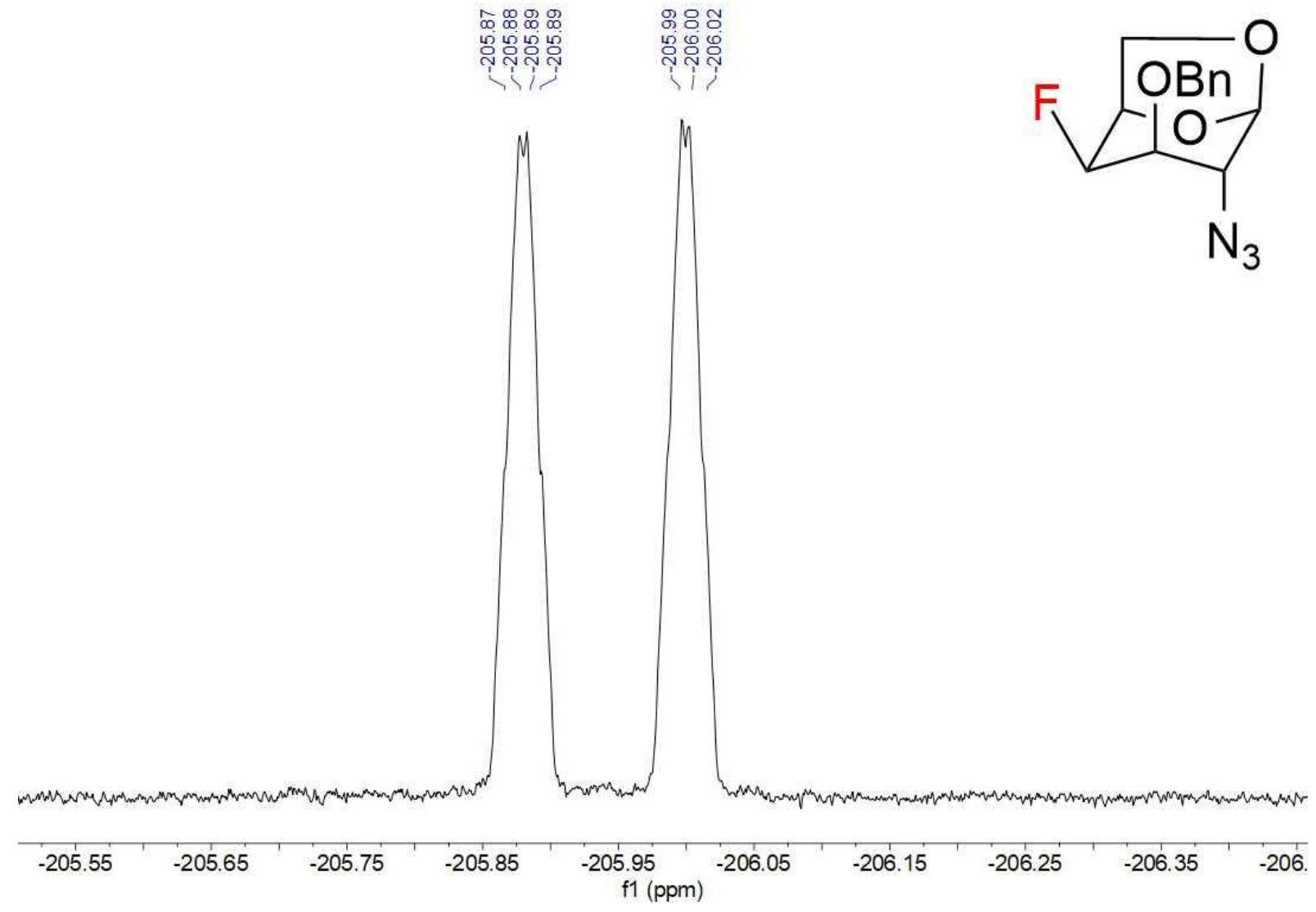


NMR $\alpha-19$

${ }^{1} \mathrm{H}$ NMR $400 \mathrm{MHz}, \mathrm{CDCl}_{3} \boldsymbol{\alpha}-19$

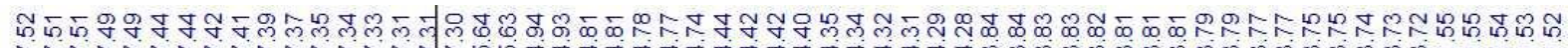
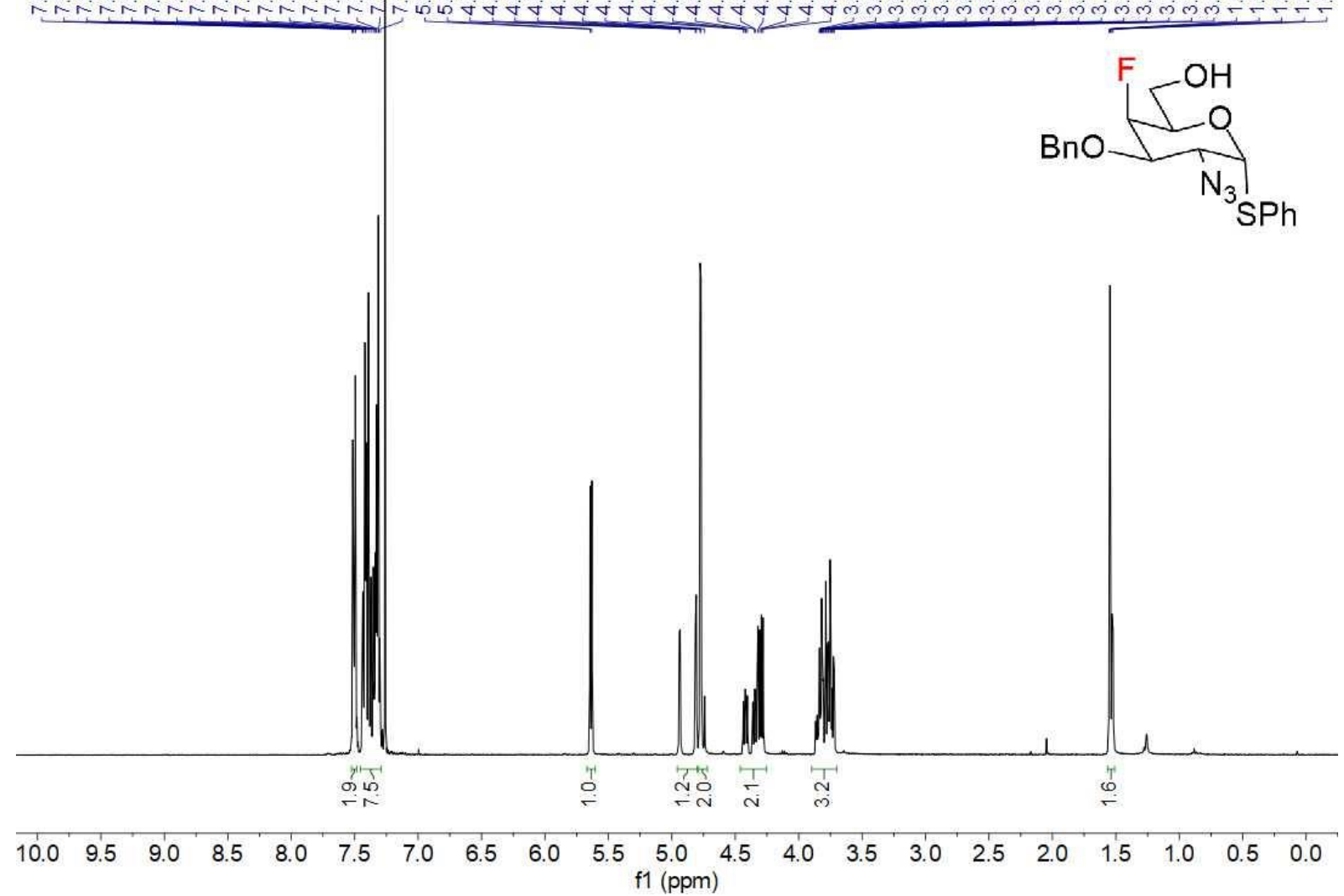

${ }^{13} \mathrm{C}\left\{{ }^{1} \mathrm{H}\right\}$ APT NMR $100 \mathrm{MHz}, \mathrm{CDCl}_{3} \boldsymbol{\alpha}-19$

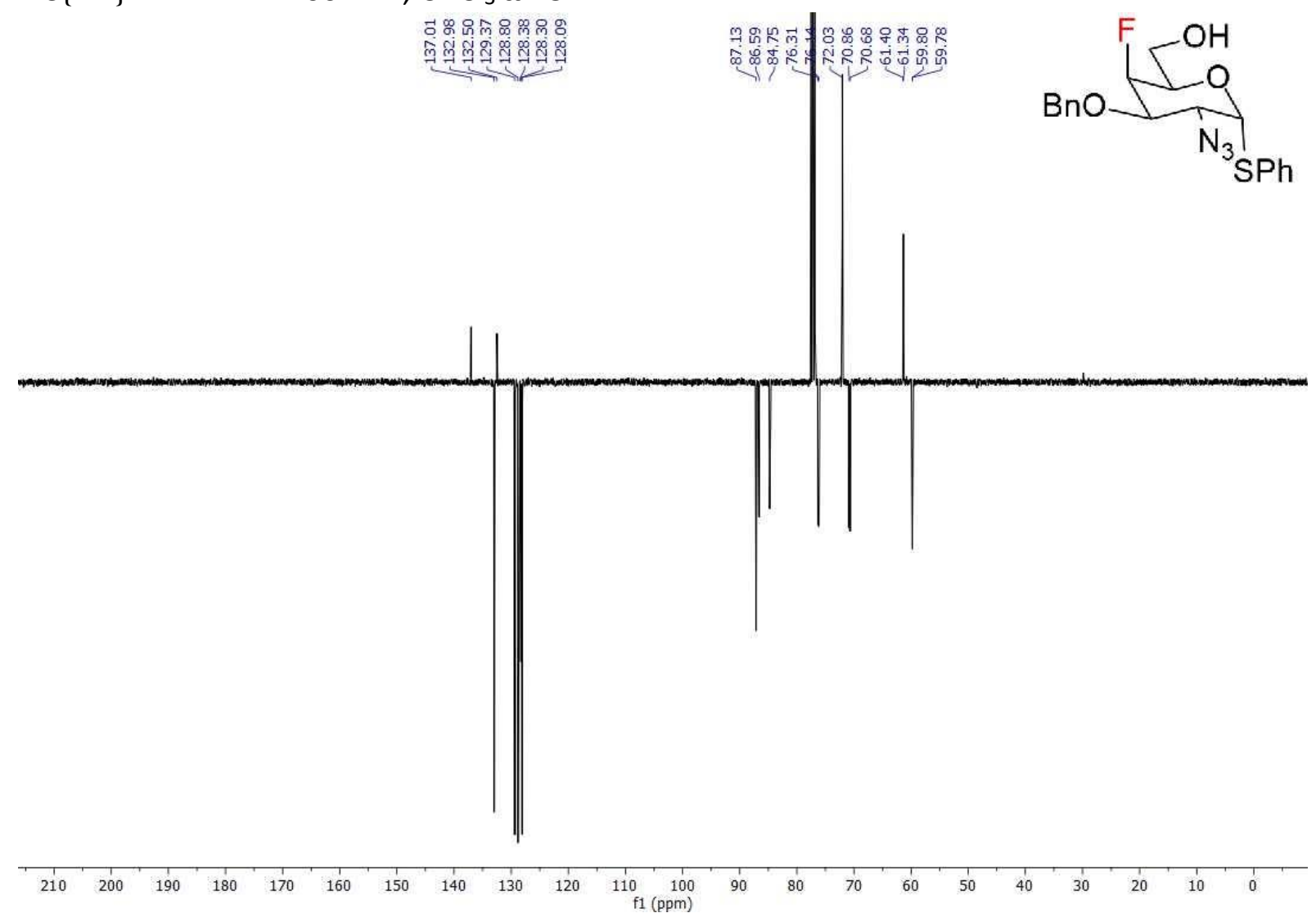


${ }^{1} \mathrm{H}-{ }^{1} \mathrm{H} \operatorname{cosY} N M R \boldsymbol{\alpha}-19$

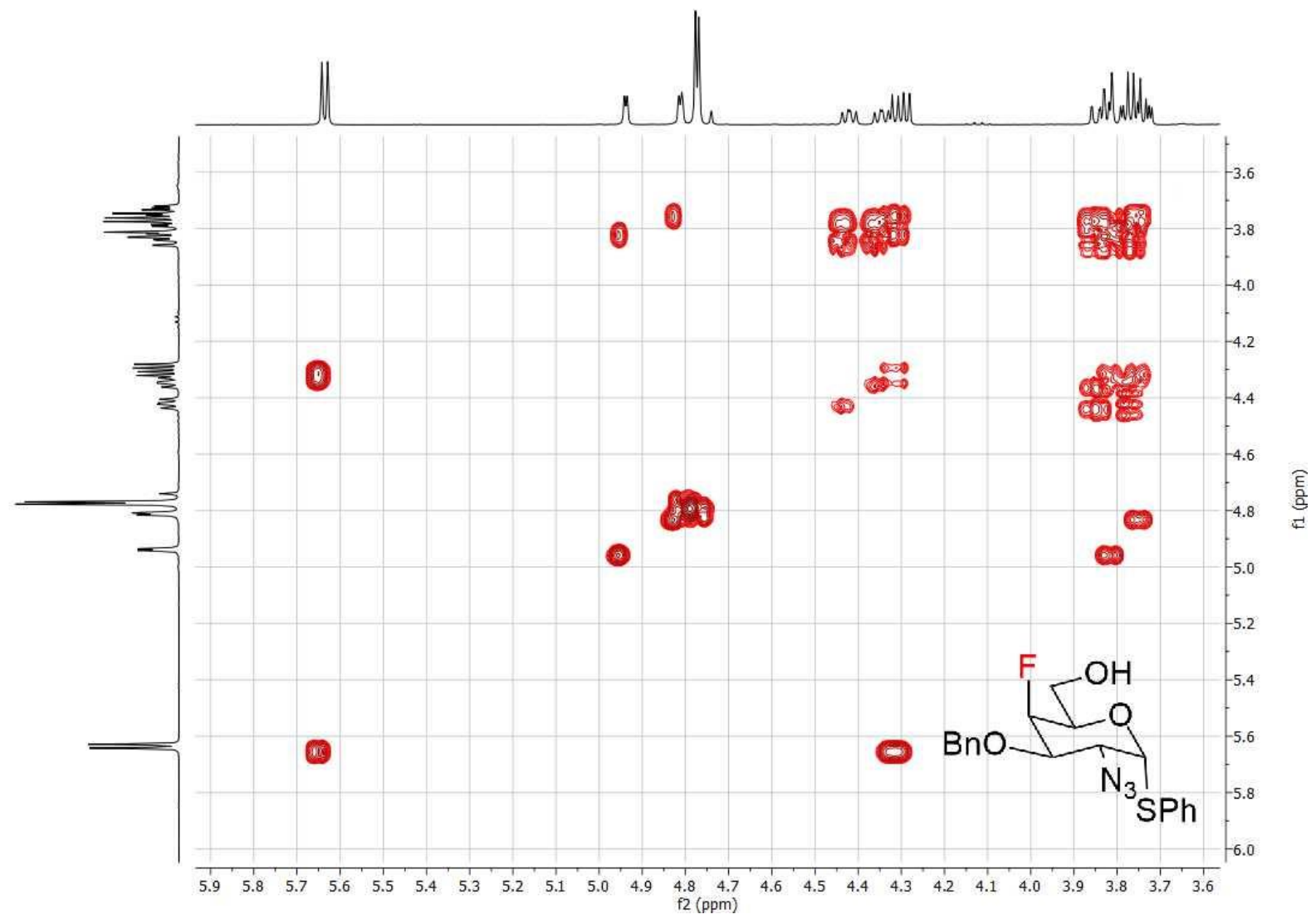

${ }^{1} \mathrm{H}-{ }^{13} \mathrm{C}$ HSQC NMR $\alpha-19$

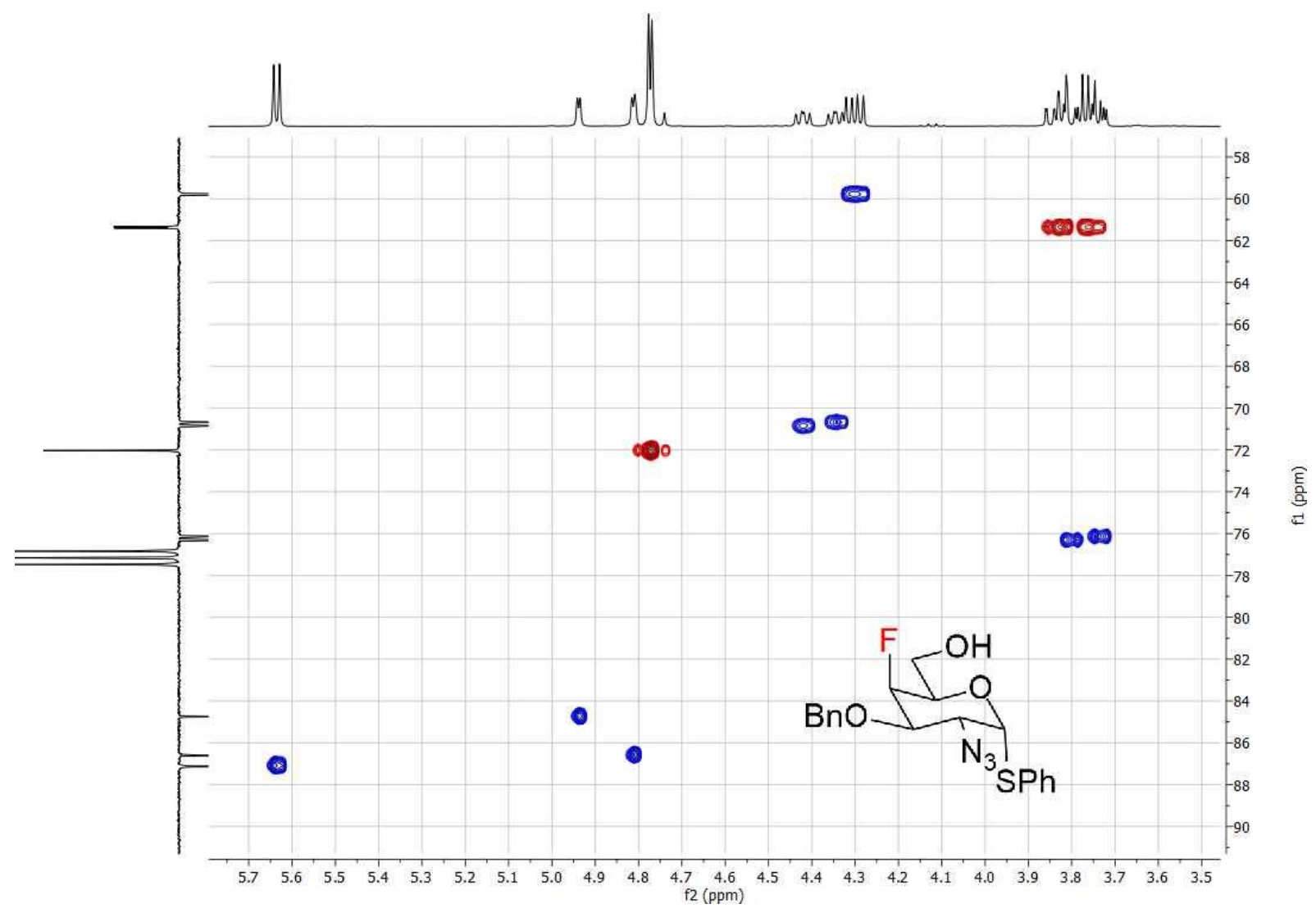


${ }^{1} \mathrm{H}-{ }^{13} \mathrm{C}$ HMBC NMR $\alpha-19$

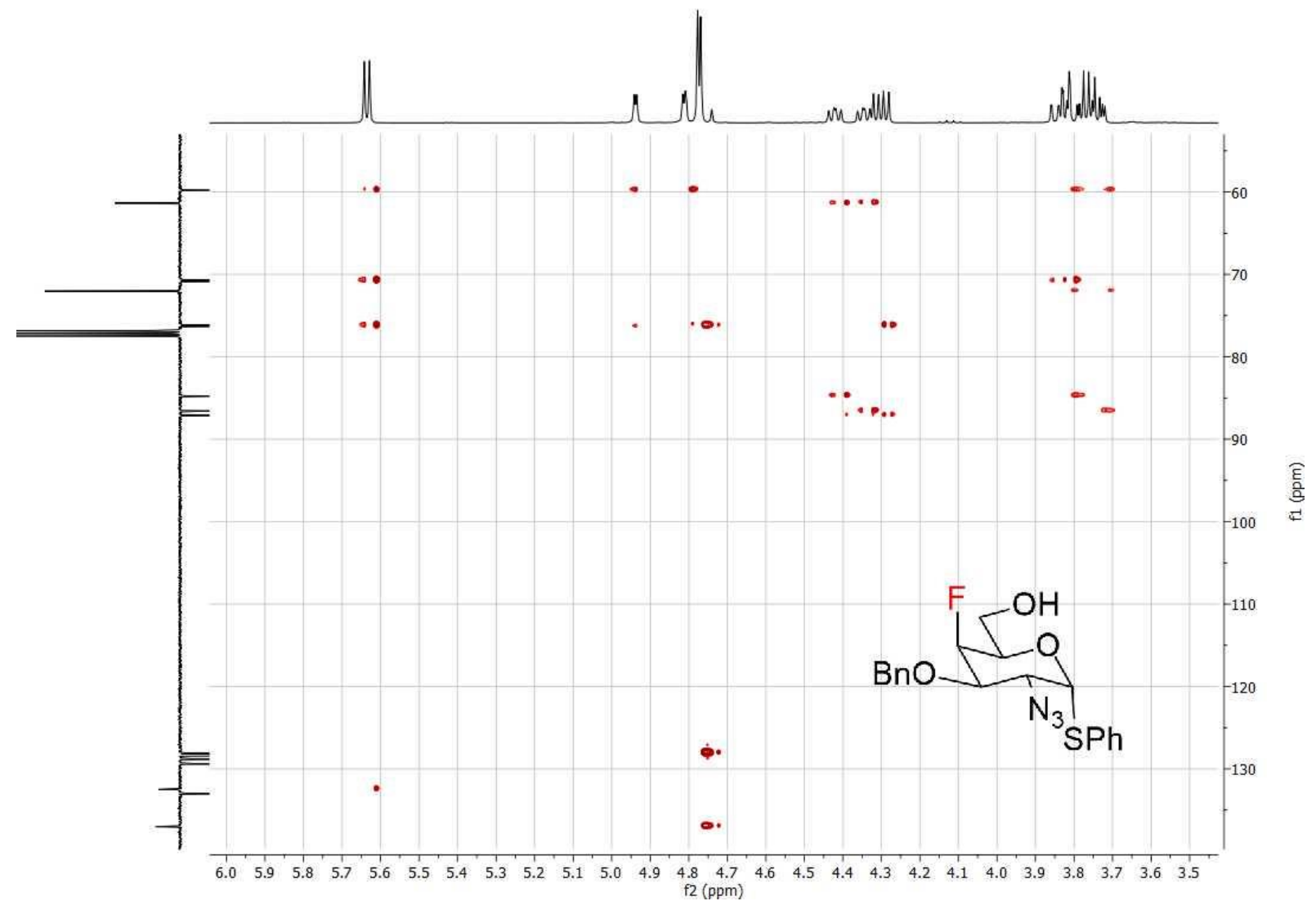

${ }^{19} \mathrm{FNMR} 376 \mathrm{MHz}, \mathrm{CDCl}_{3} \boldsymbol{\alpha}-19$

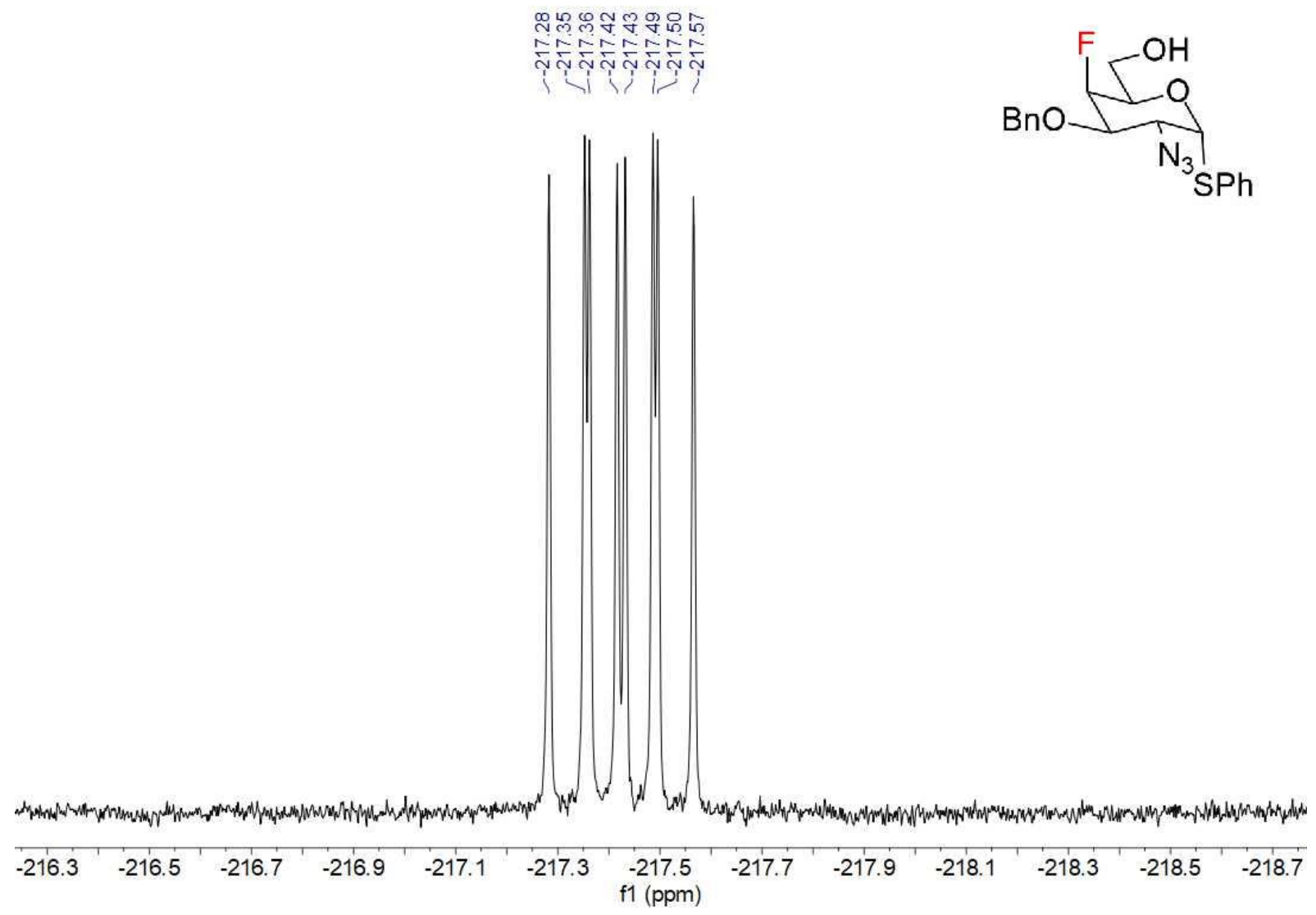


NMR $\beta-19$

${ }^{1} \mathrm{H}$ NMR $400 \mathrm{MHz}, \mathrm{CDCl}_{3} \boldsymbol{\beta}-19$

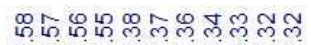

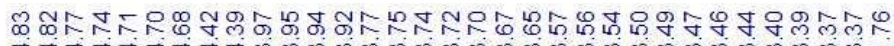
NMNNNNNNA

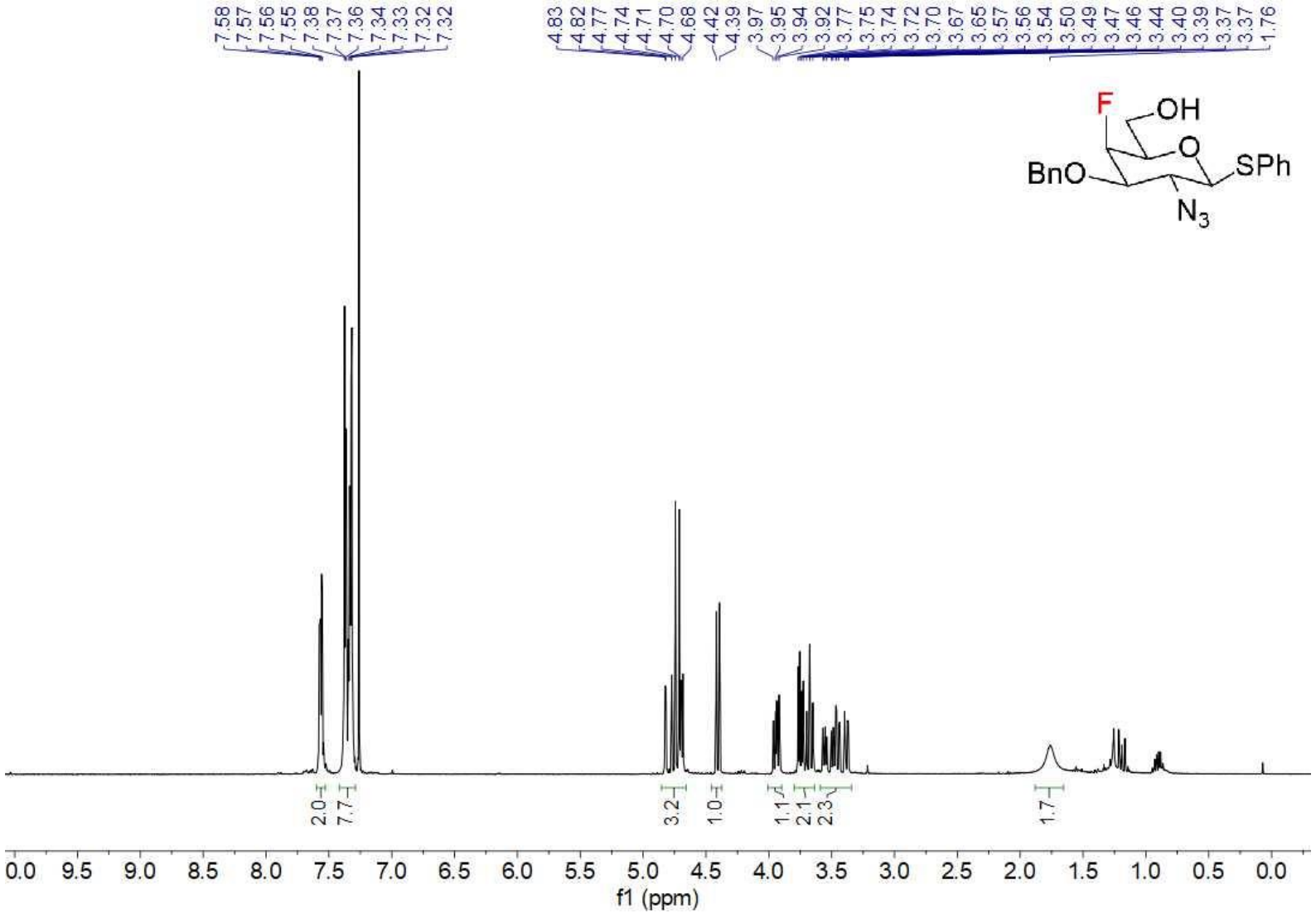

${ }^{13} \mathrm{C}\left\{{ }^{1} \mathrm{H}\right\}$ APT NMR $100 \mathrm{MHz}, \mathrm{CDCl}_{3} \boldsymbol{\beta}-19$

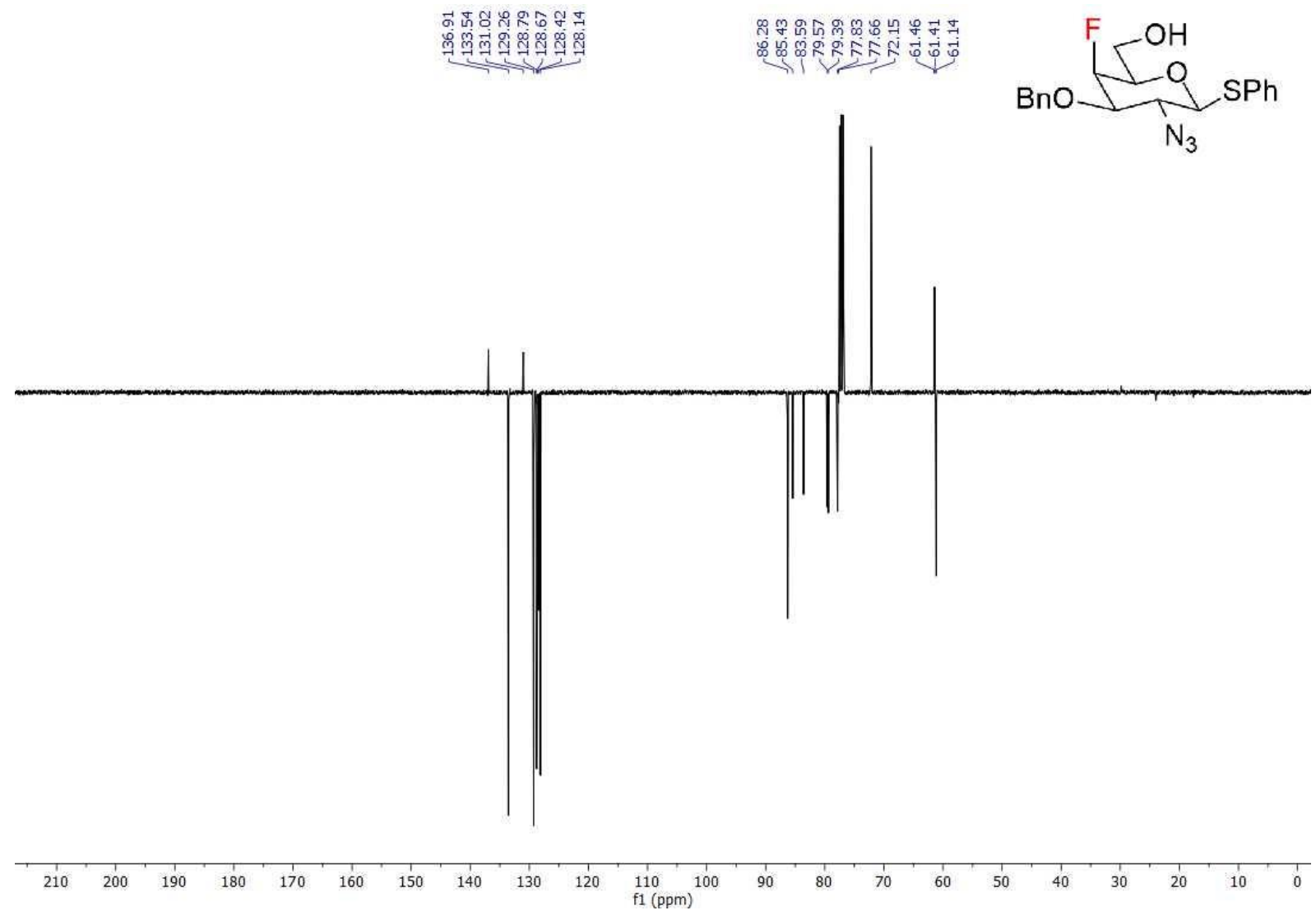


${ }^{1} \mathrm{H}-{ }^{1} \mathrm{H}$ COSY NMR $\beta-19$

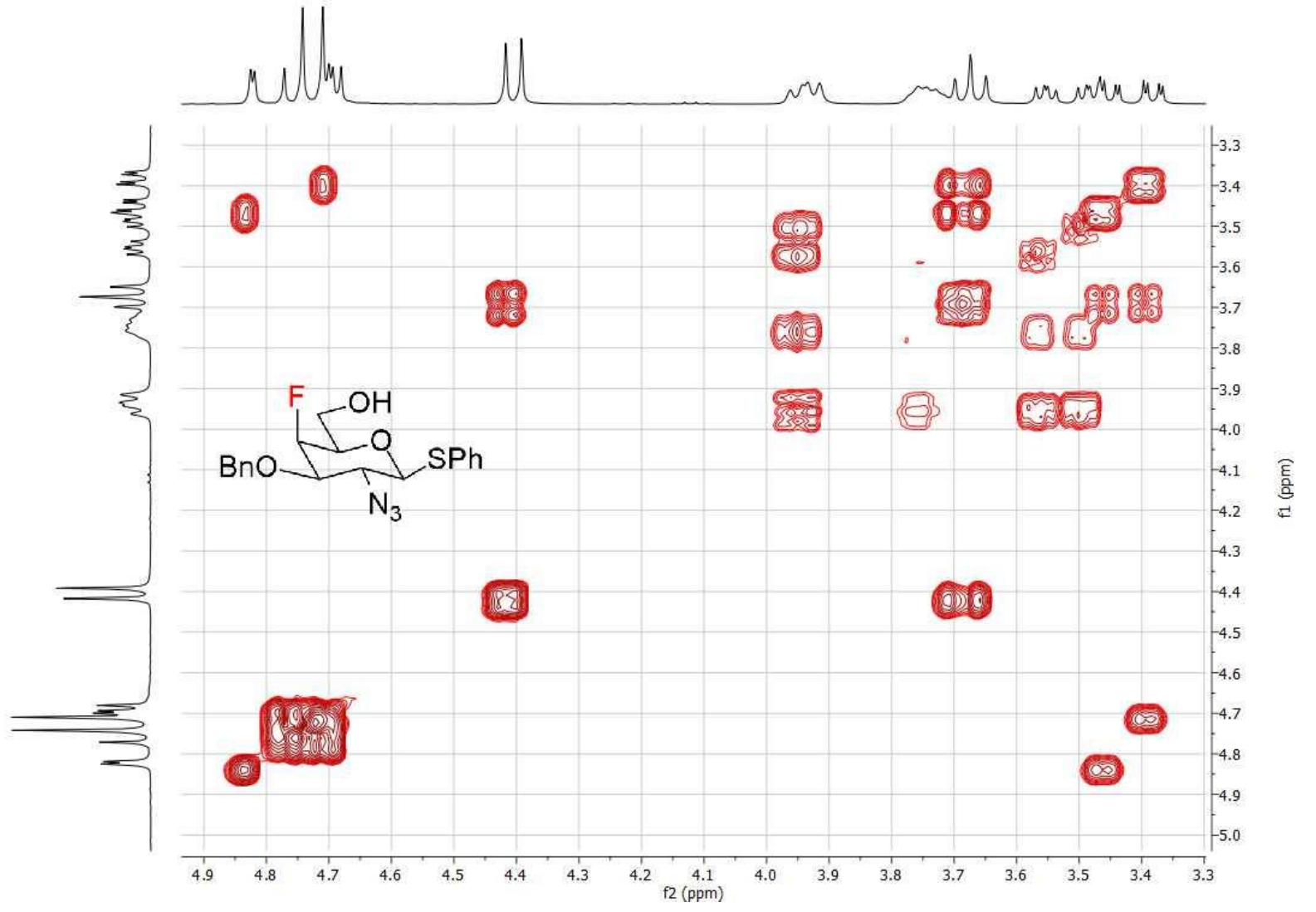

${ }^{1} \mathrm{H}-{ }^{13} \mathrm{C}$ HSQC NMR $\beta-19$

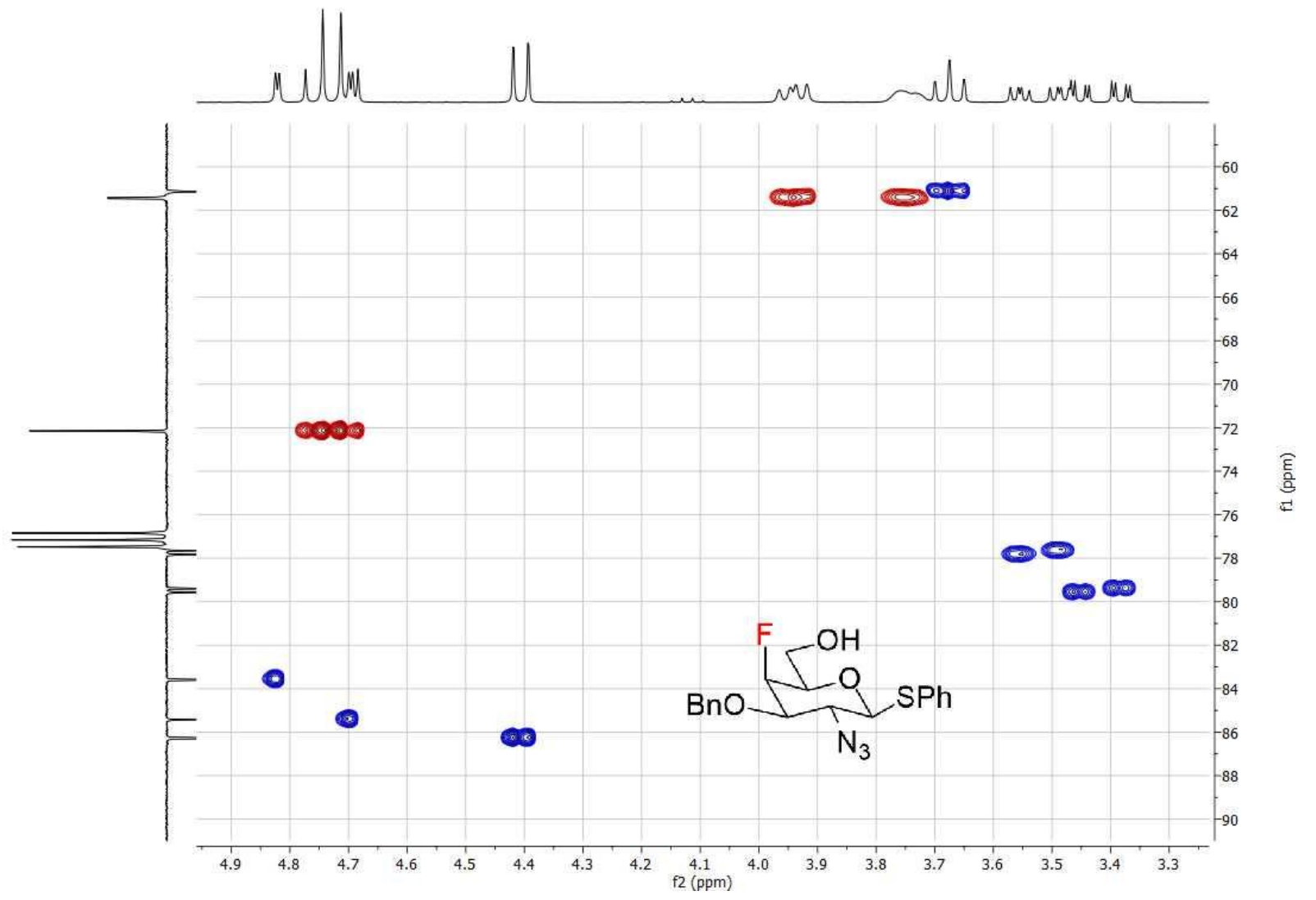


${ }^{1} \mathrm{H}-{ }^{13} \mathrm{C}$ HMBC NMR $\boldsymbol{\beta}-19$

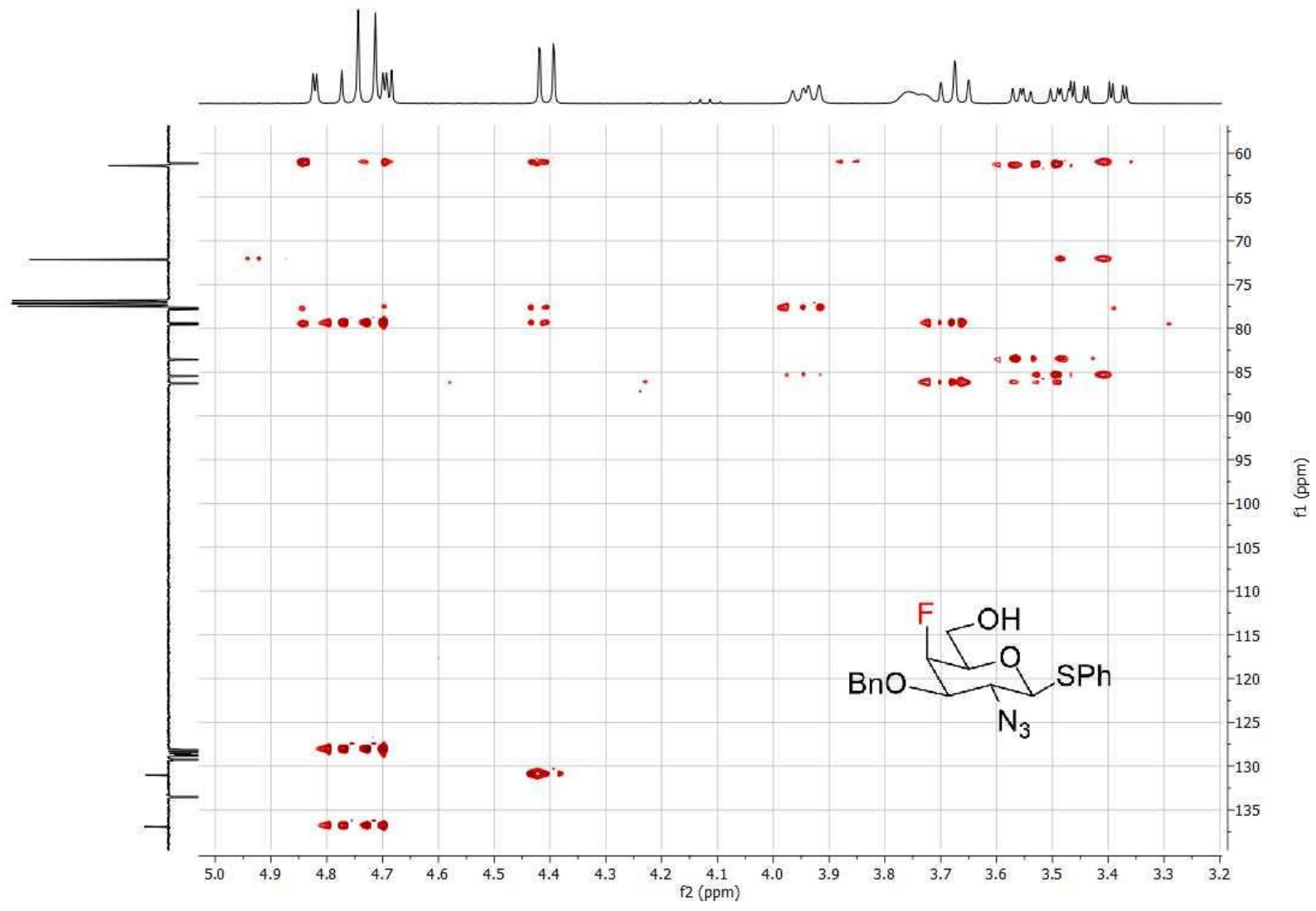

${ }^{19} \mathrm{~F} \mathrm{NMR} 376 \mathrm{MHz}, \mathrm{CDCl}_{3} \boldsymbol{\beta}-19$

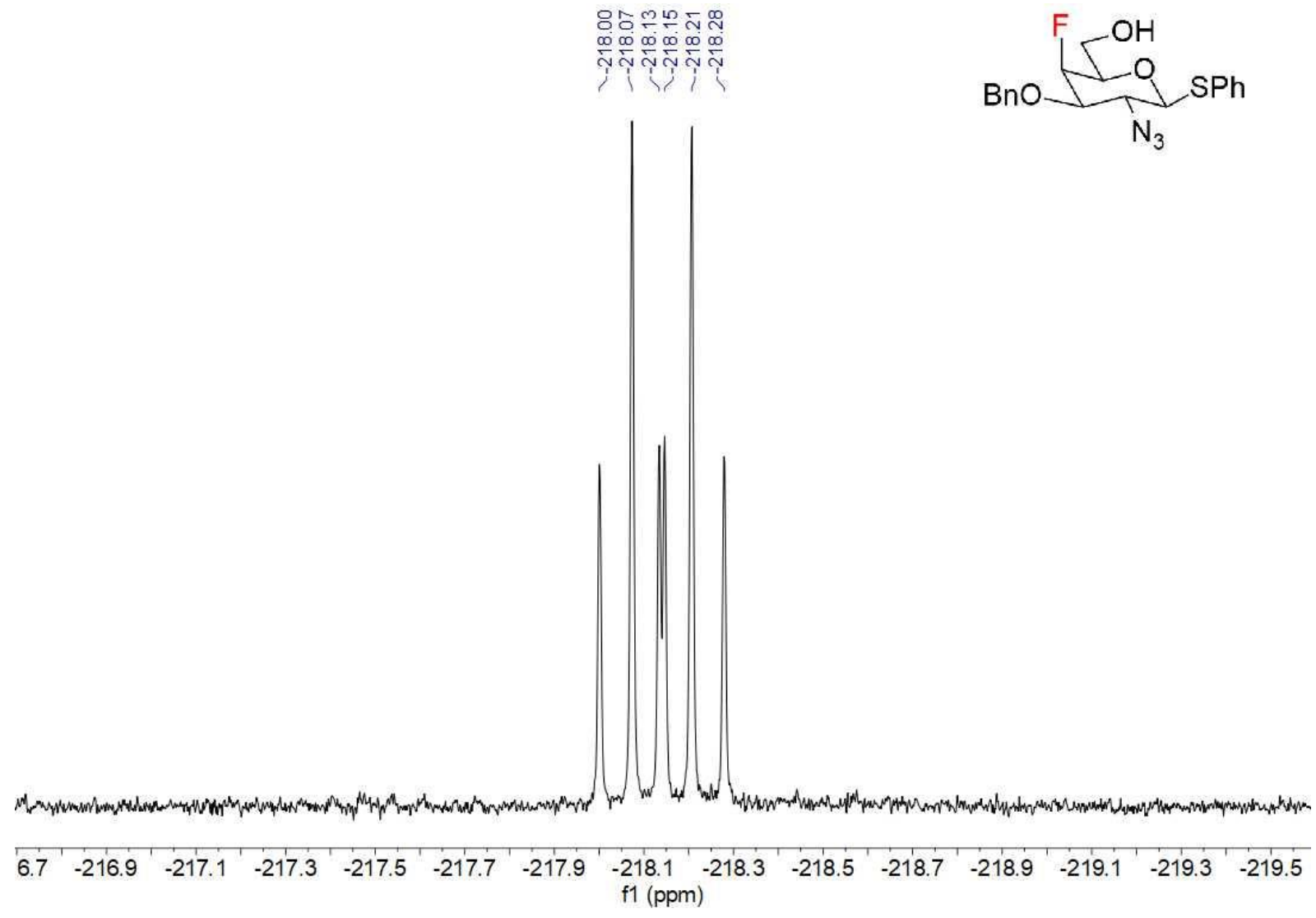


NMR $\alpha-4$

${ }^{1} \mathrm{H} \mathrm{NMR} 400 \mathrm{MHz}, \mathrm{CDCl}_{3} \boldsymbol{\alpha}-4$

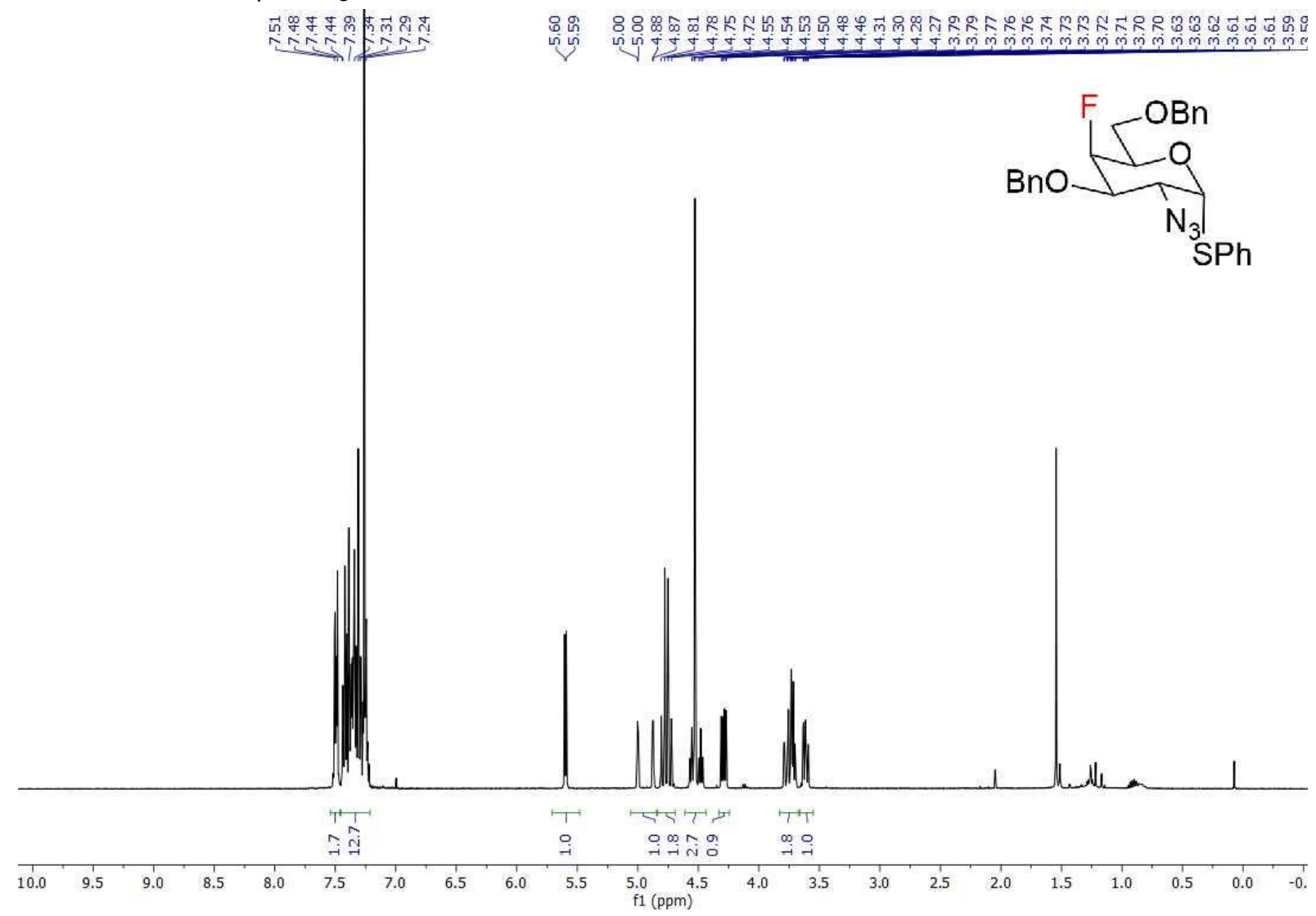

${ }^{13} \mathrm{C}\left\{{ }^{1} \mathrm{H}\right\}$ APT NMR 100MHz, $\mathrm{CDCl}_{3} \boldsymbol{\alpha}-4$

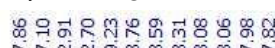

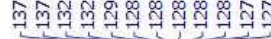

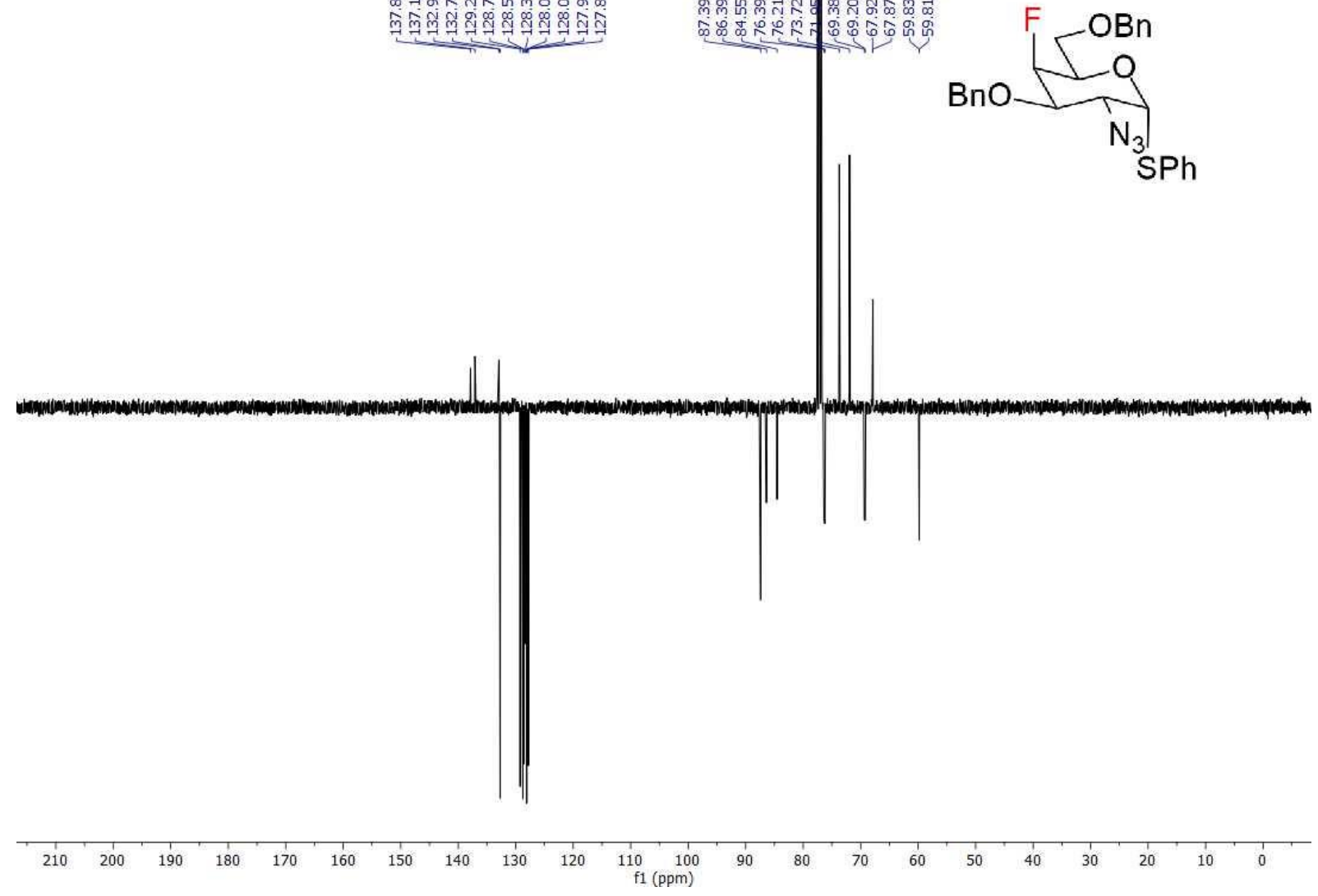


${ }^{1} \mathrm{H}-{ }^{1} \mathrm{H} \operatorname{COSY} N M R \boldsymbol{\alpha}-4$

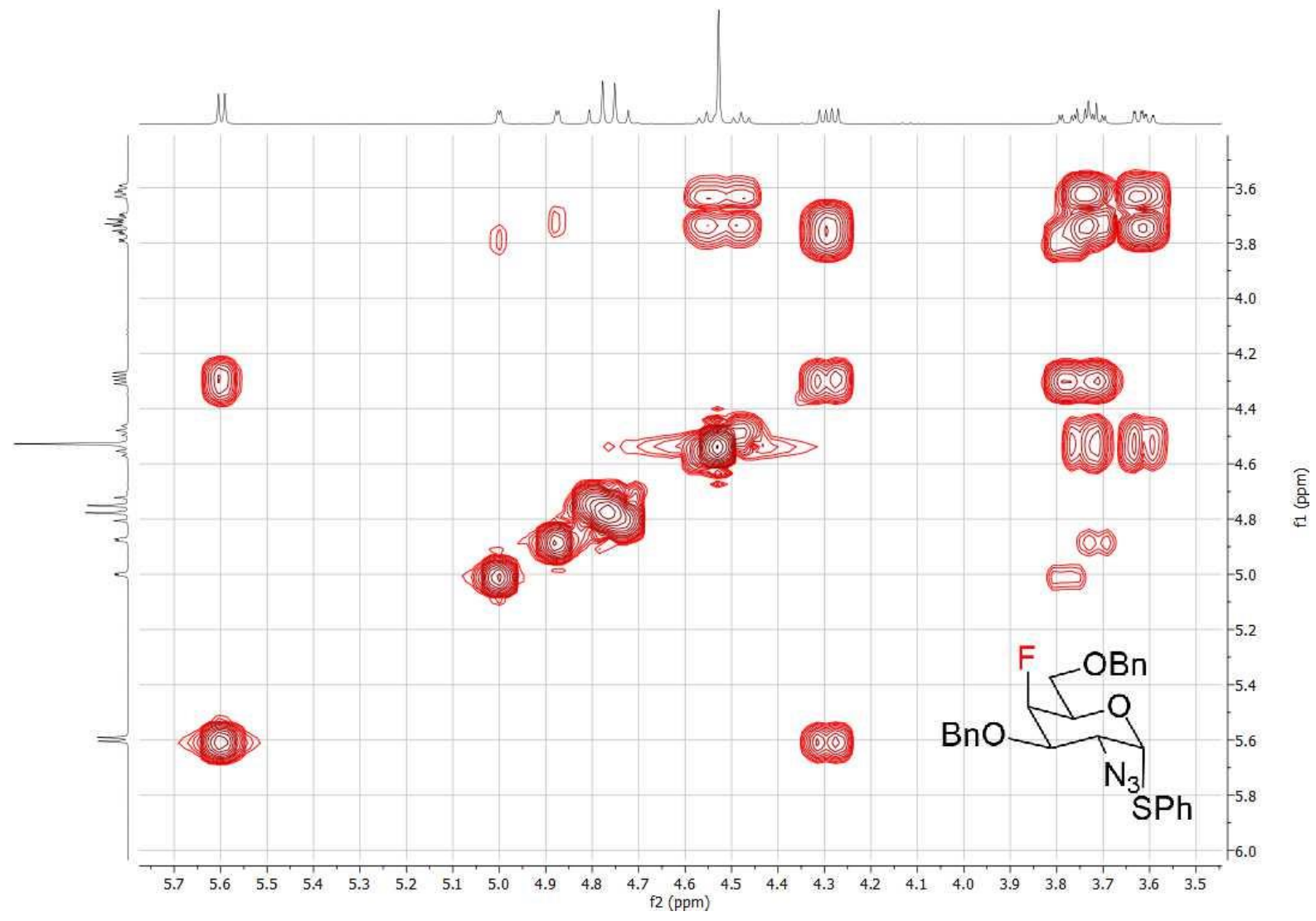

${ }^{1} \mathrm{H}^{13} \mathrm{C}$ HSQC NMR $\boldsymbol{\alpha}-4$

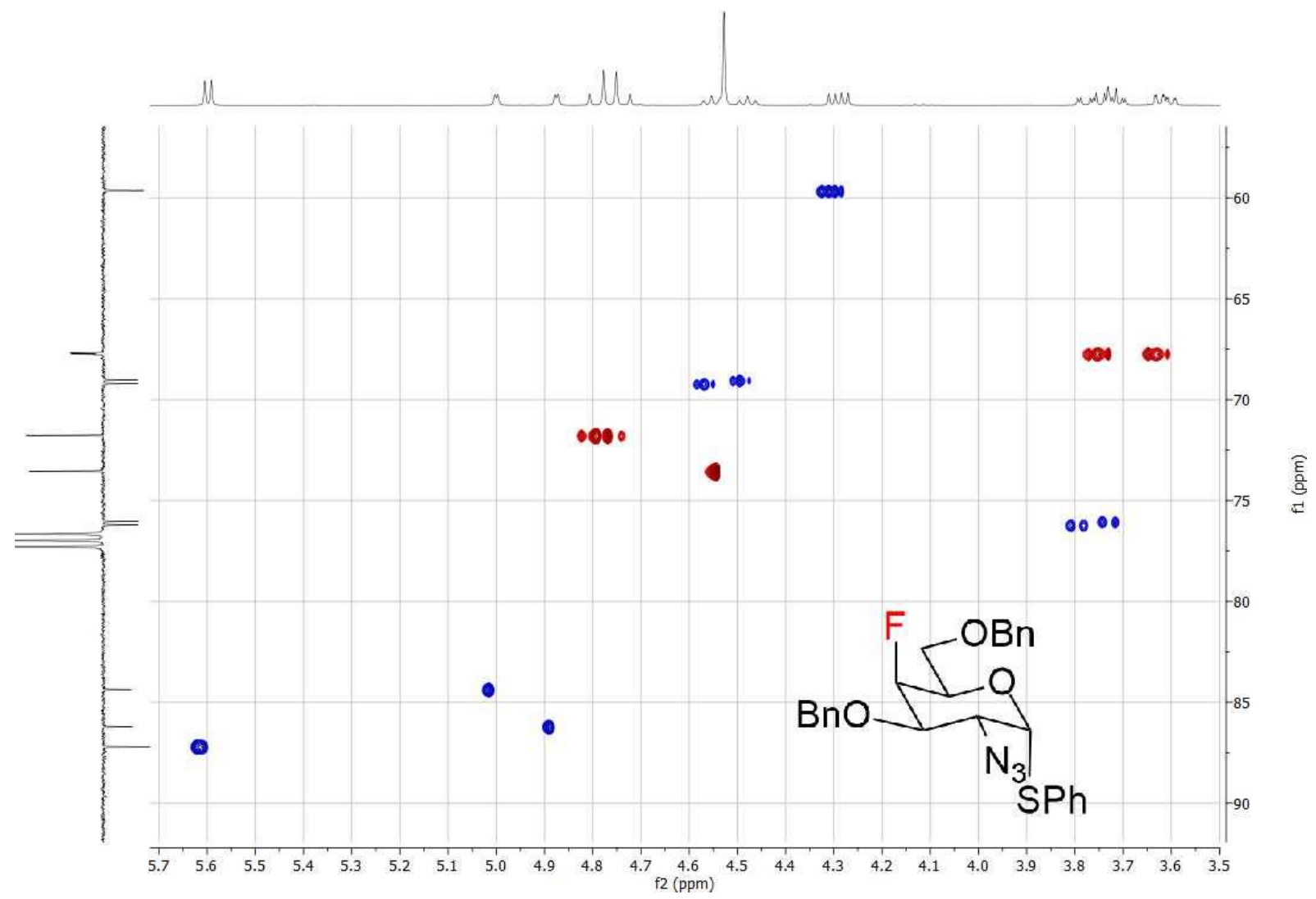


${ }^{19} \mathrm{~F} \mathrm{NMR} 376 \mathrm{MHz}, \mathrm{CDCl}_{3} \boldsymbol{\alpha}-\mathbf{4}$

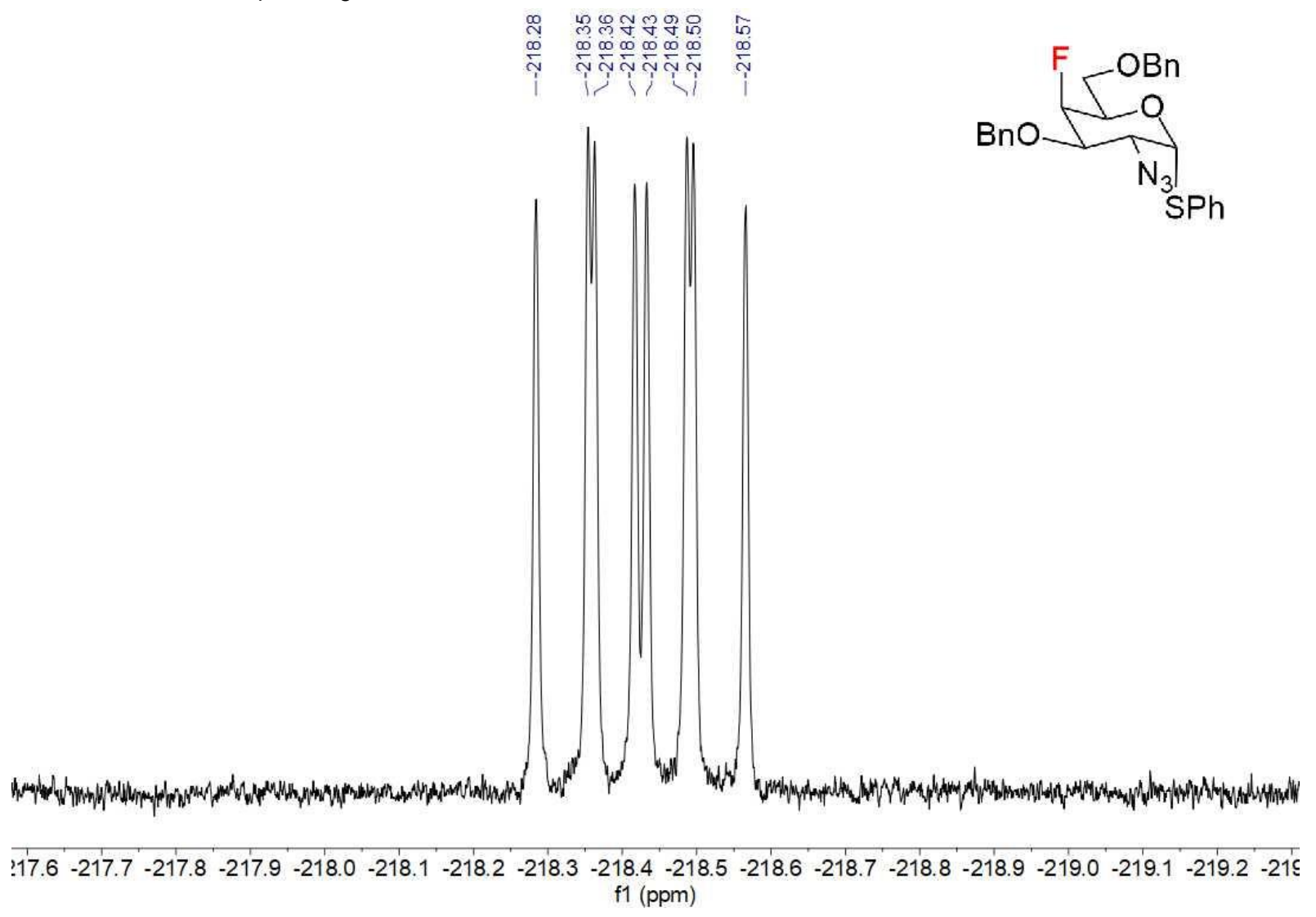

S82 
NMR $\boldsymbol{\beta}-4$

${ }^{1} \mathrm{H}$ NMR $400 \mathrm{MHz}, \mathrm{CDCl}_{3} \boldsymbol{\beta}-4$

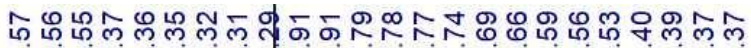

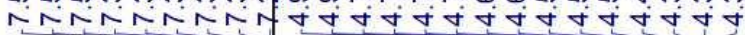

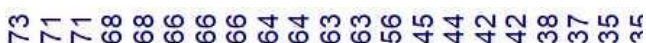

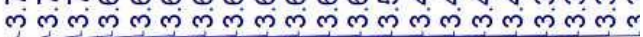

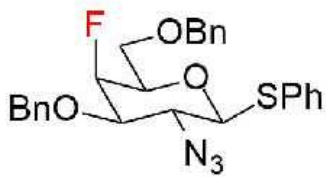

$\mathrm{N}_{3}$

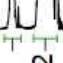

$\stackrel{1}{\stackrel{1}{+}}$

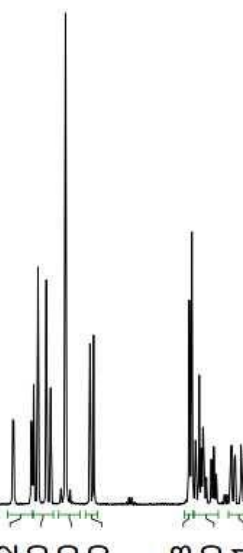

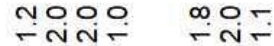

\begin{tabular}{llllllllllllllllllllllllll}
\hline 0.0 & 9.5 & 9.0 & 8.5 & 8.0 & 7.5 & 7.0 & 6.5 & 6.0 & 5.5 & $\begin{array}{l}5.0 \\
\mathrm{f} 1(\mathrm{ppm})\end{array}$ & 4.5 & 4.0 & 3.5 & 3.0 & 2.5 & 2.0 & 1.5 & 1.0 & 0.5 & 0.0 & -0
\end{tabular}

${ }^{13} \mathrm{C}\left\{{ }^{1} \mathrm{H}\right\}$ APT NMR $100 \mathrm{MHz}, \mathrm{CDCl}_{3} \boldsymbol{\beta}-4$<smiles>NC(OCc1c(F)cccc1Br)C(OCBr)c1ccccc1</smiles>

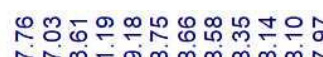

mิง

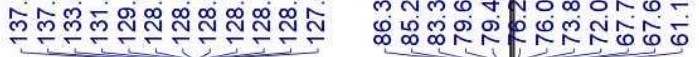

(1)

$\begin{array}{lllllllllllllllllllllllllllll}210 & 200 & 190 & 180 & 170 & 160 & 150 & 140 & 130 & 120 & \begin{array}{c}110 \\ f 1(\mathrm{ppm})\end{array} & 90 & 80 & 70 & 60 & 50 & 40 & 30 & 20 & 10 & 0\end{array}$ 
${ }^{1} \mathrm{H}-{ }^{1} \mathrm{H}$ COSY NMR $\beta-4$

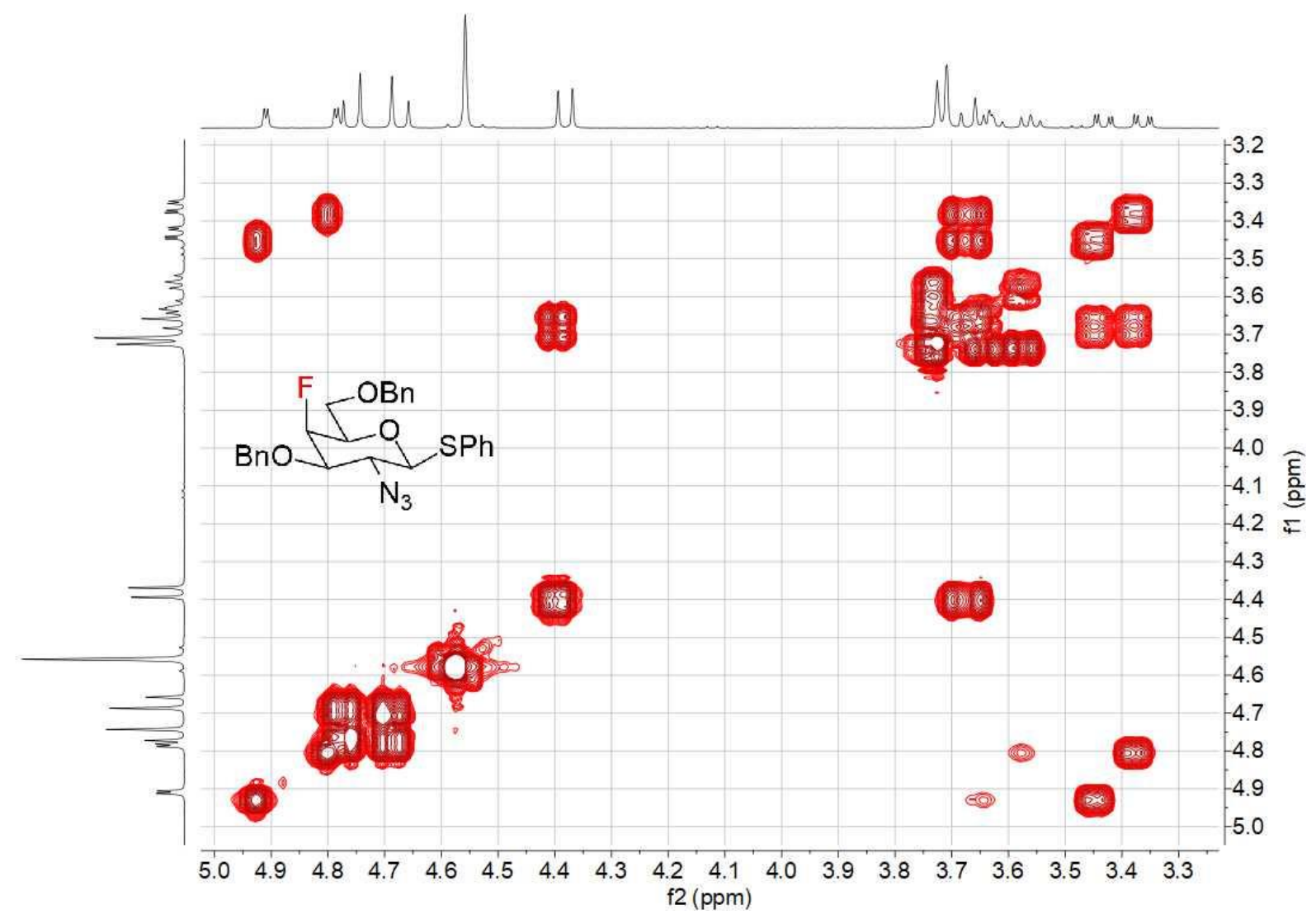

${ }^{1} \mathrm{H}-{ }^{13} \mathrm{C}$ HSQC NMR $\boldsymbol{\beta}-4$

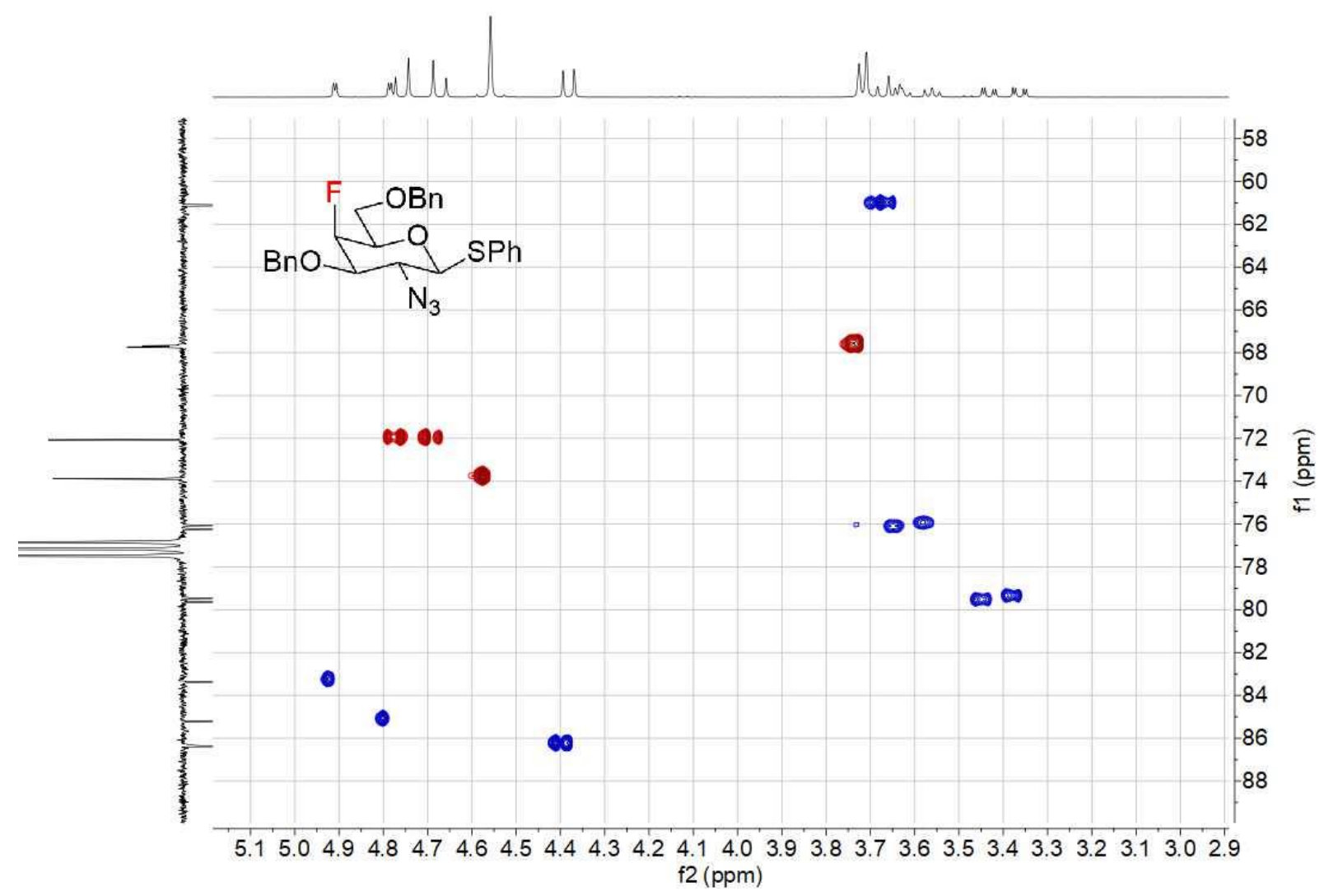




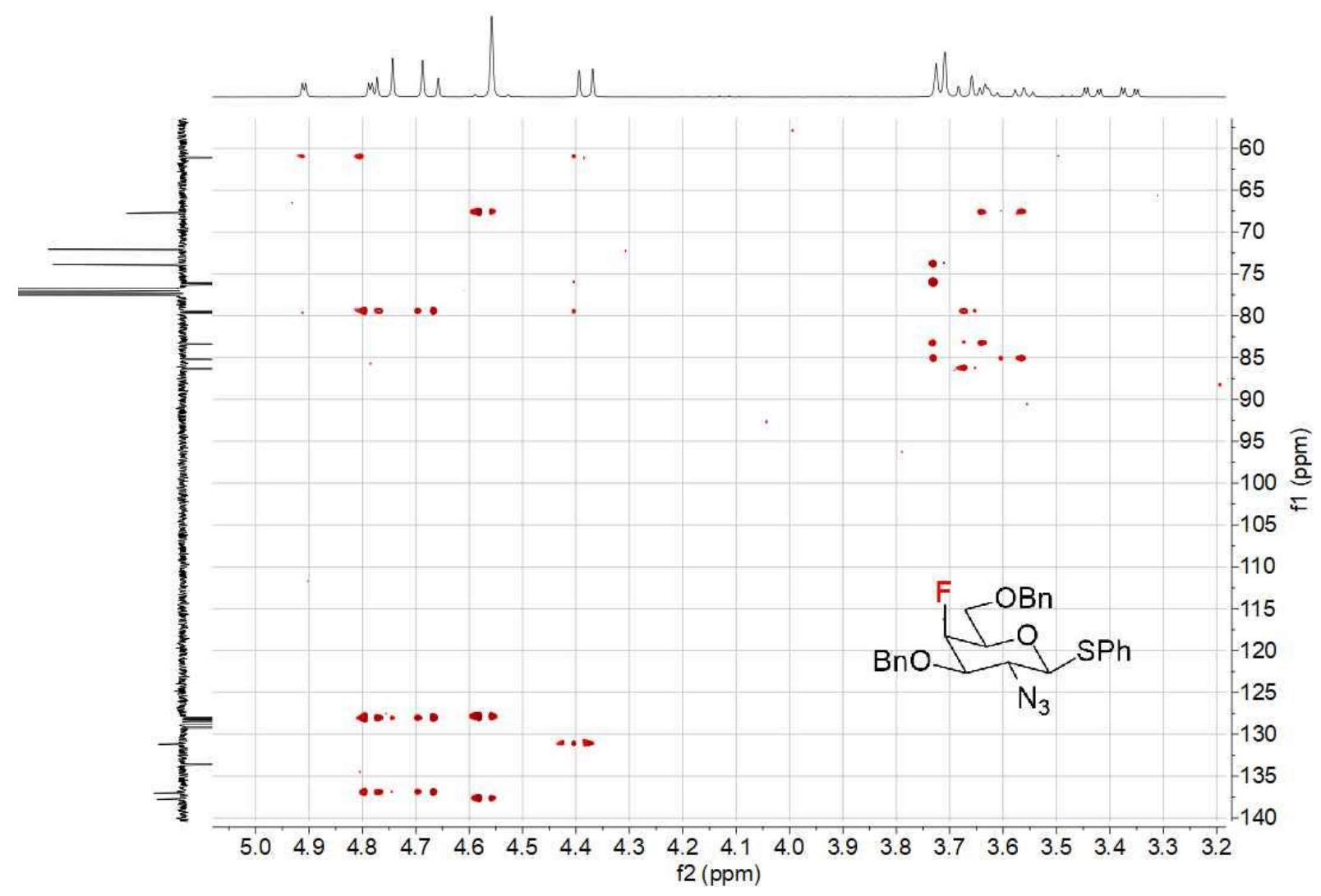

${ }^{19} \mathrm{~F} \mathrm{NMR} 376 \mathrm{MHz}, \mathrm{CDCl}_{3} \boldsymbol{\beta}-4$

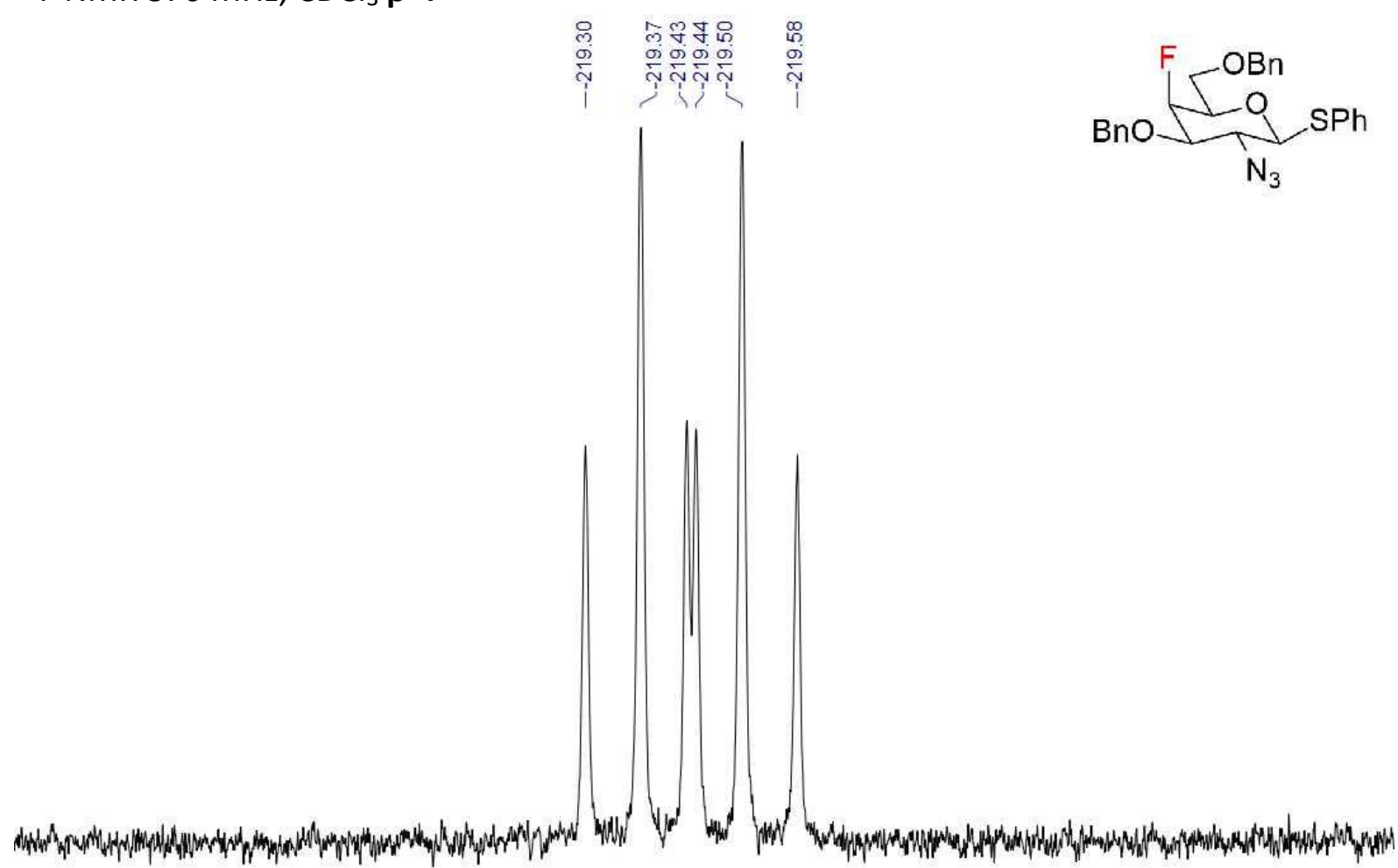


NMR D

${ }^{1} \mathrm{HNMR} 400 \mathrm{MHz}, \mathrm{CDCl}_{3}$ D

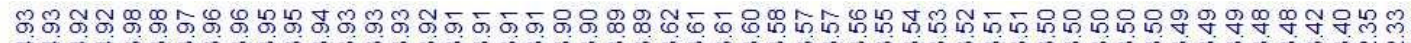
४ण

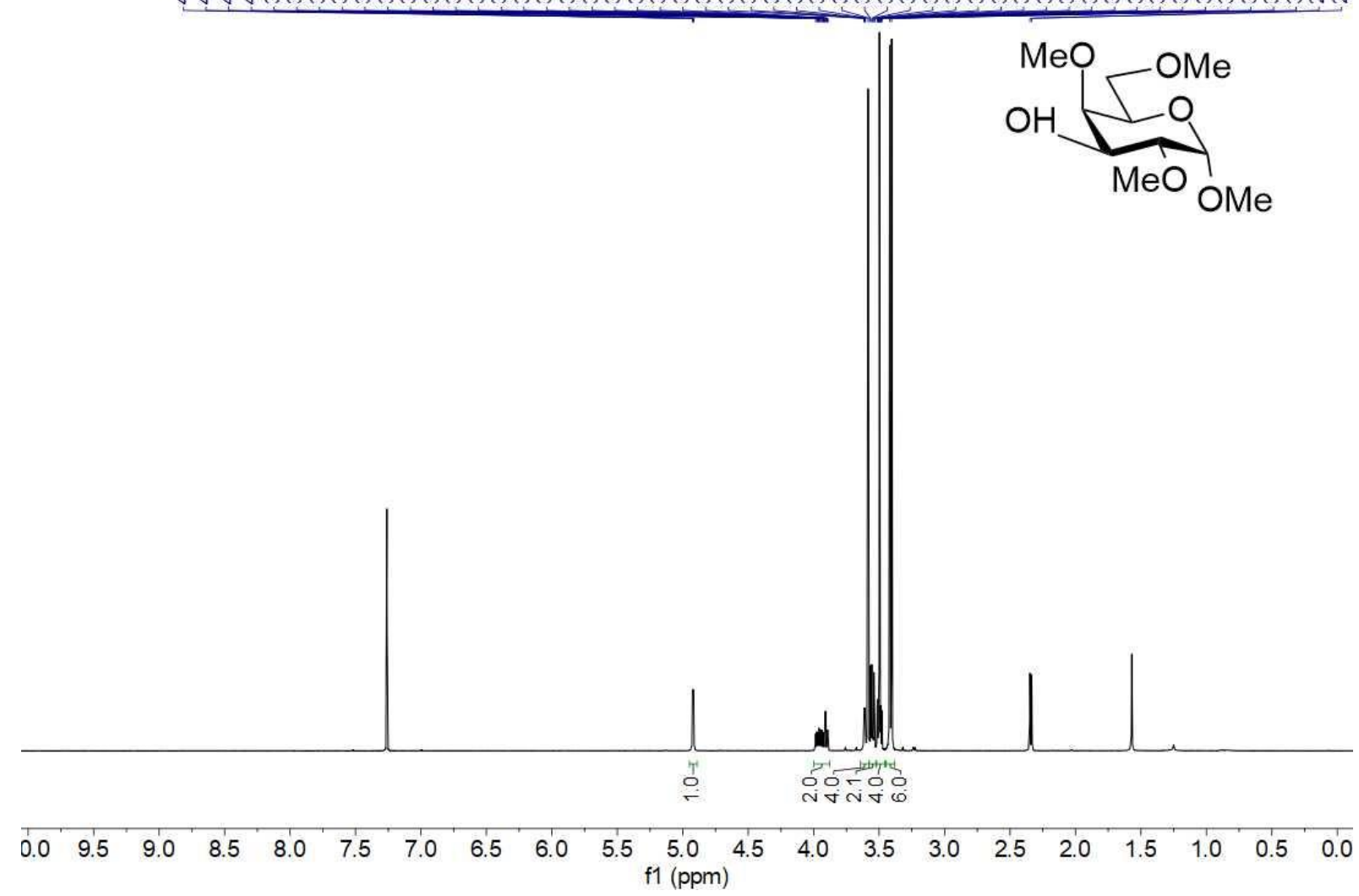

${ }^{13} \mathrm{C}\left\{{ }^{1} \mathrm{H}\right\} \mathrm{NMR} 100 \mathrm{MHz}, \mathrm{CDCl}_{3} \mathrm{D}$<smiles>COC1OC(OC)(C(OC)OC)C(OC)C1O</smiles>

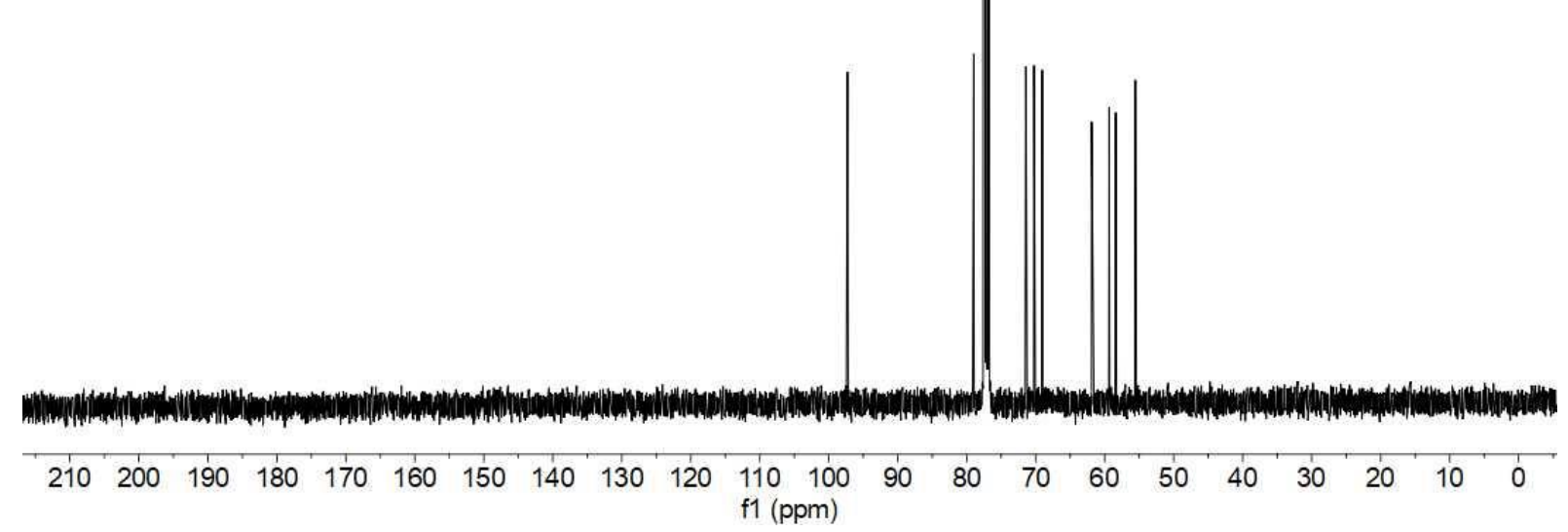


${ }^{1} \mathrm{H}^{-1} \mathrm{H}$ COSY NMR D

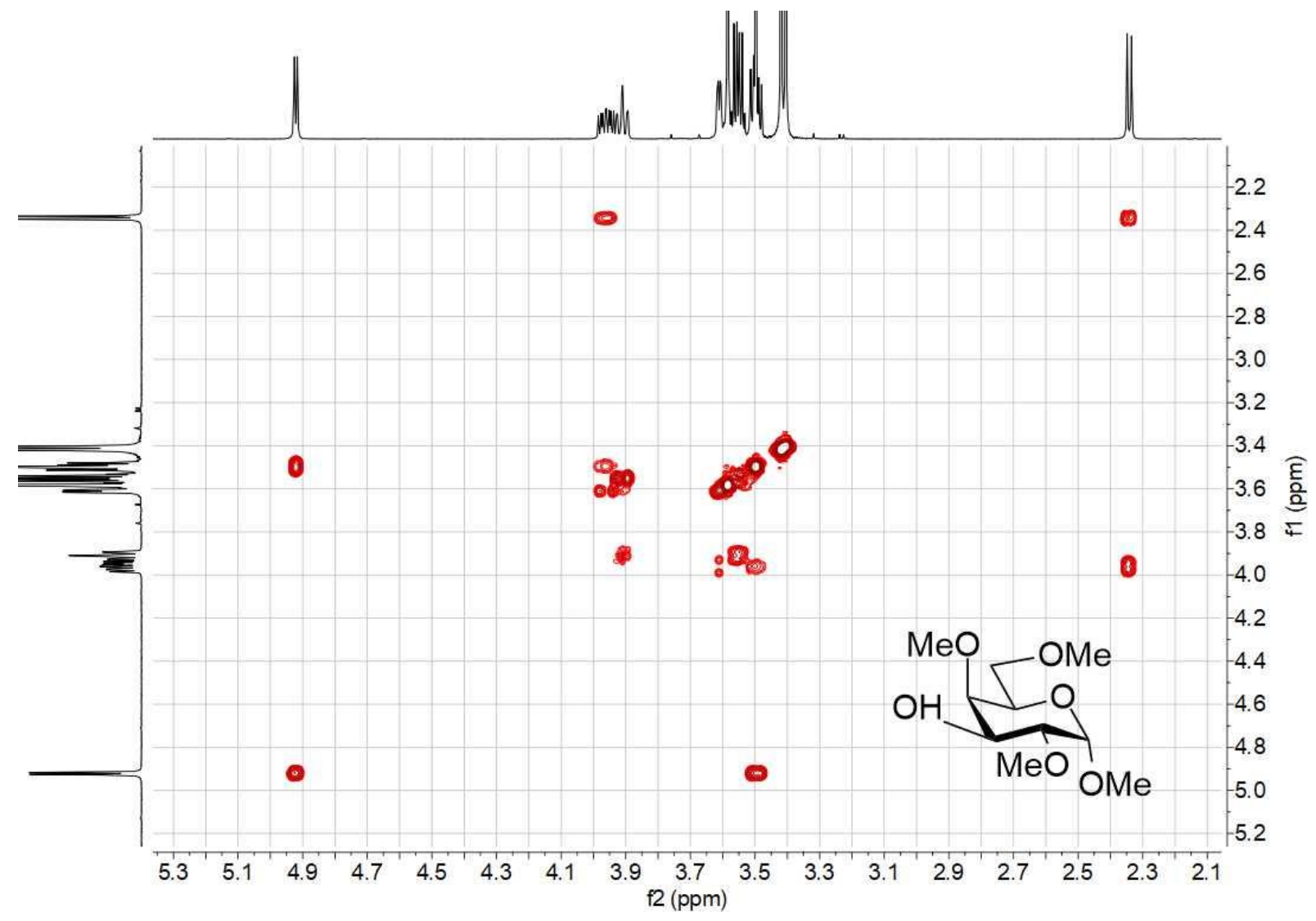

${ }^{1} \mathrm{H}^{-13} \mathrm{C}$ HSQC NMR D

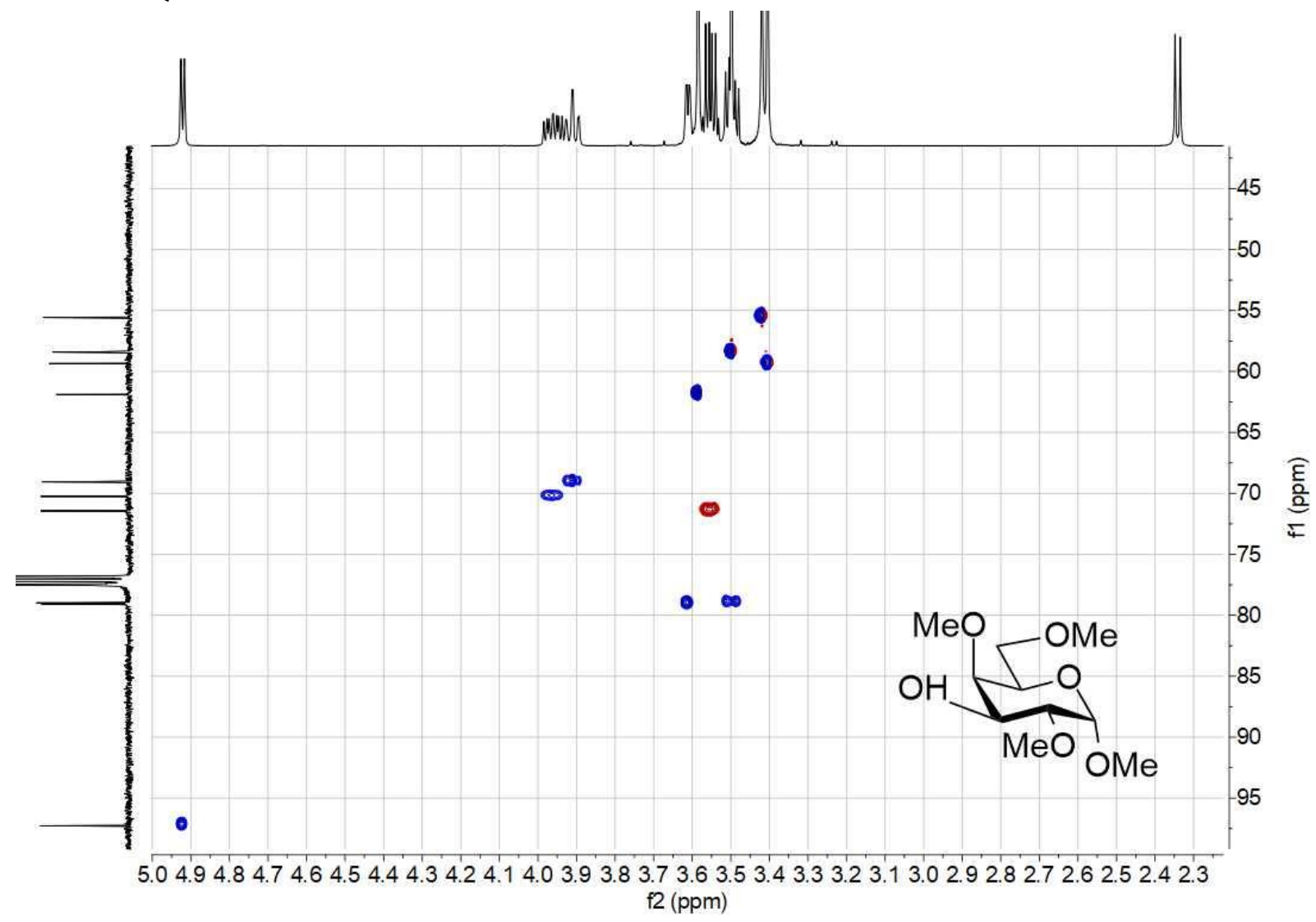


NMR 1A- $\beta$

${ }^{1} \mathrm{HNMR} 400 \mathrm{MHz}, \mathrm{CDCl}_{3} 1 \mathrm{~A}-\boldsymbol{\beta}$ and cca $5 \% 1 \mathrm{~A}-\alpha$

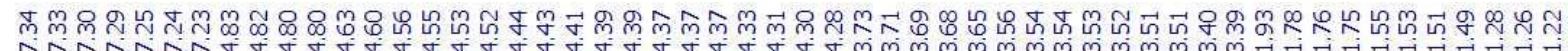

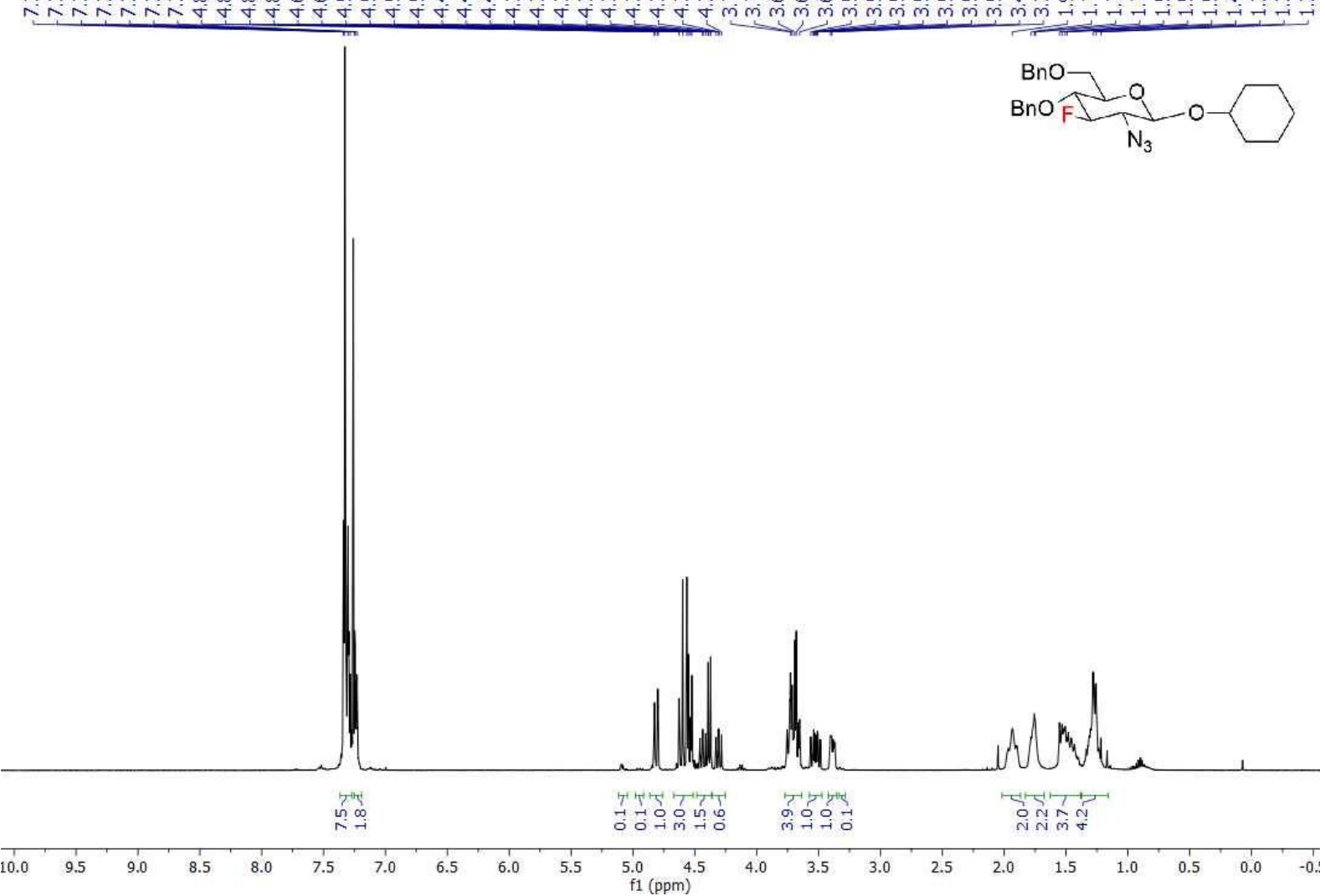

${ }^{13} \mathrm{C}\left\{{ }^{1} \mathrm{H}\right\} \mathrm{NMR} 100 \mathrm{MHz}, \mathrm{CDCl}_{3} \mathbf{1 A -} \boldsymbol{\beta}$ and cca $\mathbf{5} \% \mathbf{1 A - \alpha}$
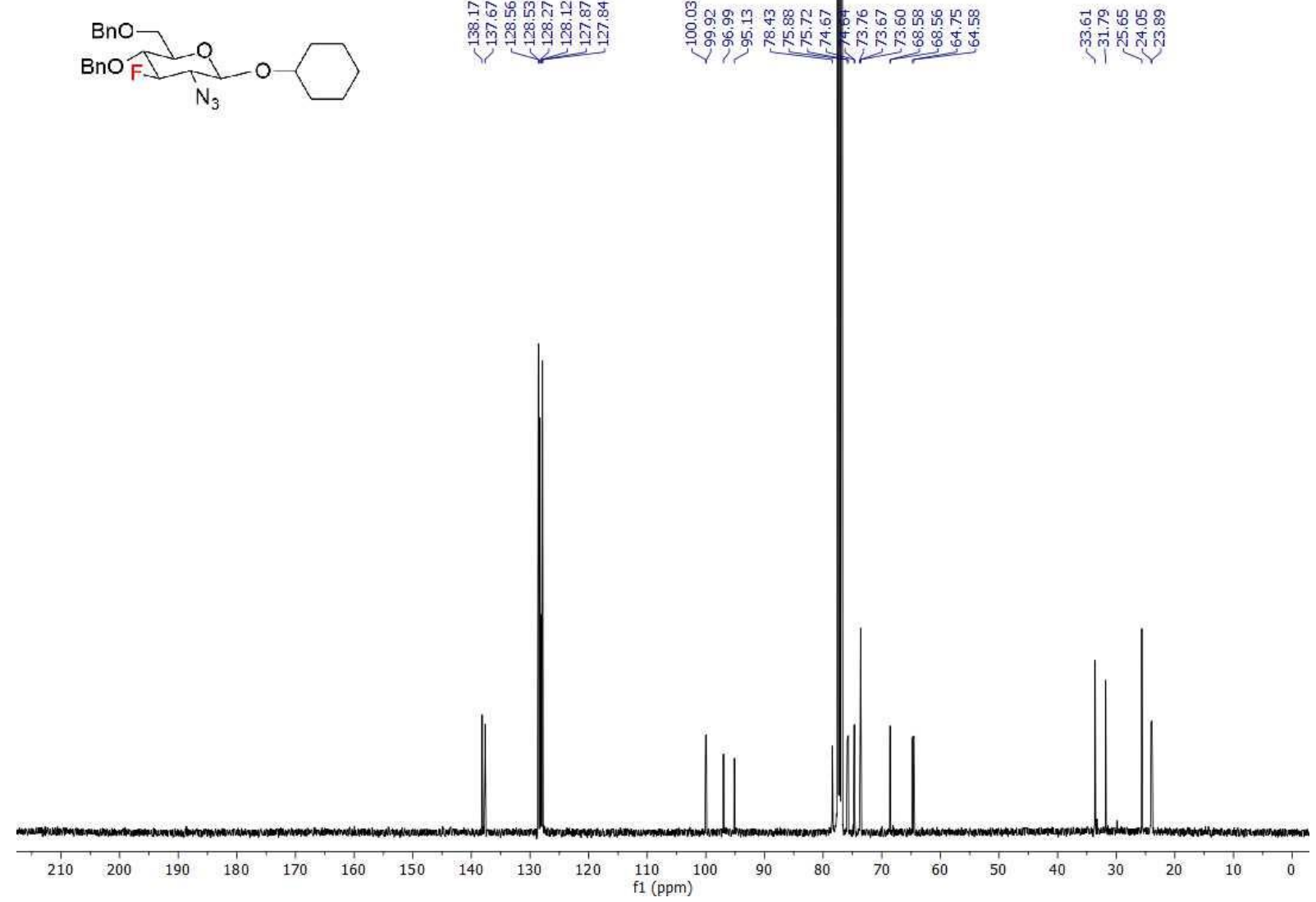
${ }^{1} \mathrm{H}-{ }^{1} \mathrm{H}$ COSY NMR $1 \mathrm{~A}-\beta$ and cca $5 \% 1 \mathrm{~A}-\alpha$

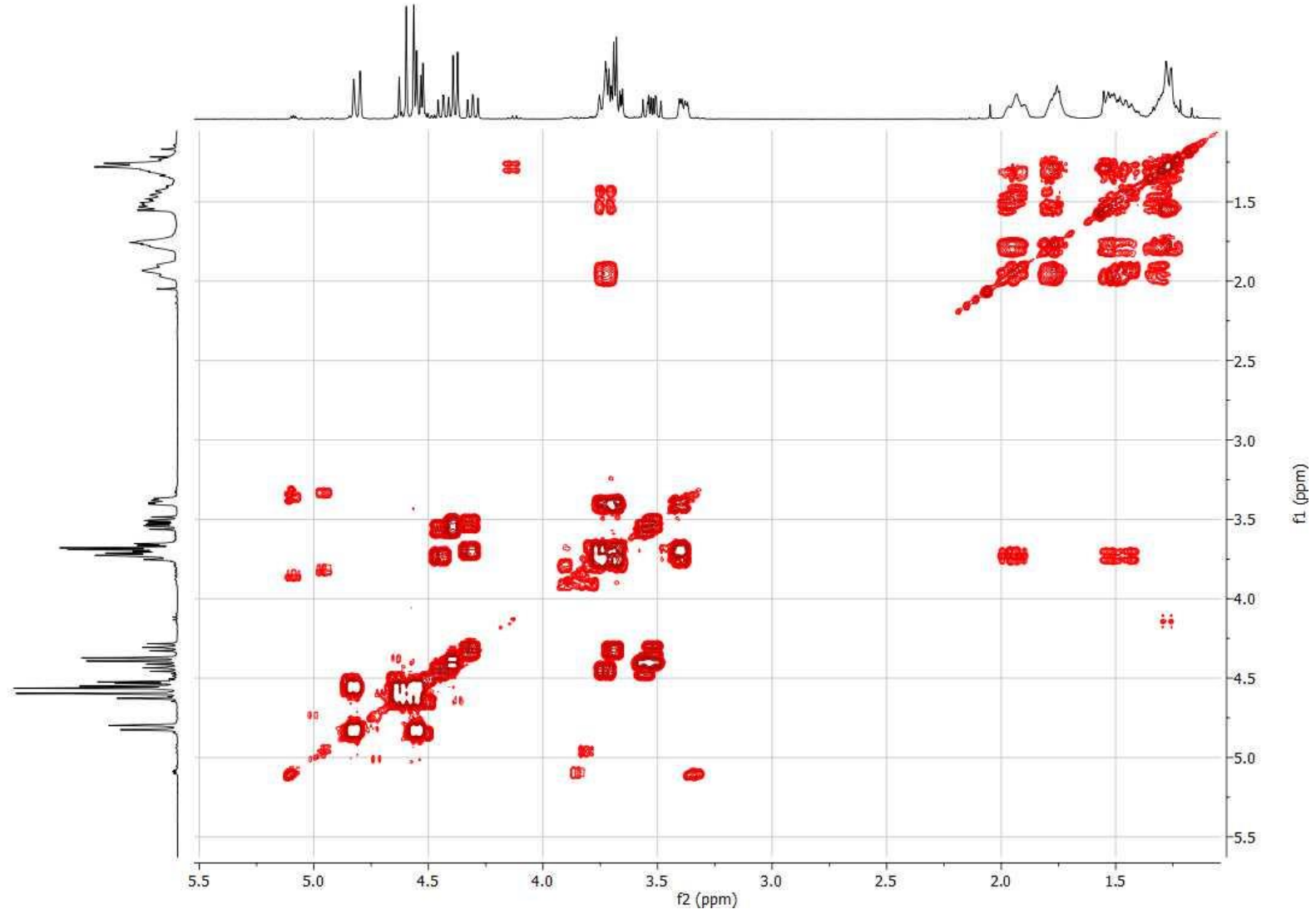

${ }^{1} \mathrm{H}-{ }^{13} \mathrm{C}$ HSQC NMR $1 \mathrm{~A}-\beta$ and cca $5 \% 1 \mathrm{~A}-\alpha$

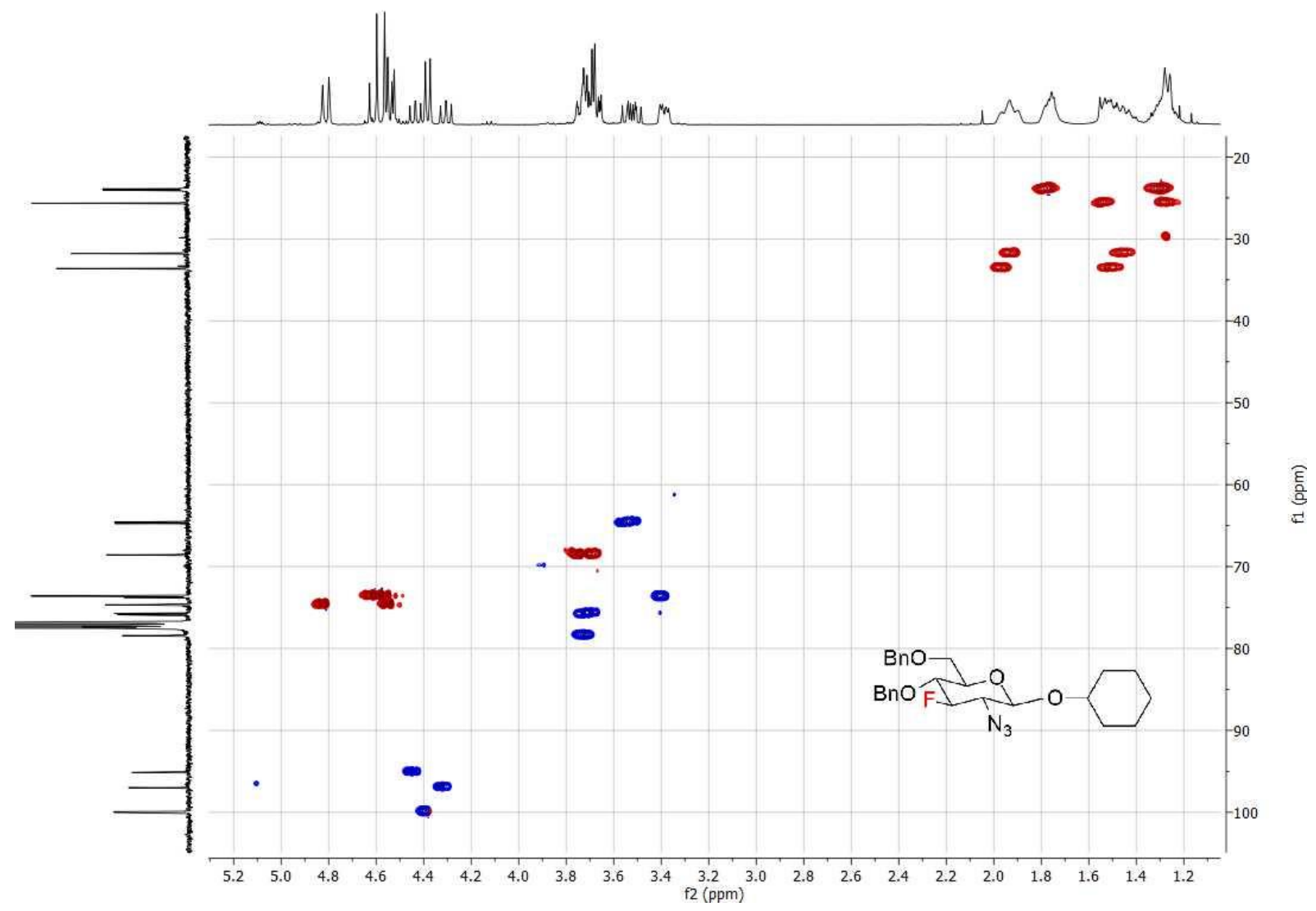


${ }^{1} \mathrm{H}-{ }^{13} \mathrm{CHMBC}$ NMR $1 \mathrm{~A}-\beta$ and cca $5 \%$ 1A- $\alpha$

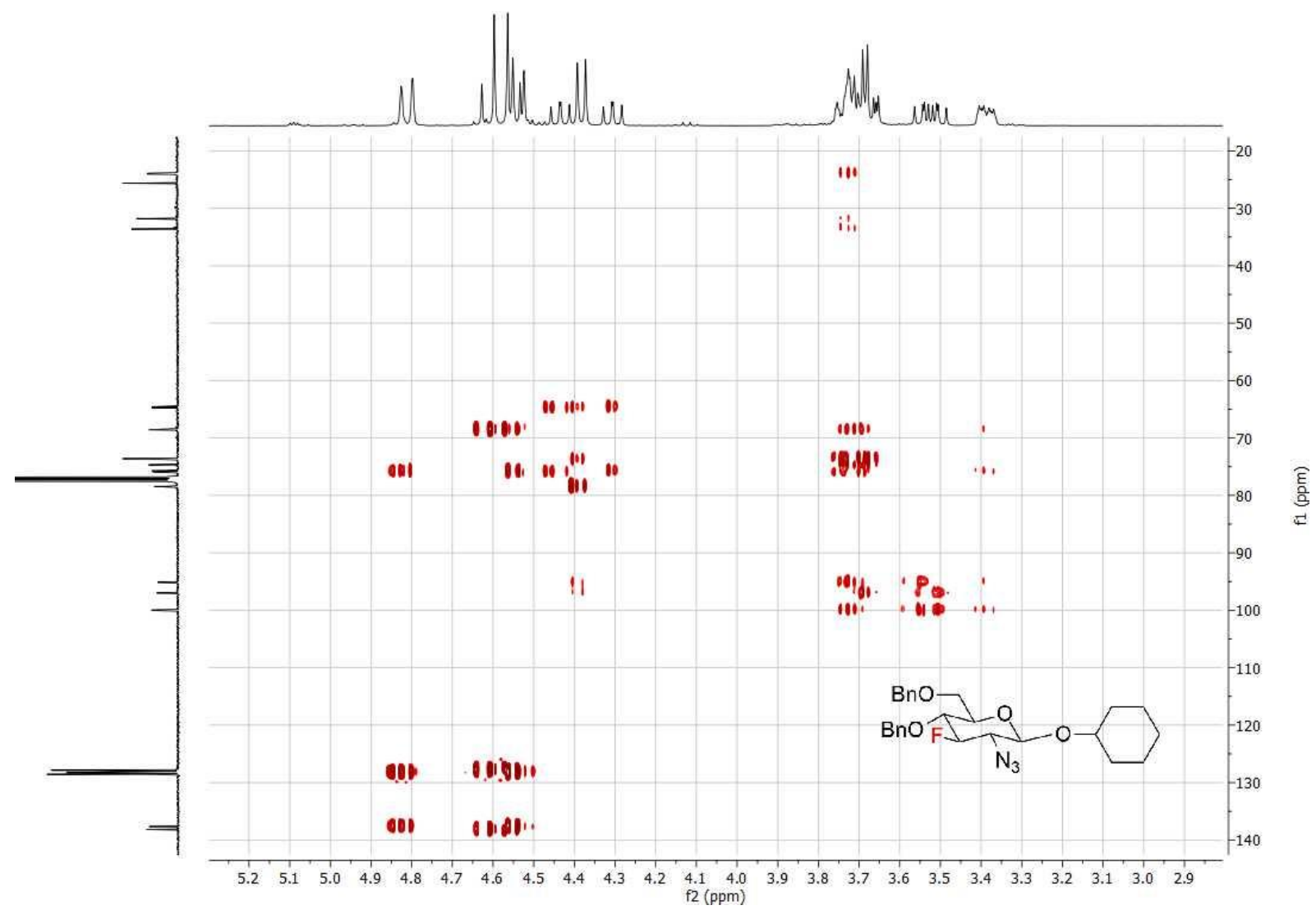

${ }^{19} \mathrm{~F} \mathrm{NMR} 376 \mathrm{MHz}, \mathrm{CDCl}_{3} \mathbf{1 A}-\boldsymbol{\beta}$

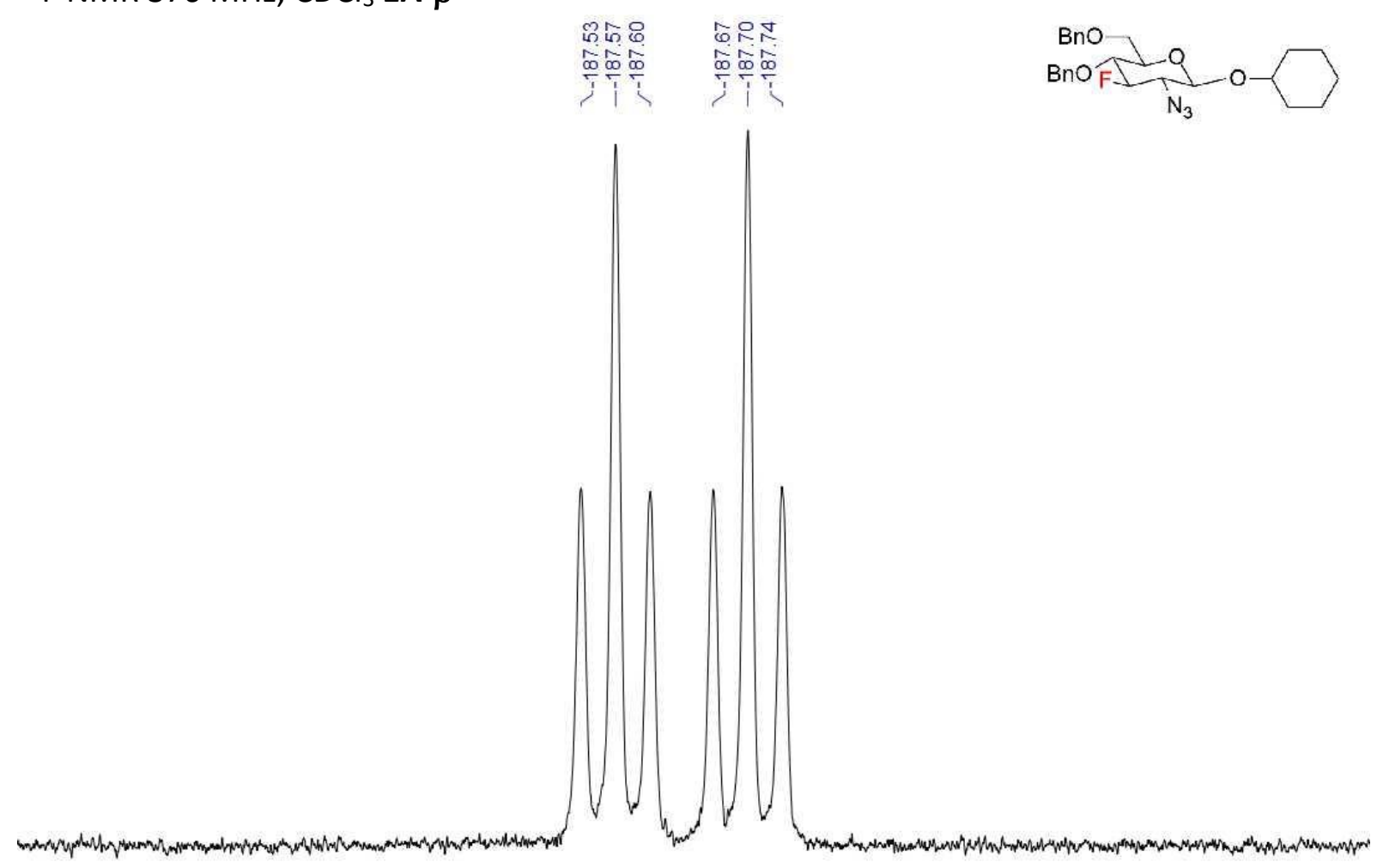

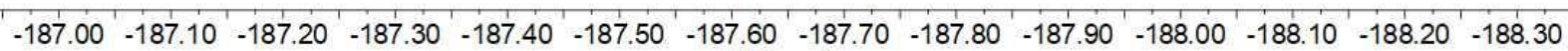
f1 (ppm) 
NMR 1B- $\beta$

${ }^{1} \mathrm{HNMR} 400 \mathrm{MHz}, \mathrm{CDCl}_{3} \mathbf{1 B}-\boldsymbol{\beta}$

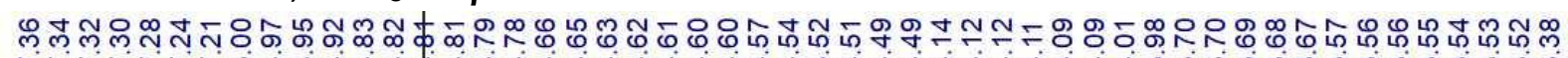
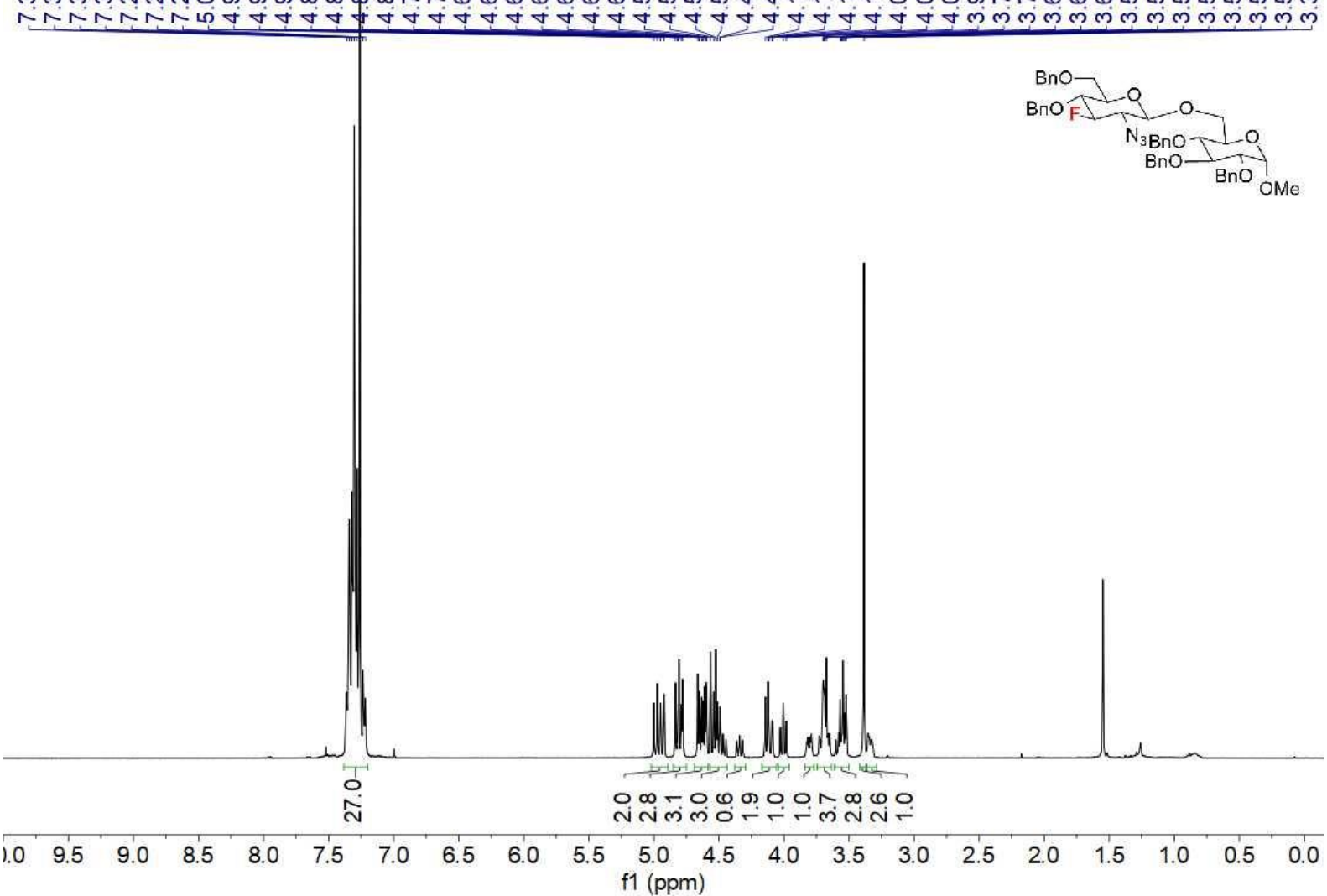

${ }^{13} \mathrm{C}\left\{{ }^{1} \mathrm{H}\right\}$ NMR 100MHz, $\mathrm{CDCl}_{3} \mathbf{1 B}-\boldsymbol{\beta}$

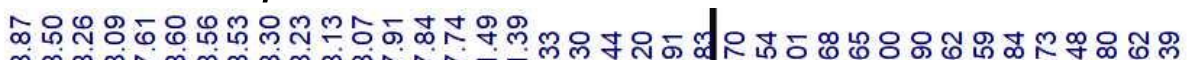

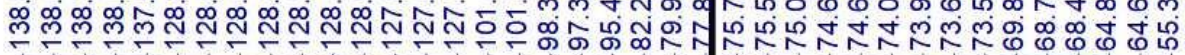

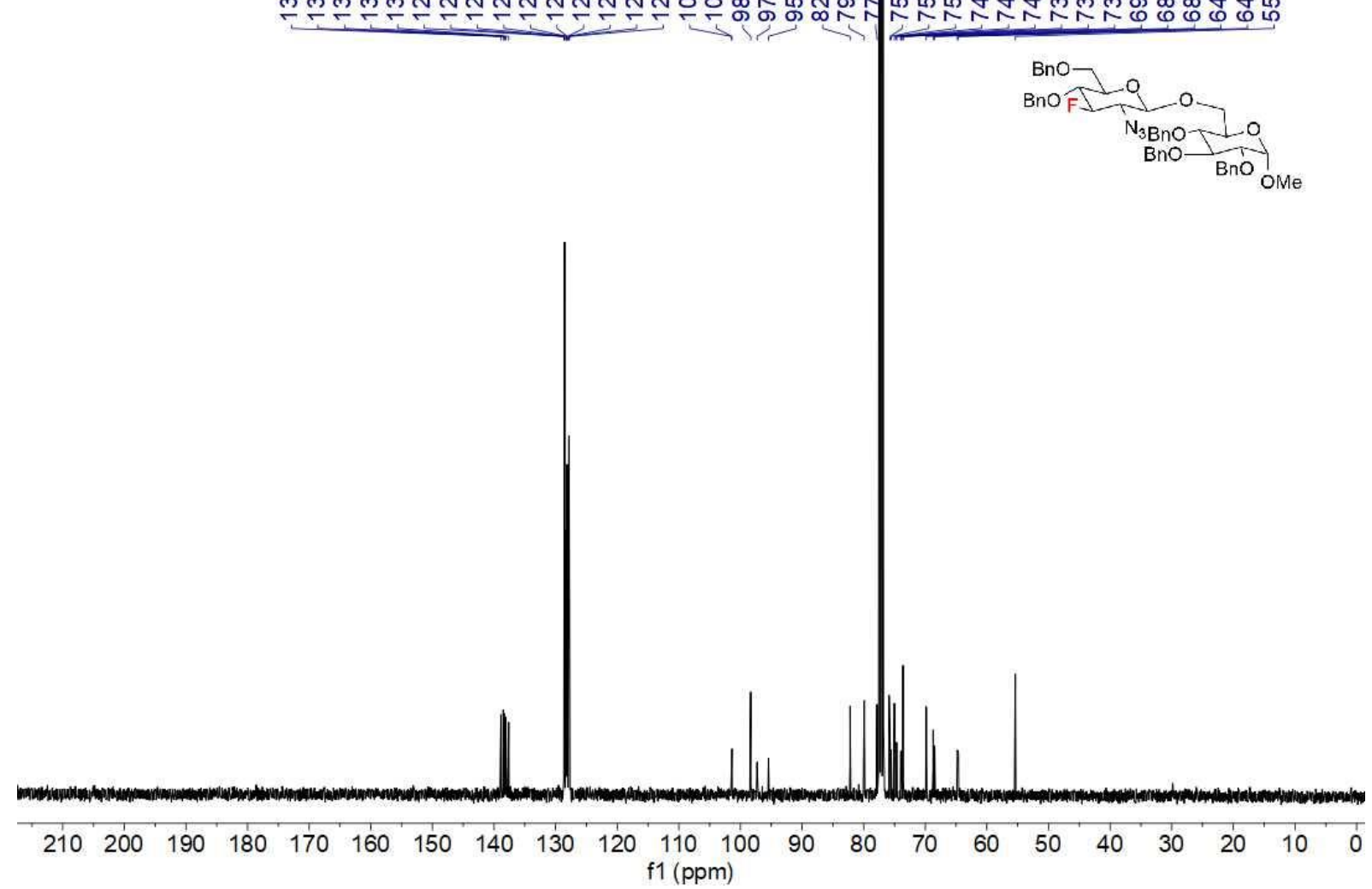


${ }^{1} \mathrm{H}-{ }^{1} \mathrm{H}$ COSY NMR $1 \mathrm{~B}-\boldsymbol{\beta}$

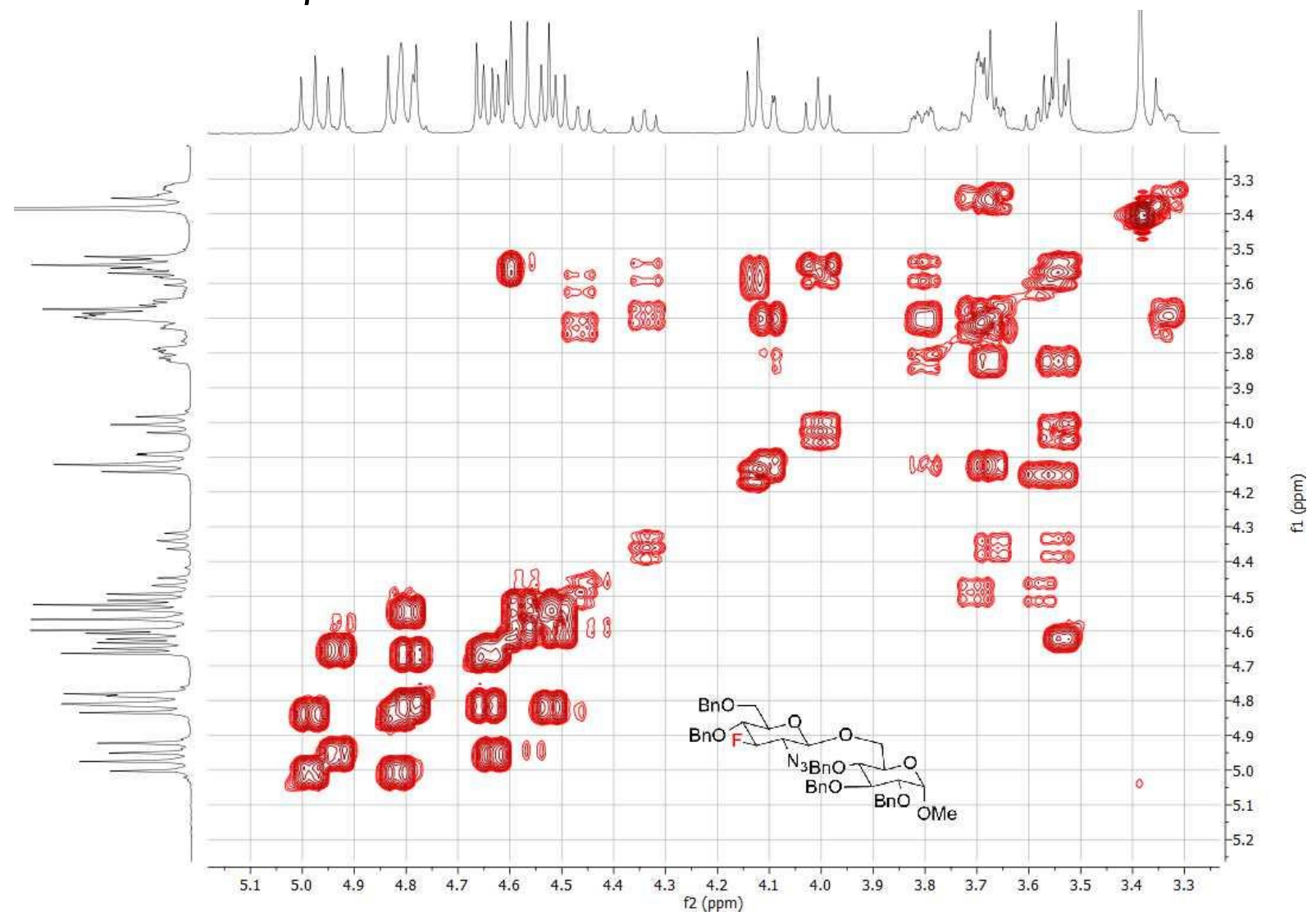

${ }^{1} \mathrm{H}-{ }^{13} \mathrm{C}$ HSOC NMR $1 \mathrm{~B}-\beta$

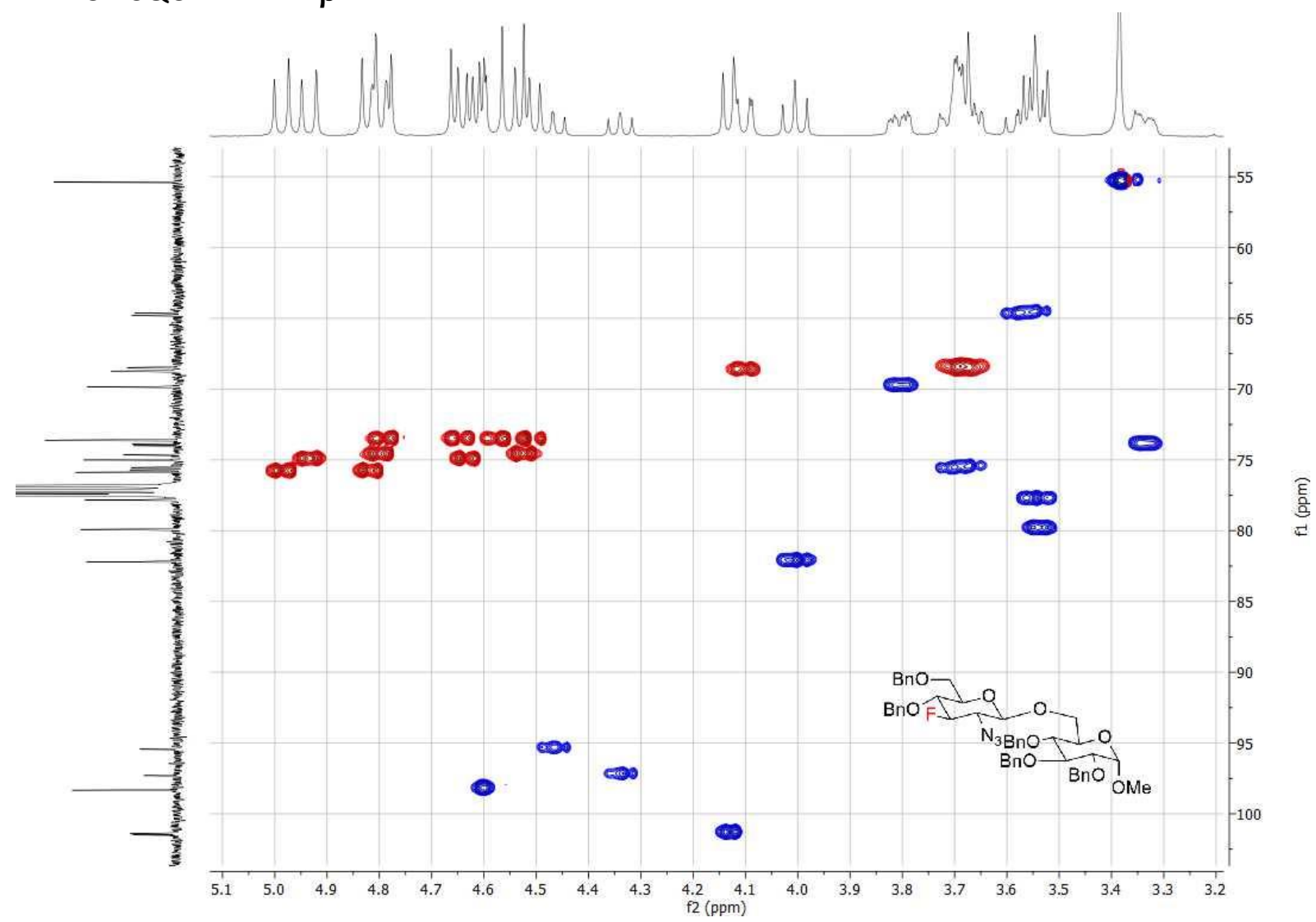




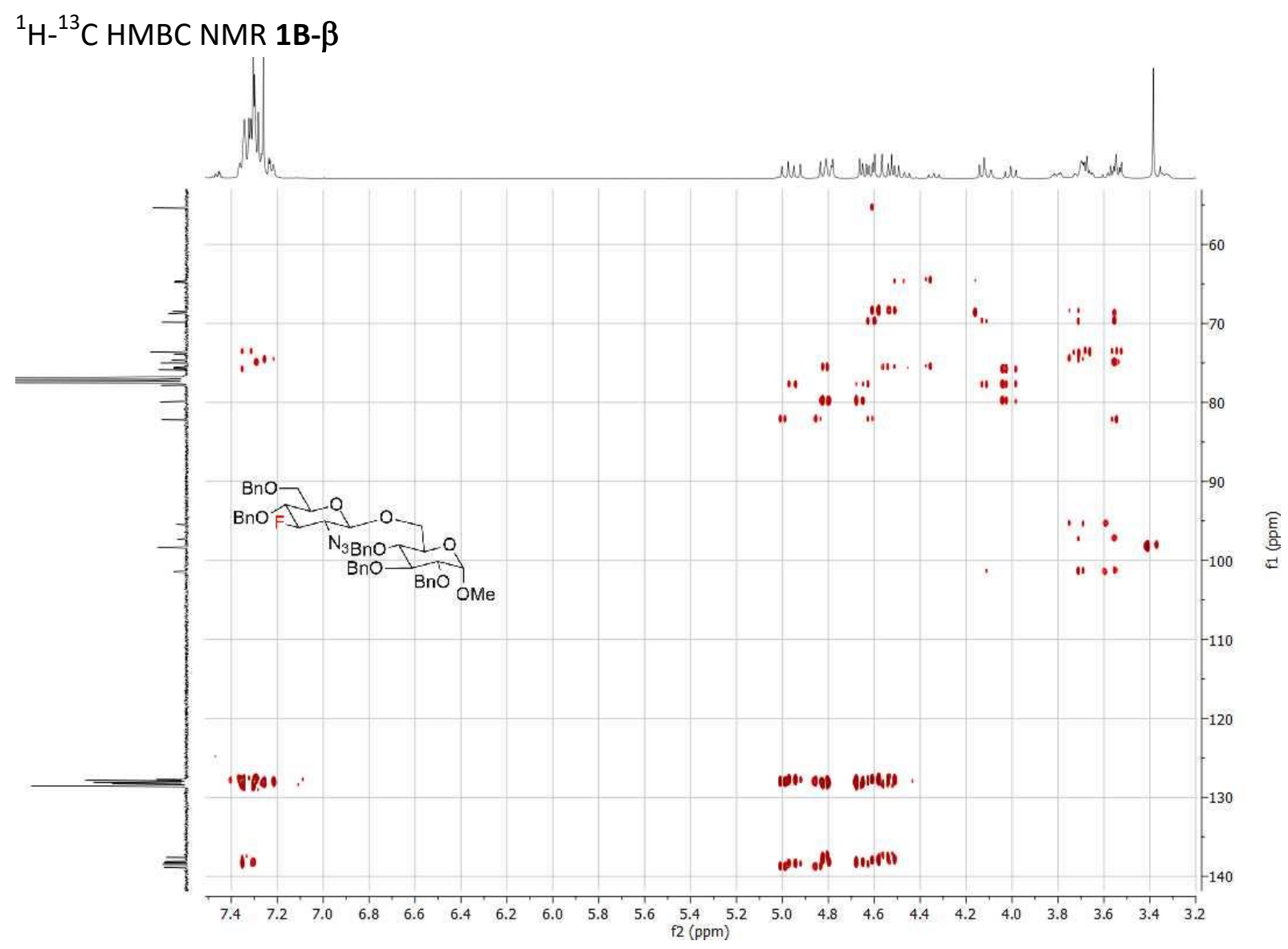

${ }^{19} \mathrm{~F} \mathrm{NMR} 376 \mathrm{MHz}, \mathrm{CDCl}_{3} \mathbf{1 B}-\boldsymbol{\beta}$

ㄷํํำ

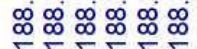

$\underset{\sim}{\longrightarrow} \div$
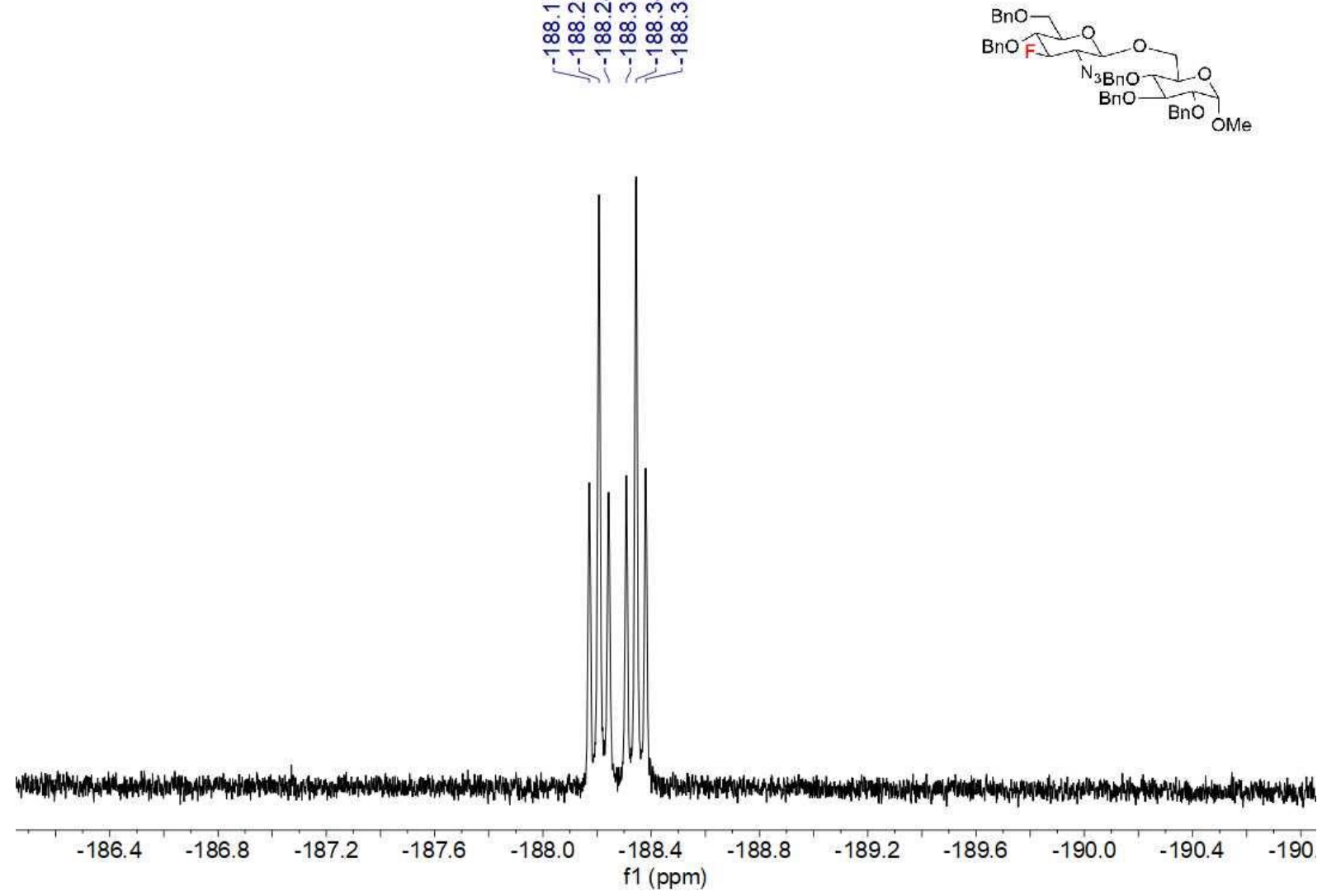
NMR 1C- $\alpha$

${ }^{1} \mathrm{HNMR} 400 \mathrm{MHz}, \mathrm{CDCl}_{3} 1 \mathrm{C}-\alpha$

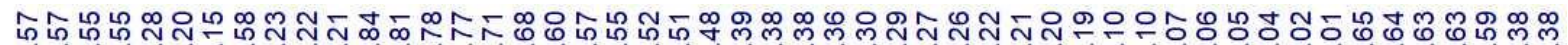

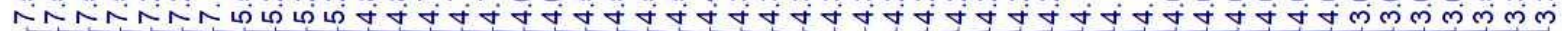

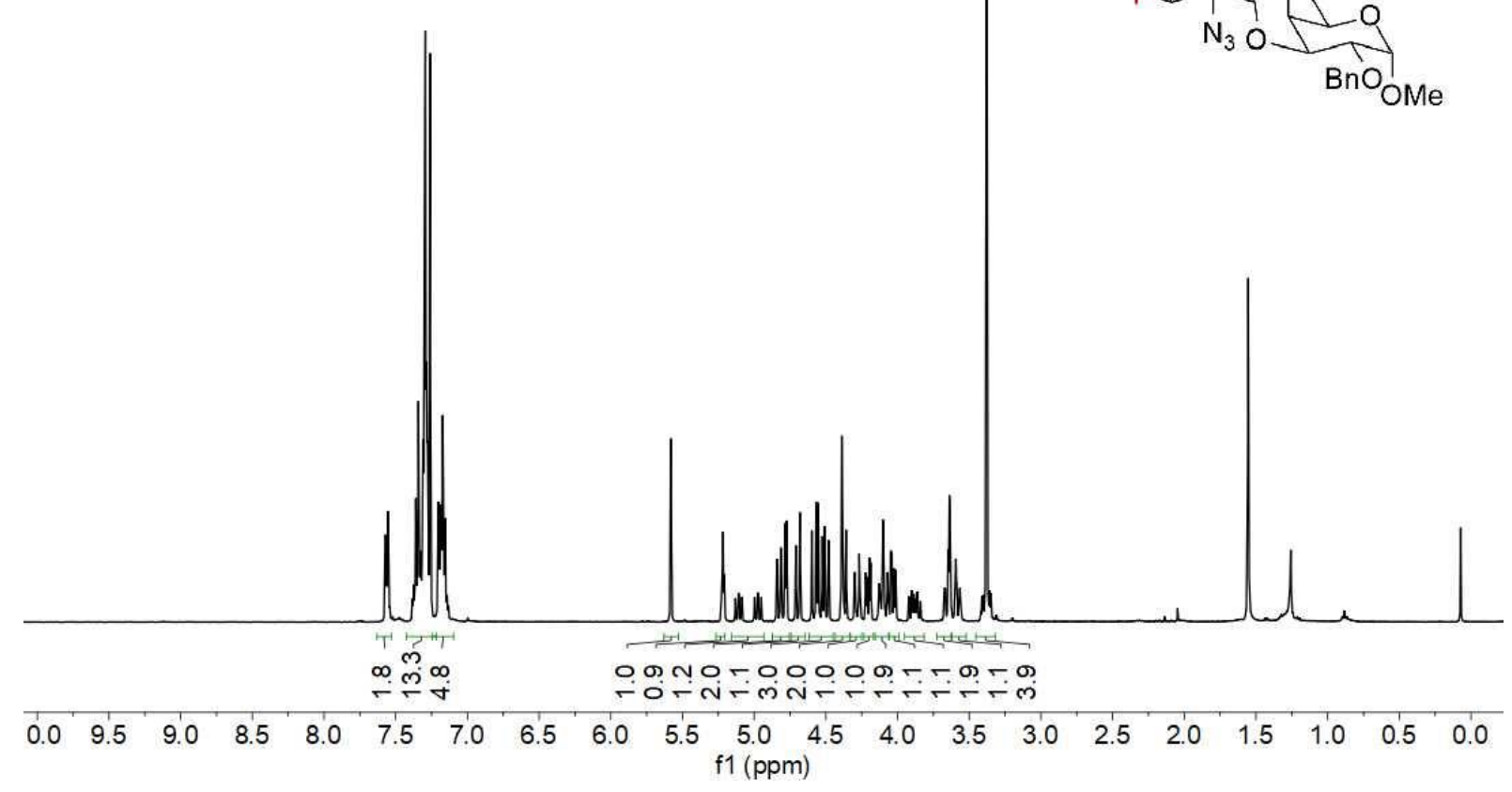


${ }^{13} \mathrm{C}\left\{{ }^{1} \mathrm{H}\right\} \mathrm{NMR} 100 \mathrm{MHz}, \mathrm{CDCl}_{3} \mathbf{1 C}-\alpha$

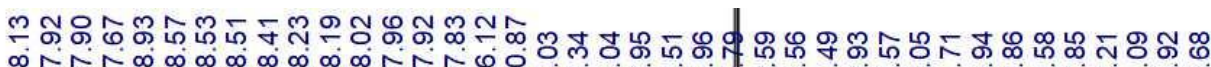

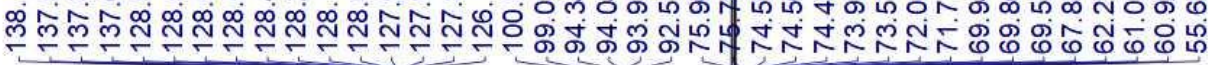

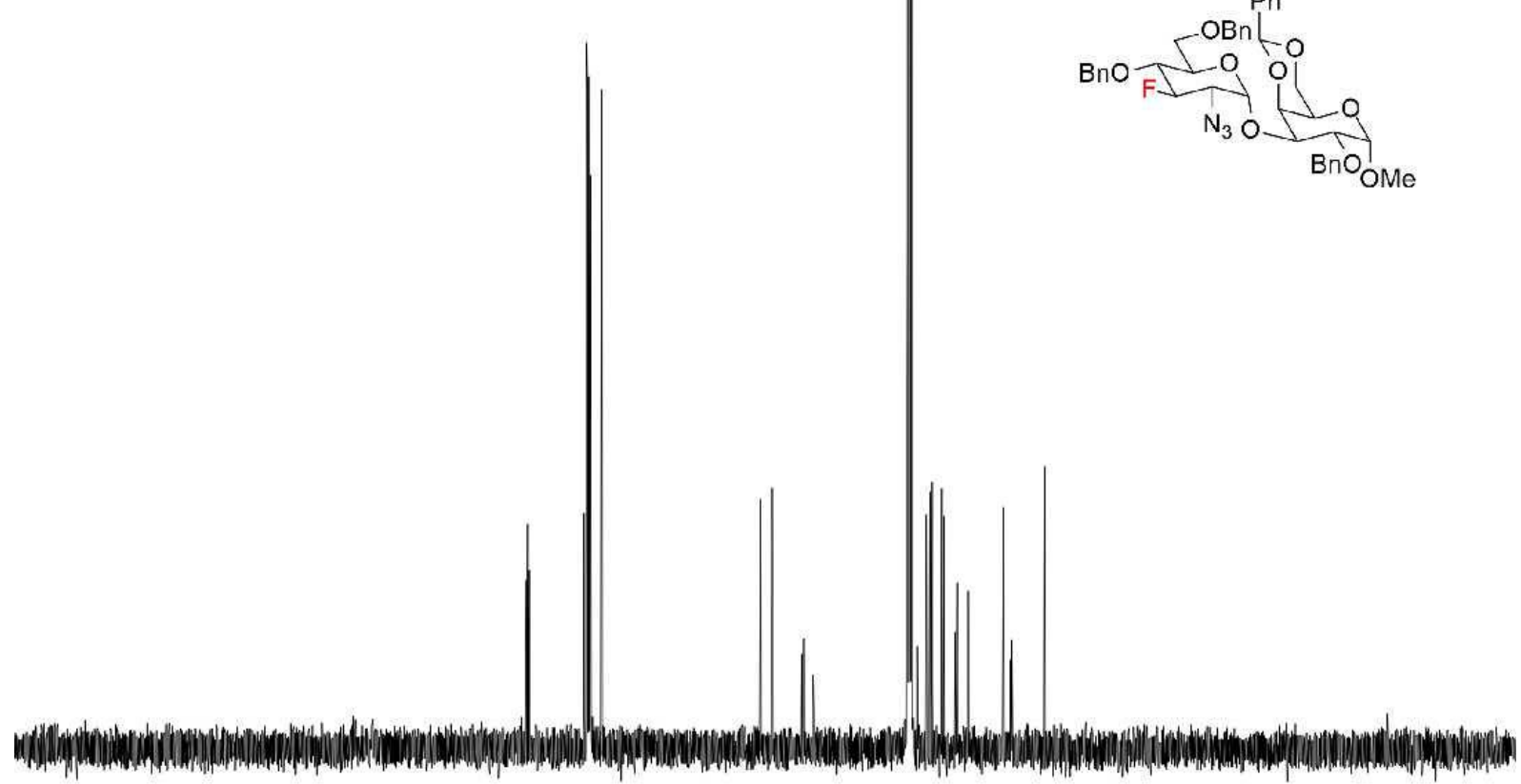

$\begin{array}{lllllllllllllllllllllll}210 & 200 & 190 & 180 & 170 & 160 & 150 & 140 & 130 & 120 & \begin{array}{c}110 \\ \mathrm{f} 1(\mathrm{ppm})\end{array} & 100 & 90 & 80 & 60 & 50 & 40 & 30 & 20 & 10 & 0 & -10\end{array}$

${ }^{1} \mathrm{H}-{ }^{1} \mathrm{H}$ COSY NMR $1 \mathrm{C}-\alpha$

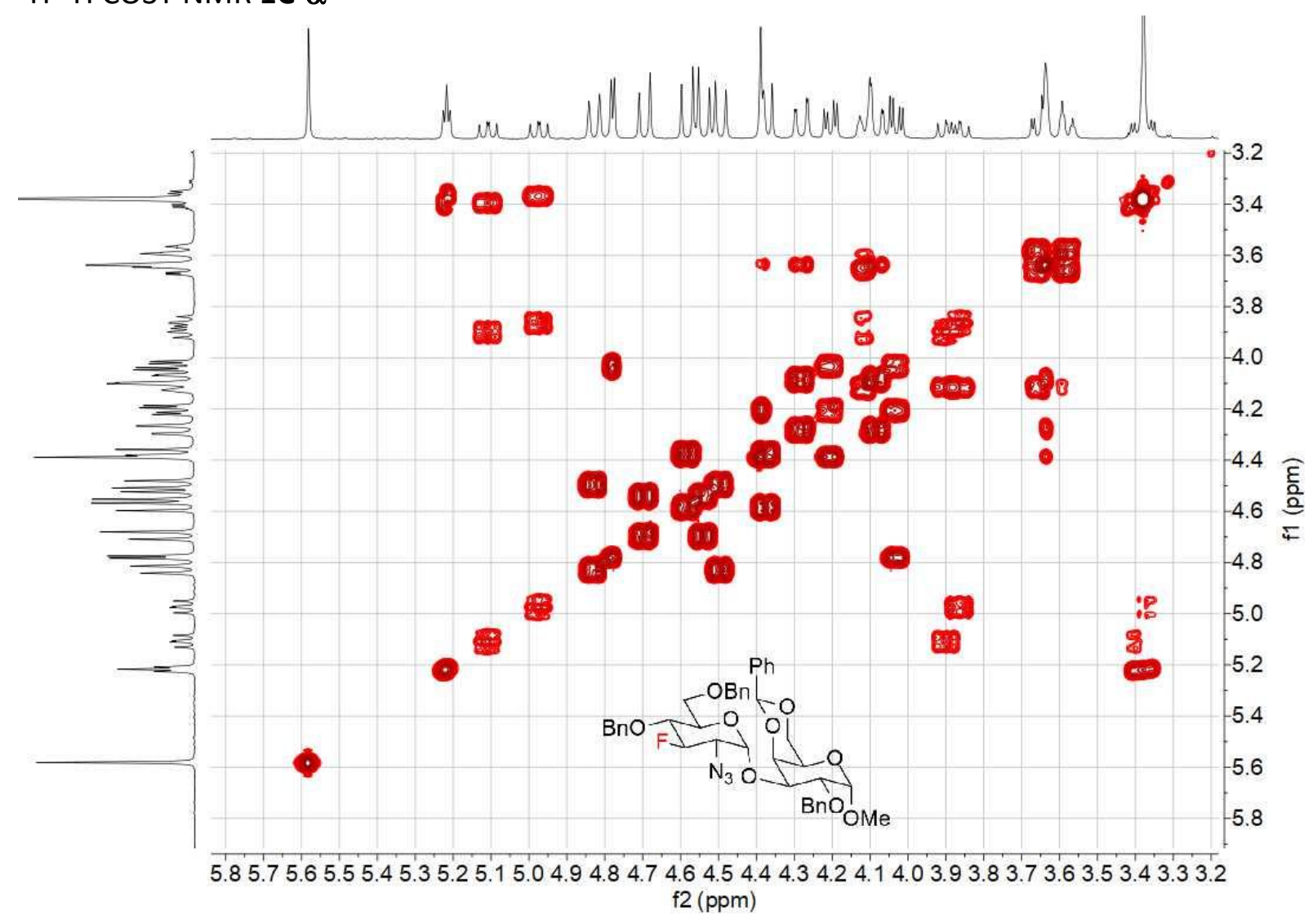


${ }^{1} \mathrm{H}-{ }^{13} \mathrm{C}$ HSOC NMR $1 \mathrm{C}-\alpha$

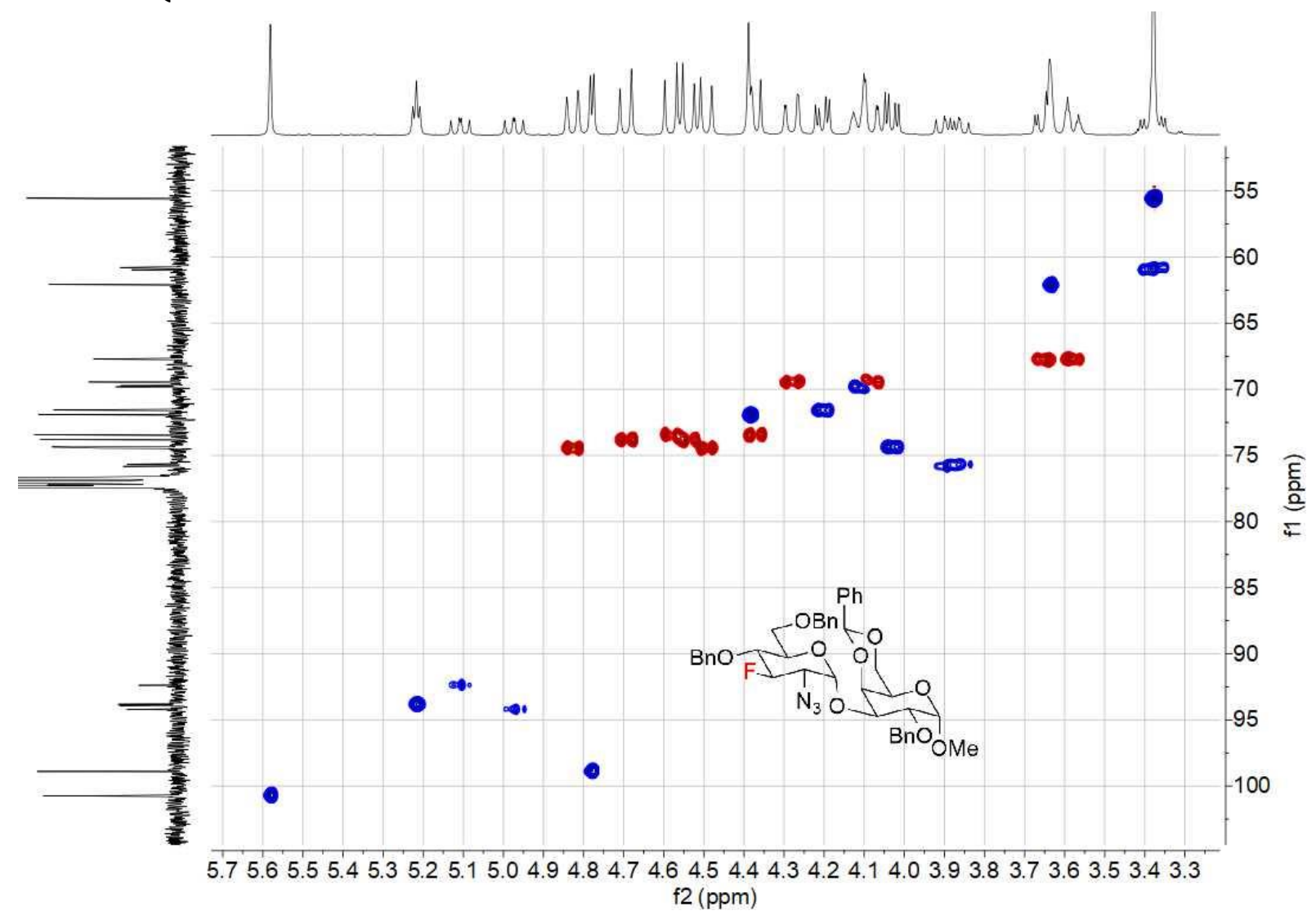

${ }^{1} \mathrm{H}-{ }^{13} \mathrm{C}$ HMBC NMR $1 \mathrm{C}-\alpha$

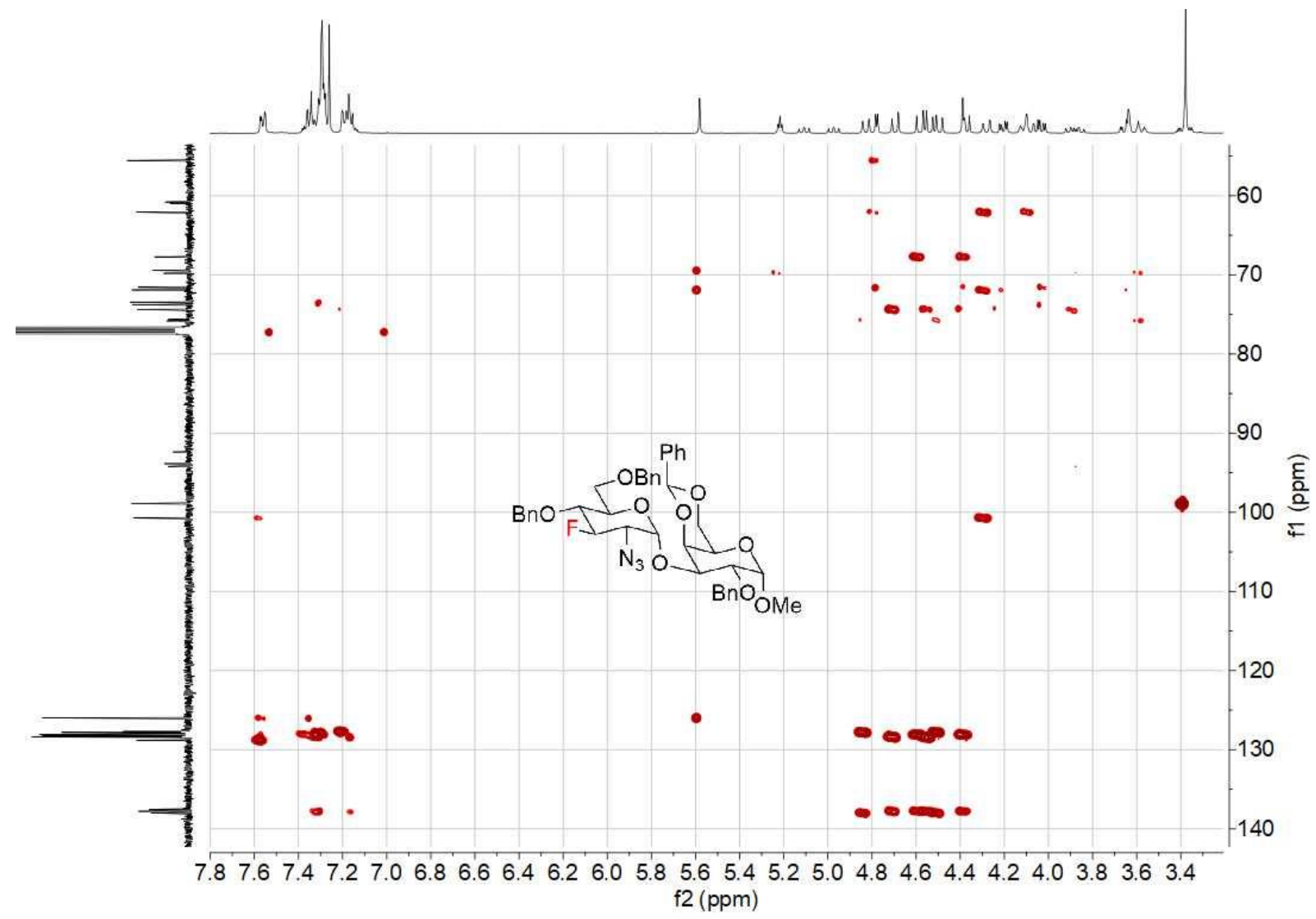


${ }^{19} \mathrm{~F}$ NMR $376 \mathrm{MHz}, \mathrm{CDCl}_{3} 1 \mathrm{C}-\alpha$

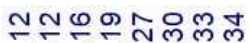

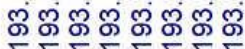

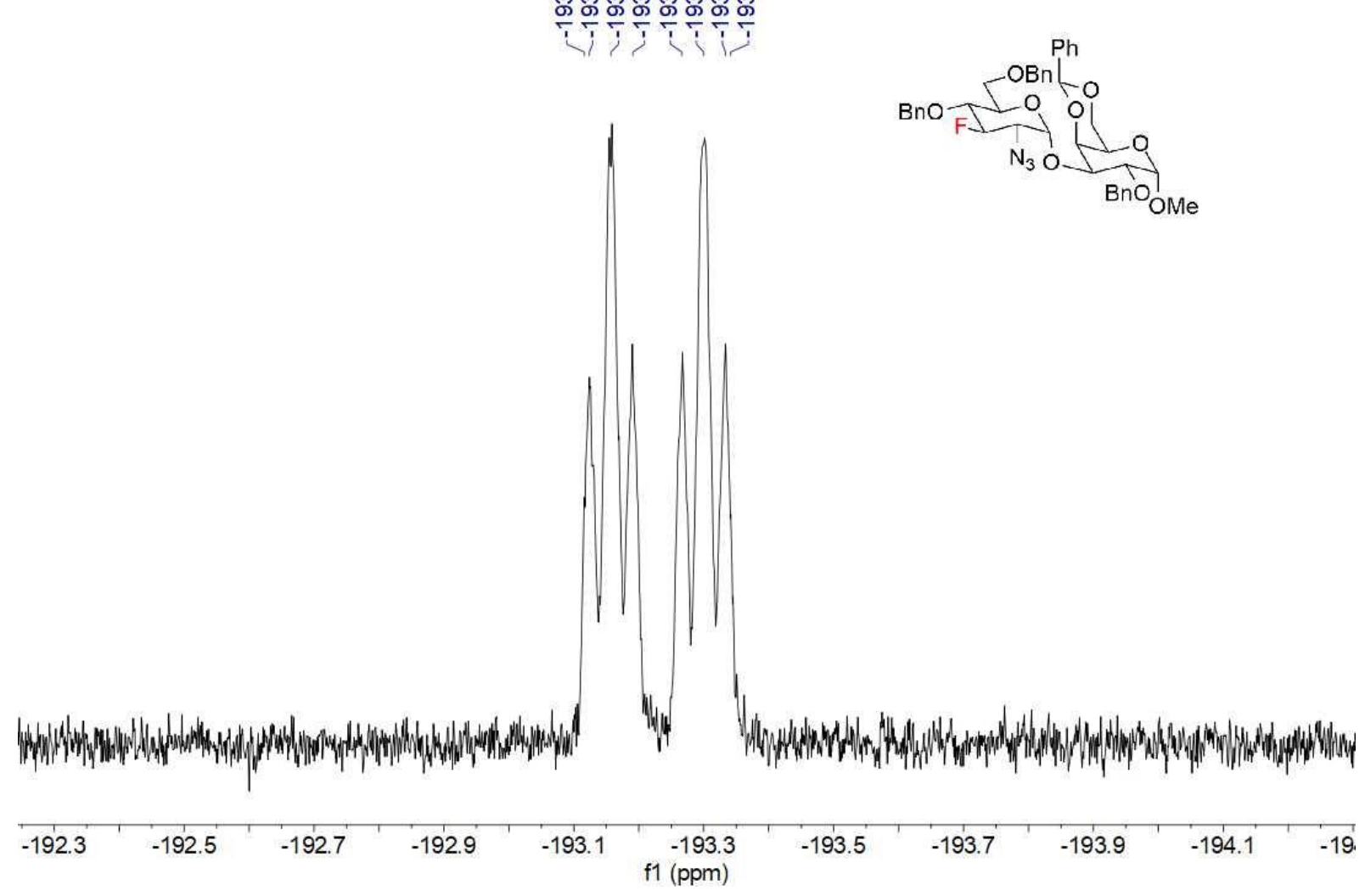

NMR 1C- $\beta$

${ }^{1} \mathrm{H}$ NMR $400 \mathrm{MHz}, \mathrm{CDCl}_{3} 1 \mathrm{C} \beta$

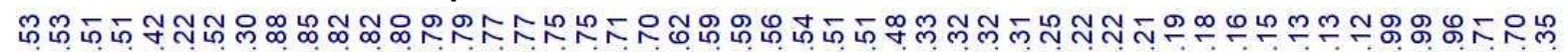

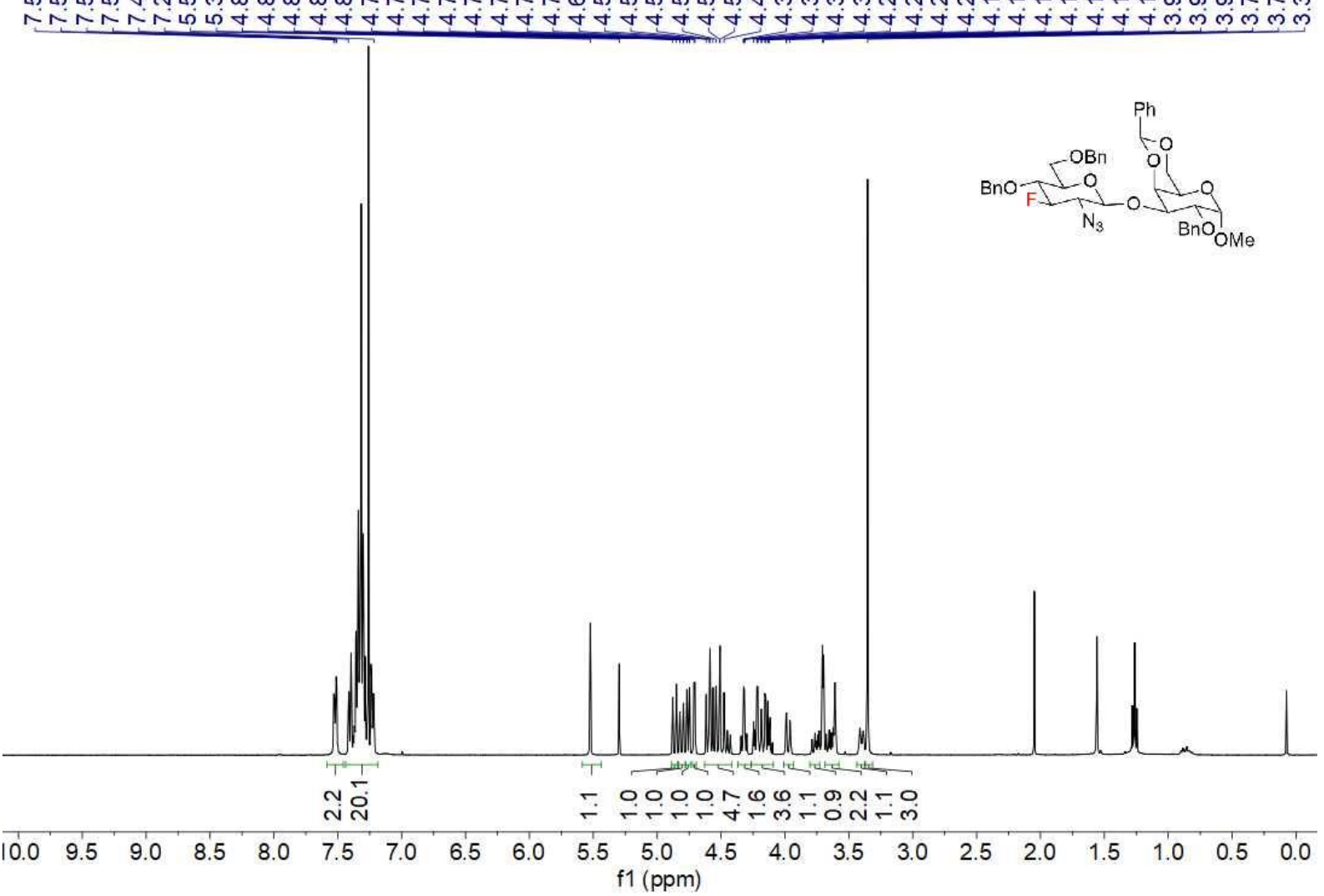




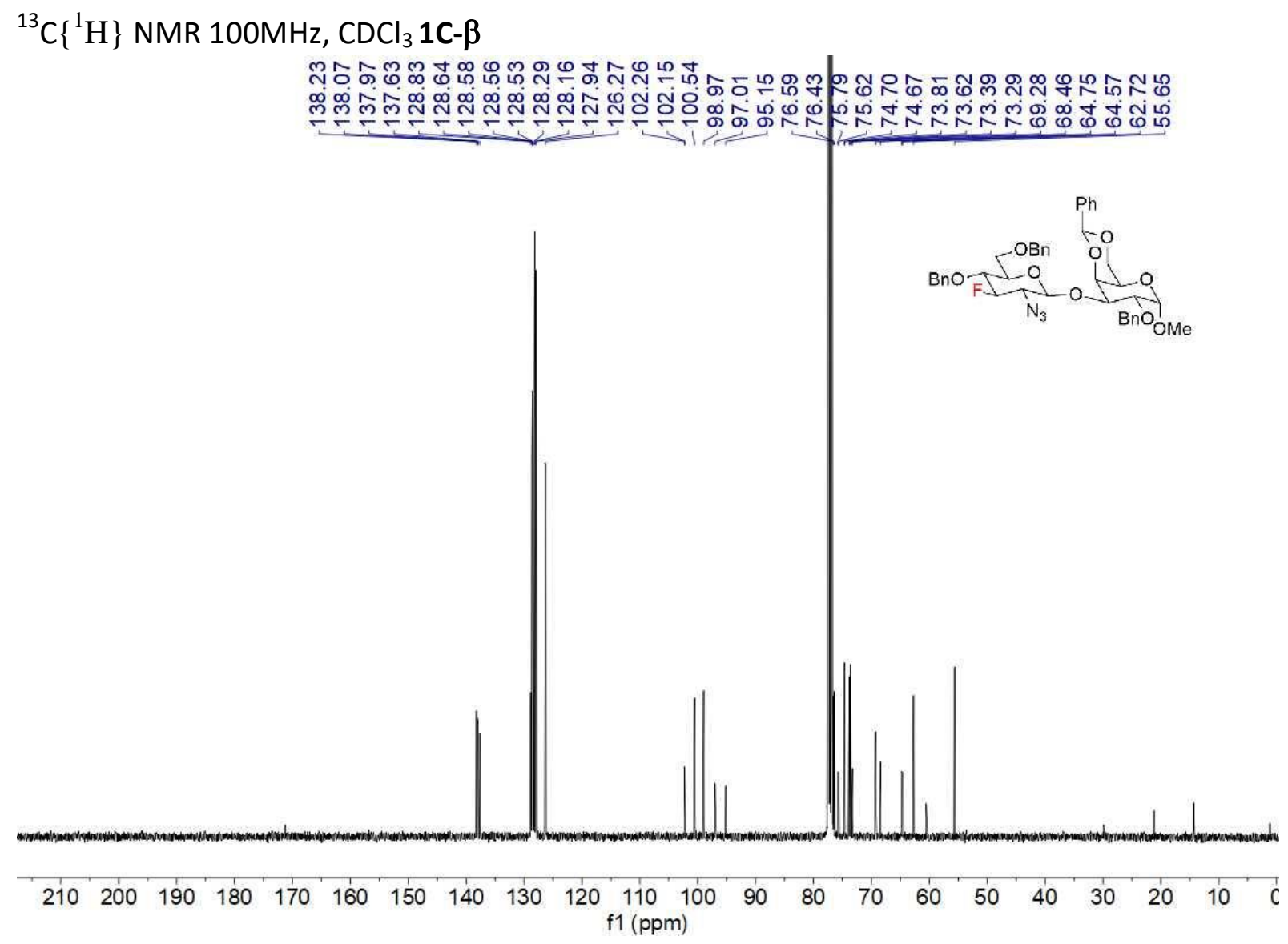

${ }^{1} \mathrm{H}-{ }^{1} \mathrm{H}$ COSY NMR $1 \mathrm{C}-\boldsymbol{\beta}$

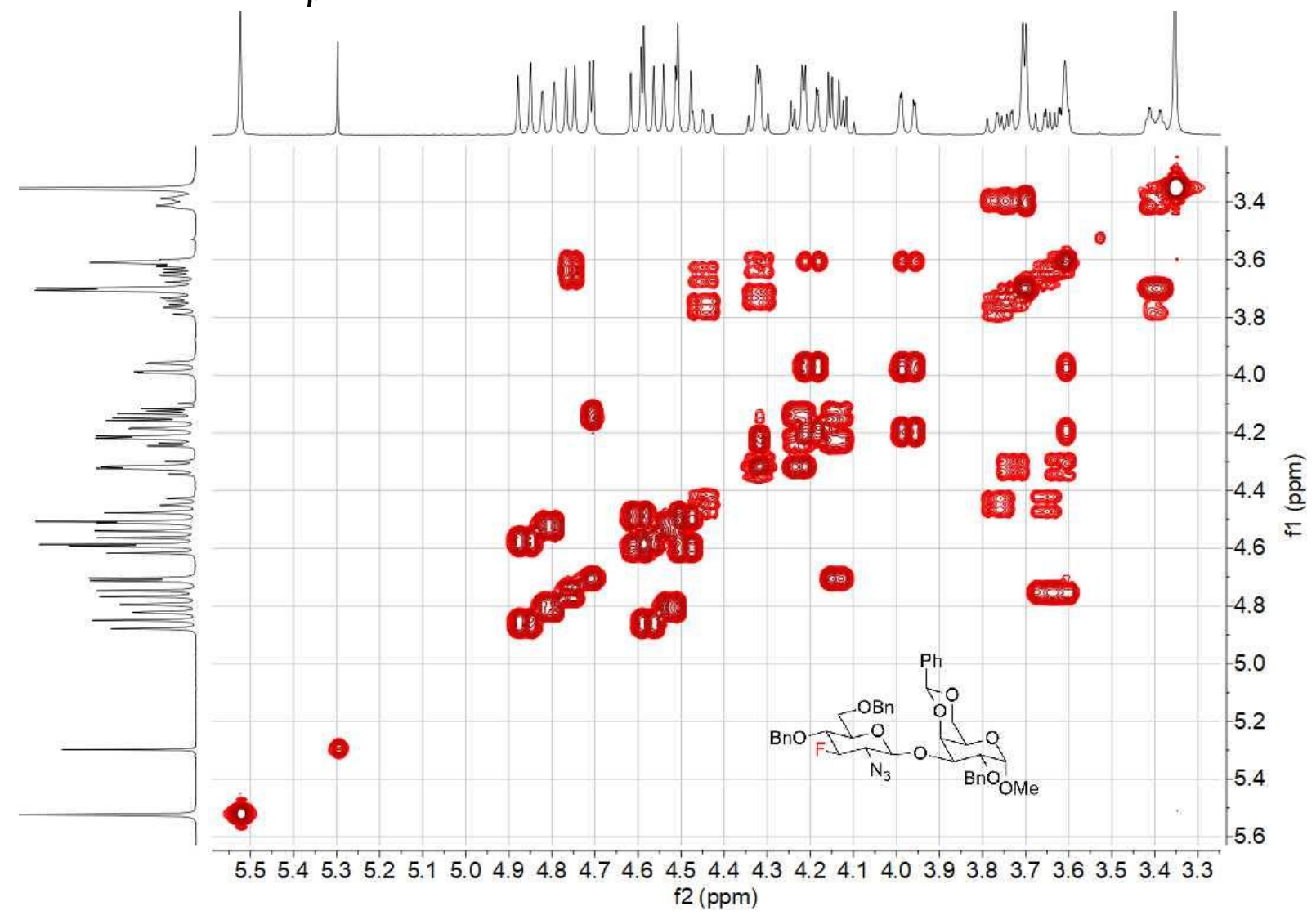




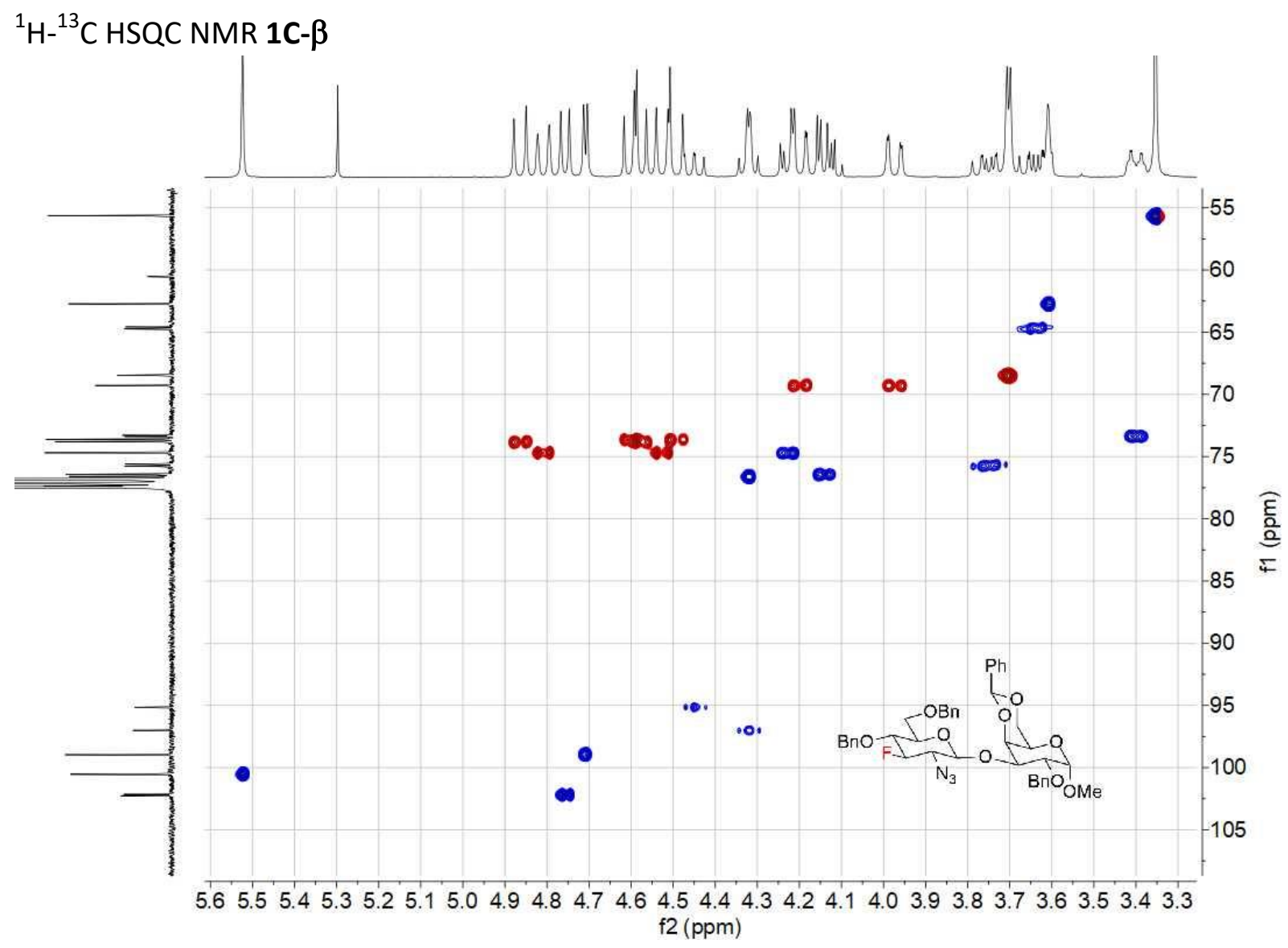

${ }^{1} \mathrm{H}^{13} \mathrm{C}$ HMBC NMR $\mathbf{1 C}-\boldsymbol{\beta}$

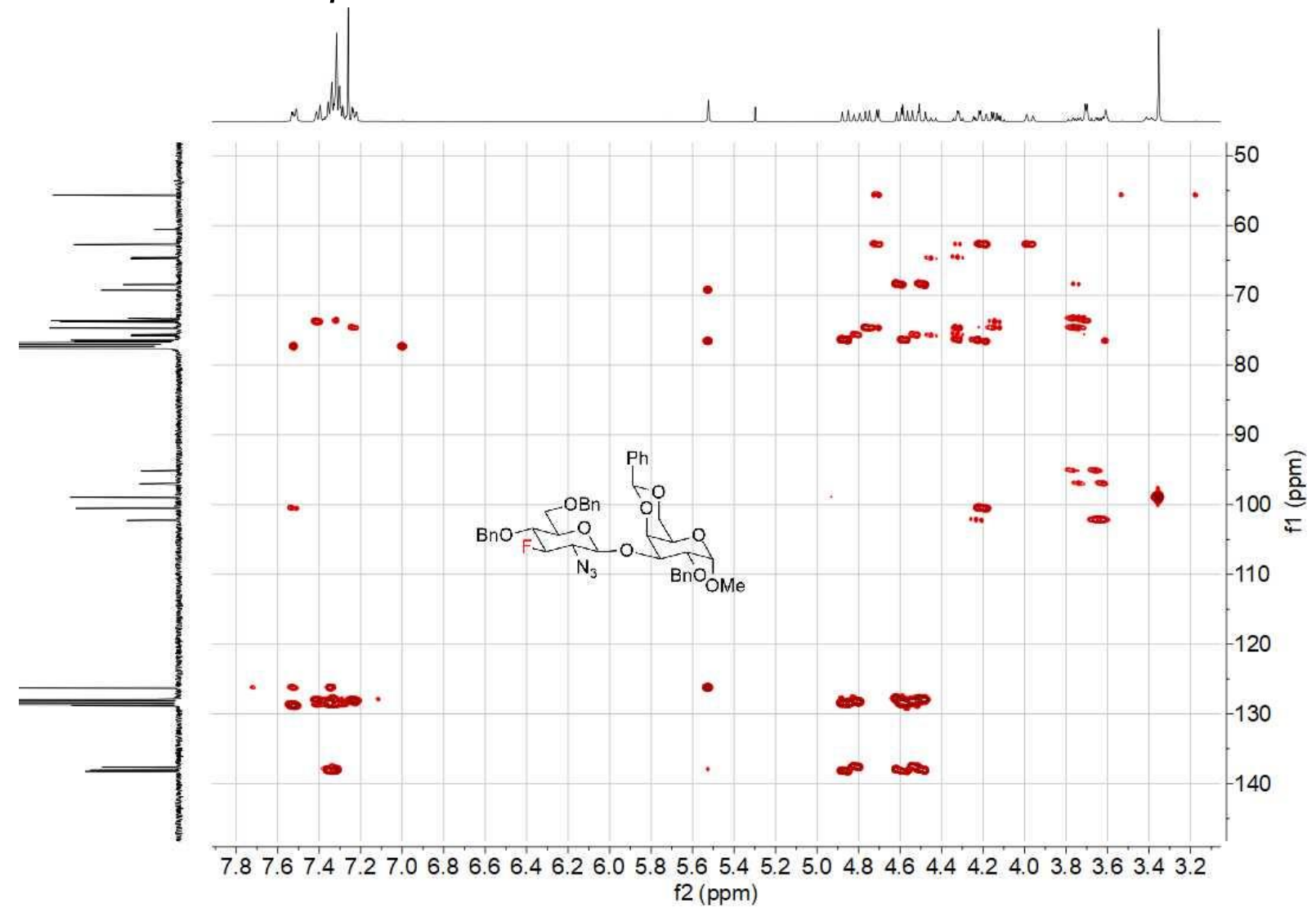


${ }^{19} \mathrm{~F} \mathrm{NMR} 376 \mathrm{MHz}, \mathrm{CDCl}_{3} \mathbf{1 C}-\boldsymbol{\beta}$

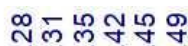

$\infty \infty_{\infty}^{\infty} \infty \infty \infty \infty$

$\div \div \div \div$
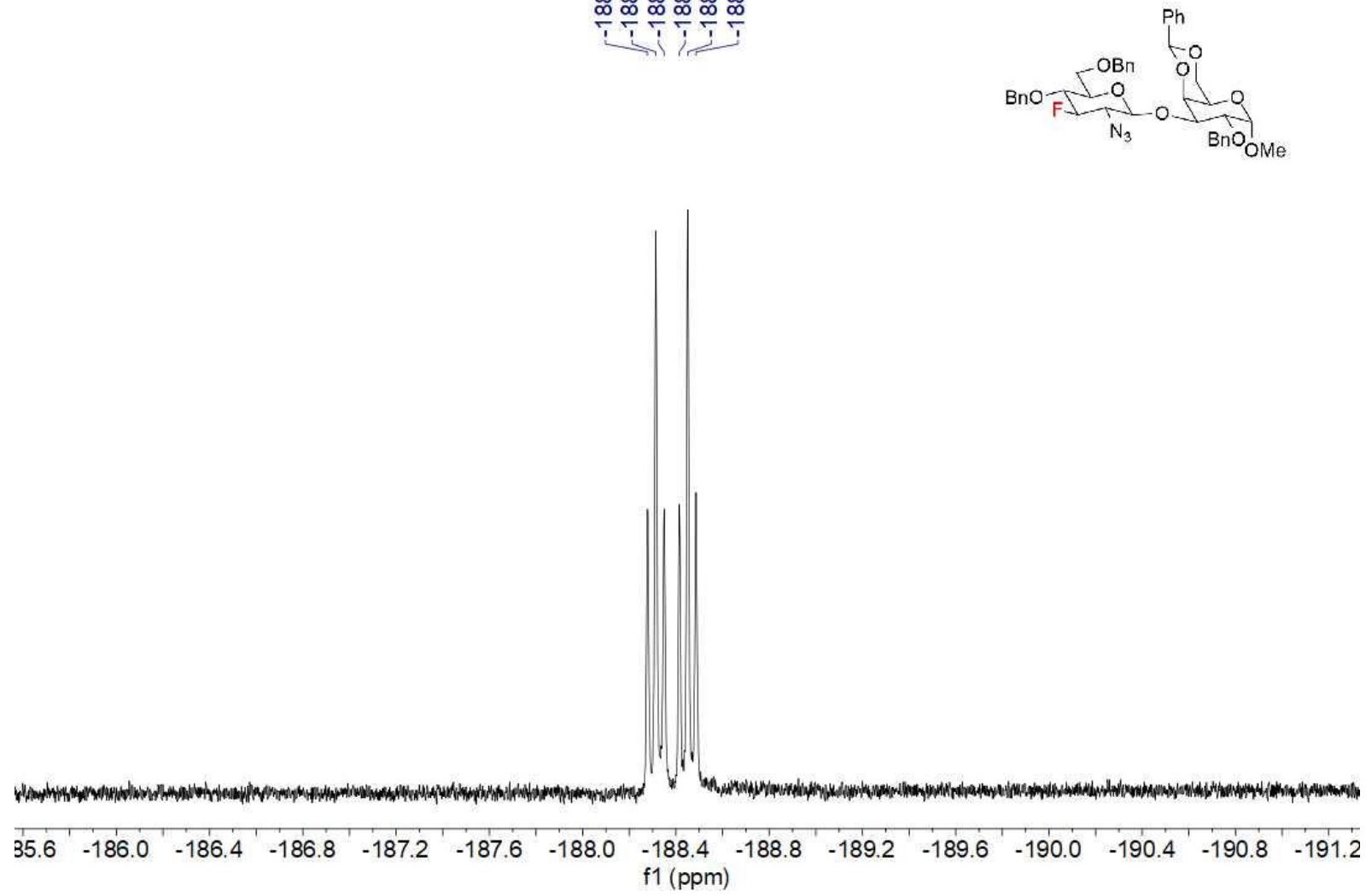

NMR 1E- $\alpha$

${ }^{1} \mathrm{HNMR} 400 \mathrm{MHz}, \mathrm{CDCl}_{3}$ 1E $\alpha$

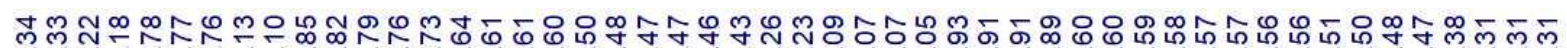

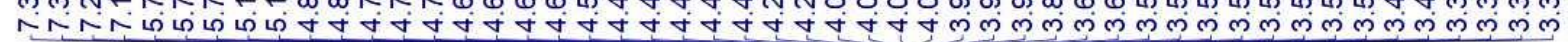

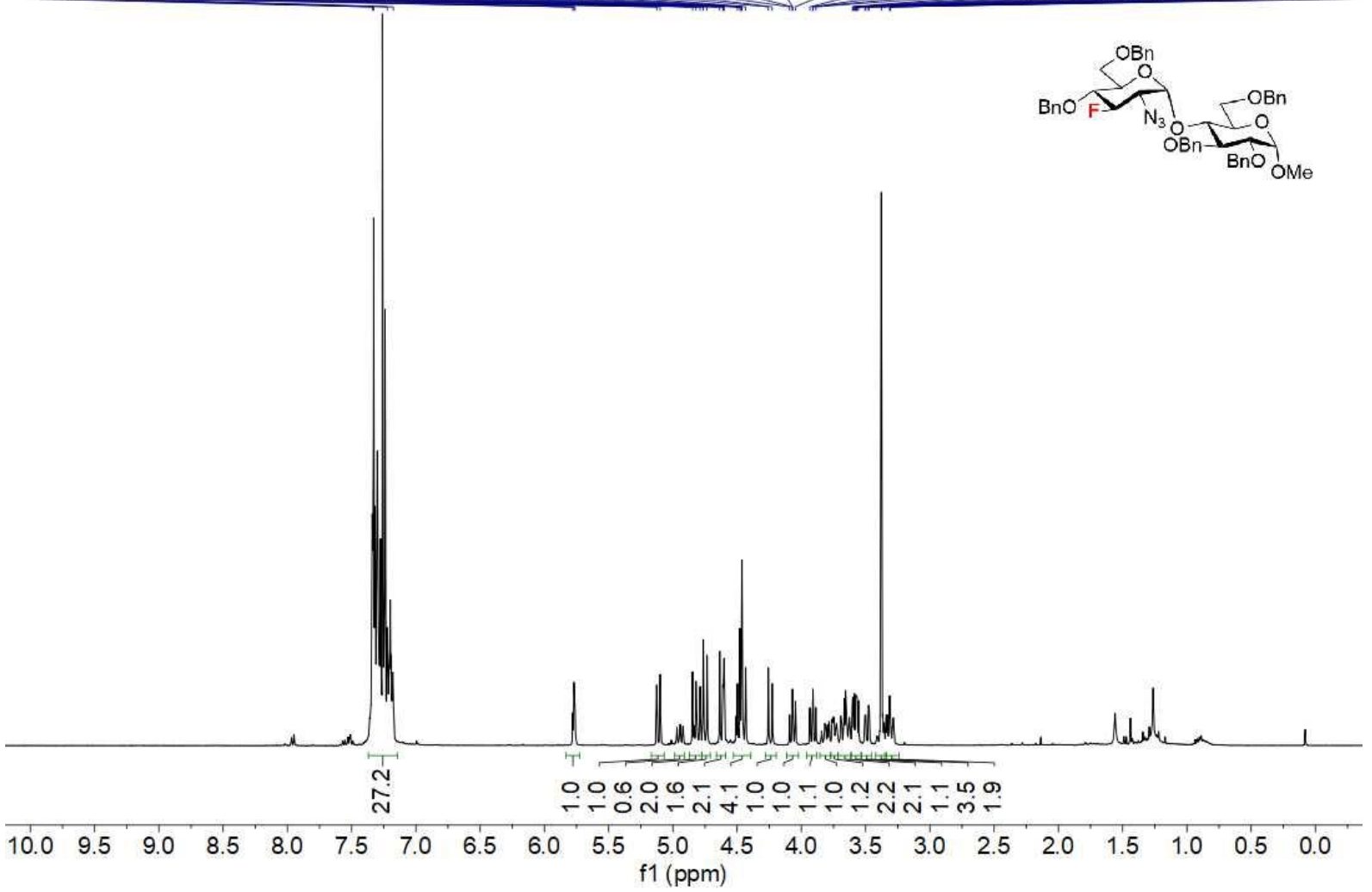


${ }^{13} \mathrm{C}\left\{{ }^{1} \mathrm{H}\right\} \mathrm{NMR} 100 \mathrm{MHz}, \mathrm{CDCl}_{3} 1 \mathrm{E}-\alpha$

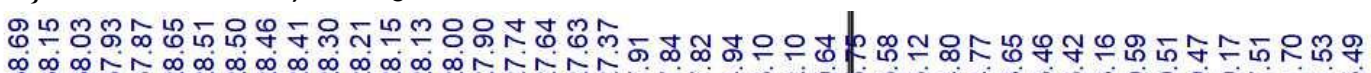
o

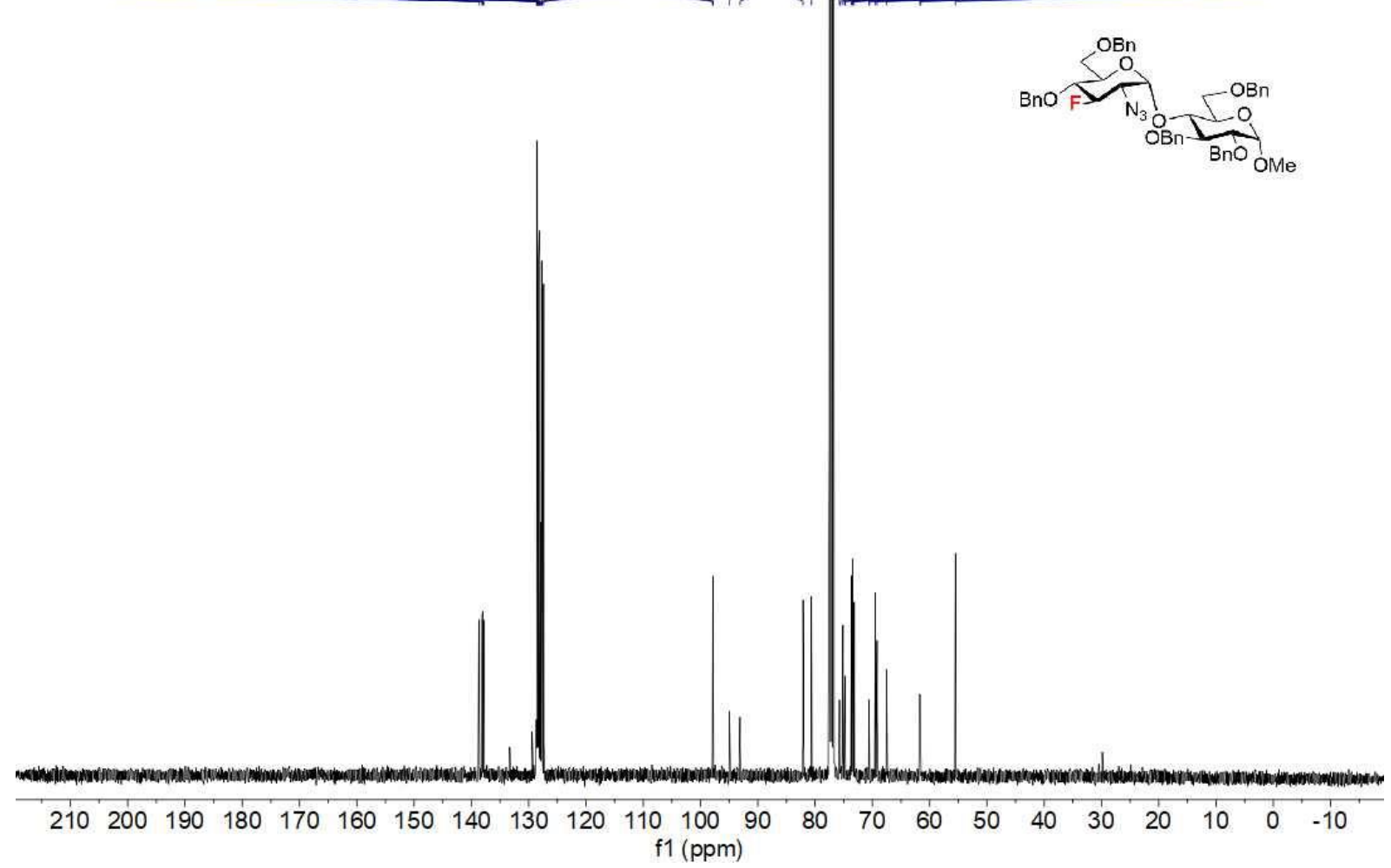

${ }^{1} \mathrm{H}-{ }^{1} \mathrm{H} \cos Y$ NMR $1 \mathrm{E}-\alpha$

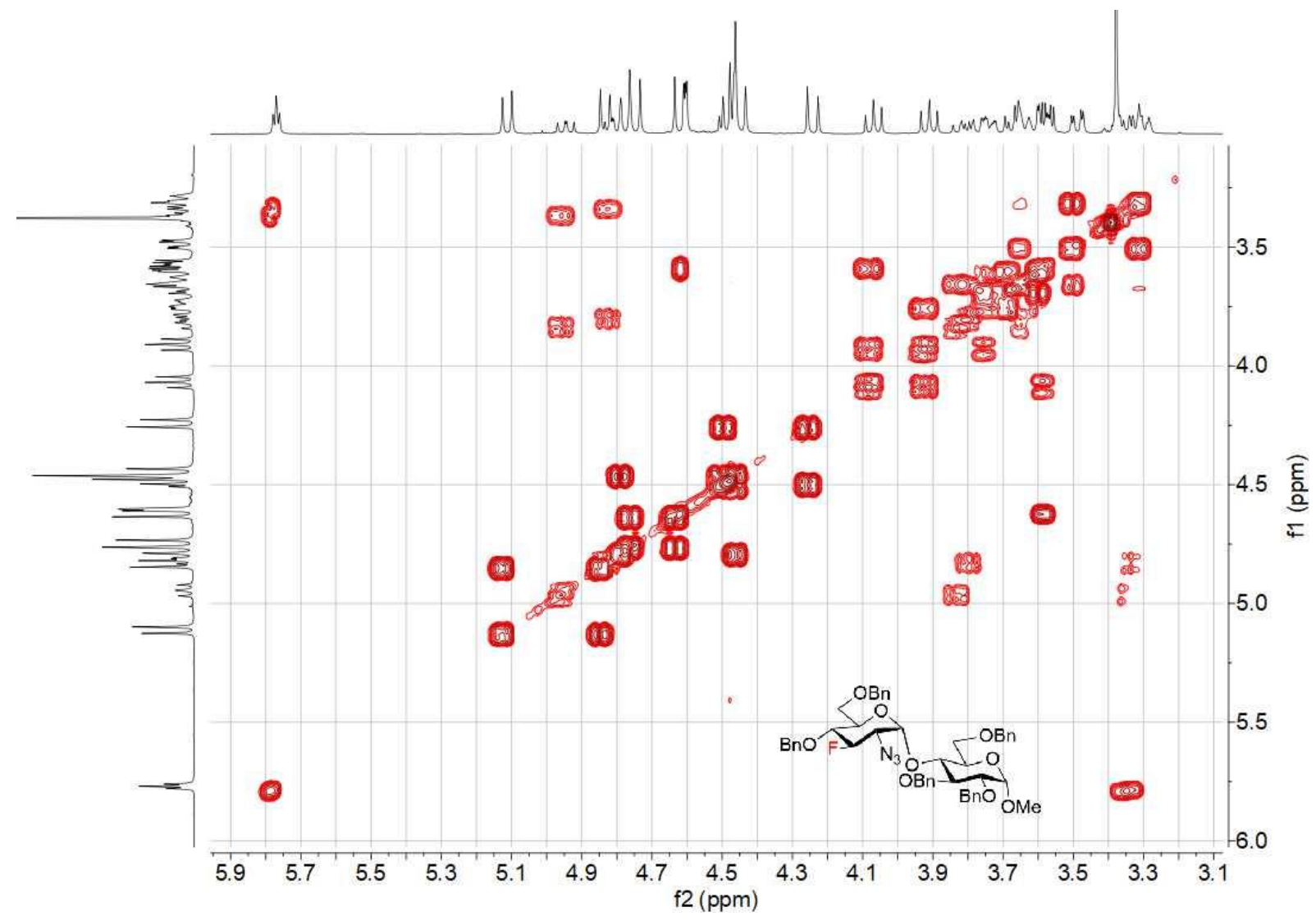


${ }^{1} \mathrm{H}-{ }^{13} \mathrm{C}$ HSQC NMR $1 \mathrm{E}-\alpha$

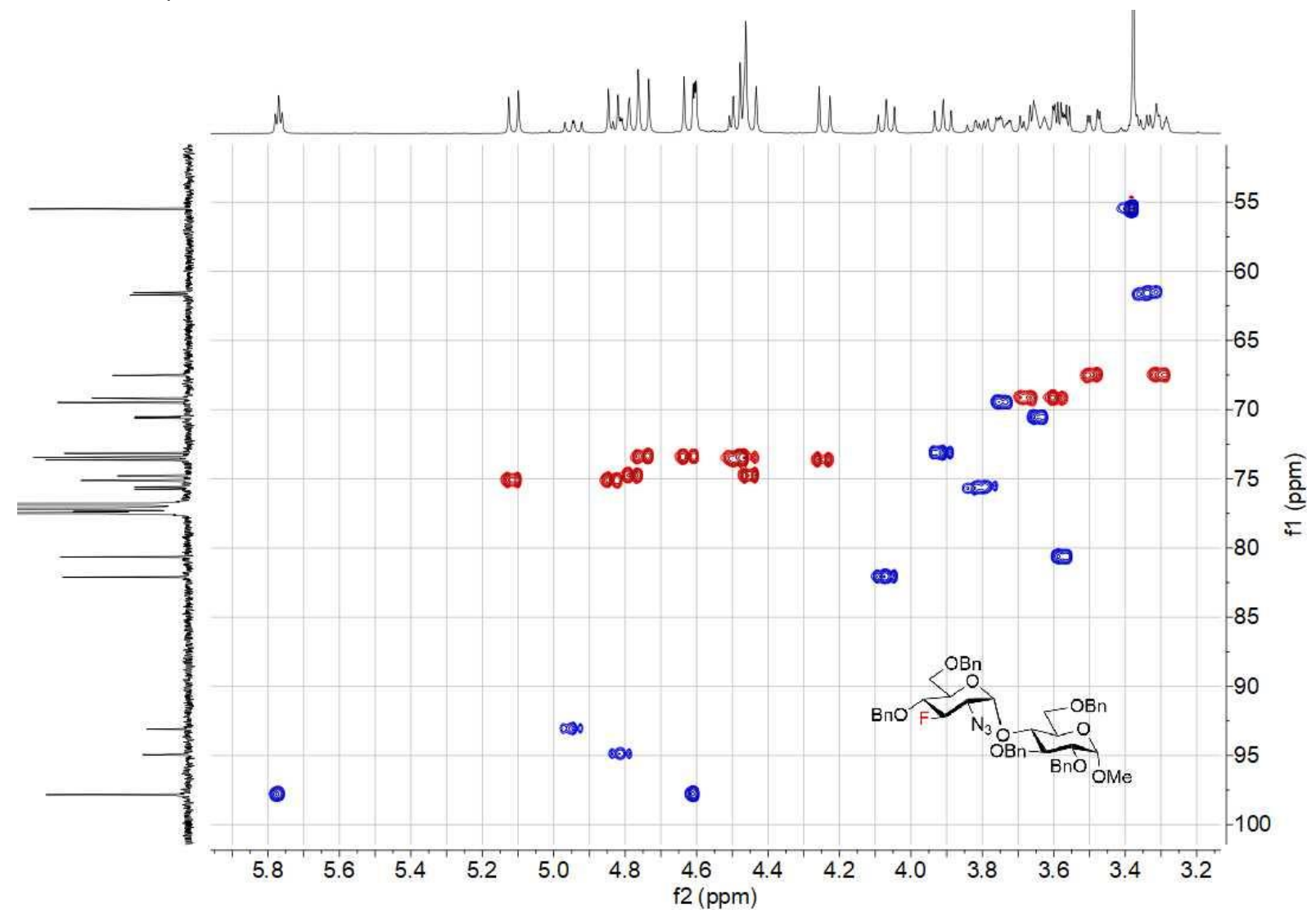

${ }^{1} \mathrm{H}-{ }^{13} \mathrm{C}$ HMBC NMR 1 E- $\alpha$

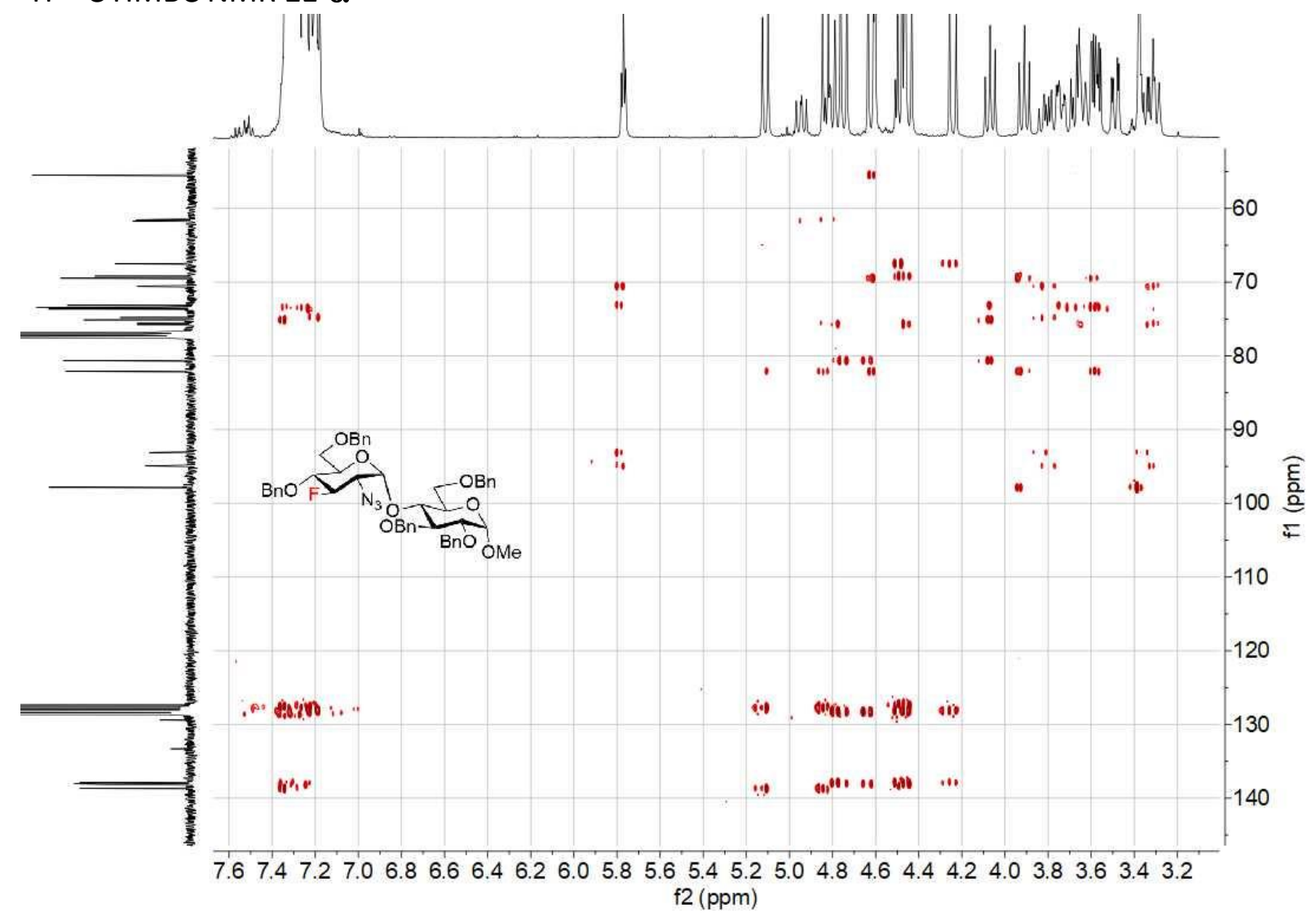


${ }^{19} \mathrm{~F} \mathrm{NMR} 376 \mathrm{MHz}, \mathrm{CDCl}_{3} 1 \mathrm{E}-\alpha$

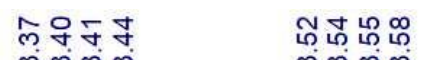

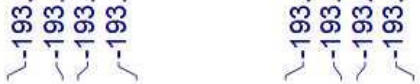
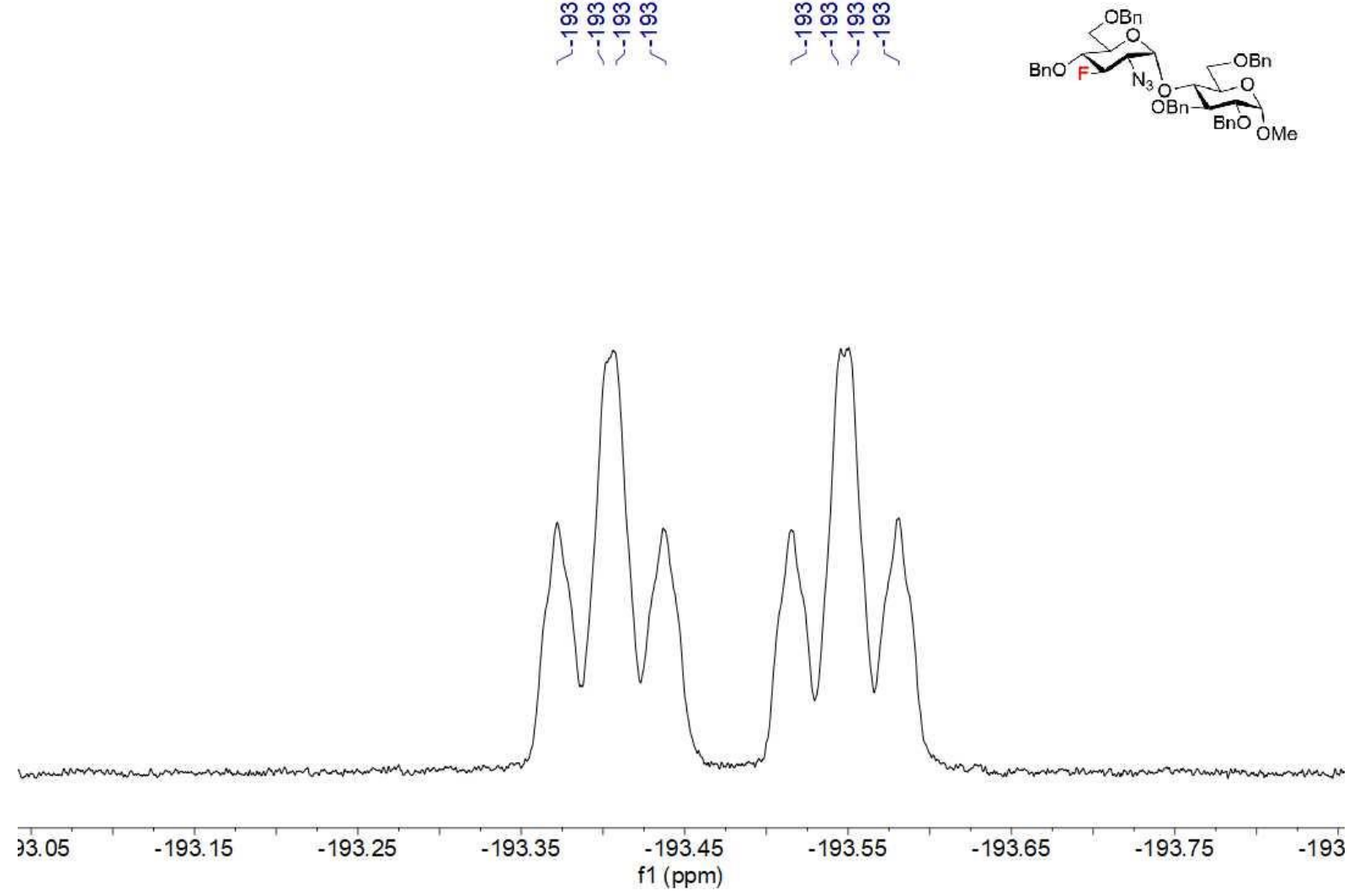

NMR 1E- $\beta$

${ }^{1} \mathrm{HNMR} 400 \mathrm{MHz}, \mathrm{CDCl}_{3} 1 \mathrm{E}-\beta$

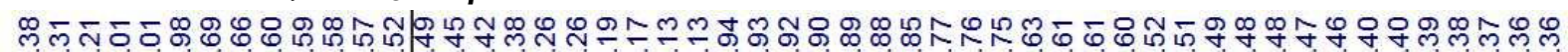
NN W

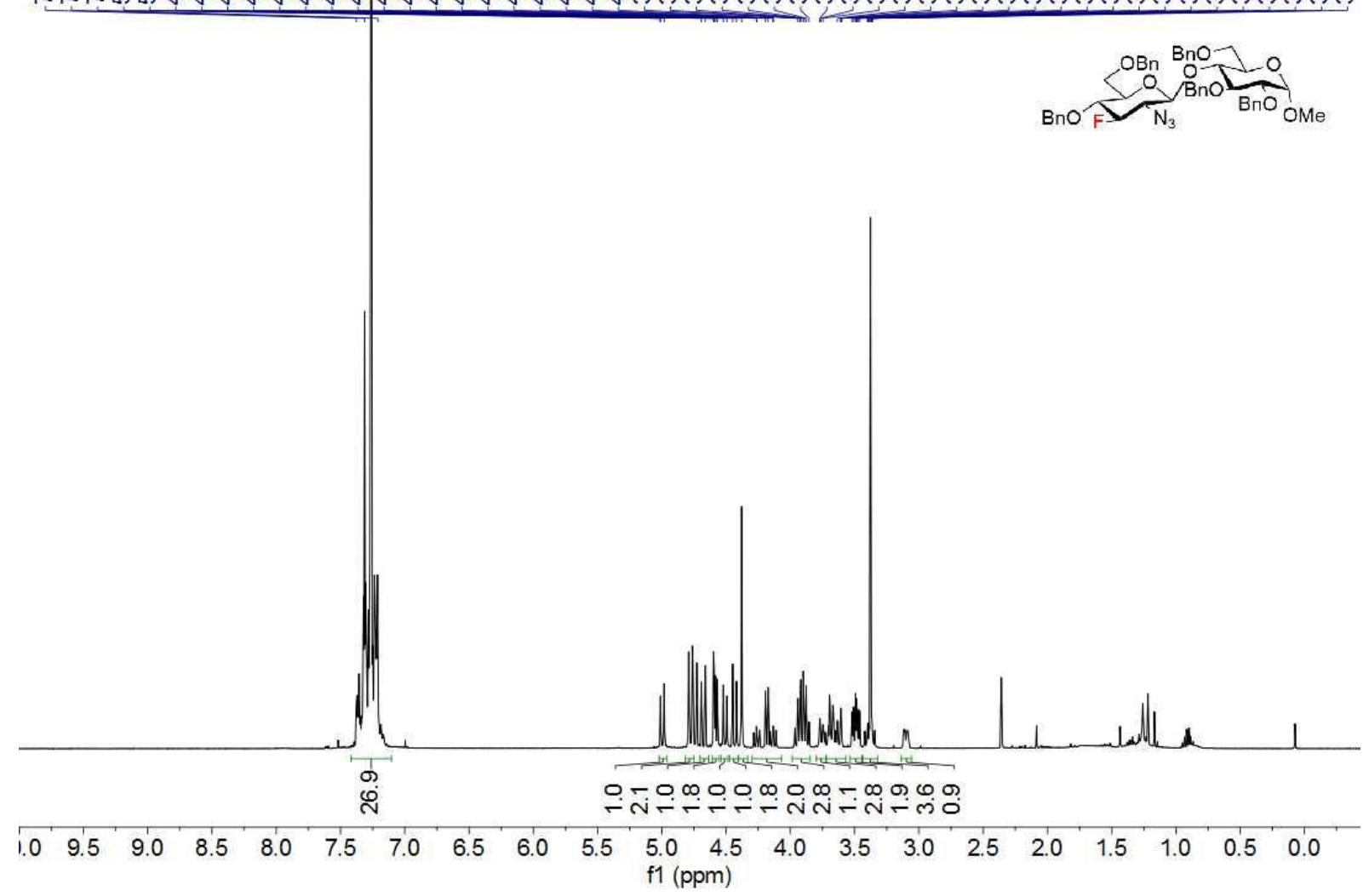


${ }^{13} \mathrm{C}\left\{{ }^{1} \mathrm{H}\right\}$ NMR 100MHz, $\mathrm{CDCl}_{3} 1 \mathrm{E}-\beta$

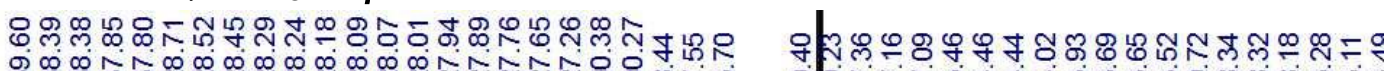
8.

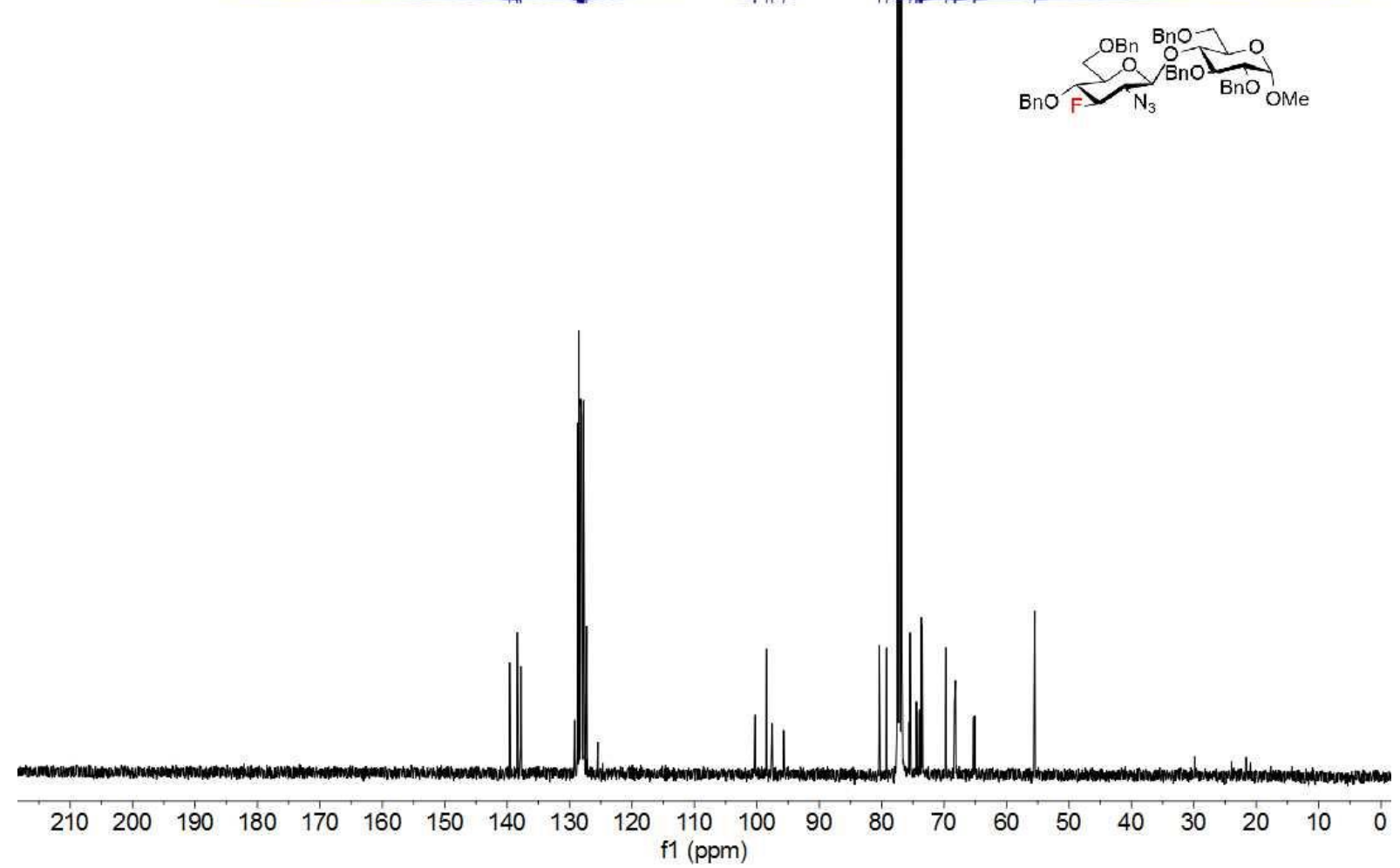

${ }^{1} \mathrm{H}-{ }^{1} \mathrm{H}$ COSY NMR $1 \mathrm{E}-\beta$

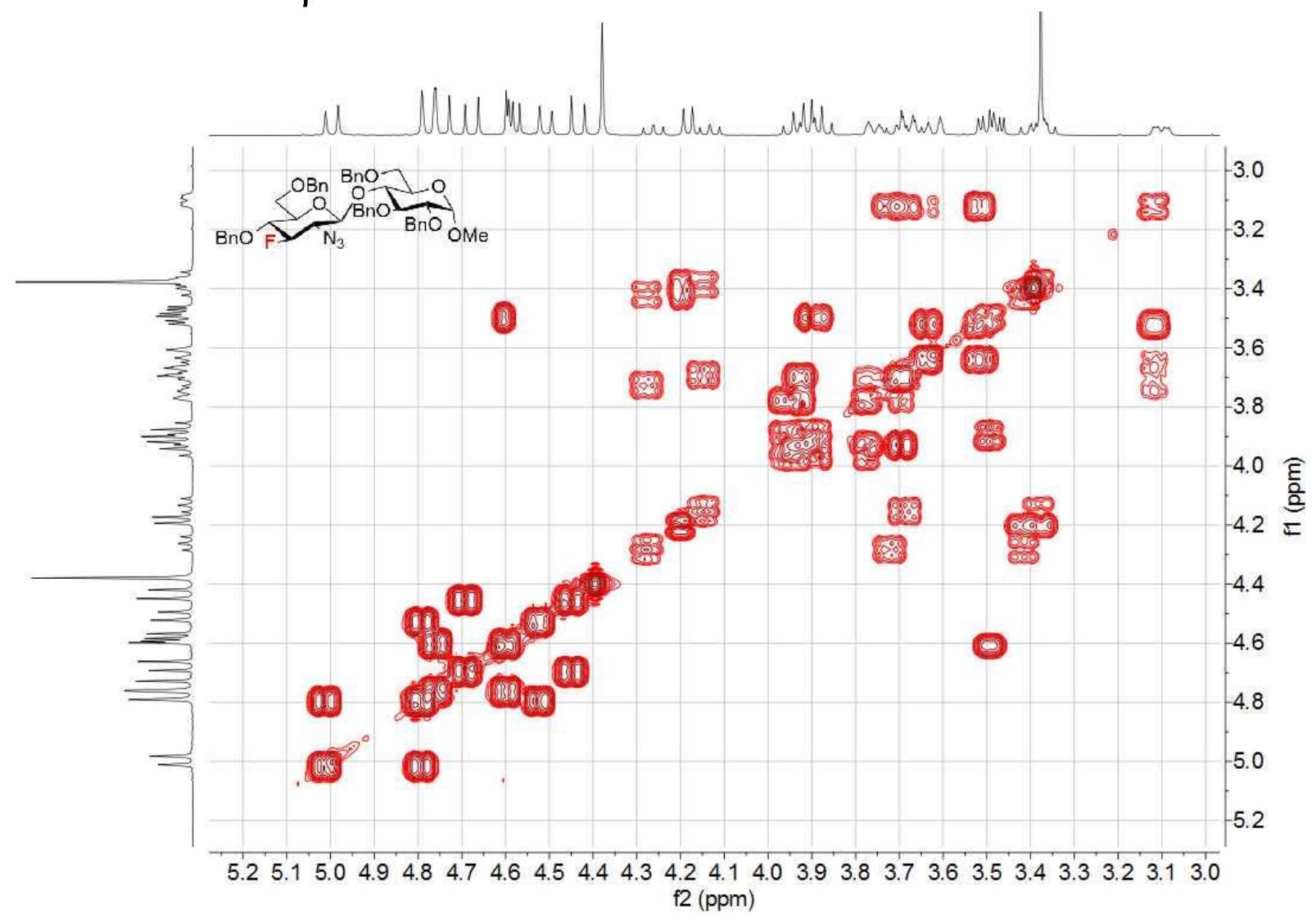




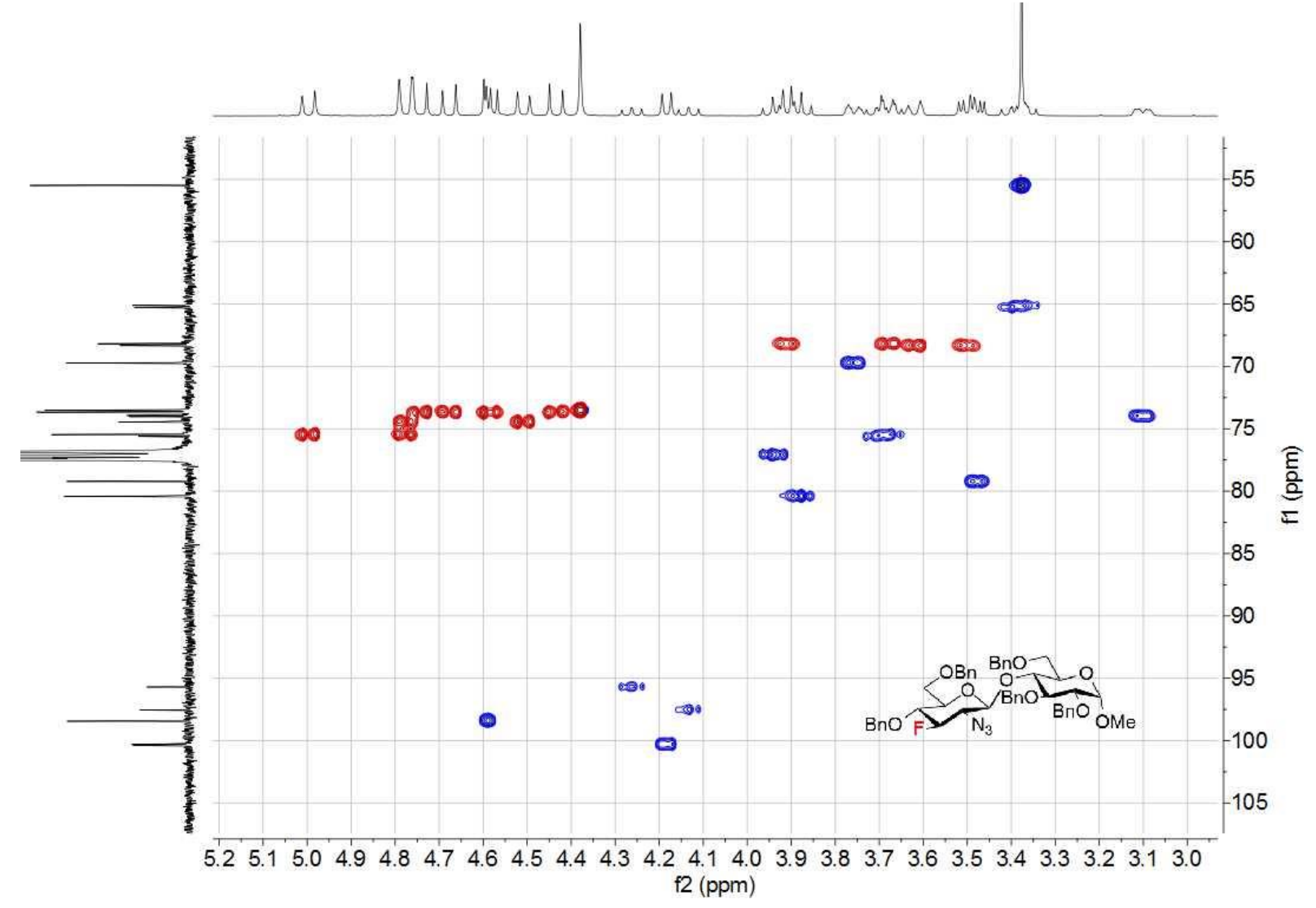

${ }^{1} \mathrm{H}-{ }^{13} \mathrm{C}$ HMBC NMR 1E- $\boldsymbol{\beta}$

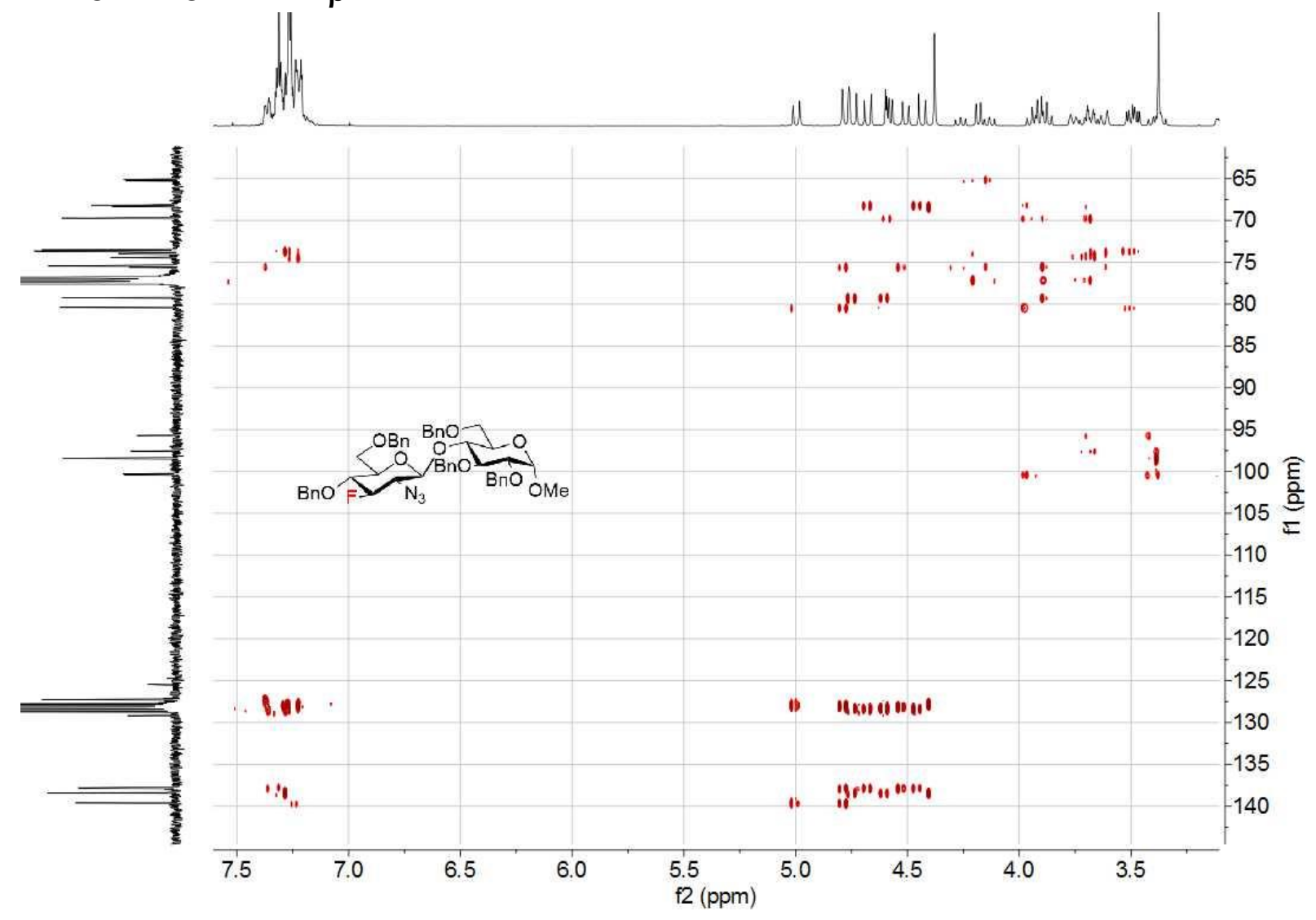


${ }^{19} \mathrm{~F} \mathrm{NMR} 376 \mathrm{MHz}, \mathrm{CDCl}_{3} \mathbf{1 E}-\boldsymbol{\beta}$

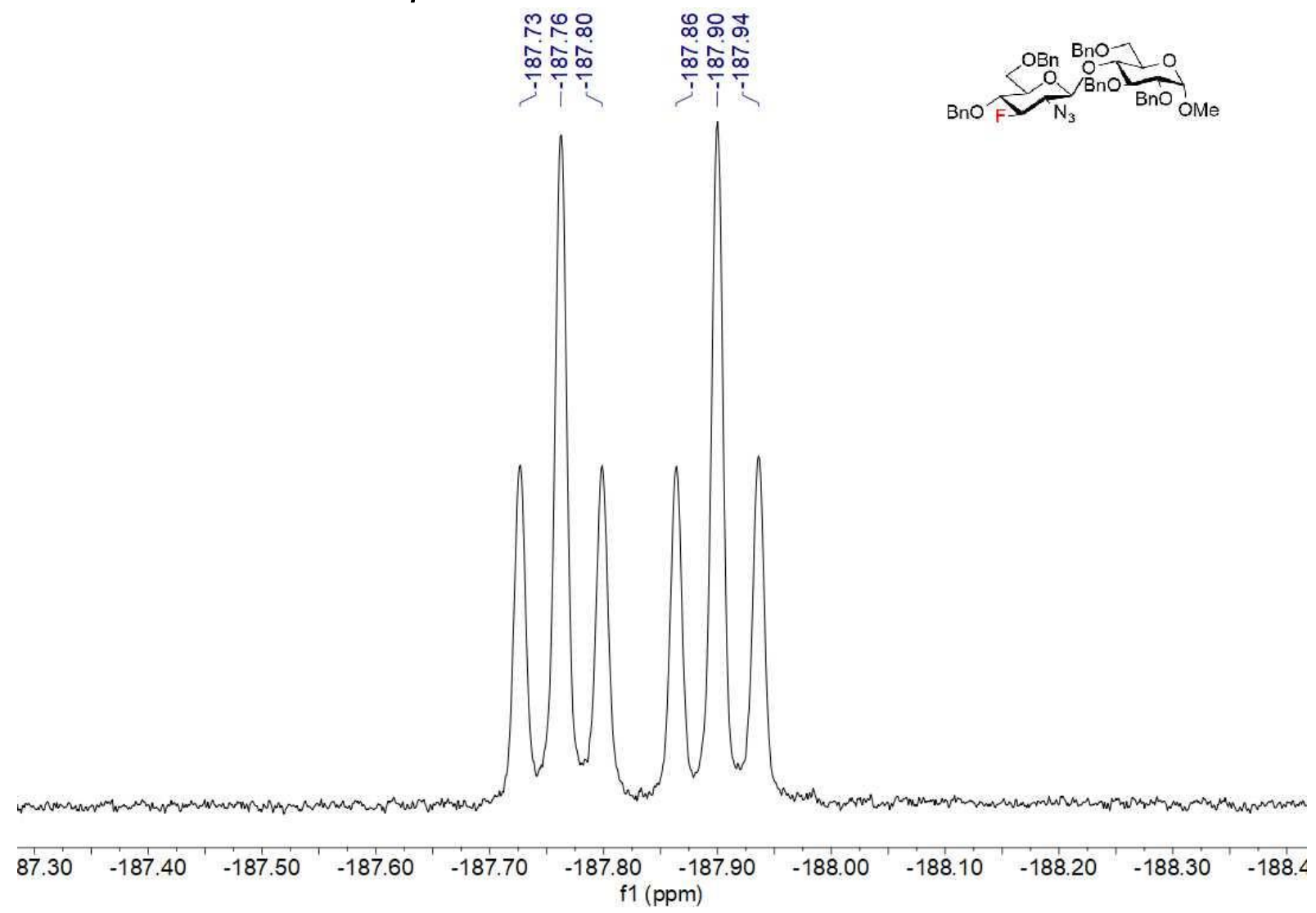

NMR 1F- $\alpha$

${ }^{1} \mathrm{HNMR} 400 \mathrm{MHz}, \mathrm{CDCl}_{3} 1 \mathrm{~F}-\alpha$

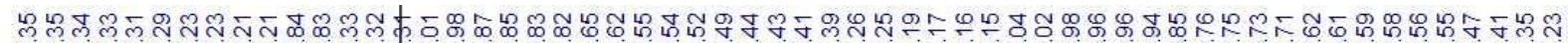

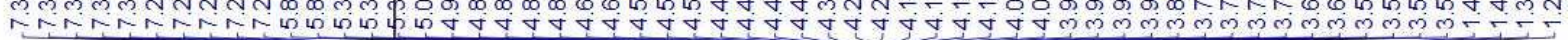

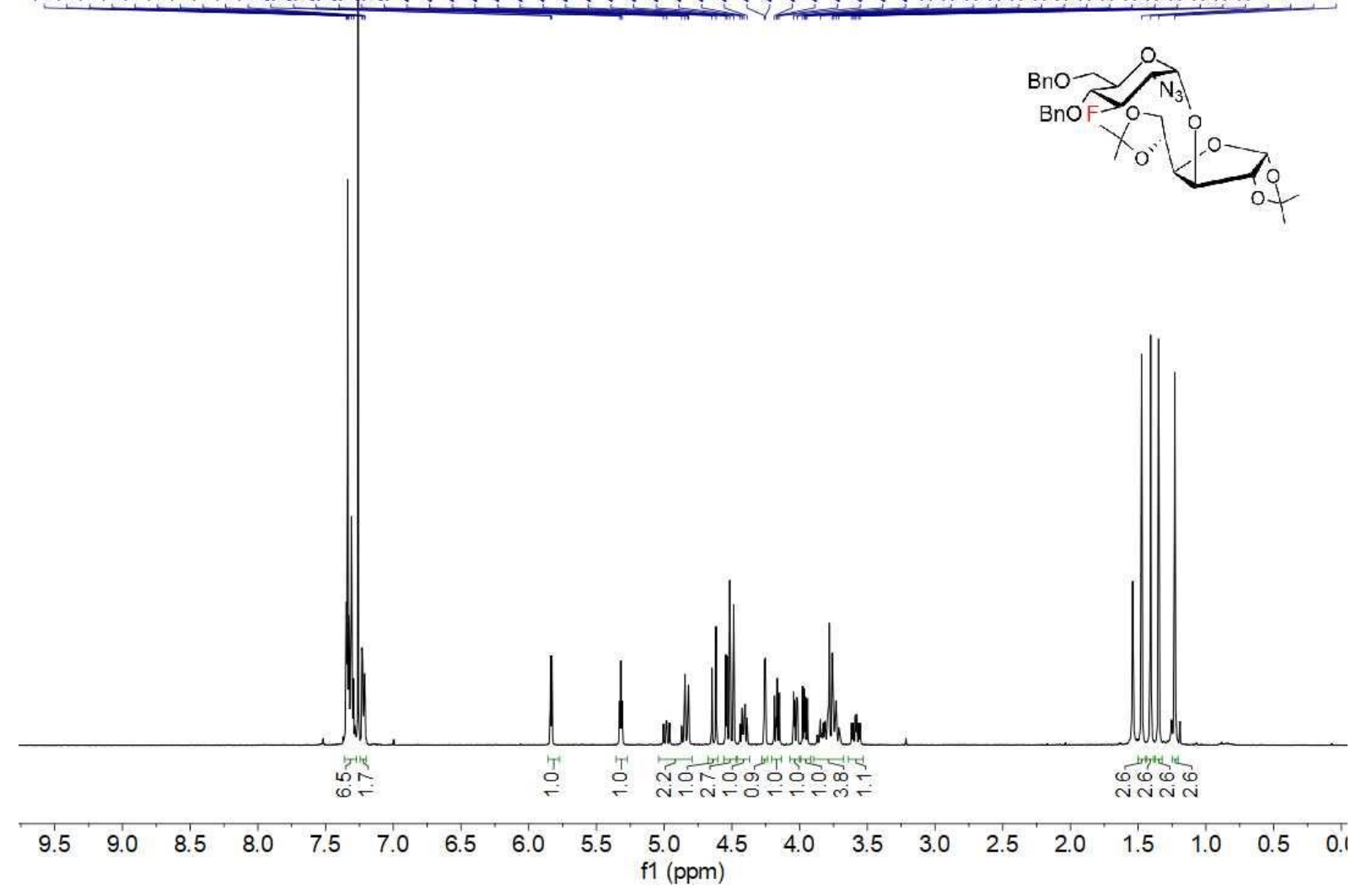




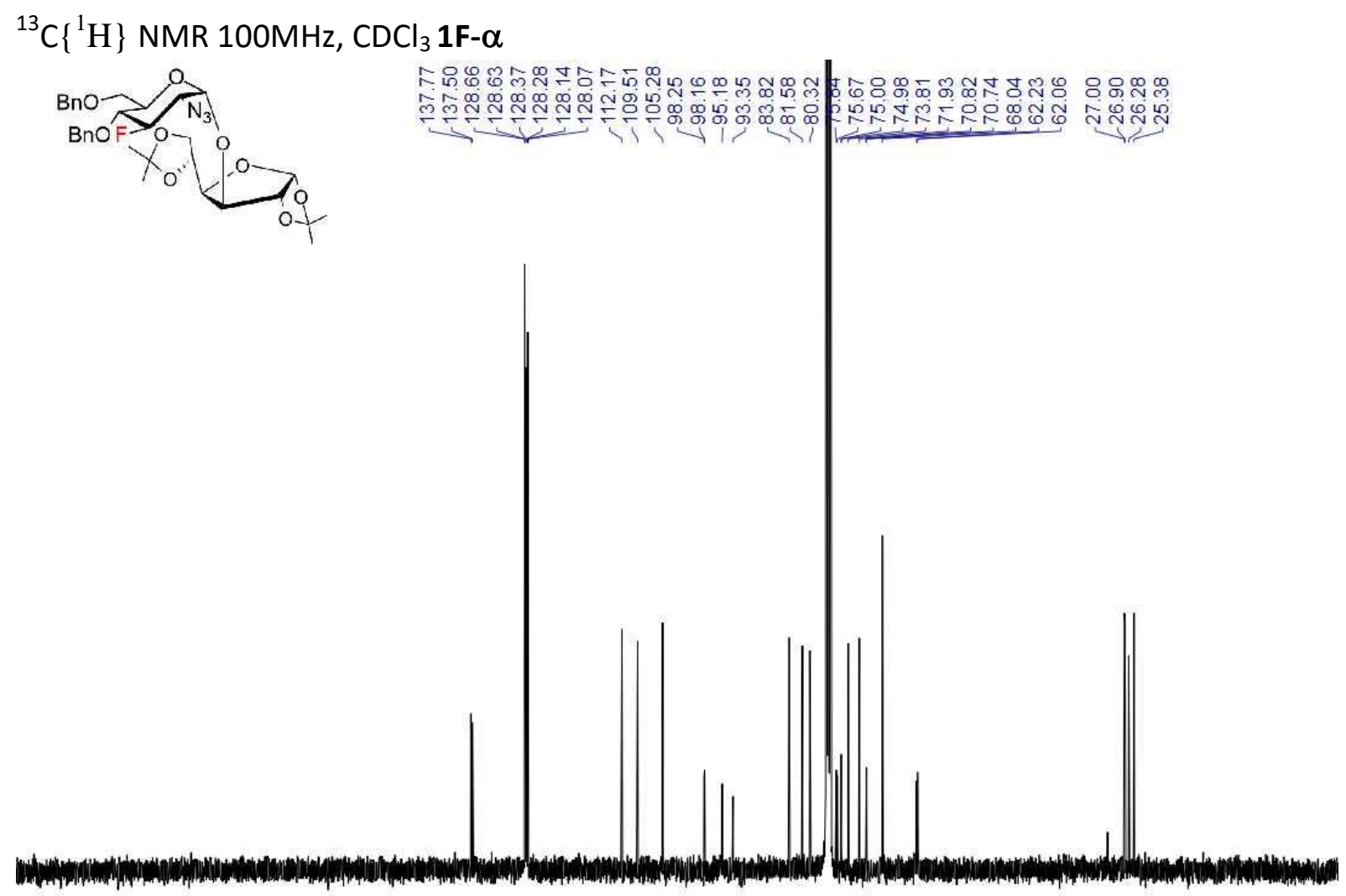

$\begin{array}{llllllllllllllllllllllll}210 & 200 & 190 & 180 & 170 & 160 & 150 & 140 & 130 & 120 & \begin{array}{r}110 \\ f 1(\mathrm{ppm})\end{array} & 90 & 80 & 70 & 60 & 50 & 40 & 30 & 20 & 10 & 0\end{array}$

${ }^{1} \mathrm{H}-{ }^{1} \mathrm{H}$ COSY NMR $1 \mathrm{~F}-\alpha$ and cca $20 \% 1 \mathrm{~F}-\beta$

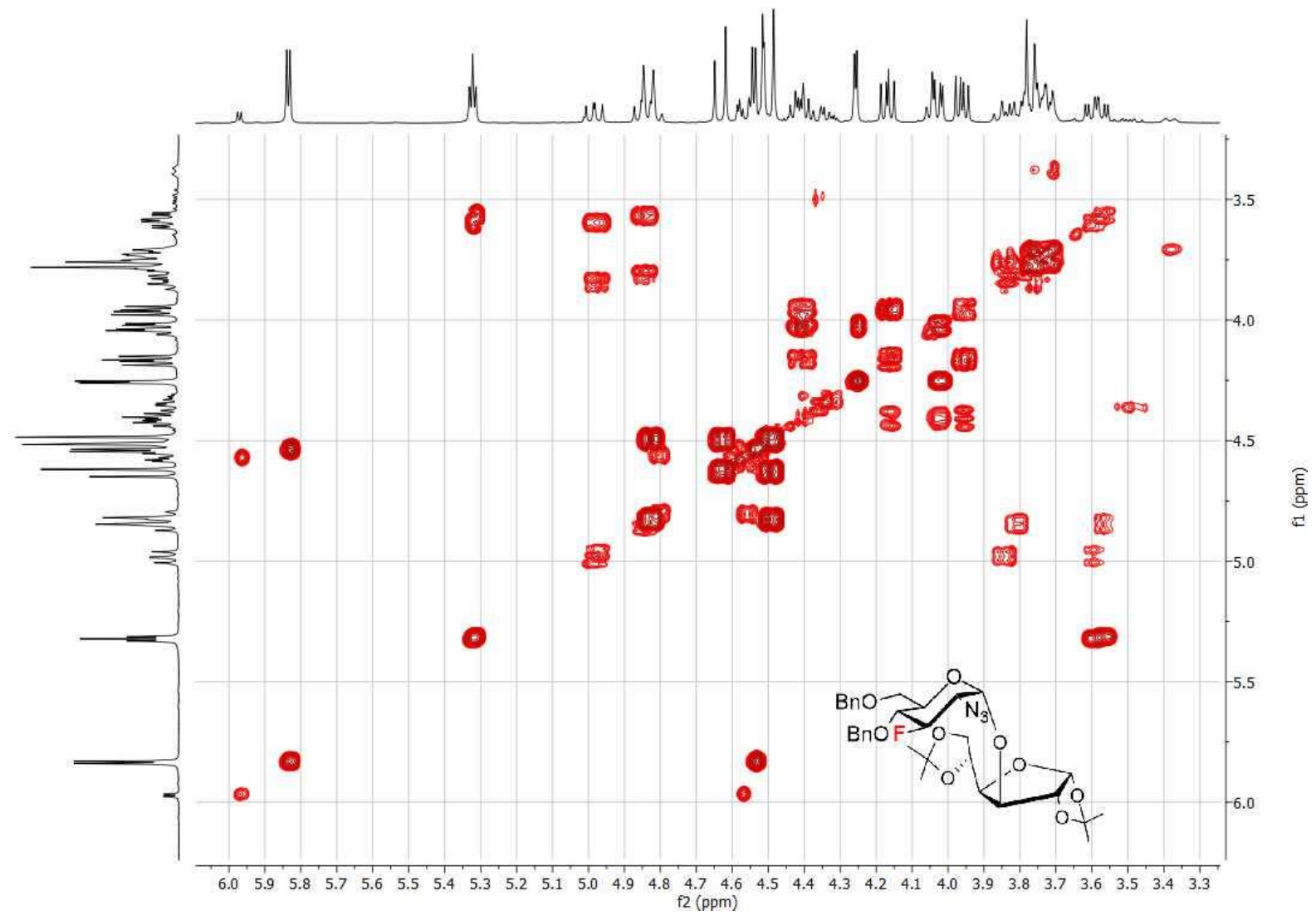


${ }^{1} \mathrm{H}-{ }^{13} \mathrm{C}$ HSQC NMR $1 \mathrm{~F}-\alpha$ and cca $20 \% 1 \mathrm{~F}-\beta$

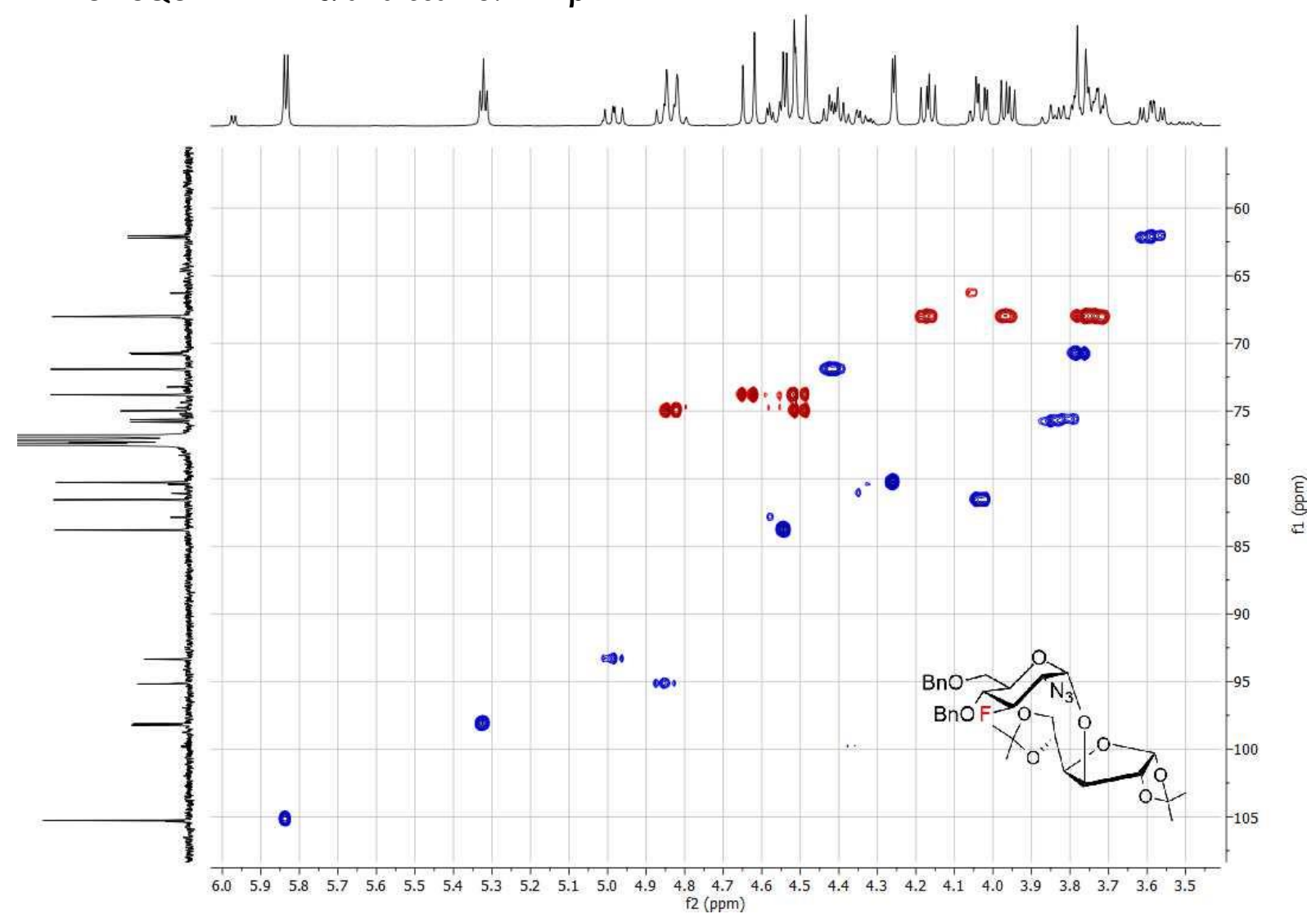

${ }^{1} \mathrm{H}-{ }^{13} \mathrm{C}$ HMBC NMR $1 \mathrm{~F}-\alpha$ and cca $20 \% 1 \mathrm{~F}-\beta$

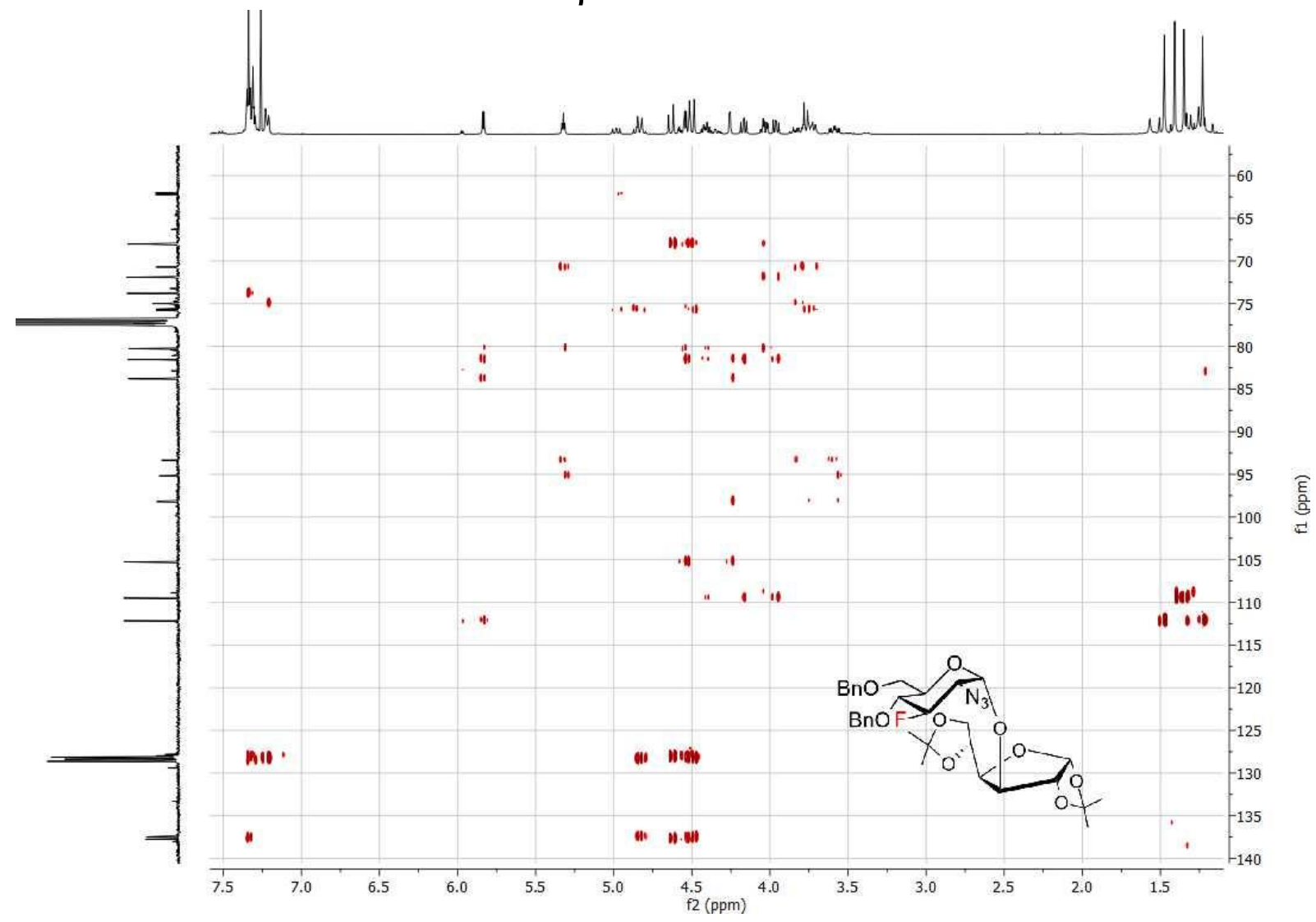


${ }^{19} \mathrm{~F} \mathrm{NMR} 376 \mathrm{MHz}, \mathrm{CDCl}_{3} \mathbf{1 F}-\boldsymbol{\alpha}$

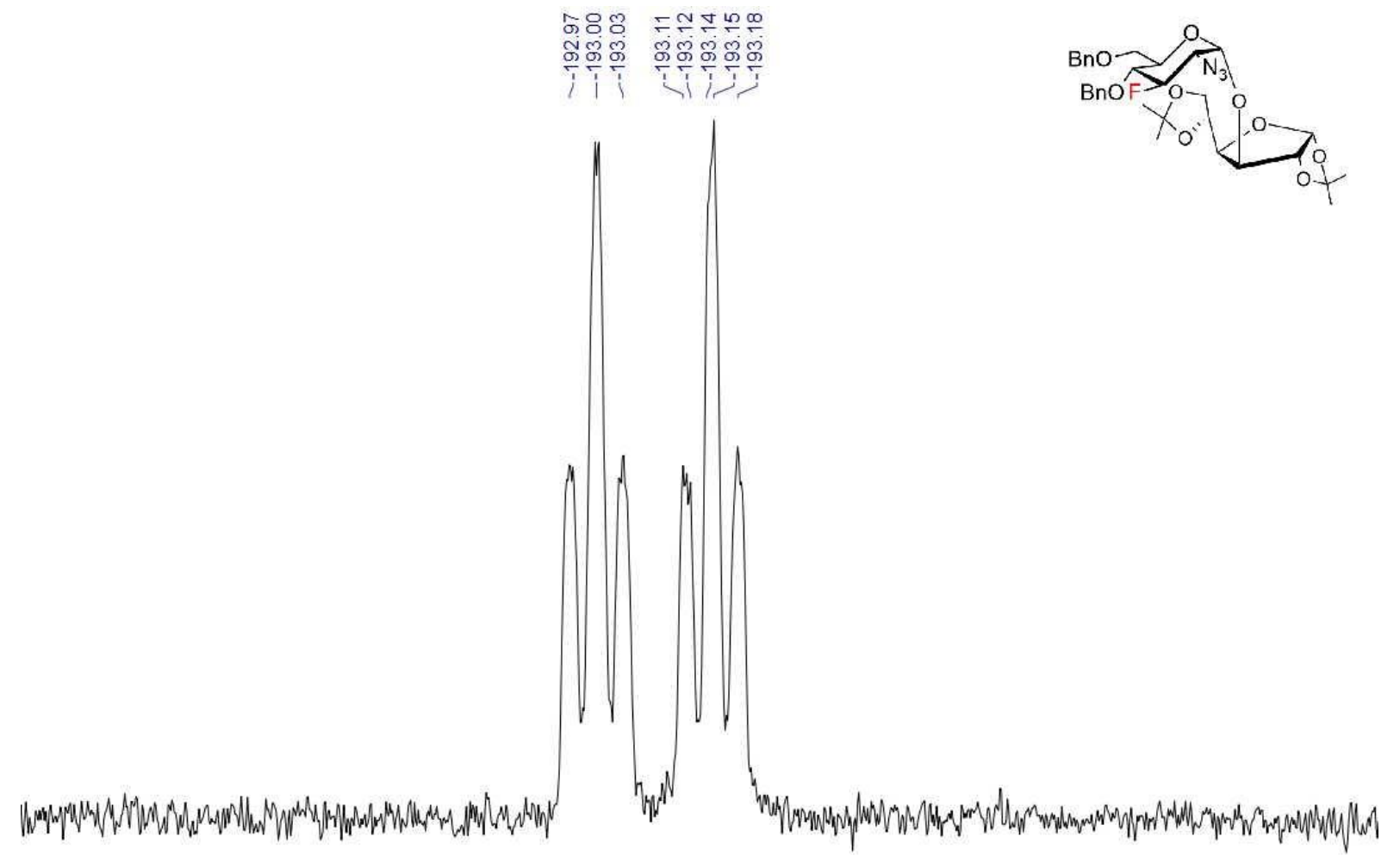

\begin{tabular}{llllllllllllllllllll}
\hline 2.3 & -192.4 & -192.5 & -192.6 & -192.7 & -192.8 & -192.9 & -193.0 & -193.1 & -193.2 & -193.3 & -193.4 & -193.5 & -193.6 & -193.7 & -193.8 & -193.9
\end{tabular} f1 (ppm) 
NMR 1F- $\beta$

${ }^{1} \mathrm{H} \mathrm{NMR} 400 \mathrm{MHz}, \mathrm{CDCl}_{3} \mathbf{1 F}-\boldsymbol{\beta}$ and cca $6 \%$ 1F- $\alpha$

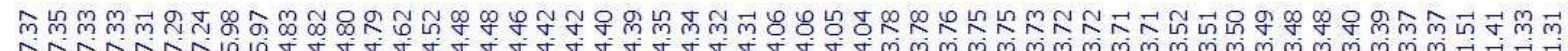

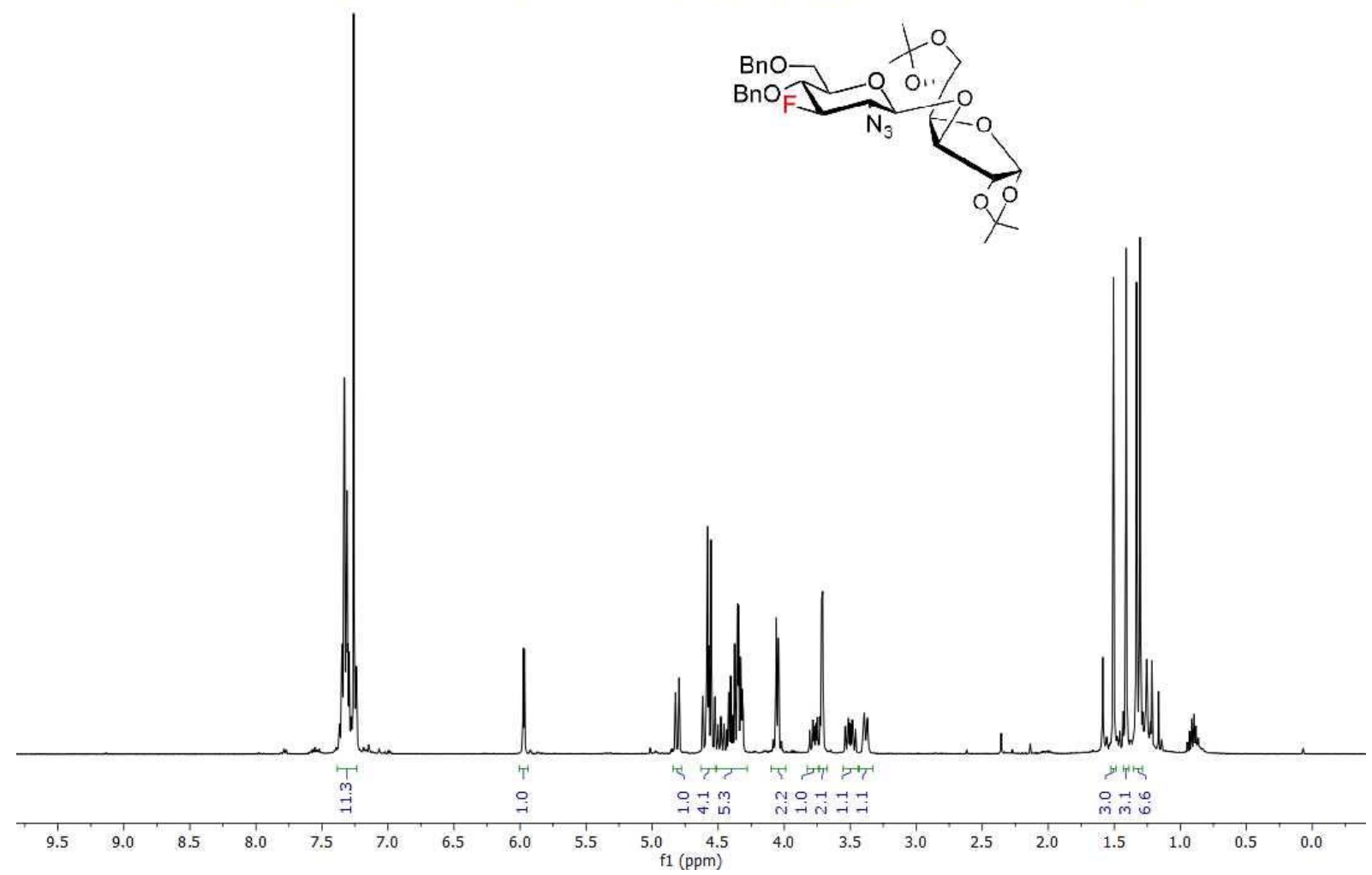

${ }^{13} \mathrm{C}\left\{{ }^{1} \mathrm{H}\right\} \mathrm{NMR} 100 \mathrm{MHz}, \mathrm{CDCl}_{3} \mathbf{1 F}-\boldsymbol{\beta}$ and cca $6 \% \mathbf{1 F}-\boldsymbol{\alpha}$

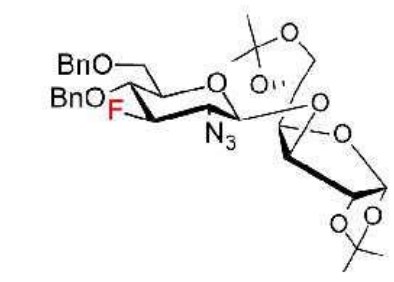

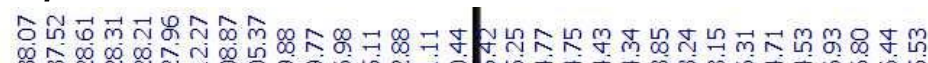

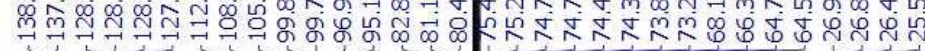

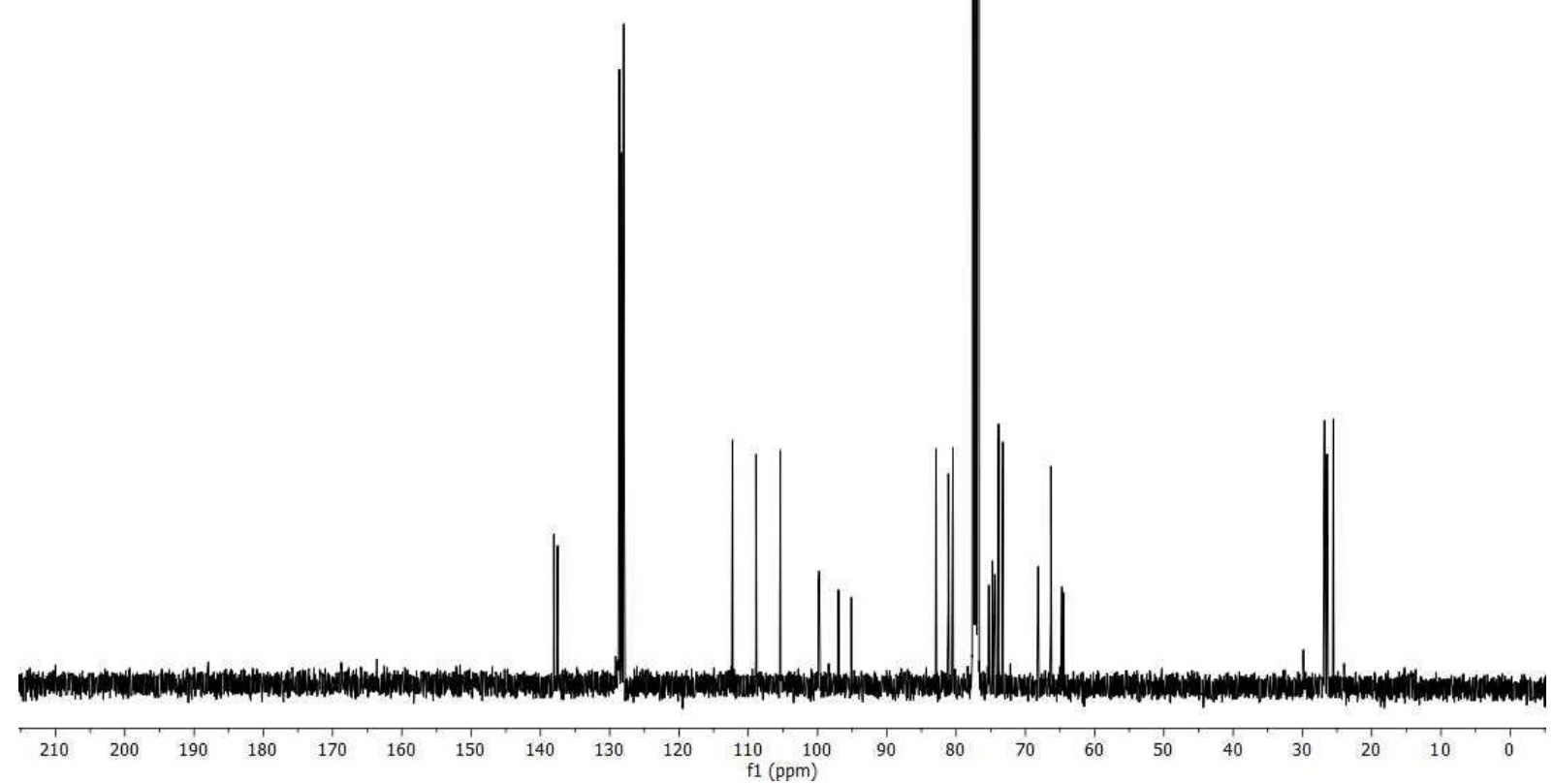


${ }^{1} \mathrm{H}-{ }^{1} \mathrm{H}$ COSY NMR $1 \mathrm{~F}-\beta$ and cca $6 \%$ 1F- $\alpha$

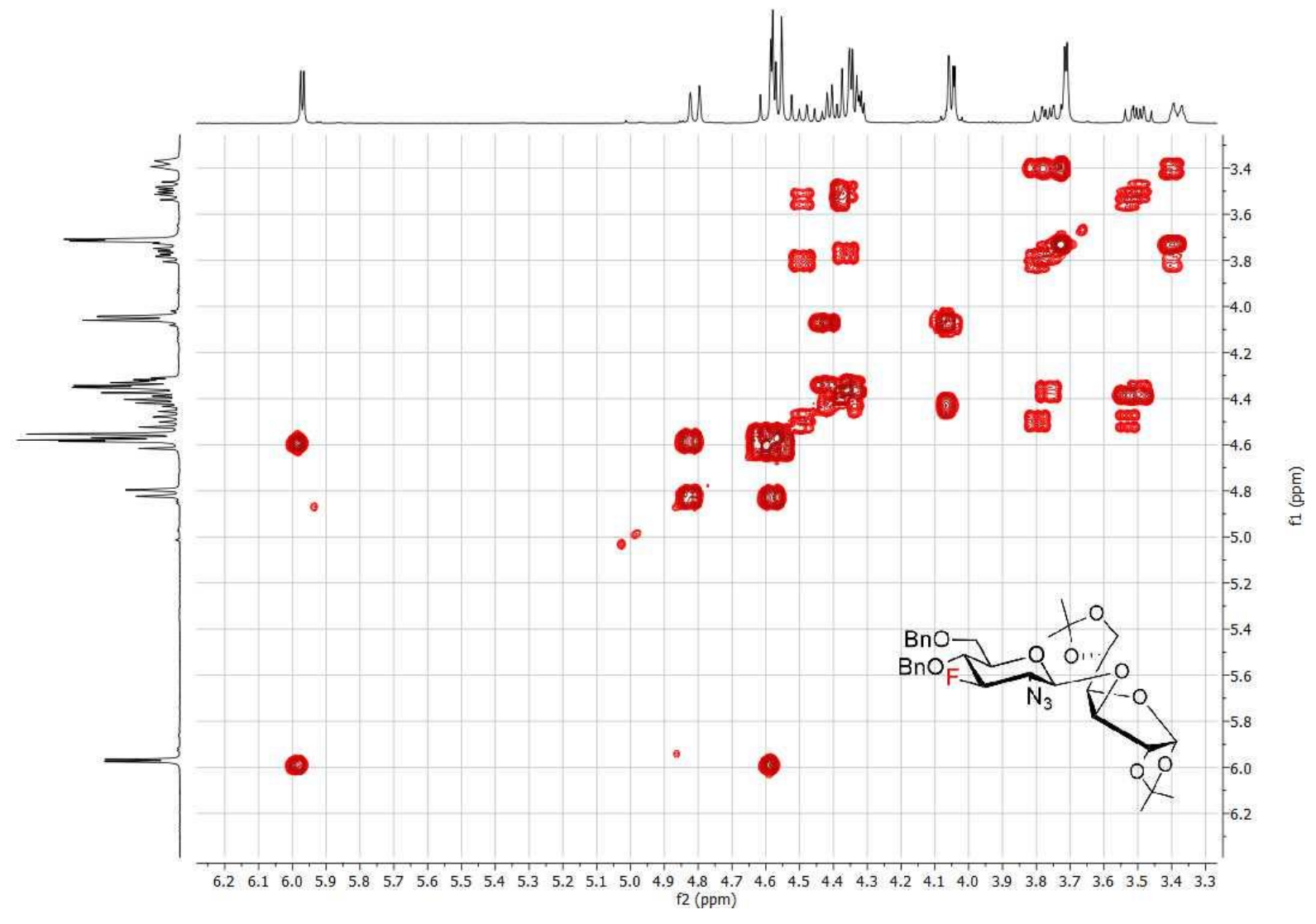

${ }^{1} \mathrm{H}-{ }^{13} \mathrm{C}$ HSQC NMR $1 \mathrm{~F}-\beta$ and cca $6 \% 1 \mathrm{~F}-\alpha$

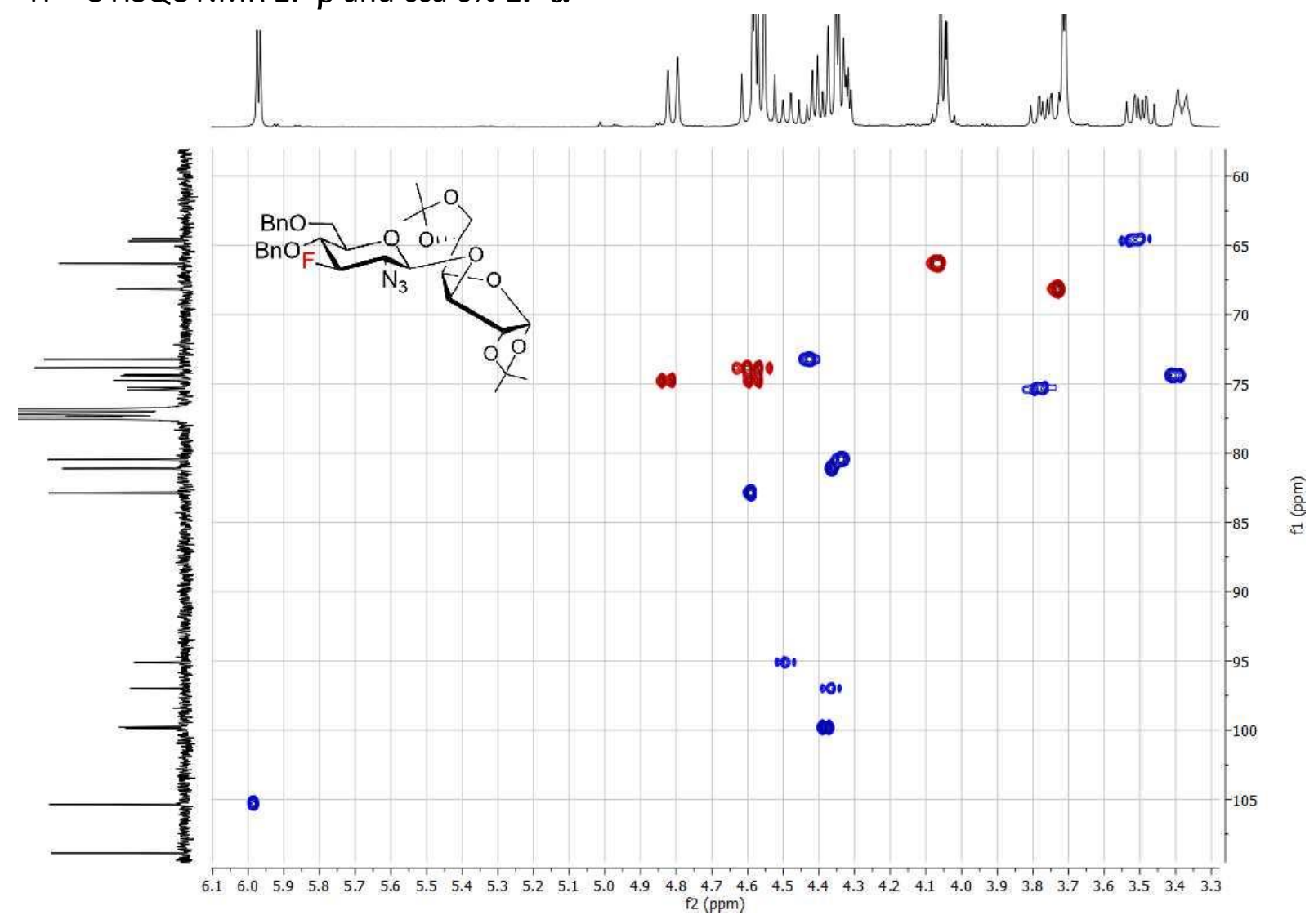


${ }^{19} \mathrm{~F} \mathrm{NMR} 376 \mathrm{MHz}, \mathrm{CDCl}_{3} \mathbf{1 F}-\boldsymbol{\beta}$

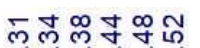

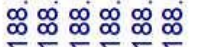

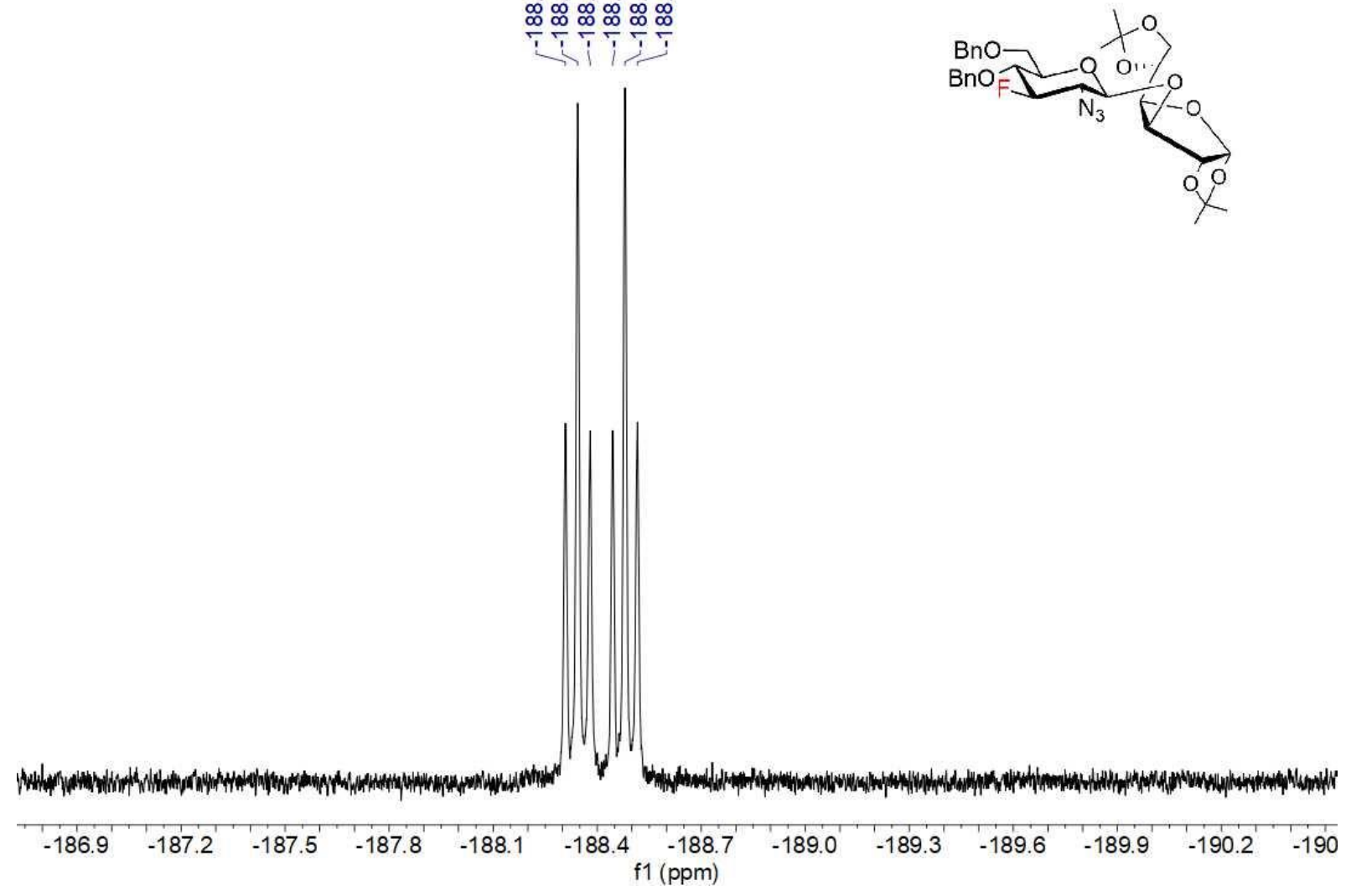




\section{NMR 1G}

\section{${ }^{1} \mathrm{HNMR} 400 \mathrm{MHz}, \mathrm{CDCl}_{3} 1 \mathrm{G}(\alpha: \beta=1: 2)$}

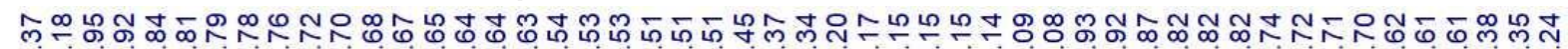

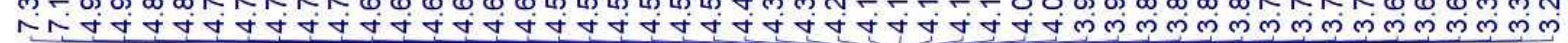

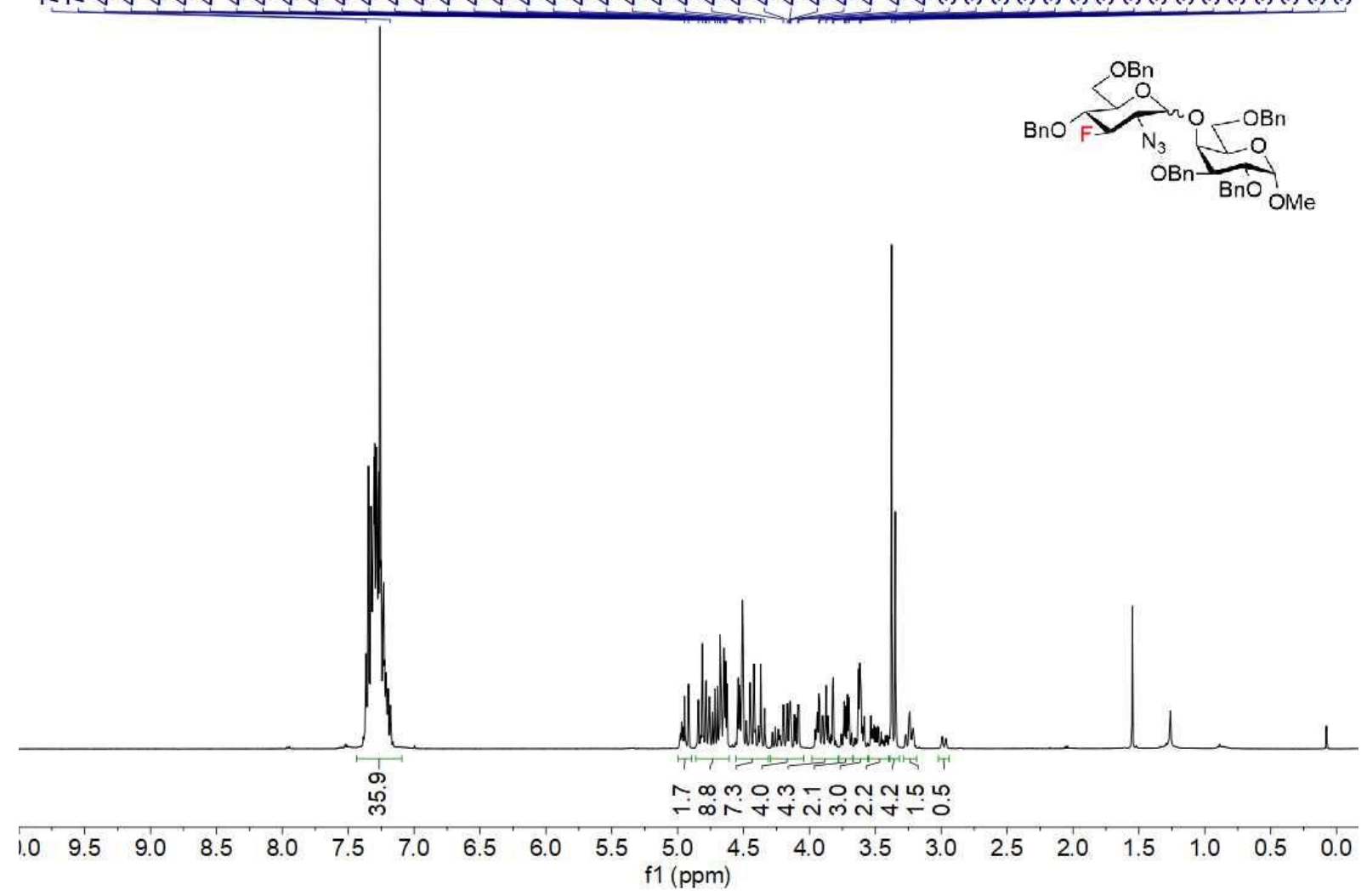

${ }^{13} \mathrm{C}\left\{{ }^{1} \mathrm{H}\right\}$ NMR $100 \mathrm{MHz}, \mathrm{CDCl}_{3} 1 \mathrm{G}(\alpha: \beta=1: 2)$

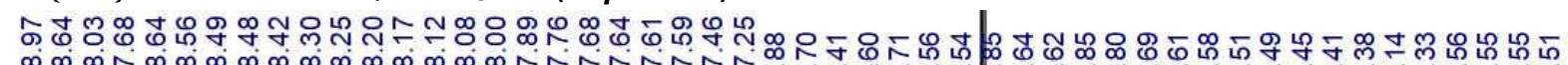

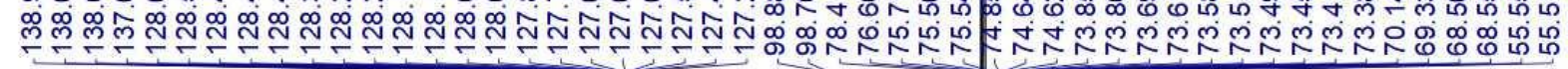

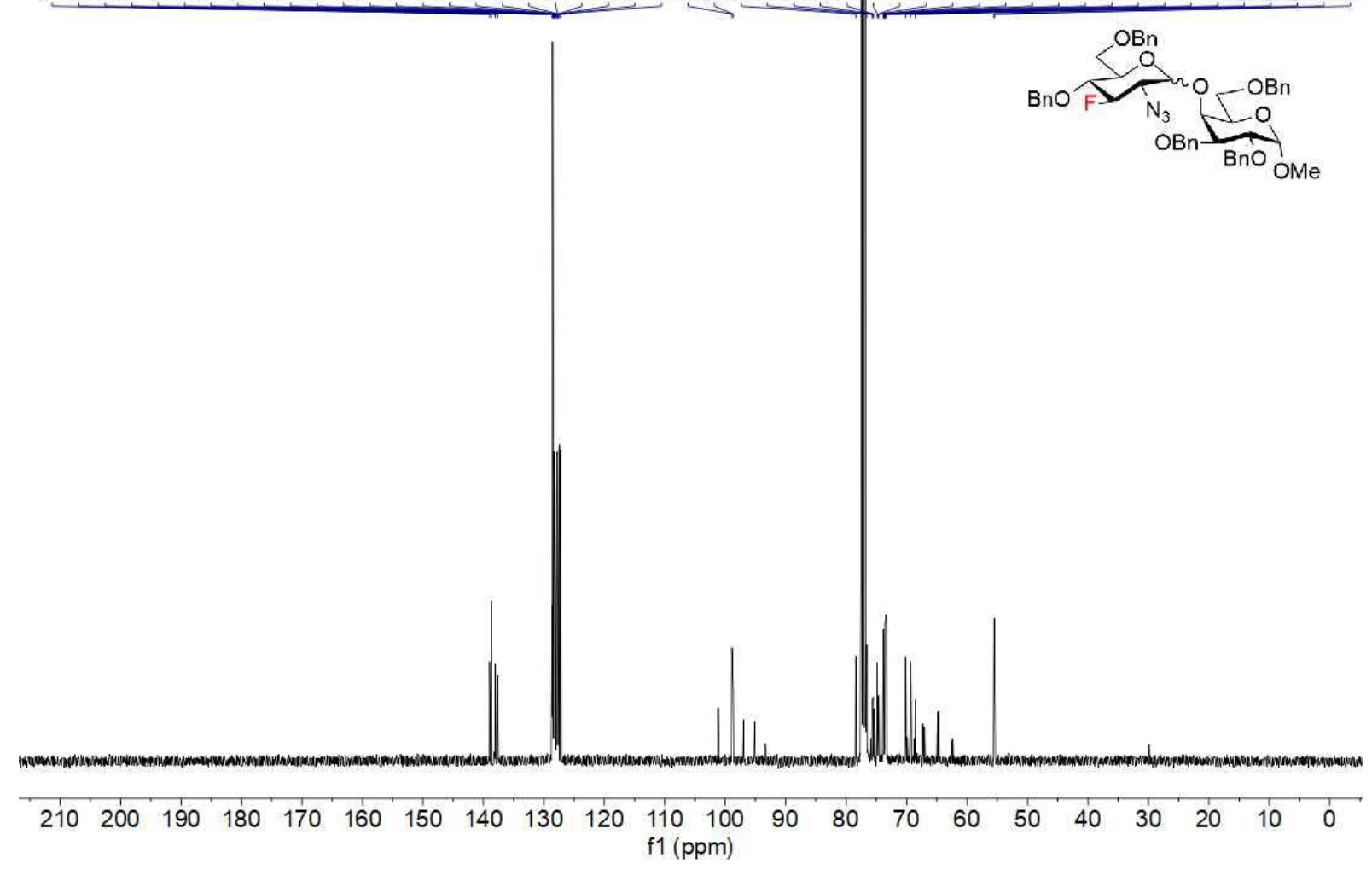


${ }^{1} H-{ }^{1} H$ COSY NMR $1 \mathbf{G}(\alpha: \beta=1: 2)$

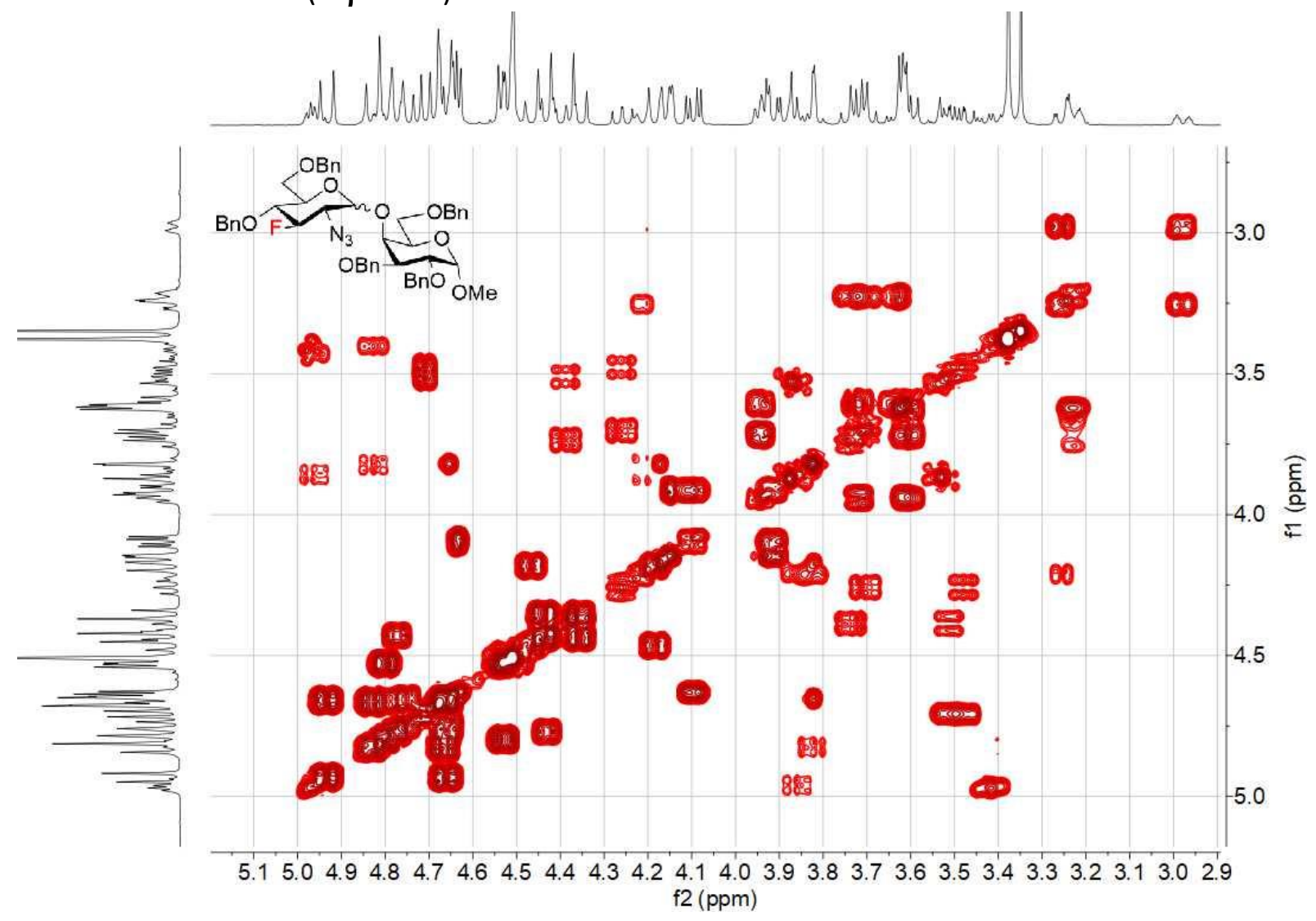

${ }^{1} \mathrm{H}-{ }^{13} \mathrm{C}$ HSQC NMR $1 \mathrm{G}(\alpha: \beta=1: 2)$

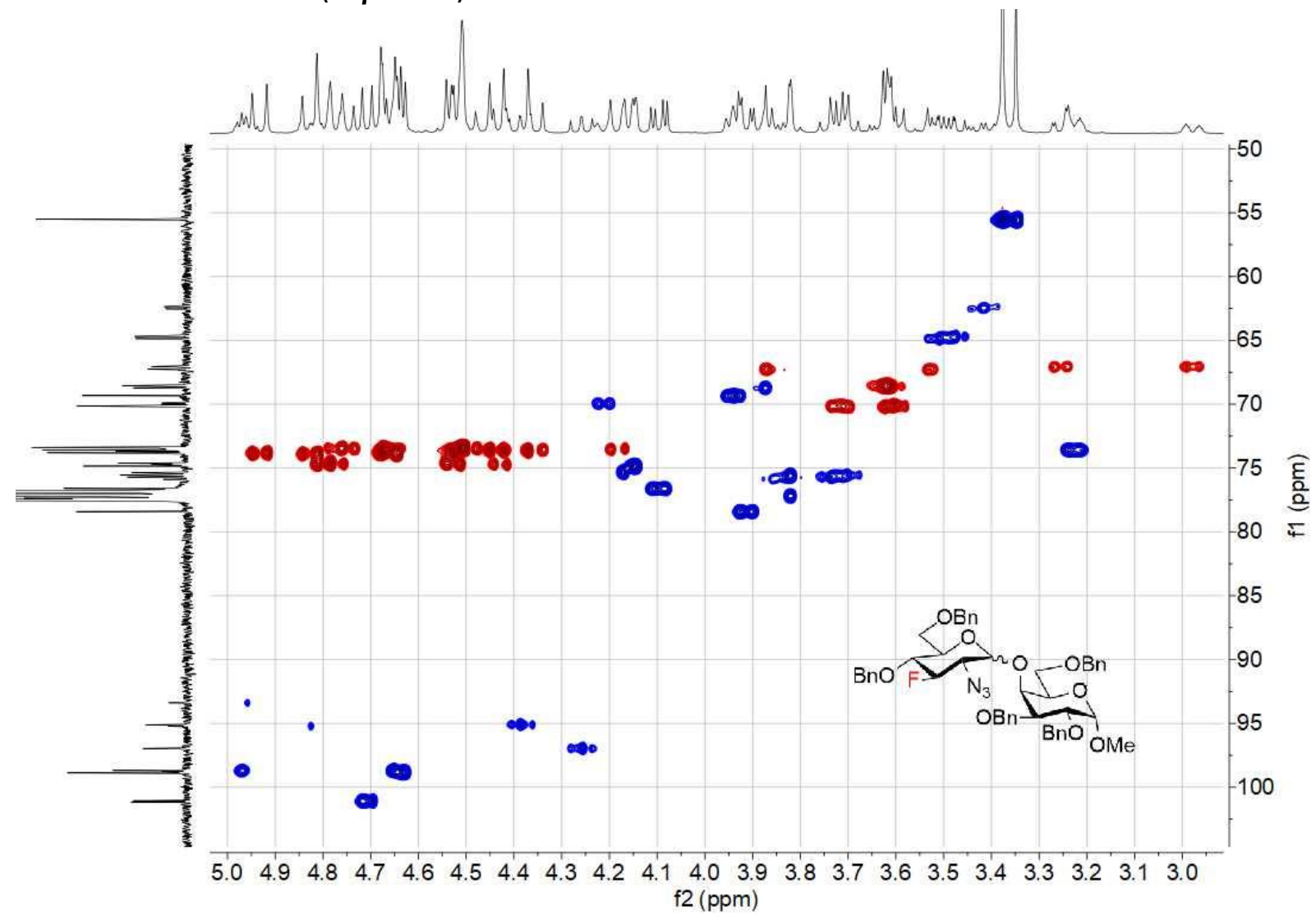




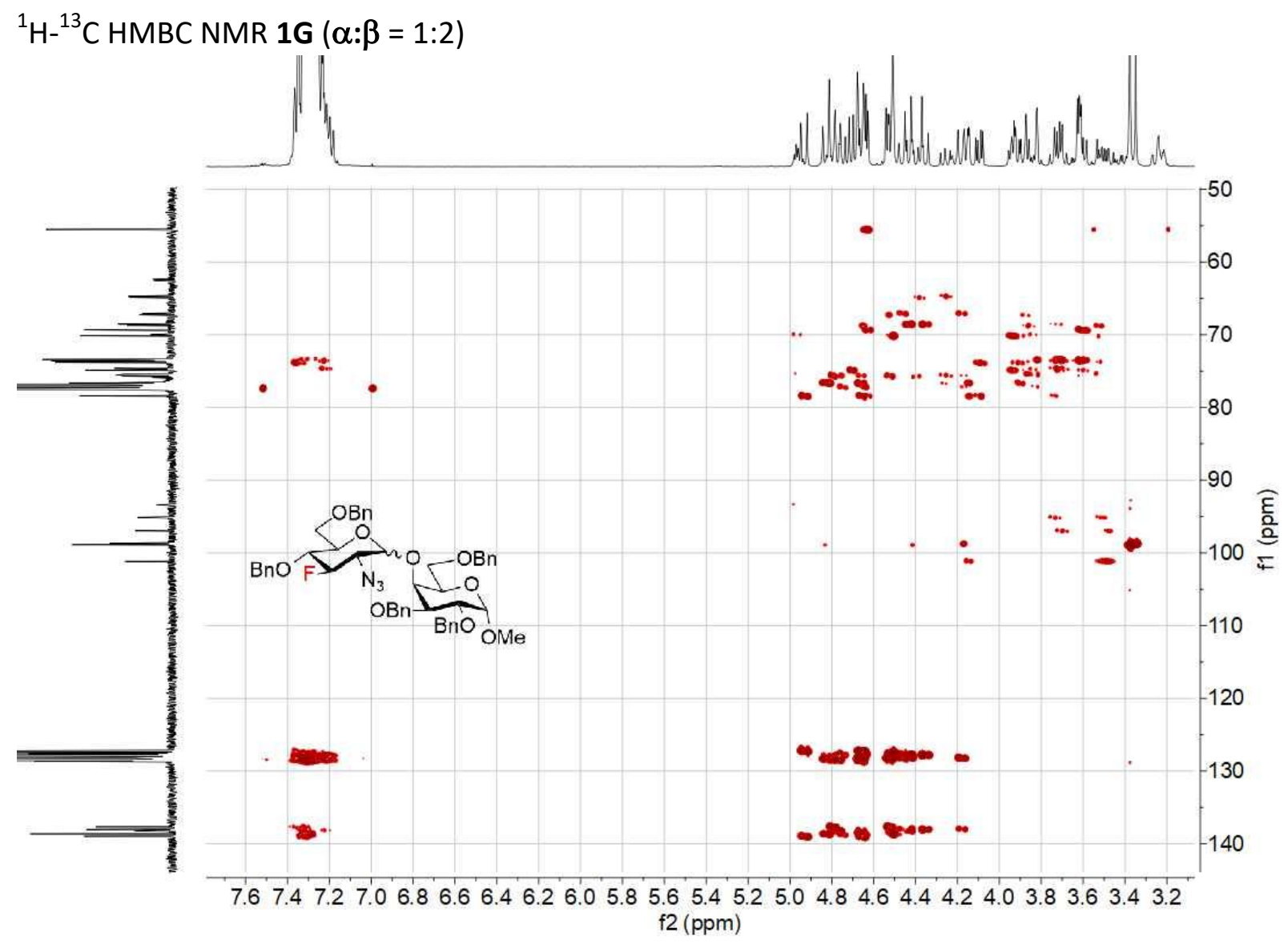

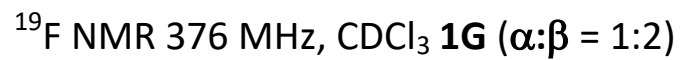

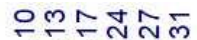

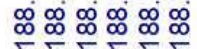

จิกิ๊กัญซ

్ఞ

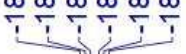
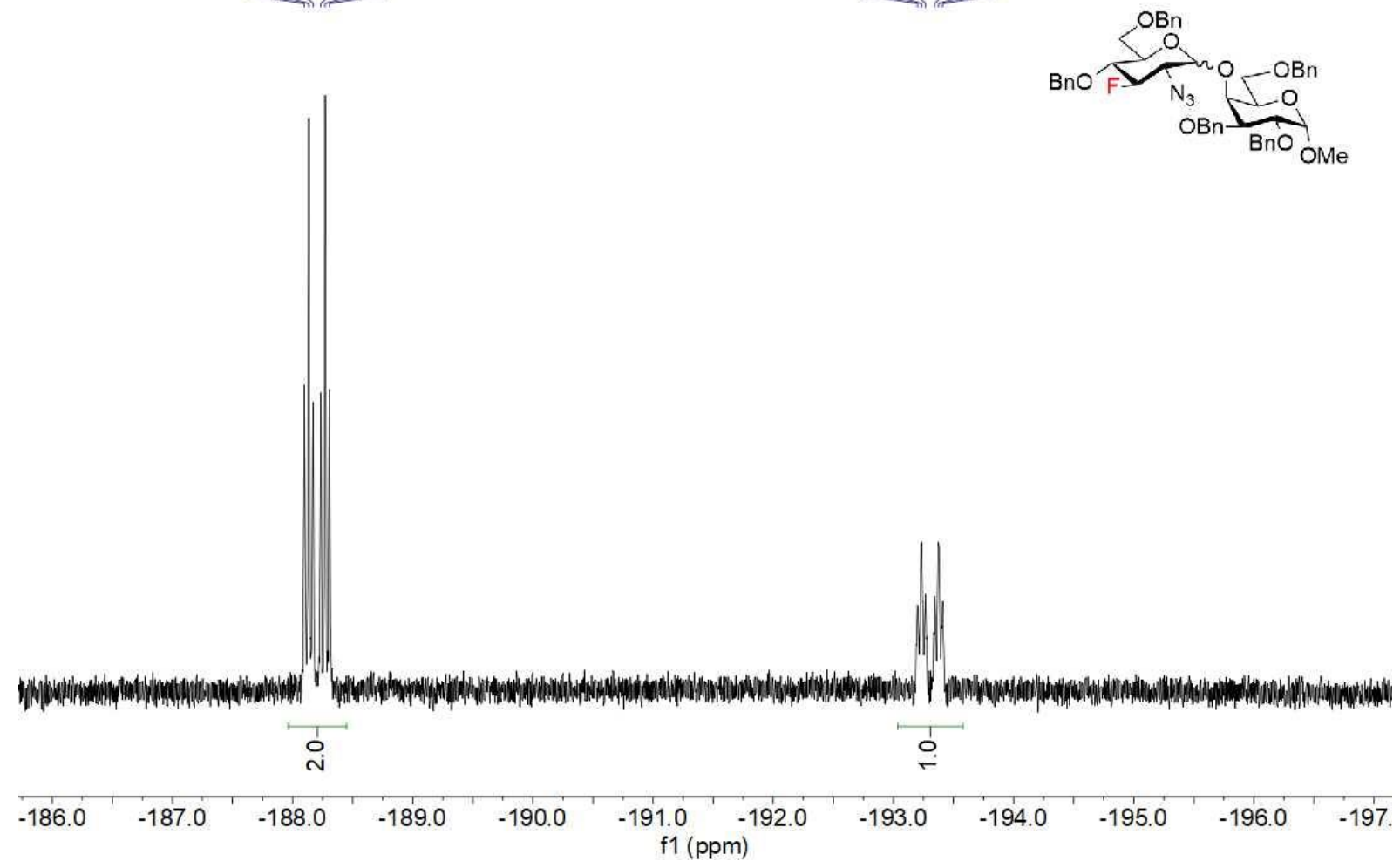


\section{NMR $1 \mathrm{H}-\alpha$}

${ }^{1} \mathrm{HNMR} 400 \mathrm{MHz}, \mathrm{CDCl}_{3} \mathbf{1 H}-\boldsymbol{\alpha}$ and ca $17 \% \mathbf{1 H}-\boldsymbol{\beta}$

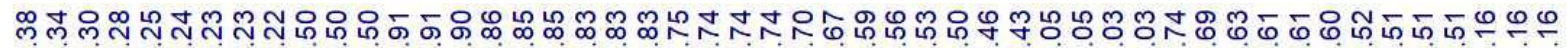

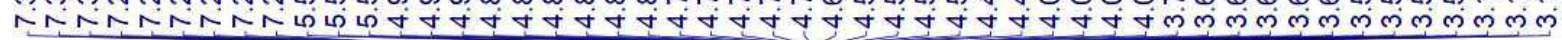

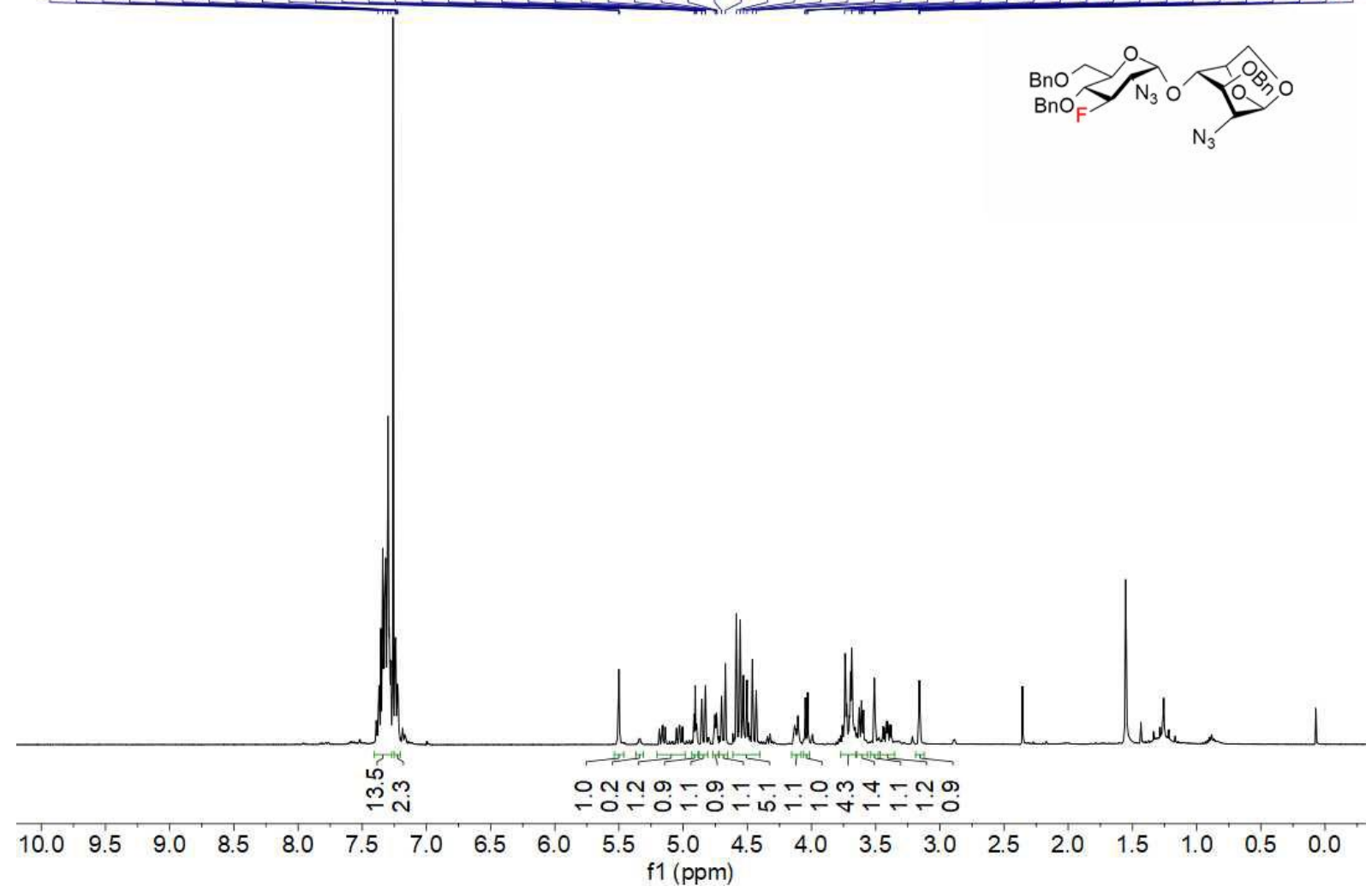

${ }^{13} \mathrm{C}\left\{{ }^{1} \mathrm{H}\right\} \mathrm{NMR} 100 \mathrm{MHz}, \mathrm{CDCl}_{3} \mathbf{1} \mathbf{H}-\boldsymbol{\alpha}$ and ca $17 \% \mathbf{1 H}-\boldsymbol{\beta}$

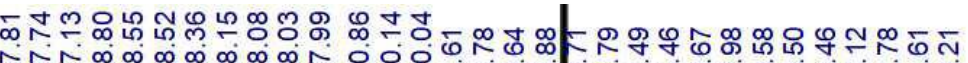

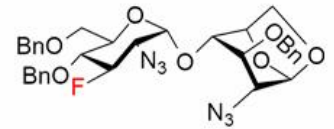

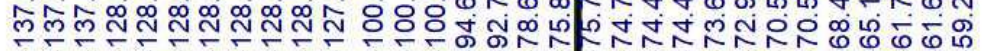

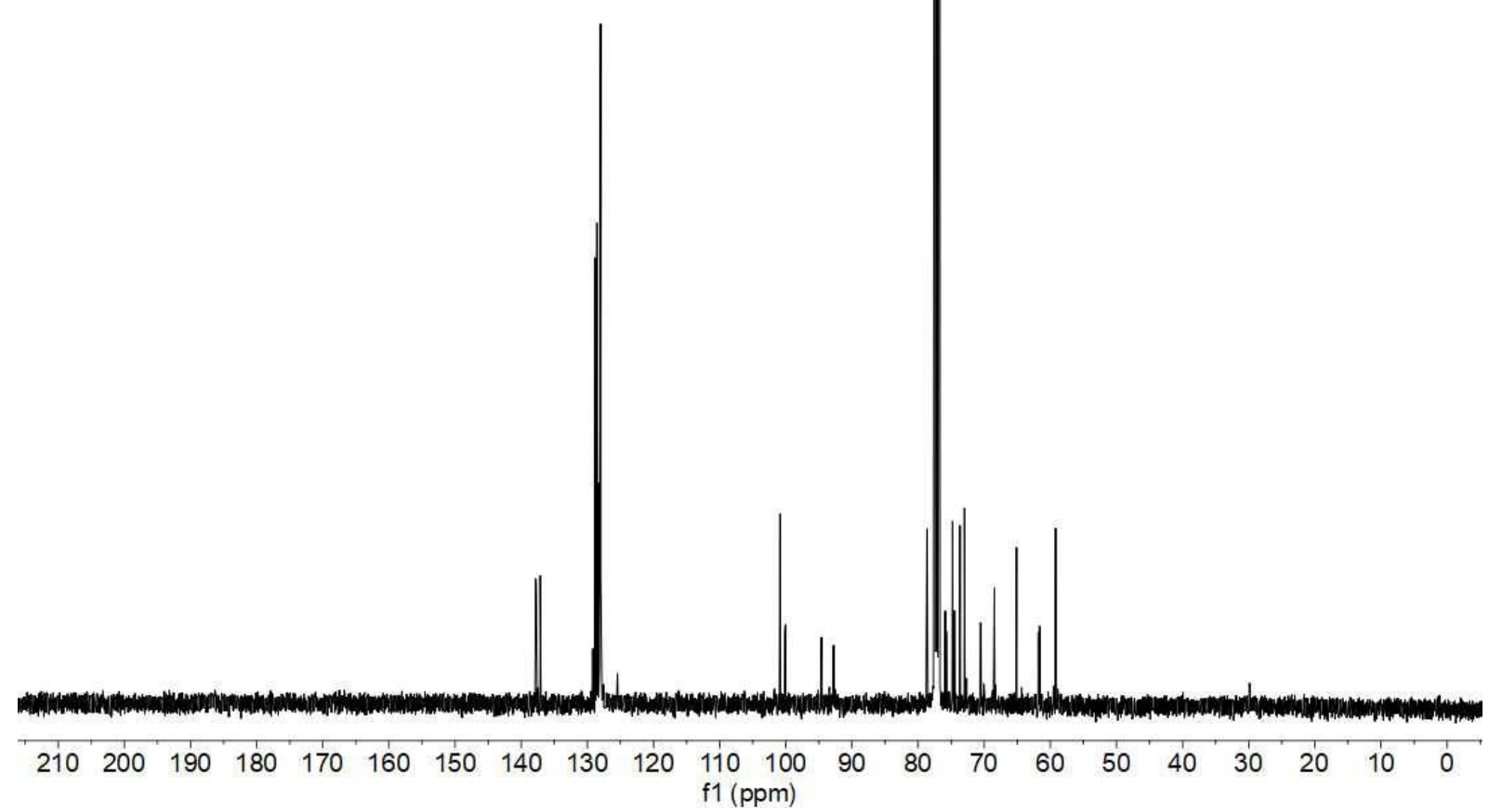


${ }^{1} \mathrm{H}-{ }^{1} \mathrm{H}$ COSY NMR $1 \mathrm{H}-\alpha$ and ca $17 \% \mathbf{1}-\boldsymbol{H}-\beta$

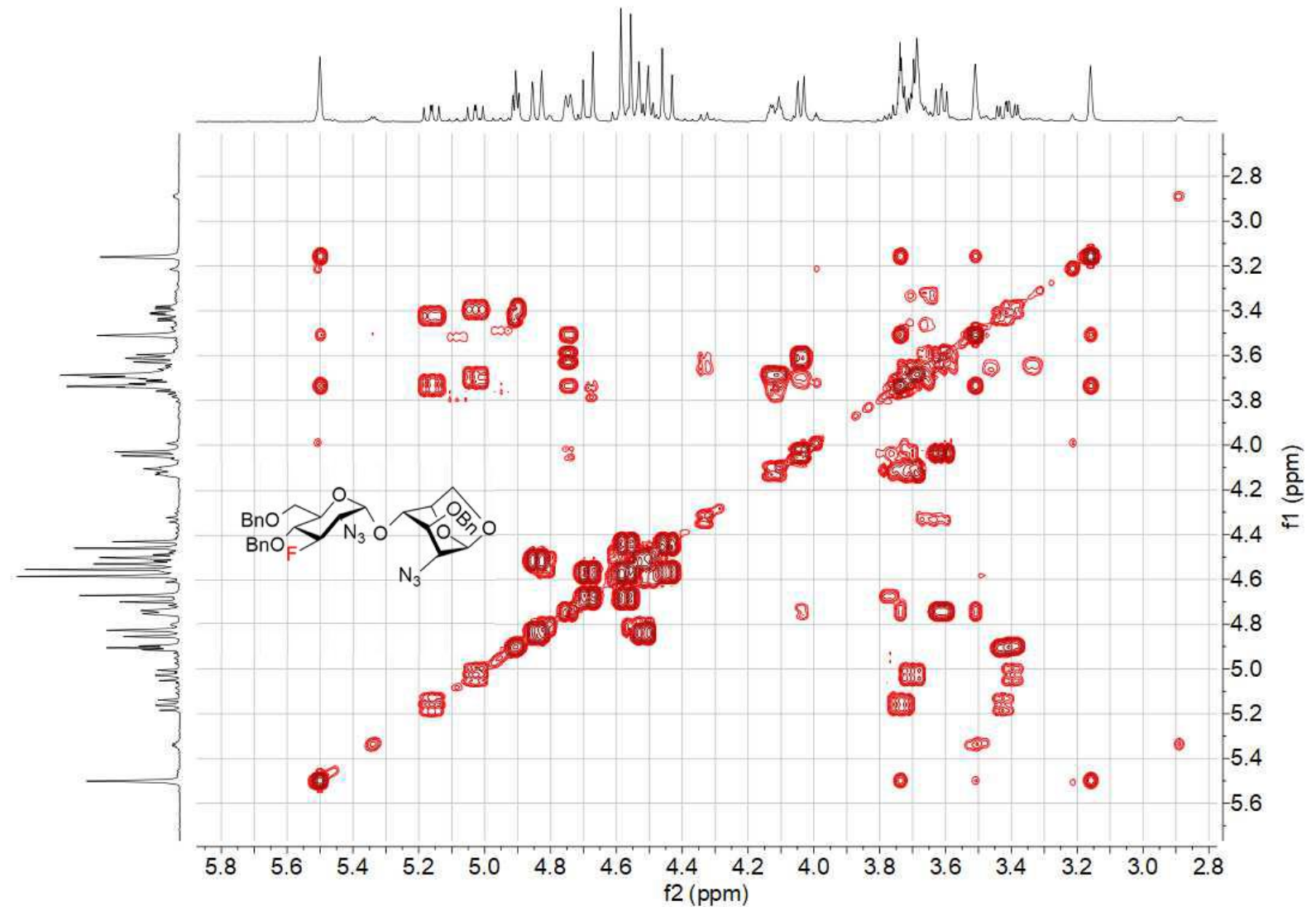

${ }^{1} \mathrm{H}-{ }^{13} \mathrm{CHSQC} N M R \mathbf{1 H}-\alpha$ and ca $17 \% \mathbf{1} \mathbf{H}-\beta$

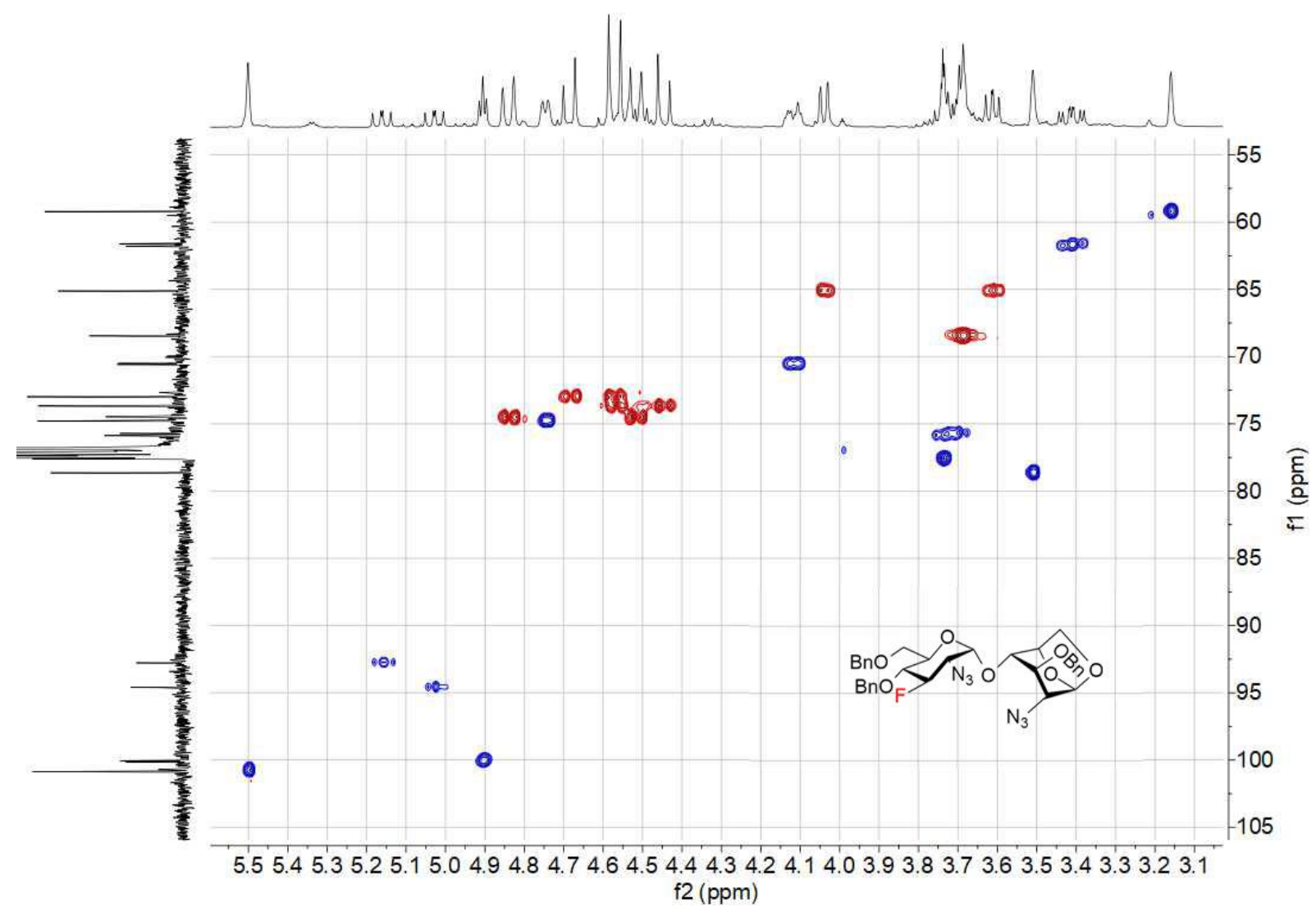




\section{${ }^{1} \mathrm{H}-{ }^{13} \mathrm{C}$ HMBC NMR $\mathbf{1 H}-\boldsymbol{\alpha}$ and ca $17 \% \mathbf{1 H}-\beta$}

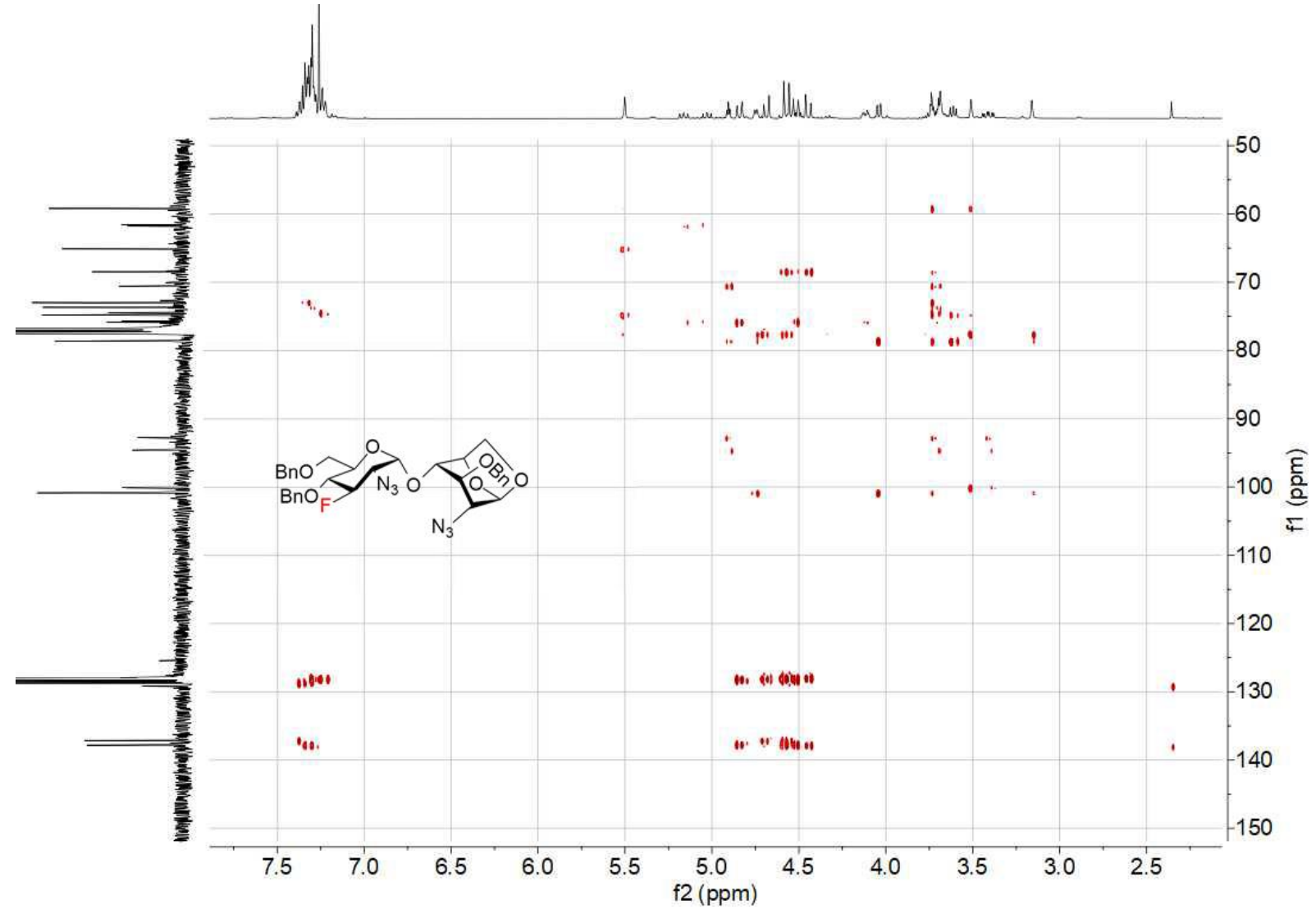

${ }^{19} \mathrm{~F} \mathrm{NMR} 376 \mathrm{MHz}, \mathrm{CDCl}_{3} \mathbf{1 H}-\boldsymbol{\alpha}$
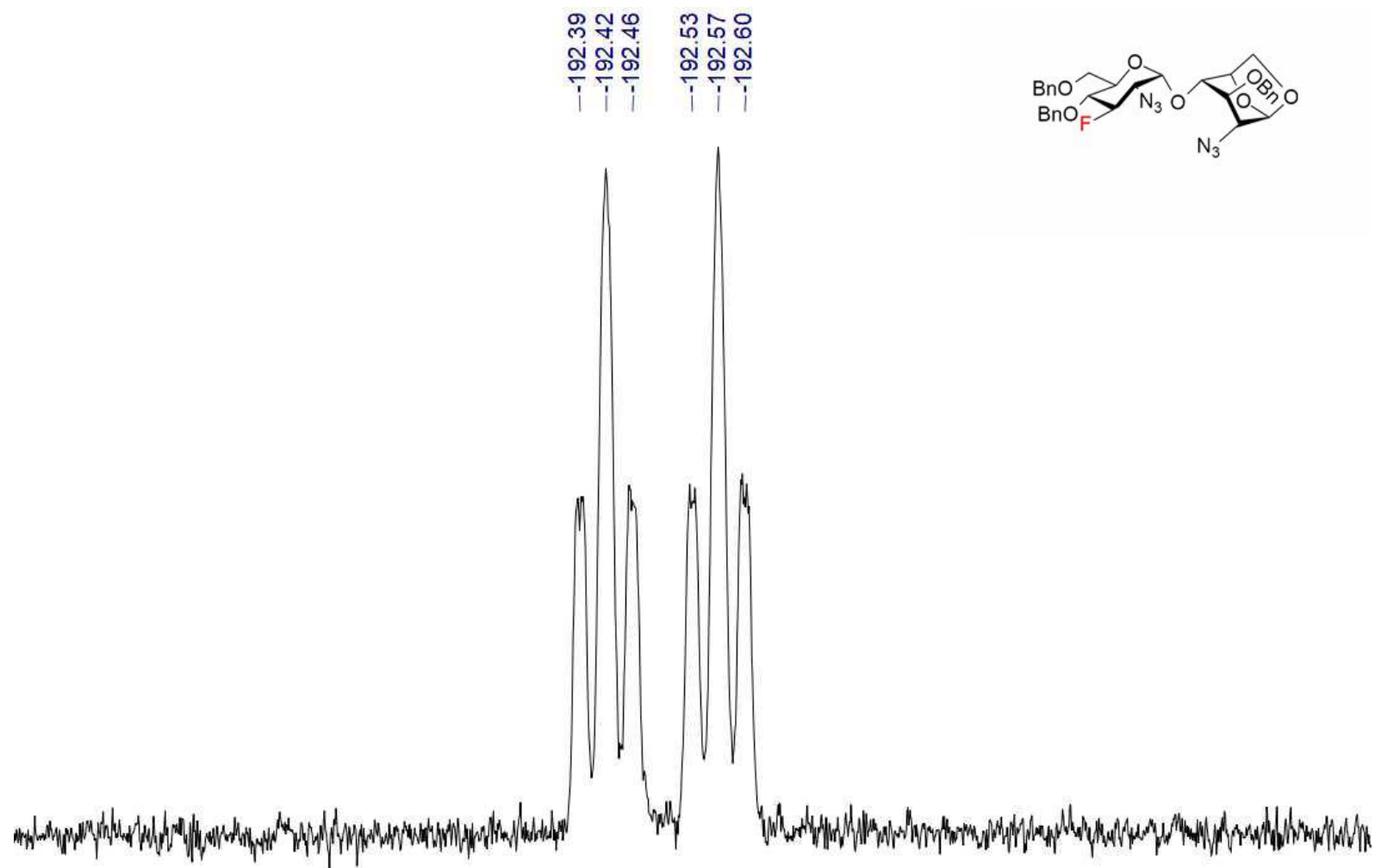

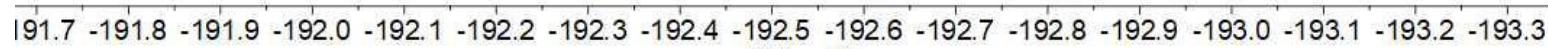
f1 (ppm) 
NMR $\mathbf{1 H}-\boldsymbol{\beta}$

${ }^{1} \mathrm{H} \mathrm{NMR} 400 \mathrm{MHz}, \mathrm{CDCl}_{3} \mathbf{1 H}-\boldsymbol{\beta}$ and ca $17 \% \mathbf{1 H}-\boldsymbol{\alpha}$

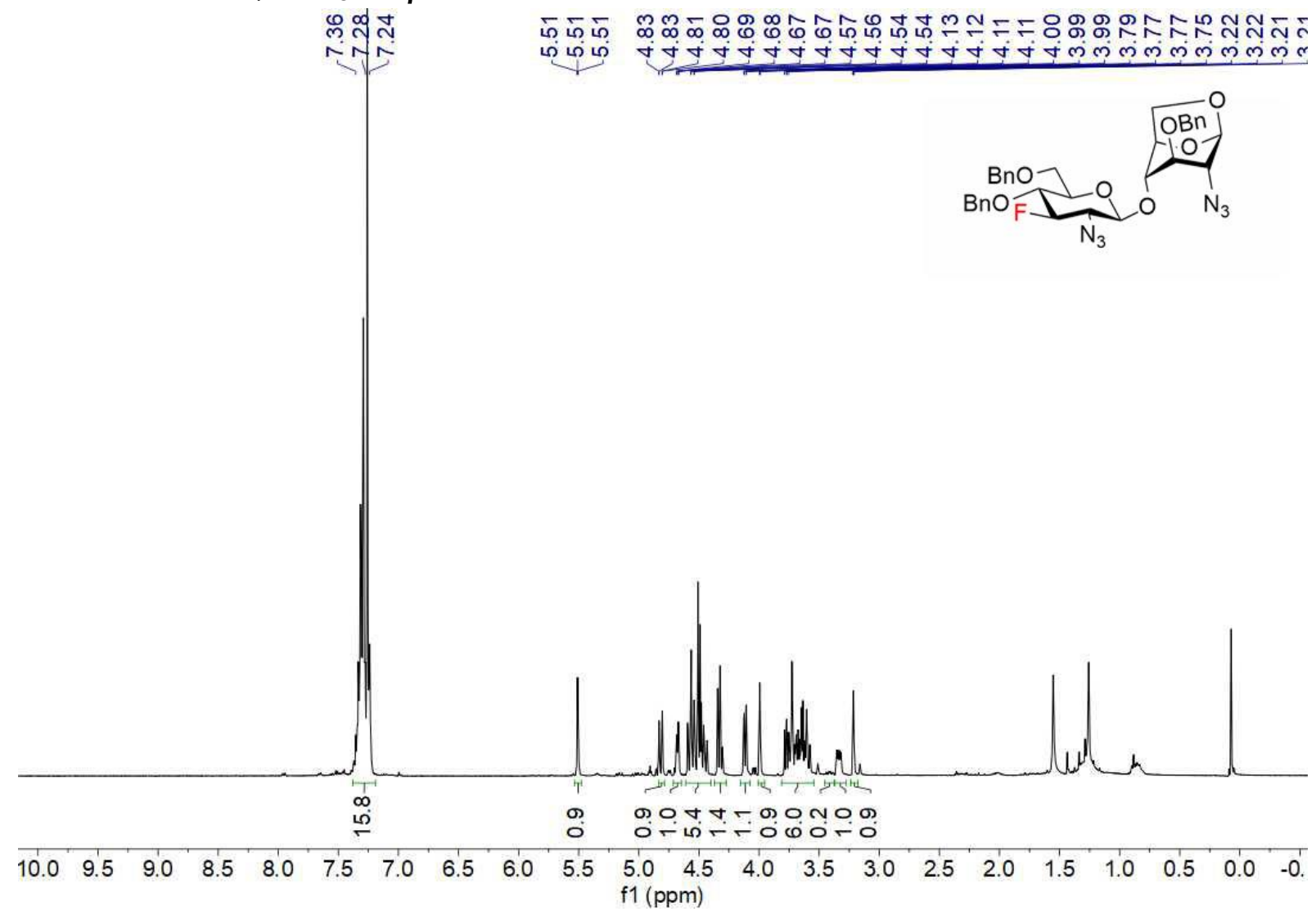

${ }^{13} \mathrm{C}\left\{{ }^{1} \mathrm{H}\right\}$ NMR $100 \mathrm{MHz}, \mathrm{CDCl}_{3} \mathbf{1 H}-\boldsymbol{\beta}$ and ca $17 \% \mathbf{1 H}-\boldsymbol{\alpha}$

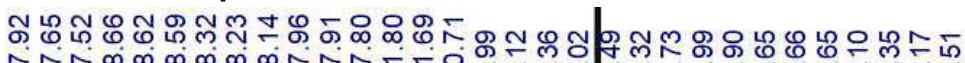

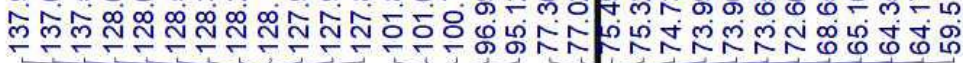

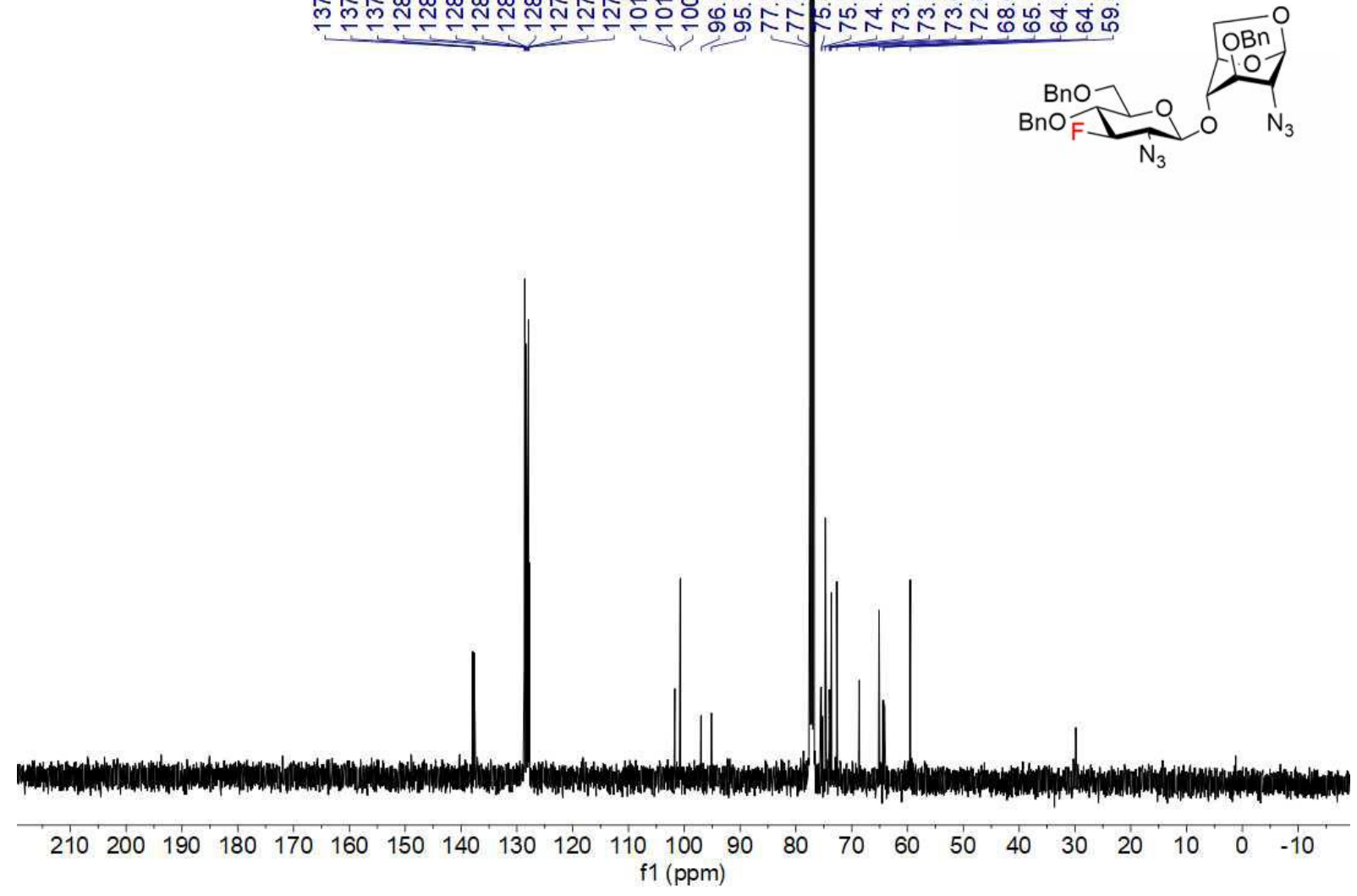


${ }^{1} \mathrm{H}-{ }^{1} \mathrm{H}$ COSY NMR $1 \mathrm{H}-\beta$ and ca $17 \% \mathbf{1 H}-\alpha$

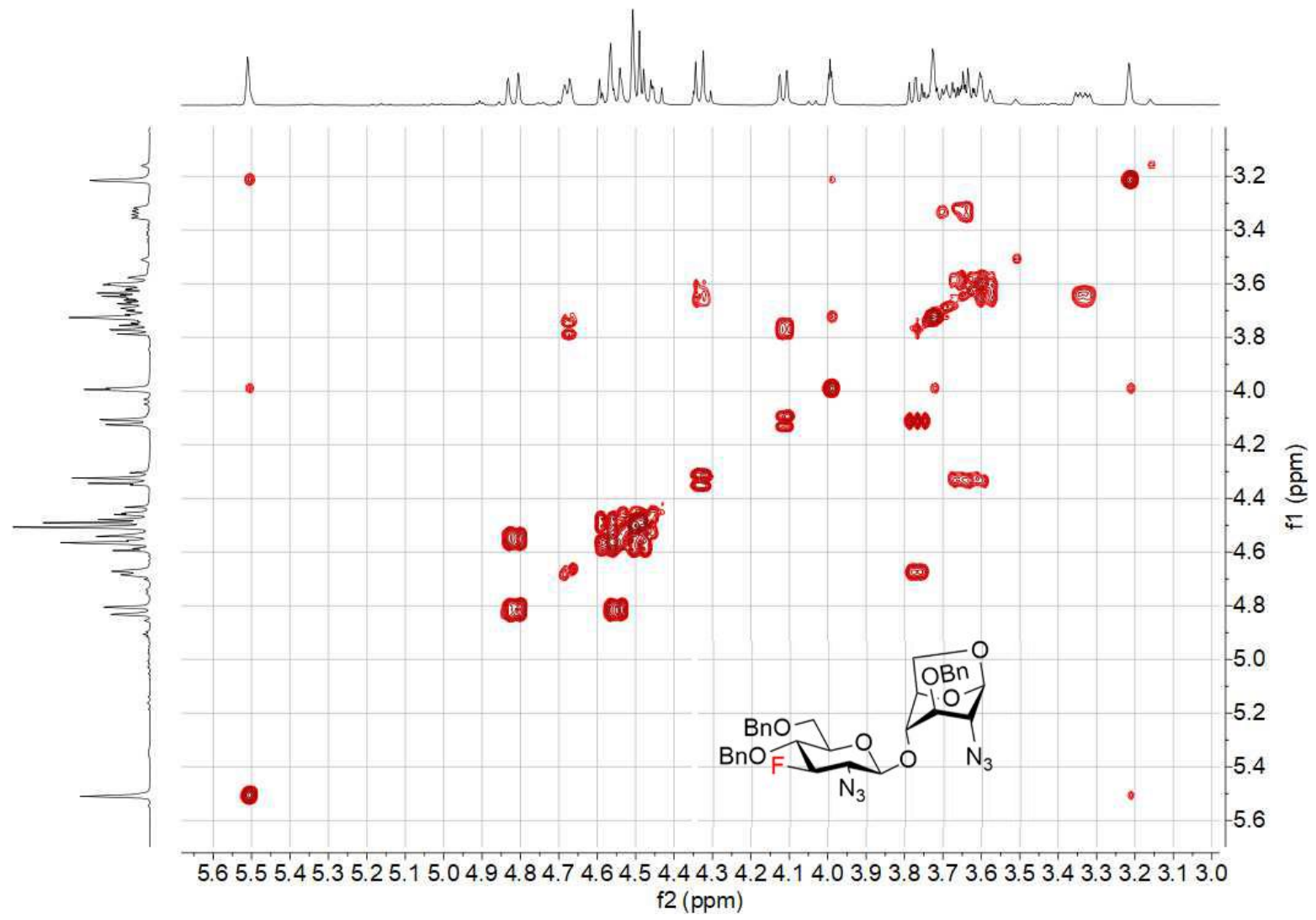

${ }^{1} \mathrm{H}-{ }^{13} \mathrm{C}$ HSQC NMR $1 \mathrm{H}-\beta$ and ca $17 \% 1 \mathrm{H}-\alpha$

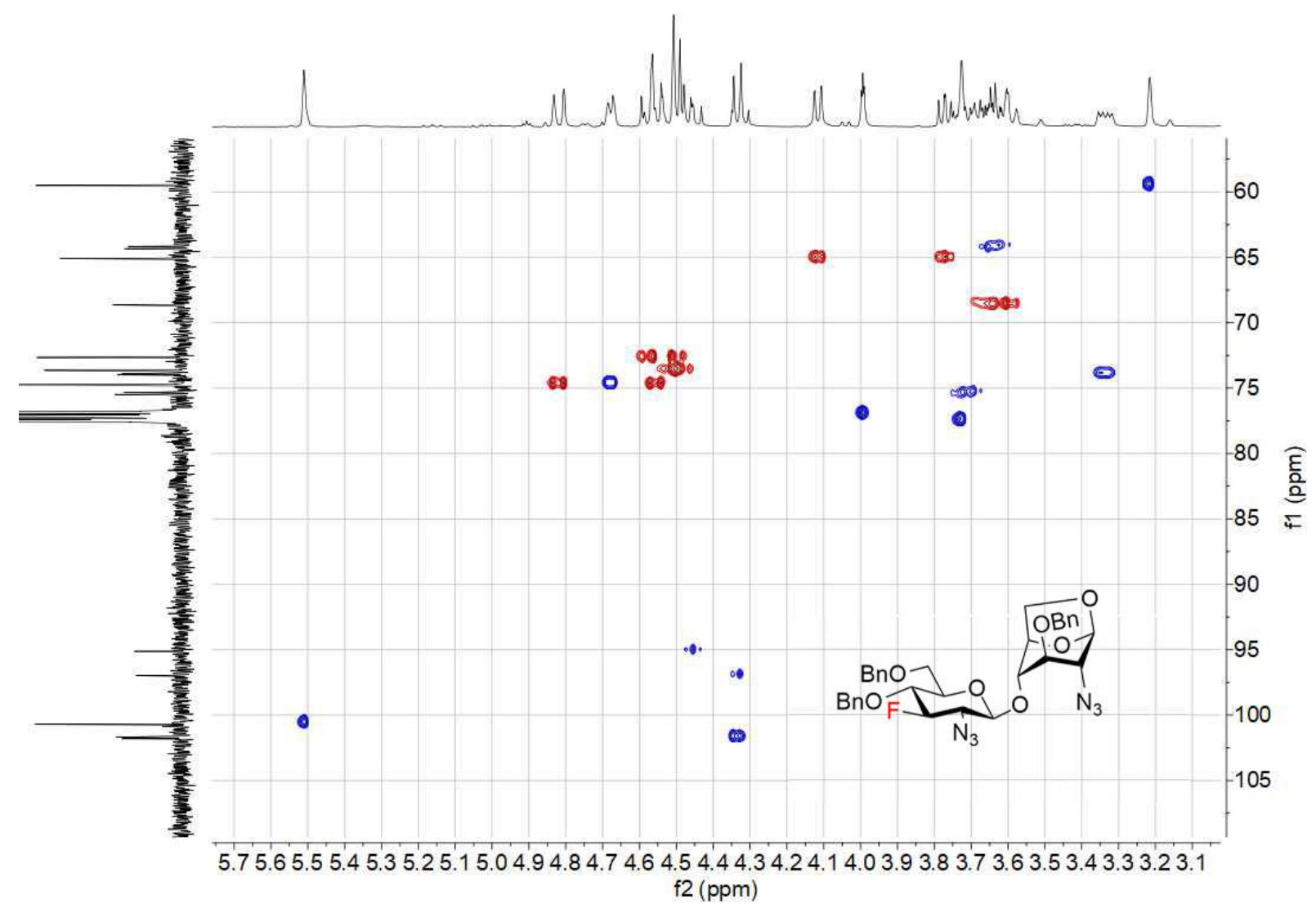


${ }^{1} \mathrm{H}-{ }^{13} \mathrm{C}$ HMBC NMR $\mathbf{1} \mathbf{H}-\boldsymbol{\beta}$ and ca $17 \% \mathbf{1} \mathbf{H}-\boldsymbol{\alpha}$

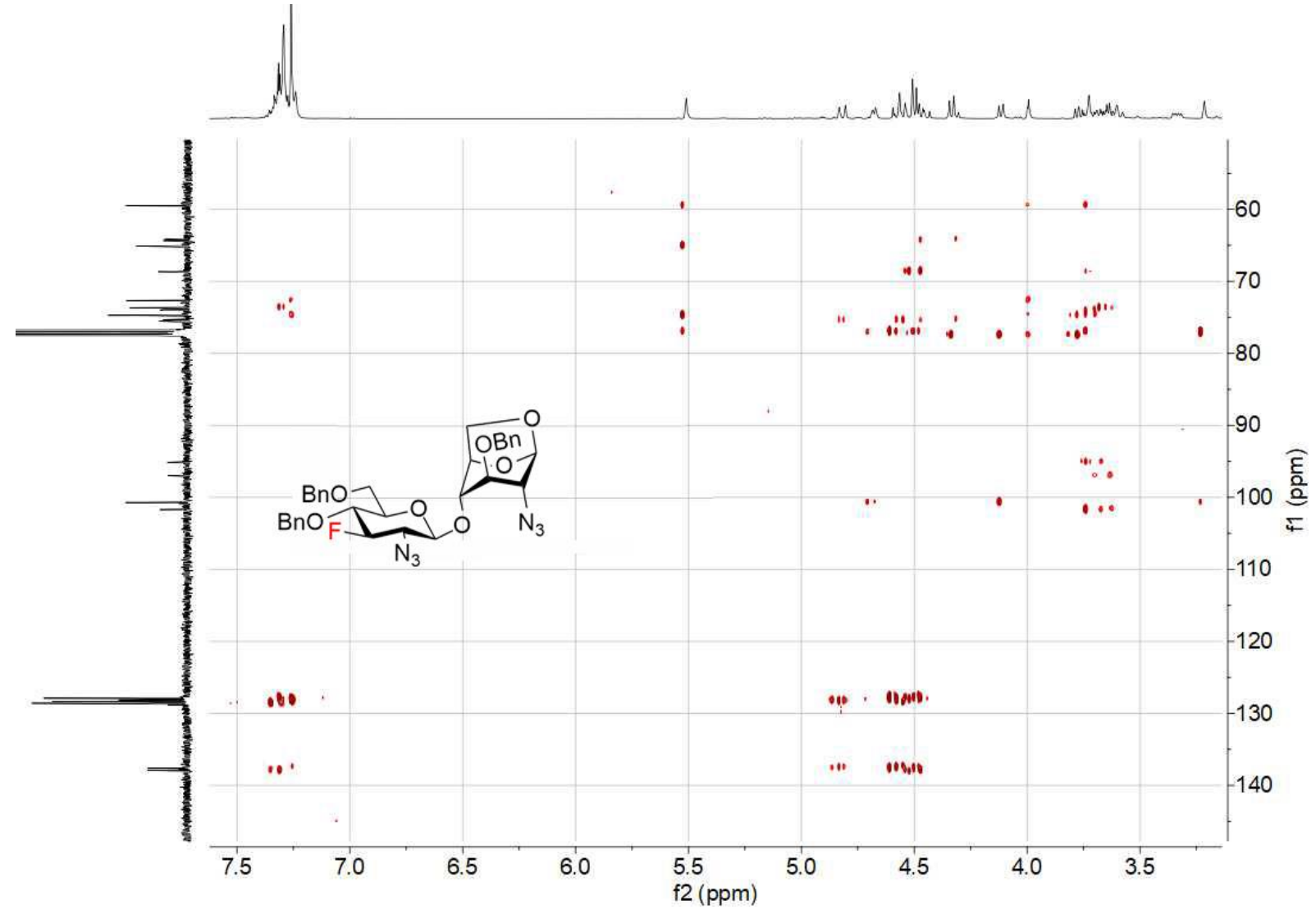

${ }^{19} \mathrm{~F} \mathrm{NMR} 376 \mathrm{MHz}, \mathrm{CDCl}_{3} 1 \mathrm{H}-\boldsymbol{\beta}$

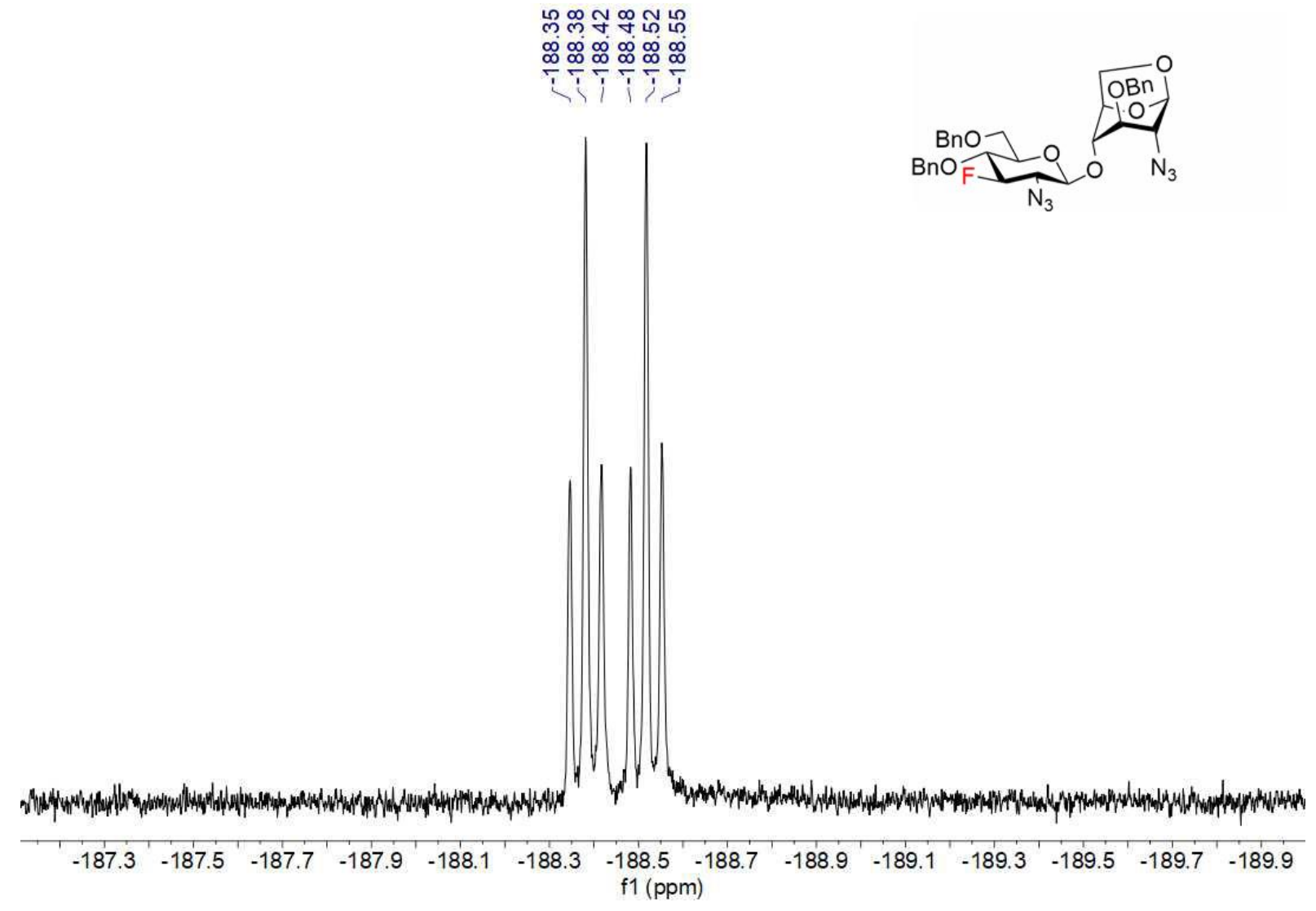




\section{NMR 1I- $\alpha$}

${ }^{1} \mathrm{H}$ NMR $400 \mathrm{MHz}, \mathrm{CDCl}_{3} 1 \mathrm{l}-\alpha$

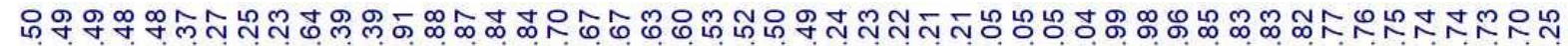

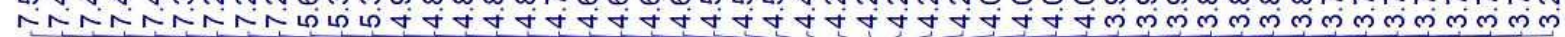

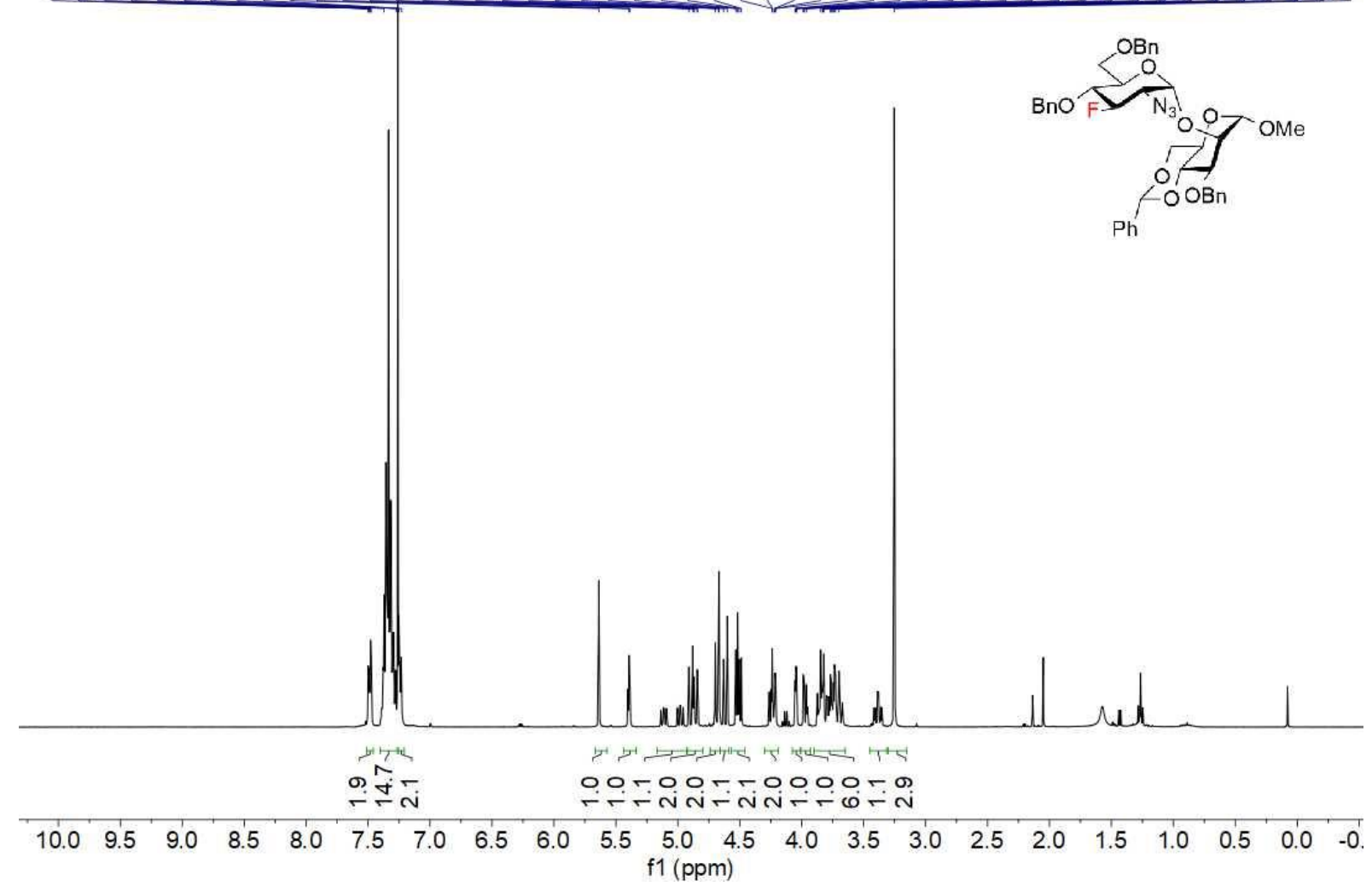

${ }^{13} \mathrm{C}\left\{{ }^{1} \mathrm{H}\right\} \mathrm{NMR} 100 \mathrm{MHz}, \mathrm{CDCl}_{3} 1 \mathrm{l}-\alpha$

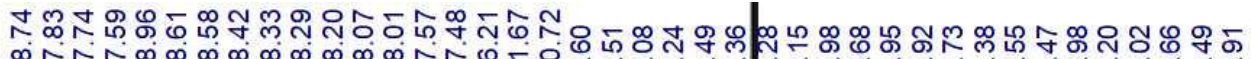

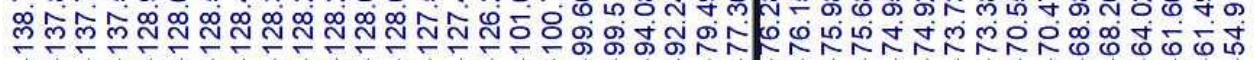

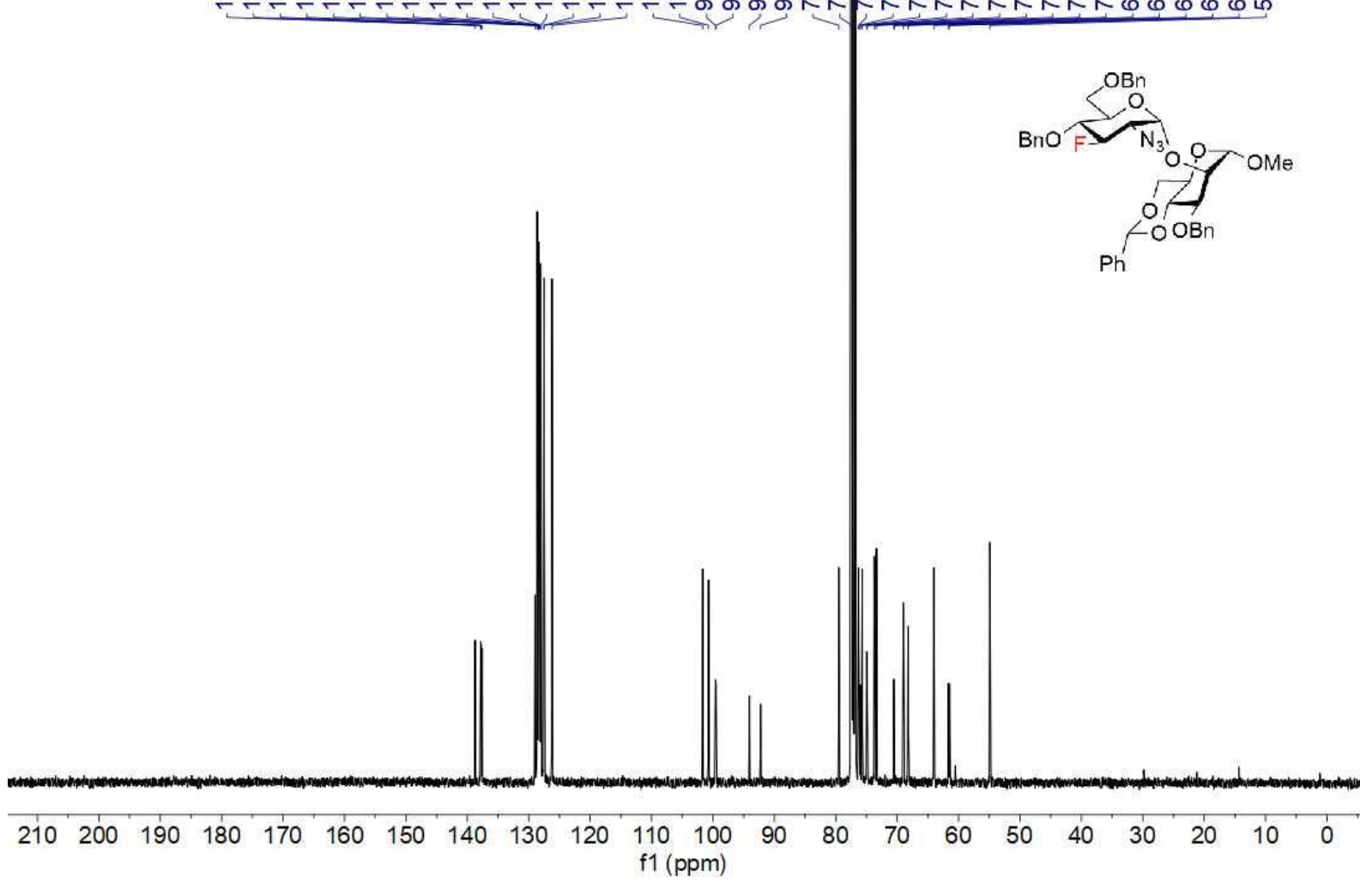


${ }^{1} \mathrm{H}-{ }^{1} \mathrm{H}$ COSY NMR $1 \mathrm{l}-\boldsymbol{\alpha}$

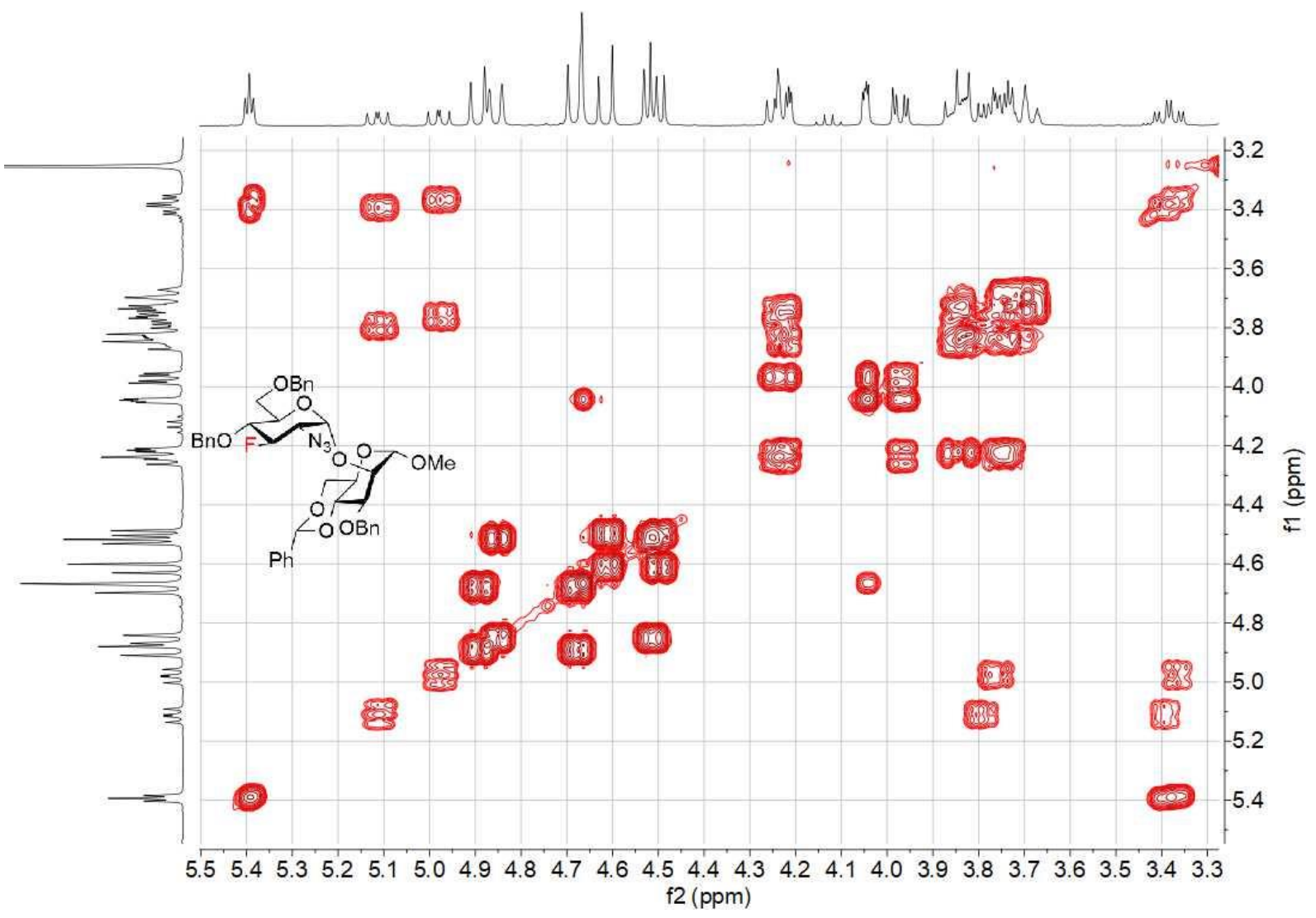

${ }^{1} \mathrm{H}-{ }^{13} \mathrm{C}$ HSQC NMR $11-\alpha$

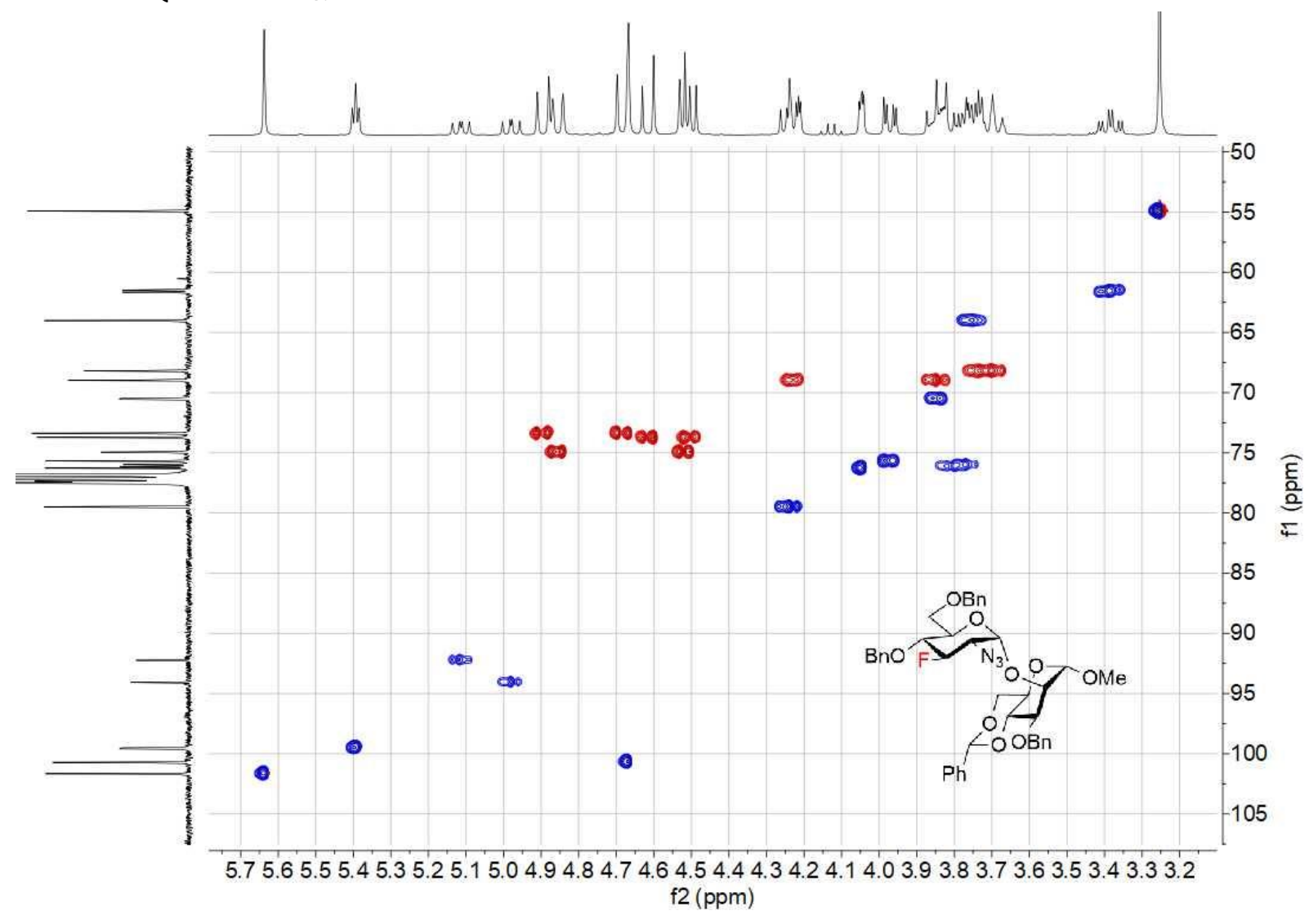


${ }^{1} \mathrm{H}-{ }^{13} \mathrm{C} H M B C N M R$ 1I- $\alpha$

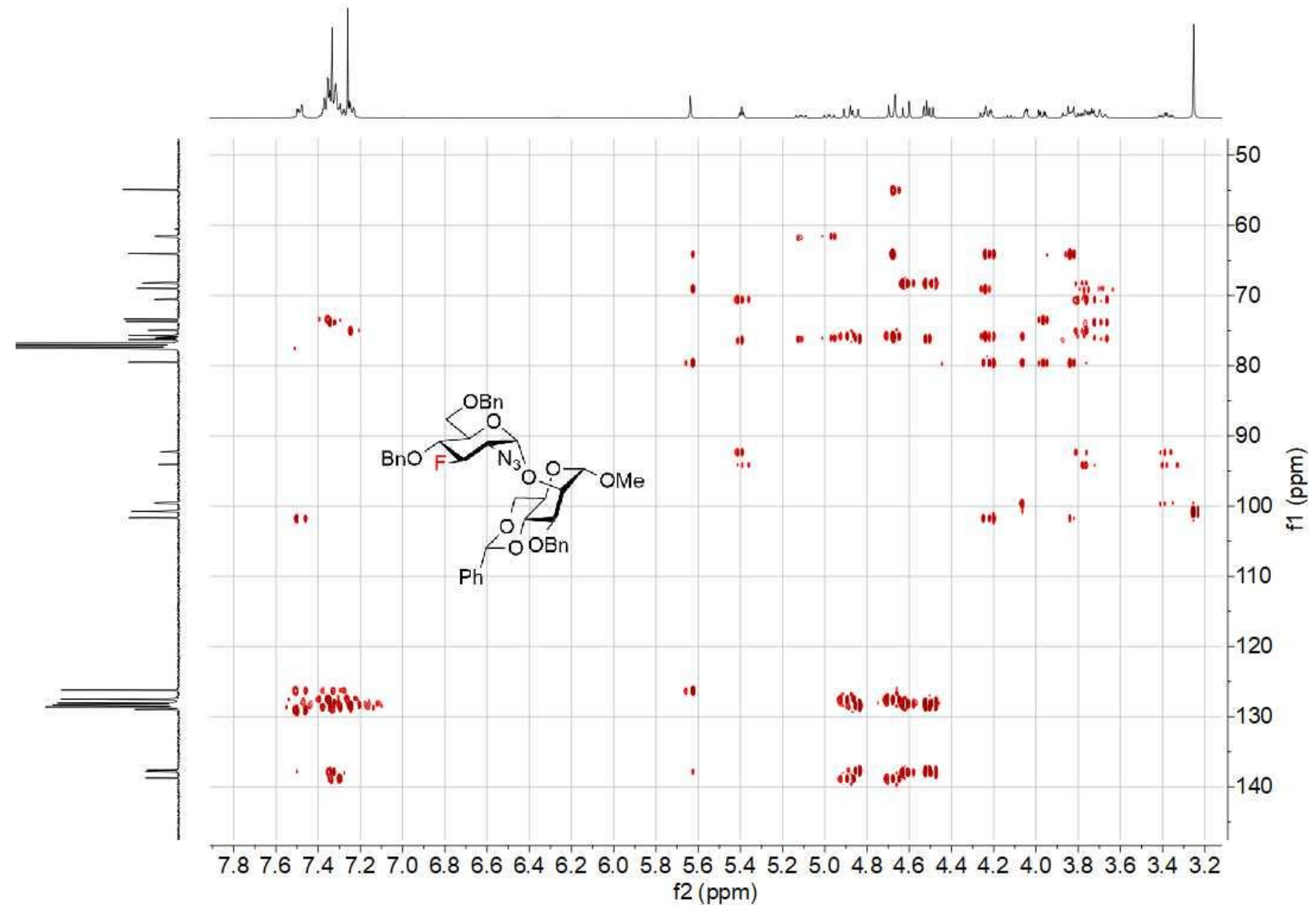

${ }^{19} \mathrm{~F} \mathrm{NMR} 376 \mathrm{MHz}, \mathrm{CDCl}_{3} 11-\alpha$

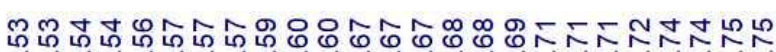

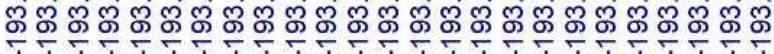

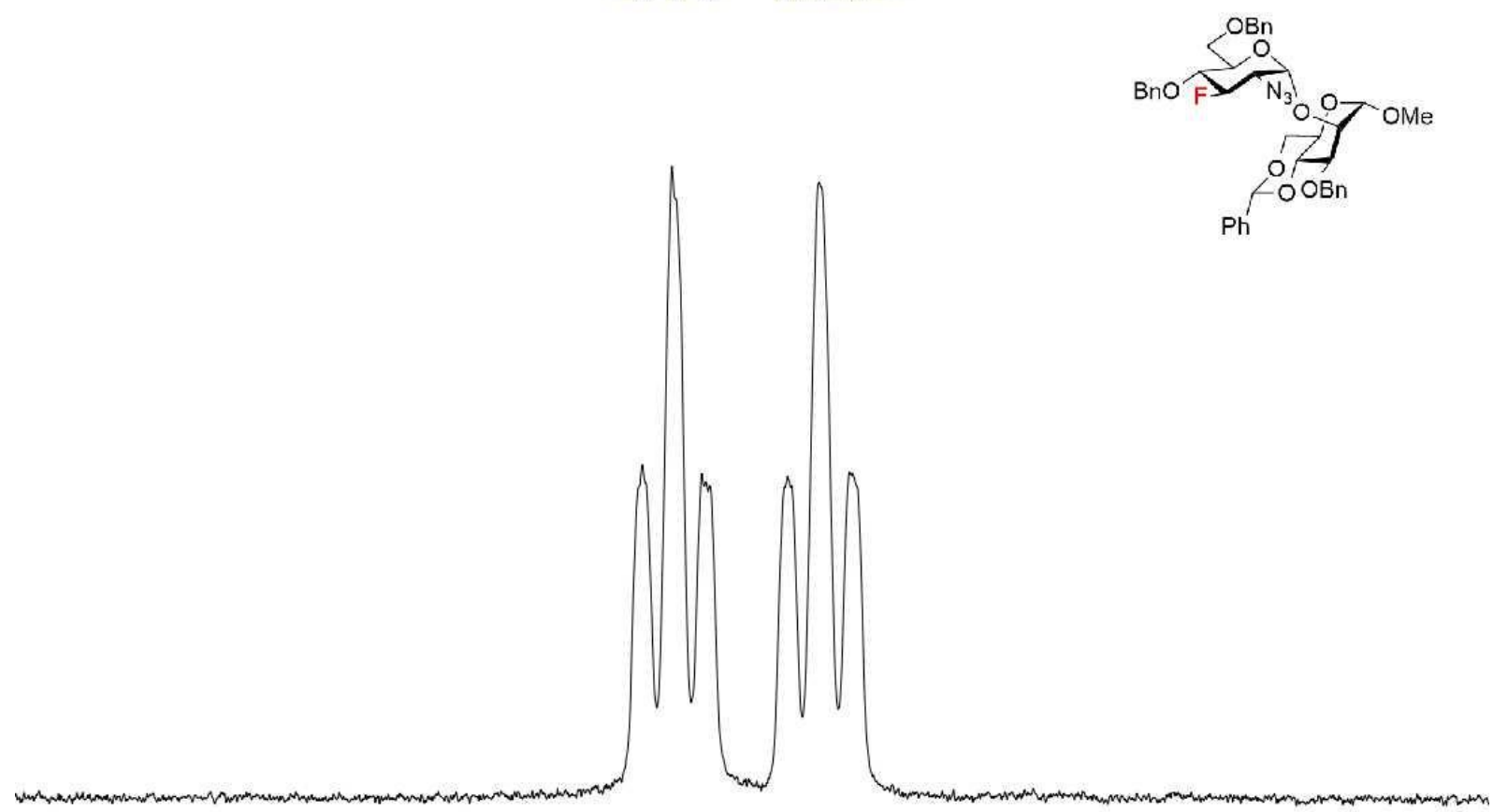

$92.95-193.05-193.15-193.25-193.35-193.45-193.55-193.65-193.75-193.85-193.95-194.05-194.15-194.25-194$ f1 (ppm) 
NMR 1I- $\beta$

${ }^{1} \mathrm{H}$ NMR $400 \mathrm{MHz}, \mathrm{CDCl}_{3} 11-\beta$

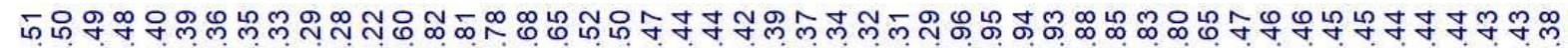

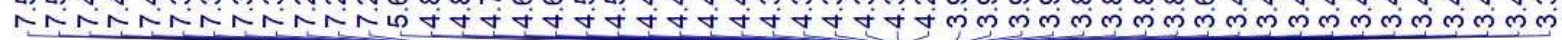

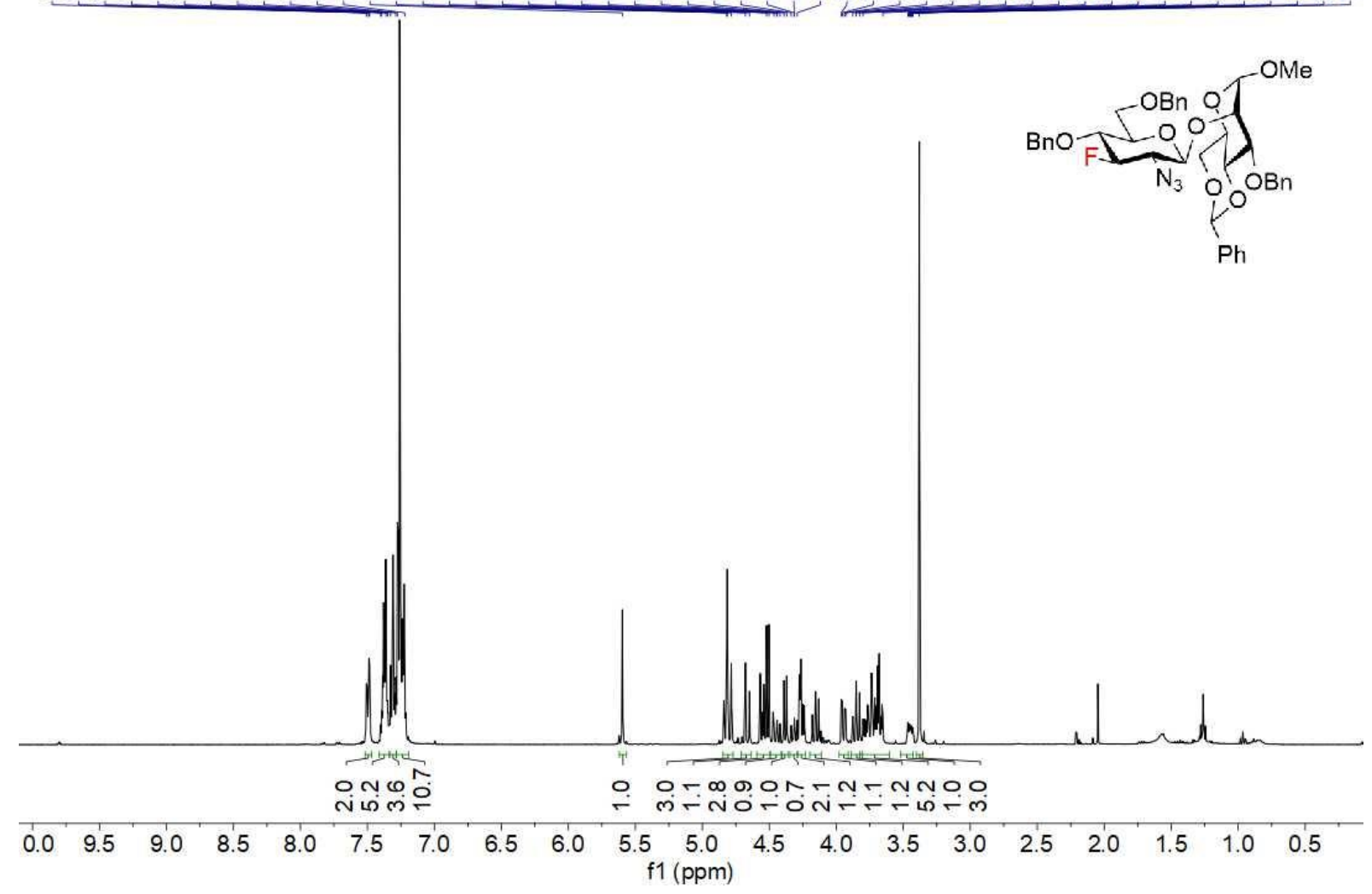

${ }^{13} \mathrm{C}\left\{{ }^{1} \mathrm{H}\right\} \mathrm{NMR} 100 \mathrm{MHz}, \mathrm{CDCl}_{3} \mathbf{1 I - \beta}$

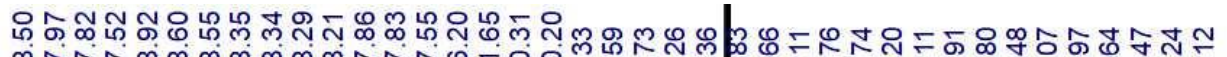

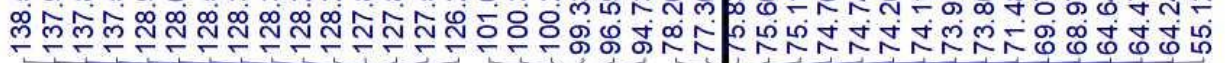

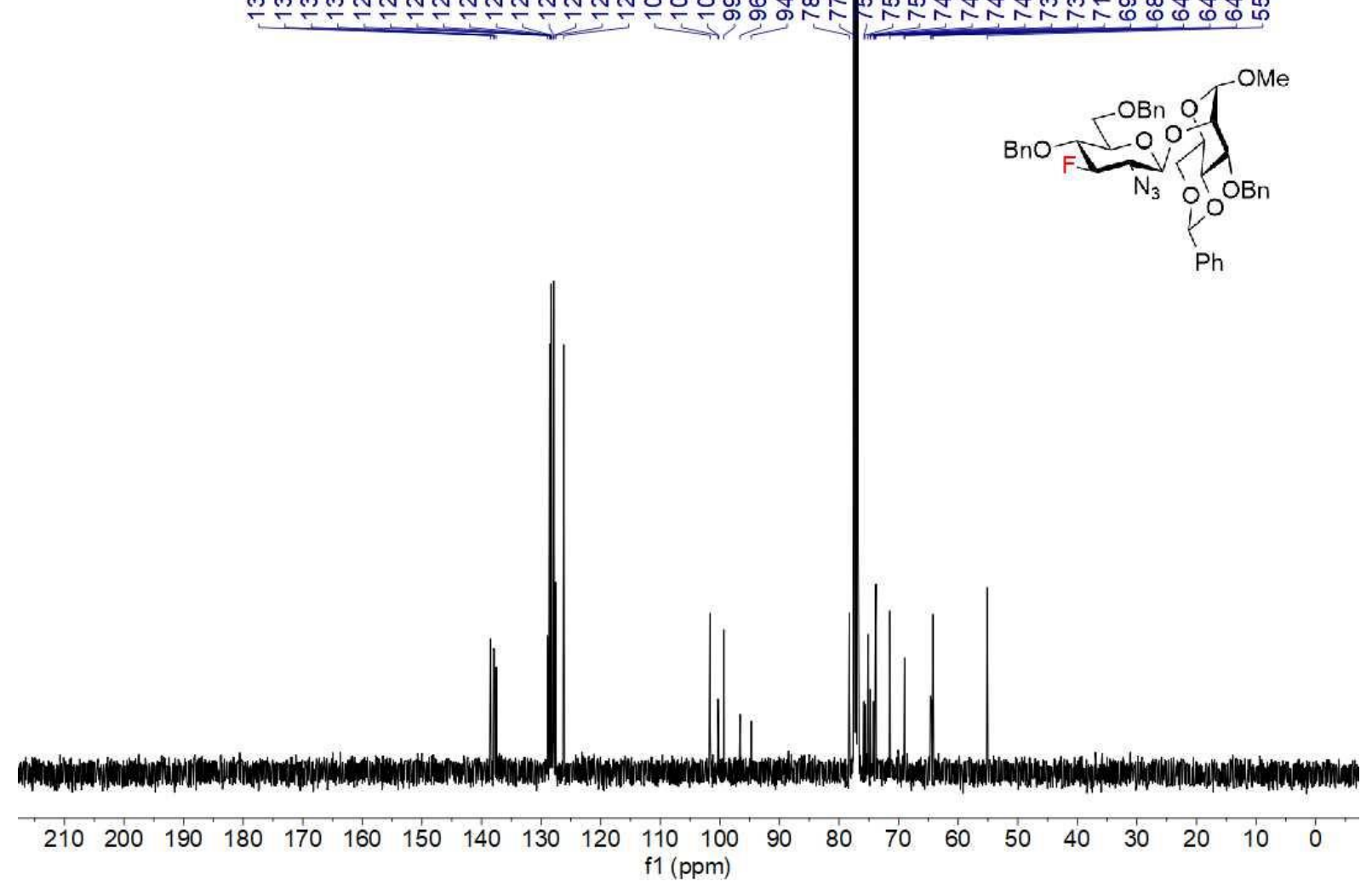


${ }^{1} \mathrm{H}-{ }^{1} \mathrm{H}$ COSY NMR $1 \mathrm{I}-\boldsymbol{\beta}$

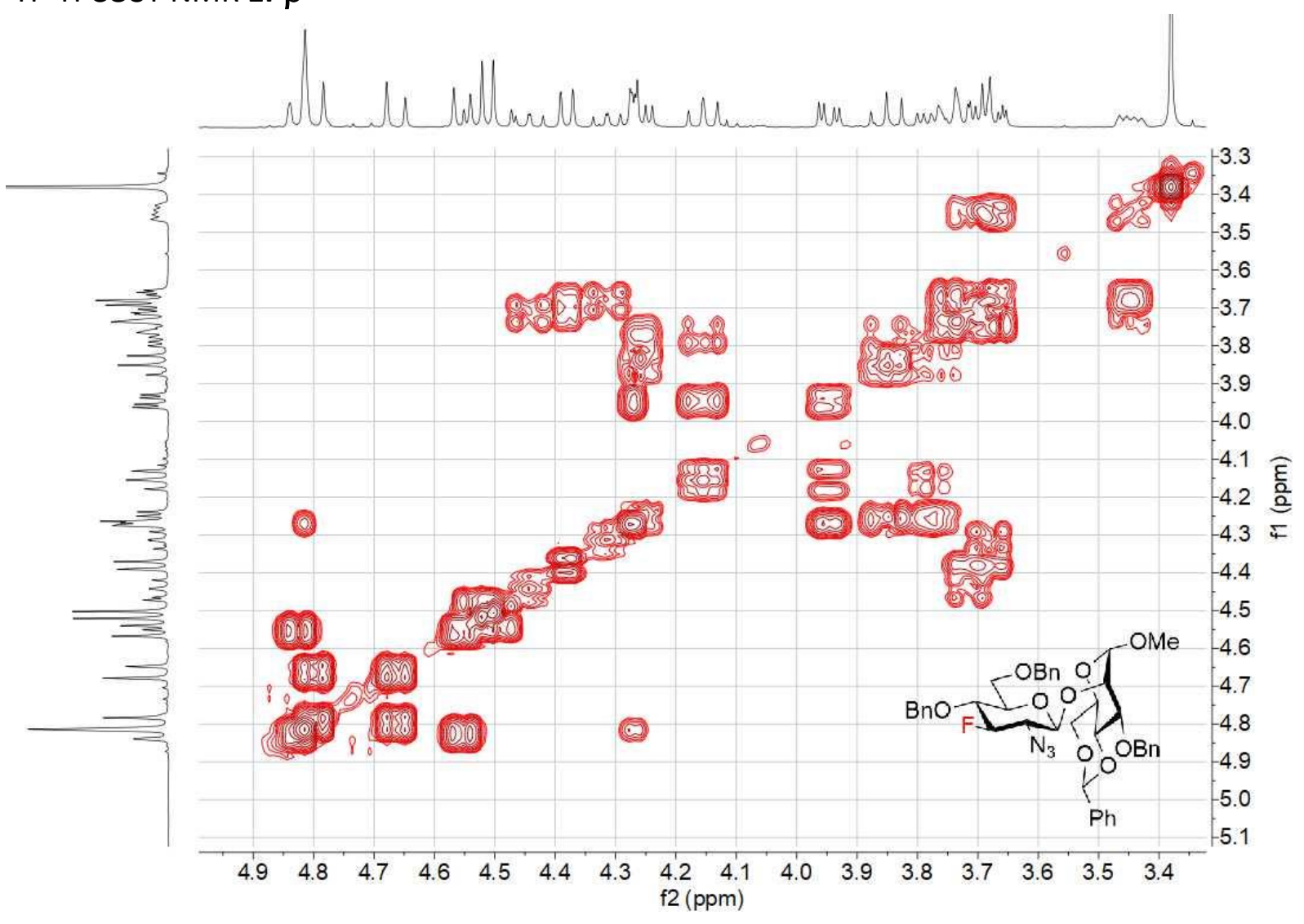

${ }^{1} \mathrm{H}-{ }^{13} \mathrm{C}$ HSQC NMR $1 \mathrm{I}-\boldsymbol{\beta}$

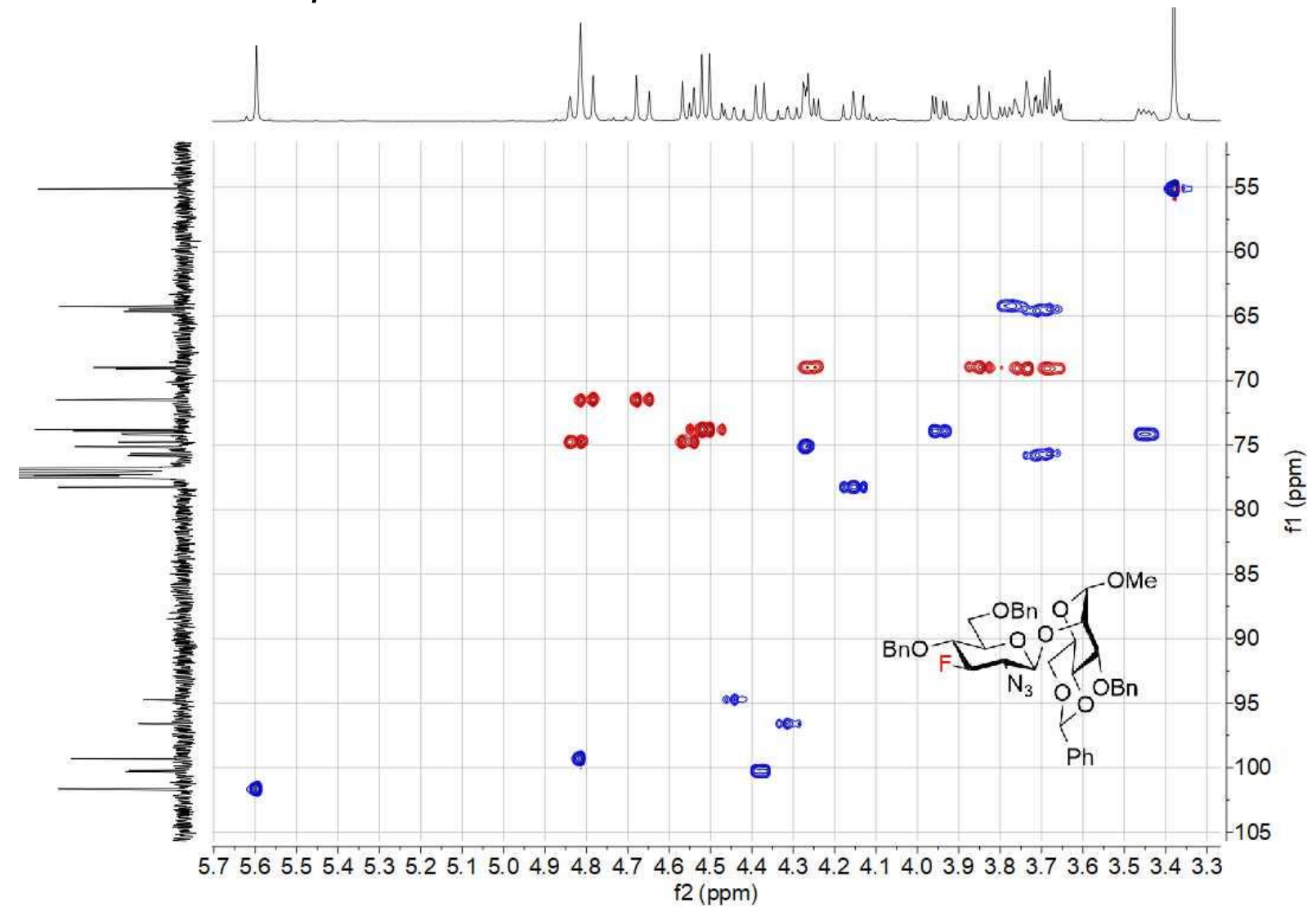


${ }^{1} \mathrm{H}-{ }^{13} \mathrm{CHMBC}$ NMR $\mathbf{1 I}-\boldsymbol{\beta}$

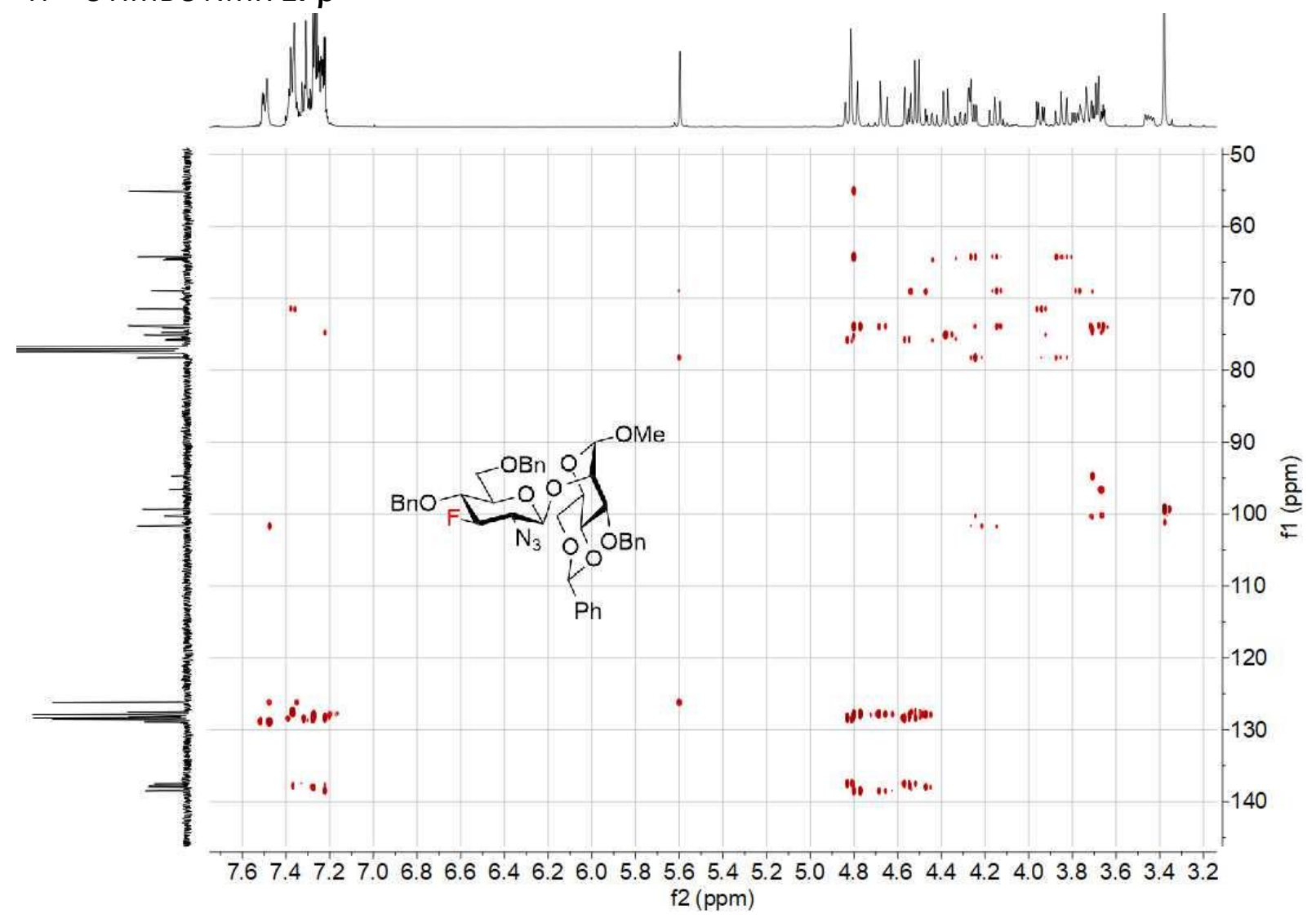

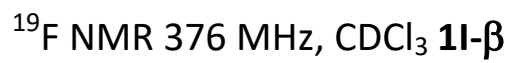

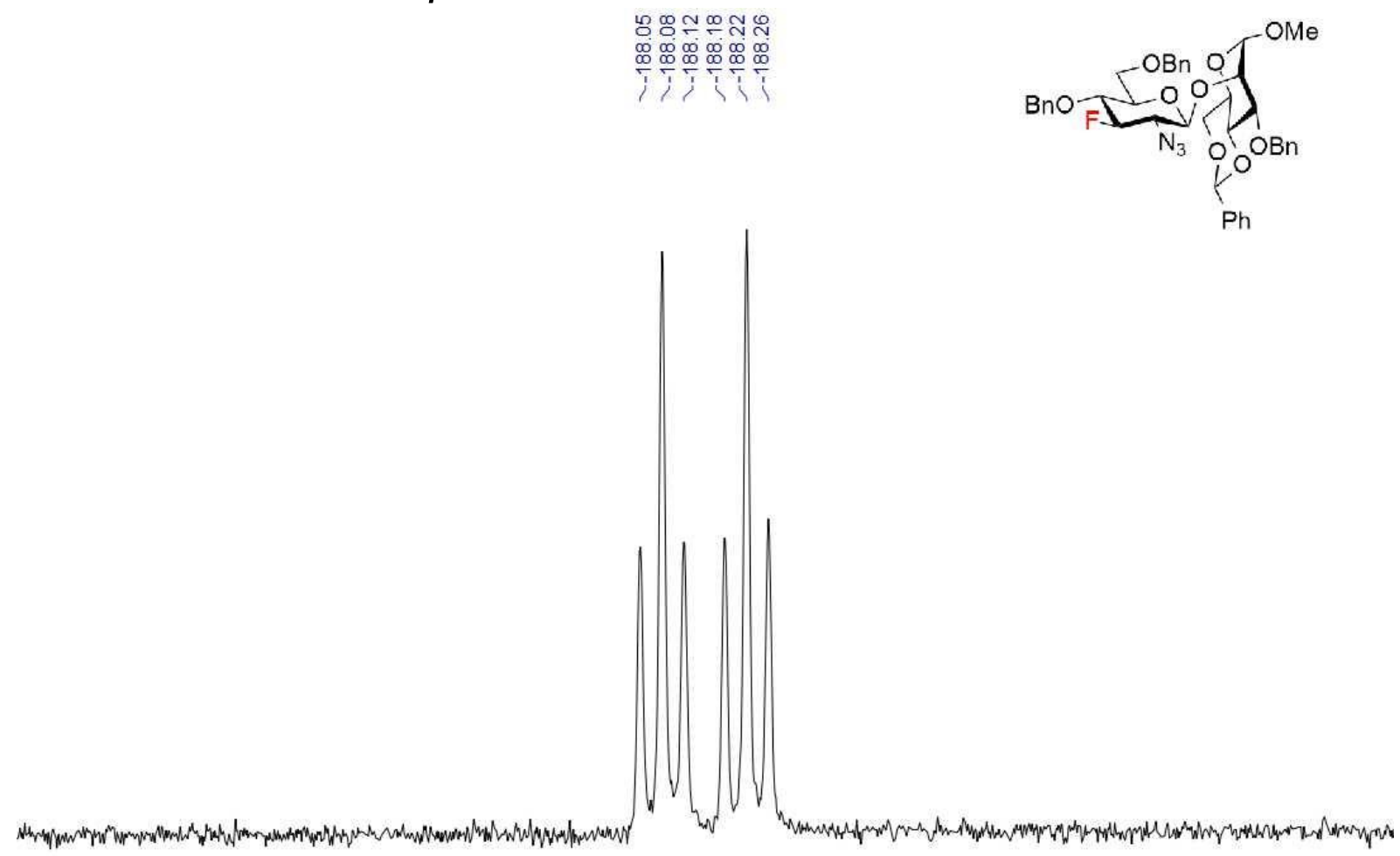

\begin{tabular}{|lllllllllllll}
\hline-187.2 & -187.4 & -187.6 & -187.8 & -188.0 & -188.2 & -188.4 & -188.6 & -188.8 & -189.0 & -189.2 \\
\hline
\end{tabular}


NMR 2A- $\alpha$

${ }^{1} \mathrm{H}$ NMR $400 \mathrm{MHz}, \mathrm{CDCl}_{3} 2 \mathrm{~A}-\alpha+2 \mathrm{~A}-\boldsymbol{\beta}$ (ca $1: 11$ )

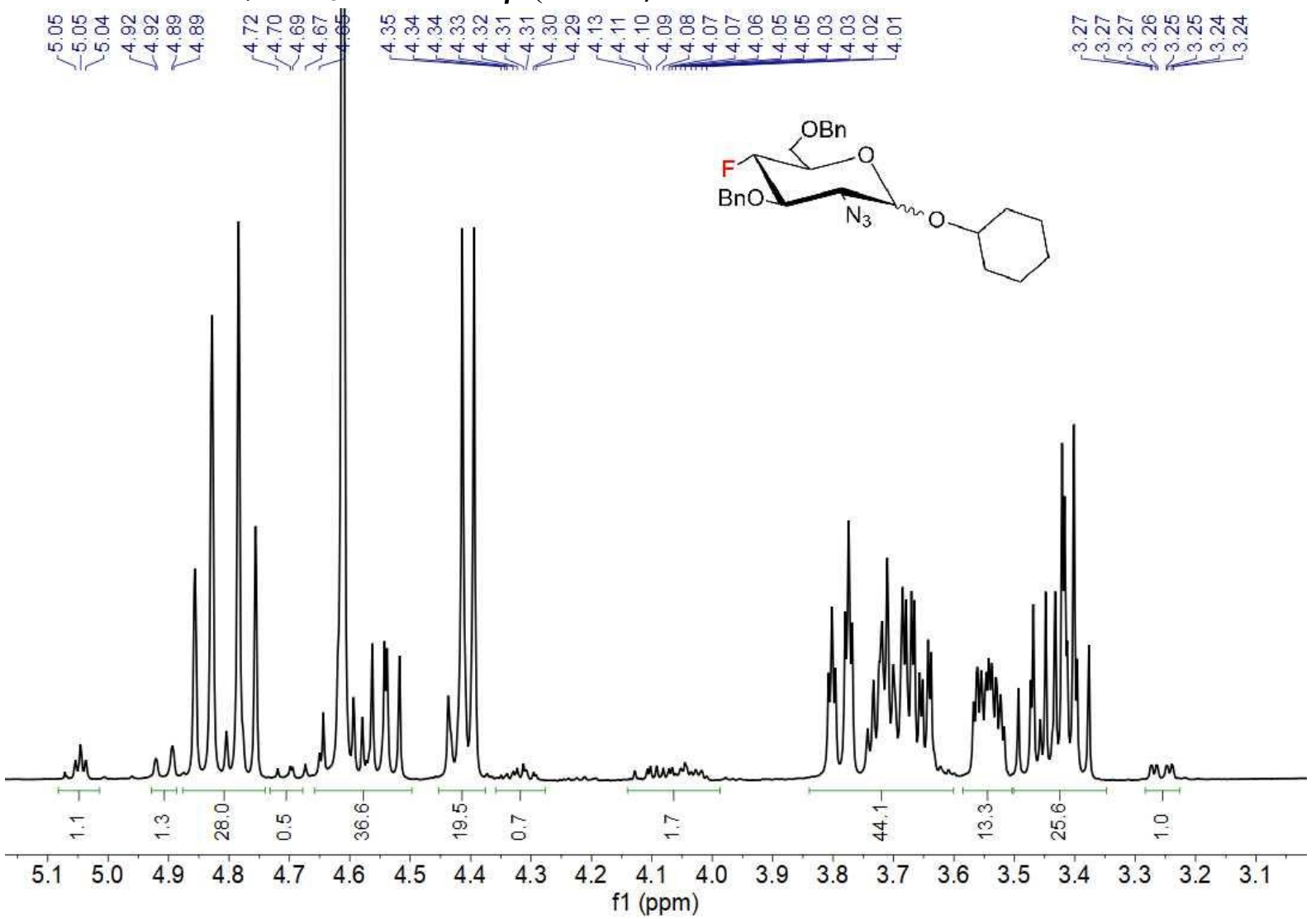

${ }^{1} \mathrm{H}-{ }^{1} \mathrm{H}$ COSY NMR $2 \mathrm{~A}-\boldsymbol{\alpha}+\mathbf{2 A}-\boldsymbol{\beta}($ ca $1: 11)$

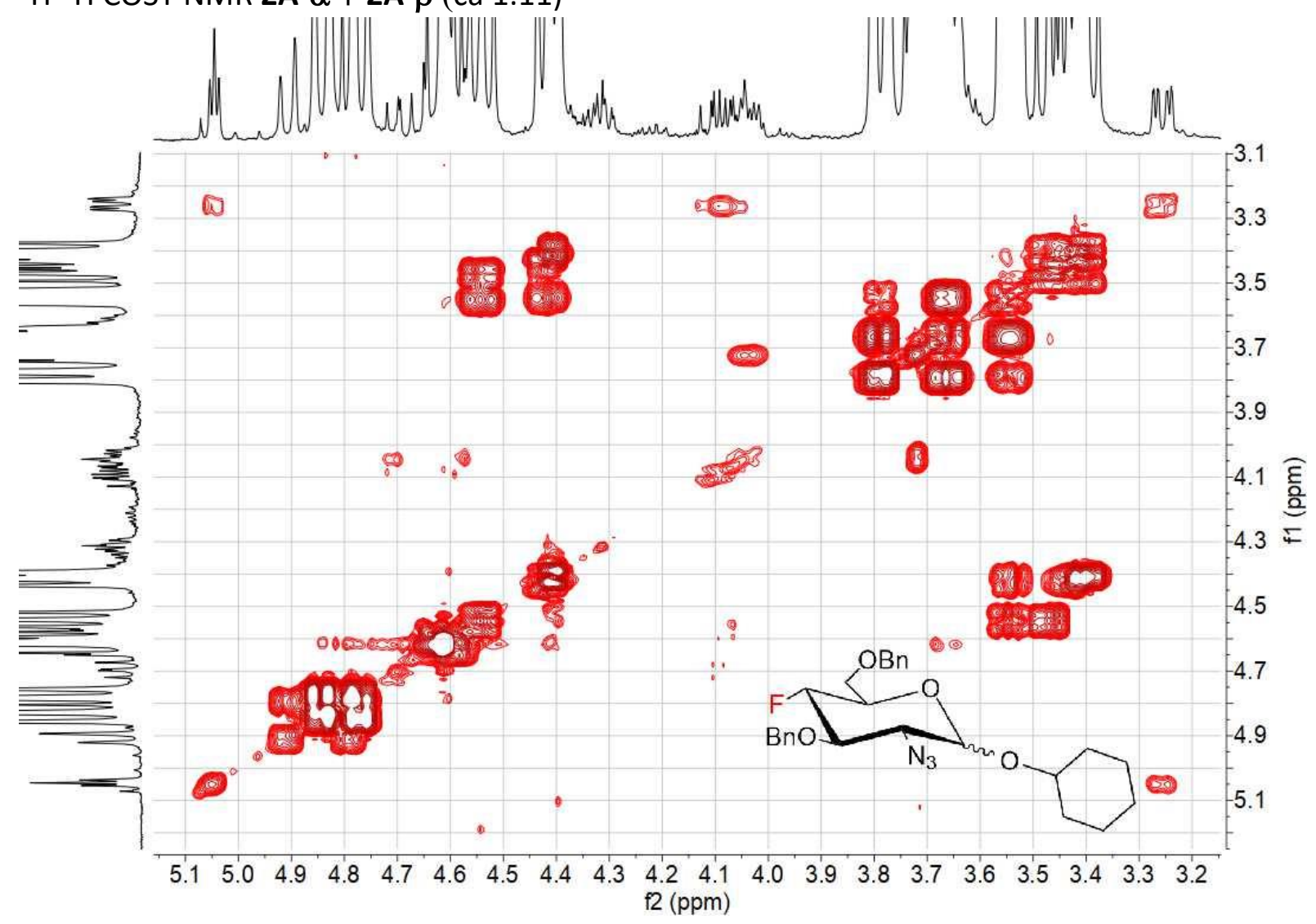


${ }^{1} \mathrm{H}-{ }^{13} \mathrm{C}$ HSQC NMR $2 \mathrm{~A}-\boldsymbol{\alpha}+\mathbf{2 A}-\boldsymbol{\beta}($ ca $1: 11)$

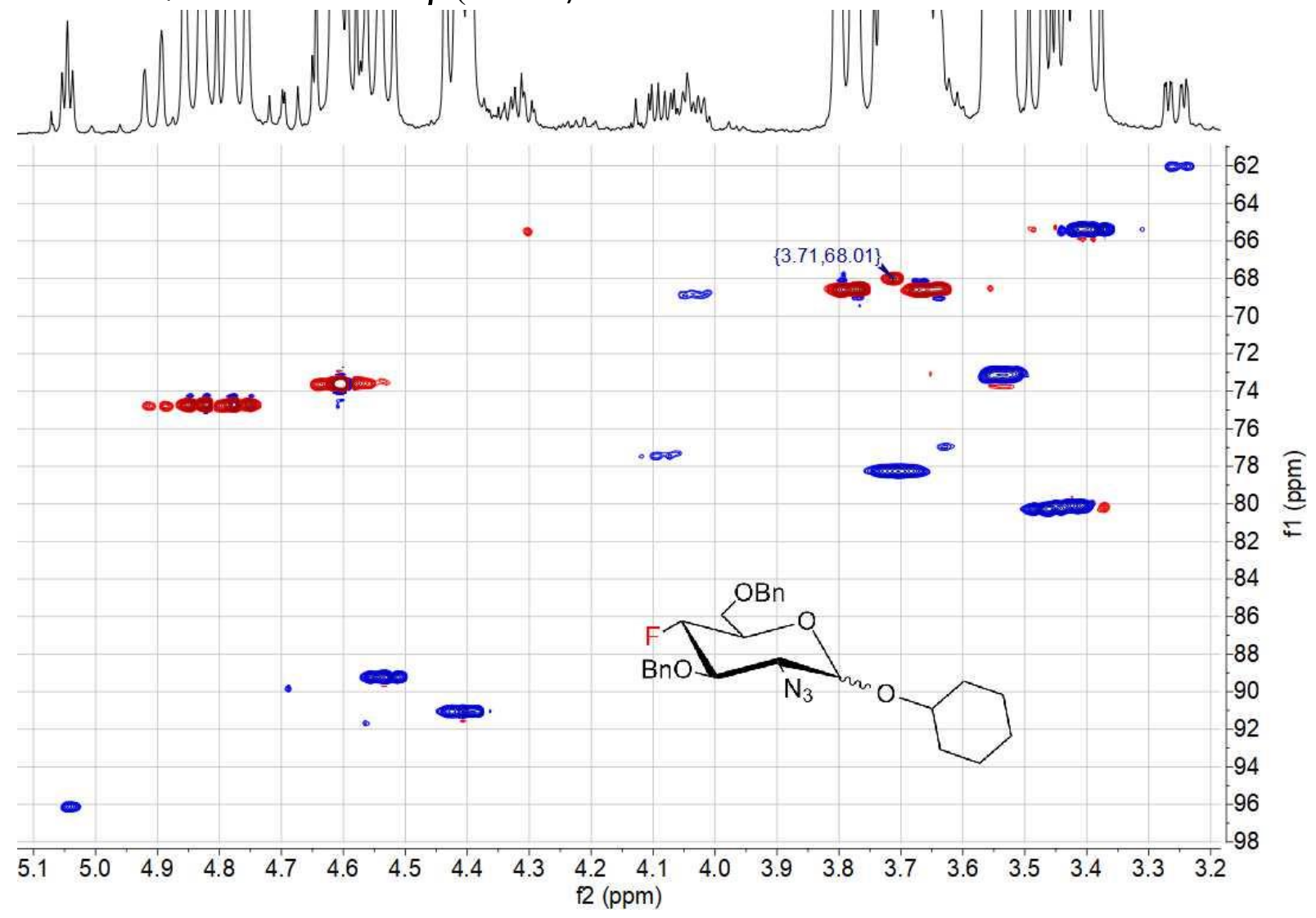

${ }^{19}$ F NMR $376 \mathrm{MHz}, \mathrm{CDCl}_{3}$ NMR $2 \mathrm{~A}-\boldsymbol{\alpha}+\mathbf{2 A}-\boldsymbol{\beta}$ (ca $\left.1: 11\right)$

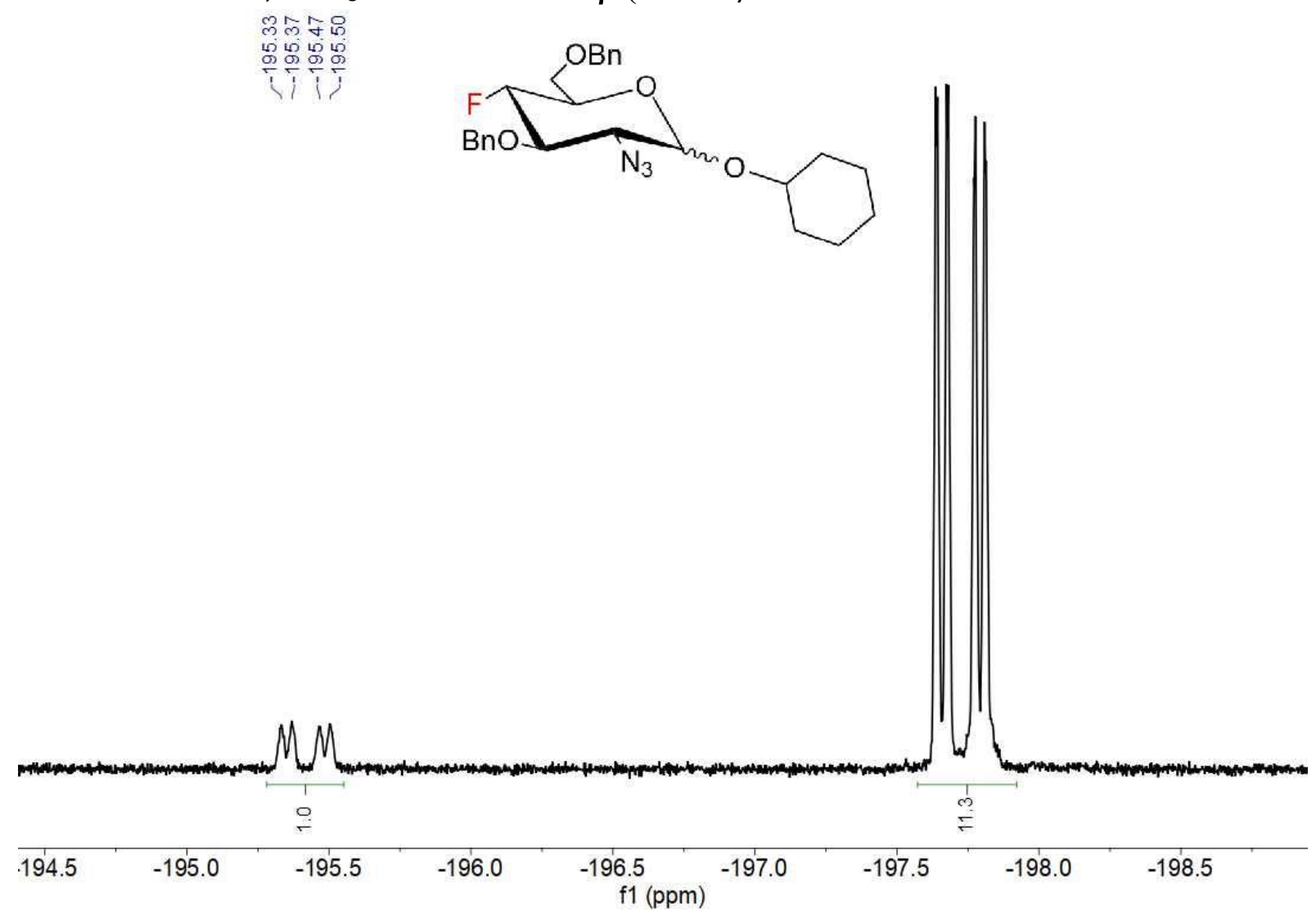


NMR 2A- $\beta$

${ }^{1} \mathrm{HNMR} 400 \mathrm{MHz}, \mathrm{CDCl}_{3} \mathbf{2 A}-\beta$

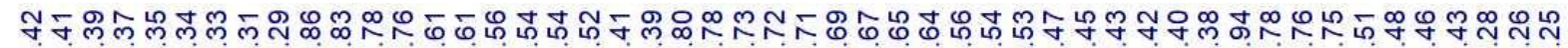

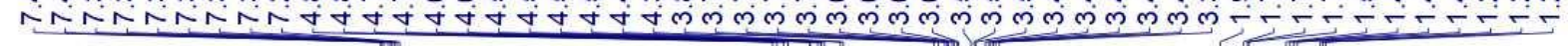

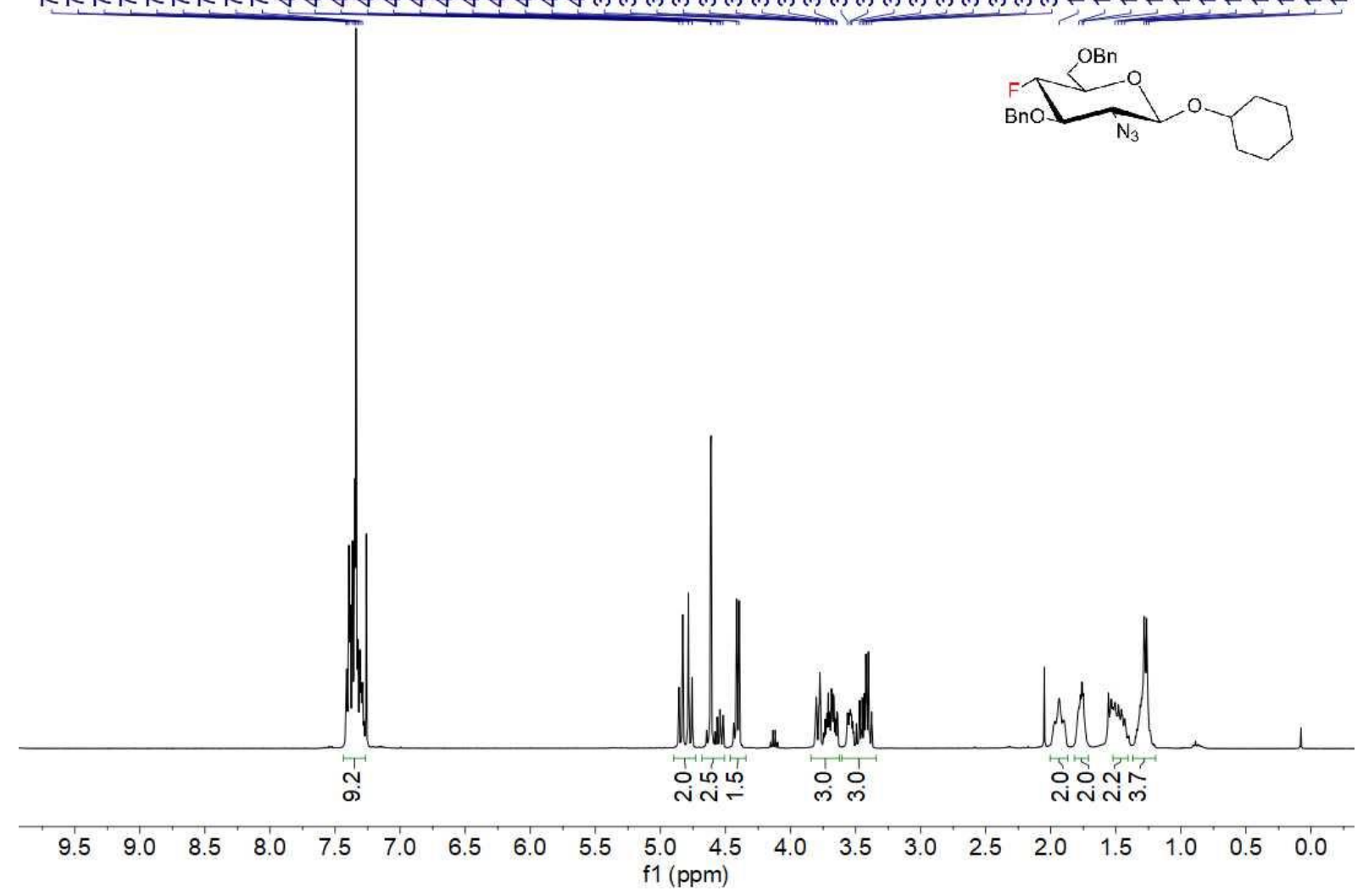

${ }^{13} \mathrm{C}\left\{{ }^{1} \mathrm{H}\right\} \mathrm{NMR} 100 \mathrm{MHz}, \mathrm{CDCl} 3 \mathbf{2 A}-\boldsymbol{\beta}$

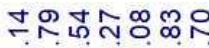

ํํ유.

๓ำ

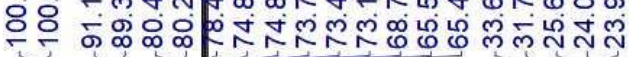

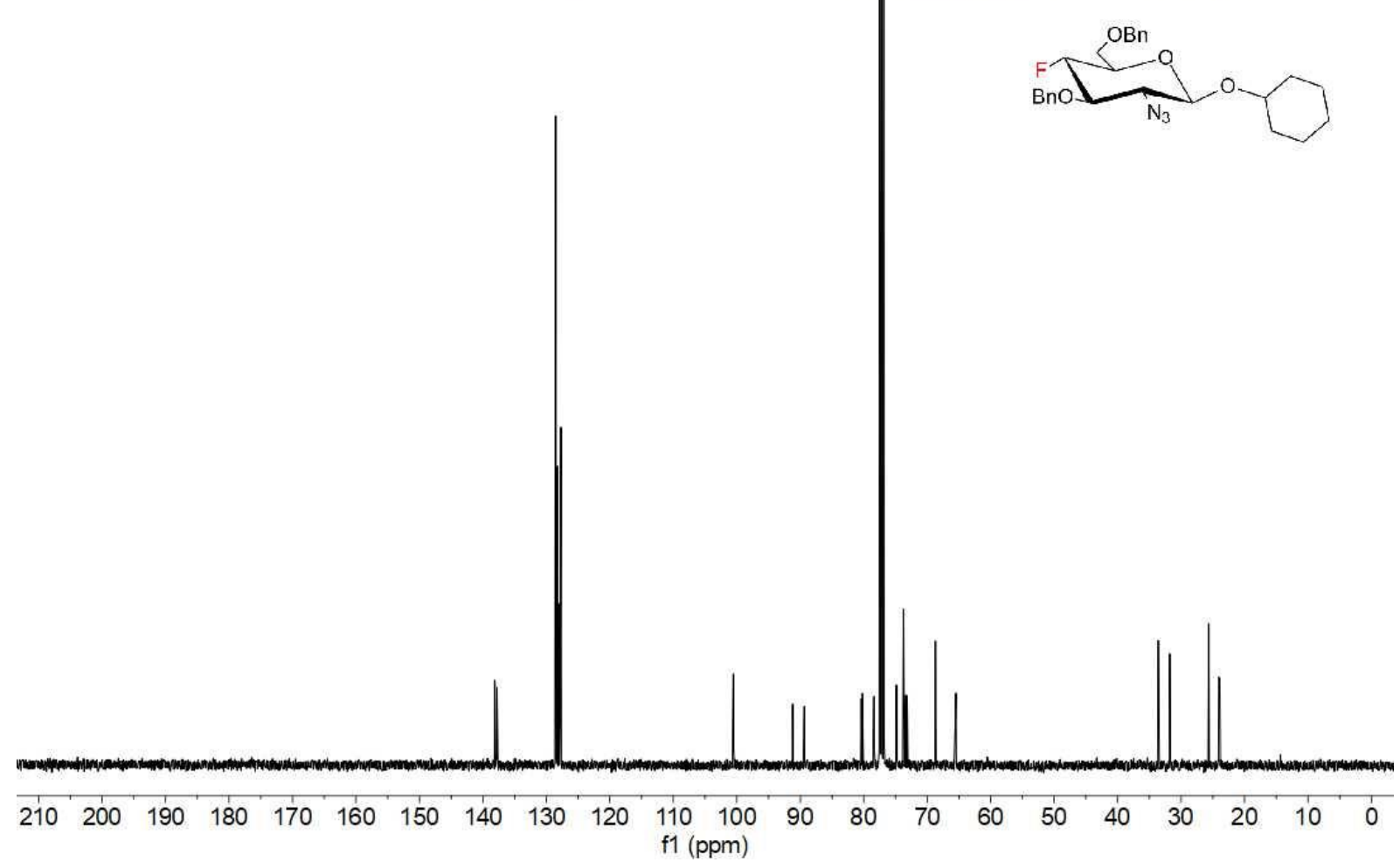


${ }^{1} \mathrm{H}-{ }^{1} \mathrm{H}$ COSY NMR $2 \mathrm{~A}-\boldsymbol{\beta}$

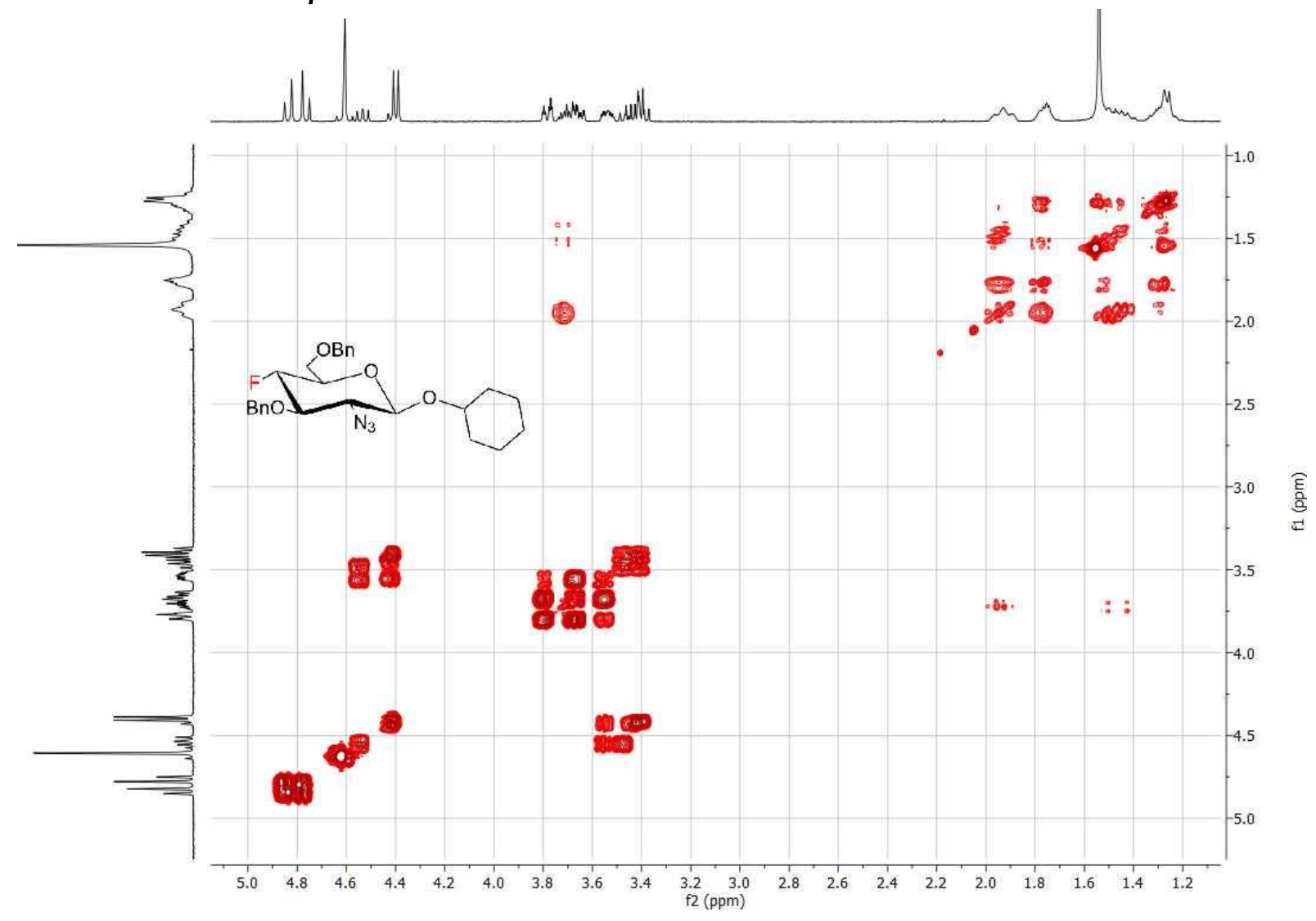

${ }^{1} \mathrm{H}-{ }^{13} \mathrm{C}$ HSQC NMR 2A- $\beta$




${ }^{1} \mathrm{H}-{ }^{13} \mathrm{C}$ HMBC NMR 2A- $\boldsymbol{\beta}$

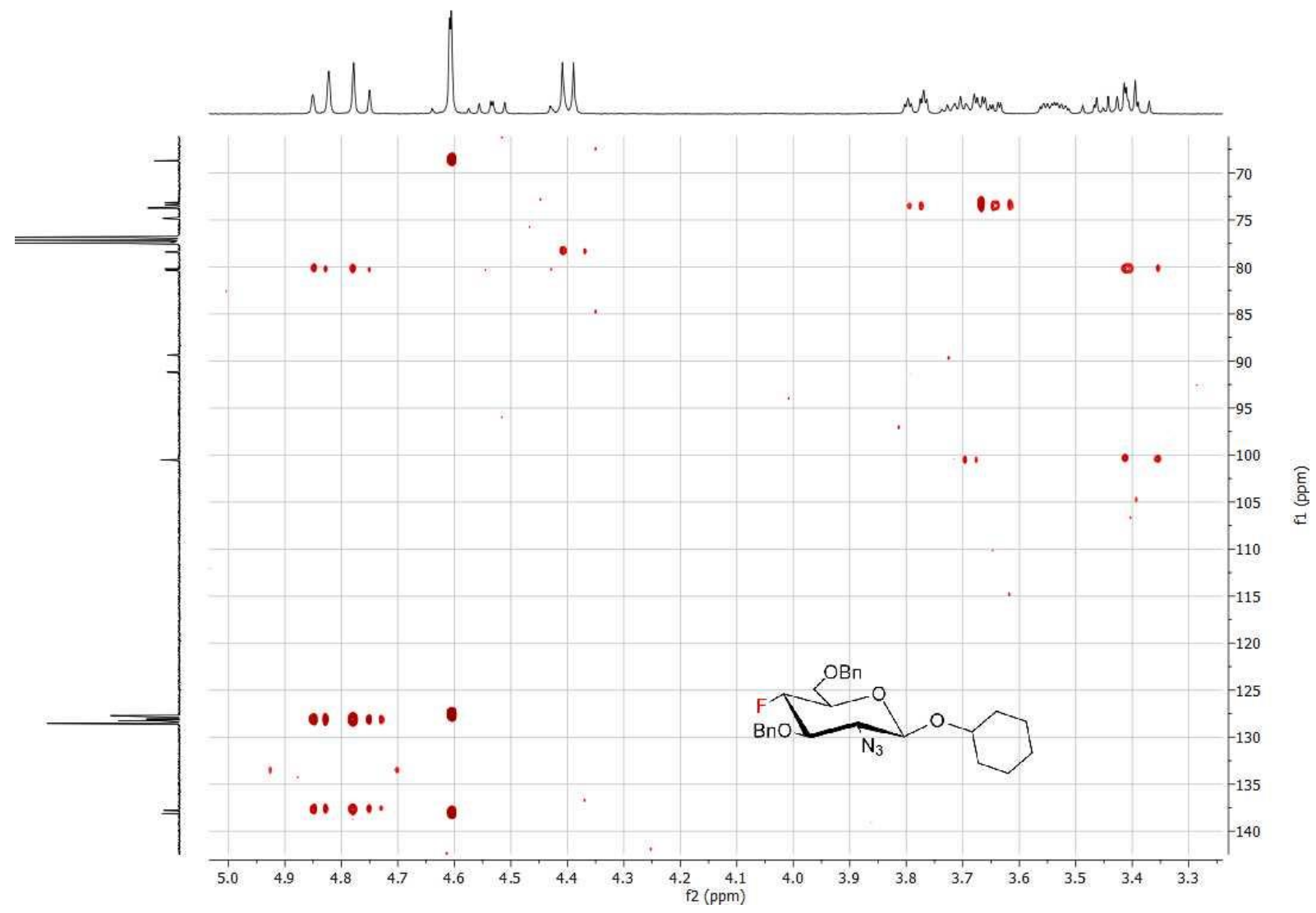

${ }^{19} \mathrm{~F}$ NMR $376 \mathrm{MHz}, \mathrm{CDCl}_{3}$ NMR $2 \mathrm{~A}-\boldsymbol{\beta}$

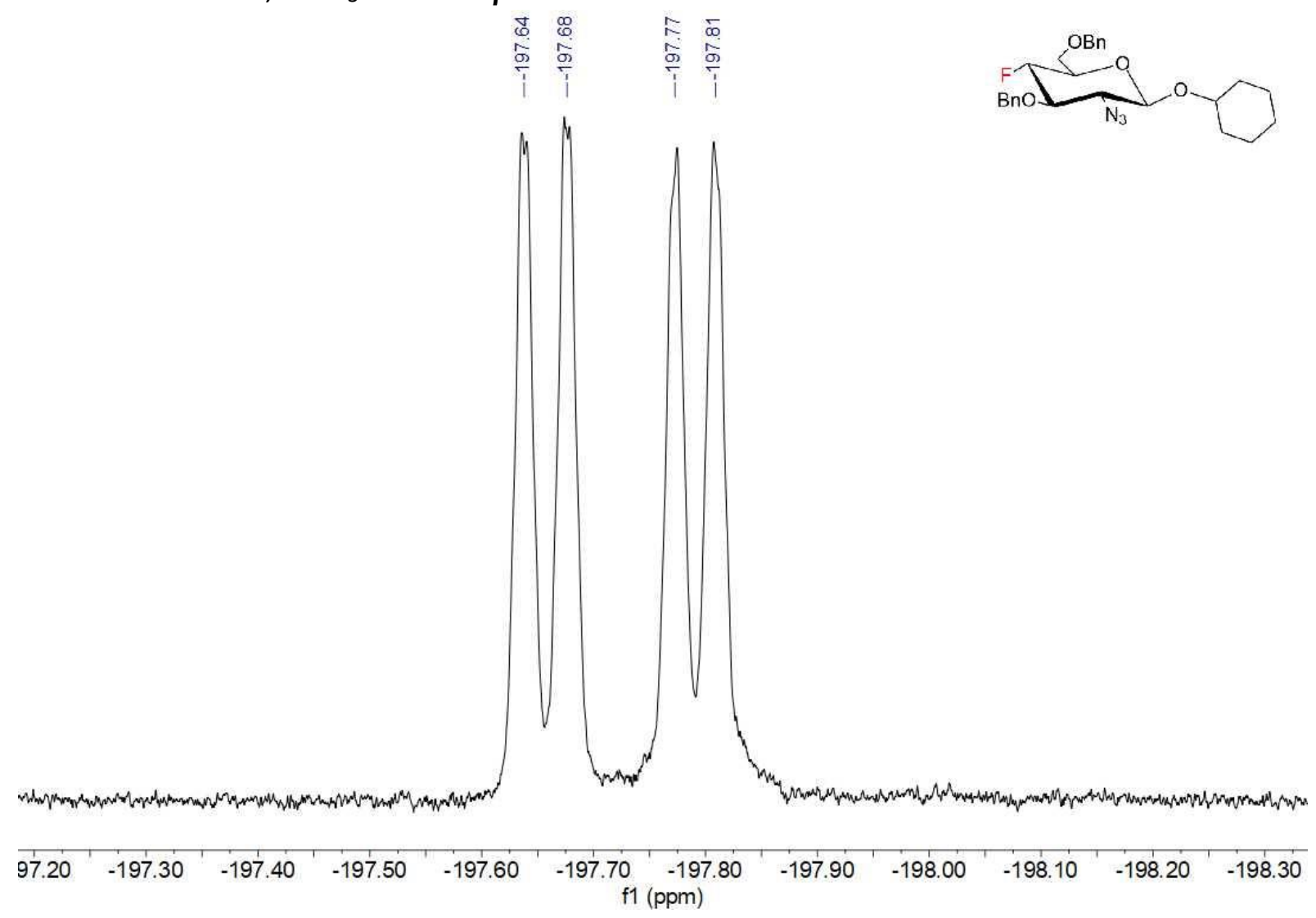




\section{NMR 2B}

${ }^{1} \mathrm{H}$ NMR $400 \mathrm{MHz}, \mathrm{CDCl}_{3} \mathbf{2 B}-\beta$ and cca $9 \% \mathbf{2 B - \alpha}$

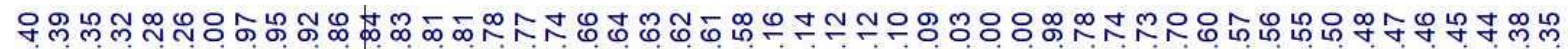

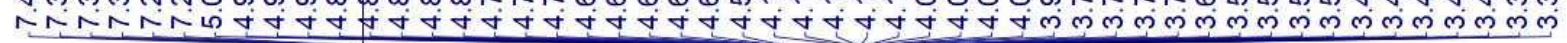

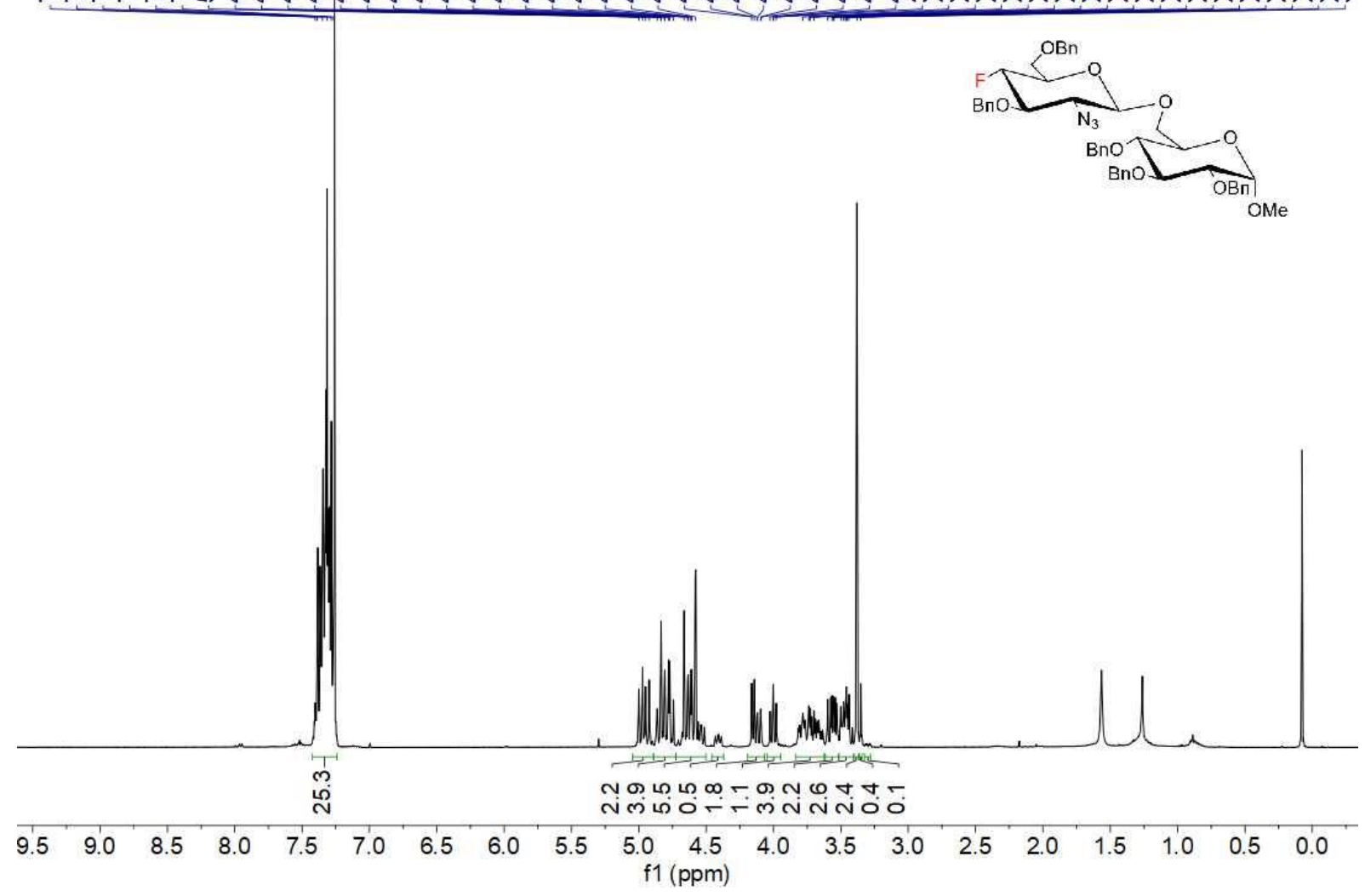

\section{${ }^{13} \mathrm{C}\left\{{ }^{1} \mathrm{H}\right\}$ NMR $100 \mathrm{MHz}, \mathrm{CDCl}_{3}$ 2B- $\boldsymbol{\beta}$ and cca $9 \% \mathbf{2 B -} \boldsymbol{\alpha}$}

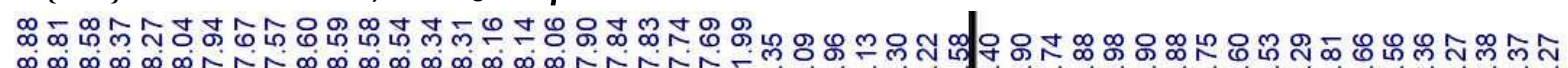

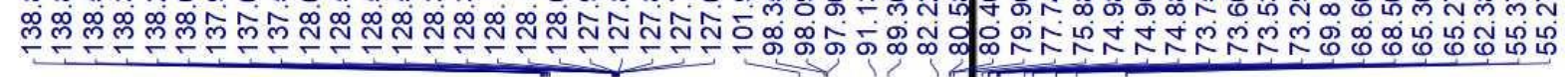

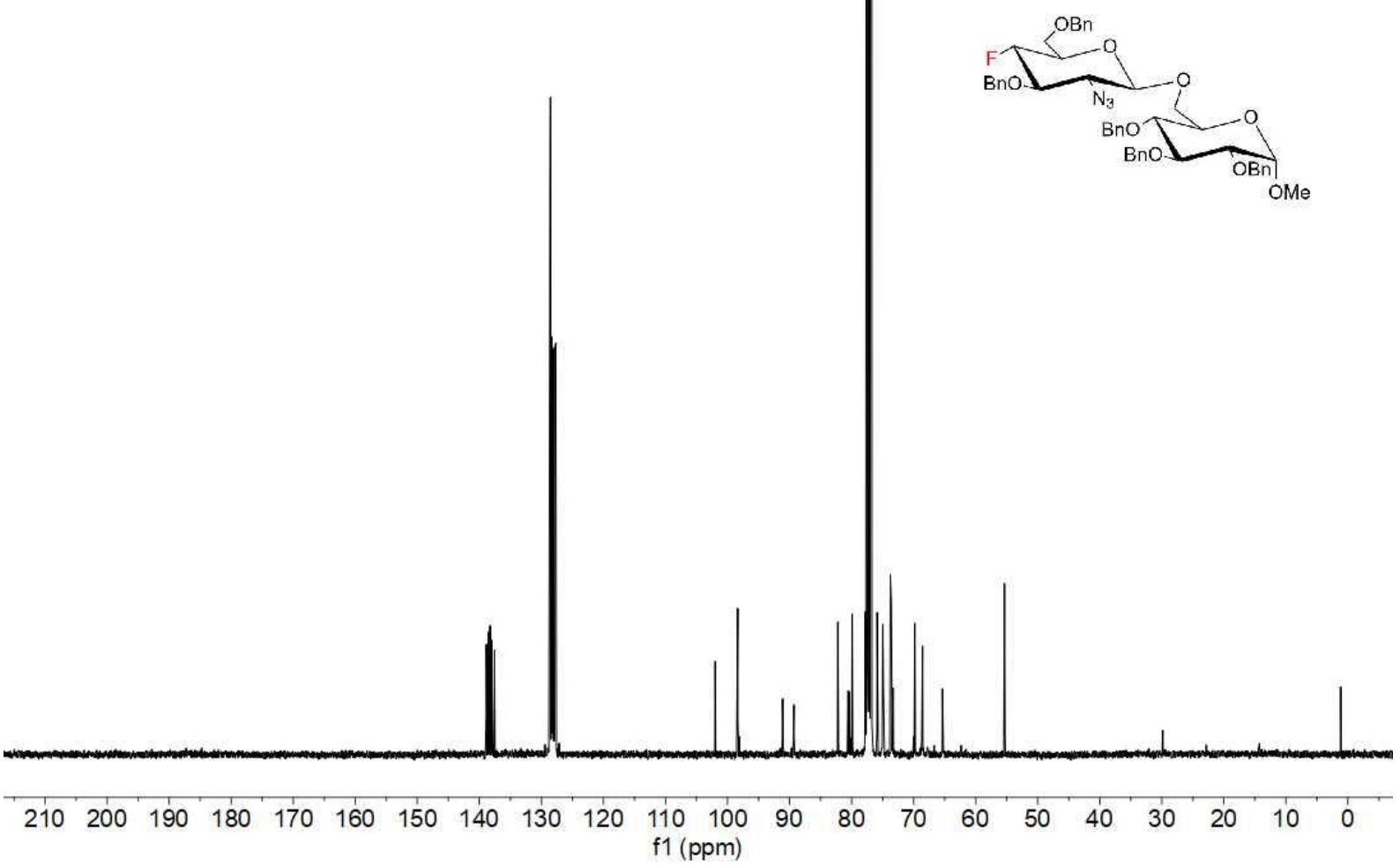


${ }^{1} \mathrm{H}-{ }^{1} \mathrm{H}$ COSY NMR 2B- $\beta$ and cca $9 \%$ 2B- $\alpha$

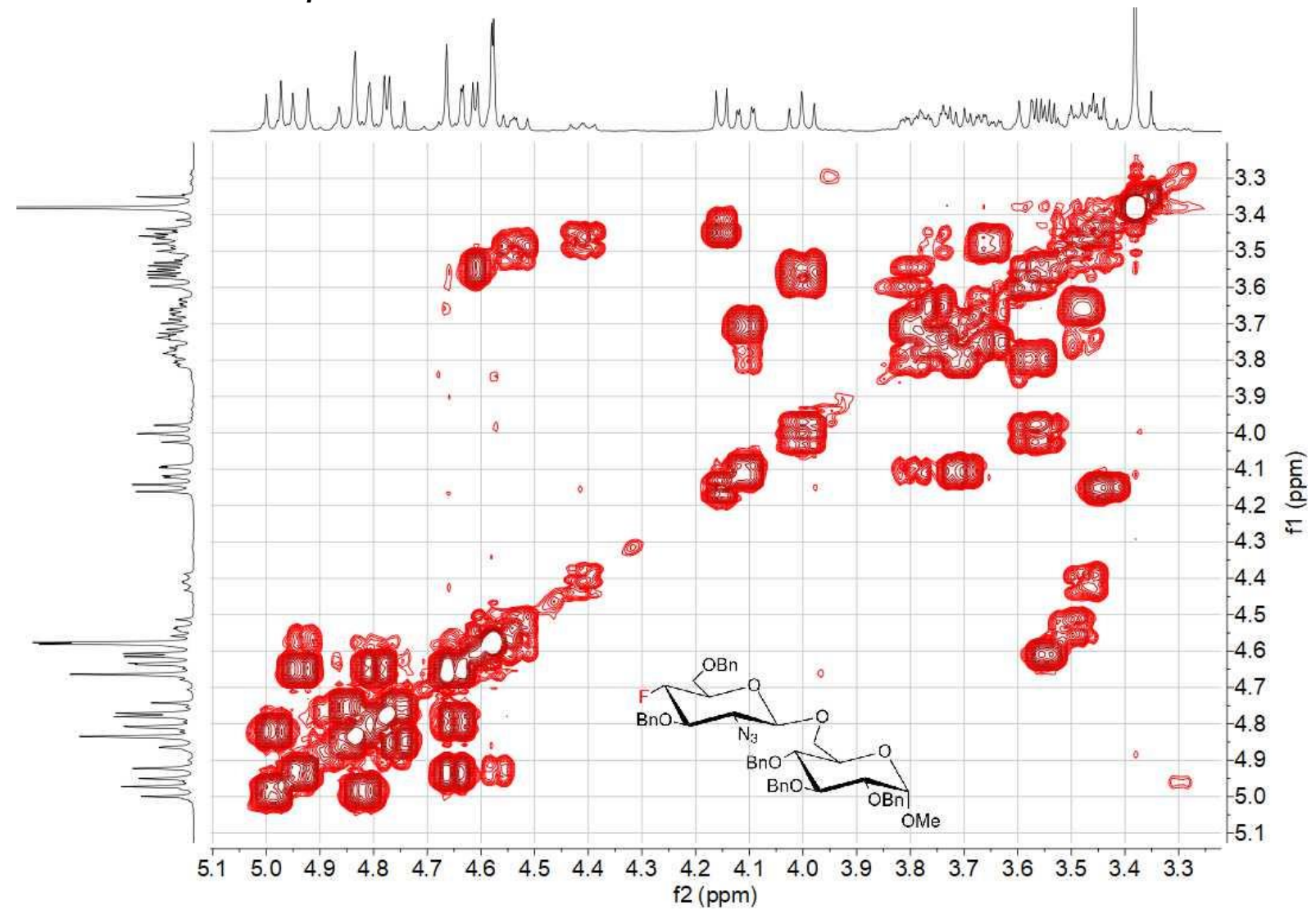

${ }^{1} \mathrm{H}-{ }^{13} \mathrm{C}$ HSOC NMR 2B- $\beta$ and cca $9 \%$ 2B- $\alpha$

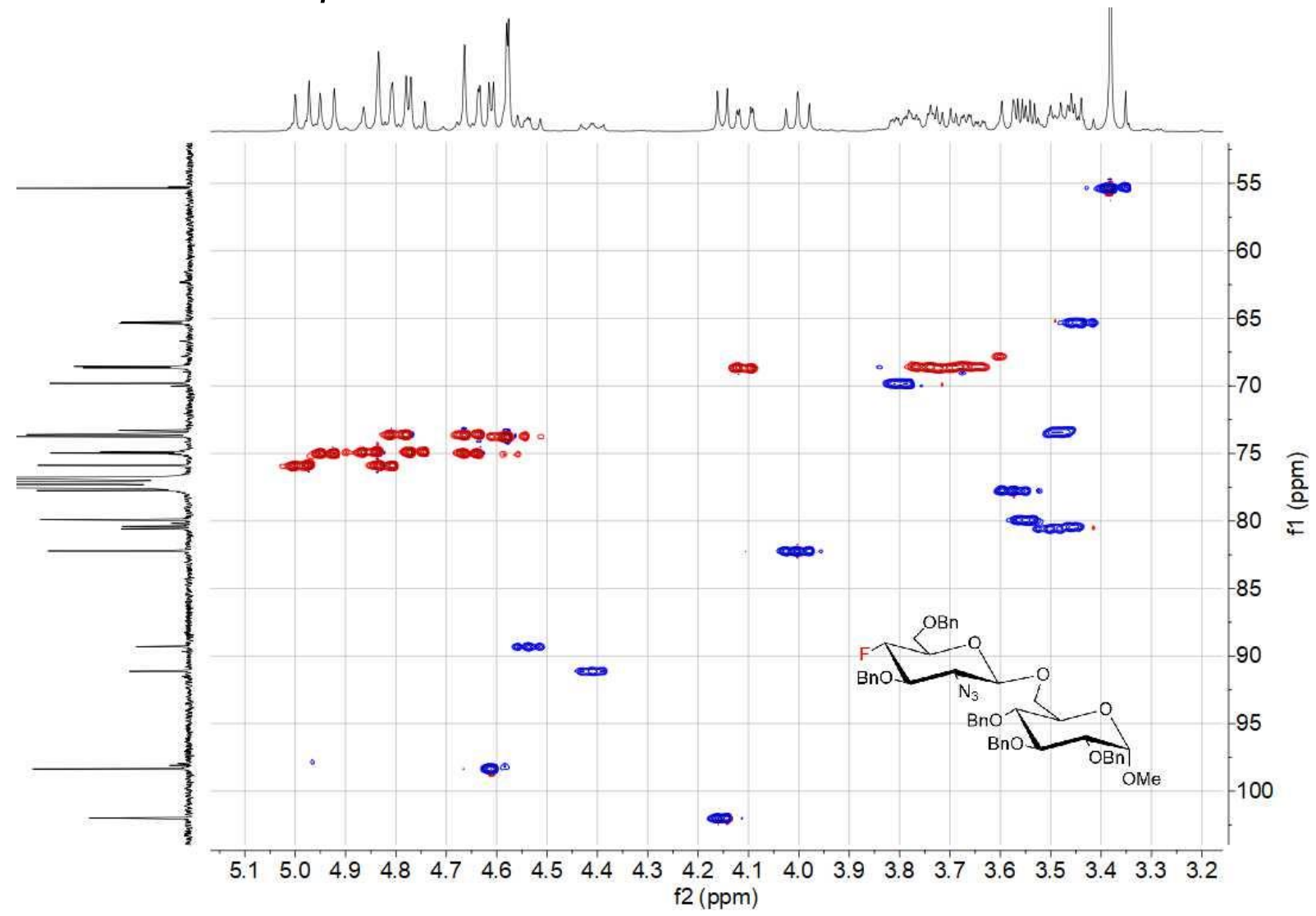


${ }^{1} \mathrm{H}-{ }^{13} \mathrm{C}$ HMBC NMR 2B- $\beta$ and cca $9 \%$ 2B- $\alpha$

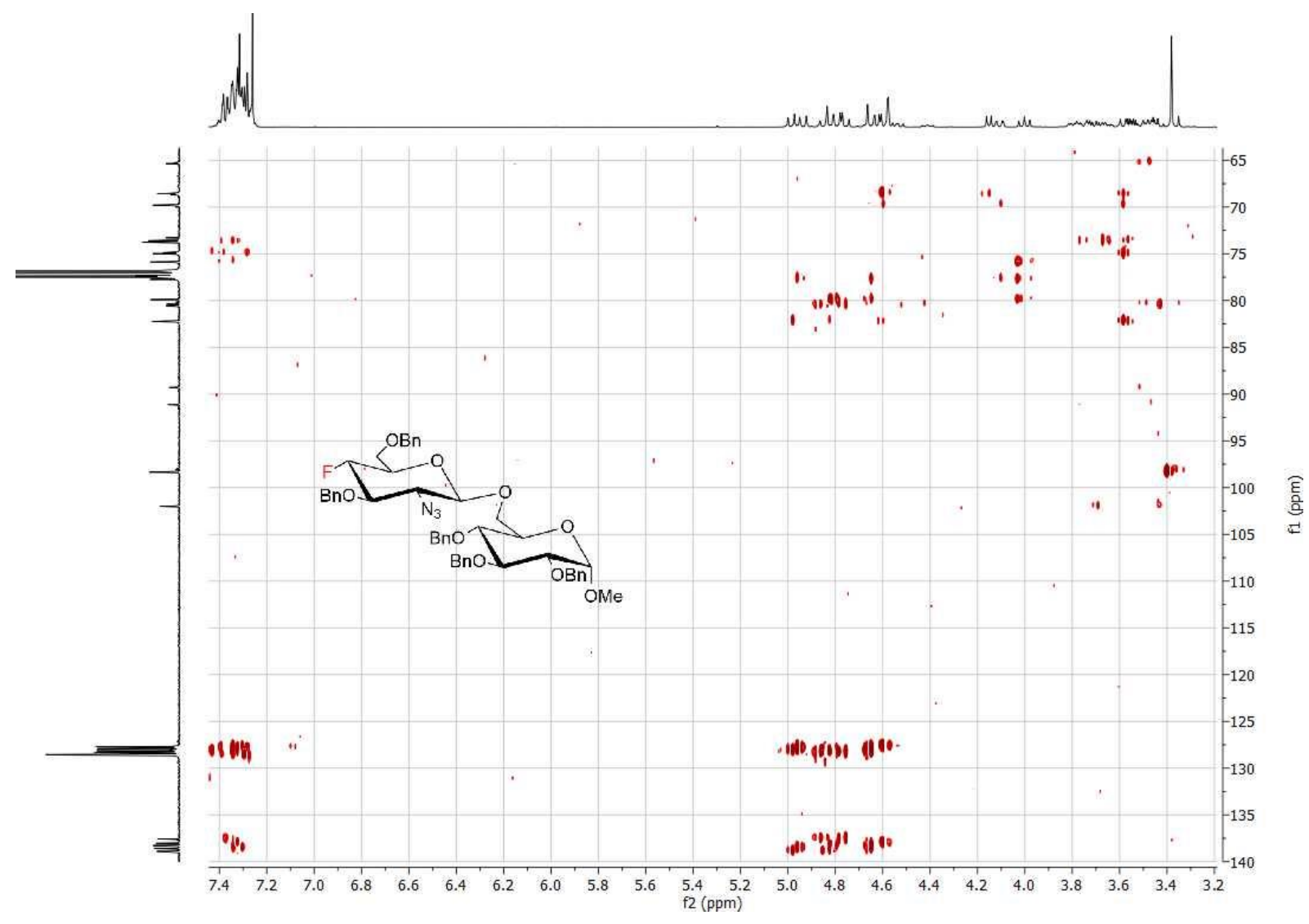

${ }^{19} \mathrm{~F} \mathrm{NMR}_{376} \mathrm{MHz}, \mathrm{CDCl}_{3}$ 2B- $\boldsymbol{\beta}$ and cca $9 \%$ 2B- $\boldsymbol{\alpha}$

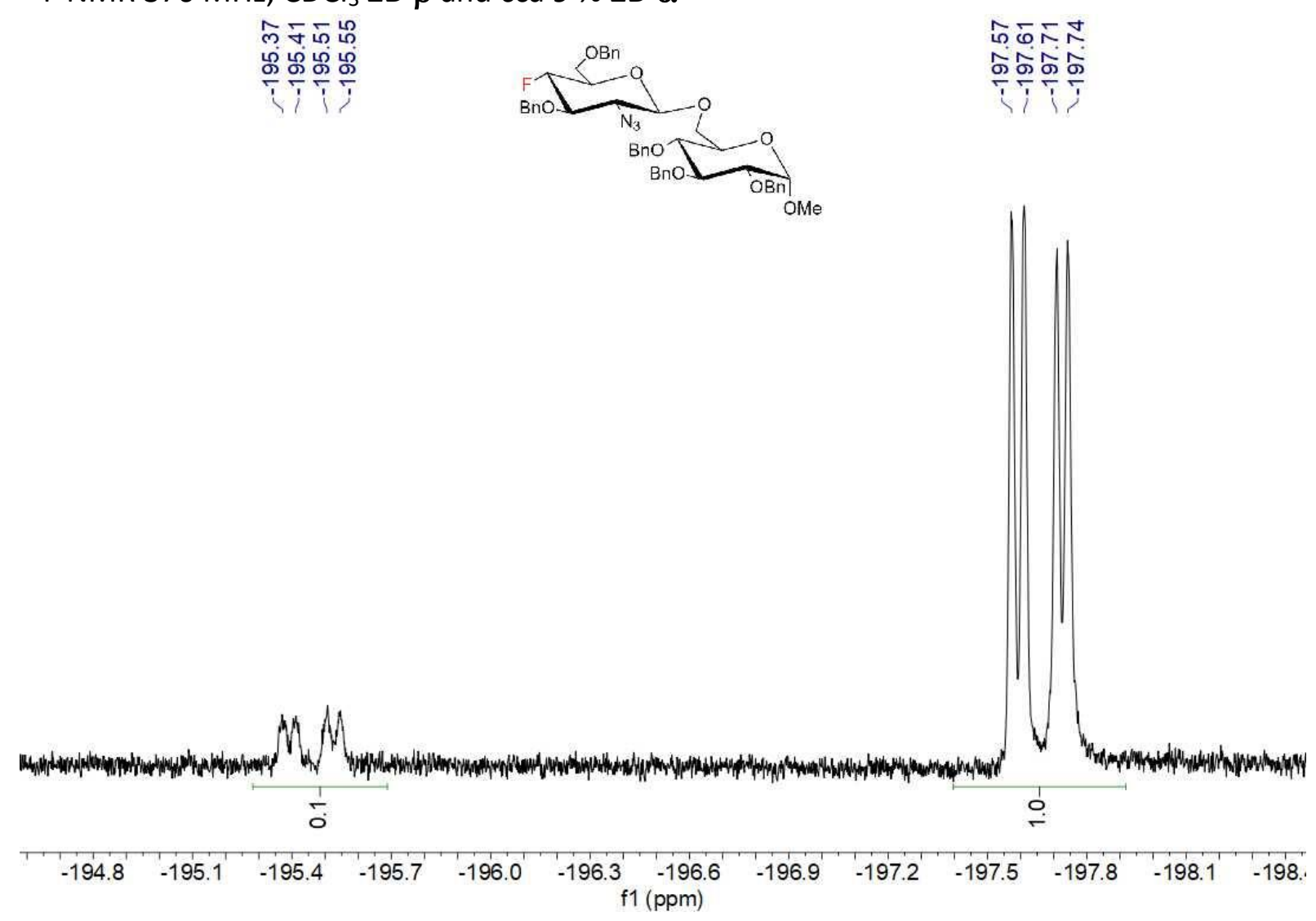




\section{NMR 2C- $\alpha$}

\section{${ }^{1} \mathrm{HNMR} 400 \mathrm{MHz}, \mathrm{CDCl}_{3} 2 \mathrm{C}-\alpha$}

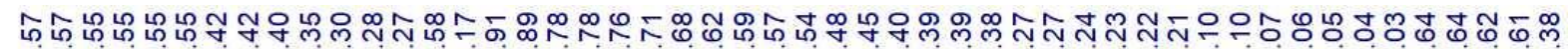

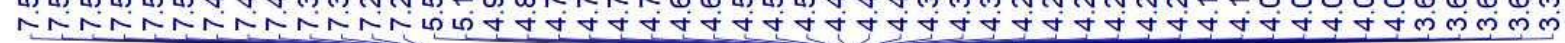

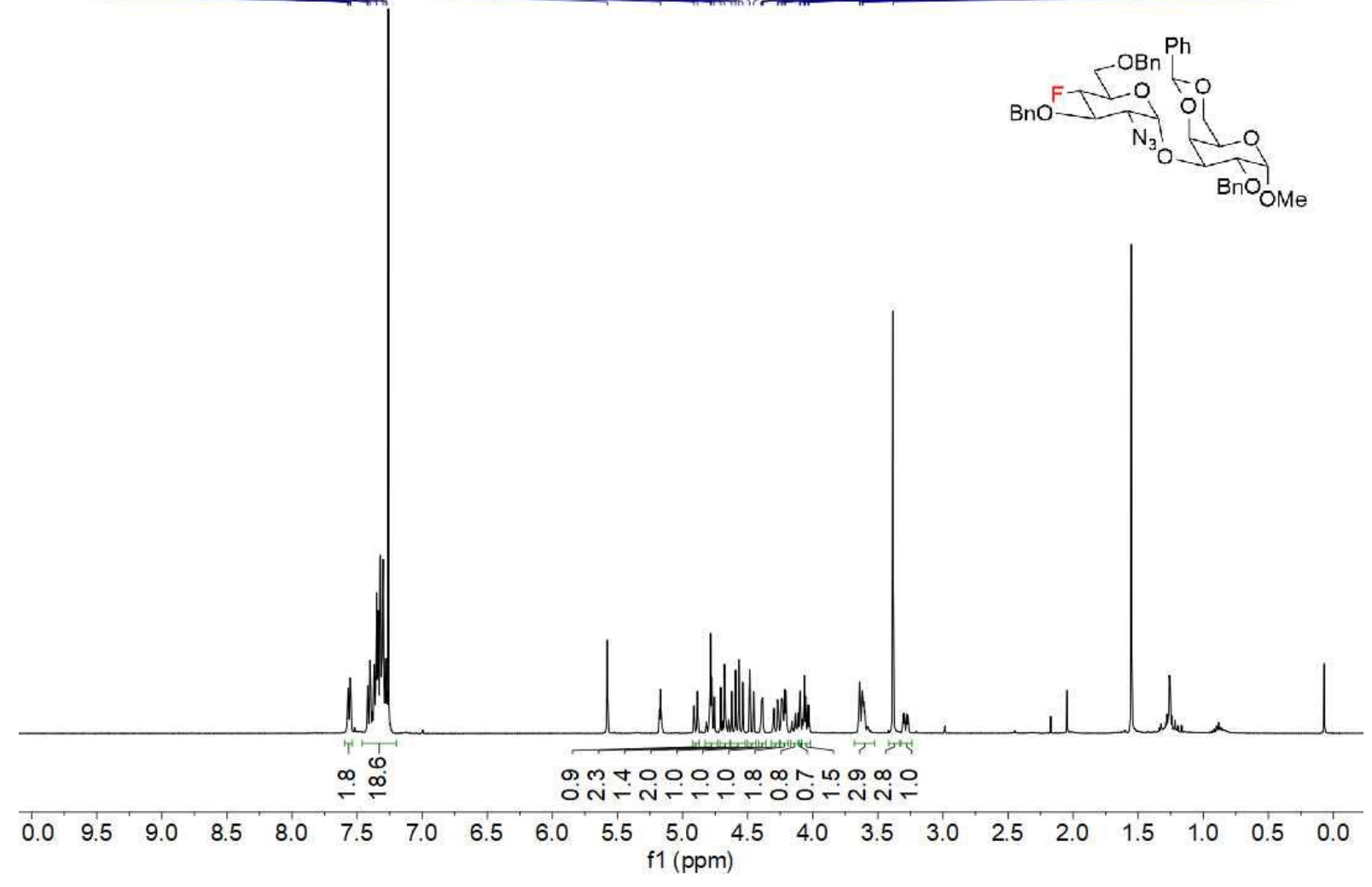

${ }^{13} \mathrm{C}\left\{{ }^{1} \mathrm{H}\right\} \mathrm{NMR} 100 \mathrm{MHz}, \mathrm{CDCl}_{3} 2 \mathrm{C}-\alpha$

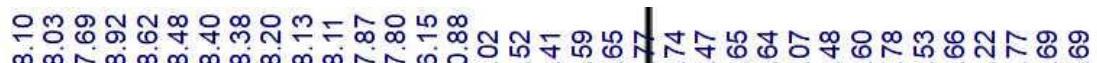

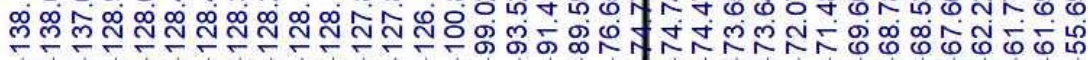

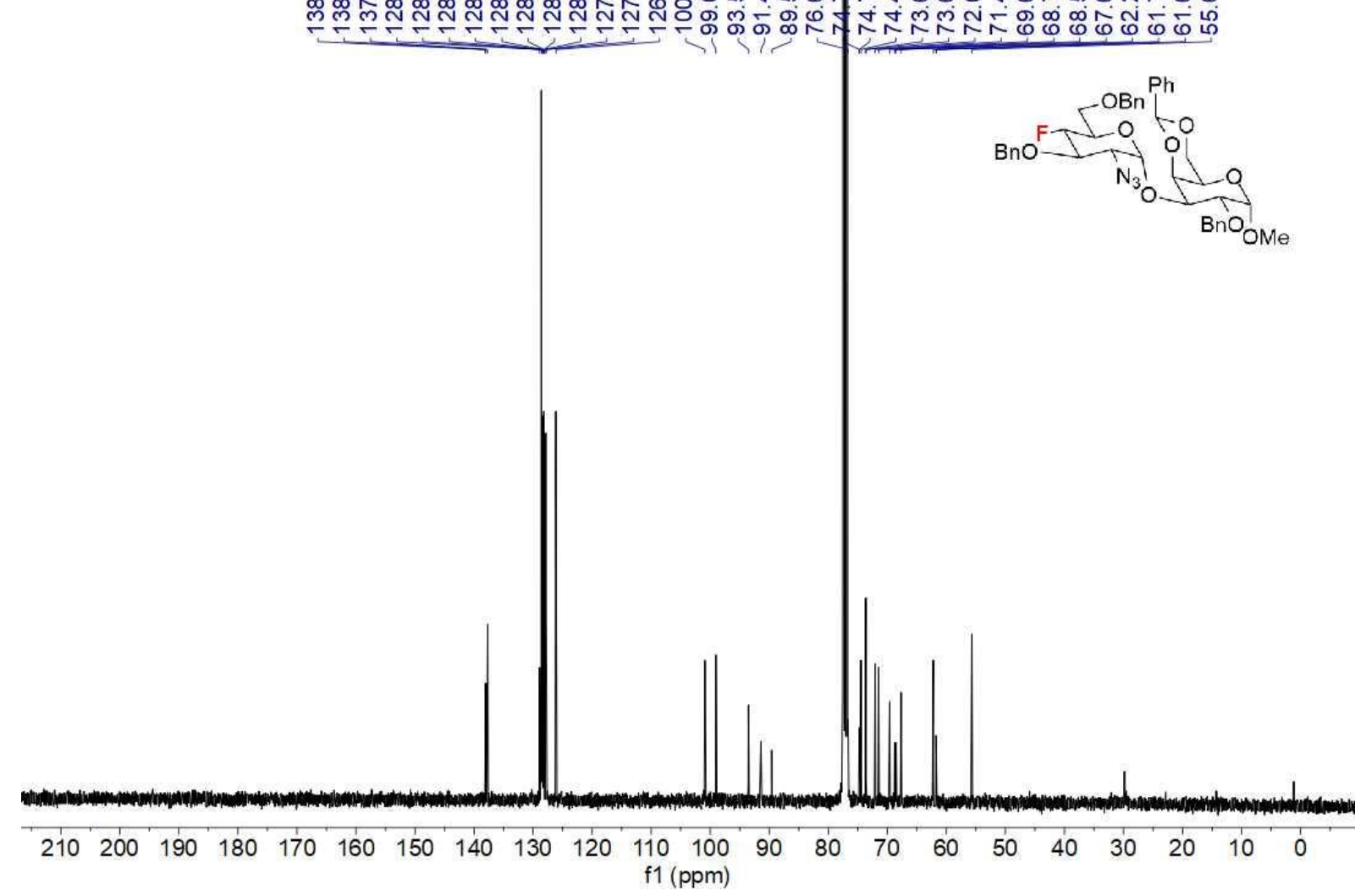


${ }^{1} \mathrm{H}-{ }^{1} \mathrm{H}$ COSY NMR $2 \mathrm{C}-\alpha$

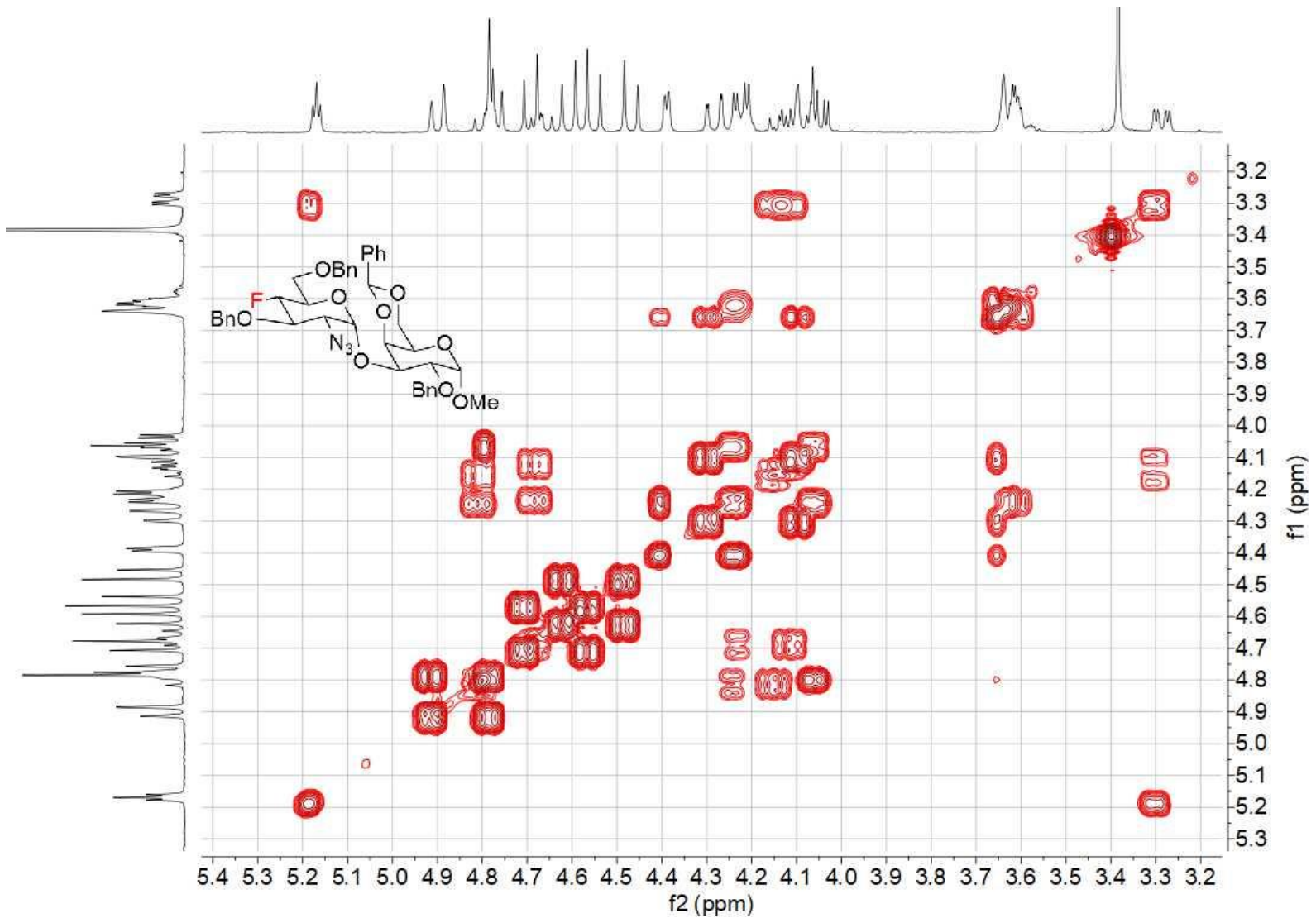

${ }^{1} \mathrm{H}-{ }^{13} \mathrm{C}$ HSQC NMR 2C- $\alpha$

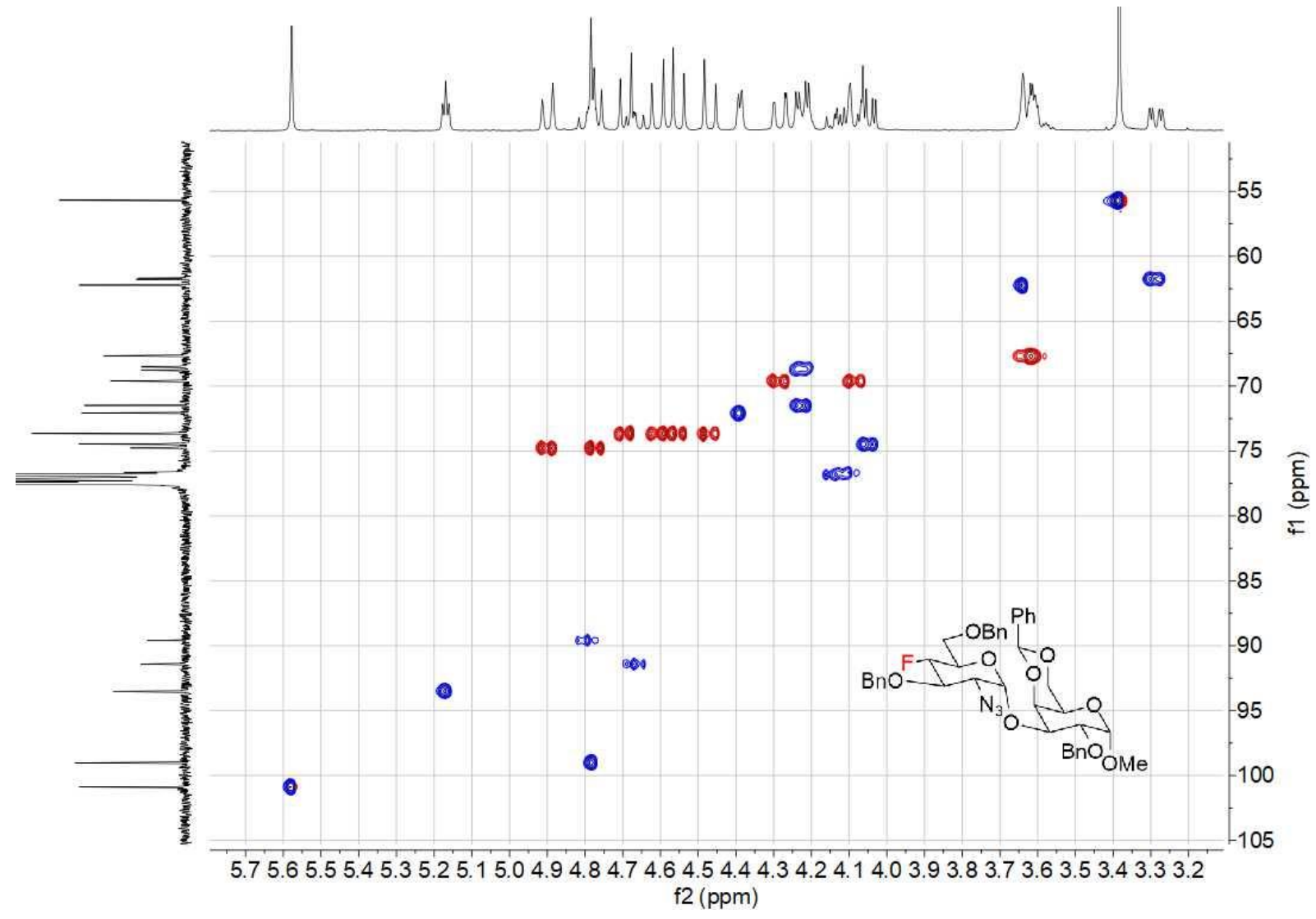




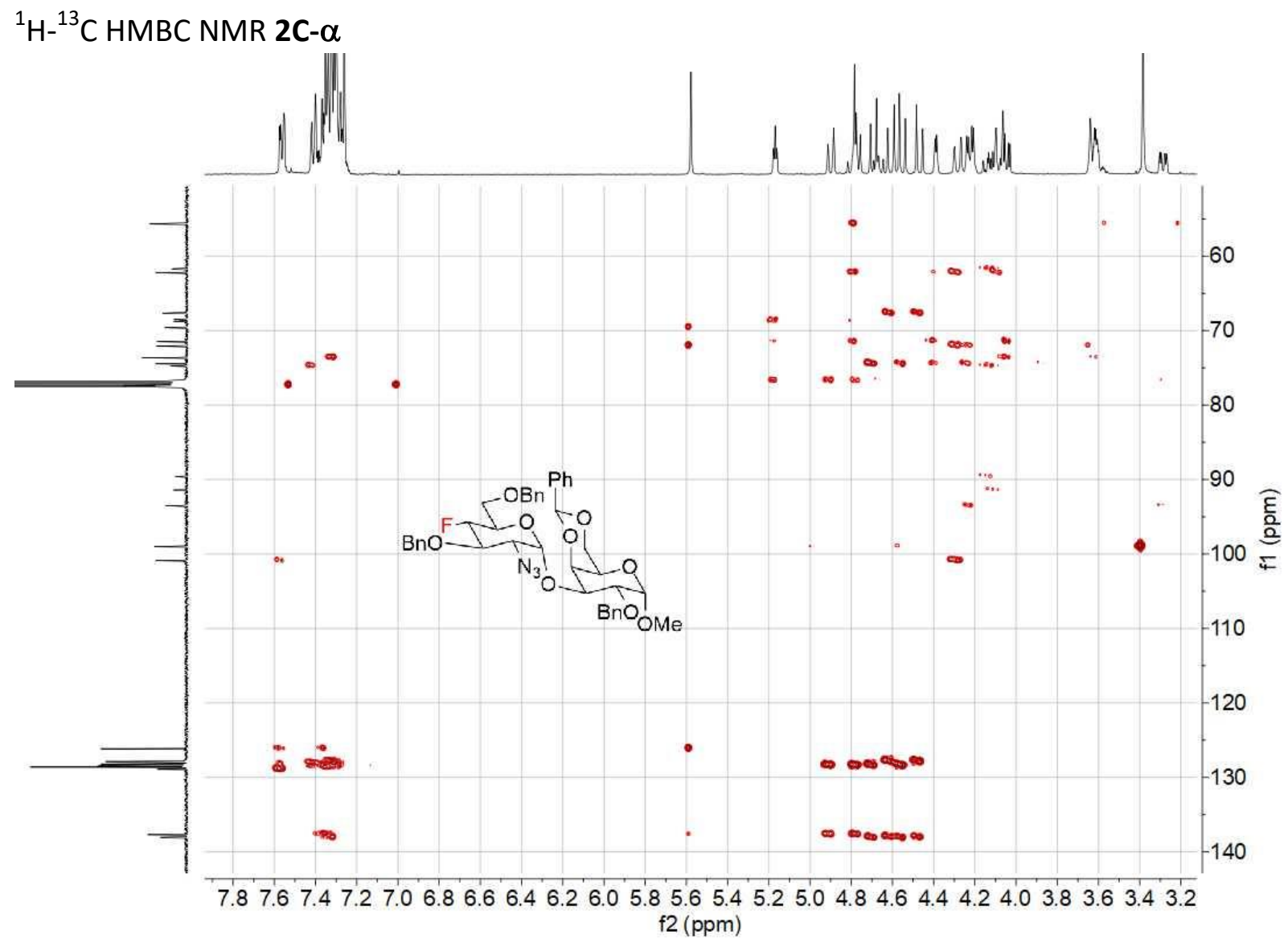

${ }^{19} \mathrm{~F}$ NMR $376 \mathrm{MHz}, \mathrm{CDCl}_{3} 2 \mathrm{C}-\alpha$

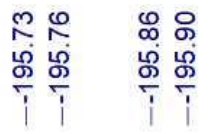
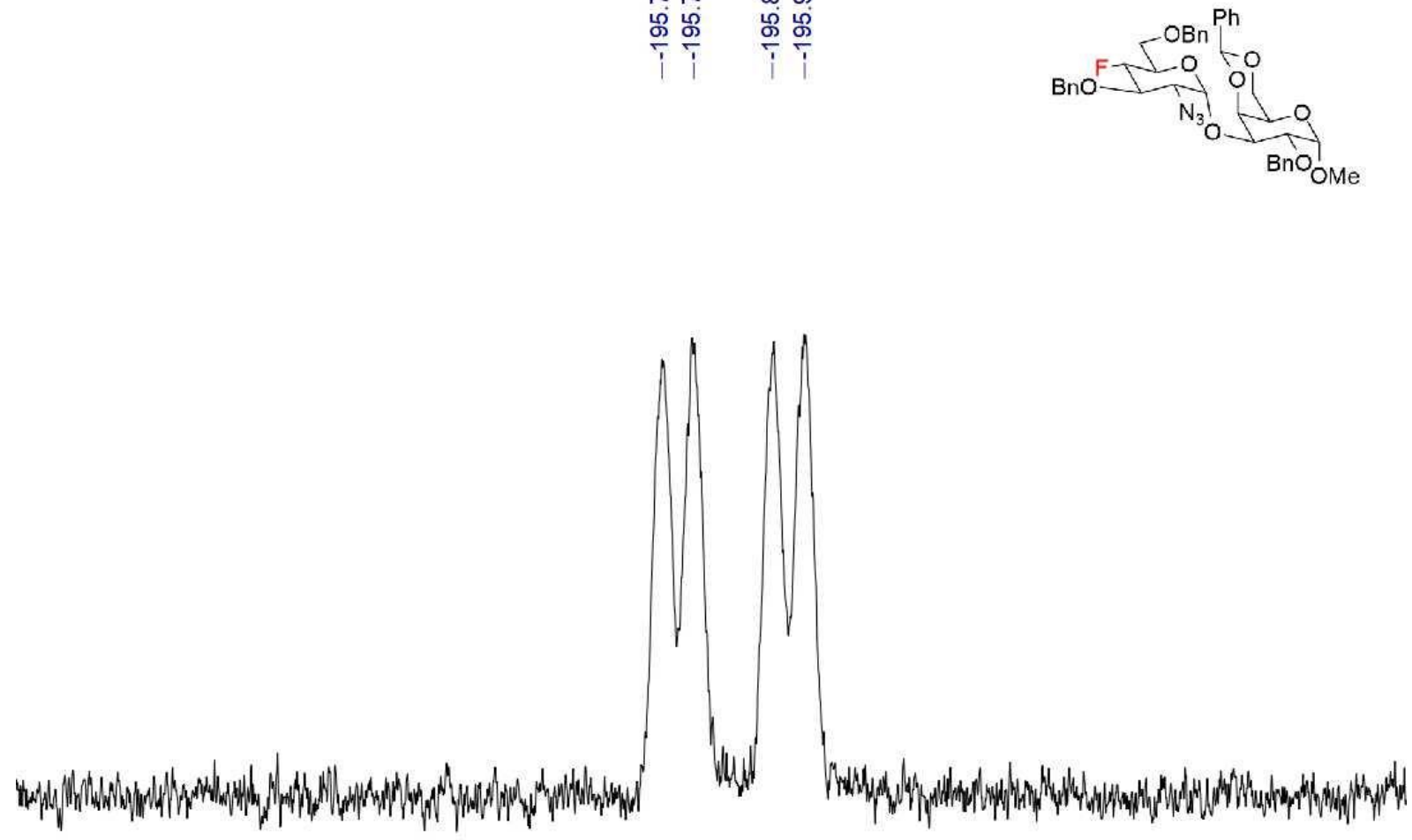

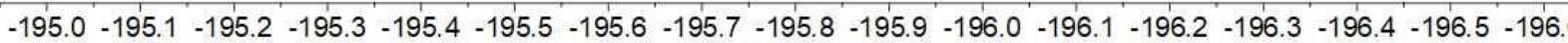
f1 (ppm) 
NMR 2C- $\beta$

${ }^{1} \mathrm{HNMR} 400 \mathrm{MHz}, \mathrm{CDCl}_{3} \mathbf{2 C}-\beta$

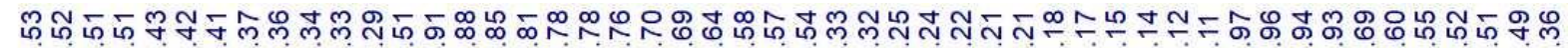

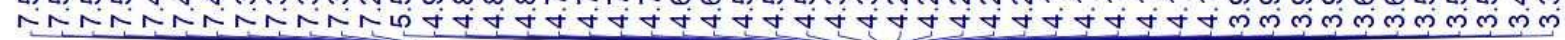

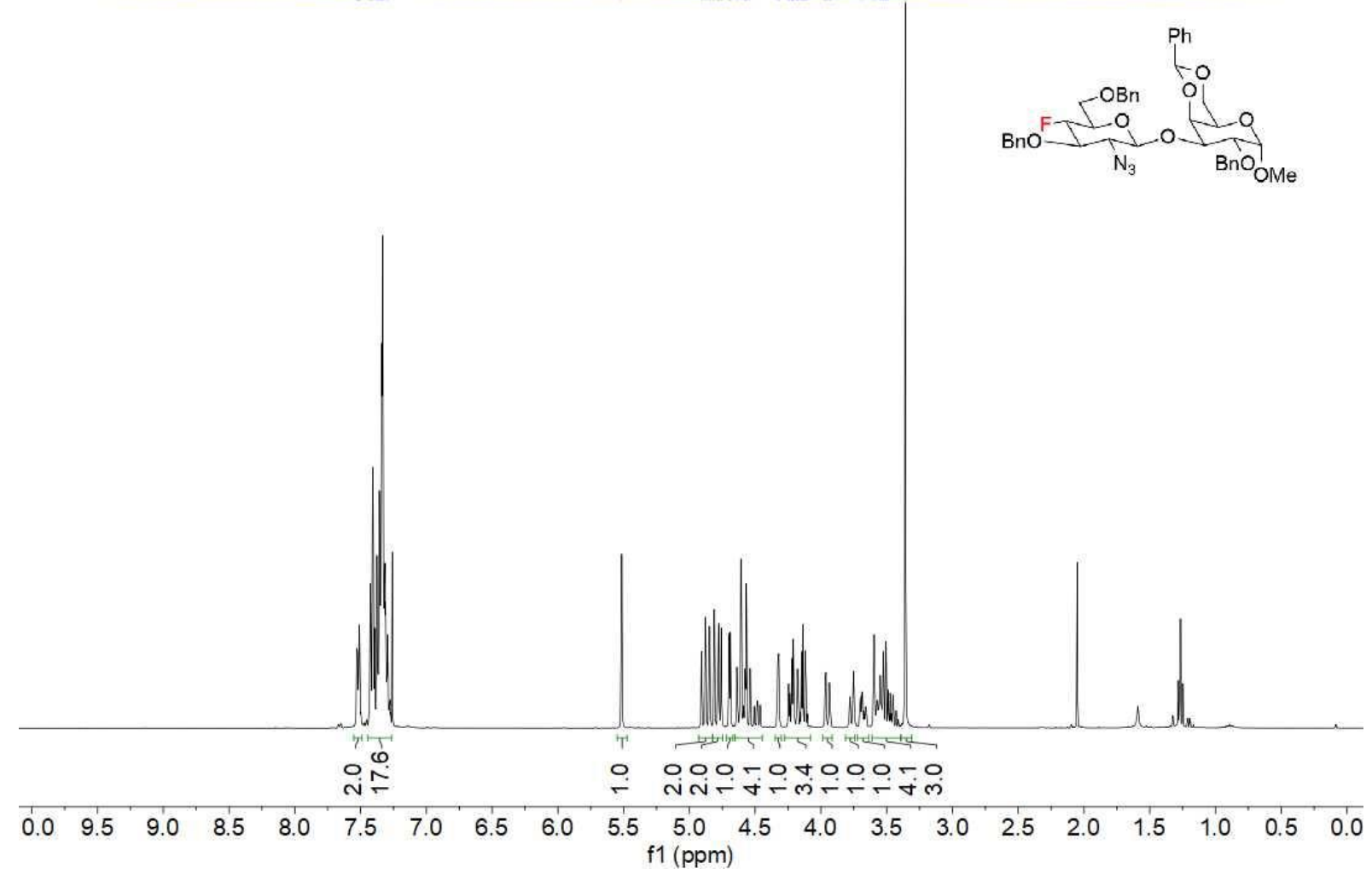

${ }^{13} \mathrm{C}\left\{{ }^{1} \mathrm{H}\right\} \mathrm{NMR} 100 \mathrm{MHz}, \mathrm{CDCl}_{3} \mathbf{2 C}-\boldsymbol{\beta}$

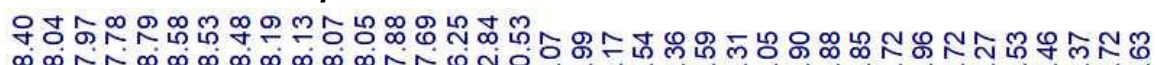

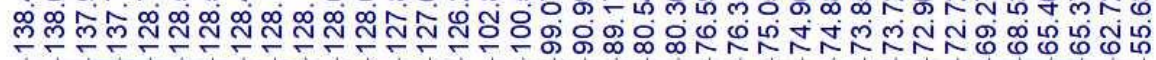

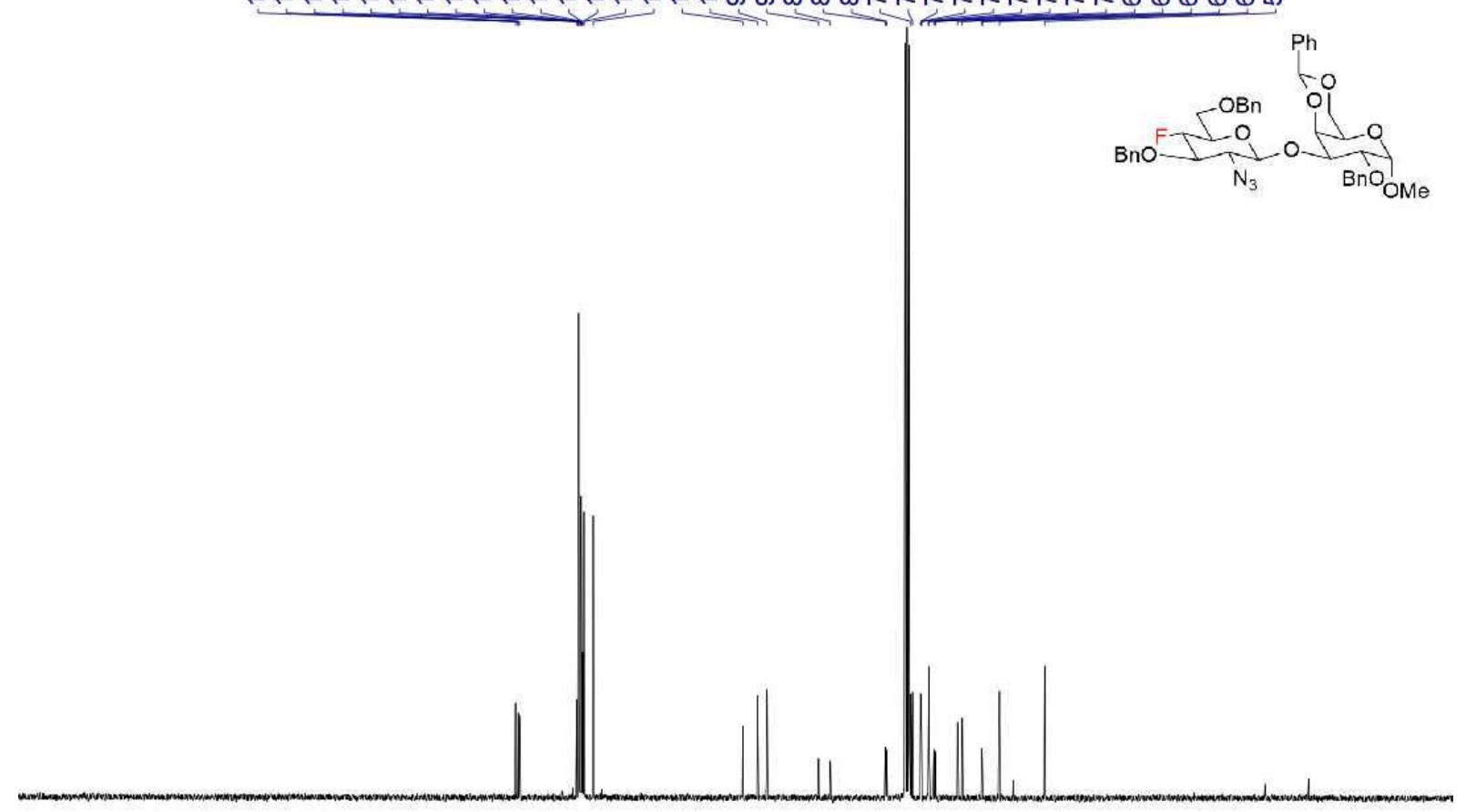

$\begin{array}{llllllllllllllllllllllllll}210 & 200 & 190 & 180 & 170 & 160 & 150 & 140 & 130 & 120 & \begin{array}{r}110 \\ \mathrm{f} 1(\mathrm{ppm})\end{array} & 100 & 90 & 80 & 70 & 60 & 50 & 40 & 30 & 20 & 10 & 0\end{array}$ 
${ }^{1} \mathrm{H}-{ }^{1} \mathrm{H}$ COSY NMR $2 \mathrm{C}-\boldsymbol{\beta}$

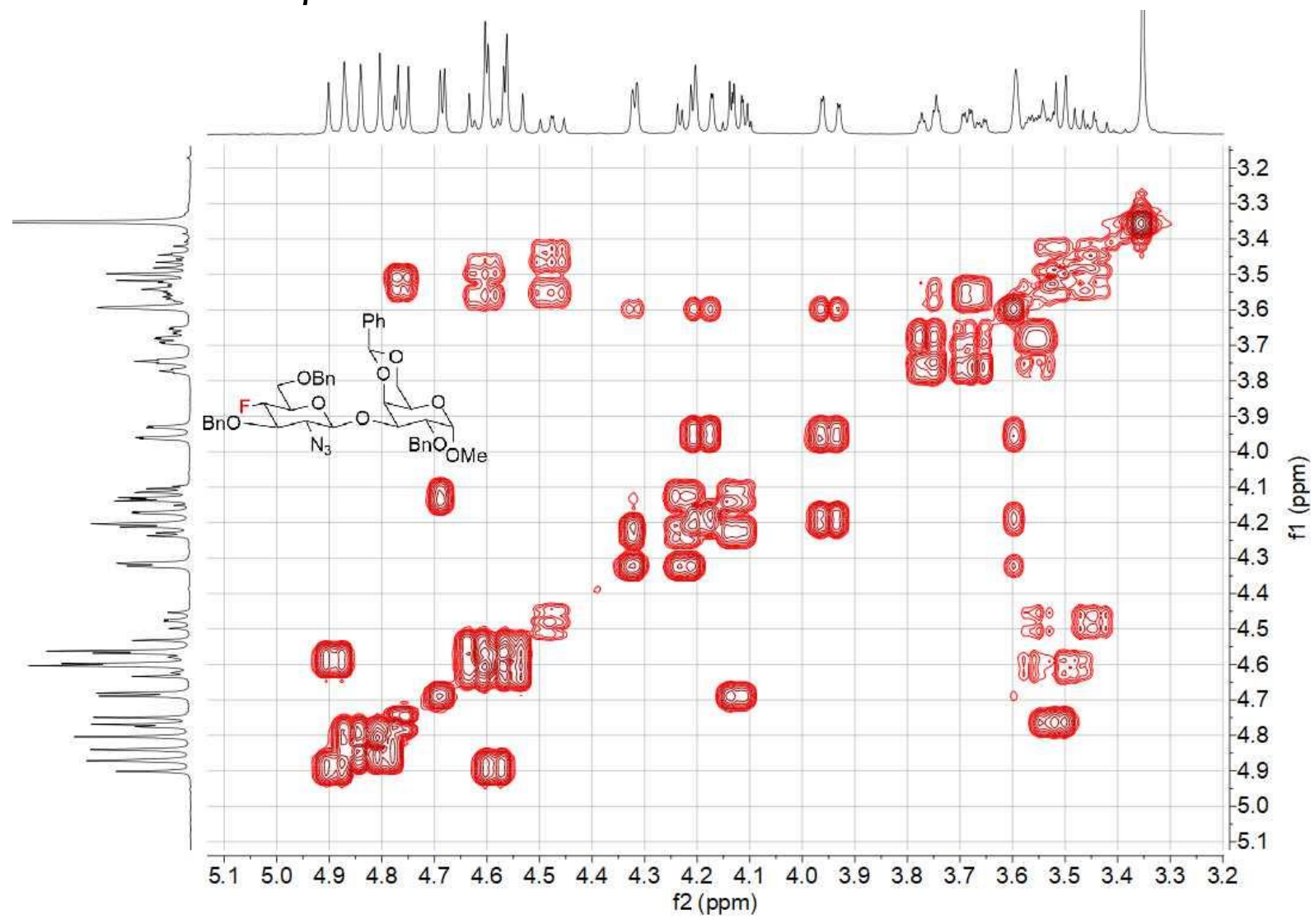

${ }^{1} \mathrm{H}-{ }^{13} \mathrm{C}$ HSQC NMR 2C- $\beta$

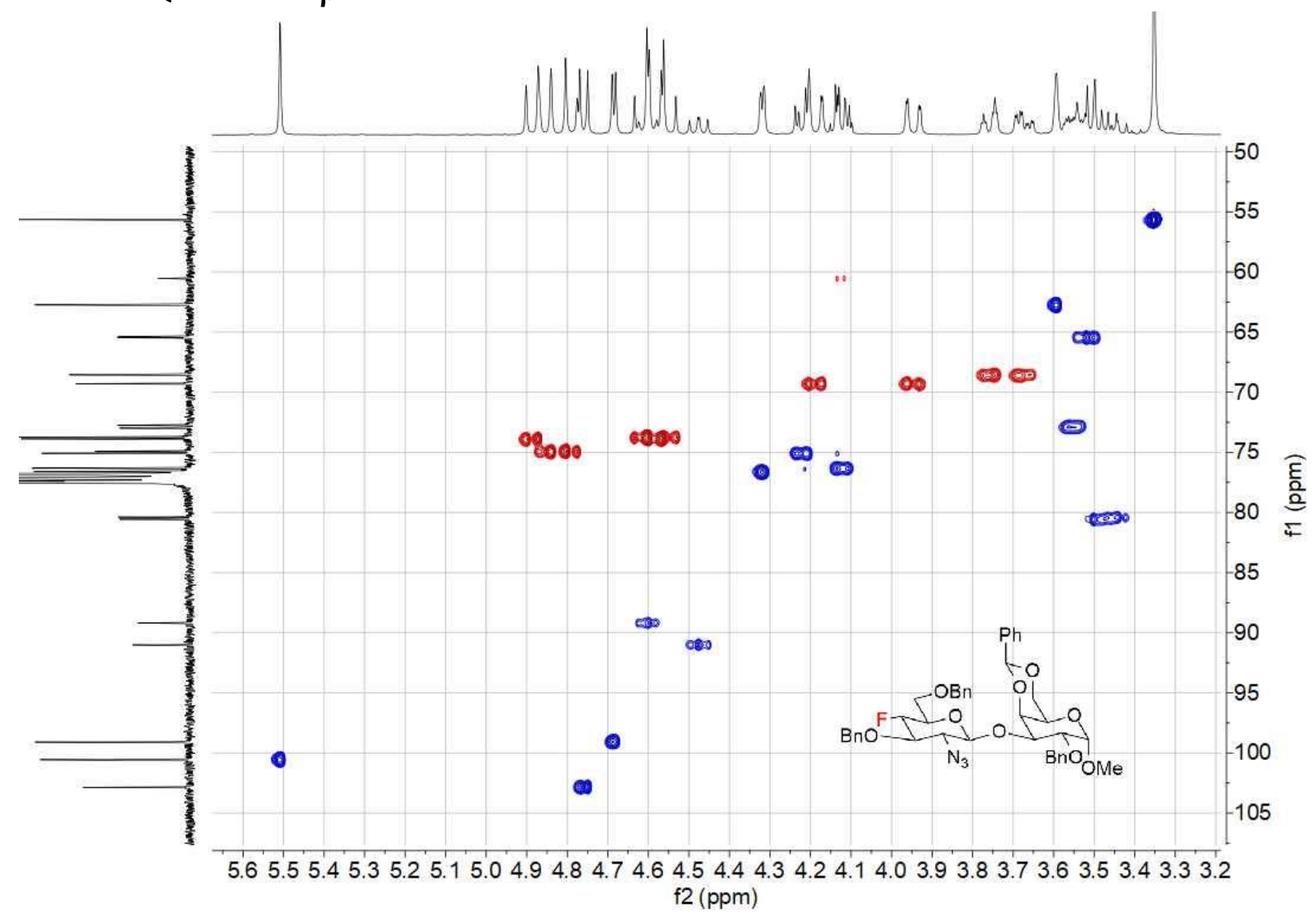


${ }^{1} \mathrm{H}-{ }^{13} \mathrm{C}$ HMBC NMR $2 \mathrm{C}-\boldsymbol{\beta}$

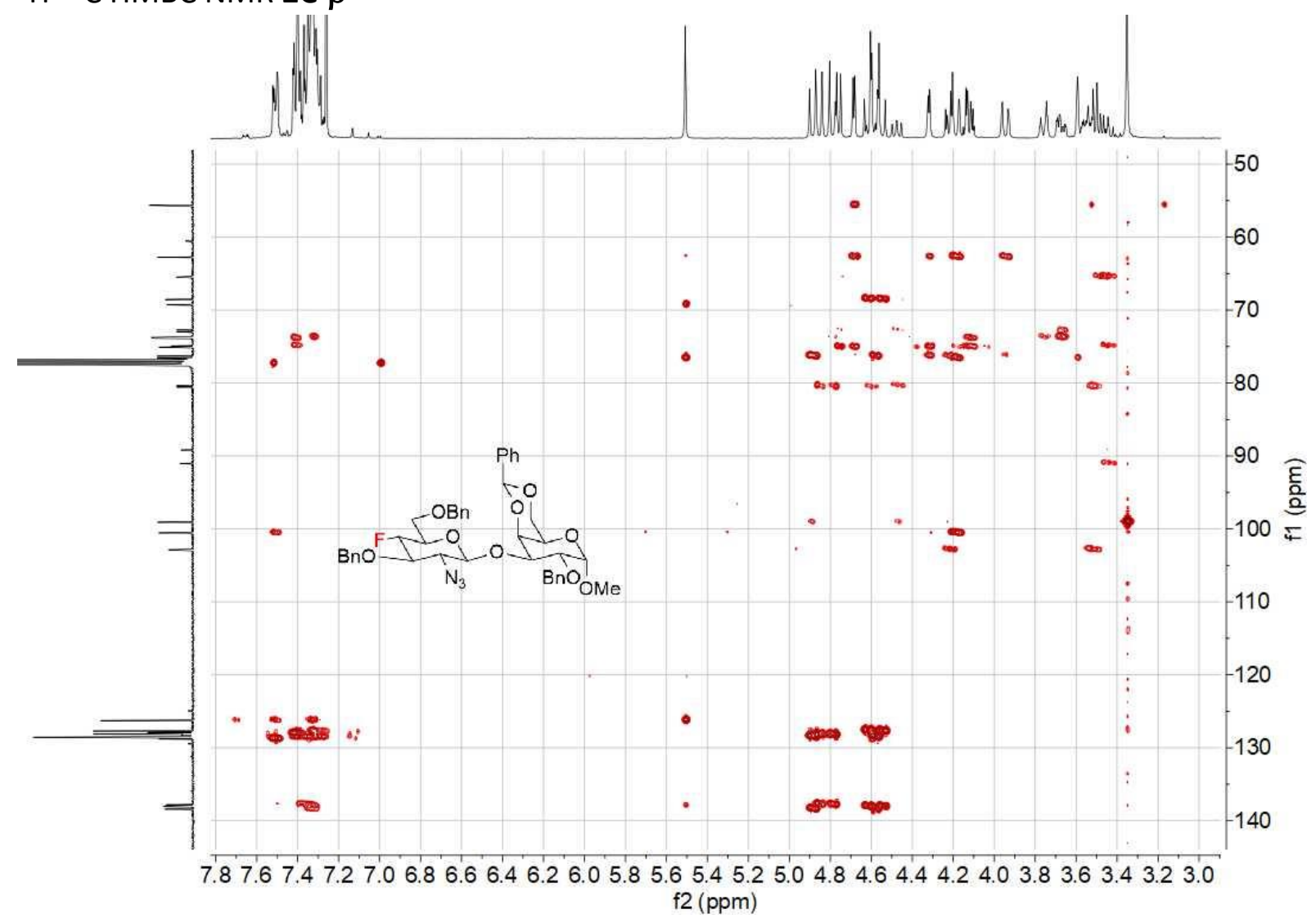

${ }^{19} \mathrm{~F} \mathrm{NMR} 376 \mathrm{MHz}, \mathrm{CDCl}_{3} \mathbf{2 C}-\boldsymbol{\beta}$

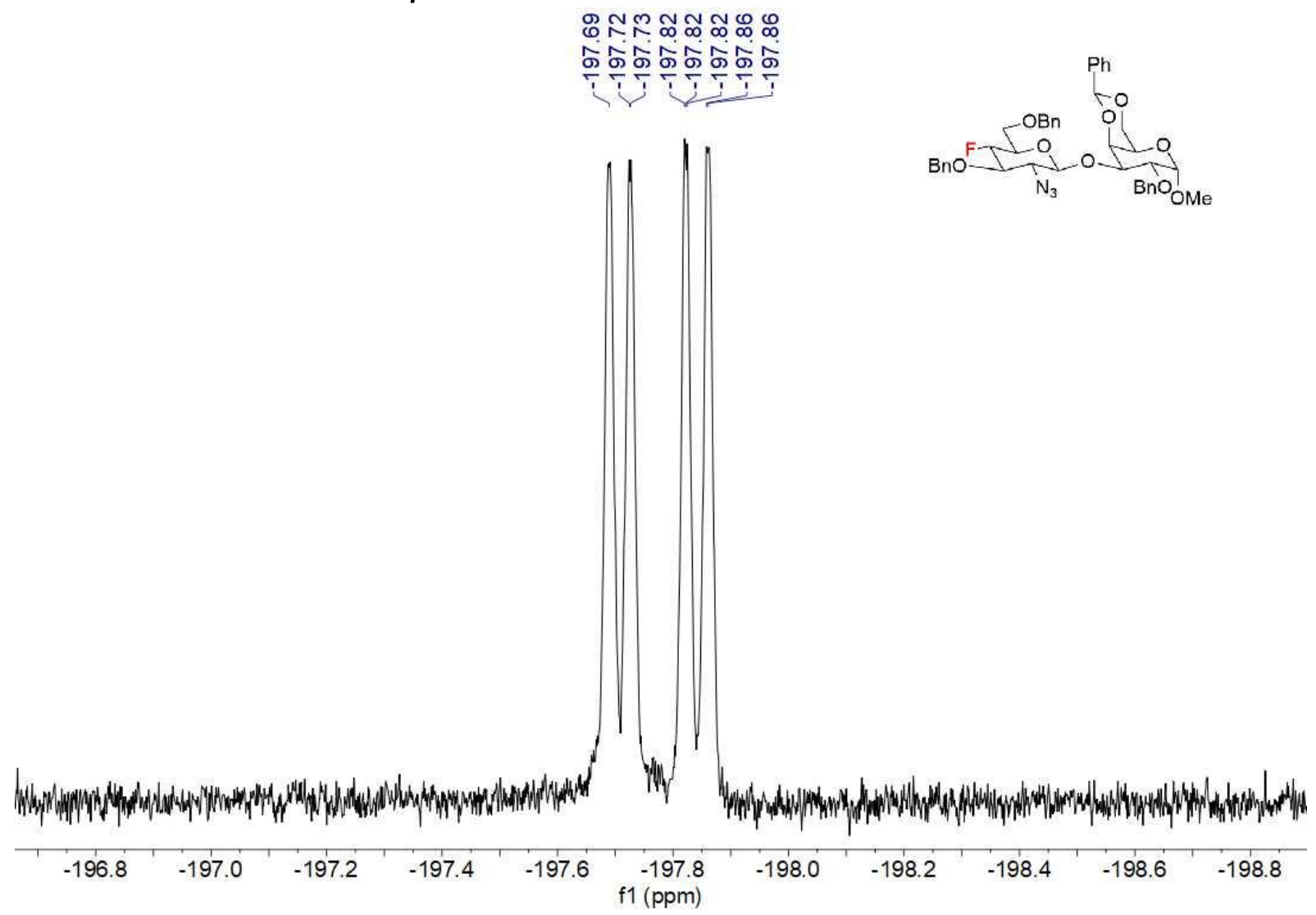




\section{NMR 2E- $\alpha$}

${ }^{1} \mathrm{HNMR} 400 \mathrm{MHz}, \mathrm{CDCl}_{3} 2 \mathrm{E}-\alpha$ and ca $17 \% 2 \mathrm{E}-\beta$

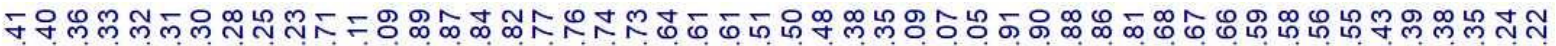

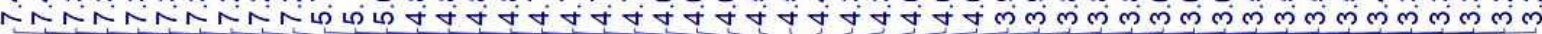

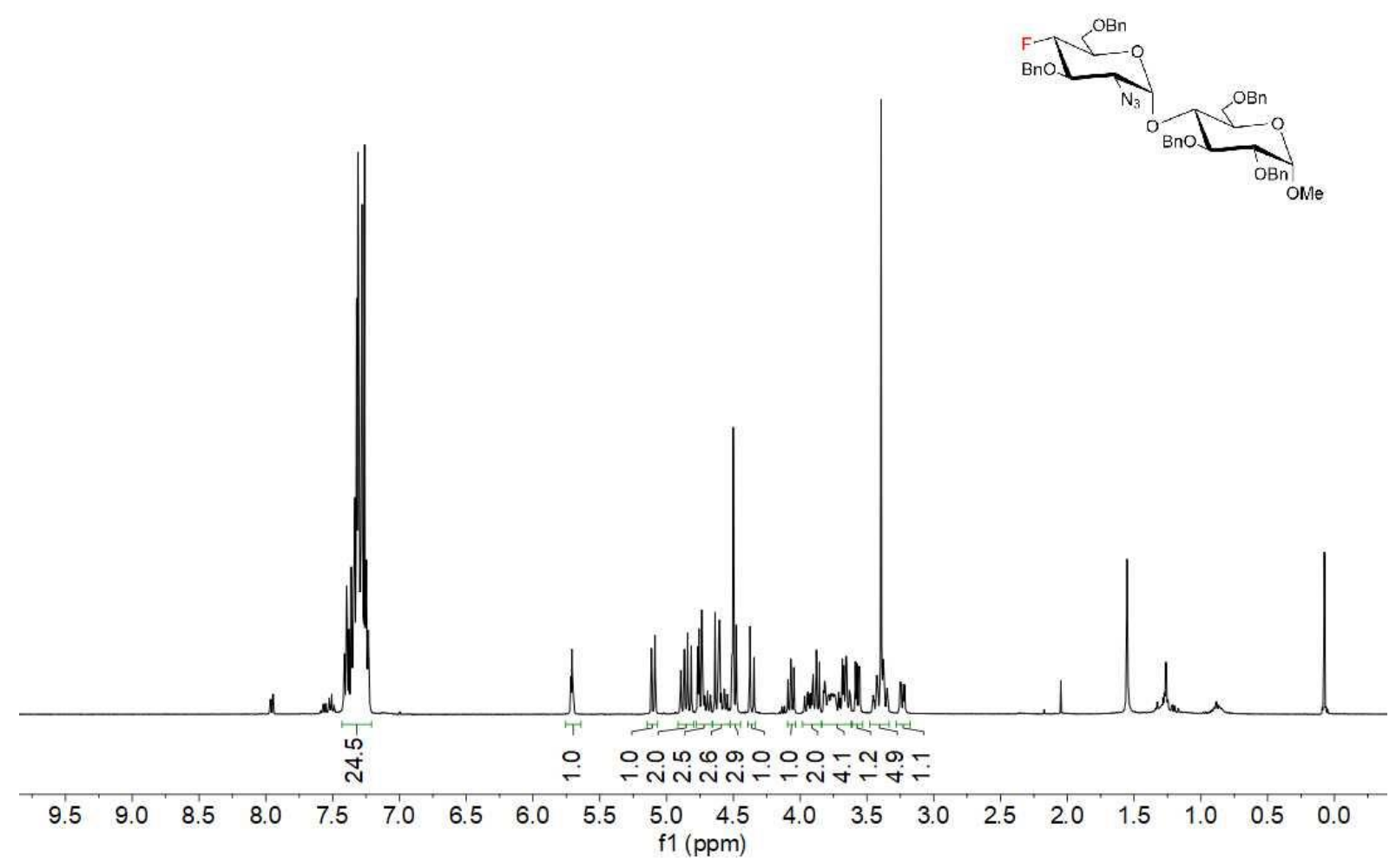

${ }^{13} \mathrm{C}\left\{{ }^{1} \mathrm{H}\right\} \mathrm{NMR} 100 \mathrm{MHz}, \mathrm{CDCl}_{3} 2 \mathrm{E}-\alpha$ and $17 \% 2 \mathrm{E}-\boldsymbol{\beta}$

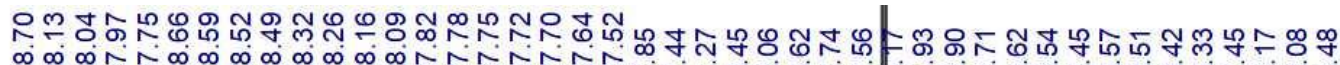

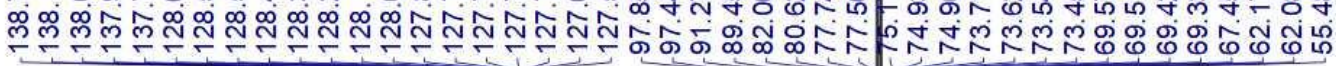

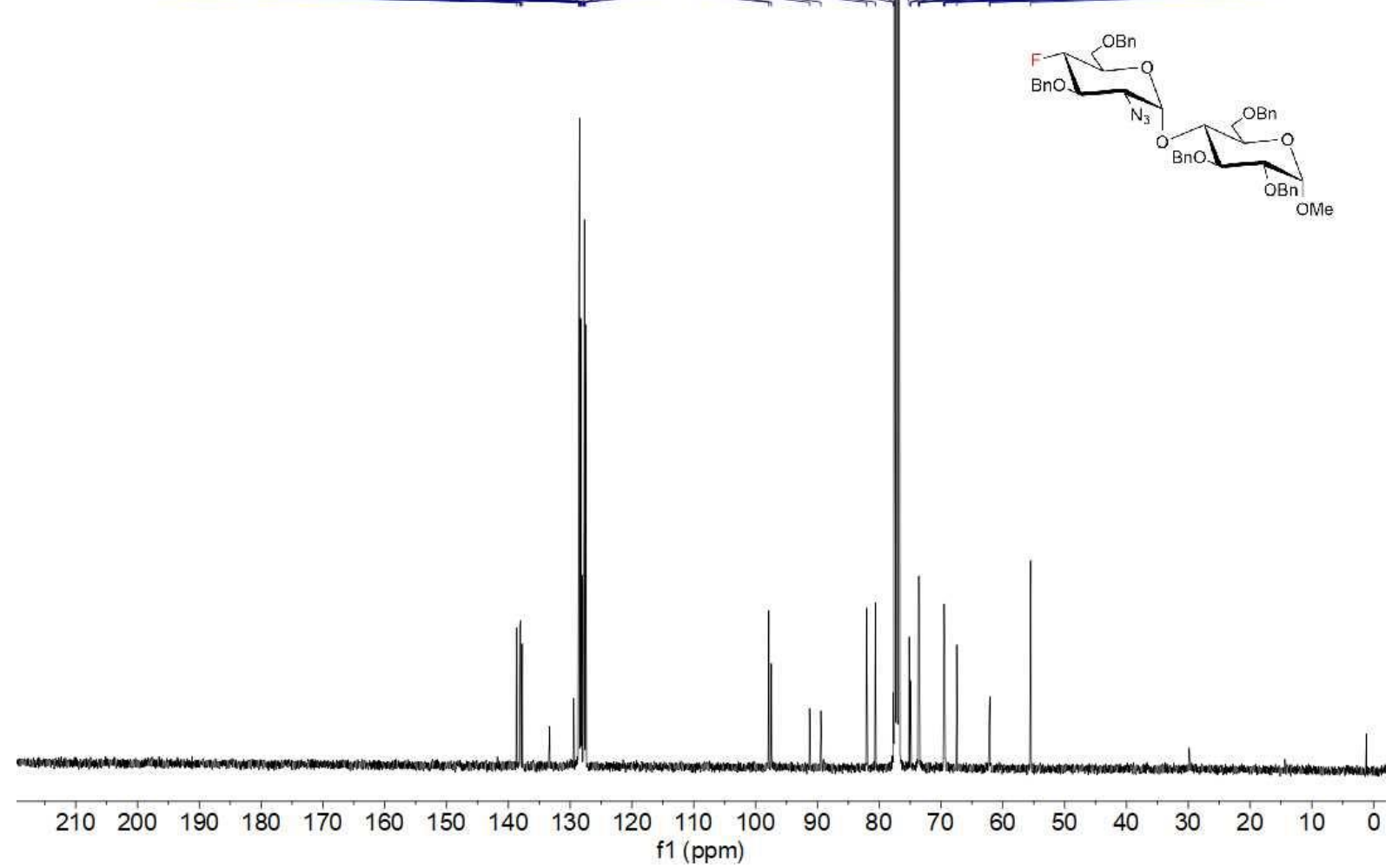


${ }^{1} \mathrm{H}-{ }^{1} \mathrm{H}$ COSY NMR $2 \mathrm{E}-\alpha$ and $17 \%$ 2E- $\beta$

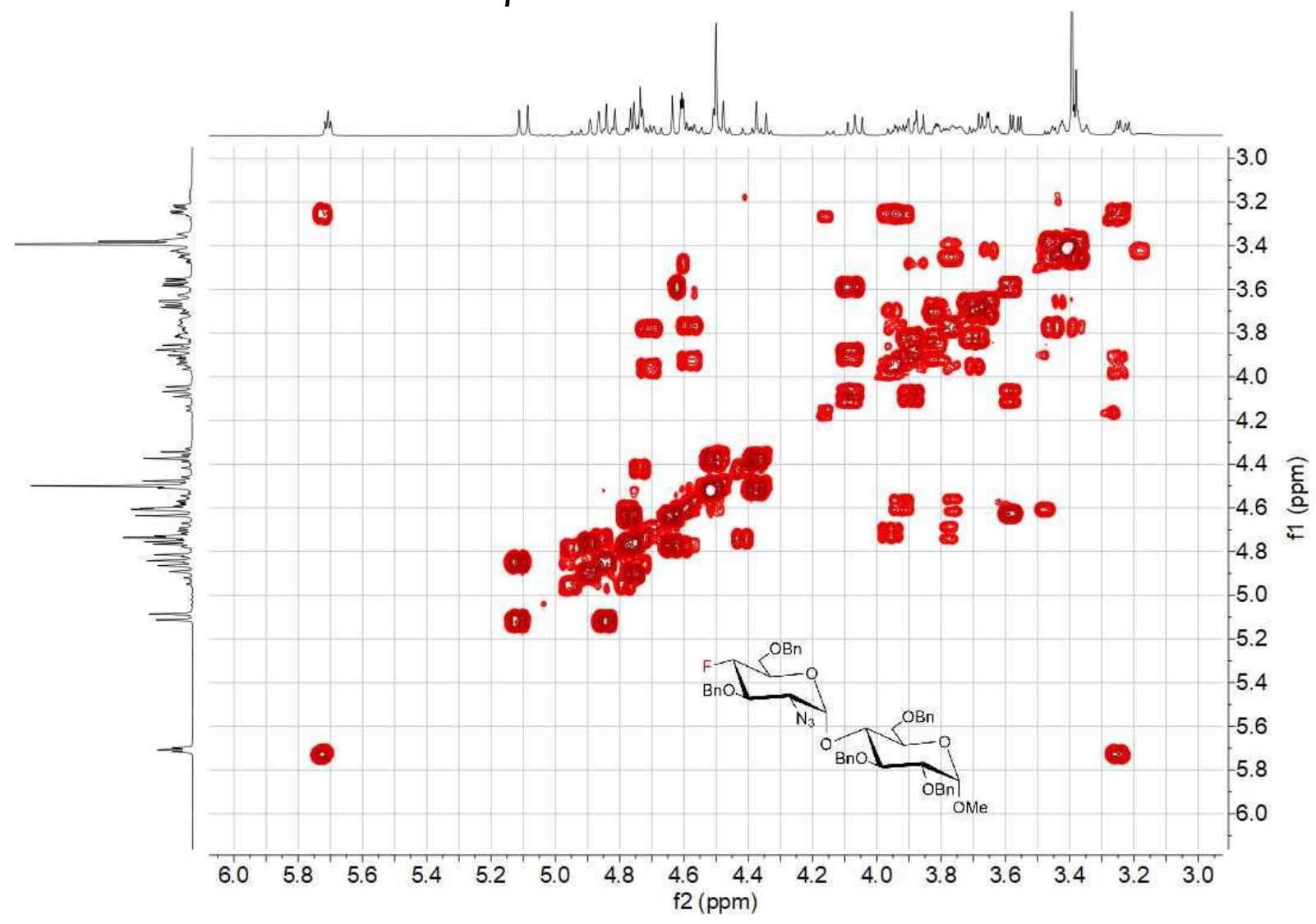

${ }^{1} \mathrm{H}-{ }^{13} \mathrm{C}$ HSQC NMR 2E- $\alpha$ and $17 \%$ 2E- $\beta$

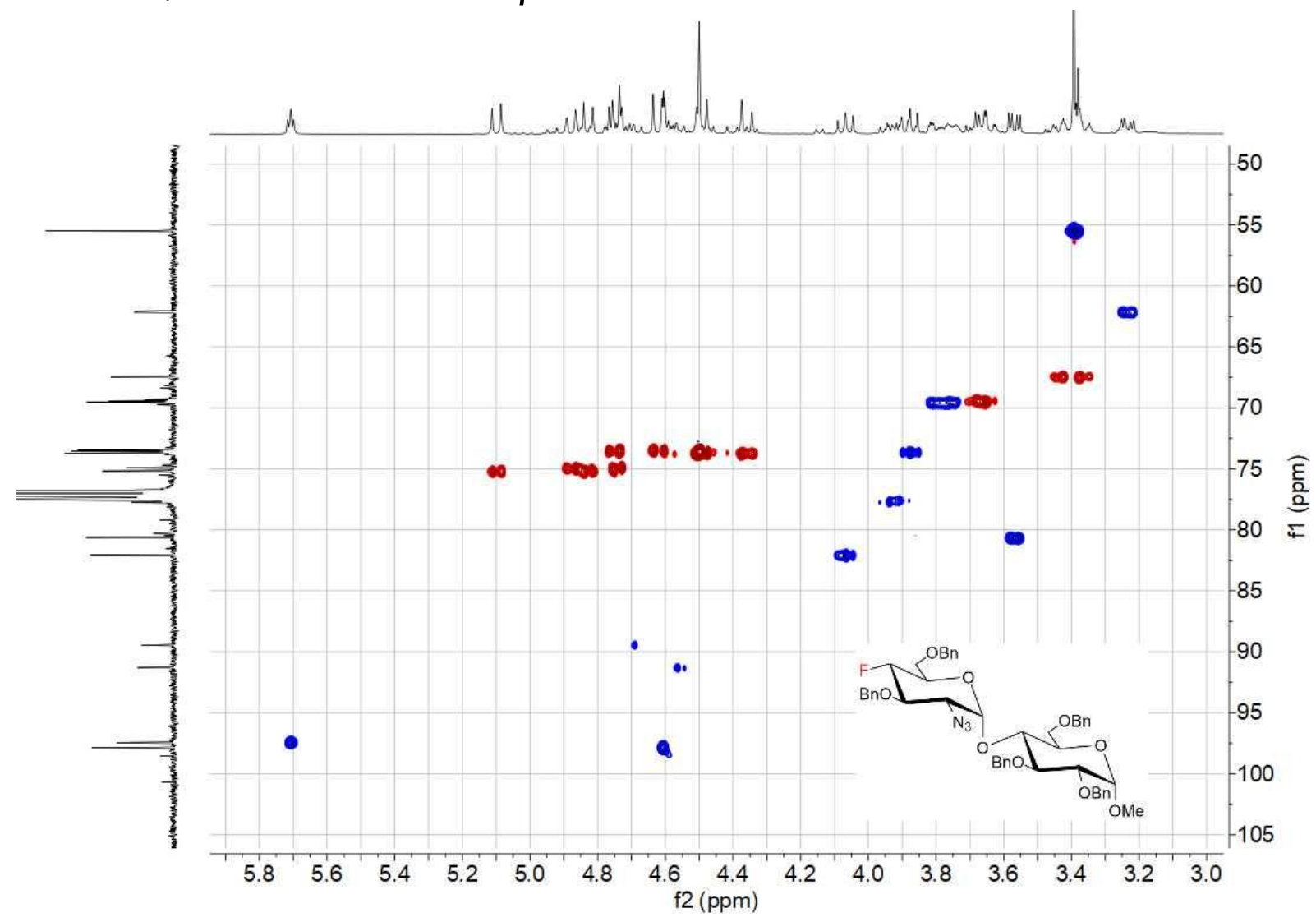


${ }^{19} \mathrm{~F} \mathrm{NMR} 376 \mathrm{MHz}, \mathrm{CDCl}_{3} \mathbf{2 E}-\boldsymbol{\alpha}$ and $17 \%$ 2E- $\boldsymbol{\beta}$

กักิร

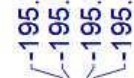

นํํ요

产产产
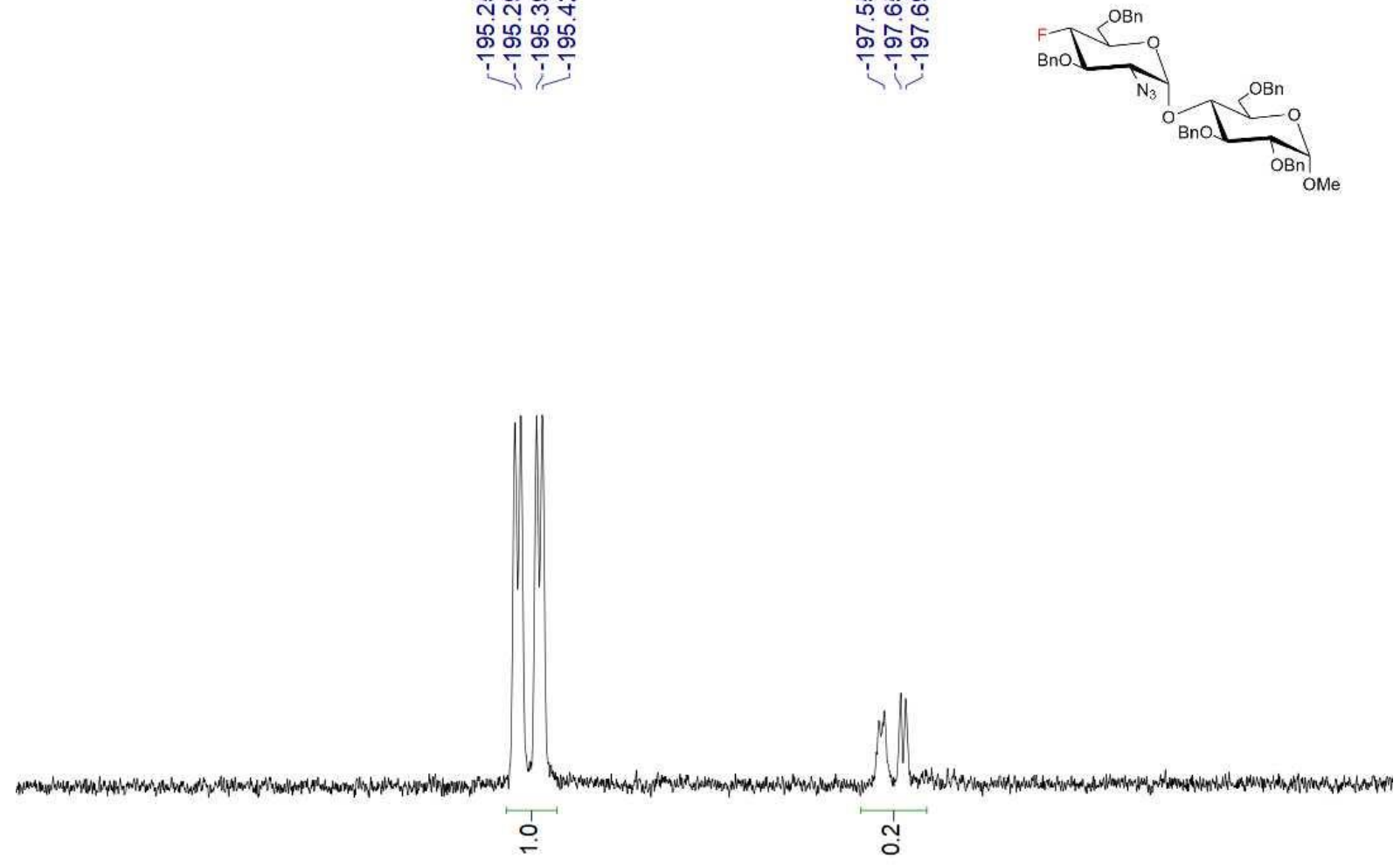

$-192.5-193.0-193.5-194.0-194.5-195.0-195.5-196.0-196.5-197.0-197.5-198.0-198.5-199.0-199.5-200.0-200.5$ f1 (ppm)

\section{NMR 2E- $\beta$}

${ }^{1} \mathrm{H} \mathrm{NMR} 400 \mathrm{MHz}, \mathrm{CDCl}_{3} 2 \mathrm{E}-\beta$ and ca $9 \% 2 \mathrm{E}-\alpha$

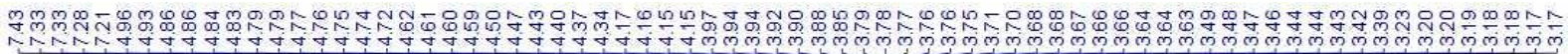

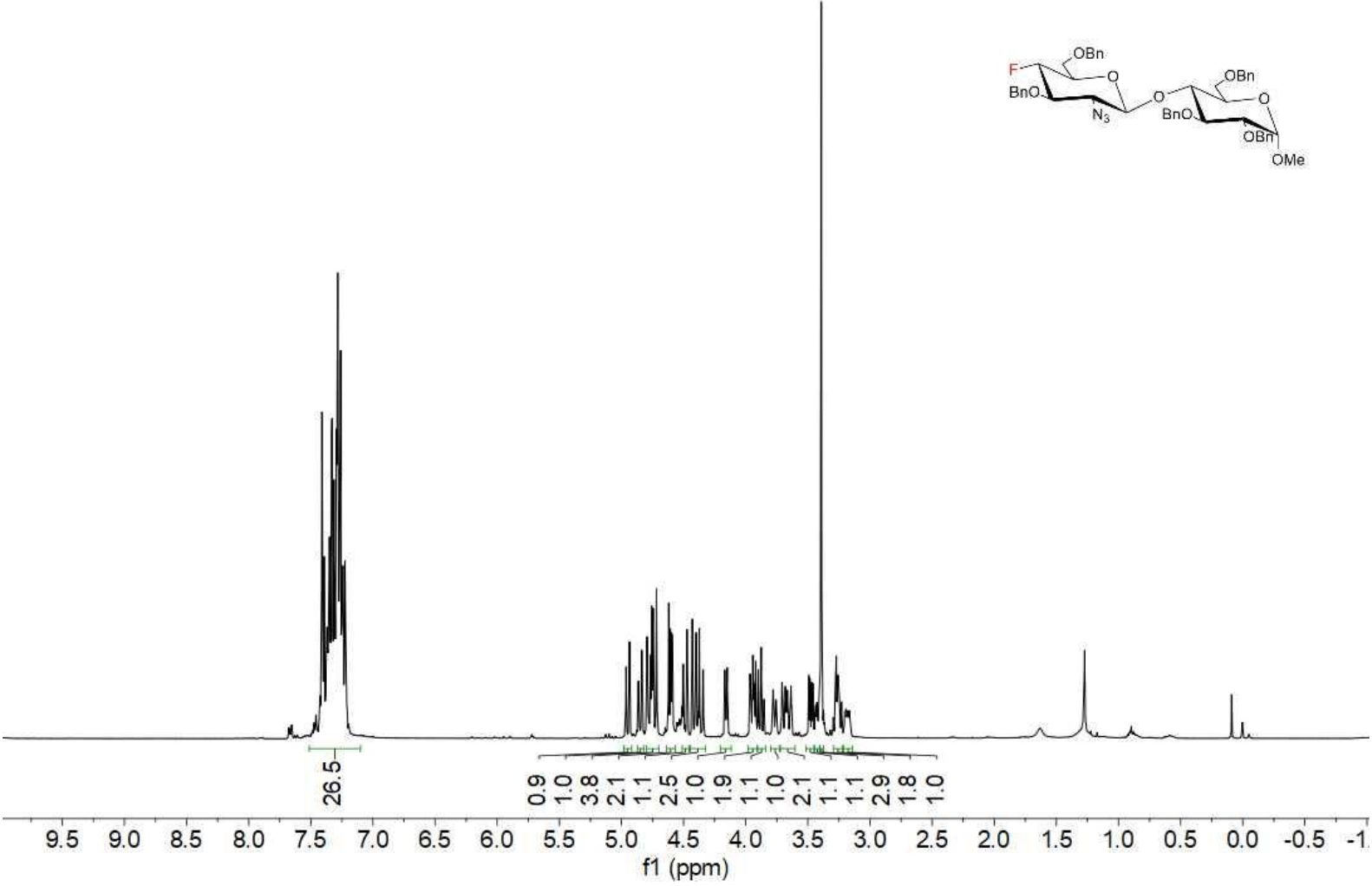


${ }^{13} \mathrm{C}\left\{{ }^{1} \mathrm{H}\right\}$ NMR $100 \mathrm{MHz}, \mathrm{CDCl}_{3} \mathbf{2 E}-\boldsymbol{\beta}$ and ca $9 \% \mathbf{2 E -} \boldsymbol{\alpha}$

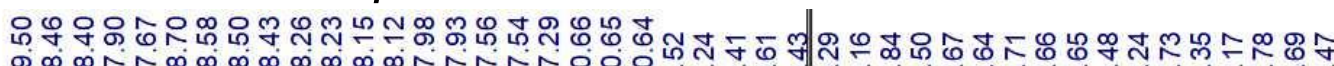
ల్m

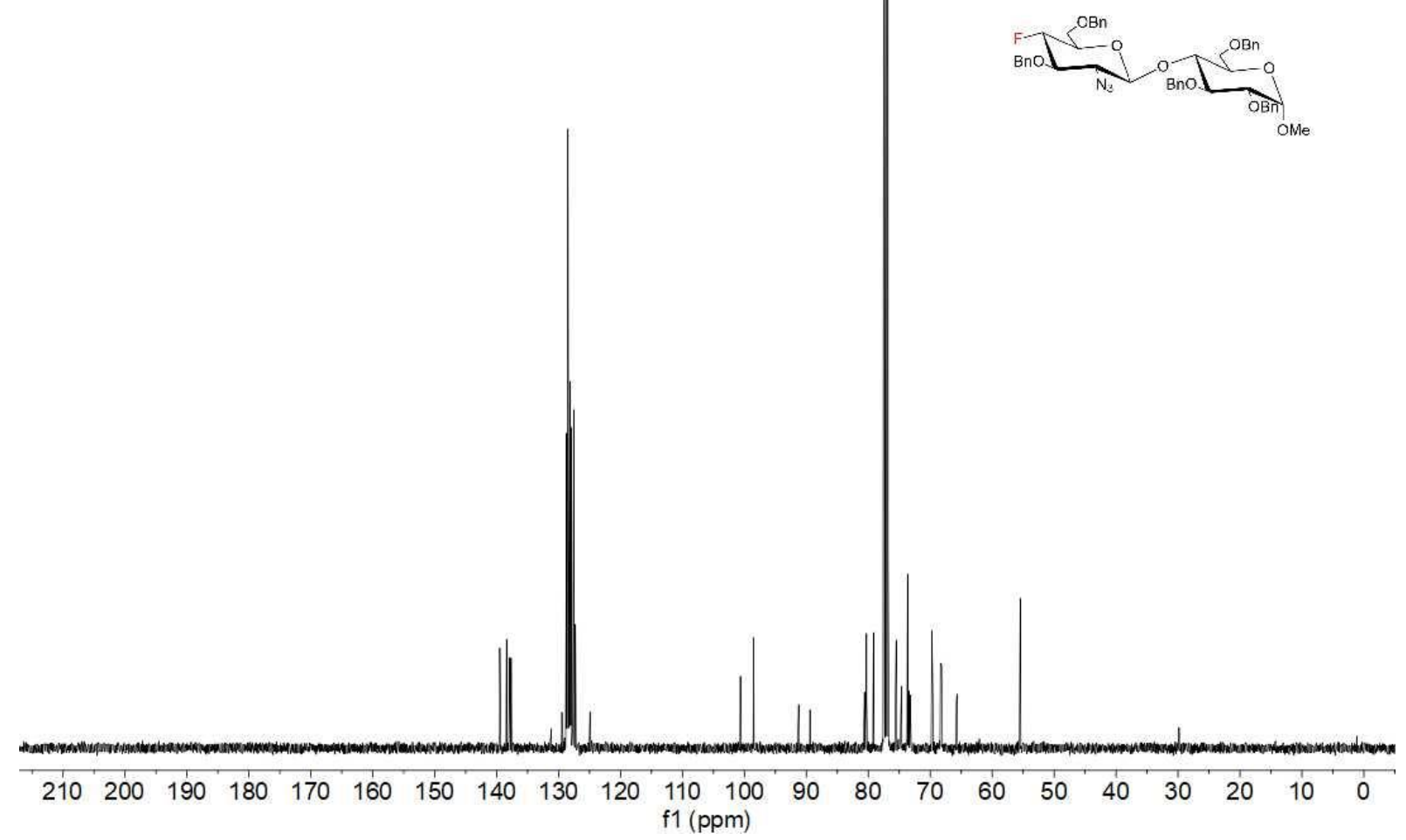

${ }^{1} \mathrm{H}-{ }^{1} \mathrm{H}$ COSY NMR $2 \mathrm{E}-\boldsymbol{\beta}$ and ca $9 \% \mathbf{2 E -} \boldsymbol{\alpha}$

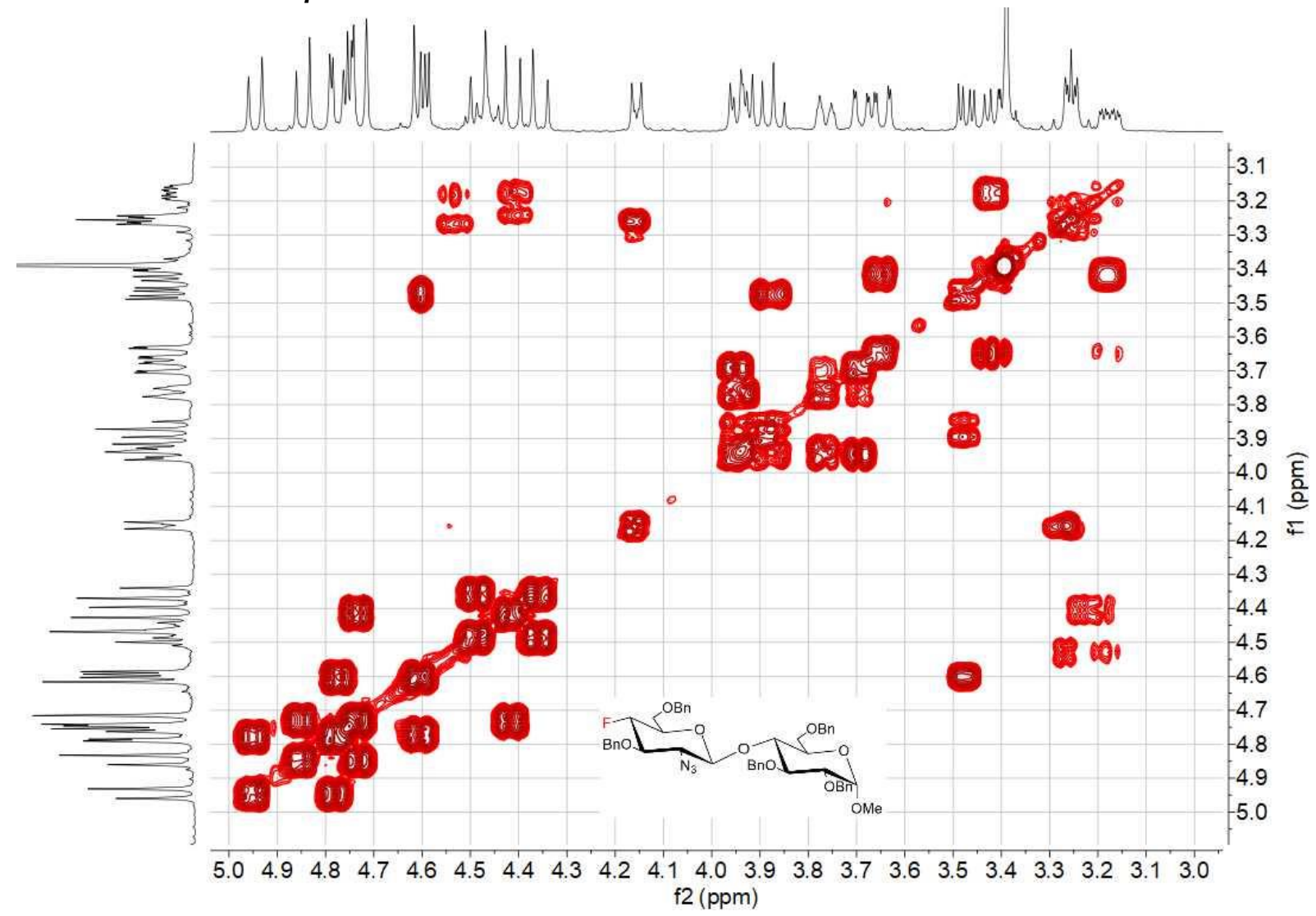


${ }^{1} \mathrm{H}-{ }^{13} \mathrm{C}$ HSQC NMR $2 \mathrm{E}-\boldsymbol{\beta}$ and ca $9 \%$ 2E- $\alpha$

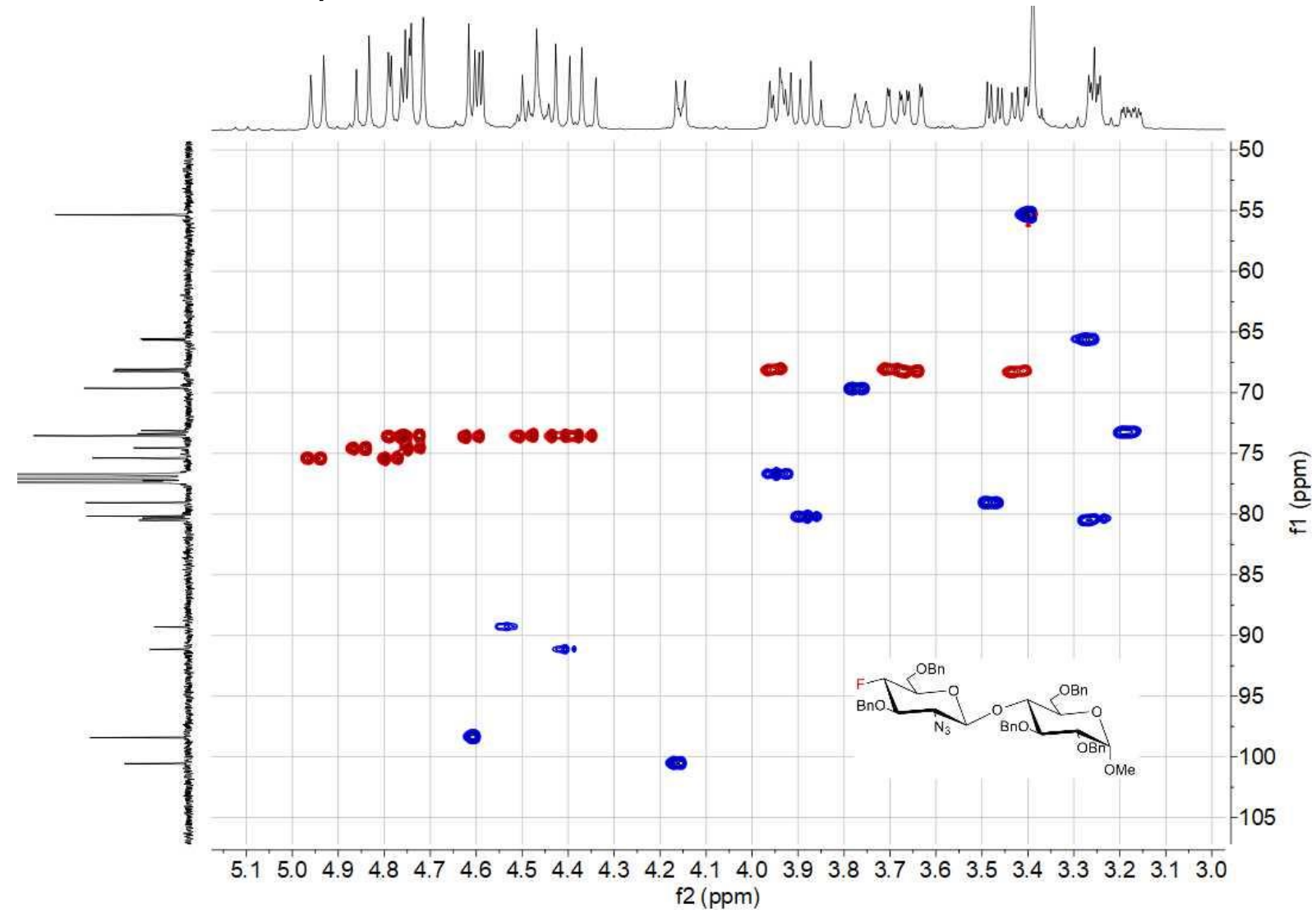

${ }^{1} \mathrm{H}-{ }^{13} \mathrm{C}$ HMBC NMR 2E- $\beta$ and ca $9 \%$ 2E- $\alpha$

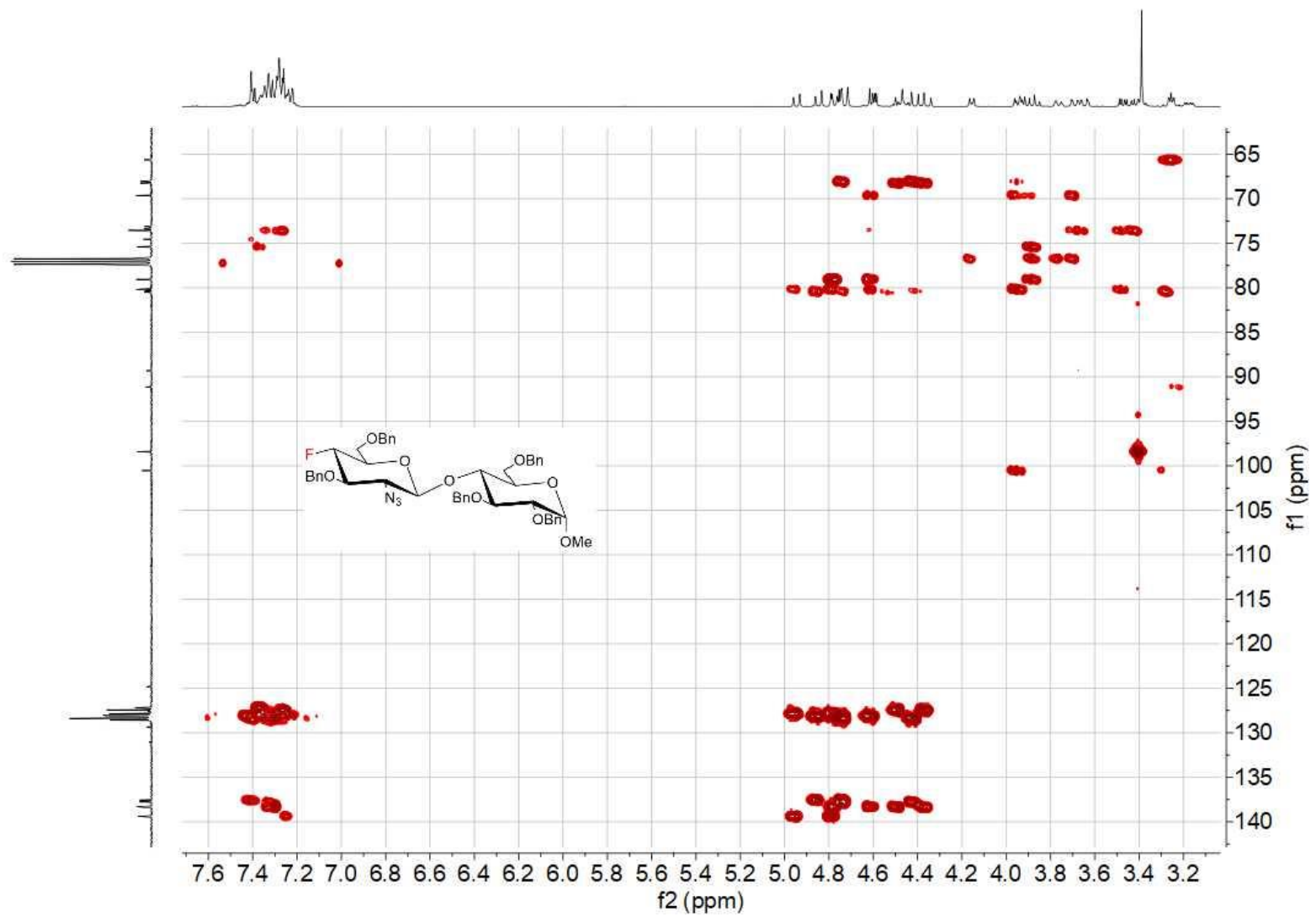


${ }^{19} \mathrm{~F} \mathrm{NMR} 376 \mathrm{MHz}, \mathrm{CDCl}_{3} \mathbf{2 E}-\beta$ and ca $9 \% \mathbf{2 E}-\alpha$

융유궈

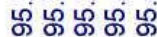

Гొน

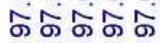

高产
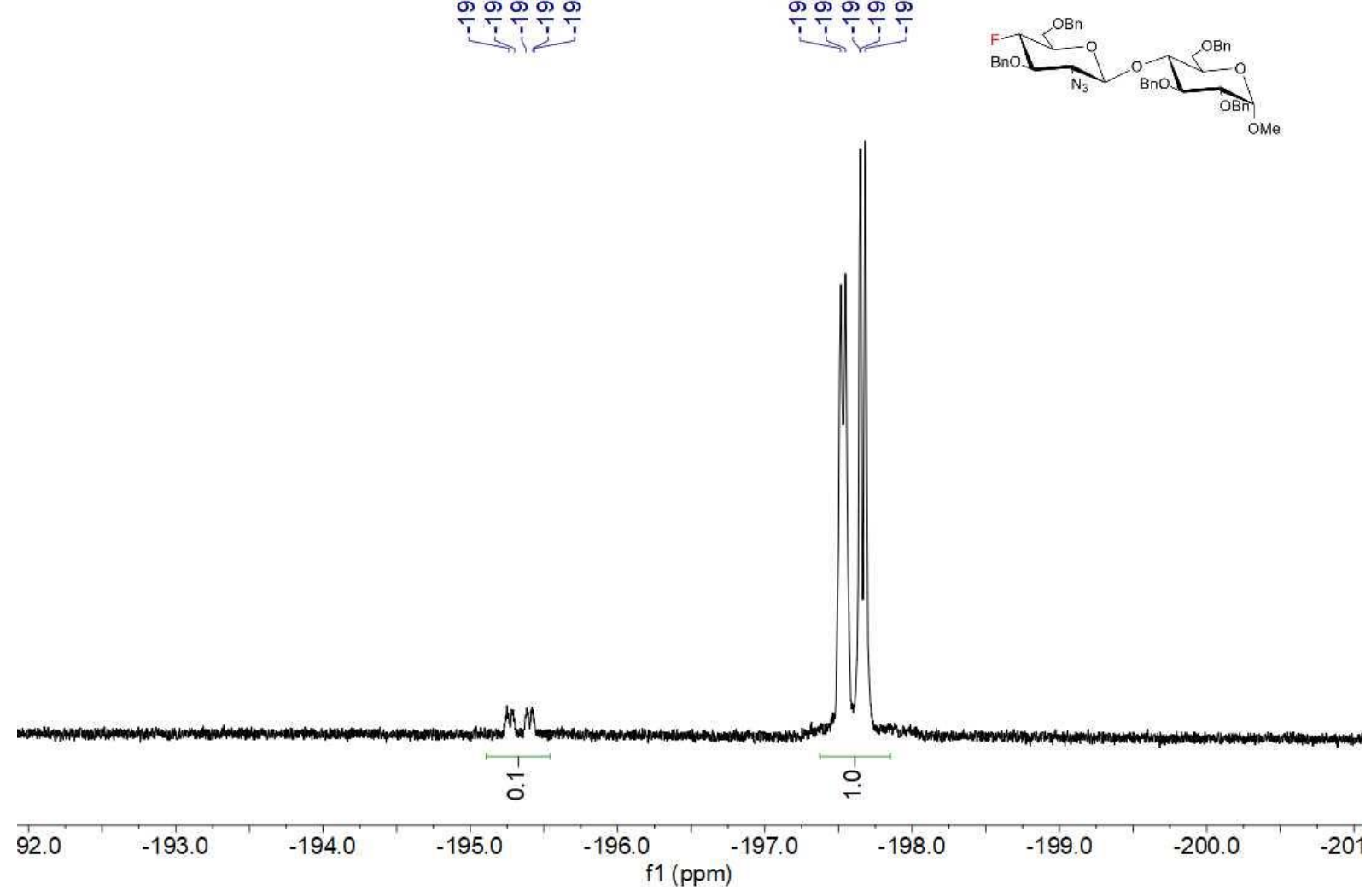

NMR 2F- $\alpha$

${ }^{1} \mathrm{HNMR} 400 \mathrm{MHz}, \mathrm{CDCl}_{3} 2 \mathrm{~F}-\alpha$

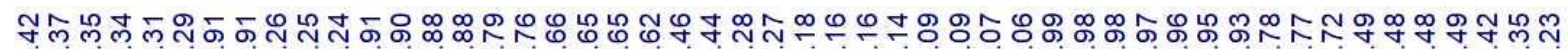

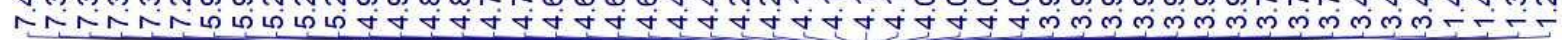

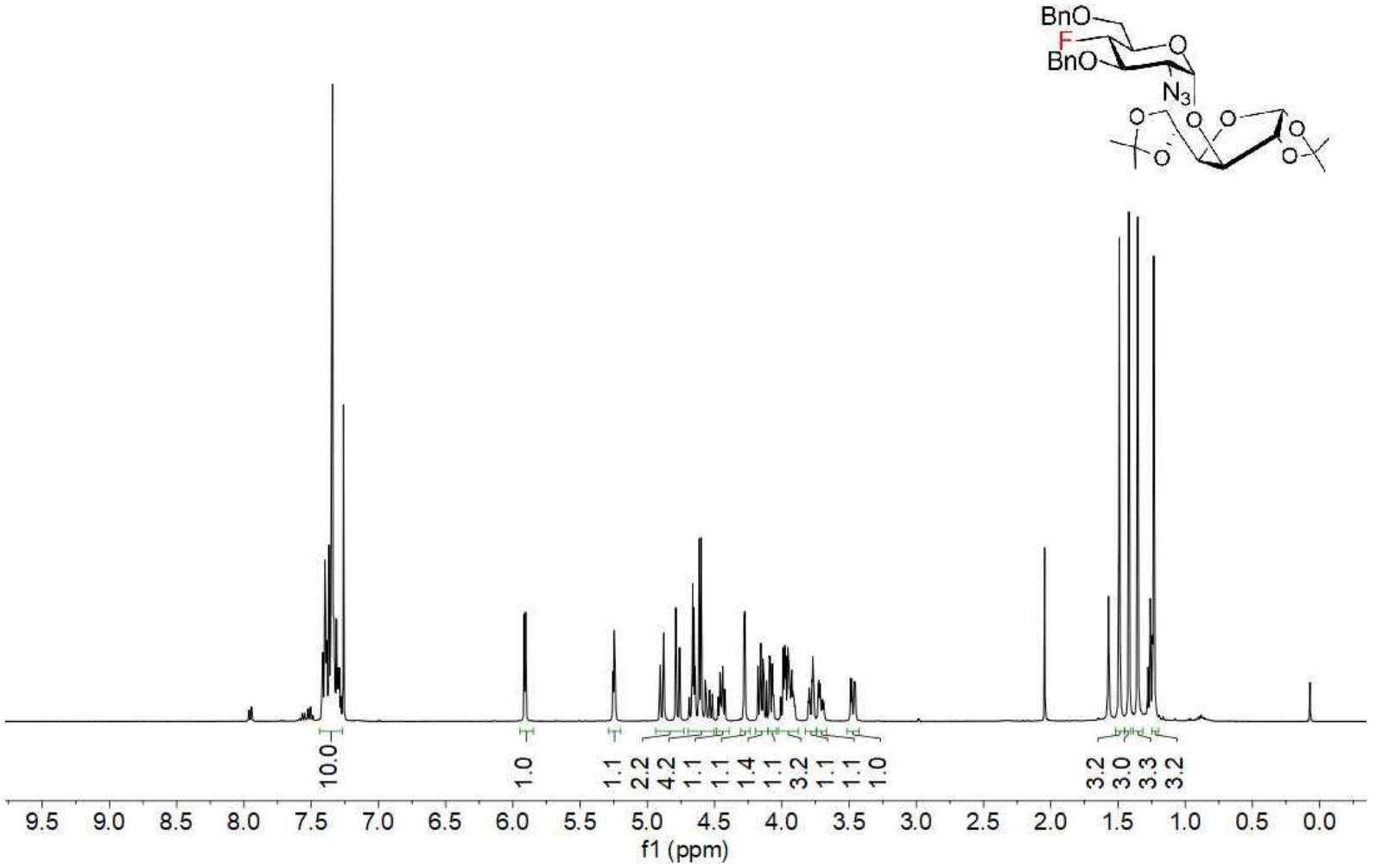


${ }^{13} \mathrm{C}\left\{{ }^{1} \mathrm{H}\right\}$ NMR $100 \mathrm{MHz}, \mathrm{CDCl}_{3} 2 \mathrm{~F}-\alpha$

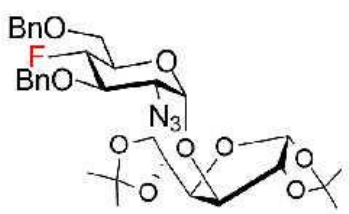

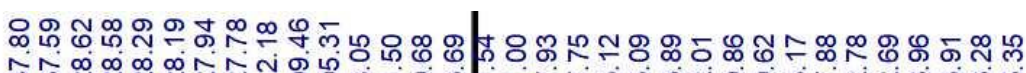

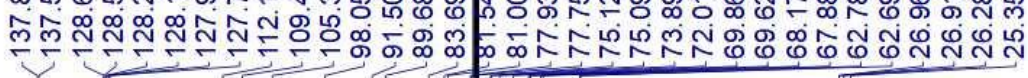

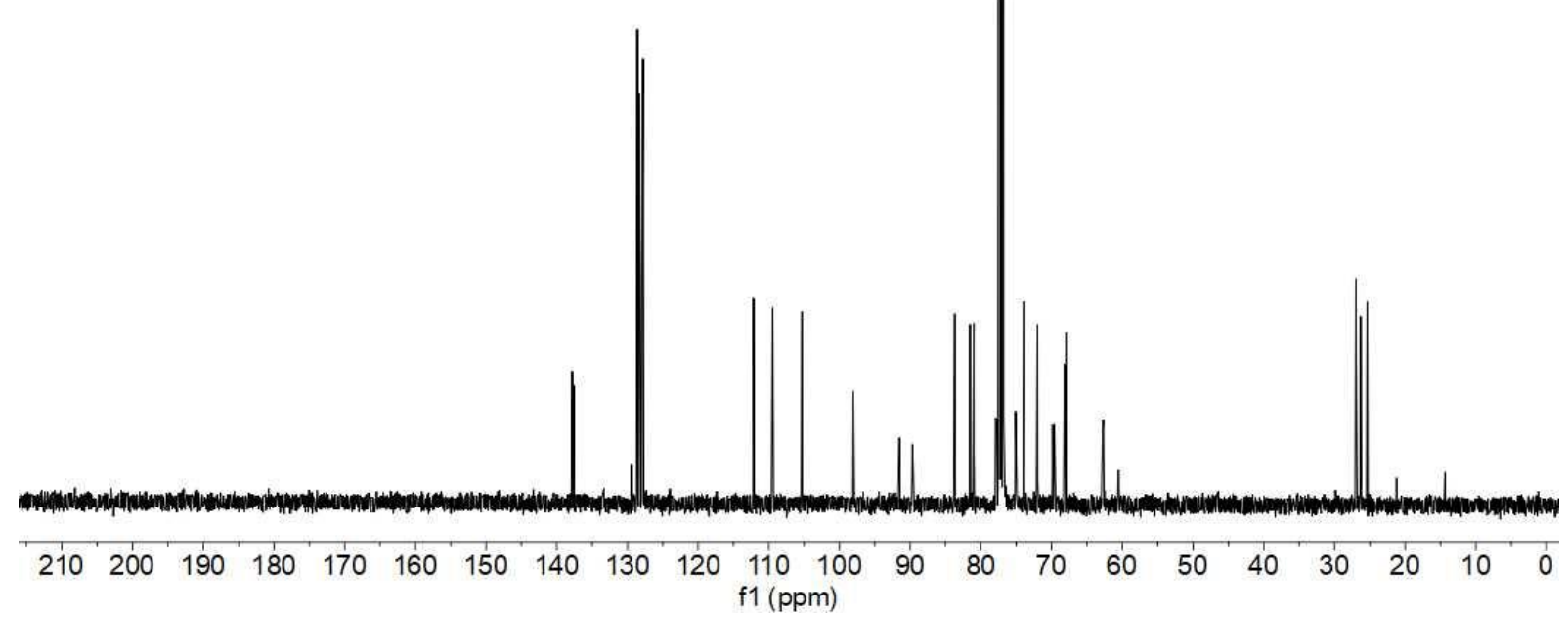

${ }^{1} \mathrm{H}-{ }^{1} \mathrm{H} \cos Y$ NMR $2 \mathrm{~F}-\alpha$

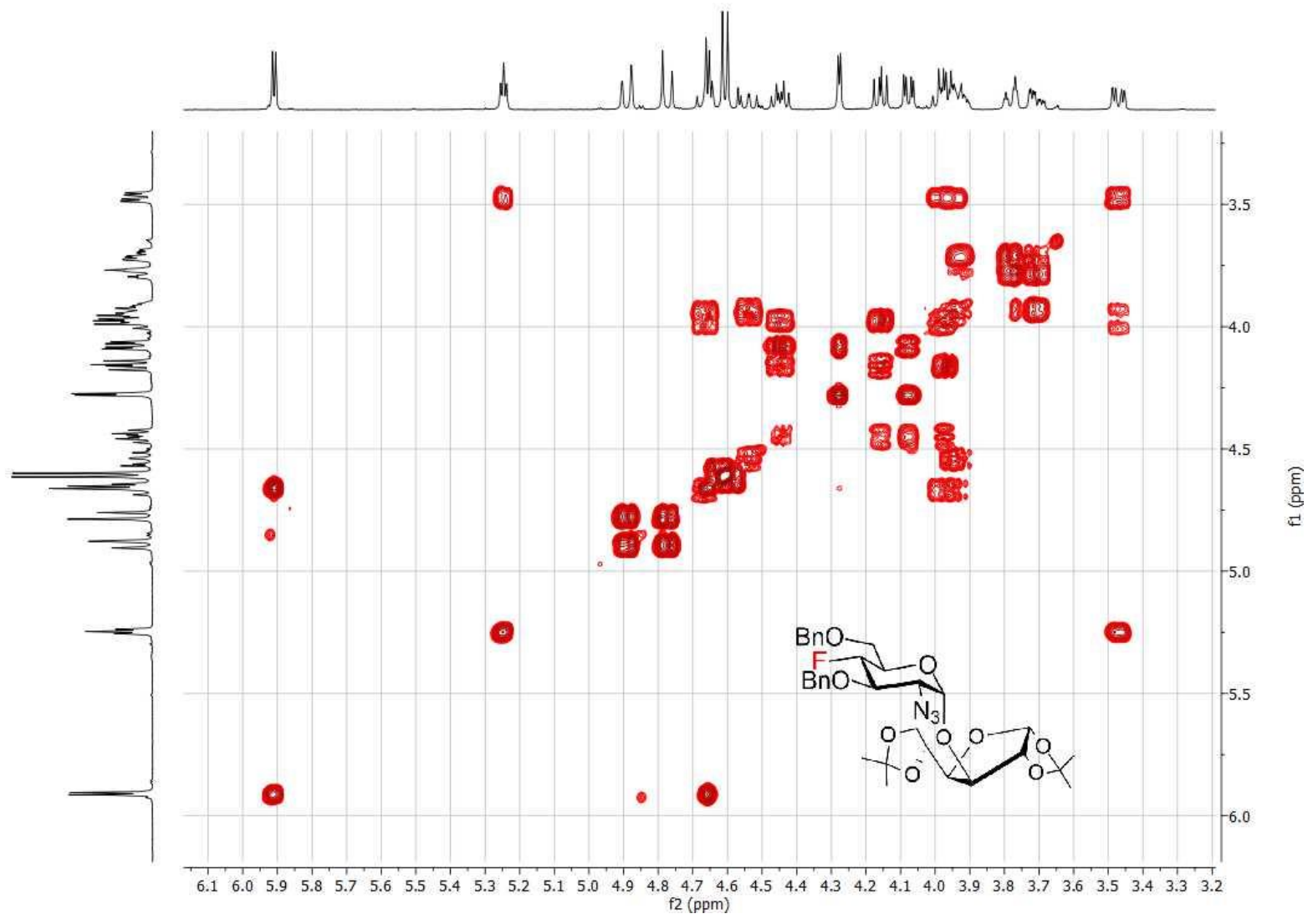


${ }^{1} \mathrm{H}-{ }^{13} \mathrm{C}$ HSQC NMR $2 \mathrm{~F}-\boldsymbol{\alpha}$

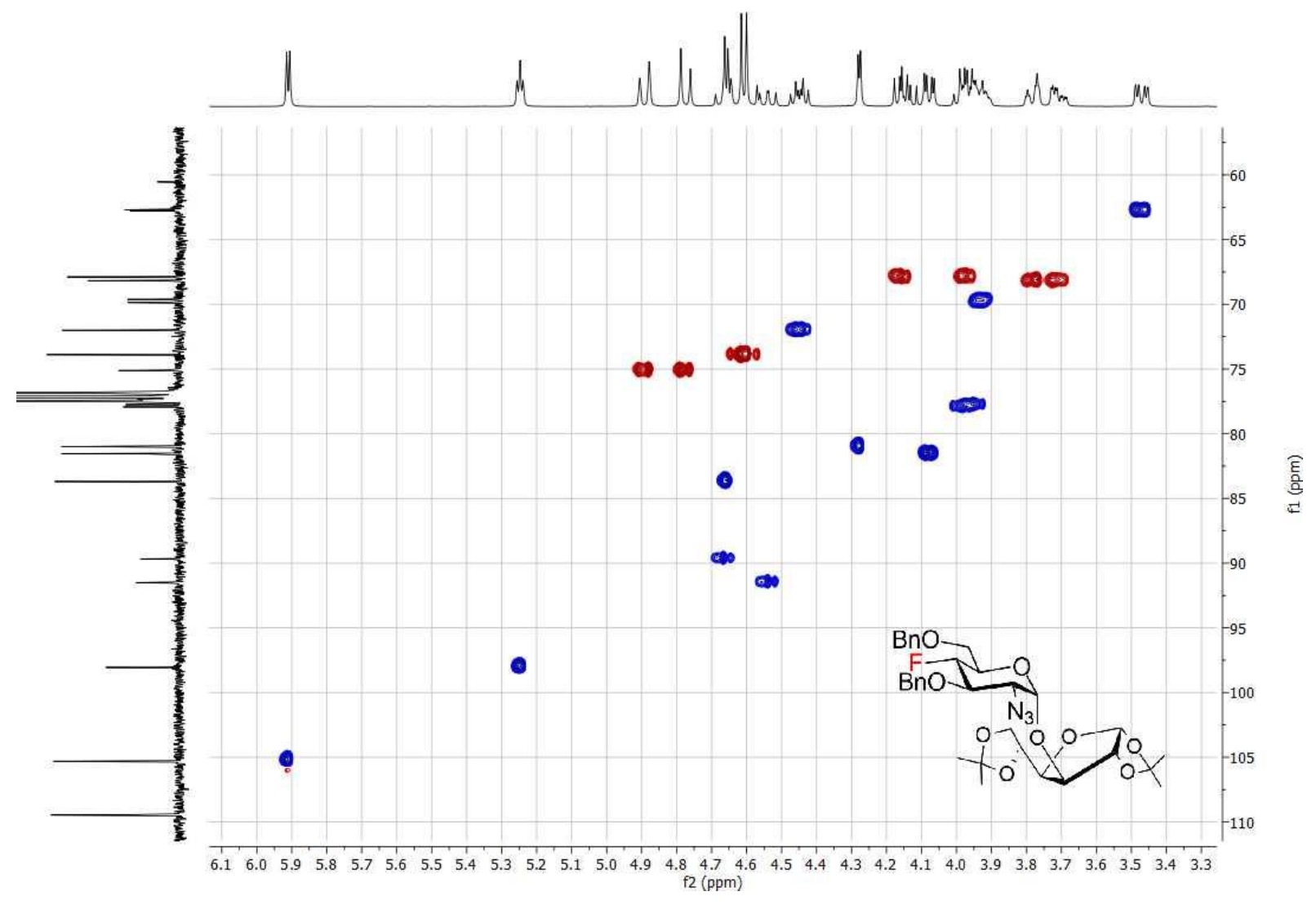

${ }^{1} \mathrm{H}-{ }^{13} \mathrm{C}$ HMBC NMR 2F- $\alpha$

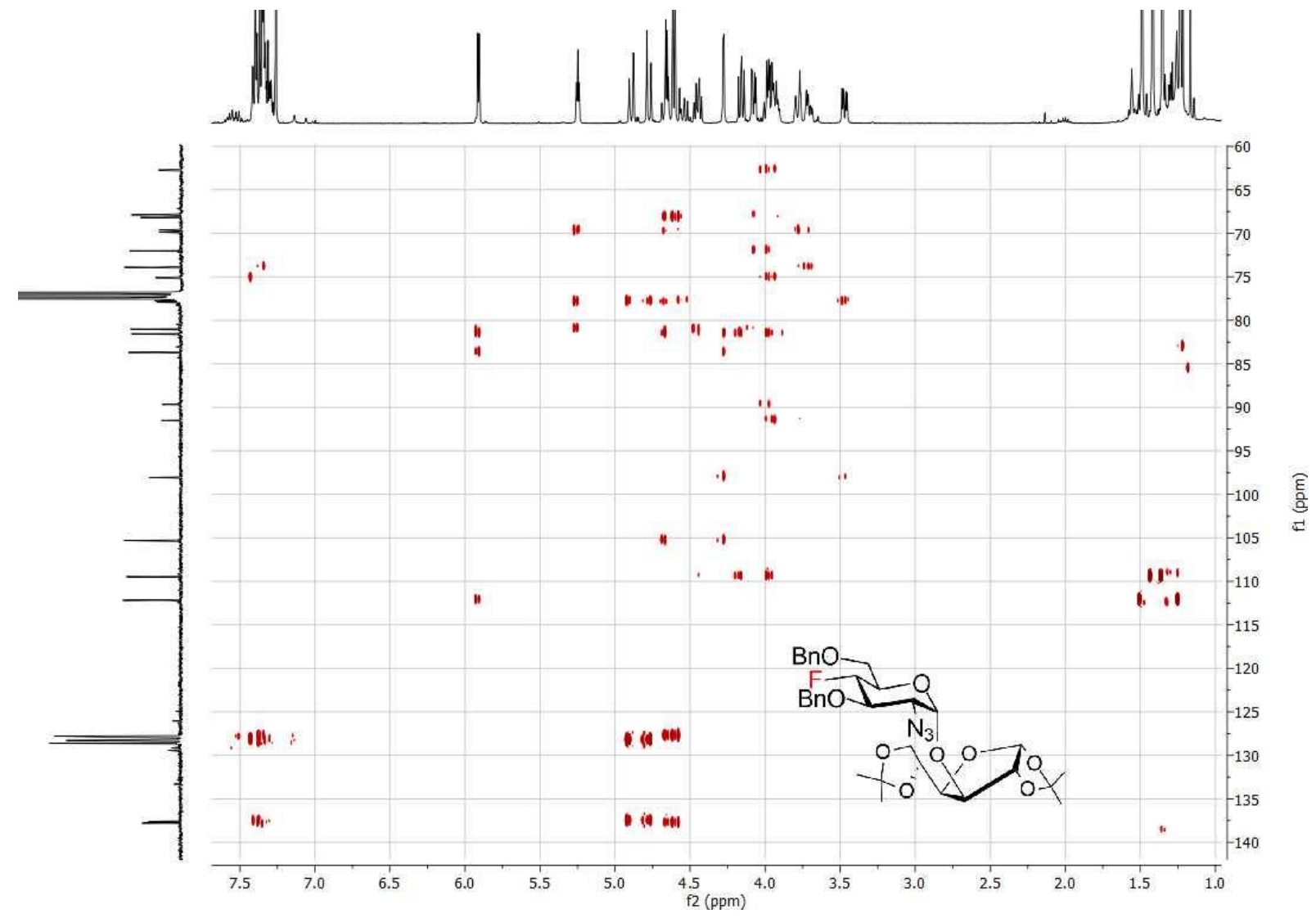


${ }^{19} \mathrm{~F} \mathrm{NMR} 376 \mathrm{MHz}, \mathrm{CDCl}_{3} 2 \mathrm{~F}-\alpha$

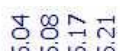

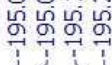

$\mathrm{BnO}-$

的

+.

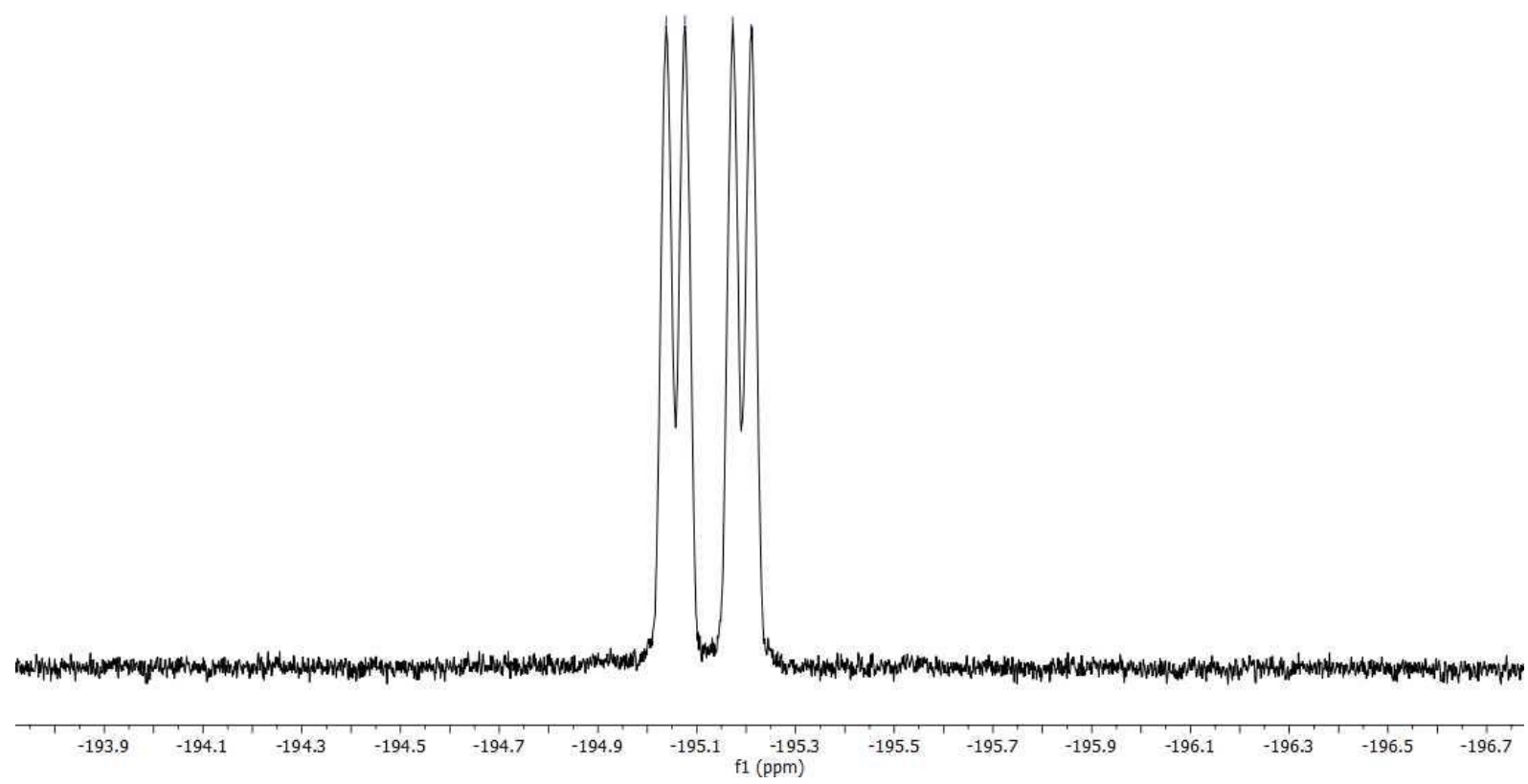

NMR $2 F-\beta$

${ }^{1} \mathrm{HNMR} 400 \mathrm{MHz}, \mathrm{CDCl}_{3} 2 \mathrm{~F}-\beta$

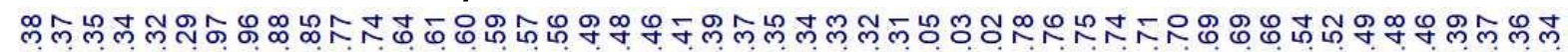

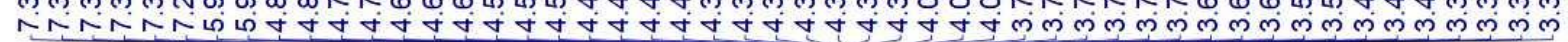

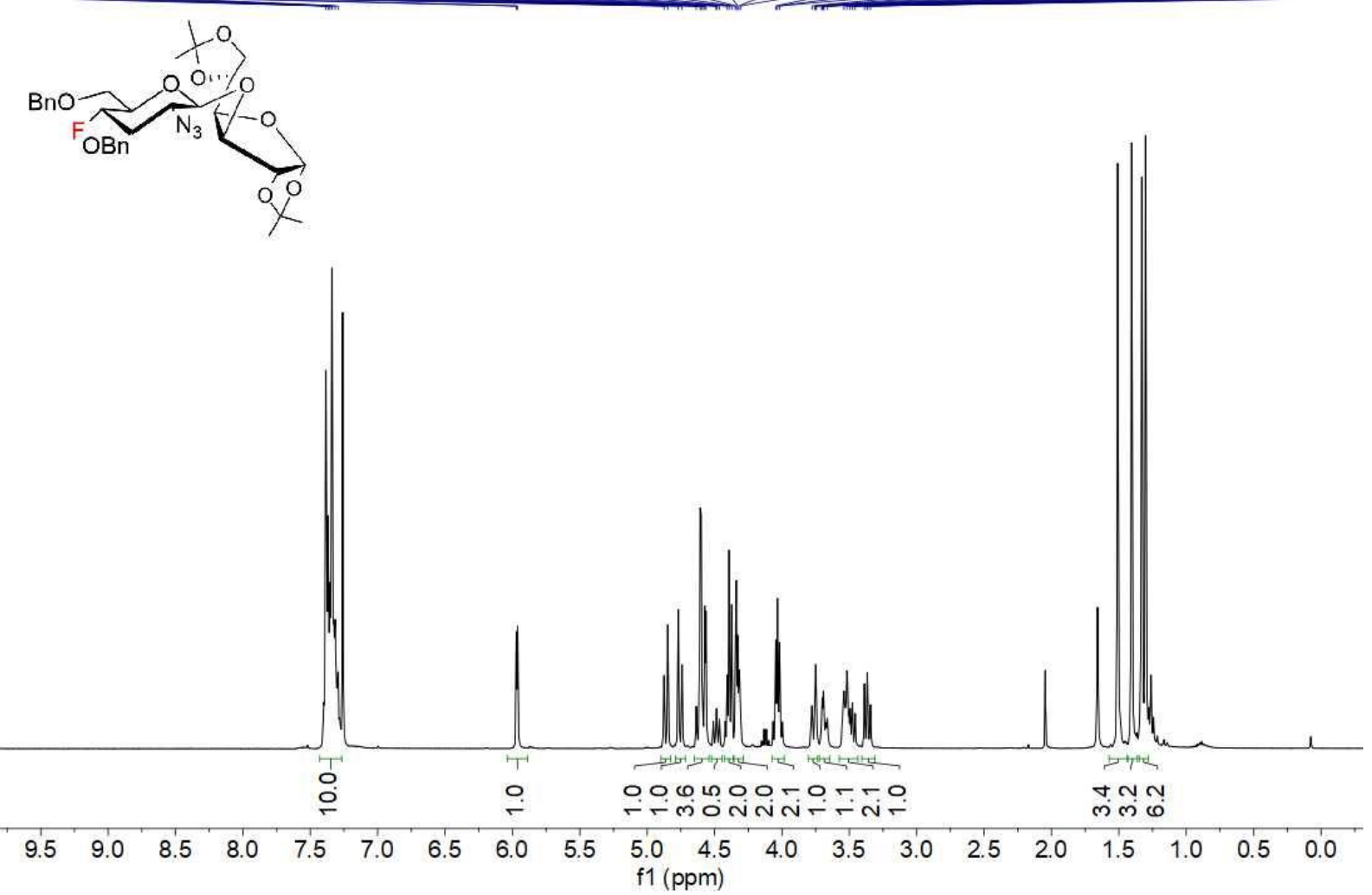


${ }^{13} \mathrm{C}\left\{{ }^{1} \mathrm{H}\right\}$ NMR $100 \mathrm{MHz}, \mathrm{CDCl}_{3} 2 \mathrm{~F}-\boldsymbol{\beta}$

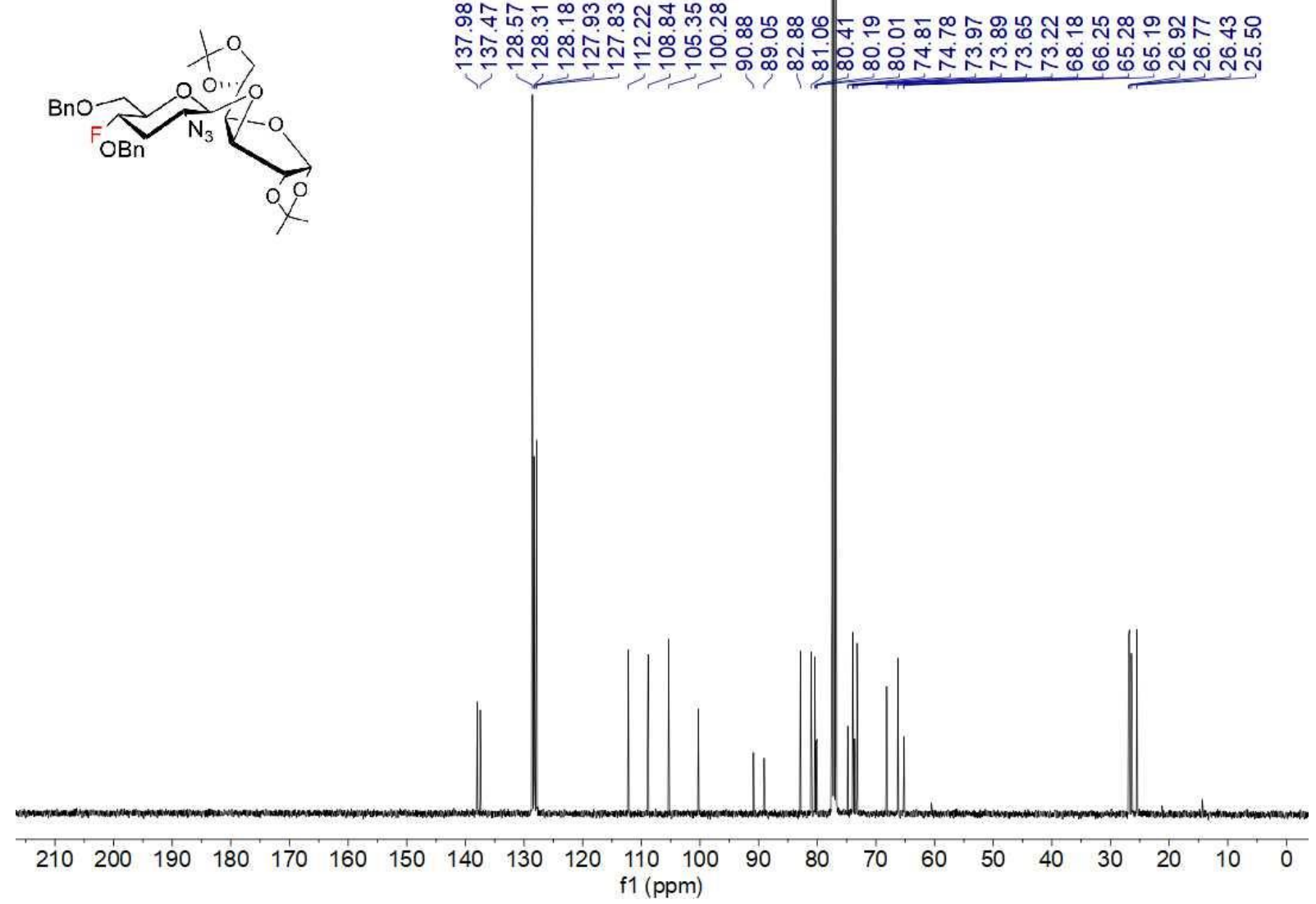

${ }^{1} \mathrm{H}-{ }^{1} \mathrm{H}$ COSY NMR $2 \mathrm{~F}-\boldsymbol{\beta}$

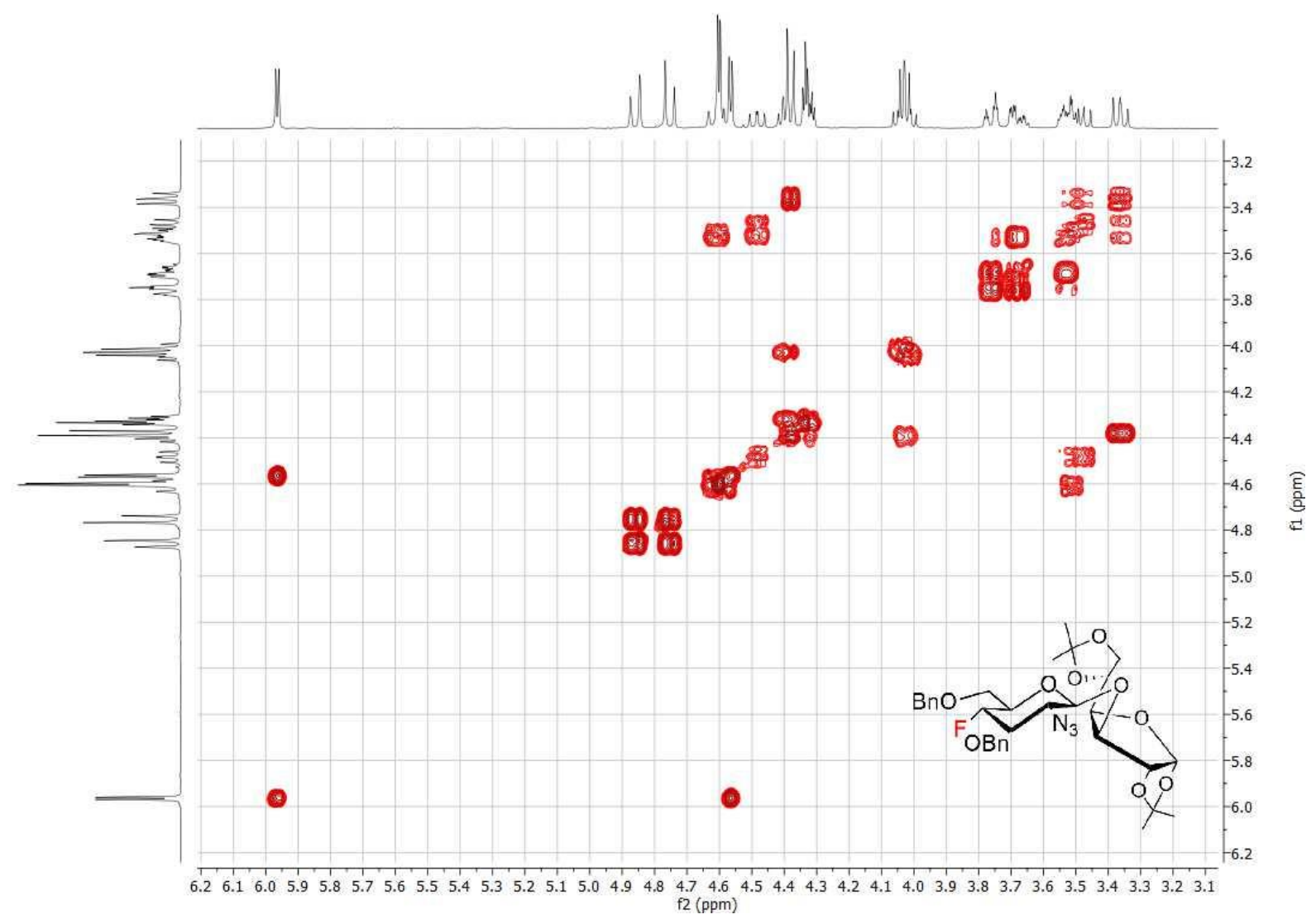


${ }^{1} \mathrm{H}-{ }^{13} \mathrm{C}$ HSQC NMR $2 \mathrm{~F}-\boldsymbol{\beta}$

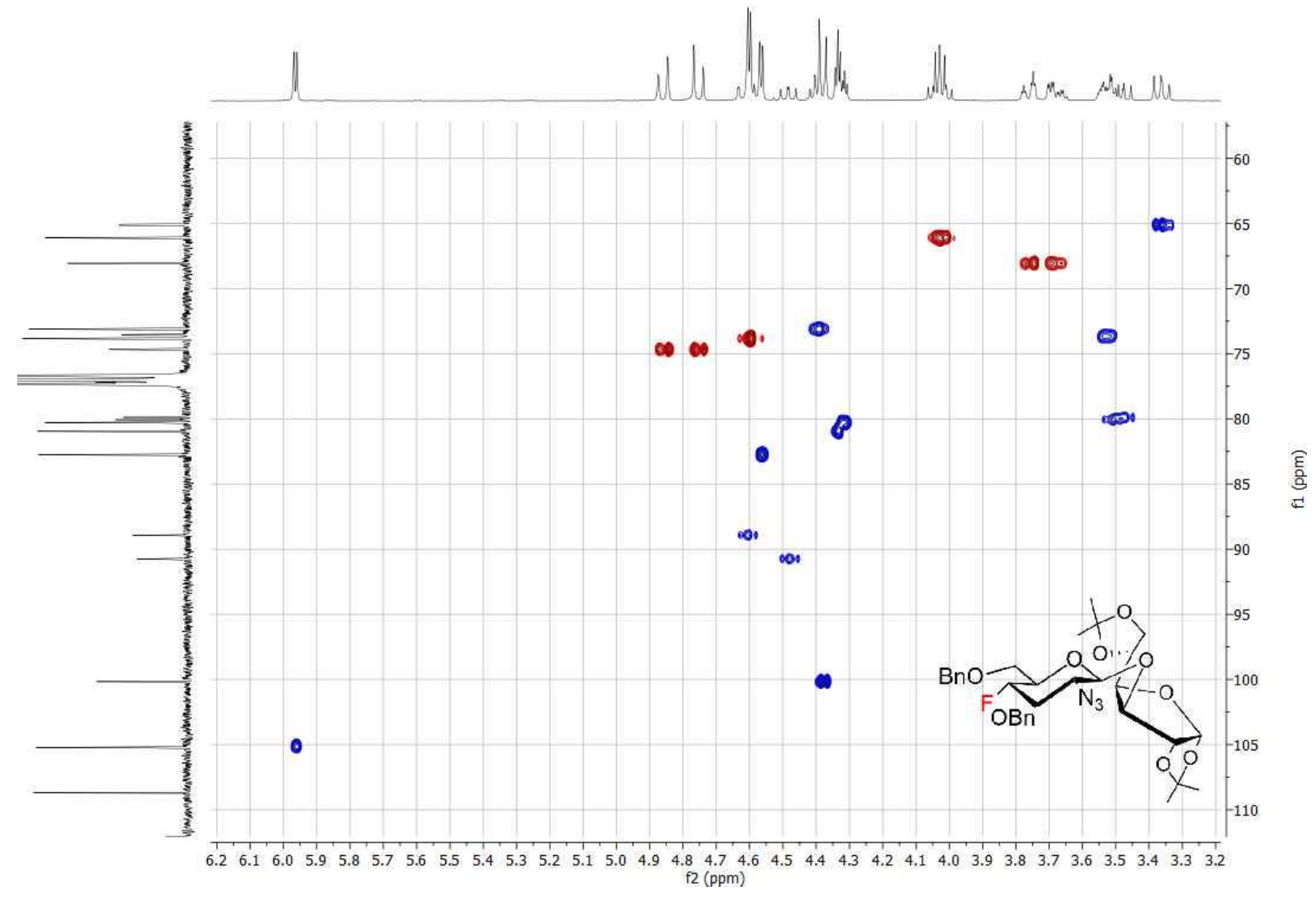

${ }^{1} \mathrm{H}-{ }^{13} \mathrm{C}$ HMBC NMR 2F- $\beta$

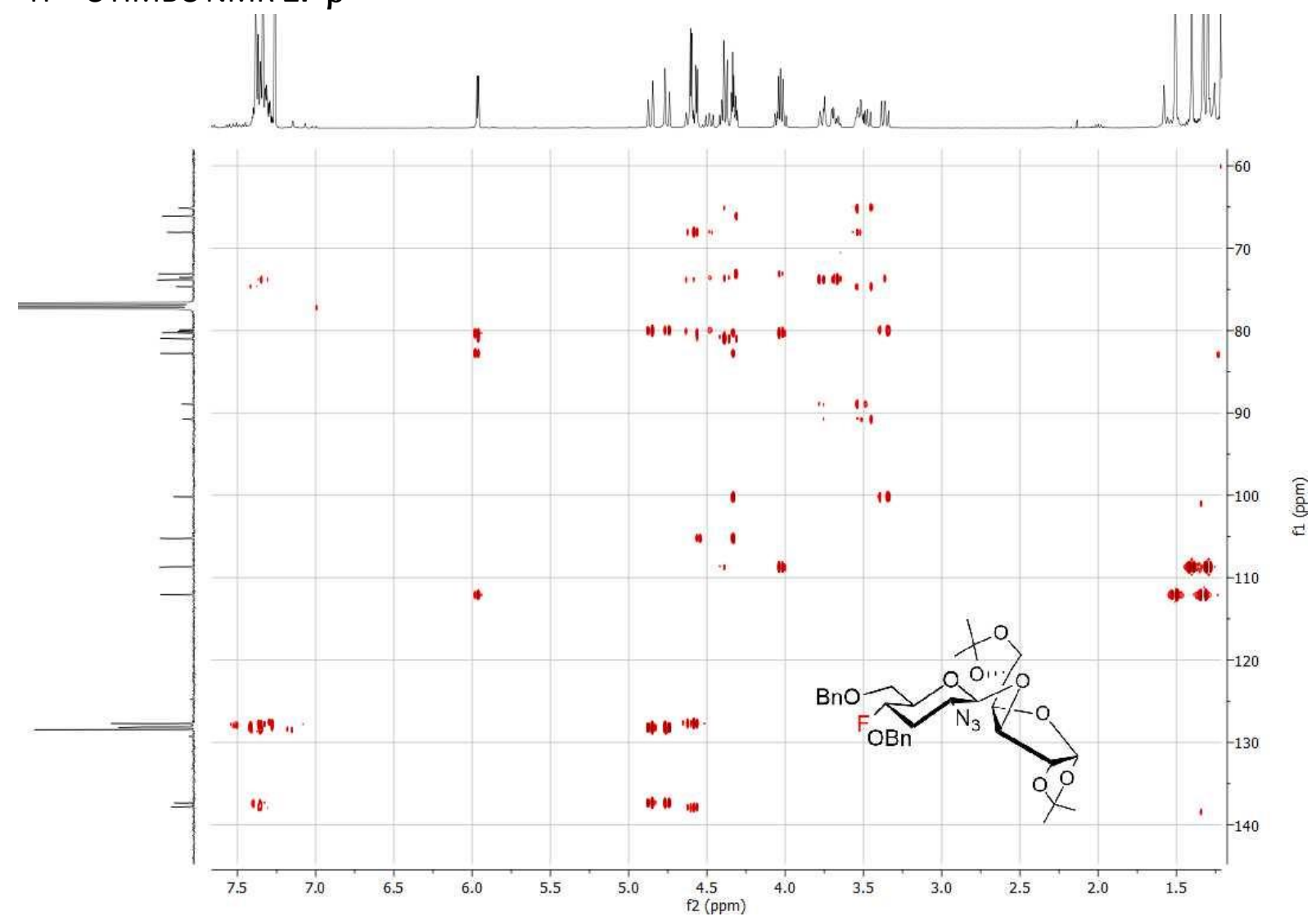


${ }^{19} \mathrm{~F} \mathrm{NMR} 376 \mathrm{MHz}, \mathrm{CDCl}_{3} 2 \mathrm{~F}-\boldsymbol{\beta}$

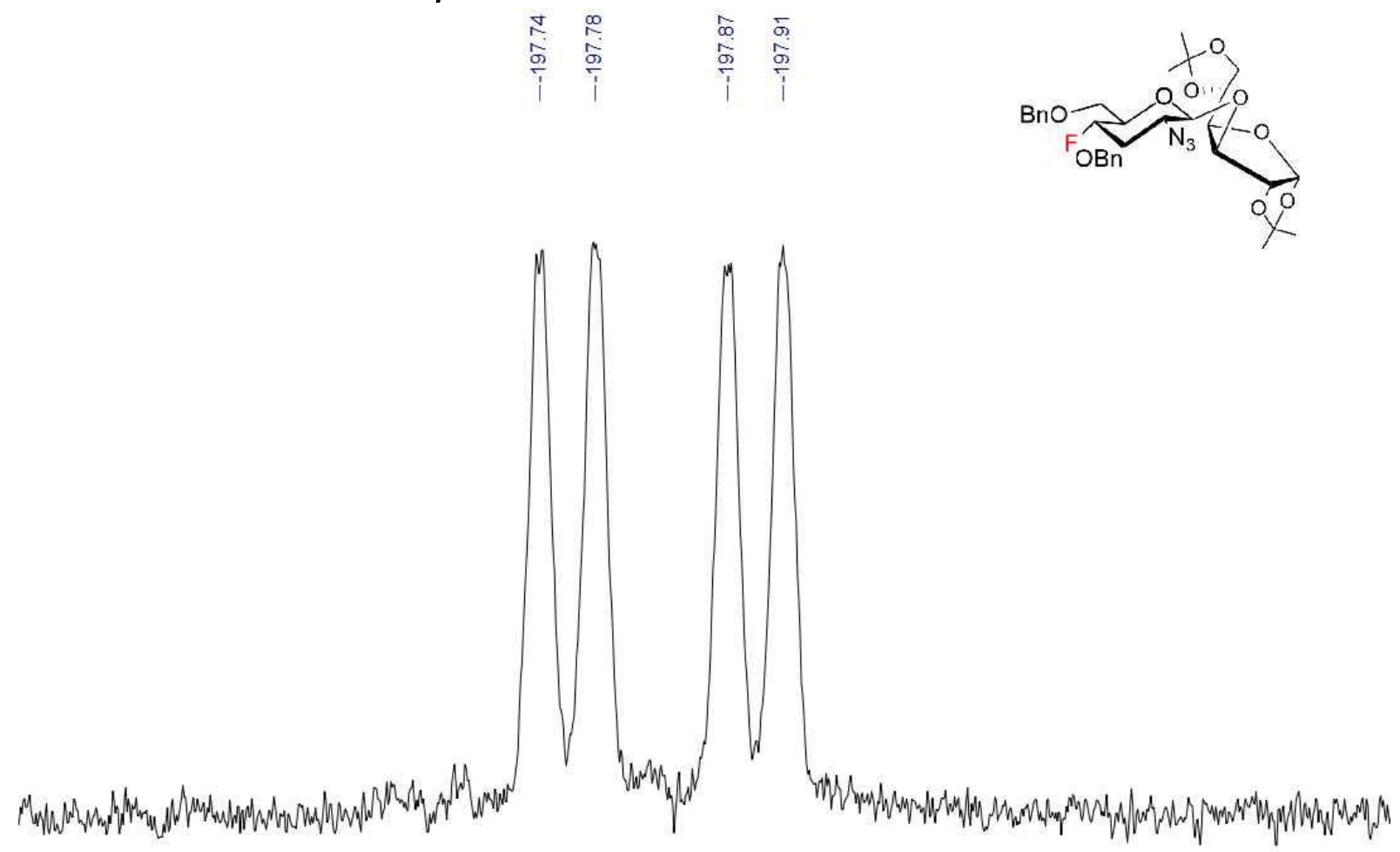

\begin{tabular}{|c|c|c|c|c|c|c|c|c|}
\hline-197.40 & -197.50 & -197.60 & -197.70 & -197.80 & $\begin{array}{l}-197.90 \\
\text { pm) }\end{array}$ & -198.00 & -198.10 & -198.20 \\
\hline
\end{tabular}

\section{NMR 2G}

${ }^{1} \mathrm{HNMR} 400 \mathrm{MHz}, \mathrm{CDCl}_{3} 2 \mathrm{G}(\alpha: \beta=1.0: 2.5)$

テ

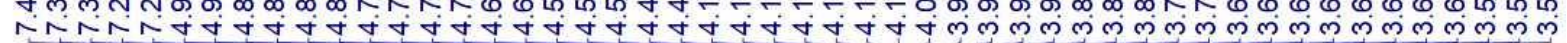

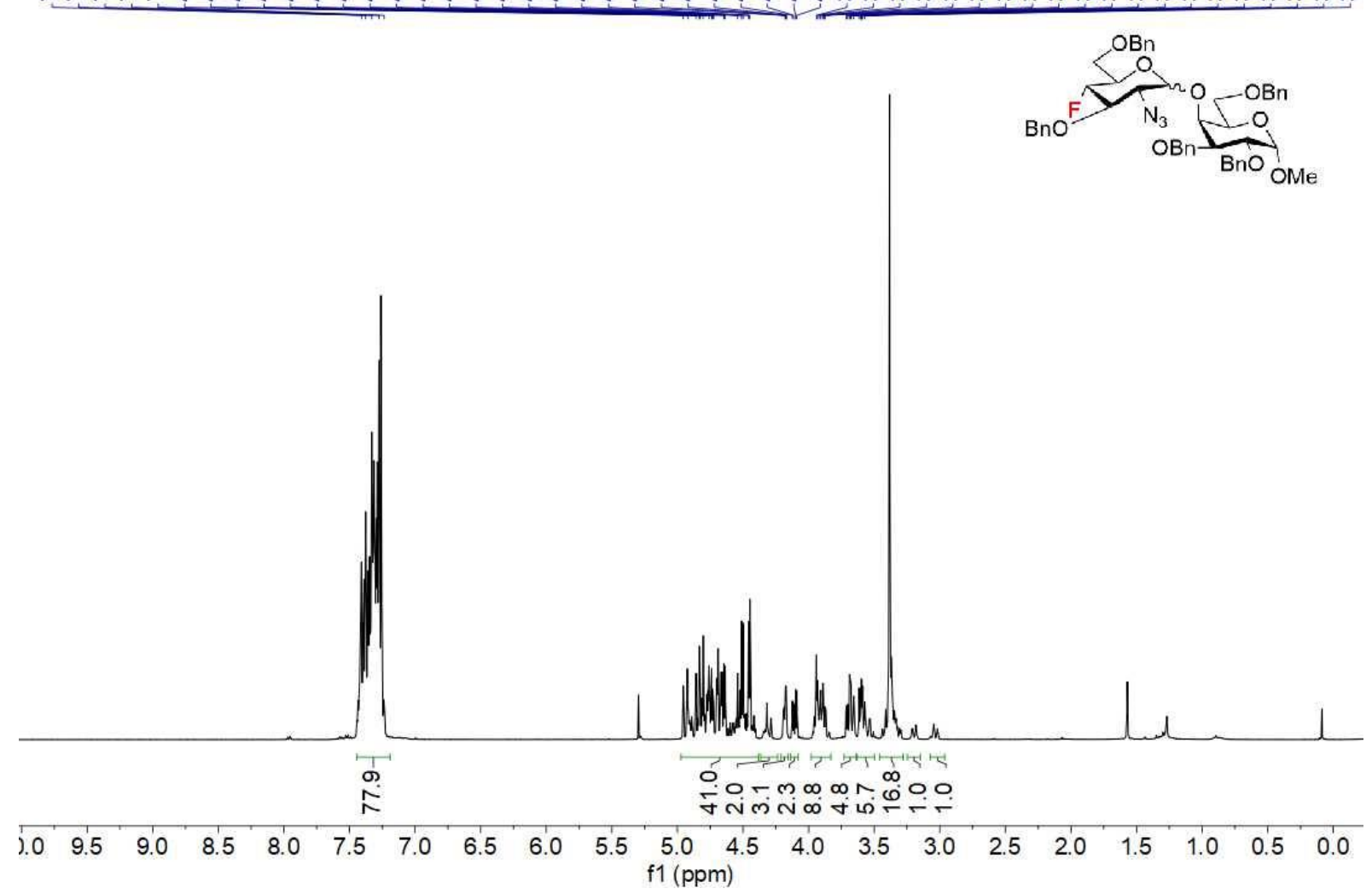


${ }^{13} \mathrm{C}\left\{{ }^{1} \mathrm{H}\right\} \mathrm{NMR} 100 \mathrm{MHz}, \mathrm{CDCl}_{3} 2 \mathrm{G}(\alpha: \beta=1.0: 2.5)$

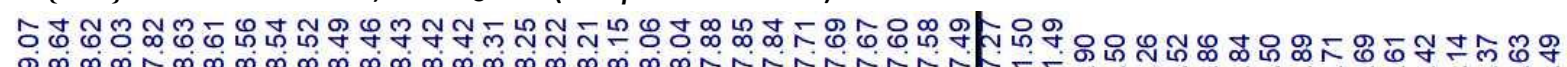

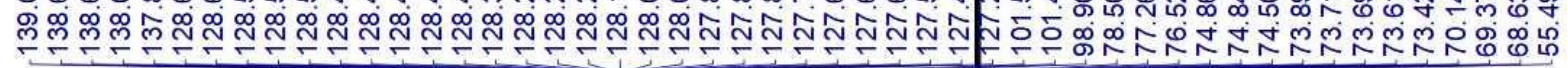

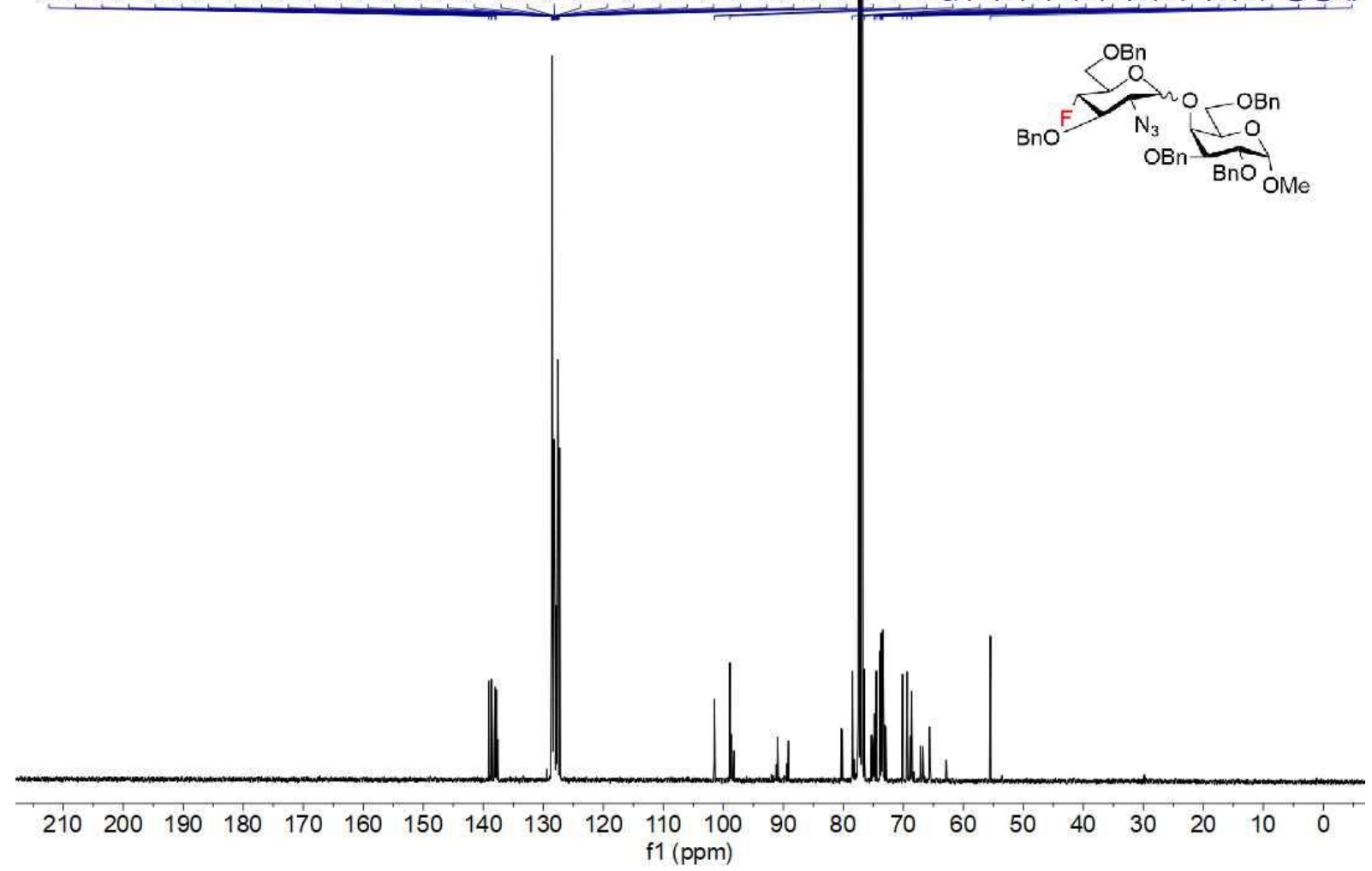

${ }^{1} \mathrm{H}-{ }^{1} \mathrm{H}$ COSY NMR $2 \mathrm{G}(\alpha: \beta=1.0: 2.5)$

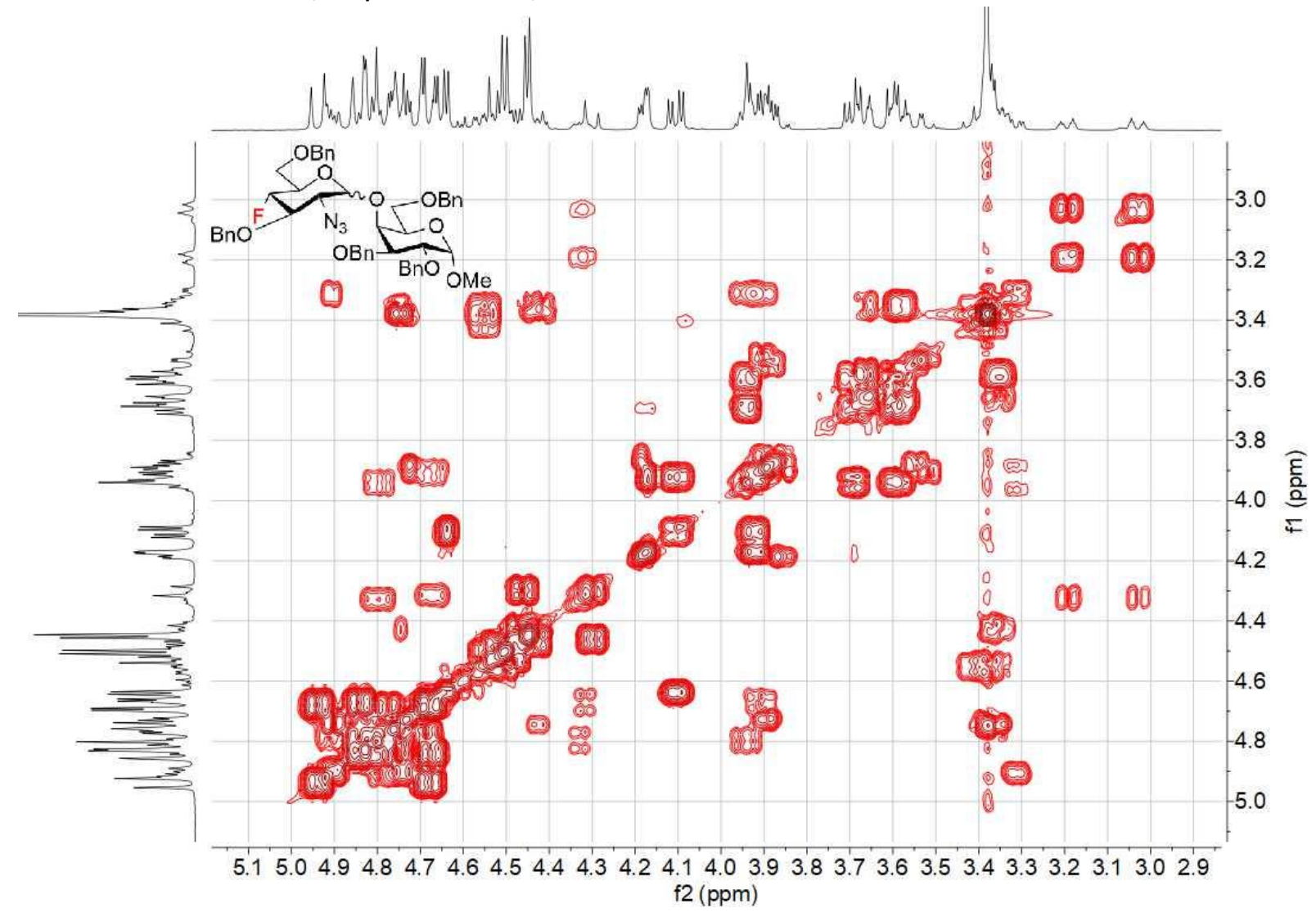


${ }^{1} \mathrm{H}-{ }^{13} \mathrm{C}$ HSQC NMR 2G $(\alpha: \beta=1.0: 2.5)$

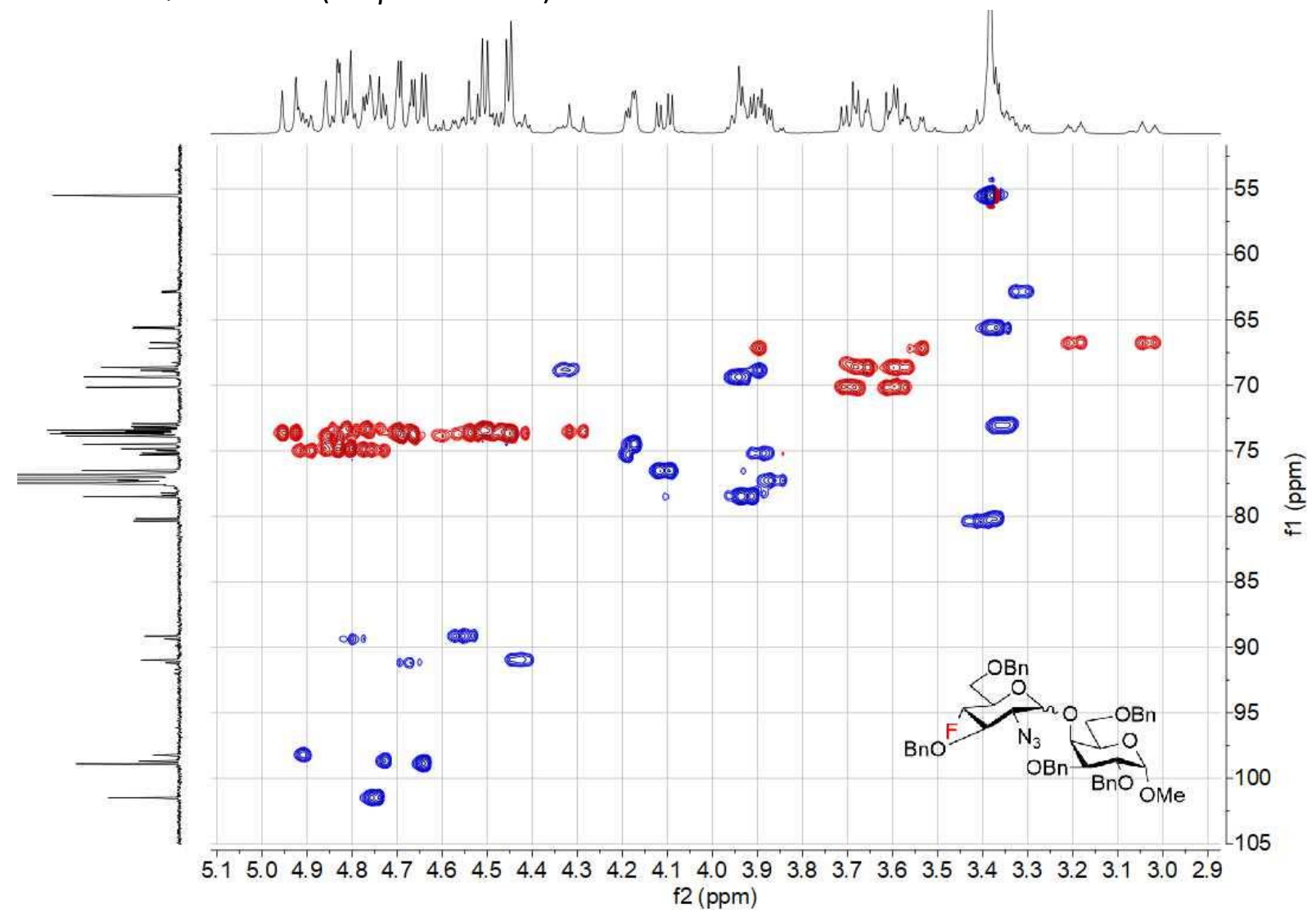

${ }^{1} \mathrm{H}-{ }^{13} \mathrm{C}$ HMBC NMR 2G $(\alpha: \beta=1.0: 2.5)$

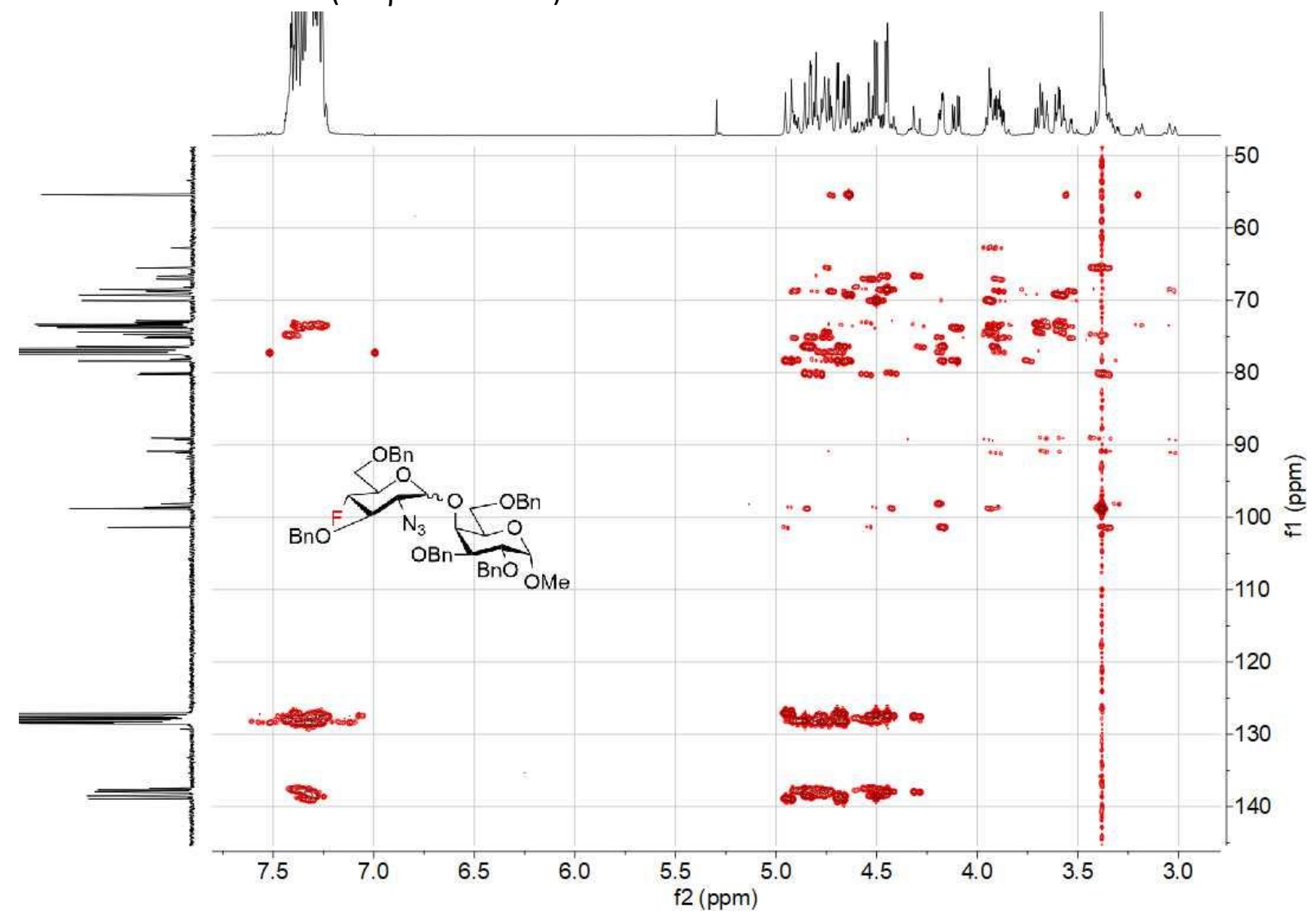




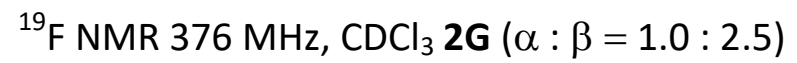

뮹주 중

ம் 붕용
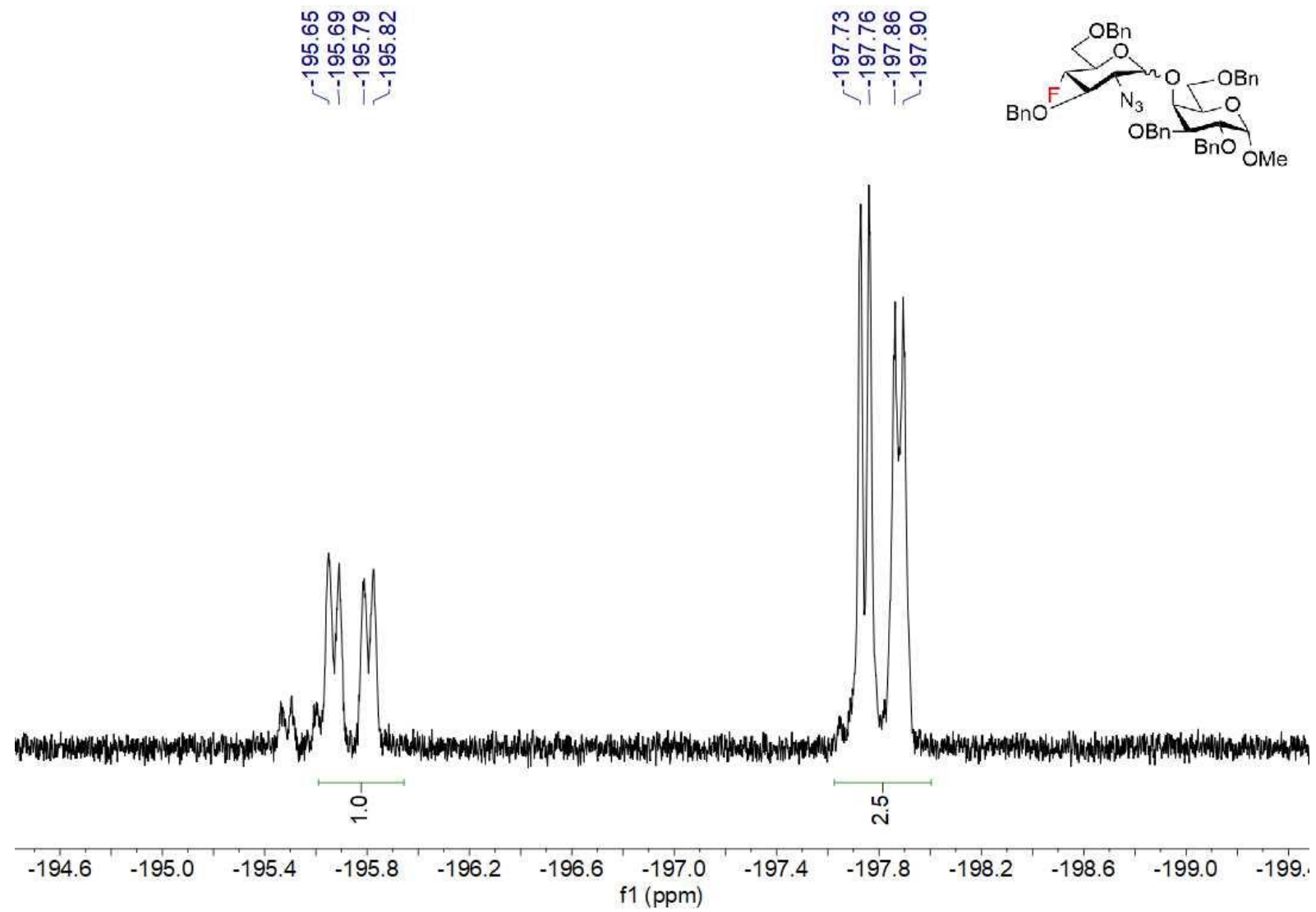

NMR $2 \mathrm{H}-\alpha$

${ }^{1} \mathrm{H} N M R 400 \mathrm{MHz}, \mathrm{CDCl}_{3} 2 \mathrm{H}-\alpha$
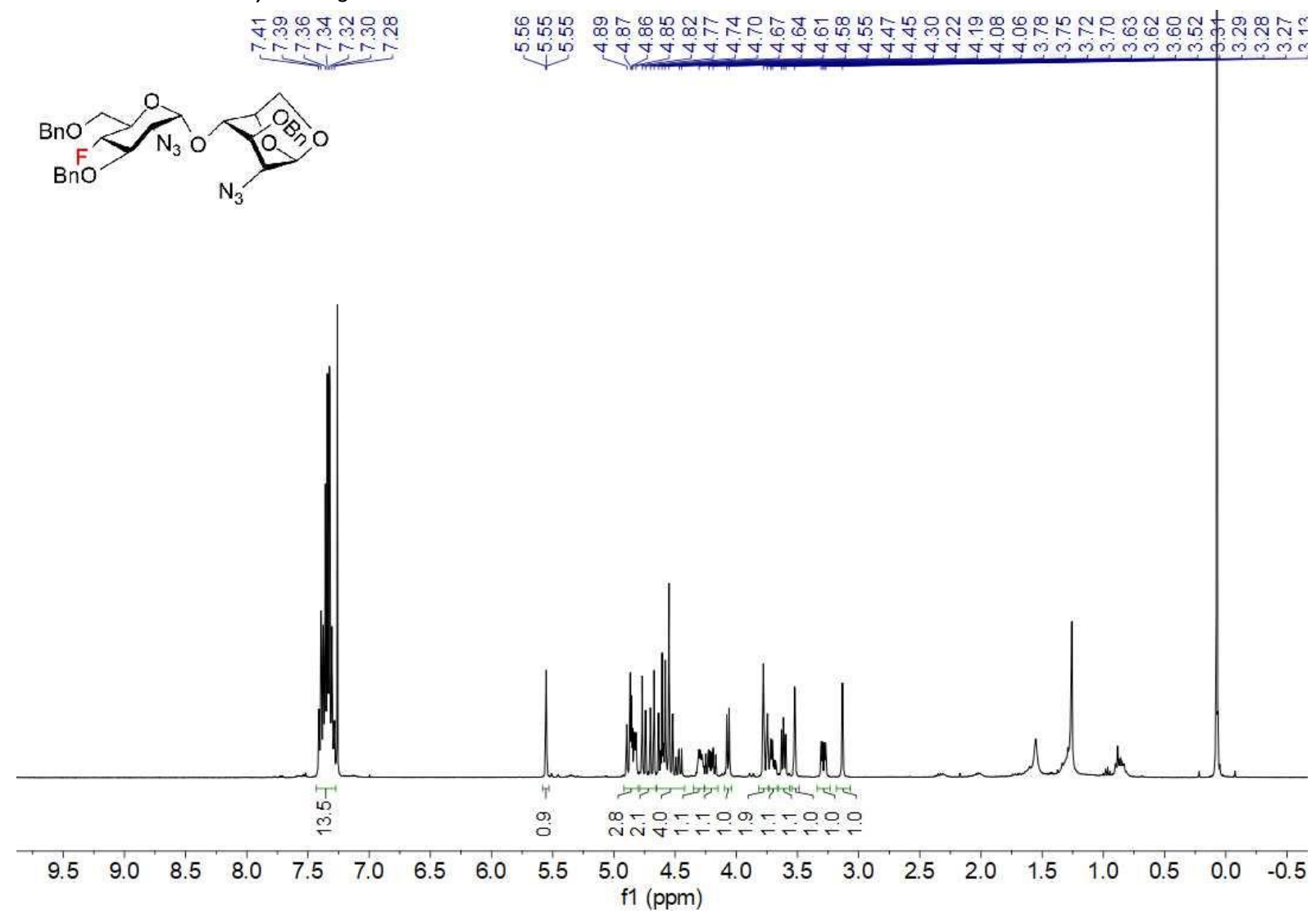


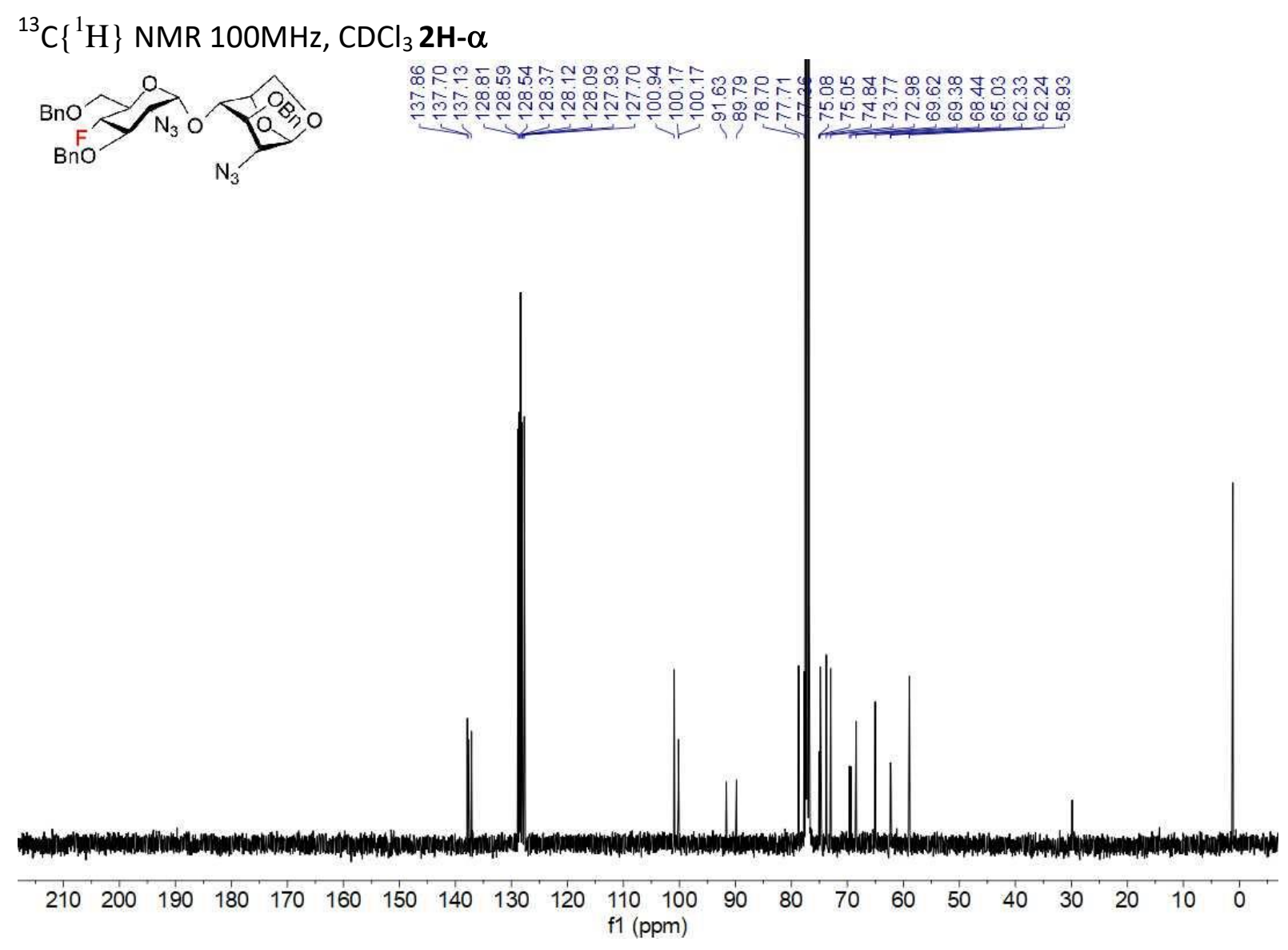

${ }^{1} \mathrm{H}-{ }^{1} \mathrm{H}$ COSY NMR $2 \mathrm{H}-\boldsymbol{\alpha}$

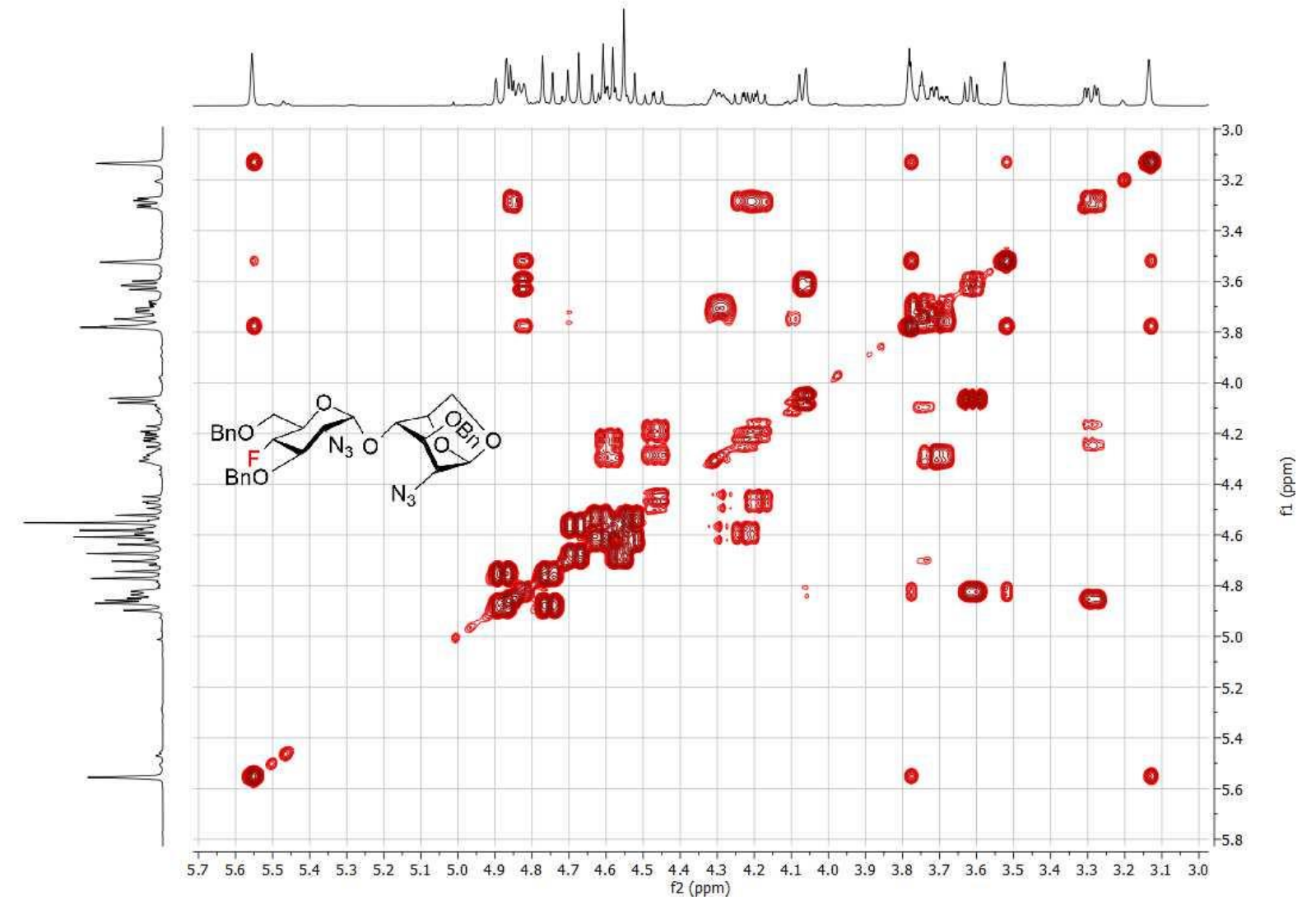


${ }^{1} \mathrm{H}-{ }^{13} \mathrm{C}$ HSQC NMR $2 \mathrm{H}-\boldsymbol{\alpha}$

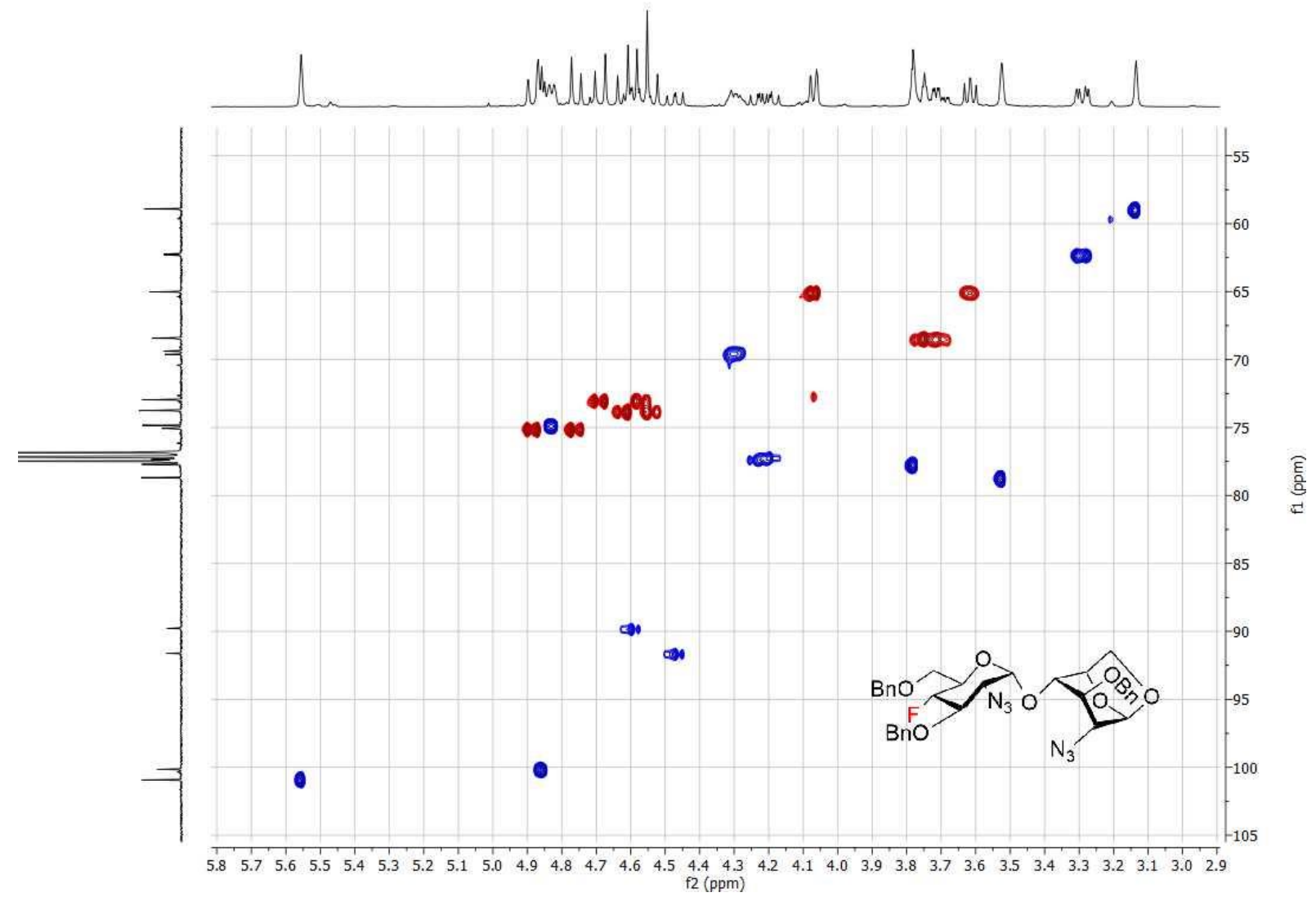

${ }^{1} \mathrm{H}-{ }^{13} \mathrm{C}$ HMBC NMR $2 \mathrm{H}-\alpha$

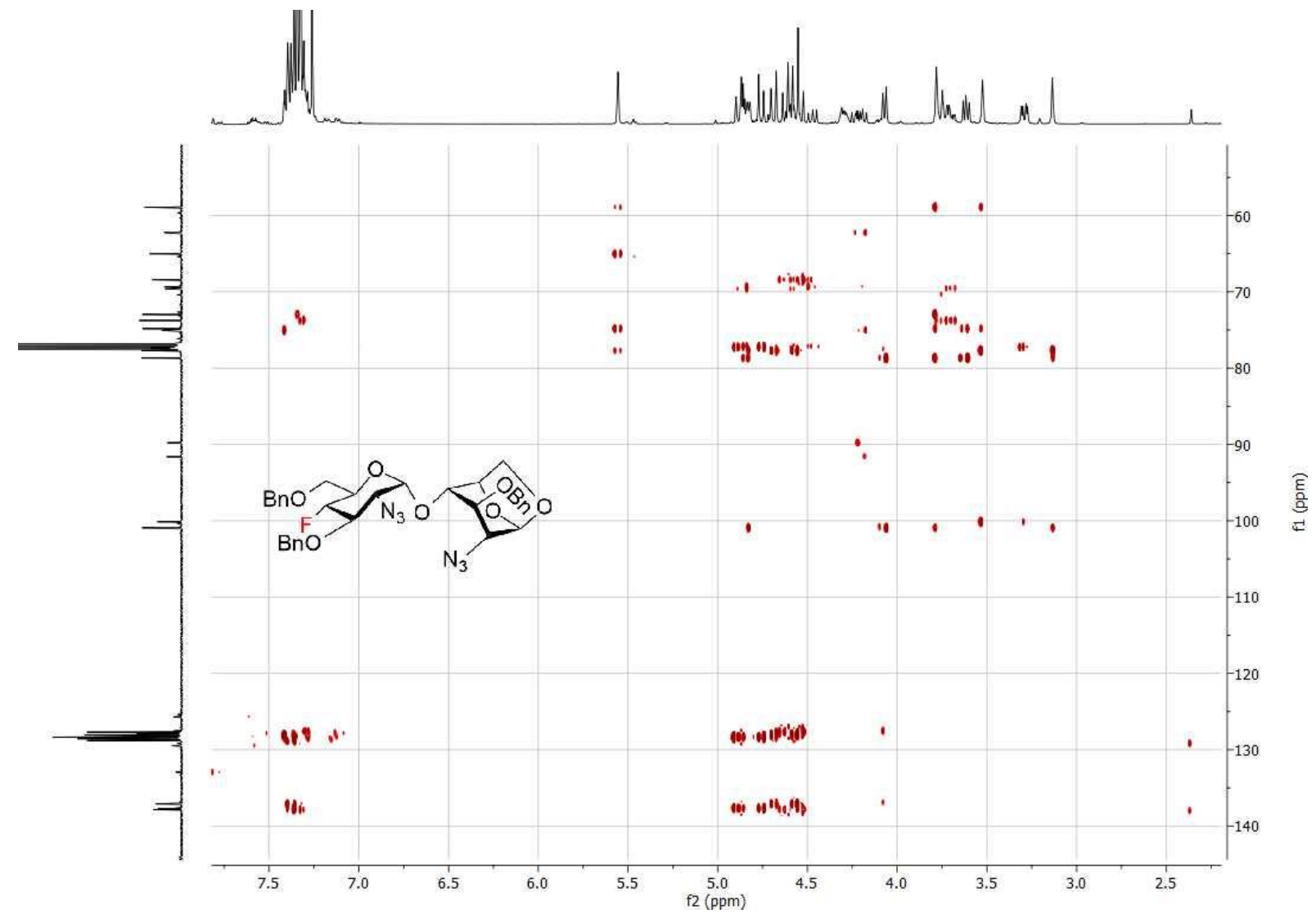


${ }^{19} \mathrm{~F}$ NMR $376 \mathrm{MHz}, \mathrm{CDCl}_{3} \mathrm{NMR} 2 \mathrm{2H}-\boldsymbol{\alpha}$

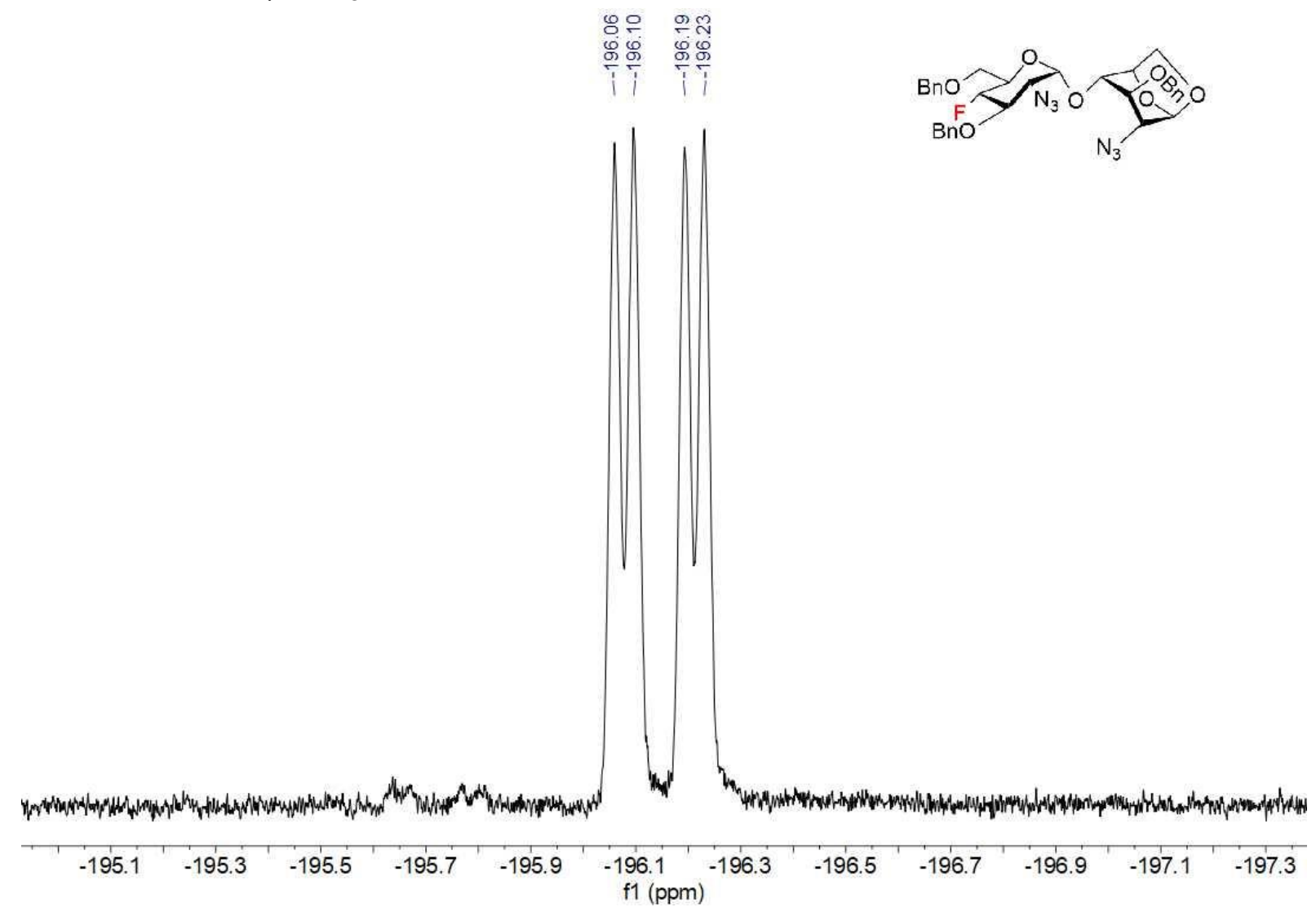

NMR $2 \mathrm{H}-\boldsymbol{\beta}$

${ }^{1} \mathrm{H} N M R 400 \mathrm{MHz}, \mathrm{CDCl}_{3} \mathbf{2 H}-\boldsymbol{\beta}$

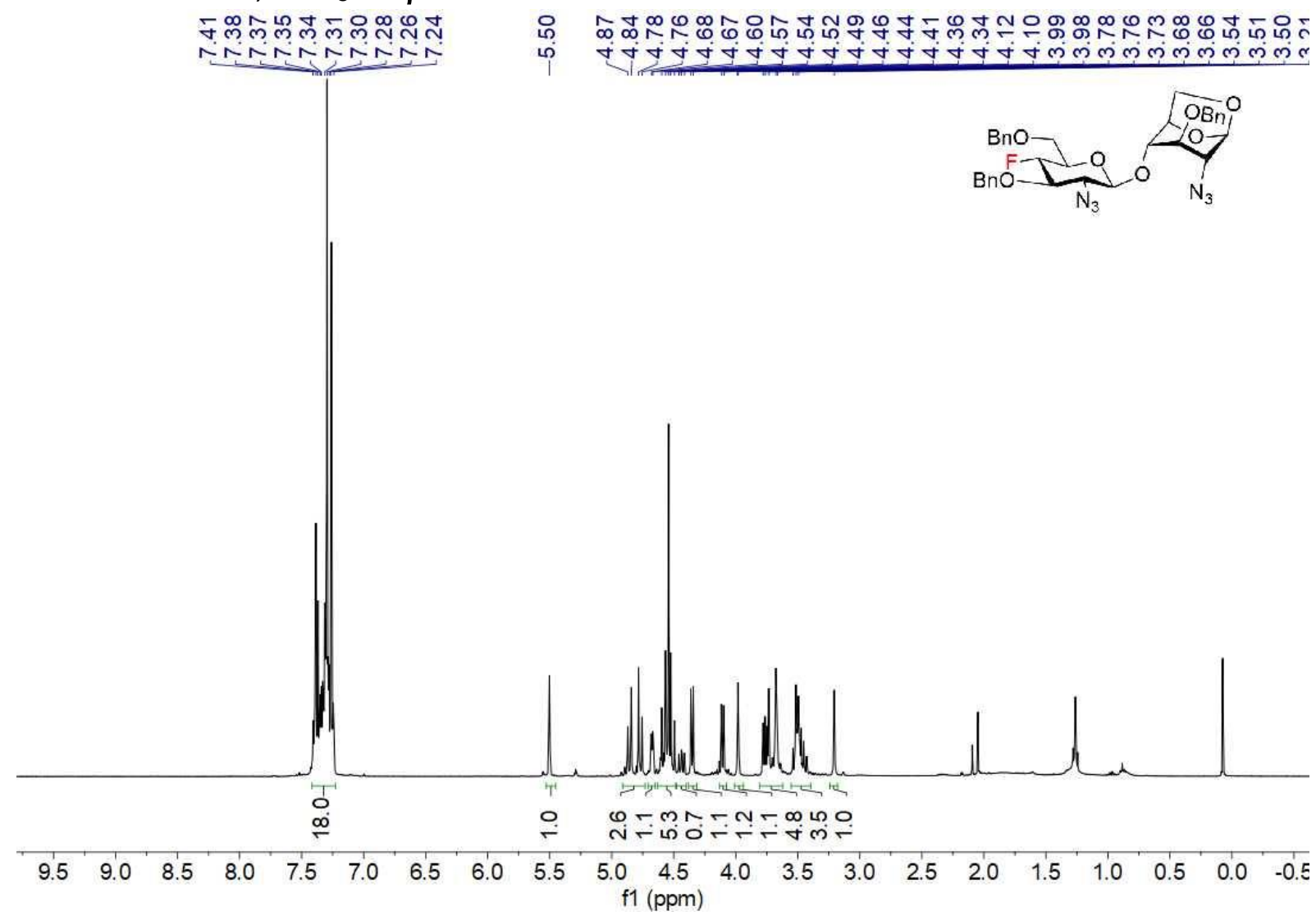


${ }^{13} \mathrm{C}\left\{{ }^{1} \mathrm{H}\right\}$ NMR $100 \mathrm{MHz}, \mathrm{CDCl}_{3} \mathbf{2 H}-\boldsymbol{\beta}$
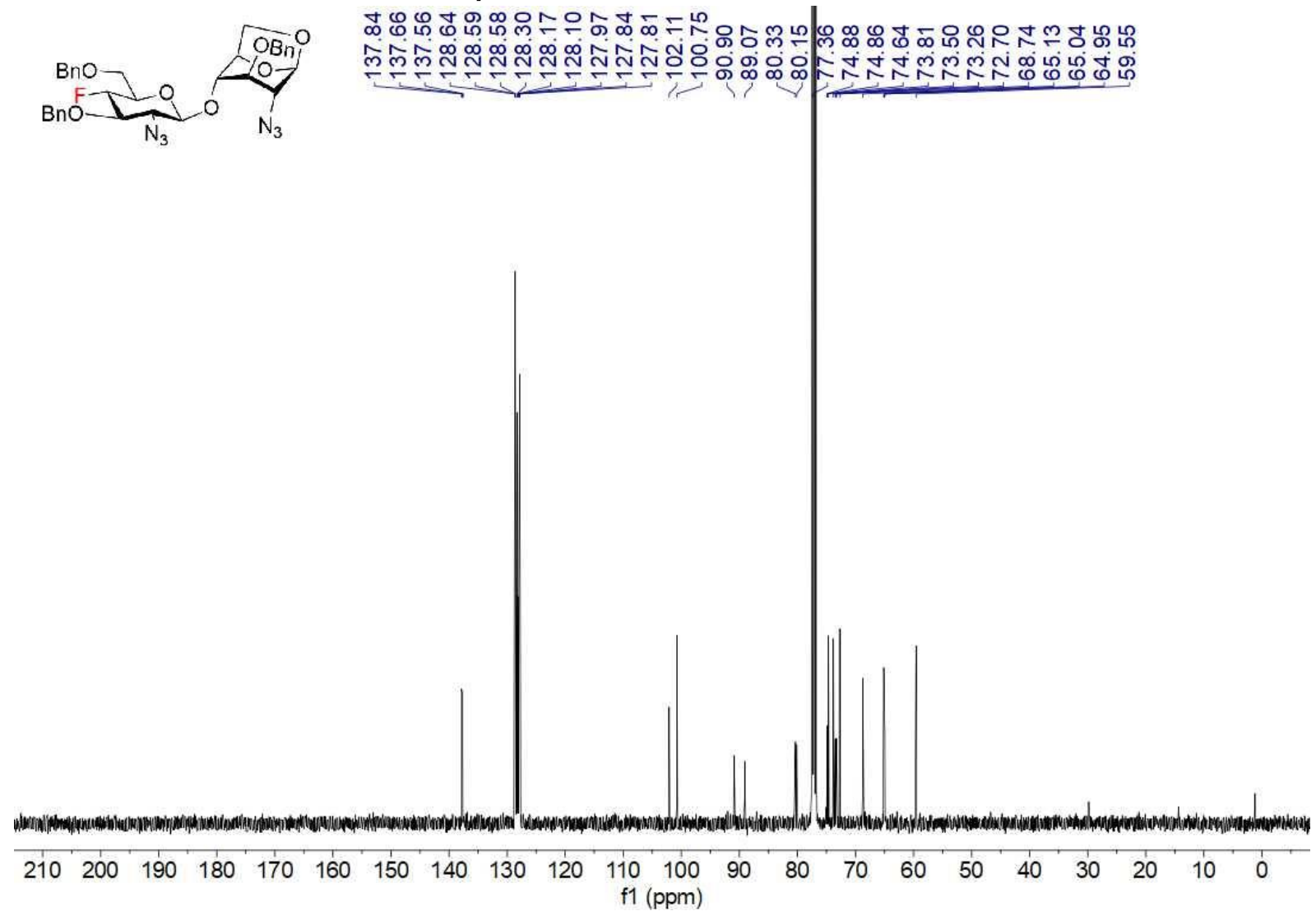

${ }^{1} \mathrm{H}-{ }^{1} \mathrm{H}$ COSY NMR $\mathbf{2 H}-\boldsymbol{\beta}$ and ca $33 \% \mathbf{2 H}-\boldsymbol{\alpha}$

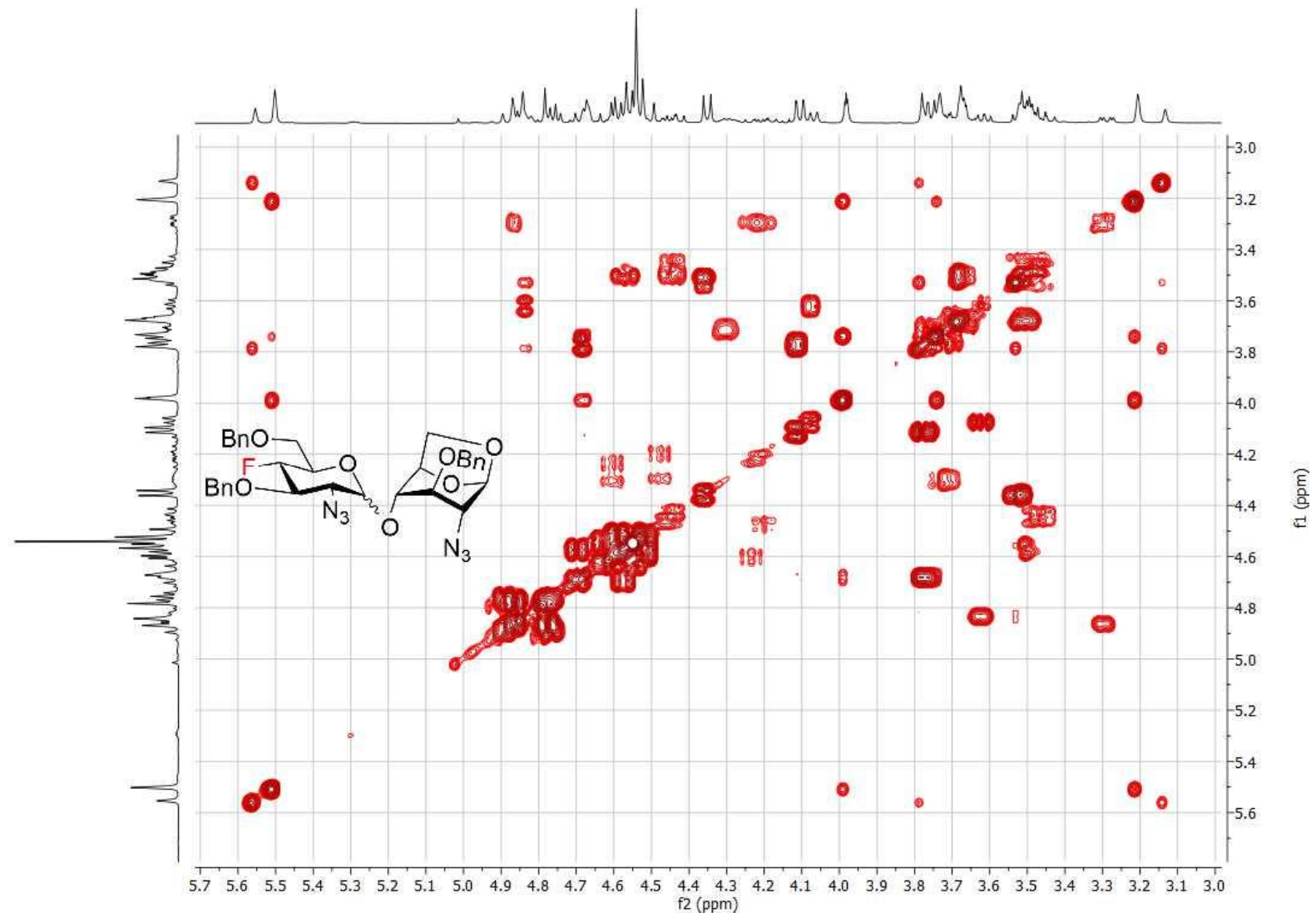


${ }^{1} \mathrm{H}-{ }^{13} \mathrm{C}$ HSQC NMR $2 \mathrm{H}-\beta$ and ca $33 \% 2 \mathrm{2H}-\alpha$

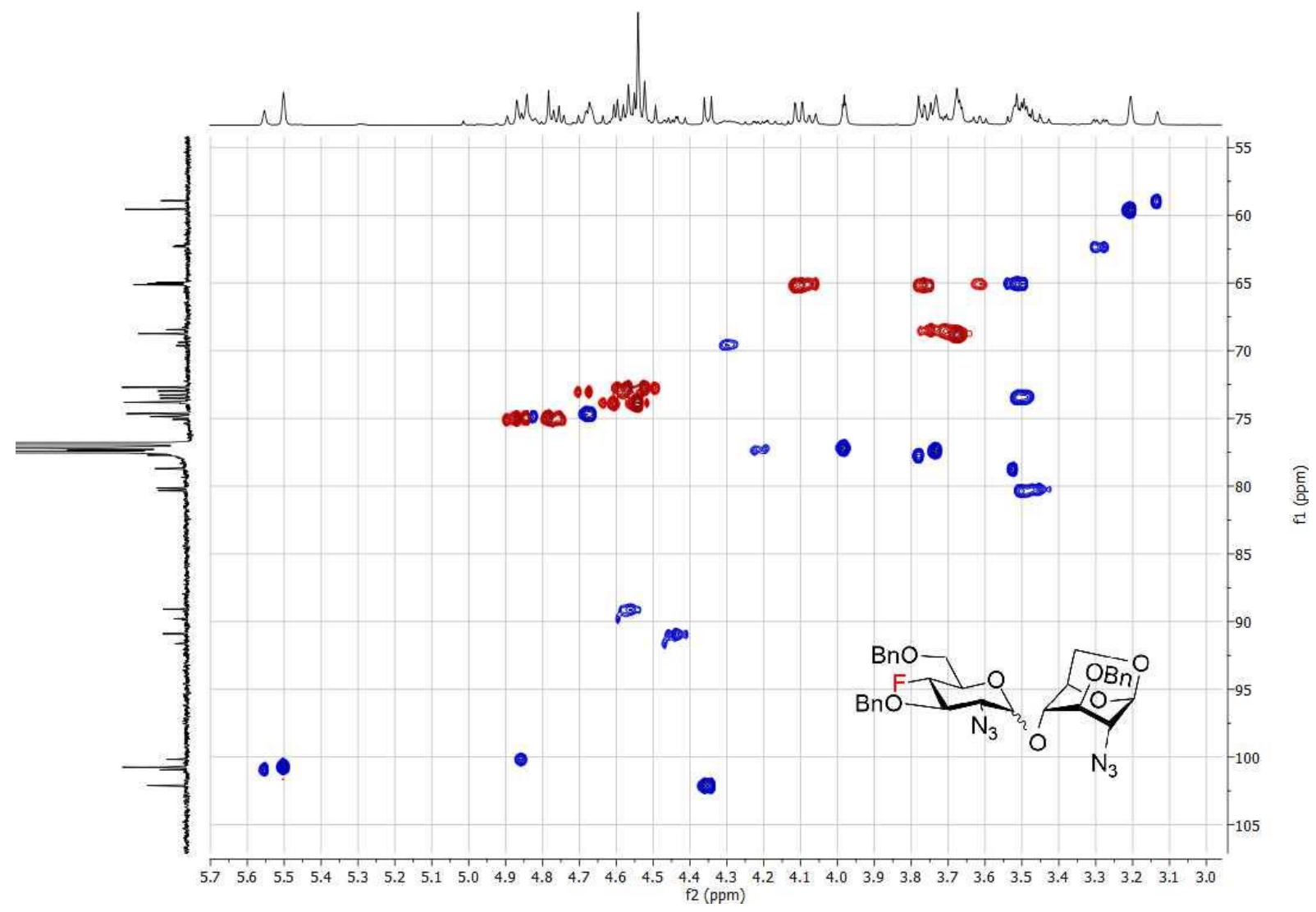

${ }^{1} \mathrm{H}-{ }^{13} \mathrm{C}$ HMBC NMR $2 \mathrm{H}-\beta$ and ca $33 \% \mathbf{2 H}-\alpha$

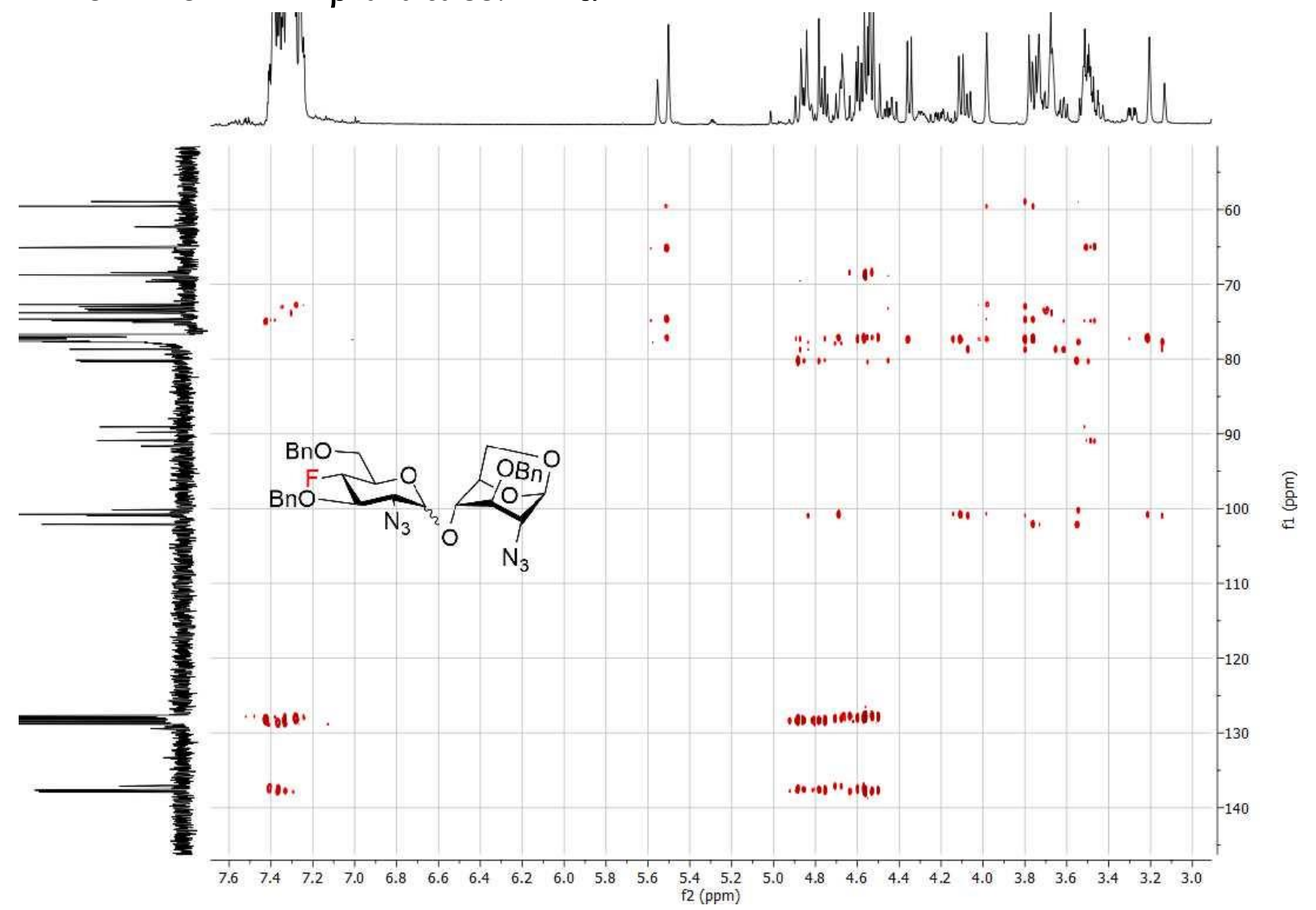


${ }^{19} \mathrm{~F} \mathrm{NMR} 376 \mathrm{MHz}, \mathrm{CDCl}_{3} \mathbf{2 H}-\boldsymbol{\beta}$

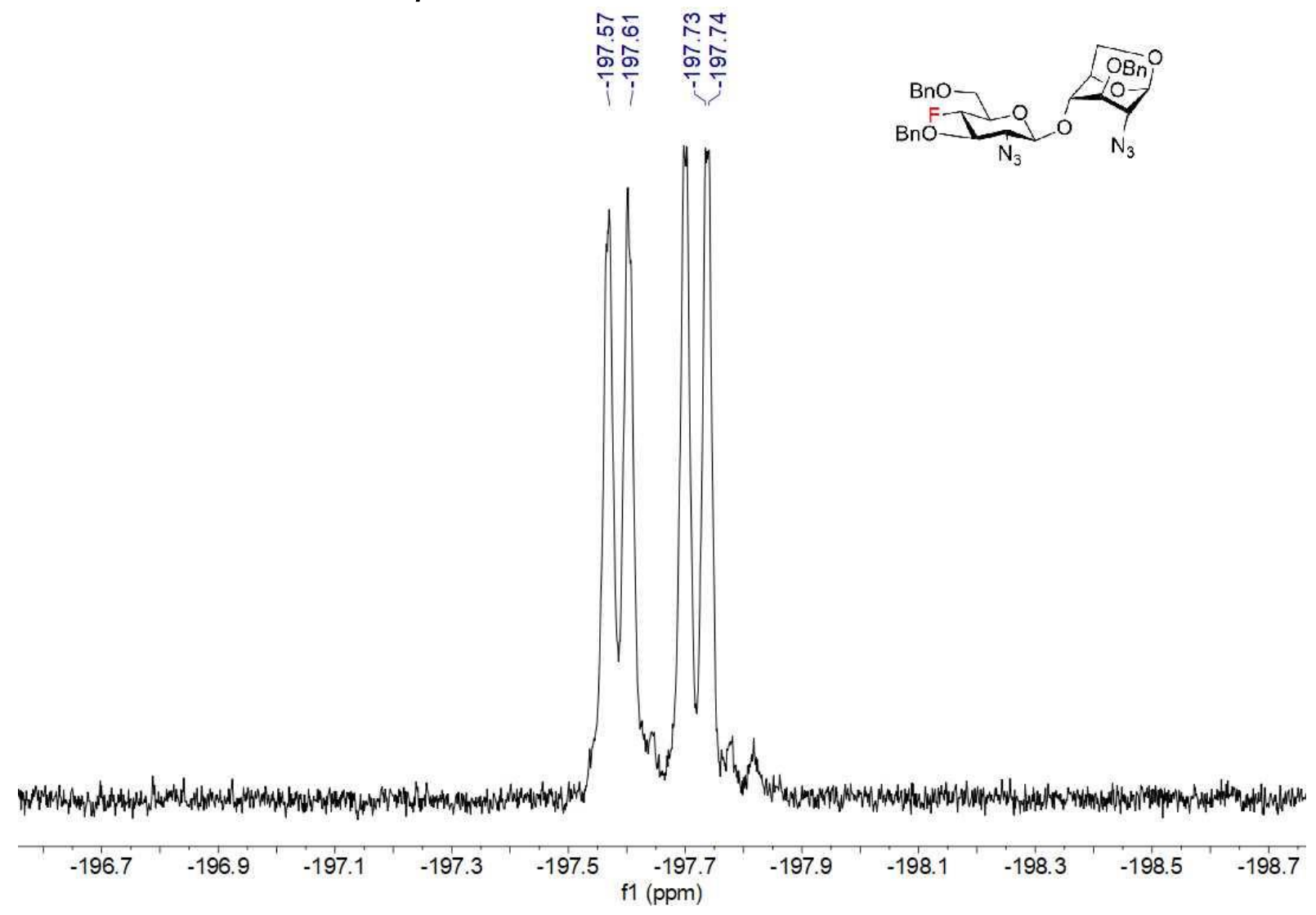

\section{NMR 2I- $\alpha$}

${ }^{1} \mathrm{H}$ NMR $400 \mathrm{MHz}, \mathrm{CDCl}_{3} 2 \mathrm{l}-\alpha$

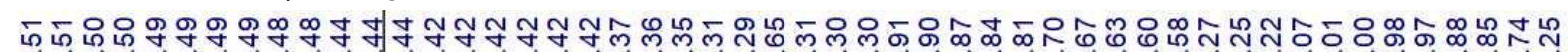

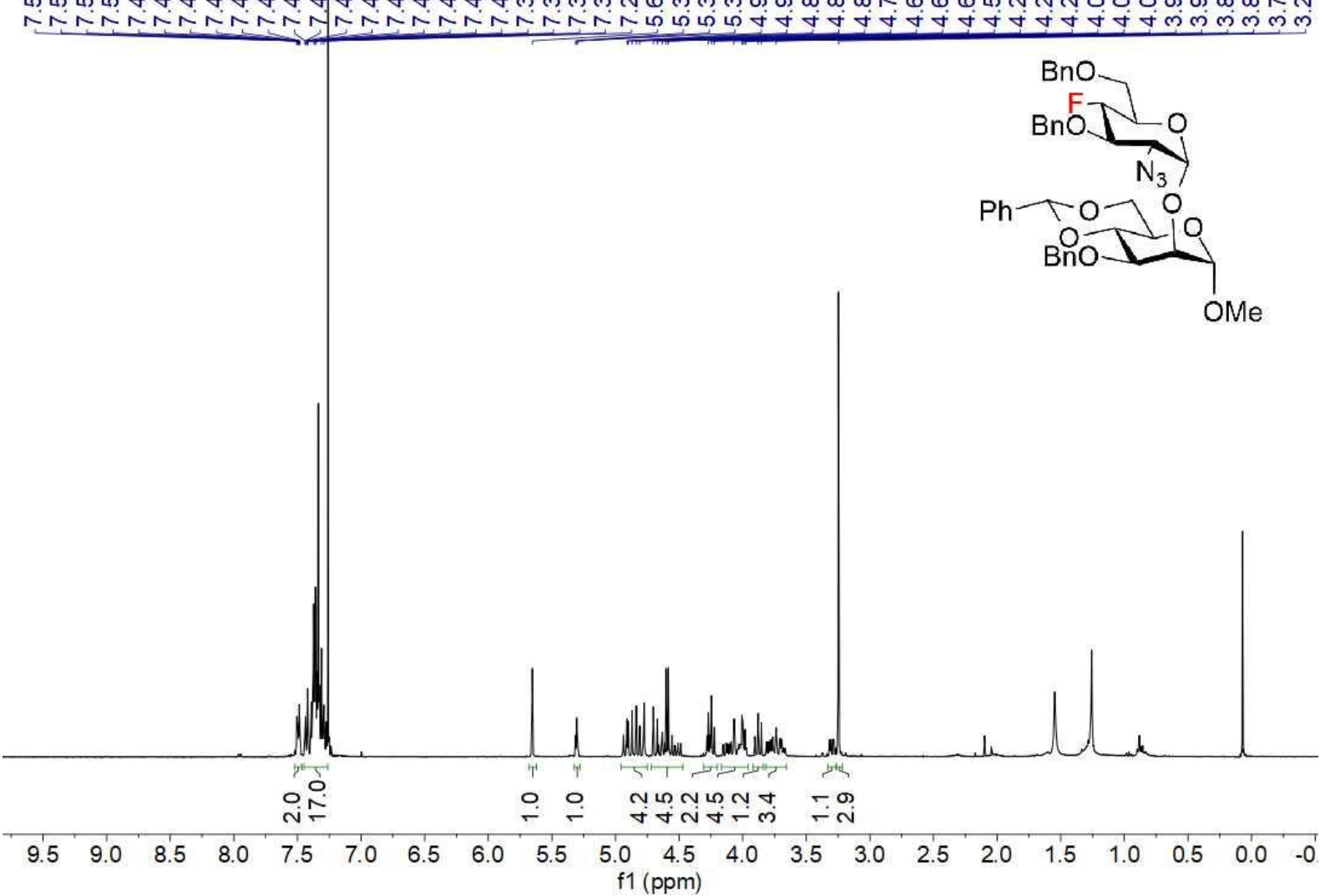




\section{${ }^{13} \mathrm{C}\left\{{ }^{1} \mathrm{H}\right\}$ NMR $100 \mathrm{MHz}, \mathrm{CDCl}_{3} \mathbf{2 l}-\alpha$}

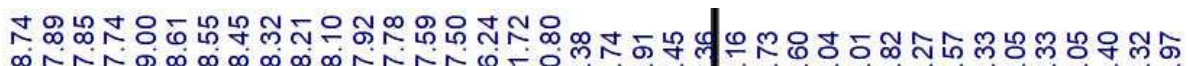

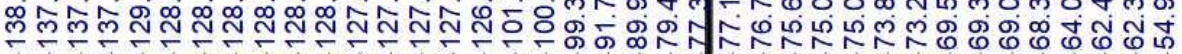
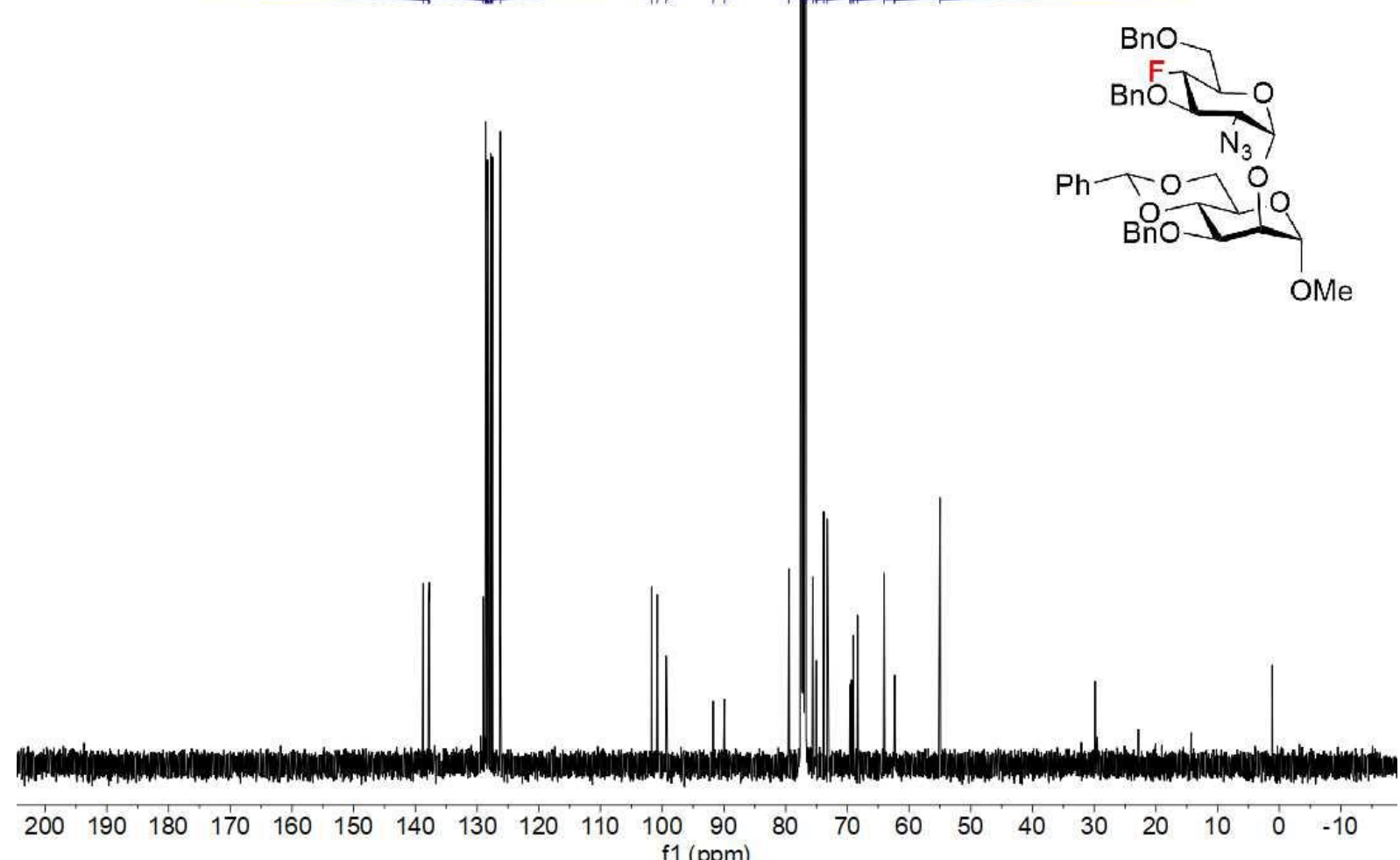

${ }^{1} \mathrm{H}-{ }^{1} \mathrm{H}$ COSY NMR $2 \mathrm{I}-\boldsymbol{\alpha}$

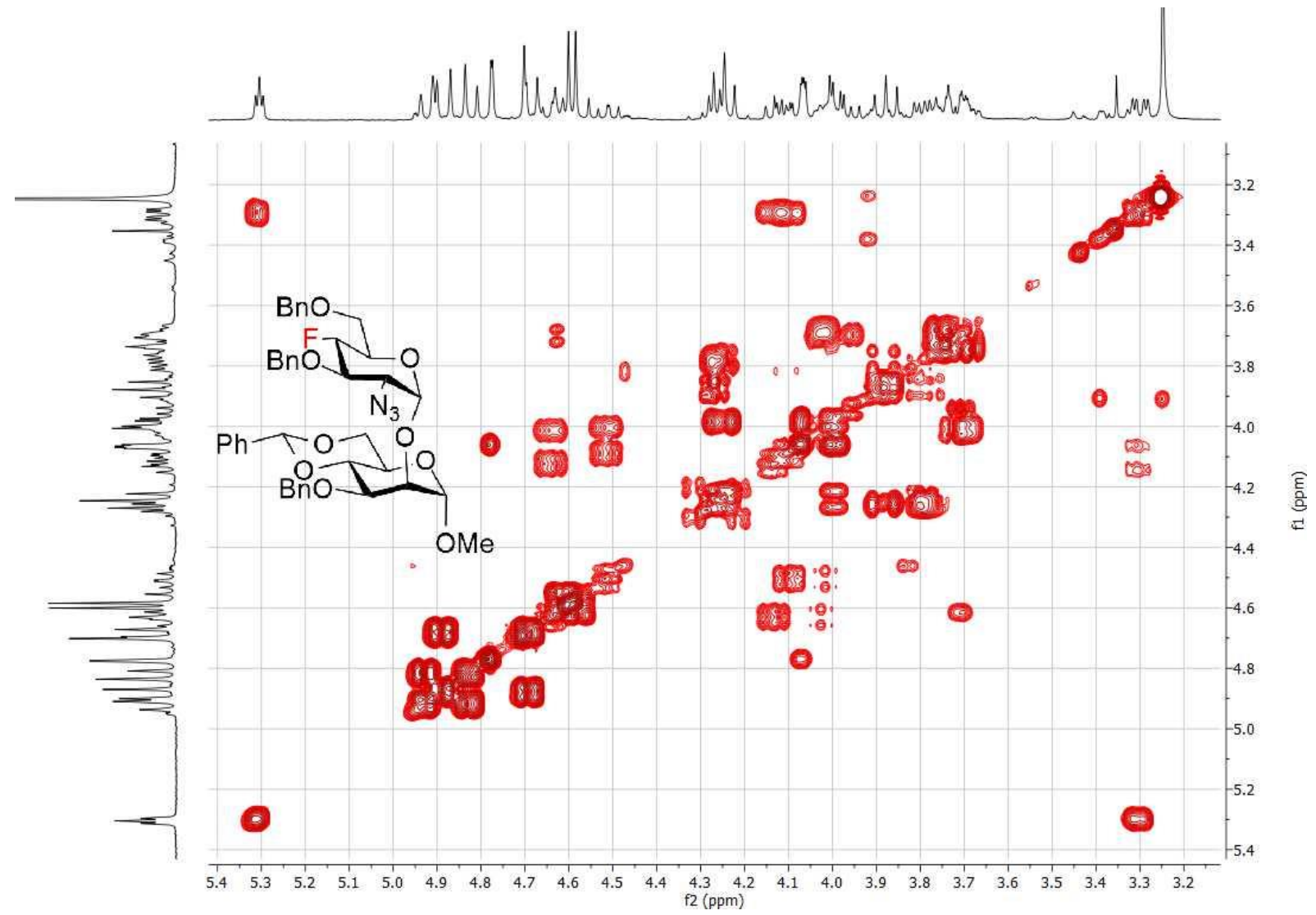


${ }^{1} \mathrm{H}-{ }^{13} \mathrm{C}$ HSQC NMR $21-\alpha$

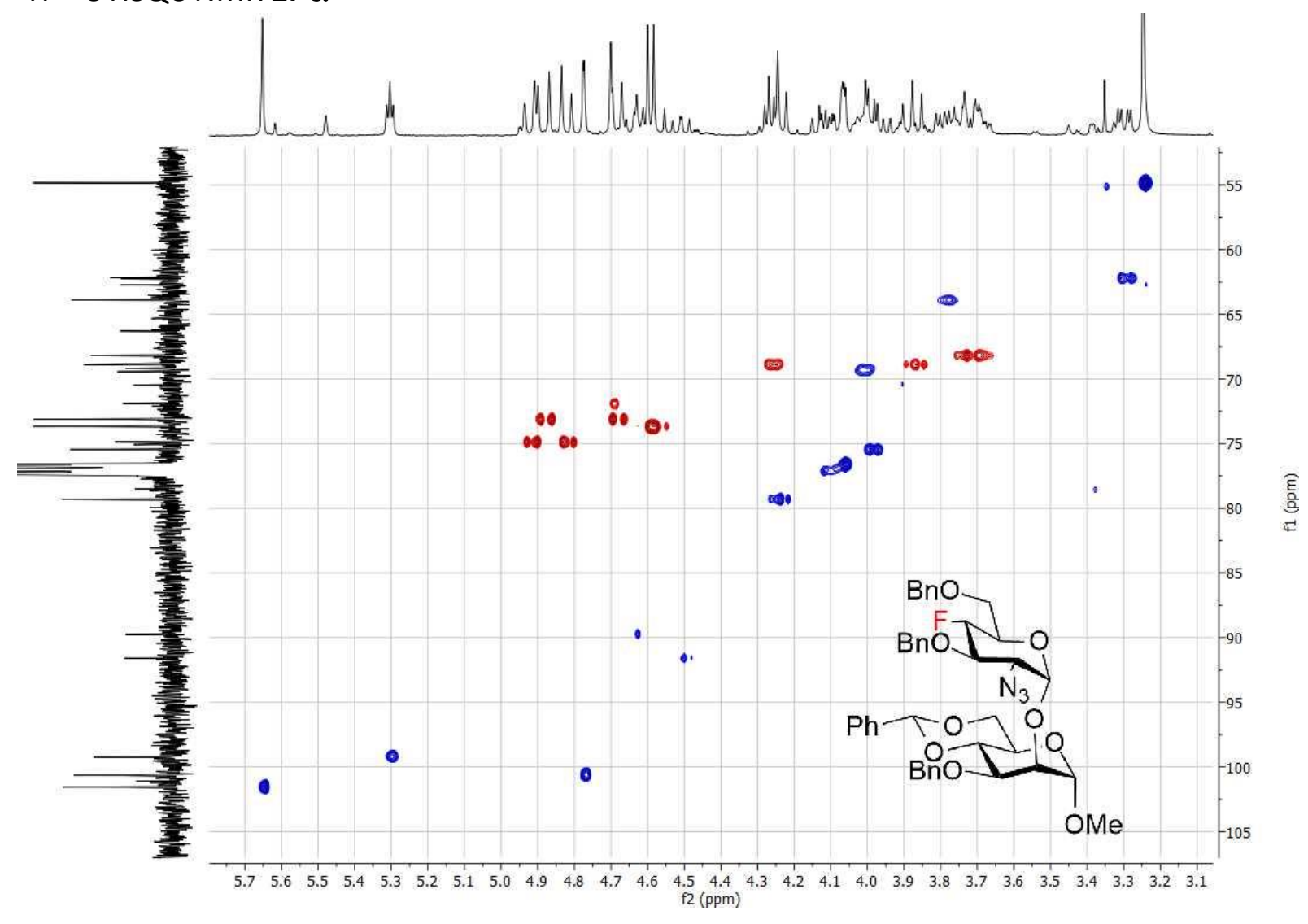

${ }^{1} \mathrm{H}-{ }^{13} \mathrm{C}$ HMBC NMR 2I- $\alpha$

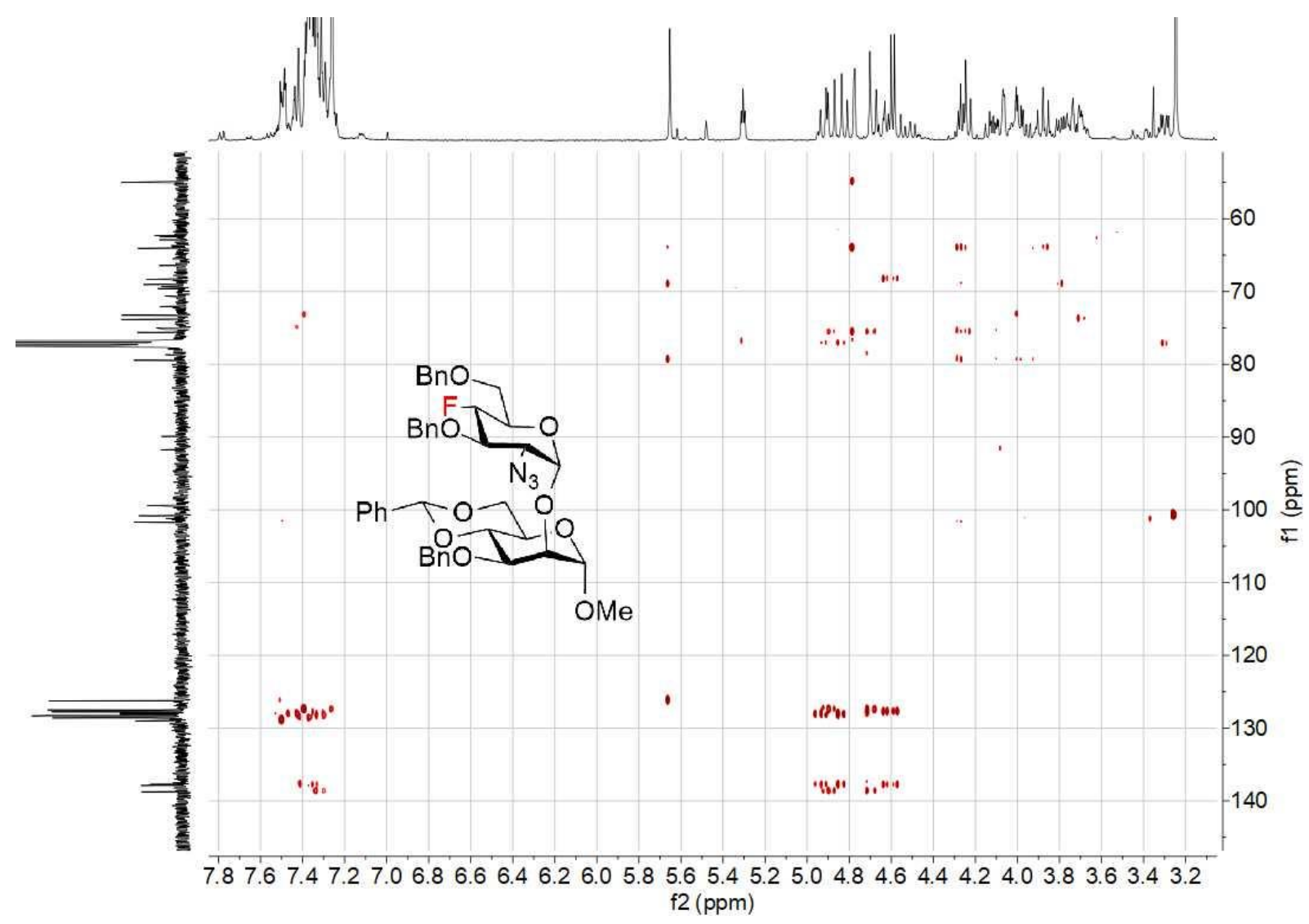


${ }^{19} \mathrm{~F} \mathrm{NMR} 376 \mathrm{MHz}, \mathrm{CDCl}_{3} 2 \mathrm{I}-\alpha$

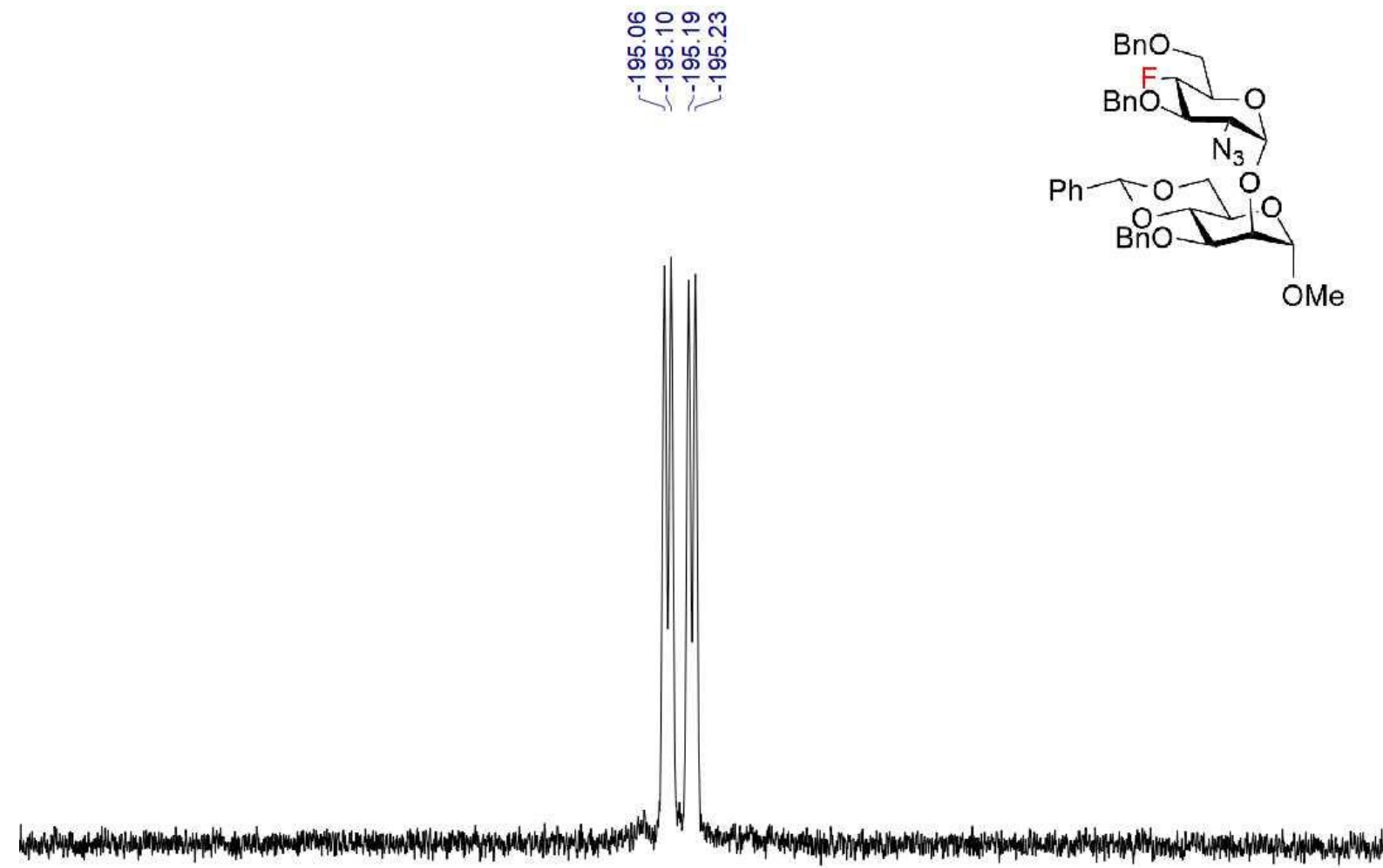

\begin{tabular}{llllllllllllllll}
\hline 1.5 & -192.0 & -192.5 & -193.0 & -193.5 & -194.0 & -194.5 & -195.0 & -195.5 & -196.0 & -196.5 & -197.0 & -197.5 & -198.0 & -198.5 & -199.
\end{tabular} f1 (ppm)

NMR 2I- $\beta$

${ }^{1} \mathrm{HNMR} 400 \mathrm{MHz}, \mathrm{CDCl}_{3} 2 \mathrm{I}-\boldsymbol{\beta}$

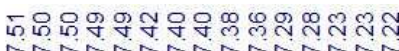

MANMNANTNANAN

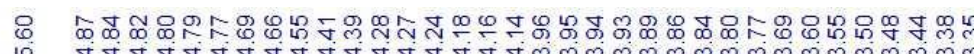

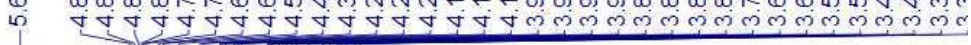

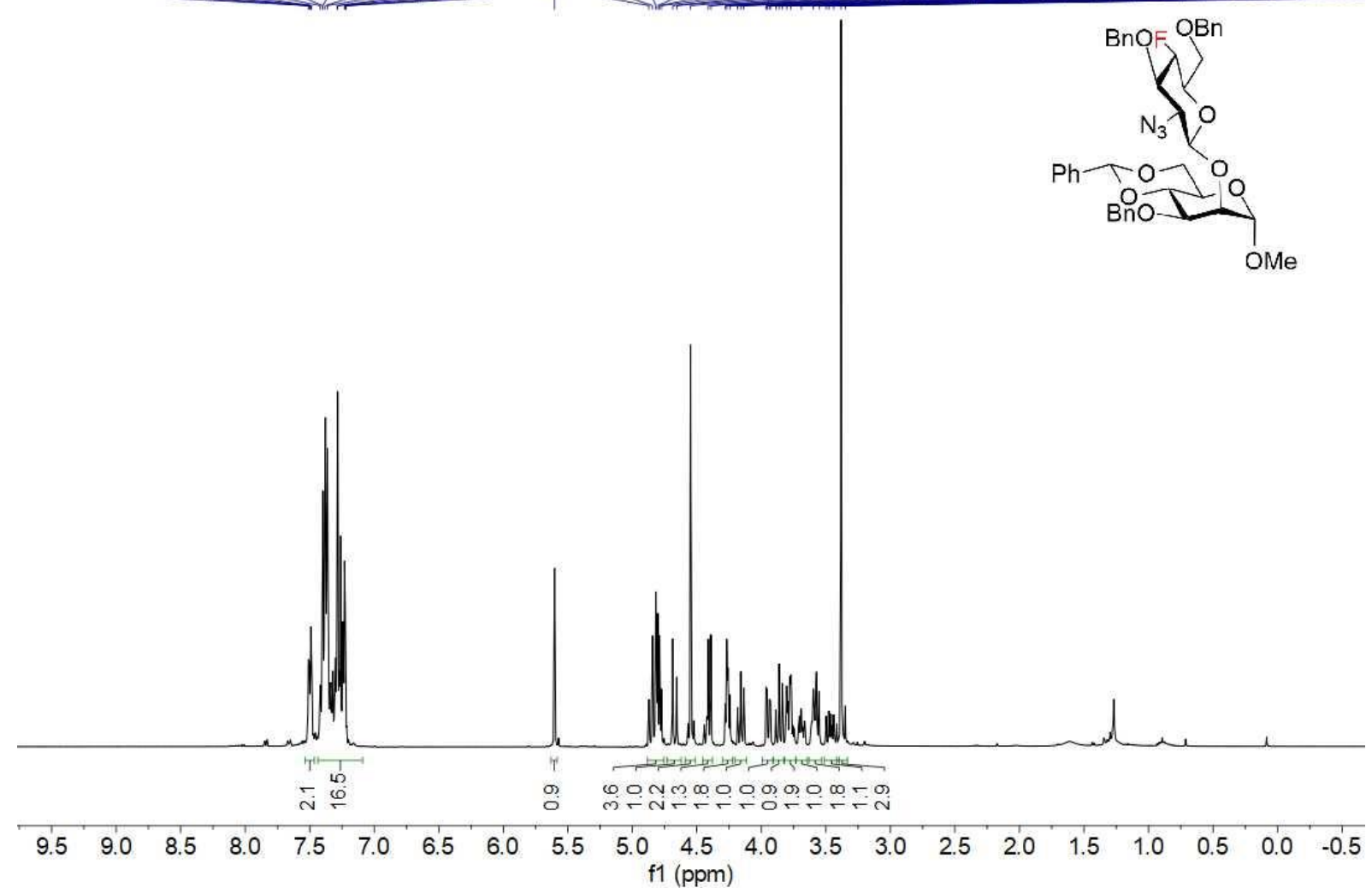




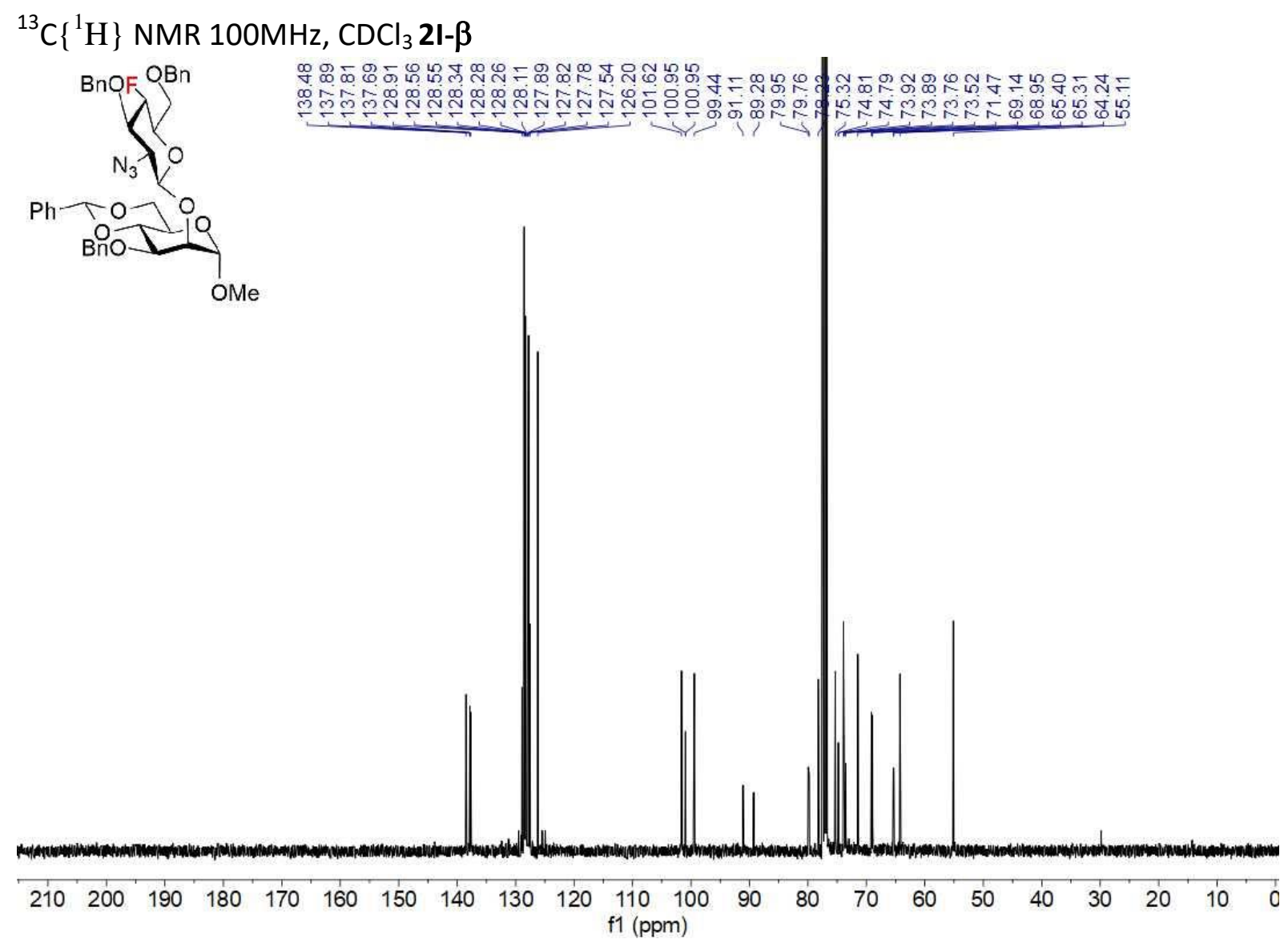

${ }^{1} \mathrm{H}-{ }^{1} \mathrm{H}$ COSY NMR $2 \mathrm{I}-\boldsymbol{\beta}$

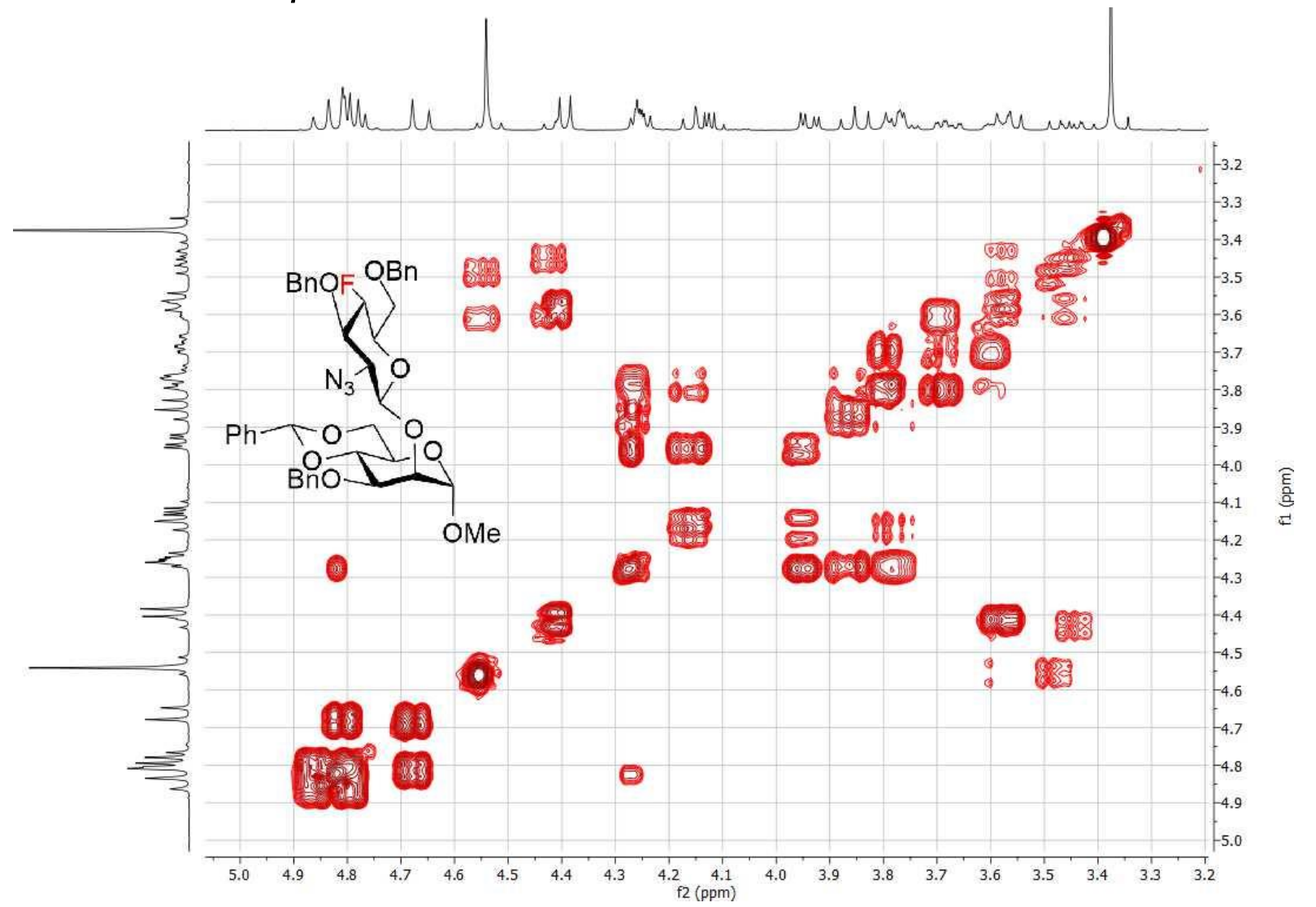


${ }^{1} \mathrm{H}-{ }^{13} \mathrm{C}$ HSQC NMR 2I- $\beta$

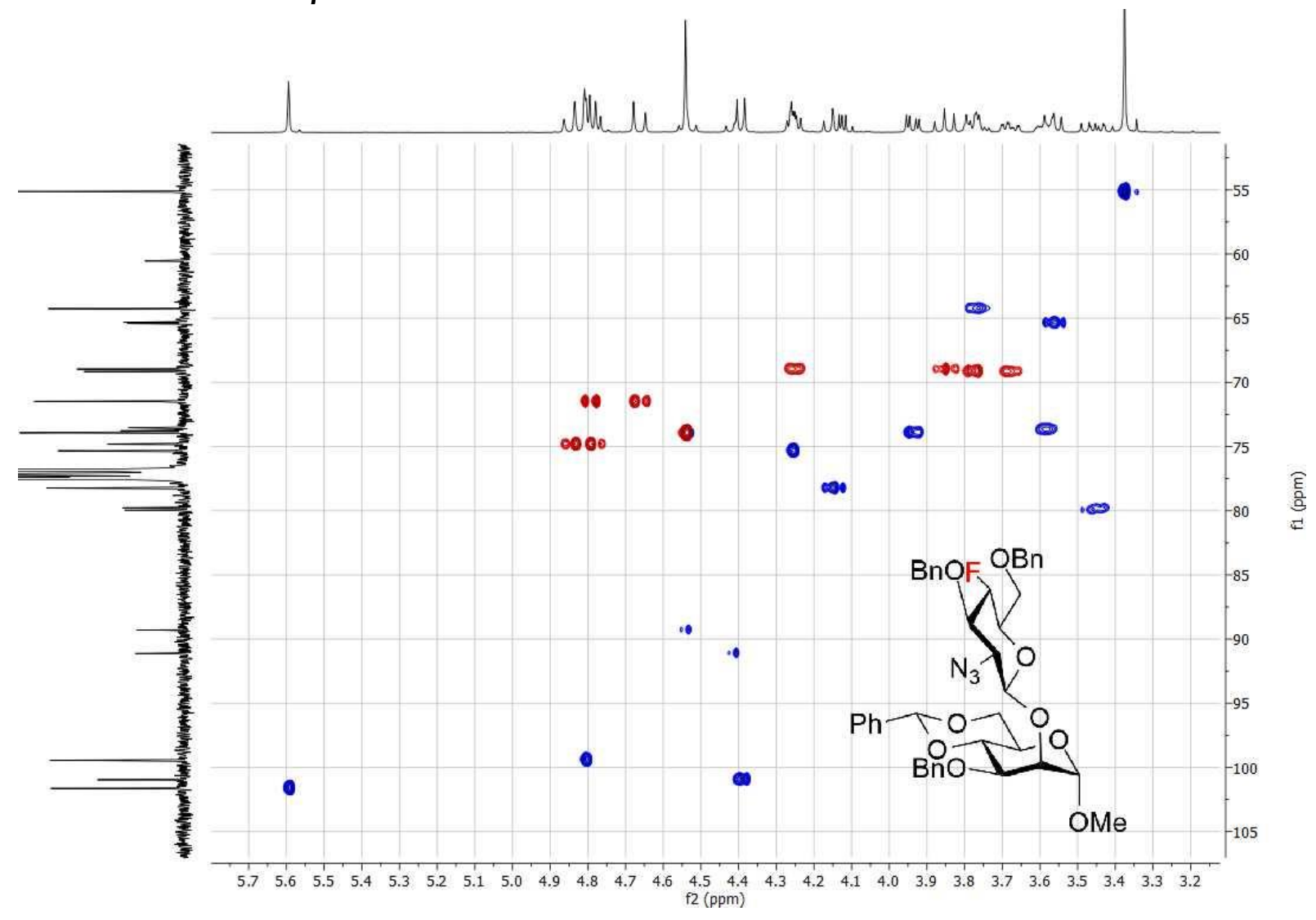

${ }^{1} \mathrm{H}-{ }^{13} \mathrm{C}$ HMBC NMR 2I- $\beta$

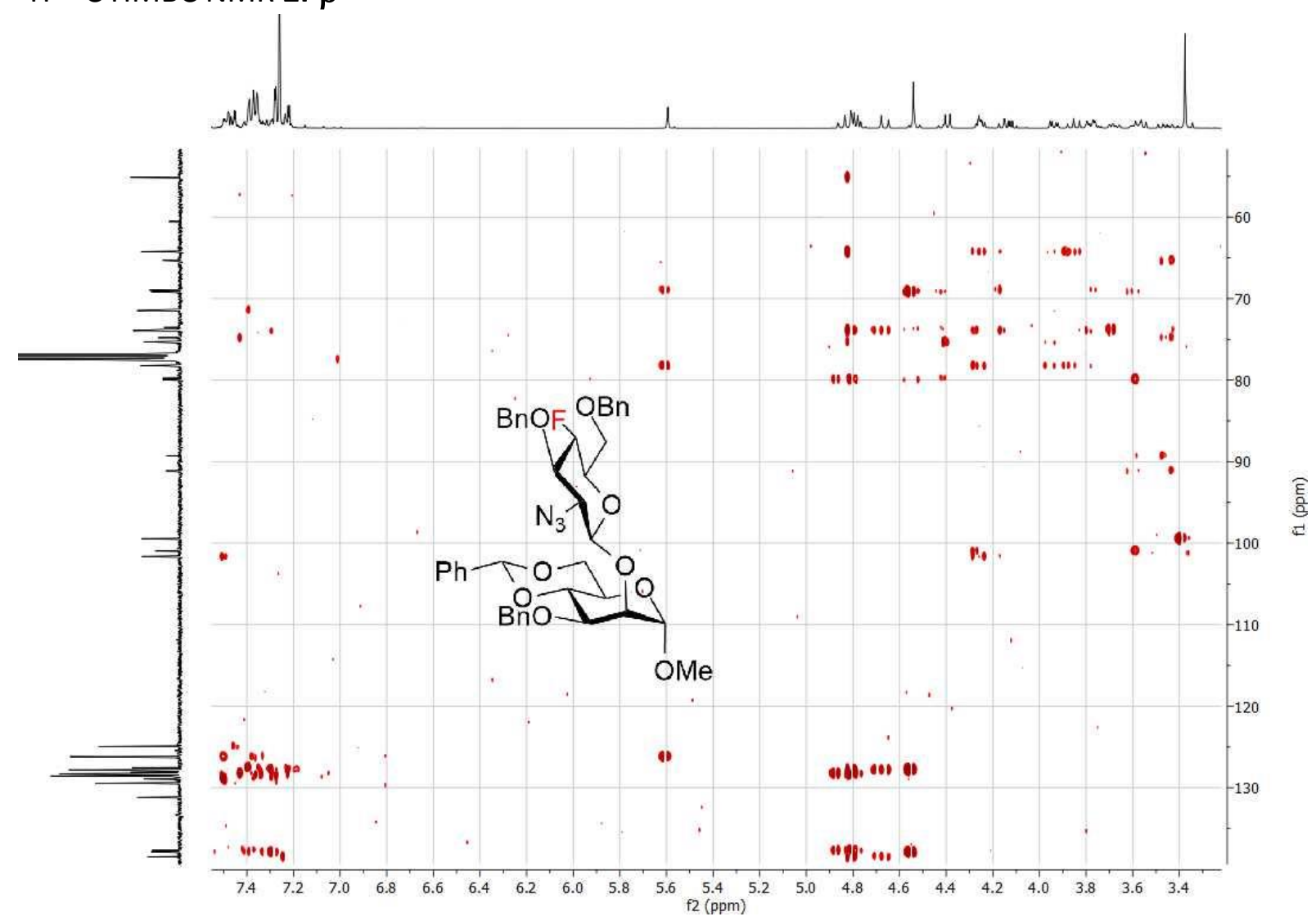


${ }^{19} \mathrm{~F} \mathrm{NMR} 376 \mathrm{MHz}, \mathrm{CDCl}_{3} \mathbf{2 I -} \boldsymbol{\beta}$

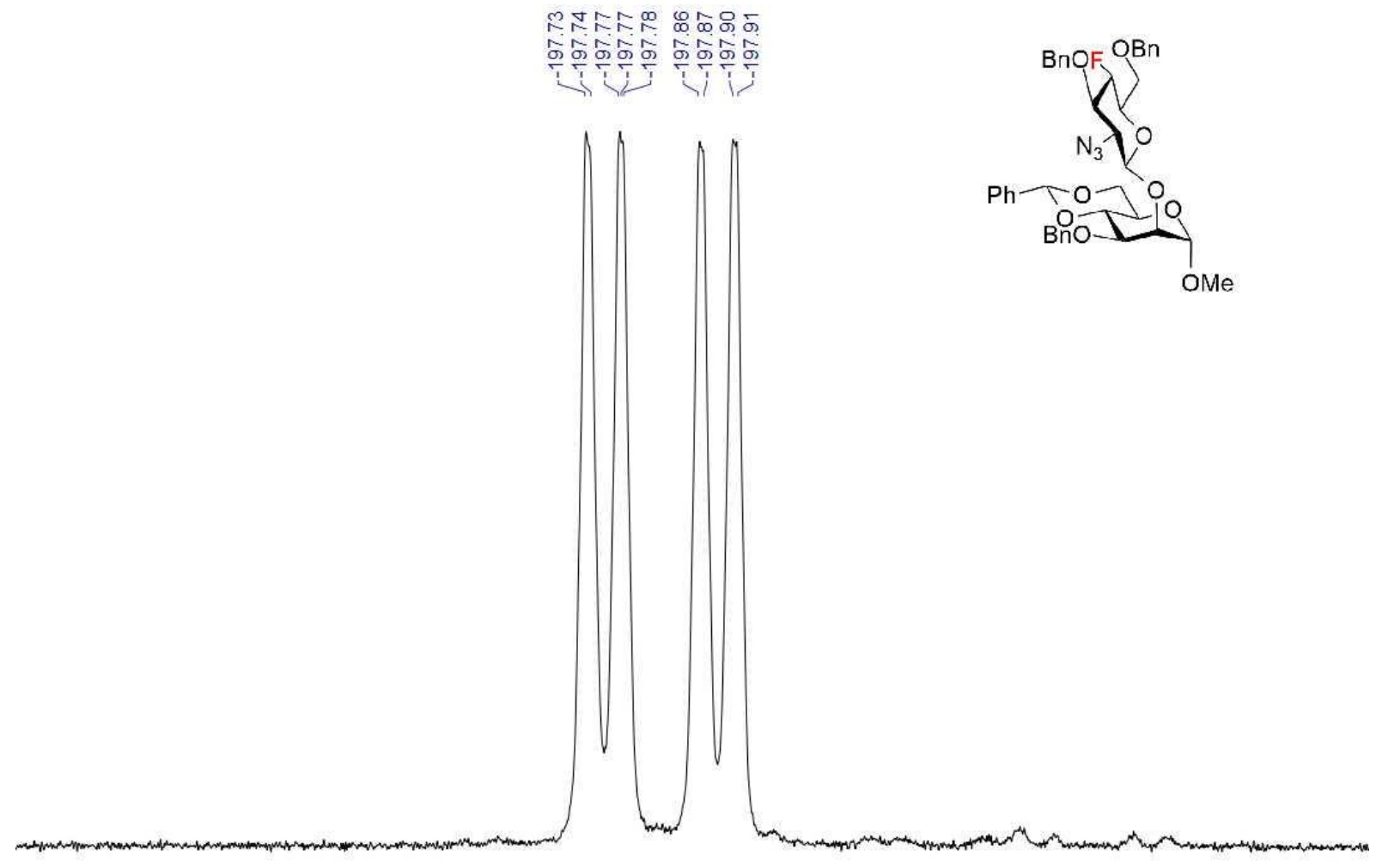

\begin{tabular}{lllllllllllllllll}
\hline-197.1 & -197.2 & -197.3 & -197.4 & -197.5 & -197.6 & -197.7 & -197.8 & -197.9 & -198.0 & -198.1 & -198.2 & -198.3 & -198.4 & -198.5 & -198.6
\end{tabular} $\mathrm{f1}(\mathrm{ppm})$

\section{NMR 3A- $\alpha$}

${ }^{1} \mathrm{H}$ NMR $400 \mathrm{MHz}, \mathrm{CDCl}_{3} 3 \mathrm{~A}-\alpha$

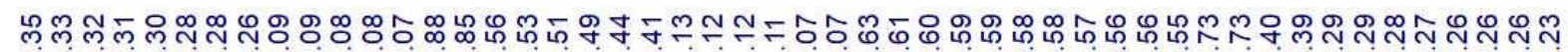

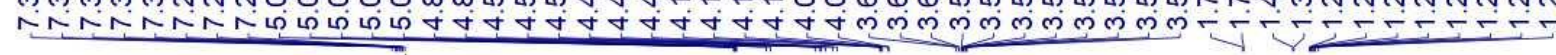

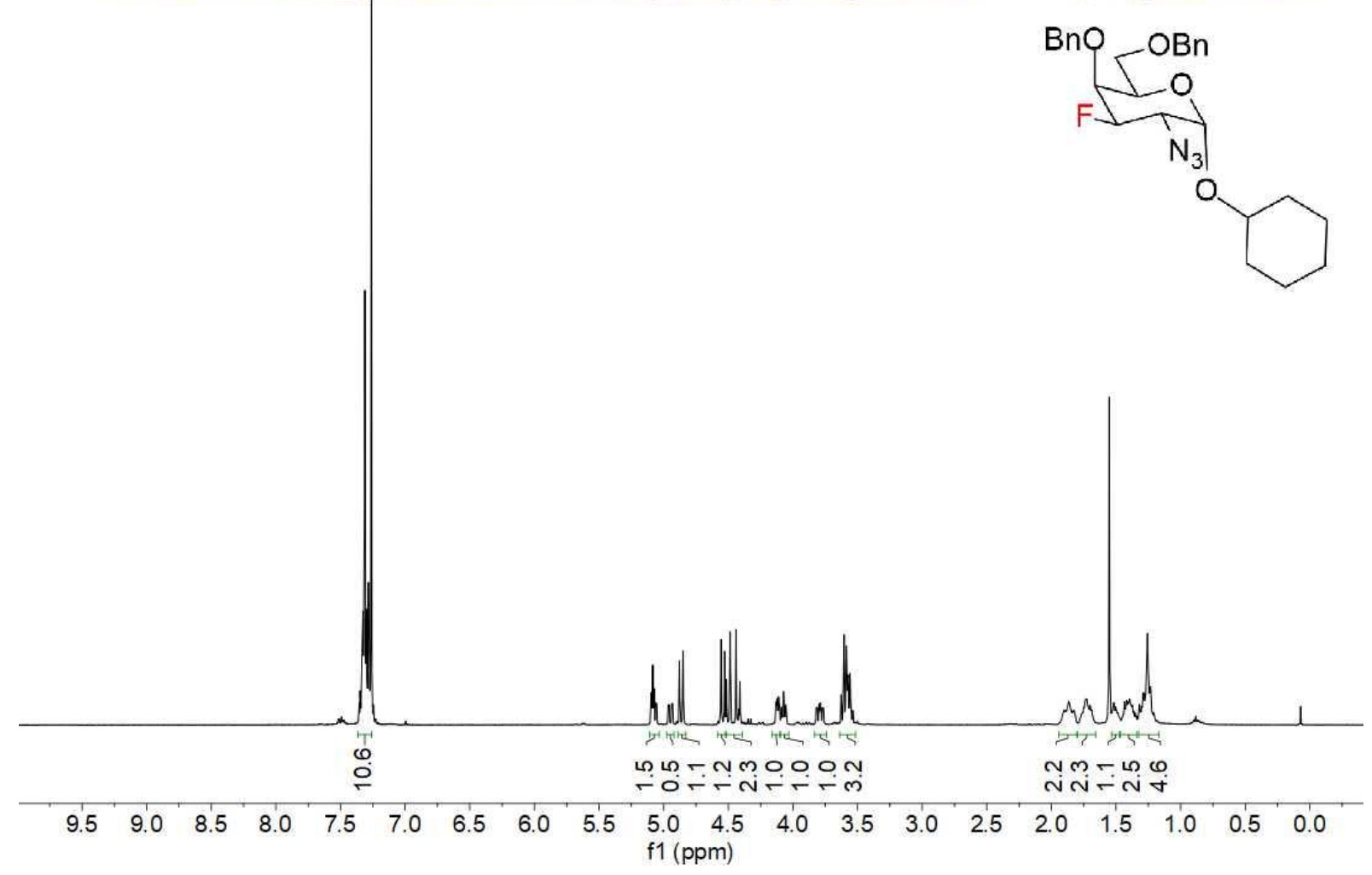




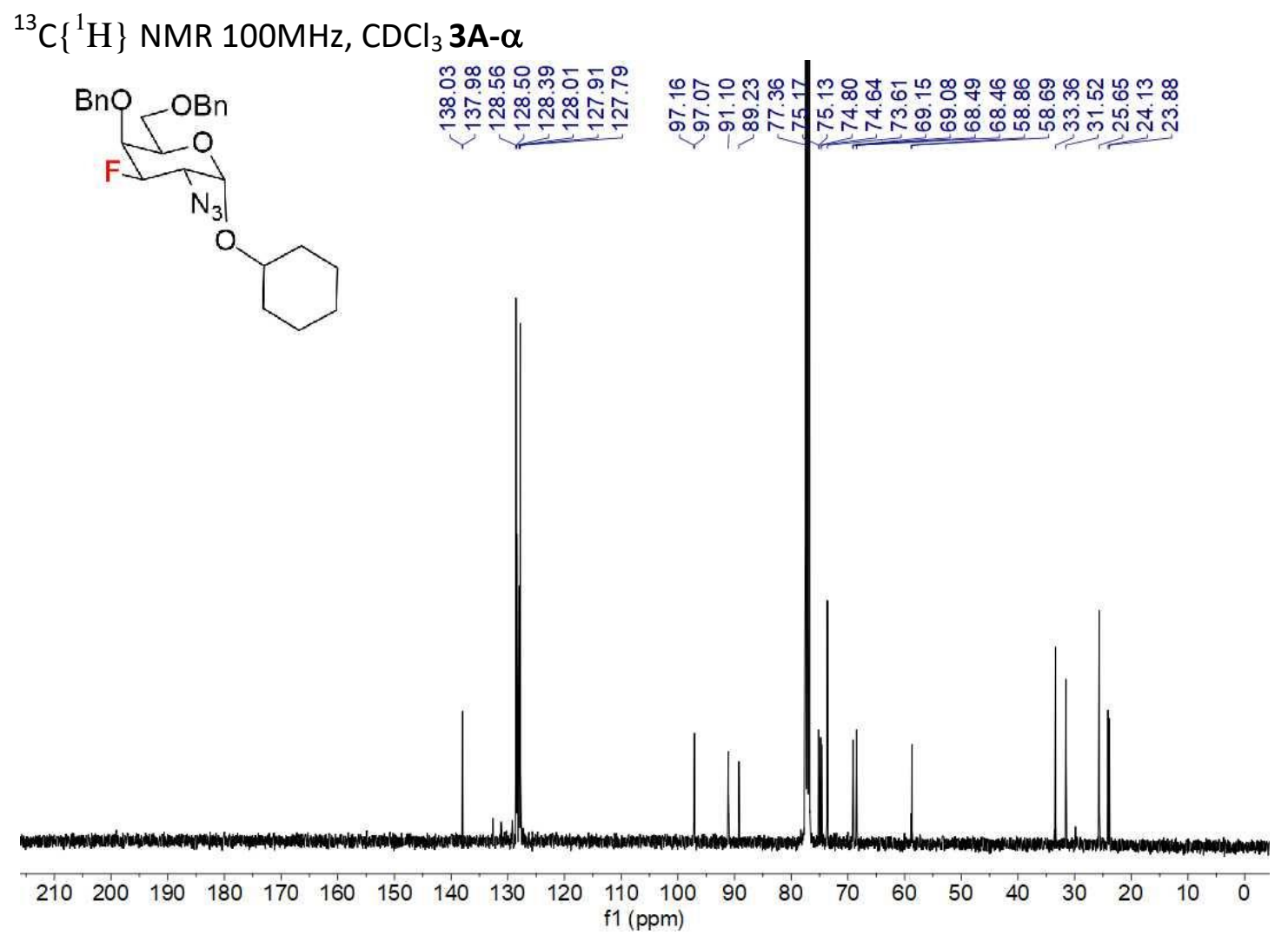

${ }^{1} \mathrm{H}-{ }^{1} \mathrm{H}$ COSY NMR $3 \mathrm{~A}-\boldsymbol{\alpha}$

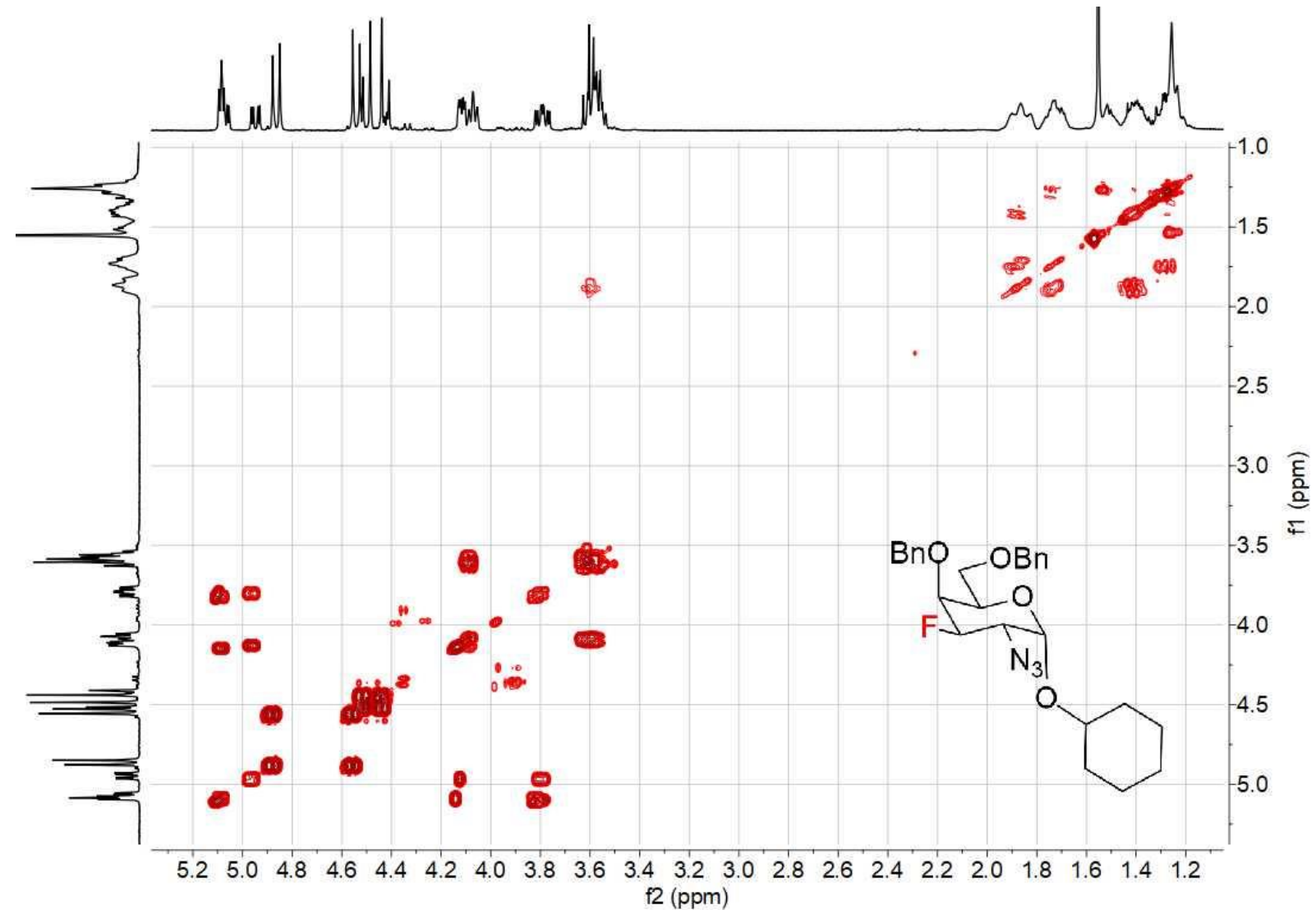


${ }^{1} \mathrm{H}-{ }^{13} \mathrm{C}$ HSQC NMR 3A- $\alpha$

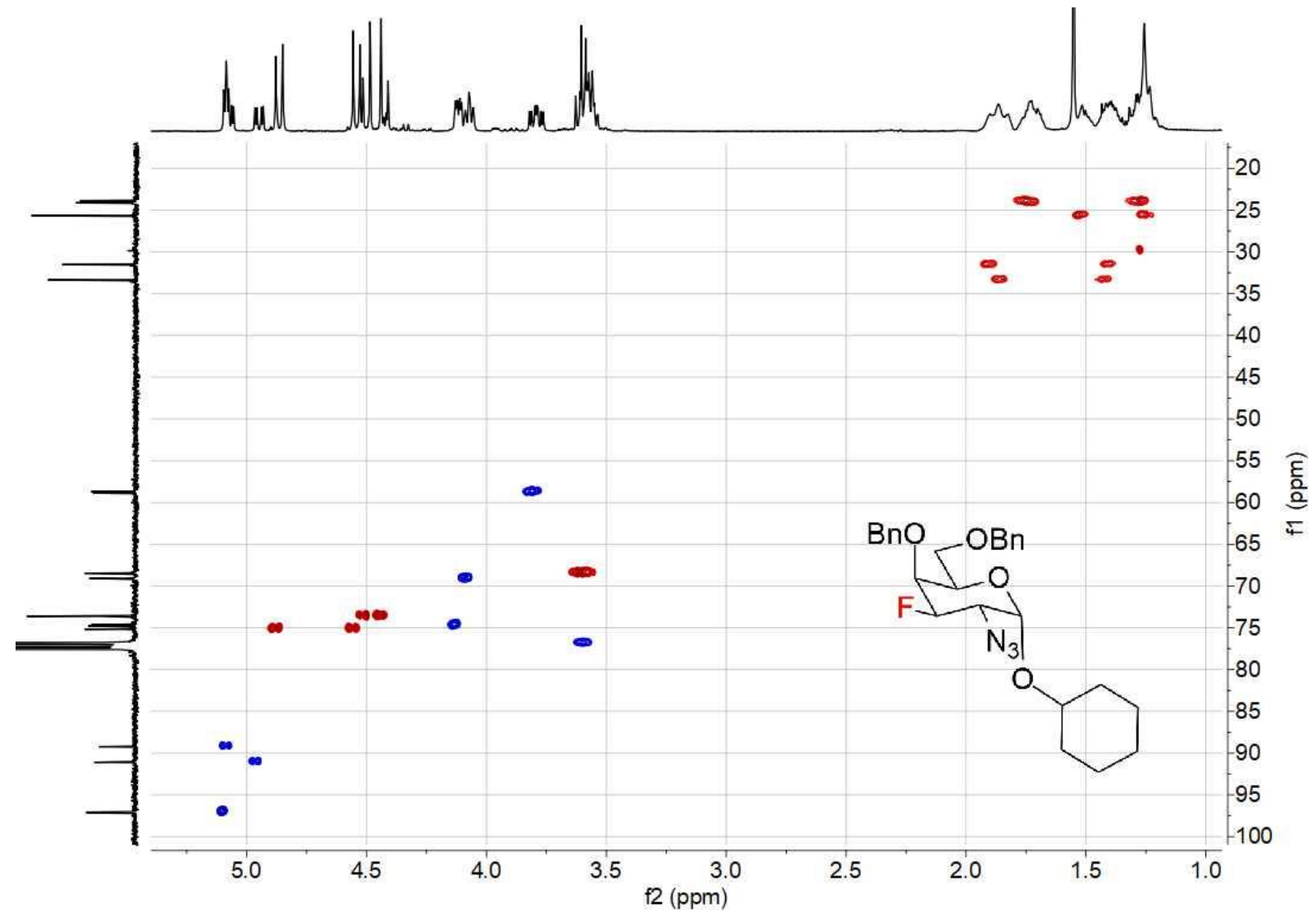

${ }^{1} \mathrm{H}-{ }^{13} \mathrm{C}$ HMBC NMR 3A- $\alpha$

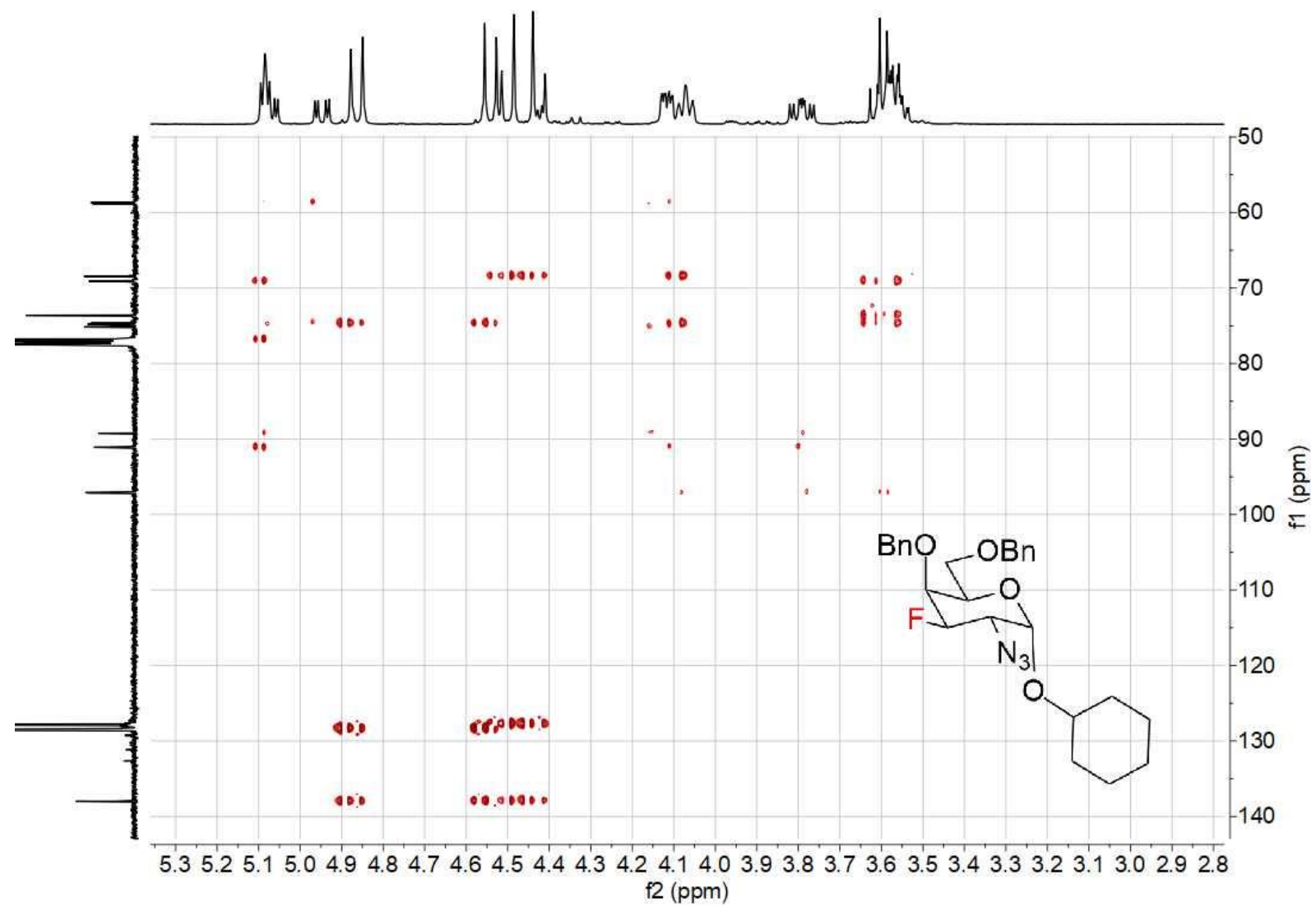


${ }^{19} \mathrm{~F} \mathrm{NMR} 376 \mathrm{MHz}, \mathrm{CDCl}_{3} 3 \mathrm{~A}-\boldsymbol{\alpha}$

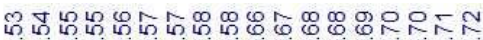

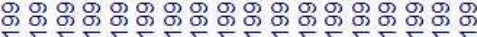
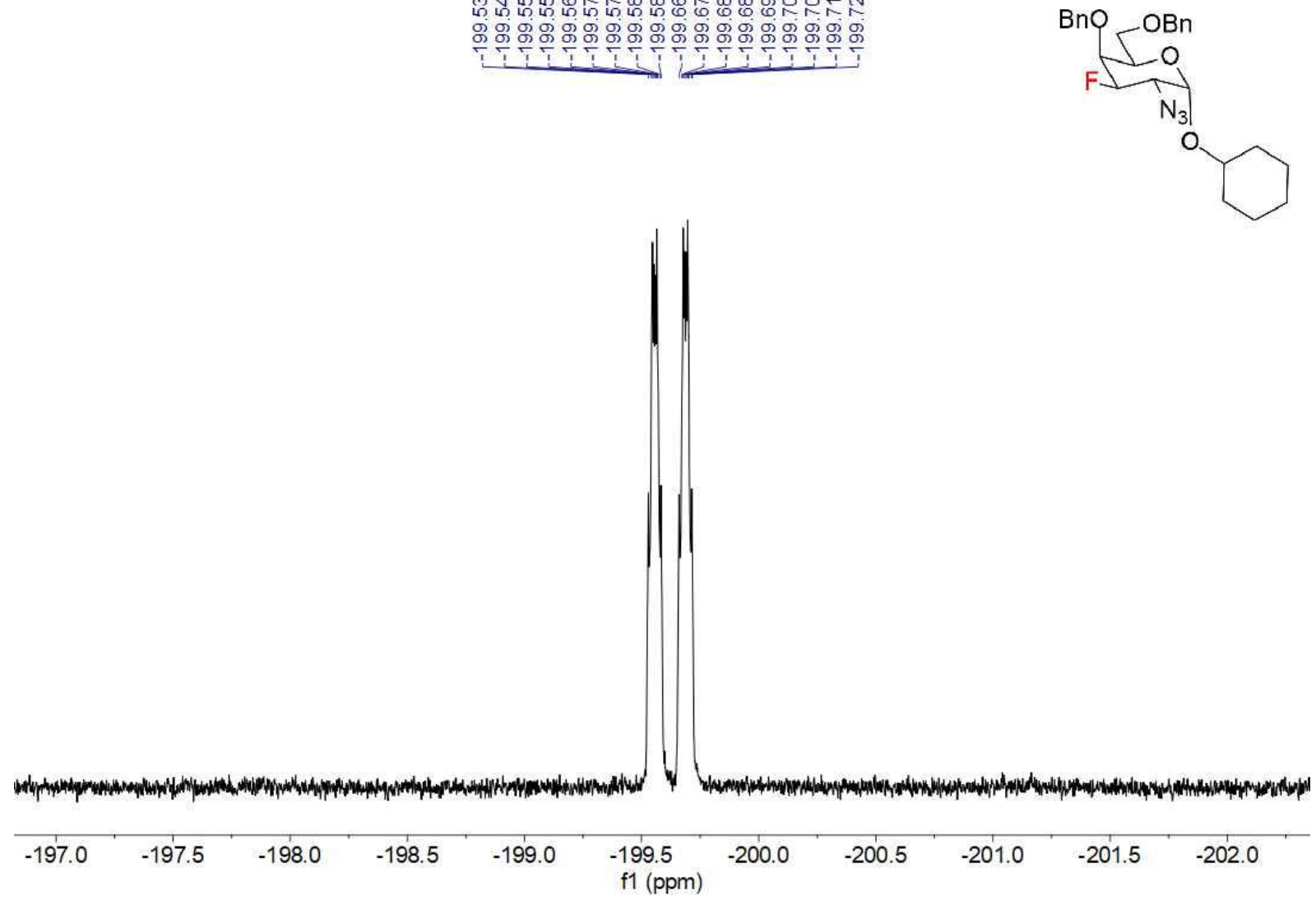

NMR 3A- $\beta$

${ }^{1} \mathrm{HNMR} 400 \mathrm{MHz}, \mathrm{CDCl}_{3} 3 \mathrm{AA}-\boldsymbol{\beta}$

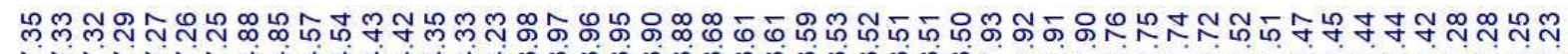

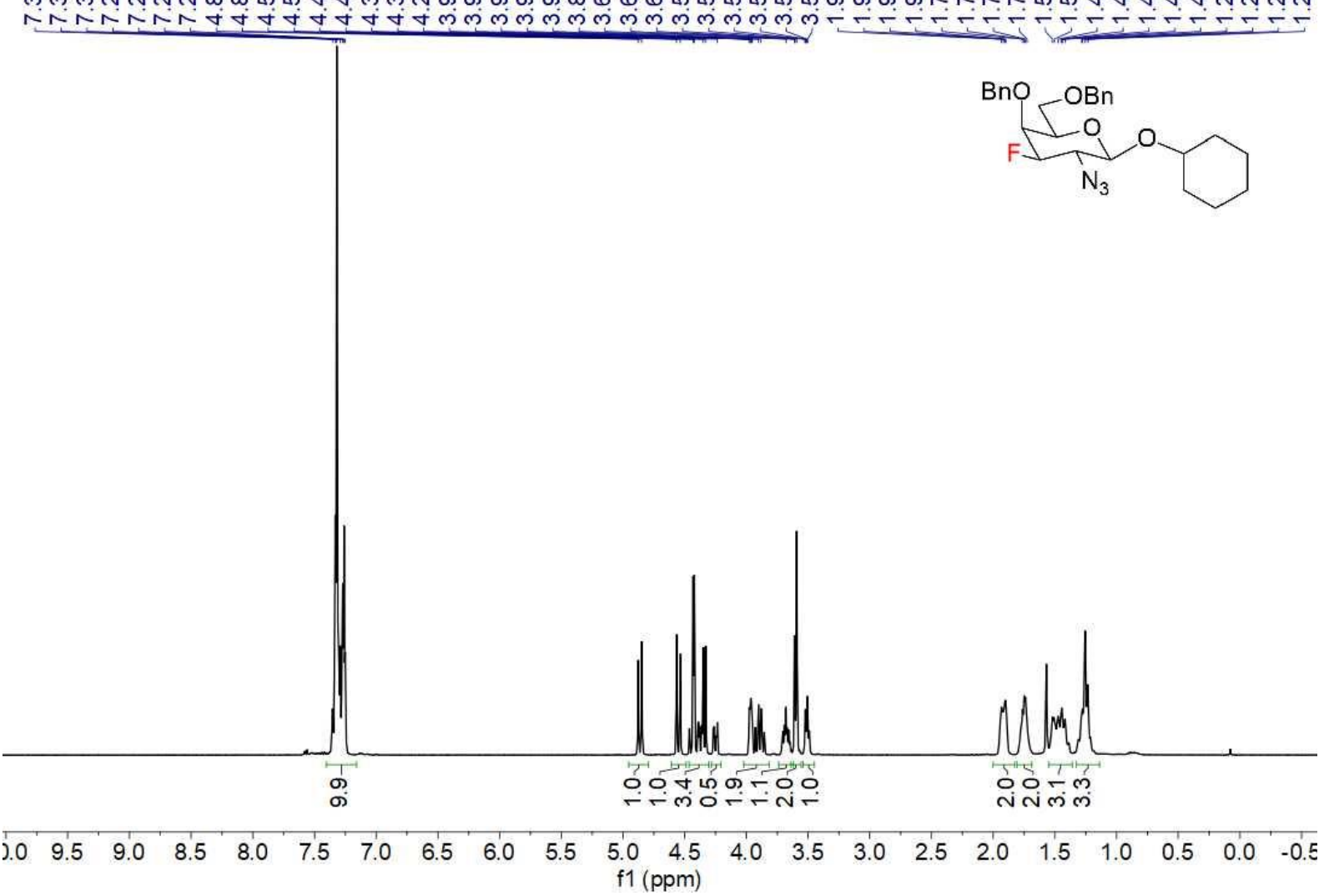


${ }^{13} \mathrm{C}\left\{{ }^{1} \mathrm{H}\right\}$ NMR $100 \mathrm{MHz}, \mathrm{CDCl}_{3} \mathbf{3 A}-\boldsymbol{\beta}$<smiles>NC(N)C(OCc1ccccc1)C1CCCCC1Oc1ccccc1</smiles>

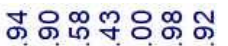

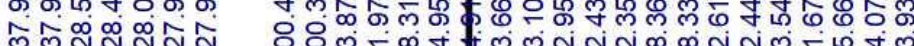

으ำ

$\mathrm{N}_{3}$

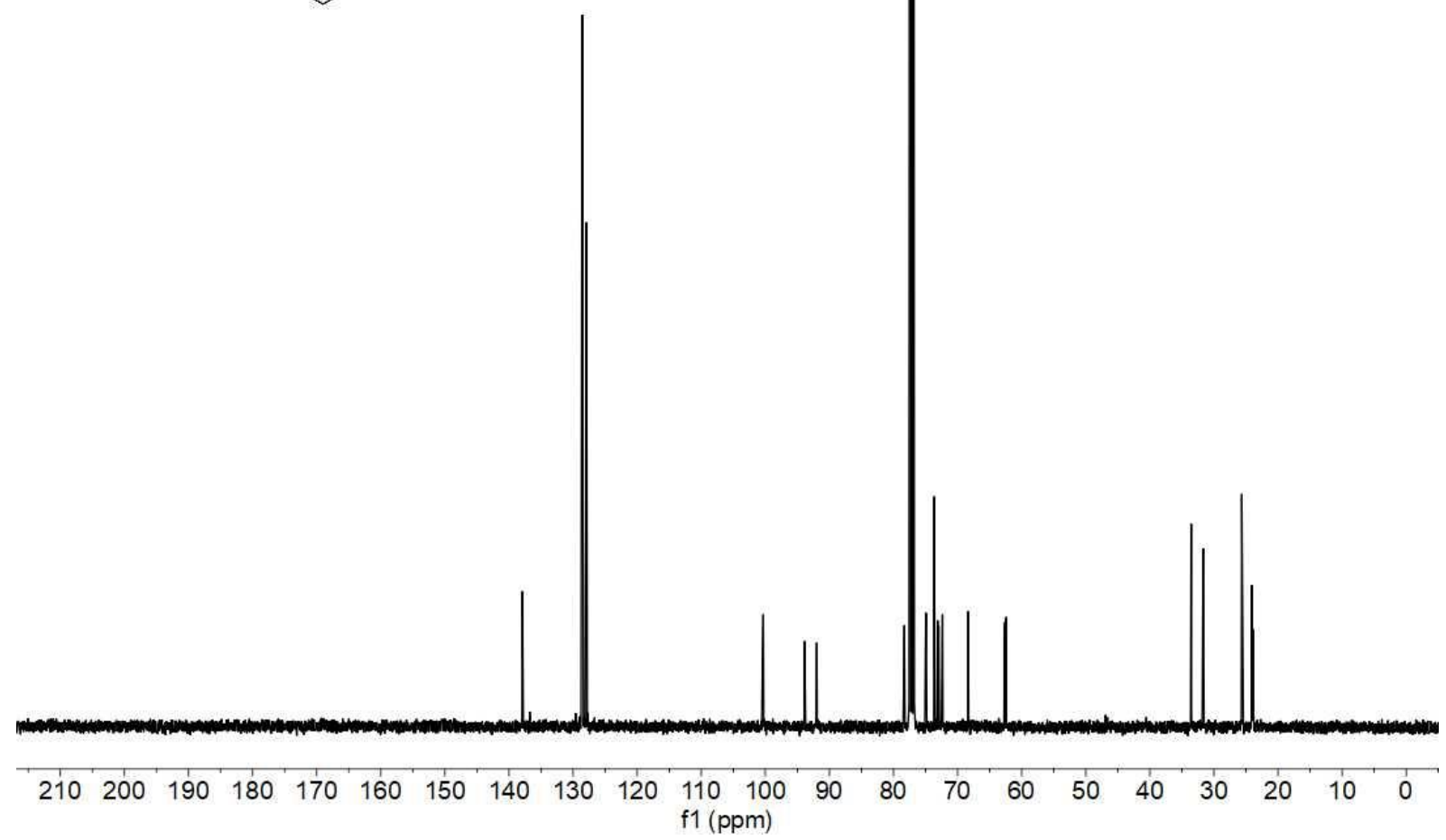

${ }^{1} \mathrm{H}-{ }^{1} \mathrm{H}$ COSY NMR $3 \mathrm{~A}-\boldsymbol{\beta}$

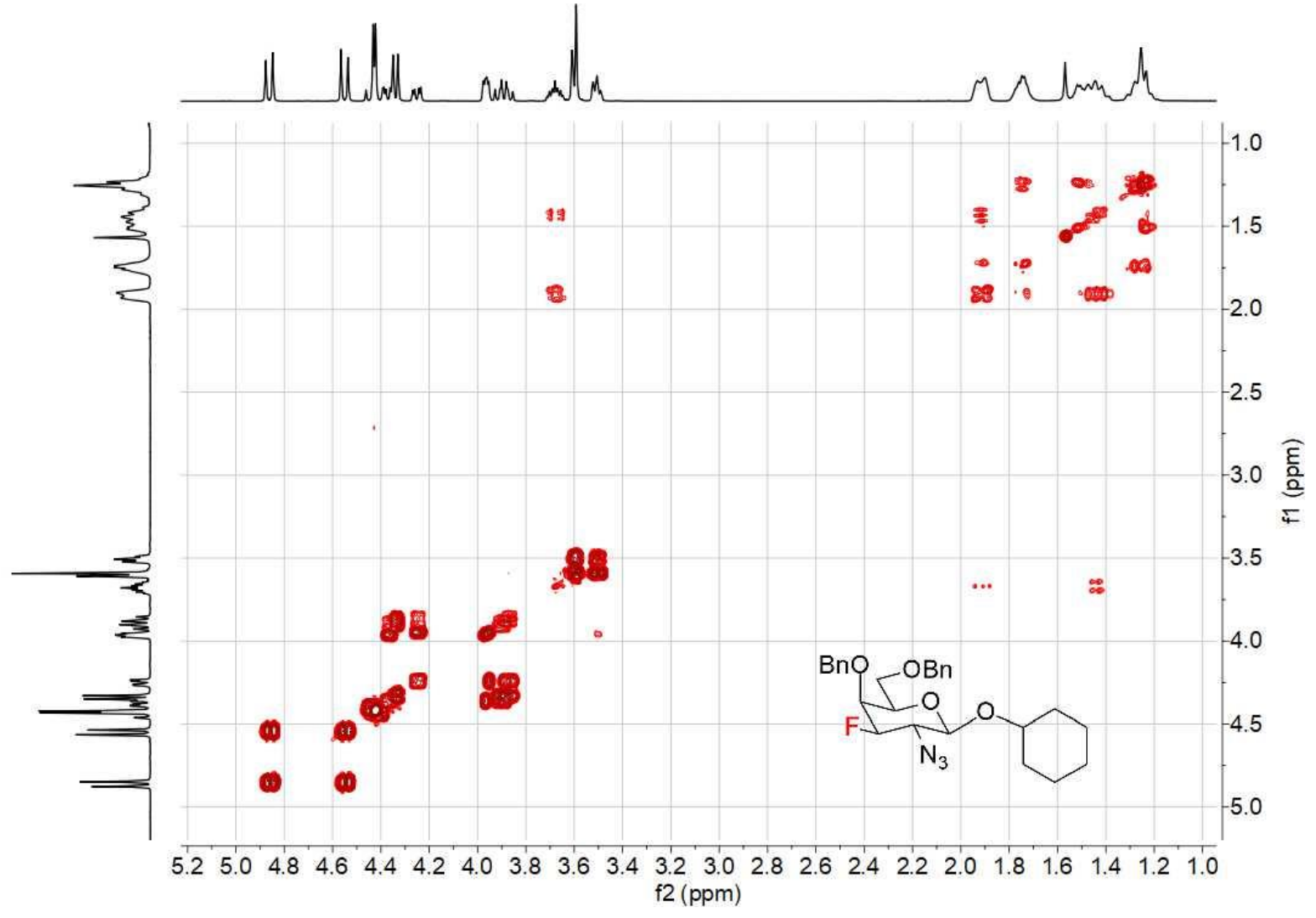


${ }^{1} \mathrm{H}-{ }^{13} \mathrm{C}$ HSQC NMR $3 \mathrm{~A}-\beta$

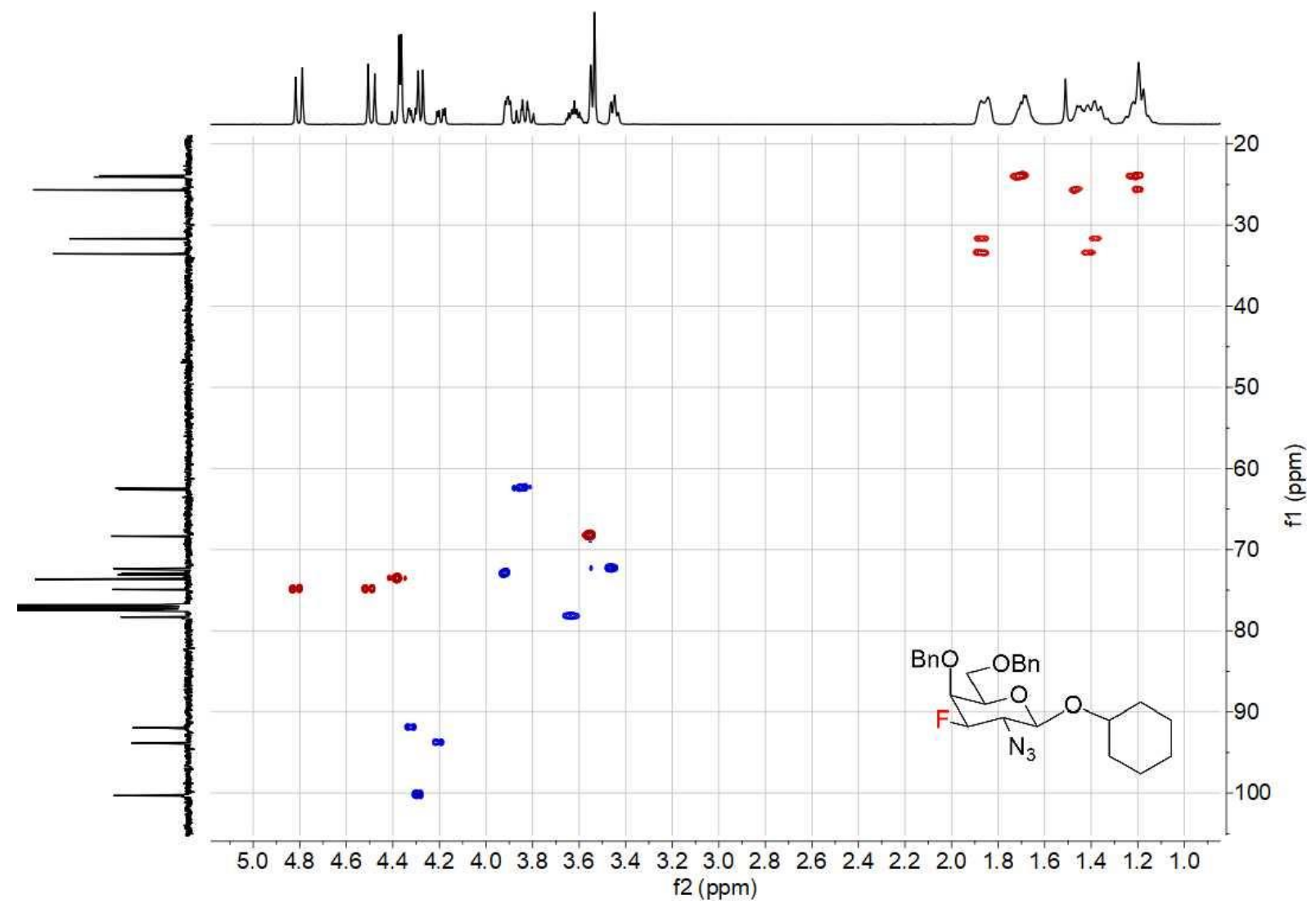

${ }^{1} \mathrm{H}-{ }^{13} \mathrm{C}$ HMBC NMR $3 \mathrm{~A}-\boldsymbol{\beta}$

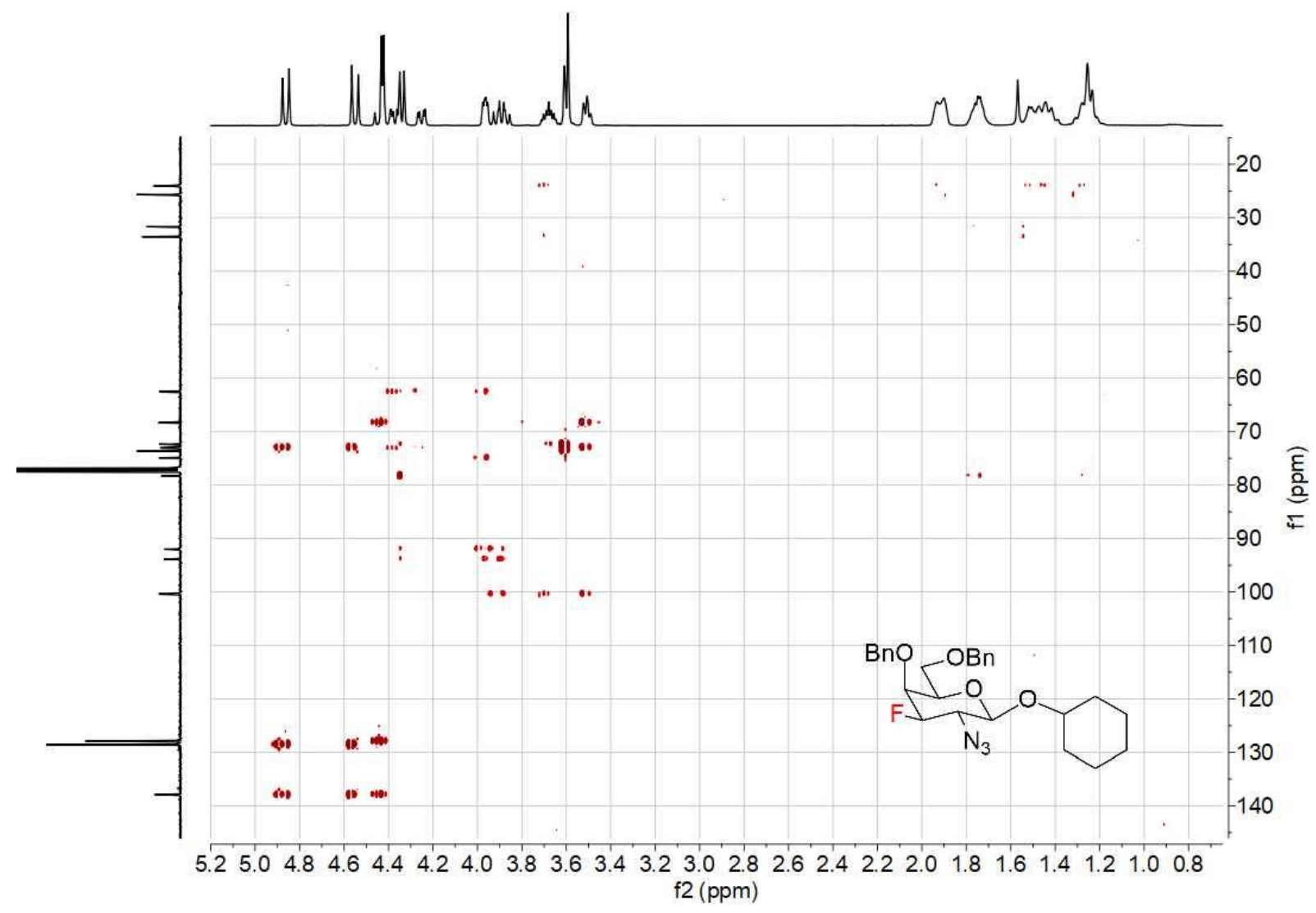



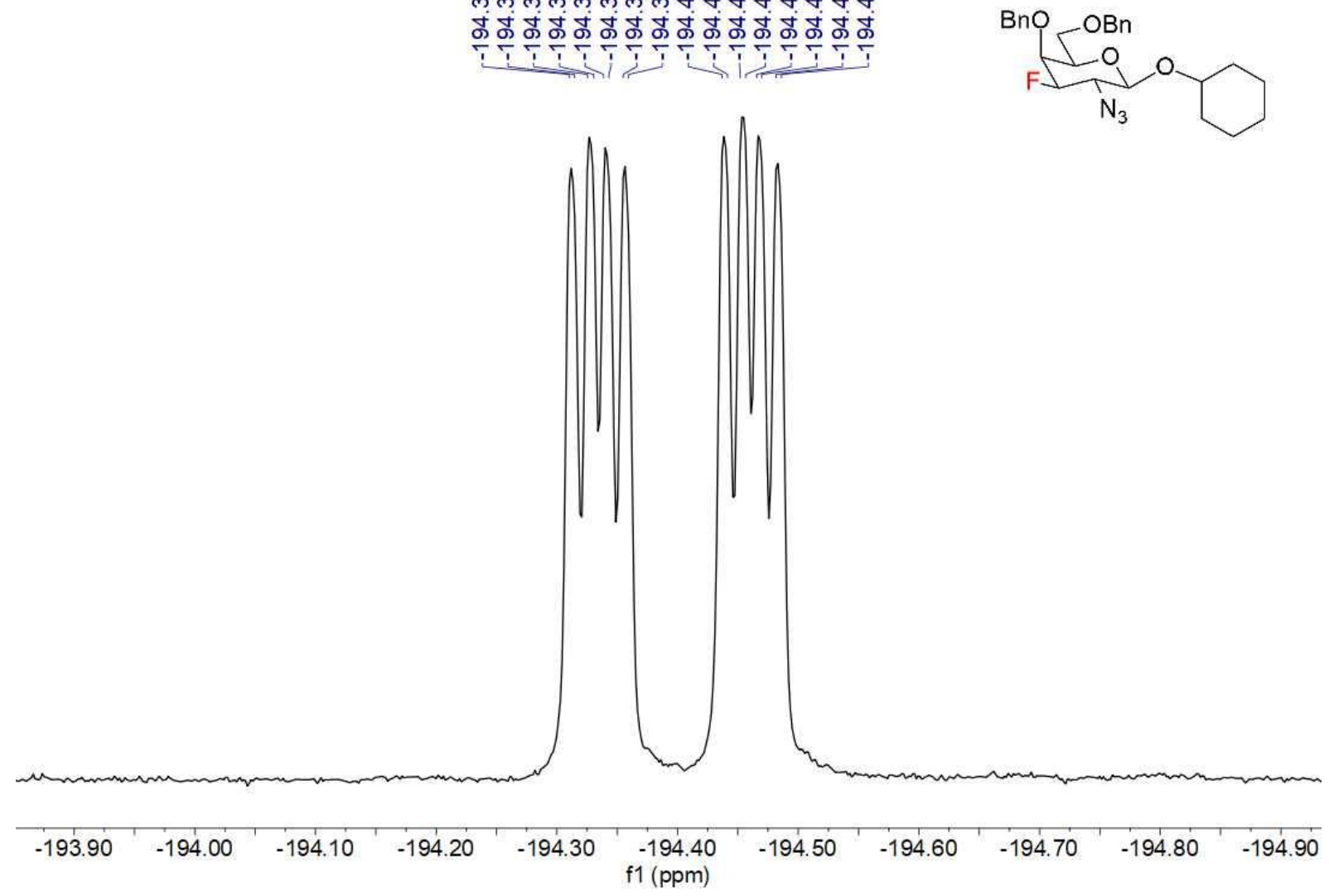

NMR 3B- $\alpha$

${ }^{1} \mathrm{HNMR} 400 \mathrm{MHz}, \mathrm{CDCl}_{3} 3 \mathrm{~B}-\alpha$

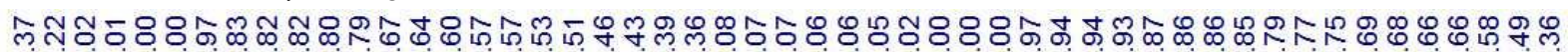
NN

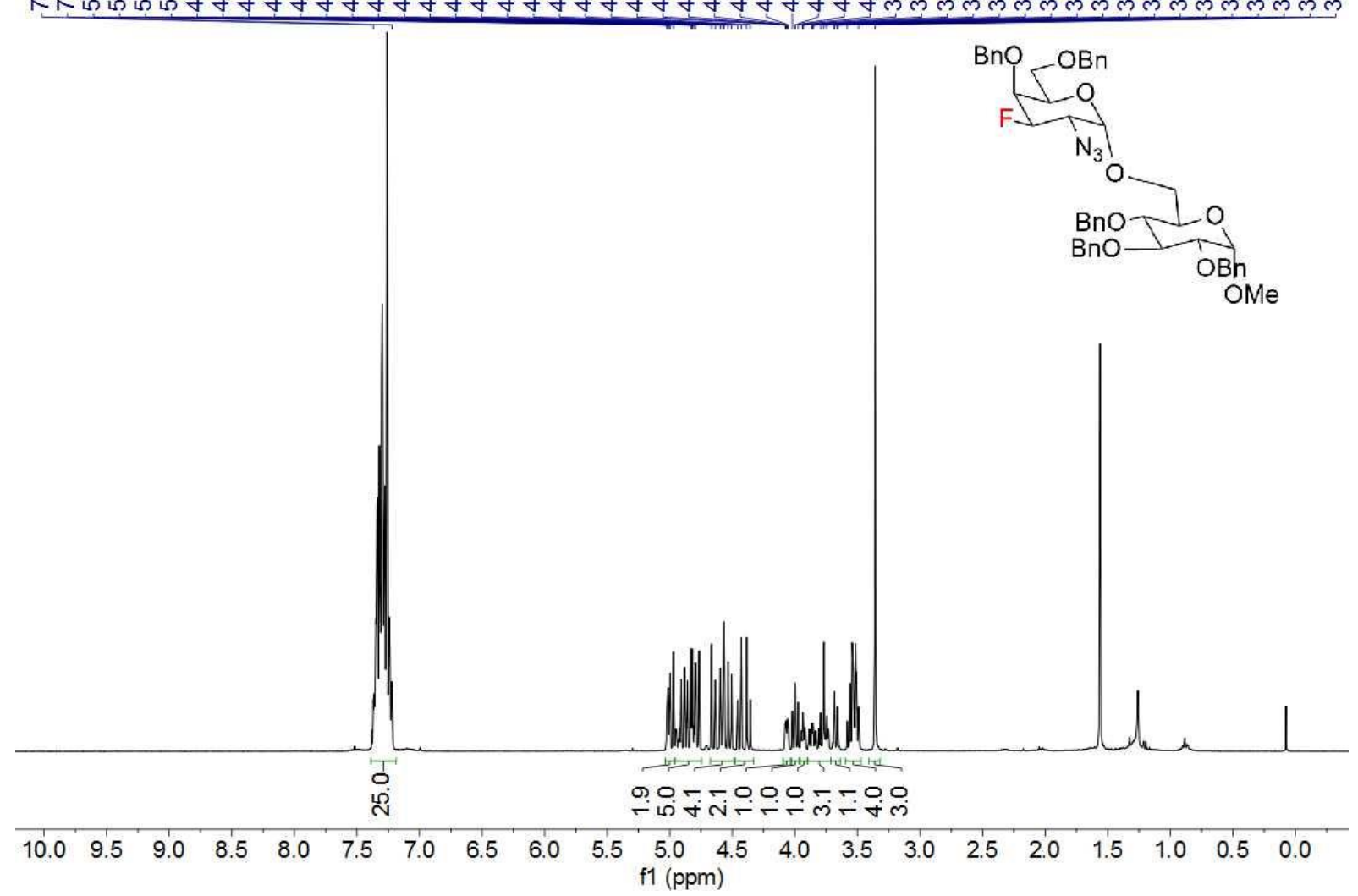


${ }^{13} \mathrm{C}\left\{{ }^{1} \mathrm{H}\right\}$ NMR $100 \mathrm{MHz}, \mathrm{CDCl}_{3} 3 \mathrm{~B}-\alpha$

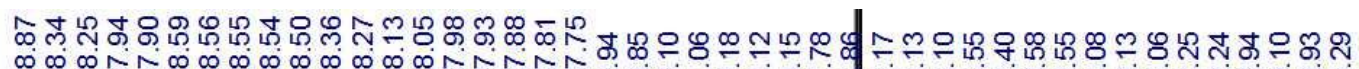

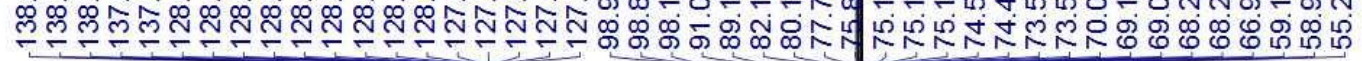

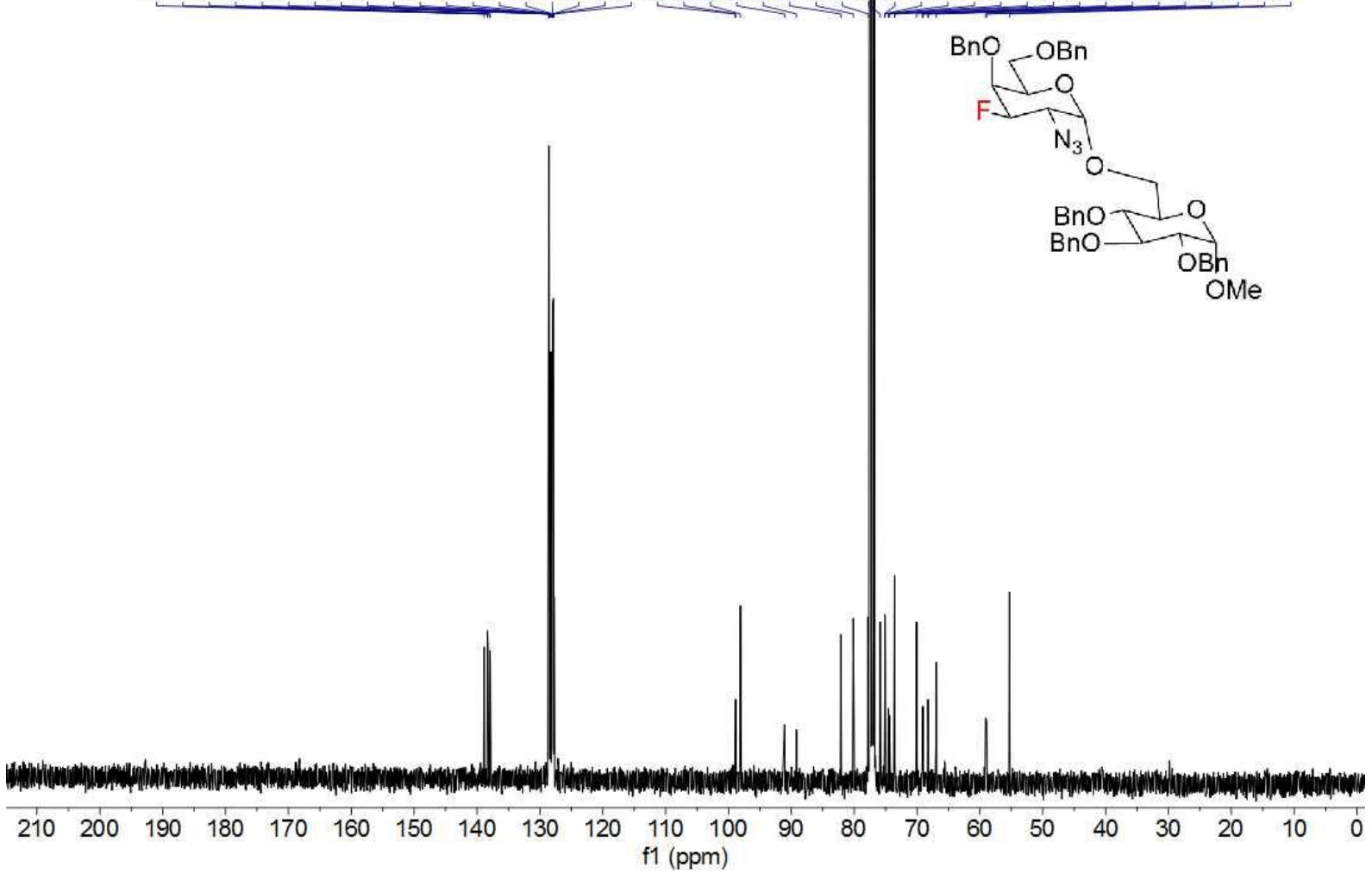

${ }^{1} \mathrm{H}-{ }^{1} \mathrm{H} \cos Y$ NMR $3 \mathrm{~B}-\boldsymbol{\alpha}$

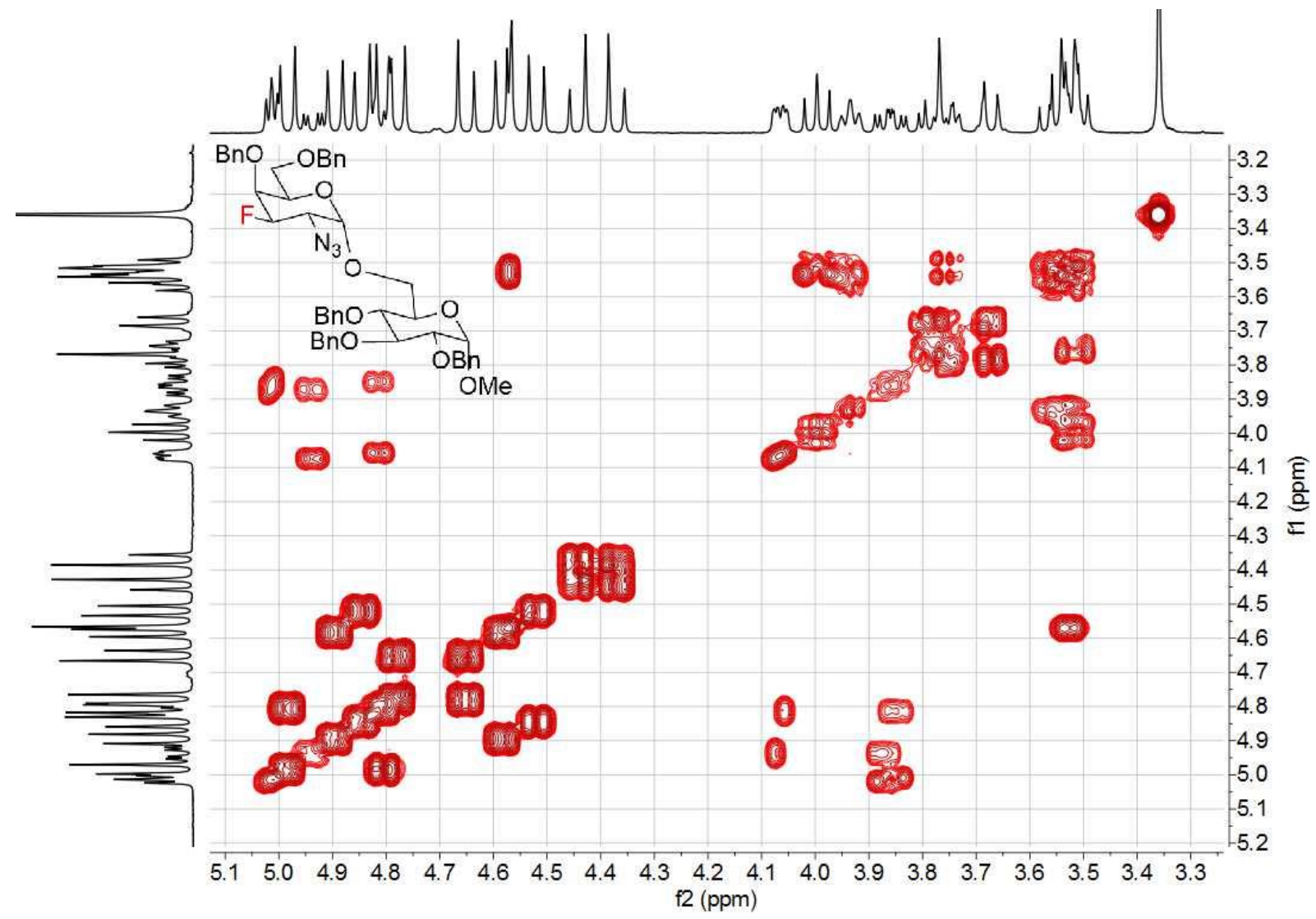


${ }^{1} \mathrm{H}^{-13} \mathrm{C}$ HSQC NMR 3B- $\alpha$

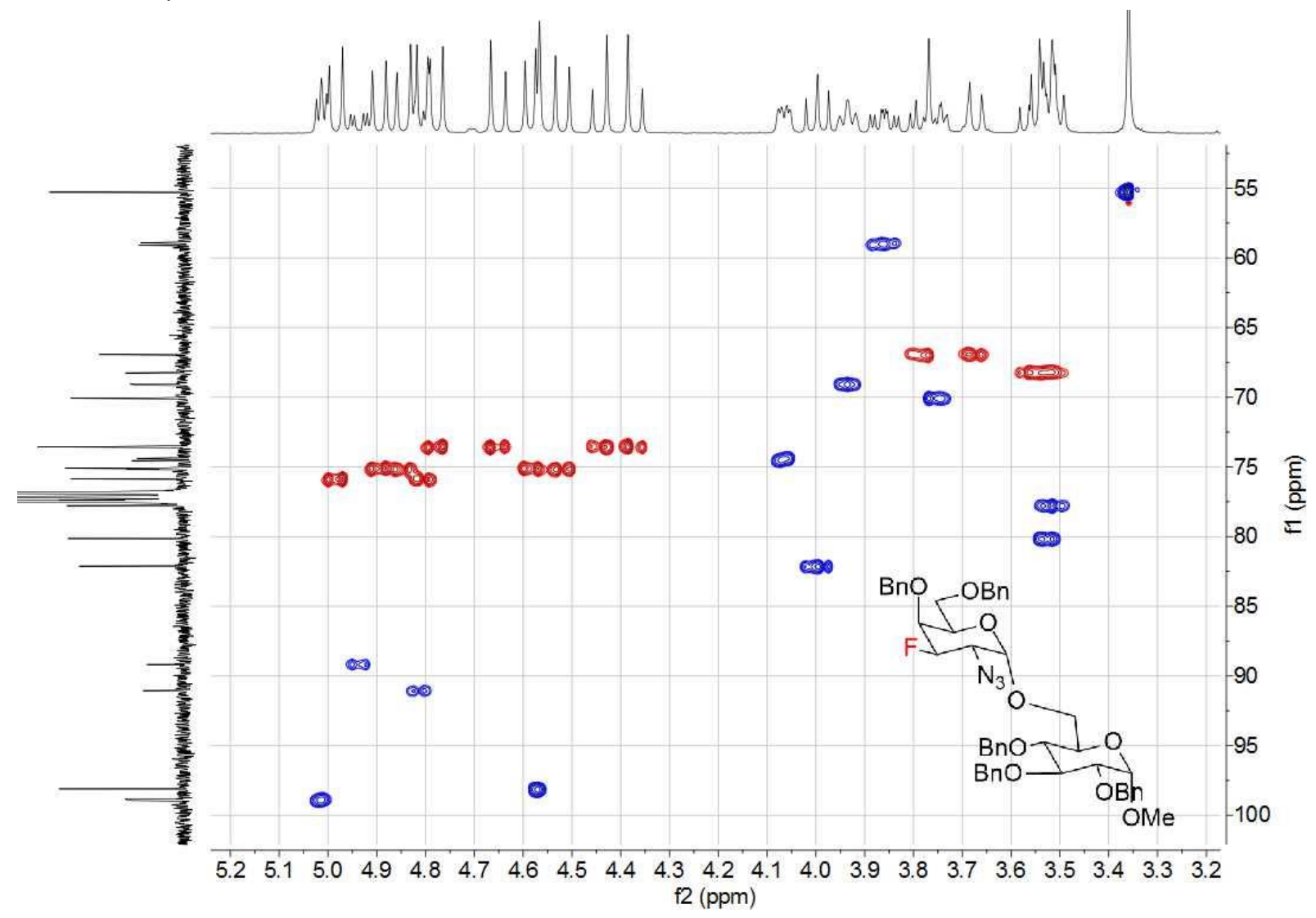

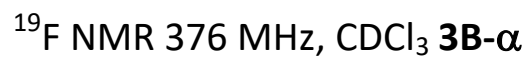

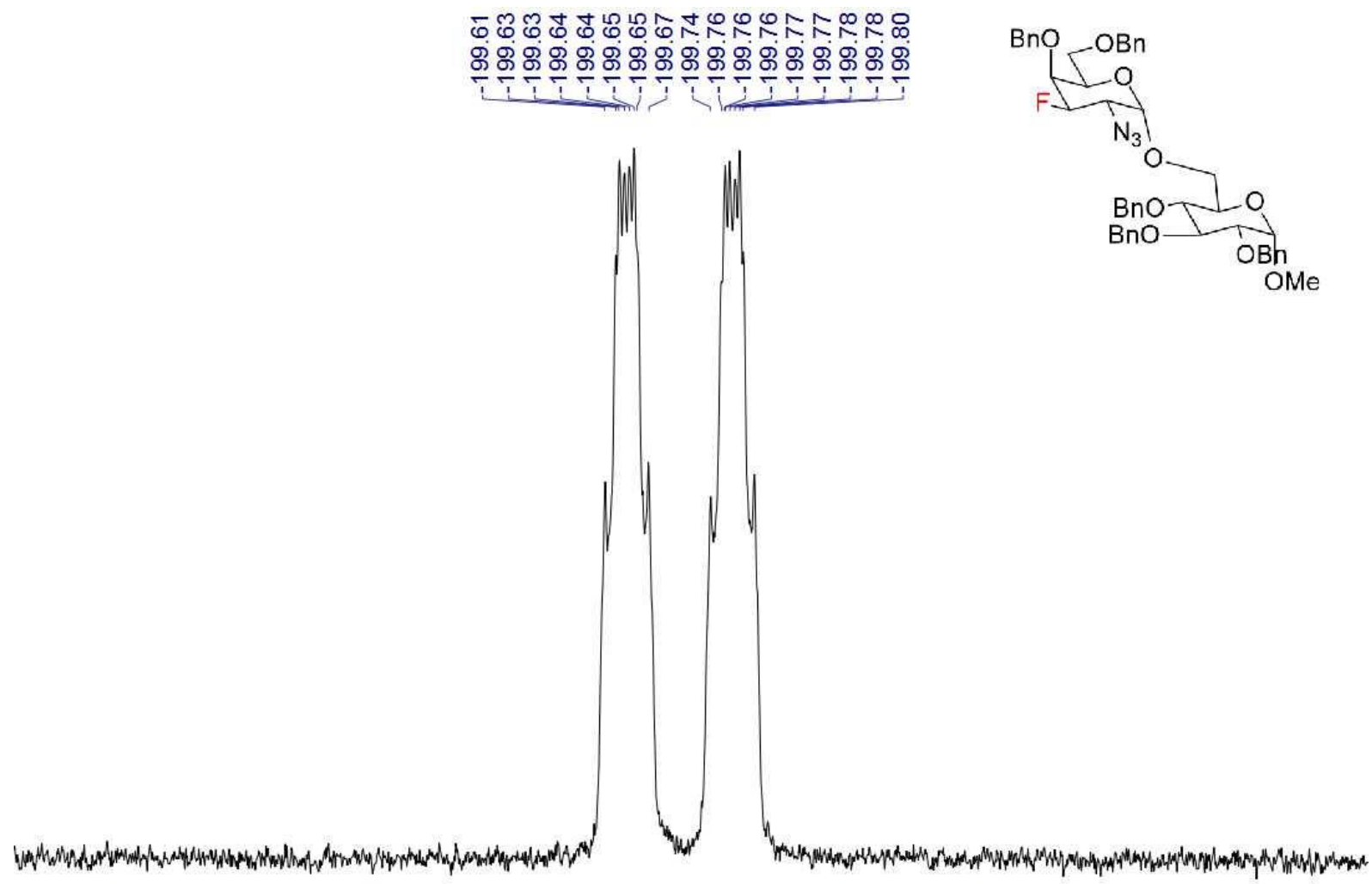
\begin{tabular}{lllllllllllllllllllll}
\hline 198.9 & -199.0 & -199.1 & -199.2 & -199.3 & -199.4 & -199.5 & -199.6 & -199.7 & -199.8 & -199.9 & -200.0 & -200.1 & -200.2 & -200.3 & -200.4 & -200.5
\end{tabular} f1 (ppm) 
NMR 3B- $\beta$

${ }^{1} \mathrm{HNMR} 400 \mathrm{MHz}, \mathrm{CDCl}_{3}$ 3B- $\beta$

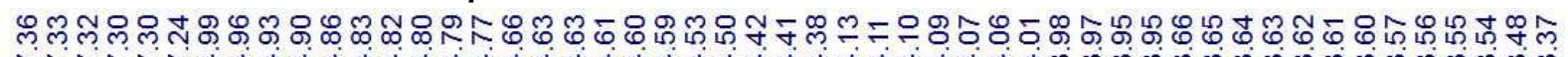

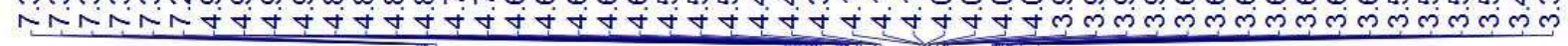

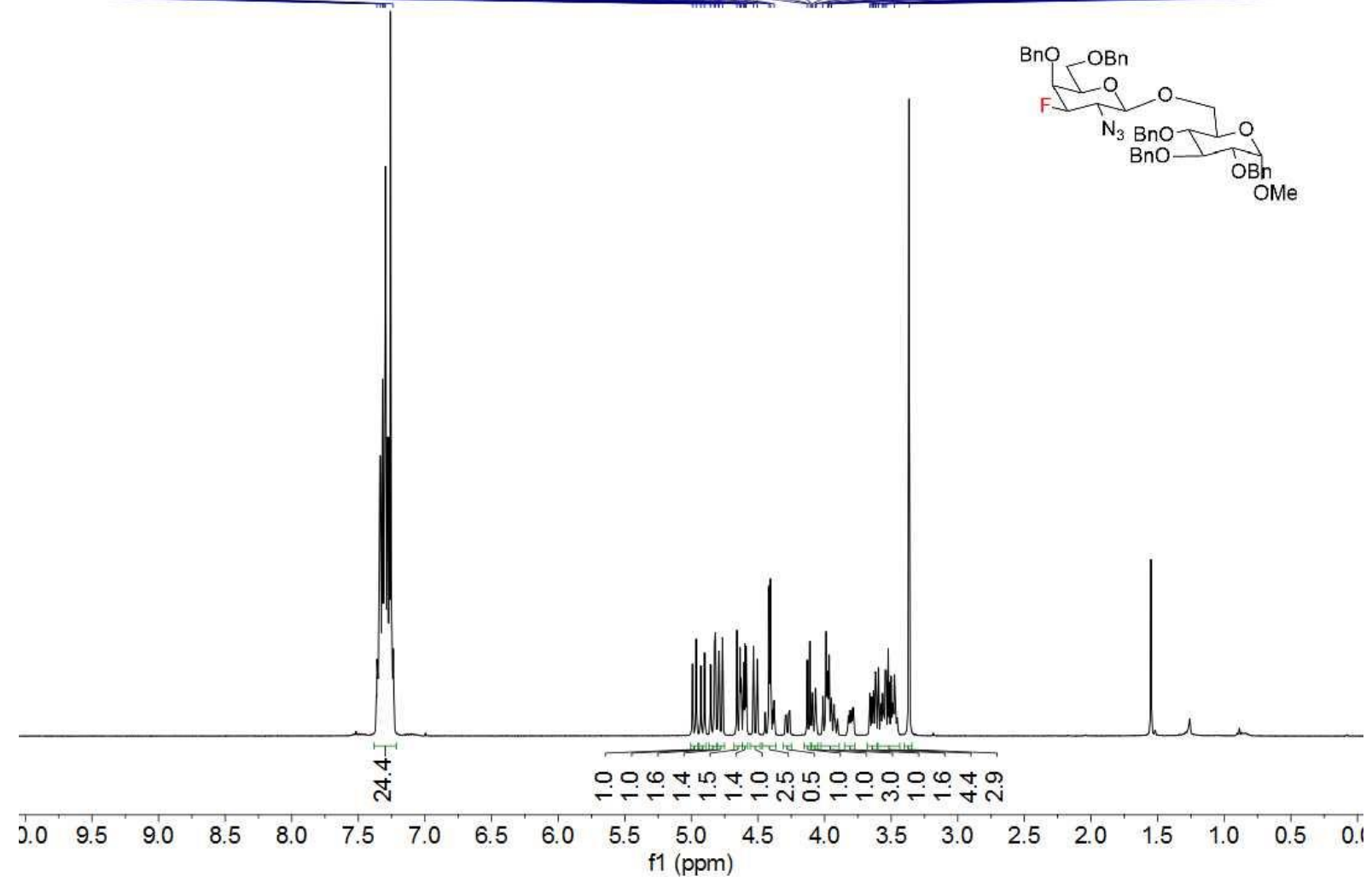

${ }^{13} \mathrm{C}\left\{{ }^{1} \mathrm{H}\right\} \mathrm{NMR} 100 \mathrm{MHz}, \mathrm{CDCl}_{3}$ 3B- $\beta$

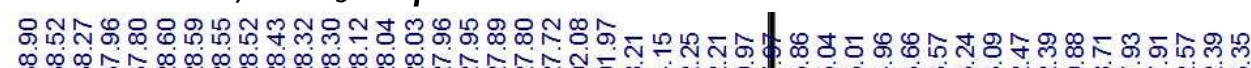

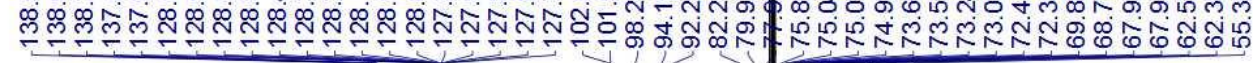

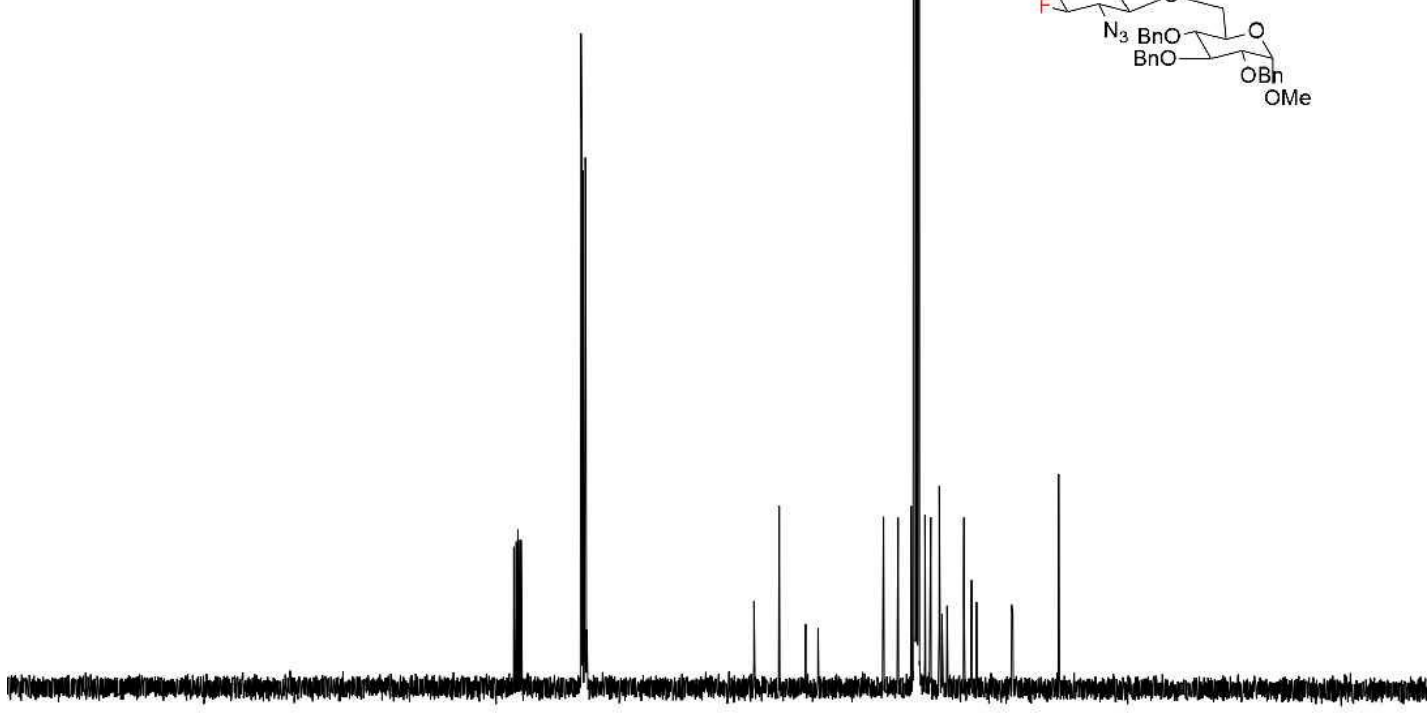

$\begin{array}{llllllllllllllllllllll}210 & 200 & 190 & 180 & 170 & 160 & 150 & 140 & 130 & 120 & \begin{array}{c}110 \\ \mathrm{f}(\mathrm{ppm})\end{array} & 90 & 80 & 70 & 60 & 50 & 40 & 30 & 20 & 10 & 0\end{array}$ 
${ }^{1} \mathrm{H}-{ }^{1} \mathrm{H}$ COSY NMR 3B- $\beta$

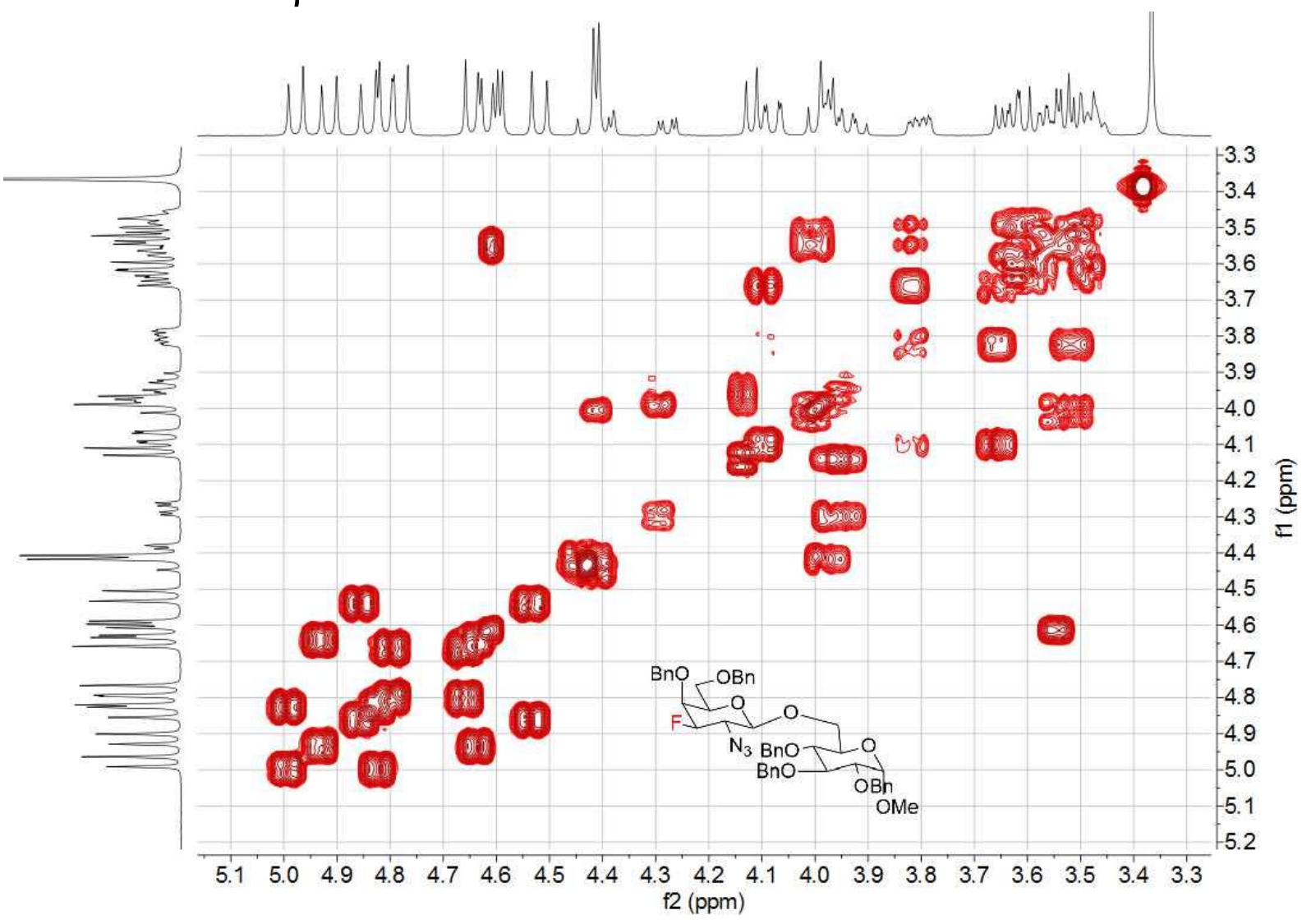

${ }^{1} \mathrm{H}^{-13} \mathrm{C}$ HSOC NMR 3B- $\beta$

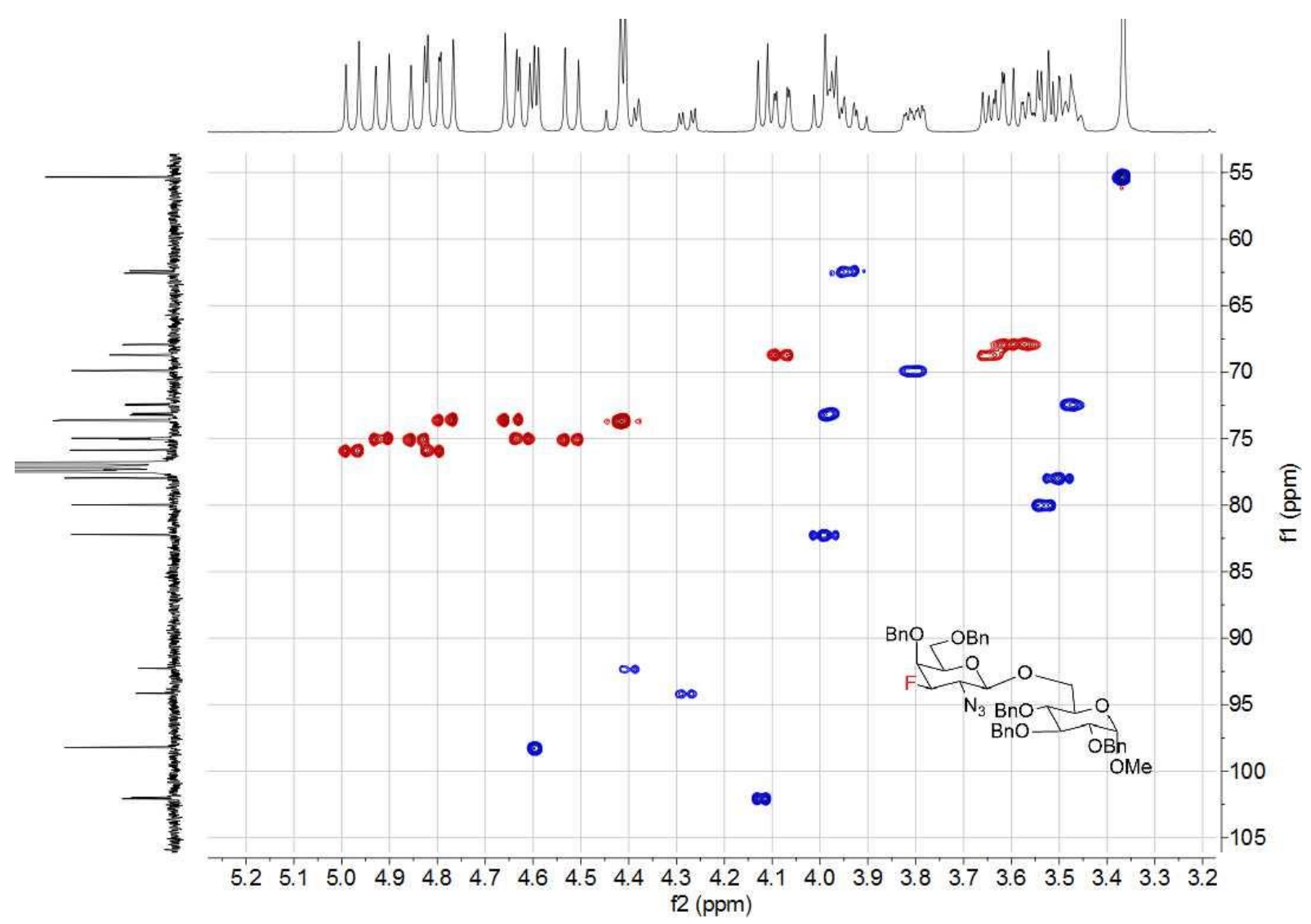


${ }^{19} \mathrm{~F} \mathrm{NMR} 376 \mathrm{MHz}, \mathrm{CDCl}_{3}$ 3B- $\boldsymbol{\beta}$

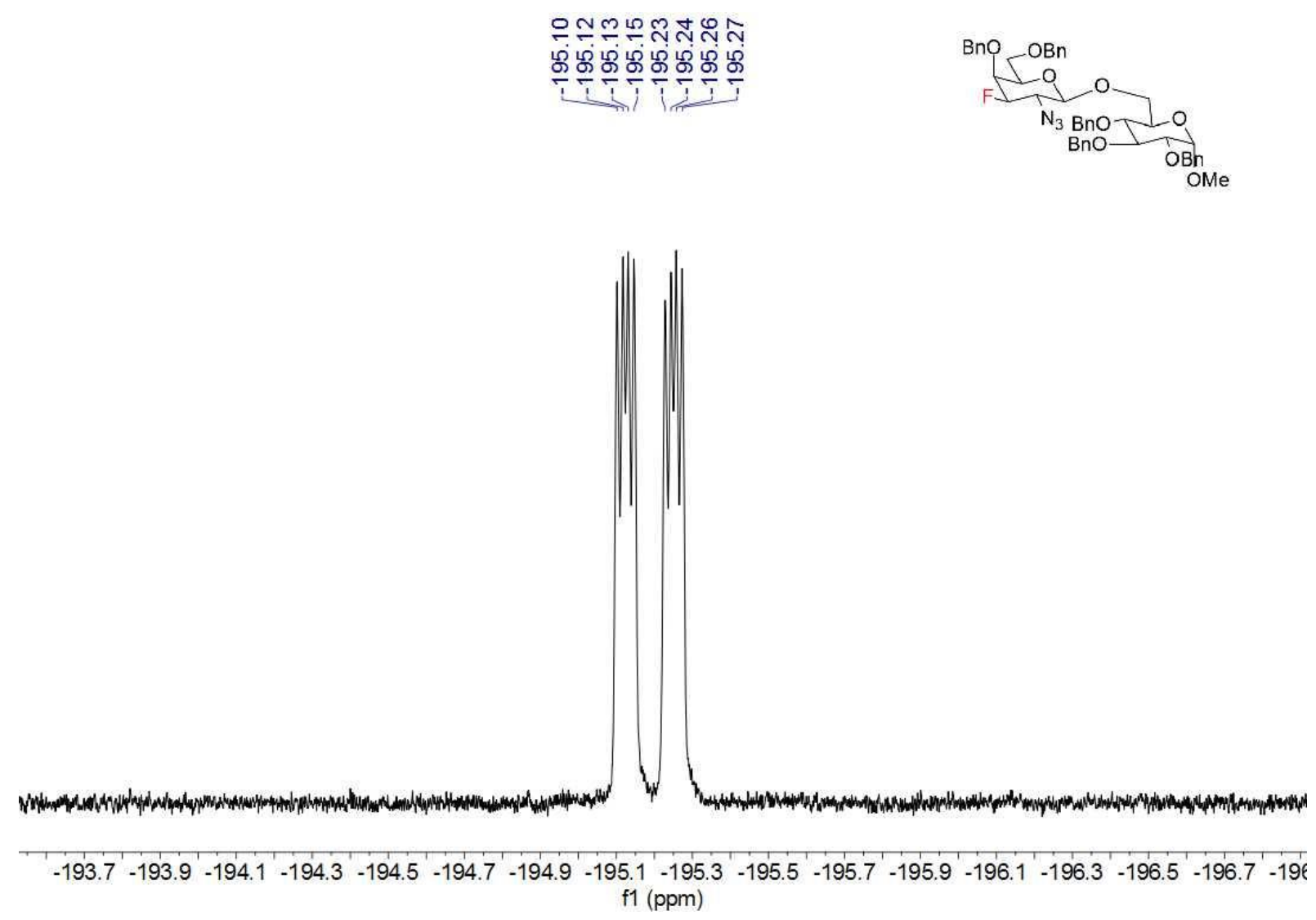




\section{NMR 3C}

${ }^{1} \mathrm{HNMR} 400 \mathrm{MHz}, \mathrm{CDCl}_{3} 3 \mathrm{C}-\alpha$

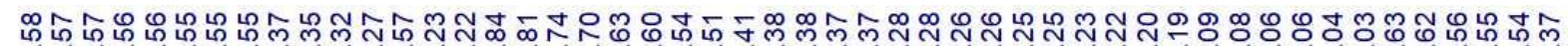

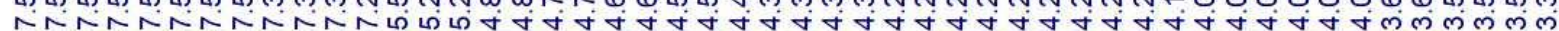

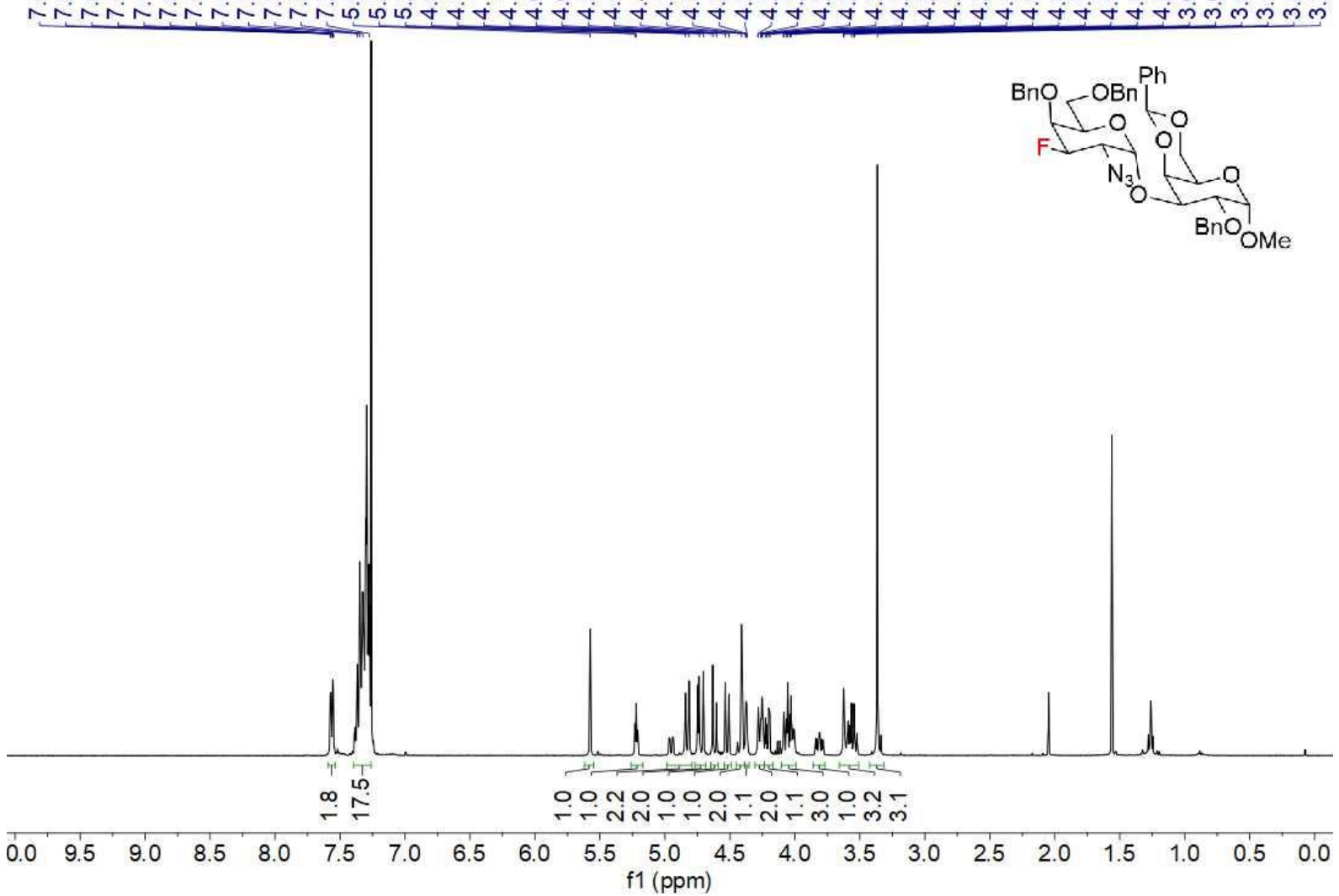

${ }^{13} \mathrm{C}\left\{{ }^{1} \mathrm{H}\right\} \mathrm{NMR} 100 \mathrm{MHz}, \mathrm{CDCl}_{3} 3 \mathrm{C}-\alpha$

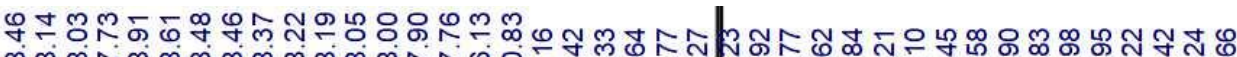

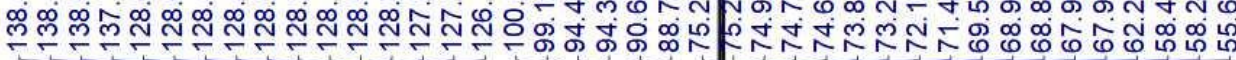

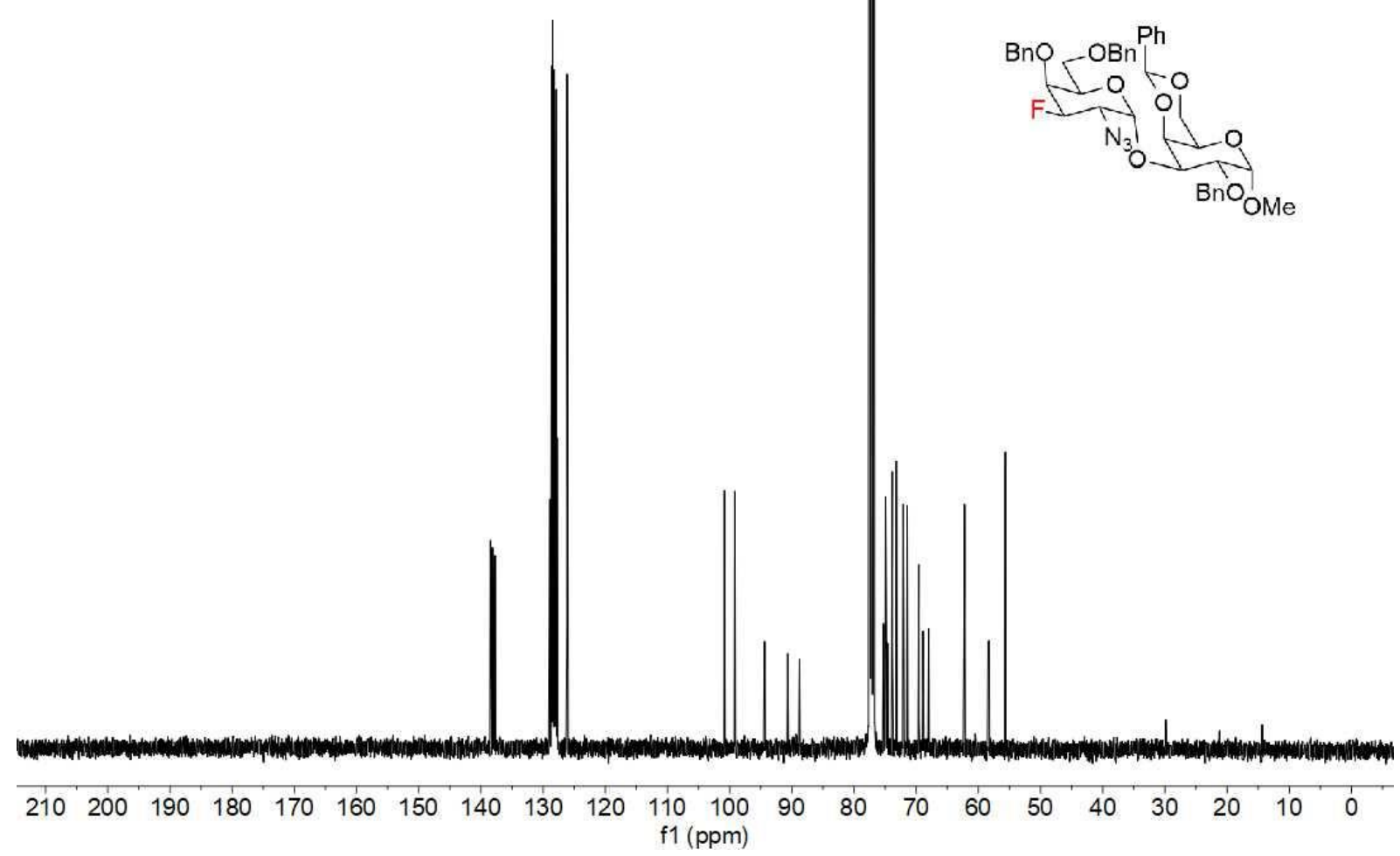


${ }^{1} \mathrm{H}-{ }^{1} \mathrm{H}$ COSY NMR $3 \mathrm{C}-\alpha$

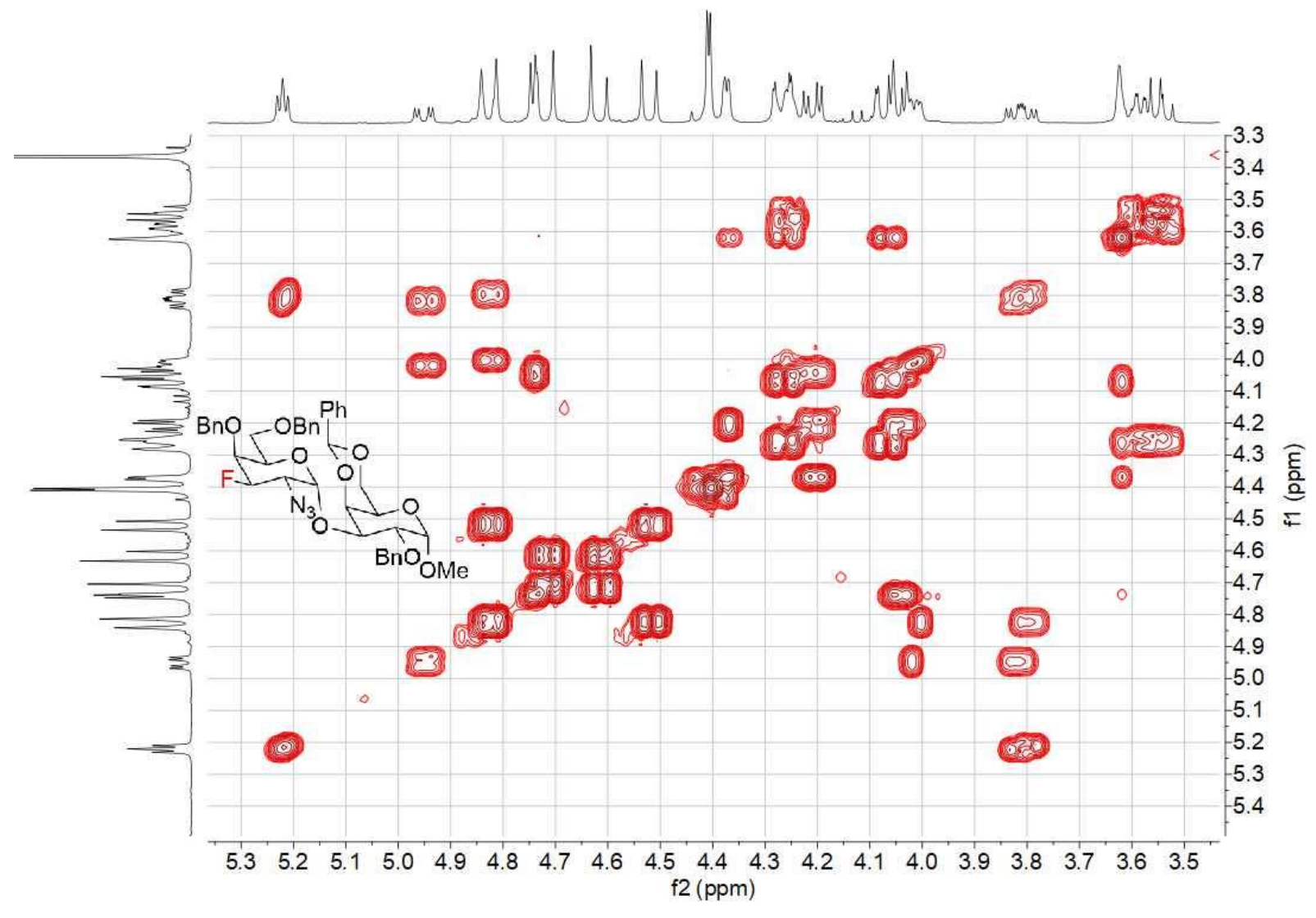

${ }^{1} \mathrm{H}-{ }^{13} \mathrm{C}$ HSQC NMR $3 \mathrm{C}-\alpha$

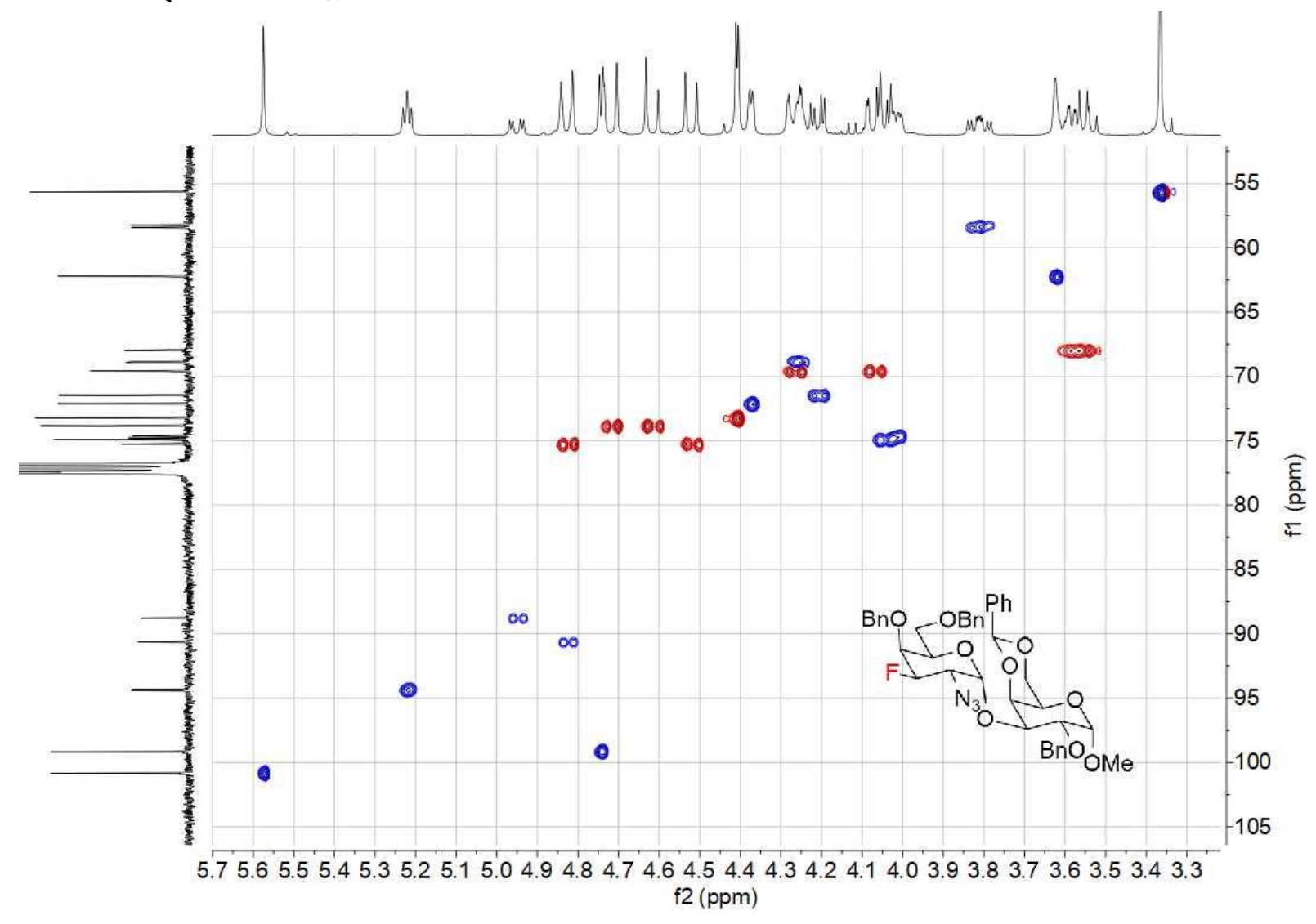




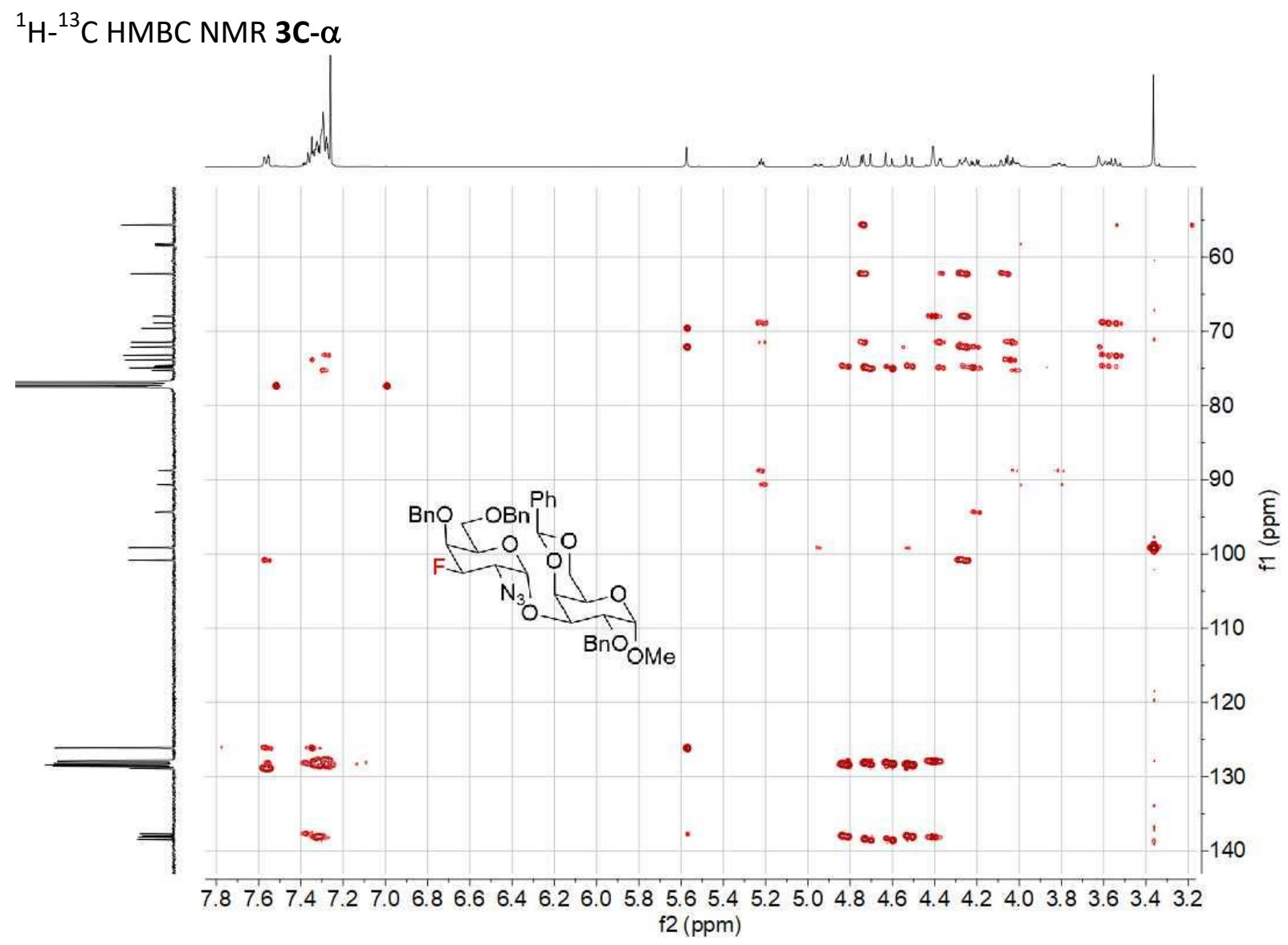

${ }^{19} \mathrm{~F}$ NMR $376 \mathrm{MHz}, \mathrm{CDCl}_{3} 3 \mathrm{C}-\alpha$
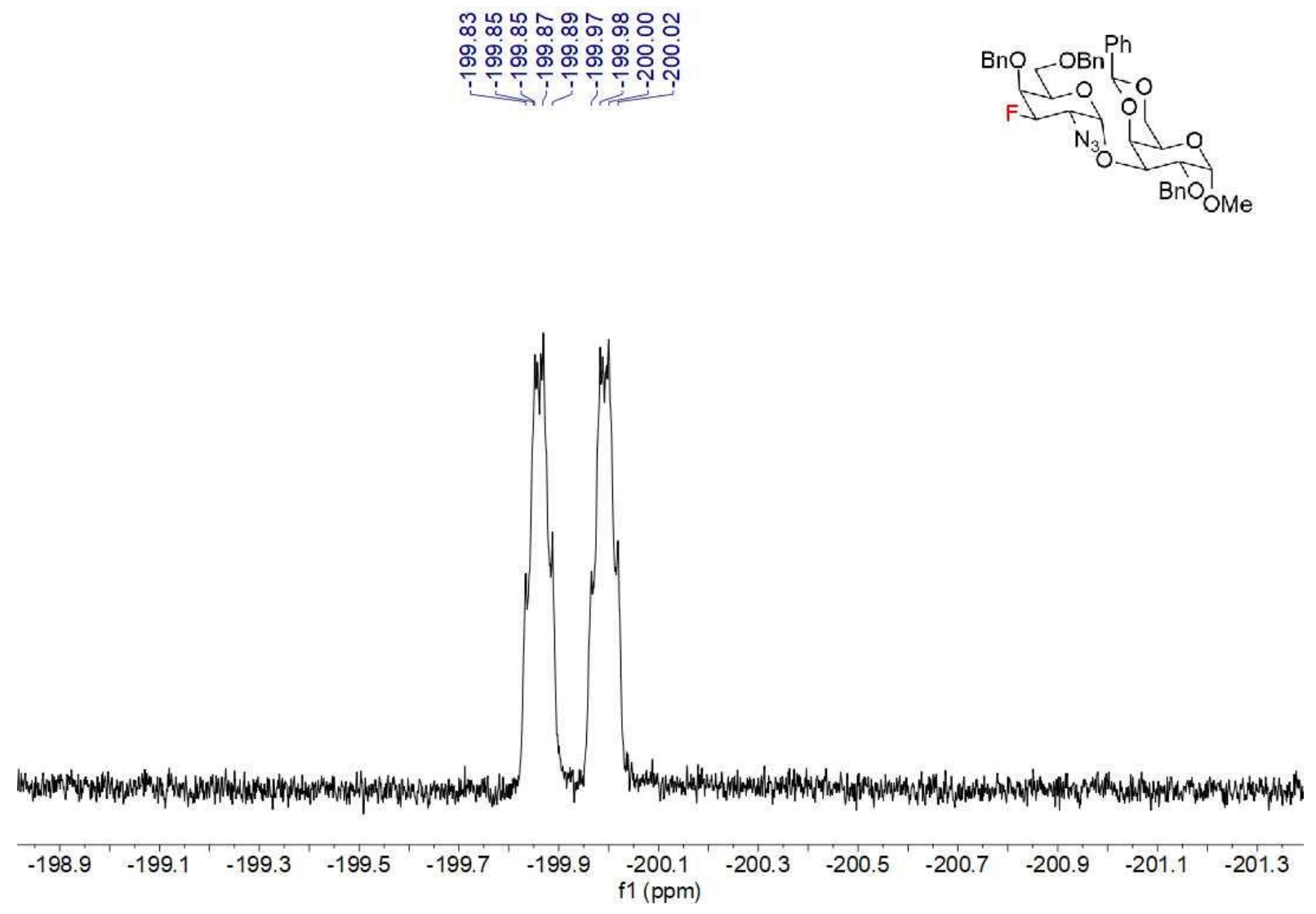
${ }^{1} \mathrm{HNMR} 400 \mathrm{MHz}, \mathrm{CDCl}_{3} 3 \mathrm{C}(\alpha: \beta$ 1.0:0.7)

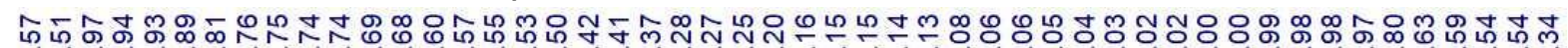
மூ
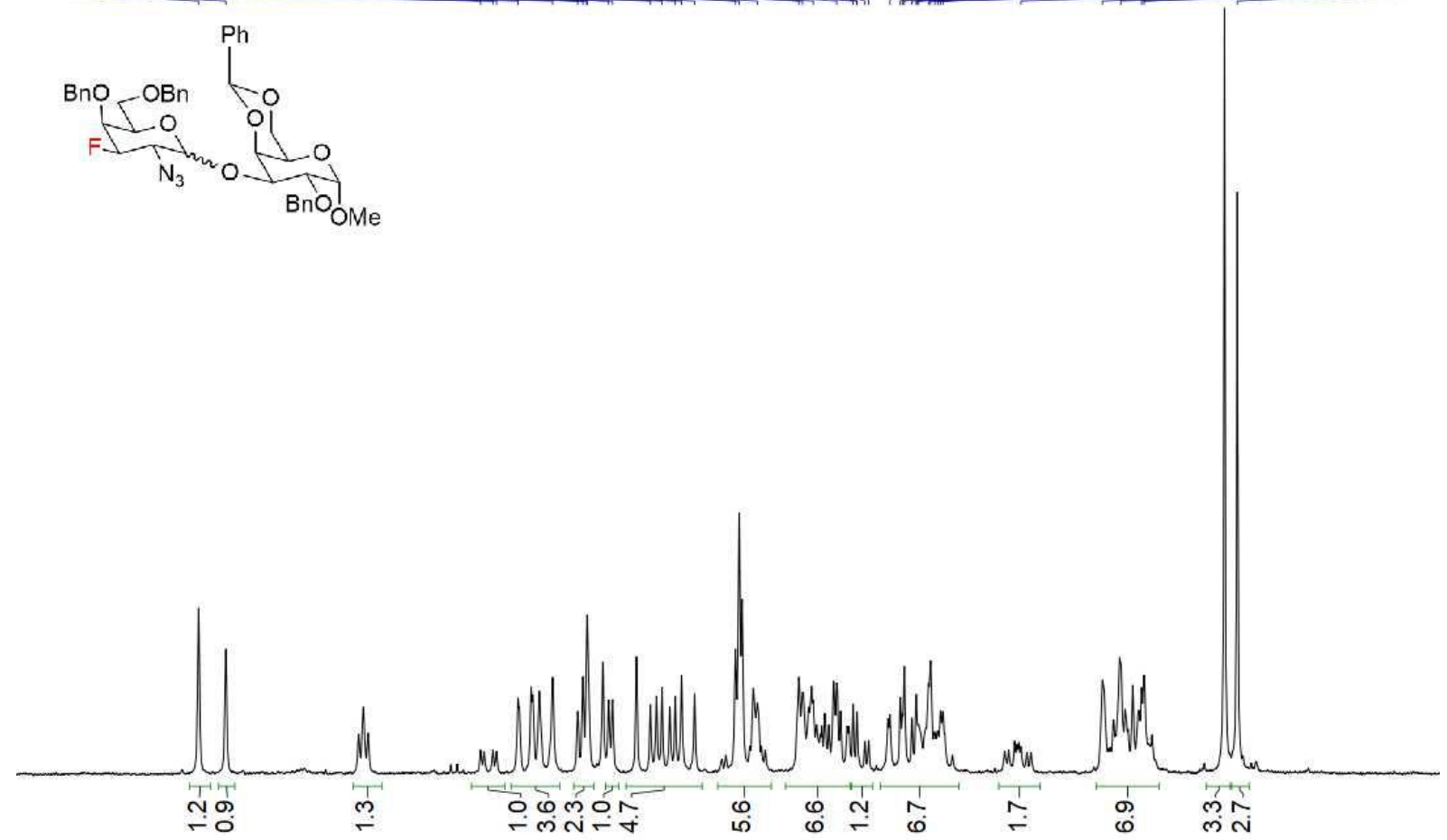

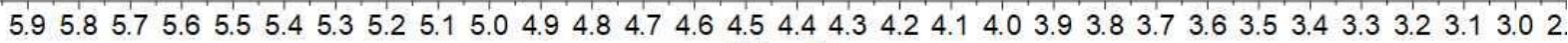
$\mathrm{f1}(\mathrm{ppm})$

${ }^{13} \mathrm{C}\left\{{ }^{1} \mathrm{H}\right\}$ NMR $100 \mathrm{MHz}, \mathrm{CDCl}_{3} 3 \mathrm{C}(\alpha: \beta$ 1:0.7)

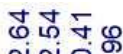

둥요용
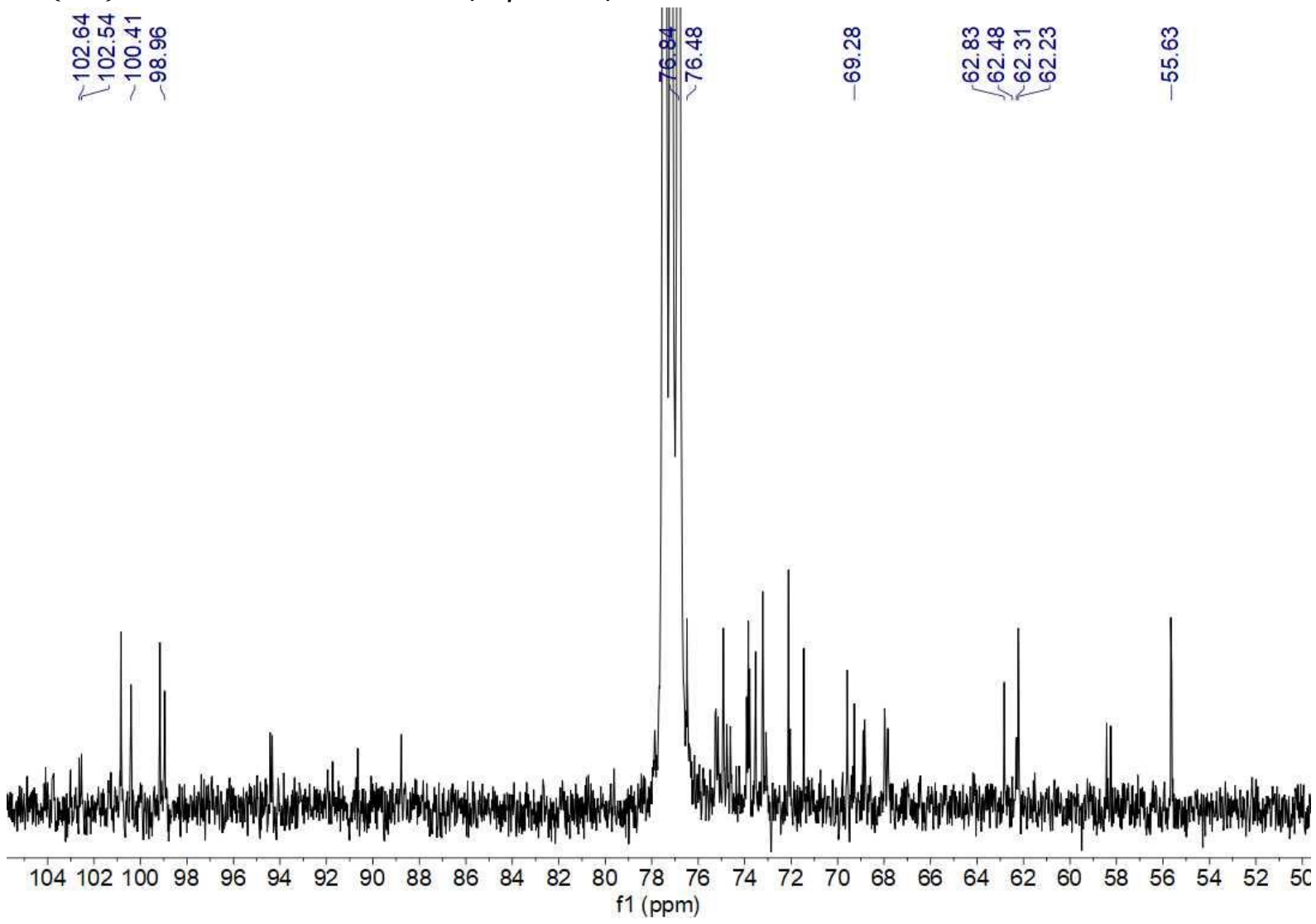
${ }^{1} \mathrm{H}-{ }^{1} \mathrm{H}$ COSY NMR $3 \mathrm{C}(\alpha: \beta 1: 0.7)$

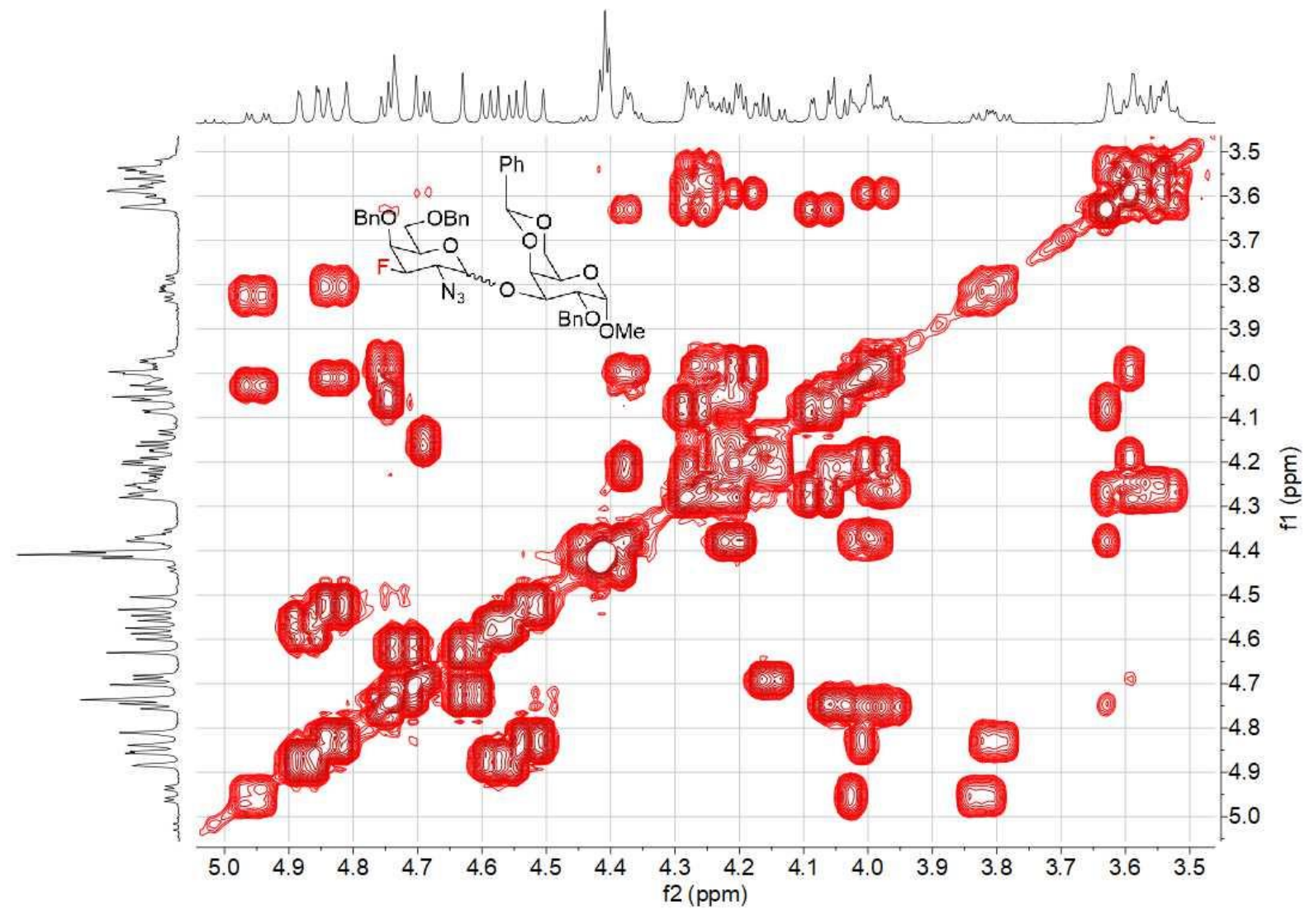

${ }^{1} \mathrm{H}-{ }^{13} \mathrm{C}$ HSQC NMR $3 \mathrm{C}(\alpha: \beta$ 1:0.7)

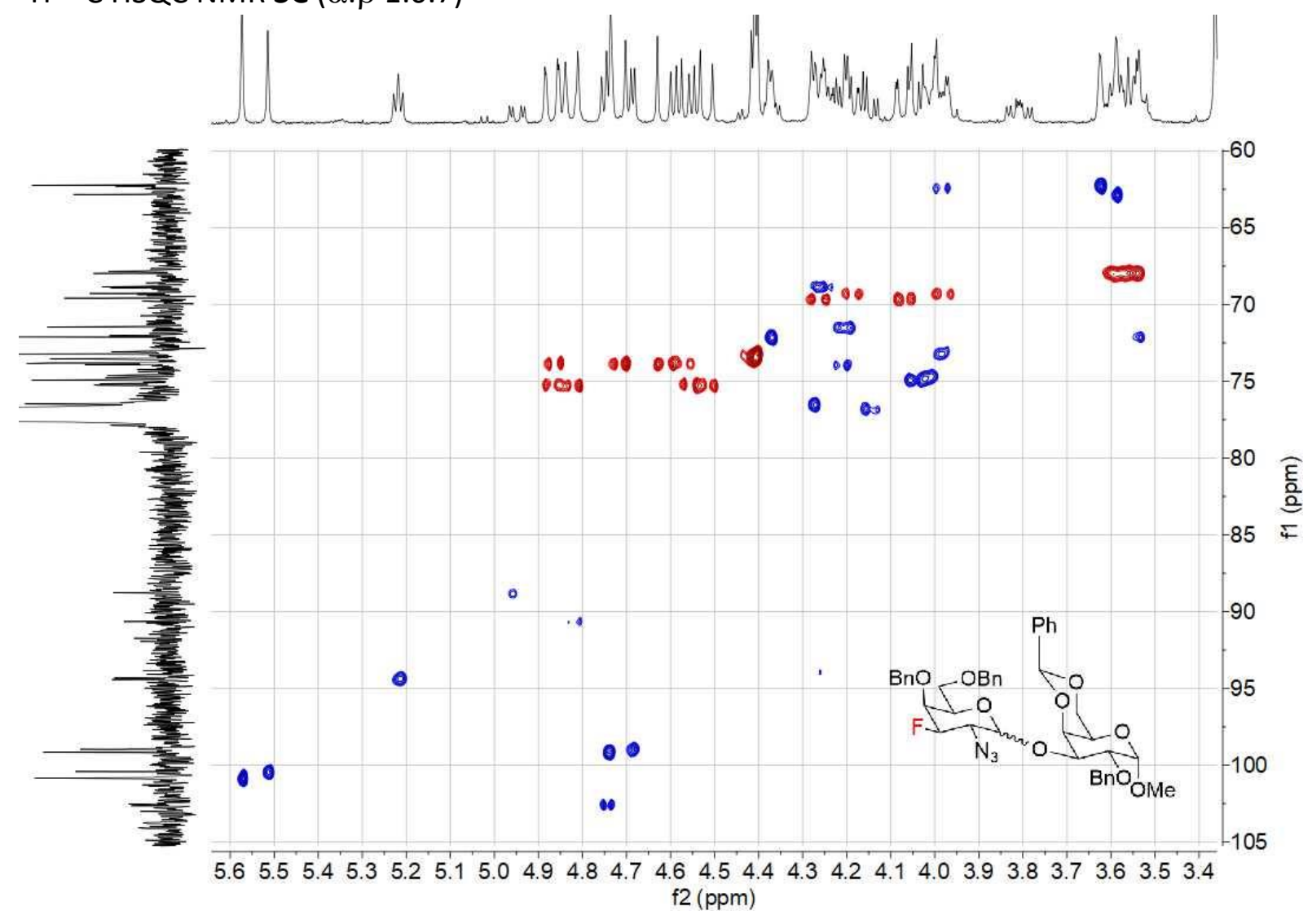


${ }^{19} \mathrm{~F} \mathrm{NMR} 376 \mathrm{MHz}, \mathrm{CDCl}_{3} 3 \mathrm{C}(\alpha: \beta$ 1:0.7)

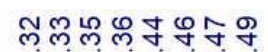

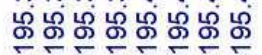

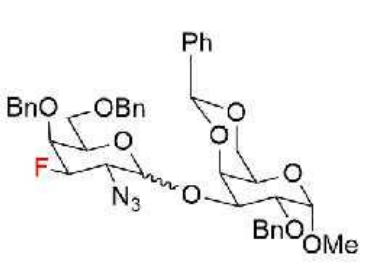

œ

ஓं

$1 T 5 T$

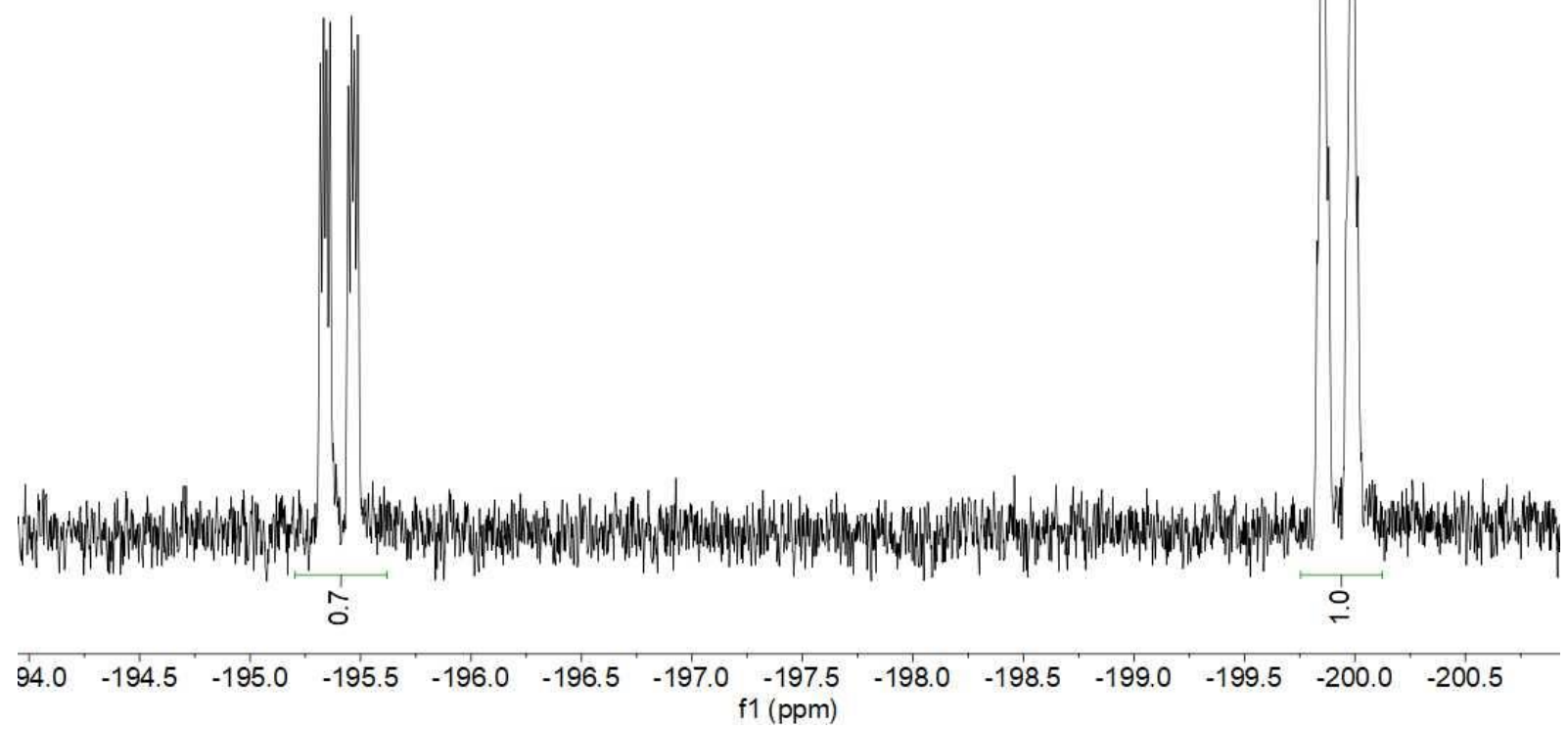

NMR 3D- $\alpha$

${ }^{1} \mathrm{HNMR} 400 \mathrm{MHz}, \mathrm{CDCl}_{3}$ 3D- $\alpha$

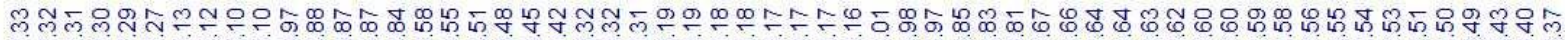

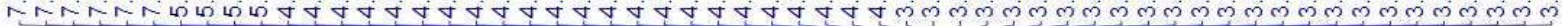

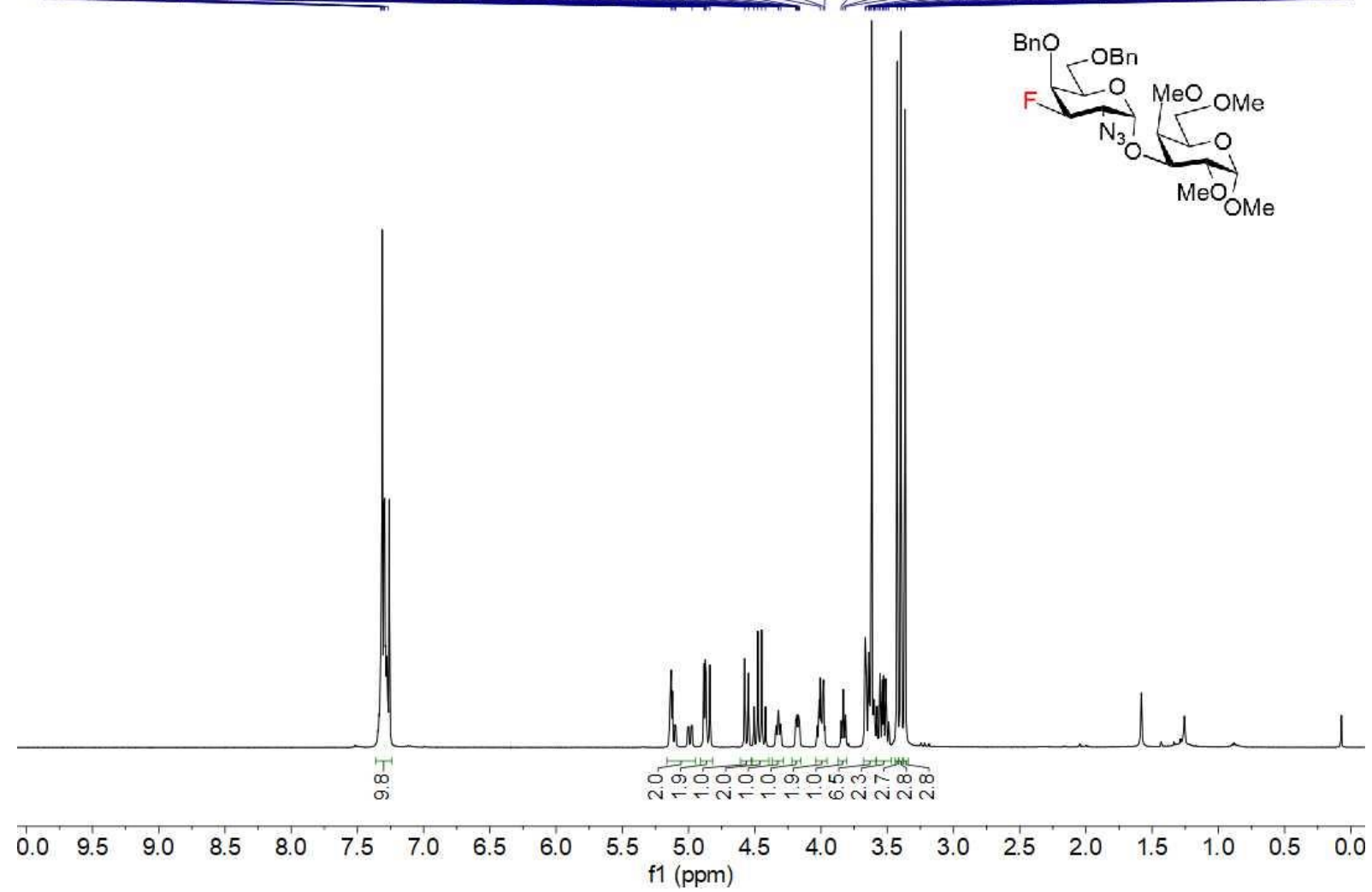


${ }^{13} \mathrm{C}\left\{{ }^{1} \mathrm{H}\right\}$ NMR 100MHz, $\mathrm{CDCl}_{3}$ 3D- $\alpha$

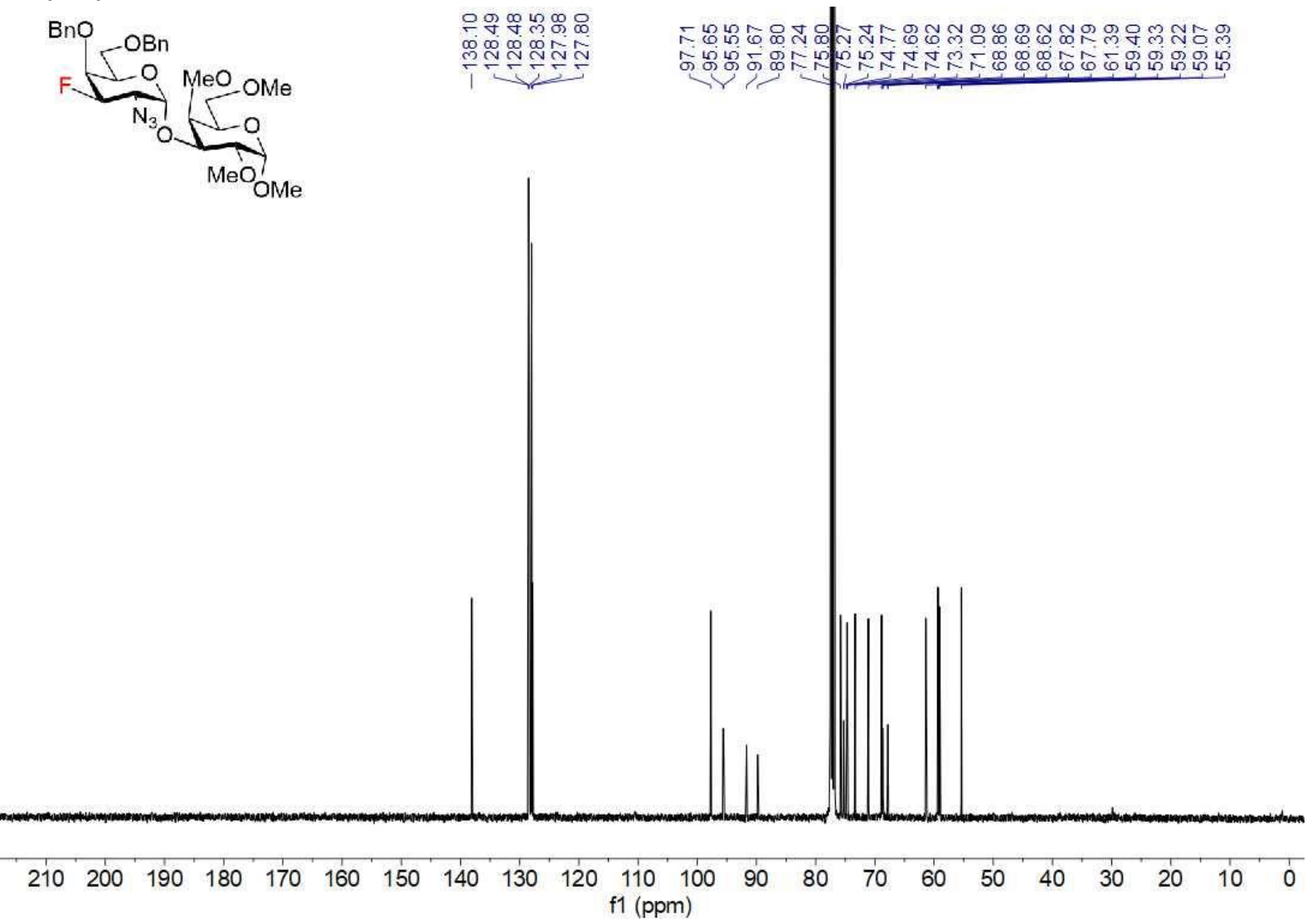

${ }^{1} \mathrm{H}-{ }^{1} \mathrm{H}$ COSY NMR 3D- $\alpha$

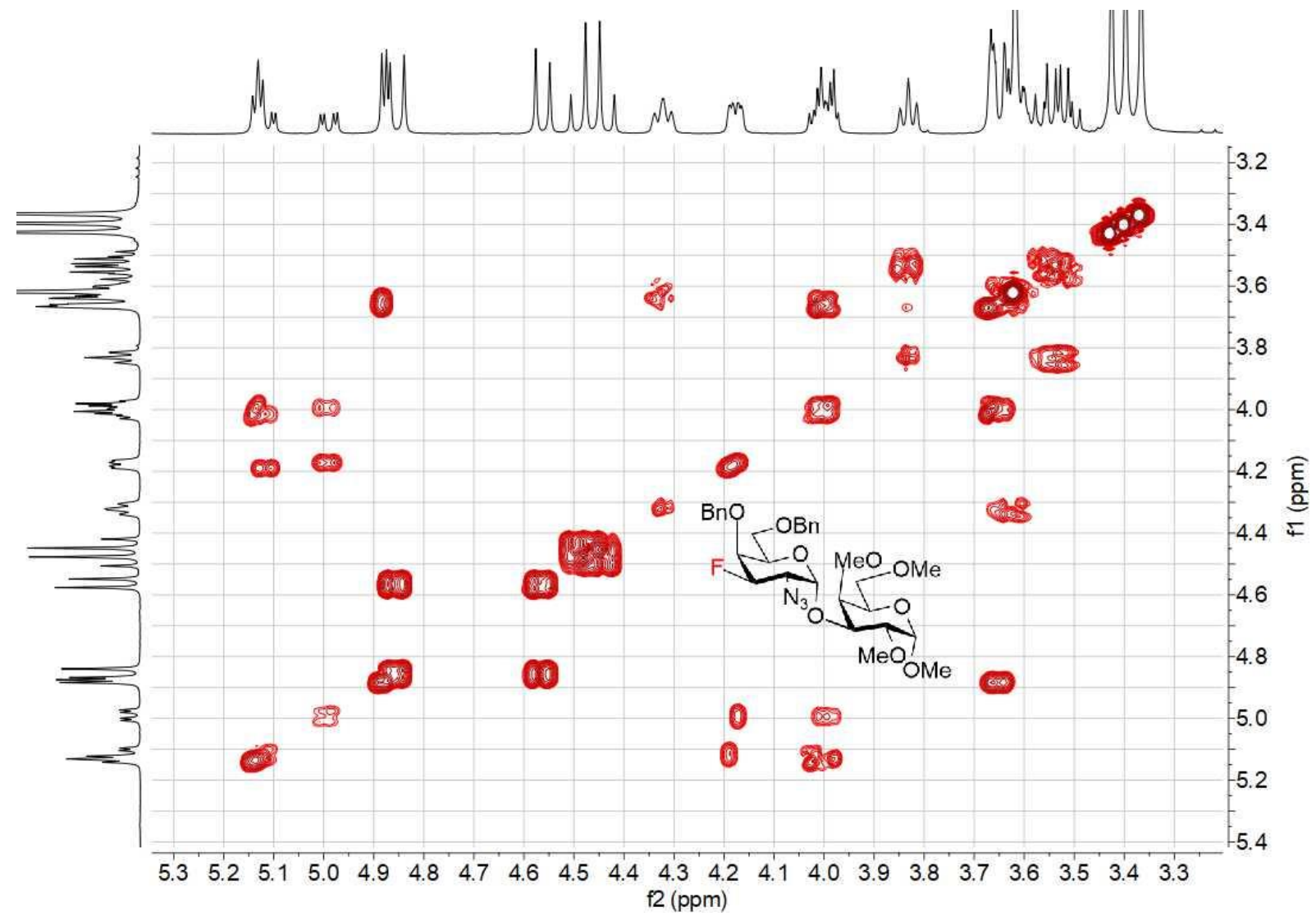


${ }^{1} \mathrm{H}-{ }^{13} \mathrm{C}$ HSQC NMR 3D- $\alpha$

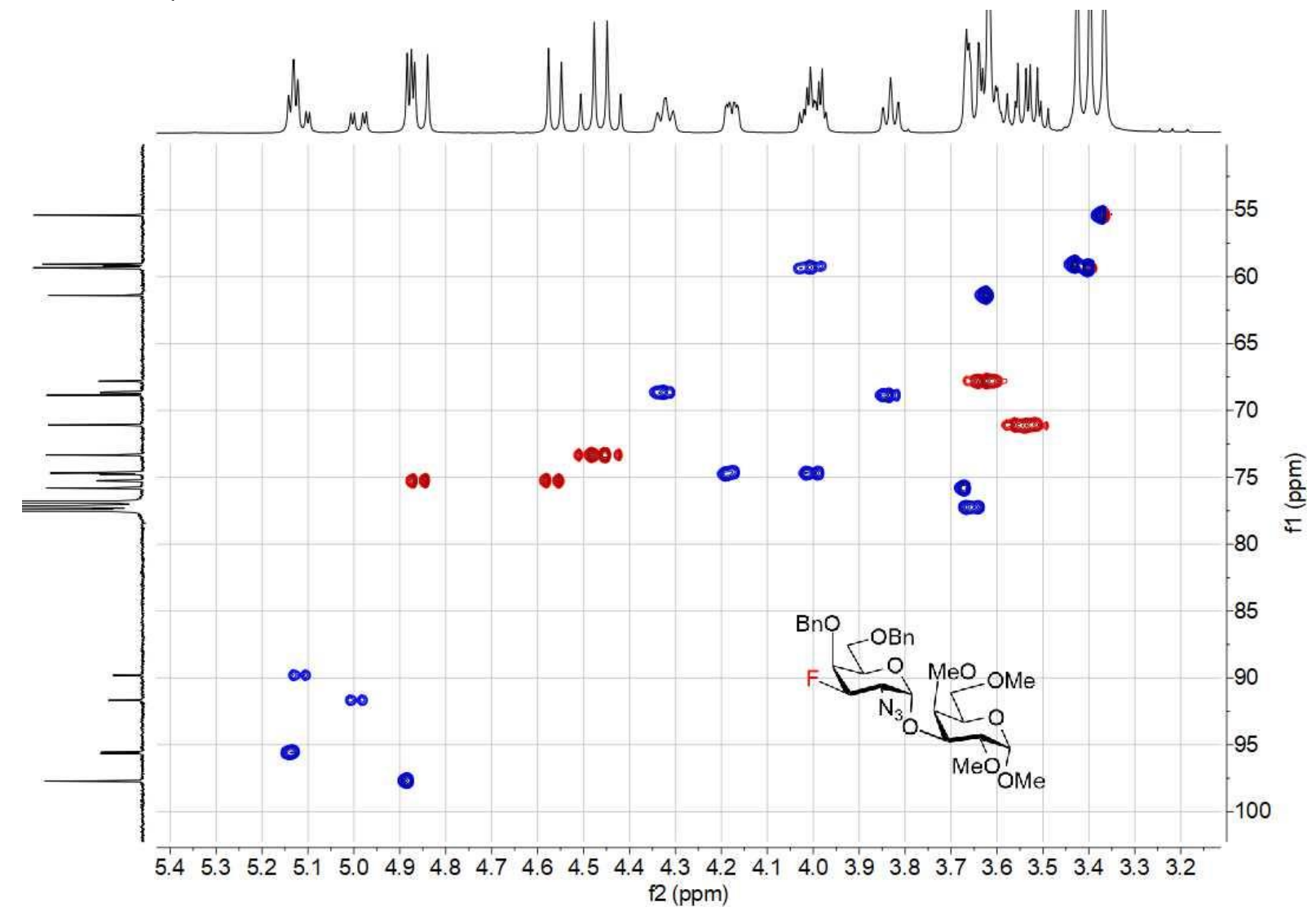

${ }^{1} \mathrm{H}-{ }^{13} \mathrm{C}$ HMBC NMR 3D- $\alpha$

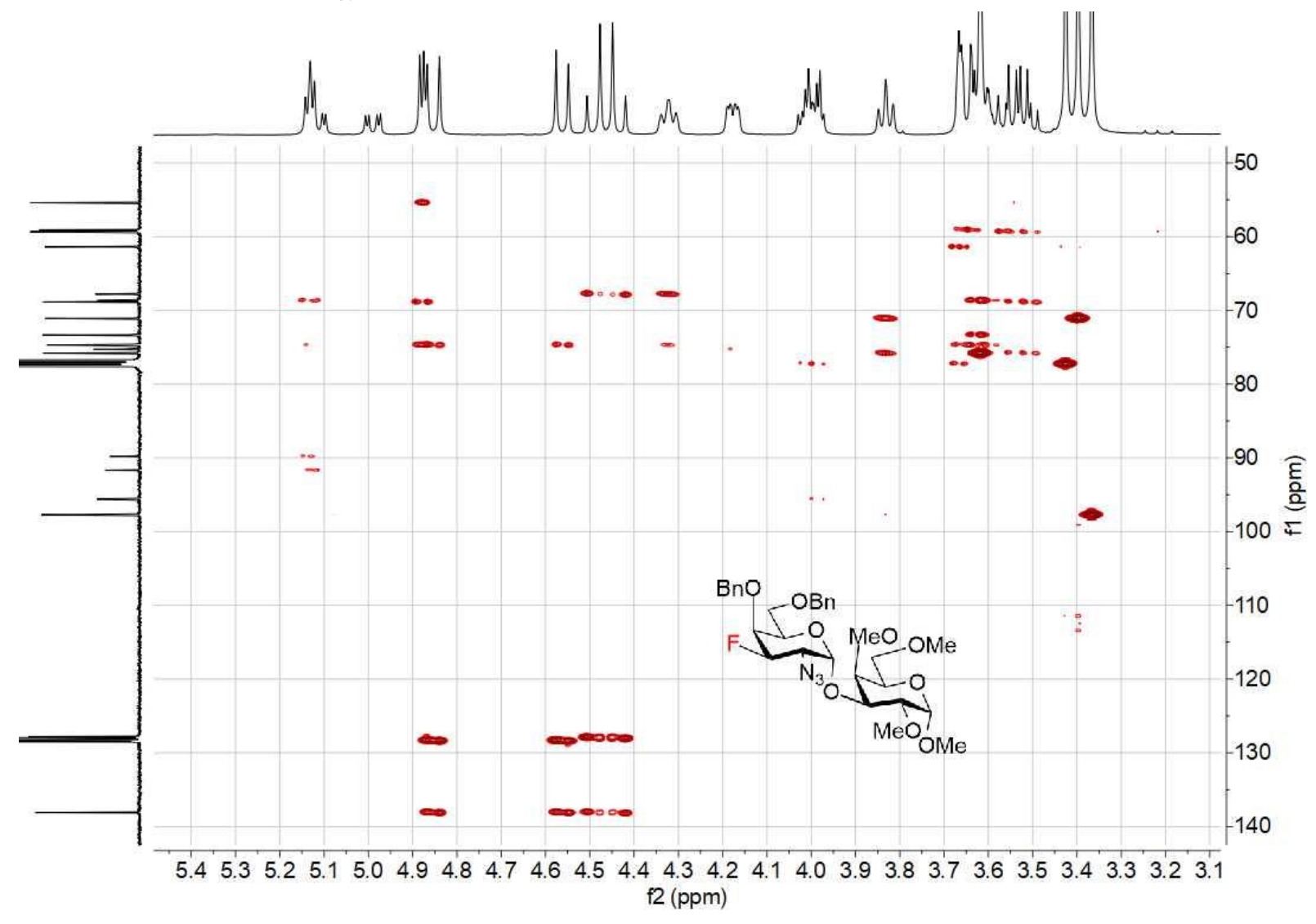



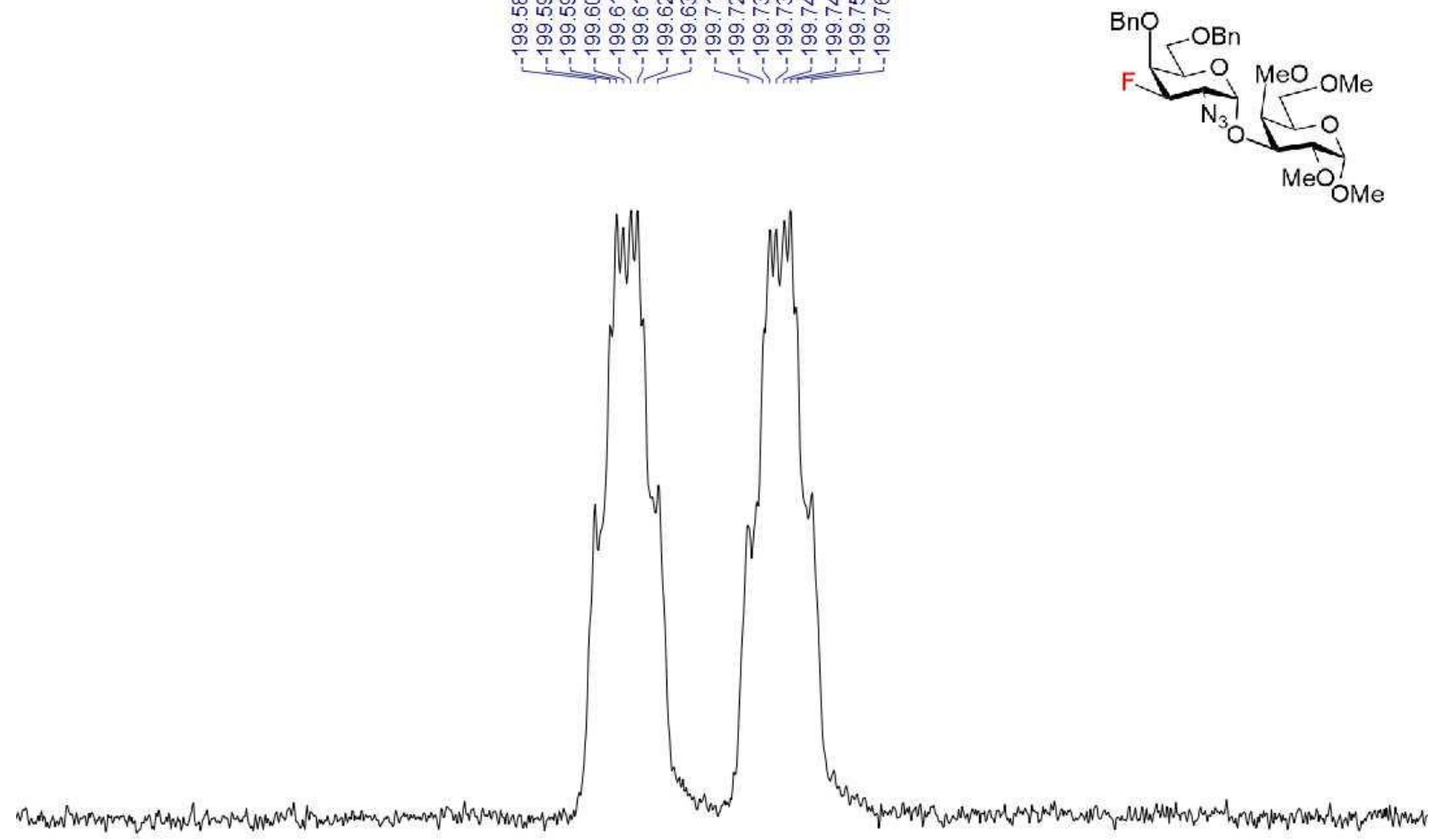

\begin{tabular}{lllllllllllll}
\hline-199.15 & -199.25 & -199.35 & -199.45 & -199.55 & -199.65 & -199.75 & -199.85 & -199.95 & -200.05 & -200.15 & -200.25 \\
$\mathrm{f}(\mathrm{ppm})$ & & &
\end{tabular}

NMR 3D- $\beta$

${ }^{1} \mathrm{HNMR} 400 \mathrm{MHz}, \mathrm{CDCl}_{3}$ 3D- $\beta$

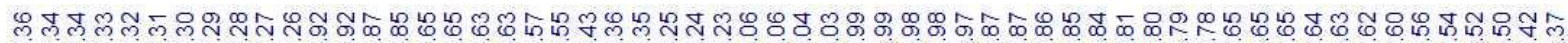

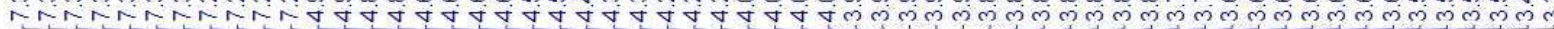

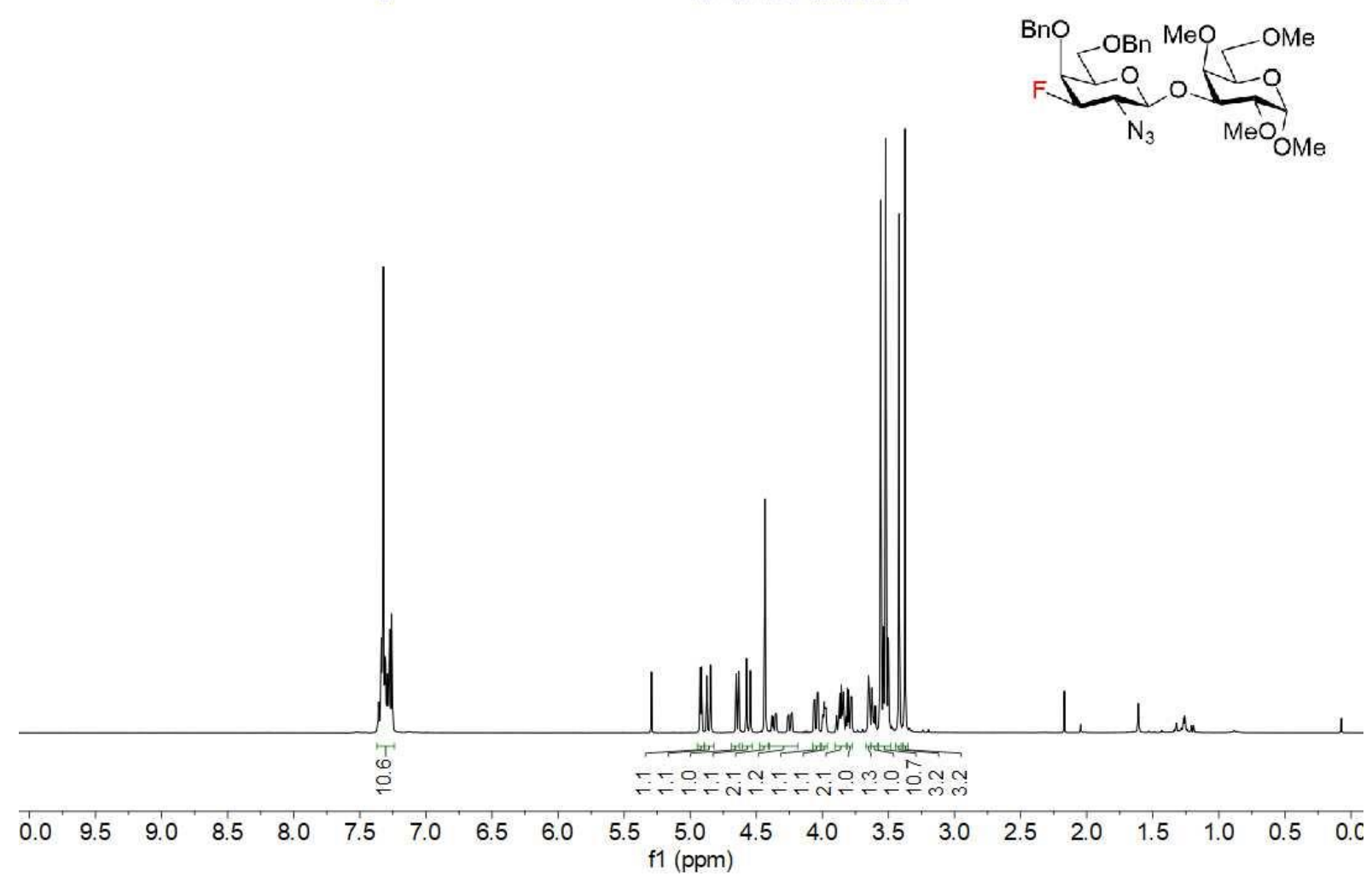




\section{${ }^{13} \mathrm{C}\left\{{ }^{1} \mathrm{H}\right\}$ NMR 100MHz, CDCl 3 3D- $\beta$}

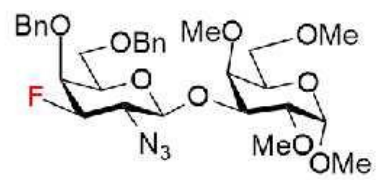

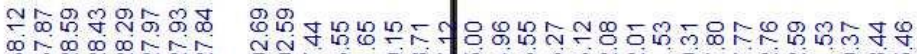

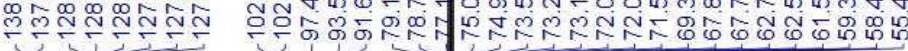

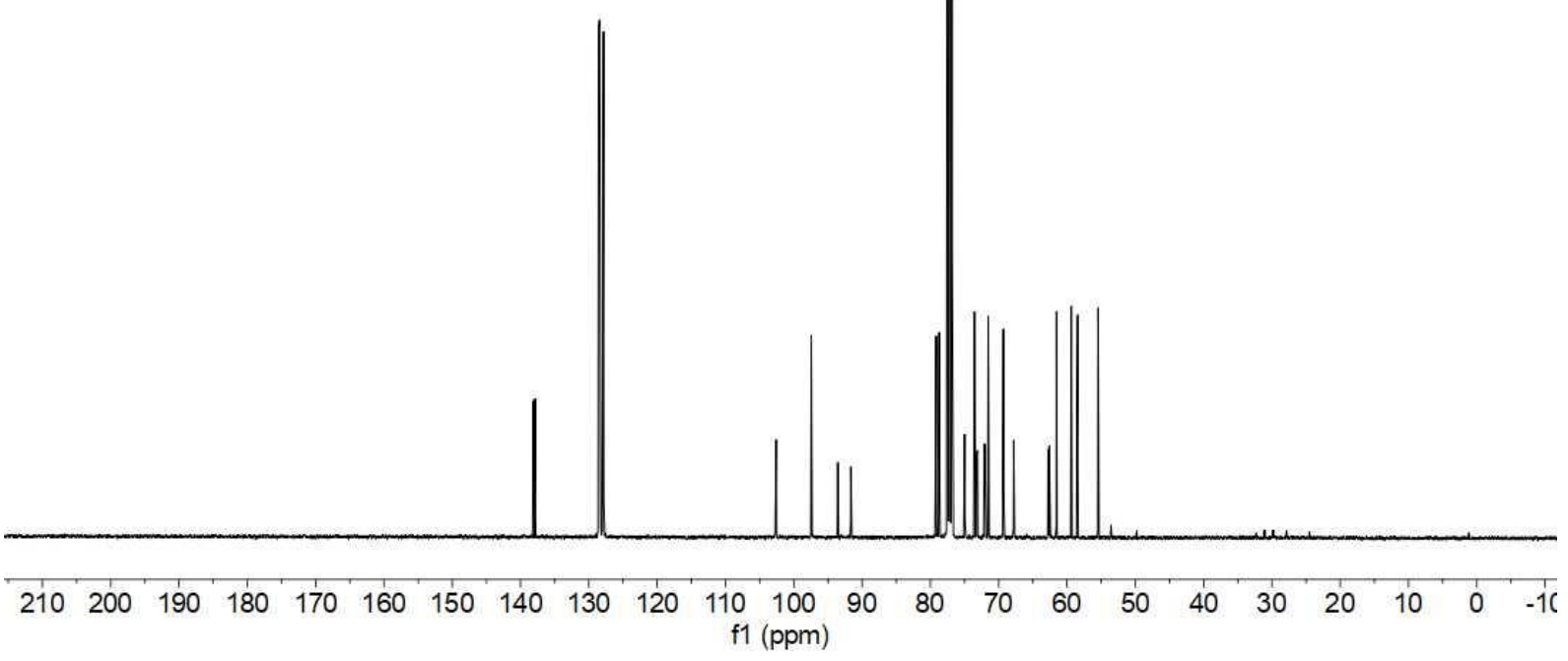

${ }^{1} \mathrm{H}-{ }^{1} \mathrm{H}$ COSY NMR 3D- $\beta$

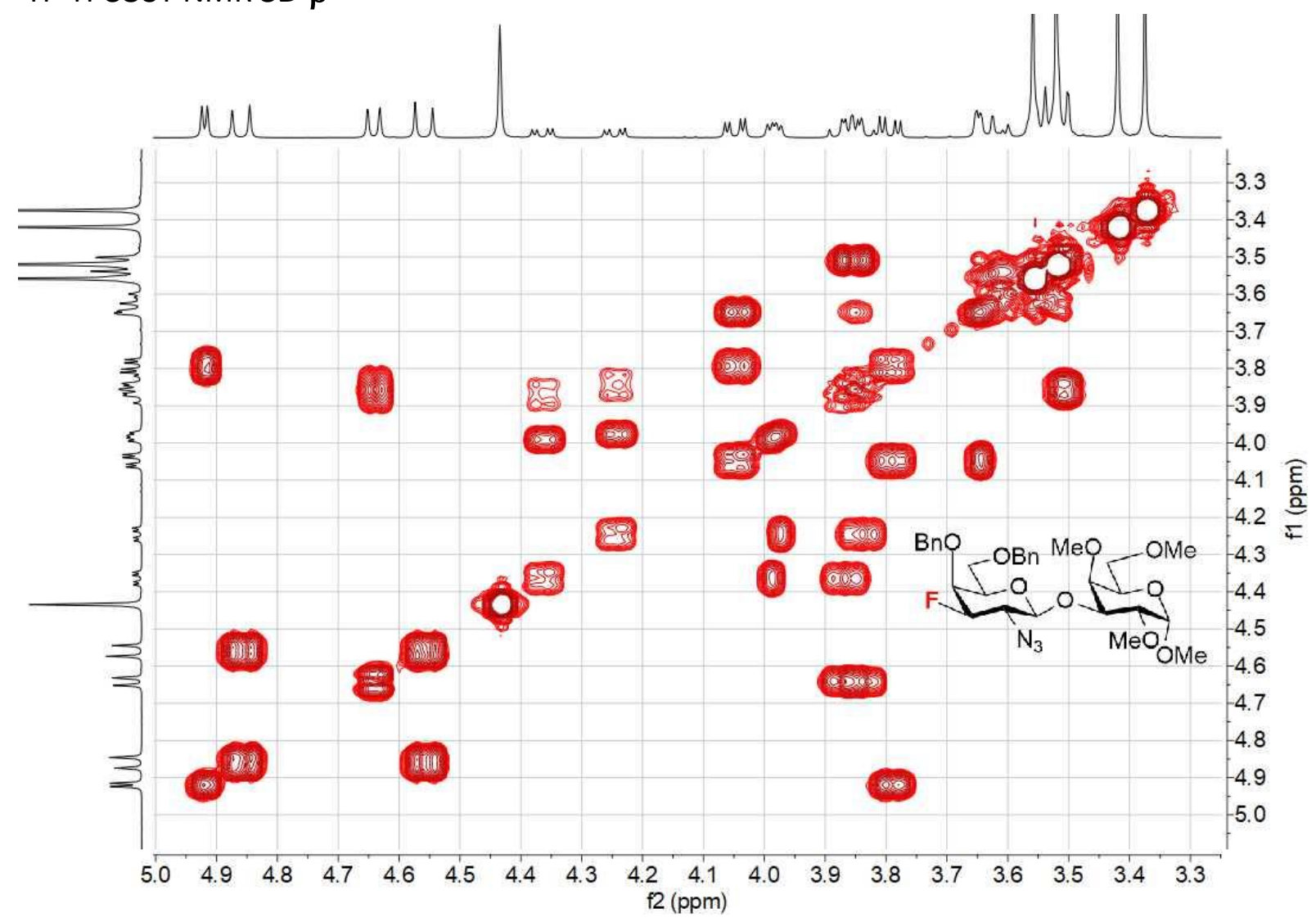




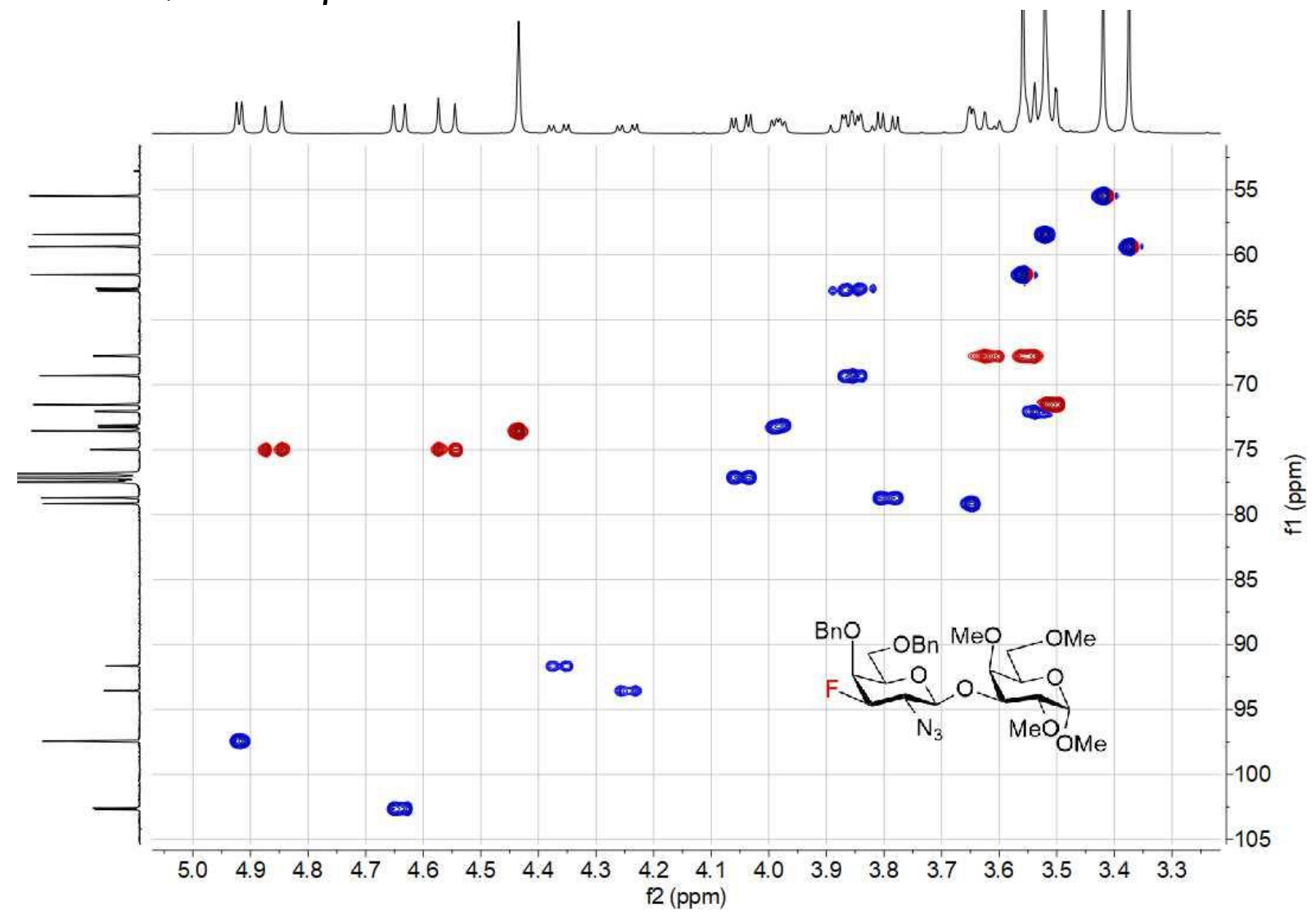

${ }^{1} \mathrm{H}-{ }^{13} \mathrm{C}$ HMBC NMR 3D- $\boldsymbol{\beta}$

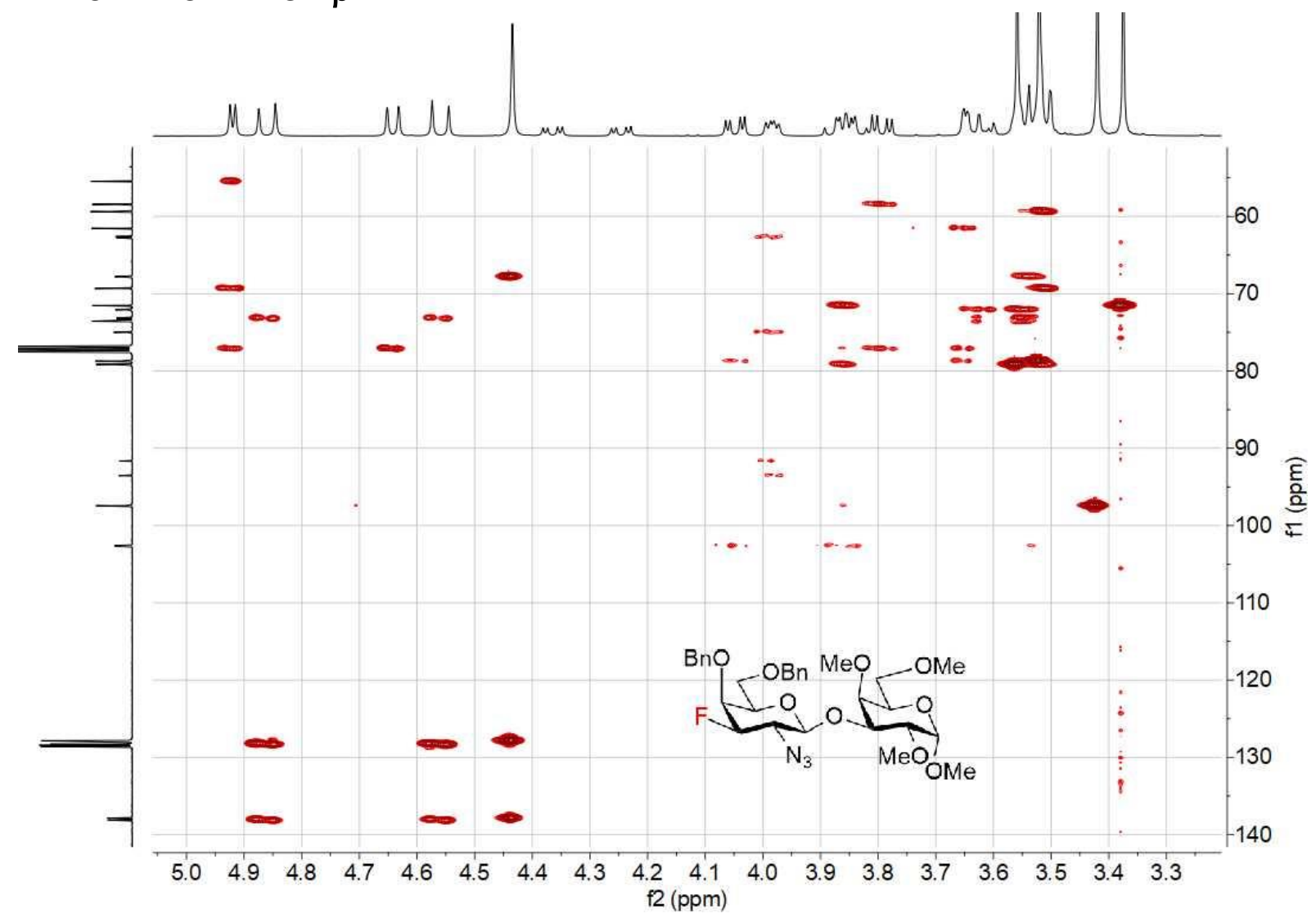


${ }^{19} \mathrm{~F} \mathrm{NMR} 376 \mathrm{MHz}, \mathrm{CDCl}_{3}$ 3D- $\beta$

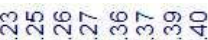

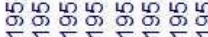
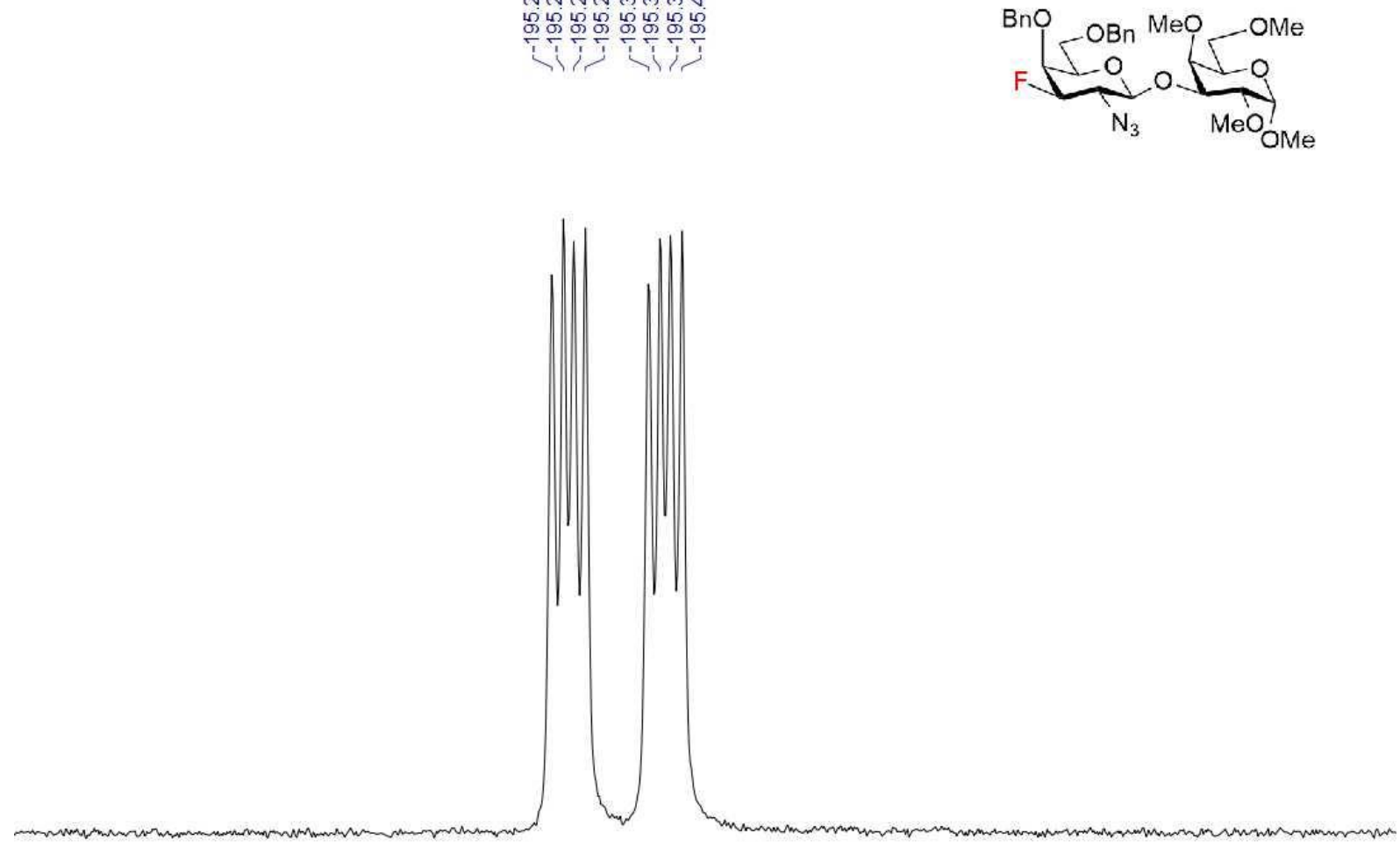

$-194.6-194.7-194.8-194.9-195.0-195.1-195.2-195.3-195.4-195.5-195.6-195.7-195.8-195.9-196.0-196.1-196.2-196.3$ $\mathrm{f1}(\mathrm{ppm})$

\section{NMR 3E- $\alpha$}

${ }^{1} \mathrm{HNMR} 400 \mathrm{MHz}, \mathrm{CDCl}_{3} 3 \mathrm{E}-\alpha$

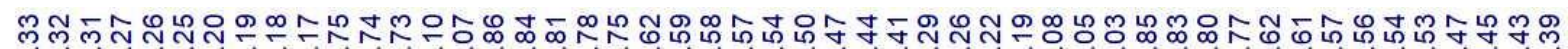

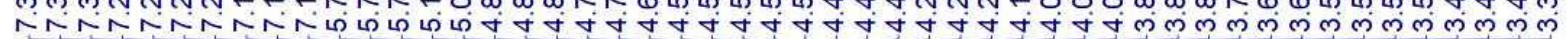

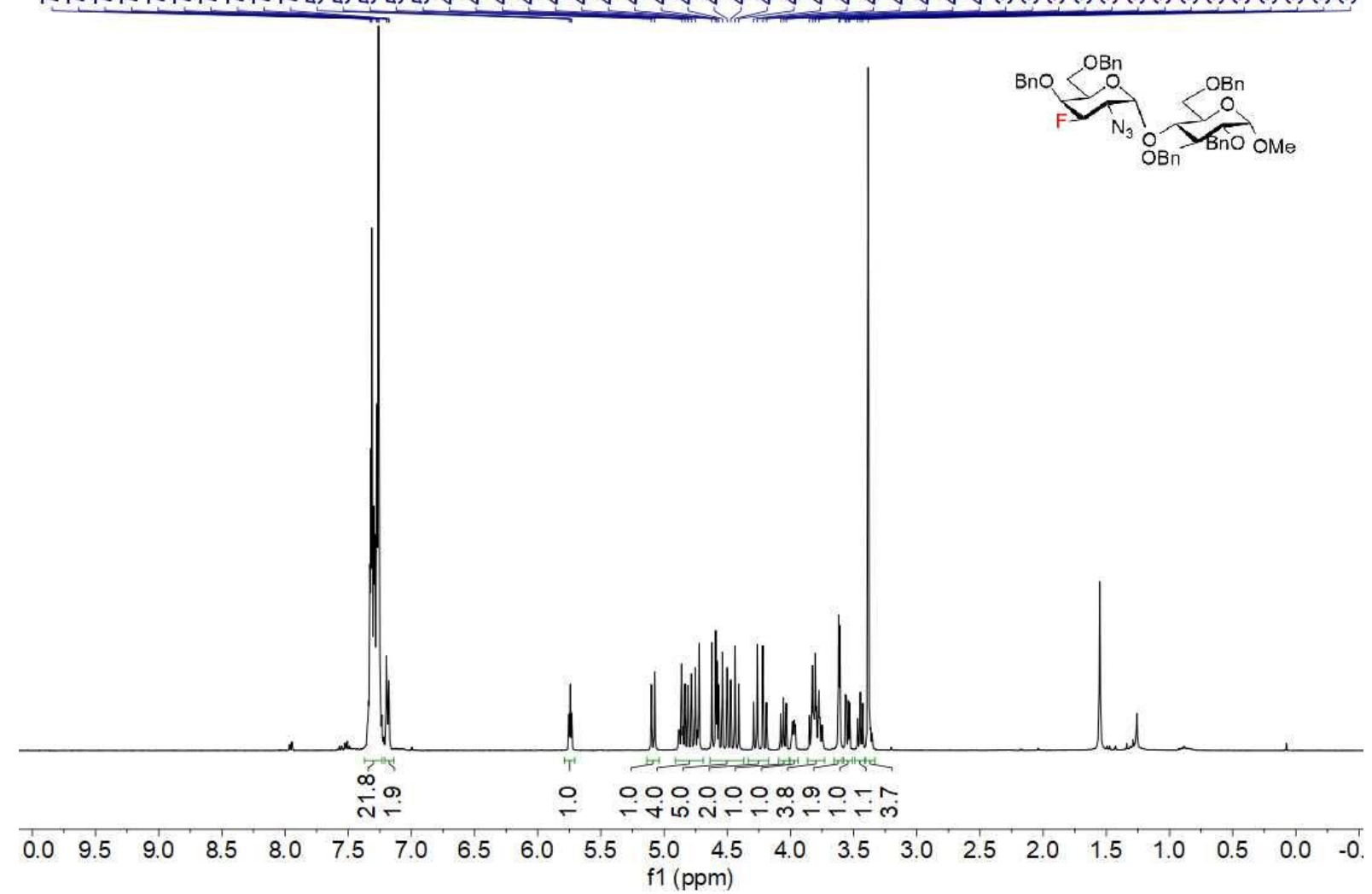




\section{${ }^{13} \mathrm{C}\left\{{ }^{1} \mathrm{H}\right\}$ NMR $100 \mathrm{MHz}, \mathrm{CDCl}_{3} 3 \mathrm{E}-\boldsymbol{\alpha}$}

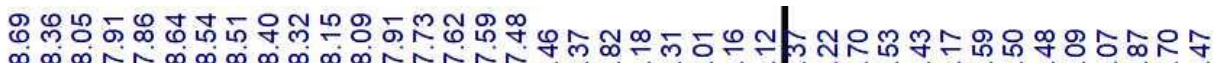

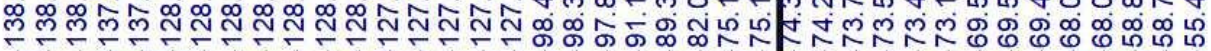

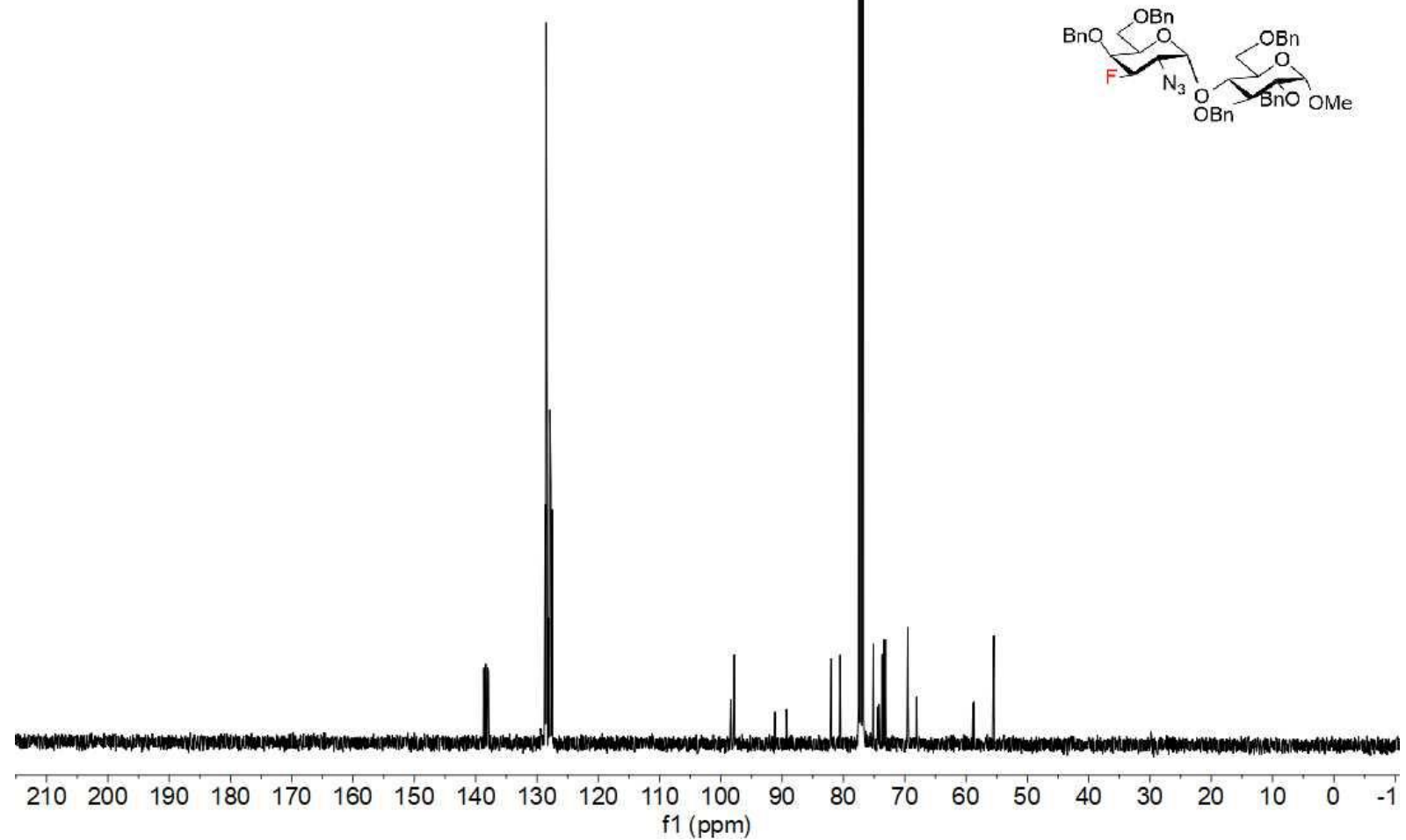

${ }^{1} \mathrm{H}-{ }^{1} \mathrm{H}$ COSY NMR $3 \mathrm{E}-\alpha$

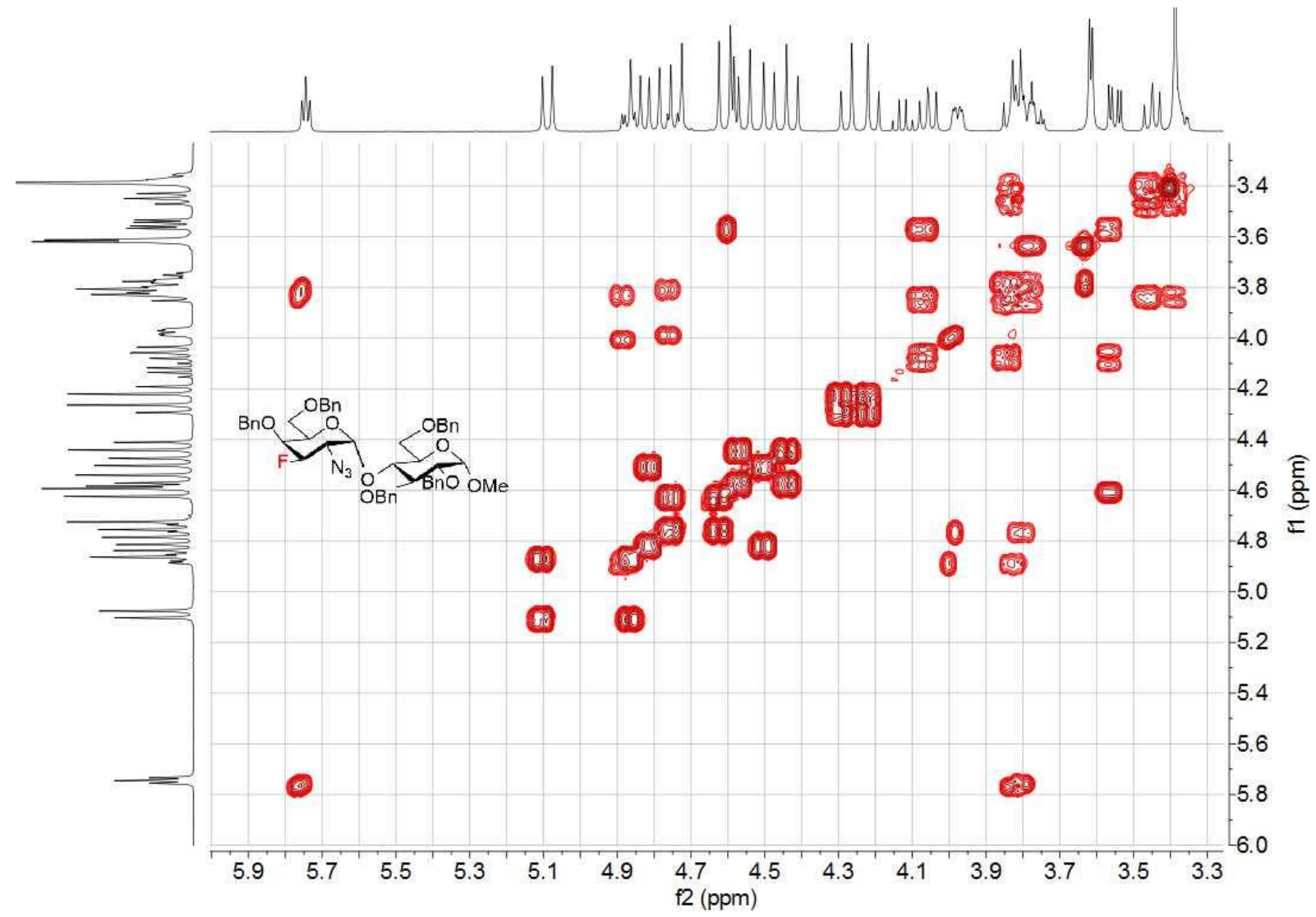


${ }^{1} \mathrm{H}-{ }^{13} \mathrm{C}$ HSOC NMR $3 \mathrm{E}-\alpha$

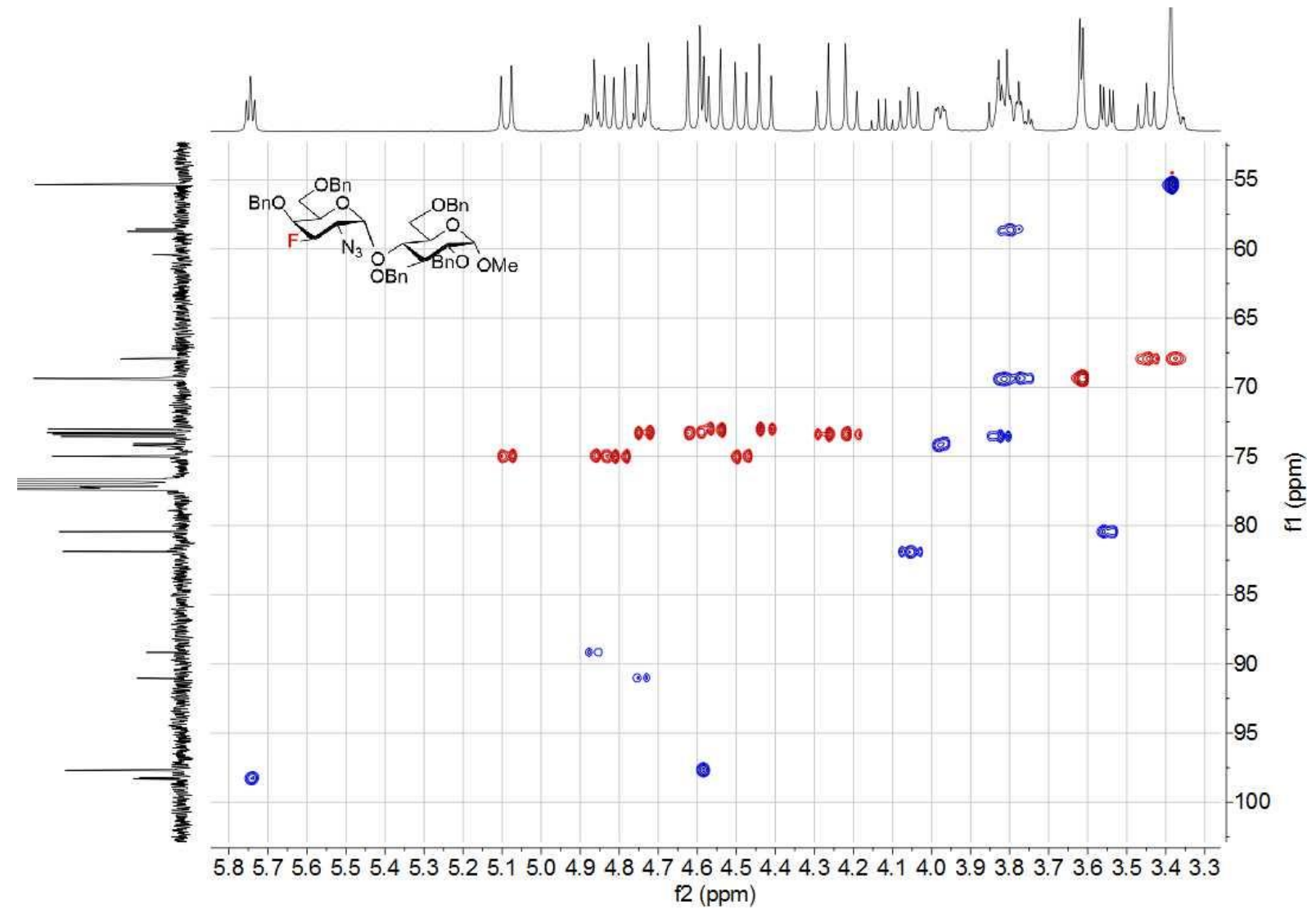

${ }^{1} \mathrm{H}-{ }^{13} \mathrm{C}$ HMBC NMR $3 \mathrm{E}-\alpha$

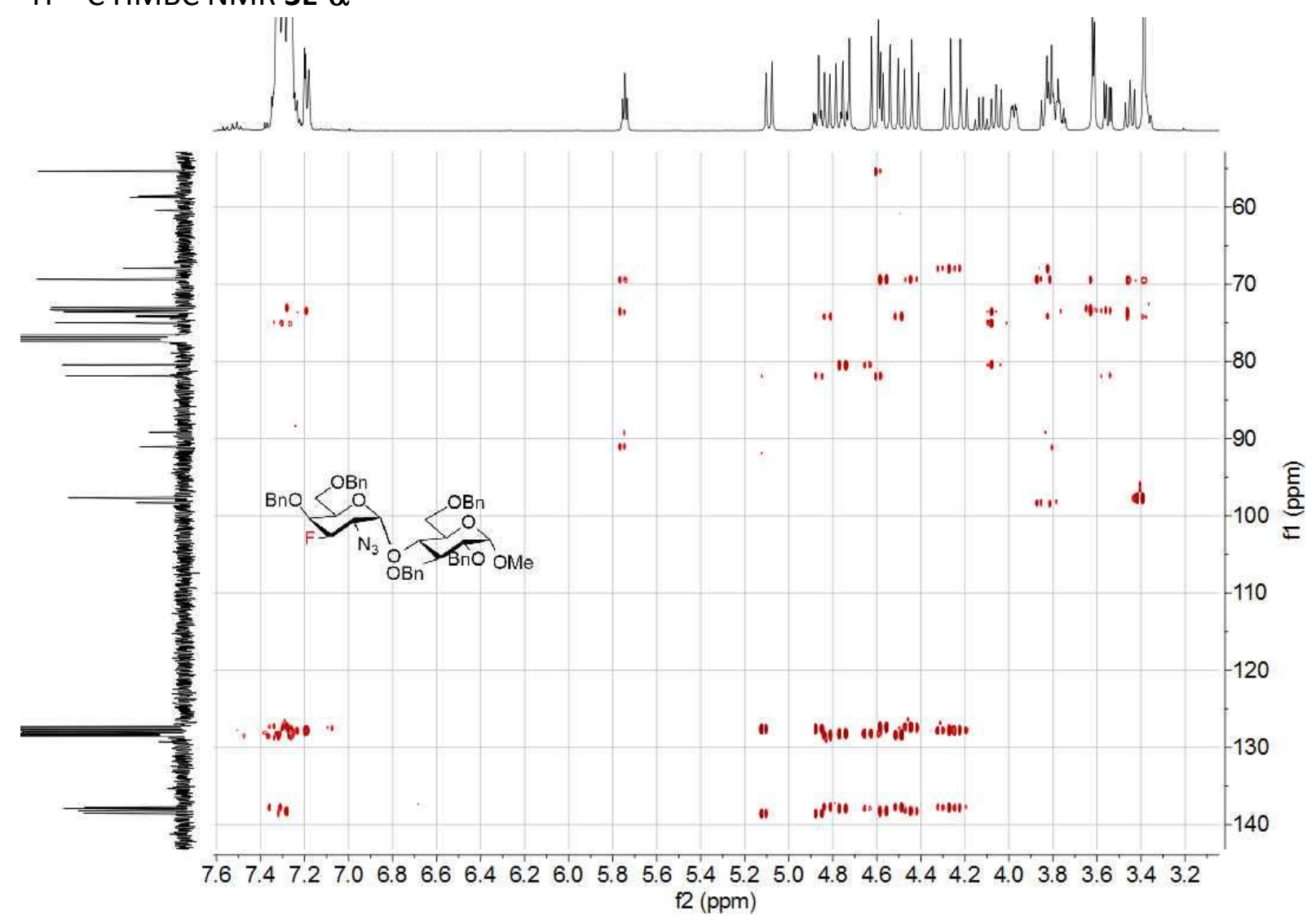




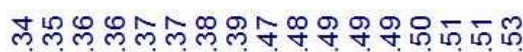

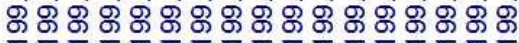

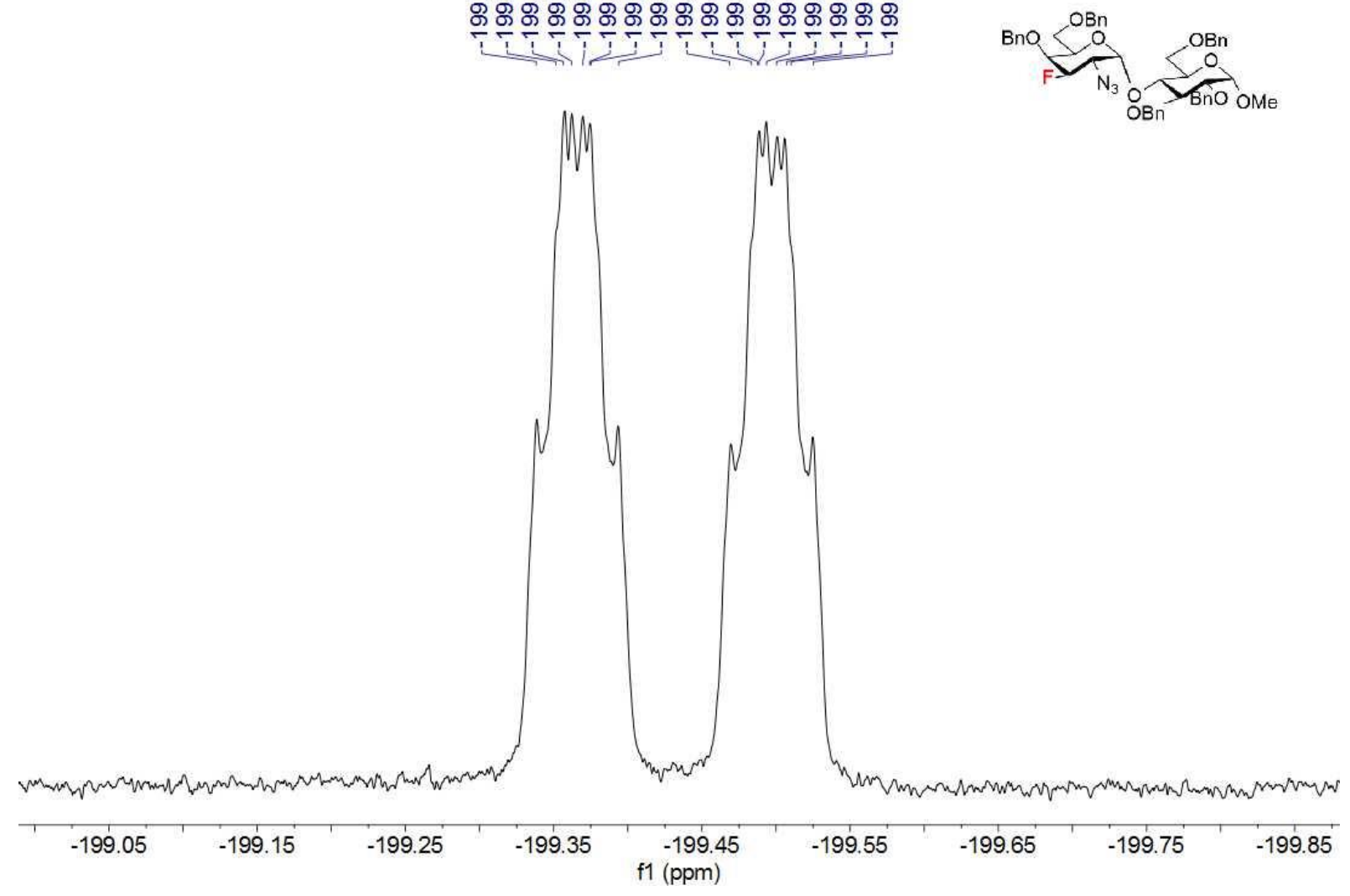

\section{NMR 3E- $\beta$}

${ }^{1} \mathrm{HNMR} 400 \mathrm{MHz}, \mathrm{CDCl}_{3} 3 \mathrm{E}-\beta$

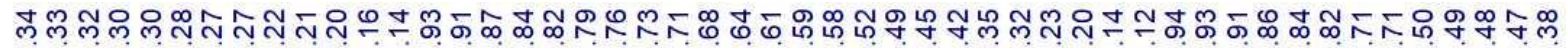

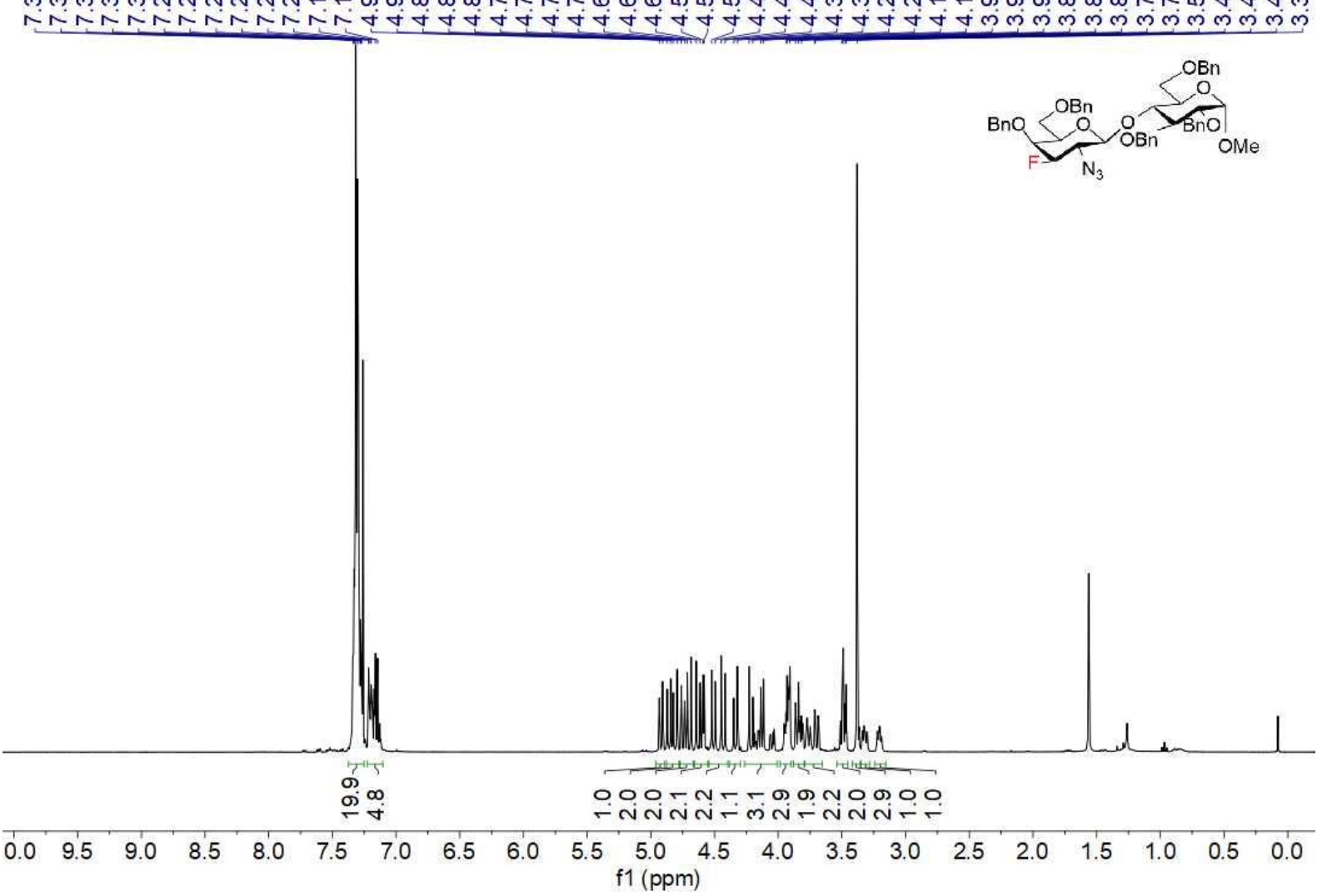


${ }^{13} \mathrm{C}\left\{{ }^{1} \mathrm{H}\right\}$ NMR $100 \mathrm{MHz}, \mathrm{CDCl}_{3} 3 \mathrm{E}-\boldsymbol{\beta}$

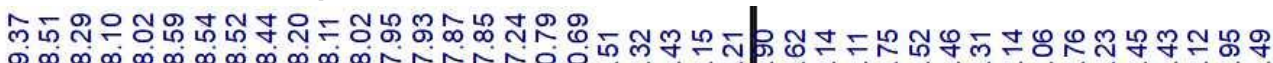

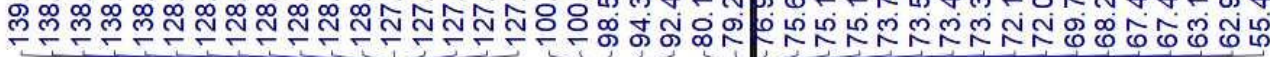

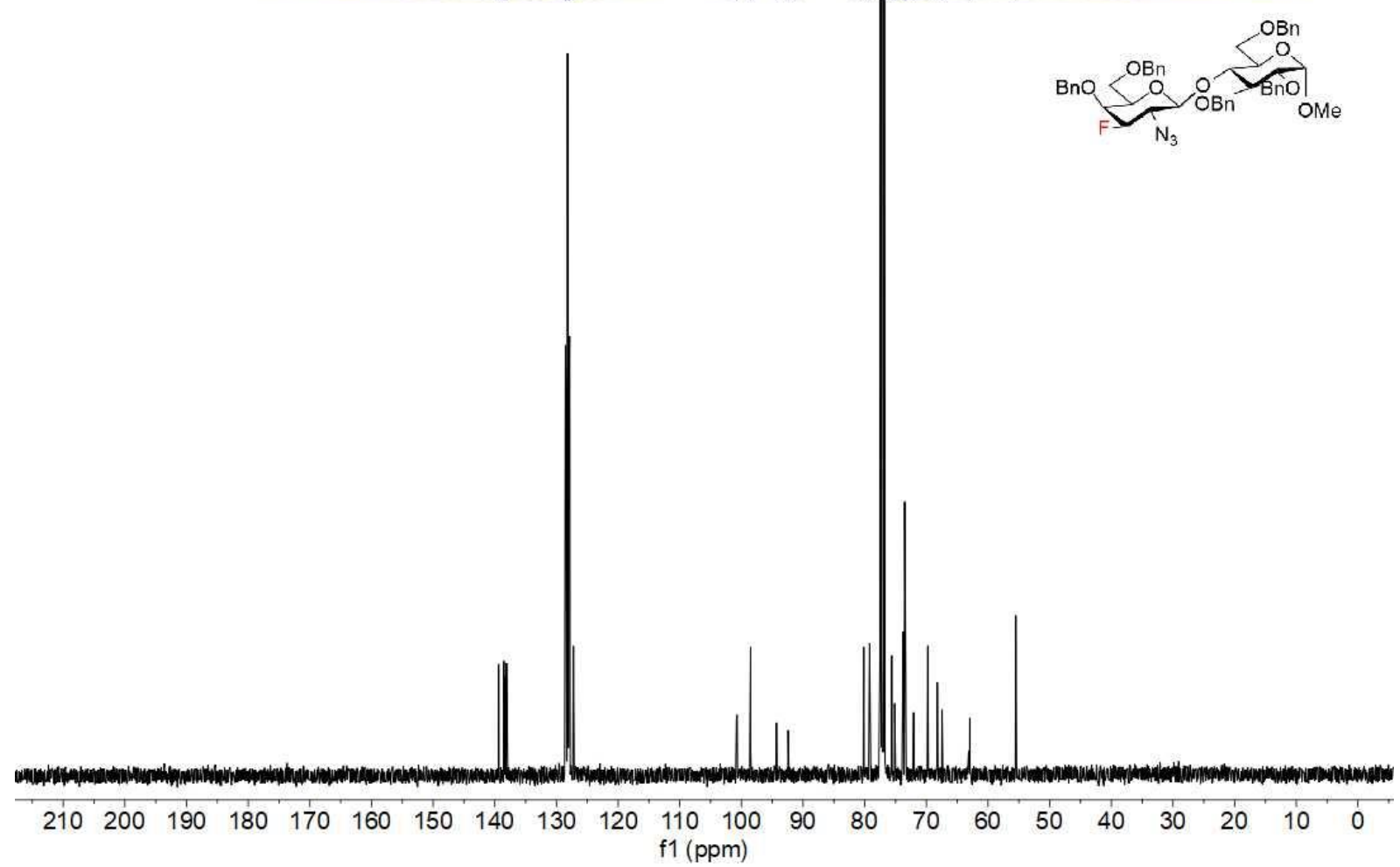

${ }^{1} \mathrm{H}-{ }^{1} \mathrm{H}$ COSY NMR $3 \mathrm{E}-\boldsymbol{\beta}$

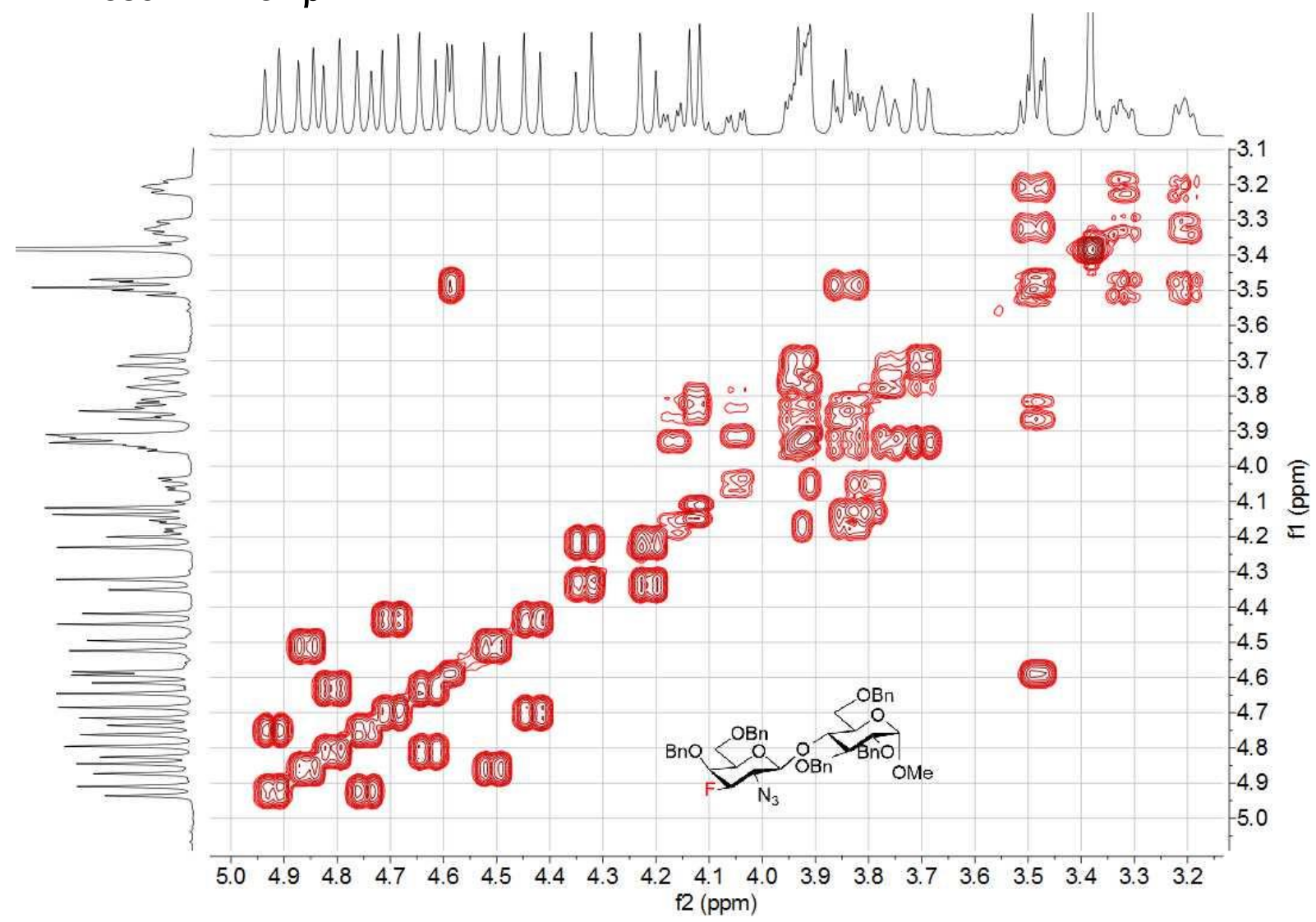


${ }^{1} \mathrm{H}-{ }^{13} \mathrm{C}$ HSQC NMR $3 E-\beta$

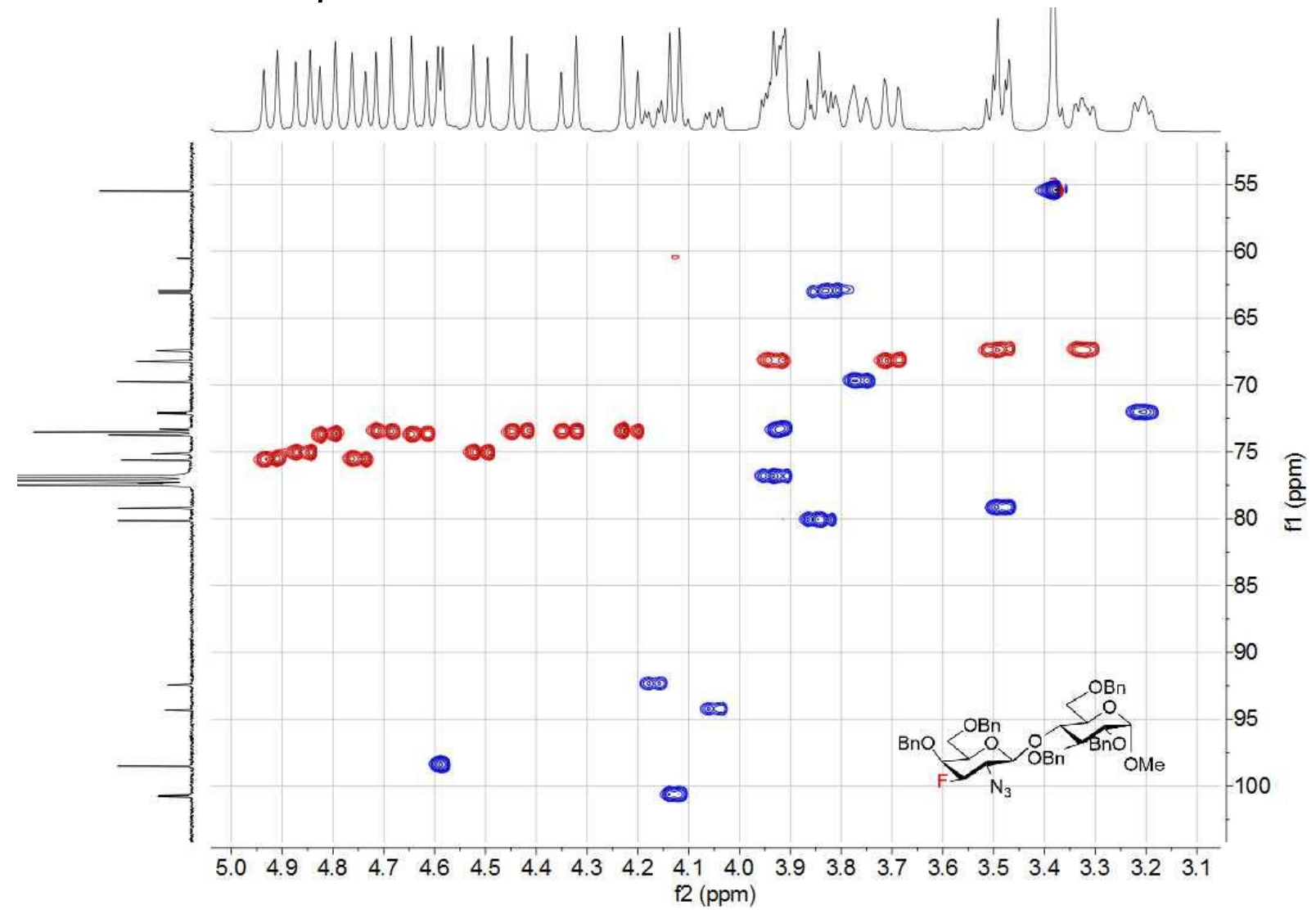

${ }^{1} \mathrm{H}-{ }^{13} \mathrm{C}$ HMBC NMR 3E- $\beta$

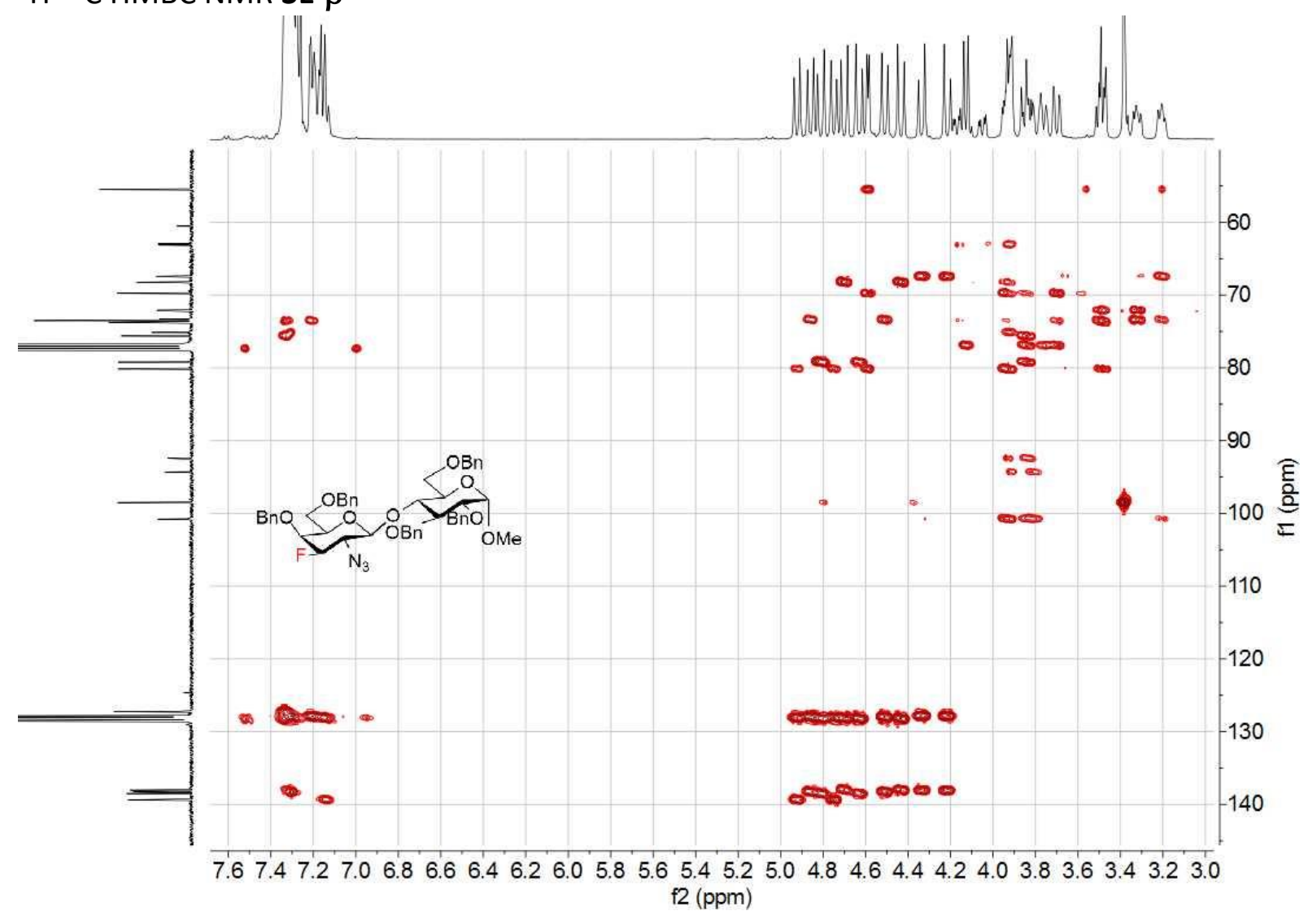


${ }^{19} \mathrm{~F} \mathrm{NMR} 376 \mathrm{MHz}, \mathrm{CDCl}_{3} \mathbf{3 E}-\boldsymbol{\beta}$

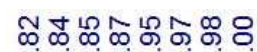

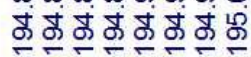

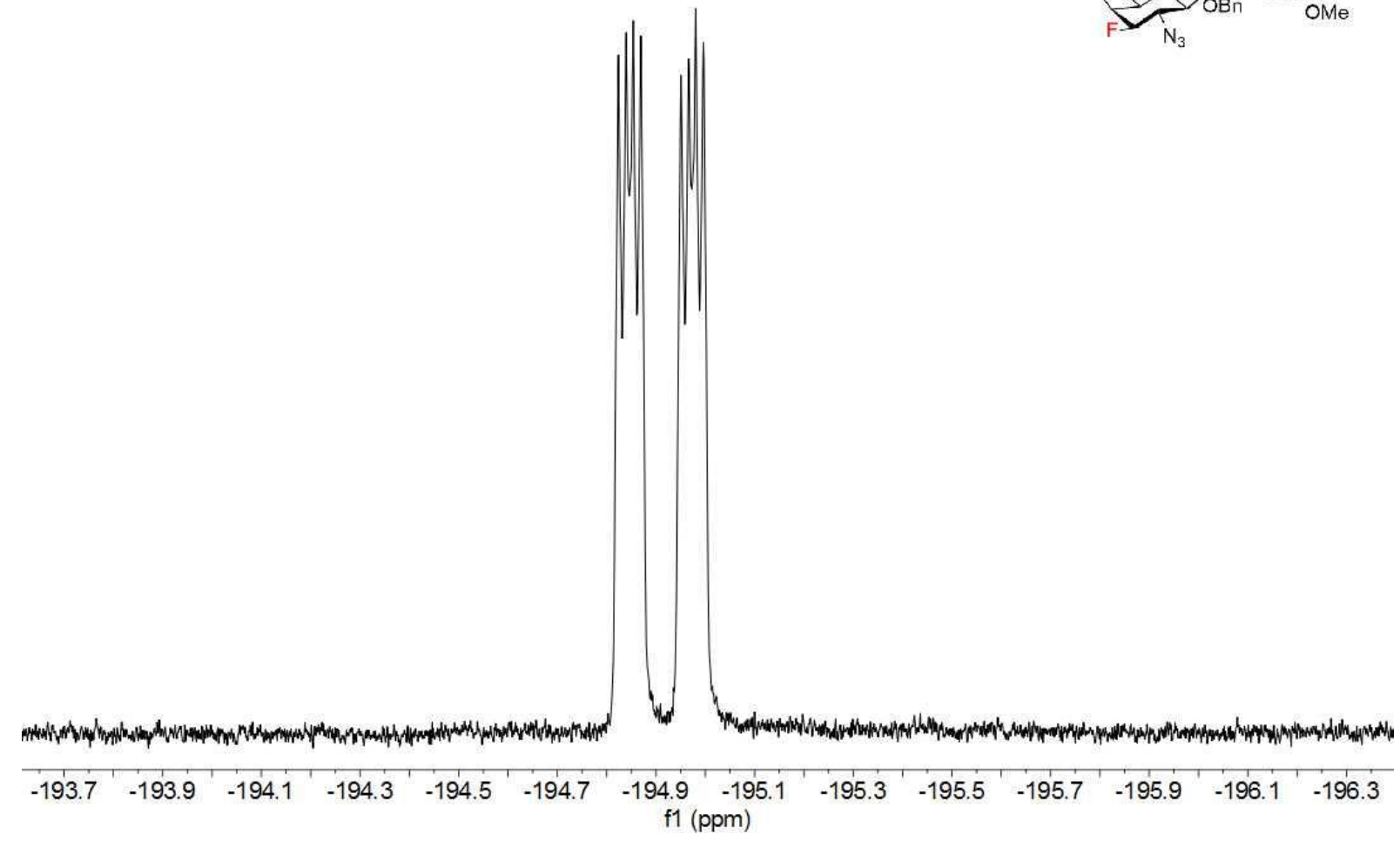

NMR 3F- $\alpha$

${ }^{1} \mathrm{H} N M R 400 \mathrm{MHz}, \mathrm{CDCl}_{3} 3 \mathrm{~F}-\alpha$

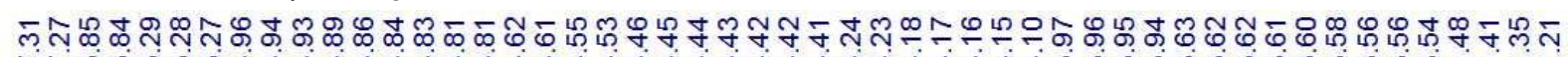

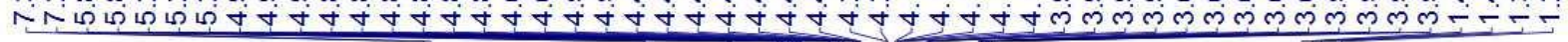

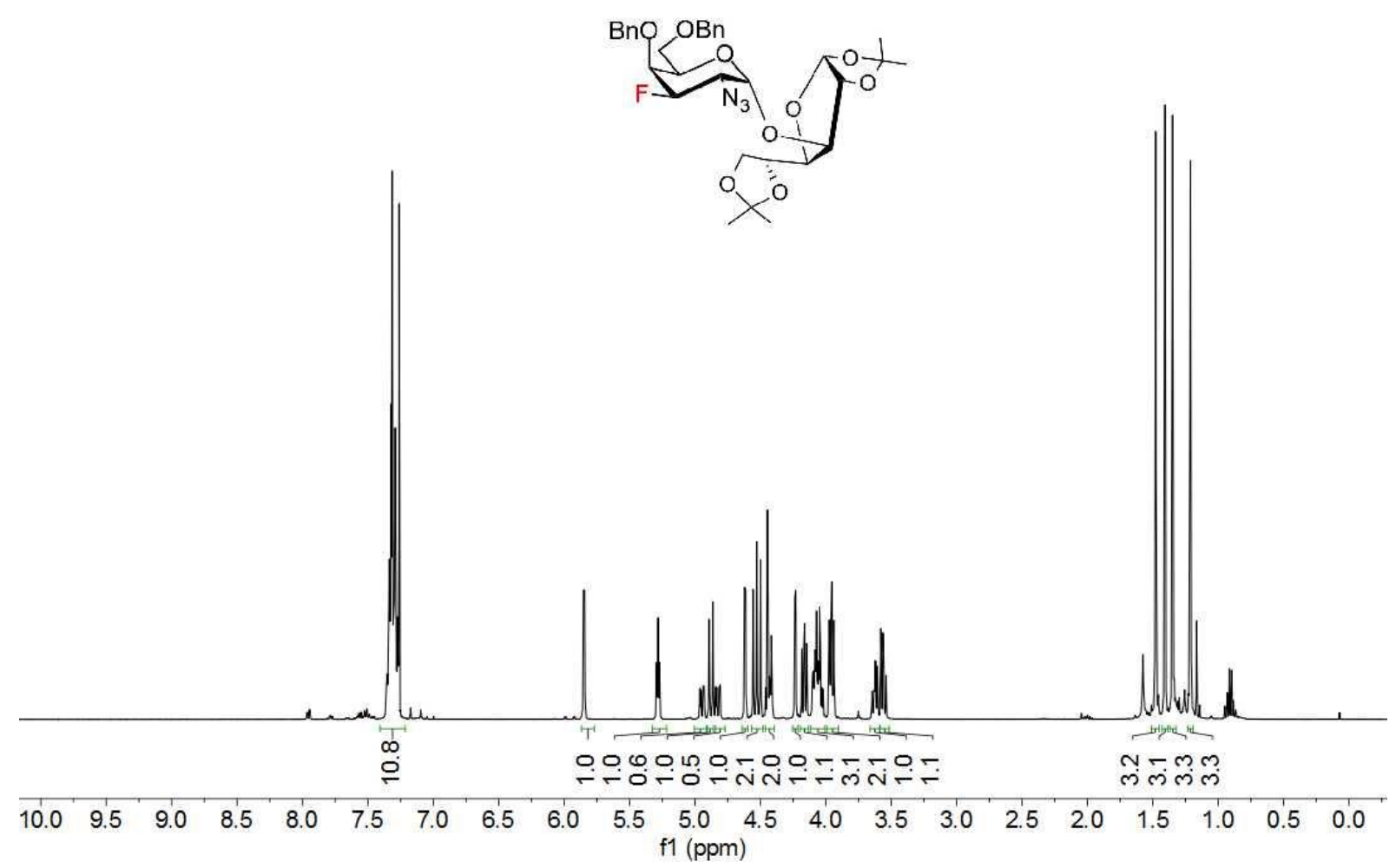


${ }^{13} \mathrm{C}\left\{{ }^{1} \mathrm{H}\right\}$ NMR 100MHz, $\mathrm{CDCl}_{3} 3 \mathrm{~F}-\alpha$

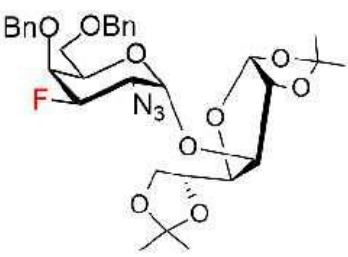

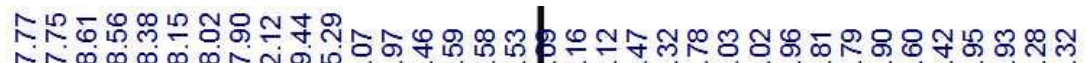

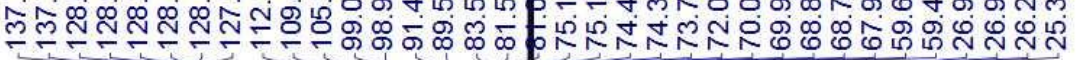

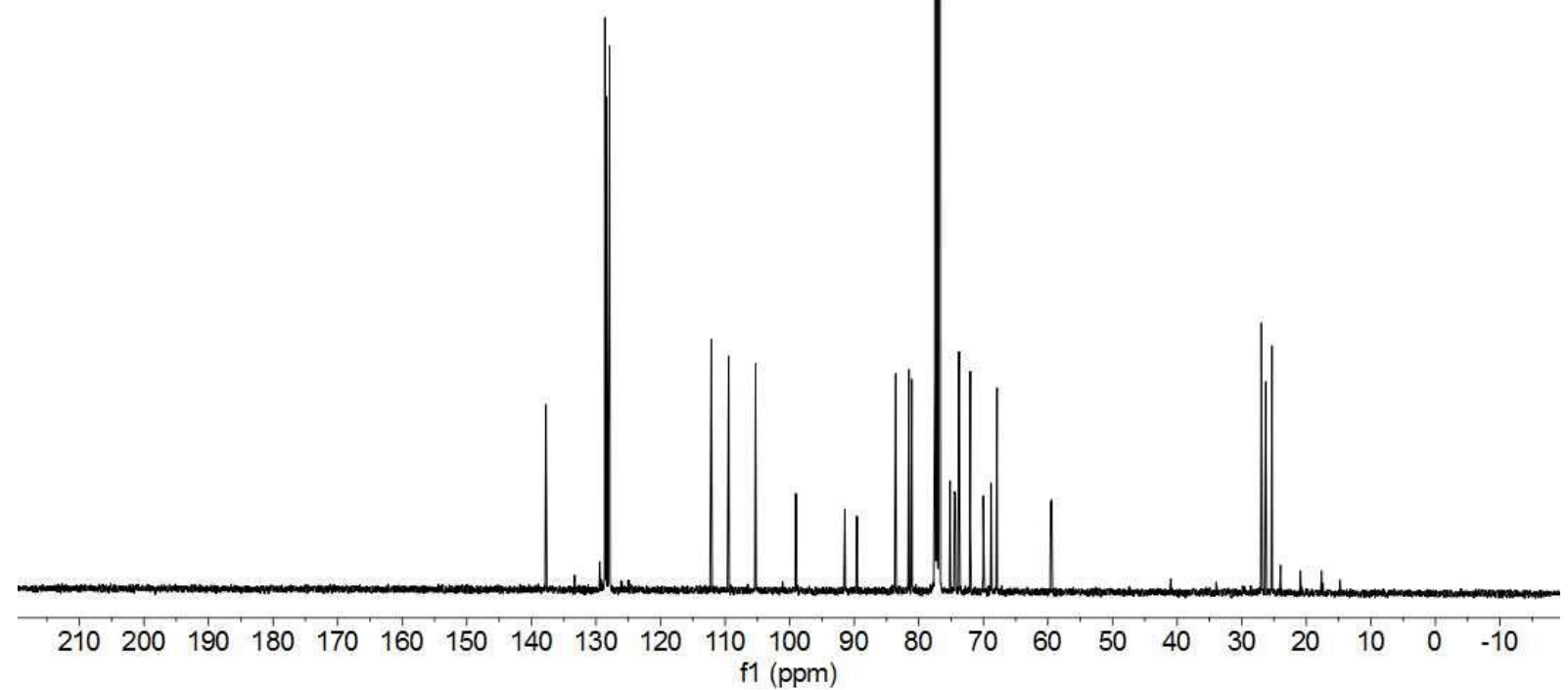

${ }^{1} \mathrm{H}-{ }^{1} \mathrm{H}$ COSY NMR $3 F-\alpha$

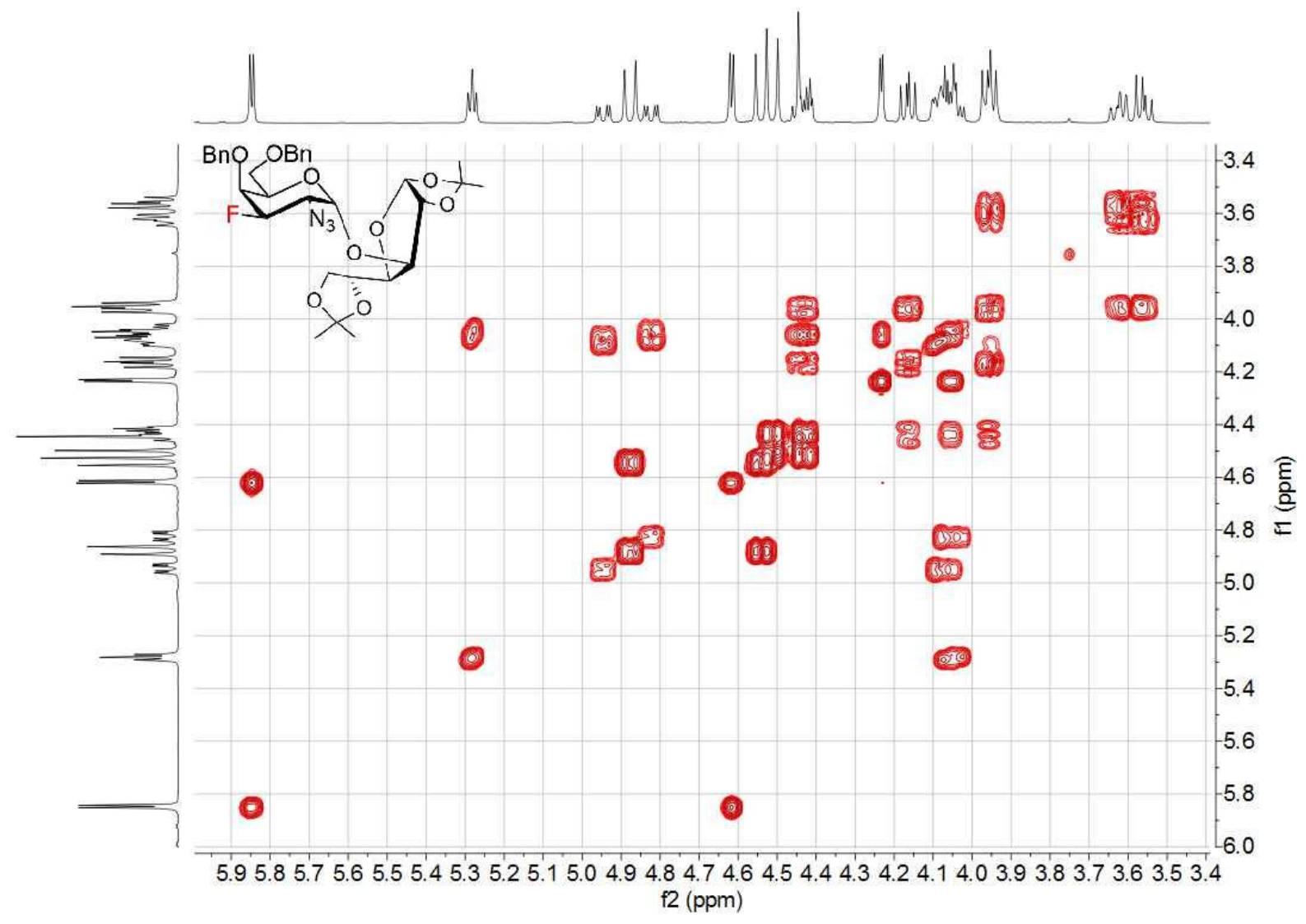


${ }^{1} \mathrm{H}-{ }^{13} \mathrm{C}$ HSOC NMR $3 \mathrm{~F}-\alpha$

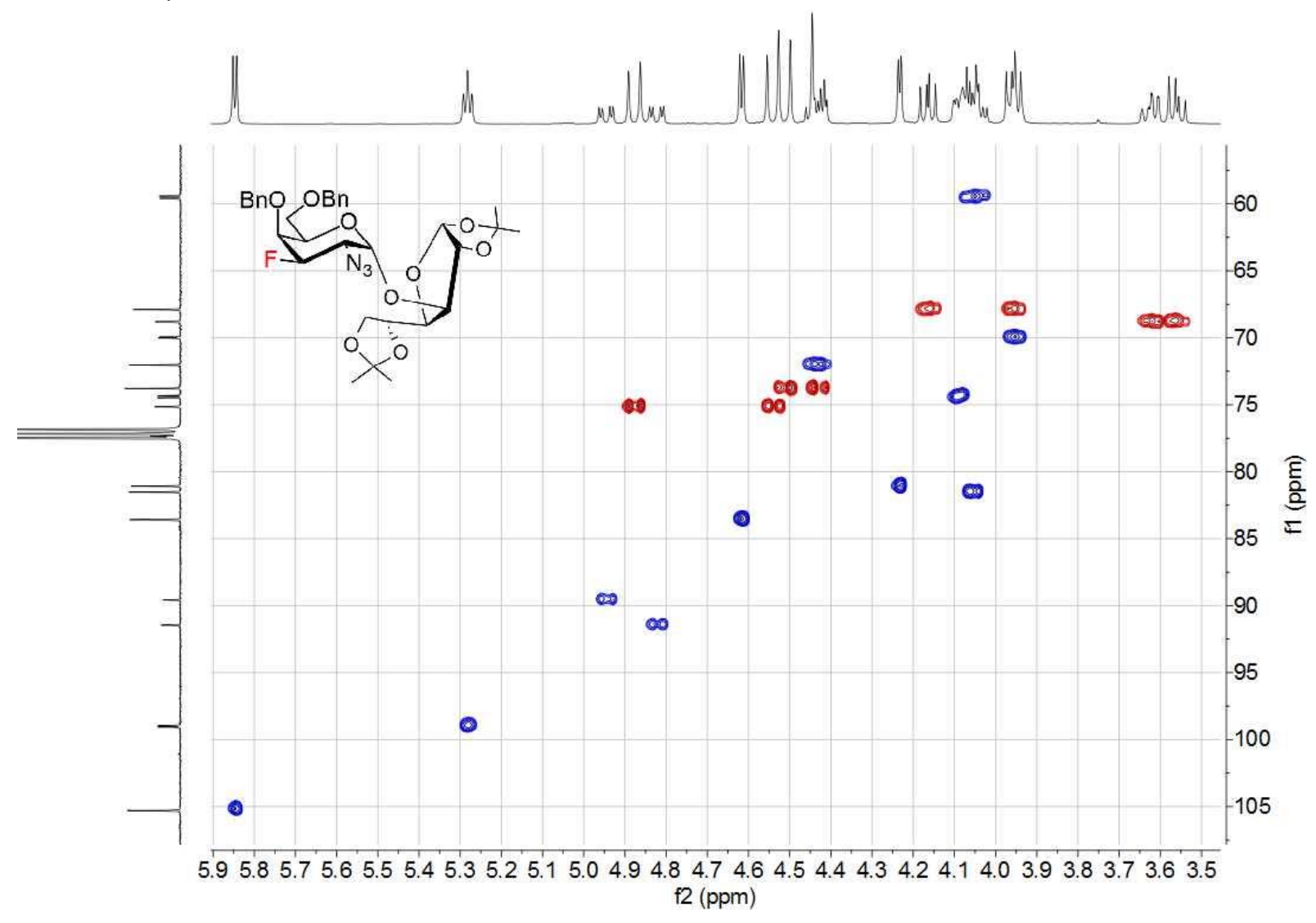

${ }^{1} \mathrm{H}-{ }^{13} \mathrm{C}$ HMBC NMR $3 \mathrm{~F}-\alpha$

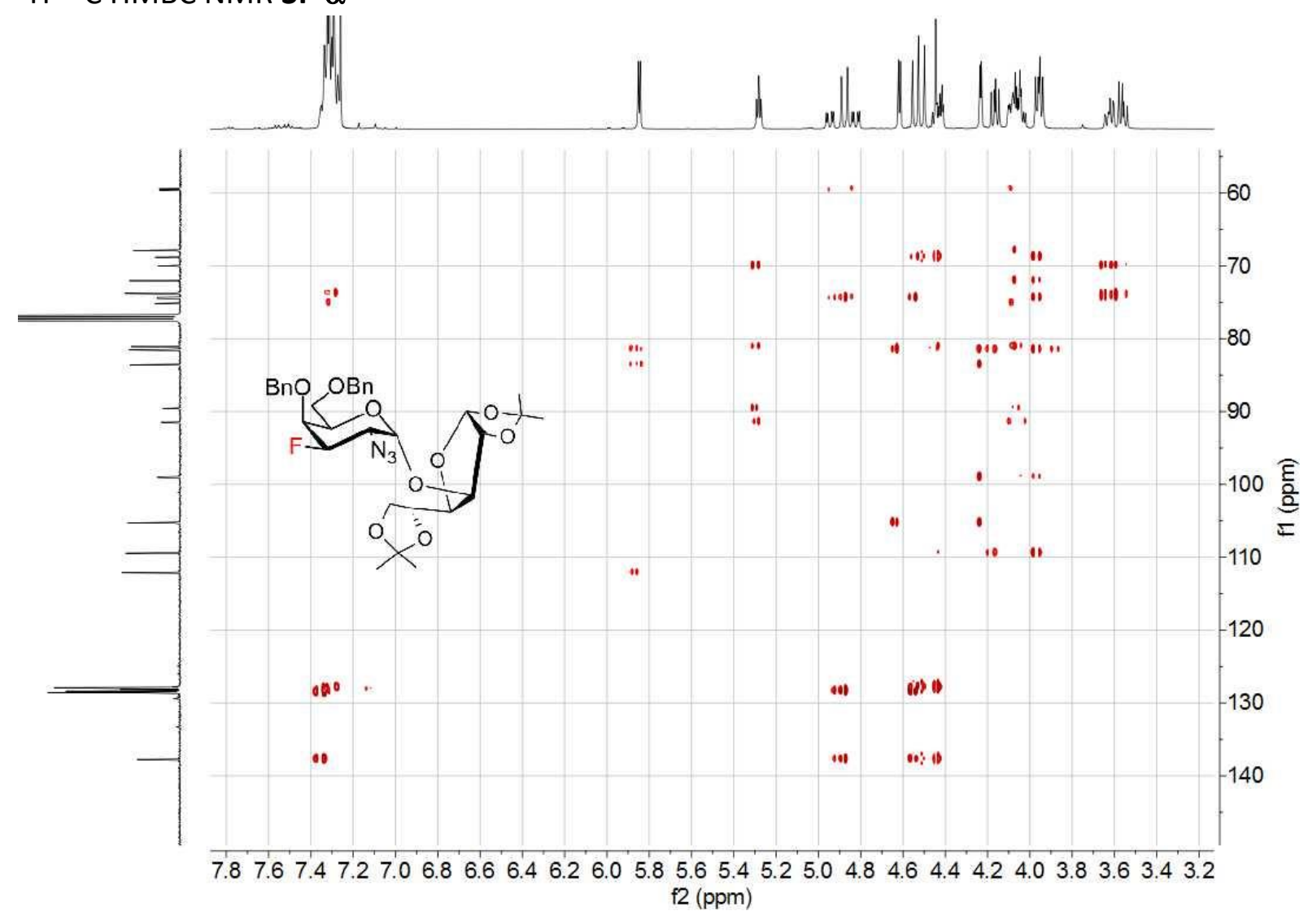


${ }^{19} \mathrm{~F} \mathrm{NMR} 376 \mathrm{MHz}, \mathrm{CDCl}_{3} 3 \mathrm{~F}-\alpha$

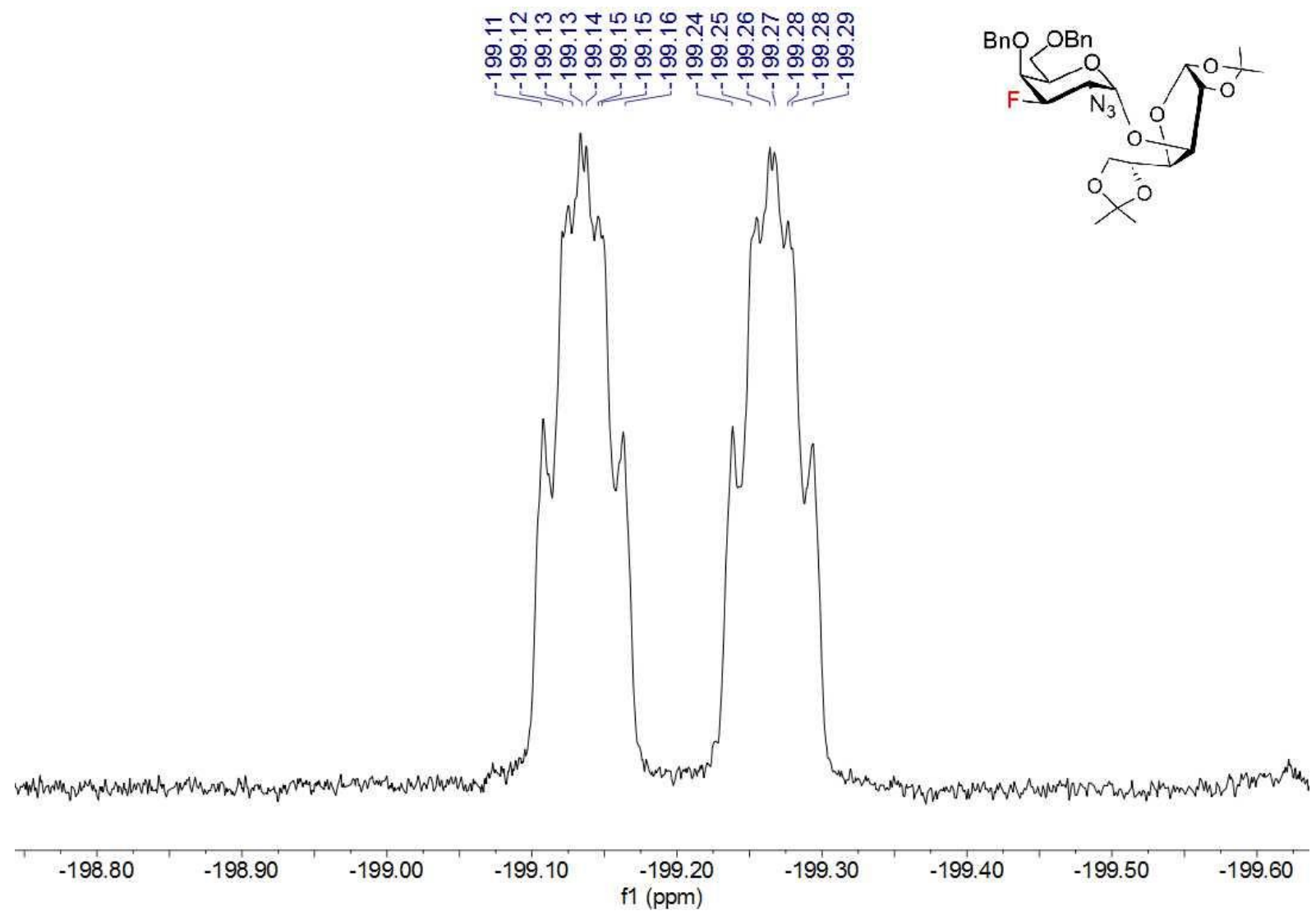

\section{NMR 3F- $\beta$}

${ }^{1} \mathrm{H}$ NMR $400 \mathrm{MHz}, \mathrm{CDCl}_{3} 3 \mathrm{~F}-\beta$

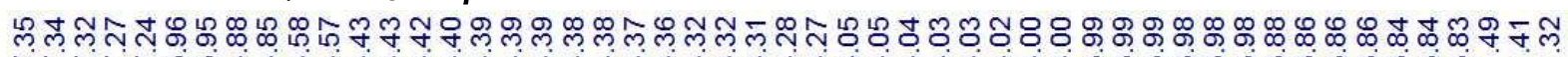

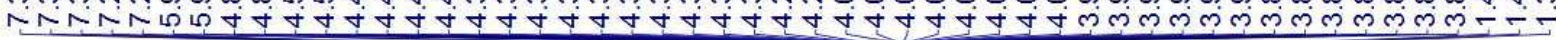
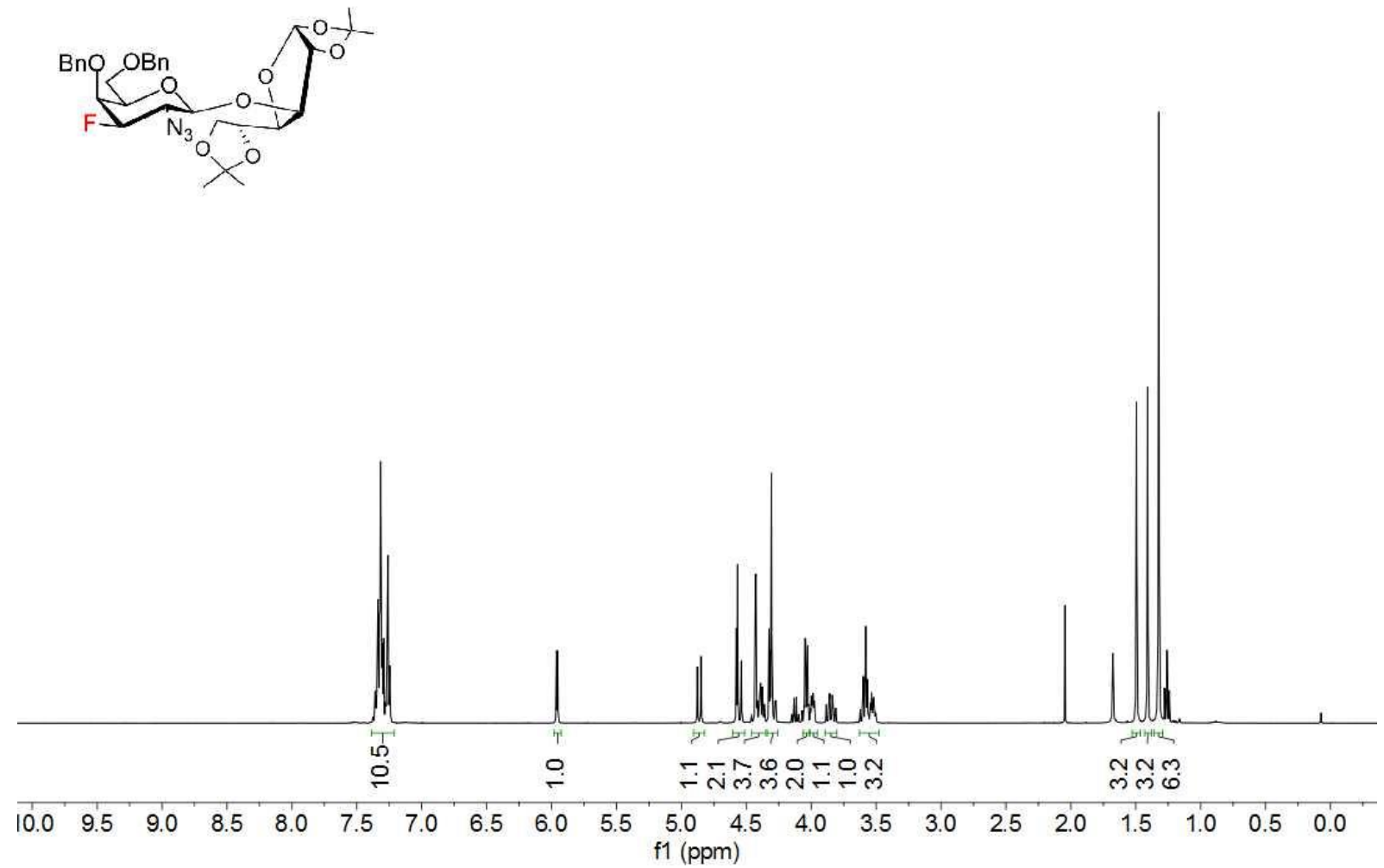


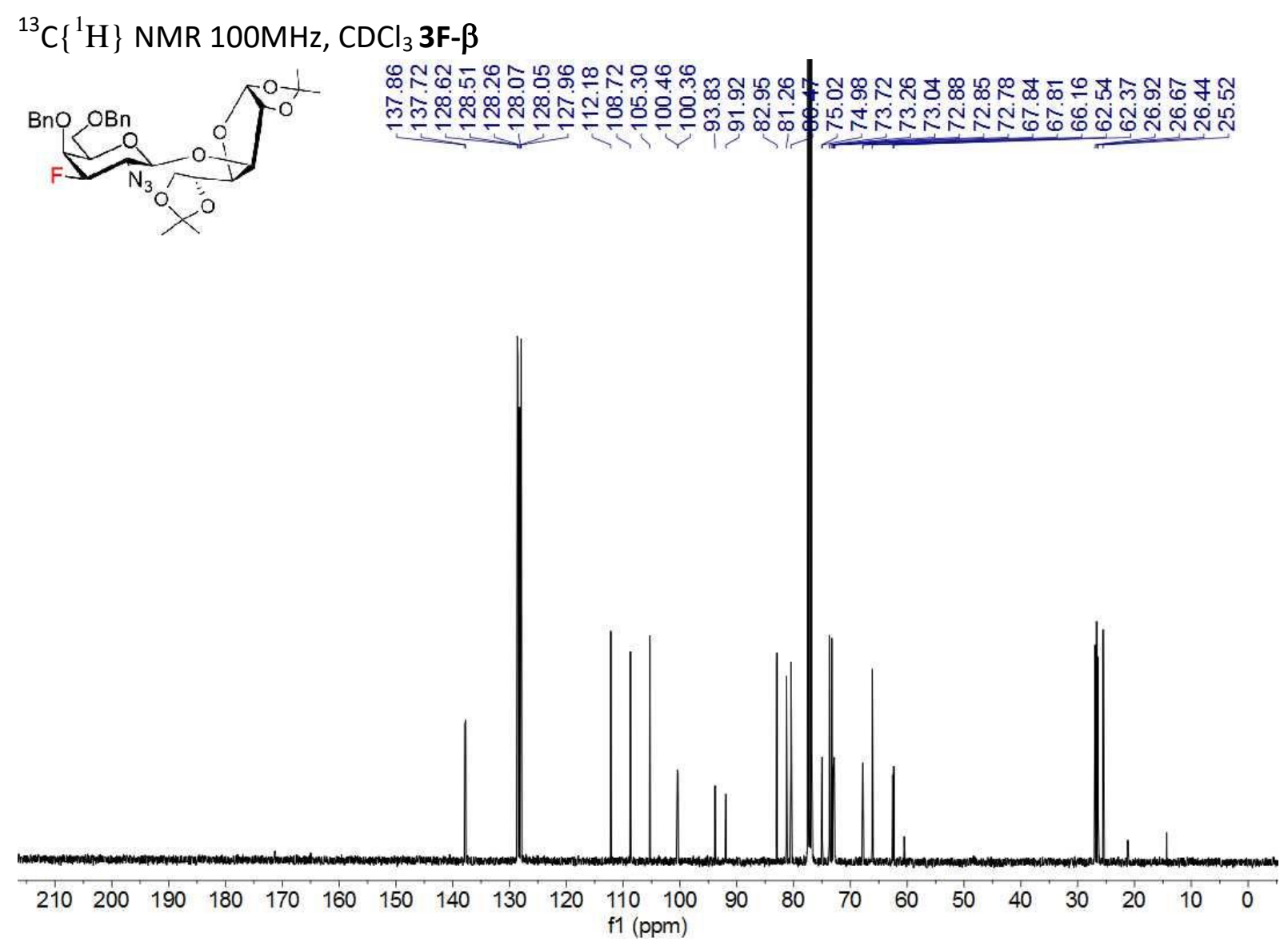

${ }^{1} \mathrm{H}-{ }^{1} \mathrm{H}$ COSY NMR $3 F-\beta$

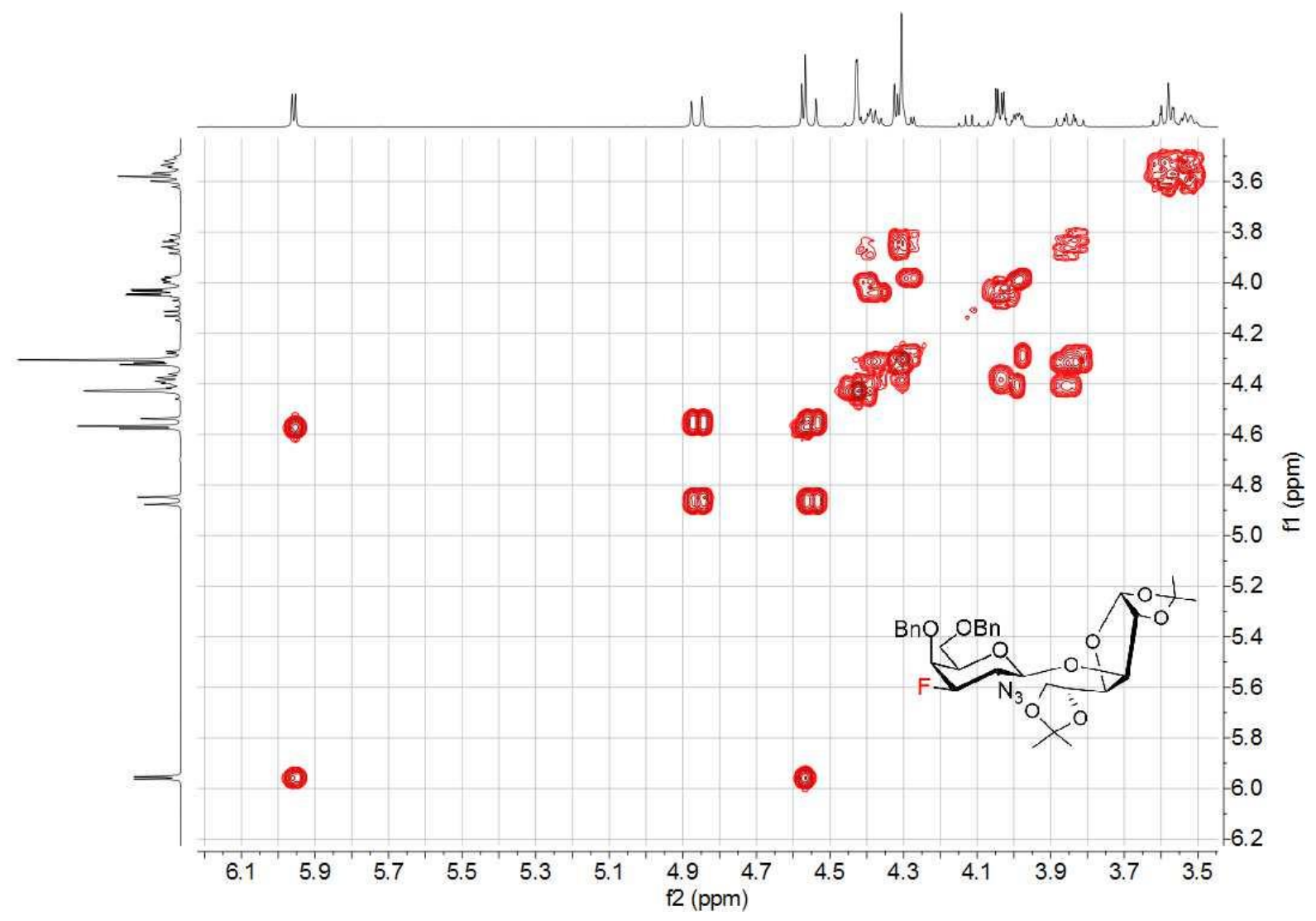


${ }^{1} \mathrm{H}-{ }^{13} \mathrm{C}$ HSQC NMR 3F- $\beta$

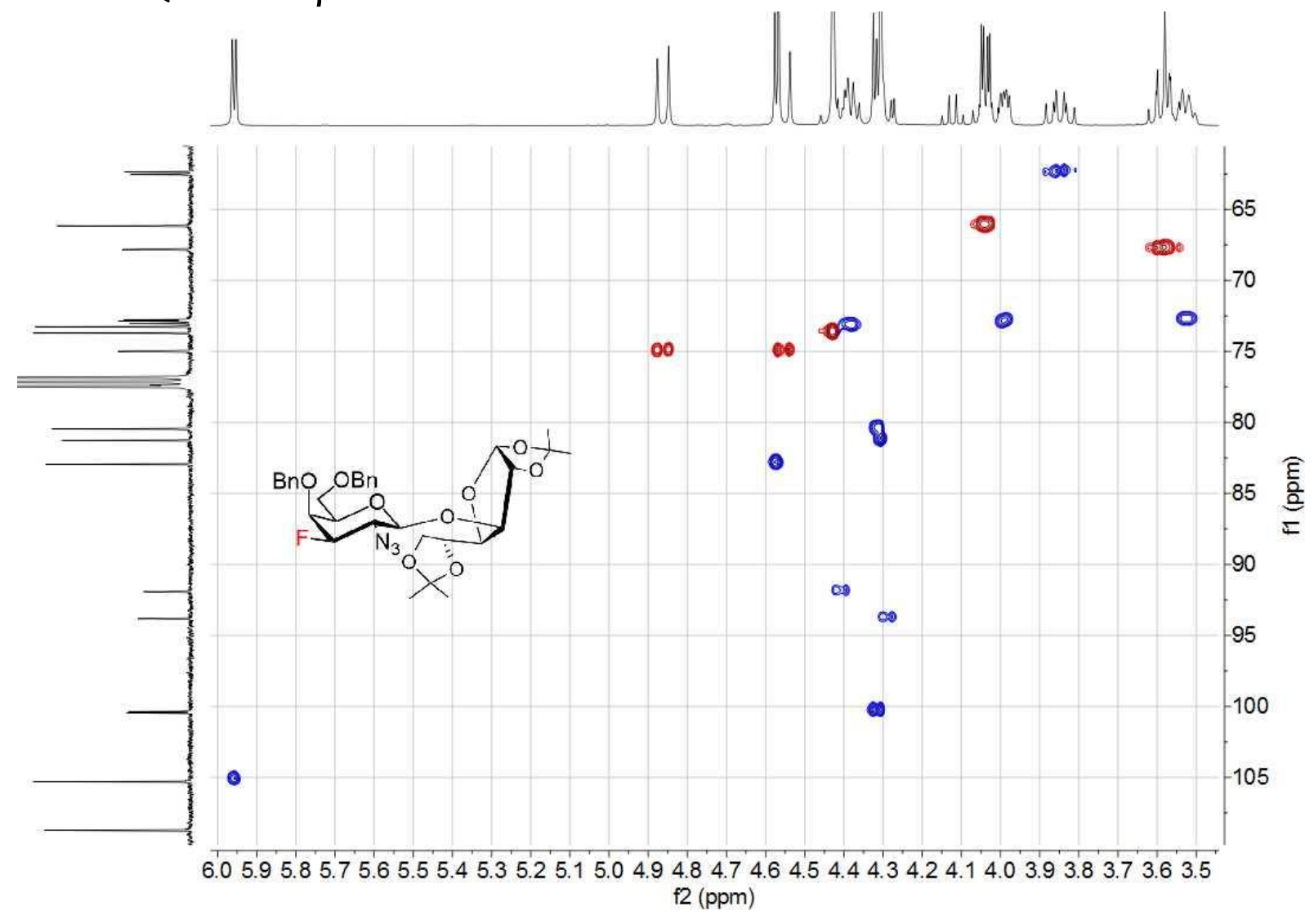

${ }^{1} \mathrm{H}-{ }^{13} \mathrm{C}$ HMBC NMR 3F- $\beta$

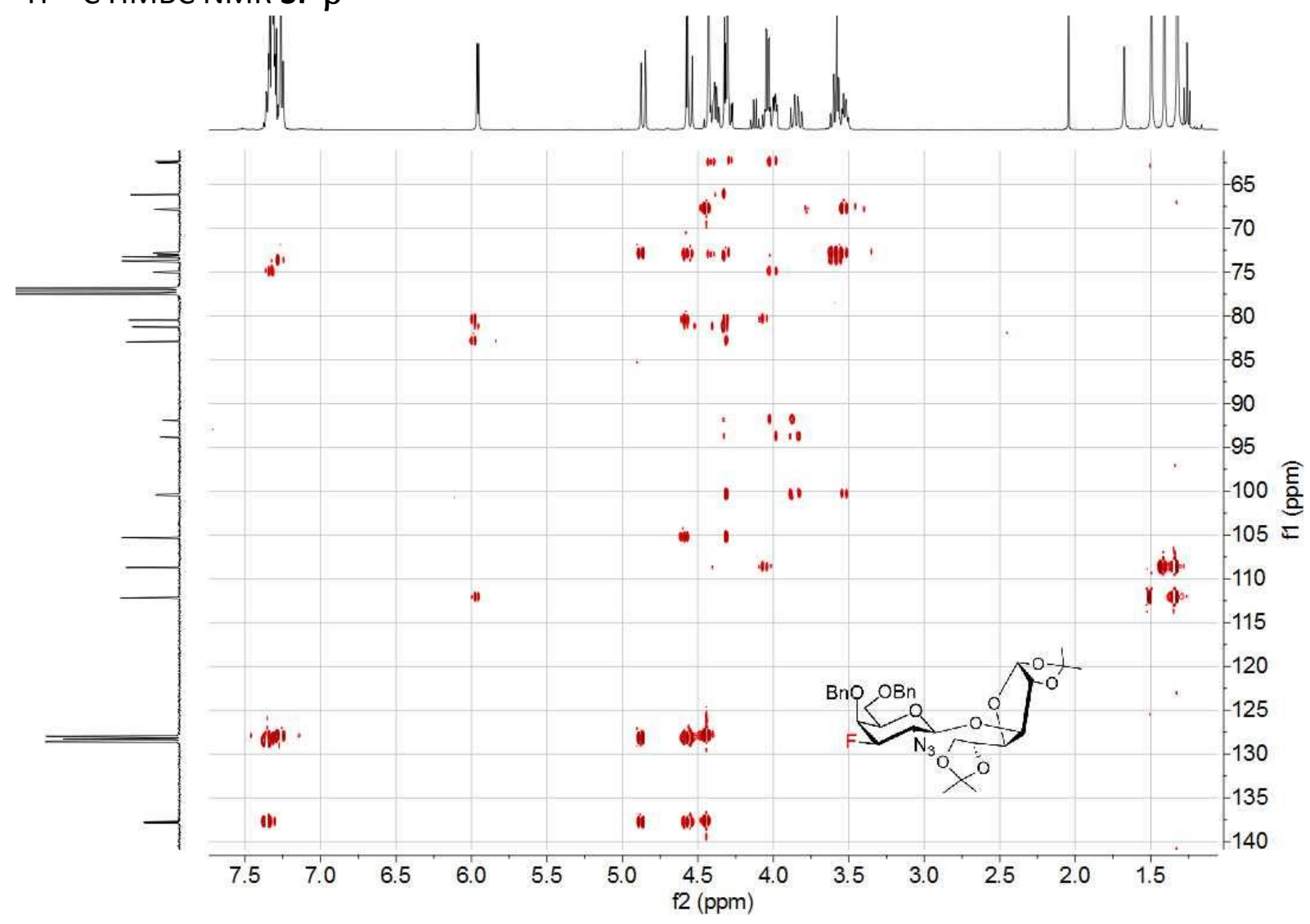


${ }^{19} \mathrm{~F} \mathrm{NMR} 376 \mathrm{MHz}, \mathrm{CDCl}_{3} \mathbf{3 F}-\boldsymbol{\beta}$

은ำกำกำ

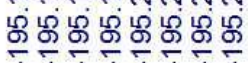

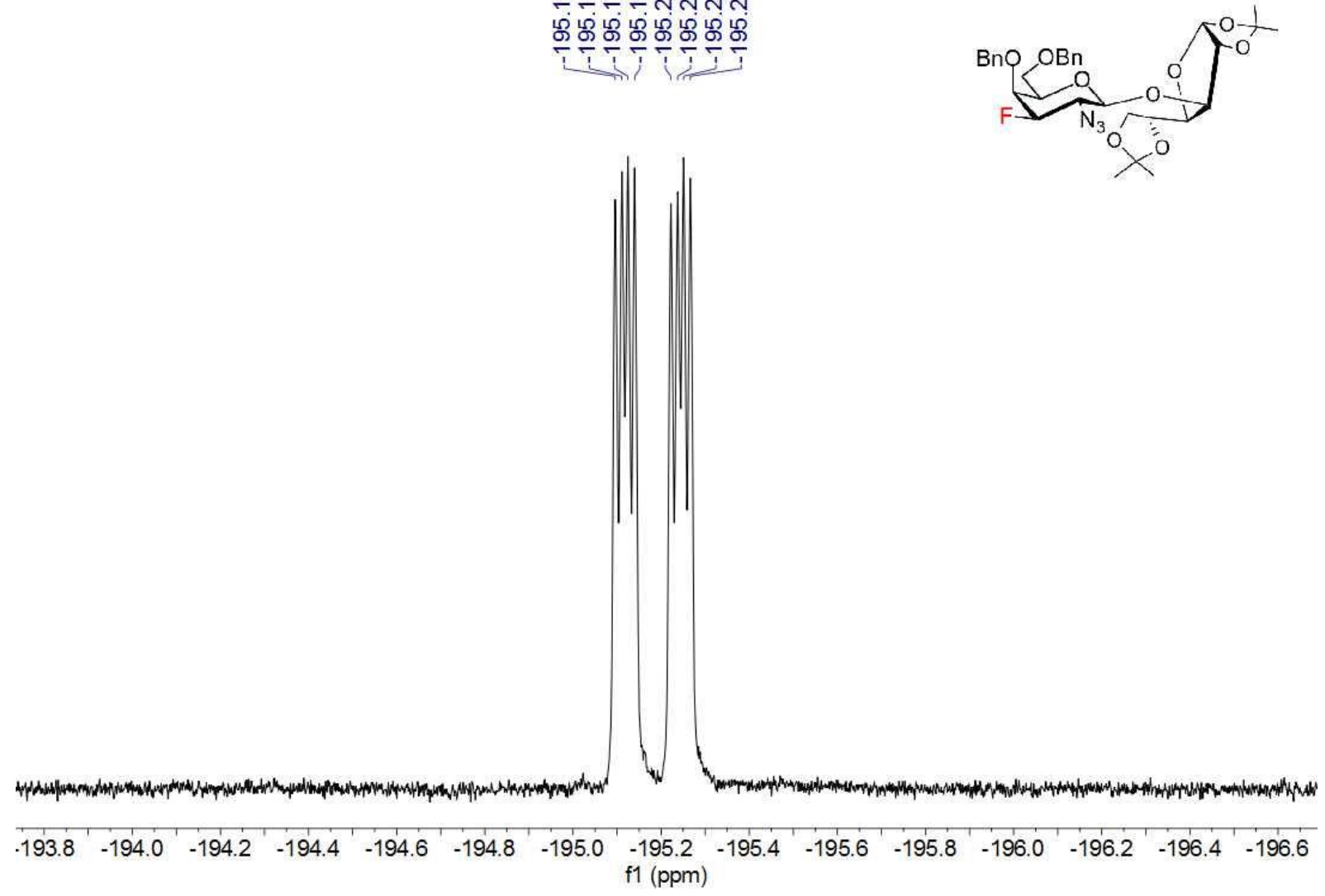

\section{NMR 3G- $\alpha$}

${ }^{1} \mathrm{HNMR} 400 \mathrm{MHz}, \mathrm{CDCl}_{3} 3 \mathrm{G}-\alpha$

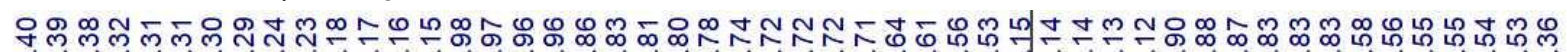

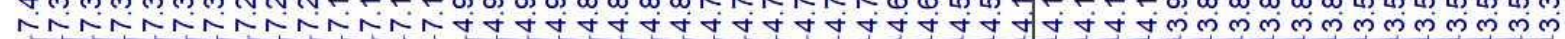

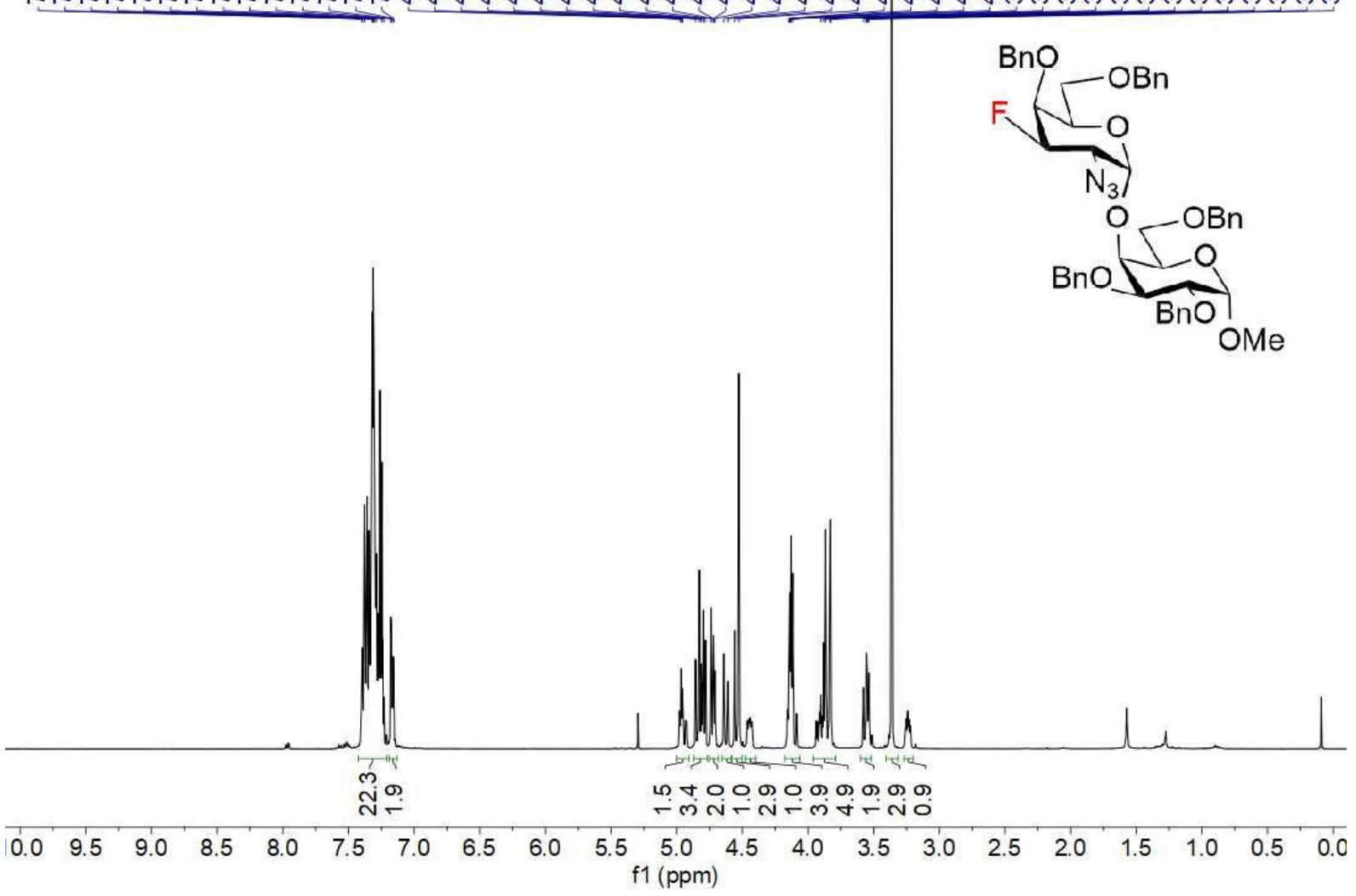


${ }^{13} \mathrm{C}\left\{{ }^{1} \mathrm{H}\right\} \mathrm{NMR}$ 100MHz, $\mathrm{CDCl}_{3} 3 \mathrm{G}-\alpha$

๓ m户ำ

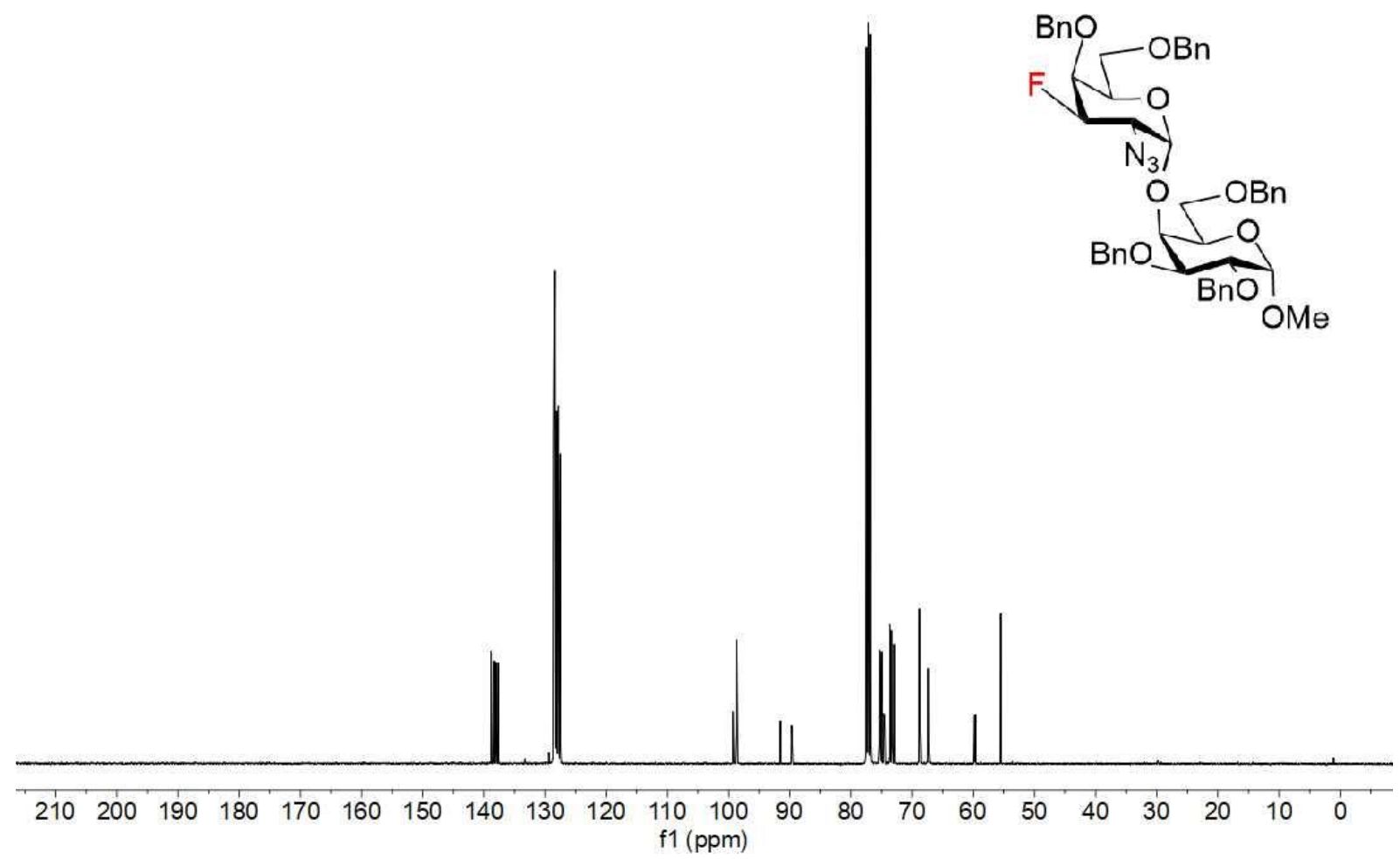

${ }^{1} \mathrm{H}-{ }^{1} \mathrm{H}$ COSY NMR 3G- $\alpha$

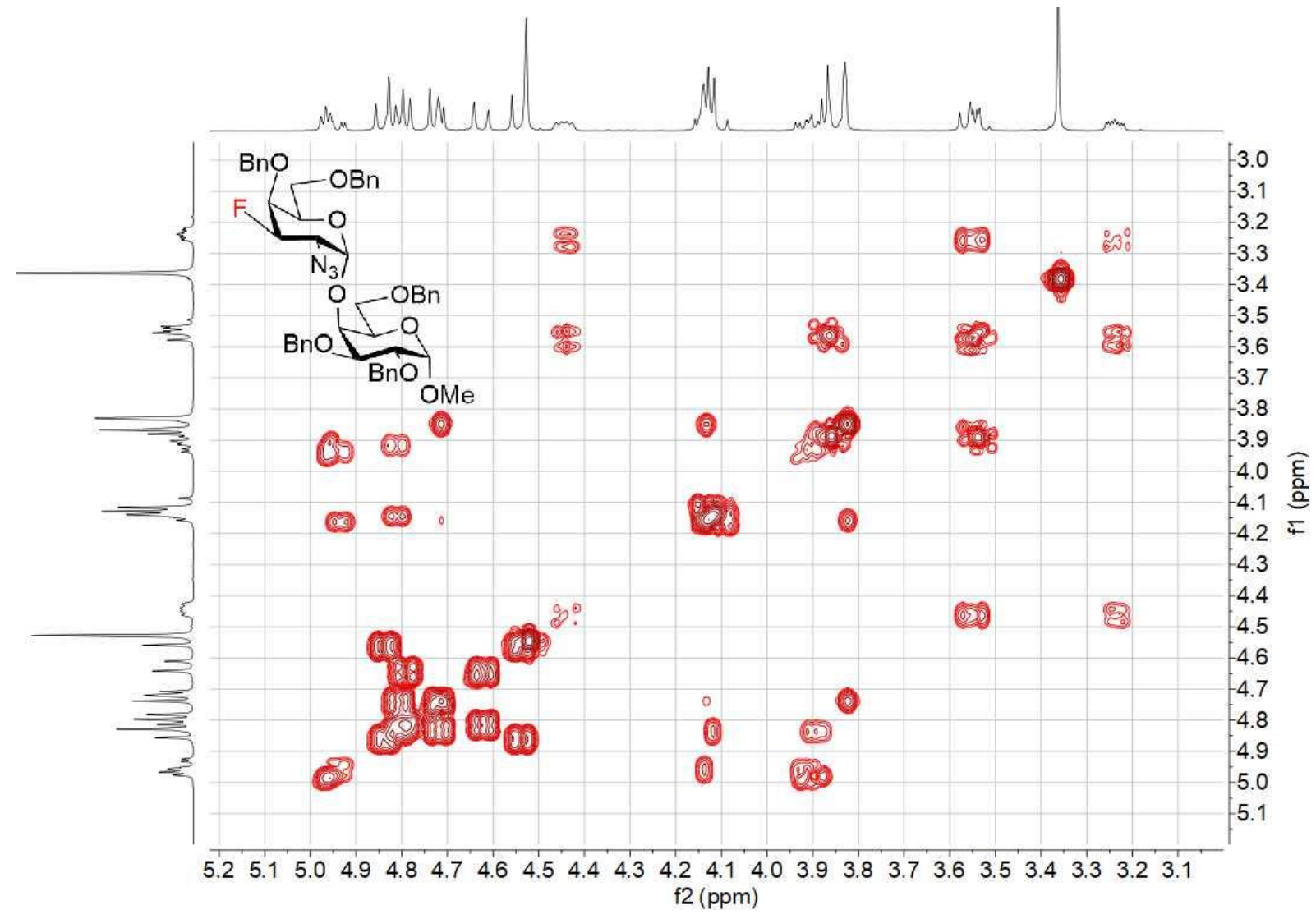


${ }^{1} \mathrm{H}^{13} \mathrm{C}$ HSQC NMR 3G- $\alpha$

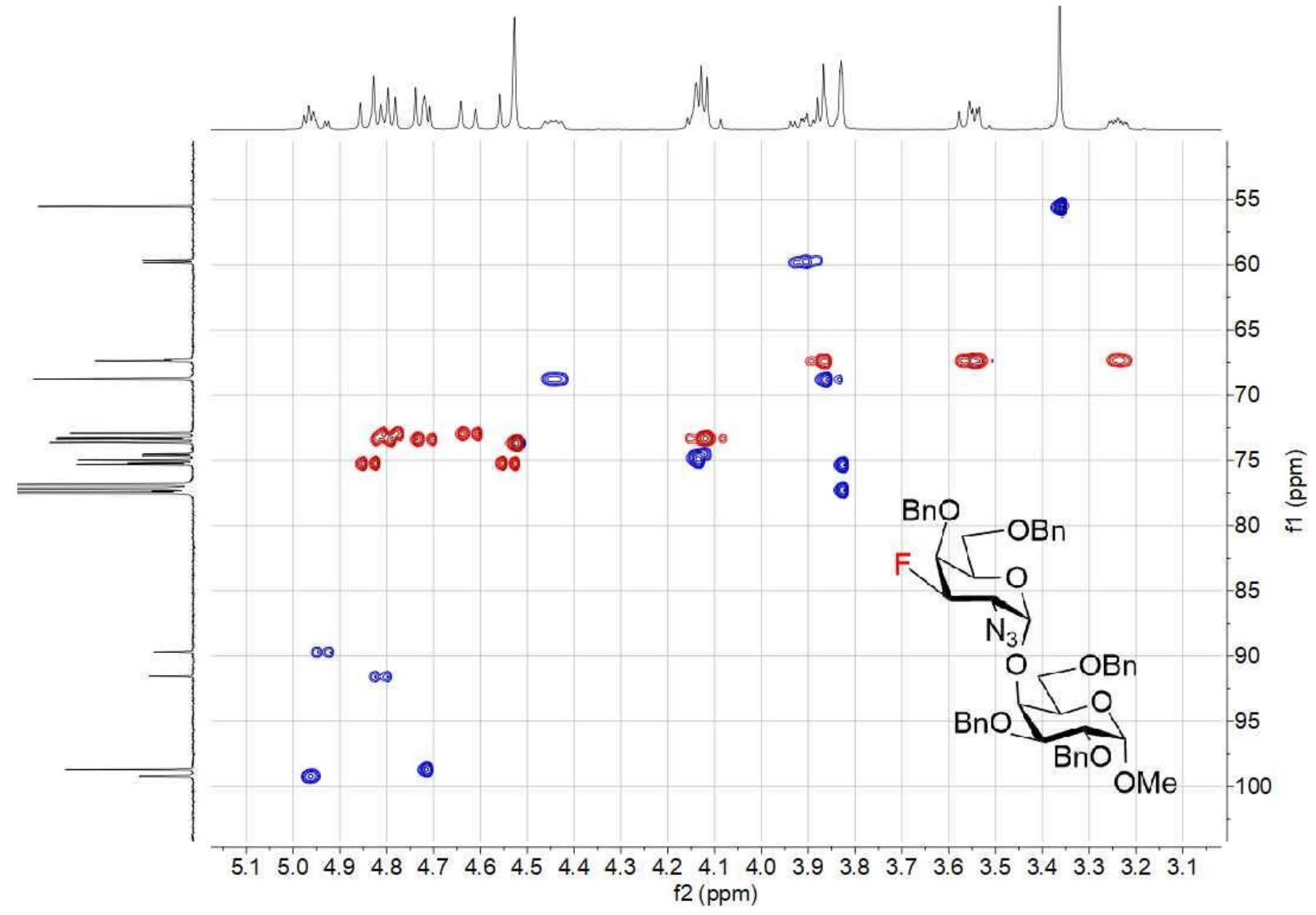

${ }^{1} \mathrm{H}-{ }^{13} \mathrm{C}$ HMBC NMR $3 \mathrm{G}-\boldsymbol{\alpha}$

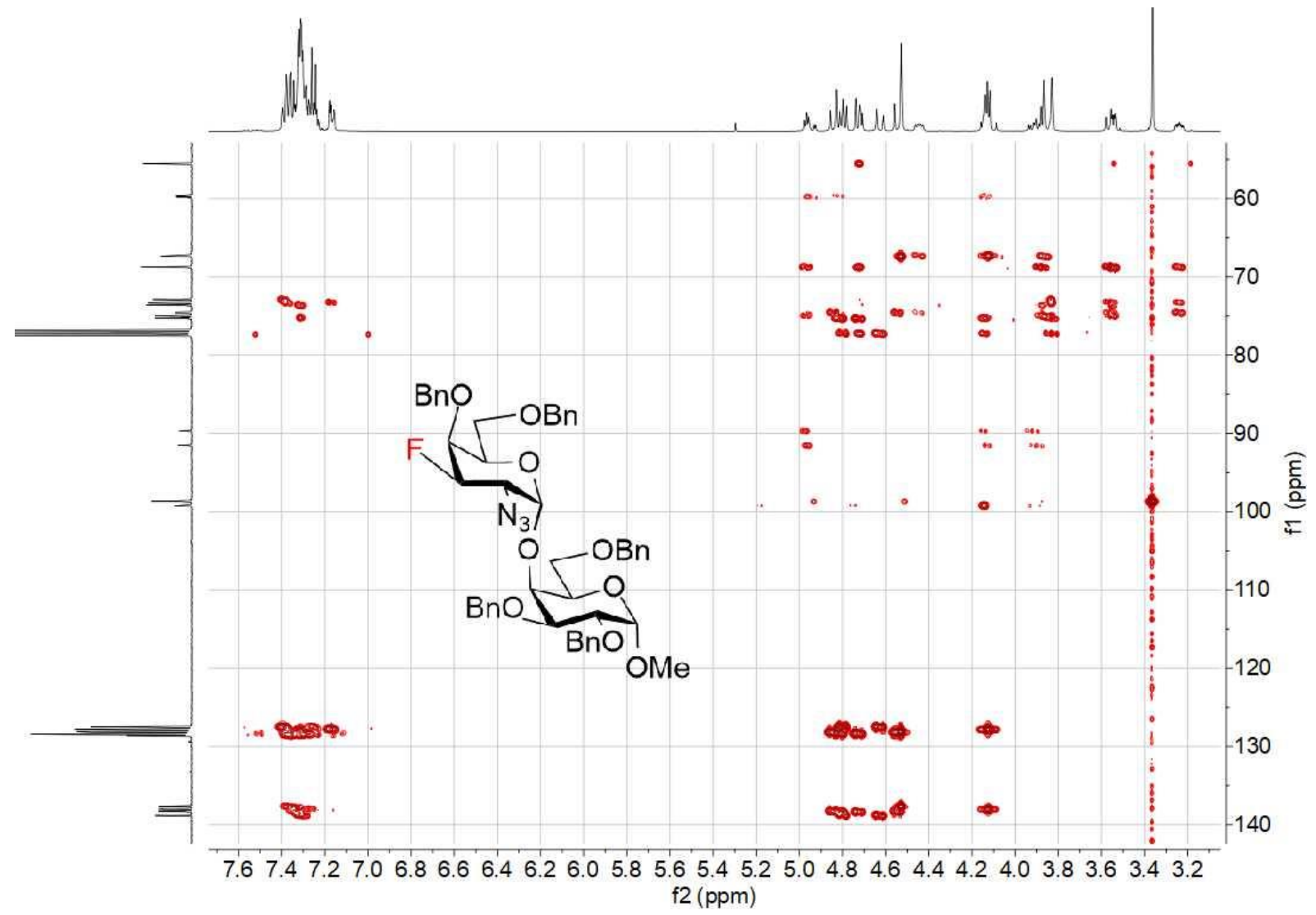




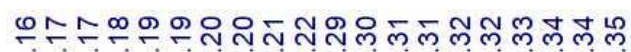

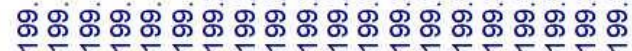
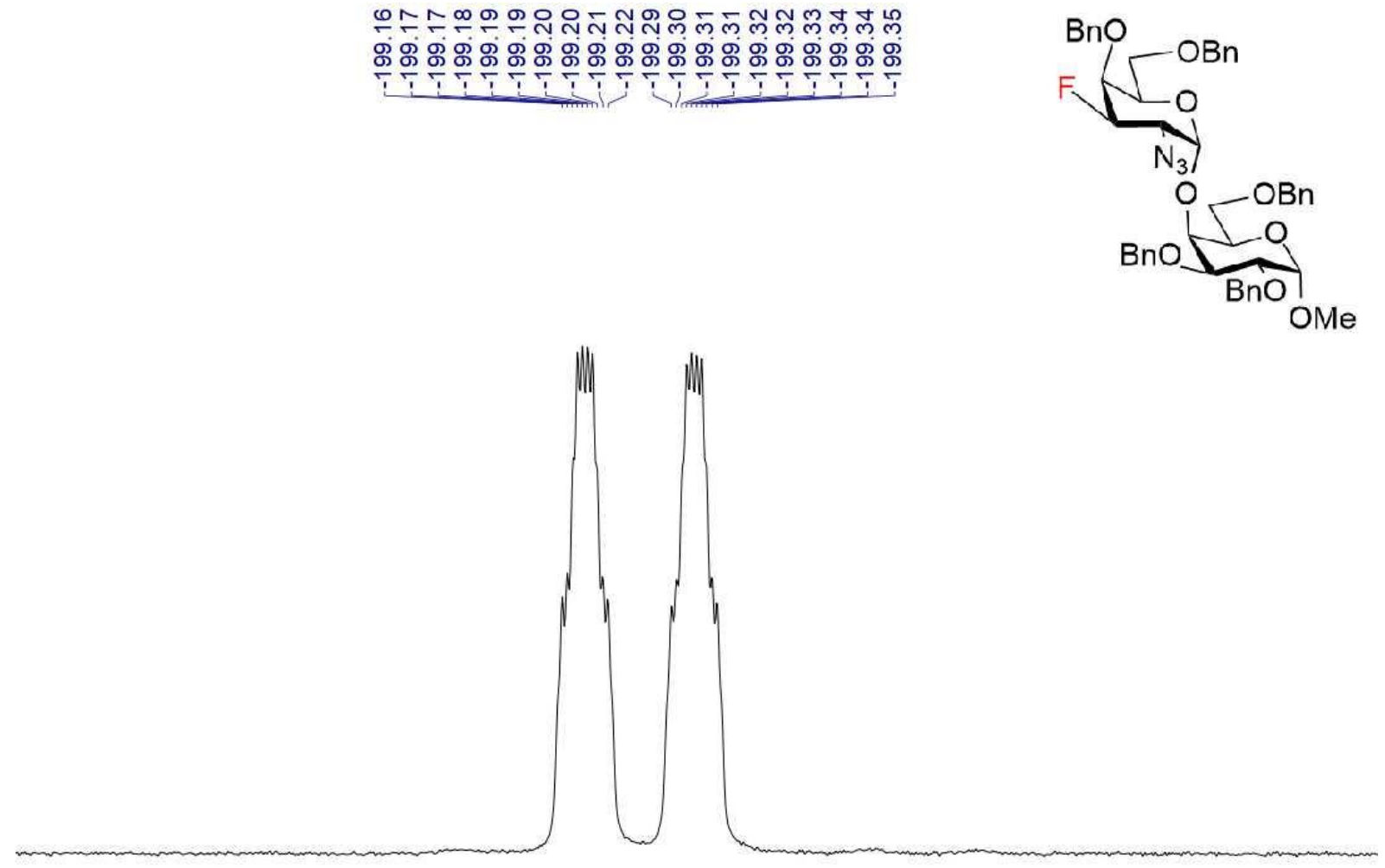

$\begin{array}{lllllllllllllllll}-198.6 & -198.7 & -198.8 & -198.9 & -199.0 & -199.1 & -199.2 & -199.3 & -199.4 & -199.5 & -199.6 & -199.7 & -199.8 & -199.9 & -200.0 & -200.1\end{array}$ f1 (ppm)

\section{NMR 3G- $\beta$}

${ }^{1} \mathrm{HNMR} 400 \mathrm{MHz}, \mathrm{CDCl}_{3}$ 3G- $\beta$

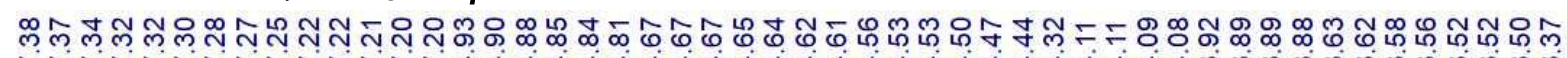

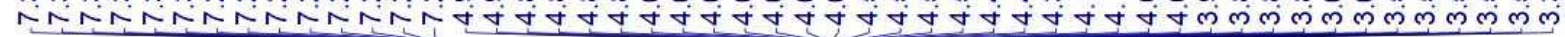

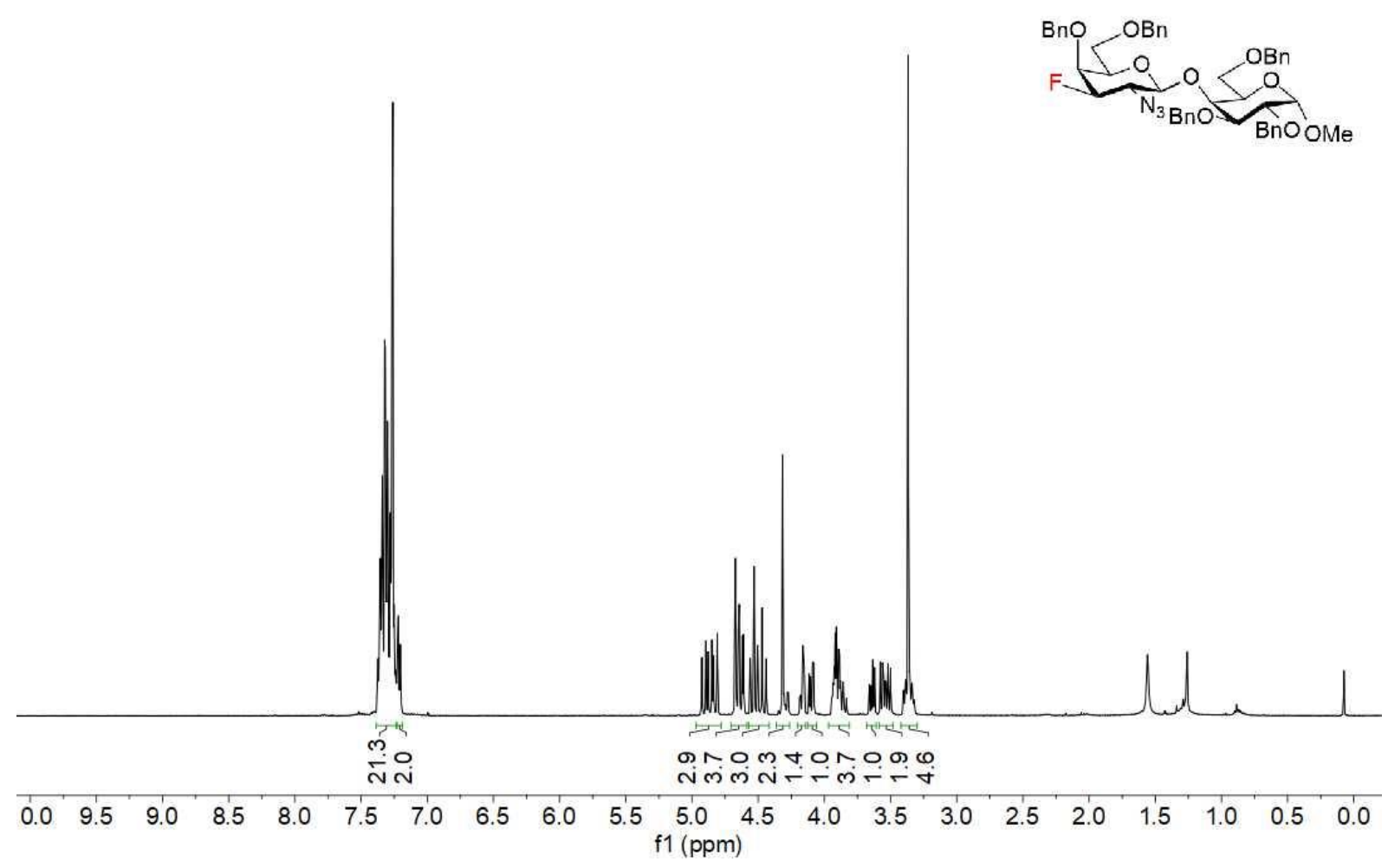




\section{${ }^{13} \mathrm{C}\left\{{ }^{1} \mathrm{H}\right\}$ NMR $100 \mathrm{MHz}, \mathrm{CDCl}_{3} 3 \mathrm{G}-\boldsymbol{\beta}$}

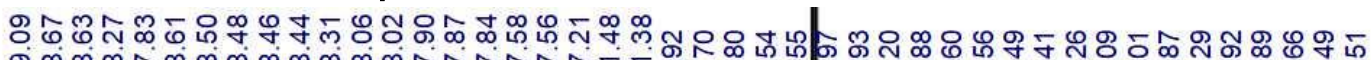
ஜு户

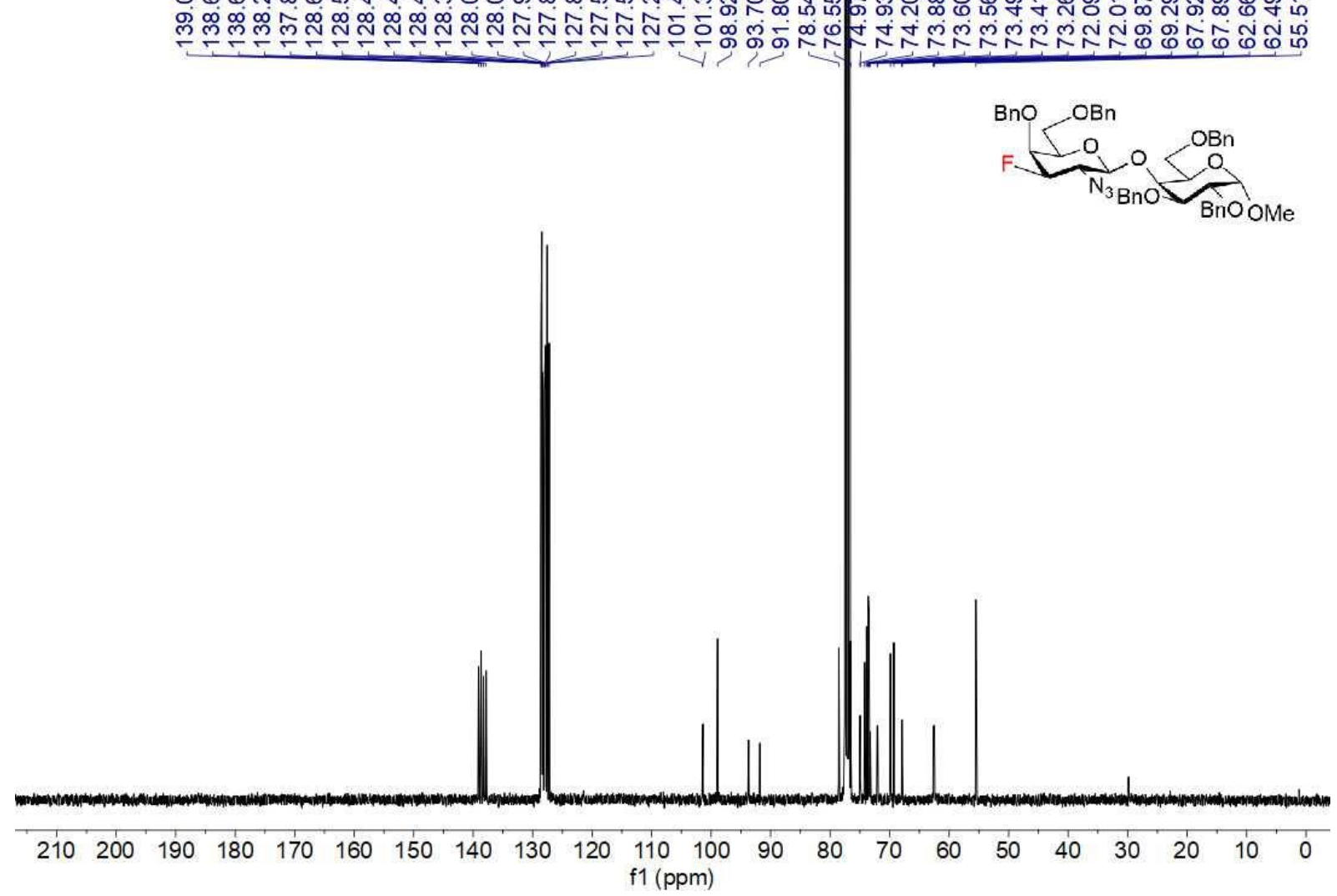

${ }^{1} \mathrm{H}-{ }^{1} \mathrm{H}$ COSY NMR 3G- $\beta$

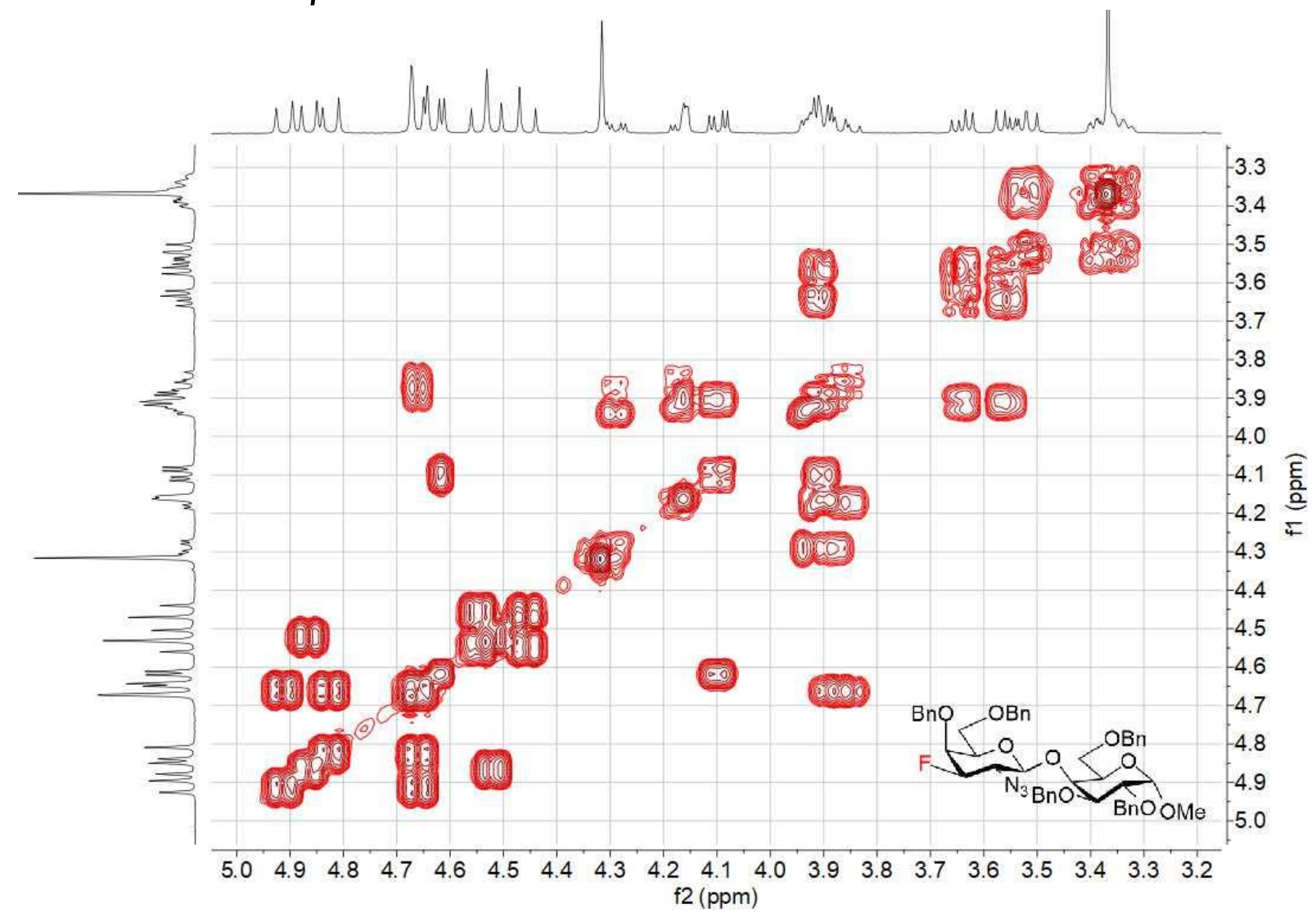


${ }^{1} \mathrm{H}-{ }^{13} \mathrm{C}$ HSOC NMR 3G- $\beta$

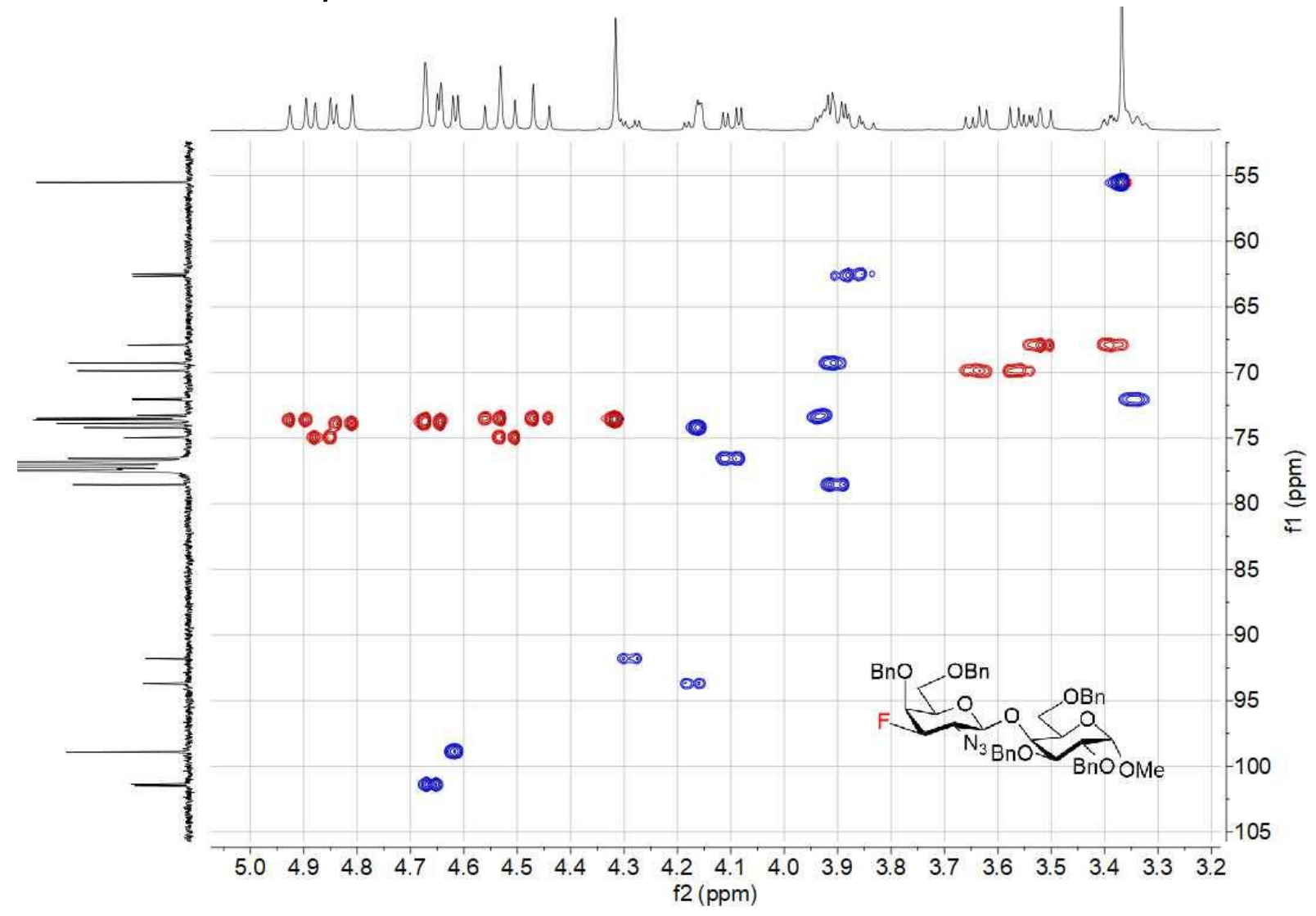

${ }^{1} \mathrm{H}^{-{ }^{13} \mathrm{C}}$ HMBC NMR 3G- $\beta$




${ }^{19} \mathrm{~F} \mathrm{NMR} 376 \mathrm{MHz}, \mathrm{CDCl}_{3} \mathbf{3 G -} \boldsymbol{\beta}$

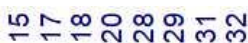

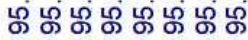

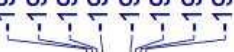

$\mathrm{BnO} \mathrm{OBn}$

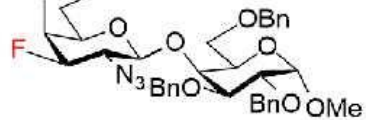

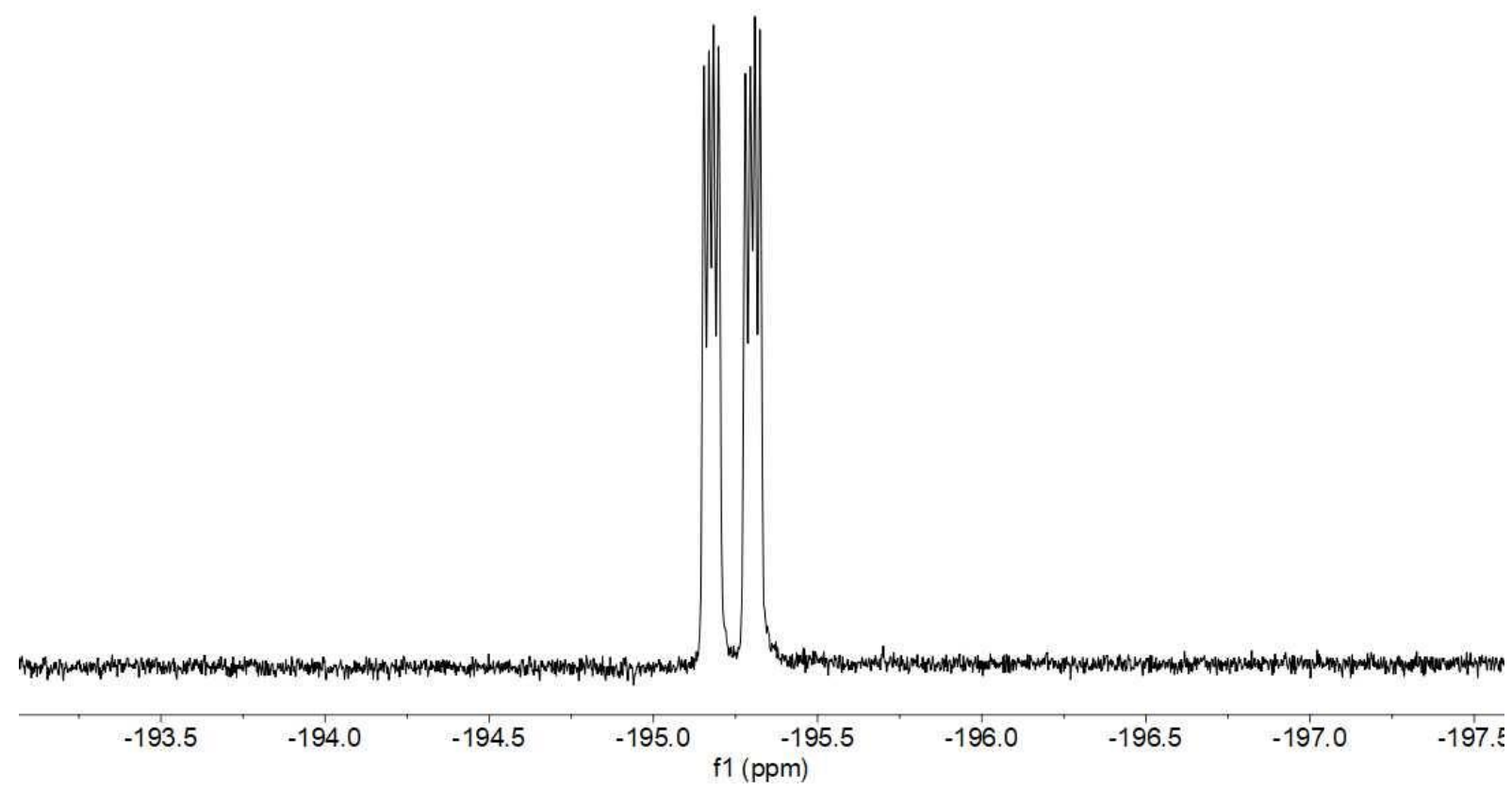




\section{NMR $3 \mathrm{H}-\alpha$}

\section{${ }^{1} \mathrm{HNMR} 400 \mathrm{MHz}, \mathrm{CDCl}_{3} 3 \mathrm{H}-\alpha$}

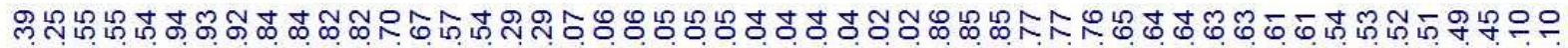

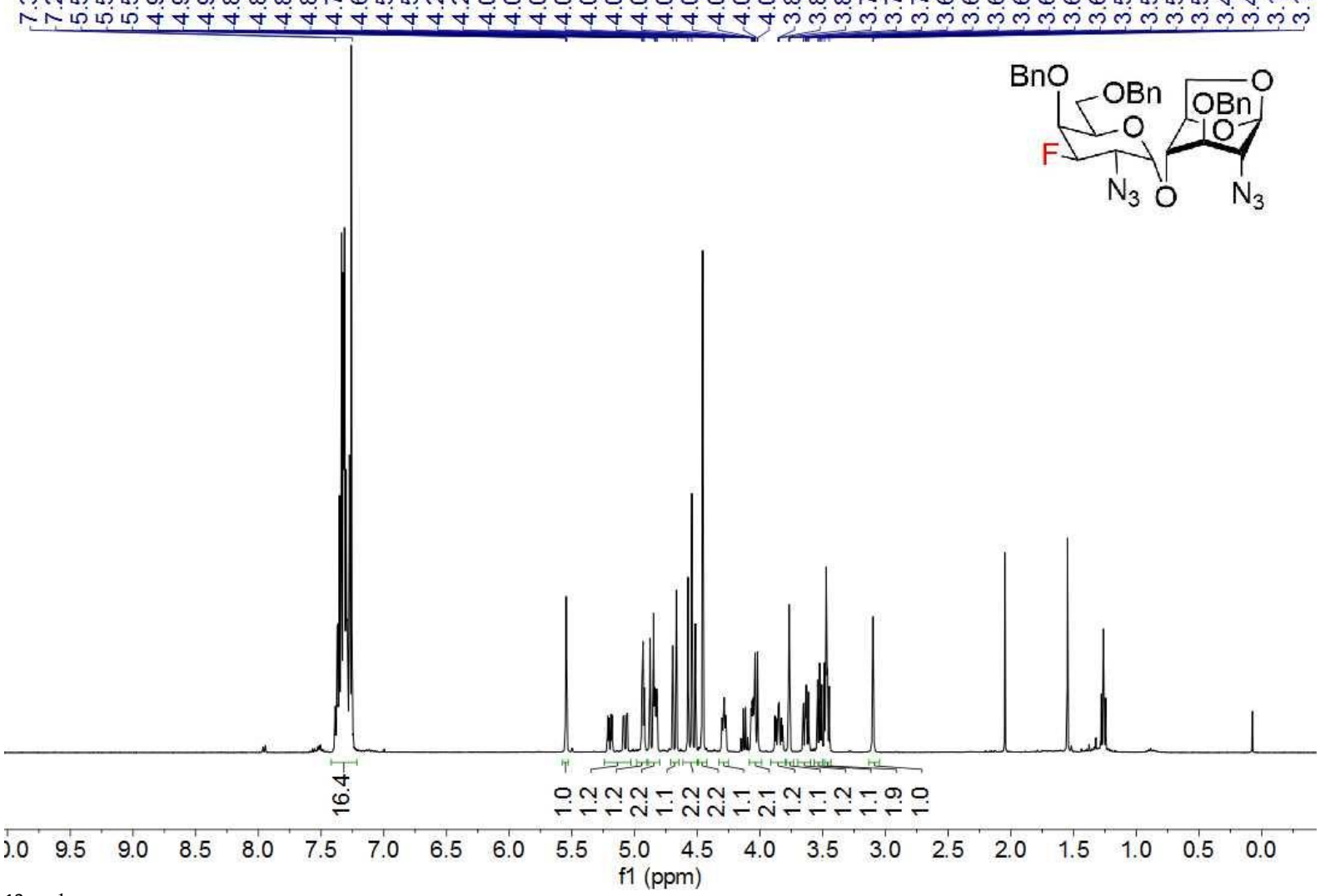

${ }^{13} \mathrm{C}\left\{{ }^{1} \mathrm{H}\right\}$ NMR $100 \mathrm{MHz}, \mathrm{CDCl}_{3} 3 \mathrm{H}-\boldsymbol{\alpha}$

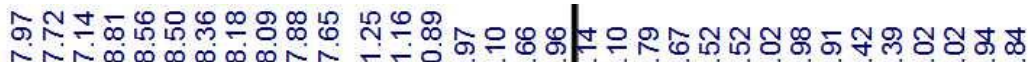

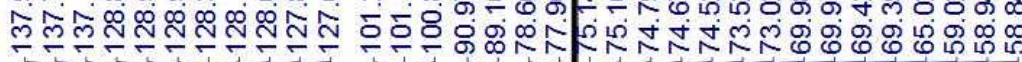
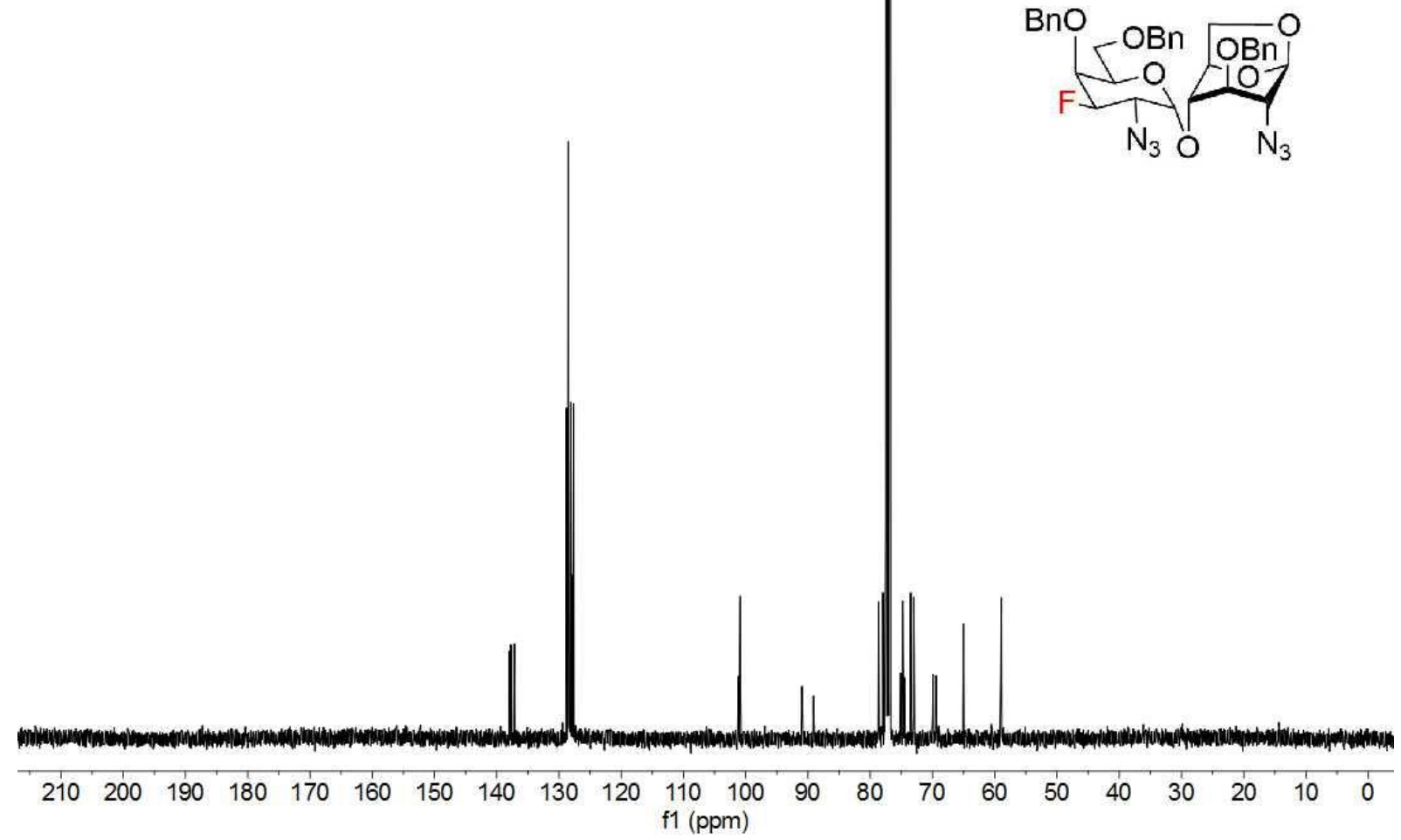
${ }^{1} \mathrm{H}-{ }^{1} \mathrm{H}$ COSY NMR $3 \mathrm{H}-\alpha$

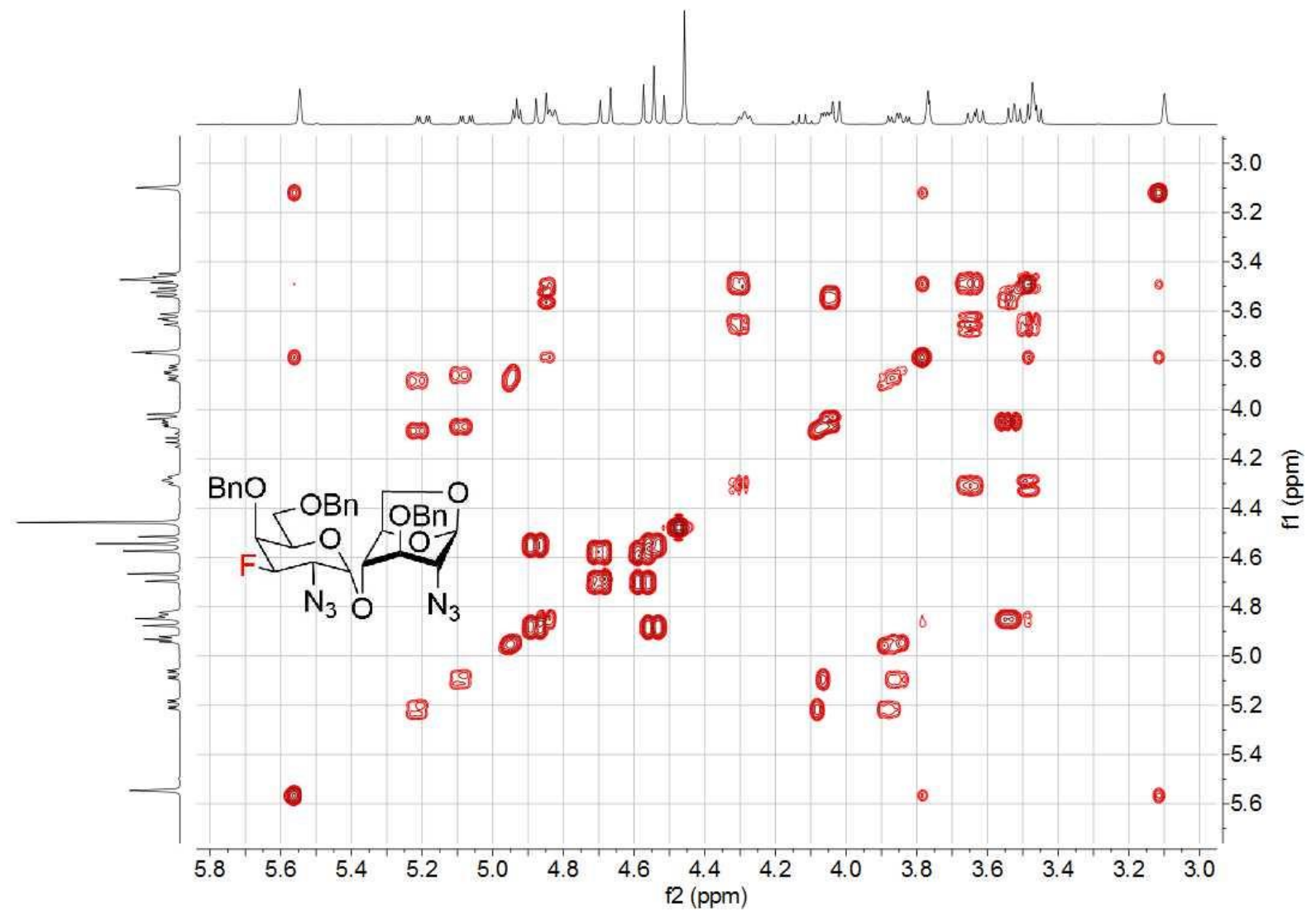

${ }^{1} \mathrm{H}^{13} \mathrm{C}$ HSQC NMR $3 \mathrm{H}-\boldsymbol{\alpha}$

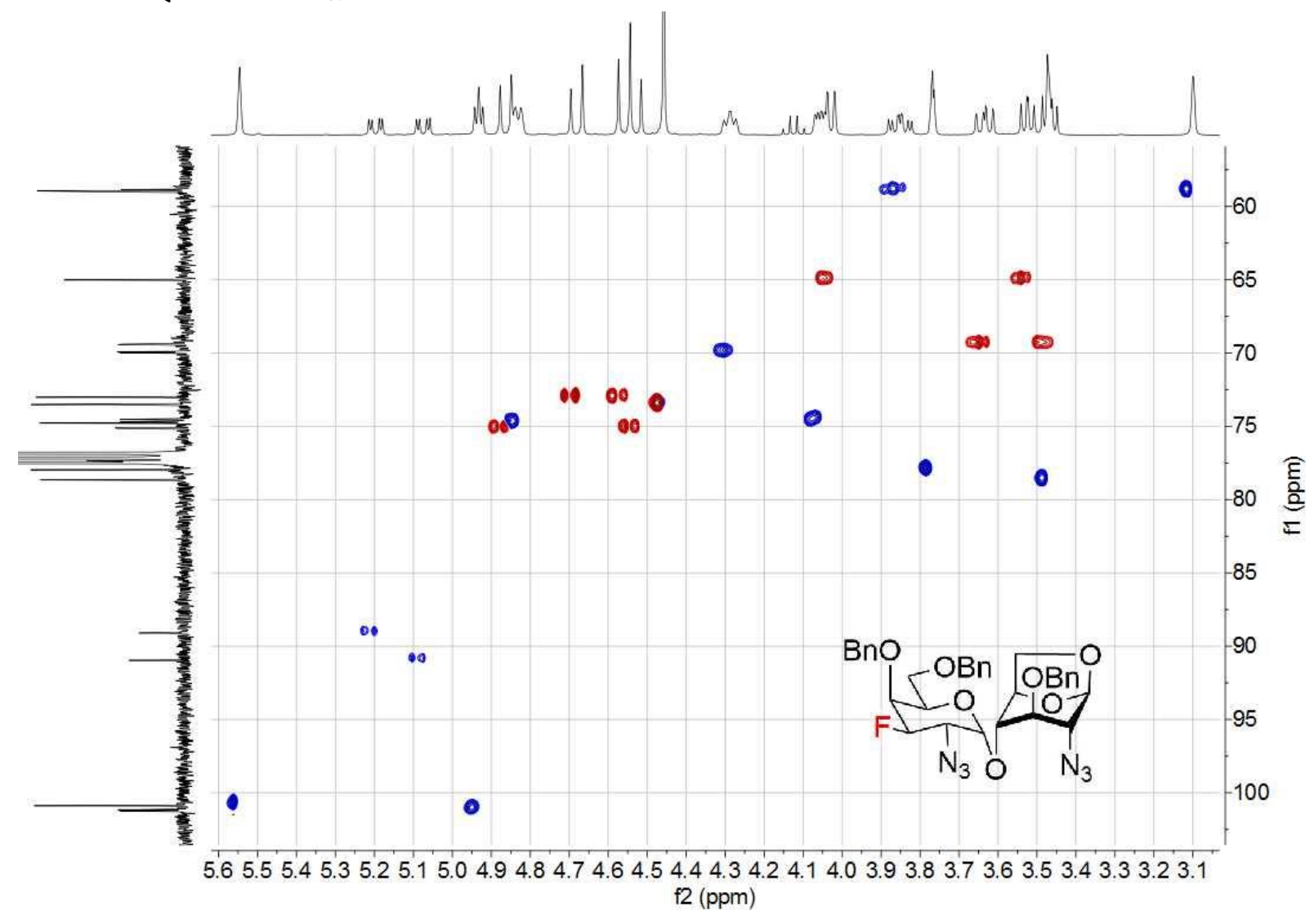


${ }^{1} \mathrm{H}-{ }^{13} \mathrm{C}$ HMBC NMR $3 \mathrm{H}-\alpha$

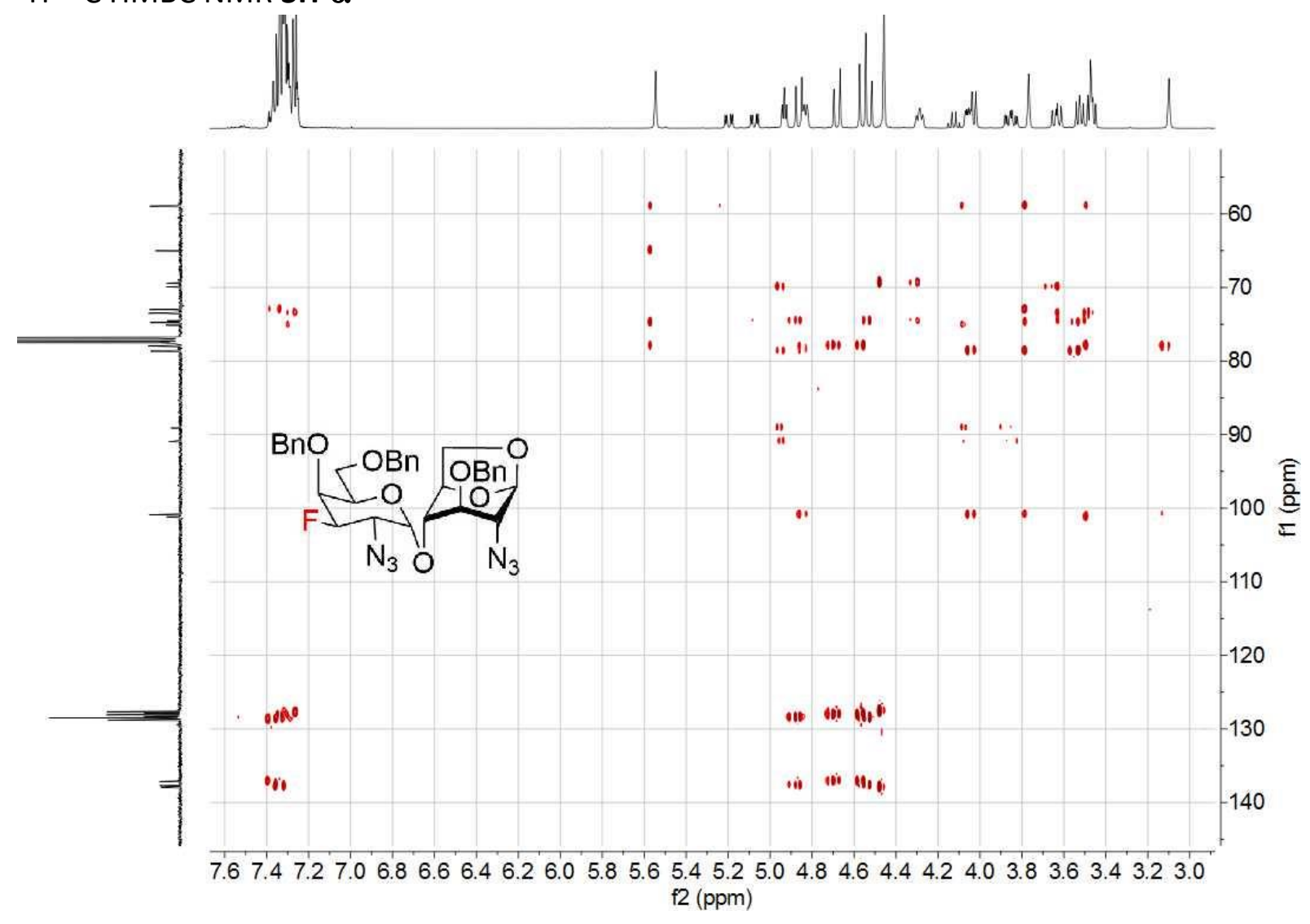

${ }^{19} \mathrm{~F} \mathrm{NMR} 376 \mathrm{MHz}, \mathrm{CDCl}_{3} 3 \mathrm{H}-\boldsymbol{\alpha}$

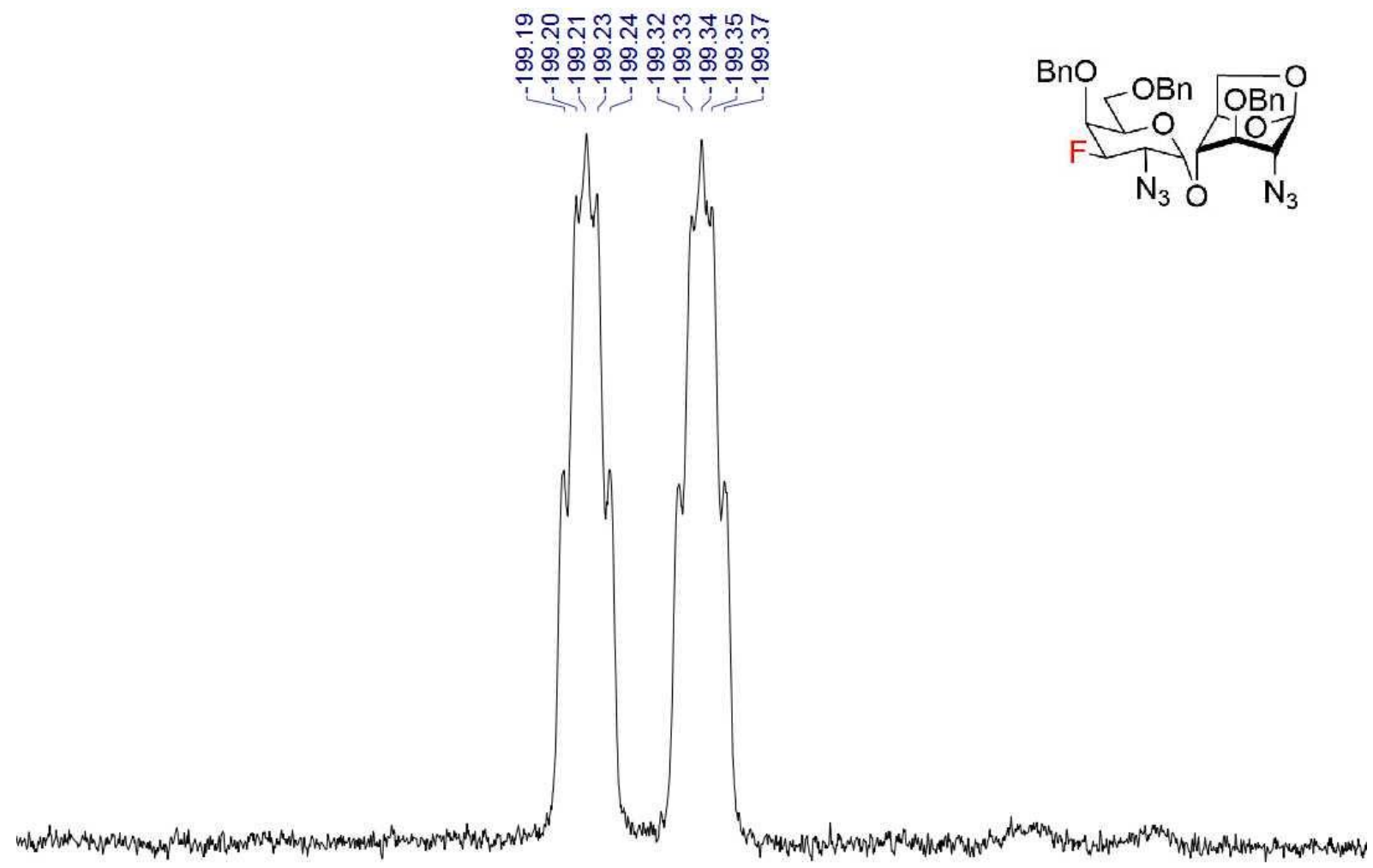

$-198.65-198.75-198.85-198.95-199.05-199.15-199.25-199.35-199.45-199.55-199.65-199.75-199.85-199.95-200.05$ f1 (ppm) 


\section{NMR $\mathbf{3 H}-\boldsymbol{\beta}$}

${ }^{1} \mathrm{HNMR} 500 \mathrm{MHz}, \mathrm{CDCl}_{3} \mathbf{3 H}-\boldsymbol{\beta}$

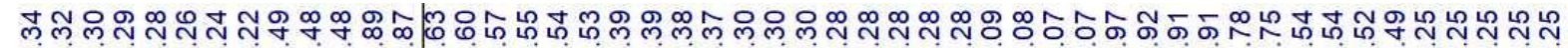

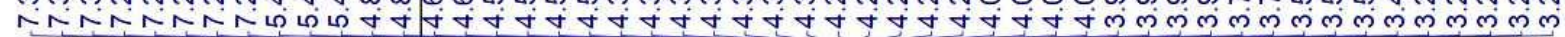

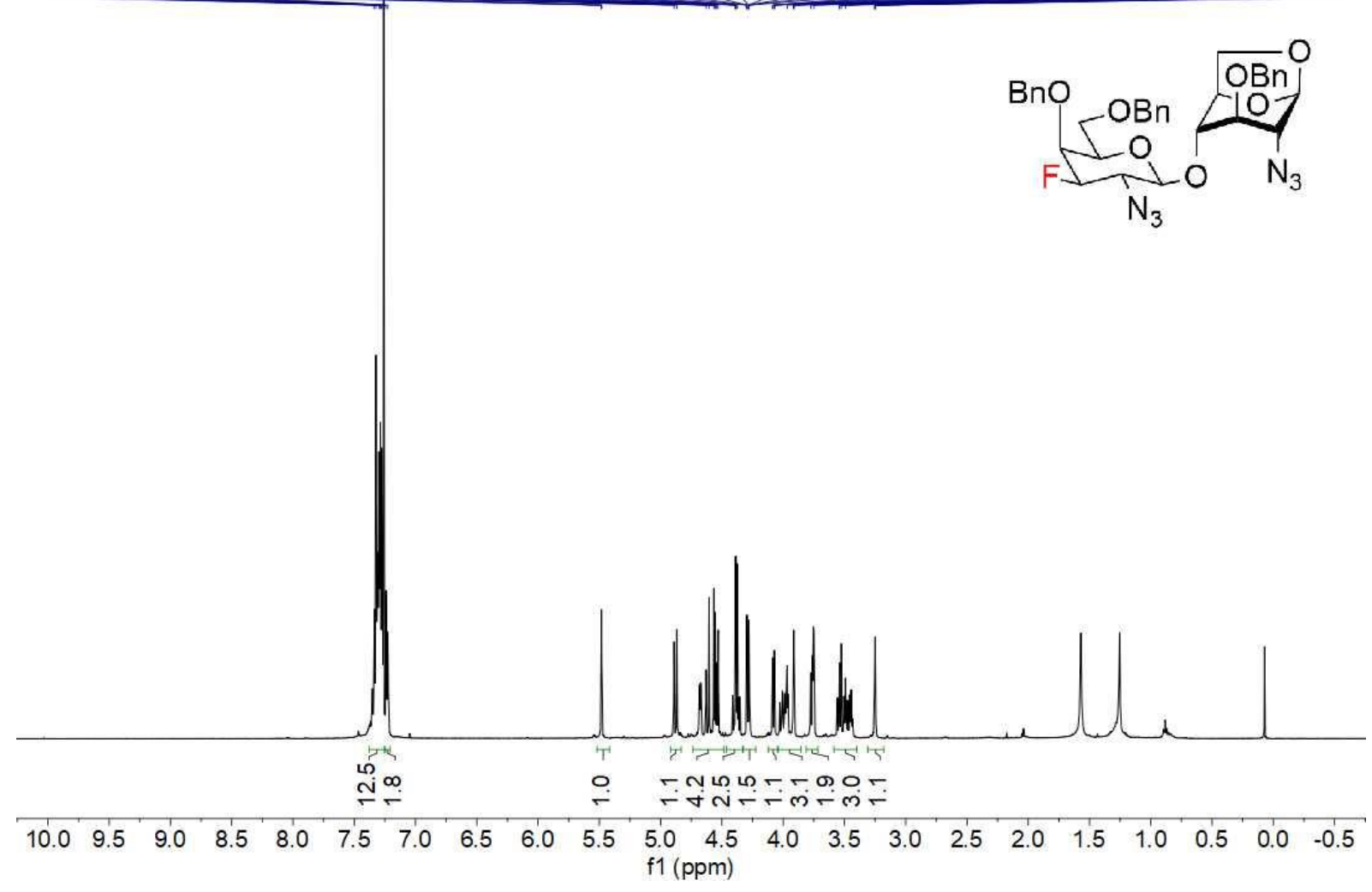

${ }^{13} \mathrm{C}\left\{{ }^{1} \mathrm{H}\right\}$ APT NMR $126 \mathrm{MHz}, \mathrm{CDCl}_{3} 3 \mathrm{H}-\boldsymbol{\beta}$

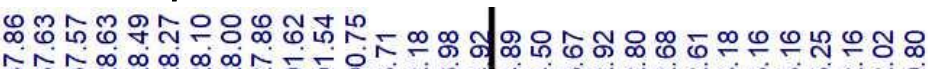

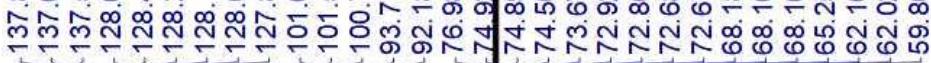

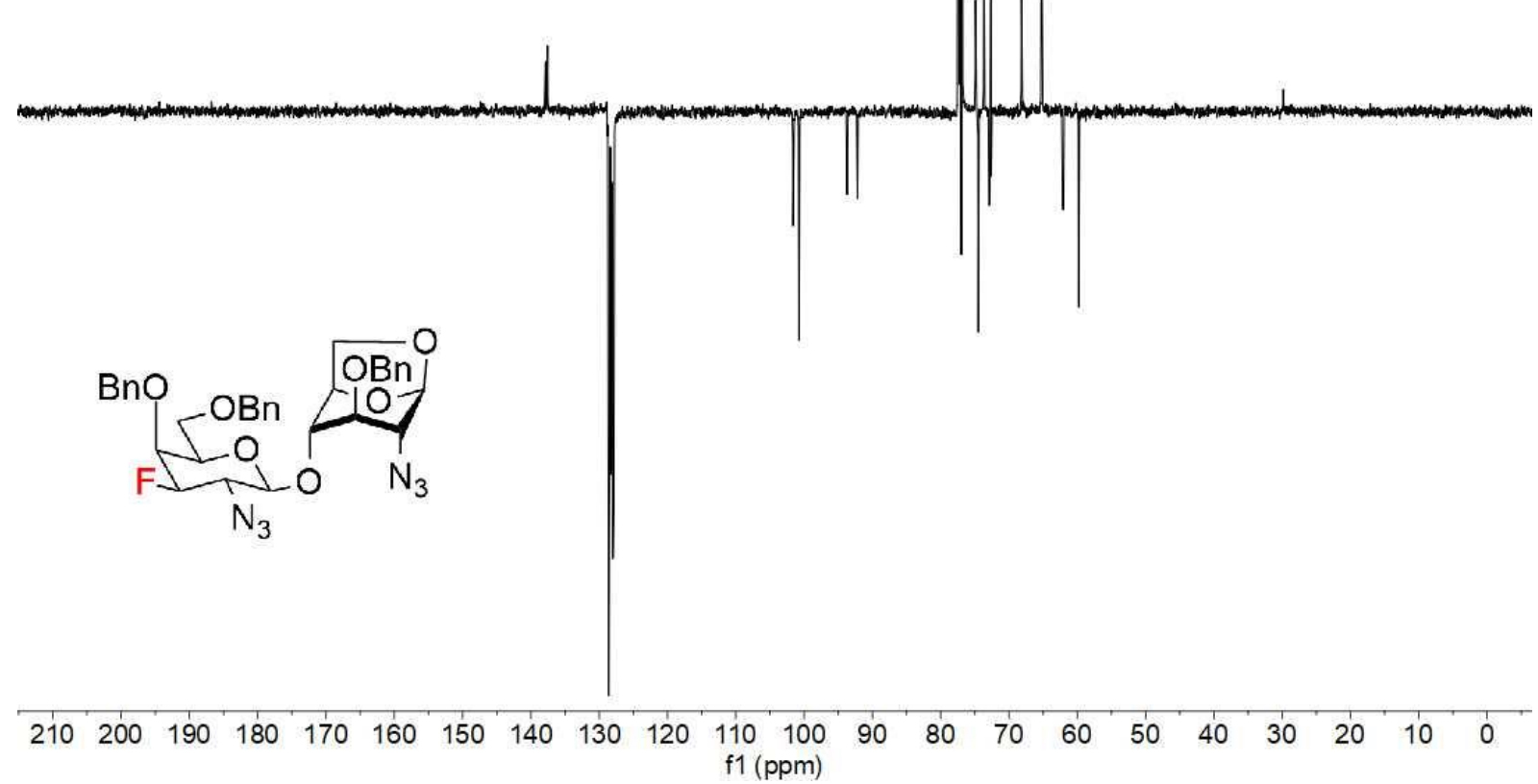

应 
${ }^{1} \mathrm{H}-{ }^{1} \mathrm{H}$ COSY NMR $\mathbf{3 H}-\boldsymbol{\beta}$

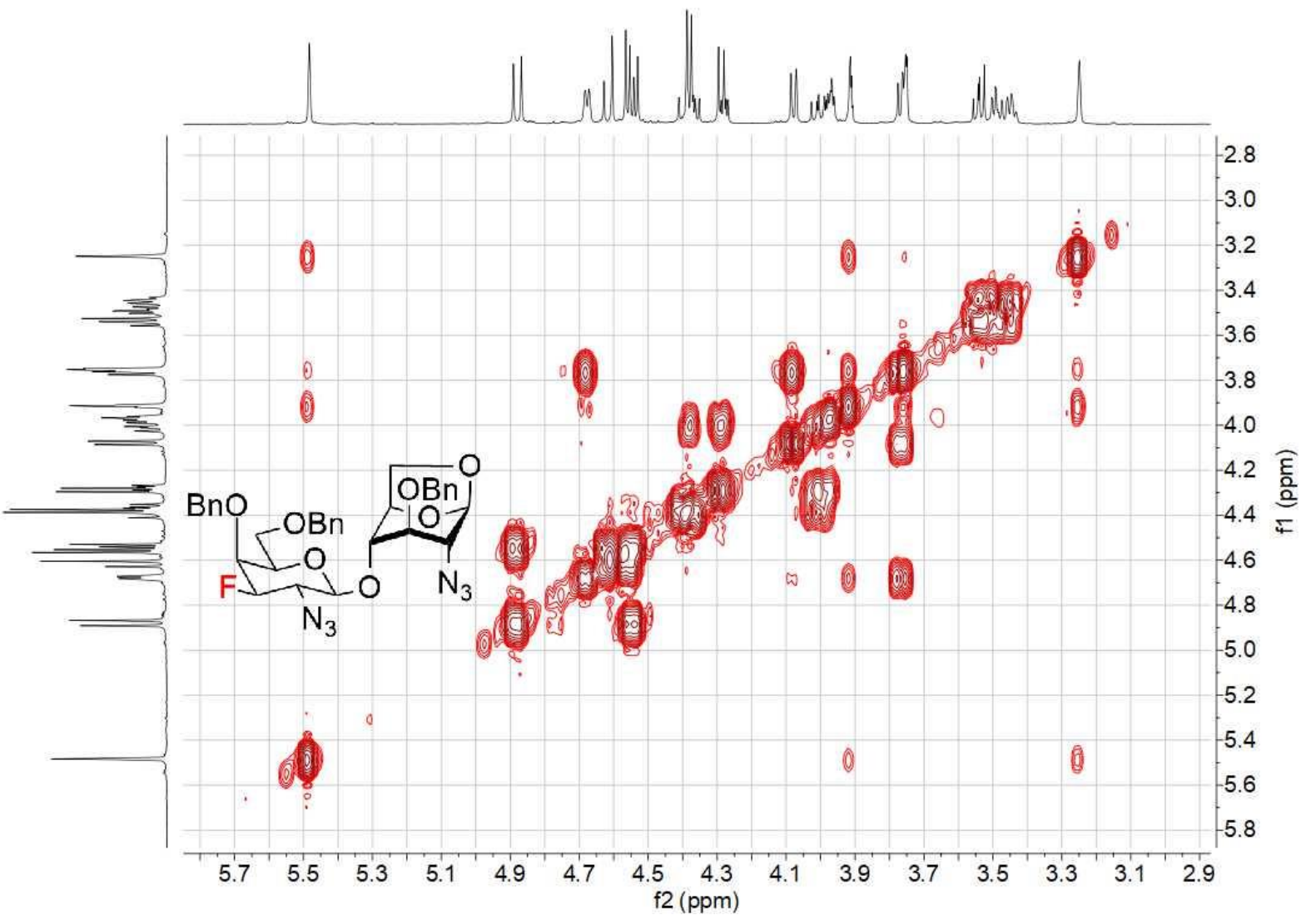

${ }^{1} \mathrm{H}-{ }^{13} \mathrm{C}$ HSQC NMR $3 \mathbf{H}-\boldsymbol{\beta}$

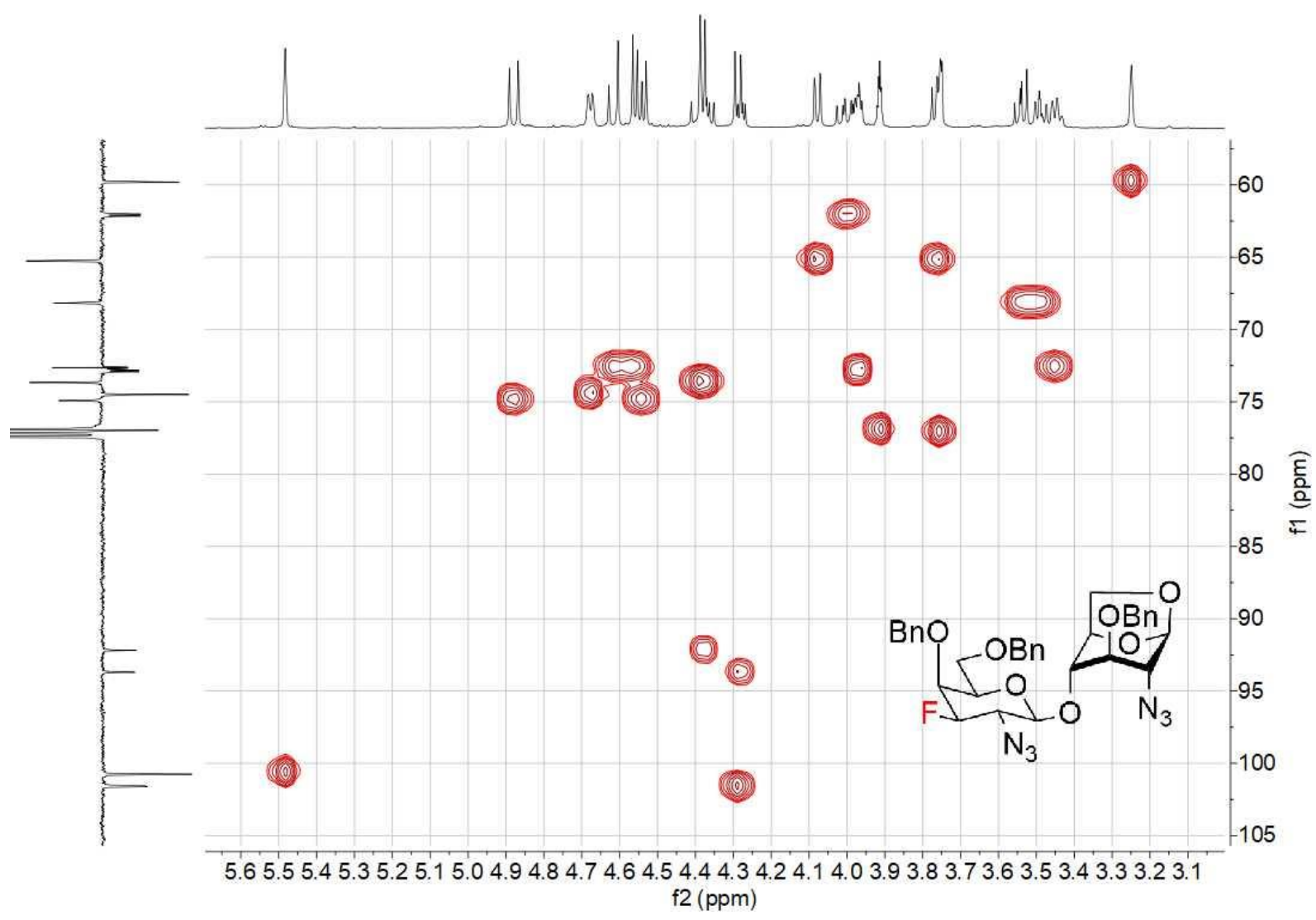

S214 
${ }^{1} \mathrm{H}-{ }^{13} \mathrm{C}$ HMBC NMR $3 \mathrm{H}-\boldsymbol{\beta}$

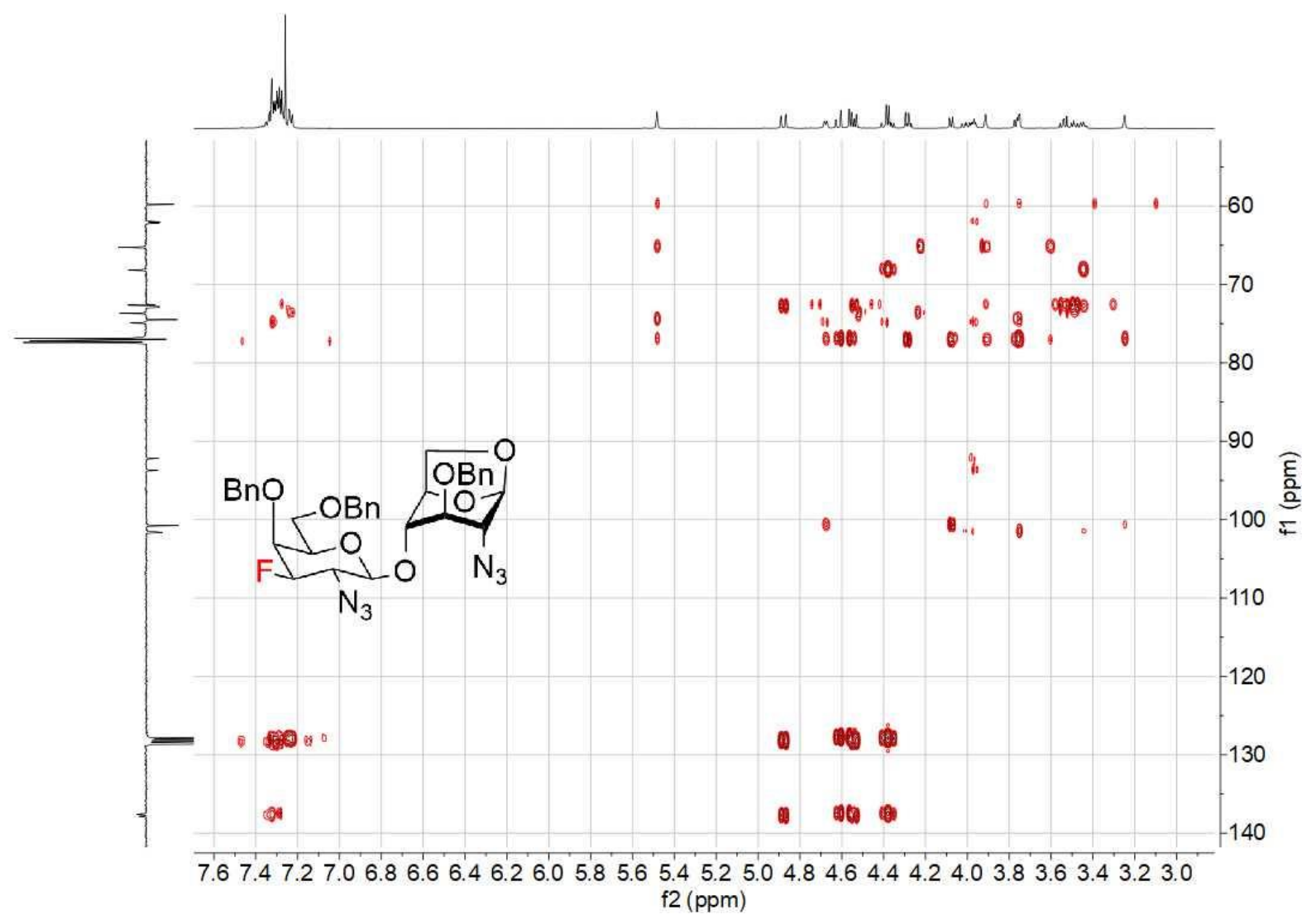

${ }^{19} \mathrm{~F} \mathrm{NMR} 376 \mathrm{MHz}, \mathrm{CDCl}_{3} 3 \mathrm{H}-\boldsymbol{\beta}$

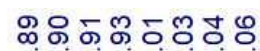

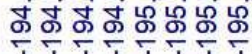
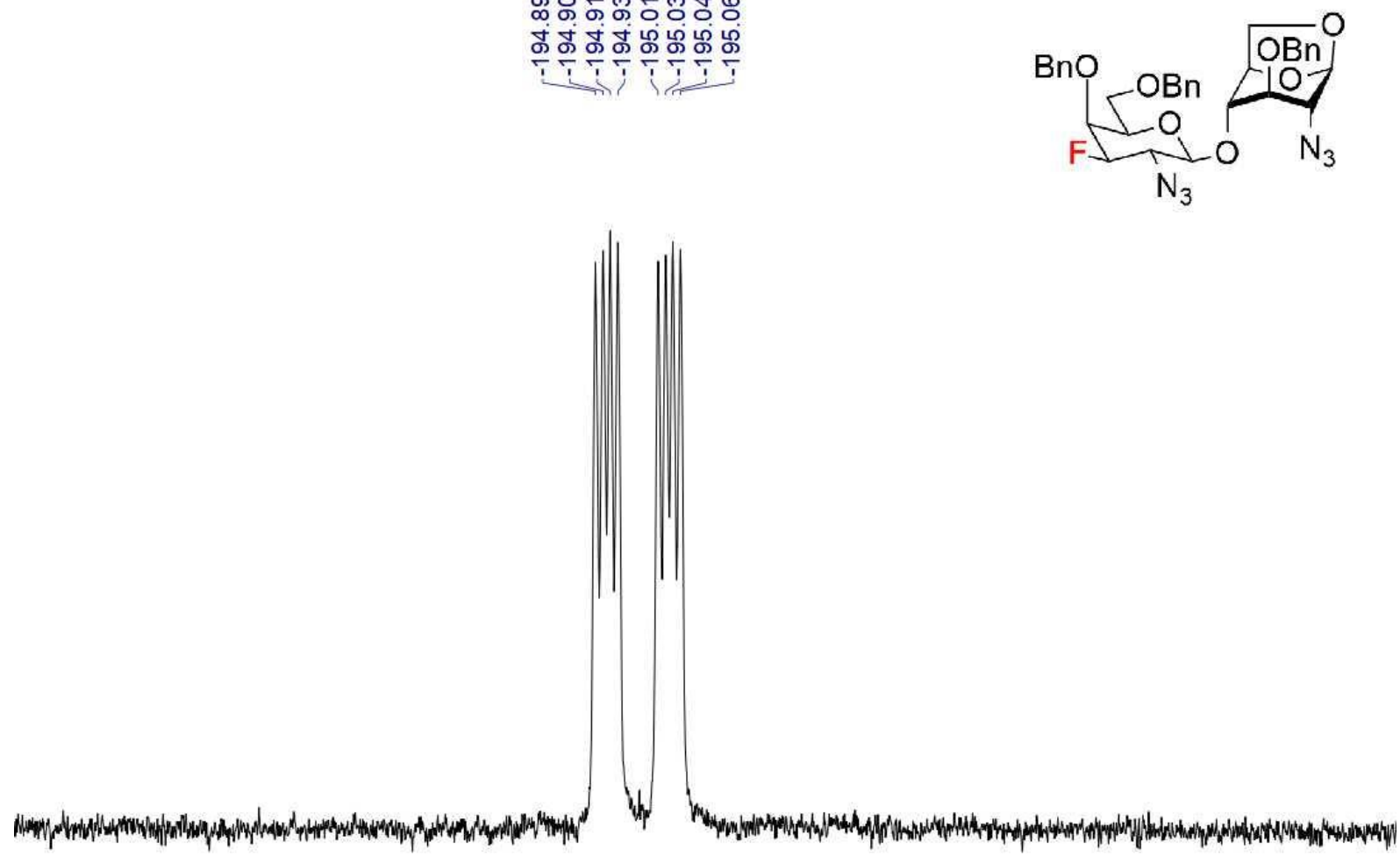

\begin{tabular}{llllllllllllll}
\hline-193.8 & -194.0 & -194.2 & -194.4 & -194.6 & -194.8 & -195.0 & -195.2 & -195.4 & -195.6 & -195.8 & -196.0 & -196.2 & -196.4
\end{tabular} f1 (ppm) 


\section{NMR S2}

${ }^{1} \mathrm{HNMR} 500 \mathrm{MHz}, \mathrm{CDCl}_{3}$ S2

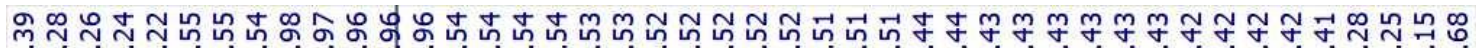

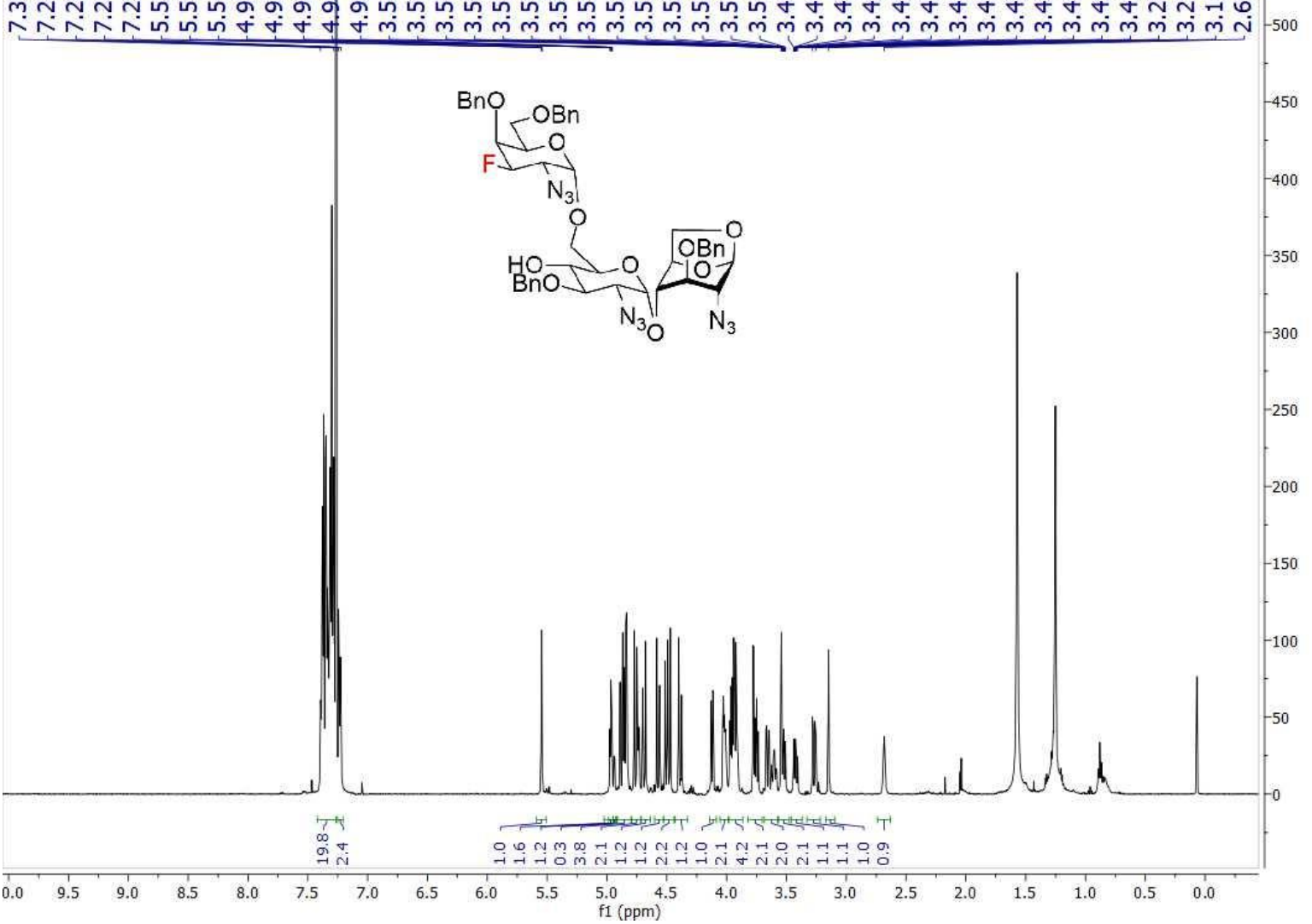

${ }^{13} \mathrm{C}\left\{{ }^{1} \mathrm{H}\right\}$ APT NMR $126 \mathrm{MHz}, \mathrm{CDCl}_{3} \mathrm{S2}$

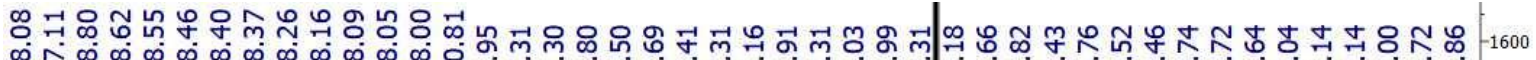
అึ.

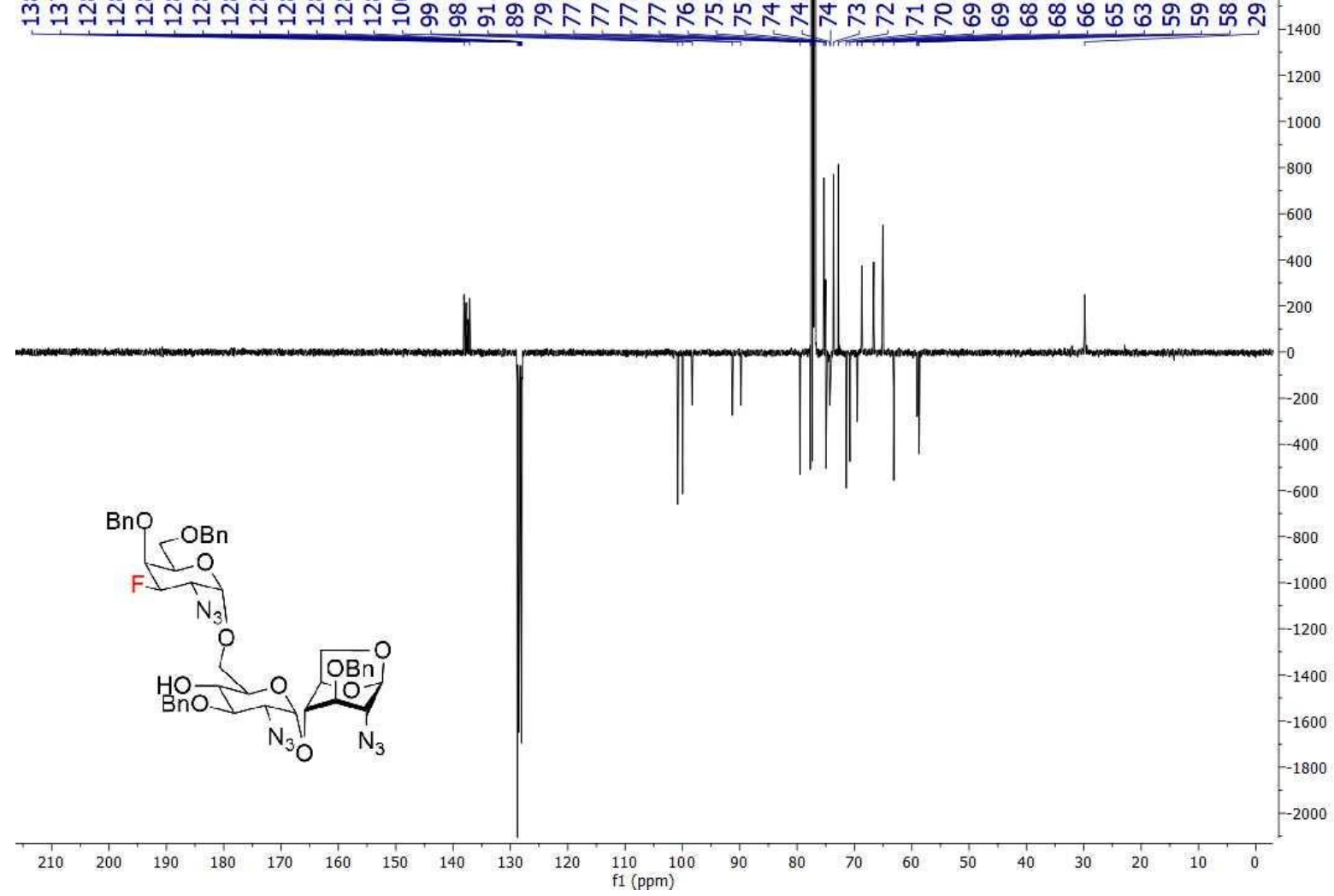


${ }^{1} \mathrm{H}-{ }^{1} \mathrm{H}$ COSY NMR SQ

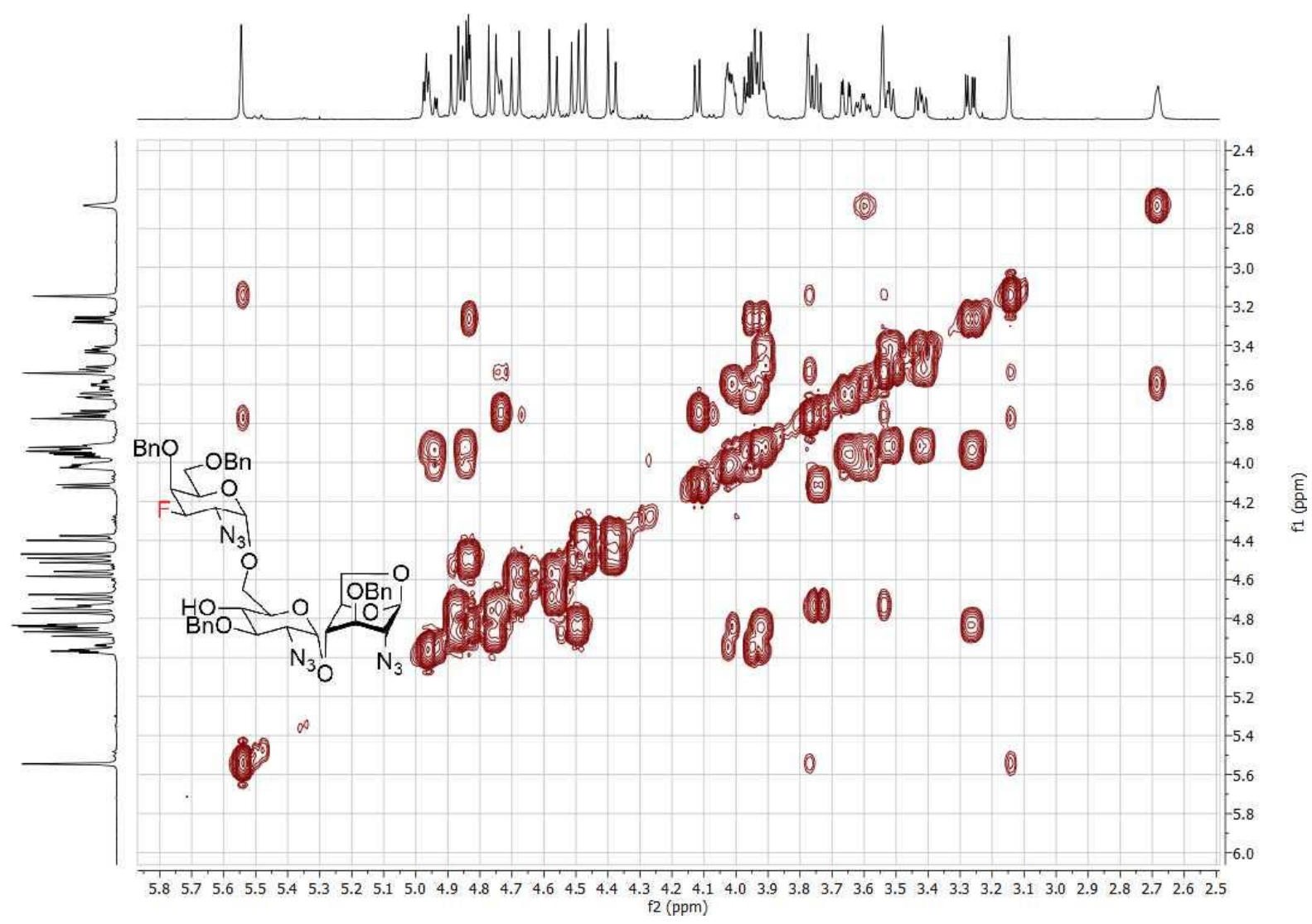

${ }^{1} \mathrm{H}-{ }^{13} \mathrm{C}$ HSQC NMR SQ

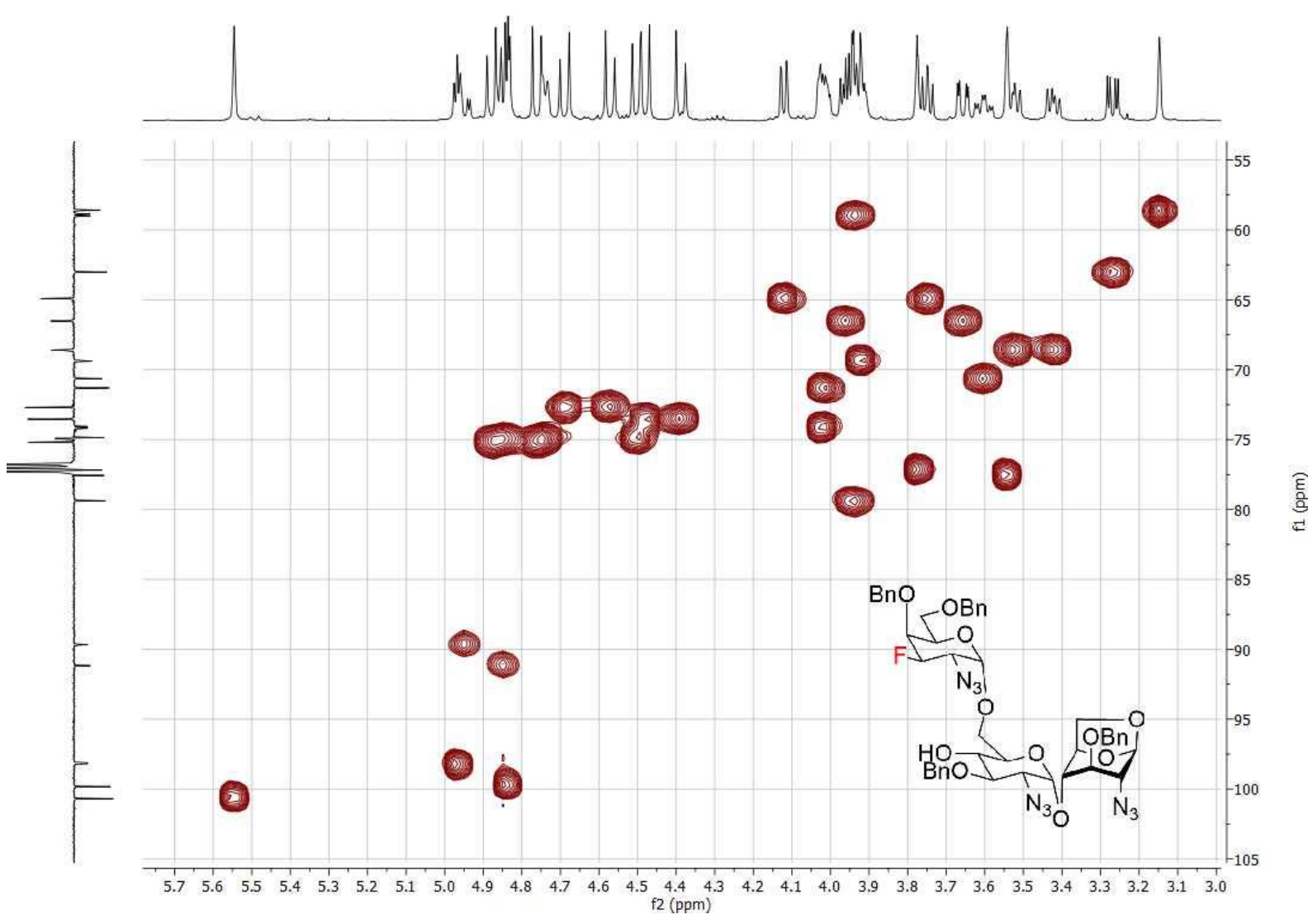

S217 
${ }^{1} \mathrm{H}^{13} \mathrm{C}$ HMBC NMR S2

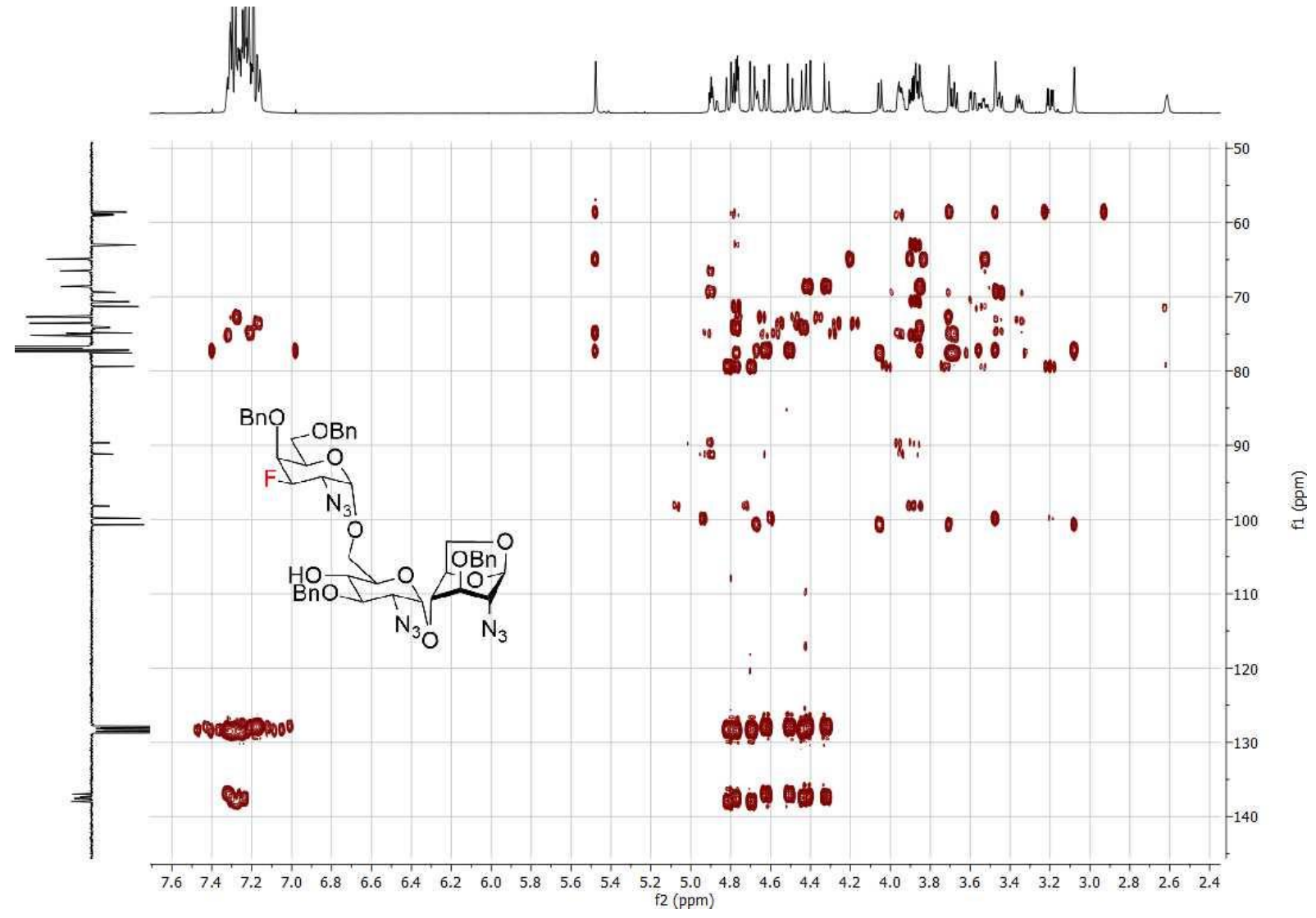

${ }^{19} \mathrm{~F} \mathrm{NMR} 376 \mathrm{MHz}, \mathrm{CDCl}_{3} \mathbf{S 2}$

ัํำำก๊ำ

ஓஃஃஃஃஃஃ

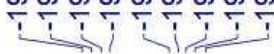
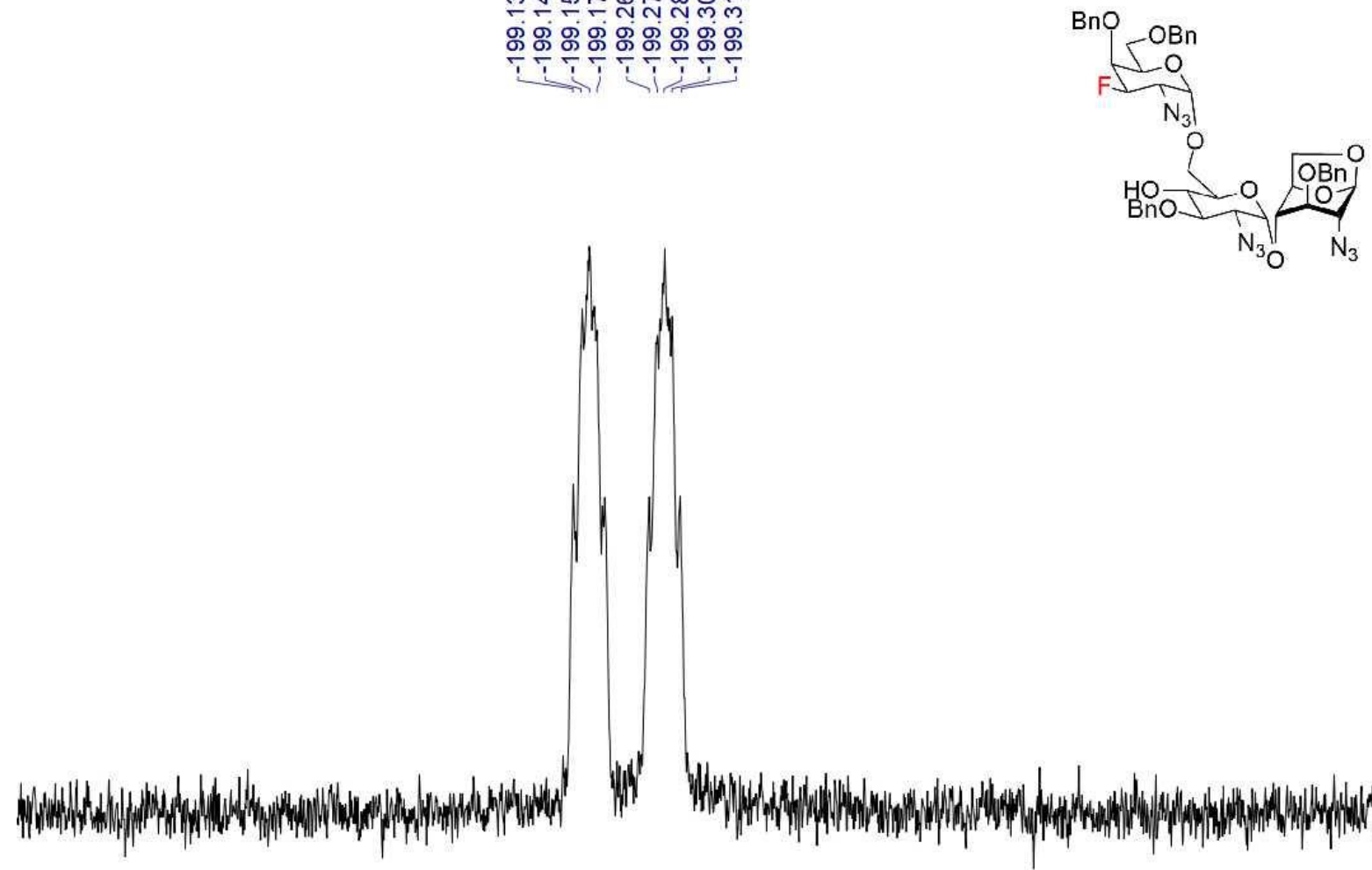

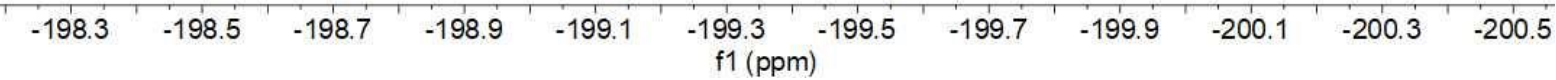




\section{NMR 3I- $\alpha$}

${ }^{1} \mathrm{H}$ NMR $400 \mathrm{MHz}, \mathrm{CDCl}_{3} 3 \mathrm{I}-\alpha$

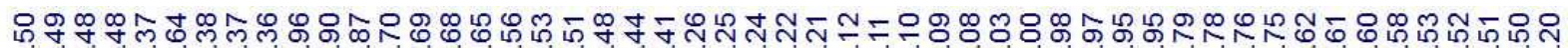

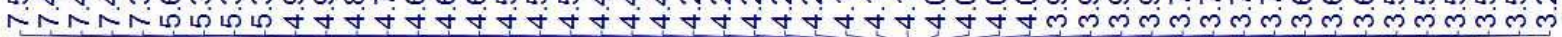

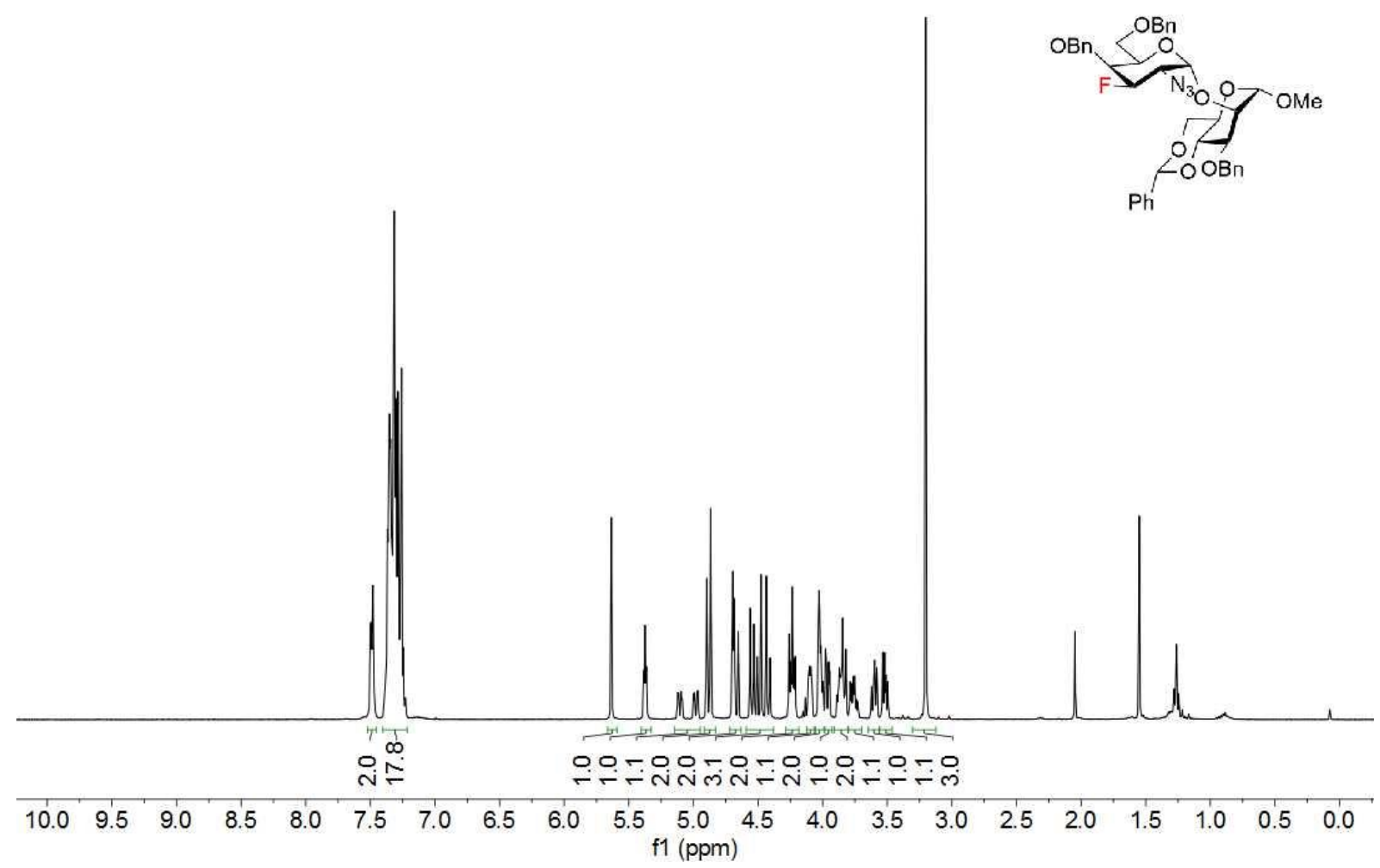

${ }^{13} \mathrm{C}\left\{{ }^{1} \mathrm{H}\right\} \mathrm{NMR} 100 \mathrm{MHz}, \mathrm{CDCl}_{3} 3 \mathrm{II} \alpha$

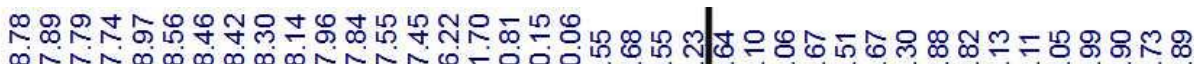

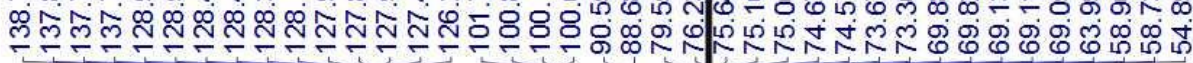

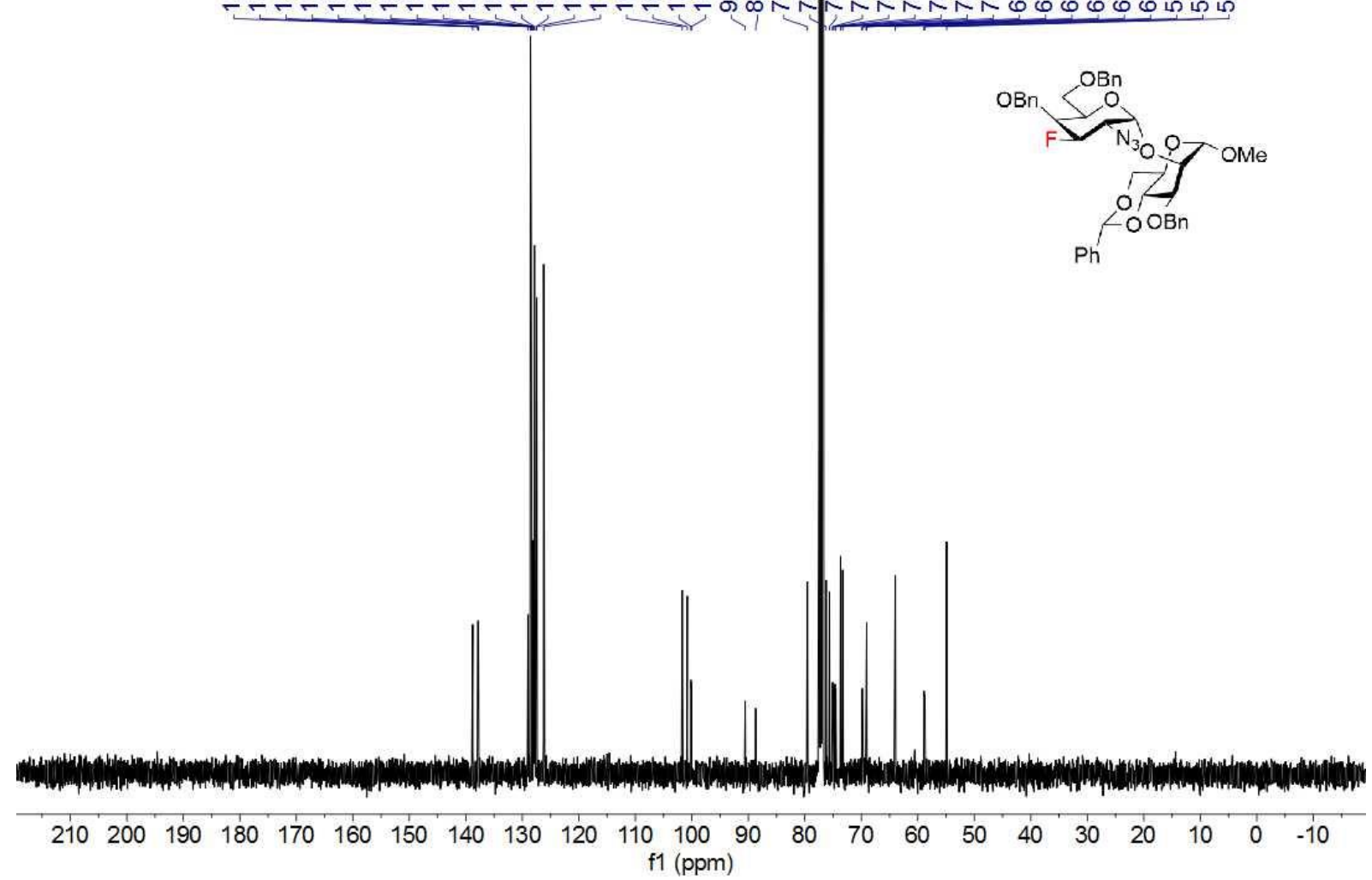


${ }^{1} \mathrm{H}-{ }^{1} \mathrm{H}$ COSY NMR $31-\alpha$

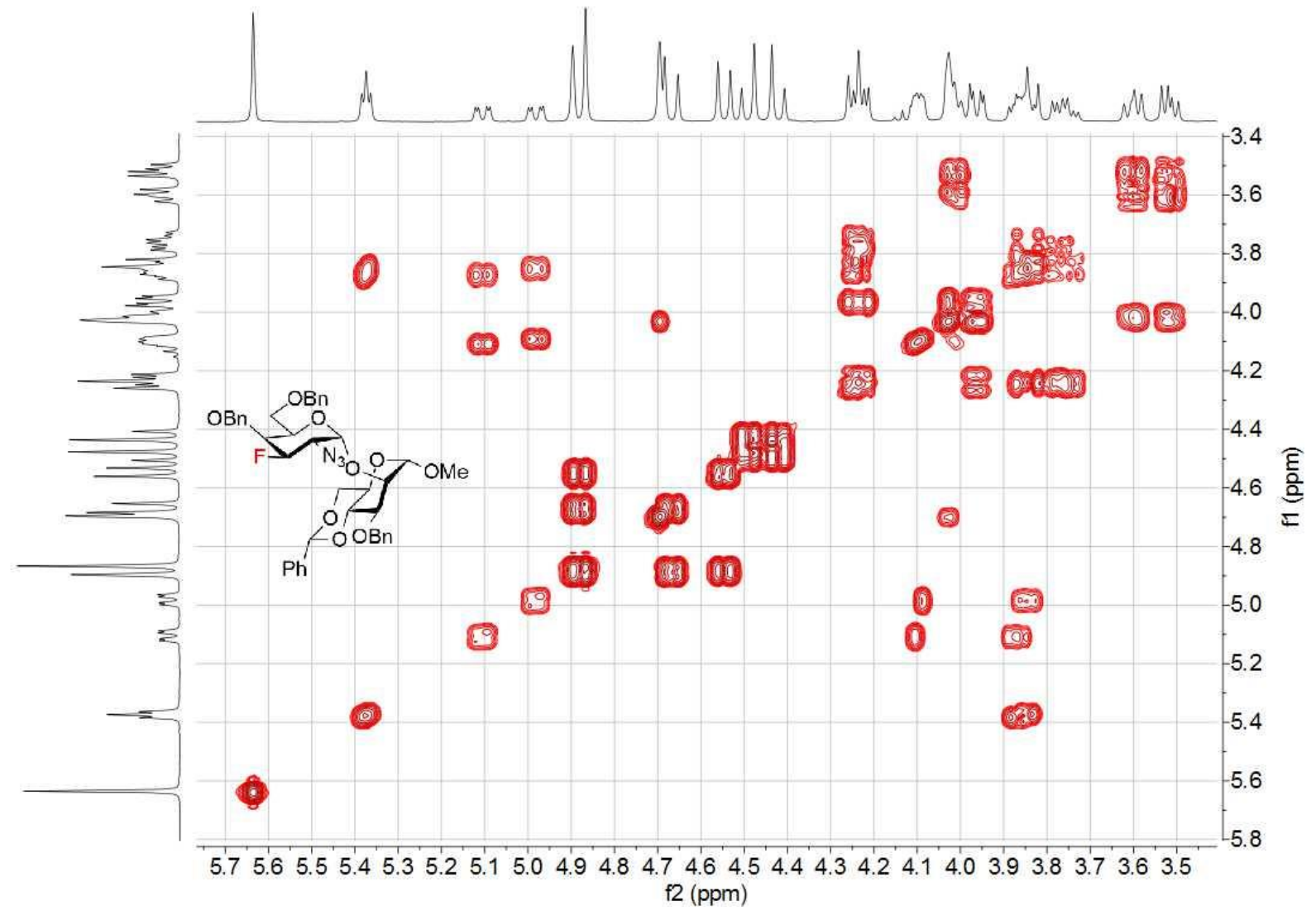

${ }^{1} \mathrm{H}-{ }^{13} \mathrm{C}$ HSQC NMR $31-\alpha$

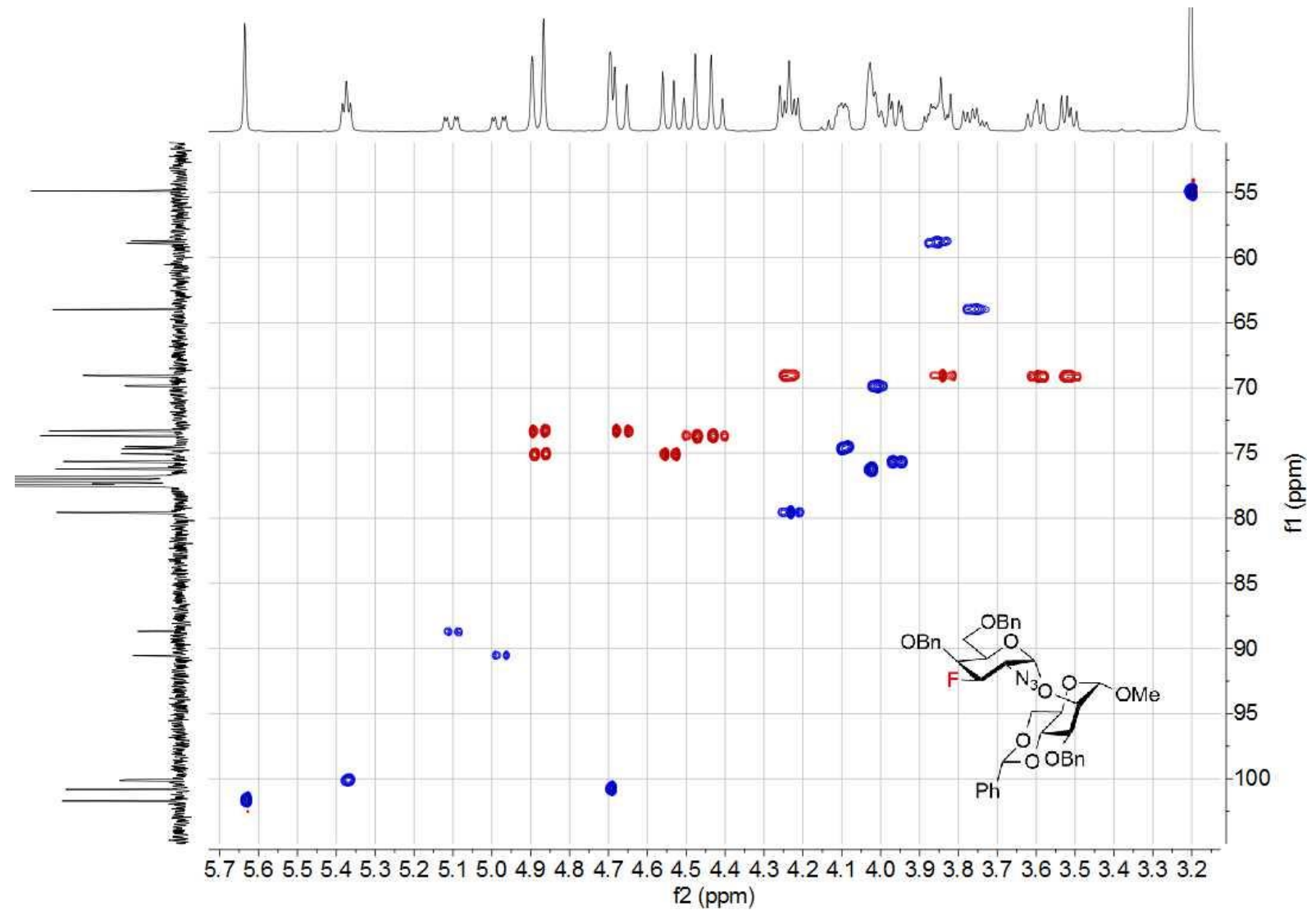


${ }^{1} \mathrm{H}-{ }^{13} \mathrm{C}$ HMBC NMR $3 \mathbf{3 l}-\alpha$

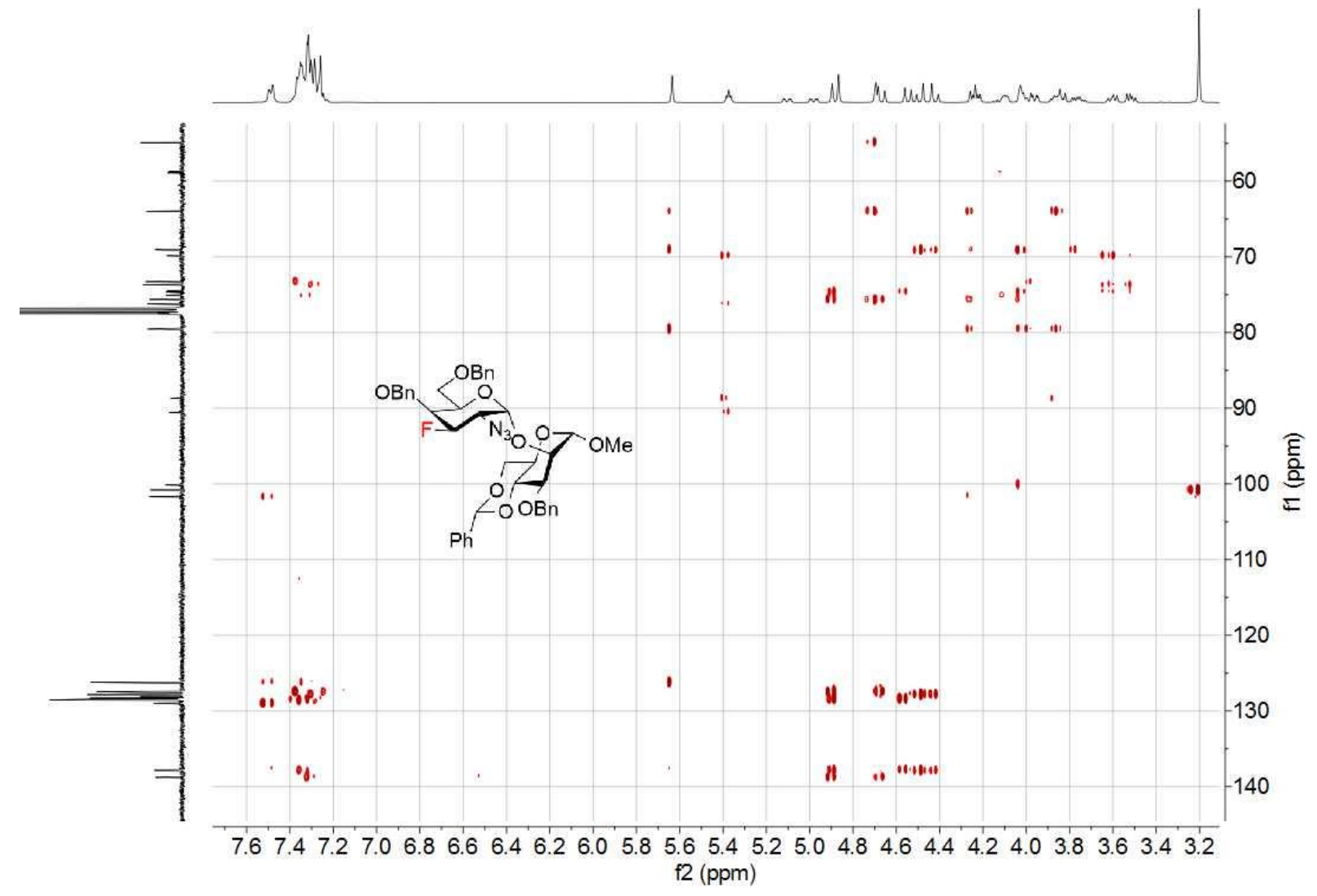

${ }^{19} \mathrm{~F} \mathrm{NMR} 376 \mathrm{MHz}, \mathrm{CDCl}_{3}$ 3I- $\alpha$

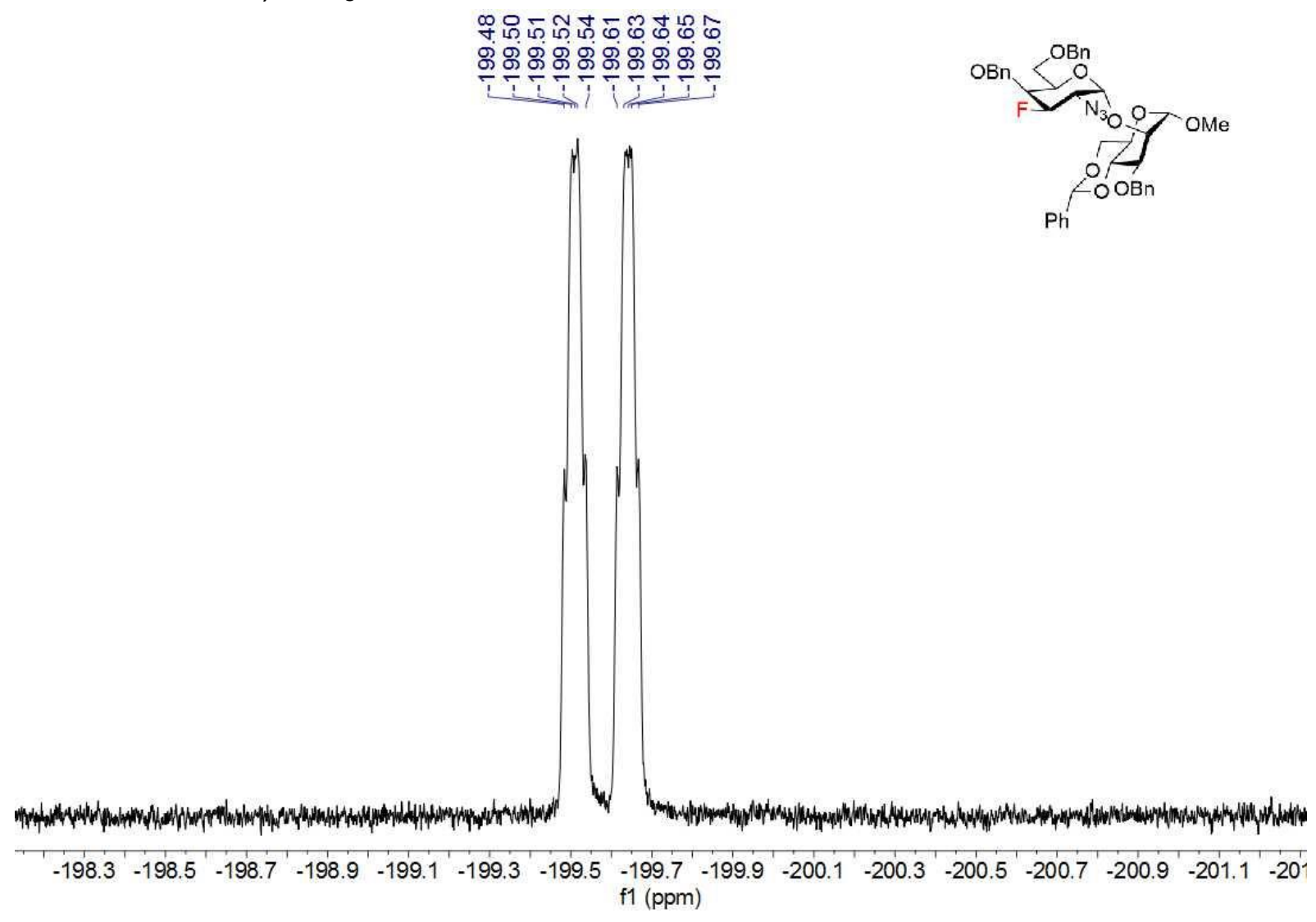


NMR 3I- $\beta$

${ }^{1} \mathrm{H}$ NMR $400 \mathrm{MHz}, \mathrm{CDCl}_{3} 3 \mathrm{I}-\beta$

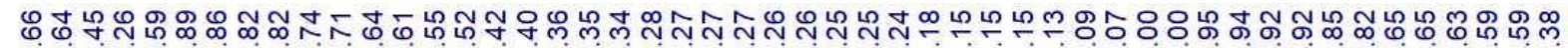

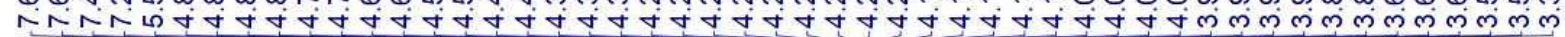

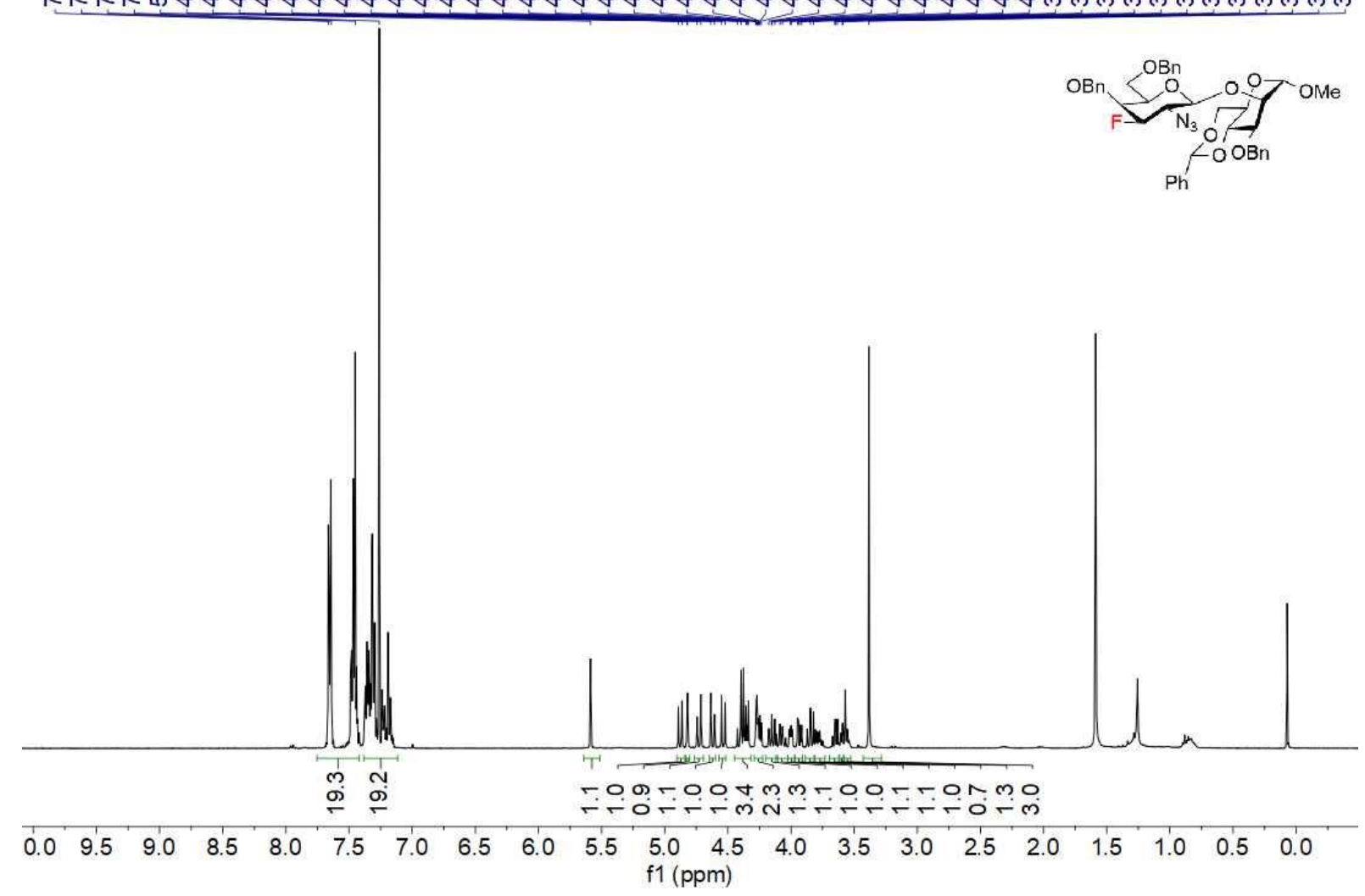

${ }^{13} \mathrm{C}\left\{{ }^{1} \mathrm{H}\right\} \mathrm{NMR} 100 \mathrm{MHz}, \mathrm{CDCl}_{3} 3 \mathrm{I}-\boldsymbol{\beta}$

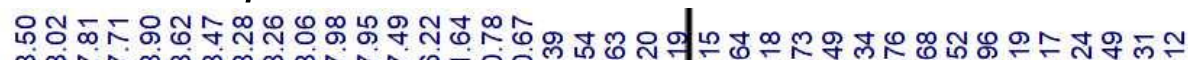

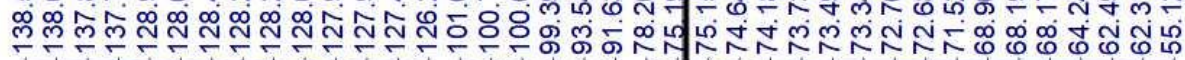

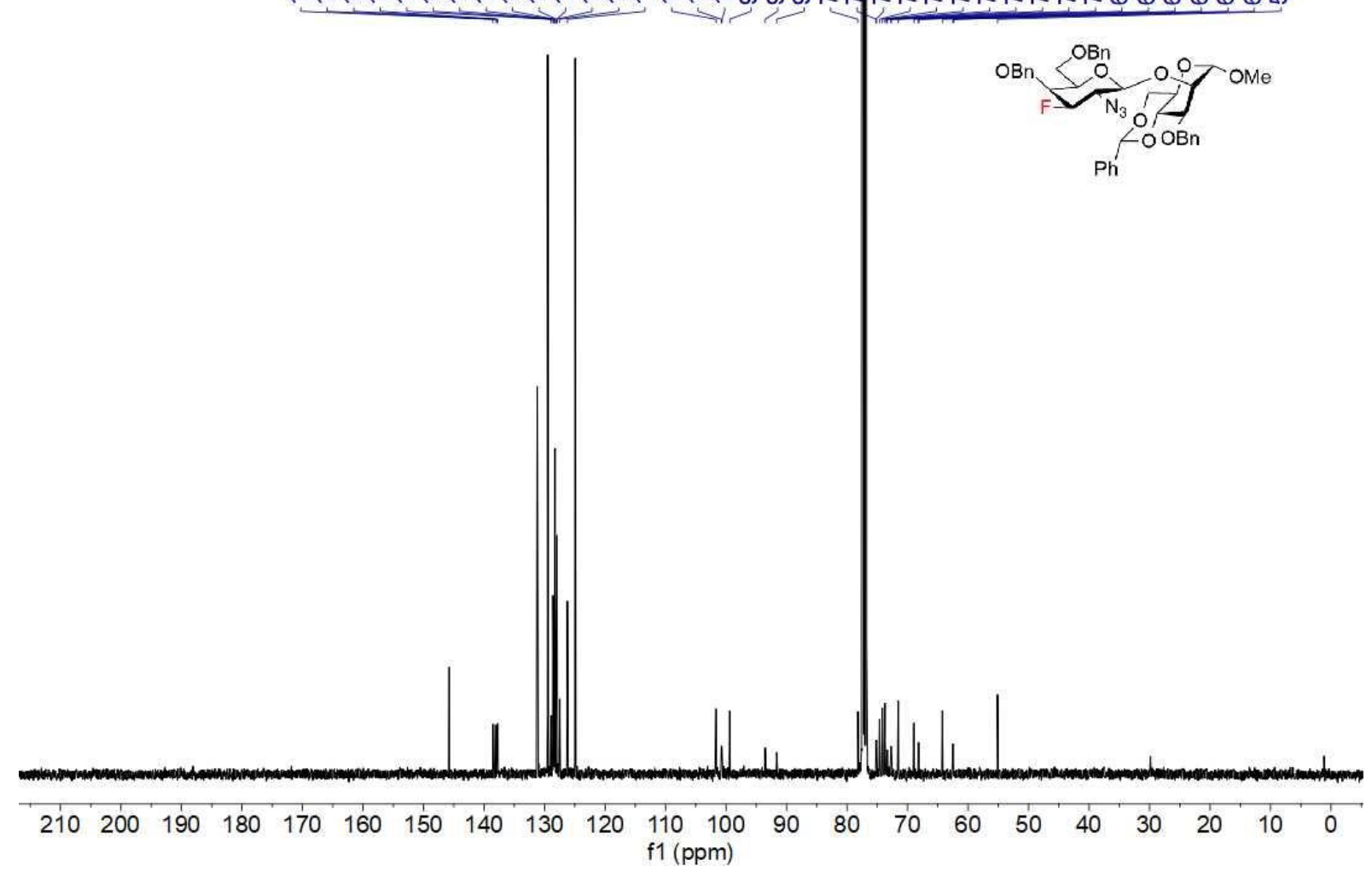


${ }^{1} \mathrm{H}-{ }^{1} \mathrm{H}$ COSY NMR 3I- $\beta$

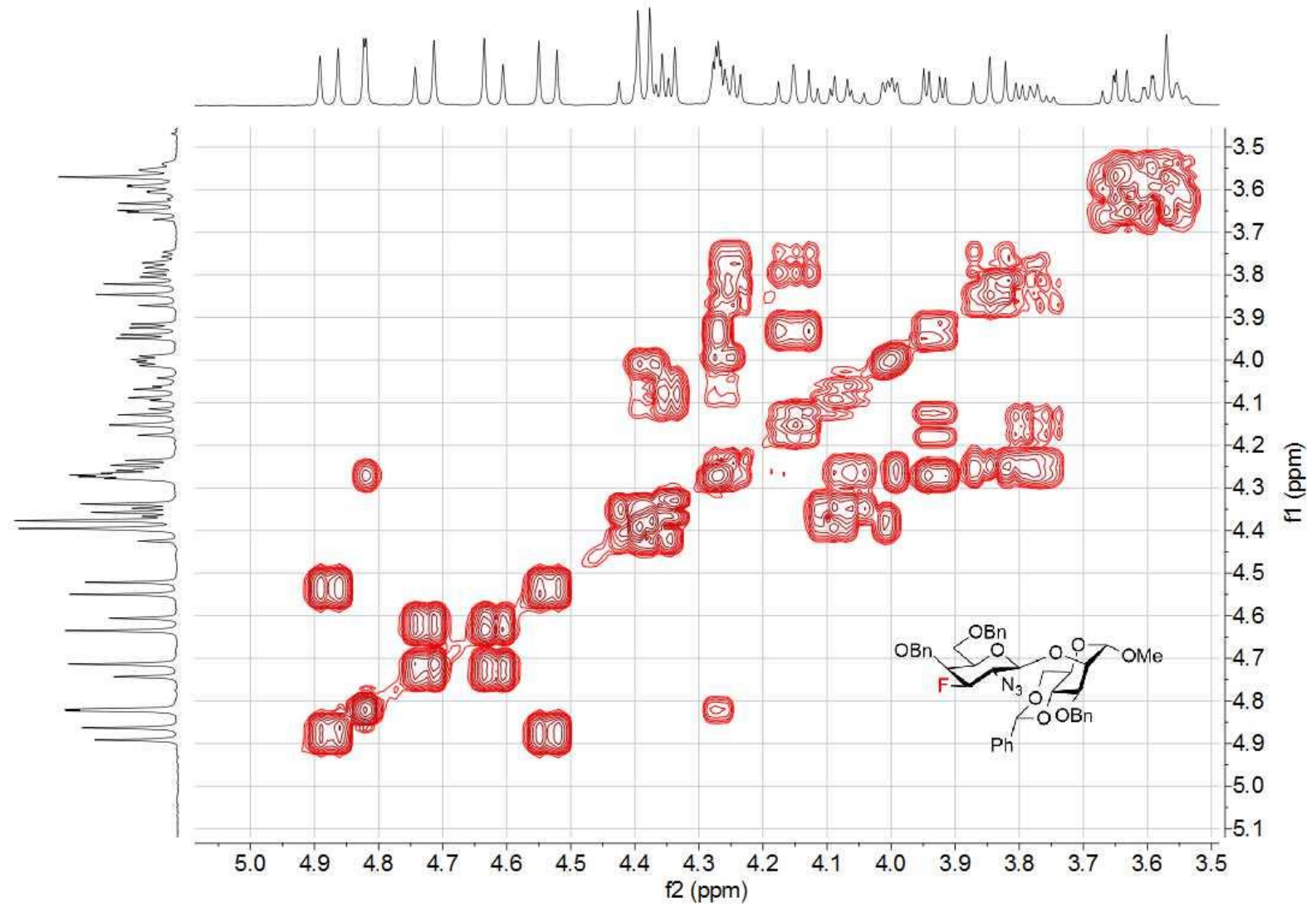

${ }^{1} \mathrm{H}-{ }^{13} \mathrm{C}$ HSQC NMR 3I- $\beta$

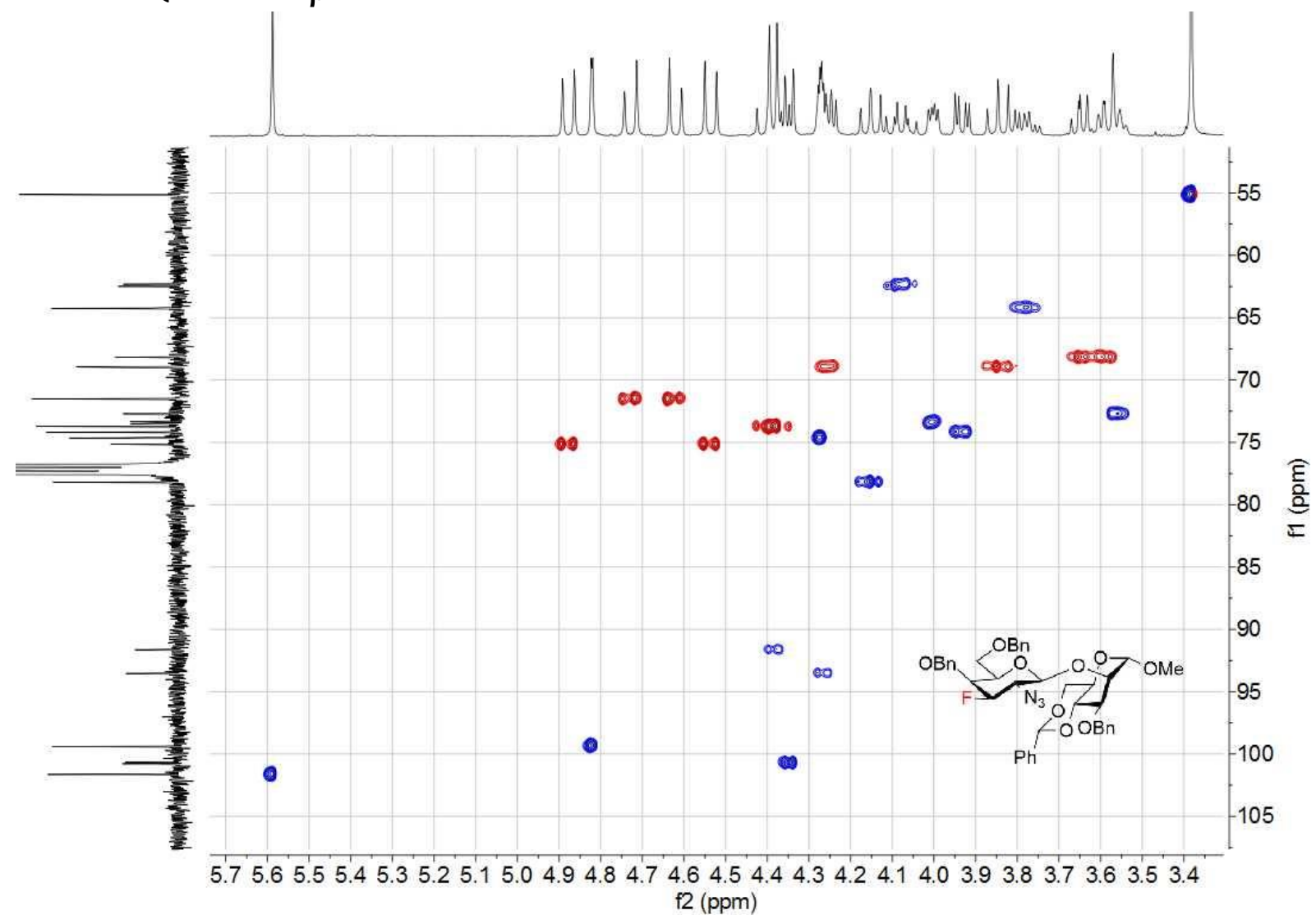




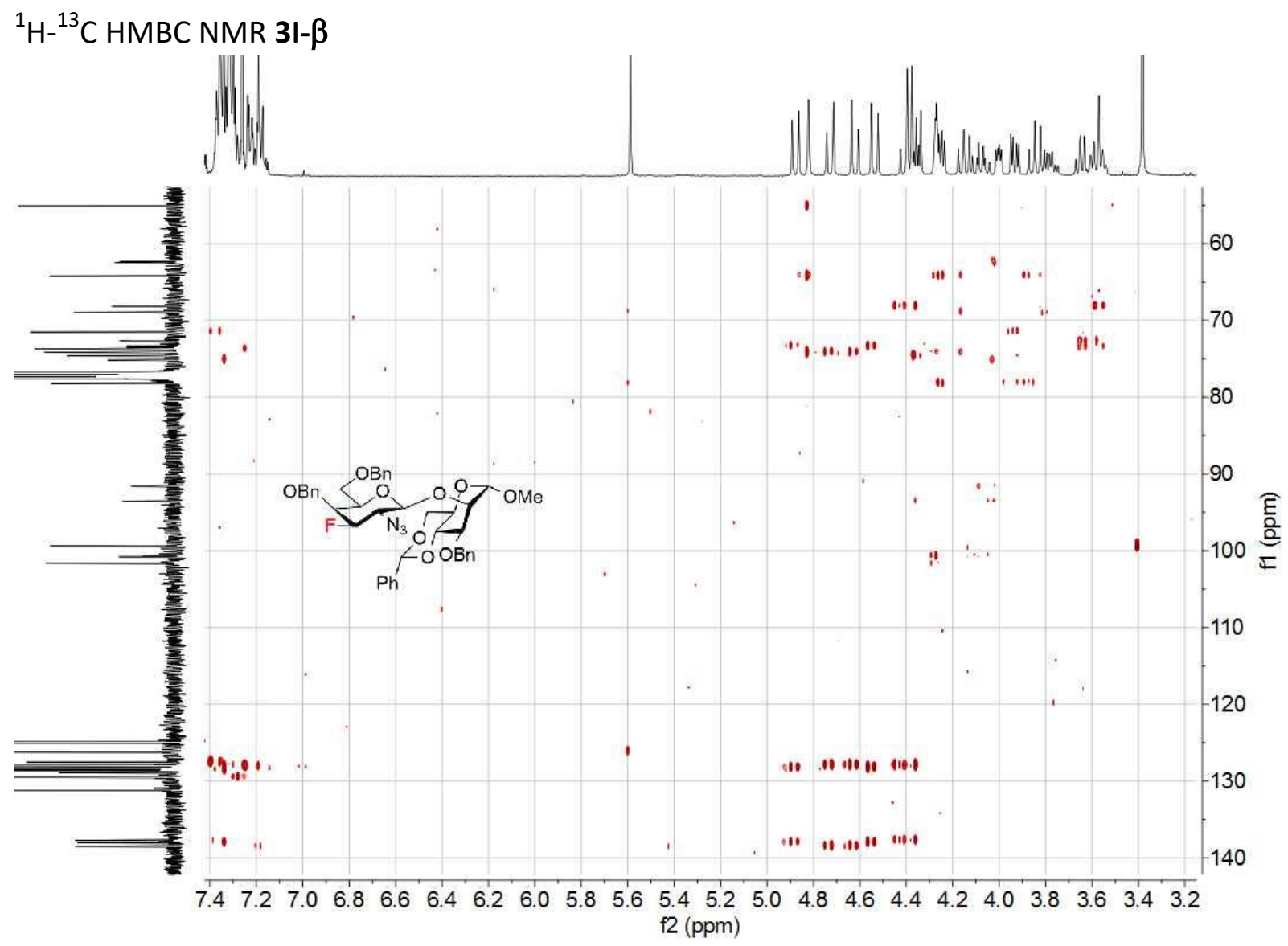

${ }^{19} \mathrm{~F}$ NMR $376 \mathrm{MHz}, \mathrm{CDCl}_{3}$ 3I- $\boldsymbol{\beta}$

으는ำกำำ

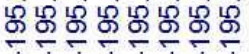
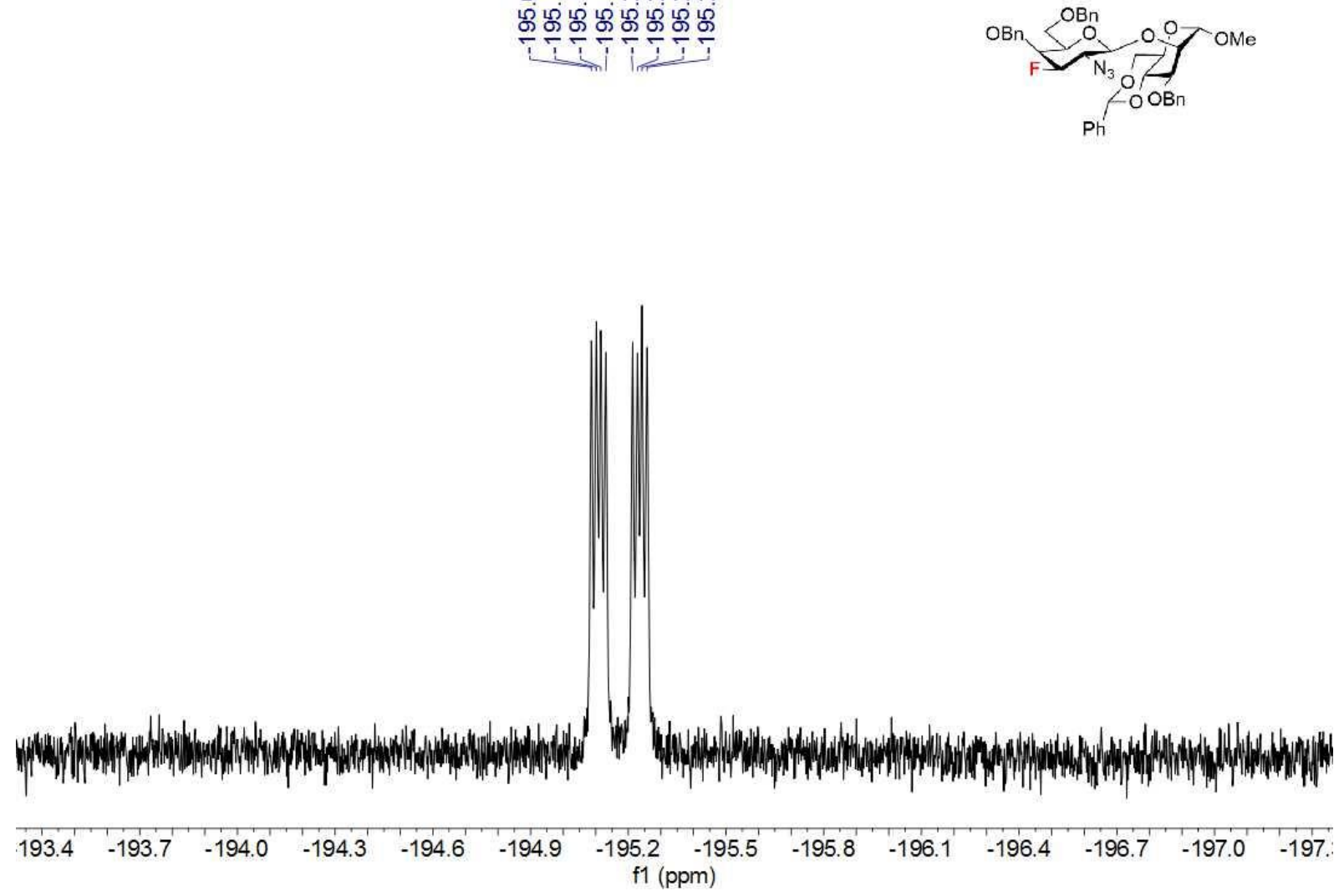
NMR 4A- $\alpha$

${ }^{1} \mathrm{HNMR} 400 \mathrm{MHz}, \mathrm{CDCl}_{3} 4 \mathrm{~A}-\alpha$

ๆ

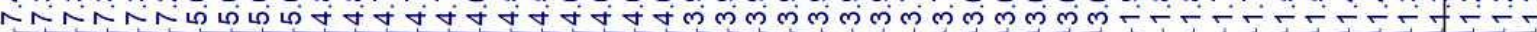

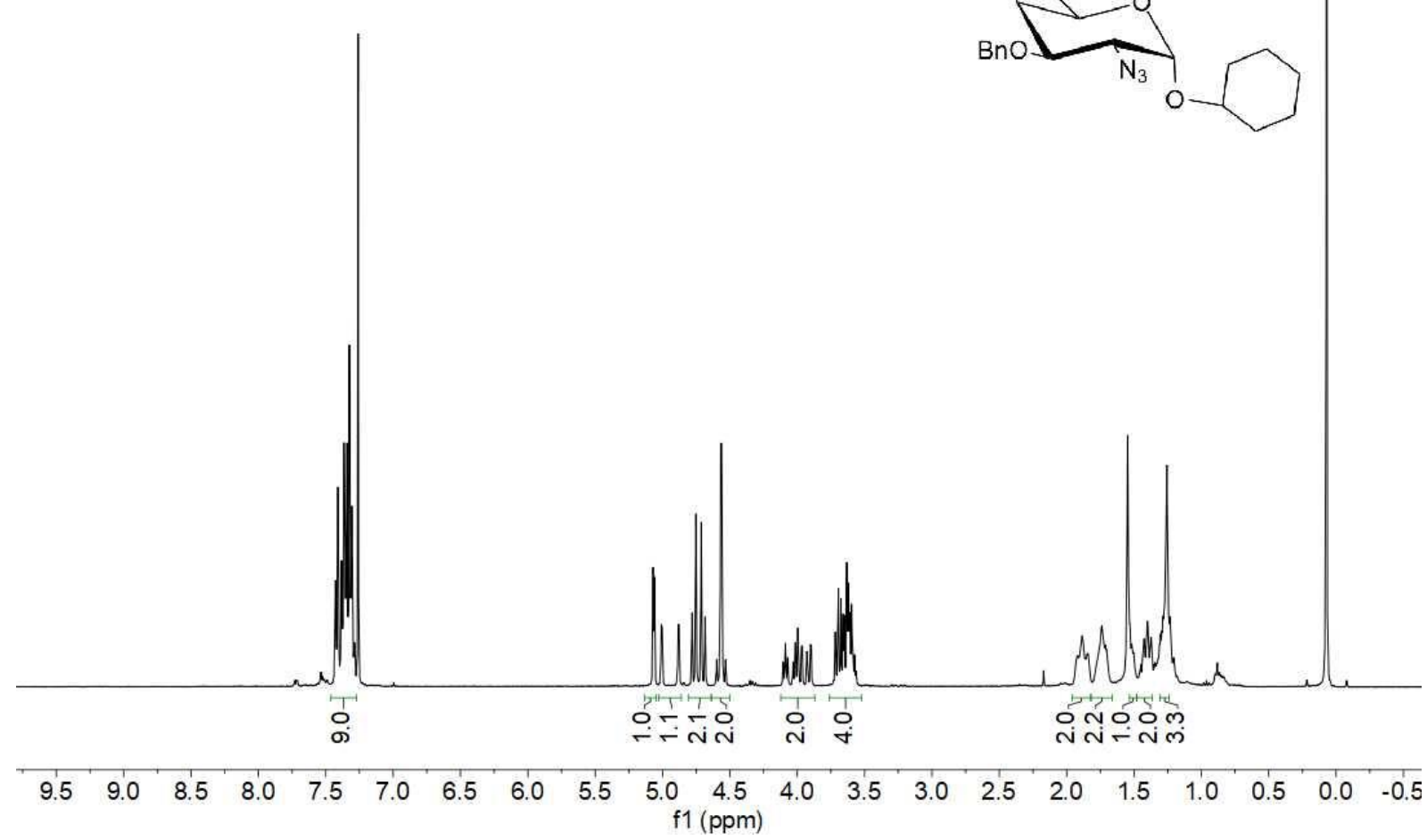

${ }^{13} \mathrm{C}\left\{{ }^{1} \mathrm{H}\right\} \mathrm{NMR} 100 \mathrm{MHz}, \mathrm{CDCl}_{3} 4 \mathrm{~A}-\alpha$

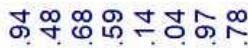

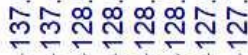

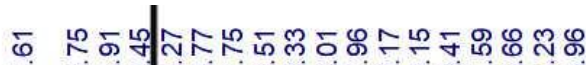

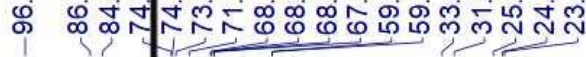

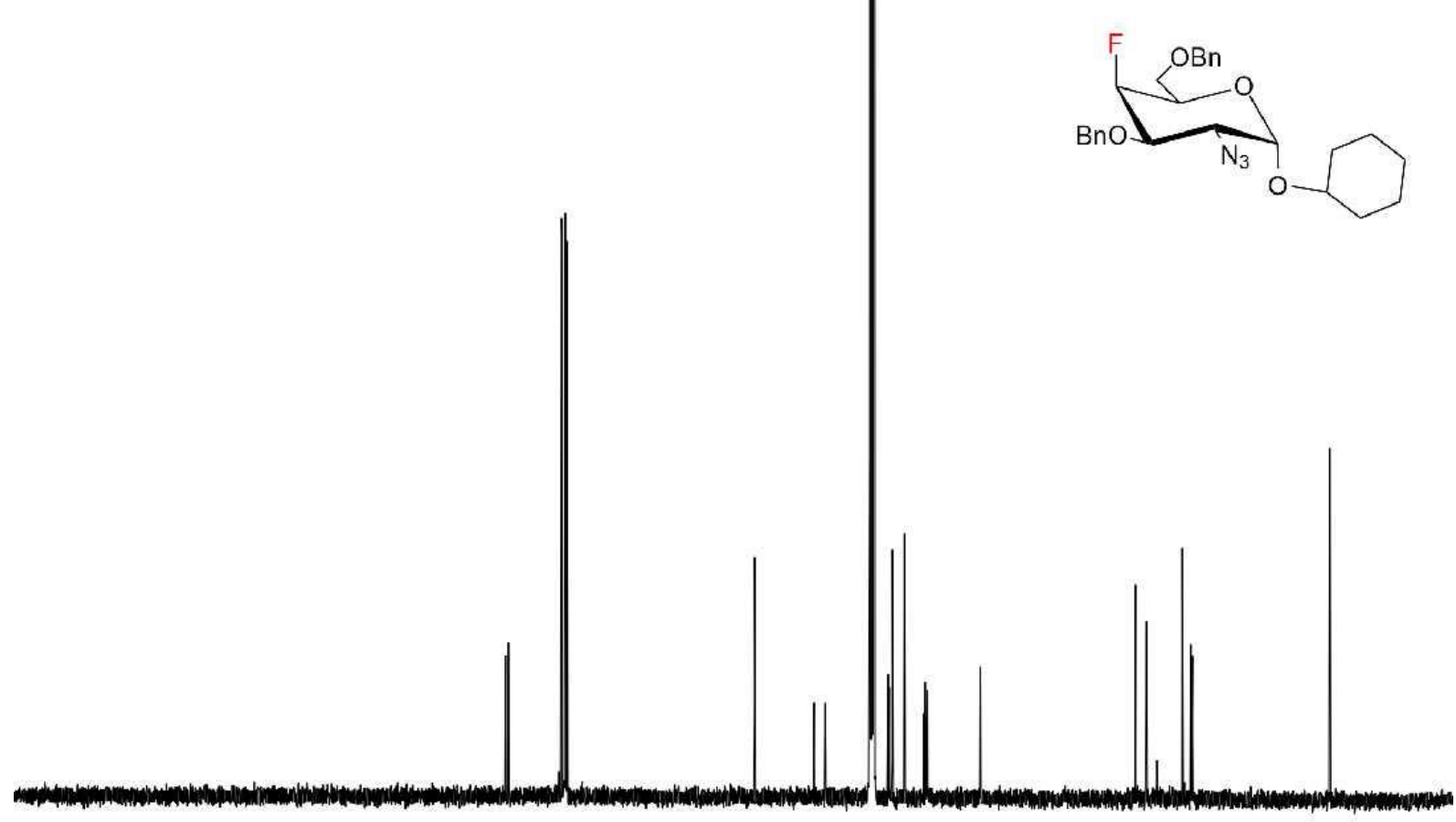

$\begin{array}{llllllllllllllllllllllll}210 & 200 & 190 & 180 & 170 & 160 & 150 & 140 & 130 & 120 & \begin{array}{c}110 \\ \mathrm{f} 1(\mathrm{ppm})\end{array} & 100 & 80 & 70 & 60 & 50 & 40 & 30 & 20 & 10 & 0 & -10\end{array}$ 
${ }^{1} \mathrm{H}-{ }^{1} \mathrm{H}$ CosY NMR 4A- $\alpha$

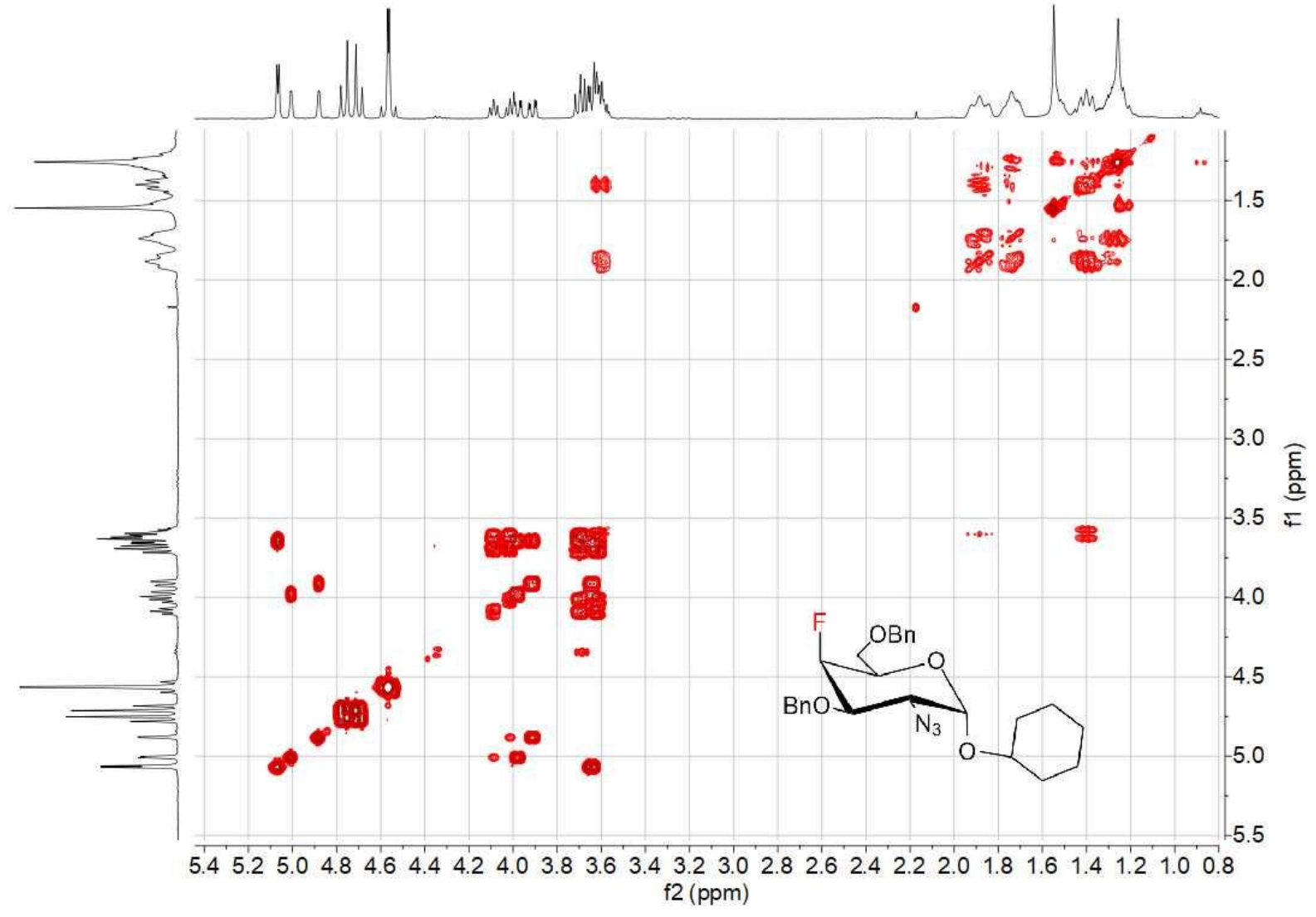

${ }^{1} \mathrm{H}^{-13} \mathrm{C}$ HSQC NMR 4A- $\alpha$

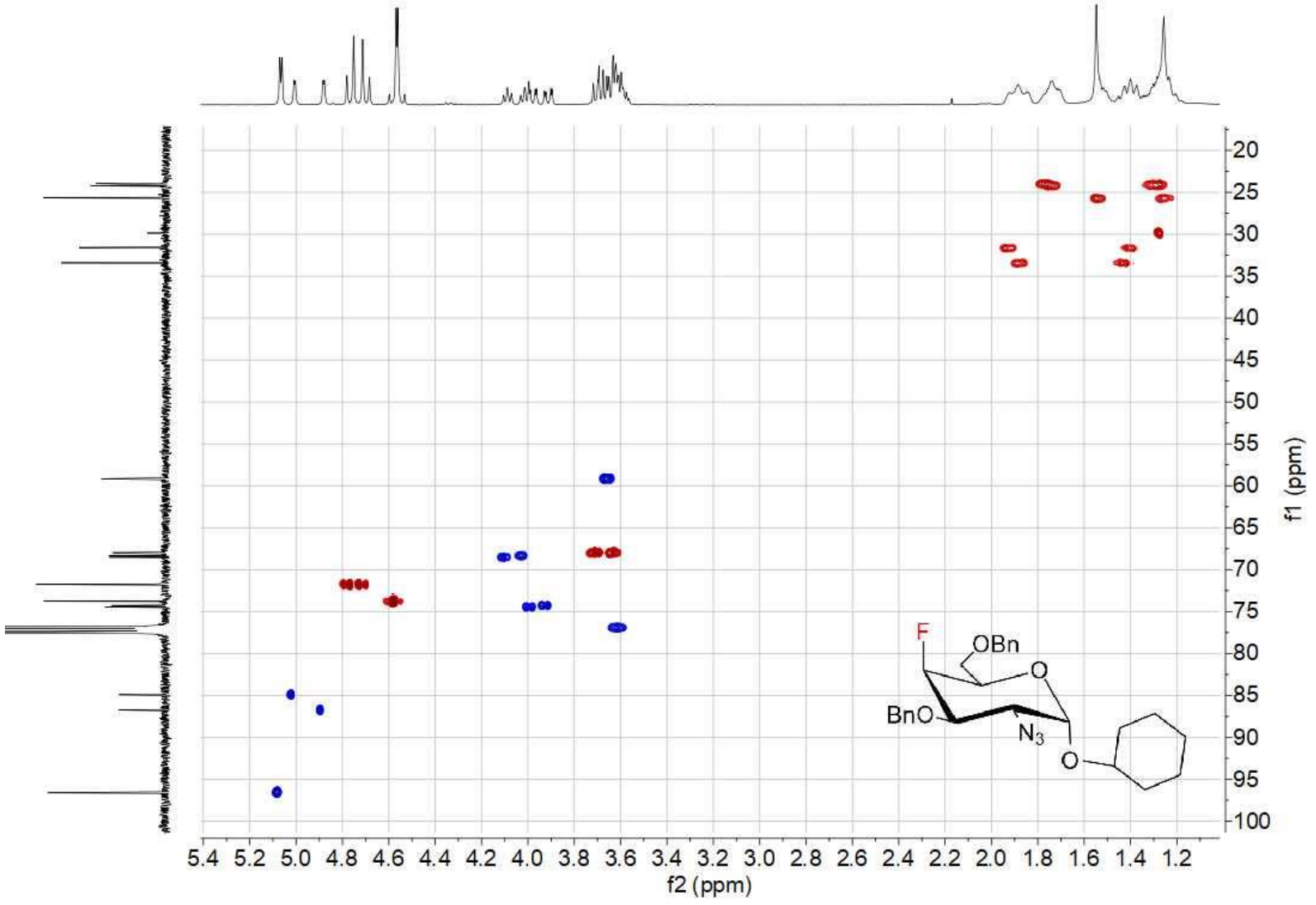


${ }^{1} \mathrm{H}-{ }^{13} \mathrm{C}$ HMBC NMR 4A- $\alpha$

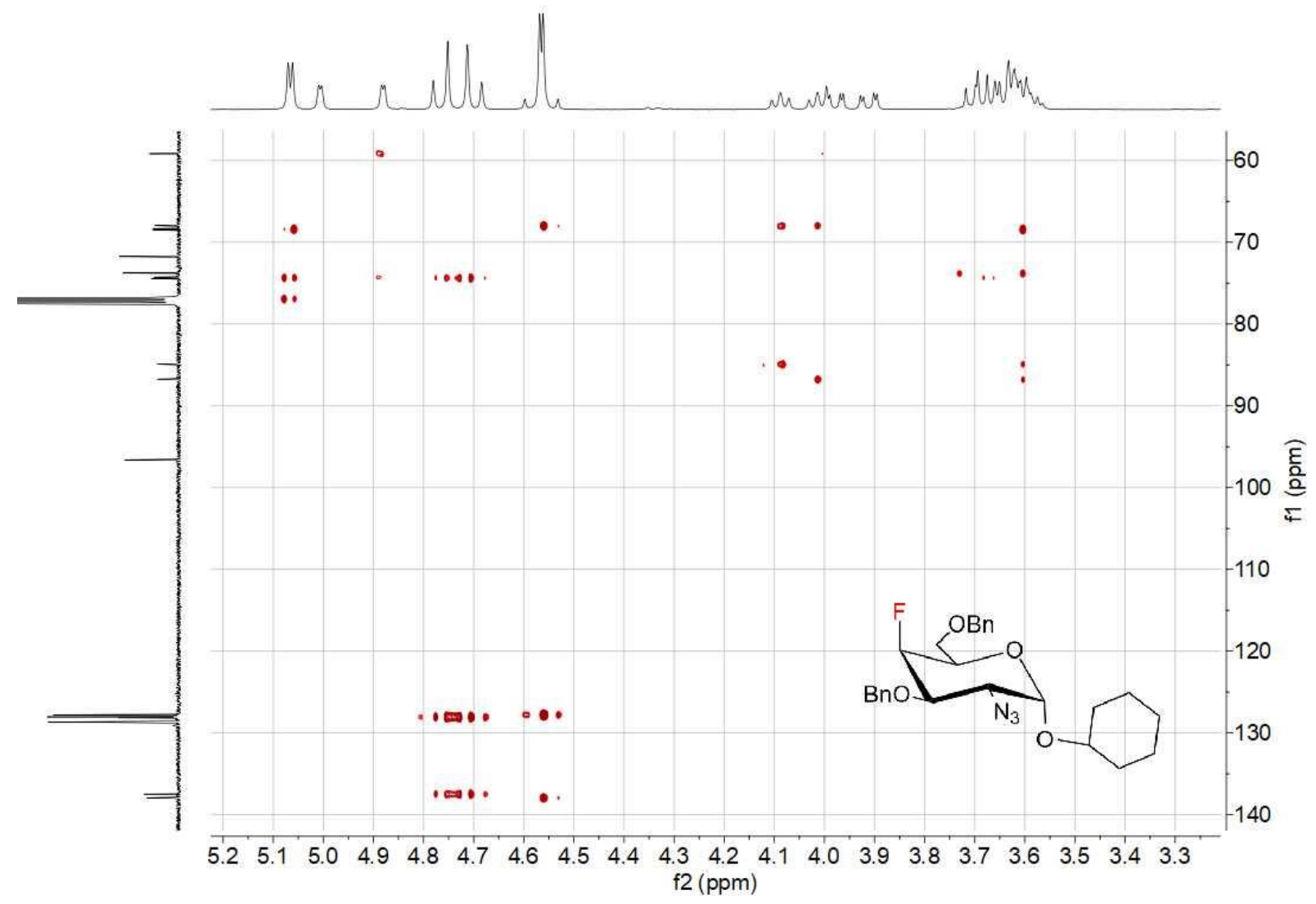

${ }^{19} \mathrm{~F} \mathrm{NMR} 376 \mathrm{MHz}, \mathrm{CDCl}_{3} 4 \mathrm{~A}-\alpha$

mำำกำ

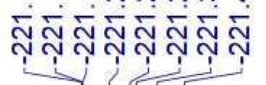
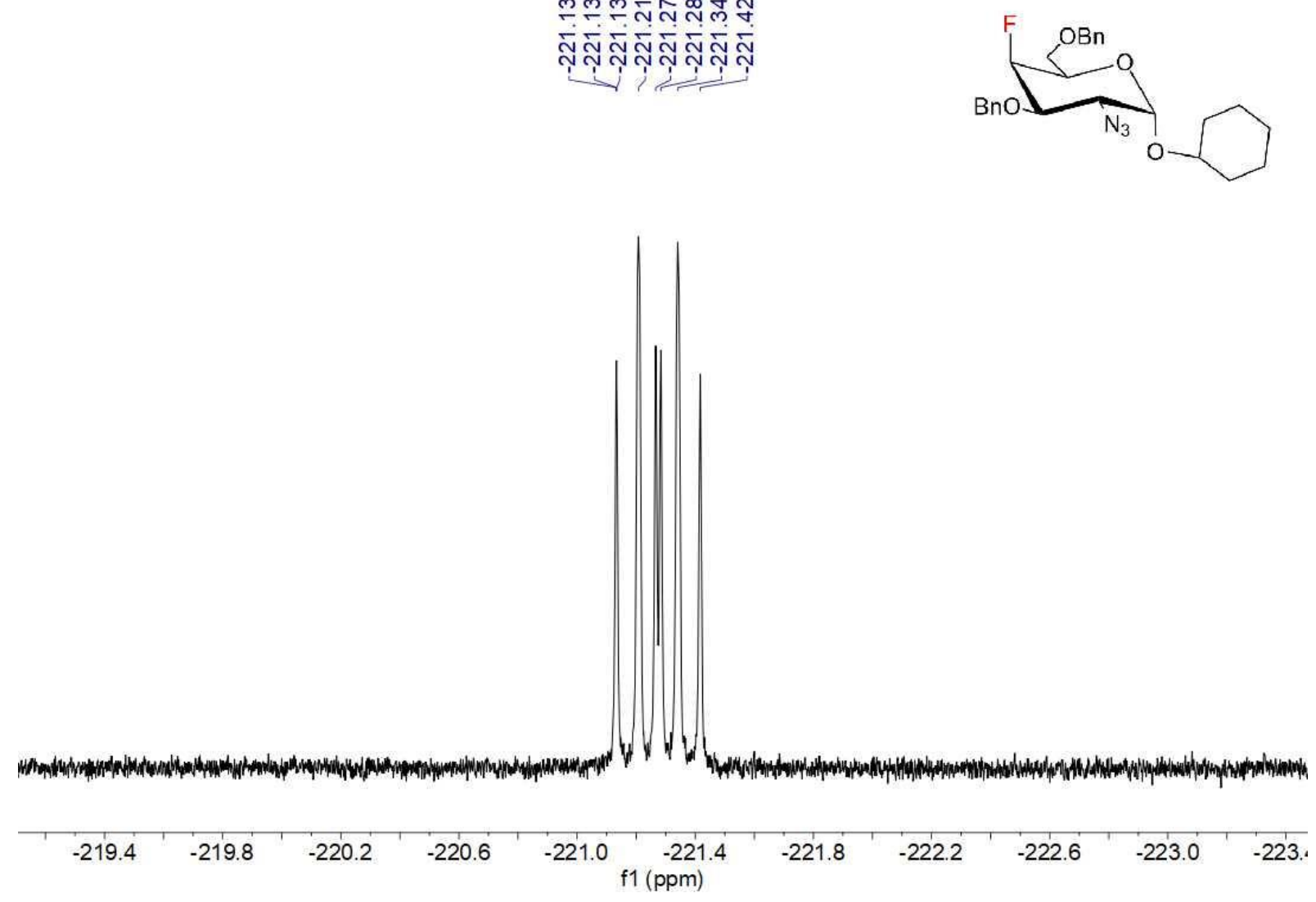
NMR 4A- $\beta$

${ }^{1} \mathrm{H} N M R 400 \mathrm{MHz}, \mathrm{CDCl}_{3} 4 \mathrm{~A}-\beta$

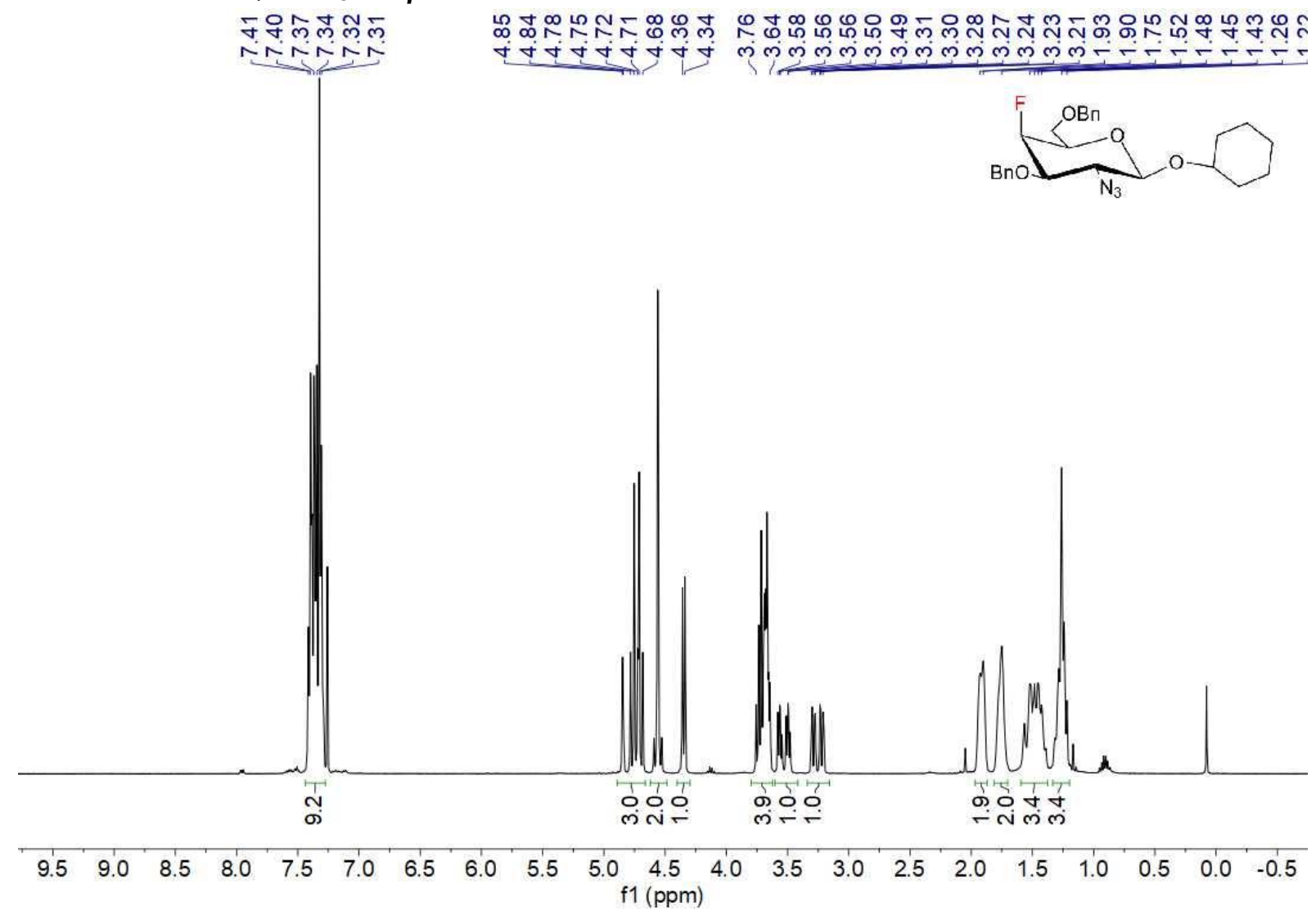

${ }^{13} \mathrm{C}\left\{{ }^{1} \mathrm{H}\right\}$ NMR $100 \mathrm{MHz}, \mathrm{CDCl}_{3} 4 \mathrm{~A}-\boldsymbol{\beta}$

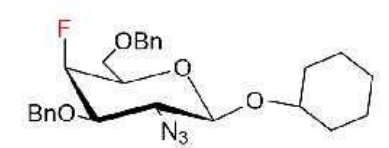

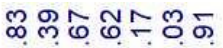

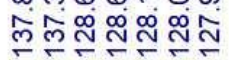

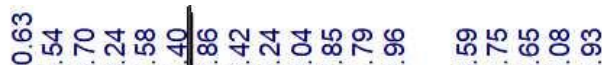

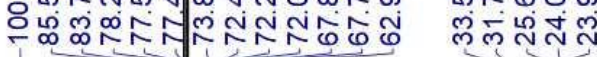

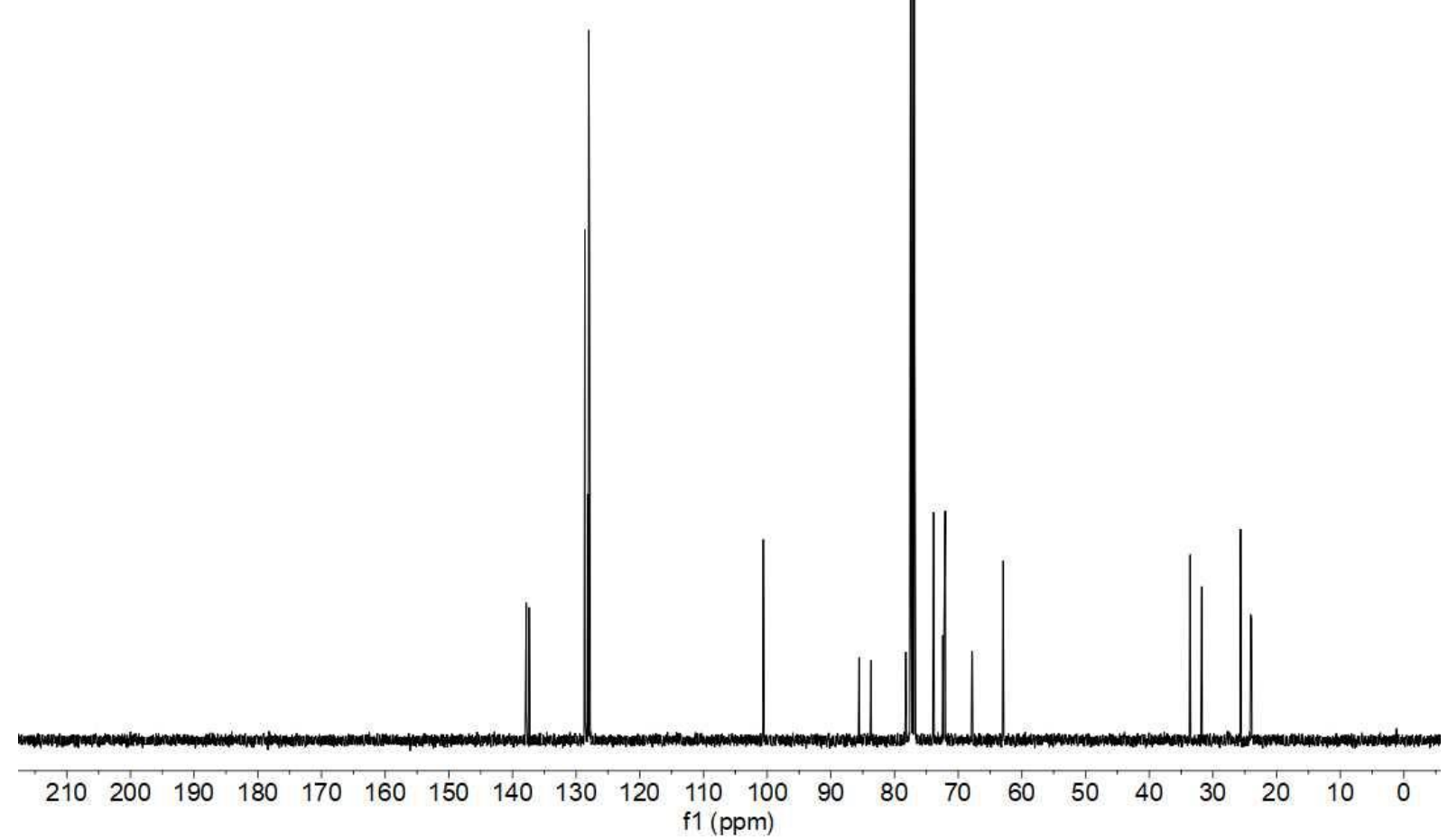


${ }^{1} \mathrm{H}-{ }^{1} \mathrm{H}$ COSY NMR $4 \mathrm{~A}-\beta$

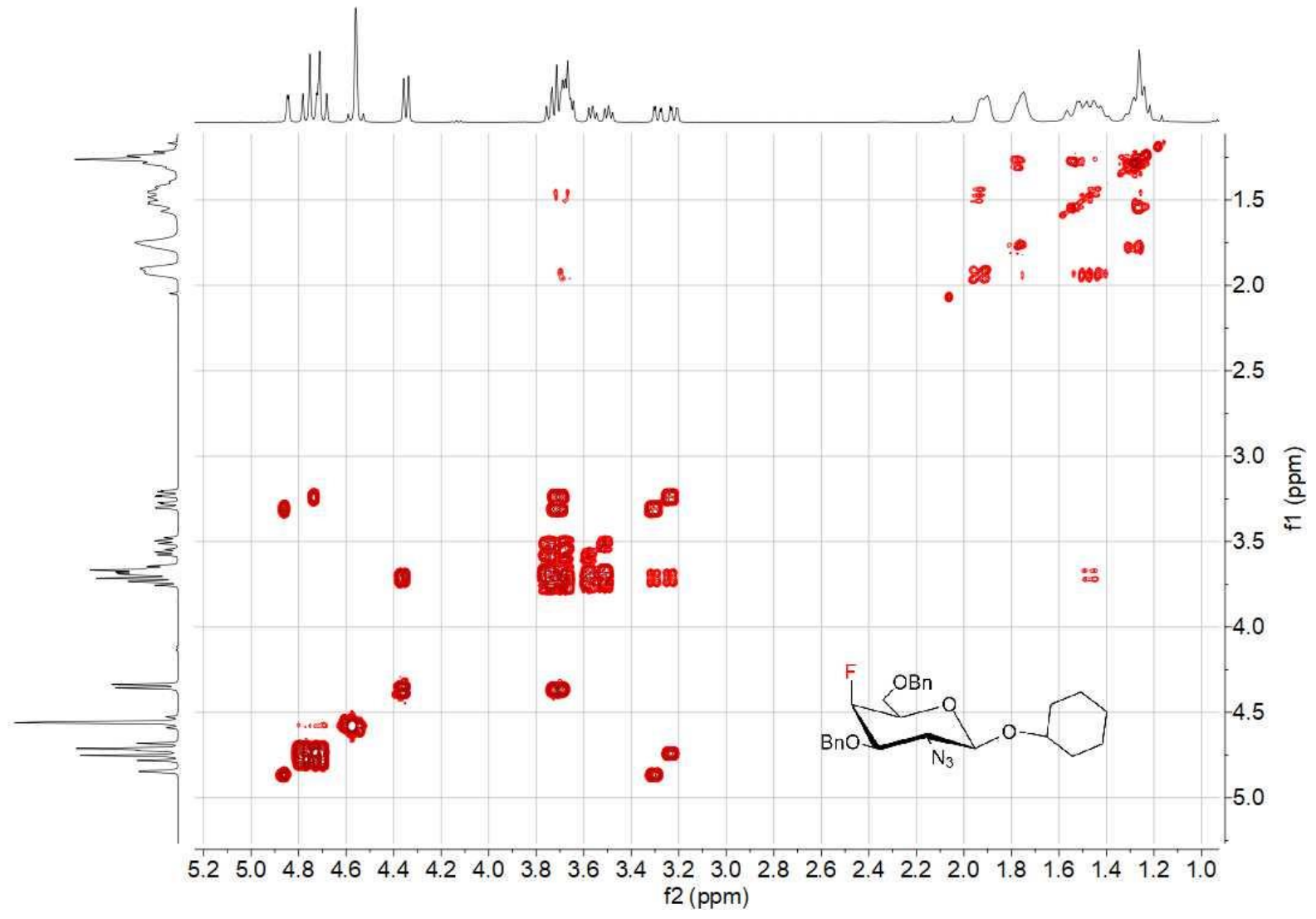

${ }^{1} \mathrm{H}-{ }^{13} \mathrm{C}$ HSOC NMR $4 \mathrm{~A}-\beta$

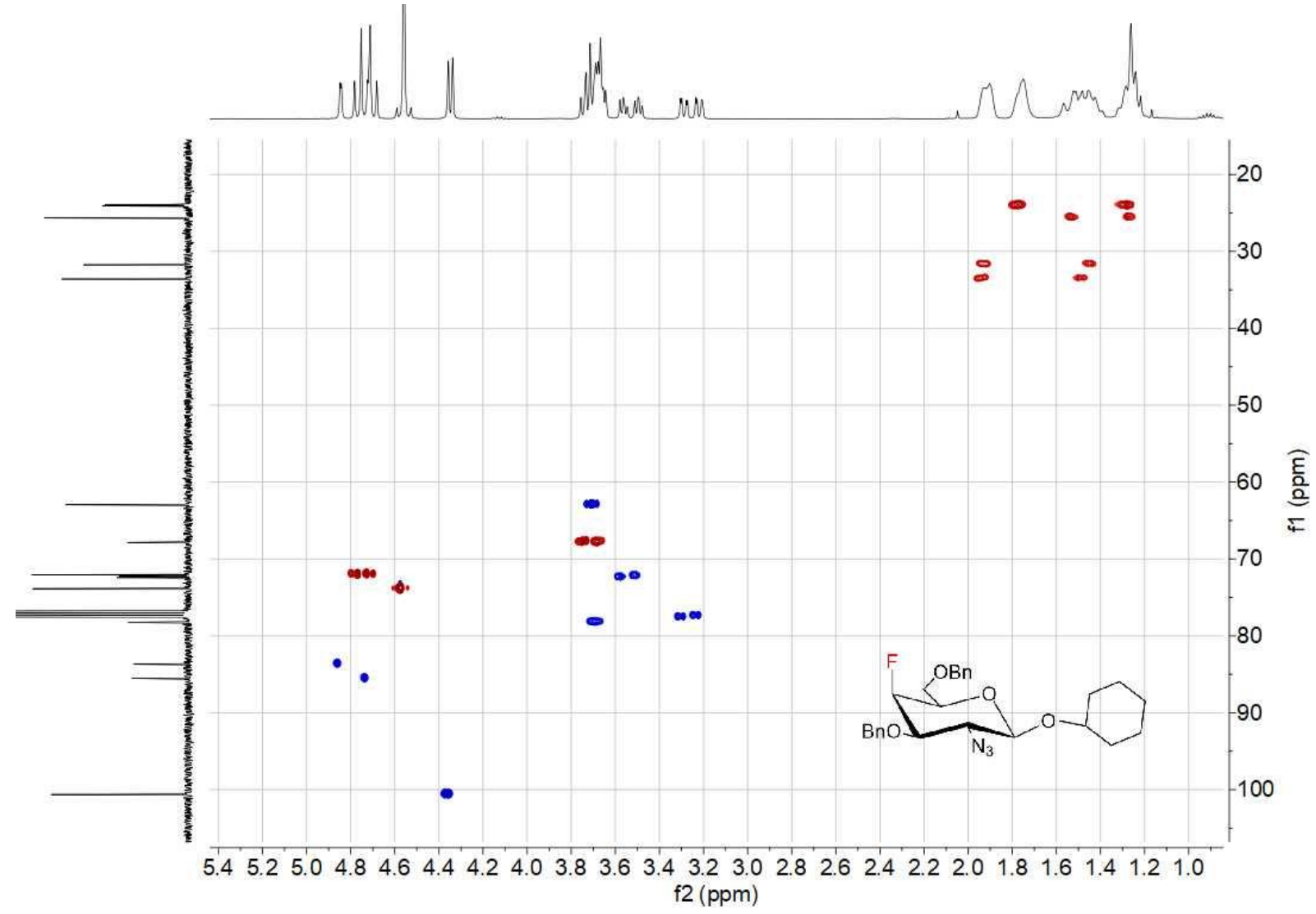


${ }^{1} \mathrm{H}-{ }^{13} \mathrm{C}$ HMBC NMR $4 \mathrm{~A}-\beta$

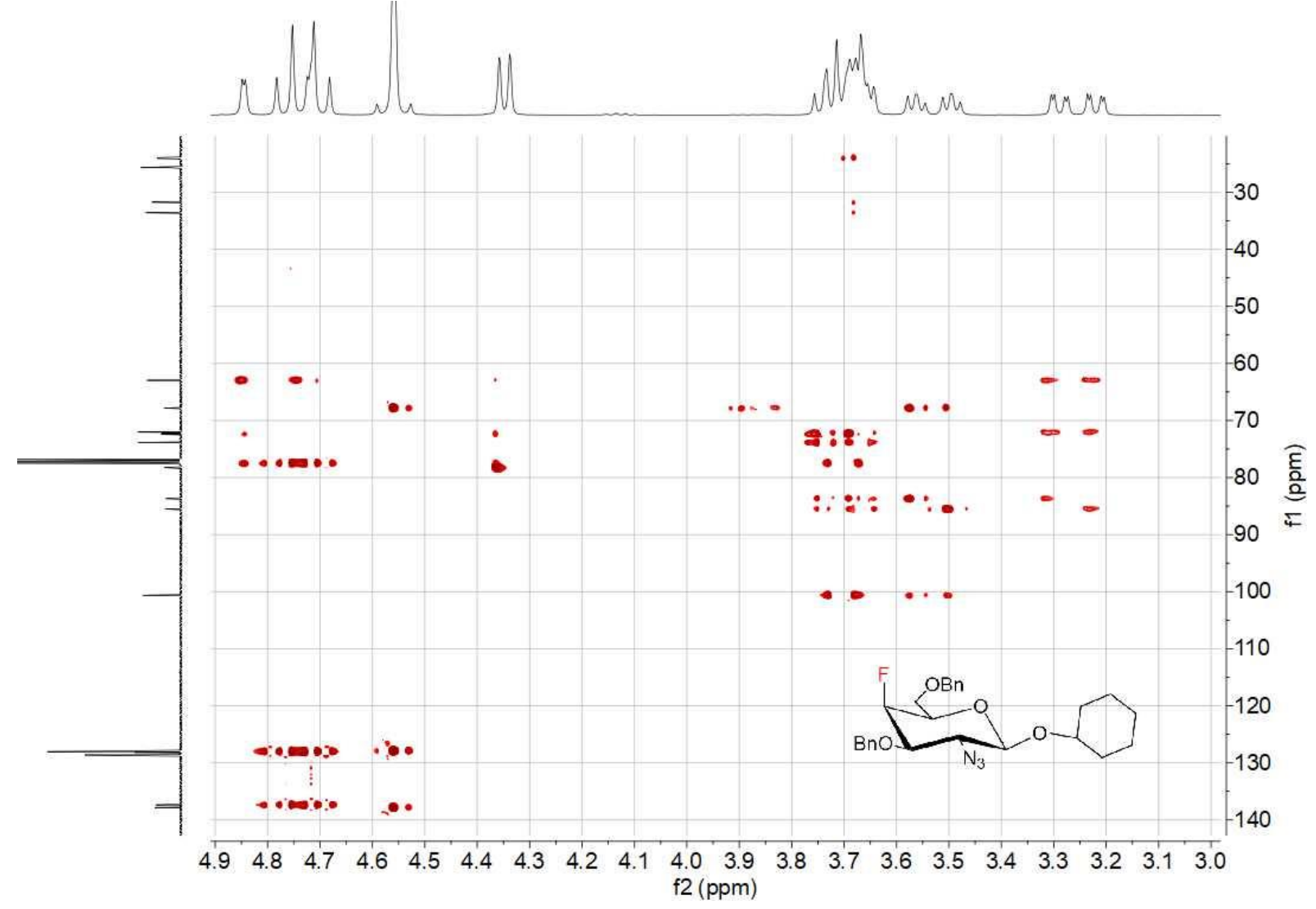

${ }^{19} \mathrm{~F}$ NMR $376 \mathrm{MHz}, \mathrm{CDCl}_{3} 4 \mathrm{~A}-\boldsymbol{\beta}$

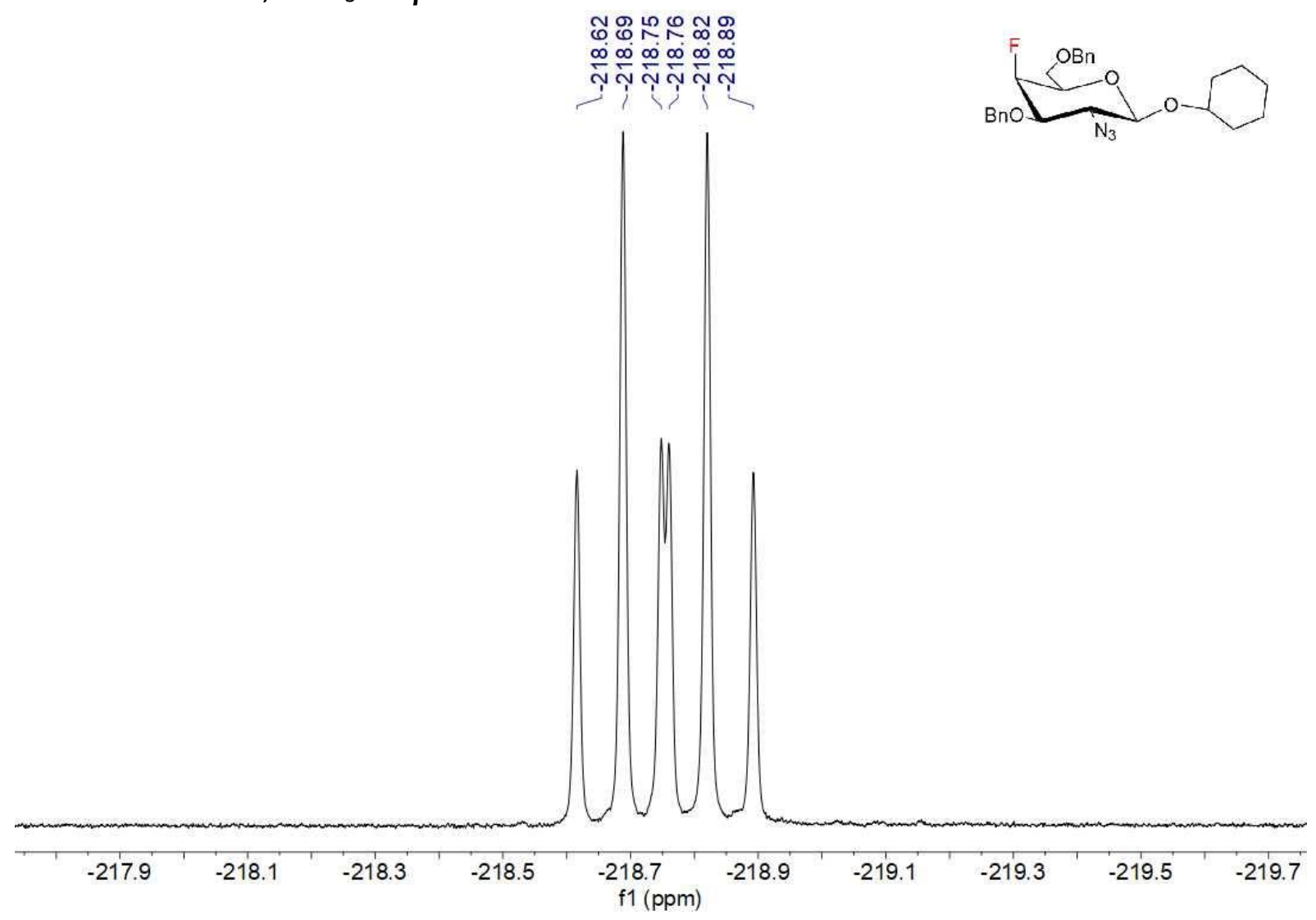


NMR 4B- $\alpha$

${ }^{1} \mathrm{HNMR} 400 \mathrm{MHz}, \mathrm{CDCl}_{3} 4 \mathrm{~B}-\alpha$

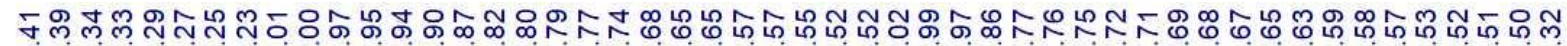

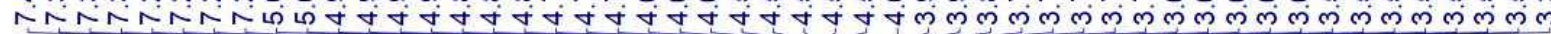

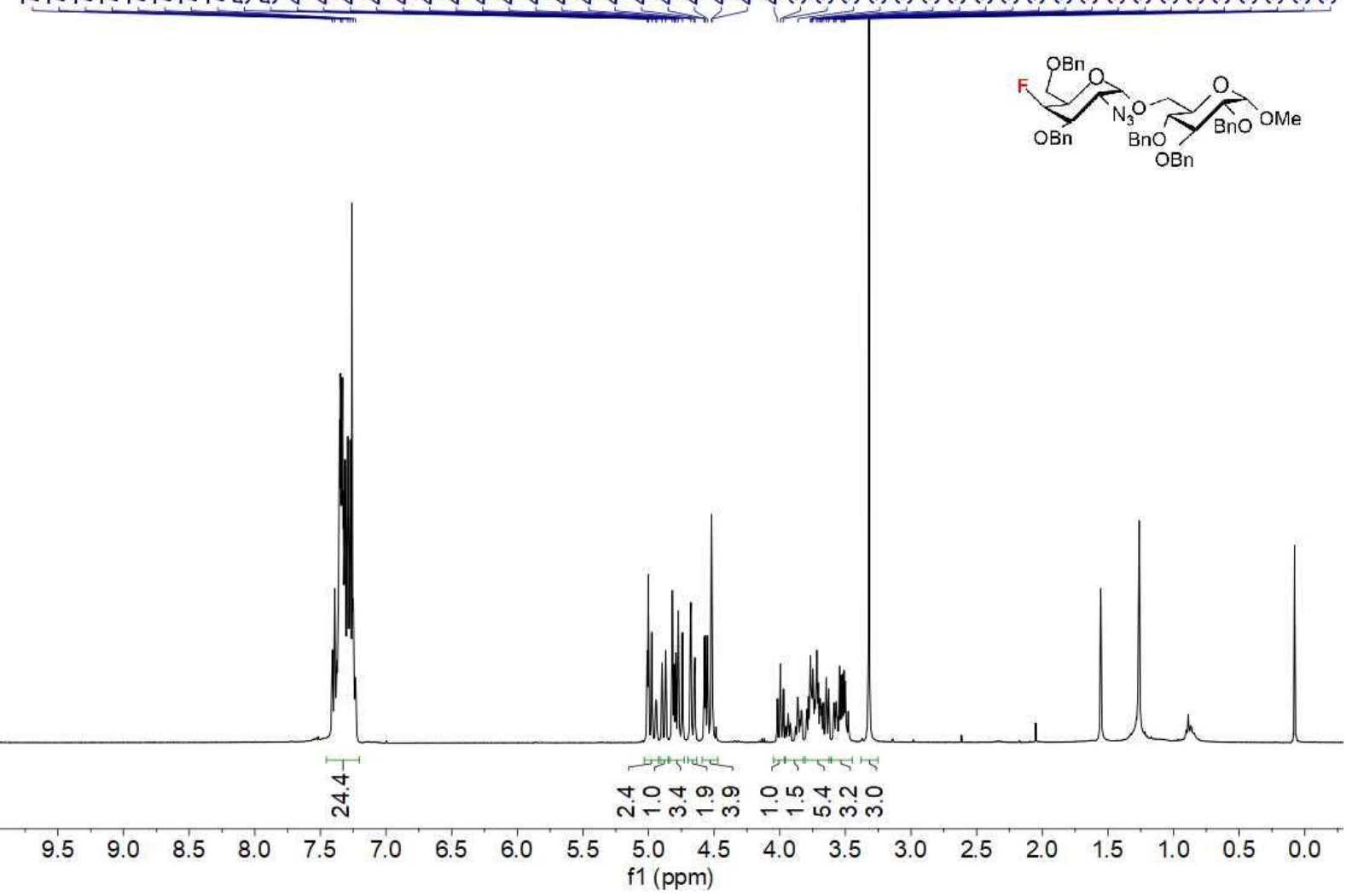

${ }^{13} \mathrm{C}\left\{{ }^{1} \mathrm{H}\right\} \mathrm{NMR} 100 \mathrm{MHz}, \mathrm{CDCl}_{3} 4 \mathrm{~B}-\alpha$

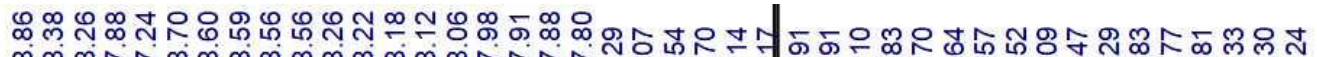

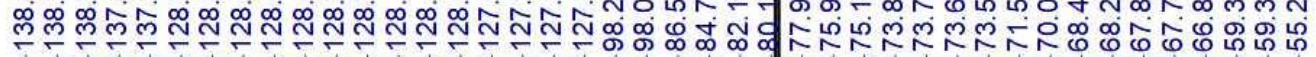

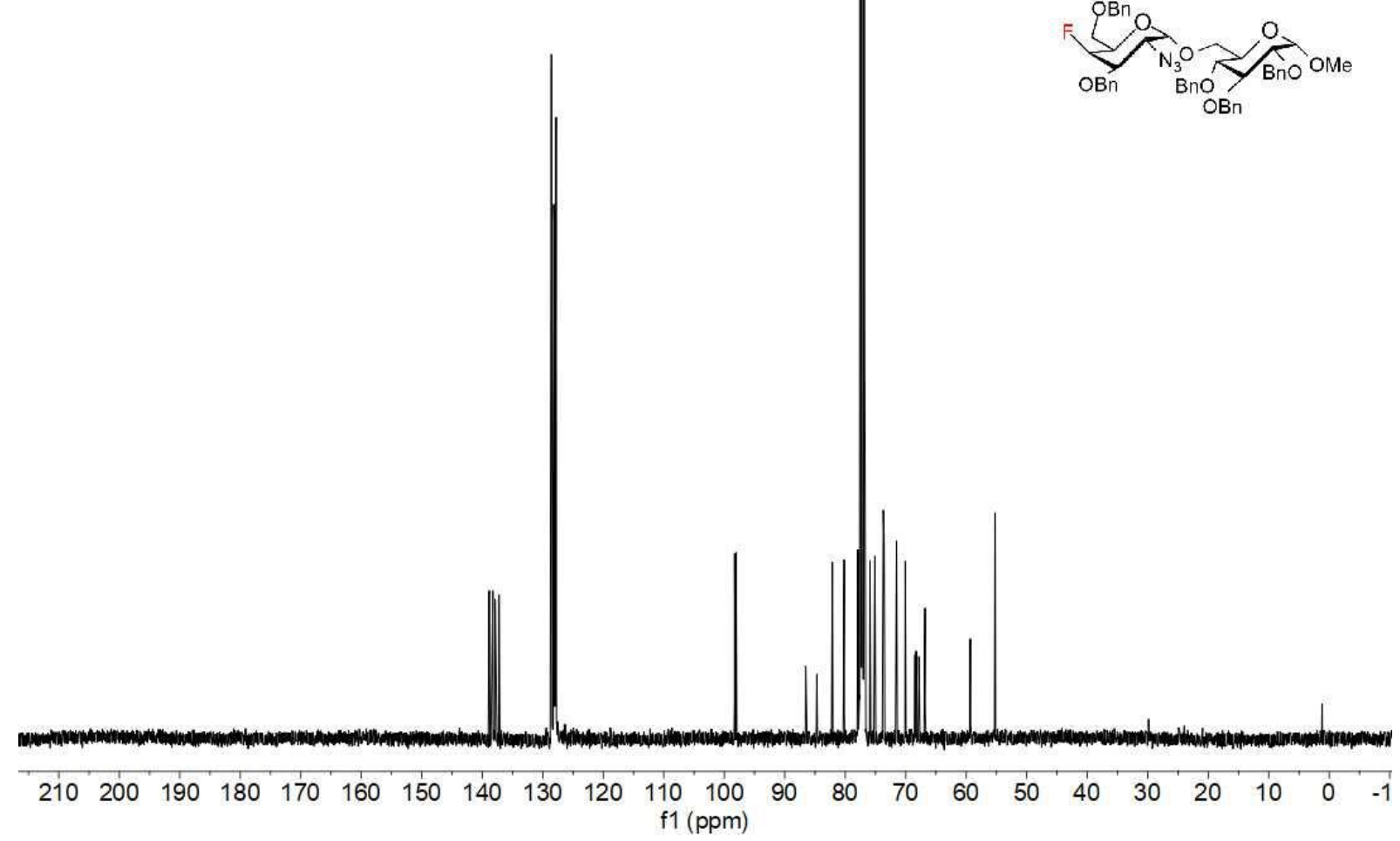


${ }^{1} \mathrm{H}-{ }^{1} \mathrm{H}$ COSY NMR 4B- $\alpha$

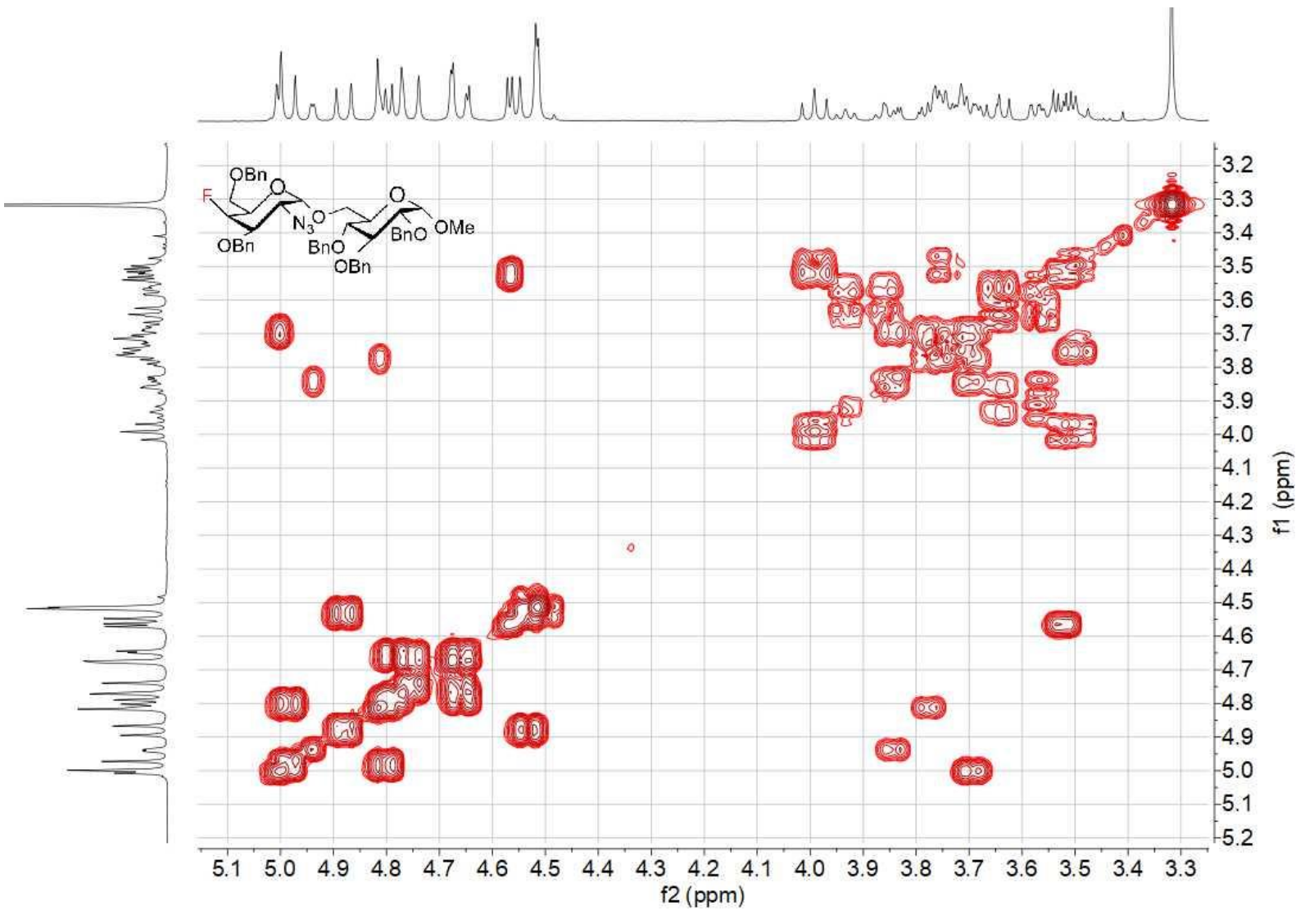

${ }^{1} \mathrm{H}-{ }^{13} \mathrm{C}$ HSQC NMR 4B- $\alpha$

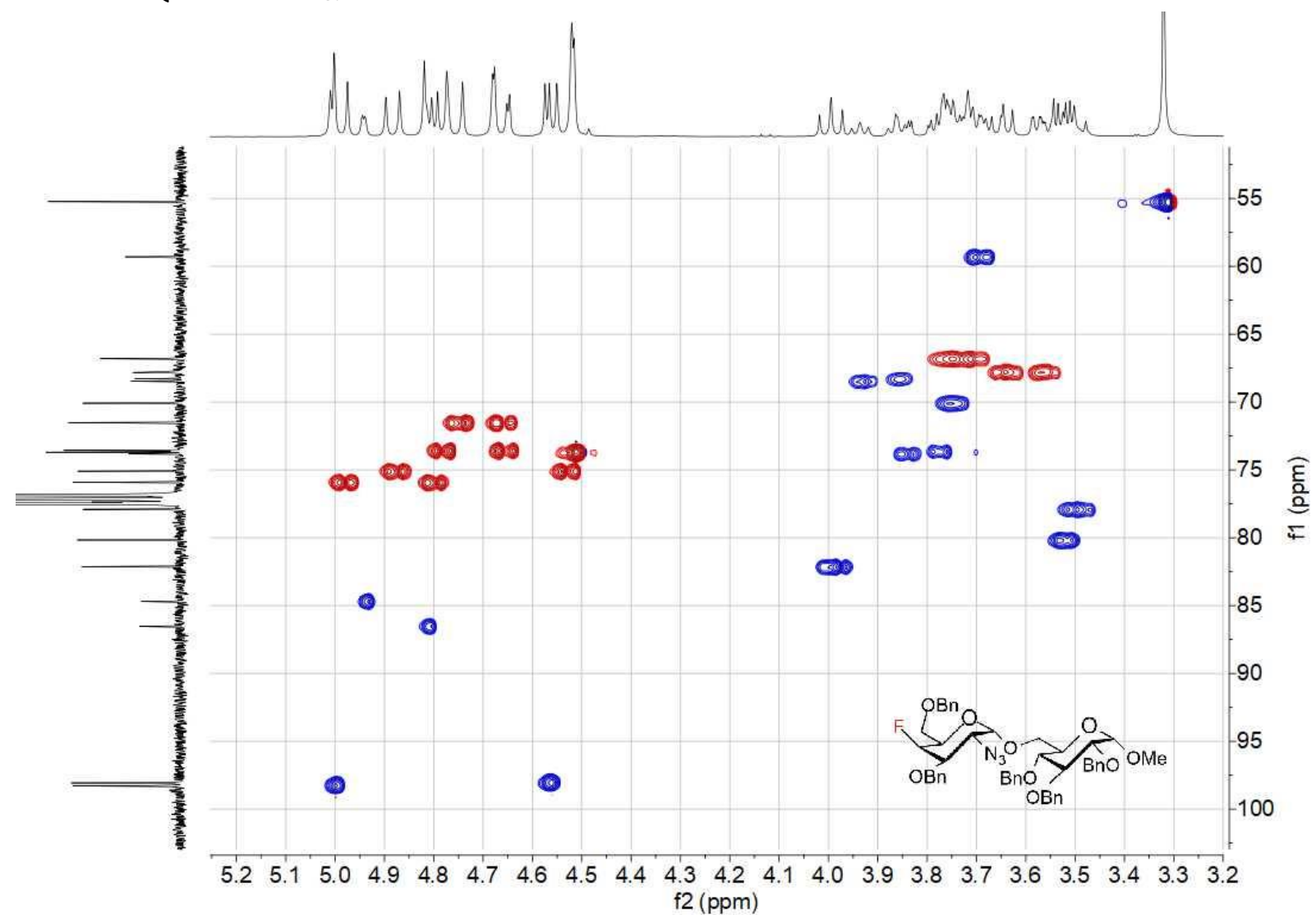


${ }^{1} \mathrm{H}-{ }^{13} \mathrm{C}$ HMBC NMR 4B- $\alpha$

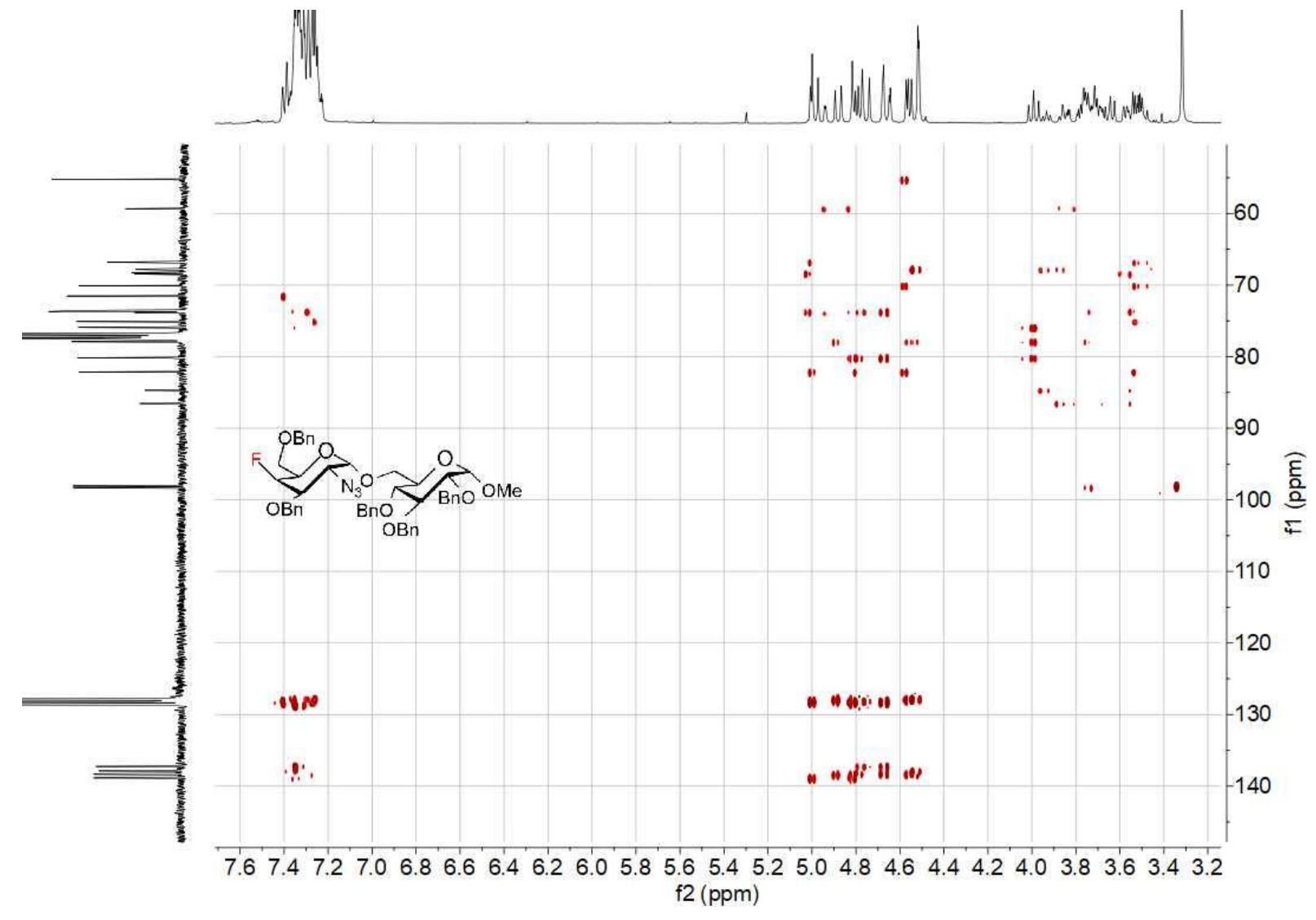

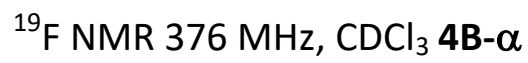

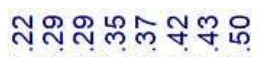

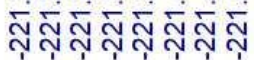
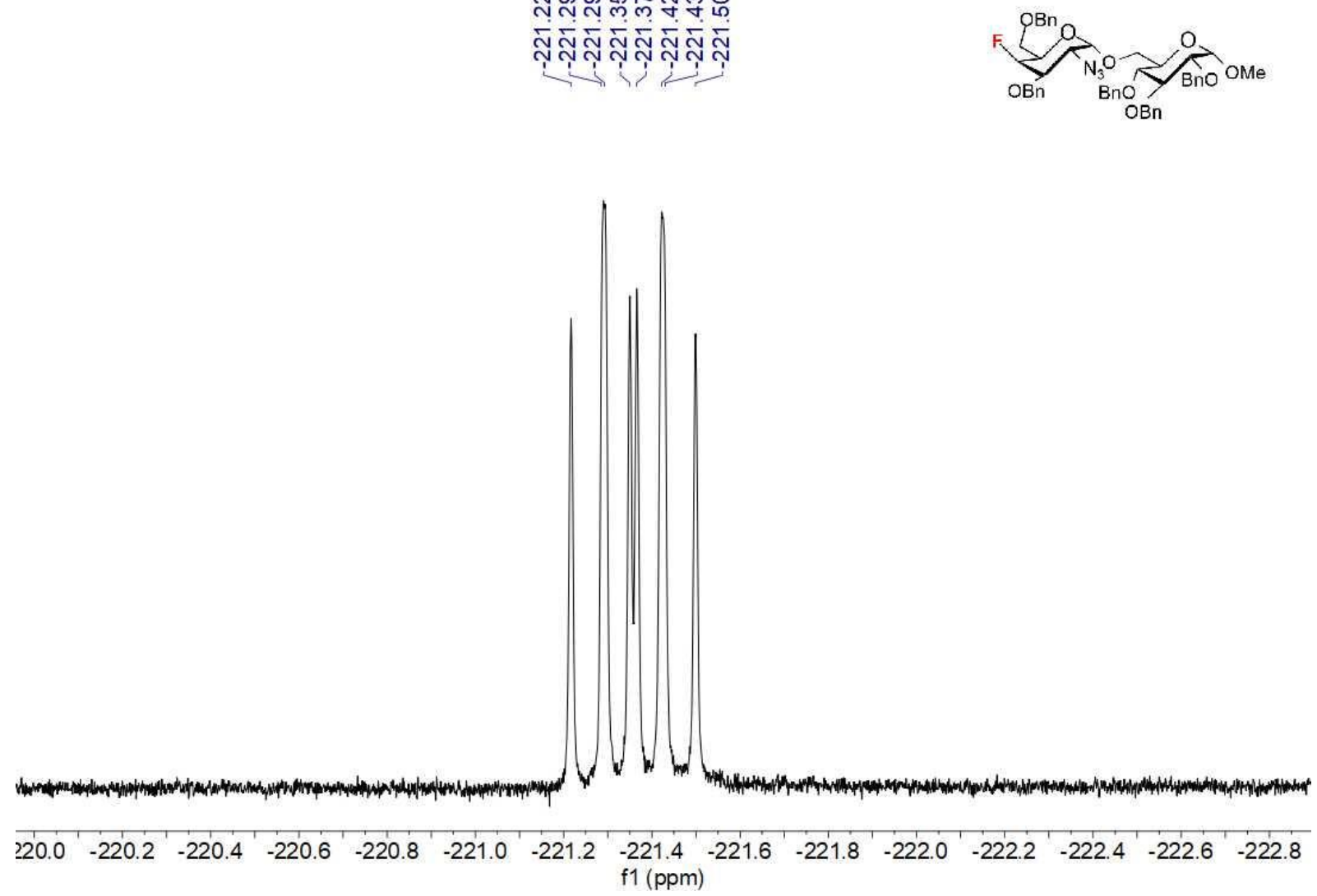
NMR 4B- $\beta$

${ }^{1} \mathrm{HNMR} 400 \mathrm{MHz}, \mathrm{CDCl}_{3} 4 \mathrm{~B}-\beta$

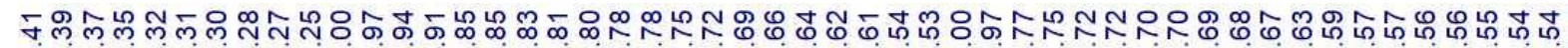

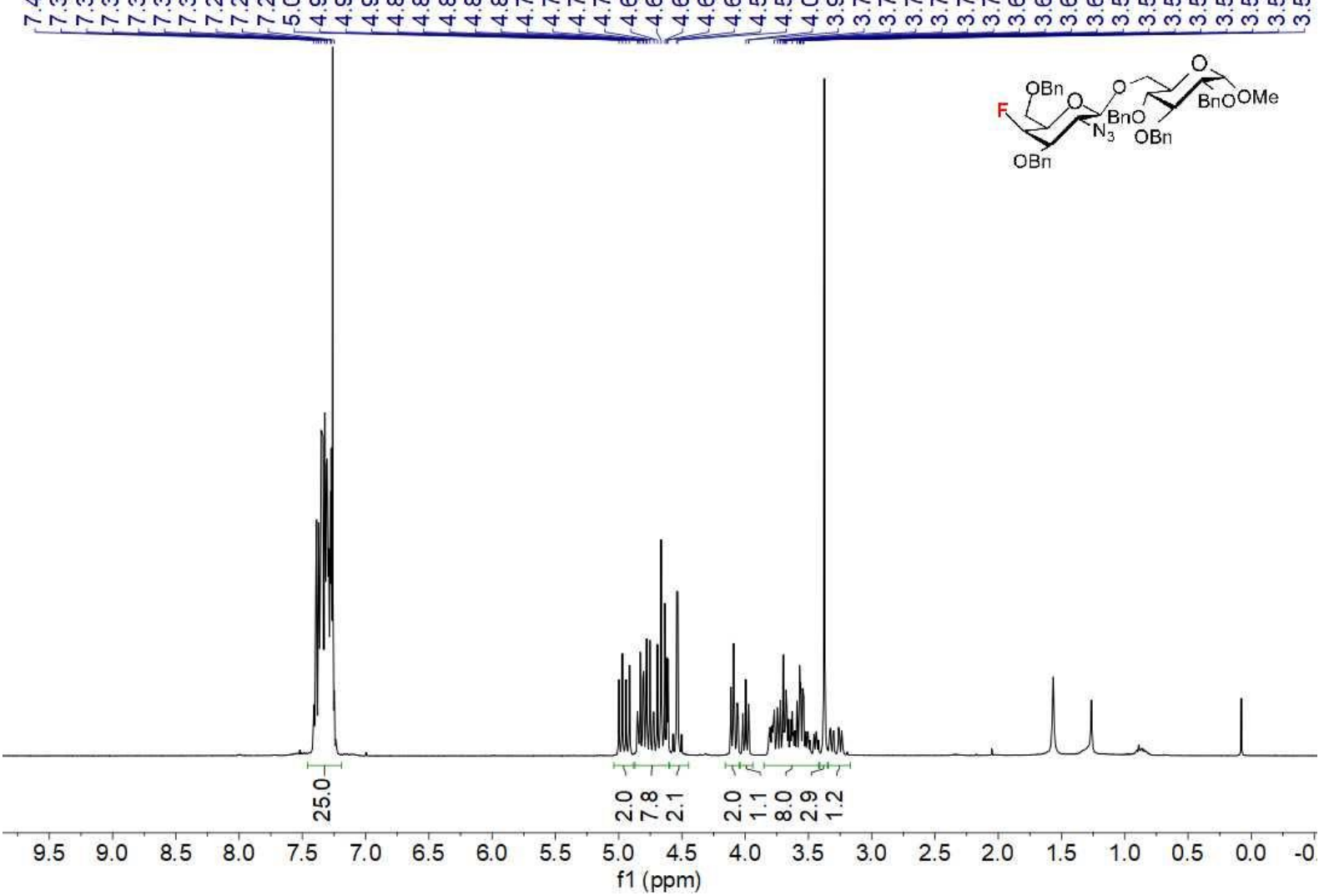

${ }^{13} \mathrm{C}\left\{{ }^{1} \mathrm{H}\right\}$ NMR 100MHz, $\mathrm{CDCl}_{3} 4 \mathrm{~B}-\boldsymbol{\beta}$

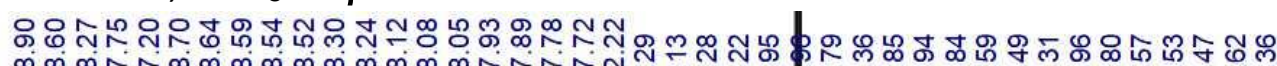

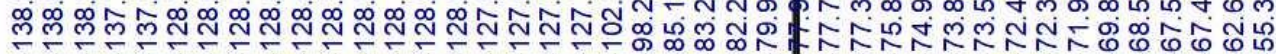

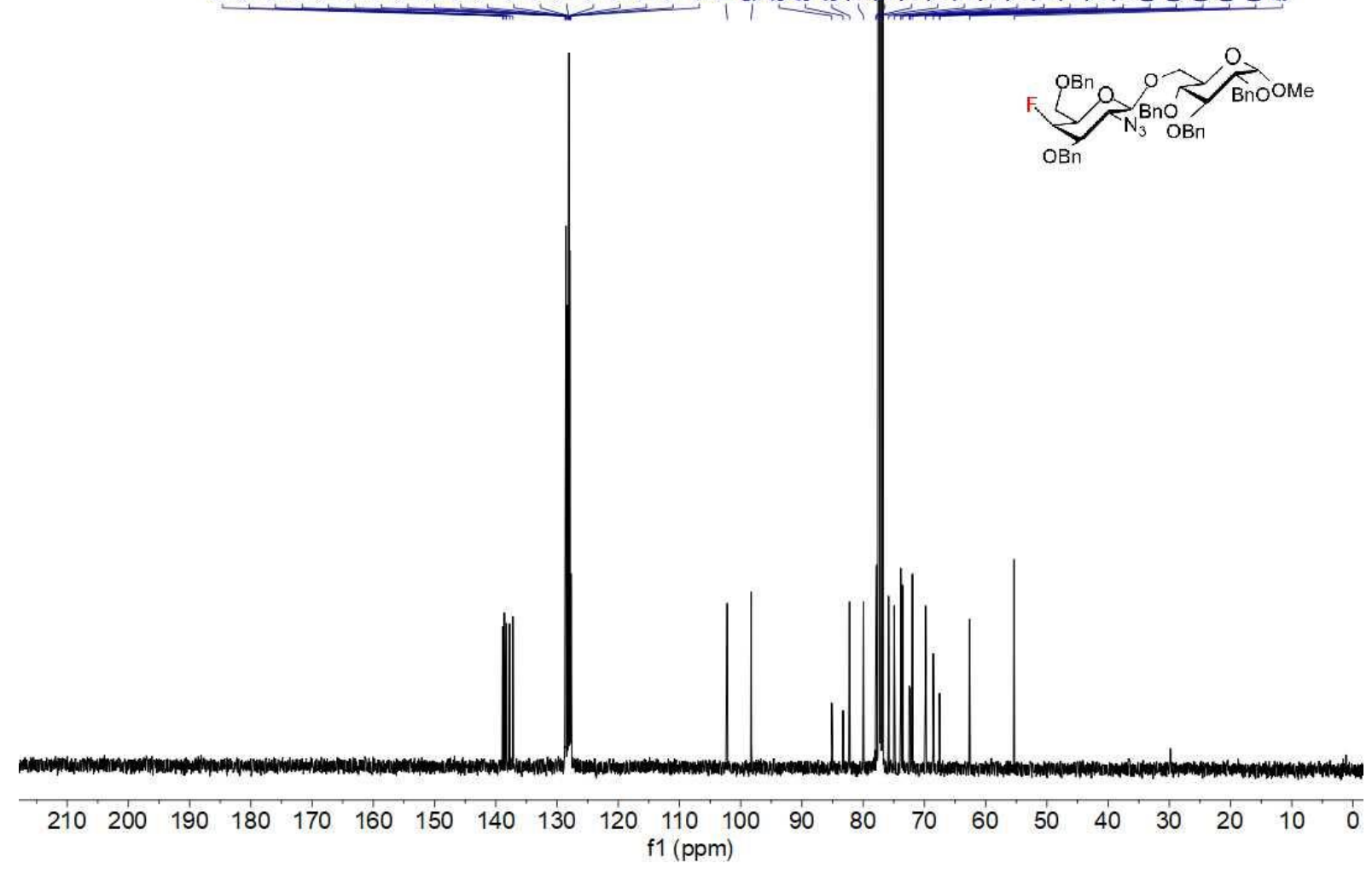


${ }^{1} \mathrm{H}-{ }^{1} \mathrm{H}$ COSY NMR 4B- $\beta$

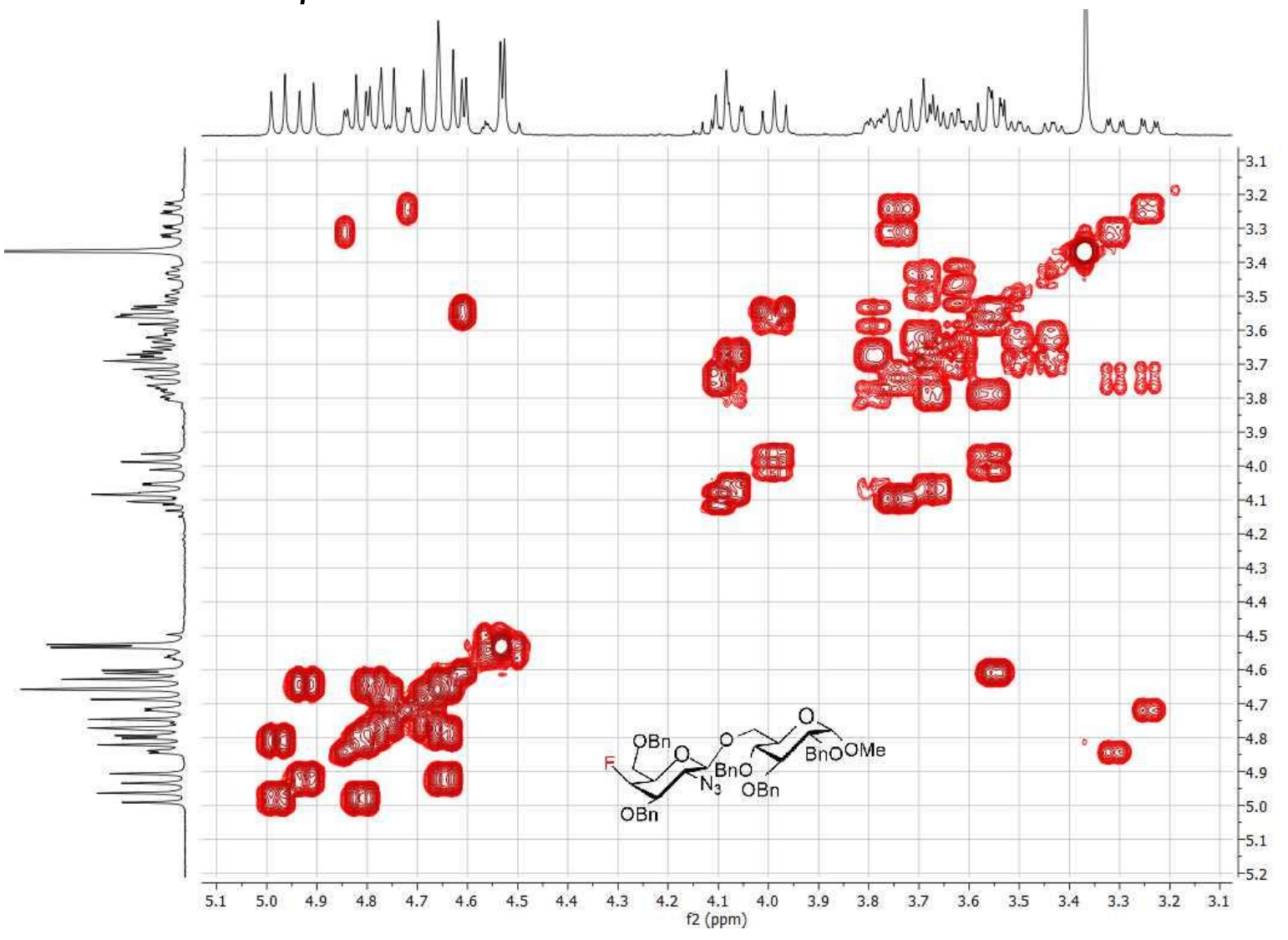

${ }^{1} \mathrm{H}-{ }^{13} \mathrm{C}$ HSQC NMR 4B- $\beta$

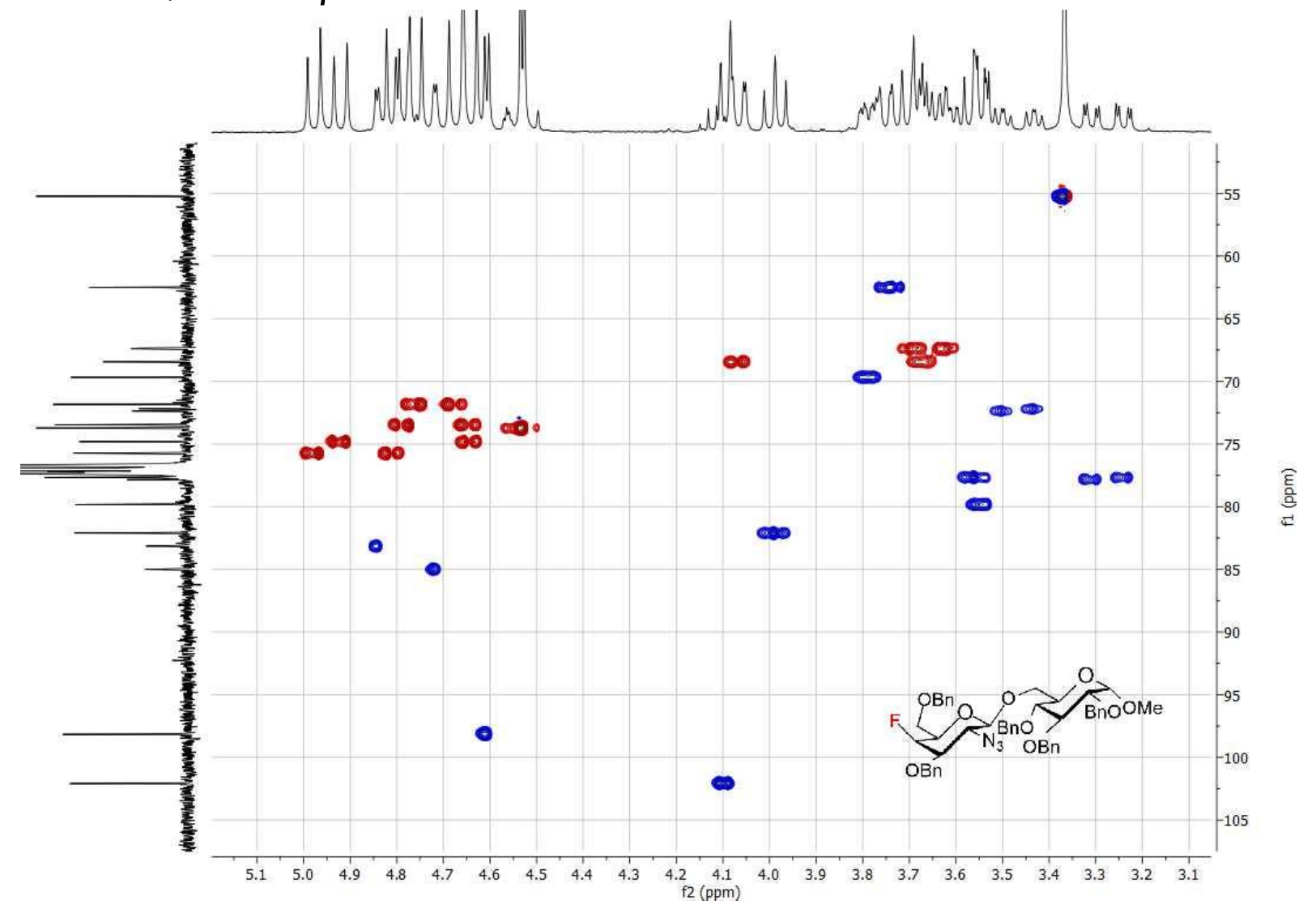


${ }^{1} \mathrm{H}-{ }^{13} \mathrm{C}$ HMBC NMR 4B- $\beta$

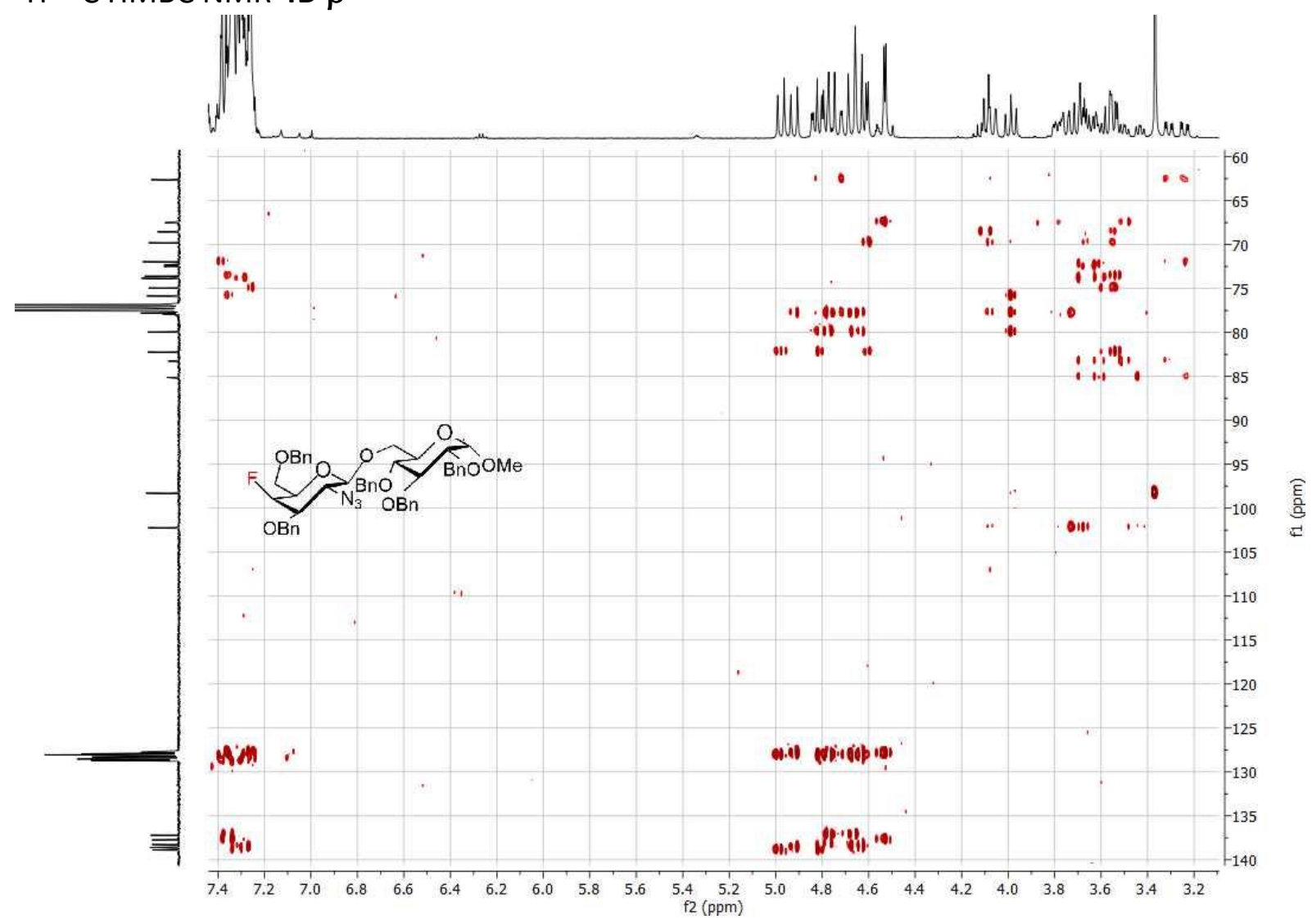

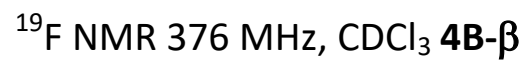

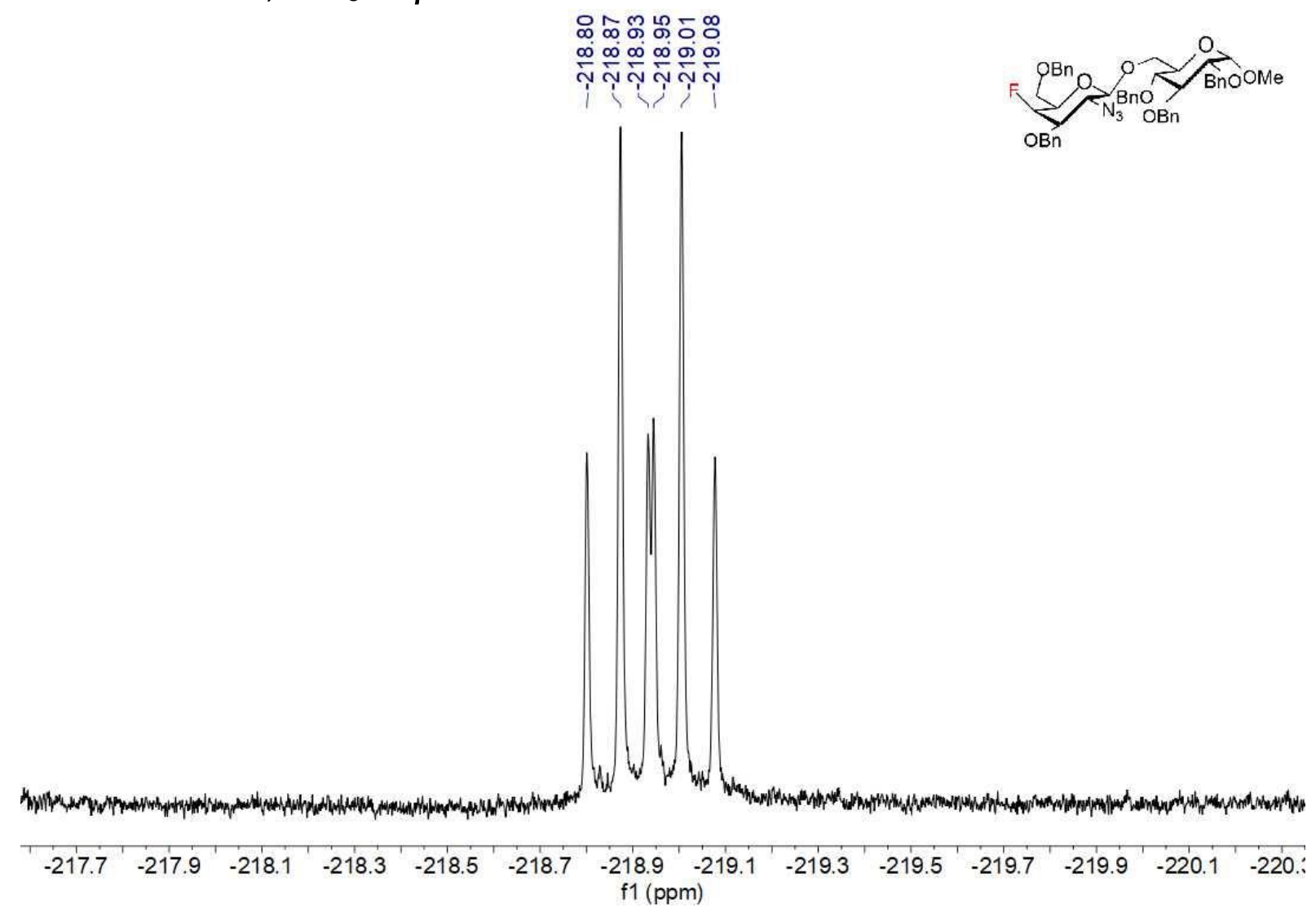


NMR 4C- $\alpha$

${ }^{1} \mathrm{HNMR} 400 \mathrm{MHz}, \mathrm{CDCl}_{3} 4 \mathrm{C}-\alpha$

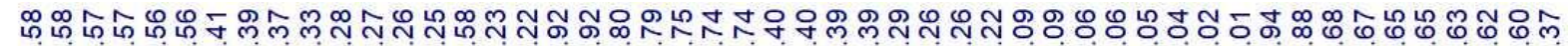

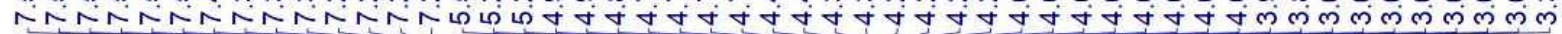

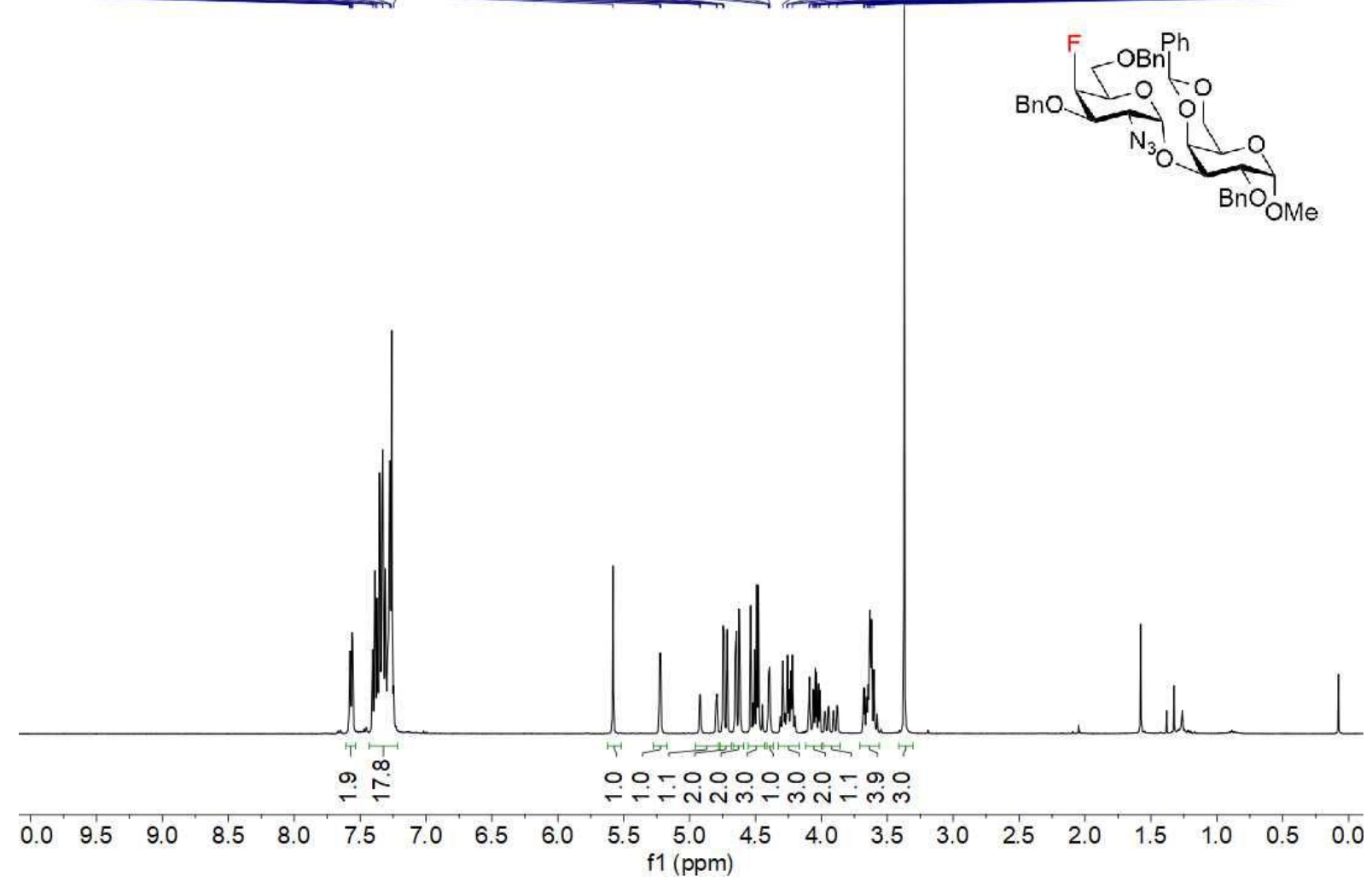

${ }^{13} \mathrm{C}\left\{{ }^{1} \mathrm{H}\right\} \mathrm{NMR} 100 \mathrm{MHz}, \mathrm{CDCl}_{3} 4 \mathrm{C}-\alpha$

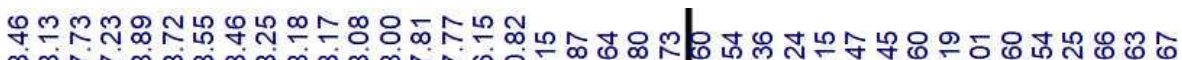

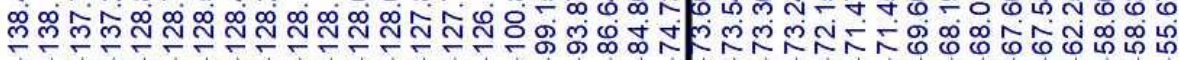

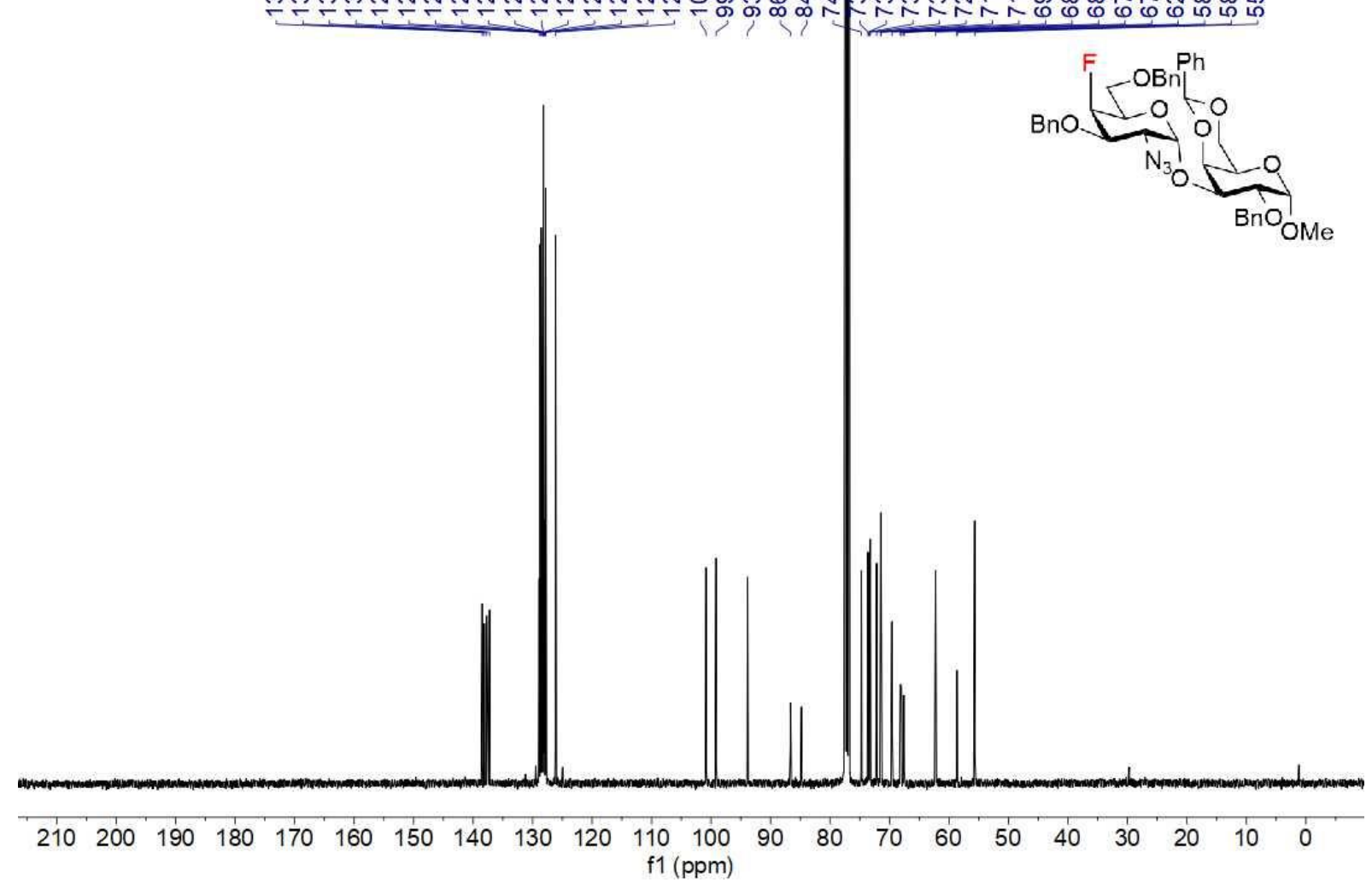


${ }^{1} \mathrm{H}-{ }^{1} \mathrm{H}$ COSY NMR $4 \mathrm{C}-\alpha$

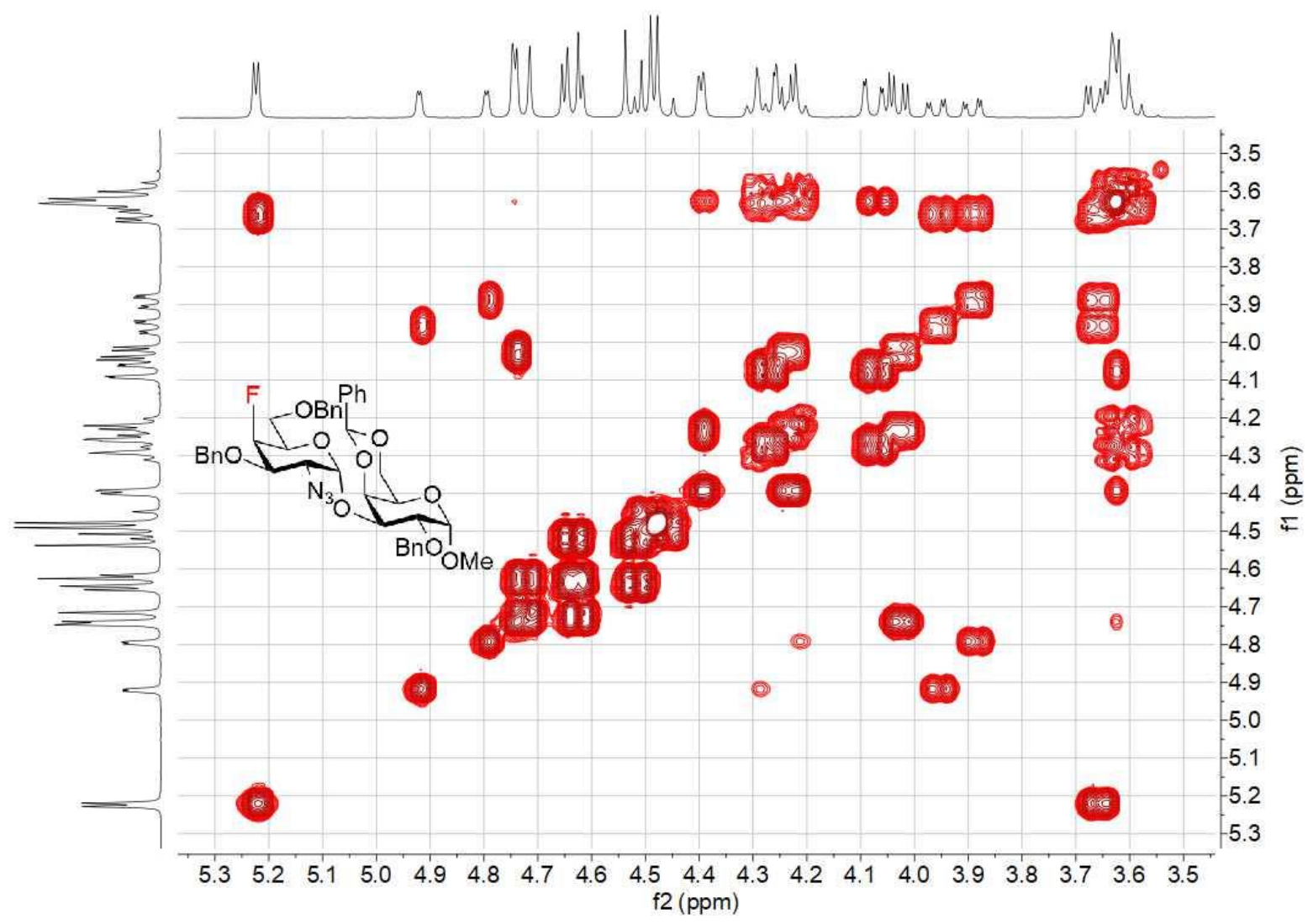

${ }^{1} \mathrm{H}-{ }^{13} \mathrm{C}$ HSOC NMR $4 \mathrm{C}-\alpha$

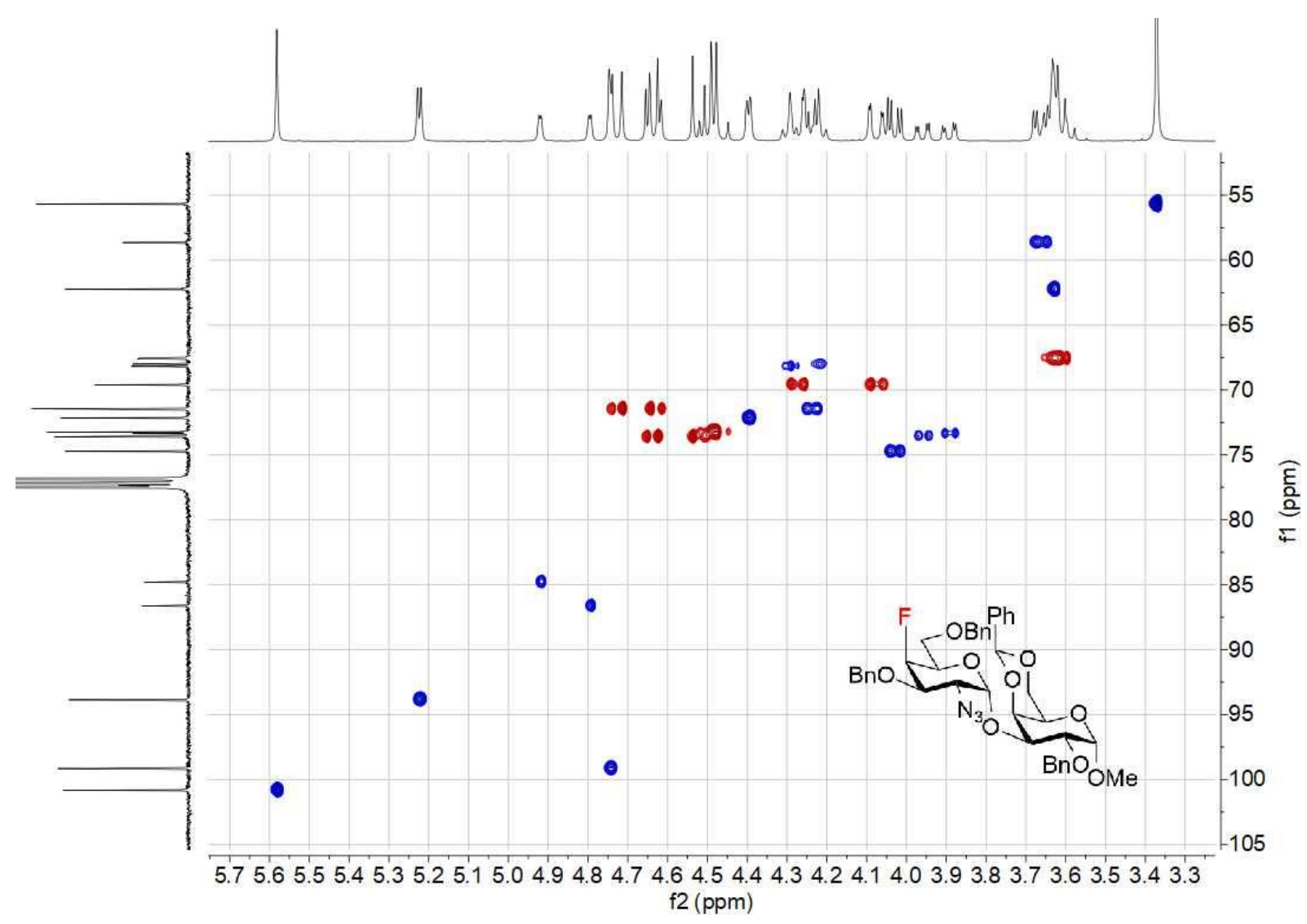


${ }^{1} \mathrm{H}-{ }^{13} \mathrm{CHMBC}$ NMR 4C- $\alpha$

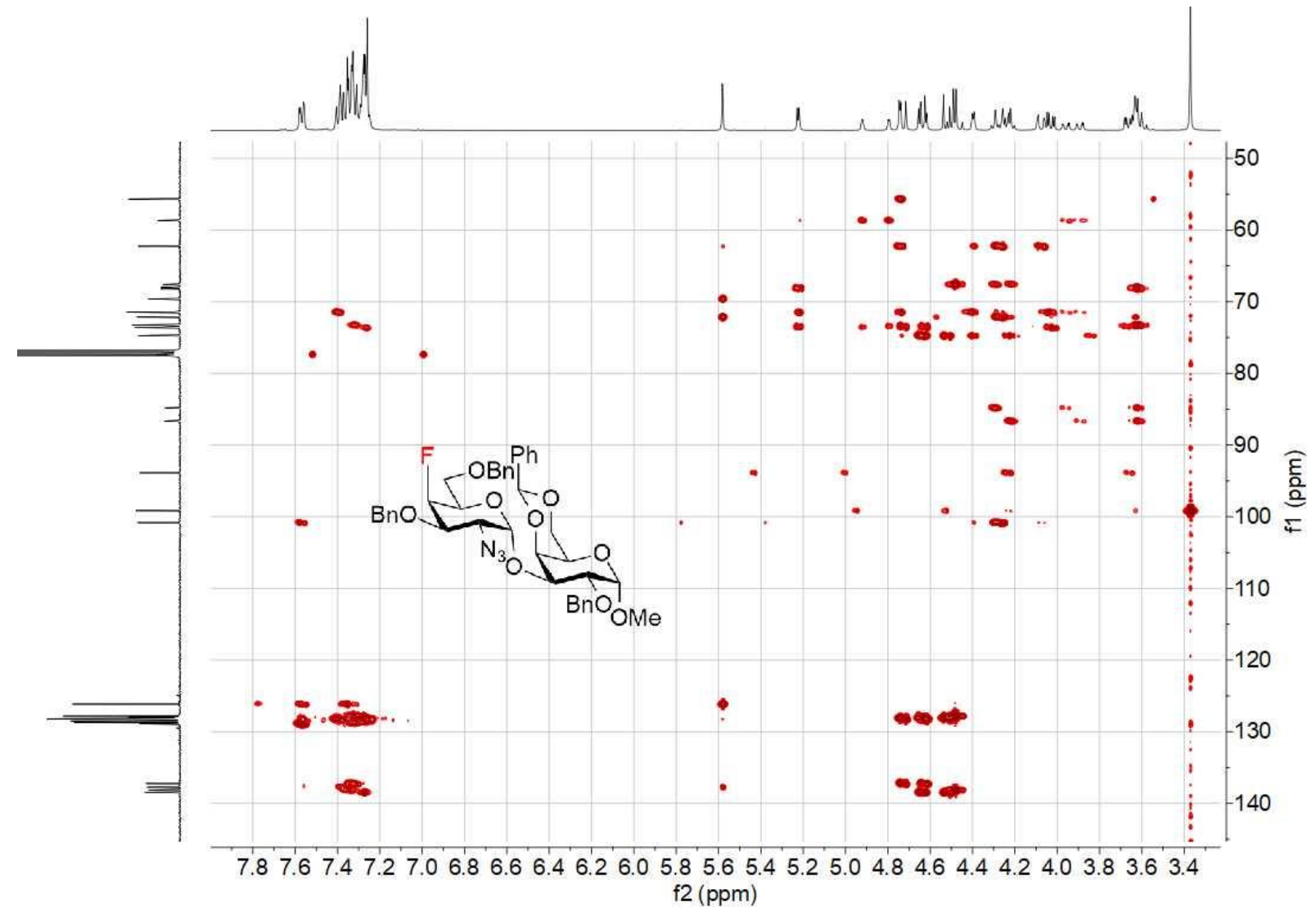

${ }^{19} \mathrm{~F}$ NMR $376 \mathrm{MHz}, \mathrm{CDCl}_{3} 4 \mathrm{C}-\alpha$

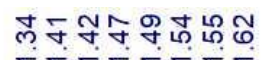

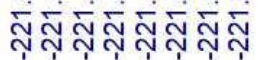
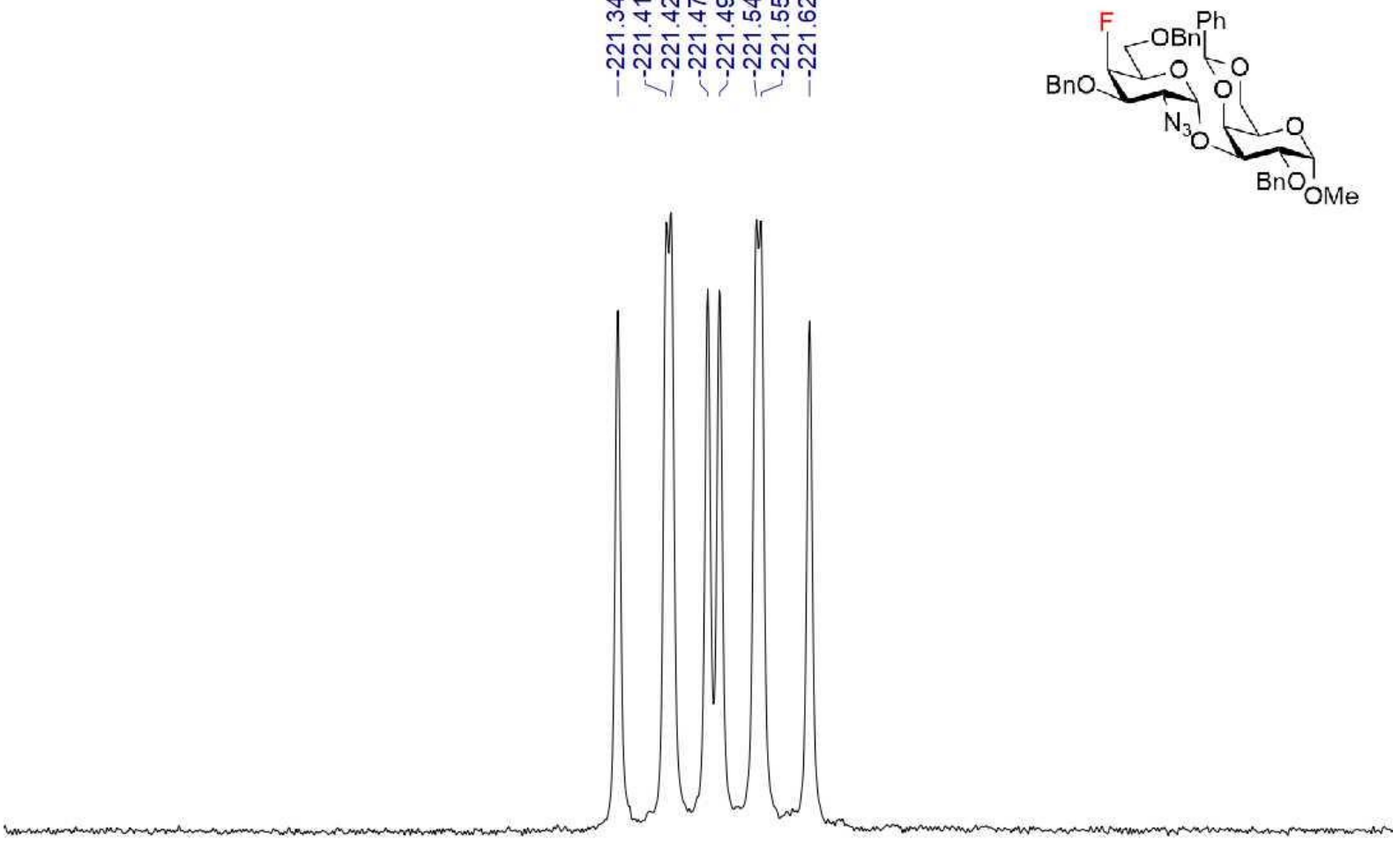
NMR 4C- $\beta$

${ }^{1} \mathrm{HNMR} 400 \mathrm{MHz}, \mathrm{CDCl}_{3} 4 \mathrm{C}-\beta$

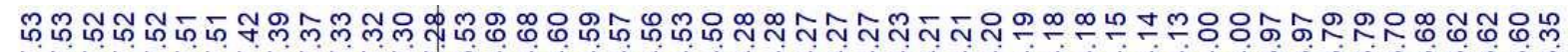

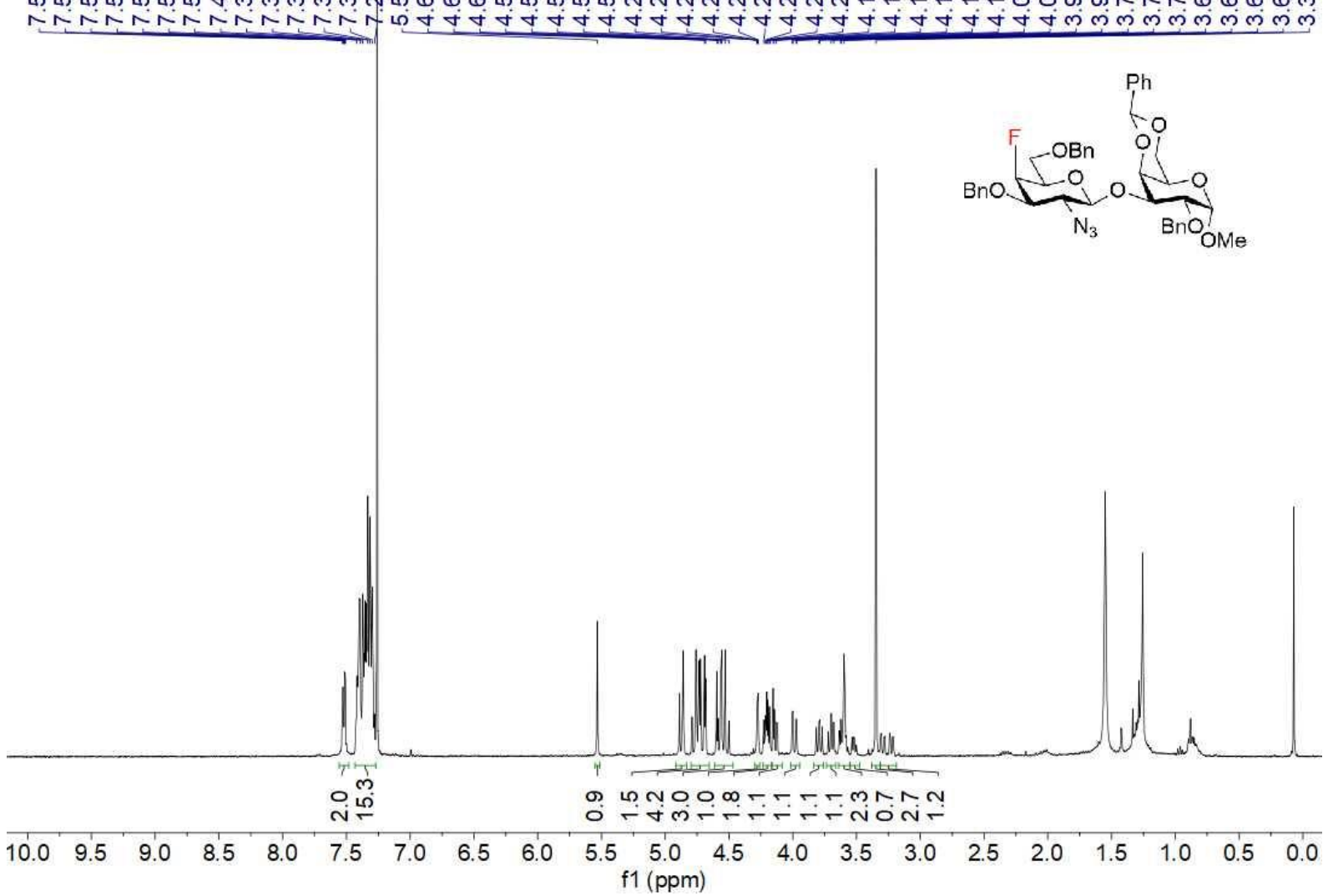

${ }^{13} \mathrm{C}\left\{{ }^{1} \mathrm{H}\right\}$ NMR 100MHz, $\mathrm{CDCl}_{3} 4 \mathrm{C}-\beta$

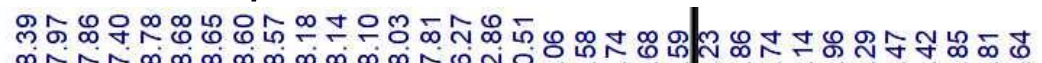

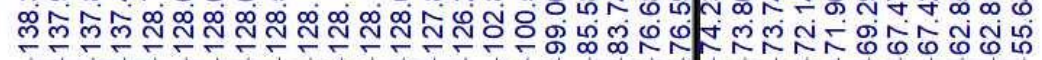

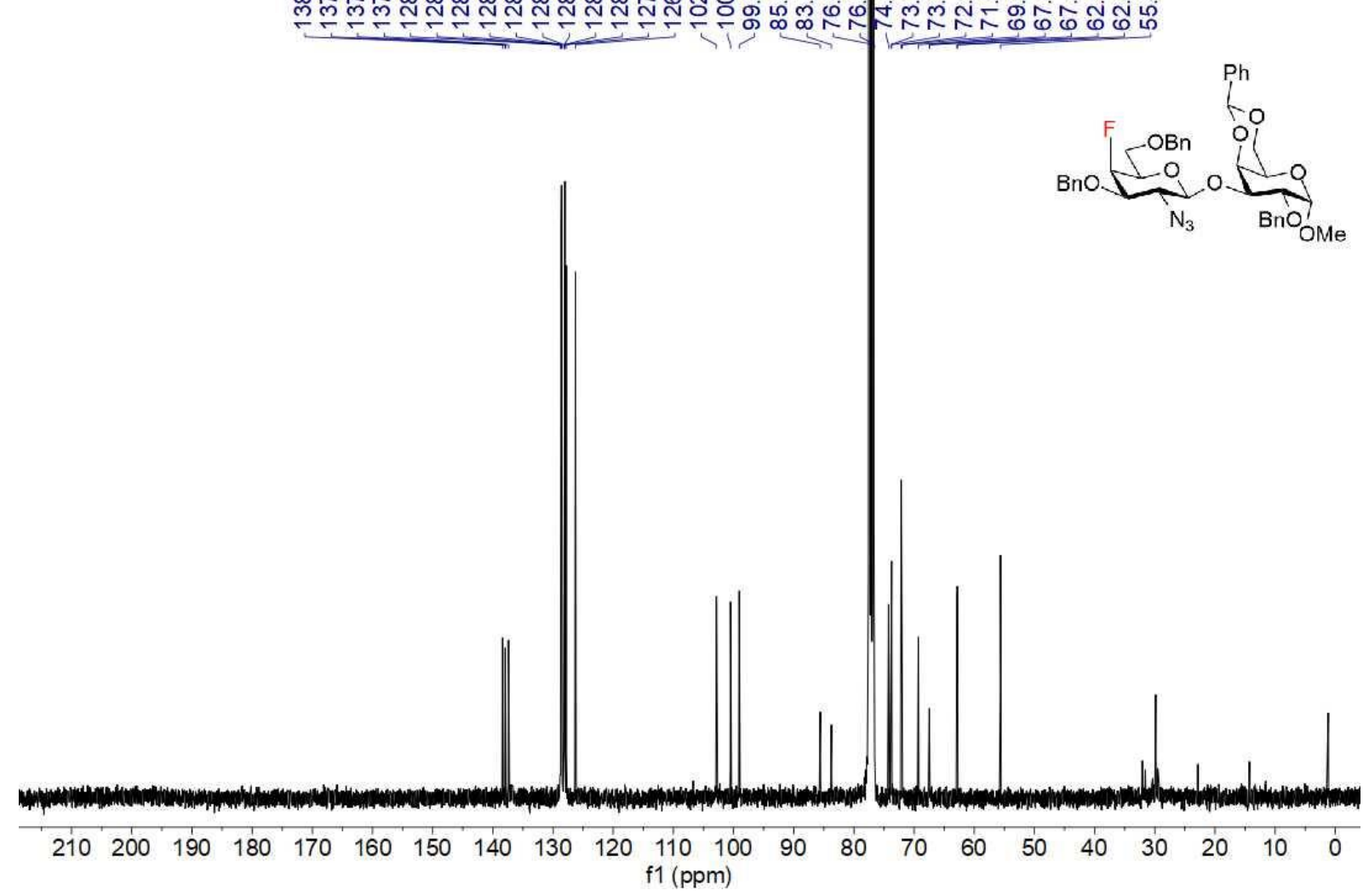


${ }^{1} \mathrm{H}-{ }^{1} \mathrm{H}$ COSY NMR $4 \mathrm{C}-\beta$

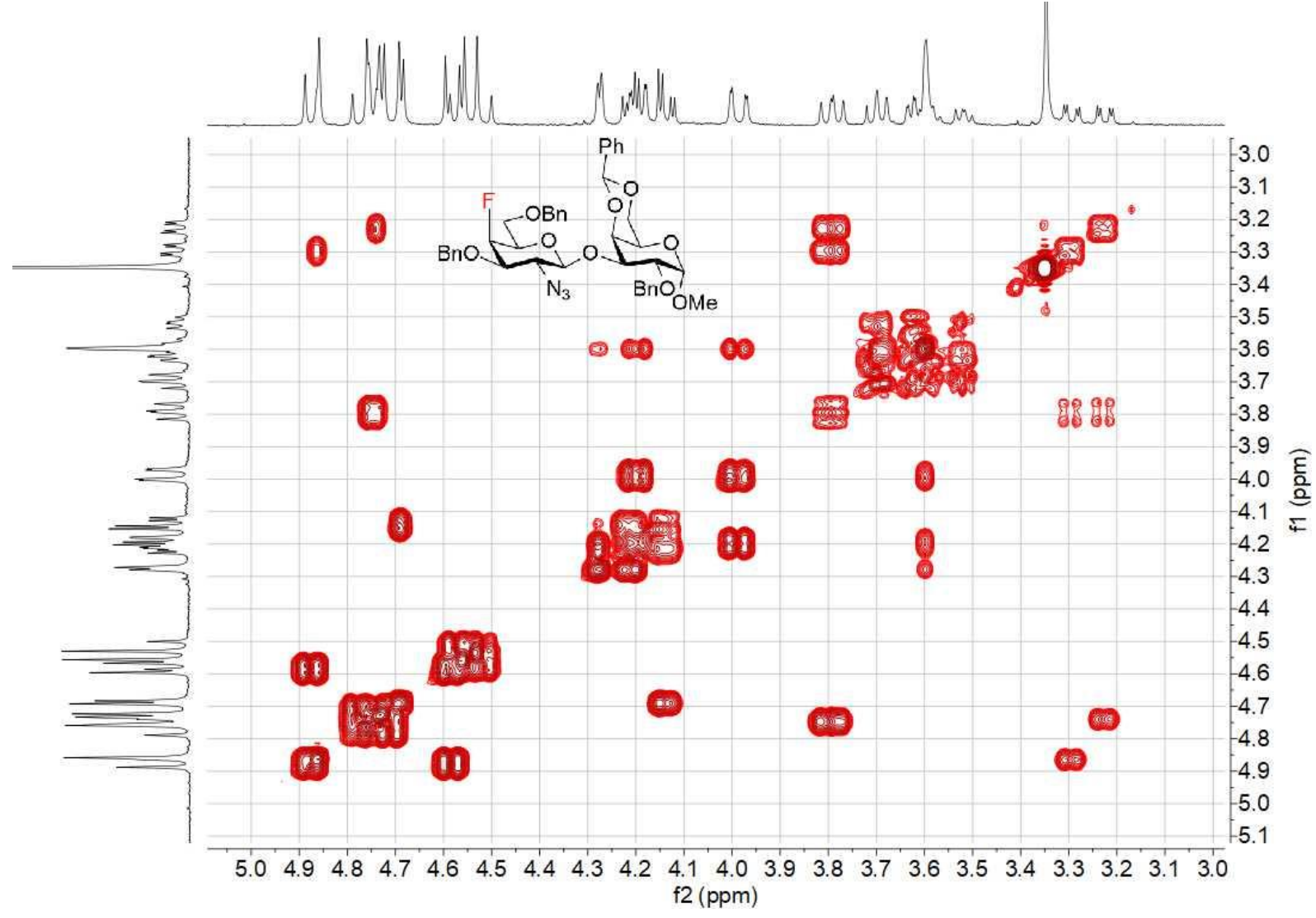

${ }^{1} \mathrm{H}-{ }^{13} \mathrm{C}$ HSQC NMR 4C $-\beta$

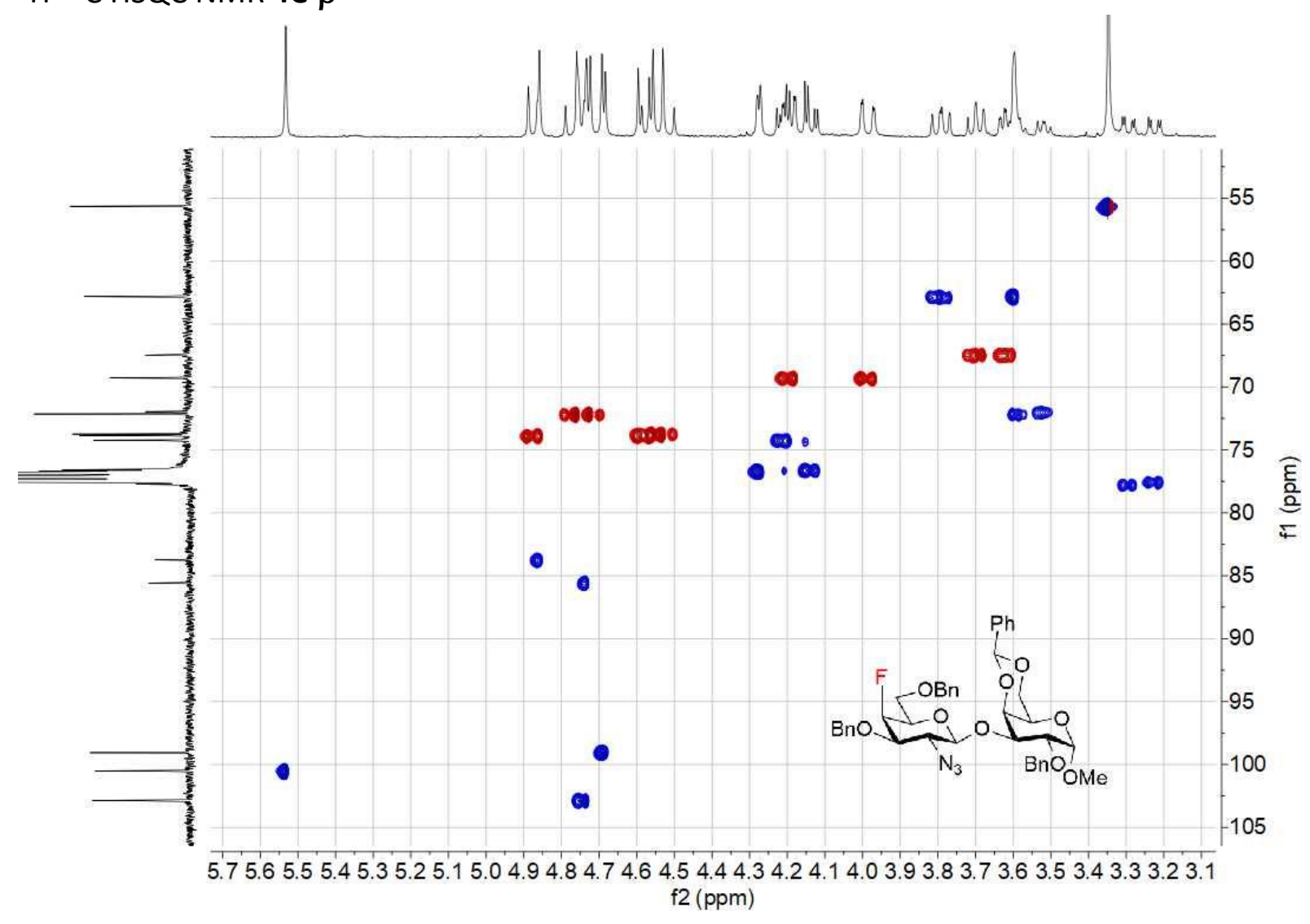


${ }^{1} \mathrm{H}-{ }^{13} \mathrm{C}$ HMBC NMR 4C- $\beta$

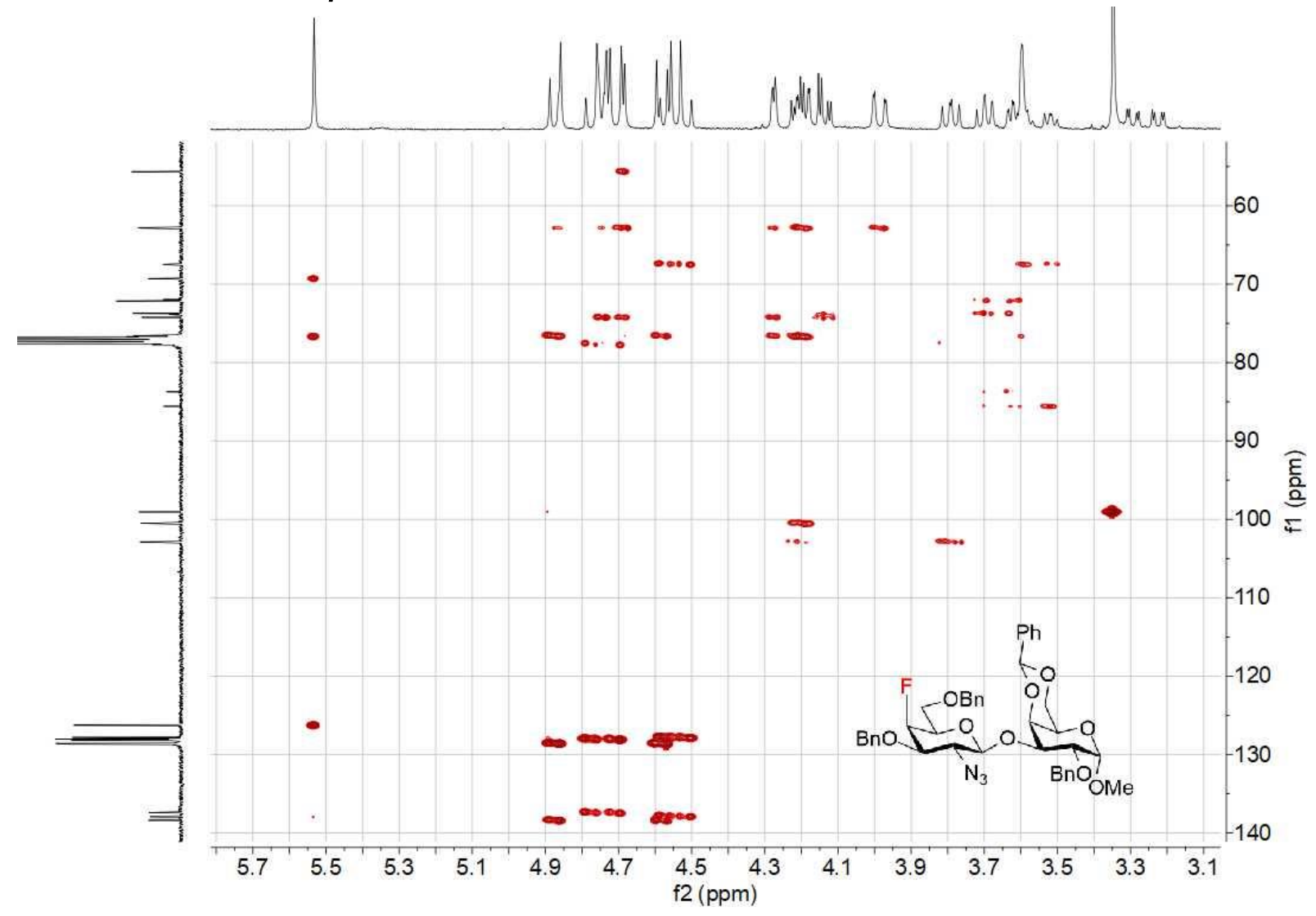

${ }^{19} \mathrm{~F} \mathrm{NMR} 376 \mathrm{MHz}, \mathrm{CDCl}_{3} 4 \mathrm{C}-\boldsymbol{\beta}$

깨윰ㅉㅀ

$\infty \infty \infty \infty \infty$

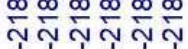

1)
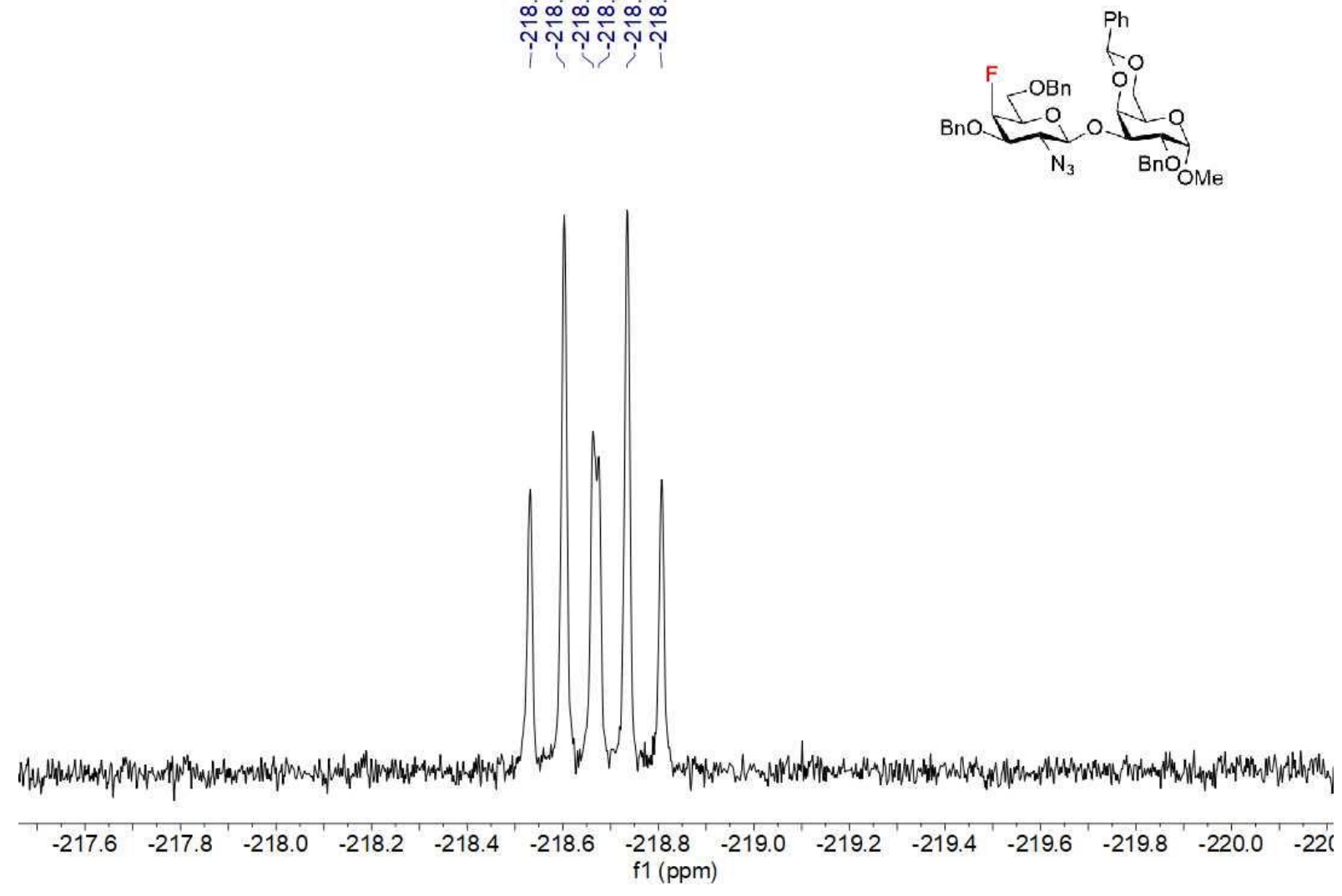
NMR 4D- $\alpha$

${ }^{1} \mathrm{H}$ NMR $400 \mathrm{MHz}, \mathrm{CDCl}_{3}$ 4D- $\alpha$

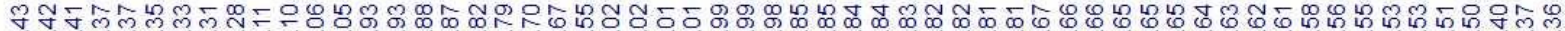

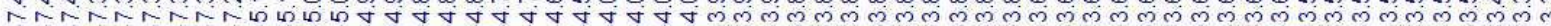

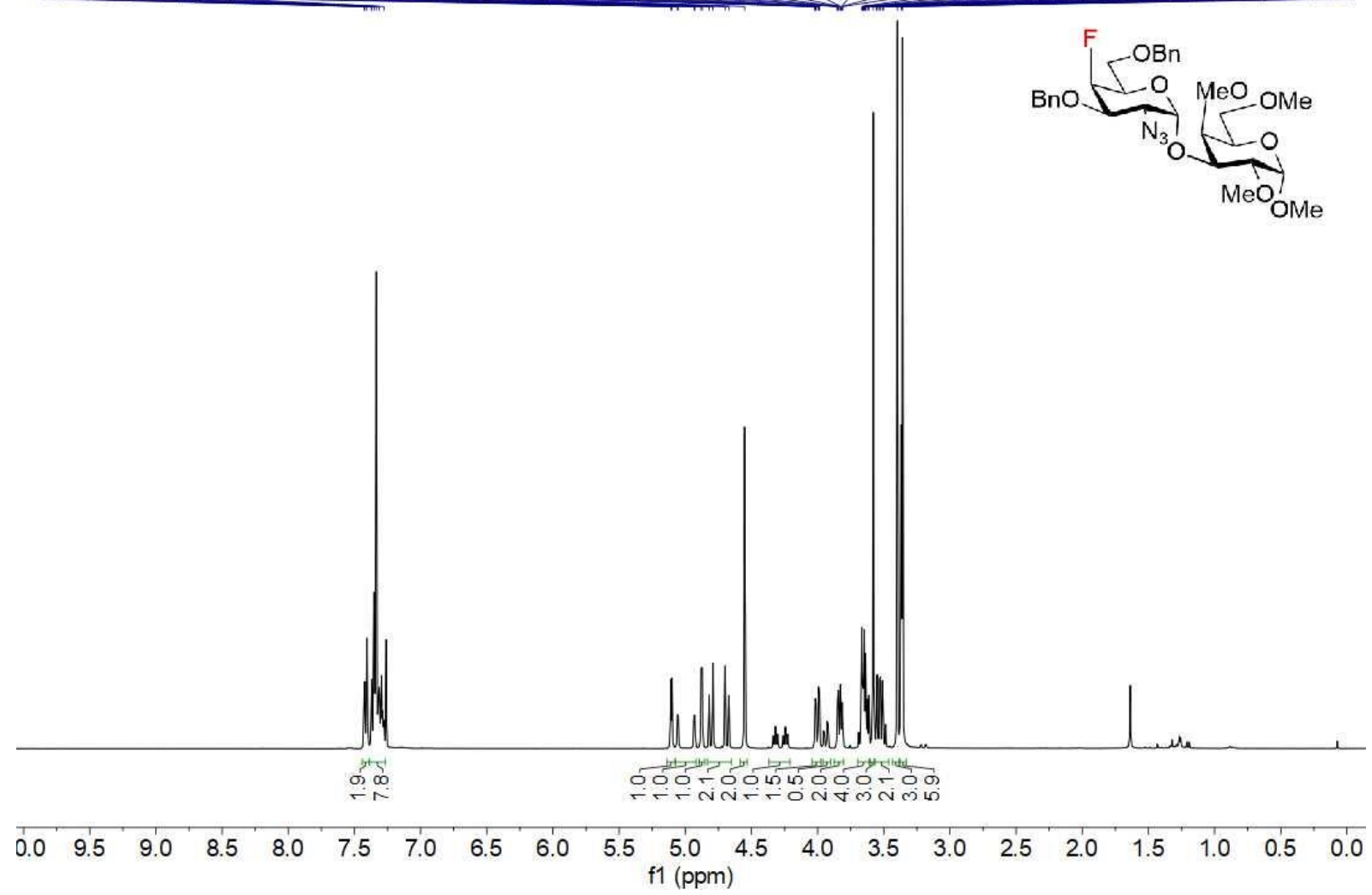

${ }^{13} \mathrm{C}\left\{{ }^{1} \mathrm{H}\right\}$ NMR $100 \mathrm{MHz}, \mathrm{CDCl}_{3}$ 4D- $\alpha$

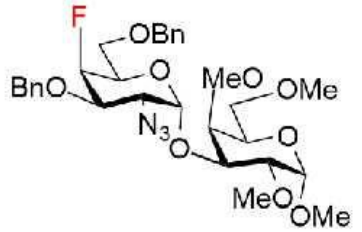

으윰요

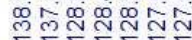

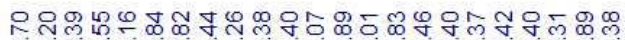

与ั

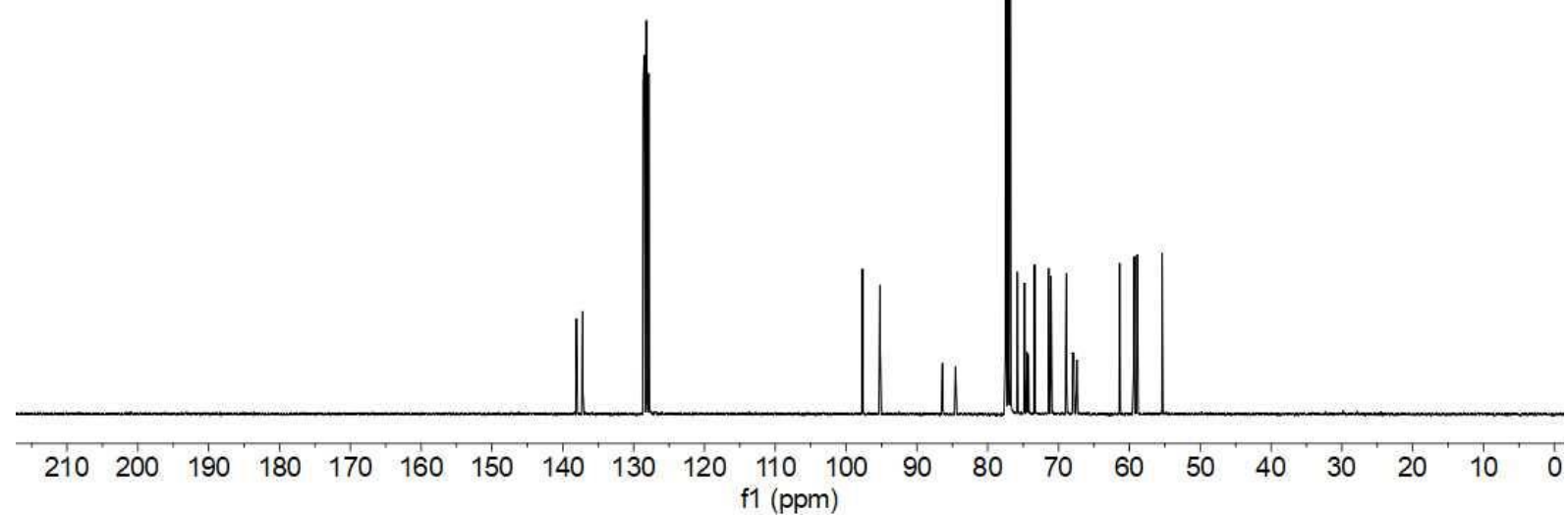


${ }^{1} \mathrm{H}-{ }^{1} \mathrm{H}$ COSY NMR 4D- $\alpha$

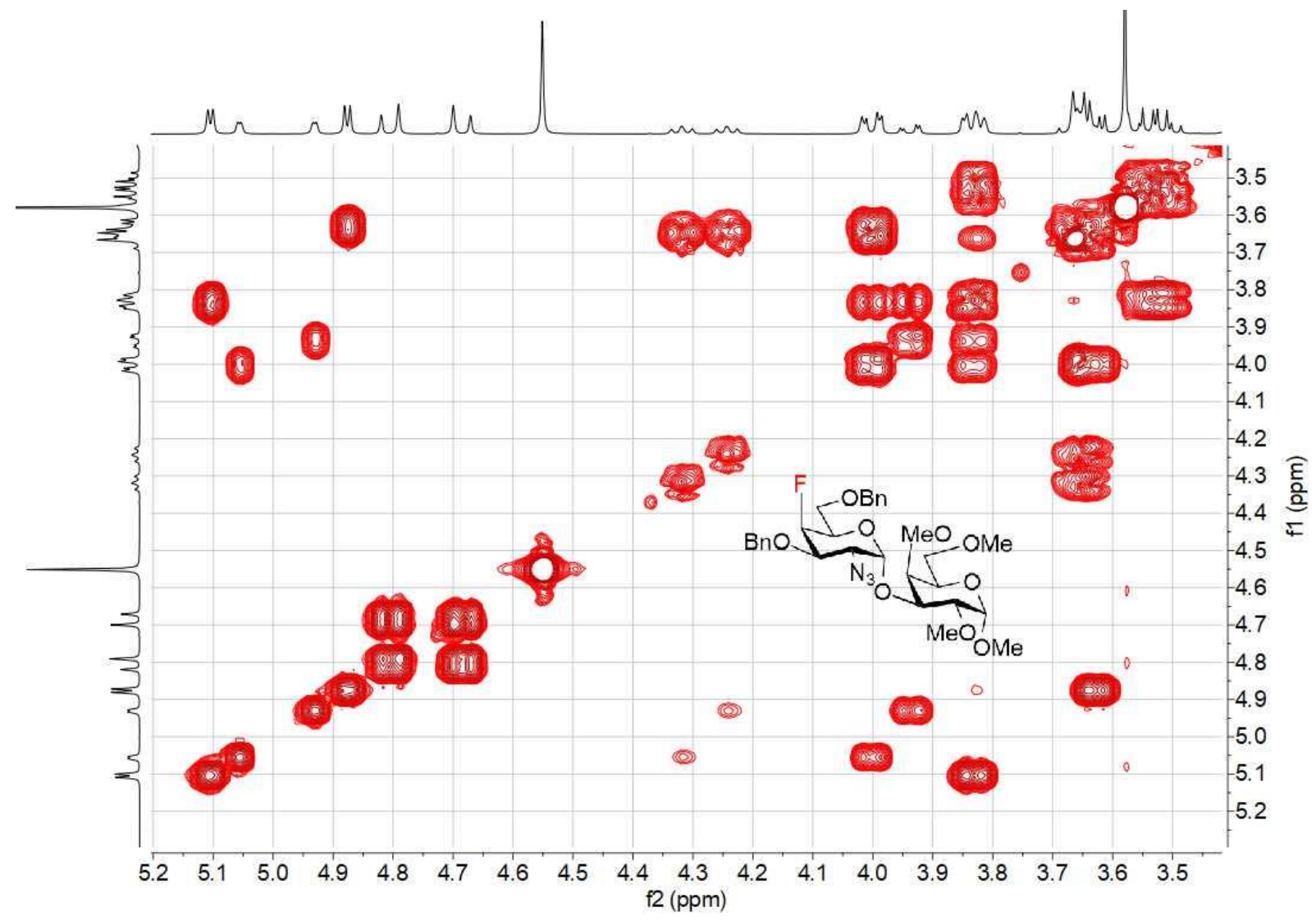

${ }^{1} \mathrm{H}-{ }^{13} \mathrm{C}$ HSQC NMR 4D- $\alpha$

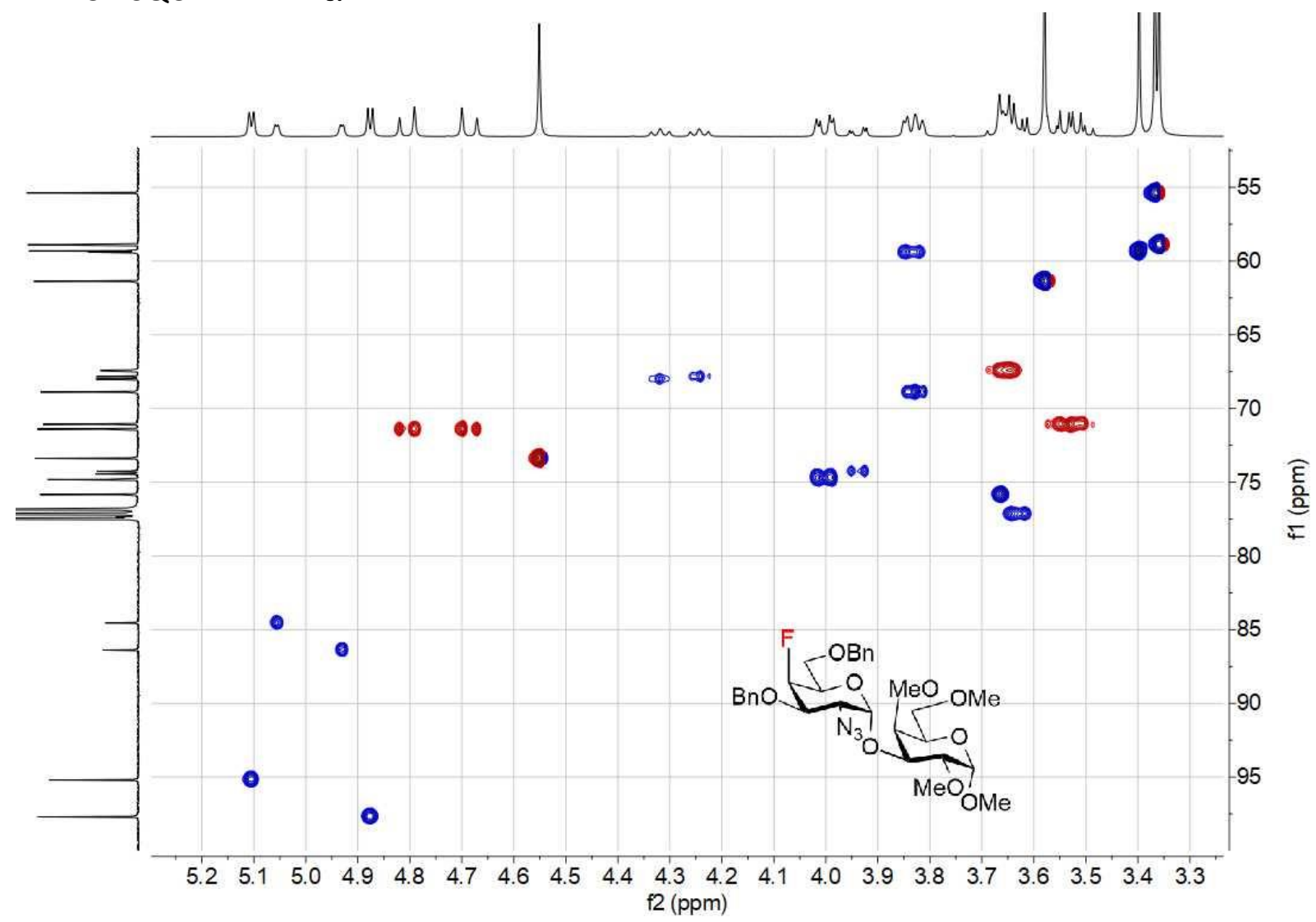




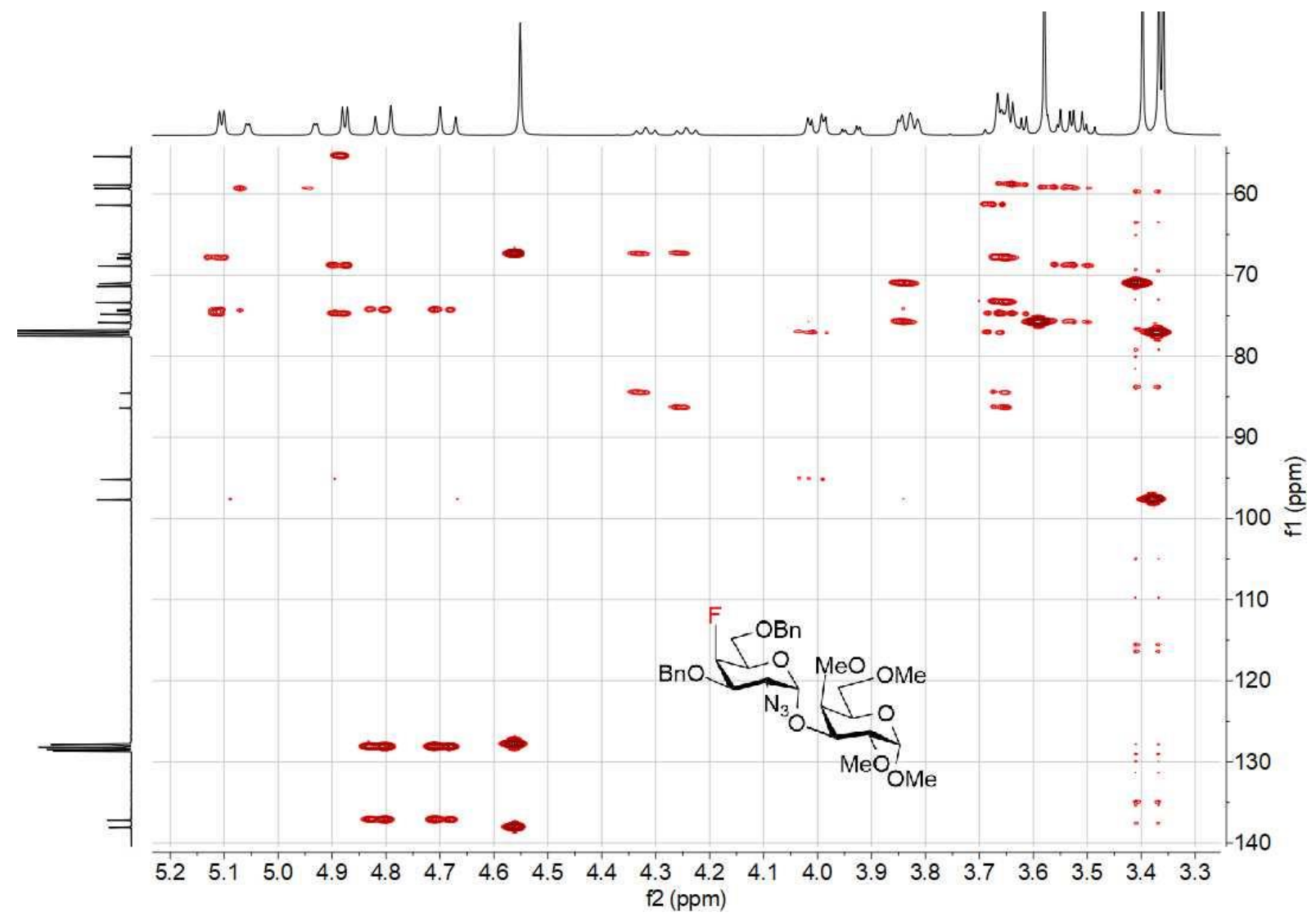

${ }^{19} \mathrm{~F} \mathrm{NMR} 376 \mathrm{MHz}, \mathrm{CDCl}_{3}$ 4D- $\alpha$

i⿱乛龰⿰
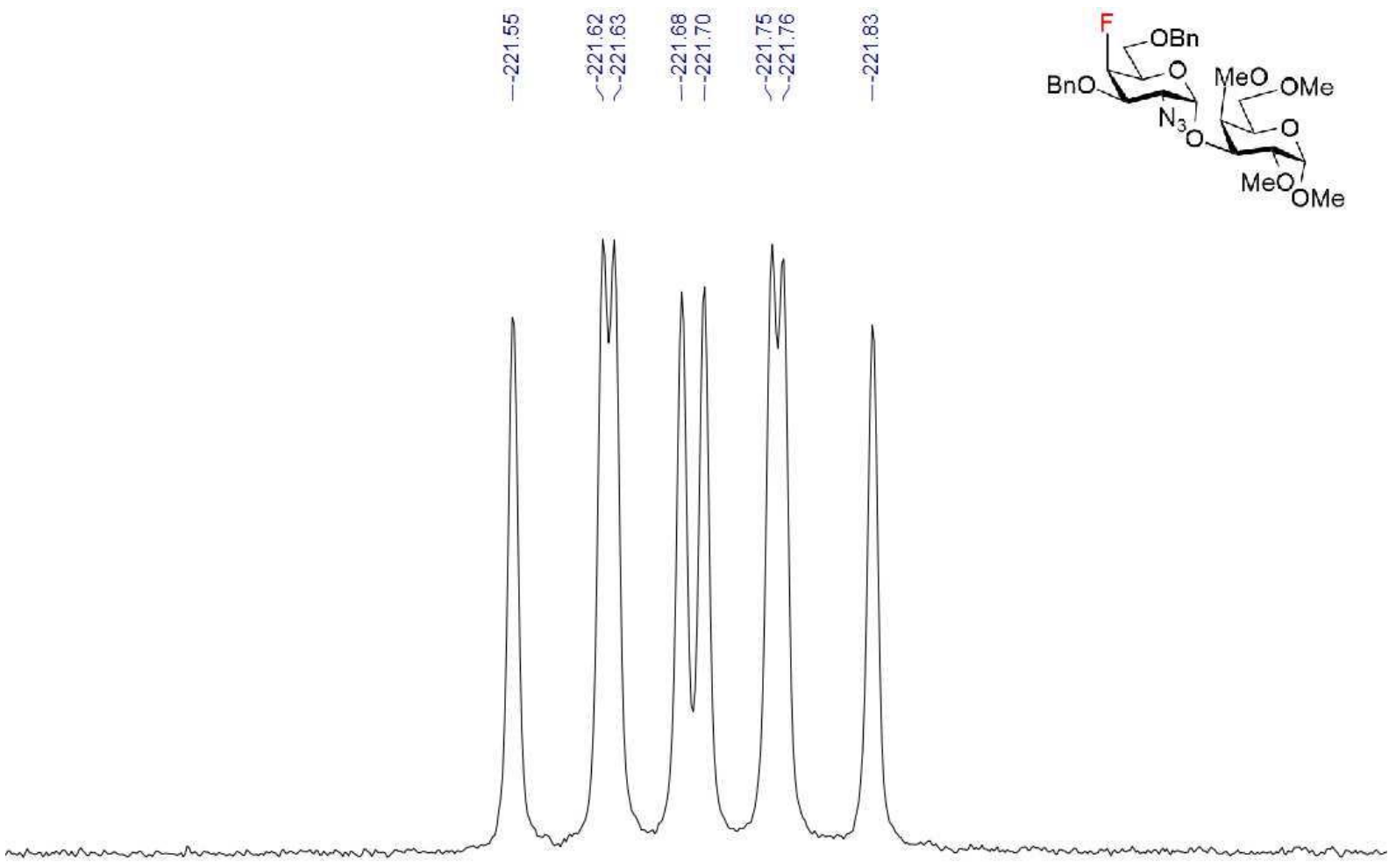

$\begin{array}{lllllllllllll}-221.20 & -221.30 & -221.40 & -221.50 & -221.60 & \begin{array}{r}-221.70 \\ f 1(\mathrm{ppm})\end{array} & -221.80 & -221.90 & -222.00 & -222.10 & -222.20\end{array}$ 
NMR D4- $\beta$

${ }^{1} \mathrm{H}$ NMR $400 \mathrm{MHz}, \mathrm{CDCl}_{3}$ 4D- $\beta$

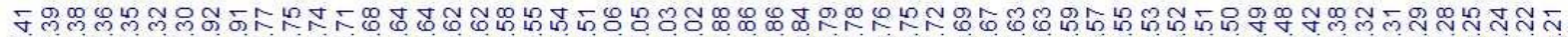

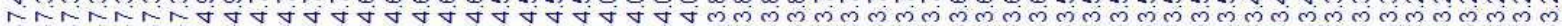

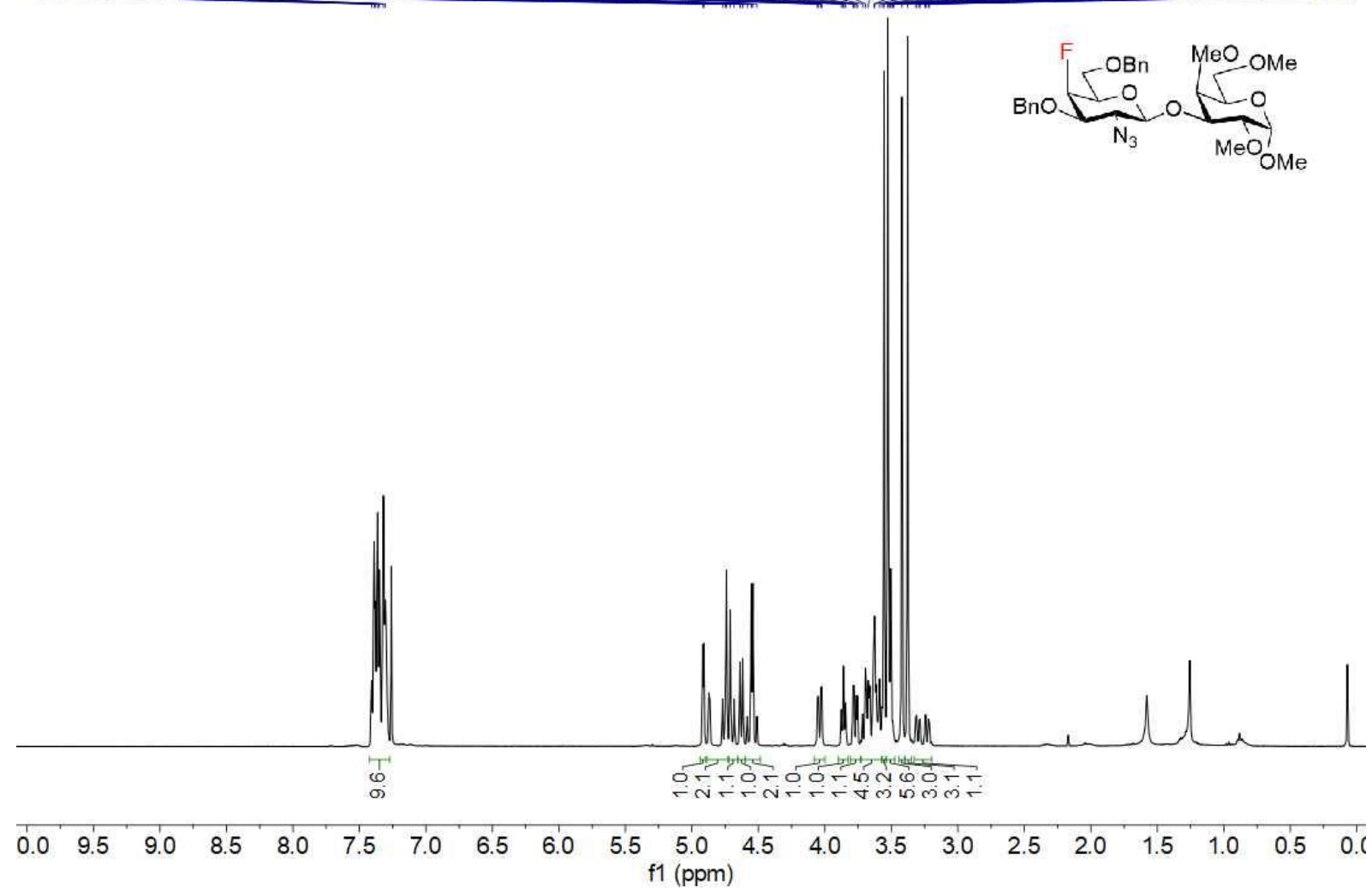

${ }^{13} \mathrm{C}\left\{{ }^{1} \mathrm{H}\right\} \mathrm{NMR}$ 100MHz, $\mathrm{CDCl}_{3} 4 \mathrm{D}-\boldsymbol{\beta}$

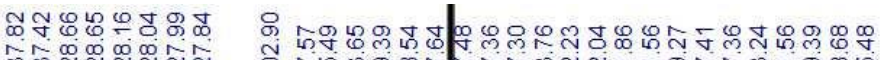

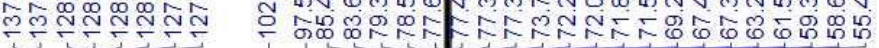

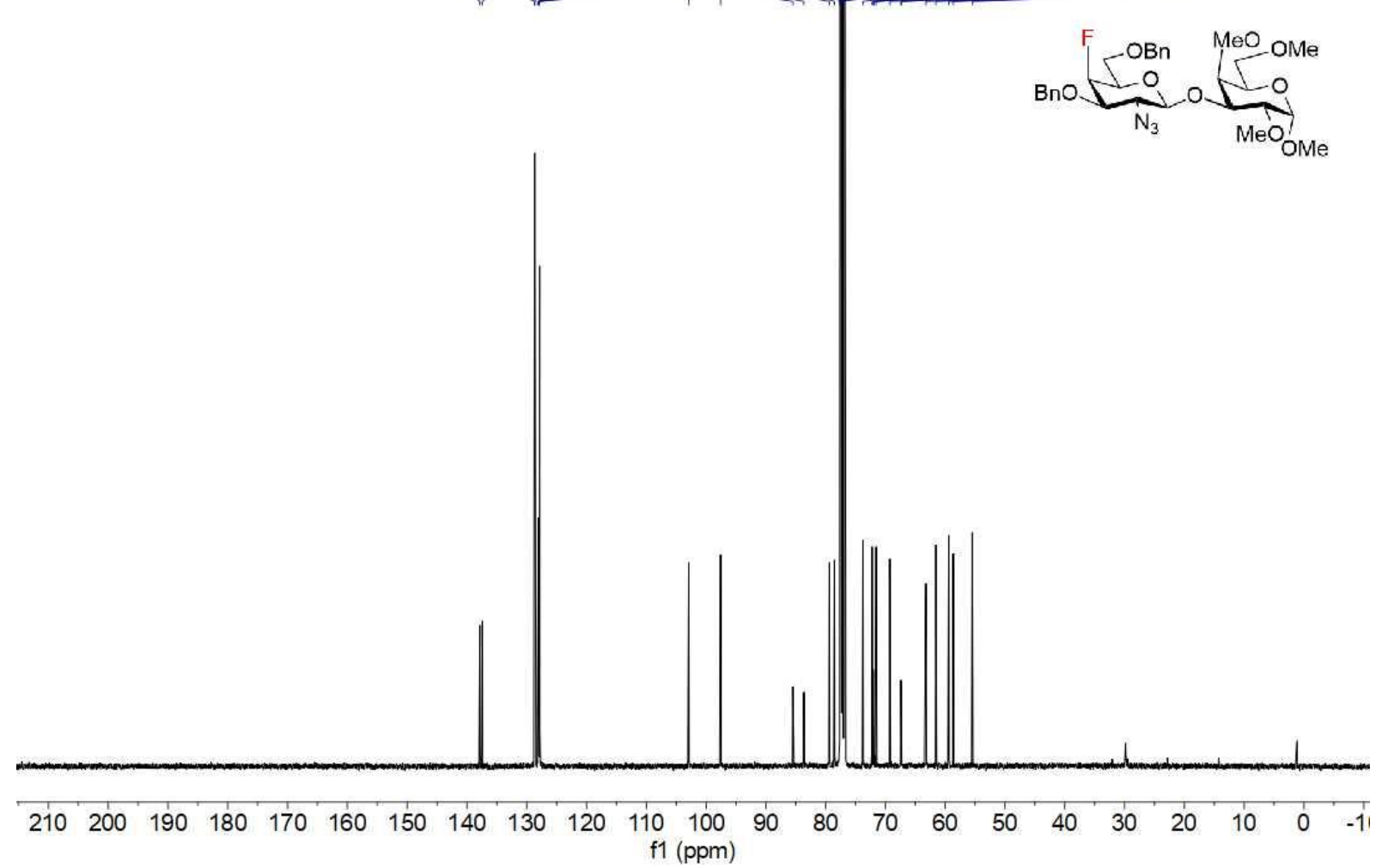


${ }^{1} \mathrm{H}-{ }^{1} \mathrm{H}$ COSY NMR 4D- $\beta$

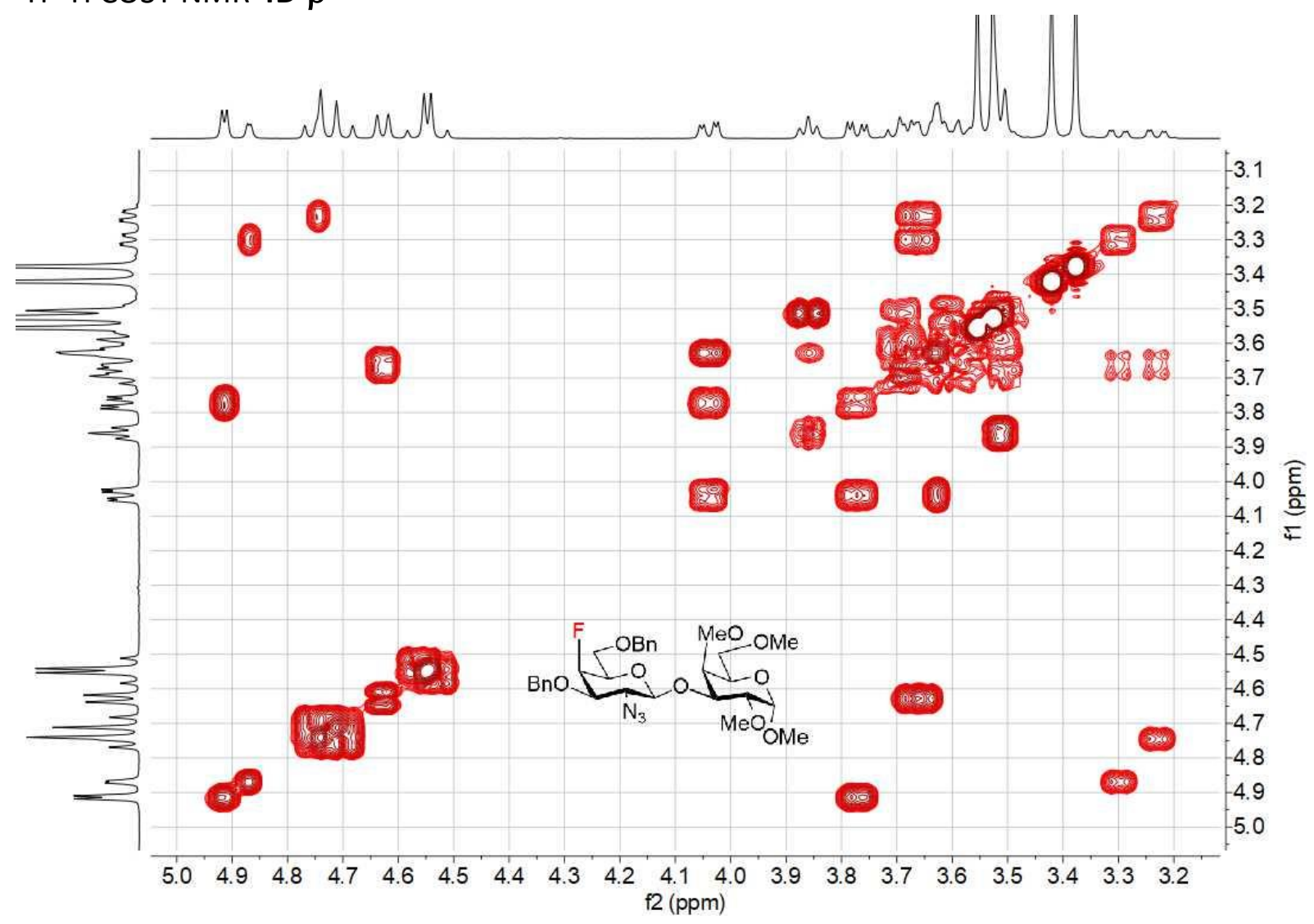

${ }^{1} \mathrm{H}^{13} \mathrm{C}$ HSQC NMR 4D- $\beta$

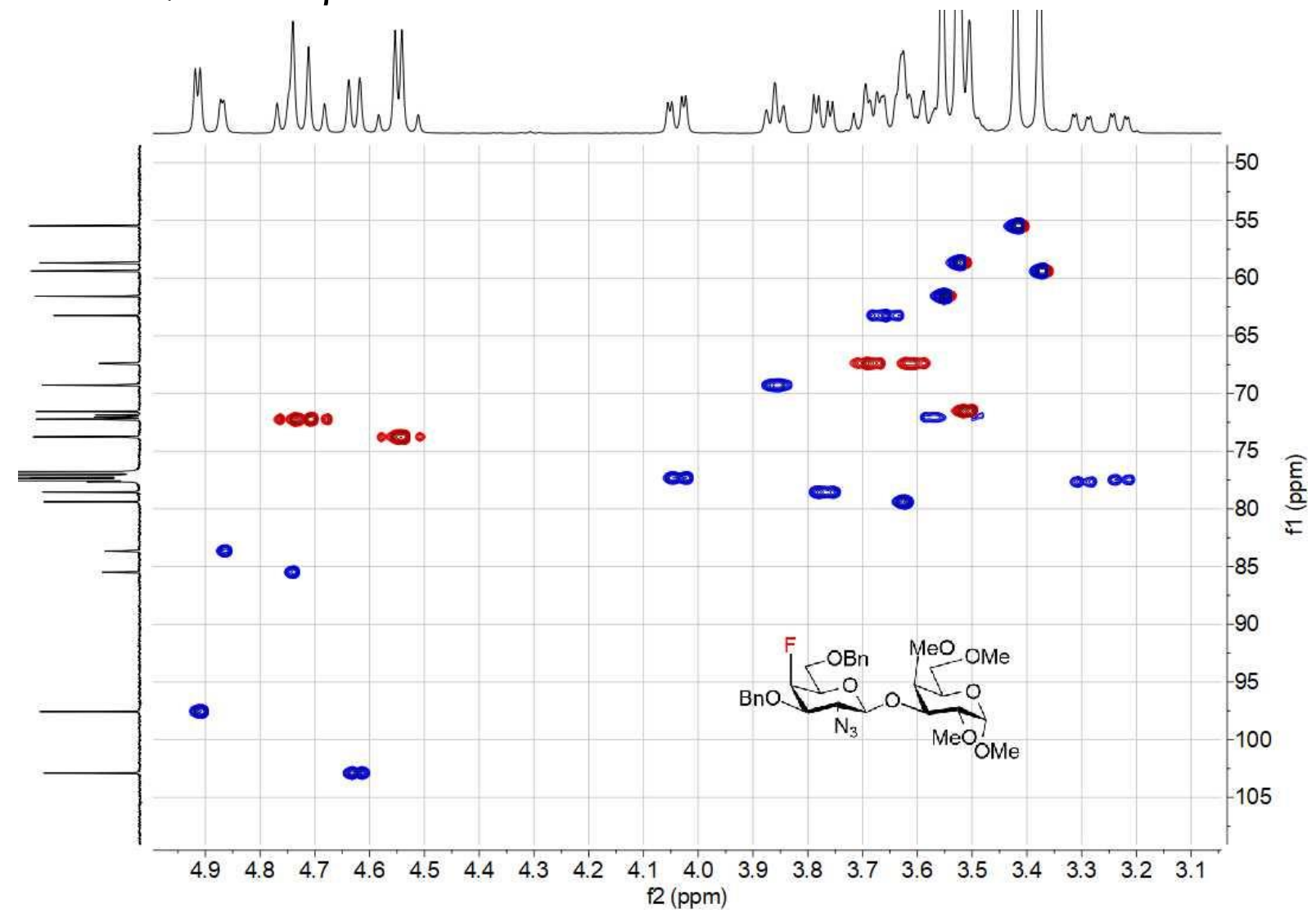


${ }^{1} \mathrm{H}-{ }^{13} \mathrm{C}$ HMBC NMR 4D- $\beta$

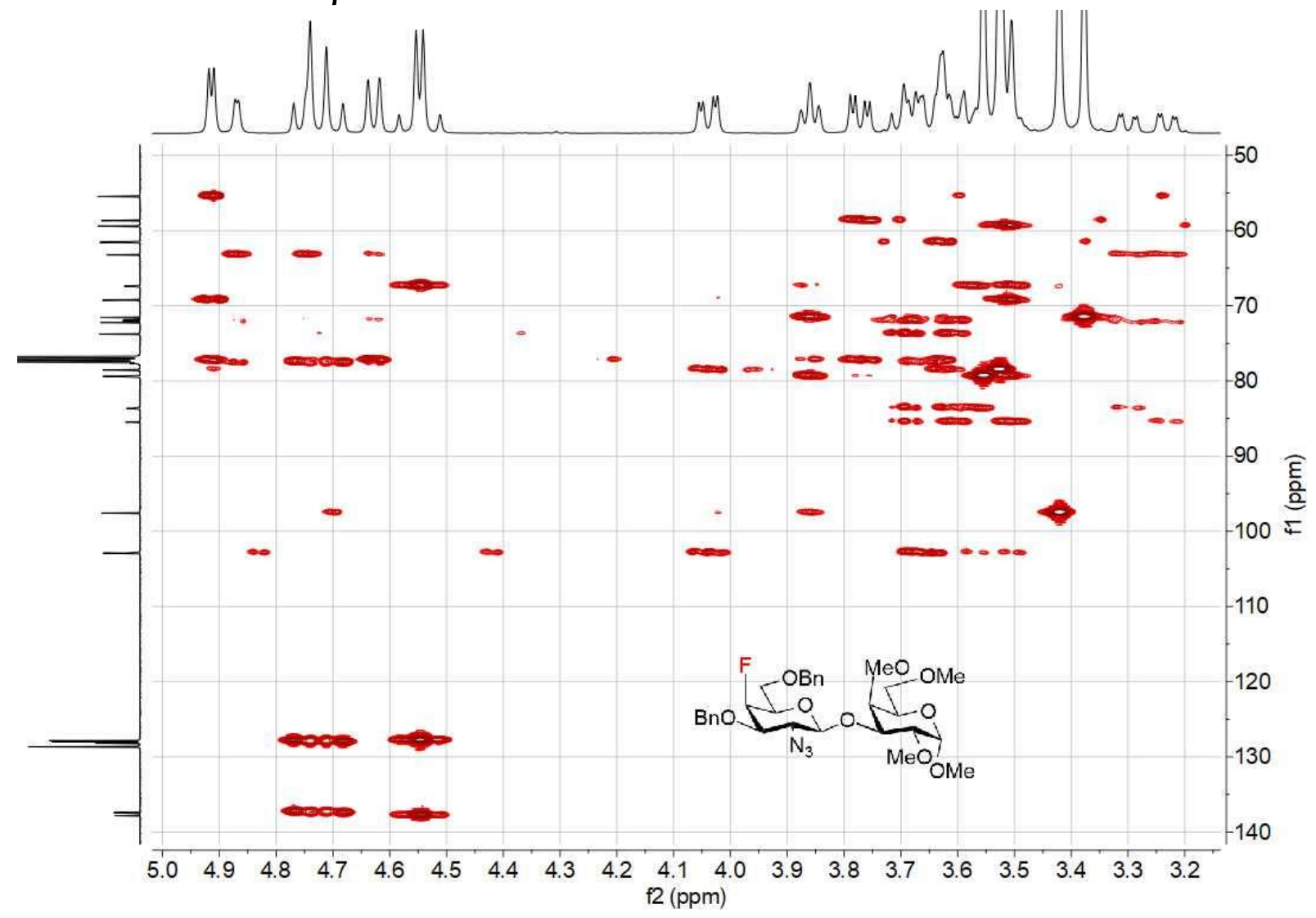

${ }^{19} \mathrm{~F} \mathrm{NMR} 376 \mathrm{MHz}, \mathrm{CDCl}_{3}$ 4D- $\beta$

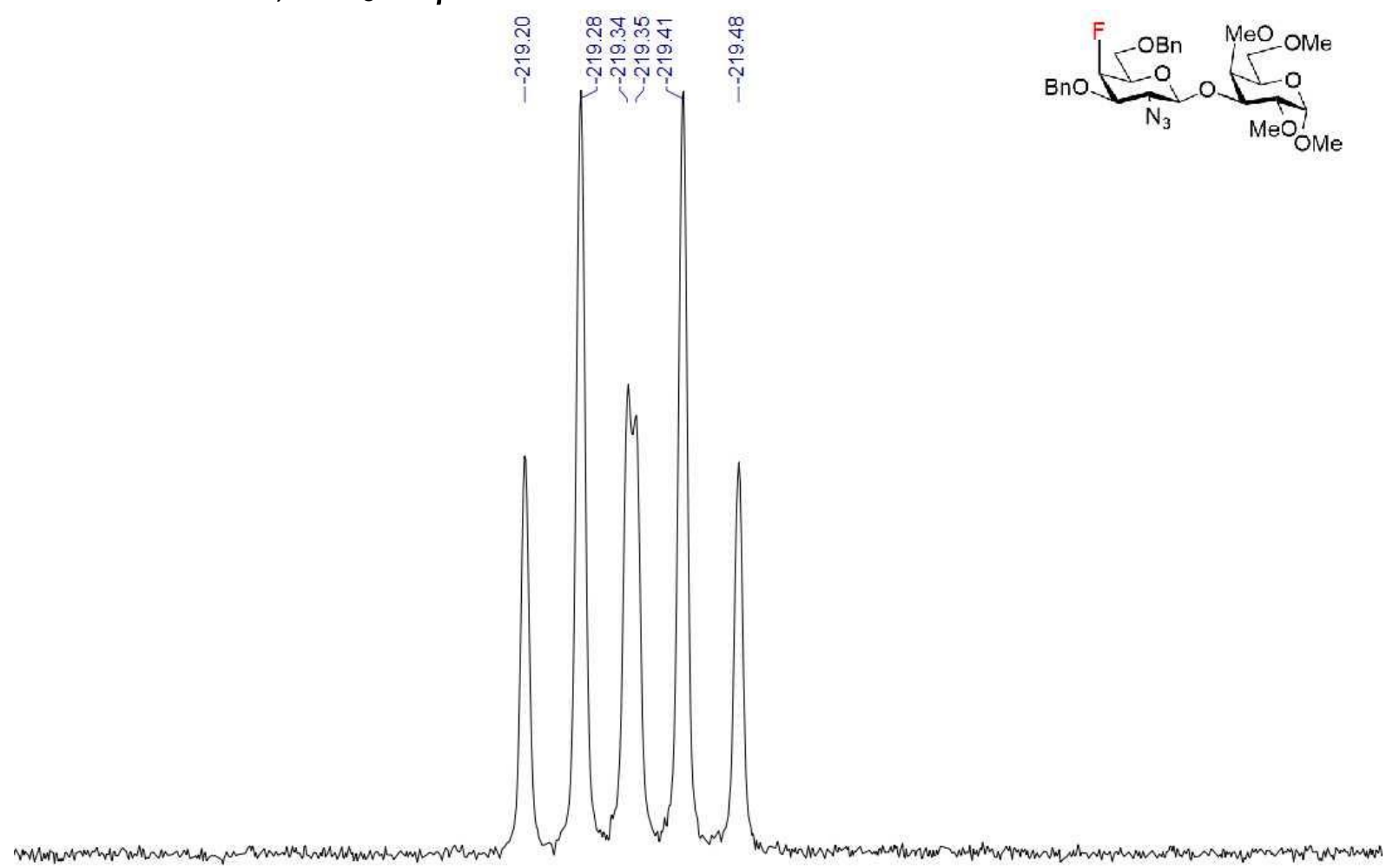

$\begin{array}{lllllllllllllllllllll}-218.6 & -218.7 & -218.8 & -218.9 & -219.0 & -219.1 & -219.2 & -219.3 & -219.4 & -219.5 & -219.6 & -219.7 & -219.8 & -219.9 & -220.0 & -220.1 & -220.2 & -22 C\end{array}$ f1 (ppm) 


\section{NMR 4E- $\alpha$}

${ }^{1} \mathrm{HNMR} 400 \mathrm{MHz}, \mathrm{CDCl}_{3} 4 \mathrm{E}-\alpha$

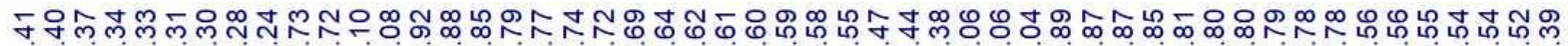

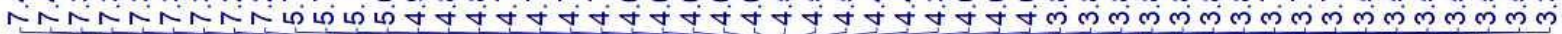

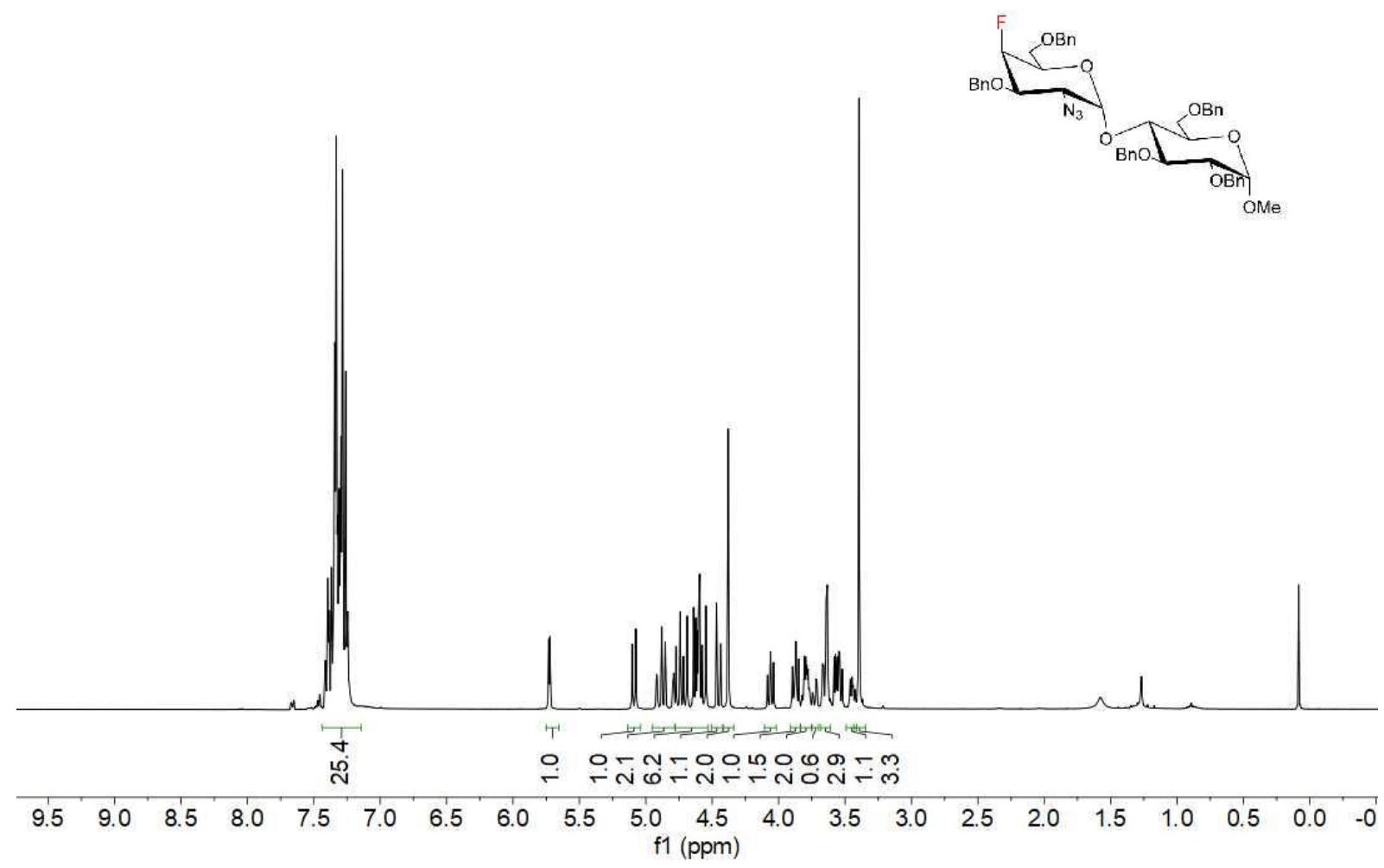

${ }^{13} \mathrm{C}\left\{{ }^{1} \mathrm{H}\right\} \mathrm{NMR} 100 \mathrm{MHz}, \mathrm{CDCl}_{3} 4 \mathrm{E}-\alpha$

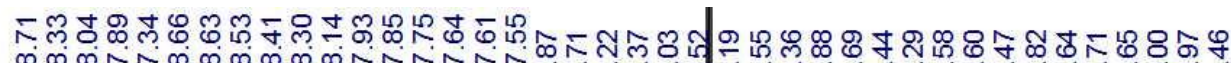

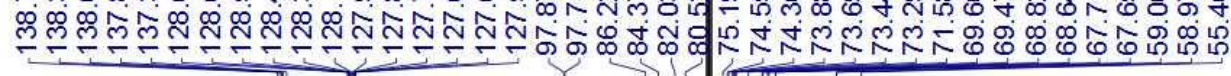

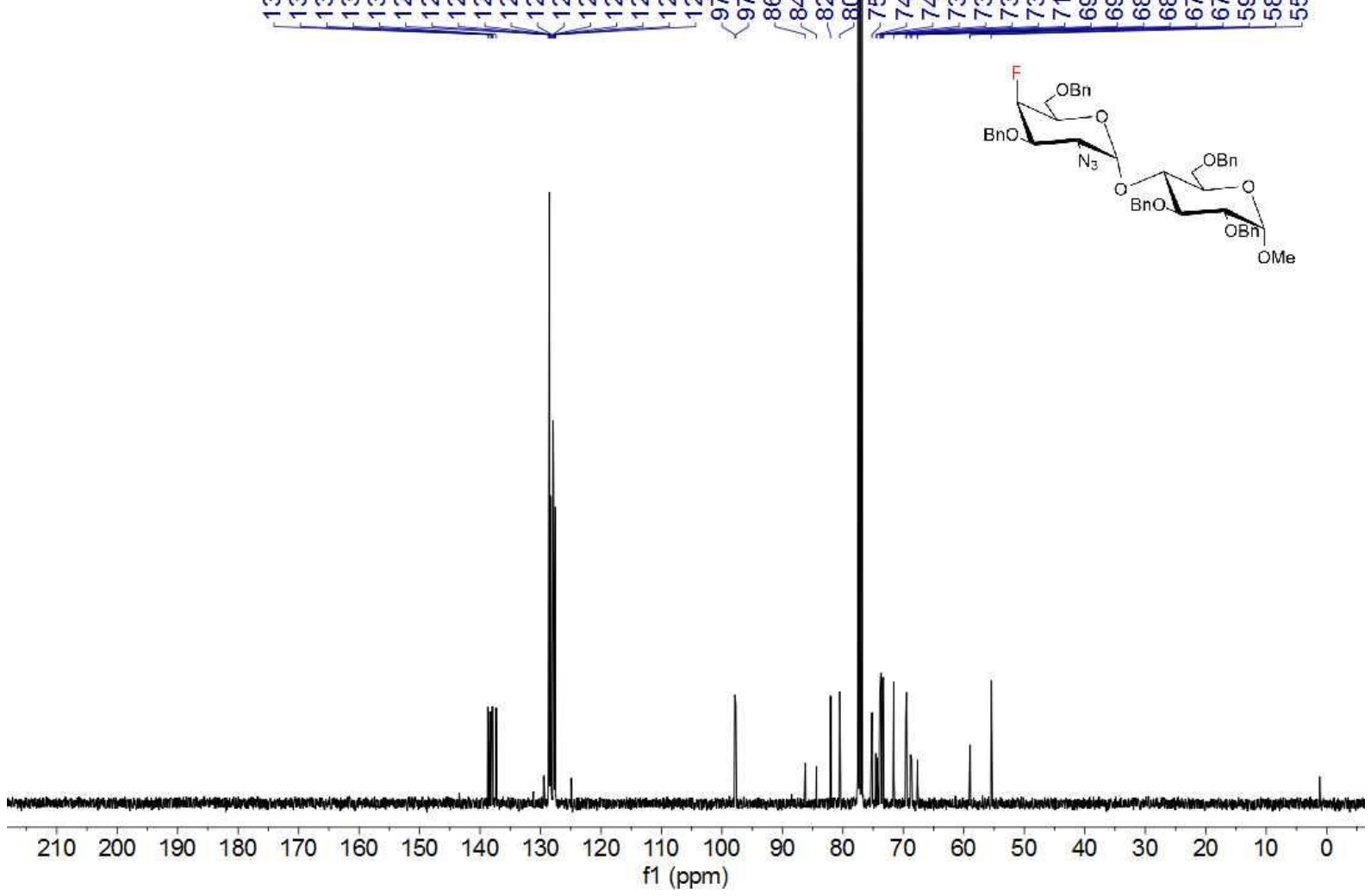


${ }^{1} \mathrm{H}-{ }^{1} \mathrm{H}$ COSY NMR $4 \mathrm{E}-\alpha$

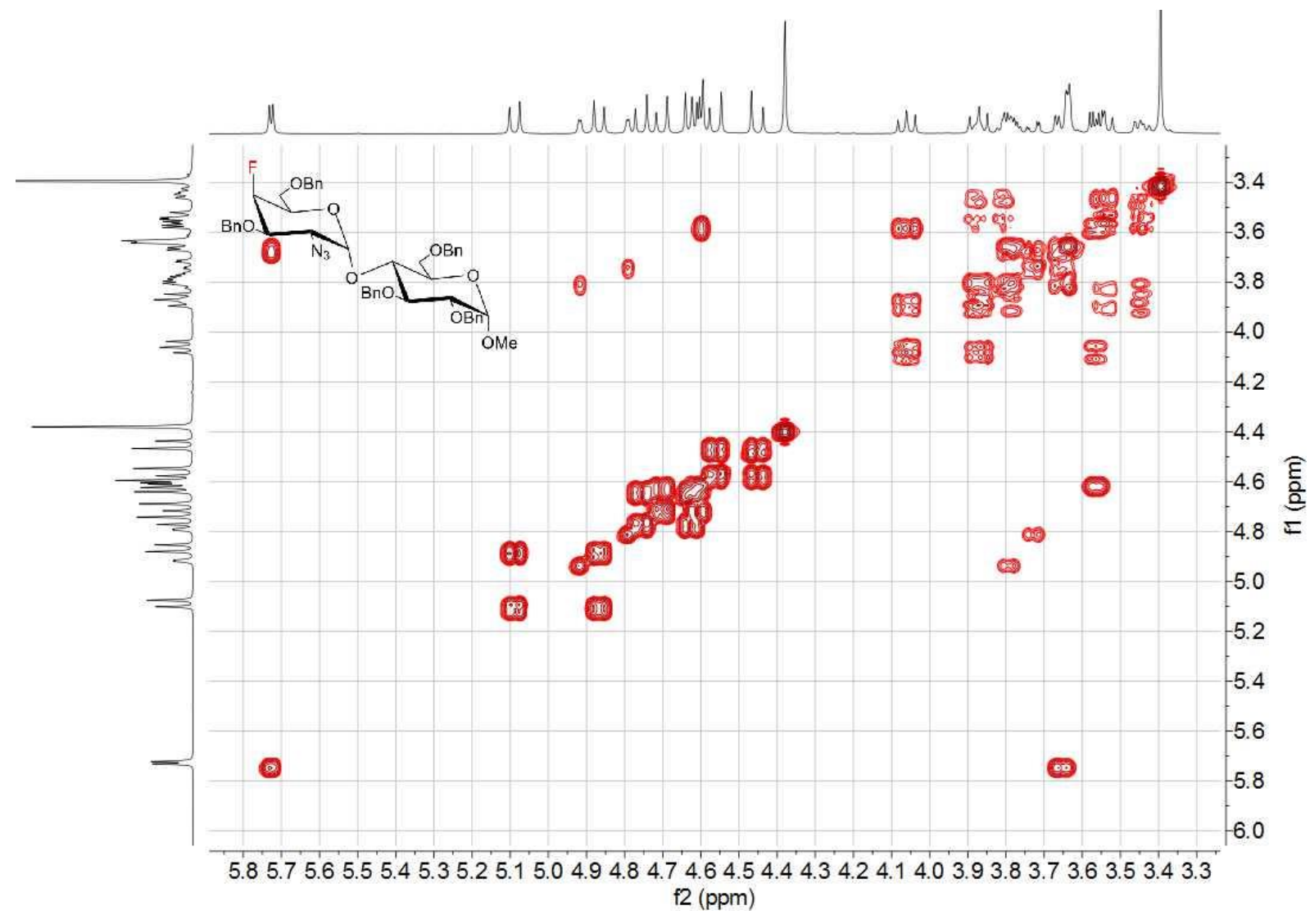

${ }^{1} \mathrm{H}-{ }^{13} \mathrm{C}$ HSQC NMR 4E- $\alpha$

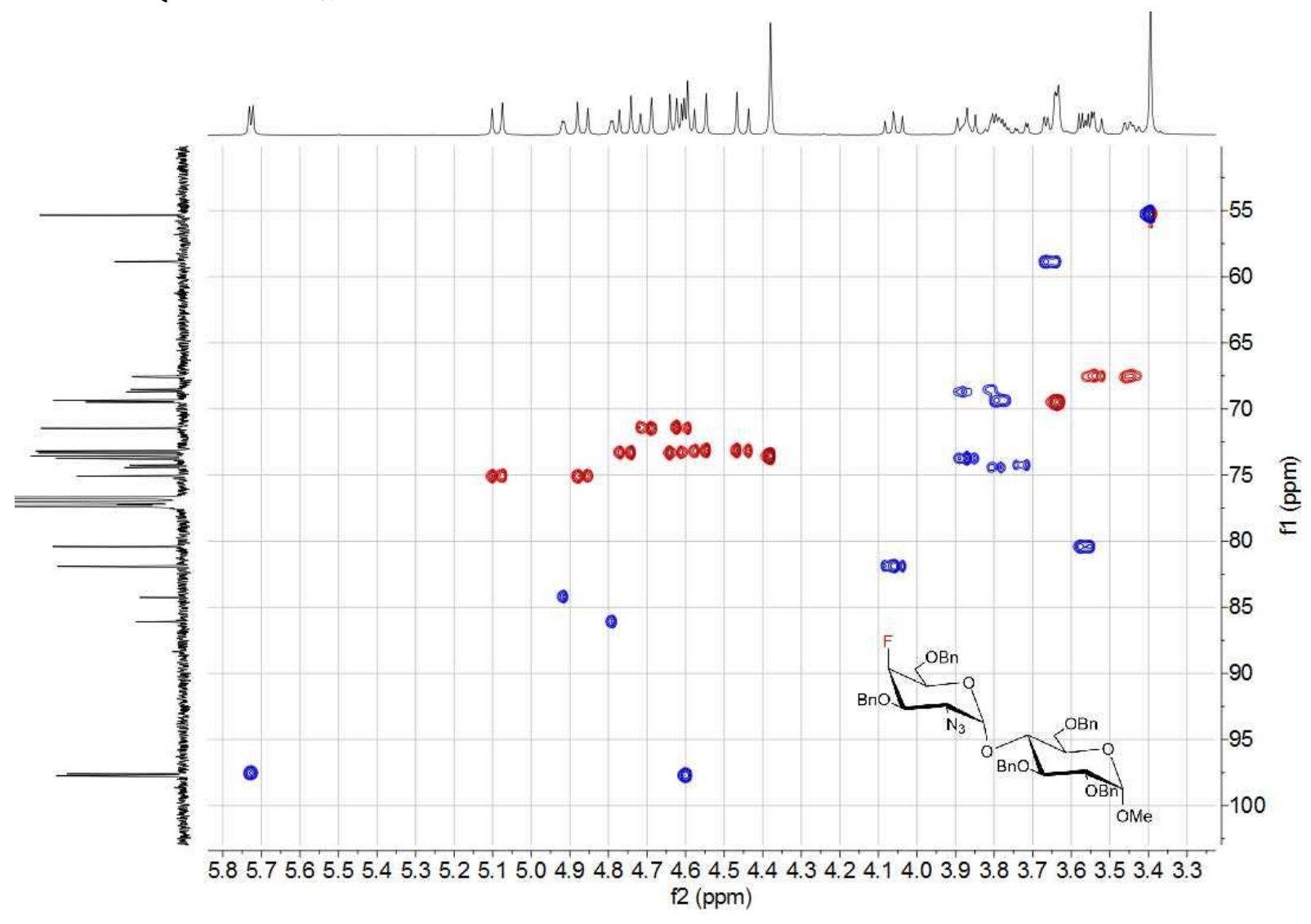


${ }^{1} \mathrm{H}-{ }^{13} \mathrm{C}$ HMBC NMR 4E- $\alpha$

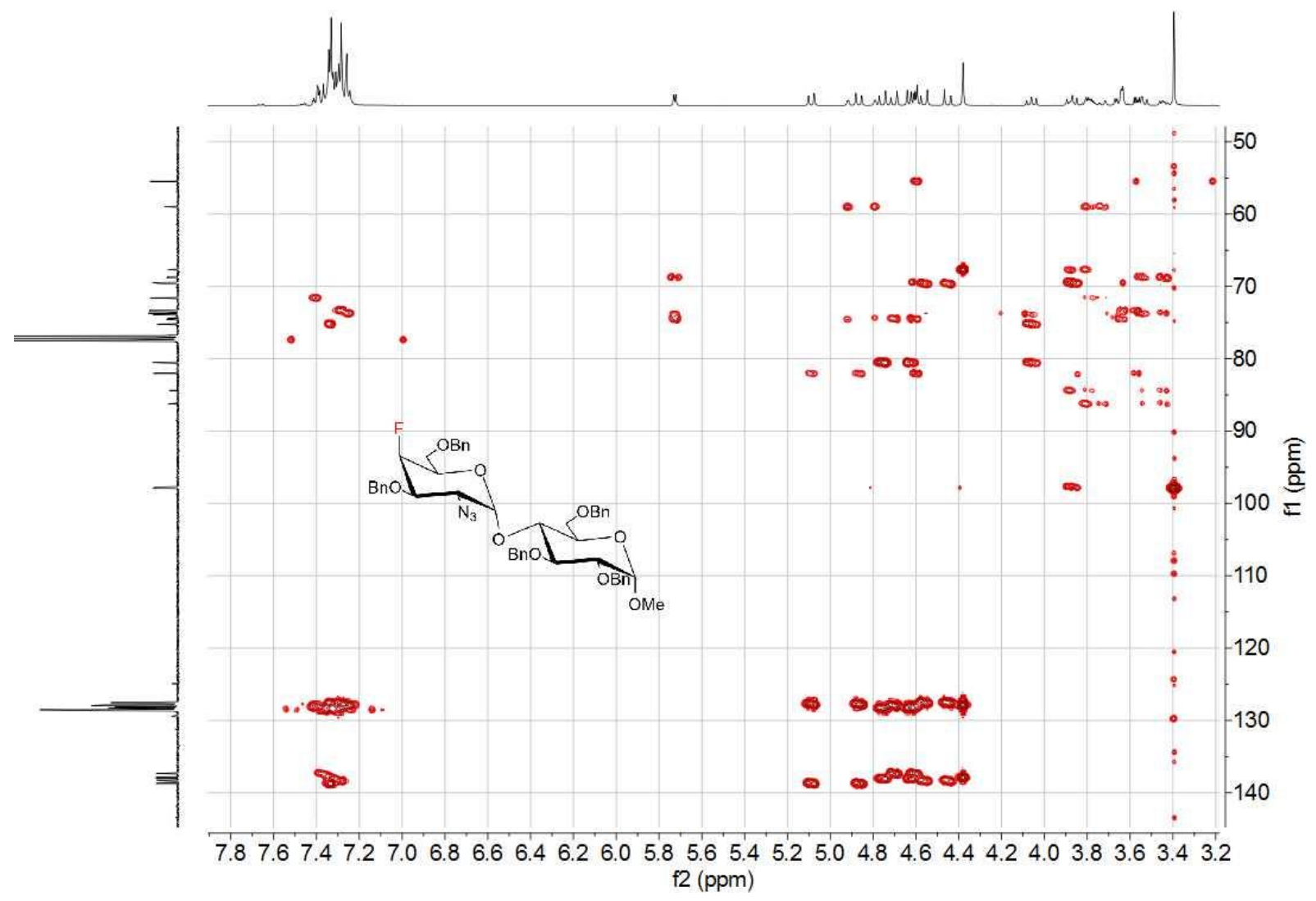

${ }^{19} \mathrm{~F}$ NMR $376 \mathrm{MHz}, \mathrm{CDCl}_{3} 4 \mathrm{E}-\alpha$

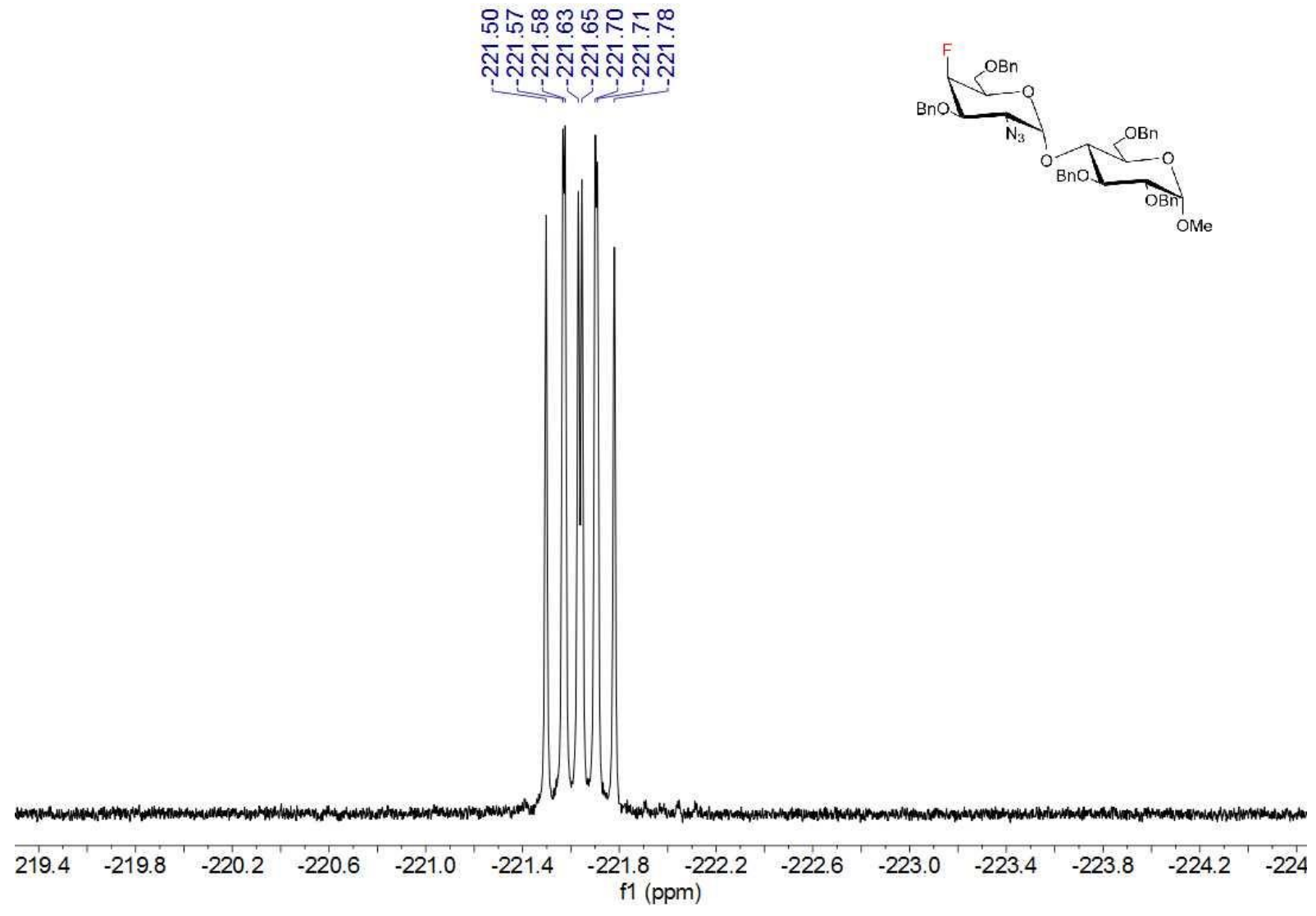


NMR 4E- $\beta$

${ }^{1} \mathrm{H} N M R 400 \mathrm{MHz}, \mathrm{CDCl}_{3} 4 \mathrm{E}-\boldsymbol{\beta}$

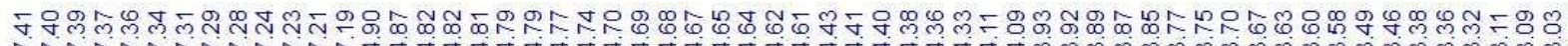

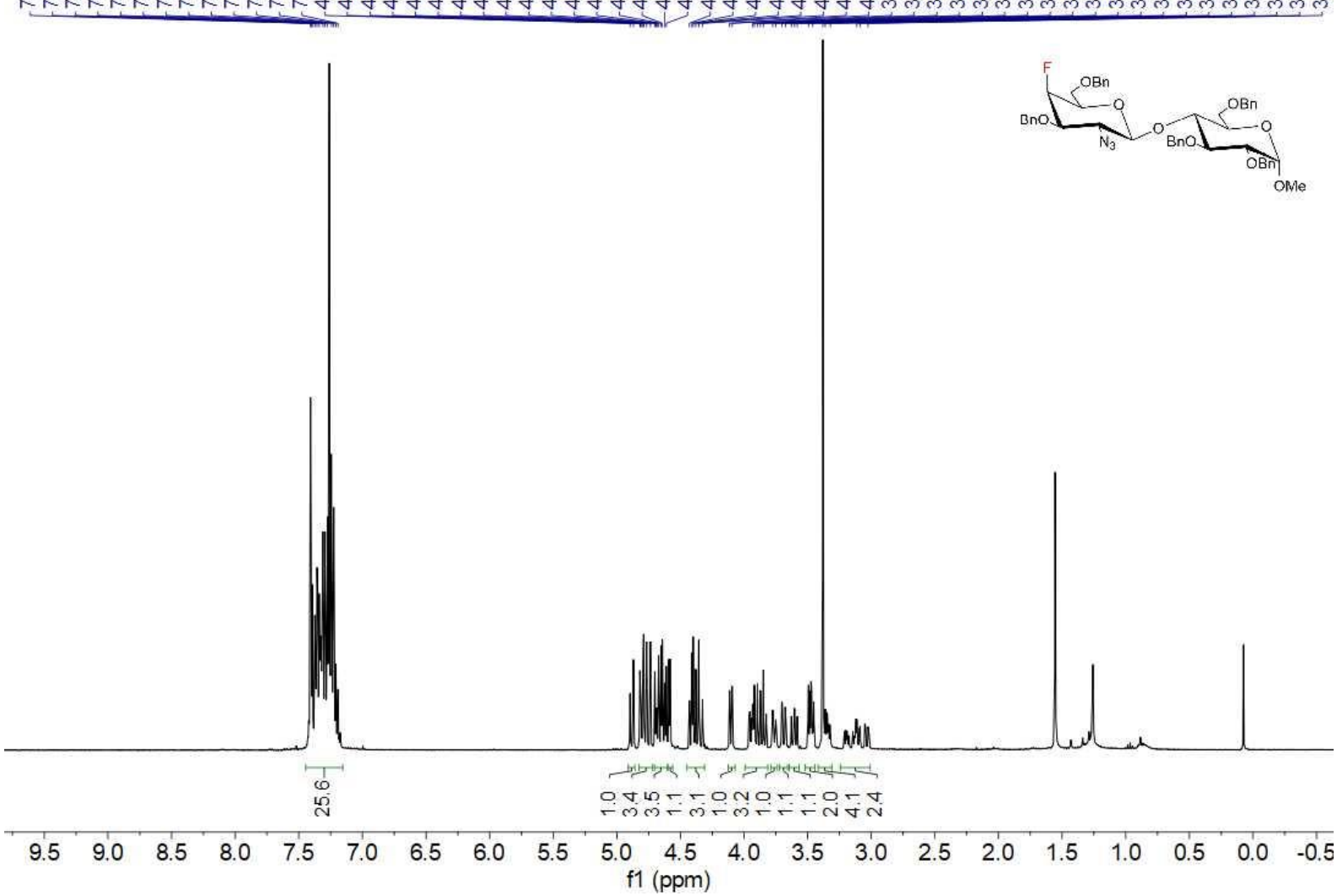

${ }^{13} \mathrm{C}\left\{{ }^{1} \mathrm{H}\right\}$ NMR 100MHz, $\mathrm{CDCl}_{3} 4 \mathrm{E}-\boldsymbol{\beta}$

ल우을

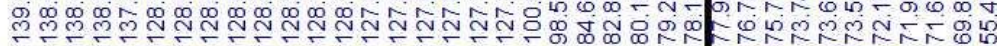

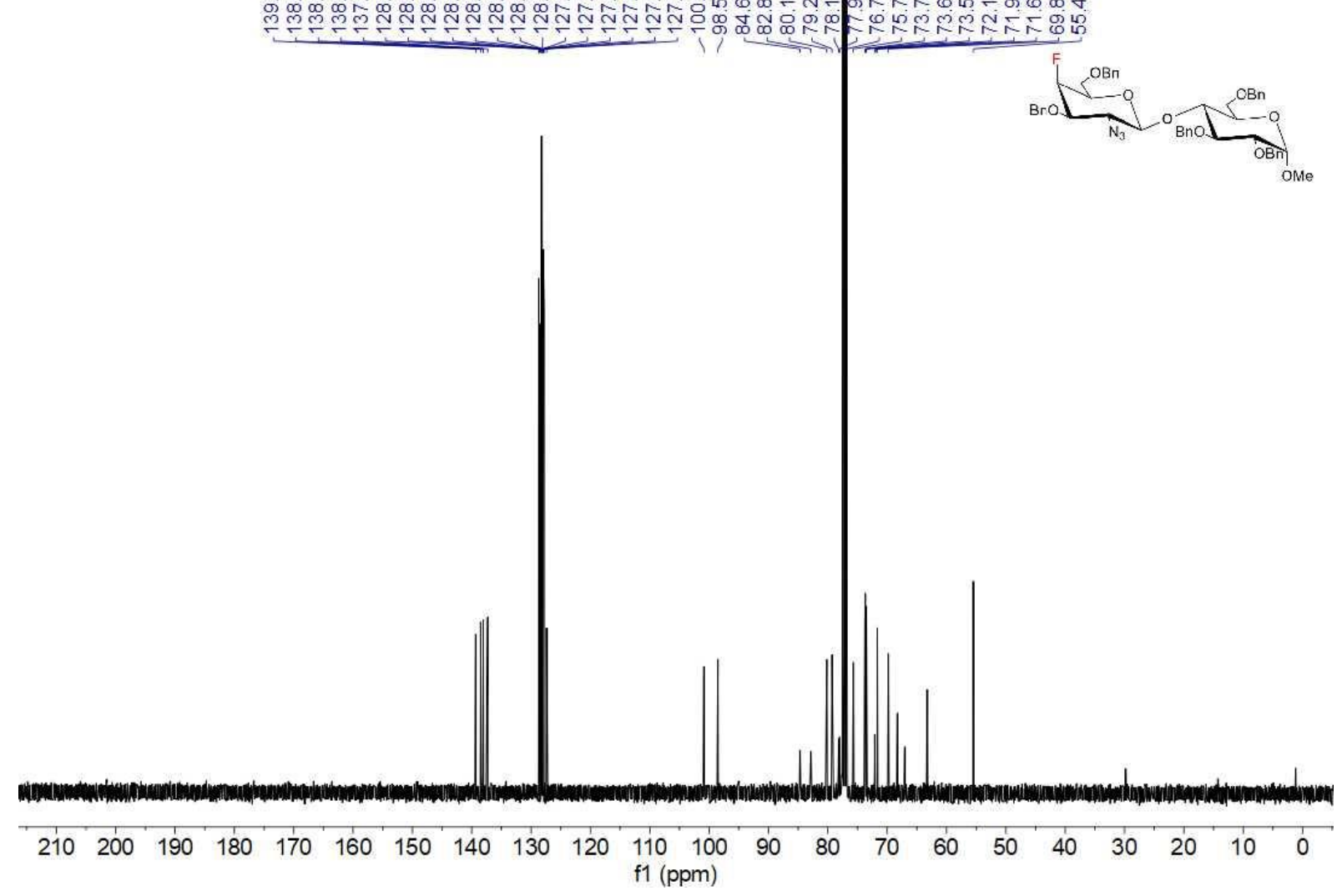


${ }^{1} \mathrm{H}-{ }^{1} \mathrm{H}$ COSY NMR $4 \mathrm{E}-\beta$

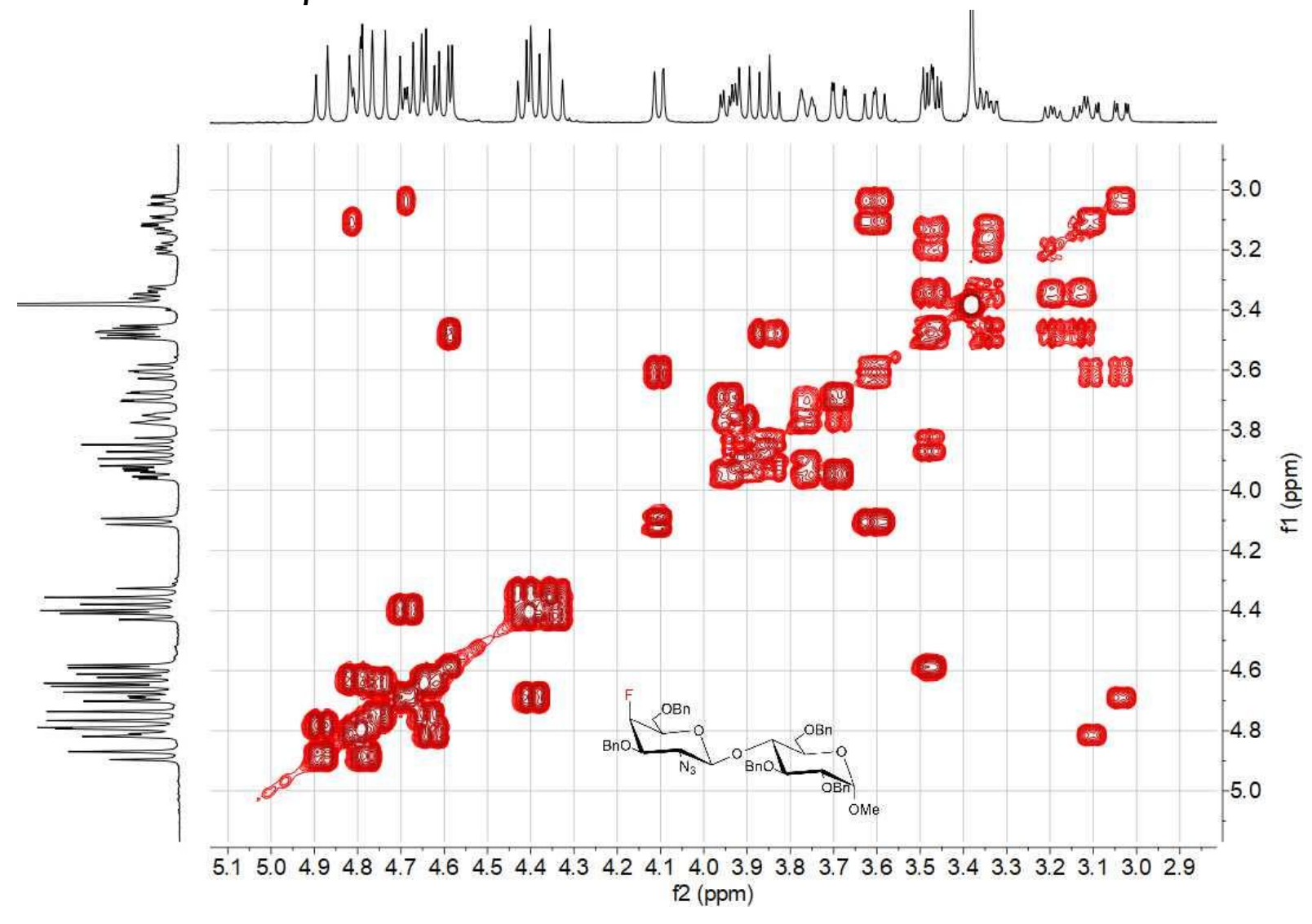

${ }^{1} \mathrm{H}-{ }^{13} \mathrm{C}$ HSQC NMR 4E- $\beta$

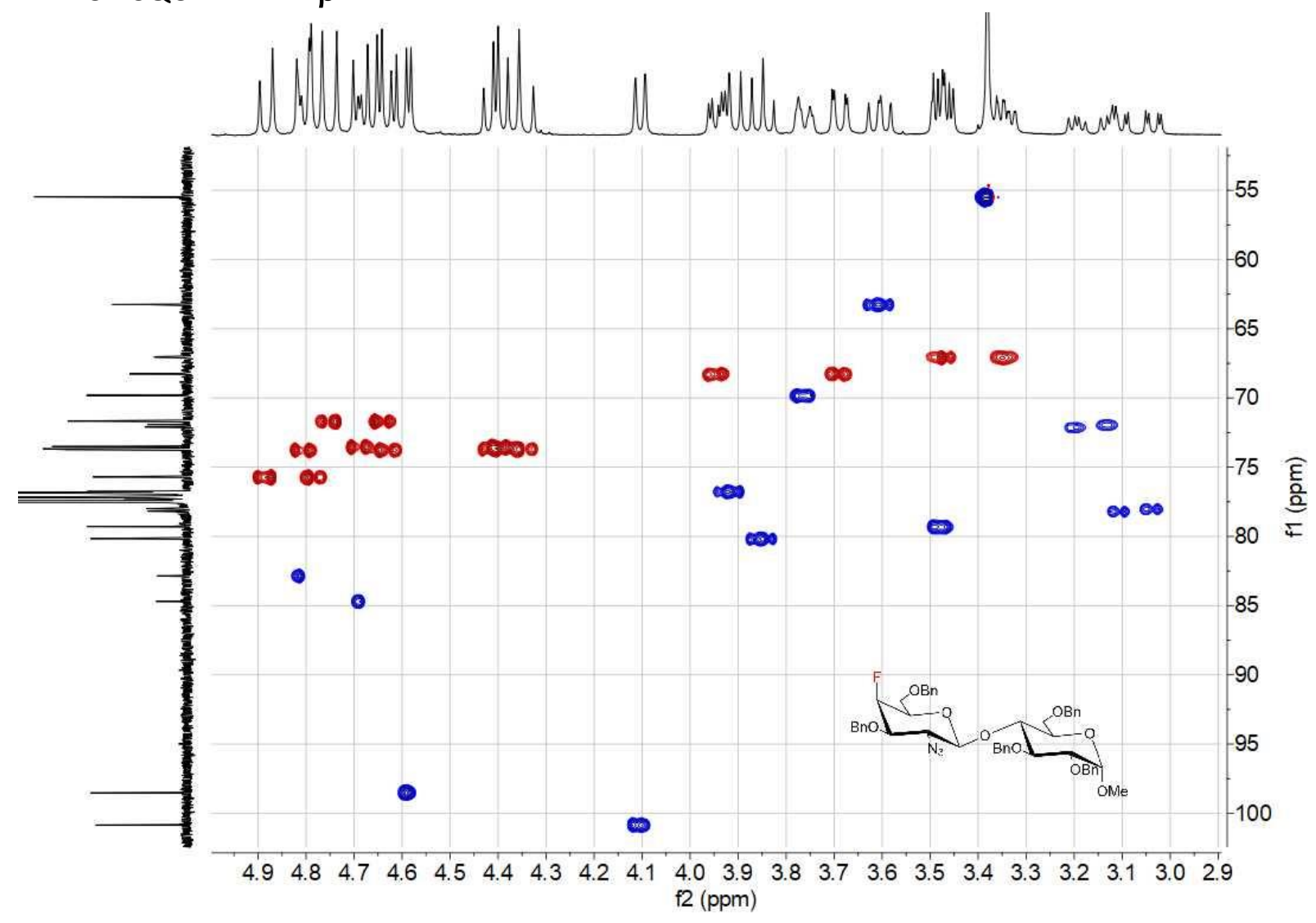


${ }^{1} \mathrm{H}-{ }^{13} \mathrm{C}$ HMBC NMR 4E- $\beta$

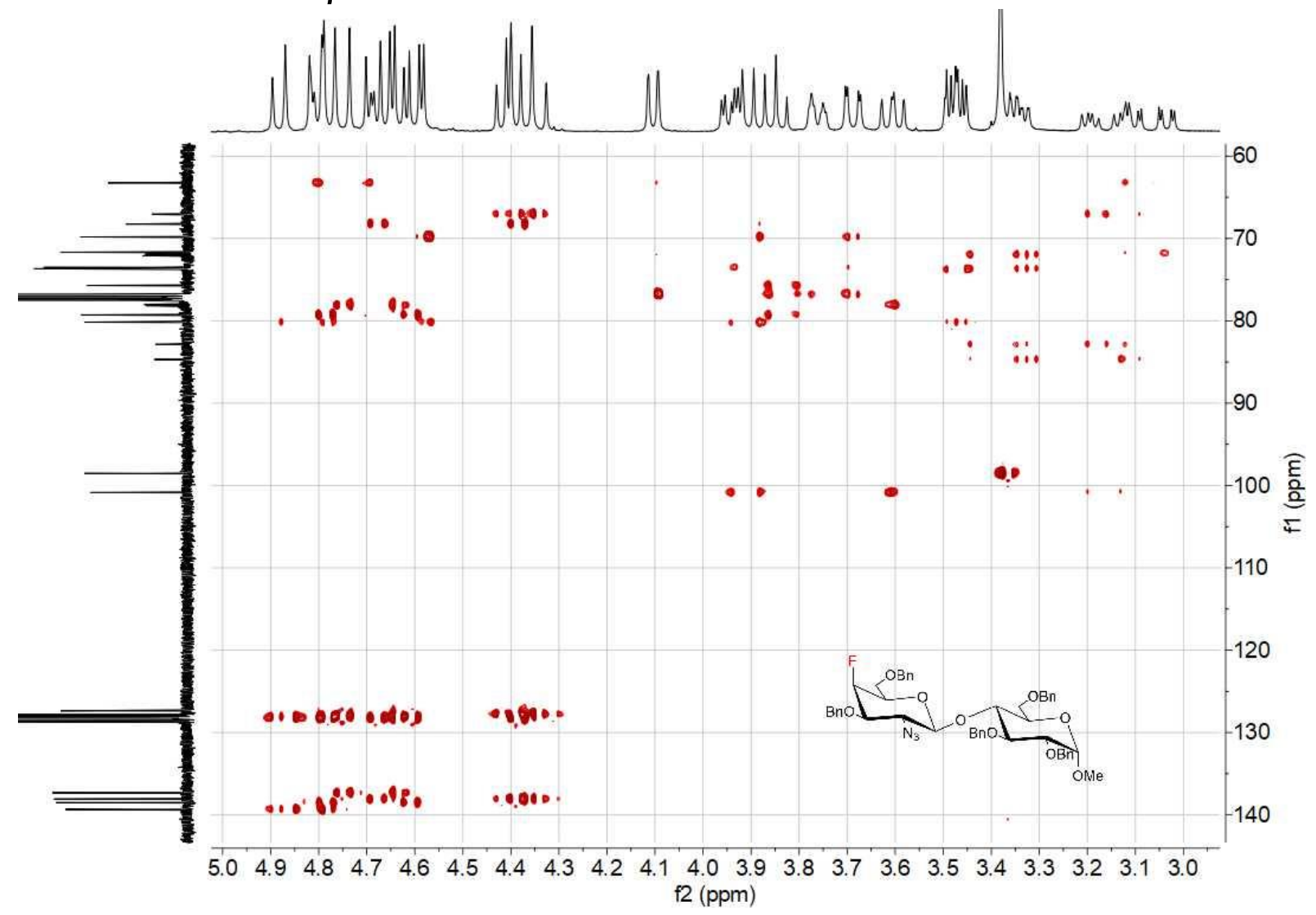

${ }^{19} \mathrm{~F} \mathrm{NMR} 376 \mathrm{MHz}, \mathrm{CDCl}_{3} 4 \mathrm{E}-\boldsymbol{\beta}$

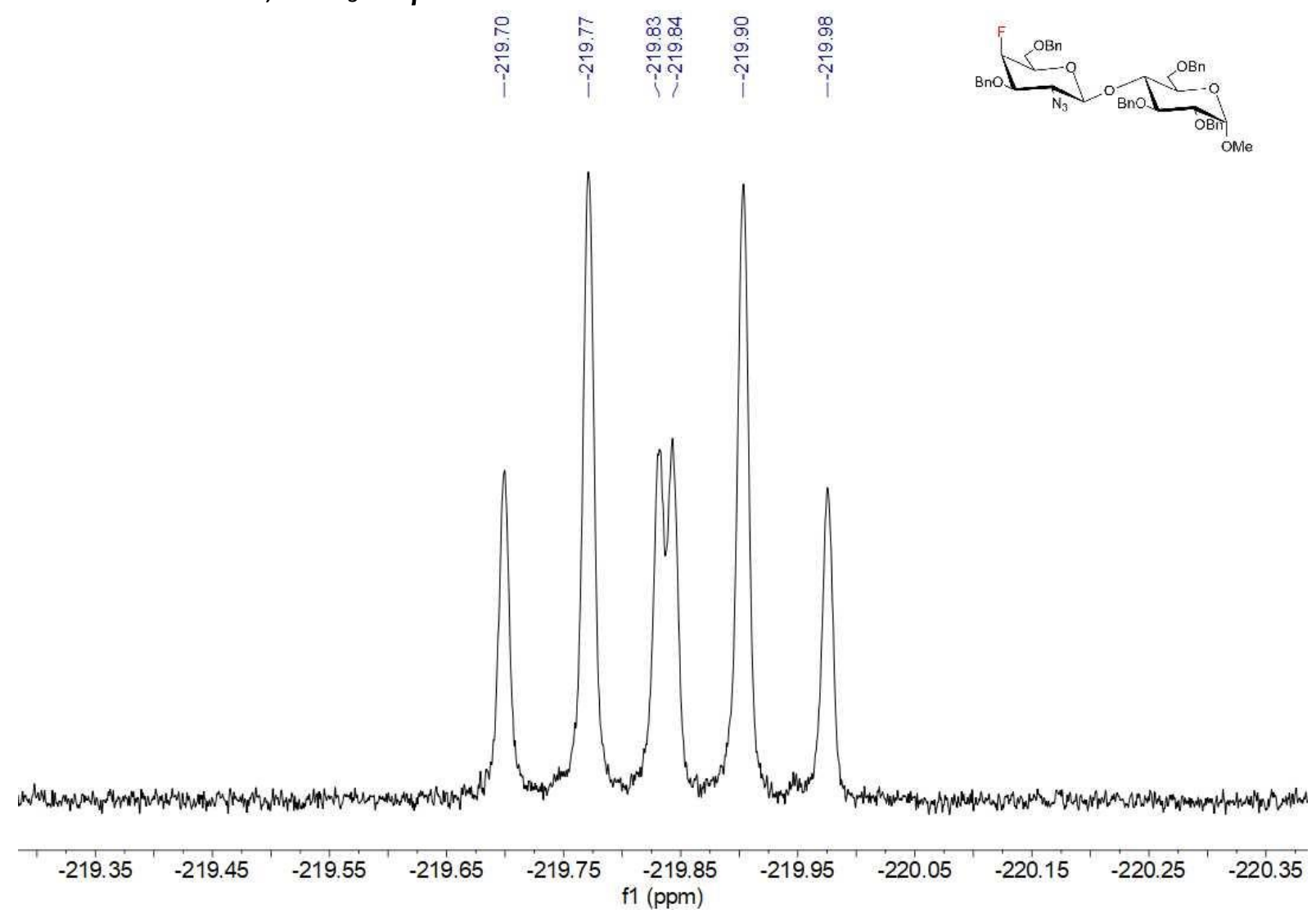




\section{NMR 4F- $\alpha$}

${ }^{1} \mathrm{HNMR} 400 \mathrm{MHz}, \mathrm{CDCl}_{3} 4 \mathrm{~F}-\alpha$

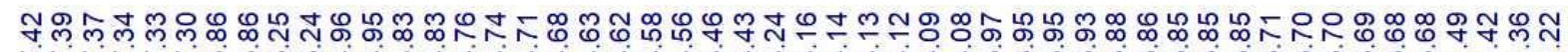

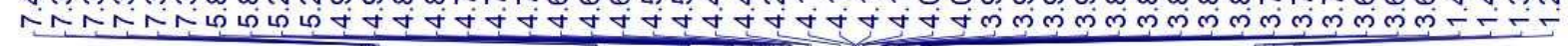

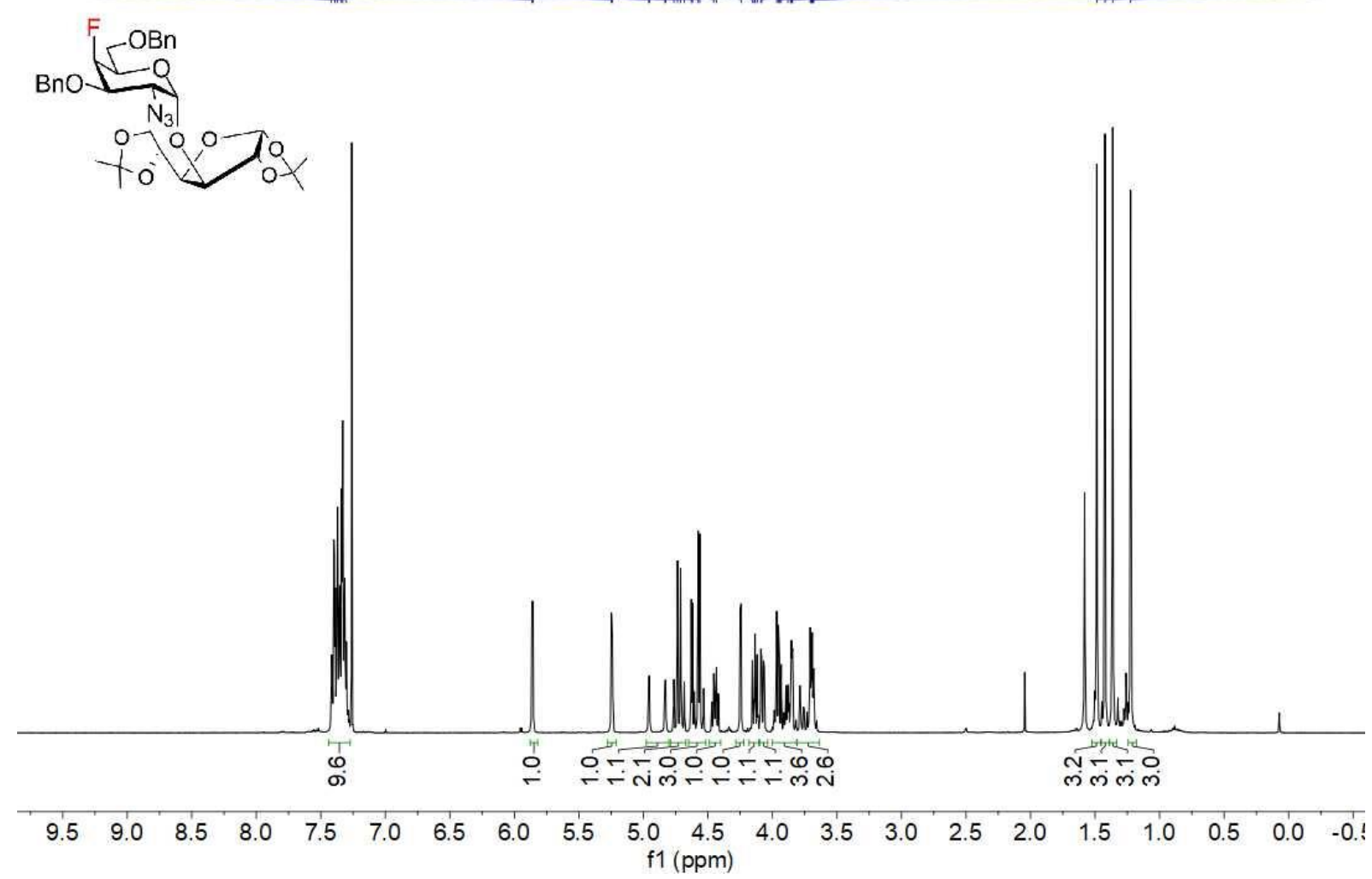

${ }^{13} \mathrm{C}\left\{{ }^{1} \mathrm{H}\right\} \mathrm{NMR} 100 \mathrm{MHz}, \mathrm{CDCl}_{3} 4 \mathrm{~F}-\alpha$

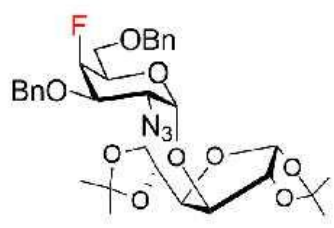

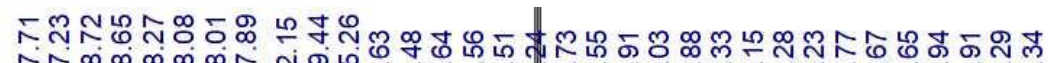

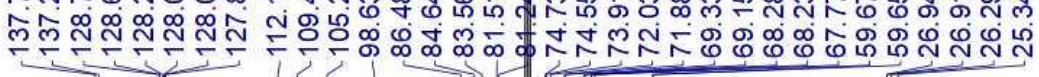

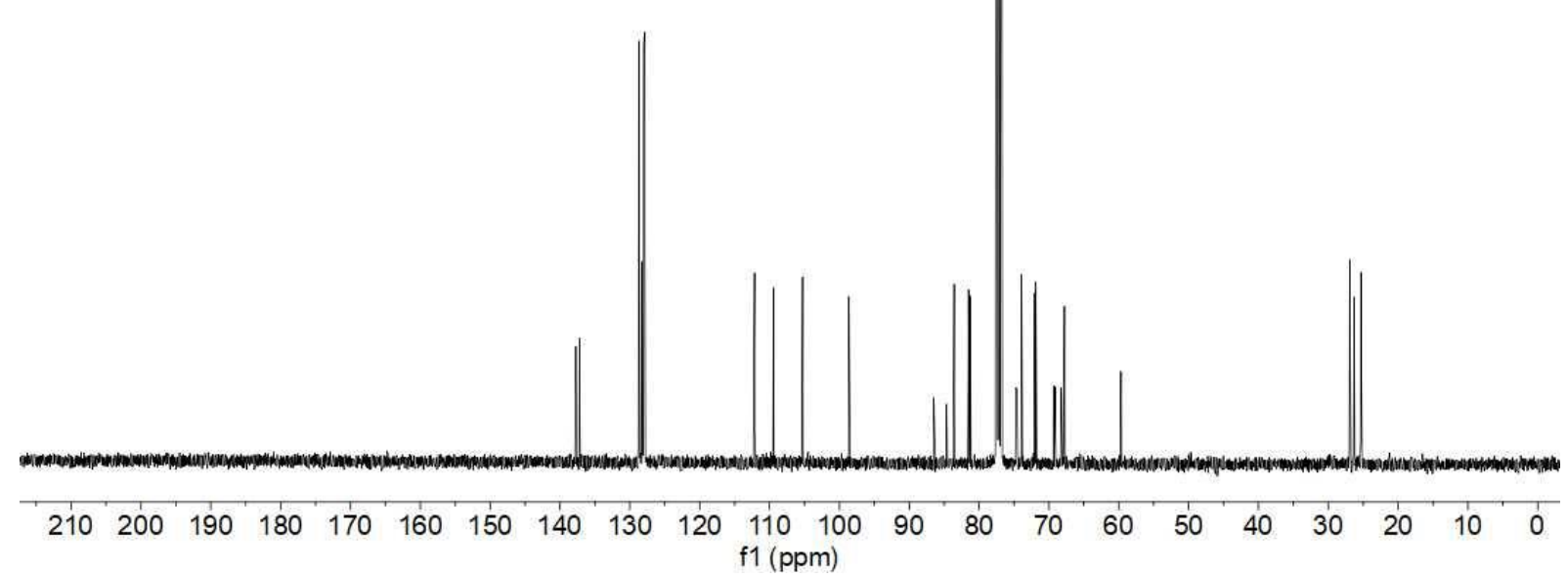


${ }^{1} \mathrm{H}-{ }^{1} \mathrm{H}$ COSY NMR $4 \mathrm{~F}-\boldsymbol{\alpha}$

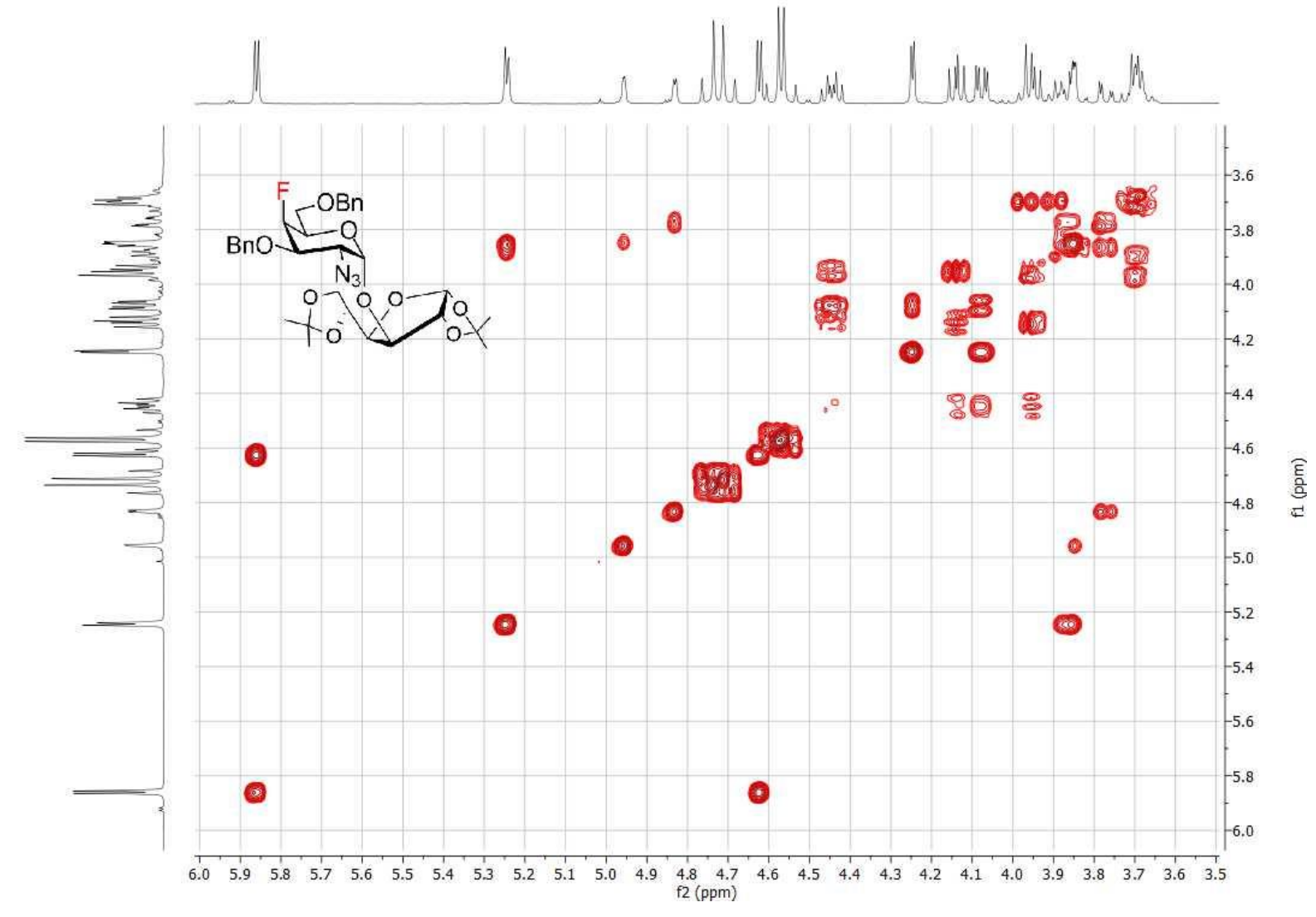

${ }^{1} \mathrm{H}-{ }^{13} \mathrm{C}$ HSOC NMR 4F- $\alpha$

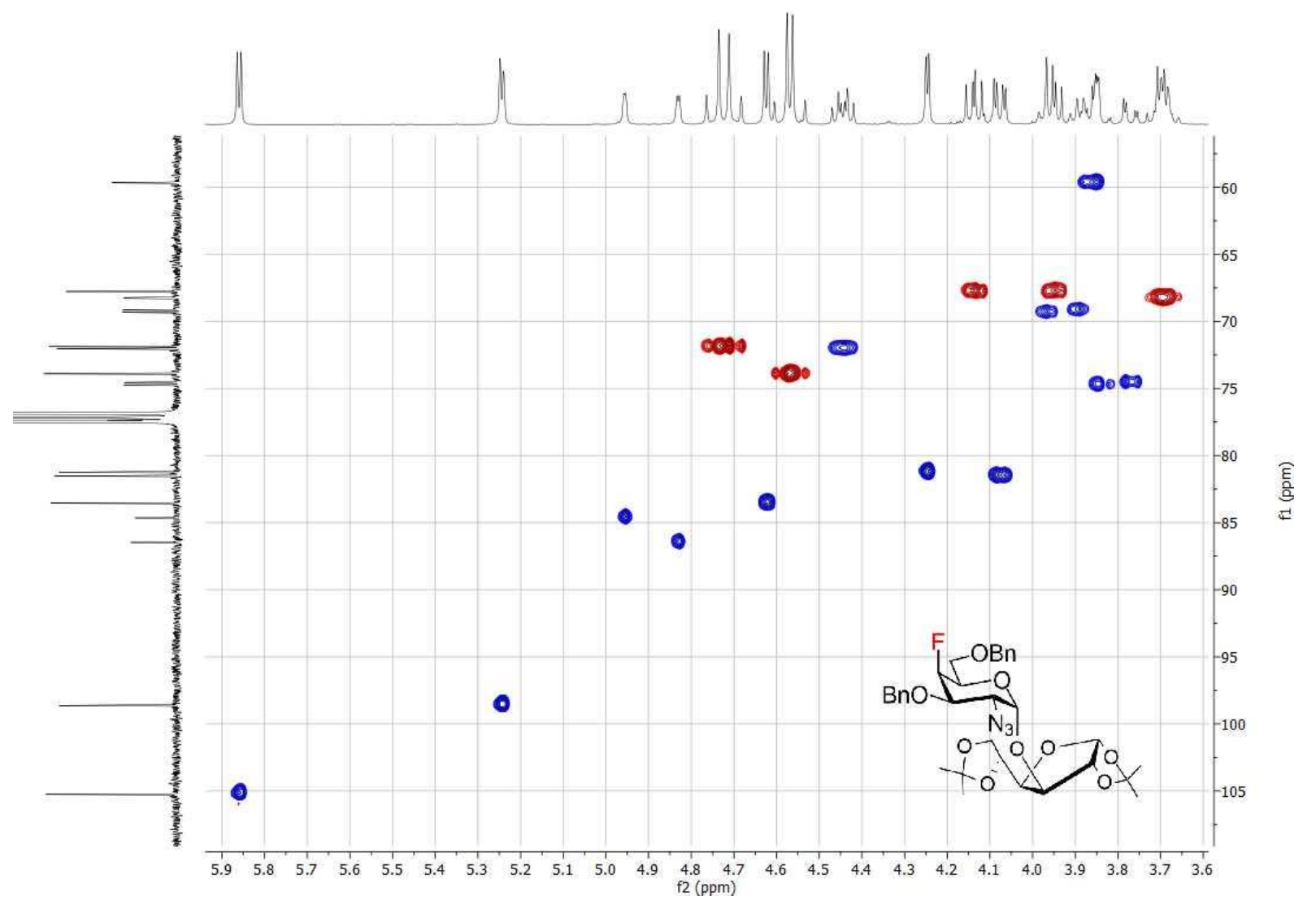


${ }^{1} \mathrm{H}-{ }^{13} \mathrm{C}$ HMBC NMR 4F- $\alpha$

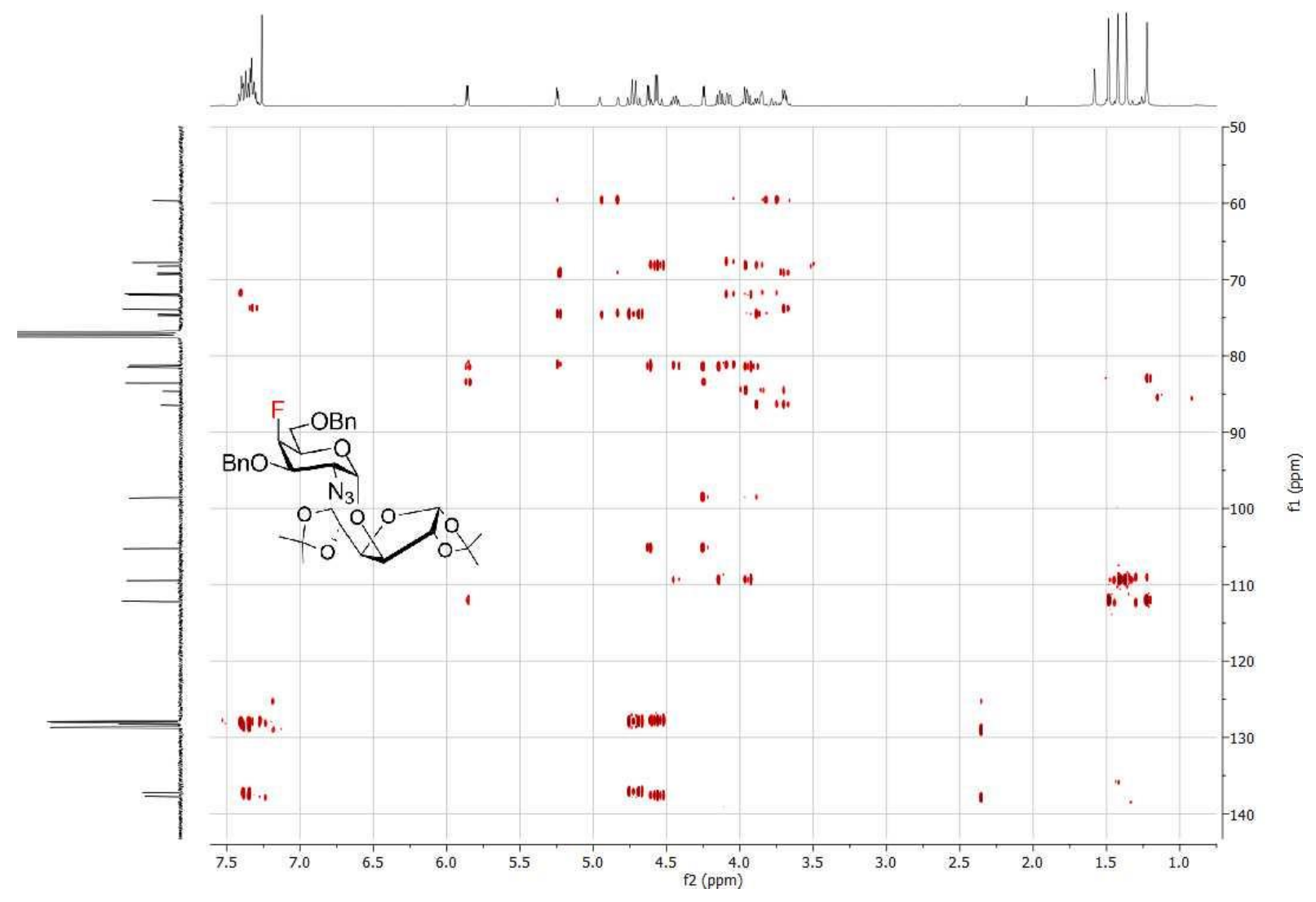

${ }^{19} \mathrm{~F}$ NMR $376 \mathrm{MHz}, \mathrm{CDCl}_{3}$ 4F- $\alpha$

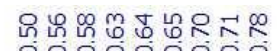

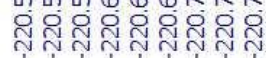

Nis
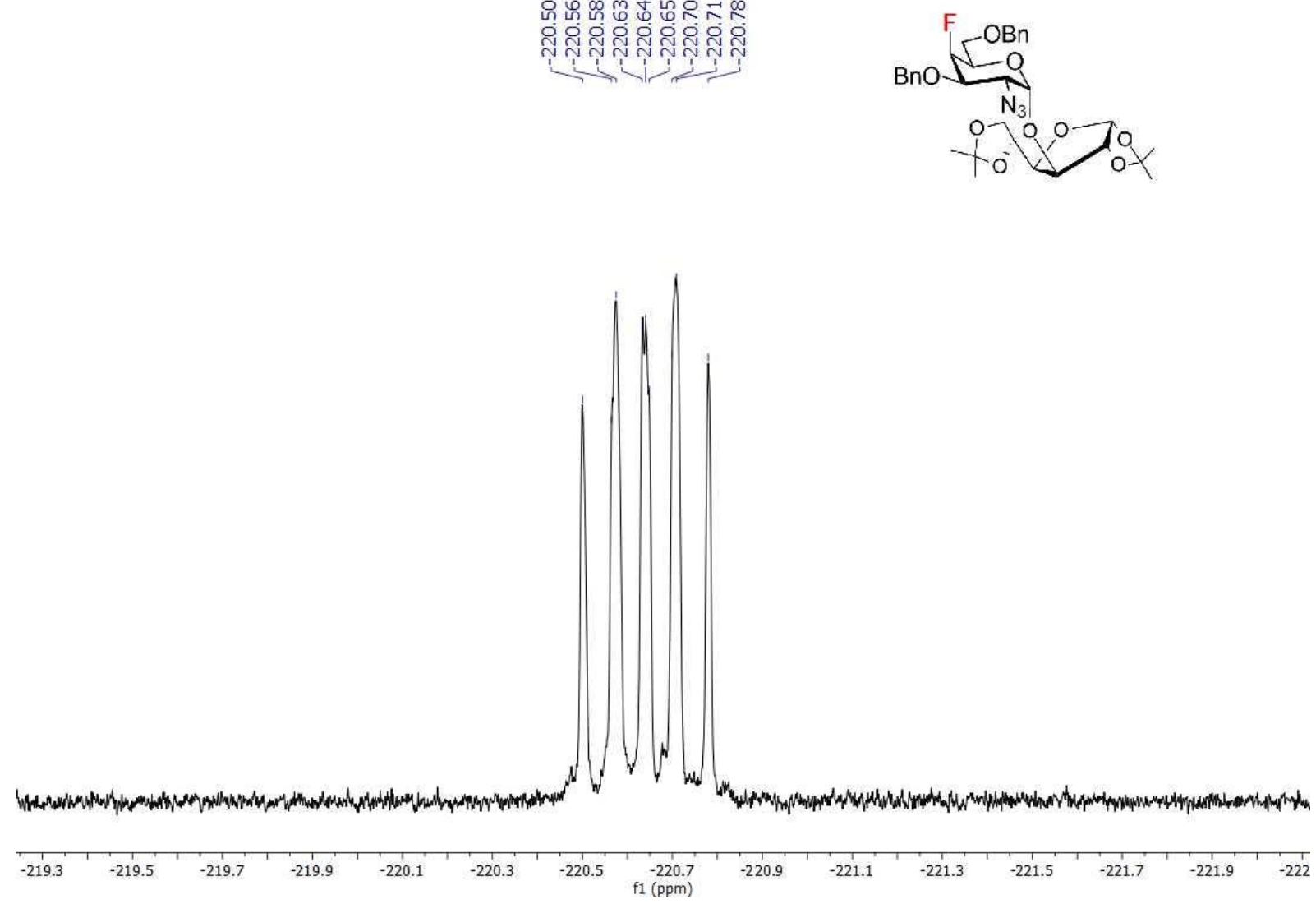
NMR 4F- $\beta$

${ }^{1} \mathrm{H} N M R 400 \mathrm{MHz}, \mathrm{CDCl}_{3} 4 \mathrm{~F}-\boldsymbol{\beta}$

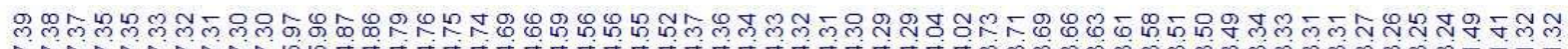

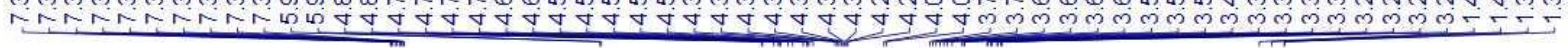

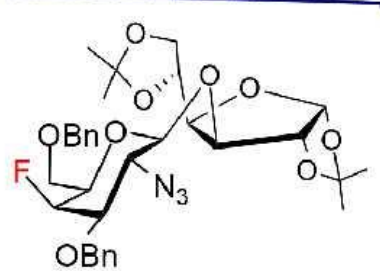

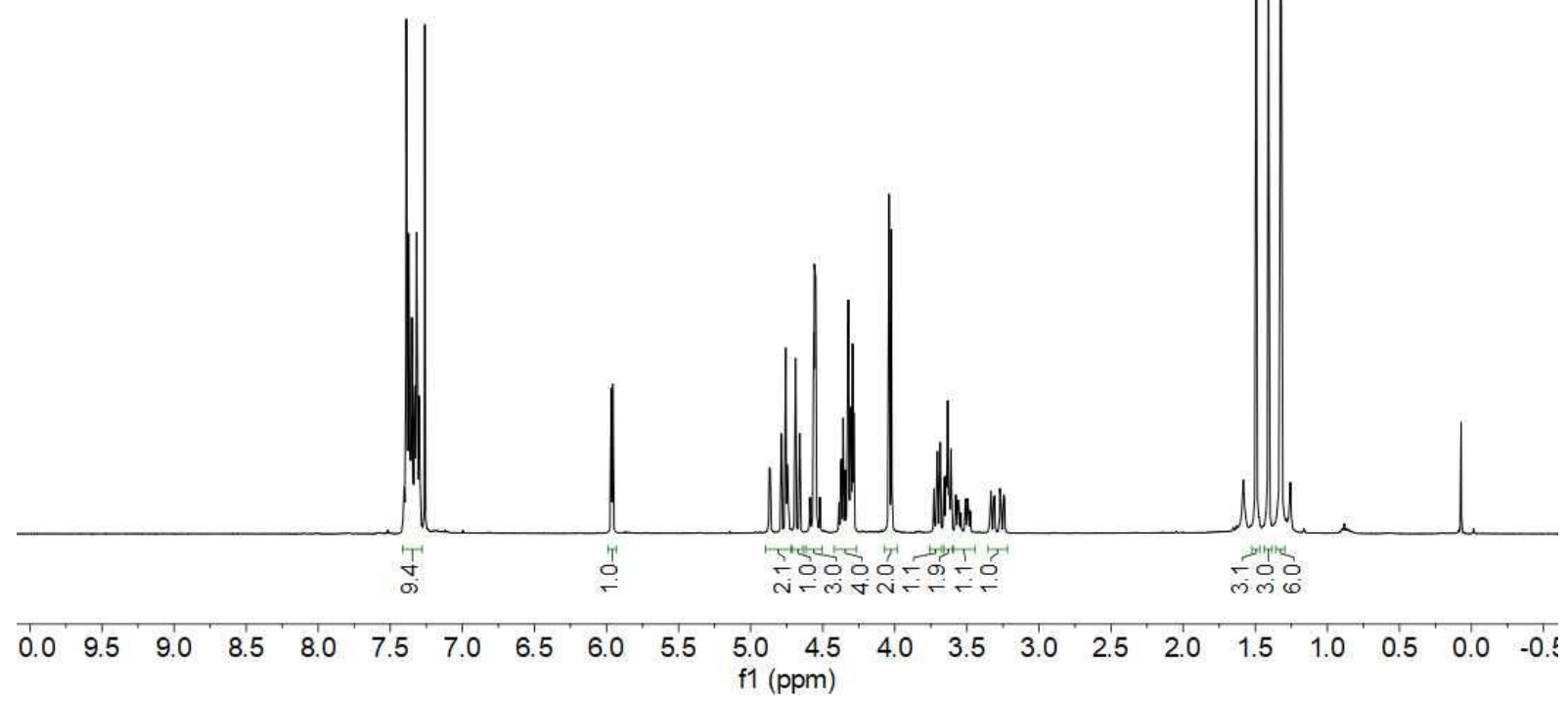

${ }^{13} \mathrm{C}\left\{{ }^{1} \mathrm{H}\right\}$ NMR $100 \mathrm{MHz}, \mathrm{CDCl}_{3} 4 \mathrm{~F}-\boldsymbol{\beta}$

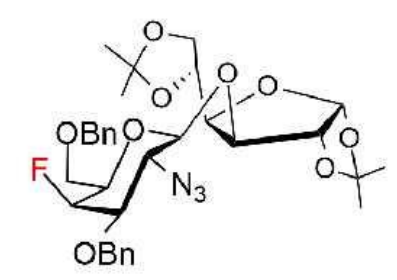

무유.

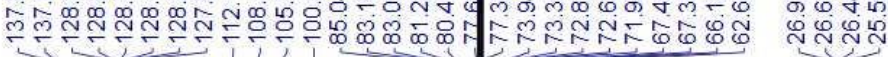

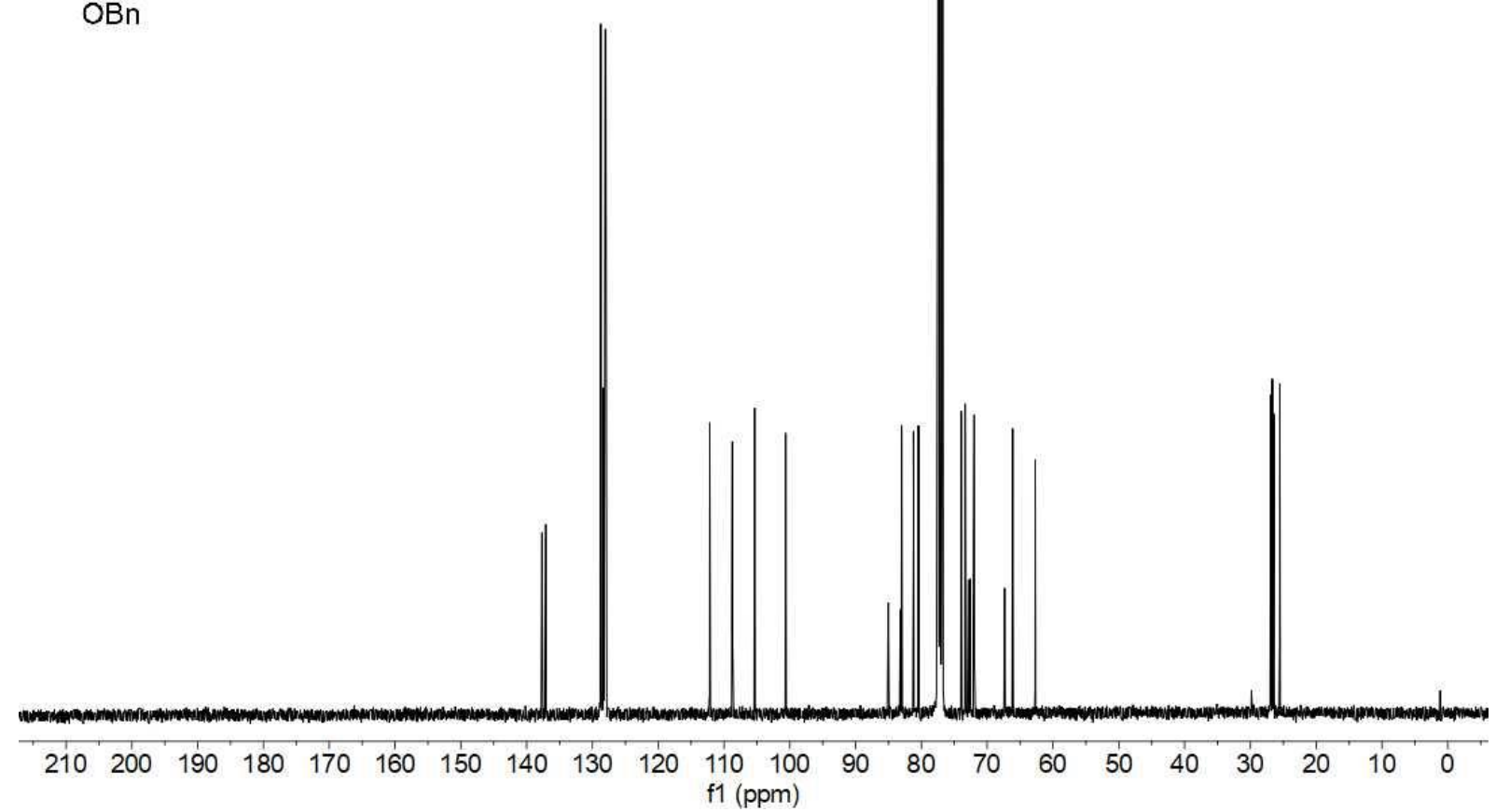


${ }^{1} \mathrm{H}-{ }^{1} \mathrm{H}$ COSY NMR 4F- $\beta$

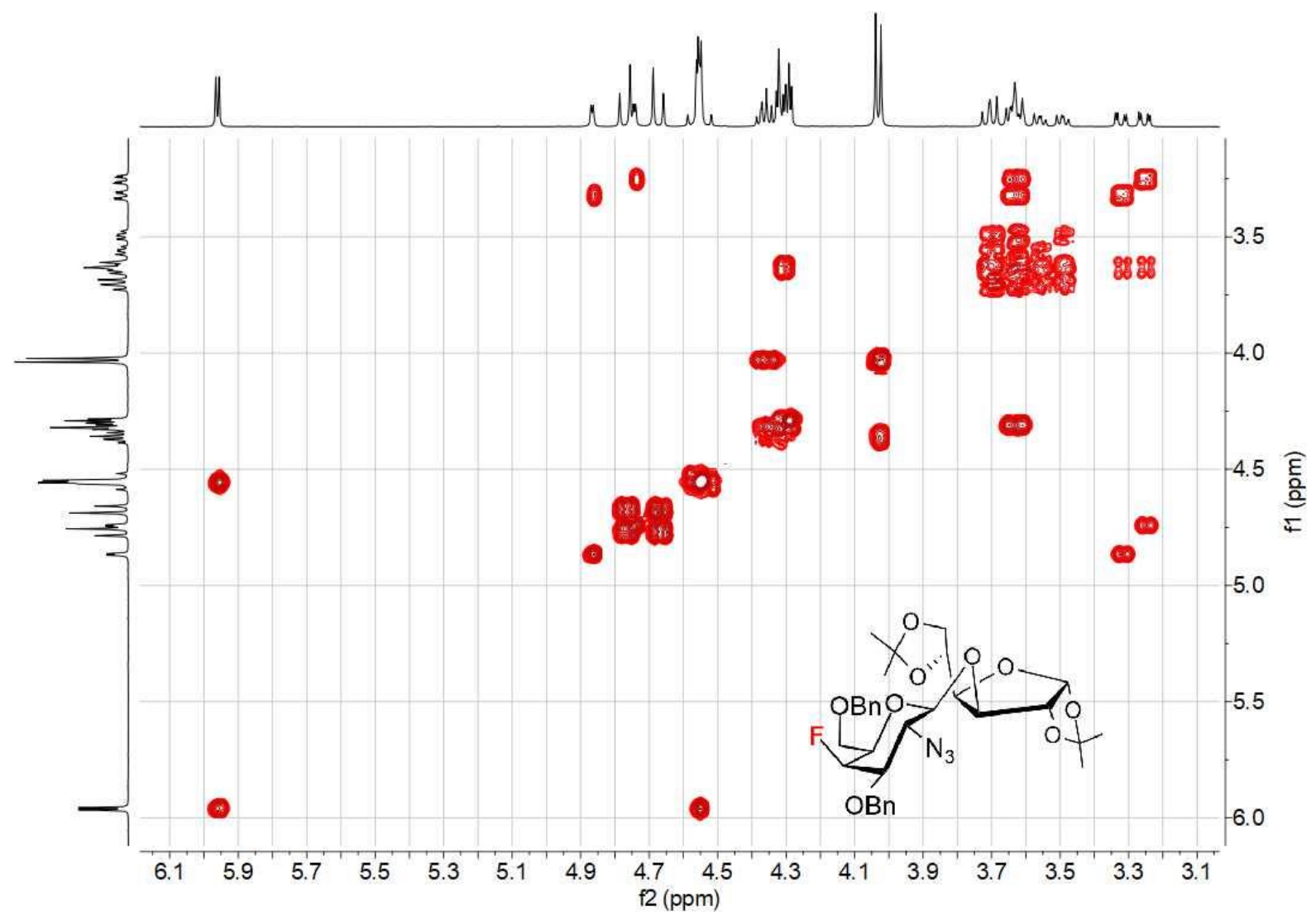

${ }^{1} \mathrm{H}-{ }^{13} \mathrm{C}$ HSQC NMR 4F- $\beta$

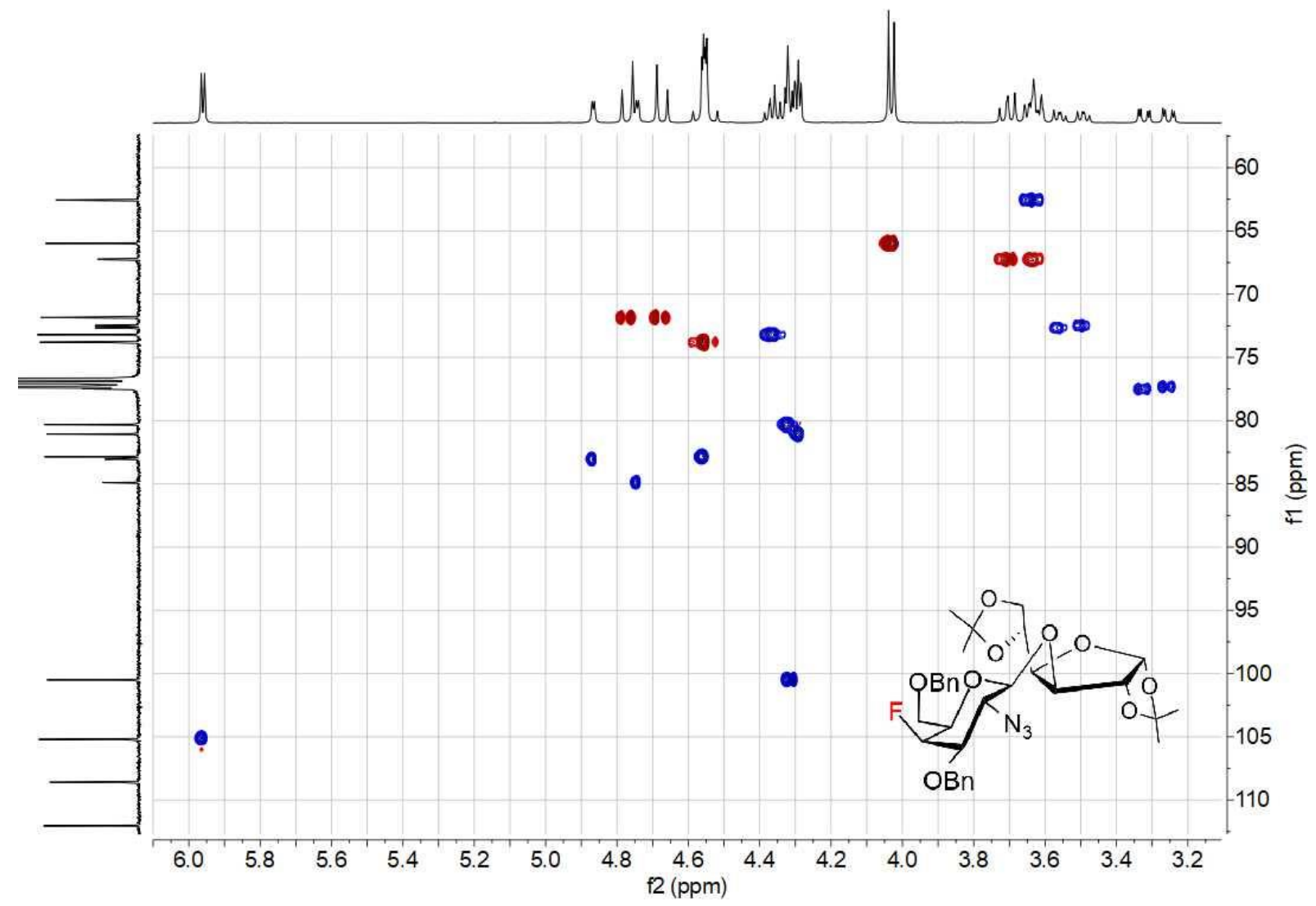


${ }^{1} \mathrm{H}-{ }^{13} \mathrm{C}$ HMBC NMR 4F- $\beta$

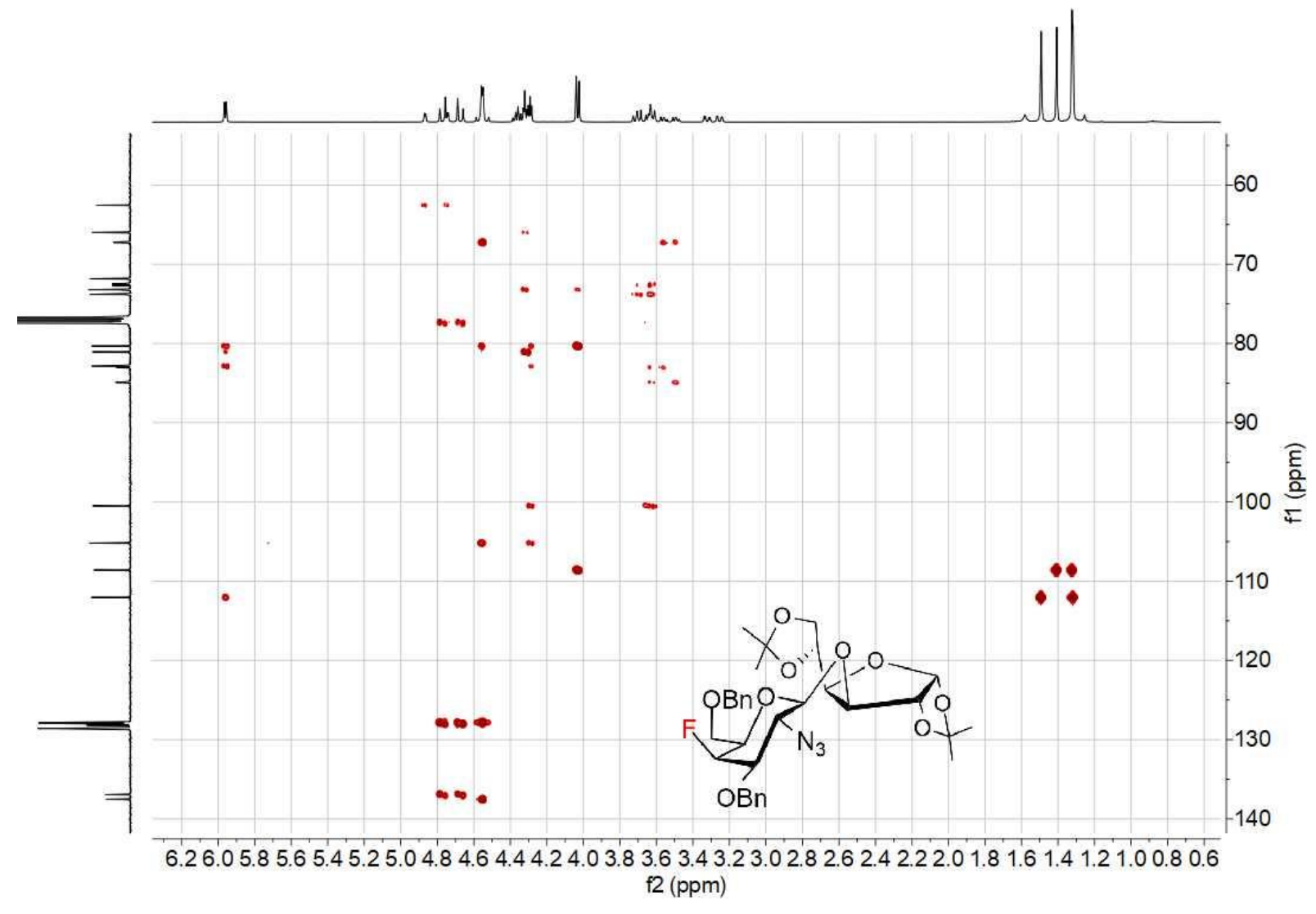

${ }^{19} \mathrm{~F} \mathrm{NMR} 376 \mathrm{MHz}, \mathrm{CDCl}_{3} 4 \mathrm{~F}-\boldsymbol{\beta}$

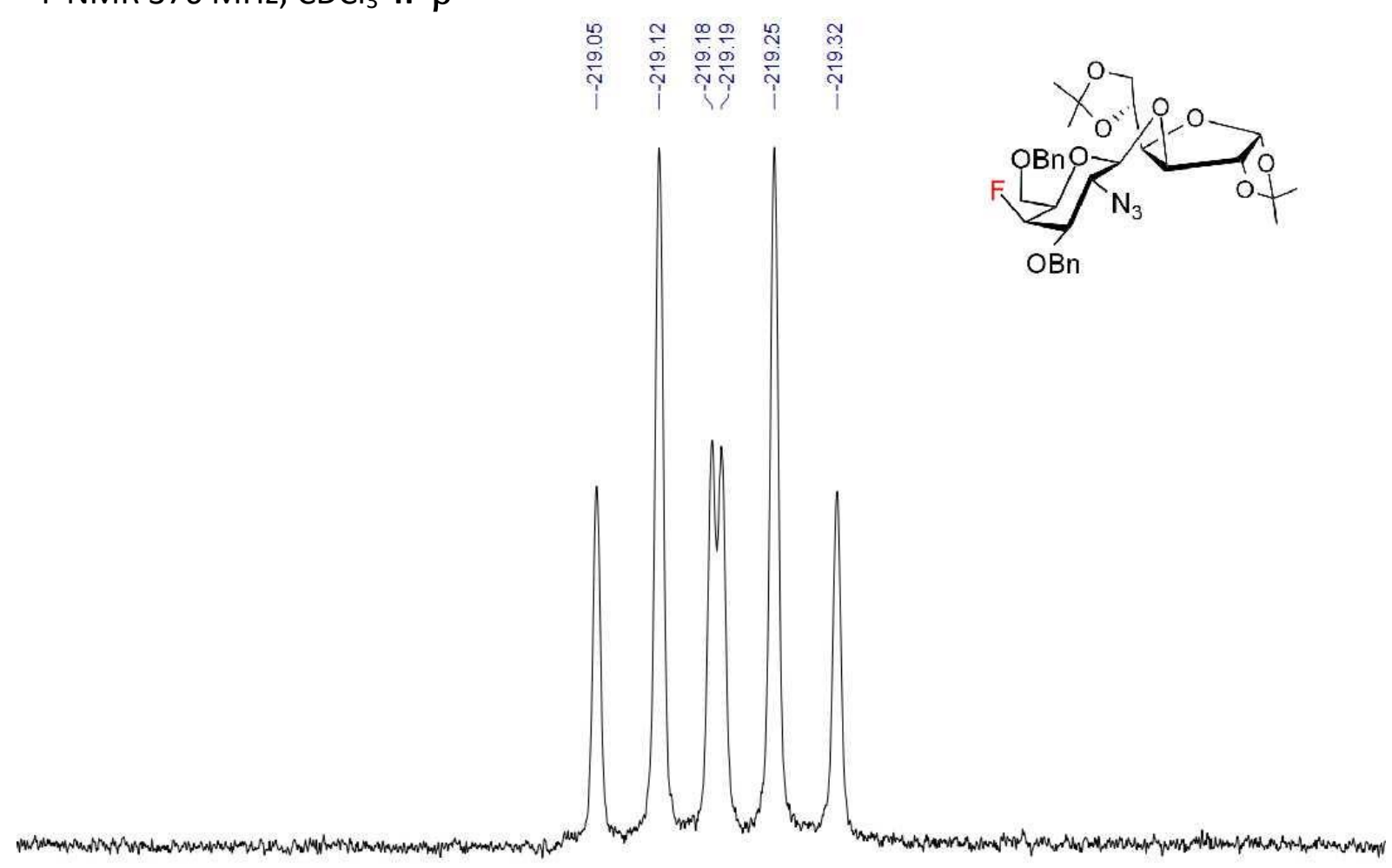

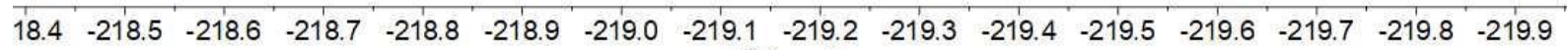
f1 (ppm) 
NMR 4G- $\alpha$

${ }^{1} \mathrm{HNMR} 400 \mathrm{MHz}, \mathrm{CDCl}_{3} 4 \mathrm{G}-\alpha$

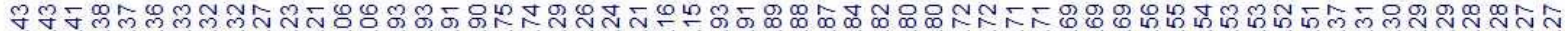

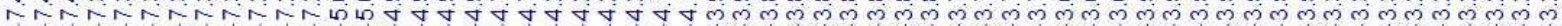

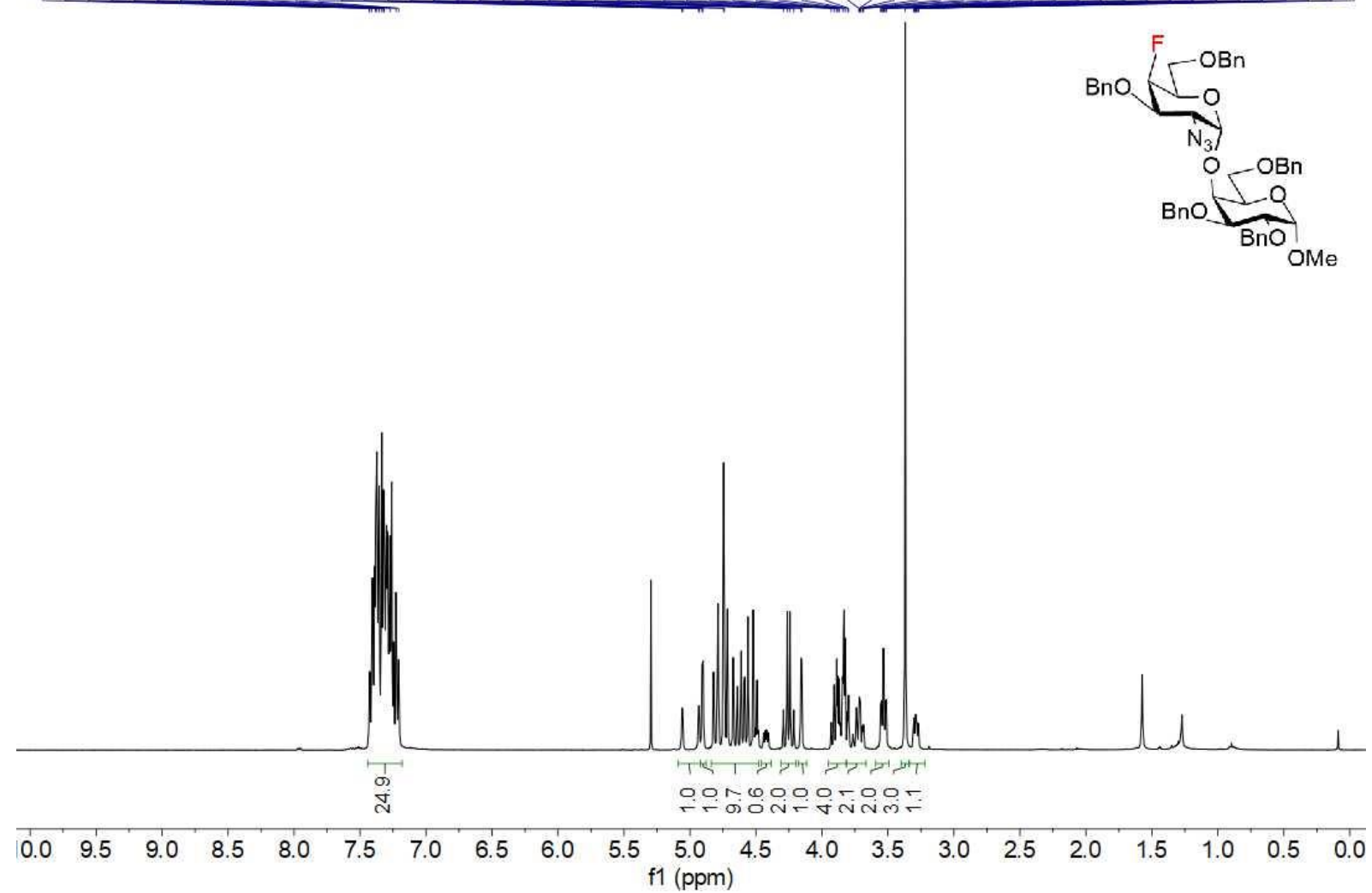

${ }^{13} \mathrm{C}\left\{{ }^{1} \mathrm{H}\right\}$ NMR $100 \mathrm{MHz}, \mathrm{CDCl}_{3}$ 4G- $\alpha$

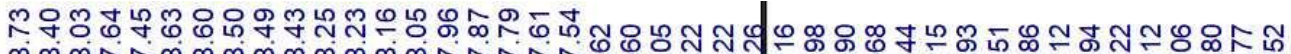

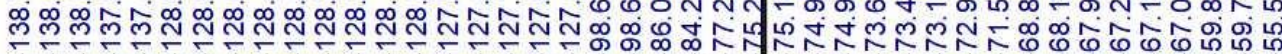

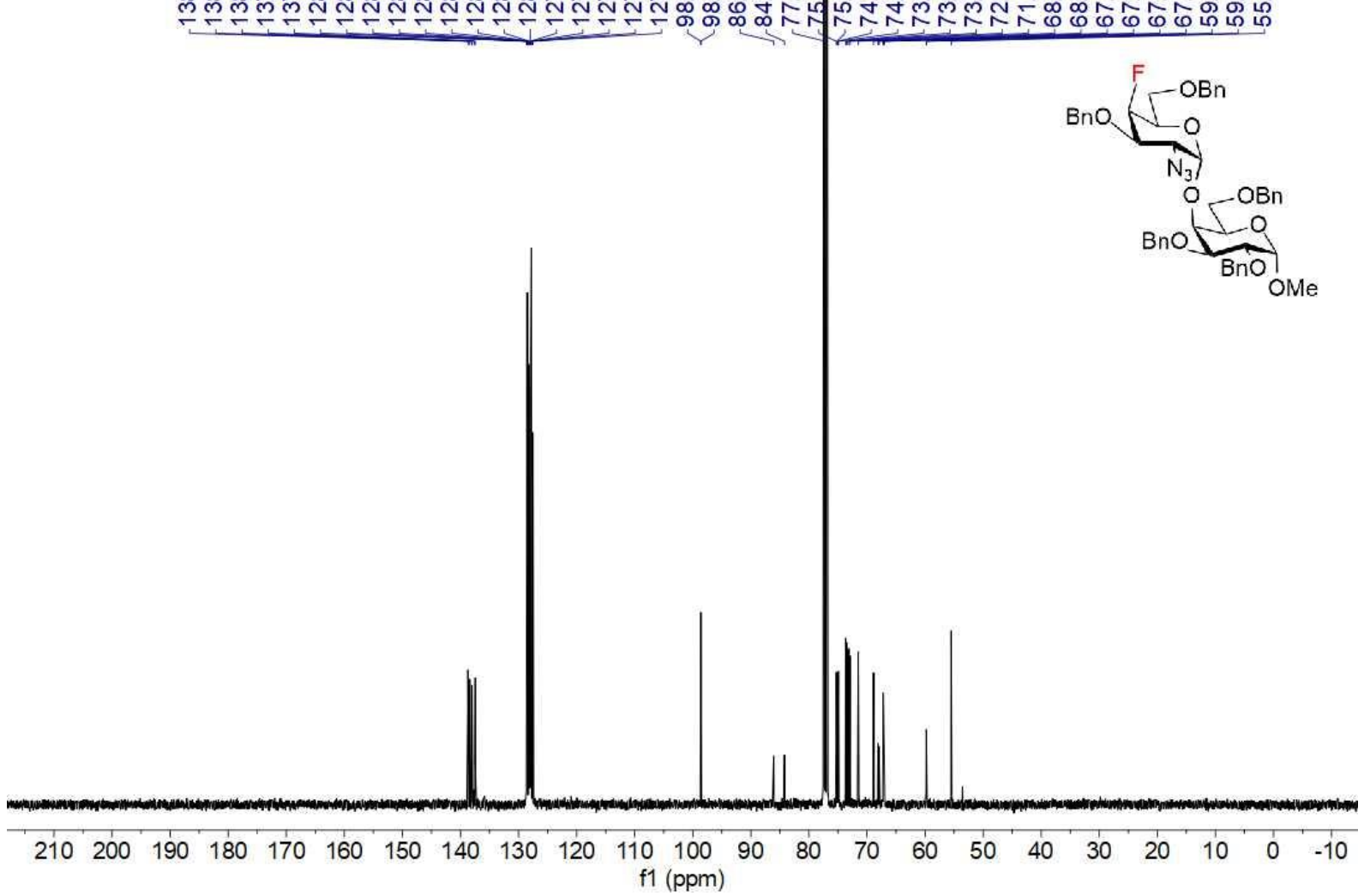


${ }^{1} \mathrm{H}-{ }^{1} \mathrm{H}$ cosY NMR 4G- $\alpha$

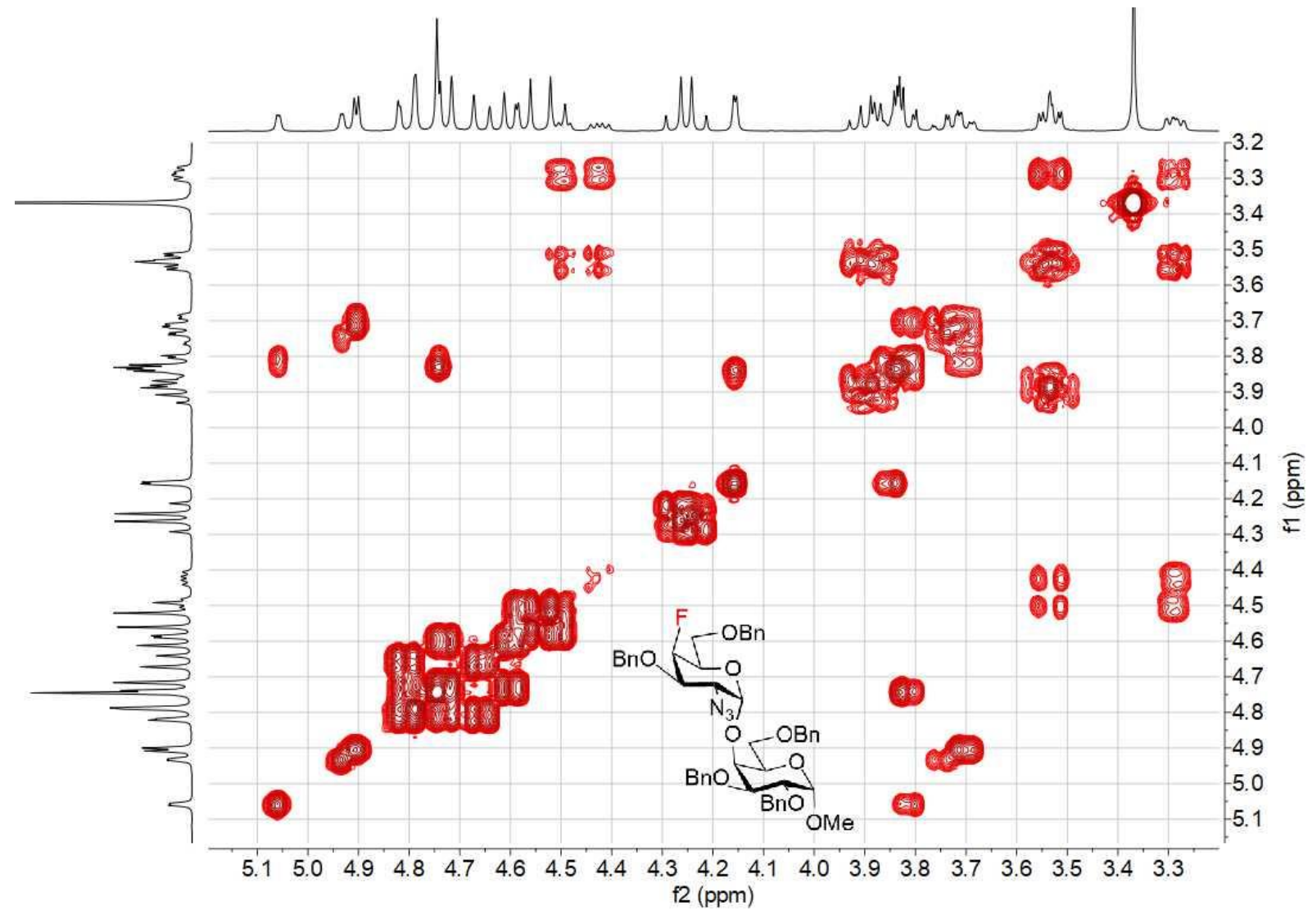

${ }^{1} \mathrm{H}^{13} \mathrm{C}$ HSQC NMR 4G- $\alpha$

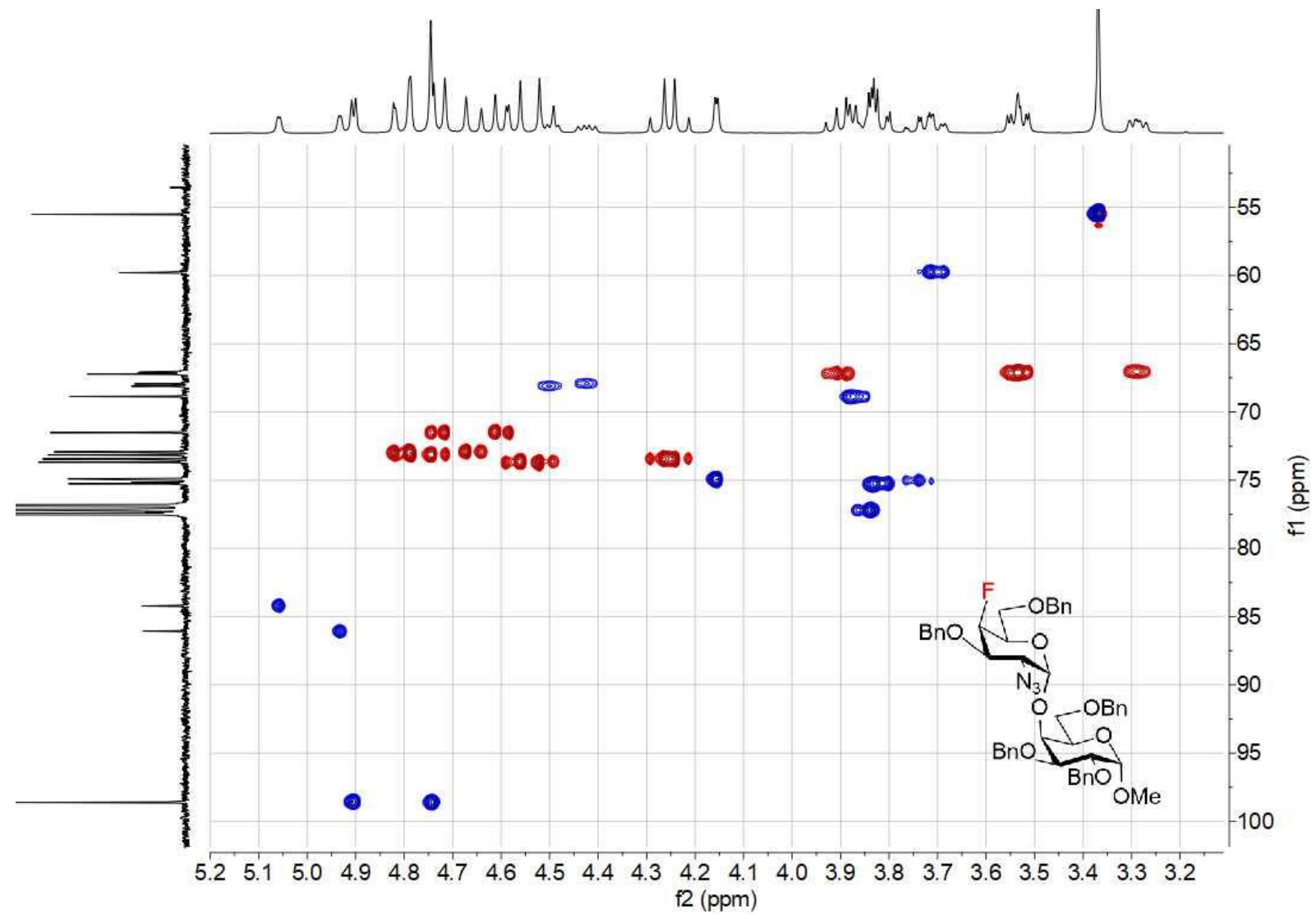




\section{${ }^{1} \mathrm{H}-{ }^{13} \mathrm{CHMBC}$ NMR 4G- $\alpha$}

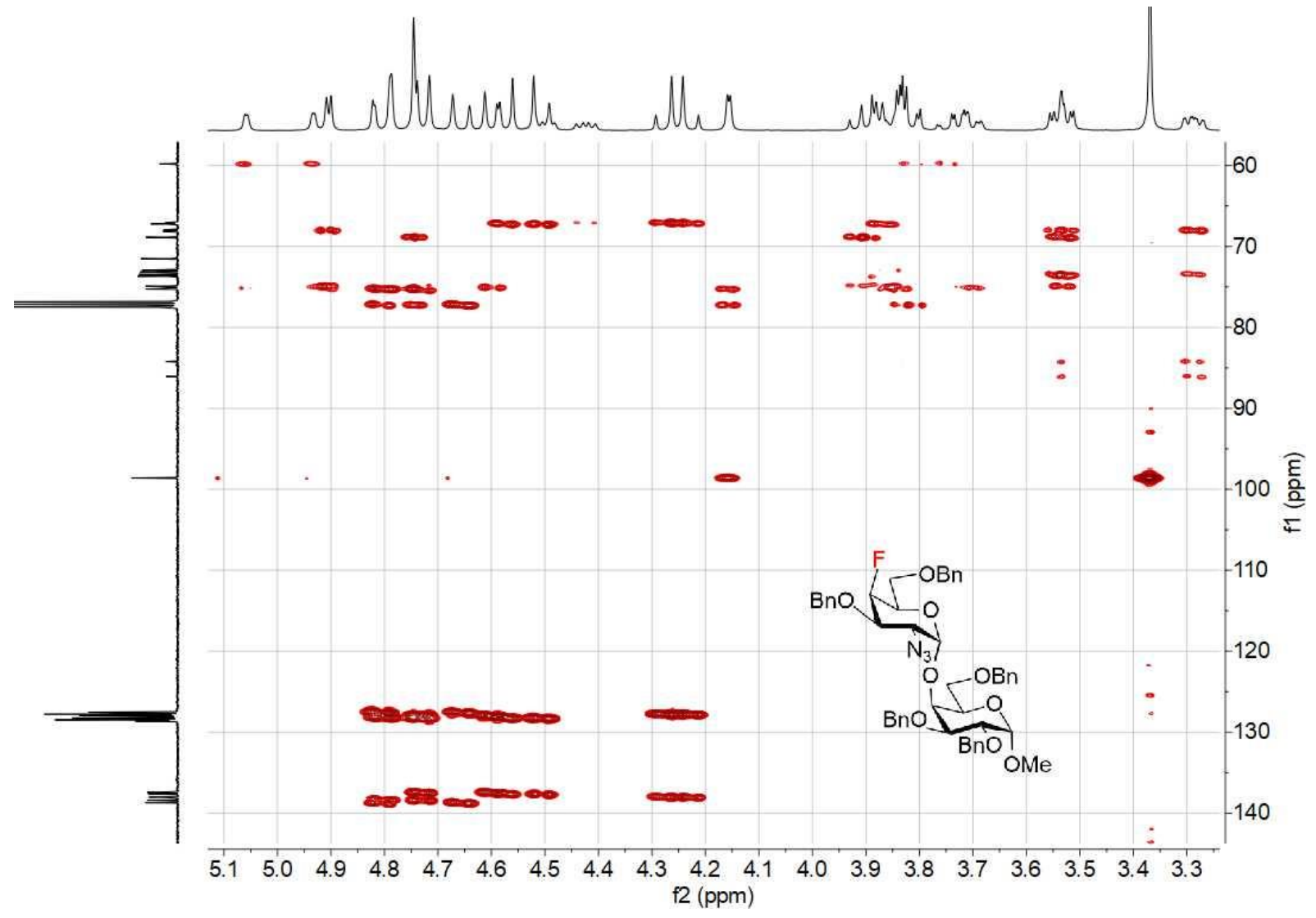

${ }^{19} \mathrm{~F} \mathrm{NMR} 376 \mathrm{MHz}, \mathrm{CDCl}_{3}$ 4G- $\alpha$

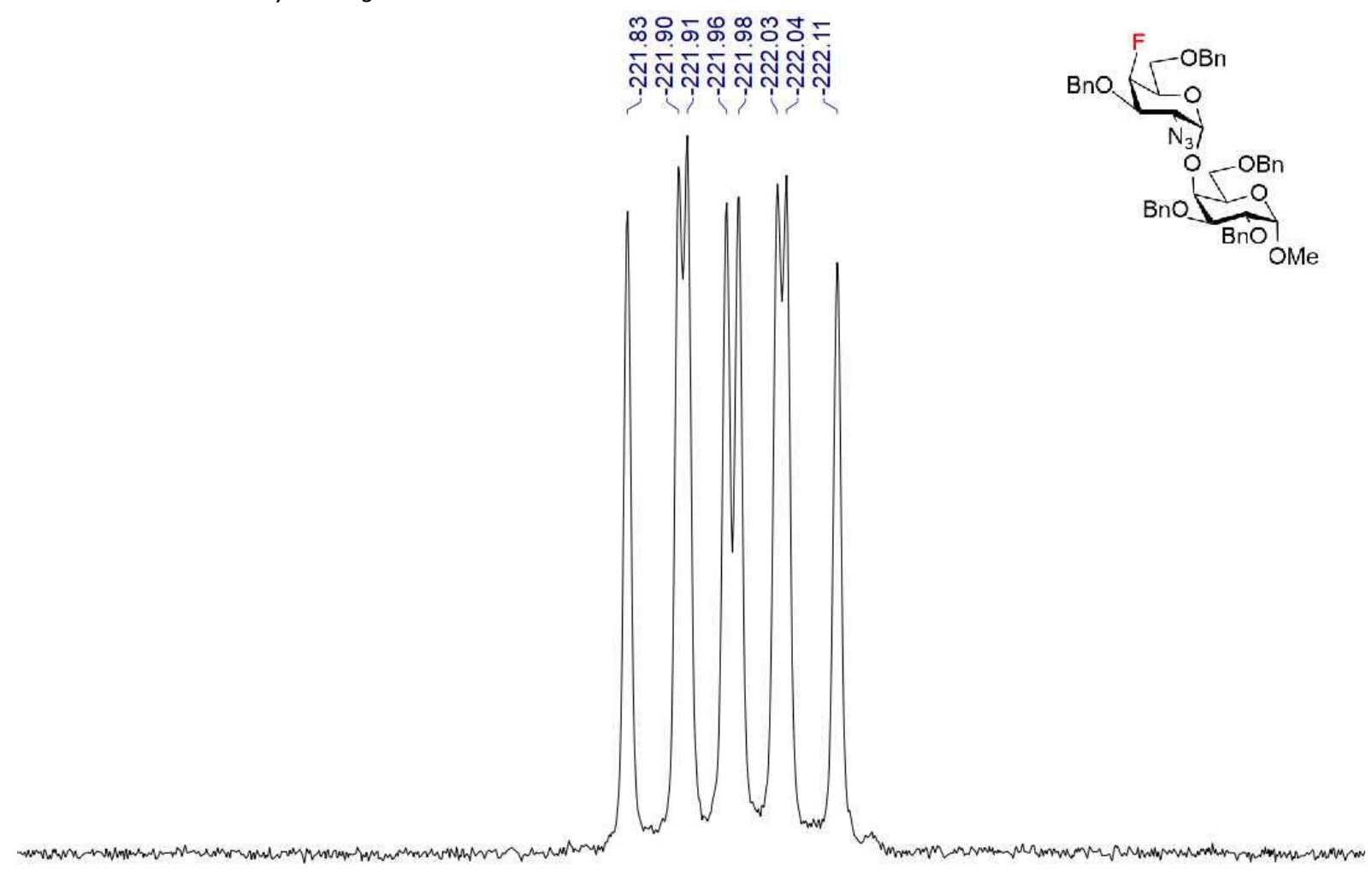

$-221.1-221.2-221.3-221.4-221.5-221.6-221.7-221.8-221.9-222.0-222.1-222.2-222.3-222.4-222.5-222.6-222.7-222$. $\mathrm{f1}(\mathrm{ppm})$ 
NMR 4G- $\beta$

${ }^{1} \mathrm{HNMR} 400 \mathrm{MHz}, \mathrm{CDCl}_{3} \mathbf{4 G}-\boldsymbol{\beta}$

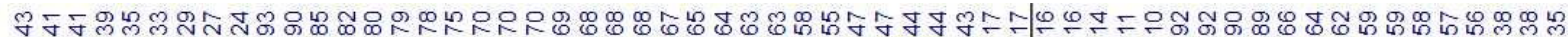

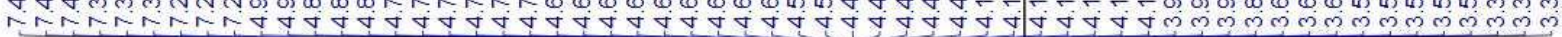

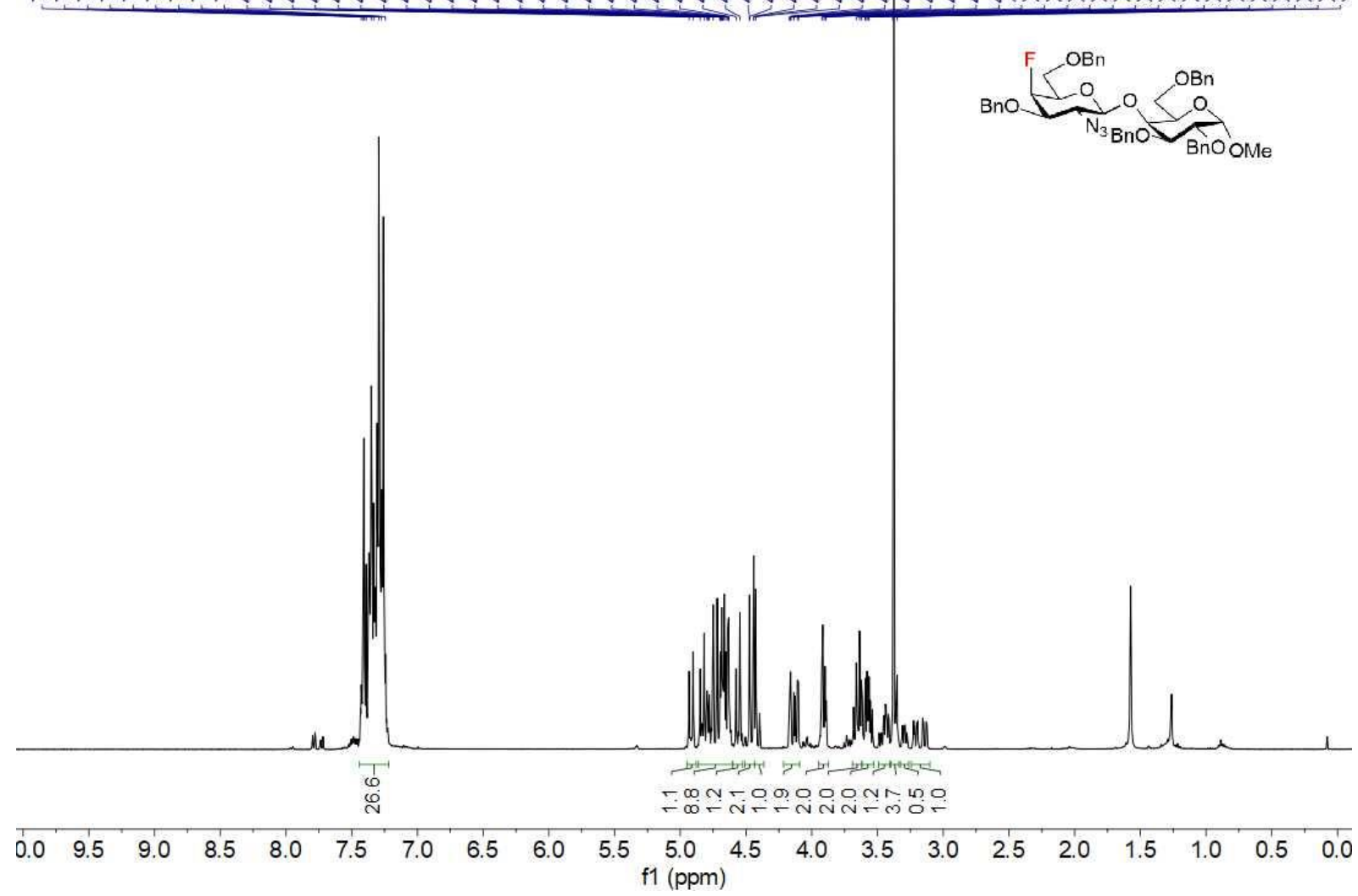

${ }^{13} \mathrm{C}\left\{{ }^{1} \mathrm{H}\right\}$ NMR 100MHz, $\mathrm{CDCl}_{3} 4 \mathrm{G}-\beta$

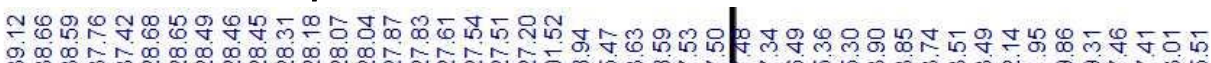

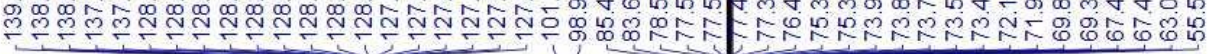

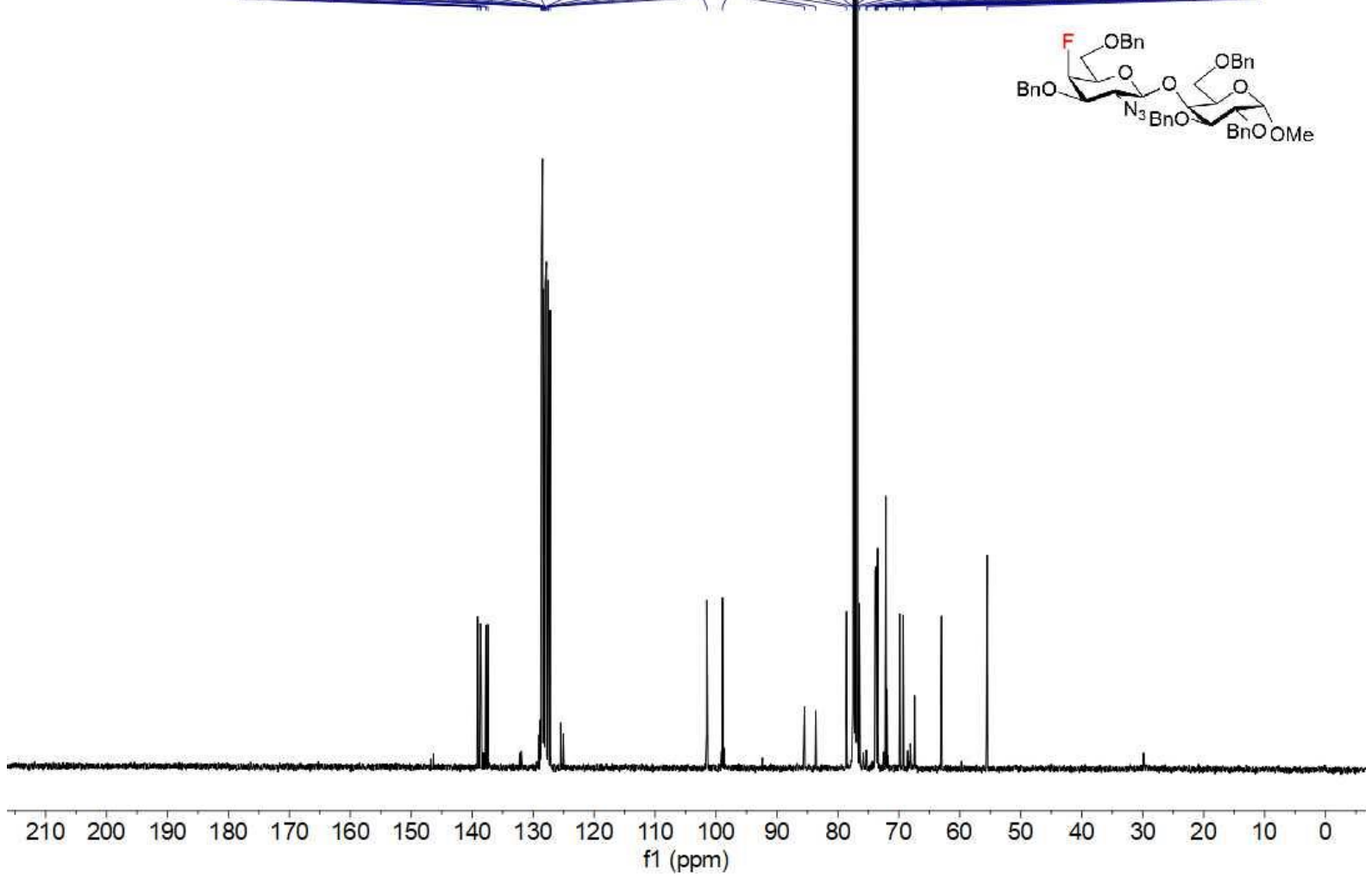


${ }^{1} \mathrm{H}-{ }^{1} \mathrm{H}$ COSY NMR 4 G- $\beta$

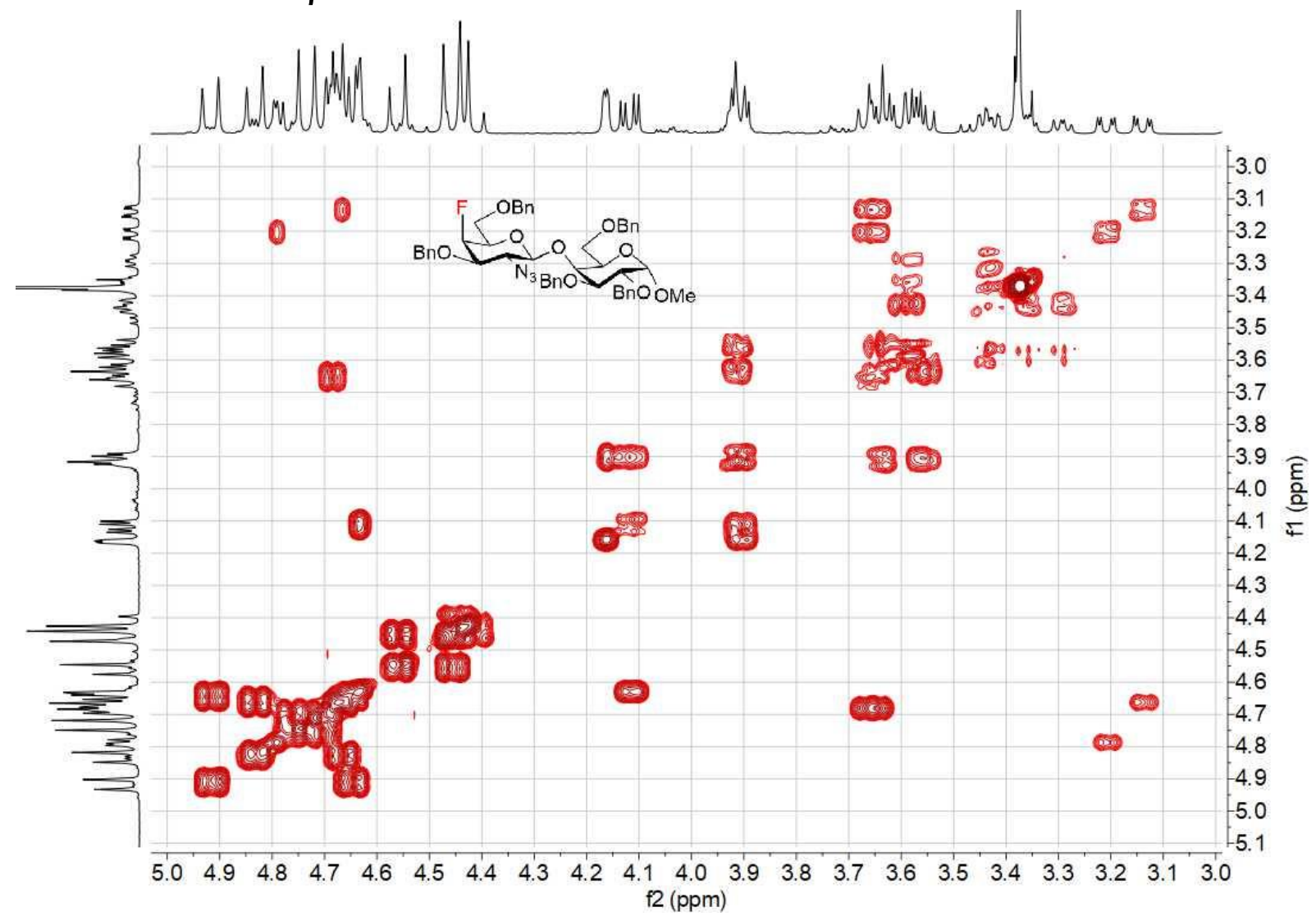

${ }^{1} \mathrm{H}-{ }^{13} \mathrm{C}$ HSOC NMR 4G- $\beta$

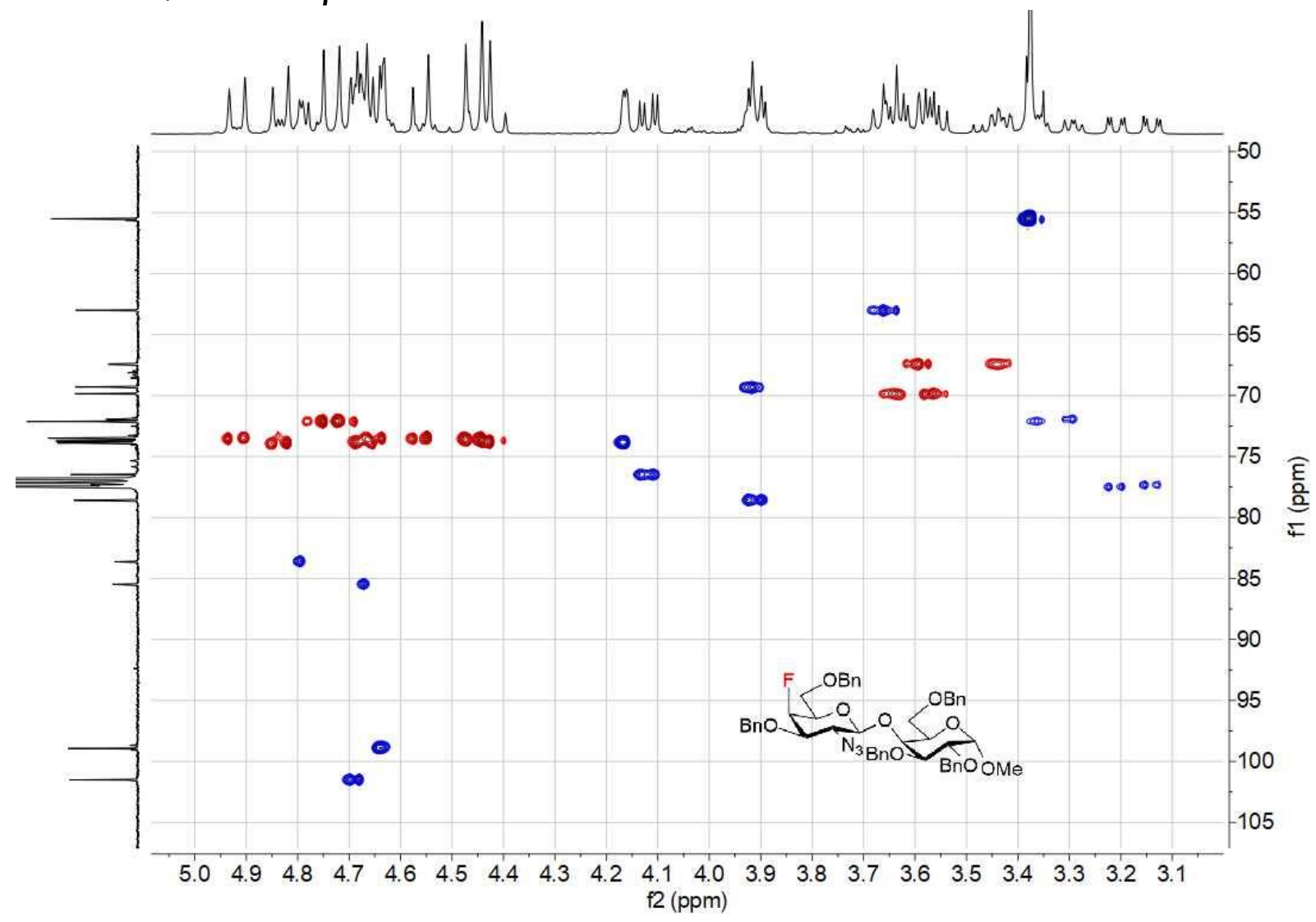


${ }^{1} \mathrm{H}^{13}{ }^{13} \mathrm{CHMBC}$ NMR 4G- $\boldsymbol{\beta}$

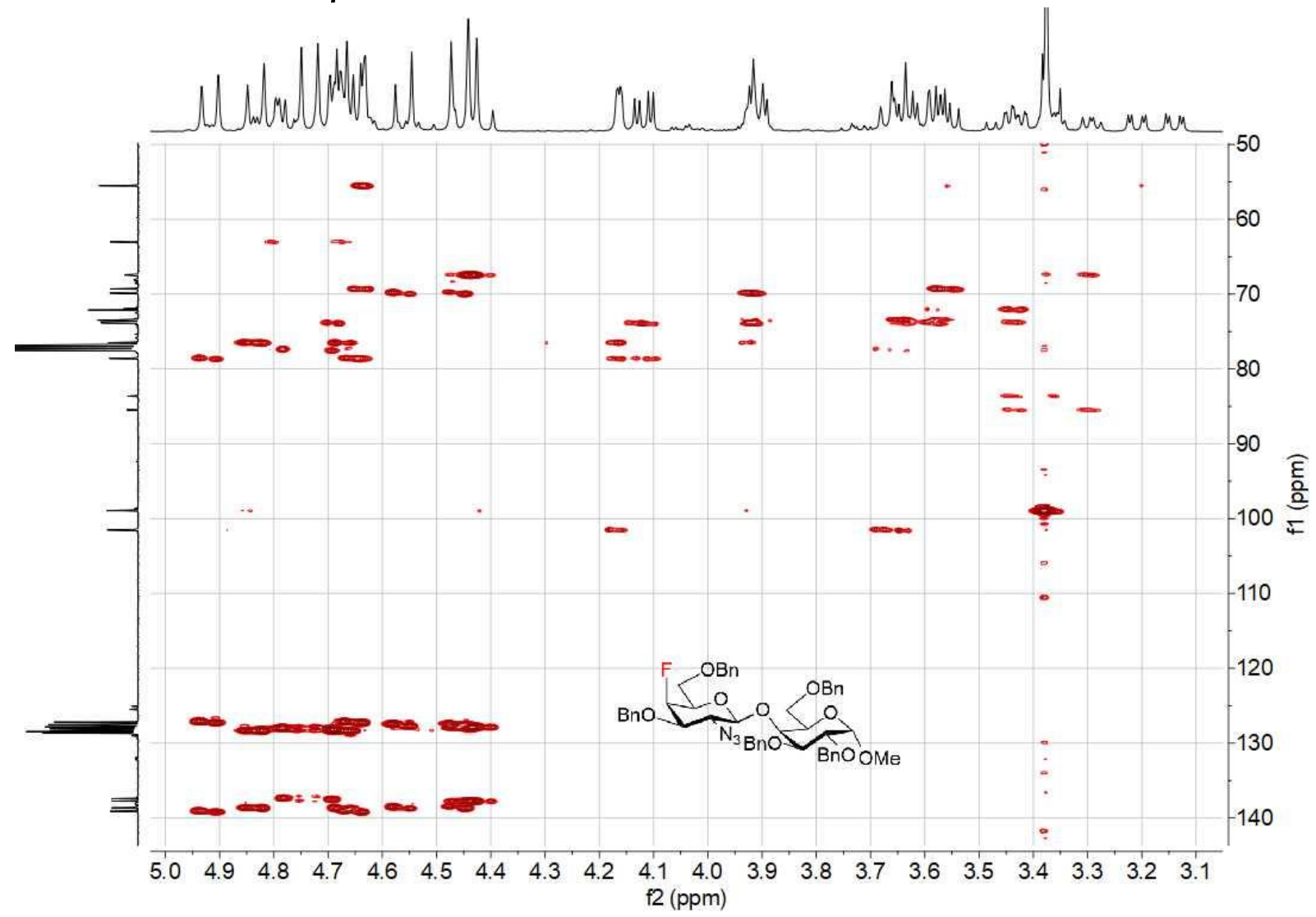

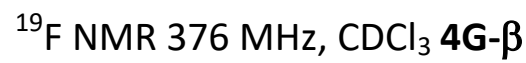

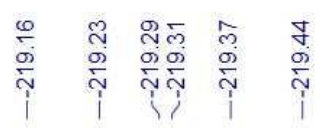
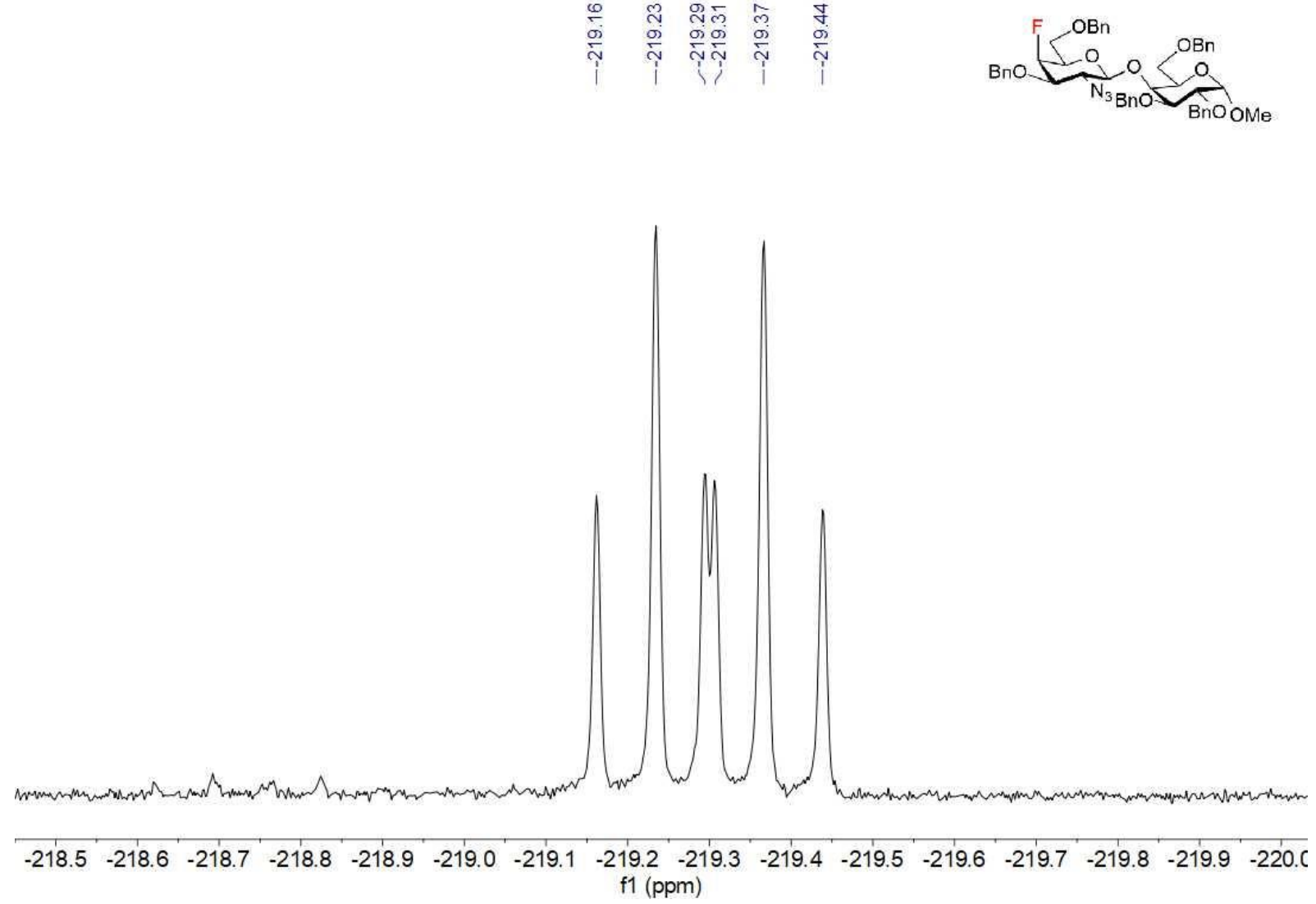
NMR $4 \mathrm{H}-\alpha$

${ }^{1} \mathrm{H}$ NMR $400 \mathrm{MHz}, \mathrm{CDCl}_{3} 4 \mathrm{H}-\alpha$

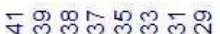

N-NMNin

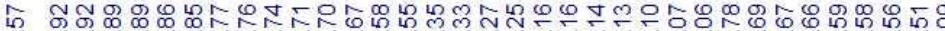

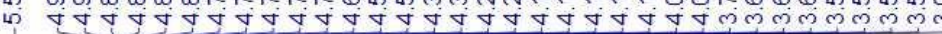

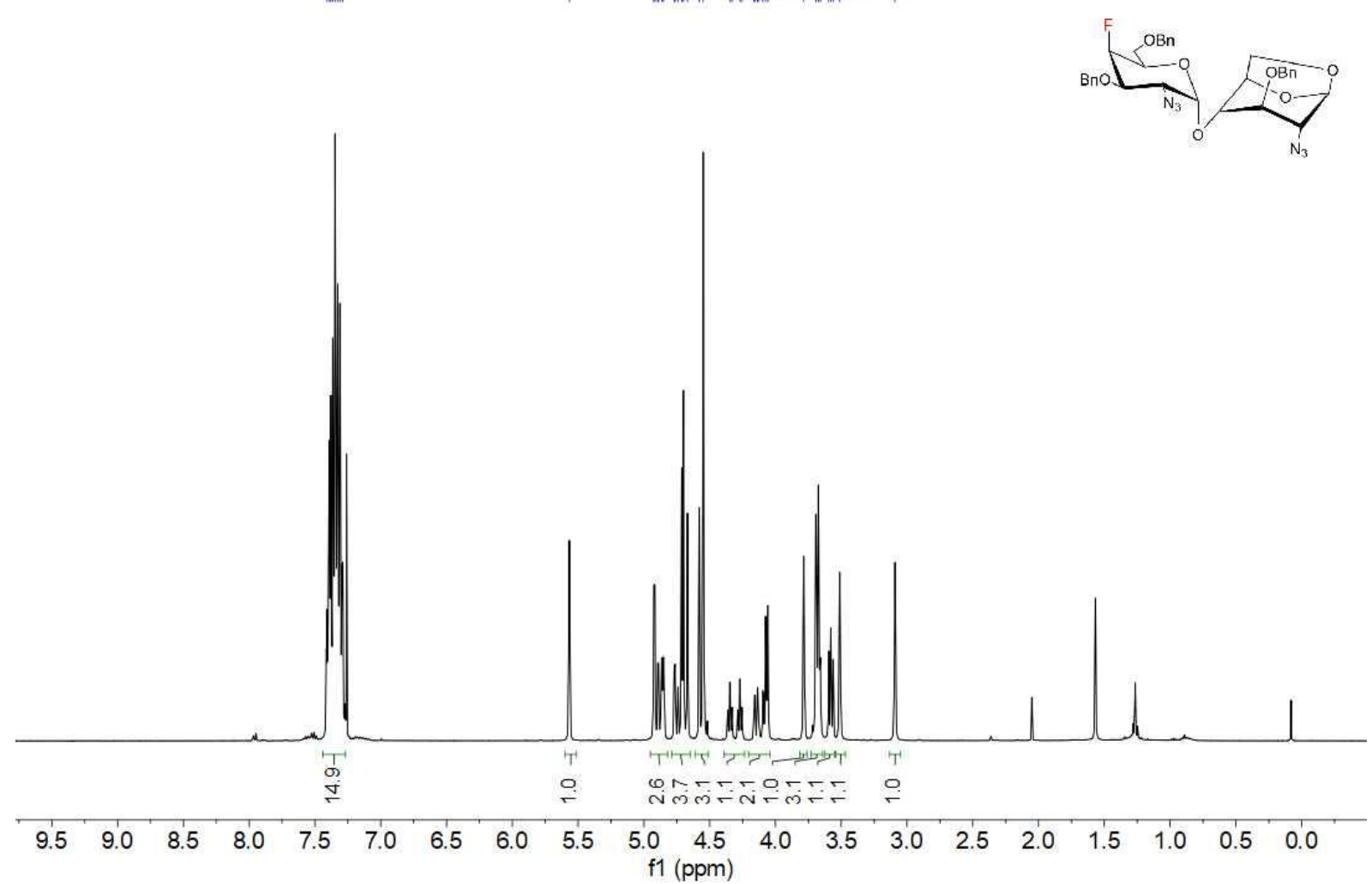

${ }^{13} \mathrm{C}\left\{{ }^{1} \mathrm{H}\right\} \mathrm{NMR} 100 \mathrm{MHz}, \mathrm{CDCl}_{3} 4 \mathrm{H}-\alpha$

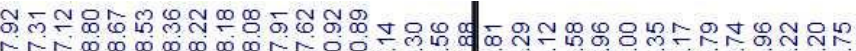

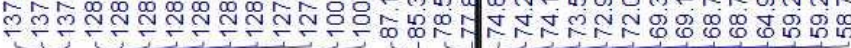

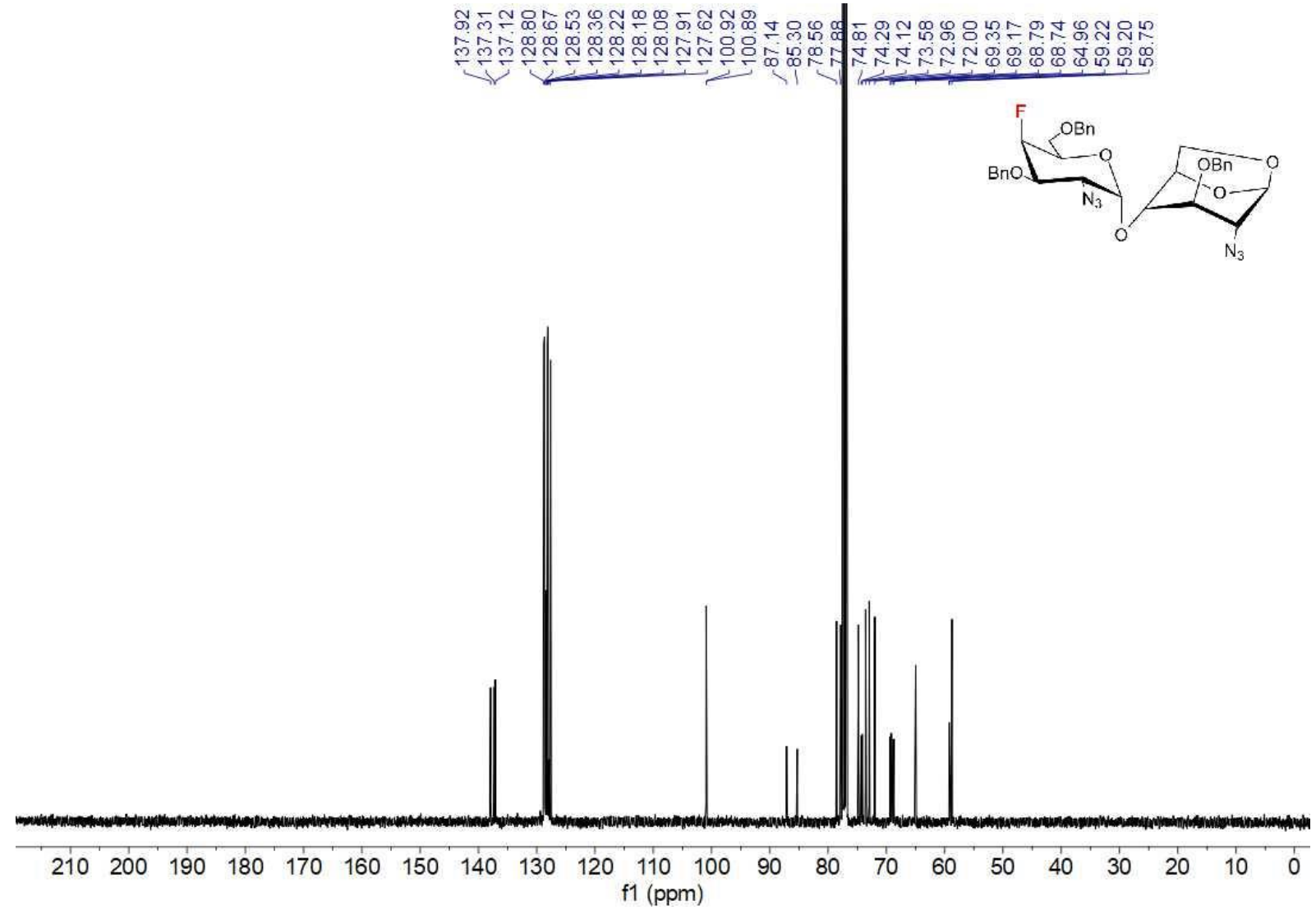


${ }^{1} \mathrm{H}-{ }^{1} \mathrm{H}$ COSY NMR $4 \mathrm{H}-\alpha$

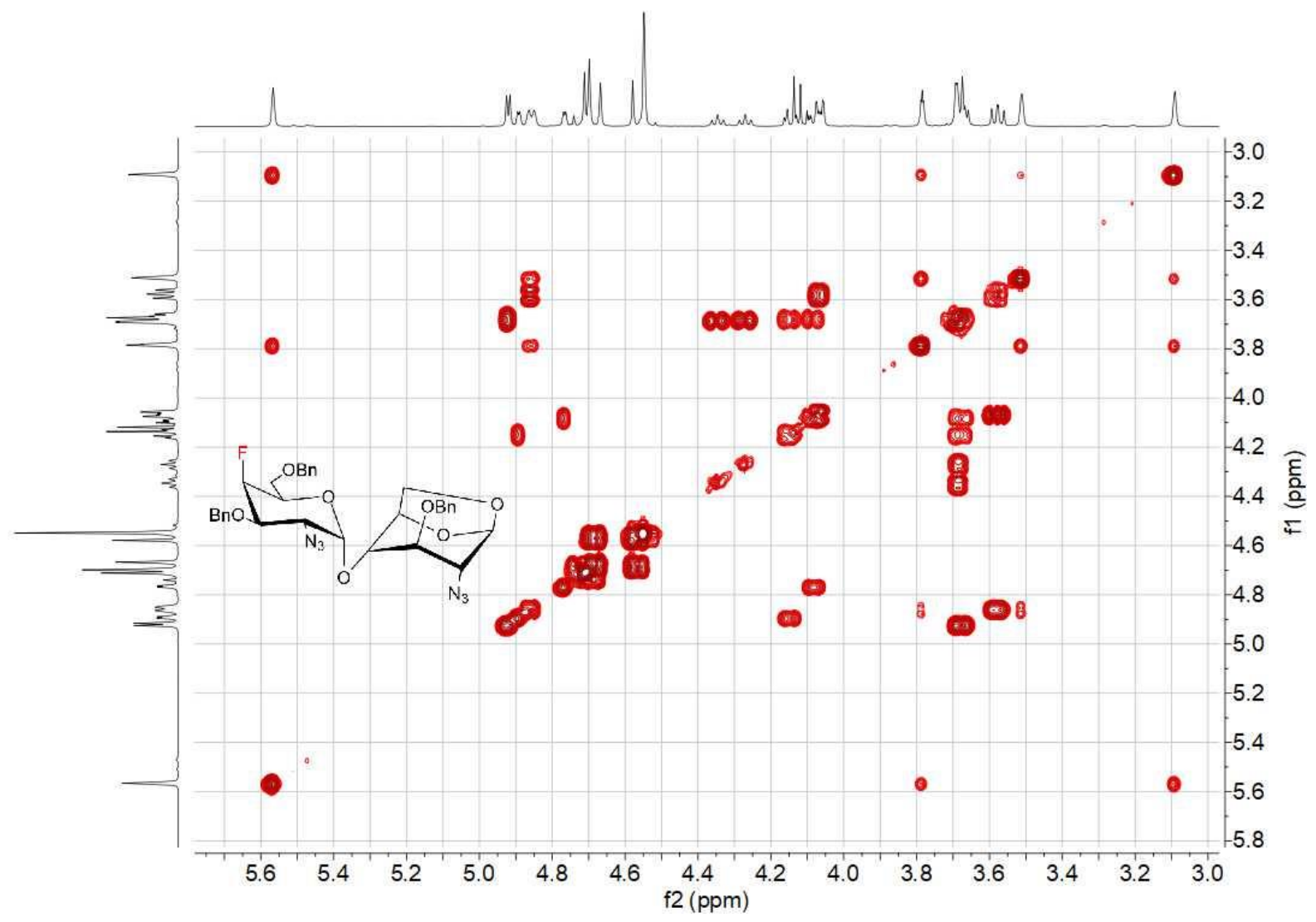

${ }^{1} \mathrm{H}-{ }^{13} \mathrm{C}$ HSQC NMR $4 \mathrm{H}-\alpha$

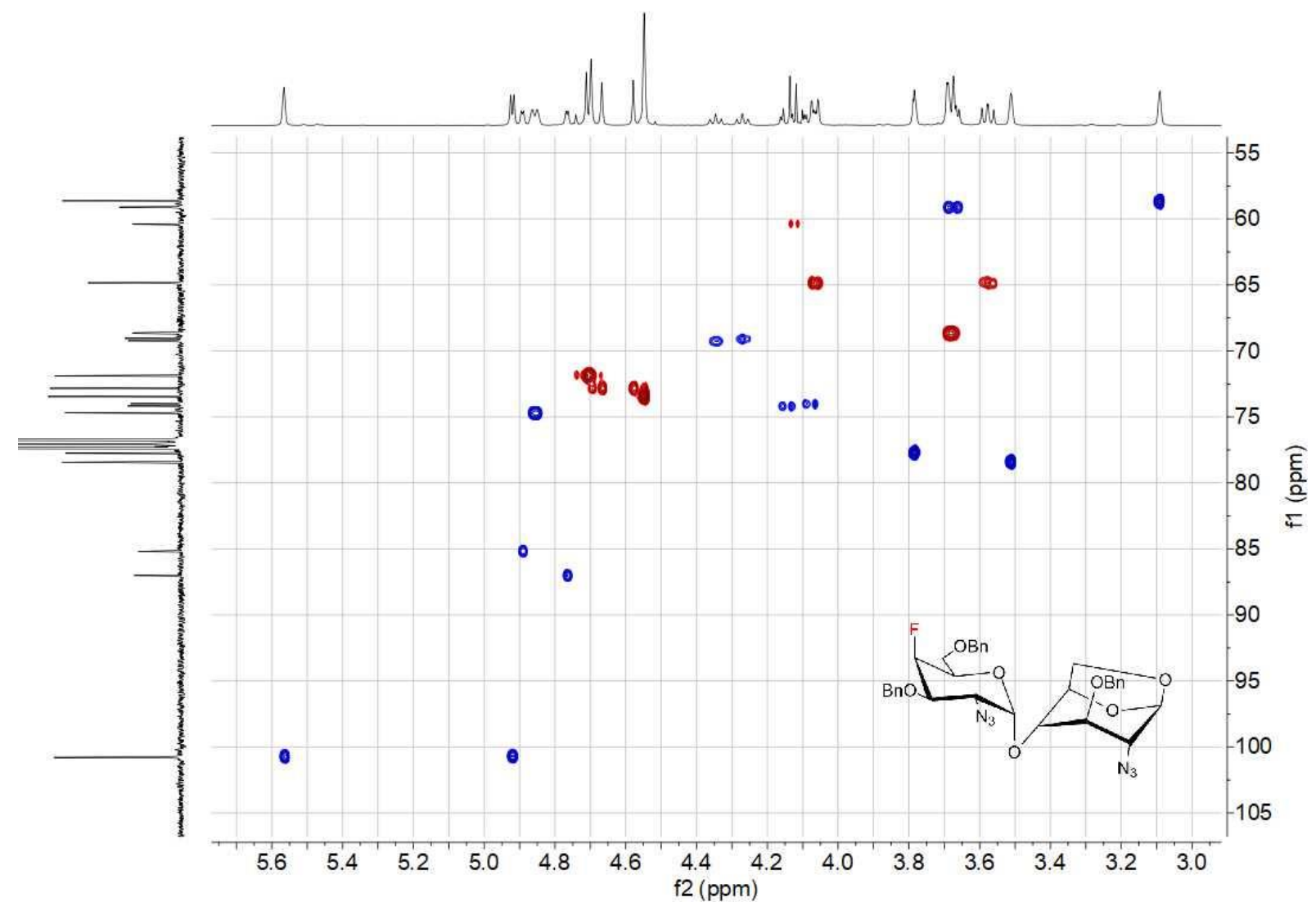


${ }^{1} \mathrm{H}-{ }^{13} \mathrm{C}$ HMBC NMR 4H- $\alpha$

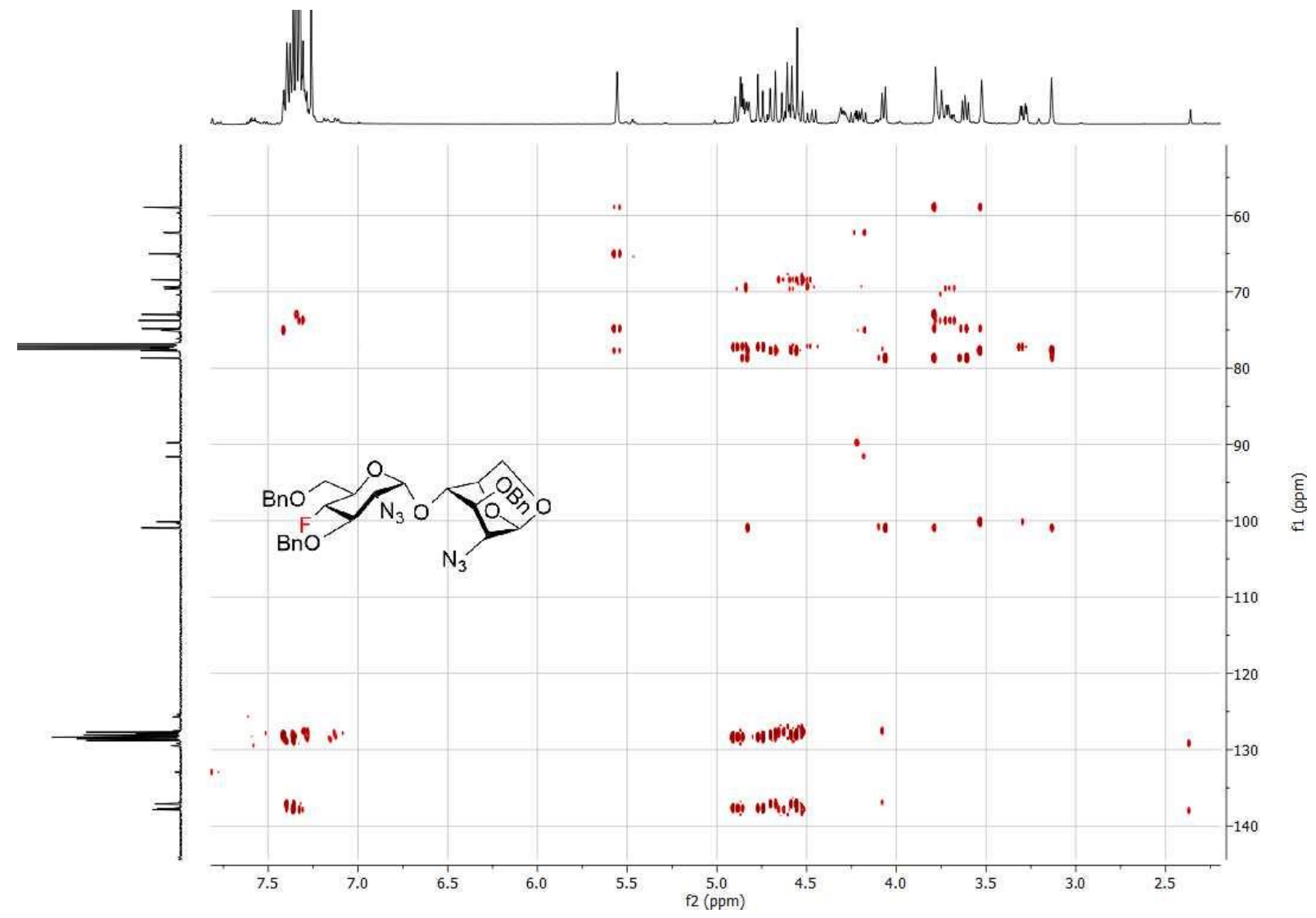

${ }^{19} \mathrm{~F} \mathrm{NMR} 376 \mathrm{MHz}, \mathrm{CDCl}_{3} 4 \mathrm{H}-\boldsymbol{\alpha}$
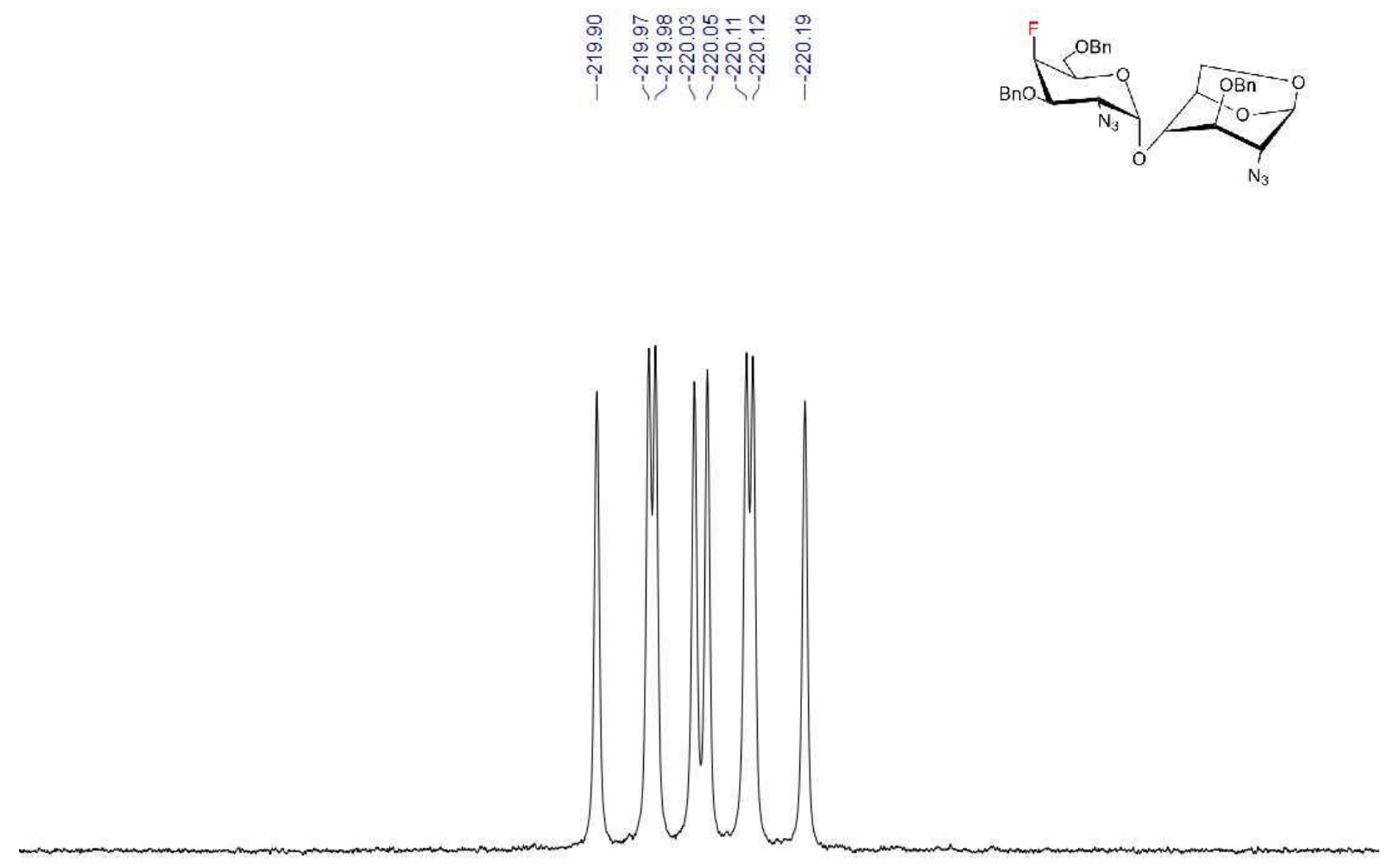

$-219.2-219.3-219.4-219.5-219.6-219.7-219.8-219.9-220.0-220.1-220.2-220.3-220.4-220.5-220.6-220.7-220.8-220.9$ f1 (ppm) 
NMR 4I- $\alpha$

${ }^{1} \mathrm{H} N M R 400 \mathrm{MHz}, \mathrm{CDCl}_{3} 4 \mathrm{I}-\alpha$

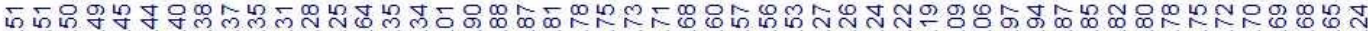

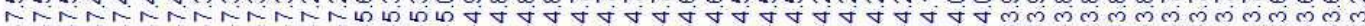

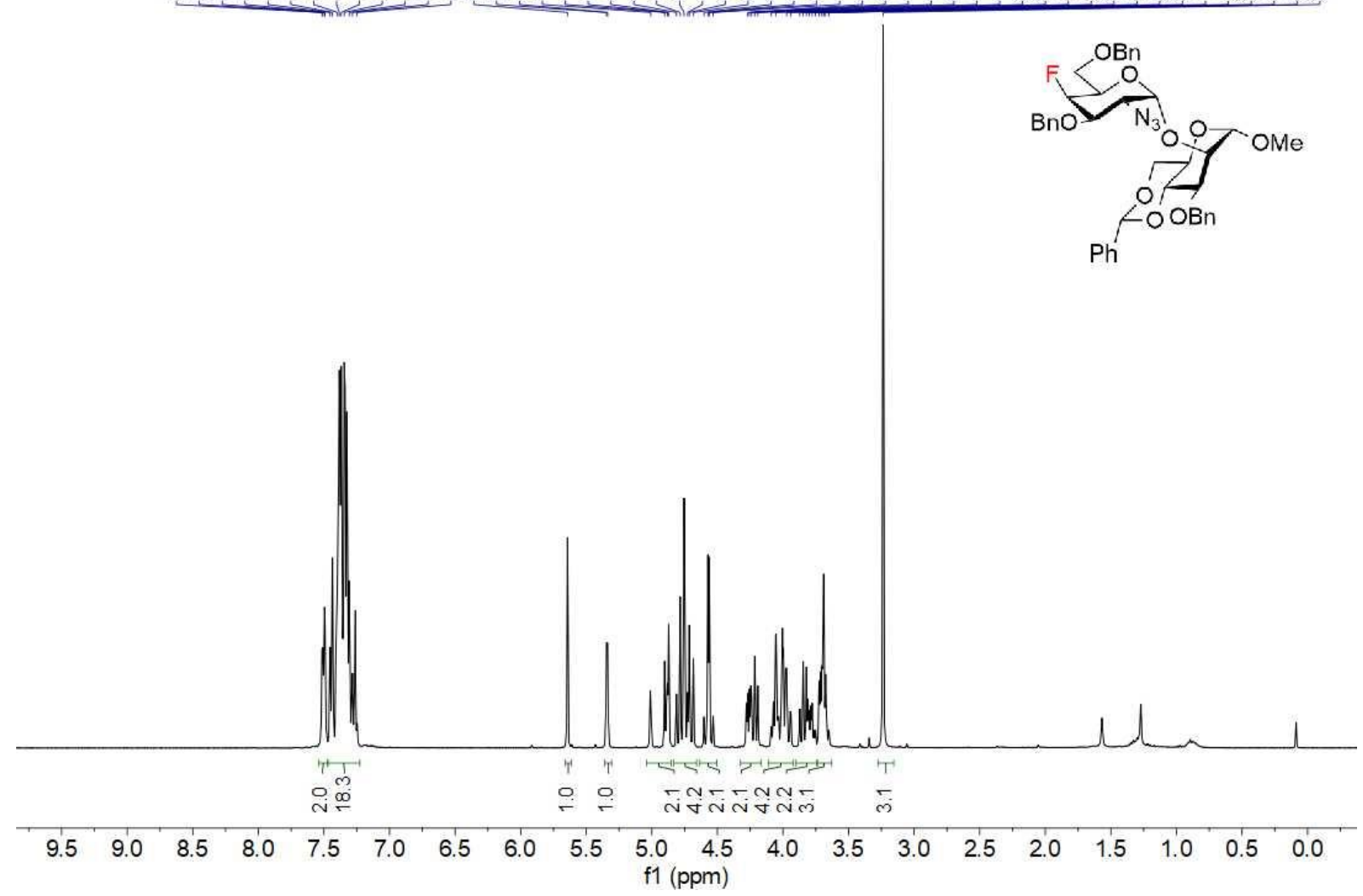

${ }^{13} \mathrm{C}\left\{{ }^{1} \mathrm{H}\right\} \mathrm{NMR} 100 \mathrm{MHz}, \mathrm{CDCl}_{3} 4 \mathrm{I}-\alpha$

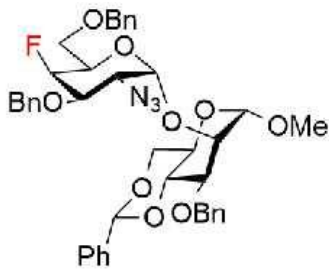

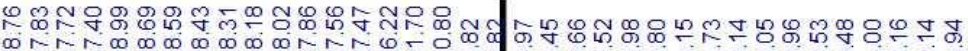

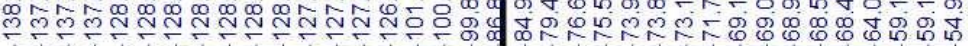

$\begin{array}{llllllllllllllllllllllllllll}210 & 200 & 190 & 180 & 170 & 160 & 150 & 140 & 130 & 120 & 110 & 100 & 90 & 80 & 70 & 60 & 50 & 40 & 30 & 20 & 10 & 0\end{array}$ 
${ }^{1} \mathrm{H}-{ }^{1} \mathrm{H}$ COSY NMR $4 \mathrm{I}-\alpha$

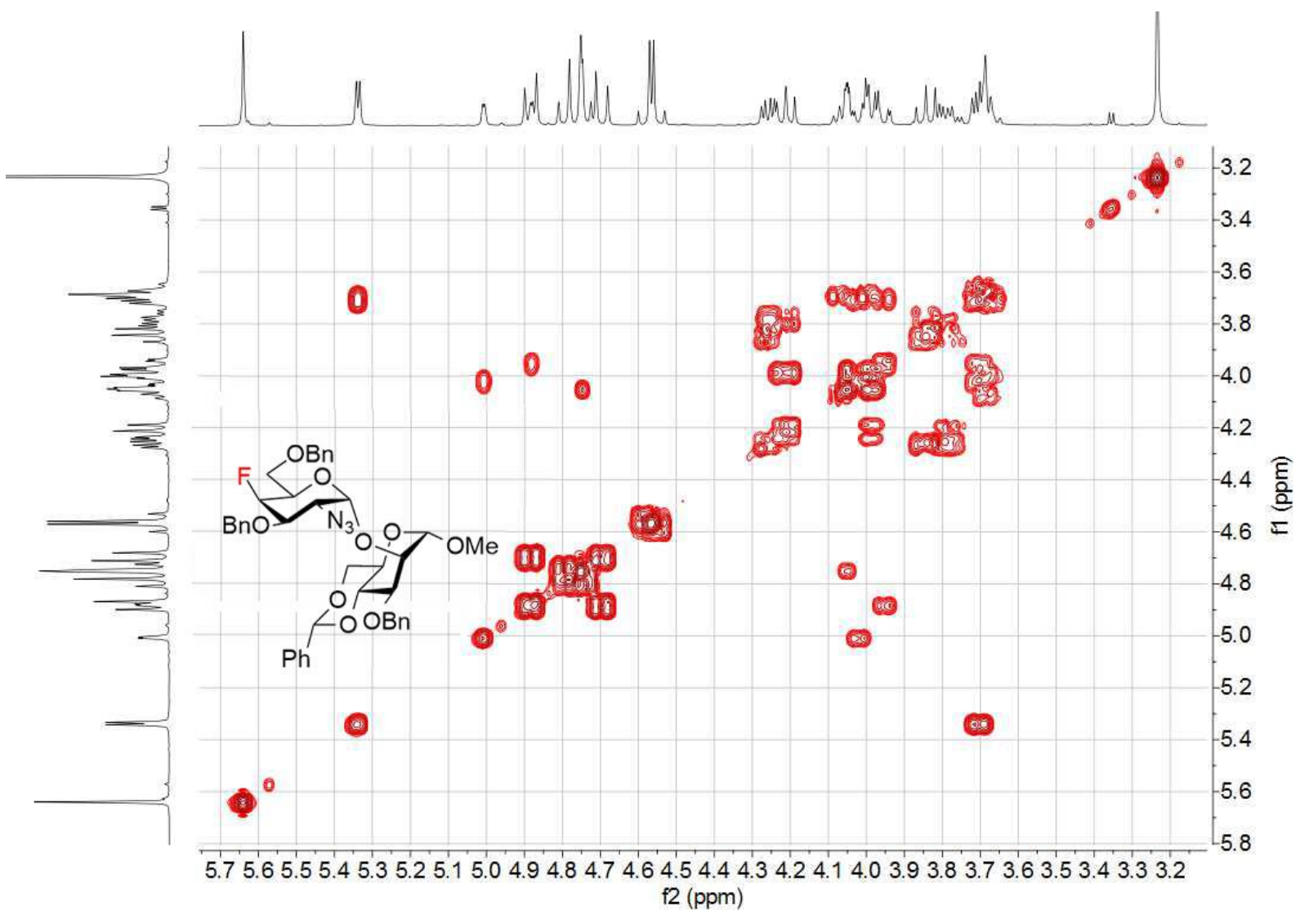

${ }^{1} \mathrm{H}-{ }^{13} \mathrm{C}$ HSQC NMR $4 \mathrm{I}-\alpha$

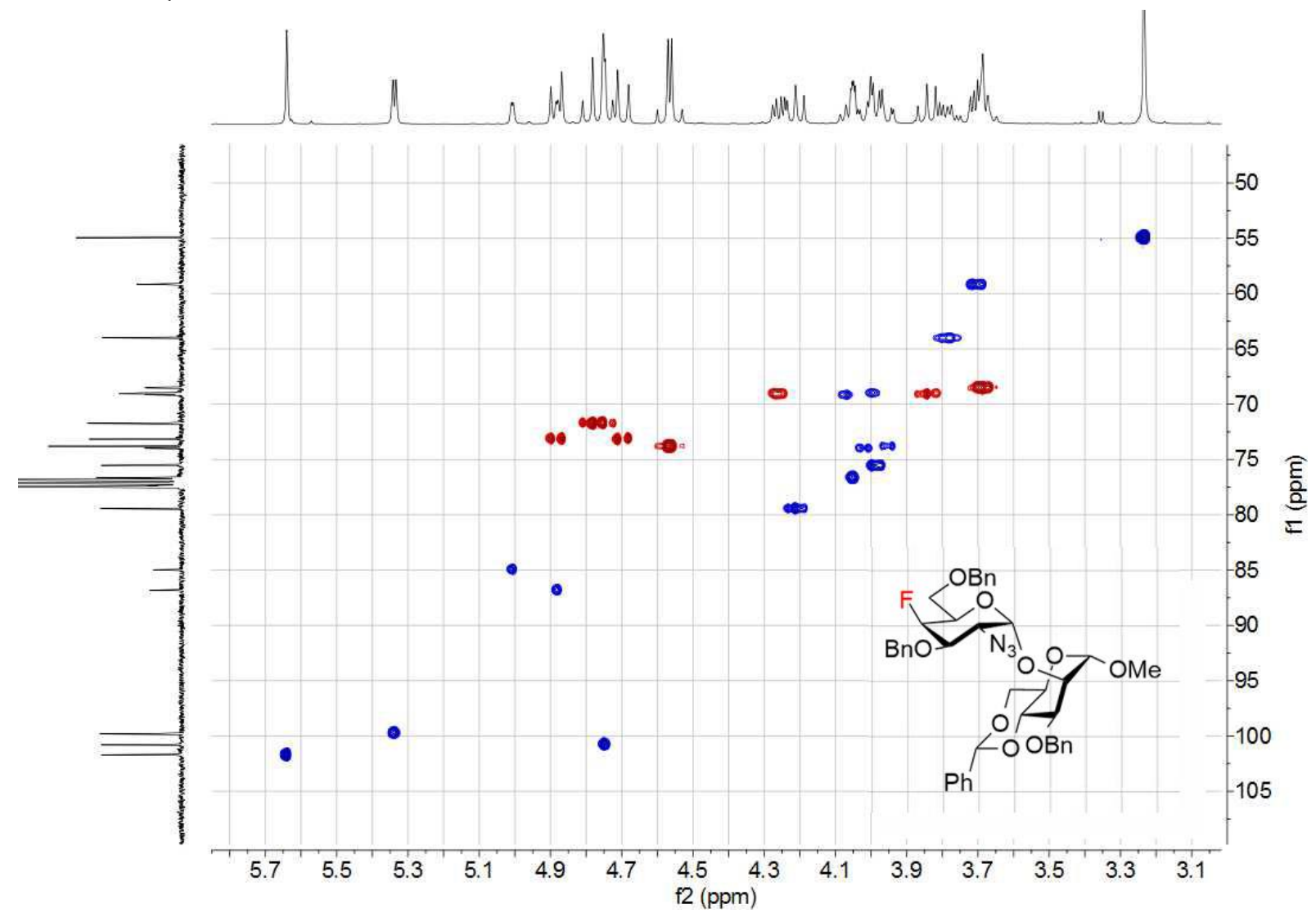


${ }^{19} \mathrm{~F} \mathrm{NMR} 376 \mathrm{MHz}, \mathrm{CDCl}_{3} 4 \mathrm{l}-\alpha$

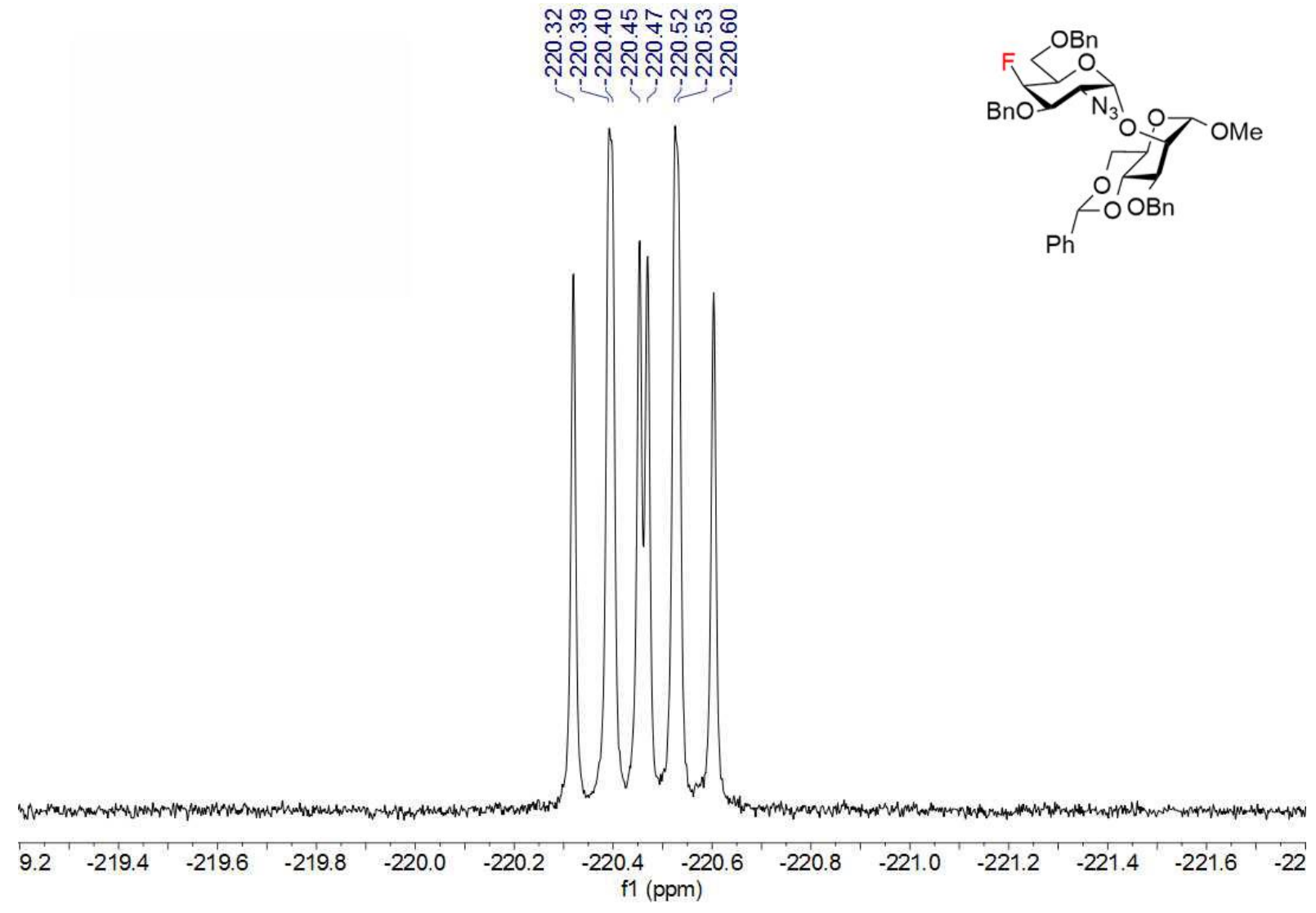

NMR $\alpha-22$

${ }^{1} \mathrm{HNMR} 400 \mathrm{MHz}, \mathrm{CDCl}_{3} \boldsymbol{\alpha}-\mathbf{2 2}$

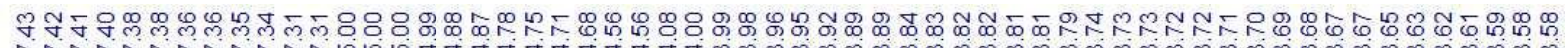

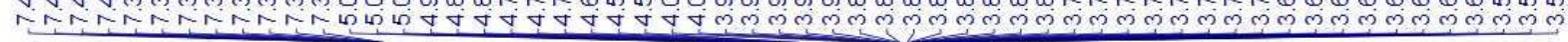

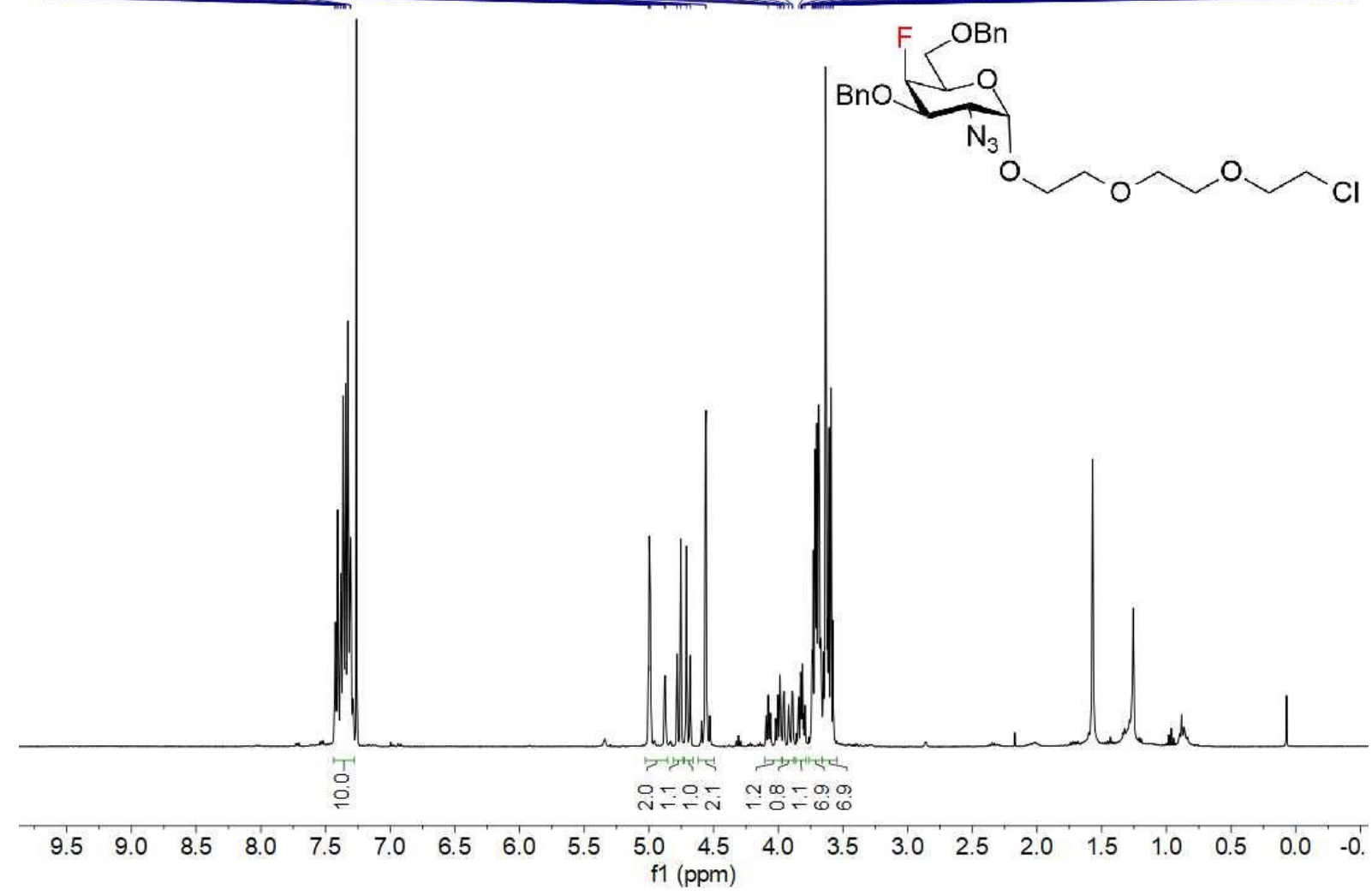



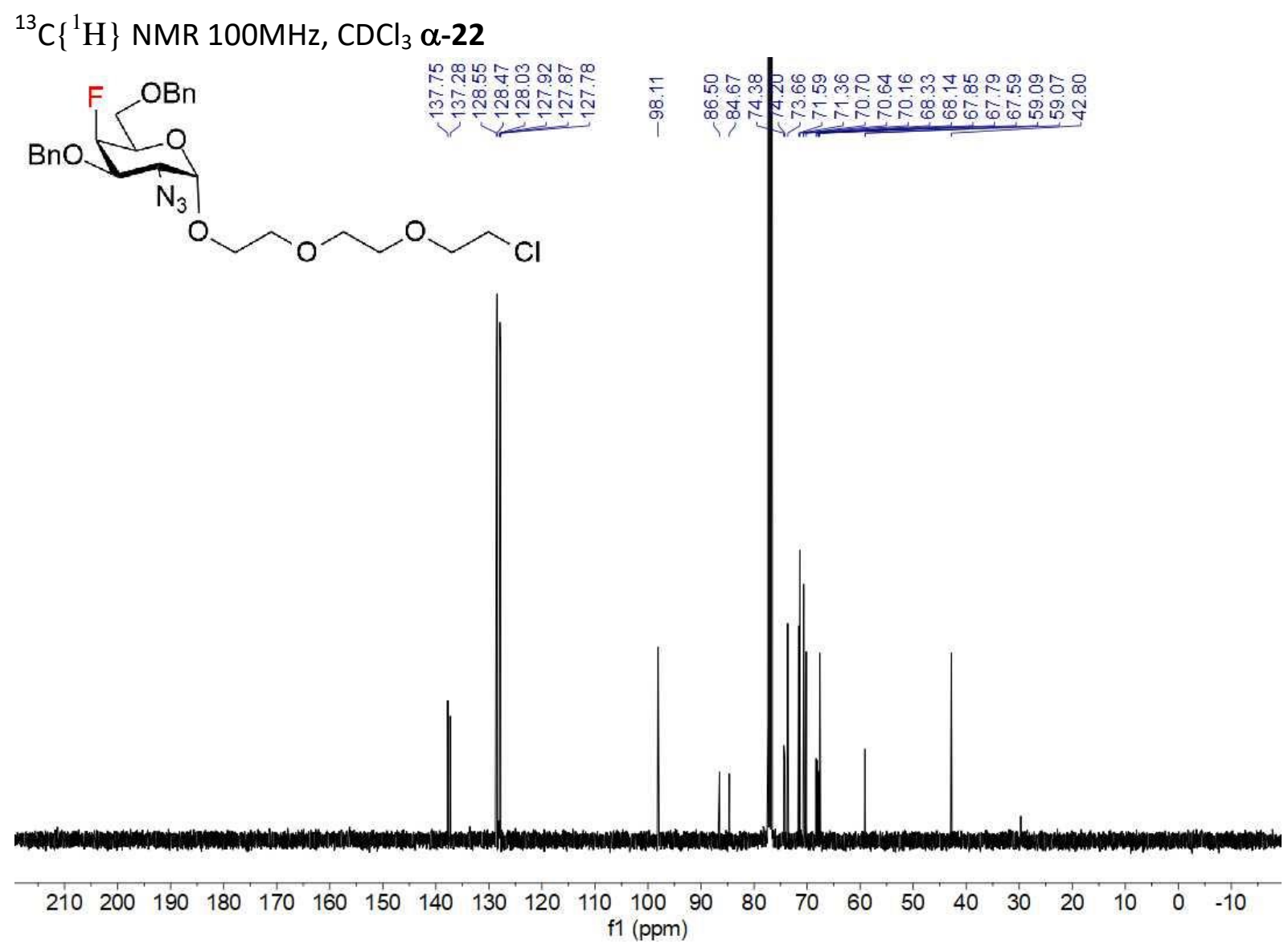

${ }^{1} \mathrm{H}-{ }^{1} \mathrm{H}$ COSY NMR $\boldsymbol{\alpha - 2 2}$

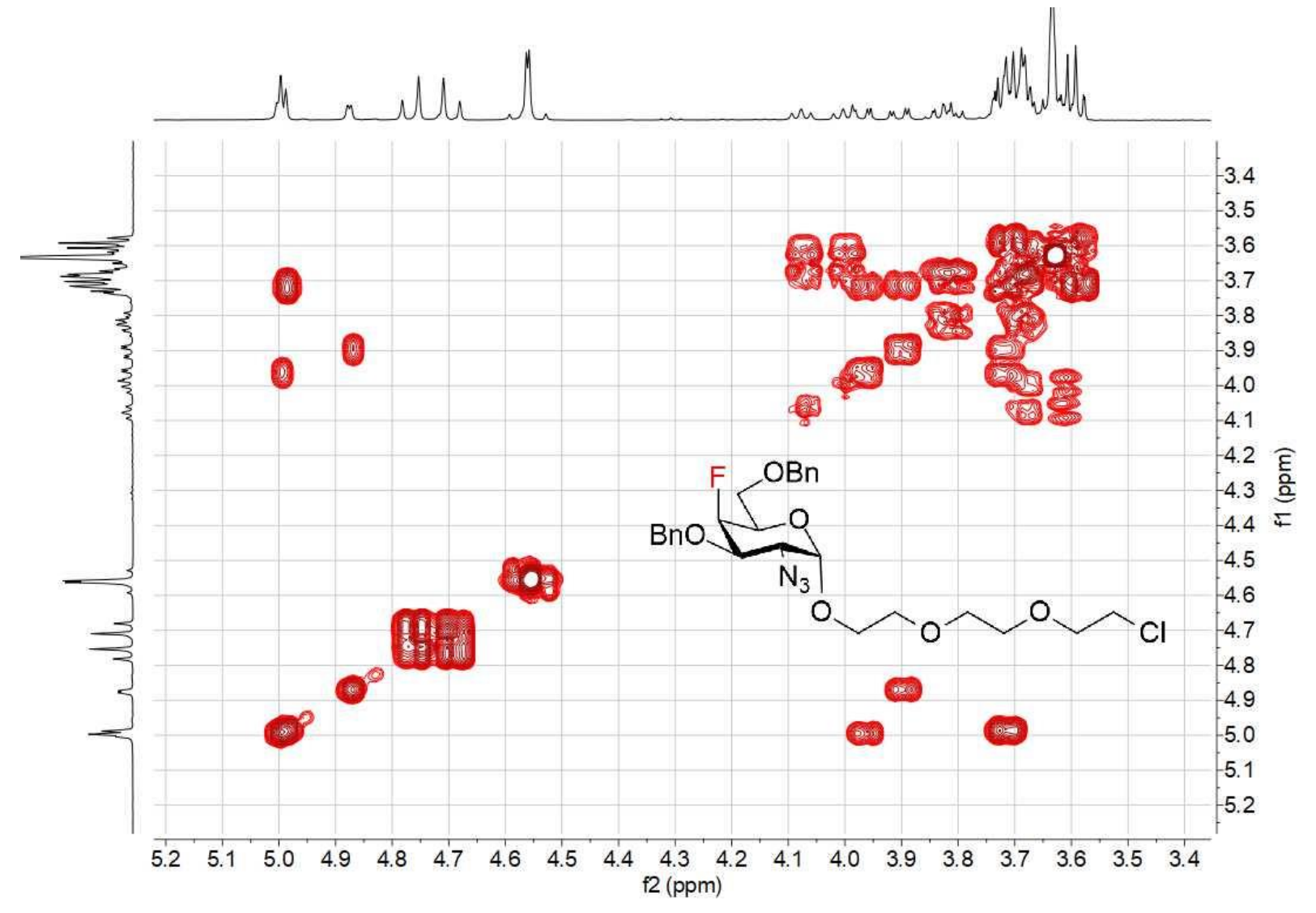


${ }^{1} \mathrm{H}-{ }^{13} \mathrm{C}$ HSQC NMR $\alpha-22$

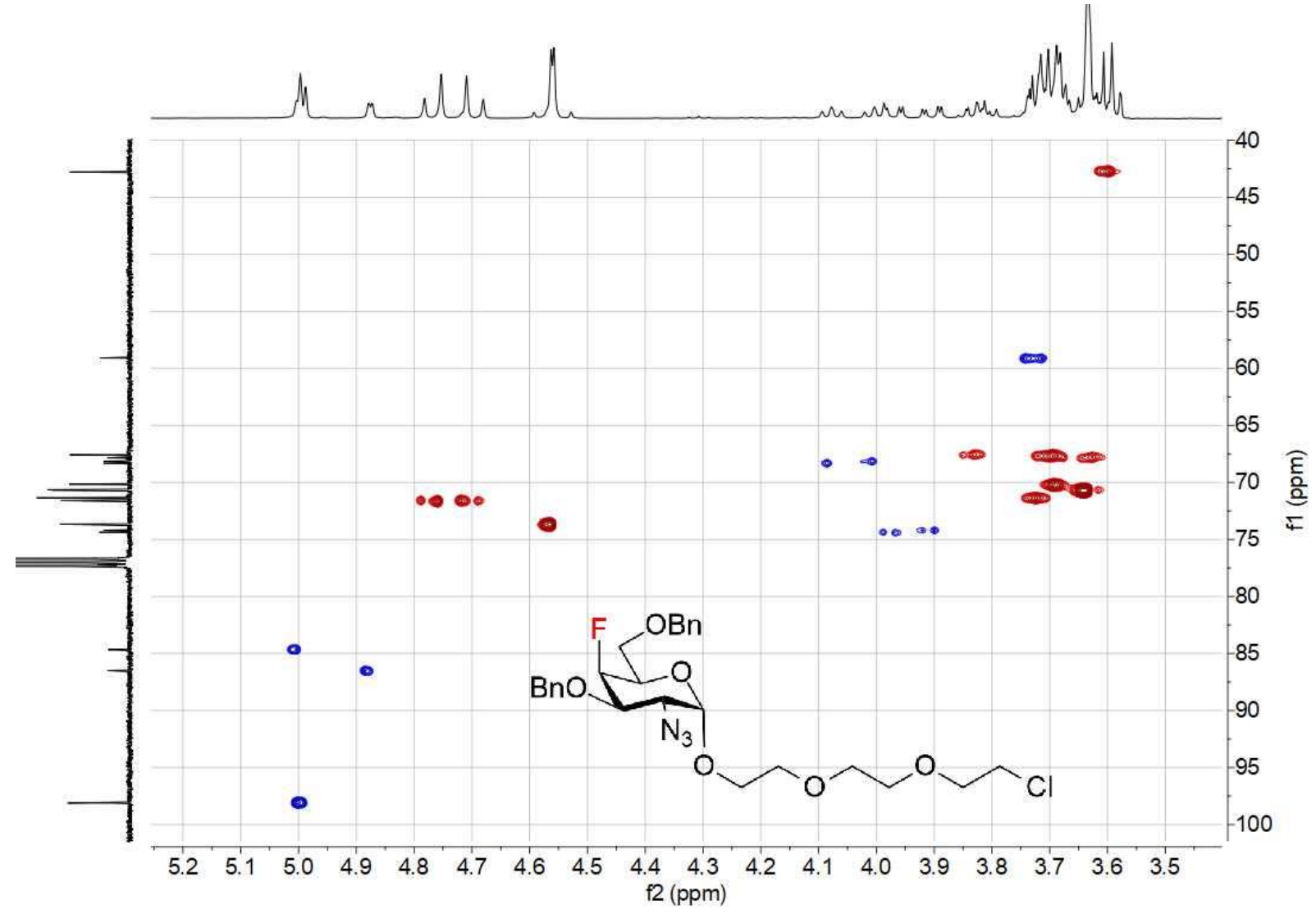




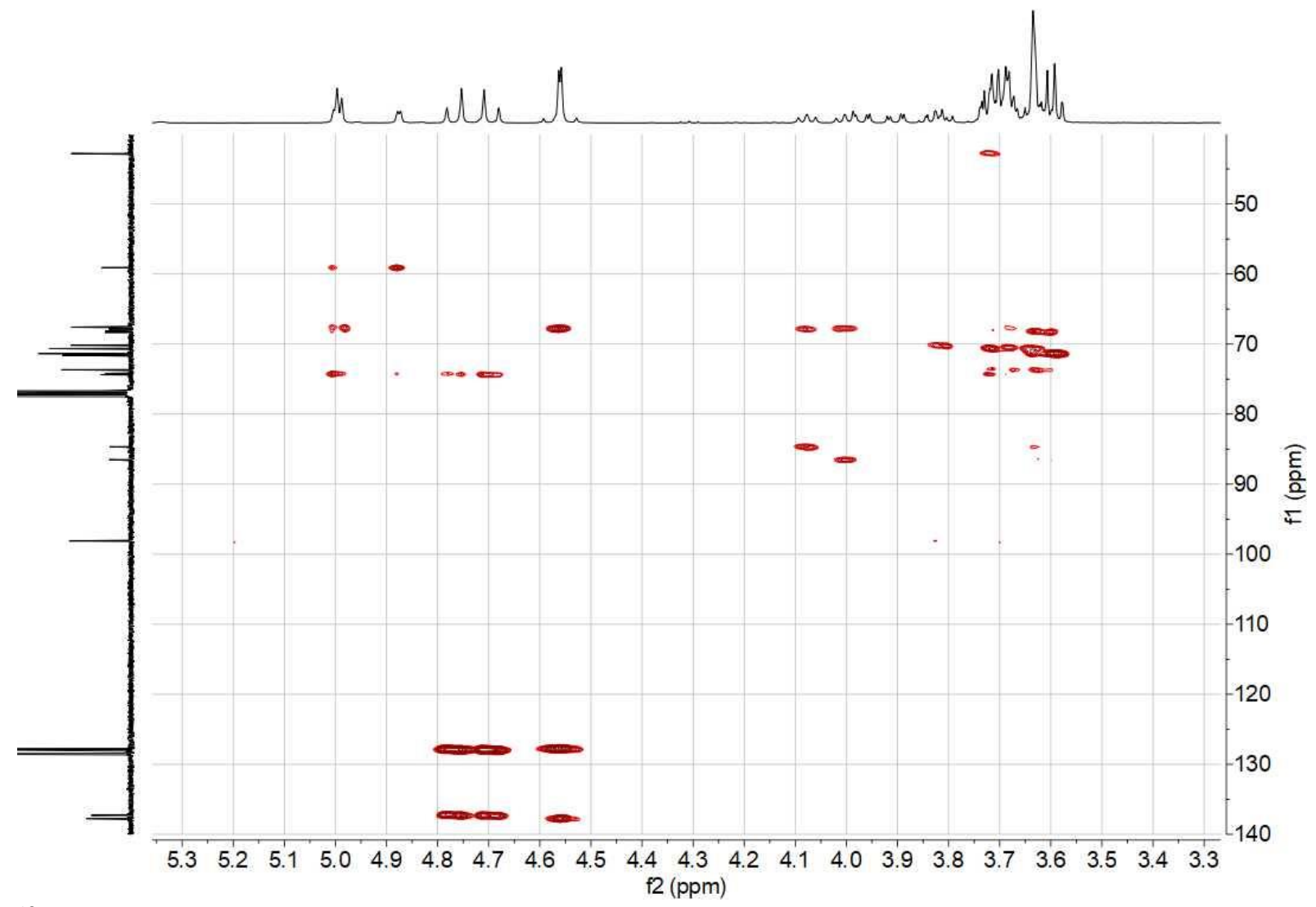

${ }^{19} \mathrm{~F} \mathrm{NMR} 376 \mathrm{MHz}, \mathrm{CDCl}_{3} \boldsymbol{\alpha}-\mathbf{2 2}$

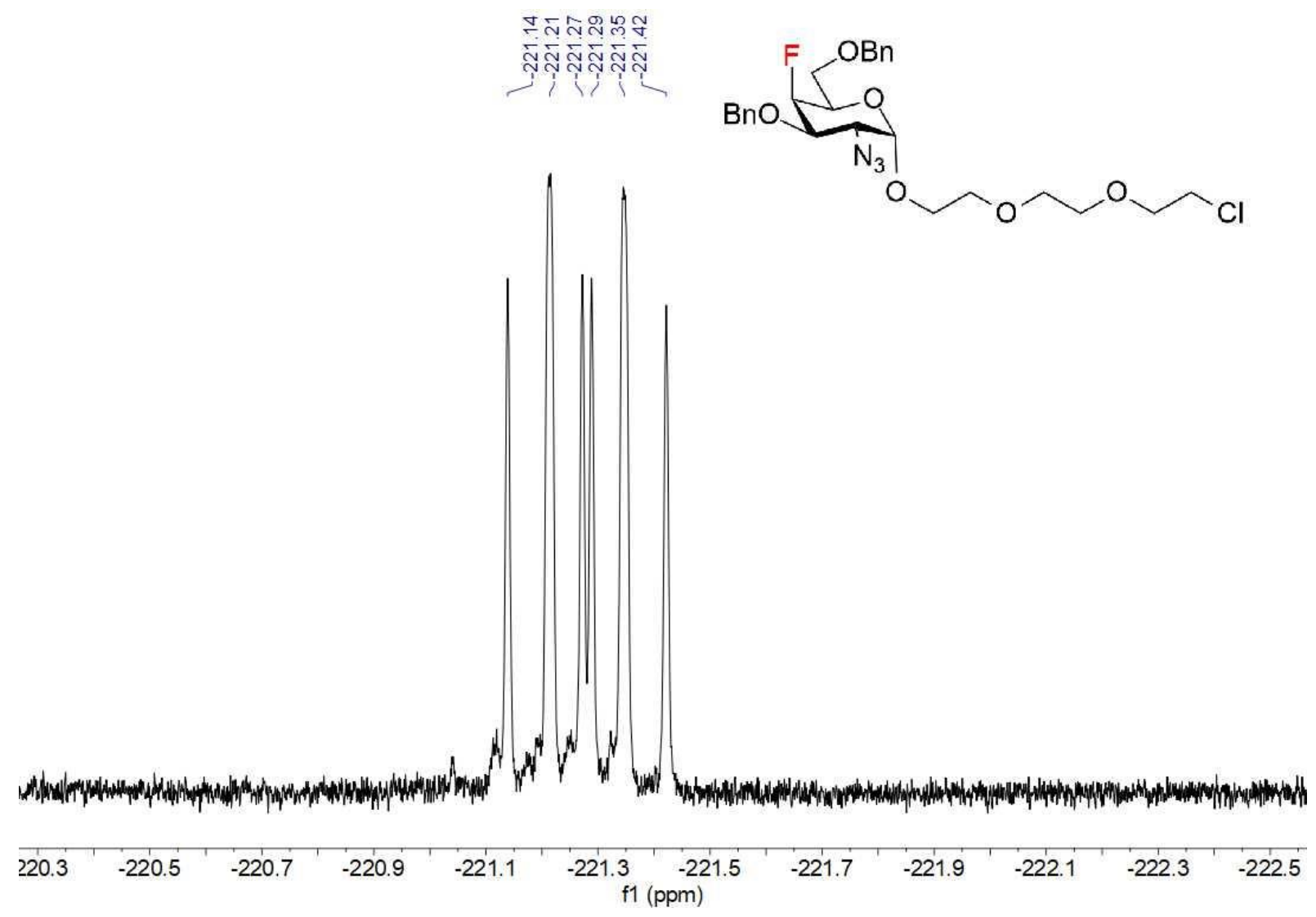


NMR $\beta-22$

${ }^{1} \mathrm{HNMR} 400 \mathrm{MHz}, \mathrm{CDCl}_{3} \beta-23$

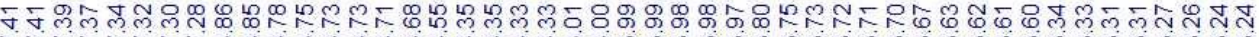

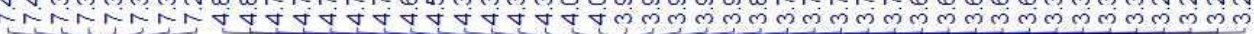<smiles>CC(C)C(OCCOCCOCCCl)C(OCc1ccccc1)C(=O)OCc1ccccc1</smiles>

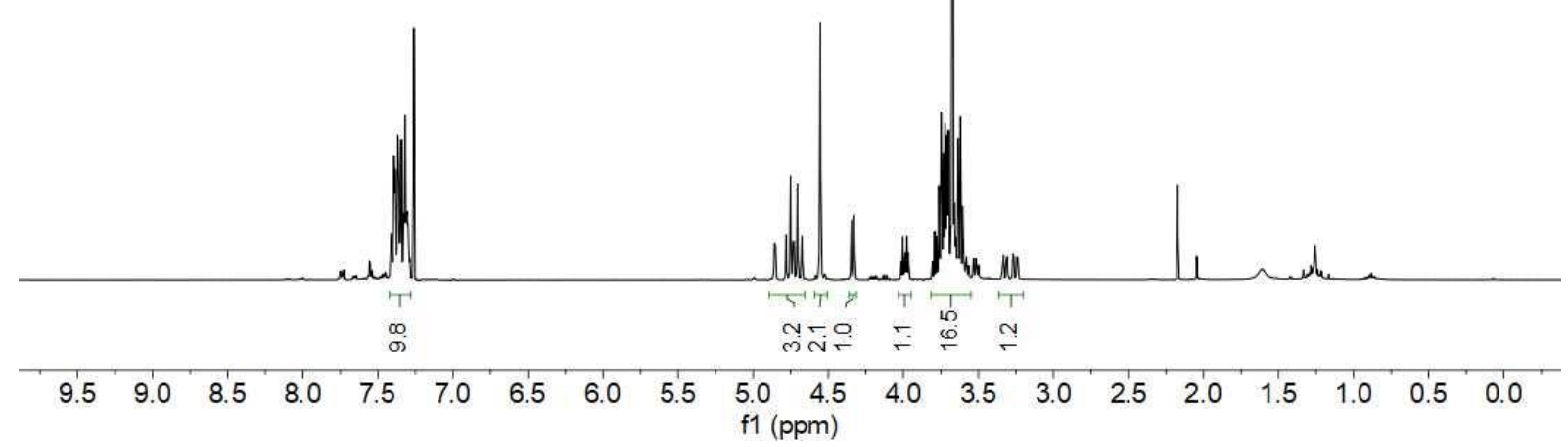

${ }^{13} \mathrm{C}\left\{{ }^{1} \mathrm{H}\right\} \mathrm{NMR}$ 100MHz, $\mathrm{CDCl}_{3} \boldsymbol{\beta}-22$

유

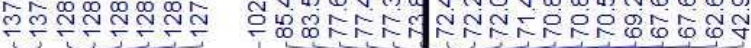

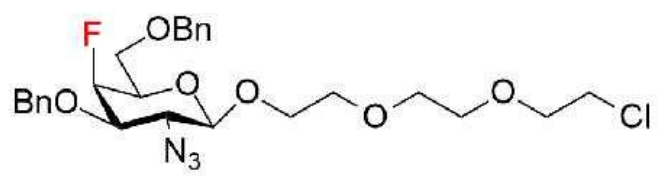

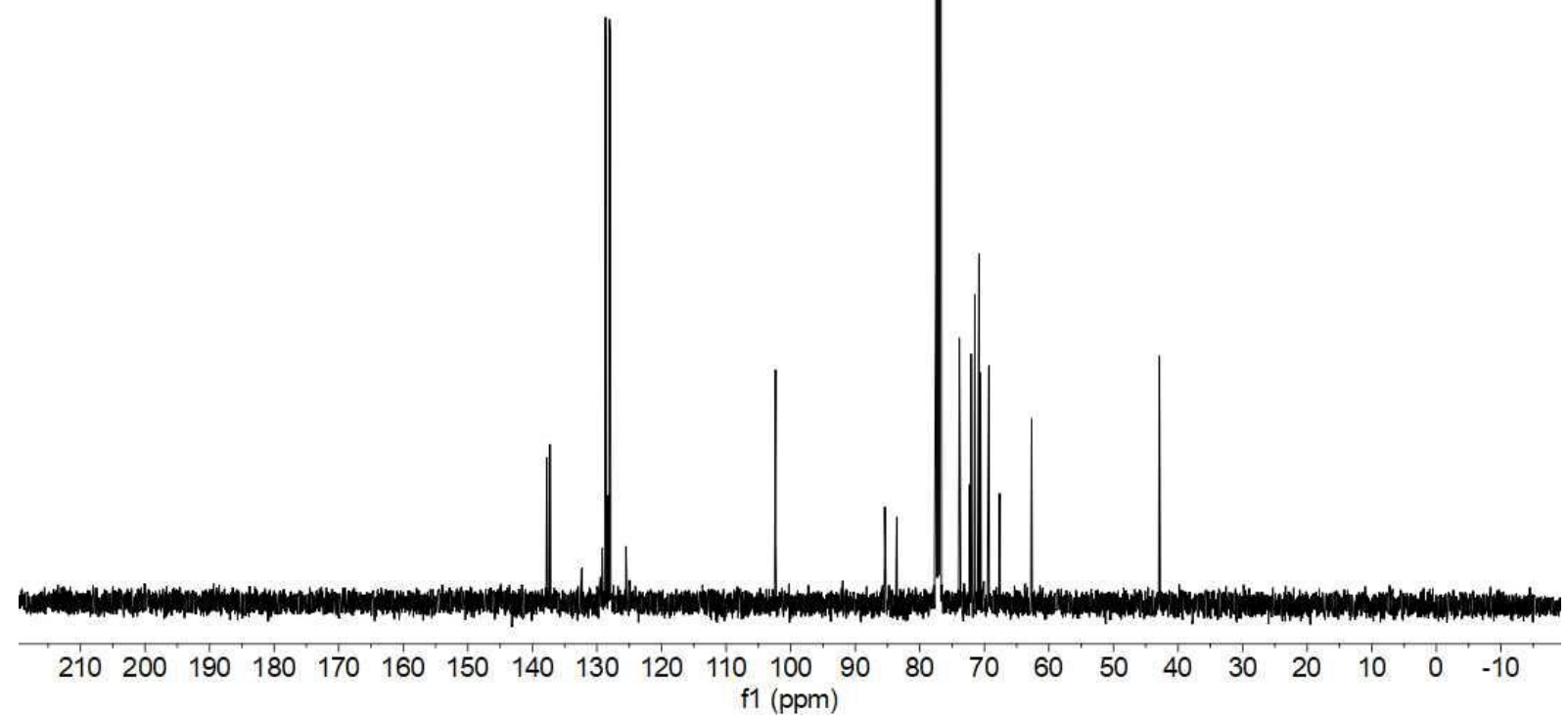


${ }^{1} \mathrm{H}-{ }^{1} \mathrm{H}$ COSY NMR $\beta-22$

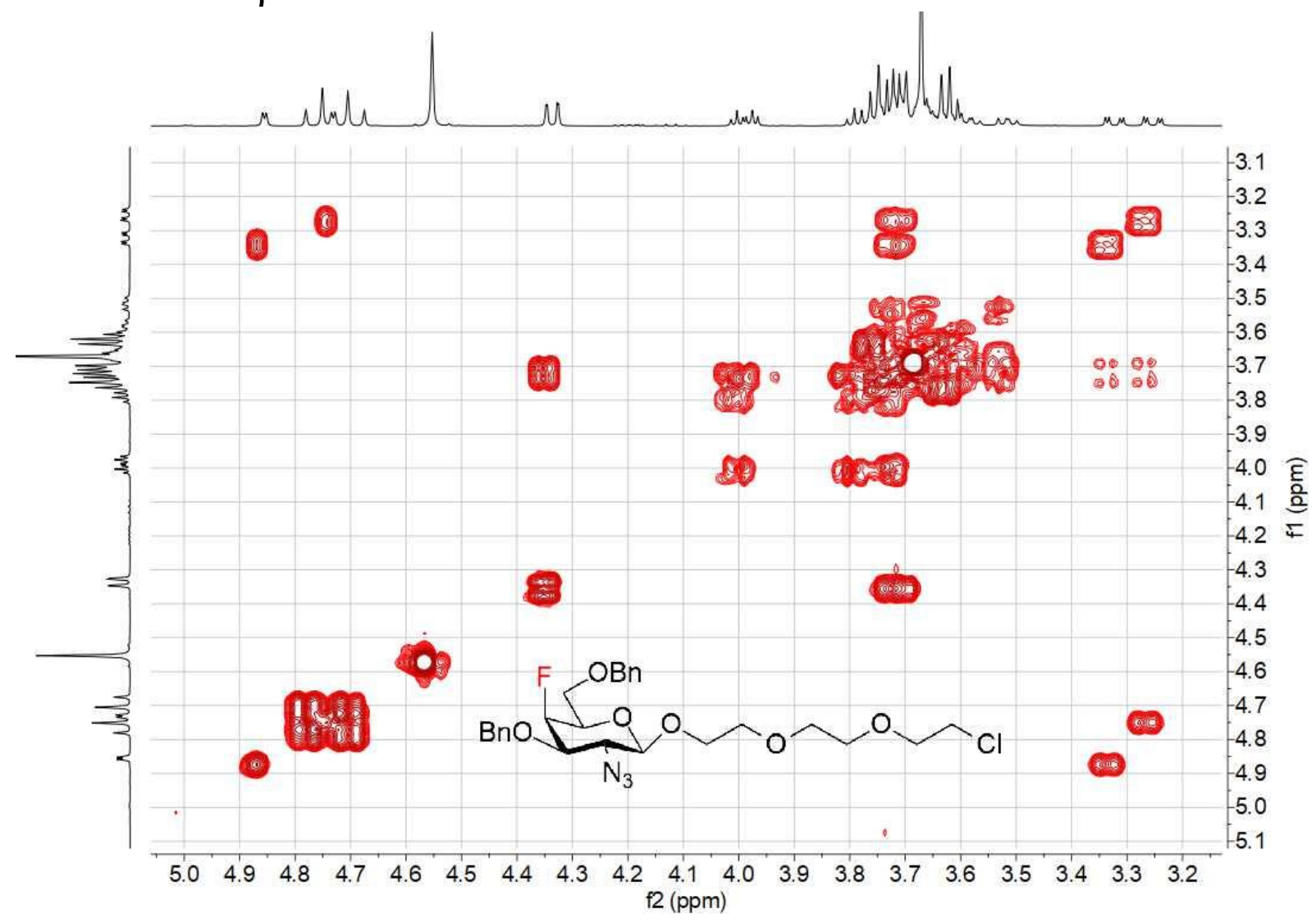

${ }^{1} \mathrm{H}-{ }^{13} \mathrm{C}$ HSQC NMR $\beta-22$

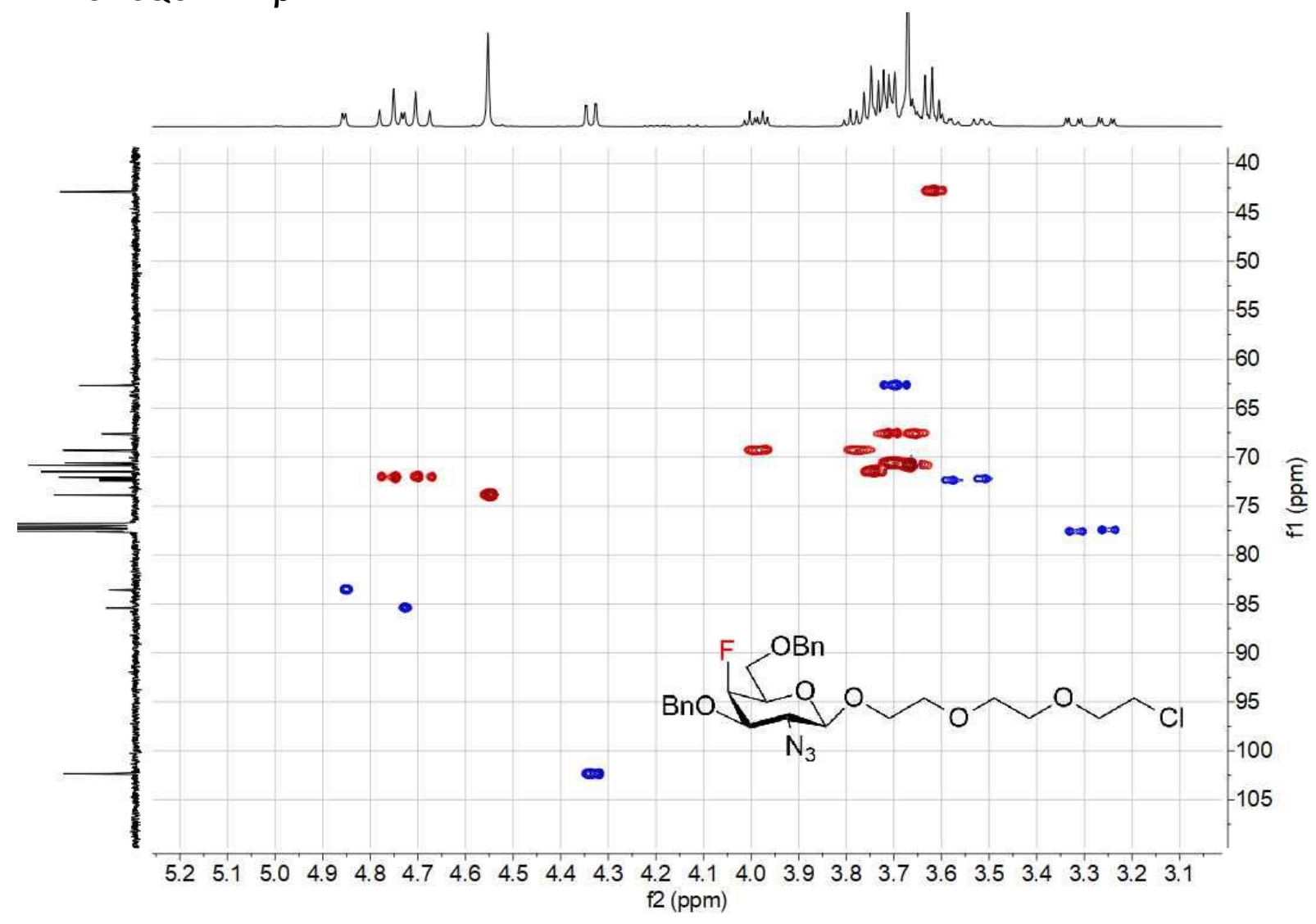


${ }^{19} \mathrm{~F}$ NMR $376 \mathrm{MHz}, \mathrm{CDCl}_{3} \boldsymbol{\beta}-\mathbf{2 2}$

ॠ

ஸे

$\mathrm{BnO} \underbrace{\mathrm{OBn}}_{\mathrm{N}_{3}} \mathrm{O}$

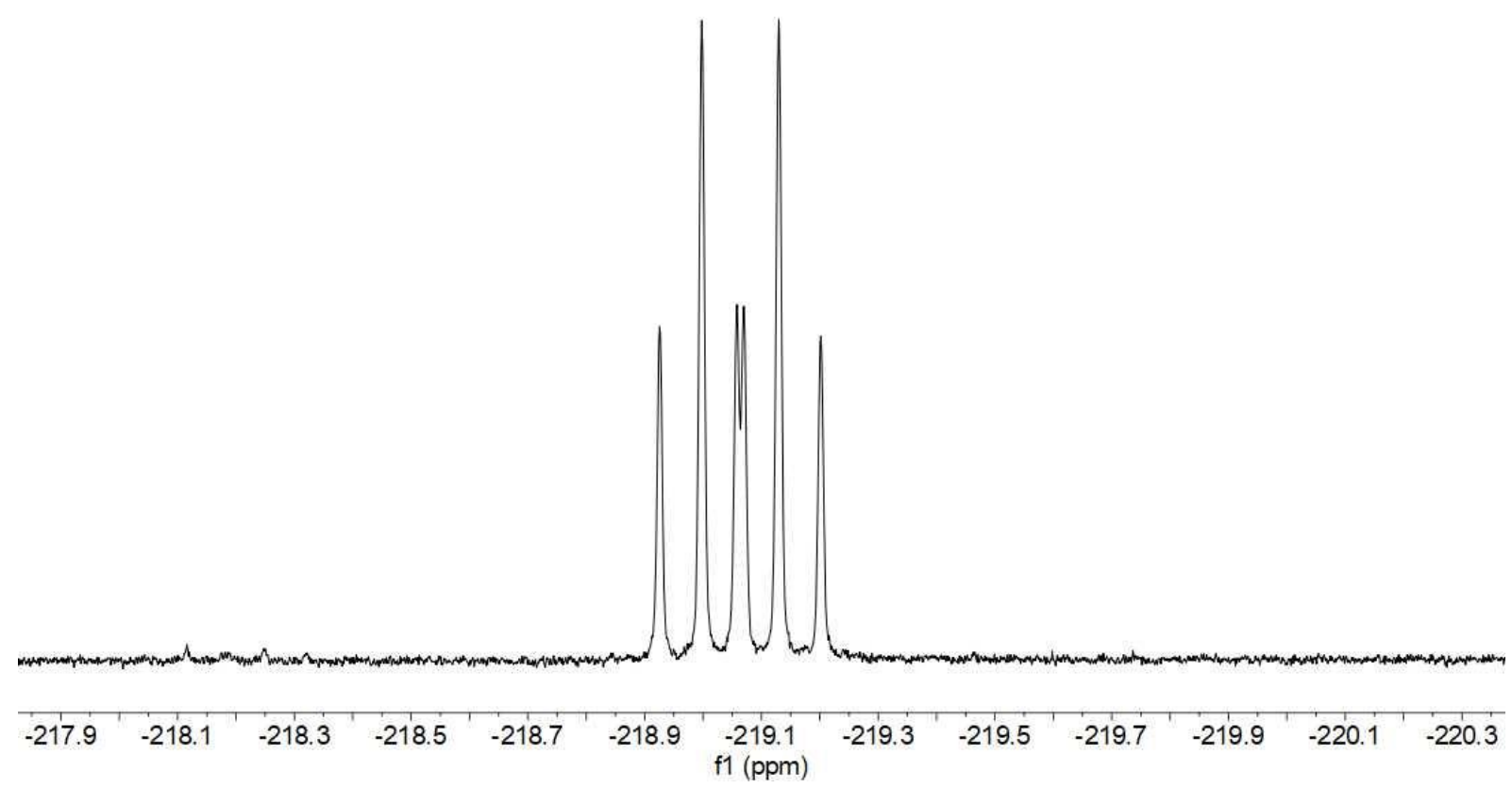

NMR 23

${ }^{1} \mathrm{HNMR} 400 \mathrm{MHz}, \mathrm{CDCl}_{3} 23$

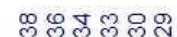

ininin

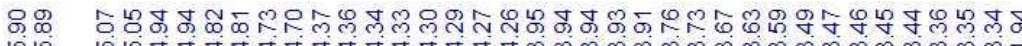

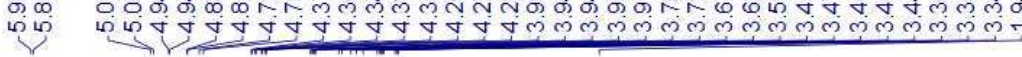

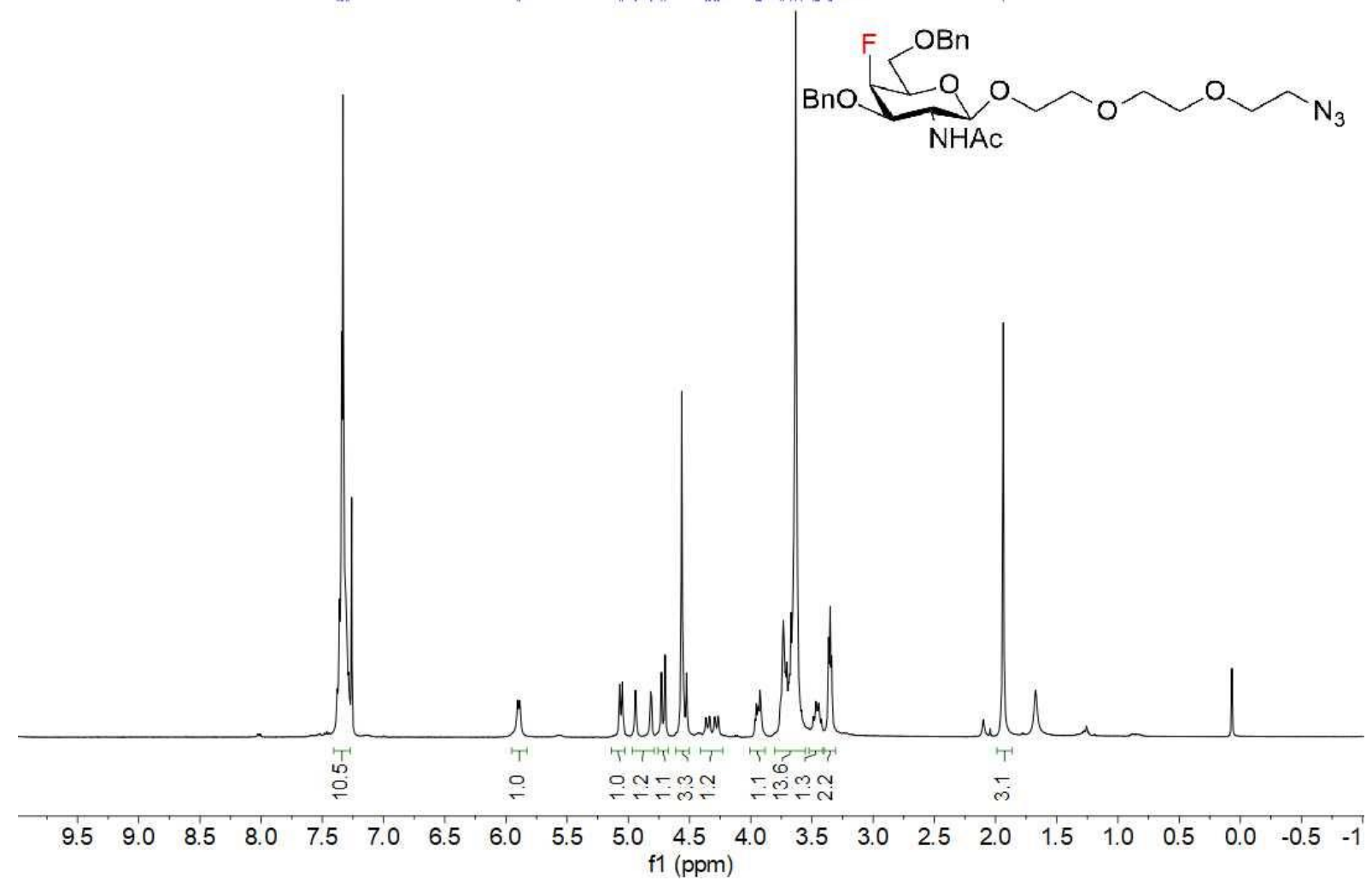


${ }^{13} \mathrm{C}\left\{{ }^{1} \mathrm{H}\right\}$ NMR 100MHz, $\mathrm{CDCl}_{3} 23$

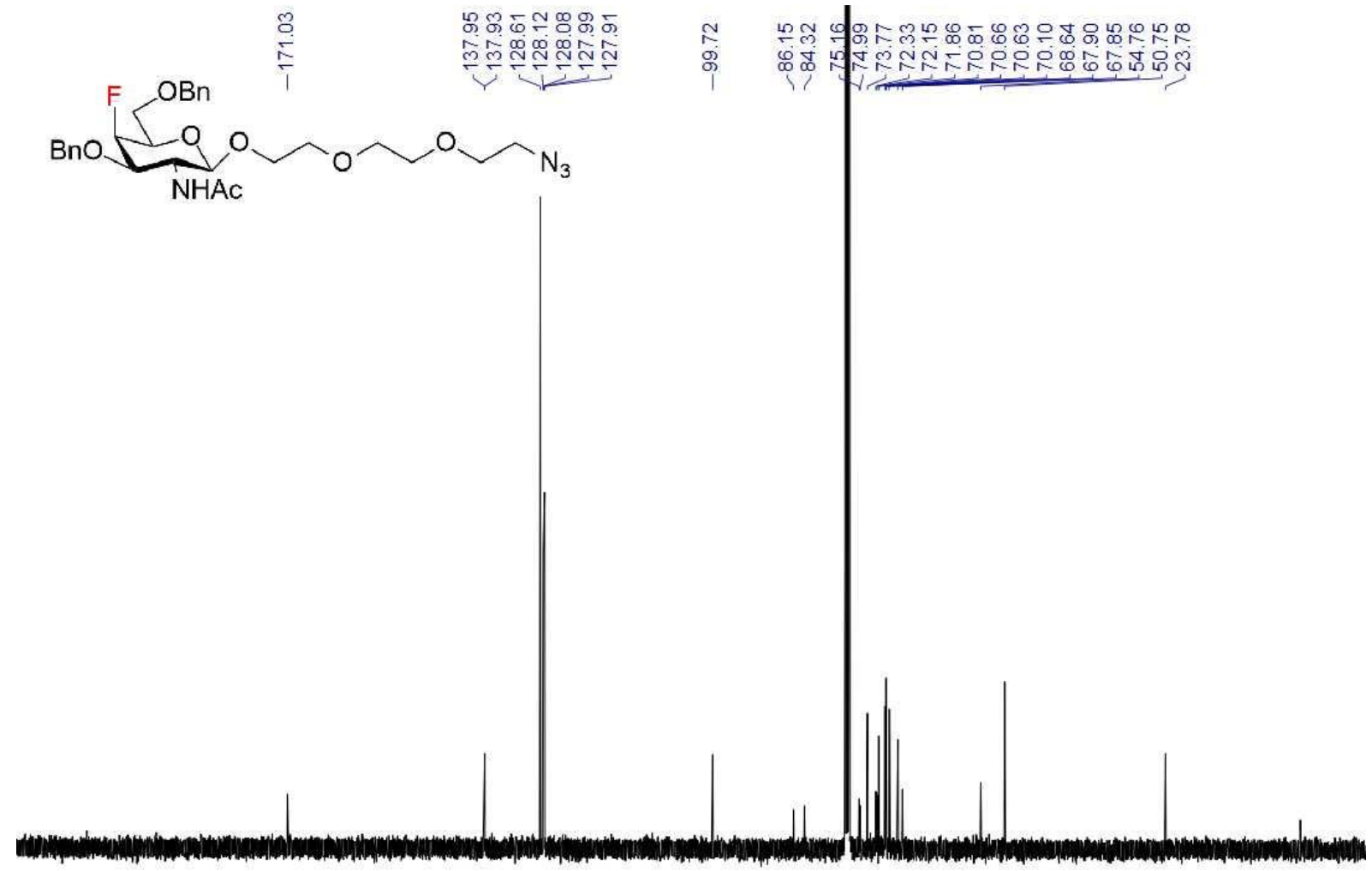

$\begin{array}{llllllllllllllllllllll}210 & 200 & 190 & 180 & 170 & 160 & 150 & 140 & 130 & 120 & \begin{array}{r}110 \\ f 1(\mathrm{ppm})\end{array} & 90 & 80 & 70 & 60 & 50 & 40 & 30 & 20 & 10 & 0 & 10\end{array}$

${ }^{1} \mathrm{H}-{ }^{1} \mathrm{H}$ COSY NMR 23

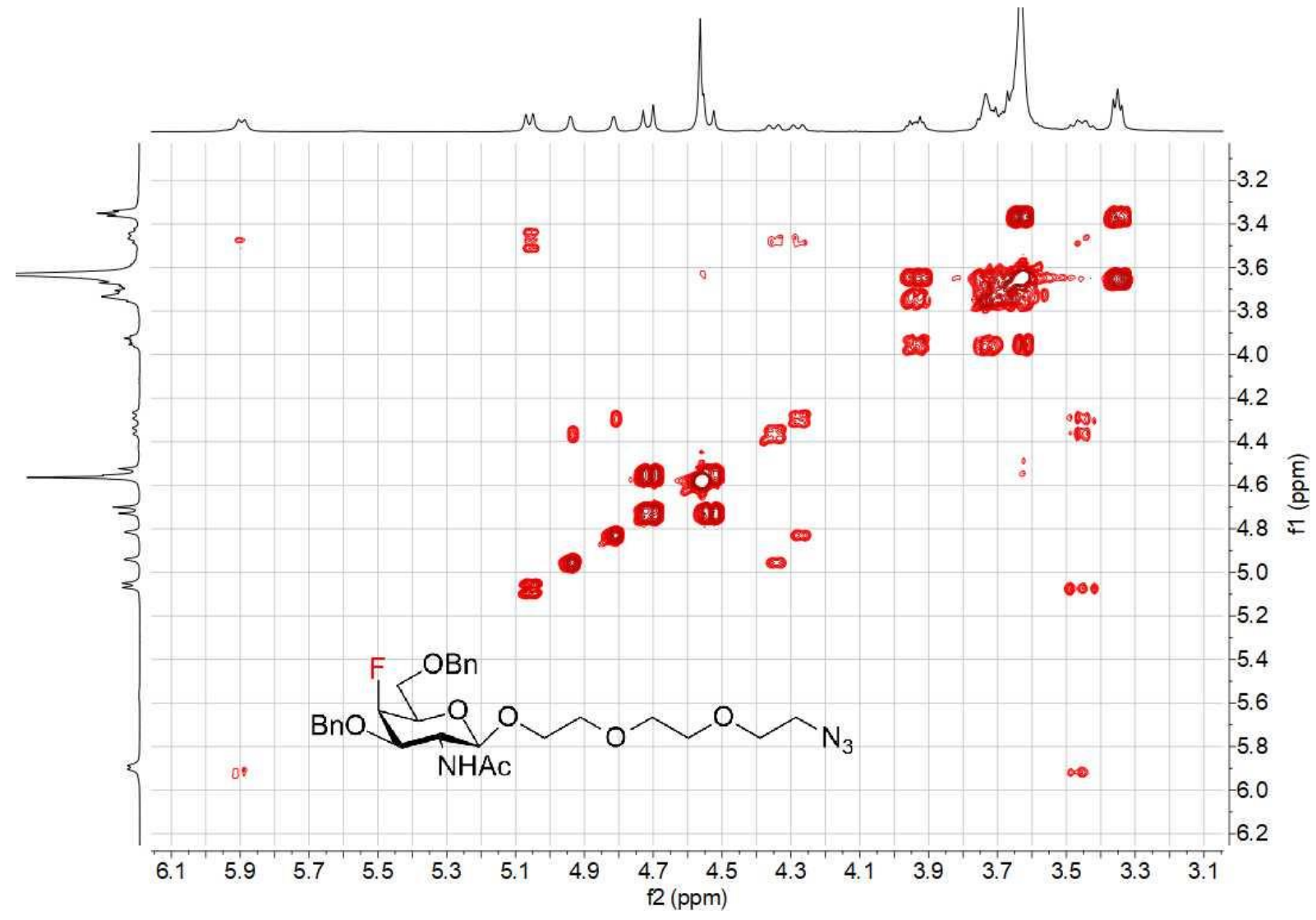


${ }^{1} \mathrm{H}-{ }^{13} \mathrm{C}$ HSQC NMR 23

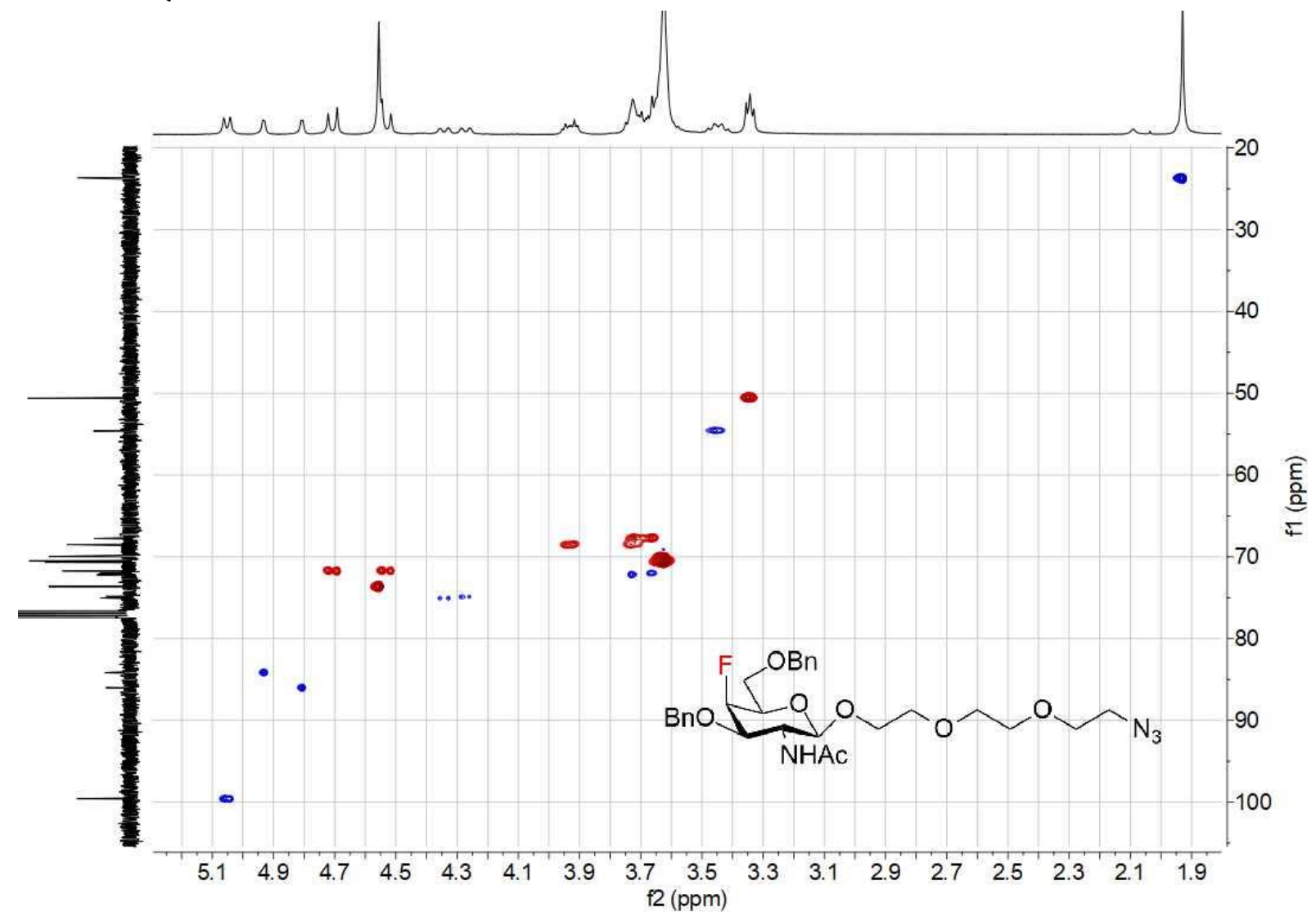

${ }^{1} \mathrm{H}^{13} \mathrm{C}$ HMBC NMR 23

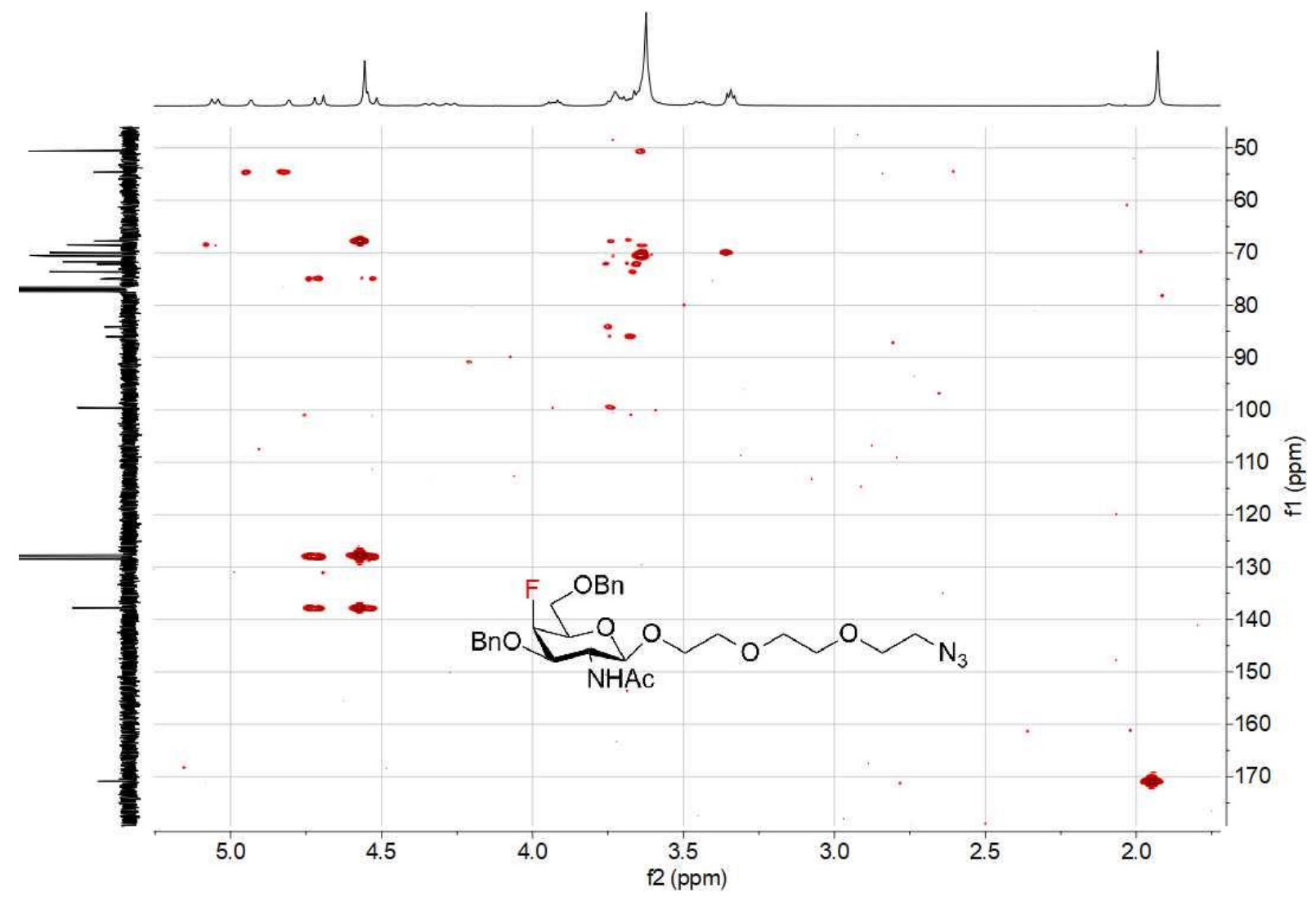


${ }^{19} \mathrm{~F} \mathrm{NMR} 376 \mathrm{MHz}, \mathrm{CDCl}_{3} 23$

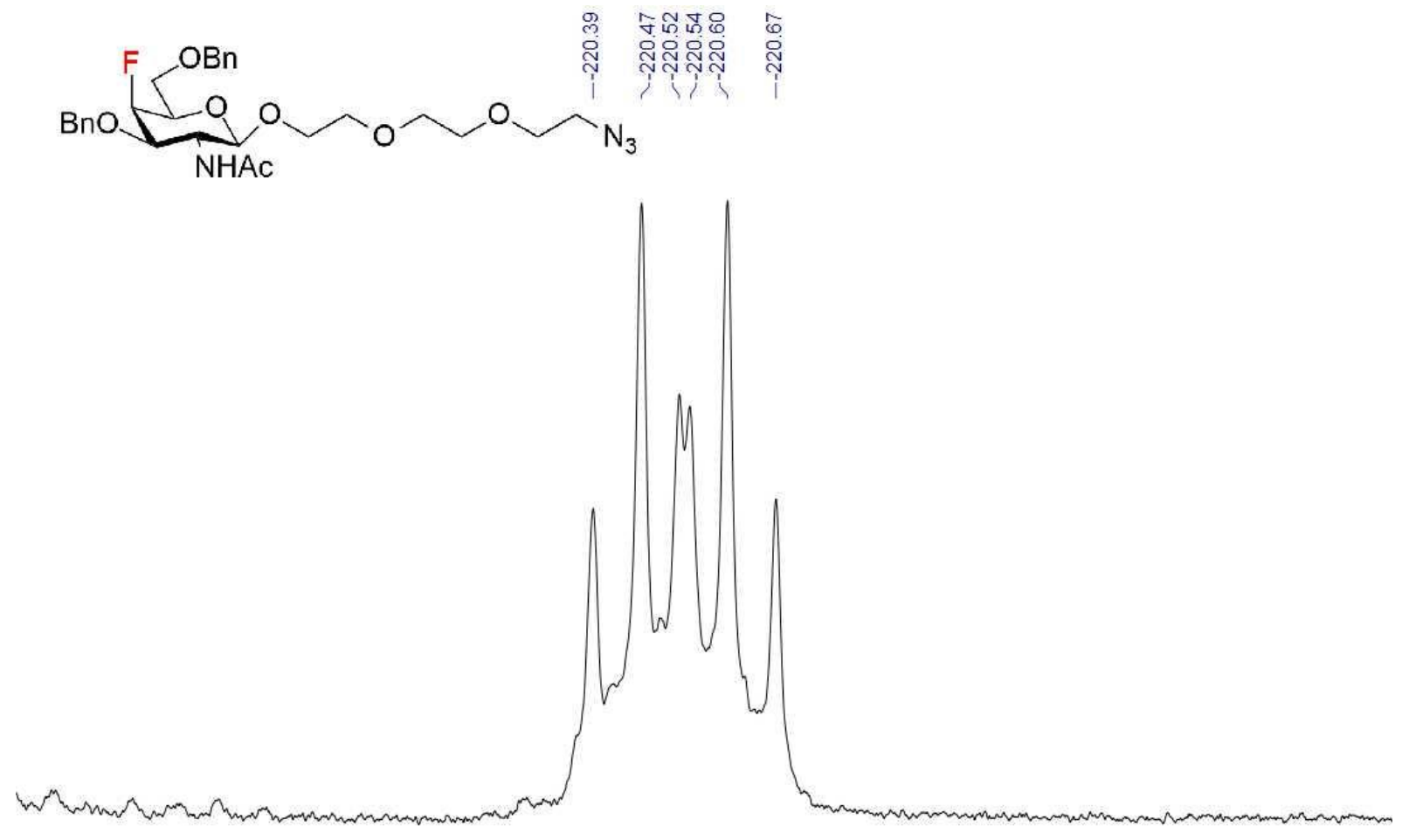

\begin{tabular}{|l|l|l|l|l|l|l|l|l|l|l|}
\hline-219.6 & -219.8 & -220.0 & -220.2 & -220.4 & -220.6 & -220.8 & -221.0 & -221.2 & -221.4 & -221. \\
\hline$f 1(\mathrm{ppm})$ & & & & &
\end{tabular}

\section{NMR 25}

${ }^{1} \mathrm{HNMR} 400 \mathrm{MHz}, \mathrm{CDCl}_{3} 25$

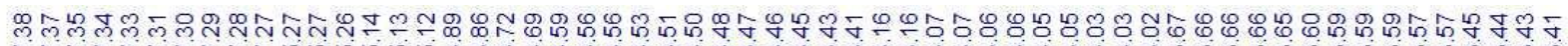

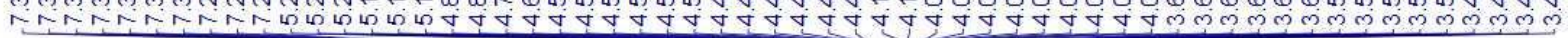

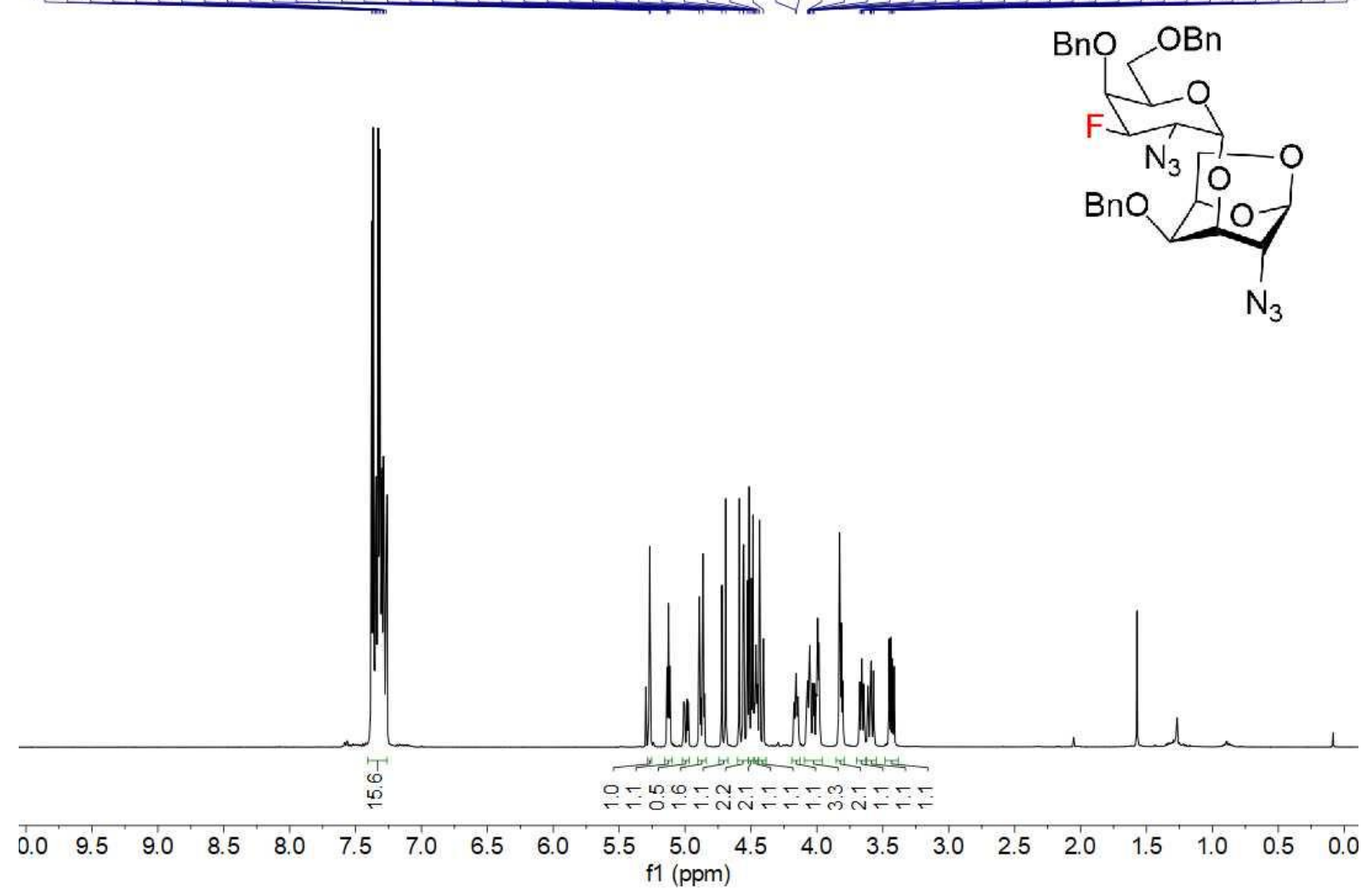


${ }^{13} \mathrm{C}\left\{{ }^{1} \mathrm{H}\right\} \mathrm{NMR} 100 \mathrm{MHz}, \mathrm{CDCl}_{3}, 25$

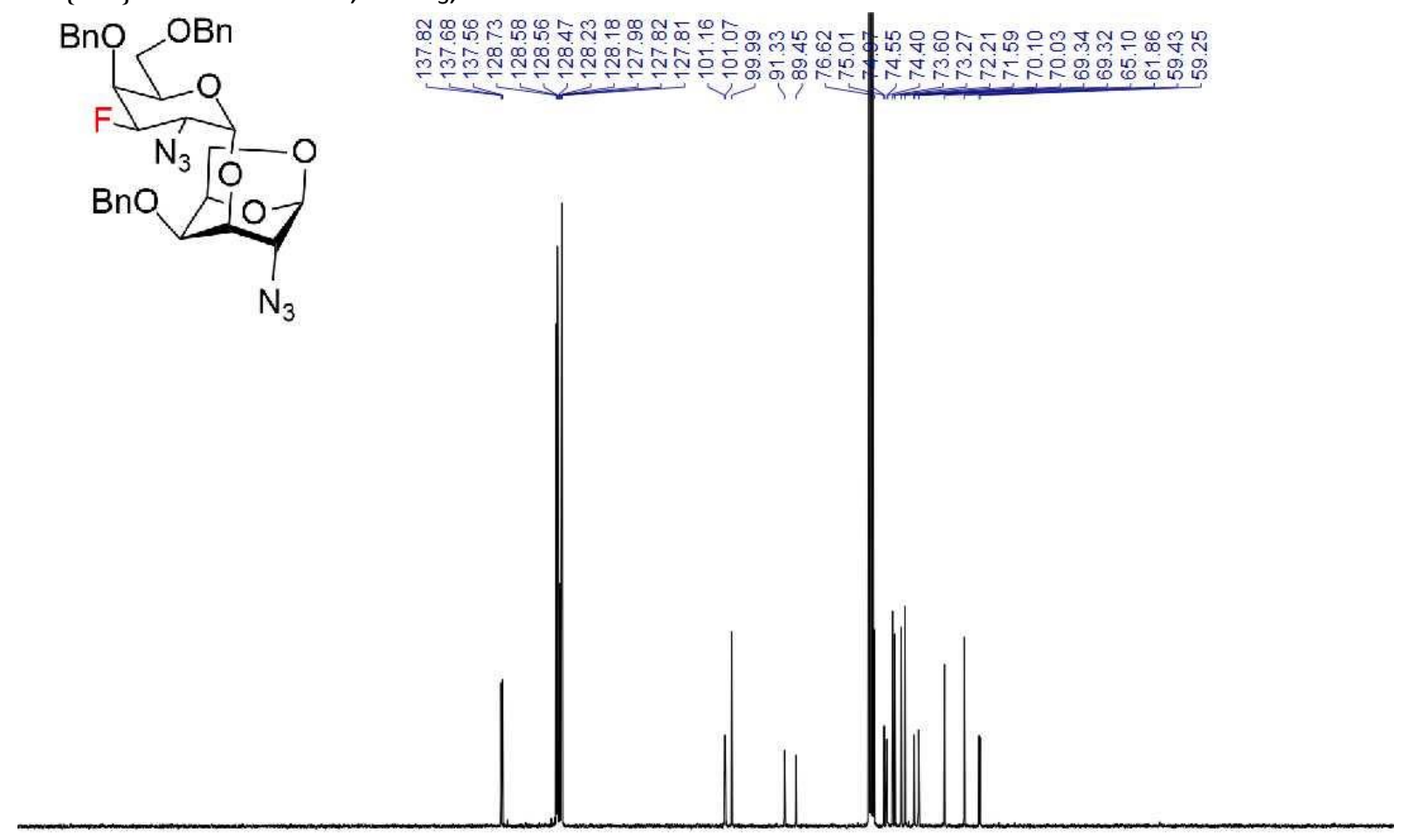

$\begin{array}{llllllllllllllllllllll}210 & 200 & 190 & 180 & 170 & 160 & 150 & 140 & 130 & 120 & \begin{array}{r}110 \\ \mathrm{f} 1(\mathrm{ppm})\end{array} & 90 & 80 & 70 & 60 & 50 & 40 & 30 & 20 & 10 & 0\end{array}$

${ }^{1} \mathrm{H}-{ }^{1} \mathrm{H}$ COSY NMR 25

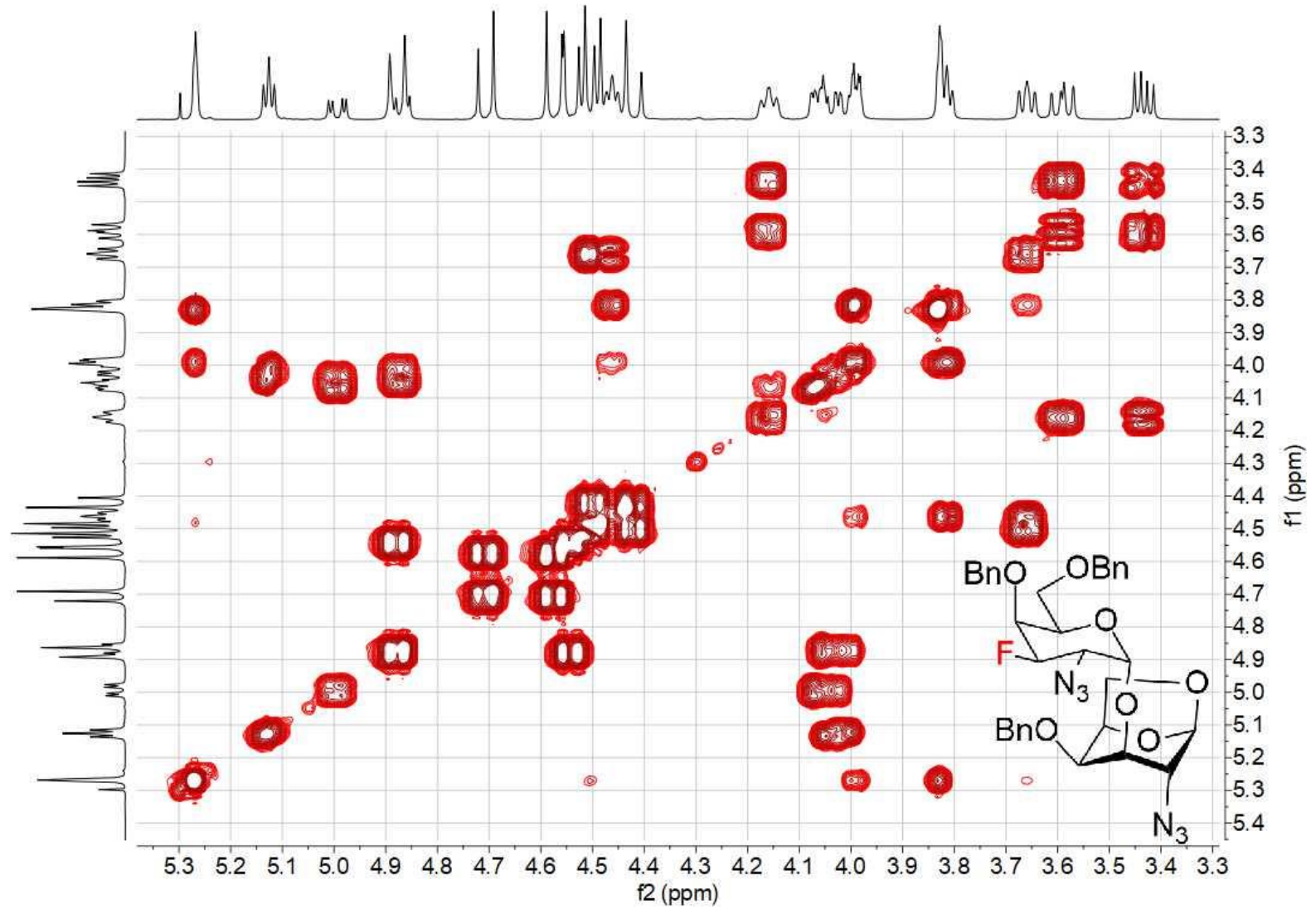


${ }^{1} \mathrm{H}-{ }^{13} \mathrm{C}$ HSQC NMR 25

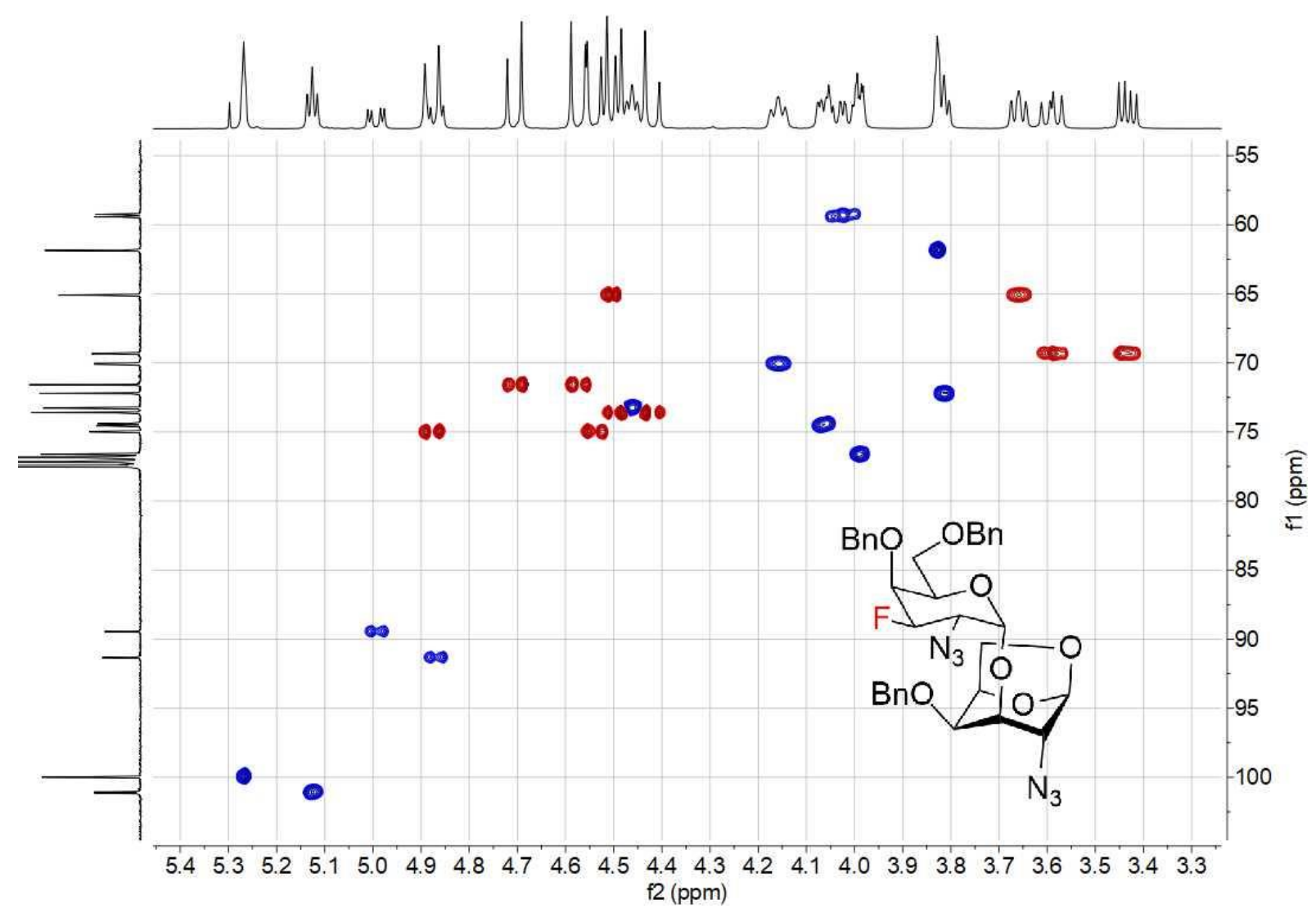

${ }^{1} \mathrm{H}^{13} \mathrm{C}$ HMBC NMR 25

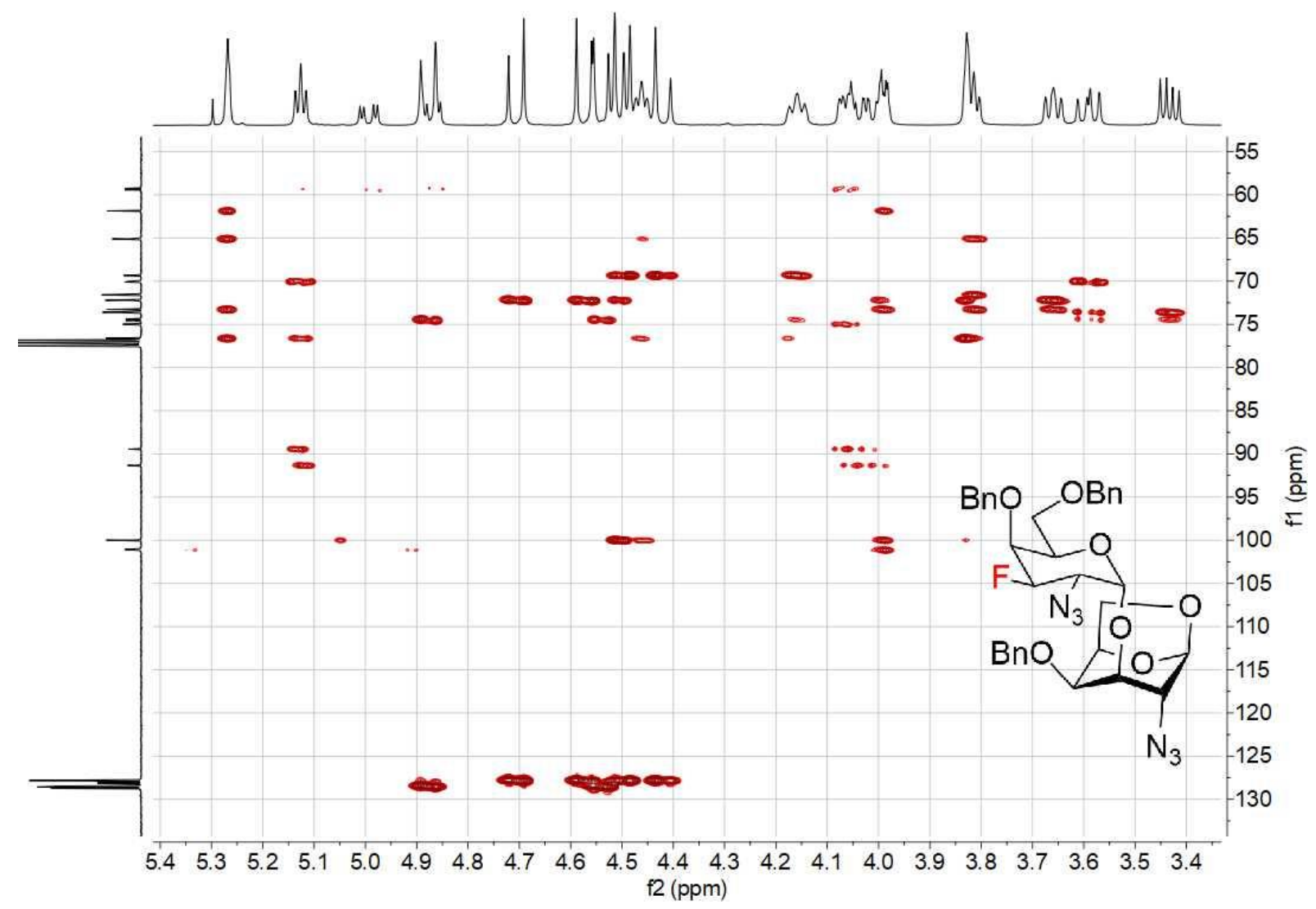


${ }^{19} \mathrm{~F} \mathrm{NMR} 376 \mathrm{MHz}, \mathrm{CDCl}_{3}, 25$

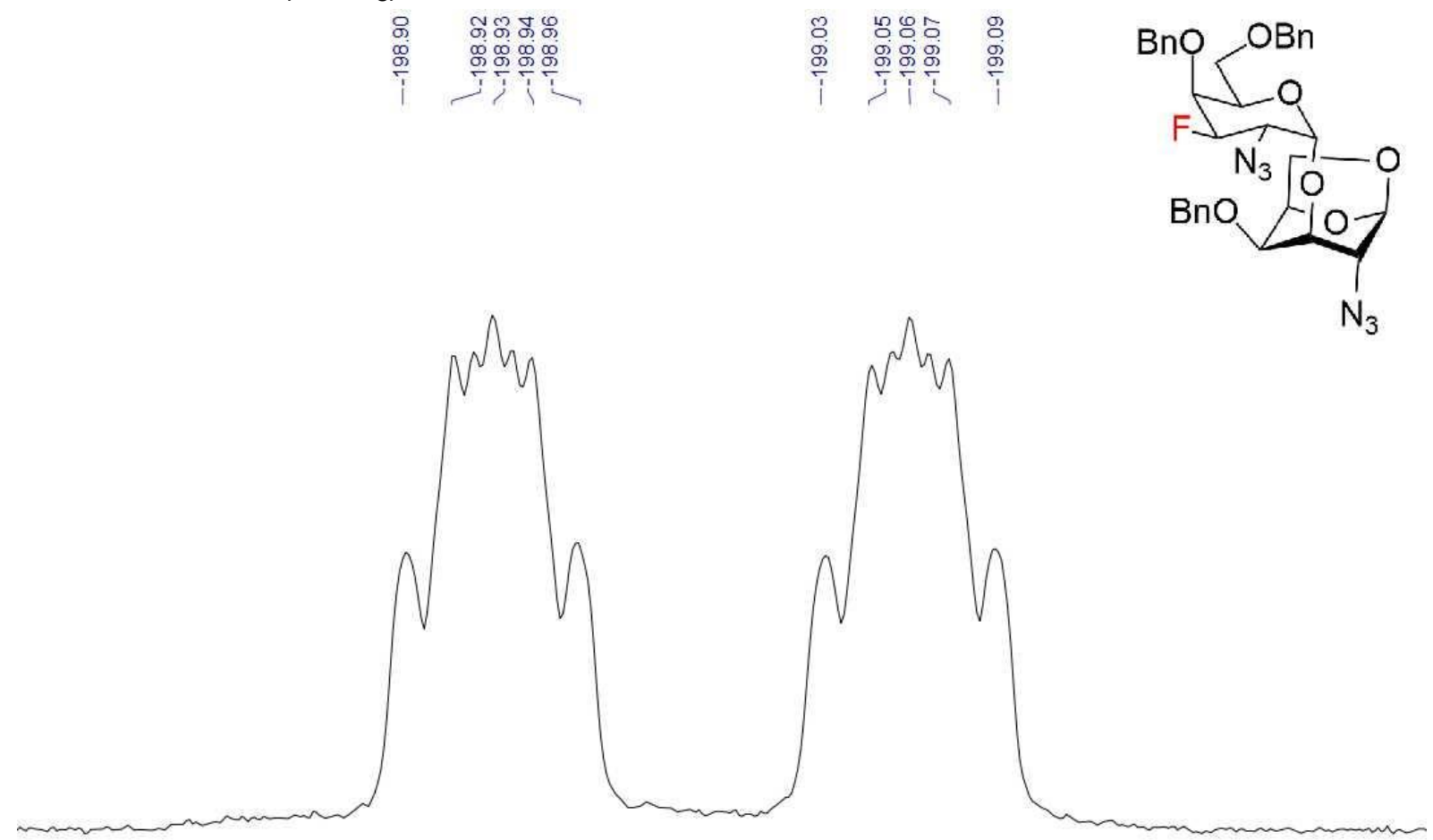

\begin{tabular}{llllll|l|l|l|l|l|l}
\hline-198.82 & -198.86 & -198.90 & -198.94 & -198.98 & -199.02 & -199.06 & -199.10 & -199.14 & -199.18 & -199 \\
$\mathrm{f} 1(\mathrm{ppm})$ &
\end{tabular}

\section{NMR $\alpha-26$}

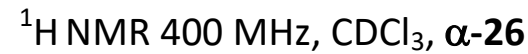

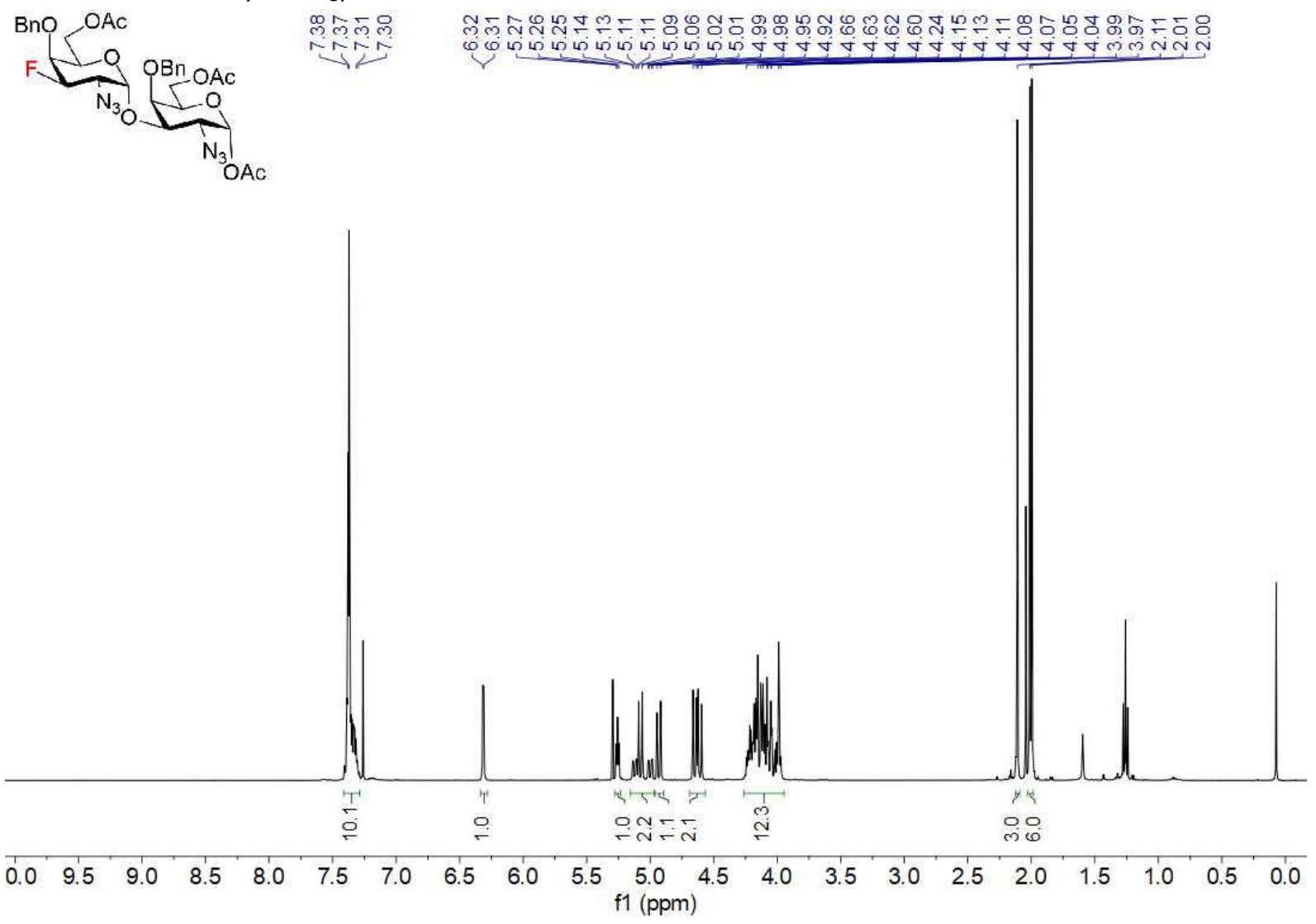


${ }^{13} \mathrm{C}\left\{{ }^{1} \mathrm{H}\right\}$ NMR $100 \mathrm{MHz}, \mathrm{CDCl}_{3}, \boldsymbol{\alpha - 2 6}$

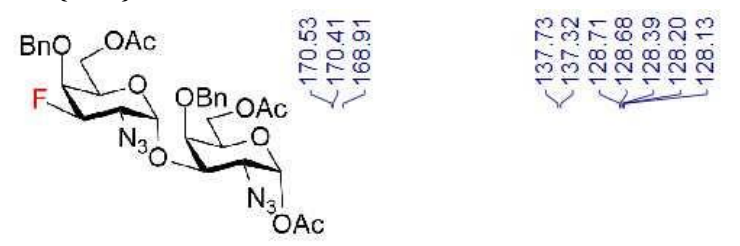

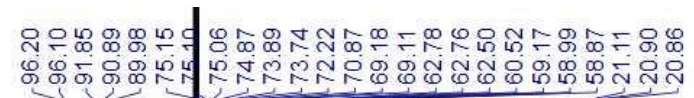

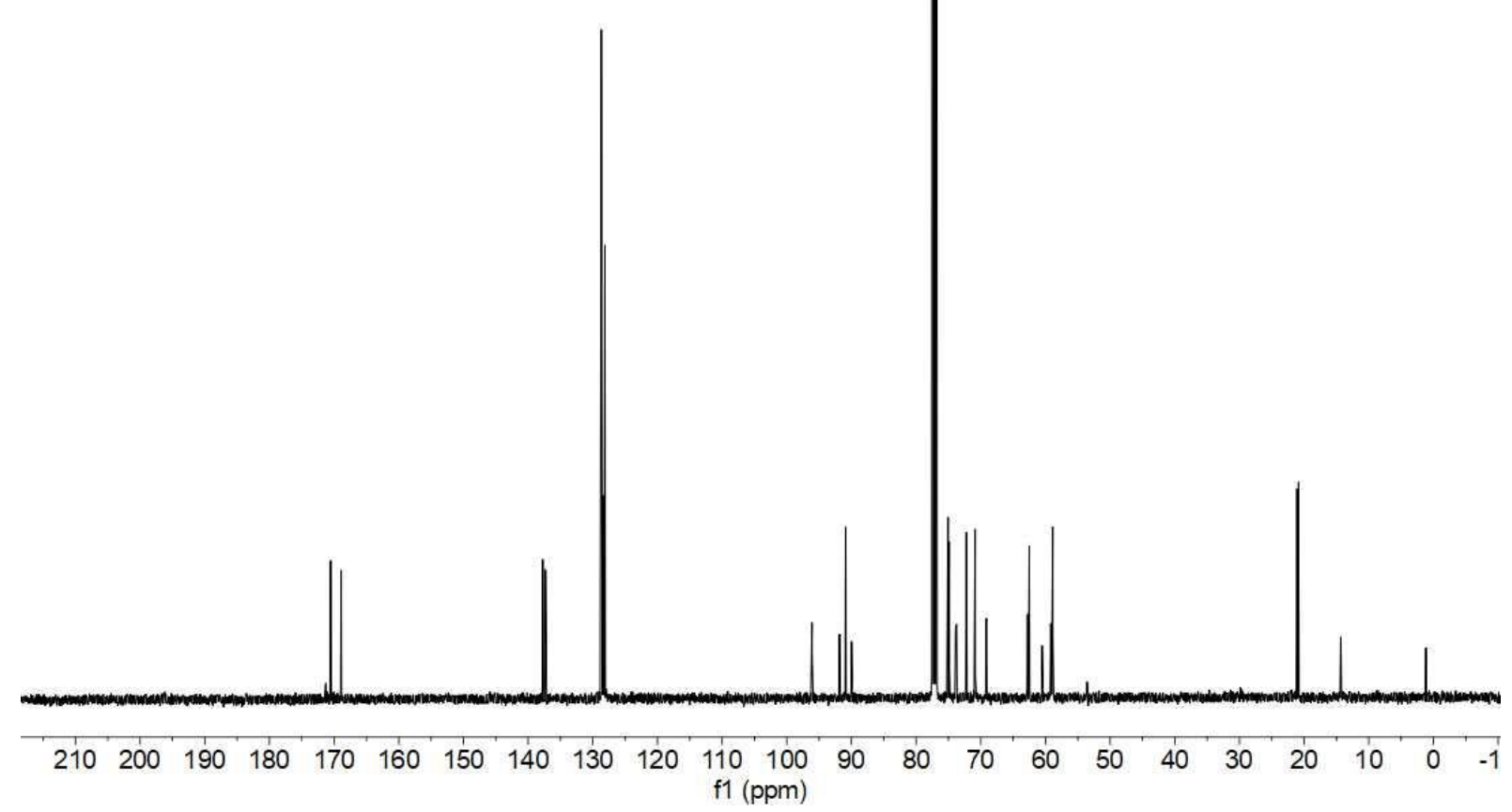

${ }^{1} \mathrm{H}-{ }^{1} \mathrm{H} \cos Y$ NMR $\boldsymbol{\alpha - 2 6}$

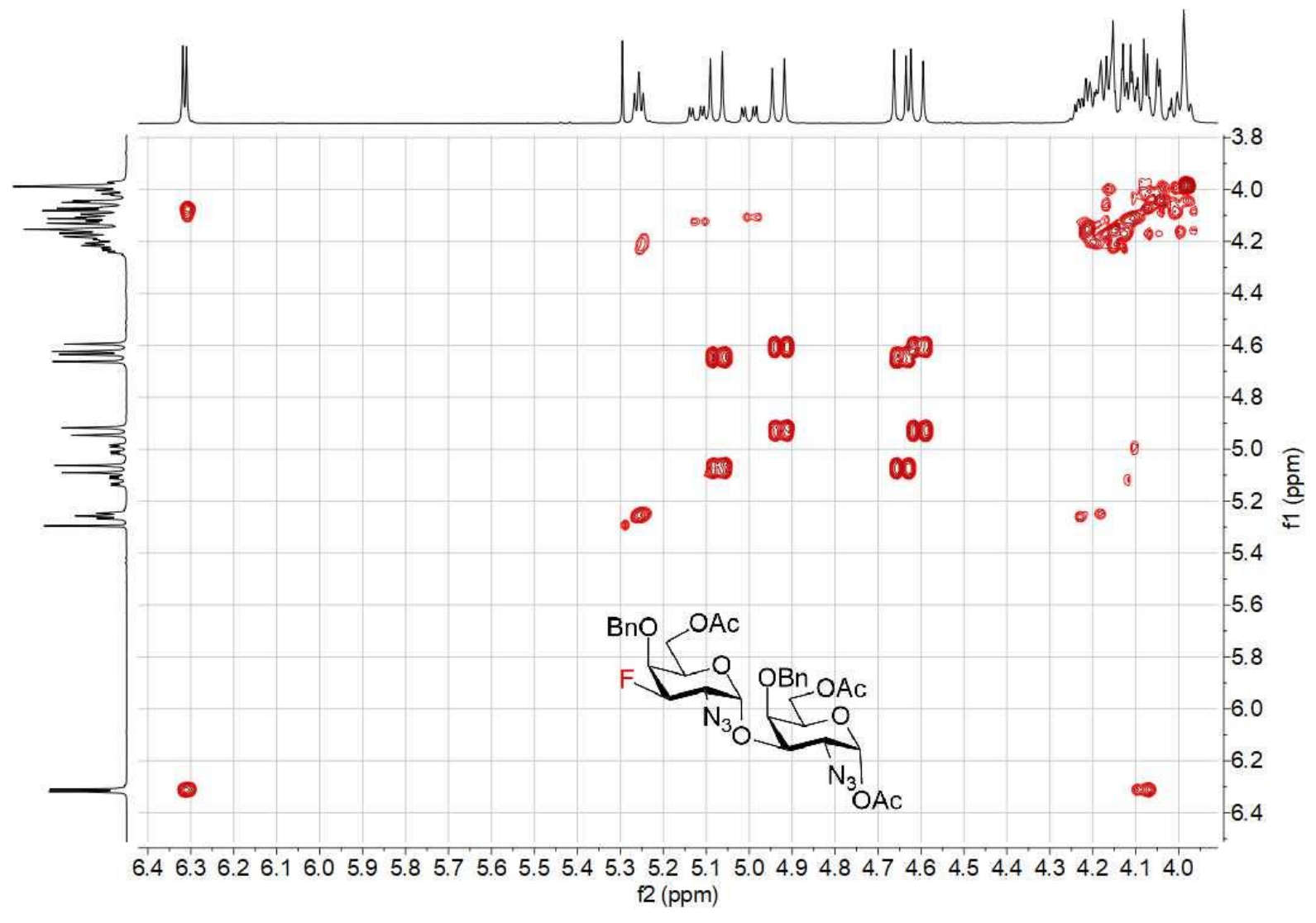




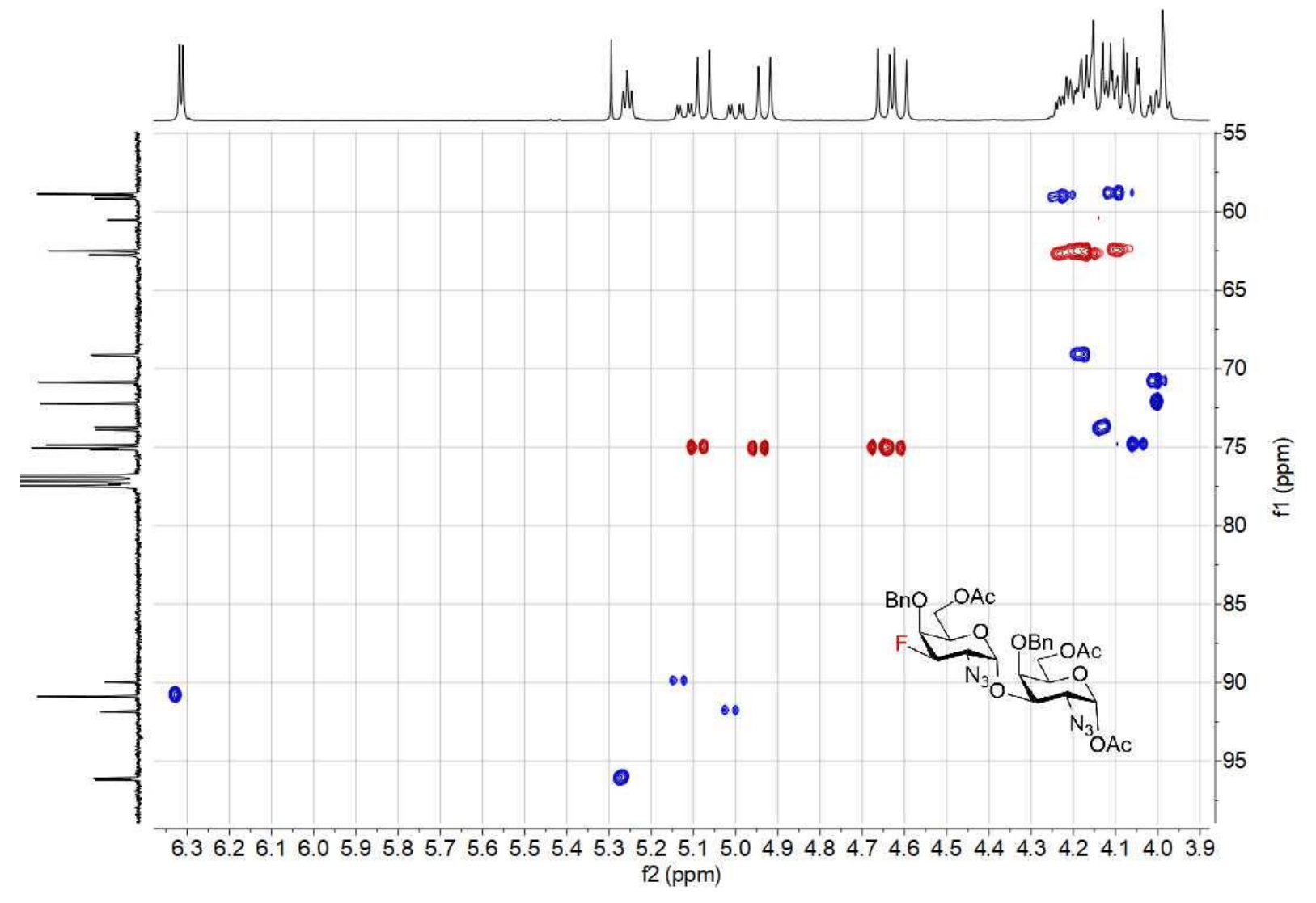

${ }^{1} \mathrm{H}-{ }^{13} \mathrm{C}$ HMBC NMR $\alpha-26$

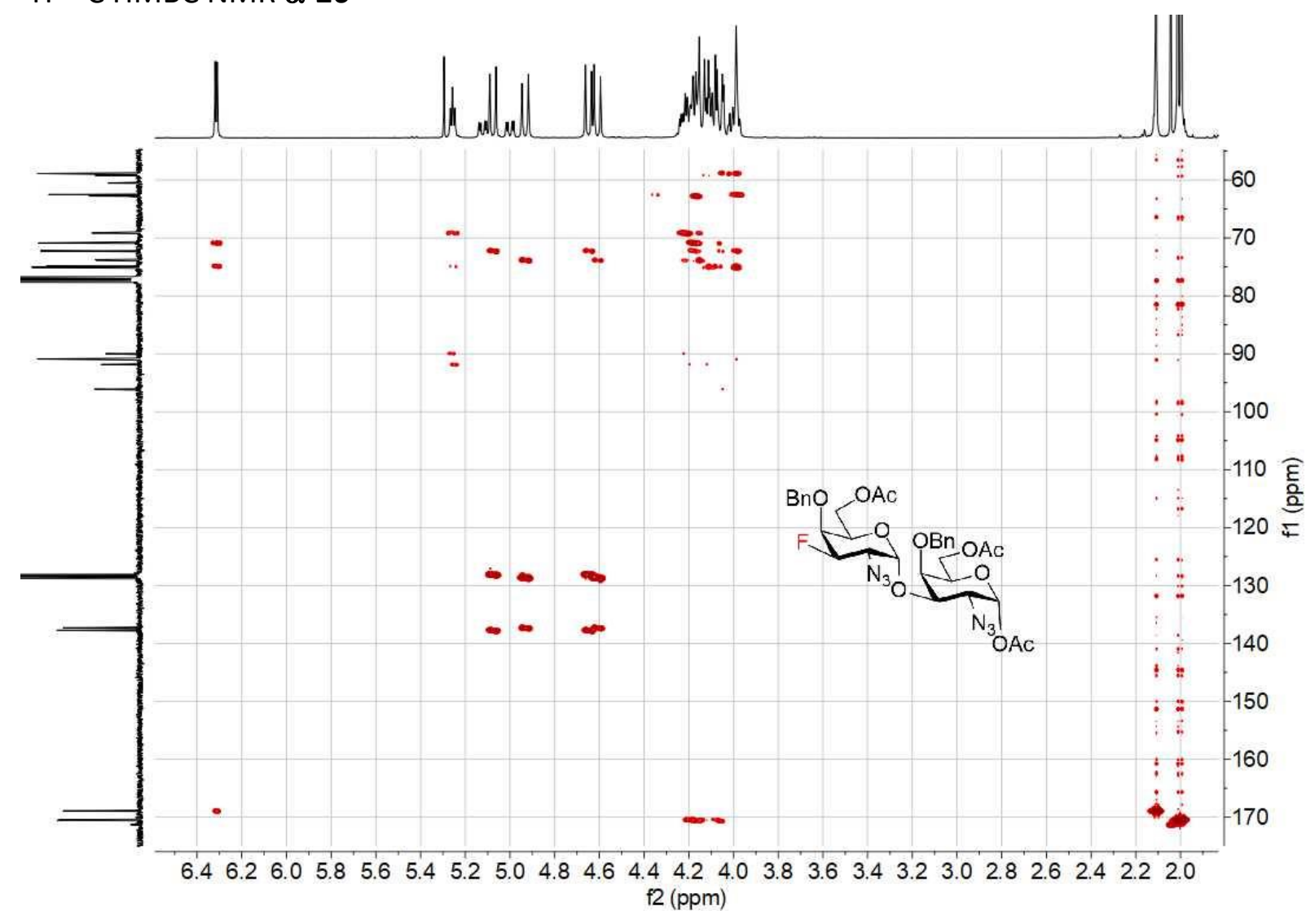


${ }^{19} \mathrm{~F} \mathrm{NMR} 376 \mathrm{MHz}, \mathrm{CDCl}_{3}, \boldsymbol{\alpha}-\mathbf{2 6}$

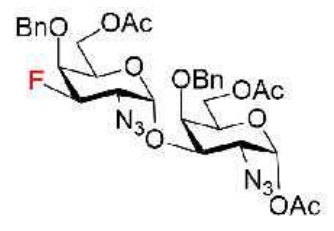

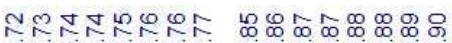

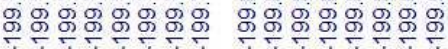

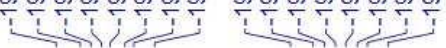

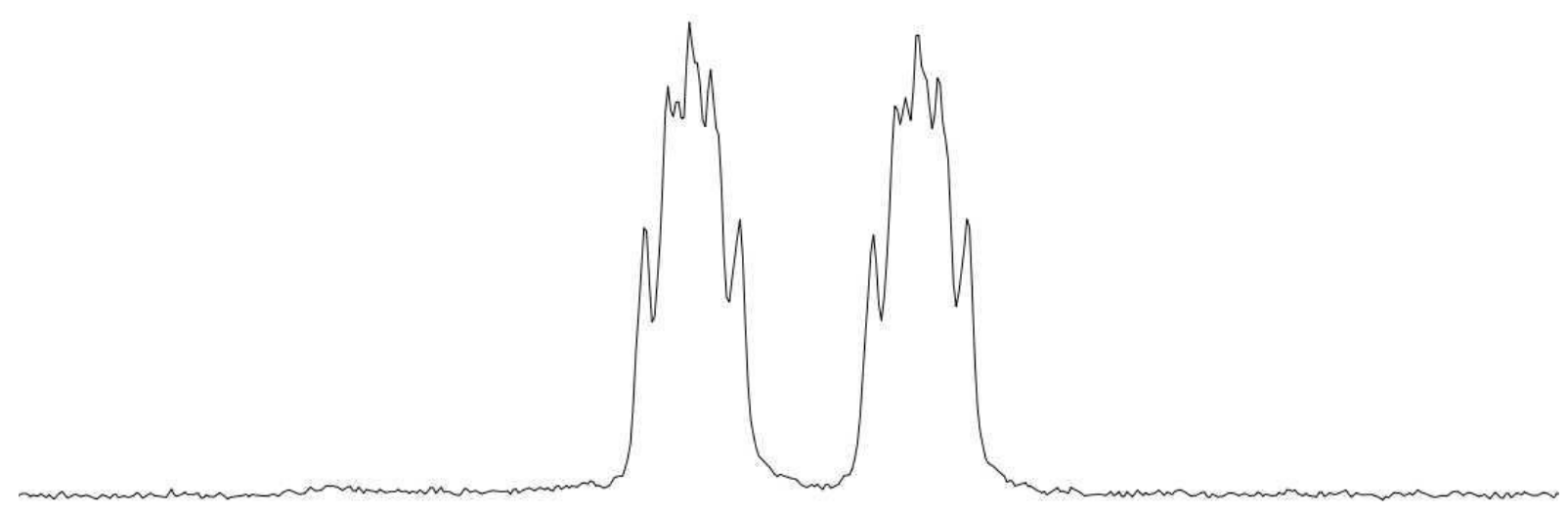

\begin{tabular}{llllllllll}
\hline-199.40 & -199.50 & -199.60 & -199.70 & $\begin{array}{l}-199.80 \\
f 1(\mathrm{ppm})\end{array}$ & -199.90 & -200.00 & -200.10 & -200.20 \\
\hline
\end{tabular}

\section{NMR $\boldsymbol{\beta}$-26}

${ }^{1} \mathrm{HNMR} 400 \mathrm{MHz}, \mathrm{CDCl}_{3} \boldsymbol{\beta}-26$

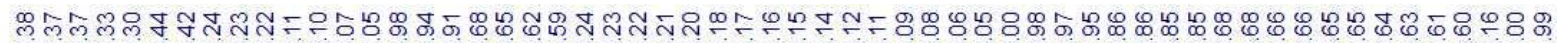

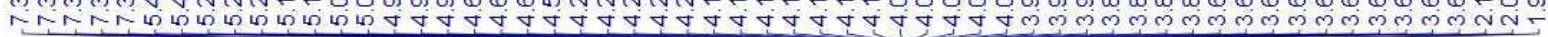

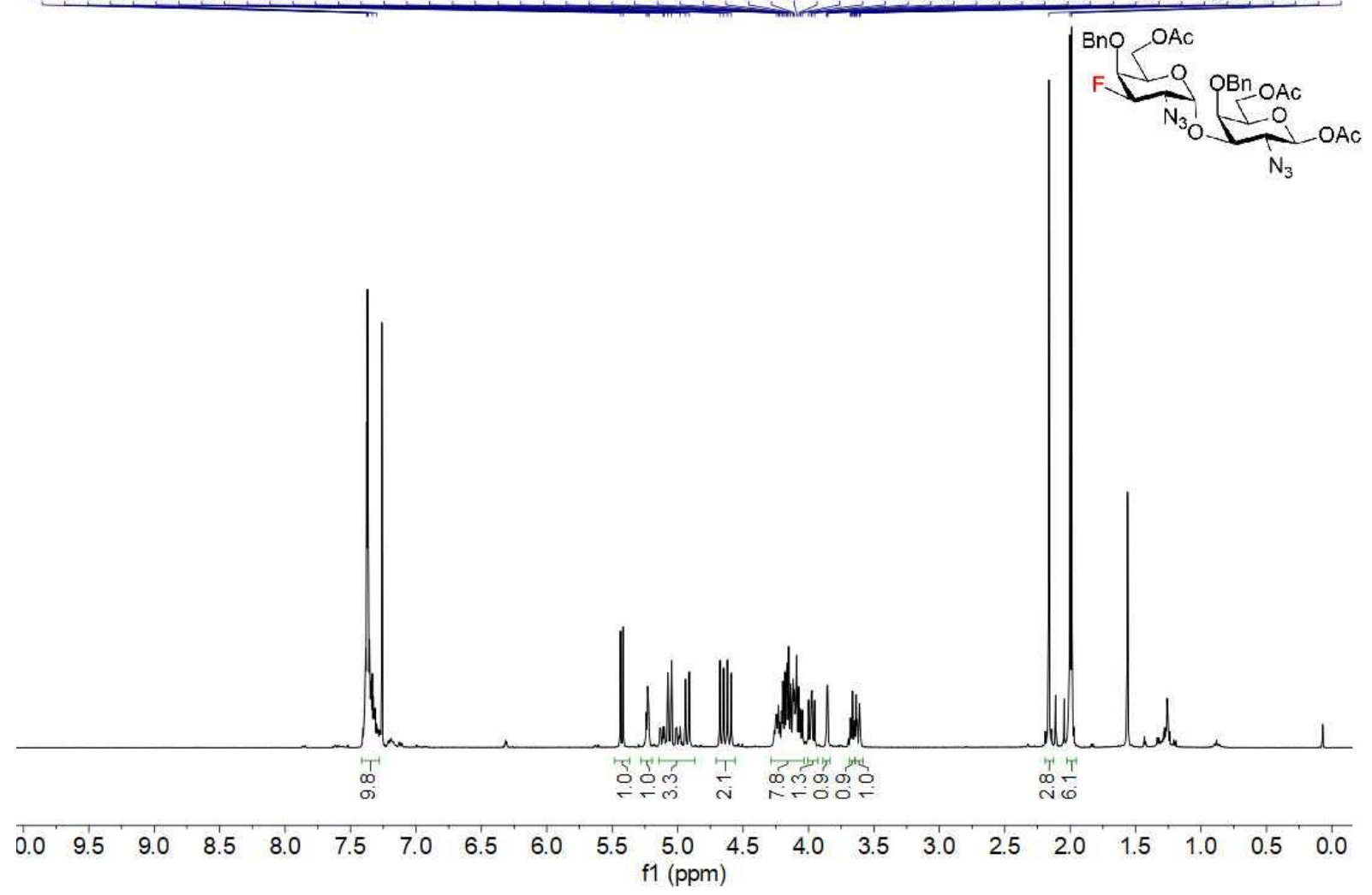


${ }^{13} \mathrm{C}\left\{{ }^{1} \mathrm{H}\right\}$ NMR $100 \mathrm{MHz}, \mathrm{CDCl}_{3} \beta-26$

ㅇำ

안윤

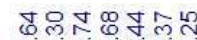

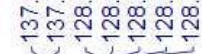

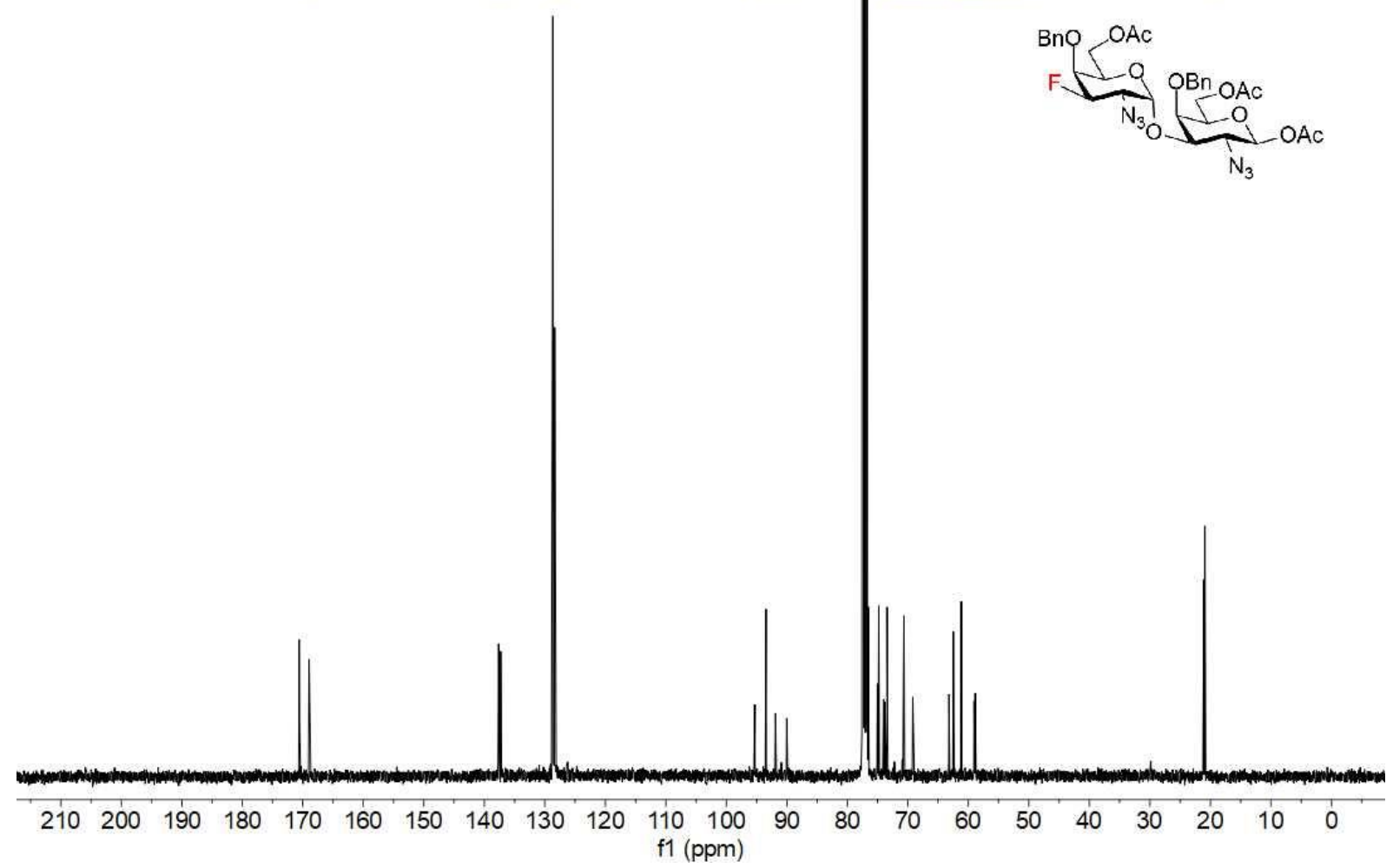

${ }^{1} \mathrm{H}-{ }^{1} \mathrm{H}$ COSY NMR $\beta-26$

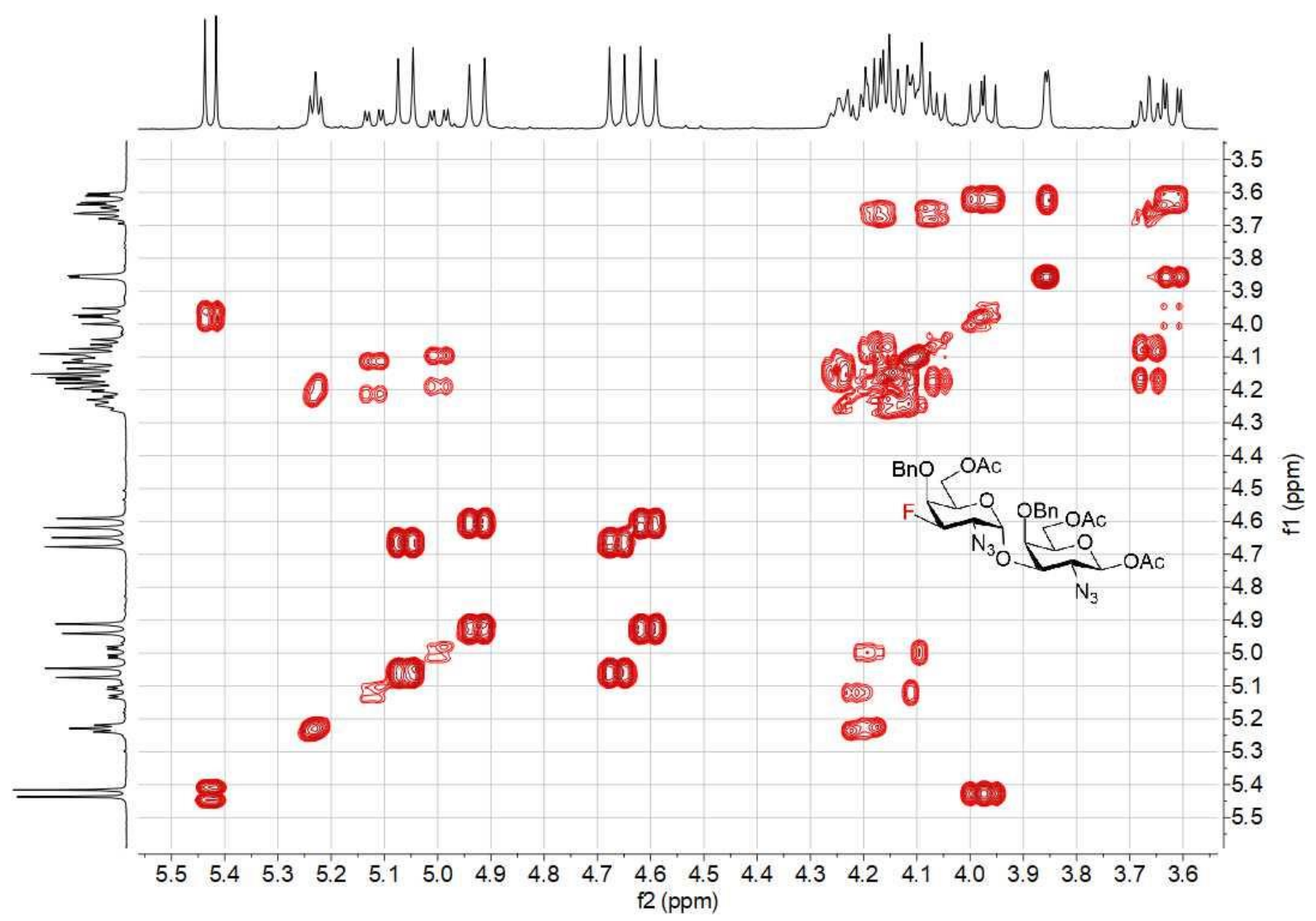

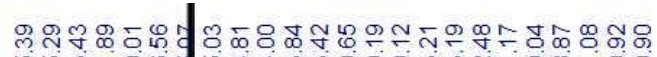

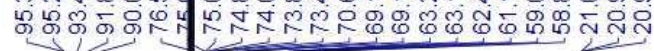

BnO OAC 


\section{${ }^{1} \mathrm{H}-{ }^{13} \mathrm{C}$ HSQC NMR $\beta-26$}

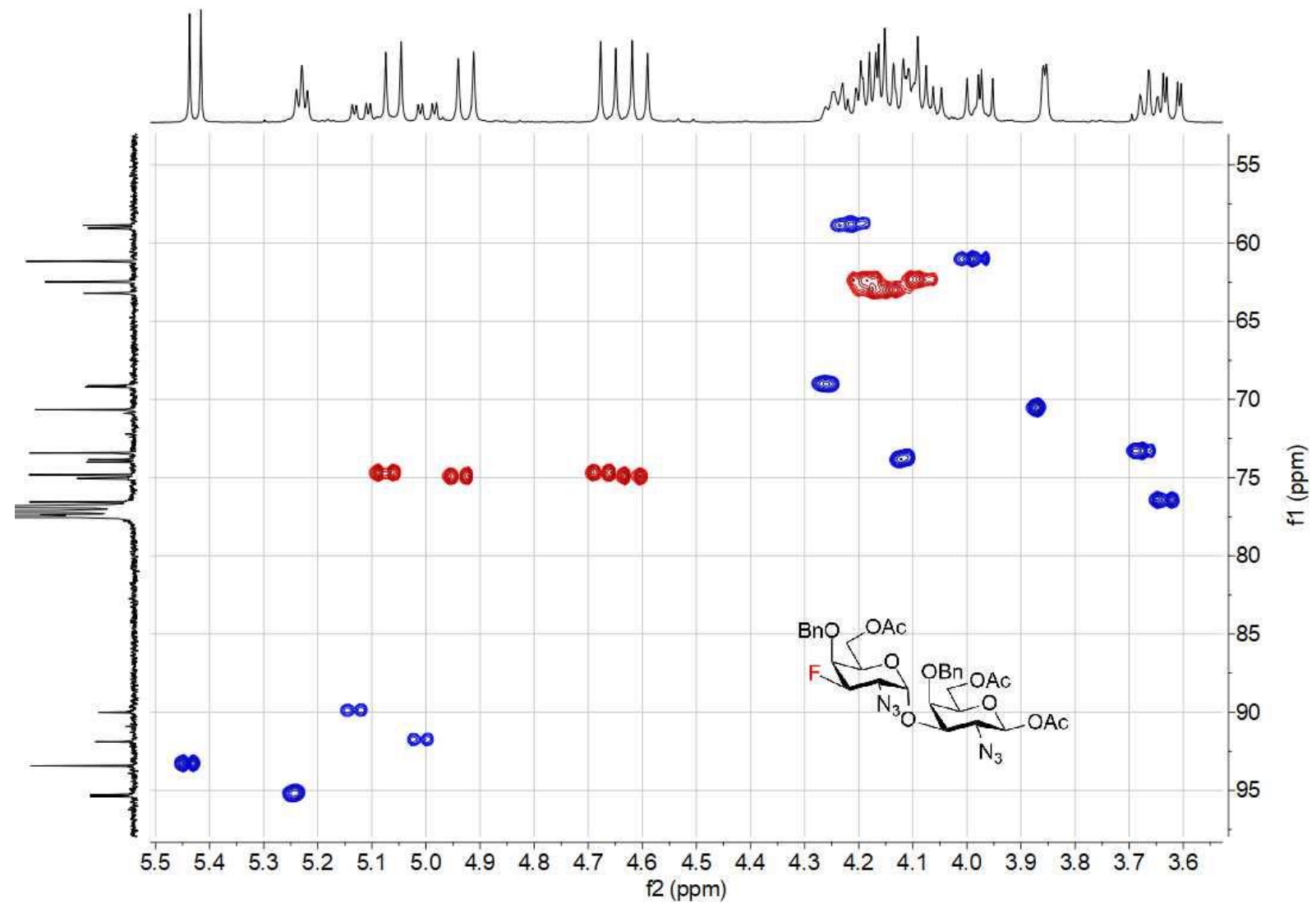

${ }^{1} \mathrm{H}-{ }^{13} \mathrm{C}$ HMBC NMR $\beta-26$

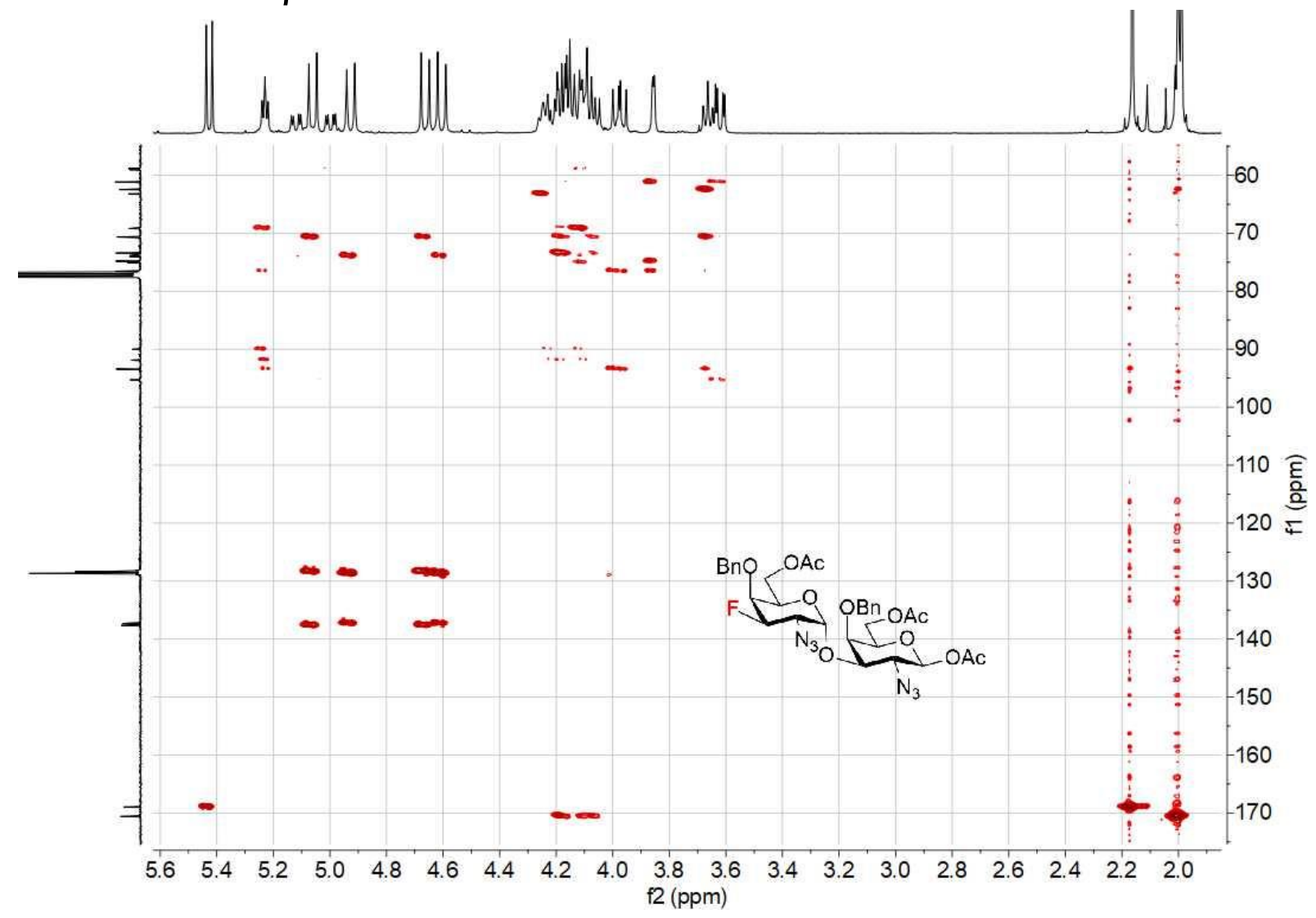


${ }^{19} \mathrm{~F} \mathrm{NMR} 376 \mathrm{MHz}, \mathrm{CDCl}_{3}, \boldsymbol{\beta}-\mathbf{2 6}$
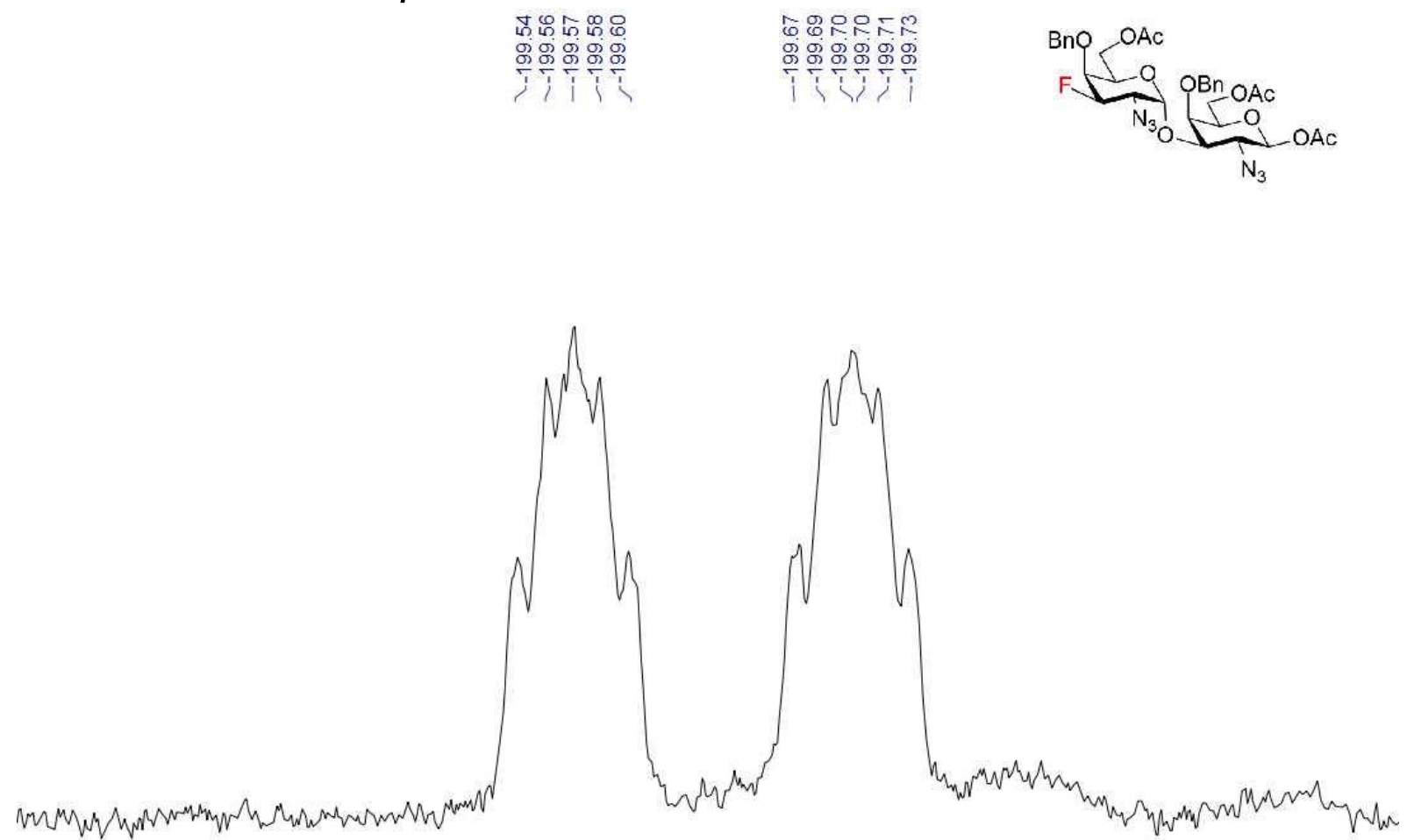

$-199.34-199.38-199.42-199.46-199.50-199.54-199.58-199.62-199.66-199.70-199.74-199.78-199.82-199.86-199.90-199.96$ f1 (ppm) 
Procedure: Starting thioglycoside donor (14.4 mg, $30 \mu \mathrm{mol})$ and $\mathrm{Ph}_{2} \mathrm{SO}(7.9 \mathrm{mg}, 39 \mu \mathrm{mol})$ were dried under vaccum overnight and then dissolved in dry $\mathrm{CD}_{2} \mathrm{Cl}_{2}$. The solution was transferred to NMR tube, capped and NMR was recorded at rt. The tube was cooled to -80 ${ }^{\circ} \mathrm{C}$ and $\mathrm{Tf}_{2} \mathrm{O}(6.6 \mu \mathrm{L}, 39 \mu \mathrm{mol})$ was added. The NMR was recorded at $-80{ }^{\circ} \mathrm{C}$ and the temperature was increased incementally until decomposition ocurred.

Figure S1. Low temperature ${ }^{1} \mathrm{H}$ NMR of $\beta-1$ activated by $\mathrm{Tf}_{2} \mathrm{O}$ (only the region of $\mathrm{H}-1$ resonances shown)

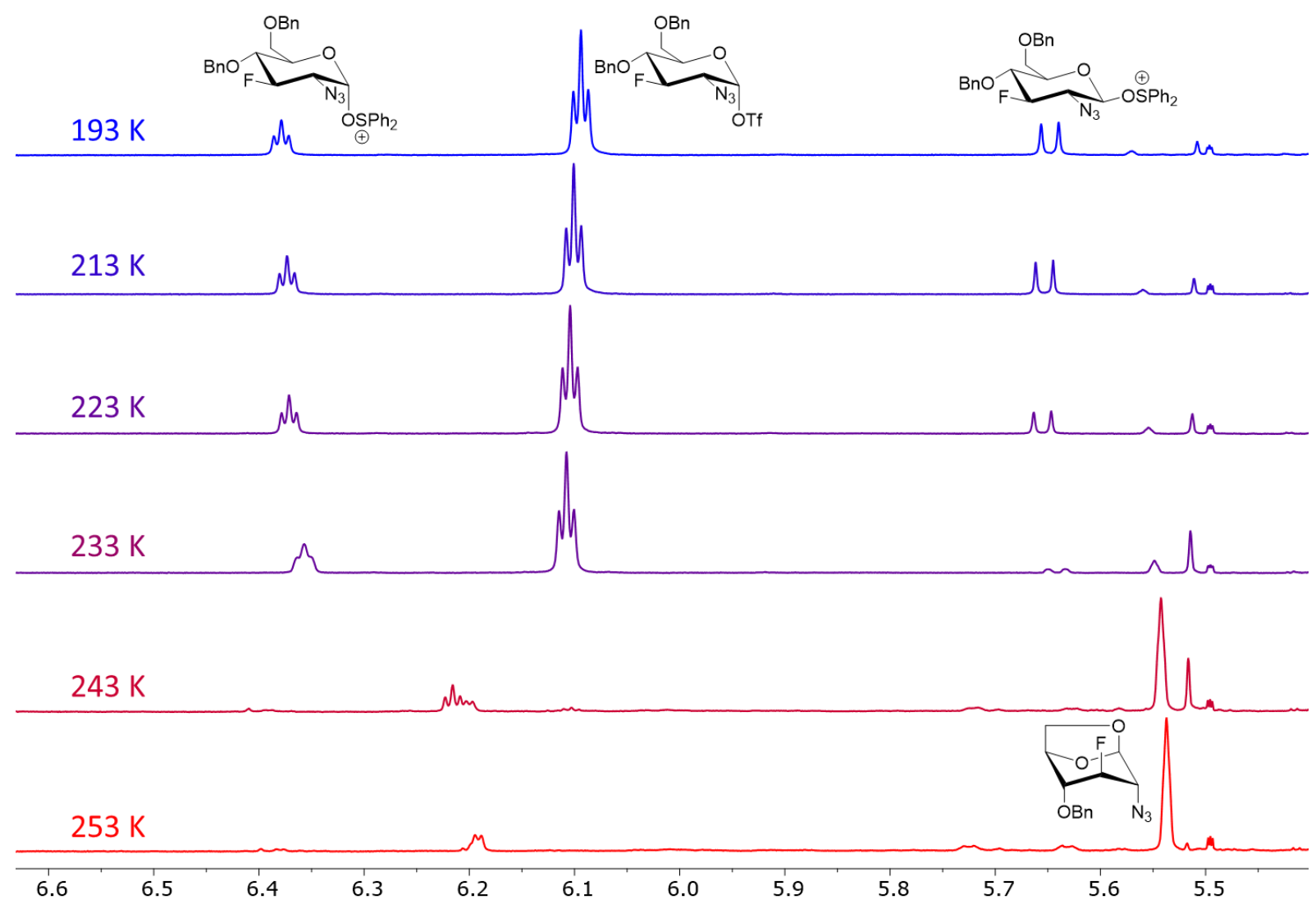


Figure S2. Low temperature ${ }^{1} \mathrm{H}$ NMR of $\alpha-1$ activated by $\mathrm{Tf}_{2} \mathrm{O}$ (only the region of $\mathrm{H}-1$ resonances shown)

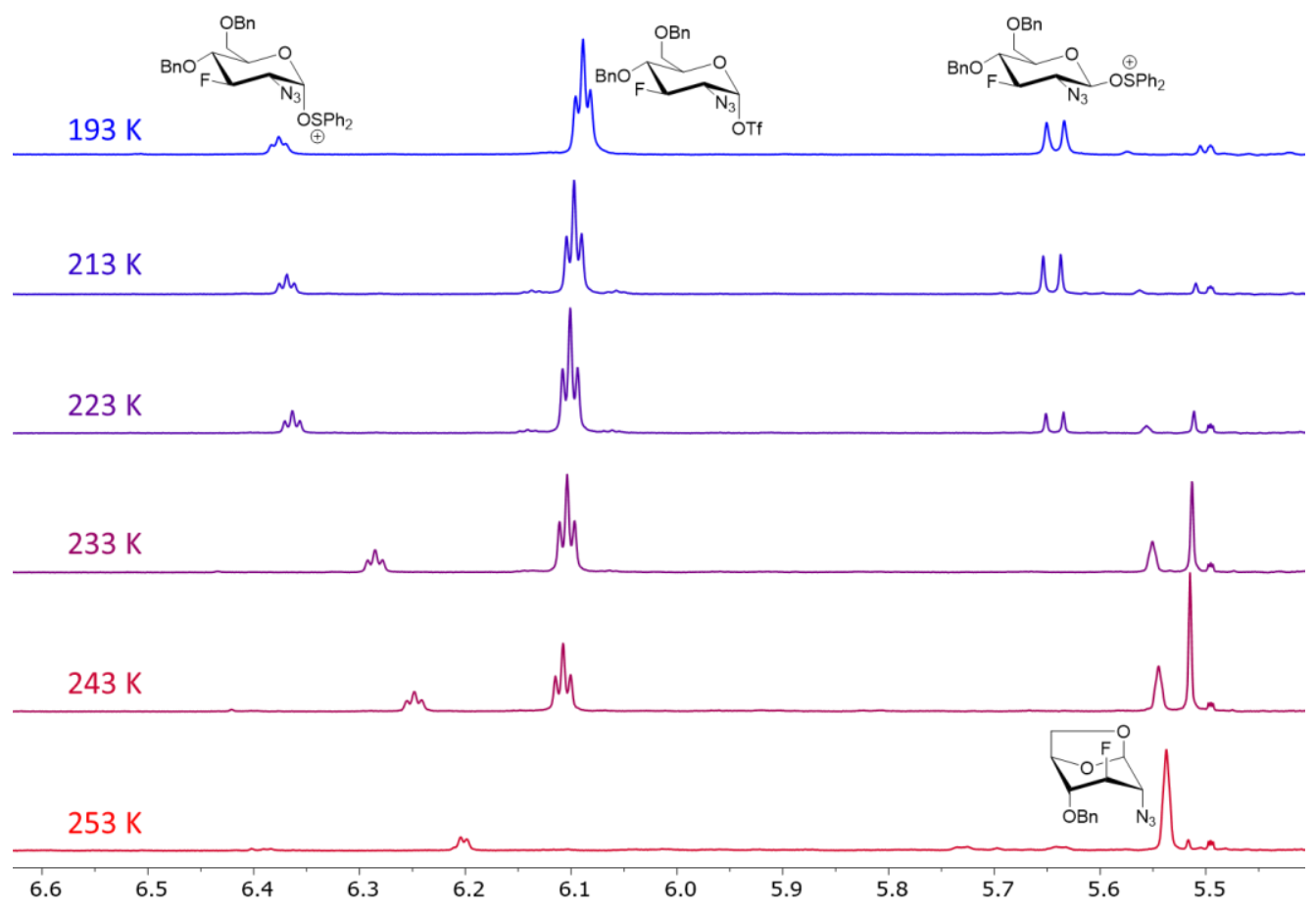

Figure S3. Low temperature ${ }^{1} \mathrm{H}$ NMR of $\alpha-1$ activated by $\mathrm{Tf}_{2} \mathrm{O}$ (increased amount of $\mathrm{Ph}_{2} \mathrm{SO}$ ) (only the region of $\mathrm{H}-1$ resonances shown)

An increased amount of $\mathrm{Ph}_{2} \mathrm{SO}$ ( $77 \mu \mathrm{mol}, 2.57$ equiv) was used
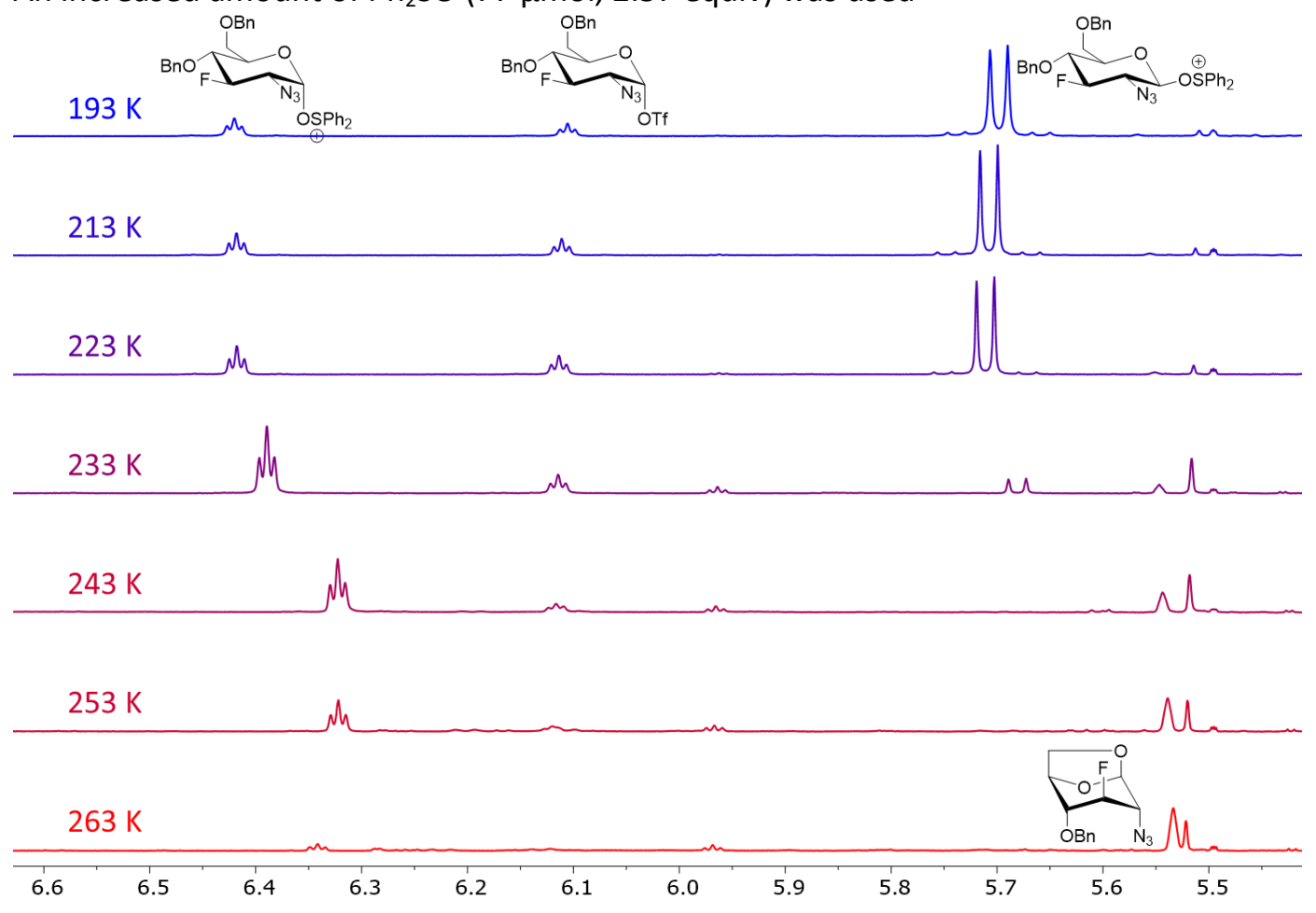

S292 
Figure S4. Low temperature ${ }^{1} \mathrm{H}$ NMR of $\alpha-3$ activated by $\mathrm{Tf}_{2} \mathrm{O}$ (only the region of $\mathrm{H}-1$ resonances shown)

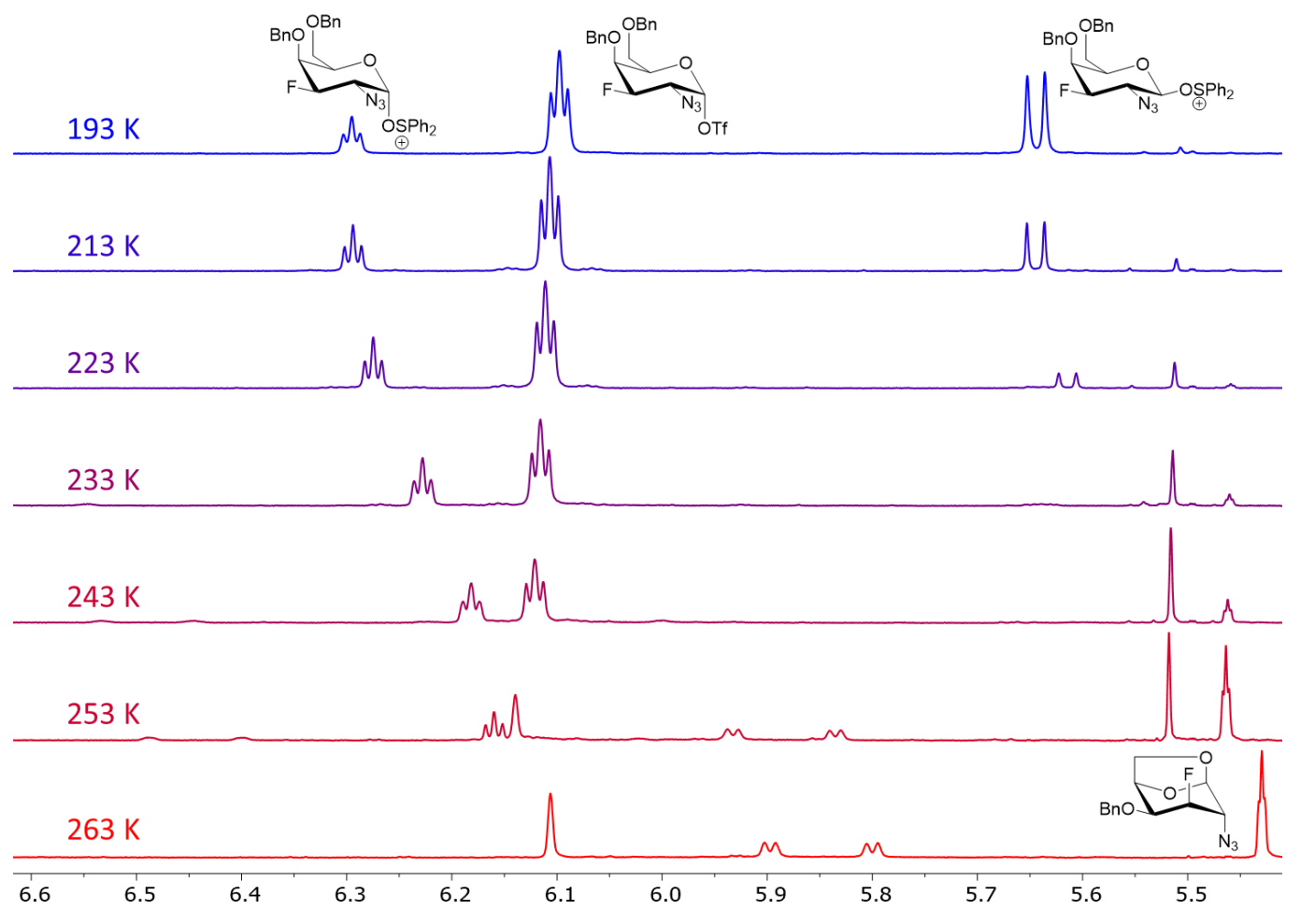

Figure S5. Low temperature ${ }^{1} \mathrm{H}$ NMR of $\beta-3$ activated by $\mathrm{Tf}_{2} \mathrm{O}$ (only the region of $\mathrm{H}-1$ resonances shown)

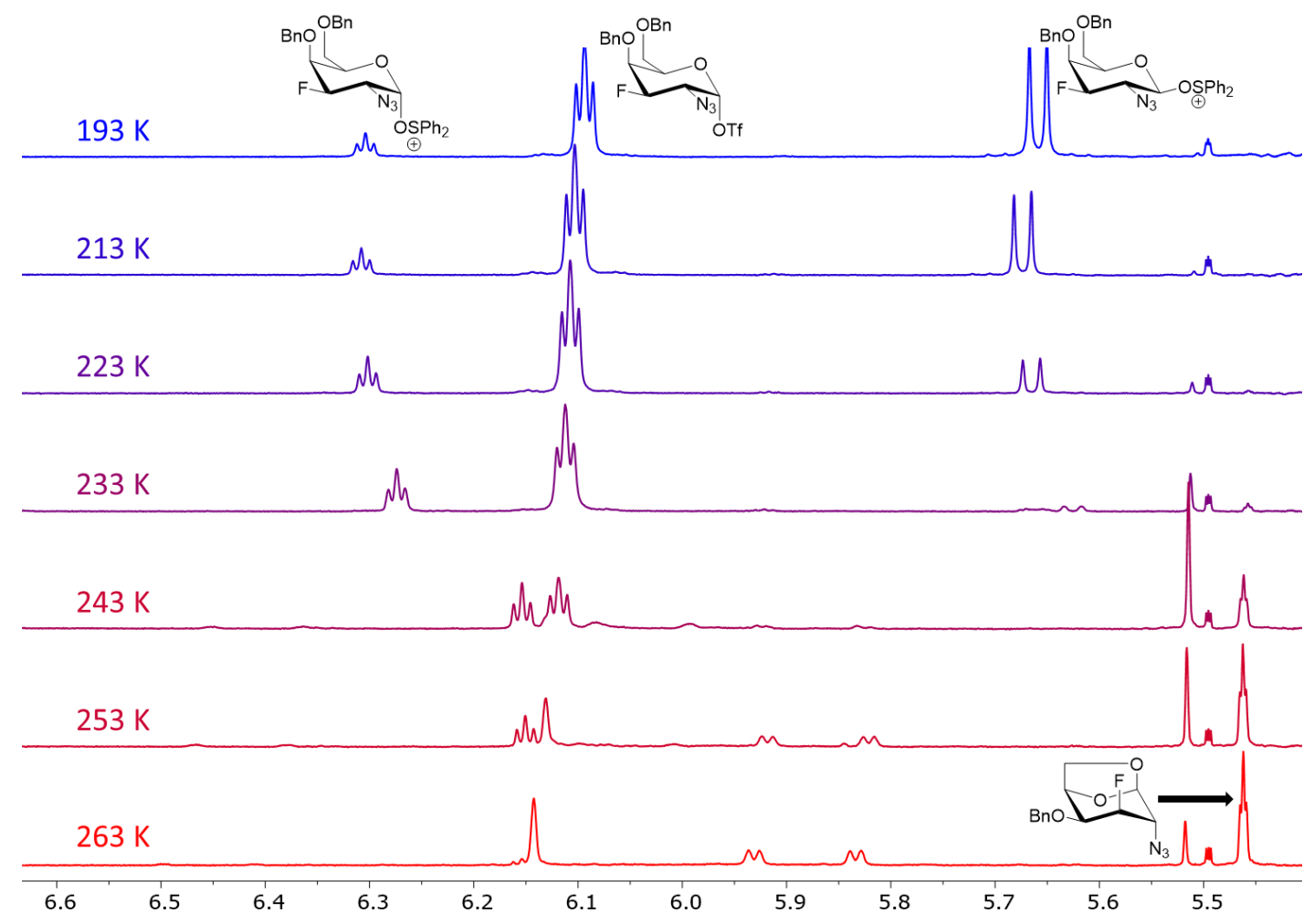

S293 
Figure S6. Low temperature ${ }^{1} \mathrm{H}$ NMR of 2 activated by $\mathrm{Tf}_{2} \mathrm{O}$ (only the region of $\mathrm{H}-1$ resonances shown)
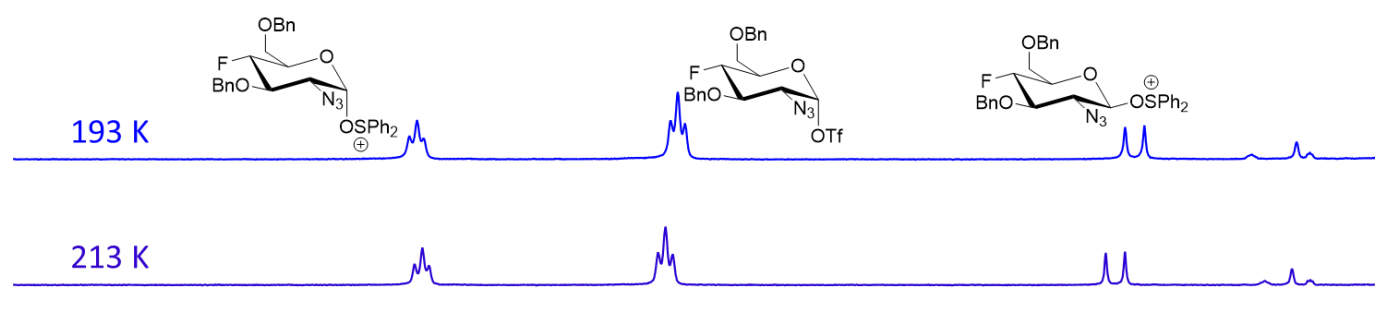

$223 \mathrm{~K}$
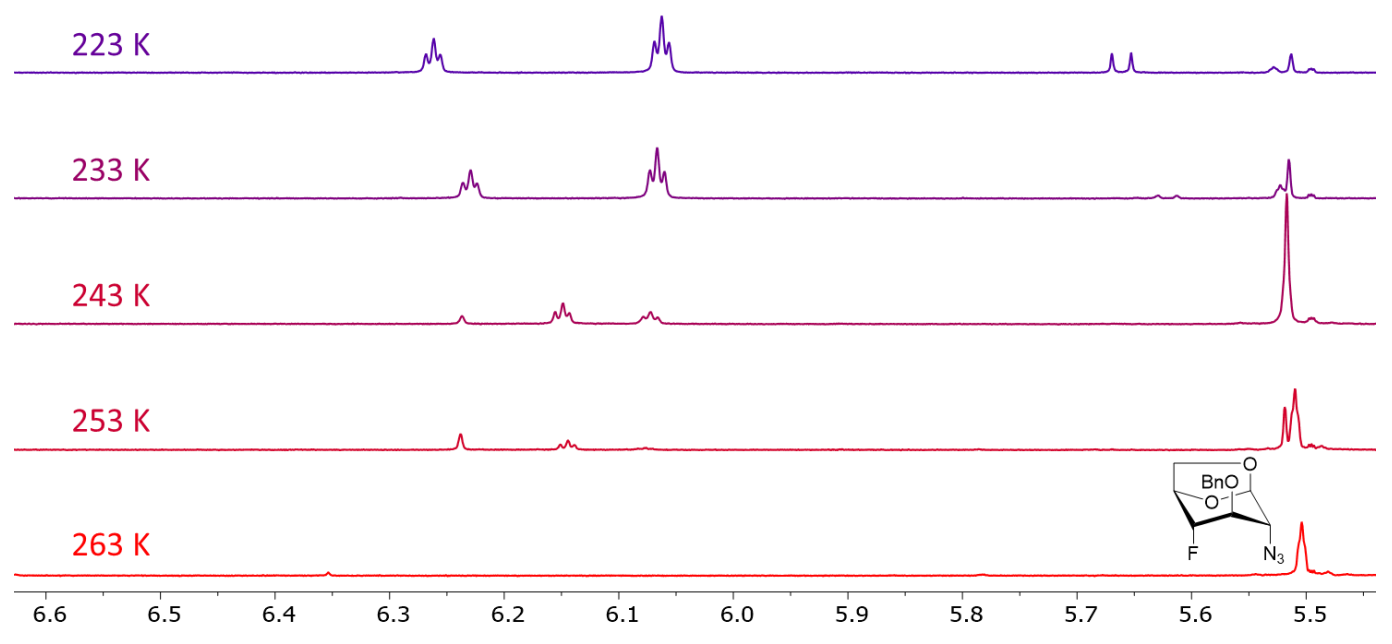

S294 
Figure S7. Low temperature ${ }^{1} \mathrm{H}$ NMR of $\alpha-4$ activated by $\mathrm{Tf}_{2} \mathrm{O}$ (only the region of $\mathrm{H}-1$ resonances shown)

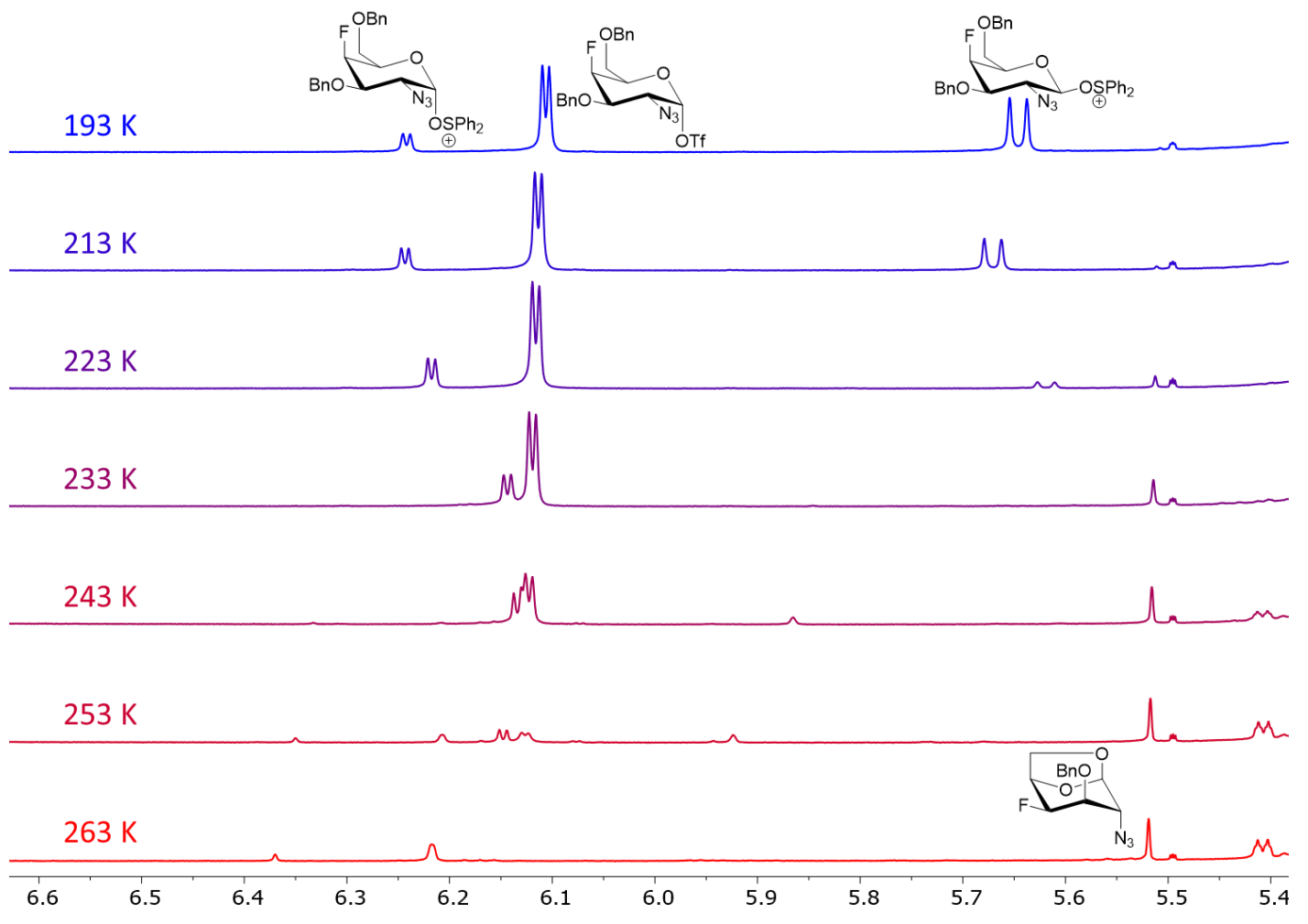

Figure S8. Low temperature ${ }^{1} \mathrm{H}$ NMR of $\beta-4$ activated by $\mathrm{Tf}_{2} \mathrm{O}$ (only the region of $\mathrm{H}-1$ resonances shown)

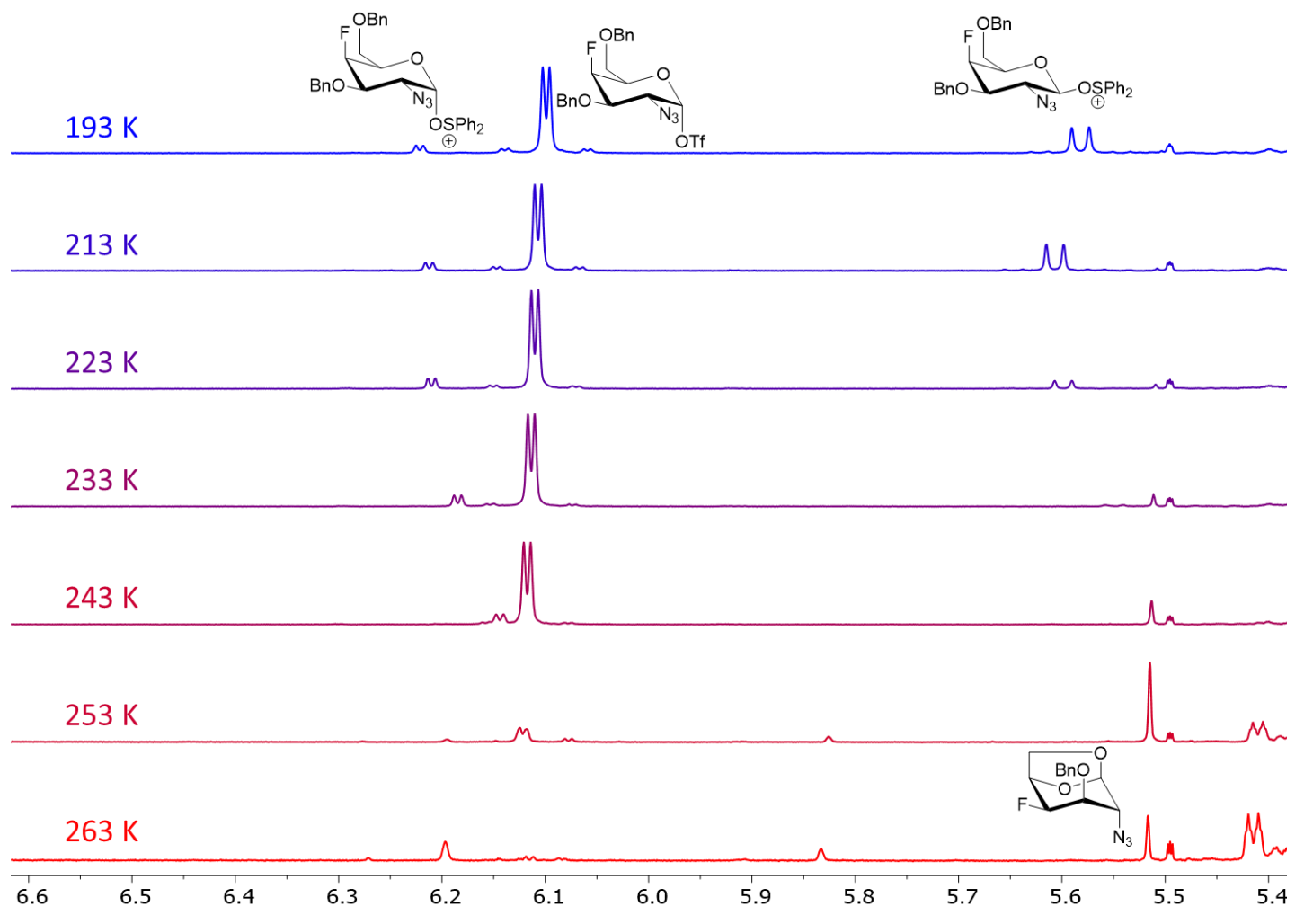

S295 